\title{
Abstracts from the 11th International Congress of Behavioral Medicine
}

\section{Wednesday \\ August 4, 2010 WORKSHOPS}

\author{
W101 \\ 9:00 AM-11:30 AM \\ CROSS CULTURAL RESEARCH IN HEALTH PROMOTION \\ AND CHRONIC DISEASE MANAGEMENT \\ Edwin B. Fisher, $\mathrm{PhD},{ }^{1,2}$ Brian Oldenburg, ${ }^{3}$ \\ Renée I. Boothroyd, ${ }^{1,2}$ Carina K. Chan ${ }^{4}$ and Marisa Finn ${ }^{5}$ \\ ${ }^{1}$ Peers for Progress, American Academy of Family Physicians \\ Foundation, Leawood, KS; ${ }^{2}$ Health Behavior \& Health Education, \\ University of North Carolina at Chapel Hill, Chapel Hill, NC; \\ ${ }^{3}$ School of Public Health \& Preventive Medicine, Monash \\ University, Melbourne, VIC, Australia; ${ }^{4}$ Tan Sri Jeffrey Cheah \\ School of Medicine, Monash University (Sunway Campus), \\ Selangor Darul Ehsan, Malaysia and ${ }^{5}$ Department of Psychology, \\ The University of Auckland, Tamaki Campus, Auckland, New \\ Zealand.
}

CORRESPONDING AUTHOR: Edwin B. Fisher, PhD, Health Behavior \& Health Education, University of North Carolina at Chapel Hill, Chapel Hill, NC, 27599-7440; edfisher@unc.edu

\section{W102}

9:00 AM-11:30 AM

DIAGNOSES AND TREATMENT OF UNSPECIFIC

COMPLAINTS

Holger Ursin, Silje Mæland and Erik L. Werner

Unifob Health, Bergen, Norway.

CORRESPONDING AUTHOR: Holger Ursin, Unifob Health, Bergen, N 5015; h.ursin@uib.no

\section{W103}

9:00 AM-11:30 AM

TRAINING HEALTH PROFESSIONALS IN HEALTH BEHAVIOUR CHANGE COMMUNICATION: DEVELOPING A MULTI-DISCIPLINARY EVIDENCE-BASED PACKAGE Jo Hart, PhD and Sarah Peters University of Manchester, Manchester, United Kingdom.
CORRESPONDING AUTHOR: Jo Hart, PhD, University of Manchester, Manchester, M14 5NP; jo.hart@manchester.ac.uk

W104

1:00 PM-3:30 PM

HOW YOU CAN CREATE INTERNET DELIVERED HEALTH BEHAVIOUR CHANGE INTERVENTIONS USING THE LIFEGUIDE

Lucy Yardley, Adrian Osmond, Sarah Williams and Leanne Morrison

University of Southampton, Southampton, United Kingdom.

CORRESPONDING AUTHOR: Lucy Yardley, University of Southampton, Southampton, SO17 1BG; L.Yardley@soton.ac.uk

W105

1:00 PM-3:30 PM

THE POWER OF EXPECTATIONS: ABOUT PLACEBO

AND NOCEBO EFFECTS

Winfried Rief, PhD

Clinical Psychology and Psychotherapy, University of Marburg, Marburg, Germany.

CORRESPONDING AUTHOR: Winfried Rief, PhD, Clinical Psychology and Psychotherapy, University of Marburg, Marburg, 35032; rief@staff.uni-marburg.de

W106

1:00 PM-3:30 PM

QUALITY DECISION MAKING IN HEALTH CARE

SETTINGS: THE CONTRIBUTION OF CONTEMPORARY SOCIAL CONFLICT THEORY

Michael Van Slyck, $\mathrm{PhD}^{1}$ and Marilyn Stern ${ }^{2}$

${ }^{1}$ Virginia Comonwealth University, Richmond, VA and ${ }^{2}$ Virginia Commonwealth University, Richmond, VA.

CORRESPONDING AUTHOR: Michael Van Slyck, PhD, Virginia Comonwealth University, Richmond, VA, 23282; mrvanslyck@vcu. edu 
W107

1:00 PM-3:30 PM

MULTILEVEL ANALYSES OF REPEATED

AND CLUSTERED OBSERVATIONS: THE GENERALISATION

FROM SIMPLE LINEAR MODELS TO LINEAR MIXED

MODELS AND VARIANCE COMPONENT MODELS

Stein Atle Lie, $\mathrm{PhD}$

Uni Research, Uni Health, Bergen, Norway.

CORRESPONDING AUTHOR: Stein Atle Lie, PhD, Uni Research, Uni Health, Christiesgate 13, Bergen, Norway, 5015; stein.lie@uni.no

\section{Wednesday August 4, 2010 POSTER SESSION (PSW) 6:30 PM-9:00 PM}

PSW-101

STEP: A HARM REDUCTIONIST APPROACH TO HIV TREATMENT ADHERENCE QUERNA, K. , LIFELONG AIDS ALLIANCE

Katie Querna, MSW

Treatment, Lifelong AIDS Alliance, Seattle, WA.

A. Introduction: Taken consistently, highly active antiretroviral therapy (HAART), has demonstrated success in inhibiting HIV viral load replication and reducing morbidity and mortality for people living with HIV. However, adherence to HAART is often suboptimal, compromising the effectiveness of treatment. The Seattle Treatment Education Program (STEP) aims to mitigate treatment adherence barriers by providing education and support through empirically driven, strengths-based, behavior change interventions.

B. Description: The STEP program is based upon a harmreductionist framework, within a larger agency which ascribes to congruent ideology. Interventions are strengths-based and grounded in Rogerian, Motivational Interviewing, and Solution Focused clinical orientations. Clients meet with a Treatment Education Coordinator (TEC) to assess their specific treatmentrelated questions, concerns, and barriers. The TEC and client strategize to create concrete steps to maintain adherence. Evidence-based tools, including symptom and barrier assessors, incentives, and regular follow ups, are utilized. Monitoring and evaluating outcomes is completed regularly through use of a specialized database, and qualitative surveys distributed to clients and referring parties.

C. Lessons Learned: Eighty percent of STEP participants maintained or achieved the 95\% HAART adherence rate suggested for optimal treatment outcomes. The literature suggests that the grounding theories in which STEP is based are efficacious in brief treatment settings, and our outcomes data supports this assertion. Limitations of the intervention include participant attrition and insufficient access to comprehensive mental health and substance use management resources.

D. Further Recommendations: For many, maintaining treatment adherence is challenging. Implementing programs that offer unlimited sessions of mental health counseling and substance use treatment or harm-reduction strategies for people living with HIV could greatly increase treatment adherence. Additionally, public health policy could be modified with the aim of de-stigmatizing those living with HIV/AIDS. Effective legislation could include acts recognizing and legitimizing same sex couples. Comprehensive, accessible health care should be provided to anyone, regardless of socioeconomic status. These changes could help mitigate the shame reported by some people living with HIV/ AIDS, potentially leading to greater help seeking behaviors and lower transmission rates. Additionally, embracing HIV testing as a routine component of primary care could help to reduce HIV incidence and minimize stigma, potentially leading to a number of positive personal and public health outcomes, including increased treatment adherence.

CORRESPONDING AUTHOR: Katie Querna, MSW, Lifelong AIDS Alliance, Seattle, WA, 98122; katieq@1laa.org

PSW-102

THE RELATIONSHIP OF ANXIETY TO MULTIPLE ASPECTS OF TREATMENT ADHERENCE IN PATIENTS WITH ESRD

Janell Schartel, $\mathrm{PhD}^{1}$ and DeAnna L. Mori, $\mathrm{PhD}^{1,2}$

${ }^{1}$ VA Boston Healthcare System, Boston, MA and ${ }^{2}$ Boston University School of Medicine, Boston, MA.

The impact of depression on treatment adherence in individuals with End Stage Renal Disease (ESRD) has been well-supported in the literature (Hailey \& Moss, 2000); however, the influence of anxiety on adherence is less clear. It has been suggested that the relationship between anxiety and adherence may be indirect and influenced by other cognitive or psychosocial variables such as locus of control or knowledge of disease (Brownbridge \& Fielding, 1994, DiMatteo et al., 2000). Anxiety has been related to adherence with antibiotic regimens (Cockburn et al., 1987), and for patients with asthma (Baiardini et al., 2006) and COPD (Dowson et al., 2004). A limitation of previous research on anxiety and adherence in ESRD is the failure to investigate the differential impact of anxiety on specific components of adherence (e.g., medication, appointment attendance, dietary guidelines).

The goal of the present study was to conduct a preliminary investigation into the relationship of anxiety and renal disease knowledge to treatment adherence. Data includes 124 veterans with ESRD referred for pre-surgical psychological evaluations for renal transplantation. Renal disease knowledge was assessed in several subcategories of diet, medication, and 
laboratory tests. Adherence was assessed with respect to diet, medication, and appointment attendance. Both knowledge and adherence were rated by clinicians based on patient self-report and renal team staff-report. Patients were divided into "anxious" and "non-anxious" groups based on self-reported presence of anxiety symptoms. Data analysis on self-reported compliance revealed that there were no group differences with regard to renal disease knowledge; however, patients in the anxious group were rated as significantly more compliant with renal diet and appointment attendance than non-anxious patients $(t>2.20, p<.05)$. In addition, regression analyses indicated that anxiety and knowledge significantly predicted compliance with appointment attendance $[F(1,116)=3.68$, $p=.05]$ and dietary adherence $[F(4,115)=5.95, p<.001]$. Knowledge, but not anxiety, predicted medication adherence $[F$ $(3,114)=3.63, p=.015]$. Results for staff-reported compliance emphasized the role of disease knowledge over anxiety in predicting adherence.

The present data support the need to consider medical adherence as a multifaceted concept by demonstrating that anxiety is related to some aspects of renal disease management. Results also highlight the need for more sophisticated models to explain the influence of anxiety on adherence in patients with ESRD. Limitations of the present study, implications, and suggestions for future research are presented.

CORRESPONDING AUTHOR: Janell Schartel, PhD, Boston VAMC, Boston, MA, 02130; janell.schartel@va.gov

PSW-103

FACTORS ASSOCIATED WITH DEFAULTING FROM COMPLETION OF CHILD FEVER TREATMENT AND REFERRAL IN URBAN ZAMBIAN COMMUNITY. A QUALITATIVE STUDY

Frederick A. Kaona, $\mathrm{PhD}$

Directorate, Mwengu Social and Health Research Centre, Ndola, Zambia.

Background: Recent studies of health care referrals among the under five children with high fever have indicated that incomplete treatment due unfulfilled referrals was the major reason for the children dying in the homes. Despite the Zambian government acknowledging malaria and fever as leading cause of morbidity and mortality among the under five children, the community behaviour and practices on care-giving at household level, have not been well documented. Identification of reasons that lead to an increased number of children dying in the homes is understudied. To try and understand risks and benefits of home management of fever a community qualitative study was conducted.

Methods: Participants consisted of 10 caregivers whose children were 5 years and below old and had died in the community, 10 parents of children 5 years and below, who were admitted in the children's hospital with severe fever, survived and discharged from the hospital and went back home and 10 participants, whose children had fever and survived within the community 14 days prior to the commencement of the study. Questions were devoted to knowledge and attitudes about health seeking behaviour at household level. Knowledge about disease transmission, treatment and prevention, perceived risks of failure to take the child to health facility for early treatment and completion of referrals, were investigated.

Results: Of the 30 key informants who participated in the study, 10 informants were of caregivers of children died before seeing the medical specialist. Majority of children (67.8\%) were between 1 and 24 months old. Over 87 and 90 percent respectively were living with biological parents at the time of death. Caretakers from the three groups feared of losing their children if admitted in the children hospital (54.3\% and 58.9\%). Fear that their children would be exposed to other disease, lack of knowledge about the disease and referral, and lack of medicines in health facilities characterized demotivation for early access of quality care. Fees and transportation resulted in delay accessing health care outside the home and unfulfilled referrals.

Conclusion: Caretakers of under five children should be educated about the benefits of referral compliance and timely treatment of fever. Behavior change interventions are strongly recommended.

CORRESPONDING AUTHOR: Frederick A. Kaona, PhD, Directorate, Mwengu Social and Health Research Centre, Ndola, -; fadkaona@gmail.com

PSW-104a

MEDICATION BELIEFS MEDIATE DEPRESSION EFFECT ON MEDICATION NON-ADHERENCE IN HYPERTENSIVE PATIENTS

Susan Galdames, MSC, $\mathrm{PhD}(\mathrm{c})$, Esperanza Muñoz, MsC and Eliana Guic, $\mathrm{PhD}$

Psychology, Universidad Catolica de Chile, Santiago, Chile.

Medication non-adherence affects health outcomes in chronic illness. Only $50 \%$ of hypertensive patients adhere to prescribed medication (World Health Organization, 2004). Depression has been considered one of the main psychosocial predictors of medication non-adherence (DiMatteo, Lepper \& Croghan, 2000). The psychological mechanisms that can explain this relationship remain uncertain.

Objective: To evaluate the role of depression as a medication nonadherence factor in hypertensive patients and the possible psychological mechanism involved in this effect.

Methods: 204 hypertensive patients (67.6 yrs old, 36.8\% men) attending Primary Health Care Centers were evaluated in their regular visit to the cardiovascular program. All patients had over 1 year of pharmacological treatment for hypertension and had blood pressure over $140 / 90 \mathrm{mmHg}$ in the last health 
control visit. Study variables included depression (POMSdepression scale), medication beliefs (Specific Beliefs about Medicine Questionnaire), and adherence to prescribed antihypertensive medication (Hill - Bone Medication Adherence Scale).

Results: Depressive patients had a higher level of specific medication concerns than non-depressive patients $(t=3,351, p<, 001)$. Multivariate linear regression shows that medication non-adherence is predicted by depression $(\mathrm{B}=.172, \mathrm{p}<.01)$, and medication beliefs have a mediating role on this effect. Mediation effects were studied using Baron and Kenny's Criteria (1986): depression predicted medication beliefs $(\mathrm{B}=-.304, \mathrm{p}<.01)$; medication beliefs predicted non-adherence $(\mathrm{B}=-.442, \mathrm{p}<.01)$, and the predictive role of depression on non-adherence decreased when medication adherence was included in the regression equation $(B=.012$, $\mathrm{p}=.863)$

Conclusion: Our results confirm, in a sample of Chilean hypertensive patients, the effect of depression and beliefs about medicine over medication adherence and suggest that concerns about medication should be included in anti-depression psychotherapy for hypertensive patients.

Acknowledgements. Grant VRAID to EG

CORRESPONDING AUTHOR: Susan Galdames, MSC, PhD(c), Psicologia, P Univ Catolica, Santiago, 000; sgaldame@uc.cl

\section{PSW-104b}

PROVIDERS AND PATIENTS IN INDIA: CONTRASTING PERCEPTIONS ON HIV MEDICATION ADHERENCE

Deborah Jones, PhD, ${ }^{1}$ Aman Sharma, MD ${ }^{2}$ Stephen M. Weiss, $\mathrm{PhD}, \mathrm{MPH},{ }^{1}$ Drenna Waldrop-Valverde, $\mathrm{PhD},{ }^{1}$ Mahendra Kumar, $\mathrm{PhD},{ }^{1}$ Ritu Nehra, $\mathrm{PhD}^{2}$ and Szonja Vamos, $\mathrm{BS}^{1}$

${ }^{1}$ Psychiatry \& Behavioral Sciences, University of Miami Miller School of Medicine, Miami, FL and ${ }^{2}$ Post Graduate Institute for Medical Education \& Research, Chandigarh, India.

Background: This study compares and contrasts patient and provider perceptions of challenges and opportunities for patients on antiretroviral therapy (ART) in India.

Methods: HIV patients ( 2 groups, $\mathrm{n}=8$ women, 8 men) and HIV providers $(n=18)$ were recruited from the Post Graduate Institute for Medical Education \& Research (PGIMER) in Chandigarh, India to participate in focus group discussions (FGDs) and key informant interviews (KIIs). KIIs included HIV peers, treatment providers, NGO stakeholders and researchers. FGDs included experienced ART users, those new to ART and HIV treatment providers; interviews and groups used semi-structured stem questions. Transcriptions were coded based on conceptual relevance; codes were expanded for common themes and grouped into categories.

Results: Provider interviews and groups reflected disparate perceptions regarding the relative importance of specific issues with respect to ART adherence, e.g., patient knowledge, karma, use of alternative medications. Providers agreed, in order of importance, that education, illiteracy, depression and alcohol and drug use negatively affect ART adherence; disagreed on the influence of income, gender and karma. Patients and providers agreed on the impact of HIV knowledge and alternative therapies (homeopathy, ayurveda, unani, yoga, reiki, astrology). Patients expressed concern about food insecurity, stigma, side effects, clinic wait time, distance from clinics and the frequency of visits required. Though medications are no-cost, patients noted the hidden costs of blood tests, travel to clinics, and days missed from work at clinics. For longer term patients, discontinuing medication following remission of symptoms occurred. Enabling migrant and traveling workers to obtain ART during time away from home, social support, increased patient morale, positive attitudes about medication and patient provider communication were identified as key elements in enhancing adherence. Conclusions: Preliminary findings highlight the need for a patient - provider collaborative approach to ART provision and enhancing adherence. Future initiatives to encourage providers and stakeholders to collectively explore existing policies for ART provision will be proposed. This study is supported by NIH grant no. R21NR011131.

CORRESPONDING AUTHOR: Deborah Jones, PhD, Psychiatry \& Behavioral Sciences, University of Miami Miller School of Medicine, Miami, FL, 33141; djones@med.miami.edu

PSW-104c

AGE DIFFERENCES IN ADJUSTMENT AND ADHERENCE IN A LARGE MULTI-SITE SAMPLE 5-10 YEARS AFTER HEART TRANSPLANTATION

Andrea M. Shamaskin, BS, ${ }^{1}$ Bruce Rybarczyk, PhD, ${ }^{1}$ Kathleen L. Grady, PhD, RN, APN, ${ }^{2}$ Connie White-Williams, PhD, RN, FNP ${ }^{3}$ and Edward Wang, $\mathrm{PhD}^{2}$

${ }^{1}$ Virginia Commonwealth University, Richmond, VA; ${ }^{2}$ Northwestern Memorial Hospital, Chicago, IL and ${ }^{3}$ University of Alabama Medical Center, Birmingham, AL.

Post-operative adherence rates among heart transplant (HT) patients remain lower than expected when considering the potential consequences, and these rates tend to decrease as more time passes following surgery. In the initial period after transplant older patients have higher rates of mortality and morbidity, but in the long-term some studies have suggested that older patients have better adjustment and quality of life compared to younger patients. The purpose of this study was: 1) to identify age differences in long-term adjustment to HT across a range of psychosocial adjustment measures, and 2) to examine other demographic and psychosocial predictors of overall adherence behavior. HT patients $(n=555$, mean age $=54)$ from multiple centers were assessed 510 years post-surgery for adherence behaviors, psychological factors and physical symptoms. Analysis of variance results showed that older adults (age $\geq 60$ ) had better adjustment on measures of quality of life, satisfaction with social support, negative affect and disease-related stress compared to younger 
adults. Linear multiple regression demonstrated that age and several psychosocial factors, including depressive cognitions, accounted for $25.7 \%$ of the variance in self-reported difficulty with adherence. Similar variables were significant in a logistic regression analysis predicting which patients would have difficulty or no difficulty with adherence. Clinical implications are discussed in terms of tailoring interventions to age groups for optimal postoperative adjustment.

CORRESPONDING AUTHOR: Andrea M. Shamaskin, BS, Virginia Commonwealth University, Richmond, VA, 23284; shamaskina@vcu.edu

\section{PSW-104d}

\section{BARRIERS TO IMMUNOSUPPRESSIVE MEDICATION} ADHERENCE IN A HIGH-RISK ADULT RENAL

\section{TRANSPLANT POPULATION}

Melissa Constantiner, $\mathrm{BA}^{1,2}$ and Daniel Cukor, $\mathrm{PhD}^{2}$

${ }^{1}$ Ferkauf Graduate School of Psychology, Yeshiva University, Bronx, NY and ${ }^{2}$ Department of Psychiatry and Behavioral Sciences, SUNY Downstate Medical Center, Brooklyn, NY.

Non-adherence to immunosuppressive medication in renal transplant recipients is a primary cause of kidney rejection. However, little is known about the reasons transplant patients are nonadherent. We previously reported the relationship between increased depressive affect and decreased medication adherence in both transplant and hemodialysis samples. We now report the specific barriers to medication adherence in ninety-four kidney transplant recipients from a major medical center in Brooklyn, New York. Overall, this primarily Black sample endorsed substantive impediment due to (1) too many dosages per day, (2) too many pills per dose, (3) not being able to tell if the medication is helping, and (4) running out of medication. The number of barriers that the sample endorsed was associated with increased depression $(\mathrm{r}=.209, \mathrm{p}=.049)$ and decreased medication selfefficacy $(r=-.300, p=.004)$. Surprisingly, there was no relationship between the degree of reported barriers and self-reported medication adherence $(\mathrm{r}=-.117 \mathrm{p}>.05)$. To determine which barriers are the most significant to medication adherence, the sample was divided between those individuals who report adequate adherence on a self-report measure (Immunosuppressive Therapy Adherence Scale) and those who report less than adequate adherence. The group with poor adherence reported the following barriers to be significantly more salient: too many doses per day $(\mathrm{t}(85)=-2.5, \mathrm{p}=.016)$; too many pills per dose $(\mathrm{t}(85)=-2.49, \mathrm{p}=.015)$; having to remember to take their medication ( $\mathrm{t}(85)=-2.2, \mathrm{p}=.03)$; perceived side effects of the medication $(\mathrm{t}(85)=-2.9, \mathrm{p}=.005)$; skipping a dose to feel good $(\mathrm{t}(85)=-2.6, \mathrm{p}=.011)$; falling out of their daily routine $(\mathrm{t}(85)=-4.5, \mathrm{p}<.001)$; and being short of money $(\mathrm{t}(85)=-3.7$, $\mathrm{p}<.001)$. This study sheds light on the barriers to immunosuppressant medication adherence in a high-risk population, which may help identify the individuals most at risk of infection, rejection episodes, and ultimately graft failure.
CORRESPONDING AUTHOR: Melissa Constantiner, BA, Ferkauf Graduate School of Psychology, Yeshiva University, New York, NY, 10011; mconstantiner@gmail.com

PSW-104e

BREAST CANCER, ILLNESS PERCEPTIONS AND NON-ADHERENCE TO AROMATASE INHIBITORS Arden Corter, PhD,${ }^{1}$ Michael P. Findlay, MBChB, MD, FRACP, ${ }^{1}$ Reuben Broom, BHB, MBChB,FRACP, ${ }^{2}$ David Porter, $\mathrm{MBChB}$ Dip Obst MD FRACP ${ }^{2}$ and Keith J. Petrie, $\mathrm{PhD}^{1}$

${ }^{1}$ Psychological Medicine, University of Auckland, Auckland, New Zealand and ${ }^{2}$ Oncology \& Regional Cancer Services, Auckland City Hospital, Auckland, New Zealand.

Purpose: Adherence to aromatase inhibitors reduces morbidity and mortality from breast cancer recurrence, but adherence is a significant problem. This study examines the relations between demographic factors, fear of cancer recurrence, illness perceptions, depression and anxiety, and self-reported adherence to aromatase inhibitors in the previous 28 days.

Methods: A sample of 155 women currently taking aromatase inhibitors completed an anonymous postal survey (57\% response rate) that included the Brief Illness Perception Questionnaire, Hospital Anxiety and Depression Scale, a measure of fear of cancer recurrence, and self reported adherence to aromatase inhibitors.

Results: Adherence was generally high but $26 \%$ of women reported missing at least one dose of medication over the past month. Preliminary analyses showed that non-adherence was unrelated to age, number of medications or time on treatment. Non-adherence was also unrelated to levels of anxiety, depression or fear of cancer recurrence. However, the number of doses missed over the previous month was significantly associated with a higher belief in personal control over the illness $(\mathrm{r}=.29, \mathrm{p}<.001)$.

Conclusion: Personal beliefs about control over breast cancer recurrence may weaken long-term adherence to aromatase inhibitors but this needs to be investigated further in longitudinal research.

CORRESPONDING AUTHOR: Arden Corter, PhD, Psychological Medicine, University of Auckland, Auckland, 1346; a.corter@ auckland.ac.nz

PSW-104f

WHY DO HIV-POSITIVE PATIENTS COMBINE

ANTIRETROVIRAL THERAPY(ART)

AND TRADITIONAL MEDICINE(TM)? EXPERIENCES

OF HEALTH WORKERS, TRADITIONAL HEALERS

AND PATIENTS. A STUDY IN 2 PROVINCES

OF SOUTH AFRICA

Thandi R. Puoane, Dr $\mathrm{PH}^{1}$ and Gail D. Hughes, $\mathrm{Dr}^{\mathrm{PH}^{2}}$

${ }^{1}$ School of Public Health, University of the Western Cape, Cape Town, South Africa and ${ }^{2}$ Natural Medicine, University of the Western Cape, Bellville, South Africa. 
Background: Despite the increase in the number of people using ART in South Africa, the use of traditional medicine in combination with ART becomes a challenge. This study explored the perceptions, knowledge and attitudes of patients, health workers and traditional healers about use of traditional medicine and ART.

Methods: Data was collected through interviews with 14 participants: six patients on ART and TM; two doctors; 2 professional nurses and 4 traditional healers. Two focus group discussions, one in each research site were held with community health workers who work with HIV positive patients. Data was analysed using a thematic content analysis approach.

Findings: When patients become sick they use traditional medicine first, then when all else fail they come to the health facility. Most patients start using traditional medicine before they are diagnosed with HIV. When they get sick the first point of entry seem to be the traditional healers. Most patients start using traditional medicine before they are diagnosed with HIV. Some people prefer using traditional medicine because they want privacy and to remain anonymous.

Conclusion: This study has revealed that patients have a history of using traditional medicine for chronic conditions prior to their diagnosis with HIV, and continue using traditional medicine in conjunction with their ART. This indicates that traditional medicine is an integral part of the "treatment modality" for patients and is used in combination with conventional medicine.

CORRESPONDING AUTHOR: Thandi R. Puoane, Dr PH, School of Public Health, University of the Western Cape, Cape Town, 7535; tpuoane@uwc.ac.za

\section{PSW-105}

PERCEIVED AND DESIRED SOCIAL SUPPORT AMONG OLDER ADULTS IN SEOUL, KOREA

Seunghyun Yoo, DrPH

Dept. of Health Science \& Services, Seoul National University, Seoul, Republic of Korea.

Learning Objectives:

1. Describe the types and sources of social support for Korean older adults.

2. Compare the received and desired social support by Korean older adults.

Background: Korea is a fast-aging country experiencing changes in social and family values. While research on social support among older adults increases, understanding the context in which they perceive and expect social support is relatively limited. This qualitative study aims to illustrate how older adults in Seoul recognize received and desired social support in what context.

Method: In-depth face-to-face interviews were conducted with 21 older adults in Seoul who were local senior center users. They were asked to describe the types and sources of received and desired social support; and the meaning of the gap between received and desired social support. Interview transcripts were analyzed using a grounded theory approach.
Results: While main sources of support perceived are spouses and adult children, local senior centers serve as supplementary source of emotional and instrumental support. Friends, neighbors, religious groups are also mentioned as social support sources. Although the respondents recognize the gap between received and desired emotional and financial support and are stressed by it, they could not identify a strategy to compromise the gap within their life circumstances. Instead, they either justify the gap as part of aging process or deny the need for support.

Conclusion: As changes in family structure and economic challenges affect the social support dependency of older adults on their children, social ecological approaches to enhance support capacities from individual to system levels are requested. Local senior center is one example that can be fostered as a social support source in the community.

CORRESPONDING AUTHOR: Seunghyun Yoo, DrPH, Seoul National University, Seoul, 110-799; syoo@snu.ac.kr

PSW-106

PROXY RATING AND SELF-REPORT ON QUALITY

OF LIFE: IS THERE CONCORDANCE?

Elizabeth W. Gonzalez, $\mathrm{PhD},{ }^{1}$ Carol F. Lippa, $\mathrm{MD}^{2}$ and Marcia Polansky, Sc D ${ }^{3}$

${ }^{1}$ College of Nursing \& Health Professions, Drexel University, Philadelphia, PA; ${ }^{2}$ College of Medicine, Drexel University, Philadelphia, PA and ${ }^{3}$ School of Public Health, Drexel University, Philadelphia, PA.

Background: Quality of life (QOL) has been recognized as a meaningful outcome measure when assessing course of illness or evaluating effectiveness of interventions. Whether individuals with dementia can evaluate their own Quality of Life (QOL) has been debated. The debate evolves on whether patient ratings or proxy ratings be used or both? The purpose of this study was to determine concordance between the patient's selfreport on QOL and caregiver's appraisal on QOL of individual with dementia.

Method: Sixty family caregivers who were living with and caring for person diagnosed with Alzheimer's disease and their care recipients participated in the study. The data is part of an ongoing intervention study to enhance resourceful skills in family caregivers. Patients have mild or moderate cognitive impairment. Quality of Life was measured using the 13-item Quality of Life for Alzheimer's Disease (QOL-AD: Logsdon et al, 2002), developed for both patient and caregivers includes patient's and caregiver's appraisal of patient's physical condition, mood, interpersonal relationships, ability to participate in meaningful activities, finances and an overall assessment of self and life quality as a whole. Overall scores were computed separately for the patient and caregiver reports by summing the 13 items, for a total possible score ranging from 13 to 52 , with higher scores indicating higher QOL. Data were analyzed using descriptive statistics, Pearson correlation and a single- measure intraclass correlation. Alpha Cronbach's coefficient for proxy QOL and patient's QOL were .71 and .85 , respectively. 
Findings: The results showed that family caregivers appraisal of patient's QOL and patient's self-report on QOL were strongly correlated $(\mathrm{r}=.71, \mathrm{p}<.002)$. This finding indicates that it is possible for persons with mild to moderate dementia to rate their own QOL and family caregivers who live with and care for a person with dementia can validly assess patient's quality of life.
Funded by NINR (R15 NR0009490-01A1)

CORRESPONDING AUTHOR: Elizabeth W. Gonzalez, PhD, College of Nursing \& Health Professions, Drexel University, Philadelphia, PA, 19102; ewg22@drexel.edu.

\begin{abstract}
TOP FIFTEEN SCORING POSTER
PSW-107

THE INFLUENCE OF VIGOR, DEPRESSIVE SYMPTOMS, AND LEUKOCYTE COUNTS ON MORTALITY? - A FIVE-YEAR FOLLOW-UP

Chaitali L. Ollars, Bachelor, ${ }^{1}$ Mimi Mehlsen, Assistant Professor, MSc, PhD, ${ }^{1}$ Dorthe Thomsen, Associate Professor, MSc, PhD ${ }^{1}$ and Robert Zachariae, Professor, Dr med, Cand, Psych ${ }^{1,2}$

${ }^{1}$ Department of Psychology, Aarhus University, Aarhus, Denmark and ${ }^{2}$ Department of Oncology, Aarhus University Hospital, Aarhus, Denmark.

Background: Ageing is associated with higher risk of infectious illnesses and mortality, which could be due to an age-related decline of immune function. There is good evidence of associations between a number of psychological factors and immunity, and several studies have shown associations between both negative and positive emotions and alterations in leukocyte counts.

Objectives: Our aim was to explore the role of depression and vigor in mortality, while controlling for leukocyte counts as a measure of immune status.

Method: We conducted a five-year follow-up to a baseline study conducted in 2000 of the significance of age, psychosocial factors (depression (BDI), vigor (POMS)), immune function, and physical activity in older healthy individuals. Participants were 314 volunteers between 70 and 85 years of age. Participants were excluded if they had any diagnosed illness or received any treatment known to influence immune function. The time of death for non-survivors was obtained from the Danish National Board of Health, and participants were censored 5 years after the last participant was included in the study.

Results: At baseline vigor was inversely correlated with depressive symptoms $(\mathrm{r}=-0.402, \mathrm{n}=$ $304, \mathrm{p}<0.01)$ as well as with leukocyte counts $(\mathrm{r}=-0.196, \mathrm{n}=306, \mathrm{p}<0.01)$. Predictors of survival were examined with a hierarchical Cox regression analysis with baseline leukocyte counts, depressive symptoms, and vigour entered as predictors, while controlling for sex and age. When analyzed separately, only baseline leukocyte counts predicted survival. However in the full model, both vigor (OR: 0.896) and depressive symptoms (OR: 0.908) were significant predictors of survival, while leukocyte counts ceased to be a significant predictor.

Conclusion: Higher levels of vigor and moderate levels of depressive symptoms were significant predictors of increased survival when analyzed together, but not when analyzed separately. Further large-scale studies are needed to validate these findings.
\end{abstract}

CORRESPONDING AUTHOR: Chaitali L. Ollars, Bachelor, Psychology, Aarhus University, Aarhus C, 8000; chaitalio@ hotmail.com

PSW-108

GENDER DIFFERENCES IN MENTAL HEALTH TRAJECTORIES AMONG ELDERLY JAPANESE PEOPLE Tomoo Nakata, MA

Hokusei Gakuen University, Sapporo, Japan.
The aim of this study is to examine the differences in mental health patterns between males and females from 60 years among Japanese people. 60 years was a key age among Japanese, because the mandatory retirement was conducted at 60 among almost all the companies and older people were oblige to deduce both their status and income at 60 . And 'male breadwinner model' comprises a large part of households in Japan. Therefore, to compare the pattern of worsening health between retired males and their housewives merit careful examination. 
This study employed the National Survey of the Japanese Elderly, from wave I (1987) to wave III (1993). Fixed / random effect models applying pooling data constructed from panel data was used. The dependent variable was mental health, which measured by the shorten version of CES-D scale which is comprised of 6 item. And duration from 60 years and its square term and, dummy variables of occupational status and marital status, the number of family cohabiters, the number of friends, and natural logarithm of household income were used as the independent variables.

At first, the results of the Hausman test, which verifies which model is better among fixed and random effect models, adopted fixed effect models for both gender. This fact reveals differences among individual was very small and there are not need to make residual term random.

As the results of fixed effect models, some differences could be seen between genders as follow; 1) For males, a linear term of age was the only significant variable. 2) For women, a square term of age was significant and this means that women worsen their health at an accelerating pace. And marital status and household income was also significant.

It may be difficult to explain the results from the situations in Japan and characteristics in later in life especially for males, because they are getting worse mentally because of ageing. On the other hand, life events in later life like loss of spouse and descent of income was the cause of mental illness for females. This seems to reflect the dependence of wives on husbands.

CORRESPONDING AUTHOR: Tomoo Nakata, MA, Hokusei Gakuen University, Sapporo, 0048631; tnakata@hokusei.ac.jp

\section{PSW-109}

\section{STRUCTURE OF LIFESTYLE-RELATED VARIABLES} AND PSYCHOSOCIAL ASPECTS -A MODEL OF YOUNGER \& OLDER GROUPS OF ELDERLY JAPANESE-

Ai Nakashima, master caurse, ${ }^{1}$ Tomohiko Muratsubaki, Doctor Course, ${ }^{2}$ Tadaaki Tomiie, Associate Professor, ${ }^{3}$ Toyohiro Hamaguchi, Associate Professor ${ }^{4}$ and Yuji Sakano, Professor ${ }^{3}$

${ }^{1}$ Graduate school Psychological science, Health Science University of Hokkaido, Sapporo, Japan; ${ }^{2}$ Tohoku University of Medicine, Sendai, Japan; ${ }^{3}$ Health Sciences University of Hokkaido, Sapporo, Japan and ${ }^{4}$ Saitama Prefectural University, Koshigaya, Japan.

Purposes: (1) To examine the relations among lifestyle, lifestyle disease, waist circumference, life satisfaction, social support, perceived physical ability, and social motivation in younger \& older groups of elderly Japanese and (2) To validate and compare two models using younger \& older groups of elderly Japanese. Method: Participants included 119 in elderly Japanese (mean age $=73.24, \mathrm{SD}=$ $6.55), 75$ in the younger group (mean age $=68.99, \mathrm{SD}=2.79$ ) and 44 in the older group (mean age $=79.45, \mathrm{SD}=0.68$ ) in the older group. The questionnaires were composed of face sheet (age, waist circumference, severity and count of lifestyle disease, Morimoto's eight Health Habit (Morimoto, 2000), Life Satisfaction Index K (Koyano, Sibata, Haga, and Suyama, 1989),
Perceptions of Physical Ability (subscale of Physical Selfefficacy; Matsuo, Takenaka, Oka, 1999 ), Social Support Scale (Iwasa, Gondou, Masui, Inagaki., Kawai., Otyuka, Ogawa, Takayama, Imuta, and Suzuki, 2007), and social motivation (social motivation for hobby, lifelong learning, sports). Data analysis were exploratory tested with a path analysis, using SPSS 16.0 and Amos 16.0 program. Result: The path models showed good model fit in the elderly Japanese (Chi square $=15.58, \mathrm{df}=15, \mathrm{p}=.41, \mathrm{BIC}=0.00, \mathrm{NFI}=0.83, \mathrm{RMSEA}=$ $0.18, \mathrm{AIC}=55.59)$ and in the younger group $(\mathrm{Chi}$ square $=5.43, \mathrm{df}=9$, $\mathrm{p}=.80, \mathrm{BIC}=0.00, \mathrm{NFI}=0.92, \mathrm{RMSEA}=0.00, \mathrm{AIC}=41.43)$ and in the older group. (Chi square $=4.80, \mathrm{df}=4, \mathrm{p}=.31, \mathrm{BIC}=0.16$, NFI $=$ 0.87, $\mathrm{RMSEA}=0.00, \mathrm{AIC}=56.07$ ) In short, lifestyle affected life satisfaction in elderly Japanese and waist circumference affected in the younger group, but lifestyle-related variables (lifestyle, lifestyle disease, waist circumference) did not affect psychosocial aspects (life satisfaction, social support, perceived physical ability, and social motivation) in the older group. Conclusion: The three path models differeded in each. In this study indicated that the older group requires more psychosocial support than the younger group.

CORRESPONDING AUTHOR: Ai Nakashima, master caurse, Graduate school Psychological science, Health Science University of Hokkaido, Sapporo, 002-8072; a-naka@hoku-iryo-u.ac.jp

PSW-110

FORGIVINGNESS AND HEALTH IN ADULTHOOD: THE MODERATING ROLE OF FUTURE TIME PERSPECTIVE

Pearl Ghaemmaghami, MSc and Mathias Allemand, PhD

Department of Psychology, University of Zurich, Zurich, Switzerland.

Previous research has shown that forgiveness is positively associated with indicators of subjective health and well-being. Building on the concept of future time perspective, the study aimed at clarifying to what extent the perception of time remaining in life moderates the association between forgivingness, defined as an enduring tendency to forgive others, and self-rated indicators of health. In order to systematically examine these associations, the present study investigated a large and representative sample of adults ( $\mathrm{N}=962$, age range: 19 to 84 years).

Results reveal that forgivingness is significantly related to selfrated indicators of health (subjective health, life satisfaction, and positive and negative affect). Moreover, the associations between forgivingness and health are largely moderated by future time perspective, in such a way that forgivingness was more strongly related to reports of subjective health and well-being when future time perspective was perceived as limited as opposed to openended. These findings emphasize the important role of future time perspective for forgivingness and health.

CORRESPONDING AUTHOR: Pearl Ghaemmaghami, MSc, University of Zurich, Zurich, 8050; p.ghaemmaghami@psychologie. uzh.ch 
PSW-111

THE INTRODUCTION OF LIFE REVIEW

AND ITS INFLUENCE ON COGNITIVE FUNCTION

OF THE ELDERLY WOMEN REQUIRING LONG-TERM CARE

Hayato Uchida, PhD, ${ }^{1}$ Yoko Kuwada, MA, ${ }^{1}$ Toshio Nishigaki, MS, ${ }^{1}$ Setsuko Ino, MA, ${ }^{2}$ Hideki Toji, $\mathrm{PhD}^{1}$ and Kaori Araki, $\mathrm{PhD}^{1}$ ${ }^{1}$ School of Human Science and Environment, University of Hyogo, Himeji, Japan and ${ }^{2}$ Geriatric division, Ino Hospital, Himeji, Japan.

The aim of this study was to clarify whether the life review which we continued for a month affected the physical and mental functions of the elderly women requiring long-term care. The participants were seven elderly women (age 85.7+/-8.3) in a geriatric health services facility, Himeji, Hyogo, Japan. We selected the control group consisting of seven women $(88.0+/-2.6)$ in the same facility. The life reviews were conducted for ten times in February 2008. Each session lasted for fifteen to thirty minutes. The contents of the interview were about the events and memories in her childhood, adolescence and adulthood until today. We carried out the surveys to evaluate their physical and mental functions using the questionnaires such as Mini Mental State Examination, modified Barthel Index, and Dementia Behavior Disturbance scale. The surveys were done for three times, on the beginning of December 2007, the end of January 2008, and the beginning of March 2008. From the comparisons of the average scores of the questionnaires before and after the life review, MMSE score significantly increased in the life-reviewed group $(\mathrm{P}<0.05)$. In particular, the score of "Recall of 3 words, in which the participants recalled three words we previously asked her to remember", significantly increased $(\mathrm{P}<0.05)$. It was showed that the approach of life review could contribute to improve cognitive function of the elderly women requiring long-term care.

CORRESPONDING AUTHOR: Hayato Uchida, PhD, School of Human Science and Environment, University of Hyogo, Himeji, 6700092; uchida@shse.u-hyogo.ac.jp

PSW-112a

DEVELOPMENT OF COGNITIVE BEHAVIORAL THERAPY FOR OLDER ADULTS WITH DEPRESSION IN JAPAN

Mamiko Igo, Master ${ }^{1}$ and Nobuo Furuta, MD $^{2}$

${ }^{1}$ Graduate School of Human Sciences, Waseda University, Kokubunji, Japan and ${ }^{2}$ Yokufukai Geriatric Hospital, suginami, Japan.

In Japan, there is little evidence about the effect of Cognitive Behavioral Therapy (CBT) for older adults compared to Europe and the United States. The purpose of this study was to develop CBT for older adults with depression which fit in with Japanese culture and research its effectiveness in outpatient care. By randomized clinical trial recruiting, 15 older adults were chosen. All the participants had a diagnosis of Major depression. The participants were randomly allocated to receive either Treatment as usual (TAU) group $(n=10)$ or
CBT group ( $n=5)$. We assessed the degree of depression before the treatments (baseline), at the 6th week and at the 12th week. Measures used in this study were the Zung Self-Rating Depression Scale (SDS), the Geriatric Depression Scale Japanese version (GDS), and the Montgomery Asberg Rating Scale for Depression - Japanese (MADRS-J).In CBT intervention, the psychoeducation about the typical cognitive characteristics of older adults' depression and the reminiscence based hearing of subjects' compliant were conducted in addition to basic CBT strategy.

The results suggested that the score of SDS was significantly decreased in both CBT group and TAU group at 12th week (CBT; SDS baseline $\mathrm{M}=54.4,12$ th week $\mathrm{M}=40.6$, TAU; SDS baseline $\mathrm{M}=$ $58.5,12$ th week $M=46.7)$. The CBT developed in the present study was effective for improving depressive symptoms. However, the difference between CBT group and TAU group in the score of depression was nonsignificant. The results of GDS and MADRS-J showed the same tendency as SDS(CBT; GDS baseline $\mathrm{M}=10.8$, 12th week $\mathrm{M}=8.6$, MADRS-J baseline $\mathrm{M}=24.8$, 12th week $\mathrm{M}=11.2$, TAU; GDS baseline $\mathrm{M}=11.4,12$ th week $\mathrm{M}=9.7$, MADRS-J baseline $\mathrm{M}=25.2,12$ th week $\mathrm{M}=10.6$ ).

The limitation of this study is that the number of CBT session, which was 6 , might have not been enough to get the result which would make the differentiate it from TAU. Since CBT alone was effective for the patients to whom the medication of antidepressant had been stopped, the effectiveness of this treatment has been suggested.

Future studies need long-term intervention of CBT in a large number of participants.

CORRESPONDING AUTHOR: Mamiko Igo, Master, Graduate School of Human Sciences, Waseda University, Kokubunji, 1850022; m-igo@moegi.waseda.jp

PSW-112b

WHEN I'M 64: FINDINGS FROM THE UNC ALUMNI HEART STUDY

Ilene C. Siegler, PhD, MPH, ${ }^{1}$ Redford B. Williams, MD, ${ }^{1}$ Barbara K. Rimer, Dr $\mathrm{PH}^{2}{ }^{2}$ David C. Rubin, $\mathrm{PhD},{ }^{3}$ Beverly H. Brummett, $\mathrm{PhD},{ }^{1}$ John C. Barefoot, $\mathrm{PhD}^{1}$ and Paul T. Costa, $\mathrm{PhD}^{1}$

${ }^{1}$ Psychaitry and Behavioral Sciences, Duke University, Durham, NC; ${ }^{2}$ School of Public Health, University of North Carolina, Chapel HIll, NC and ${ }^{3}$ Psychology and Neurosciences, Duke Universtiy, Durham, NC.

The UNC Alumni Heart Study started in 1986 with data from college entry in 1964-66. We now have 46 years of postcollege follow-up and 12 waves of data collected during midlife from ages 40-60. The majority of the cohort were born in the 1940s and represent the beginning of the Baby Boom generation.

We have a large variety of findings on the role of hostility and other MMPI based personality traits from college which have been shown to predict survival, CHD risk factors and hypertension and NEO Personality data from the decade of the 40 s that are associated with behavioral risk indicators and life events. 
To date, we have observed 1708 cases of hypertension, 841 cases of depression, 341 cases of Type 2 diabetes, 645 cancers of multiple types, 318 cases of CHD and 43 strokes. The cohort included 7007 persons (1213 females and 5794 males). We have recorded 573 deaths and 4270 are continuing participants. By age $64,673(55.5 \%)$ of the women and $4228(60.3 \%)$ of the men have reported none of the disorders. We have college personality predictors on 6,959 persons.

A second cohort of spouses without college personality data joined the study in the 1992 has 18 years of follow-up and includes 1354 persons (1037 females and 317 males) and adds 339 cases of hypertension, 281 cases of depression, 54 cases of diabetes, 154 cancers, $43 \mathrm{CHD}, 8$ strokes and 42 deaths.

When we combine the two cohorts, we have 4 repeated measures of hostility from age 18 to age 60 on approximately 3000 persons (mean hostility is 18.8 in college, 14.0 at age $40,11.3$ at age 60 and 10.6 at age 60) and NEO personality data - with repeated measures of the Five Factors and 30 facets on 4346 persons at age 42 and 3676 persons at age 50. Mean values for the Five factors at the two times of measurement are Neuroticism 49.7,48.7; Extraversion 49.4,48.6; Openness 53.5,52.7; Agreeableness 47.9,48.1 and Conscientiousness 52.0,51.5.

This extraordinarily rich data set allows us to test complex, integrative models on the impact of stability and change in multiple personality dimensions and stability and change in multiple risk behaviors as related to premature mortality and morbidity at midlife and provide an excellent picture of who survives to become elderly.

CORRESPONDING AUTHOR: Ilene C. Siegler, PhD, MPH, Psychaitry and Behavioral Sciences, Duke University, Durham, NC, 27705; ilene.siegler@duke.edu

\section{PSW-112c \\ COMPARISON OF CHARACTERISTICS AND PROPORTION OF CERTIFICATION FOR LONG-TERM CARE BETWEEN PARTICIPANTS AND NON-PARTICIPANTS IN HEALTH CHECK-UPS OVER A 3-YEAR FOLLOW-UP Emiko Saito, PhD, ${ }^{1}$ Chikako Kaneko, $\mathrm{MD},{ }^{2}$ Shouzoh Ueki, $\mathrm{PhD}^{3}$ and Seiji Yasumura, DMSc ${ }^{2}$ \\ ${ }^{1}$ Tokyo Metropolitan University, Tokyo, Japan; ${ }^{2}$ Fukushima Medical University, Fukushima, Japan and ${ }^{3}$ Tohoku Bunka Gakuen University, Miyagi, Japan.}

Background: a mandatory social long-term care insurance of Japan was reformed in 2006, though there are still few data about the certification for long-term care onset in geriatric health examination. Objective: to compare the care-needs certification proportion of participants and non-participants in a geriatric health examination targeting community-dwelling seniors aged 70 years and older over 3 years.

Design: population-based cohort study

Setting and participants: 838 seniors aged 70 years and older living in a village of Japan in 2004.
Methods: We followed the occurrence of new care-needs certification for 3 years in 443 subjects who participated in the health examination and in the 395 non-participants we were able to interview during home visits (838 total subjects).

Results: A total of 443 subjects participated in the examination. Home visiting for 449 older adults (of 904 non-participants) living in 8 of 16 districts in the village were conducted. We followed the occurrence of new care-needs certification for 3 years in 443 subjects who participated in the health examination and in the 395 non-participants we were able to interview during home visits (838 total subjects). Among the 838 subjects, there were 94 new certifications (11.2\%) during the observation period. Starting in the second year, the occurrence rate of new care-needs certifications for non-participants was significantly higher than that of participants. The rate of care-needs certification over 3 years was significantly higher for non-participants $(63 / 395,16.0 \%)$ than for participants $(31 / 443,7.0 \%)$.

Conclusion: Non-participants have a higher risk of care-needs certification. Projects aimed at understanding older adults are often performed during health examinations; however, it is necessary to investigate current data gathering methods for elderly patients who do not undergo these examinations.

CORRESPONDING AUTHOR: Emiko Saito, PhD, Tokyo Metropolitan University, Tokyo, 116-8551; saito@hs.tmu.ac.jp

PSW-112d

AGE- AND GENDER-RELATED DIFFERENCES

IN THE PHYSICAL, COGNITIVE, AND PSYCHOLOGICAL FUNCTIONS OF ELDERLY LIVING AT HOME

Shin Murata, Doctor

Nishikyushu University, Saga, Japan.

To create basic data for the effective health guidance of elderly living at home, this study was conducted involving 291 adults aged 65 or older, with a comparison of physical, cognitive, and psychological functions between young-old and old-old adults, and by gender. Certain physical functions such as muscular strength of the extremities and the dexterity of digits seemed to be affected by gender. Overall, however, their physical functions were significantly affected by aging, and had decreased to a greater degree in old-old than young-old adults. Especially, it was suggested that balance while standing and the walking ability as well as cognitive functions were more significantly affected by aging than by gender. On the other hand, psychological aspects such as their subjective view of their own health and satisfaction with life showed no significant differences by gender or between age groups, suggesting their lower susceptibility to gender and aging. These findings demonstrated that both men and women showed a high risk of falling in the old-old adults, suggesting the importance of training to improve balance and walking ability as well as cognitive training in preventive care and health guidance for people in this age group.

CORRESPONDING AUTHOR: Shin Murata, Doctor, Nishikyushu University, Saga, 842-8585; muratas@nisikyu-u.ac.jp 
PSW-113

ACCUMULATED DISADVANTAGE RELATED TO HISTORY OF LABOUR MARKED ATTACHMENT AND COHABITATION IS ASSOCIATED WITH ATYPICAL DRINKING PATTERNS IN DANISH MEN BORN IN 1953

Margit Kriegbaum, Msc, ${ }^{1}$ Ulla Christensen, $\mathrm{PhD},{ }^{1}$ Merete Osler, $\mathrm{DrMSc}^{1,2}$ and Rikke Lund, $\mathrm{PhD}^{1}$

${ }^{1}$ Institute of public Helath, University of Copenhagen, Copenhagen, Denmark and ${ }^{2}$ Glostrup University Hospital, Research Centre for Prevention and Health, Copenhagen, Denmark.

Objective: to investigate how history of employment and cohabitation affects alcohol drinking behaviour, and to study joint exposure to both.

Methods: birth-cohort study of 6,112 Danish men born in 1953 with follow-up in 2004 on drinking behaviour at age 51.

Results: Risk of heavy drinking increase with number of joblosses (OR 1.72 to 2.48) and duration of unemployment (OR 1.34 to 4.17). Heavy drinking was also associated with number of broken partnerships (OR 1.44 to 2.68) and with living alone above five years (OR 1.59 to 2.55). Abstention from alcohol was associated with number of job-losses (OR 1.63 to 3.42) and duration of unemployment (OR 1.56 to 6.70). Abstention was associated with number of broken partnerships (OR 1.55 to 1.89 ) and with time living alone beyond five years (OR 1.65 to 2.66). We found an interaction between the number of job-losses and of broken partnerships in relation to heavy drinking.

Conclusions: heavy drinking and abstaining from alcohol intake are both related to number of job-losses, broken partnerships, and time without employment and living alone.

CORRESPONDING AUTHOR: Margit Kriegbaum, Msc, Institute of public Helath, University of Copenhagen, Copenhagen, 1014; makr@sund.ku.dk

\section{PSW-114}

CHAT: SOS - WHAT DO ADOLESCENTS AND COUNSELORS WANT IN AN ONLINE RELAPSE PREVENTION PROGRAM?

Kimberlee J. Trudeau, PhD, Sadaf Charity, MBA and Jessica L. Ainscough, BA

Inflexxion, Inc., Newton, MA.

Approximately $75 \%$ of adolescents who receive substance abuse treatment relapse within one year. To test the feasibility of developing an online relapse prevention program for adolescents to provide ongoing adjunct support during aftercare, we conducted a two phase research project. In the first phase we interviewed counselors $(\mathrm{N}=16)$ about the content and features this program should include, compiled those responses, then asked counselors to sort and rate the responses using an online concept mapping program. The eight-cluster map provided the most meaningful break out of content: Health Education $(M=4.73)$, Setting Boundaries in Peer Relationships $(\mathrm{M}=4.38)$, How to Follow Through with Decisions $(\mathrm{M}=4.23)$, Figuring Out What You Really Want $(\mathrm{M}=4.18)$, How to Achieve and Maintain Healthy Relationships $(M=4.06)$, Working with a Counselor or Sponsor $(M=4.01)$, Developing Peer Support for Recovery $(M=3.97)$ and
Educational Activities $(\mathrm{M}=3.68)$ [i.e., how to present content from the other clusters]. These findings were used to develop the interactive demonstration version of the proposed program. In the second phase of this project, we presented the demonstration program to these counselors and adolescents for feedback and discussion. Twenty-four adolescents $(\mathrm{M}=15.8$ years) in substance abuse treatment participated in the study. We exceeded our preestablished acceptability criteria ( $80 \%$ of participants will rate their satisfaction with the demo program as "good" (5), "very good" (6), or "excellent" (7) on the Likert scale questions) with both adolescents $(\mathrm{M}=84 \%)$ and counselors $(\mathrm{M}=86 \%)$. In addition, all items received a mean of 5 or better (adolescent sample had 21 items, overall $\mathrm{M}=5.74$; counselors sample had 27 items, $M=5.75$ ). Lastly, these adolescents participated in focus groups in which they discussed their use of technology and challenges in early recovery. This two phase project indicated acceptance of the program concept, as well as provided content ideas from key stakeholders to inform the development of the full program.

CORRESPONDING AUTHOR: Kimberlee J. Trudeau, PhD, Inflexxion, Inc., Newton, MA, 02464; ktrudeau@inflexxion.com

PSW-115

TEXTING FOR SMOKING CESSATION: IDENTIFYING NEEDS OF POTENTIAL USERS

Beth Bock, PhD, ${ }^{1,3}$ Kathleen Morrow, PhD, ${ }^{1,3}$ Christopher Deutsch, $\mathrm{BA}^{2}$ and Robert Foster, $\mathrm{BA}^{2}$

${ }^{1}$ Behavioral Medicine, Miriam Hospital, Providence, RI; ${ }^{2}$ I Live Inspired, LLC, Alexandria, VA and ${ }^{3}$ Psychiatry, Brown Medical School, Providence, RI.

Young adults are more likely to smoke and less likely to seek treatment compared to older smokers. They are also heavy users of communications technology. We developed initial designs for a smoking-cessation program to be delivered primarily through SMS text messaging and presented them in focus groups.

Adults, age 18-35, who smoked or recently quit ( $<6$ months) and used text messaging participated in focus groups. A 3-week recruitment period yielded 46 calls and screened 38 individuals. Eligible callers $(n=30)$ netted 21 participants (ppts) who completed surveys and a 2-hour focus group.

Results: Ppts ( $M=25.6$ years of age; range 20-33), were primarily nonHispanic white (76.2\%); the remainder were Hispanic, Black, Native American (4.8\% each), or bi-racial (9.4\%); 69\% were female. Most had completed $2(47.6 \%)$ or $4(23.8 \%)$ years of college, were currently employed full time $(n=18)$; the median annual income was $25-50,000$ USD.

Nearly all $(n=20)$ were current smokers, smoking an average of 12.8 cigarettes/day $(\mathrm{SD}=6.4)$. Most $(76 \%)$ had at least one previous quit attempt (lasting 3 days to 24 months). The most common method used was "cold turkey" $(n=15)$.

Texting: Nearly all ppts (81\%) had unlimited text plans and $47 \%$ had no land-line (non-mobile phone). Ppts reported speaking by phone an average of 5 times/day (range $=1-20$ ), however they sent/received an average of 70 text messages/day (range $=5-600$ ). 
All had internet access at home, most (75\%) accessing it multiple times daily, nearly $70 \%$ through their mobile phone. Nearly all also used social networking sites.

Qualitative Data: Most ppts liked the idea of a smoking cessation program offered through text messages. Many proposed features were viewed positively, and several significant modifications were recommended by ppts. Social networking functions and control of program output through an online profile were deemed particularly important. Interestingly, ppts challenged a common procedure of cessation programs by suggesting that a TXT 2 Quit program could begin on Quit Day, not preparatory to QD.

CORRESPONDING AUTHOR: Beth Bock, PhD, Behavioral Medicine, Miriam Hospital, Providence, RI, 02903; bbock@lifespan.org

\section{EARLY CAREER AWARDEE}

PSW-116

SMOKING PREVALENCE, KNOWLEDGE, AND ATTITUDES AMONG 3RD YEAR MEDICAL STUDENTS IN LIBYA. DR AHMED MOHAMED BUNI COMMUNITY MEDICENE DEPRTMENT - UNIVERSITY OF EL-FATEH - TRIPOLI -LIBYA

A. Buni, EDD

${ }^{1}$ Community Medicine, University of El-Fateh -- Ahmed m Buni, Tripoli, Libyan Arab

Jamahiriya and ${ }^{2}$ University of El-Fateh -Ahmed m Buni, Tripoli, Libyan Arab Jamahiriya.

Objective: - This paper summarizes data from the global health professions student survey (GHPSS) conducted in Libya concerning smoking prevalence, knowledge and attitudes among 3rd year medical students in three health professions disciplines namely dentistry, medicine, and pharmacy in year 2006 .Method: The GHPSS is a university - based survey of 3rd year students developed by the WHO, with the cooperation with the U.S Center for Disease Control and prevention, and the Canadian public Health Association. The survey was administered to students pursuing their degrees in dentistry, medicine, nursing and pharmacy .. EPI Info 2003 was used for the data analysis. The school response rates to the GHPSS in Libya were $80 \%$ for medical school and $50 \%$ for each of dental and pharmacy schools. The student response rates were more than $72 \%$ in all 3 schools of a total of 2126 students

Results: The prevalence of current smokers among third year medical students in the three disciplines in the year 2006 ranged from $1.2 \%$ for pharmacy students to $2.6 \%$ for dental students and $10.8 \%$ for medical students

Almost half of medical students (48.1\%), 37.3\% of dental student and $23.4 \%$ pharmacy student reported that they were exposed to second hand smoke (S H S) from others in their home during the past week, and $45.1 \%$ of dental student, $64.7 \%$ of pharmacy student and $62.1 \%$ of medical student were exposed to S H S from others in public place during the past week.

Finding from GHPSS data also show that less than 4 in 10 students in all 3 disciplines reported that they learned cessation approaches to use with their patients, even though more than 8 in10students in all 3 disciplines believed that health professionals should get specific training on cessation techniques. Conclusion: Discouraging or preventing tobacco use among health professionals is an essential and necessary step in any comprehensive tobacco control

CORRESPONDING AUTHOR: Ahmed M. Buni, MA,EDS,EDD, Community Medcine Department, University of El-Fateh for meical Sciences, Tripol -Tajora, 00218; Ahmedmombuni@hotmail.com

\section{PSW-117}

DEPRESSIVE SYMPTOMS AND BENEFITS ASSOCIATED WITH CIGARETTE USE AMONG CHILEAN YOUTH: DO CHILEAN YOUTH SMOKE TO IMPROVE THEIR MOOD? Paula Repetto, PhD, Yerko Molina, BA, Eliana Guic, PhD, Lilian Ferrer, PhD and Sebastian Sanhueza, BA

P. Universidad Catolica de Chile, Santiago, Chile.
According to the World Youth Tobacco Survey, Chile has one of the highest rates of tobacco use among youth around the world. Also, data from Chile suggest that rates of tobacco use between females and males are similar. Researchers have long discussed the role of negative affect on cigarette use among youth. They have proposed that adolescents may use cigarettes to self-medicate and improve their mood. Most of these studies, however, have not explored perceived beliefs associated with cigarette use that may also play a role in explaining the relationship between depressive symptoms and cigarette smoking. In the present study we explored the influence of depressive symptoms and perceived benefits of smoking cigarettes on mood in a sample of 737 chilean youth (mean age $=16,05$, range 12 to $25,54,5 \%$ females). 
Participants completed a self-administered questionnaire from a study that has been examining determinants of cigarette use among adolescents. We explored the relationship using a stepwise regression analyses and entered first demographics, then depressive symptoms variables and finally perceived beliefs about benefits associated with cigarette use. We also included in the model other determinants that have been previously associated with cigarette use as parental smoking and peer influences as control. Our findings suggest that negative affect $($ Beta $=.116, t(566)=2.607, p=.009)$ does predict current cigarette smoking. Once we entered perceived benefits, depressive symptoms continues to predict cigarette use $(\operatorname{Beta}=.108, \mathrm{t}(566)=2.456, \mathrm{p}=.014)$. Perceived benefits on mood of cigarette smoking $($ Beta $=.115, \mathrm{t}(556)=$ 2.543, $\mathrm{p}=.014$ ) also appear to predict this behavior. Our findings suggest that depressive symptoms influences cigarette use directly and indirectly through the perceived benefits youth perceive have that smoking cigarettes may improve their mood. We also report sex differences and implications of these findings to further understand cigarette use among adolescents.

CORRESPONDING AUTHOR: Paula Repetto, PhD, P. Universidad Catolica de Chile, Santiago, 0000; prepetto@uc.cl

\section{PSW-118}

\section{SMOKING BEHAVIOR AMONG ADOLESCENTS}

IN THAILAND AND MALAYSIA

Tawima Sirirassamee, MD, ${ }^{1}$ Buppha Sirirassamee, $\mathrm{PhD},{ }^{2}$ Maizurah Omar, $\mathrm{PhD},{ }^{3}$ Ron Borland, $\mathrm{PhD},{ }^{4}$ Geoffrey T. Fong, $\mathrm{PhD}^{5}$ and Pete Driezen, $\mathrm{MSc}^{5}$

${ }^{1}$ Srinakarinwirot university, Bangkok, Thailand; ${ }^{2}$ Institute for Population and Social Research, Mahidol university, Bangkok, Thailand; ${ }^{3}$ National Poison Center, Penang, Malaysia; ${ }^{4}$ The Cancer Council Victoria, Melbourne, VIC, Australia and ${ }^{5}$ University of Waterloo, Waterloo, ON, Canada.

Background: Tobacco use is the leading preventable cause of death. Half of the regular smokers die prematurely of a tobaccorelated disease. Although the prevalence of youth smoking in some Western countries has been in steady decline, smoking prevalence in many developing countries is increasing. Thailand and Malaysia are in Southeast Asia, one of the critical regions for tobacco consumption.

Objective: To compare the smoking behavior among adolescents in Thailand and Malaysia.

Design/Methods: A population-based, national surveys were conducted among 1,694 adolescents with ages between 13 and 18 from Thailand $(n=917)$ and Malaysia $(n=777)$. Respondents were selected using multistage cluster sampling. Respondents were asked to complete self-administered questionnaires. Data were analyzed using descriptive statistics, Chi-Square tests and t tests.

Results: Approximately 5 percents of Thai and Malaysian adolescents were current smokers, with an additional 9.6 percents of Thai and 7.8 percents of Malaysian adolescents reported being a beginner smoker. On average, Thai smoker reported to have first smoked a whole cigarette at 14.6 years of age $(\mathrm{SD}=1.93)$, while Malaysian smoker at age $13.9(\mathrm{SD}=2.16)$. About half of Thai smoker $(56.9 \%)$ reported that they bought cigarettes for them- selves and 31.5 percents got cigarettes from friends. In Malaysia, most of smoker $(71.4 \%)$ reported that they bought cigarettes for themselves, only 19.8 percents got cigarettes from friends. Sixty seven percents of Thai adolescent smokers smoked factory-made cigarettes as their usual brand compare to 29.9 percents of Malaysian adolescent smokers $(p<0.01)$. Eight percents of Thai adolescents and 13.6 percent Malaysian adolescents reported smoking hand-rolled cigarettes. Eighty four percents of Thai and 76.1 percent of Malaysian adolescent smokers reported that they tried to quit.

Conclusions: Smoking prevalence of Thai adolescents was closed to that of Malaysian adolescents. Factory-made cigarette consumption is an important problem in Thai adolescents and needs to be targeted.

CORRESPONDING AUTHOR: Tawima Sirirassamee, MD, Srinakarinwirot university, Nakornnayok, 26120; tawima_s@ yahoo.com

PSW-119

PROTECTING CHILDREN AND YOUNG PEOPLE FROM TOBACCO INDUSTRY EXPLOITS: THE CASE FOR PLAIN PACKAGING IN MALAYSIA

Yen L. Tan, MA

Southeast Asia Tobacco Control Alliance, Bangkok, Thailand.

Objectives: To monitor tobacco industry's marketing tactics pre and post implementation of pictorial health warnings in Malaysia. Methods: An observational study was conducted to monitor new marketing strategies and changes in product and packaging designs prior and post to implementation of pictorial health warnings on cigarette packs effective 1 January 2009. A survey instrument was used to collect data in selected point-of-sales in Penang, Malaysia. All new product and pack designs of the most popular cigarette brands were purchased and scrutinized for promotional features. Observations of point-of-sales displays were also made. Data were analyzed using qualitative methods.

Results: Since the banning of advertising at point-of-sales the tobacco industry invested more resources into packaging design and point-of-sale display in order to communicate brand imagery and increase sales primarily targeting children and young people. With the implementation of pictorial health warnings and ban on descriptors, the industry continues to promote its products with introduction of a variety of innovative pack and product designs. Textual descriptors such as light and mild are replaced with varied colour tones (silver and blue) to shape consumer perceptions of risk and have the misleading effect as light and mild. Colours and pack designs are also used by the industry to dilute the effects of pictorial health warnings.

Conclusions: Tobacco control policies have to be water-tight to prevent the tobacco industry from exploiting the loopholes in these policies. The Malaysian government should implement plain packaging which would increase the effectiveness of health warnings, reduce misconceptions about smoking risks and prevent the use of bran variants as a promotional tool. This will help reduce smoking uptake amongst children and young people. 
Learning Objectives:

At the end of this presentation, participants will be able to

1) Describe types of tobacco industry tactics and strategies in circumventing tobacco control law on pictorial health warnings

2) Discuss lessons learned of having water-tighted pictorial health warnings regulation to avoid tobacco industry exploiting the loopholes

CORRESPONDING AUTHOR: Yen L. Tan, MA, National Poison Center, Universiti Sains Malaysia, Georgetown, 11800; yenlian_1121@yahoo.com

PSW-120

CUE-INDUCED CRAVING PROSPECTIVELY PREDICTS CESSATION FAILURE FOLLOWING AN UNTREATED, COLD-TURKEY SMOKING CESSATION ATTEMPT

Joel Erblich, $\mathrm{PhD}, \mathrm{MPH}^{1}$ and Dana $\mathrm{H}$. Bovbjerg, $\mathrm{PhD}^{2}$

${ }^{1}$ Department of Oncological Sciences, Mount Sinai School of Medicine, New York, NY and ${ }^{2}$ University of Pittsburgh Cancer Institute, Pittsburgh, PA.

Cigarette smoking is one of leading preventable causes of morbidity and mortality in the world. Clinical reports and empirical data have highlighted the importance of environmental cues (e.g., the sight or smell of a cigarette) in triggering cravings that may lead to relapse following a quit attempt. This 'cue-induced craving' has been reliably modeled under laboratory conditions, in which smokers exposed to smoking-related cues report higher levels of cigarette cravings than following exposure to neutral cues. Whether or not these craving responses predict smoking cessation failure is a matter of some debate in the scientific literature. Retrospective studies have provided mixed results, and prospective studies are generally lacking. Initial results from our longitudinal study of smokers making a cold-turkey untreated smoking cessation attempt are reported here. Briefly, participants $(n=20,50 \%$ male, $50 \%$ female, mean age $=37.5$ yrs, $60 \%$ African American, $15 \%$ Hispanic, $20 \%$ Caucasian, mean cigarettes/day=19.1) were exposed to smoking (unlit cigarette) and neutral (stapler) cues one week prior to their quit date and reported cravings (0-100) before and after each exposure. Exposures were counterbalanced and separated by a 90 -second rest. Time until relapse (Mean= 5.6 days) was assessed at 30 days post quit. Findings indicated that higher pre-quit levels of smoking cue-induced cravings predicted quicker time to relapse $(\mathrm{p}<0.0006)$. These preliminary results provide initial support for the validity of modeling cueinduced craving in the laboratory and suggest that cravings triggered by exposure to cues may play an important role in cessation failure.

CORRESPONDING AUTHOR: Joel Erblich, PhD, MPH, Department of Oncological Sciences, Mount Sinai School of Medicine, New York, NY, 10029; joel.erblich@mssm.edu
PSW-121

RELATIONSHIP BETWEEN THE SMOKING STATUS AND LIFESTYLE CHOICES OF JUNIOR-HIGH SCHOOL STUDENTS

Daisuke Matsui, Student, ${ }^{1}$ Isao Watanabe, student, ${ }^{1}$ Masako Shigeta, MD, ${ }^{1}$ Nagato Kuriyama, MD, ${ }^{1}$ Satoko Mitani, MD, ${ }^{1}$ Etsuko Ozaki, MD, ${ }^{1}$ Kotaro Ozasa, $\mathrm{MD}^{2}$ and Yoshiyuki Watanabe, $\mathrm{MD}^{1}$

${ }^{1}$ Epidemiology for Community Health and Medicine, Kyoto Prefectural University of Medicine, Kyoto, Japan and ${ }^{2}$ Radiation Effects Research Foundation, Hirosima, Japan.

Background: The smoking rate among adolescents has been decreasing in Japan. According to the National Survey, the current smoking rates of 7 th grade students decreased from $7.5 \%$ (males) and 3.8\% (females) in 1996 to $3.2 \%$ and $2.4 \%$ in 2004 . In Kyoto, workshop-style tobacco-free classes based on theory of behavioral science have been conducted since 2002, in cooperation with a medical university, a medical association and the local government.

Objective: During 2009, we conducted tobacco-free workshops for 3562 students at 26 municipal junior-high schools. In order to clarify the smoking status of these students, we investigated the perception of smoking and the relationship between smoking and other life-style habits. Subjects : There were 1644 students at 13 schools who agreed to participate in the survey. The response rate was $98.1 \%$. Males: 820 , Females: 721 , not specified: 108 .

Methods: The questionnaire was anonymous and self-completed. We made sure that teachers would not check the questionnaire responses. Questions about lifestyle including smoking and drinking, were asked mainly in fourfold choice style. After analyzing the smoking status by school and sex, we examined the relationship between lifestyle and smoking.

Results: The current smoking rate was less than $1 \%$ at half of the schools, but $3 \sim 7 \%$ at 3 schools. Overall, $5.5 \%$ of the subjects had tried smoking, and $1.9 \%$ were current smokers. $52.9 \%$ were living with smokers; $46.7 \%$ had tried alcohol; $50.5 \%$ watched TV more than 3 hours a day and $4.4 \%$ shopped at convenience stores every day. Current smoking and previous smoking showed significant association with smoking by cohabiters, drinking experience, study time, attending school club activities and shopping at convenience stores.

Discussion: The smoking prevalence of subjects was low compared with that in the National Survey, possibly because our subjects were not randomly selected and Kyoto has the lowest smoking prevalence among adults. This is the first report in Japan indicating that smoking habits may be related to shopping at convenience stores. The students are mainly buying cigarettes at convenience stores, where cigarettes companies place a large number of advertisements. Tobacco sales at convenience stores should be strictly monitored.

CORRESPONDING AUTHOR: Daisuke Matsui, Student, Epidemiology for Community Health and Medicine, Kyoto Prefectural University of Medicine, Kyoto, 602-0841; d-matsui@koto.kpu-m. ac.jp 
PSW-122

AGE AT SMOKING ONSET AND SMOKING LEVEL AMONG ADOLESCENT SMOKERS IN KOREA

Soon-Woo Park, PhD and Jong-Yeon Kim, PhD

Preventive Medicine, Catholic University of Daegu School of Medicine, Daegu, Republic of Korea.

The aim of this study was to explore the relationship between the age at cigarette smoking onset and the smoking level among adolescent current smokers. In 2007, one or two classes of 10th and 11th grade were randomly selected from the high schools sampled by PPS (probability proportional to size) sampling after all the schools in a large city in Korea were stratified by grade, gender, and region. A total of 743 current smokers among a sample of 4,602 students were analyzed with a self-administered questionnaire. The smokers were defined as those who had smoked at least one cigarette during past one month. The age of smoking onset was measured as the grade when the respondents first tried a cigarette even one or two puffs. The odds ratios (ORs) and 95\% confidence intervals (CIs) were calculated by multiple logistic regression analysis and they were adjusted for potential confounders. Compared with those who tried smoking first after entrance into high school, the OR of being a frequent smoker (i.e. those who smoked on 20 days or more during the past one month) among those who tried during middle school period was 2.40 (CI 1.53-3.78), that of those who tried smoking during late elementary school period was 3.70 (CI 1.67 8.17), that of those who tried smoking early elementary school period or earlier was 3.91 (CI: 1.74 8.78). The OR of being a regular smoker (i.e. those who smoked everyday) among those who tried during middle school period was 1.77 (CI: 1.14-2.76), that of those who tried smoking during late elementary school period was 2.34 (CI: 1.14 4.78), that of those who tried smoking early elementary school period or earlier was 4.83 (CI: 2.19 10.69). The OR of being a heavy smoker (i.e. those who smoked one-half pack or more per day on the average) among those who tried during middle school period was 3.61 (CI: 1.84-7.08), that of those who tried smoking during late elementary school period was 6.67 (CI: 2.87 15.50), that of those who tried smoking early elementary school period or earlier was 5.65 (CI: 2.34 13.62). This study showed the importance of smoking prevention or delaying smoking onset among children and adolescents.

CORRESPONDING AUTHOR: Soon-Woo Park, PhD, Preventive Medicine, Catholic University of Daegu School of Medicine, Daegu, 705-718; parksw@cu.ac.kr

\section{PSW-123}

THE ASSOCIATION BETWEEN PAID MATERNAL EMPLOYMENT IN CHILDHOOD AND ADOLESCENT SMOKING IN BHPS STUDY

Jitka Pikhartova, MD, Anne McMunn, PhD, Annie Britton, $\mathrm{PhD}$, Tarani Chandola, $\mathrm{PhD}$ and Yvonne Kelly, PhD

Epidemiology and Public Health, UCL, London, United Kingdom.
Background: Maternal employment has been shown to influence health behaviors of children and adolescents such as overweight and obesity. The evidence related to adolescent smoking is limited as well as results using UK data. In this analysis, we assessed the influence of maternal employment in three periods of childhood on smoking of young people aged 16-21 years in British Household Panel Survey (BHPS).

Methods: BHPS is annual panel survey that has started in 1991 and completed its 17 th wave in 2007. Response rate to wave 1 was $74 \%$, and response between waves 2 and 17 varied between $84 \%-89 \%$. There are 3,615 young individuals with available data on smoking at age 16-21 and maternal employment data from age 0-16 years. Covariates, such as gender, maternal age, maternal education and marital status, household income or maternal smoking were used as explanatory variables in logistic regression analysis in STATA 10.

Results: $29 \%$ of young adults aged 16-21 reported being current smoker. Approximately $40 \%$ of mothers worked at some point during age $0-4$ (preschool age) of their child. This proportion increased to $59 \%$ at age 5-11 (primary school age) and $68 \%$ at age 12-16 (secondary school age). Children of mothers who were not employed were more likely to smoke (OR of smoking 1.18, 1.19 and 1.60 for 3 periods of exposure), although these effects were almost entirely explained when adjusted for maternal education and household income.

Conclusions: The associations between maternal employment in childhood and young adults' smoking exist at least partly because of the generally higher social position and more stable family structure of households with working mothers. Maternal education and household income seem to be stronger social predictors of adolescent smoking than maternal employment status.

CORRESPONDING AUTHOR: Jitka Pikhartova, MD, Epidemiology and Public Health, UCL, London, WC1E 6BT; j.pikhartova@ ucl.ac.uk

PSW-124

RESEARCH ON SMOKING PREVALENCE

IN ALTERNATIVE HIGH SCHOOLS

Isao Watanabe, Student, ${ }^{1}$ Daisuke Matui, Student, ${ }^{1}$ Etuko Ozaki, Associate Professor, ${ }^{1}$ Satoko Mitani, Associate Professor, ${ }^{1}$ Nagato Kuriyama, Associate Professor, ${ }^{1}$ Masako Shigeta, Associate professor, ${ }^{1}$ Kotaro Ozasa, Chief ${ }^{1,2}$ and Yoshiyuki Watanabe, Professor ${ }^{1}$

${ }^{1}$ Epidemiology for Community Health \& Medicine, Kyoto Prefectual University of Medicine, Kyoto, Japan and ${ }^{2}$ Radiation Effects Research Foundation, Hirosima, Japan.

Background: In order to ensure the availability of high school curriculum, there are alternative high schools (evening high schools that are generally between 18 o'clock to 21 o'clock) in Japan, for students who cannot attend ordinary schools due to economic or academic reasons. The ratio of alternative high school students has been increasing. According to the National Survey, the smoking prevalence of adolescents has been decreasing; however, alternative high schools were not included in this survey. 
Objective: We conducted research into the smoking prevalence among alternative high school students in order to review the success and feasibility of anti-tobacco education.

Subjects and Methods: We conducted an anonymous selfcompleted questionnaire survey and workshop-style smoking prevention classes, among 691 students in 6 alternative high schools in the Kansai area. We compared the smoking prevalence to those in 3 ordinary high schools.

Results: In alternative high schools, $47.6 \%$ of the students had a history of smoking. Current smokers comprised 31.6\%. The prevalence of both a smoking history and current smoking was significantly higher than those in ordinary high schools, among both sexes. Alternative high school students tended to have worse lifestyle habits than ordinary high school students, such as poor sleep, poor diet, and poor study habits. The smoking preventive classes were successful, as the students showed an interest in the lecture part, and the attendance to the workshops was acceptable. We observed that the students had gained accurate knowledge of tobacco and that the consciousness of smoking prevention had been strengthened.

Discussion: The pervasiveness of smoking at alternative high schools is a serious problem and early intervention is necessary. We consider that anti-tobacco programs are effective and need to be implemented urgently at alternative high schools, where intervention is possible among students attending schools.

CORRESPONDING AUTHOR: Isao Watanabe, Student, Kyoto Prefectual University of Medicine, Kyoto, 602-8566; ricky@koto. kpu-m.ac.jp

\section{PSW-125}

THE STATUS OF DRINKING ALCOHOL AND THE IMPACT OF INTERVENTION AMONG HEALTHY JAPANESE MALES Masako Shigeta, MD, ${ }^{1,2}$ Atsuko Nakazawa, MD, ${ }^{1,2}$ Miho Ueda, $\mathrm{MD}^{2}{ }^{2}$ Katsura Yamakado, $\mathrm{Ns}^{2}{ }^{2}$ Daisuke Matsui, DT, ${ }^{1}$ Isao Watanabe, DT, ${ }^{1}$ Etsuko Ozaki, NT, ${ }^{1}$ Satoko Mitani, PhD, ${ }^{1}$ Nagato Kuriyama, $\mathrm{MD}^{1}$ and Yoshiyuki Watanabe, $\mathrm{MD}^{1}$

${ }^{1}$ Kyoto Prefectural University of Medicine, Kyoto, Japan and ${ }^{2}$ Kyoto First Red Cross Hospital, Kyoto, Japan.

Background: Many researchers have demonstrated that inappropriate drinking has various adverse effects. Japanese national survey have pointed out that people have not been sufficiently educated enough about the dangers of drinking, although the amount of alcohol consumed by Japanese people has been increasing.

Objective: Since 2002, we have been asking detailed questions about the amount and the frequency of drinking by healthy people undergoing routine medical checkups and giving them advice based on their responses. We analyzed our findings in order to clarify the drinking status of these subjects and the effects of the intervention.
Subjects and Methods: Subjects were 3633 males who under-

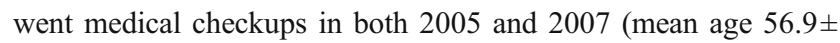
11.2). Among these subjects, 959 were non-drinkers (ND), 1574 were light drinkers (LD: less than $140 \mathrm{~g}$ of alcohol/week), 943 (26.0\%)were moderate drinkers (MD: 140-420 g/week), and $157(4.3 \%$ ) were heavy drinkers (HD:over $420 \mathrm{~g} /$ week). We examined the relationship between the amount of alcohol intake and laboratory data by analysis of variance. We provided these subjects motivational support using medical data on the checkup day, and sent further advice 2 weeks later. We analyzed changes in the amount of alcohol intake and laboratory data 2 years later.

Result: $\gamma$-GTP (IU/L) values in 2005 were ND $(34.9 \pm 1.2)$, LD (44.5 \pm 1.2$), \mathrm{MD}(66.7 \pm 5.4)$, and HD (96.5 \pm 5.8$)$. There were significant differences among the groups, except for that between ND and LD. The amount of alcohol intake was decreased in both MD\&HD 2 years later. The $\gamma$-GTP, GPT, and TG values of 1100 subjects of MD or HD decreased significantly.

Conclusions: One third of the subjects had inappropriate drinking behaviors, although they were sufficiently health-conscious to come for a medical checkup. After receiving motivational support, the amount of alcohol intake decreased and the laboratory data improved.

CORRESPONDING AUTHOR: Masako Shigeta, MD, Kyoto Prefectural University of Medicine, Kyoto, 6028566; mshige@ koto.kpu-m.ac.jp

PSW-126a

CHARACTERISTICS OF THE INITIAL INTERVIEW DETERMINE ADOLESCENTS' ADHERENCE TO AN OUTPATIENT SUBSTANCE ABUSE TREATMENT PROGRAM. B. GONZáLEZ, E. GUIC, A.M. VILLARINO, X. SANTANDER. HEALTH PSYCHOLOGY UNIT, PSYCHOLOGY DEPARTMENT, CATHOLIC UNIVERSITY OF CHILE, SANTIAGO, CHILE AND SERVICIO DE SALUD METROPOLITANO SUR-ORIENTE

Beatriz Gonzalez, MSc, ${ }^{1}$ Eliana Guic, $\mathrm{PhD},{ }^{1}$ Ana Maria Villarino, Psychologist $^{2}$ and Ximena Santander, Psychologist ${ }^{1}$

${ }^{1}$ Health Psychology, UC, Santiago, Chile and ${ }^{2}$ Servicio Salud Metropolitano Sur-Oriente, Santiago, Chile.

An adolescents' outpatient substance abuse treatment program is available free of charge to Chilean youth under 20 years old at secondary health centers. One of the main purposes of the initial interview is to increase patients' treatment motivation. In this study we describe health provider's motivational interviewing (MI) consistent and inconsistent responses during the first interview and we study the effects of these responses on adherence to treatment.

Method: Participants were 142 adolescents with substance use disorders seeking admission to the program during 2009 (64\% 
men; 15 . yrs old, 11 to $19 ; 79.6 \%$ with substance abuse for 6 month or more) and their mental health providers $(n=18)$, from 10 health centers. All of them volunteered to participate according to the informed assents and consents. We audiotaped the initial interviews and analyzed their content using the MISC 2.1 coding system. We also collected data related to the patients and their context. Adherence to treatment was defined as attendance to at least one planned activity in the program.

Results: Adherence to treatment was $54.9 \%$ after the initial interview; $50.1 \%$ at month $1 ; 46 \%$ at month 2 ; and $36.6 \%$ at month 3 after admission. Using multivariate logistic regression analysis, therapist specific behaviors, considered to potentially predict adherence, were assessed. Number of reflections $(\mathrm{OR}=.926$; $95 \% \mathrm{CI}=.862-.995 ; \mathrm{p}<.037)$, confrontations $(\mathrm{OR}=.920 ; 95 \%$ $\mathrm{CI}=.847-.999 ; \mathrm{p}<, 048)$ and total number of question asked during initial interview $(\mathrm{OR}=.991 ; 95 \% \mathrm{CI}=.985-.998 ; \mathrm{p}<.012)$, were significant predictors of adherence one month after the initial visit and at month 3 after admission, adjusting for adolescents' demographics and abuse variables.

Conclusions: These results confirm, in a sample of Chilean participants, that MI consistent and inconsistent responses are important factors determining retention rates in adolescents' drug treatment programs.

Acknowledgments: FONIS Grant to EG.

CORRESPONDING AUTHOR: Eliana Guic, PhD, Health Psychology, PUC, Santiago, 1; eguic@uc.cl

PSW-126b

SMOKING ONSET AMONG ROMANIAN JUNIOR HIGH SCHOOL STUDENTS

Lucia M. Lotrean, $\mathrm{MD}, \mathrm{PhD},{ }^{1}$ Floarea Mocean, $\mathrm{MD}, \mathrm{PhD}^{1}$ and Hein De Vries, $\mathrm{PhD}^{2}$

${ }^{1}$ University of Medicine and Pharmacy, Cluj Napoca, Romania and ${ }^{2}$ Maastricht University, Maastricht, Netherlands.

The objective of this study is to assess predictors of smoking onset among Romanian junior high school students aged 1314 years old.

The data were obtained from a two-wave, 9 months longitudinal study carried out among 504 junior high school non-smokers from Cluj county, Romania. Questionnaires assessed smoking behaviour, attitudes, social influence, self-efficacy and intention regarding smoking (motivational variables), as well as different sociodemographic features.

The results from the logistical regression analysis reveal that baseline lower self-efficacy in refraining from smoking in several social situation, baseline pressures to smoke from peers, and baseline intentions to smoke significantly increased the risk of non-smokers to become smokers at follow up nine months after.

These findings underline that a smoking prevention approach for this age group that aimed at reinforcing social self-efficacy, resisting peer pressures and maintaining negative intentions are essential ingredients for smoking prevention programs among Romanian junior high school students

CORRESPONDING AUTHOR: Lucia M. Lotrean, MD, PhD, University of Medicine and Pharmacy, Cluj Napoca, 400340; llotrean@umfcluj.ro

PSW-126c

OPINIONS OF CURRENT AND FORMER SMOKERS ON TOBACCO CONTROL

Hanna Ollila, M Soc Sci, ${ }^{1}$ Ulla Broms, PhD, ${ }^{1,2}$ Tellervo Korhonen, $\mathrm{PhD},{ }^{1,2}$ Jaakko Kaprio, $\mathrm{MD}, \mathrm{PhD}^{2,3}$ and Kristiina Patja, $\mathrm{MD}, \mathrm{PhD}^{4}$

${ }^{1}$ National Institute for Health and Welfare, Helsinki, Finland; ${ }^{2}$ University of Helsinki, Helsinki, Finland; ${ }^{3}$ Institute for Molecular Medicine Finland FIMM, Helsinki, Finland and ${ }^{4}$ The Association for Medical Continuous Professional Development in Finland (Pro Medico), Helsinki, Finland.

Objectives: The Finnish tobacco legislation pursues towards a tobacco-free society with a large number of bans: e.g. advertising ban and smoking ban in workplaces and restaurants. We studied opinions of daily (D), occasional (O) and former (F) smokers on tobacco control and on societal support for smoking cessation.

Methods: Cross-sectional data from national FINRISK-study 2007. The tobacco subsample $(\mathrm{N}=1746)$ consists of eversmokers answering to statements on tobacco control.

Results: Two thirds of smokers and ex-smokers viewed smoking ban in restaurants as a good decision. However, former (OR 4.14; $95 \%$ CI 3.24-5.29) and occasional (2.19; 1.58-3.05) smokers were more likely to view it positively compared to daily smokers. Occasional smokers were $1.58(1.12-2.25)$ times more likely to see the smoking ban in workplaces working well compared to daily smokers. Former (.47; .37-.61) and occasional (.62; .44-.88) smokers were less likely to see the smoking restrictions being supervised enough. Former smokers were 2.31 (1.63-3.27) times more likely to see tobacco use as approved behavior in society compared to daily smokers. Nine out of ten subjects agreed that youth smoking needs to be restricted, yet quitters agreed more likely $(1.67 ; 1.18-2.37)$ than daily smokers. $61 \%$ of former, $52 \%$ of occasional and $34 \%$ of daily smokers agreed that tobacco sales points should be reduced [F: 2.98 (2.38-3.72), O: 2.33 (1.70 $3.20)$ ]. Former $(6.24 ; 4.69-8.32)$ and occasional $(4.85 ; 3.34-7.04)$ smokers were more likely to agree with smoking ban in balcony. Former and occasional smokers were less likely to agree that 
society should support quitters with tobacco-related disease (F: $.57 ; .46-.70, \mathrm{O}: .56 ; .41-.76)$ or all who quit smoking (F: .63; $.51-.79$, O: .51;.38-.70).

Conclusions: Most former and current smokers hold positive opinions towards tobacco control. Former and occasional smokers are more likely than daily smokers to support stronger restrictions, such as reducing the number of tobacco sales points, but less likely to demand societal support for smoking cessation.

CORRESPONDING AUTHOR: Hanna Ollila, M Soc Sci, National Institute for Health and Welfare, Helsinki, 00271; hanna.ollila@thl.fi

\section{TOP FIFTEEN SCORING POSTER}

PSW-127

INFLUENCE OF SMOKING CESSATION ON PROGNOSIS IN EARLY STAGE LUNG CANCER: A SYSTEMATIC REVIEW WITH META ANALYSIS

Amanda Parsons, MPhil, Amanda Daley, PhD, Rachna Begh, MRes and Paul Aveyard, PhD

Primary Care Clinical Sciences, University of Birmingham, Birmingham, United Kingdom.

Background

Although the effect of smoking on lung cancer incidence is well known, it is not yet known if continued smoking after diagnosis affects prognosis.

Methods

We systematically searched for studies that measured the effect of quitting smoking after diagnosis of lung cancer on prognosis. Two researchers independently identified studies to include and extracted data. Estimates were combined using a random effects model and I2 statistic was calculated to examine heterogeneity. Using life tables, we modelled 5-year survival for non-small cell lung cancer (NSCLC) and small cell lung cancer (SCLC) using death rates for continuing smokers and quitters obtained from the review.

Data sources

We searched CINAHL (from 1981), EMBASE (from 1980), MEDLINE (from 1966), Web of science (from 1966) and CENTRAL (from 1977) to December 2008 and the reference lists of included studies.

Results

Continued smoking was associated with an increased risk of all-cause mortality and recurrence in early stage NSCLC ((HR 2.94 95\% CI $(1.15,7.54)$, HR $1.86(1.01,3.41))$ and with an increased risk of all-cause mortality, development of second primary and recurrence in limited stage SCLC ((HR 1.86 (1.33, 2.59), HR $4.31(1.09,16.98)$ and HR $1.26(1.06,1.50)$. Life table modelling estimated $41 \%$ 5-year survival in 65-year old NSCLC continuing smokers compared to $75 \%$ in quitters. In SCLC, an estimated $36 \%$ of continuing smokers survive for five years compared to $68 \%$ quitters.

Conclusions

There is evidence that smoking cessation after diagnosis of lung cancer is associated with improved prognosis. Assuming causality, the number of deaths prevented is larger than would be expected due to reduction of cardio-respiratory deaths after smoking cessation and it is likely the majority of the mortality gain is due to reduced cancer progression.

CORRESPONDING AUTHOR: Amanda Parsons, MPhil, Primary Care Clinical Sciences, University of Birmingham, Birmingham, B17 8EE; a.c.parsons@bham.ac.uk

\section{PSW-128}

THE HIDDEN COSTS OF CANCER: BARRIERS

\section{TO ACCESSING FINANCIAL ASSISTANCE}

Joanne S. Buzaglo, PhD, Kasey R. Dougherty, BA, Ellyn Micco, MS, Melissa Miller, $\mathrm{PhD}$ and Mitch Golant, $\mathrm{PhD}$

Research \& Training Institute, Cancer Support Community, Philadelphia, PA.
Many questions remain about the direct and indirect costs to families incurred during the course of cancer care. Despite availability, many patients fail to access co-pay assistance programs. The Cancer Support Community and Genentech piloted a national survey investigating psychosocial barriers to accessing co-pay assistance and the hidden costs of care. Methods: Cancer patients $(n=79)$ diagnosed over 6 months ago or had active treatment within 2 years and caregivers $(n=29)$ of patients who met the above criteria, completed a survey that included the Revised Impact of Event Scale to assess intrusive and avoidant ideation around the cost of care. 
Results: Respondents $(\mathrm{N}=108)$ were ethnically diverse; $25 \%$ patients and $18 \%$ caregivers had household incomes less than $\$ 20$ K. Patients reported high levels of intrusion (18.5 \pm 9.2$)$ and avoidance (20.2 \pm 8.8$)$. Caregiver levels were also high for intrusion (15.0 \pm 10.0$)$ and avoidance $(17.8 \pm 10.1)$. Based on clinical cut-offs, $82 \%$ reported stress within moderate to severe levels. Due to care costs, $74 \%$ patients depleted savings, $44 \%$ downsized living accommodations; $32 \%$ caregivers worked extra jobs to help pay for care, $60 \%$ reported extra stress at work. To reduce costs, $49 \%$ patients delayed seeking psychological counseling. Among patients, increased intrusion correlated with having no one to help with insurance questions $(p<.0001)$. Increased avoidance was associated with delay in seeking psychological counseling $(\mathrm{p}<.0001)$ and less awareness of co-pay assistance $(p<.0001) .33 \%$ patients and $40 \%$ caregivers felt too overwhelmed to apply for financial help.

Discussion: These data suggest that managing cancer costs is associated with clinically significant stress levels. Those with greatest need may not seek help, underscoring the need for programs that help families manage the stress related to the cost of care.

CORRESPONDING AUTHOR: Joanne S. Buzaglo, PhD, Research \& Training Institute, Cancer Support Community, Philadelphia, PA, 19131; joanne@cancersupportcommunity.org

\section{EARLY CAREER AWARDEE}

PSW-129

DEVELOPMENT OF EMPATHY AMONG MEDICAL SCHOOL STUDENTS TOWARDS PSYCHOSOCIAL NEEDS OF CANCER PATIENTS AND THEIR RELATIVES THROUGH MICRO SKILLS INTERVIEW TRAINING

Sanghamitra Pati, MD DNHE MPH

Medical Education, SCB Medical College, Bhubaneswar, India.

The patient-provider relationship and specifically physicians' ability to communicate empathy greatly impacts chronic disease management. Empathetic communication is significant in navigating difficult circumstances like dealing with oncology patients. Unfortunately, research demonstrates that empathy declines in $75 \%$ of medical students as they advance in their training. There is growing recognition that medical education must address this decline in empathy and properly prepare physicians to work with diverse patients. However traditional educational techniques in India are unlikely to meet this need. Experiential learning may be used to address this problem.

The purpose of this study was to investigate the effectiveness of faculty-supervised and selfinstructional listening micro skills training in increasing the responsiveness of medical students to the psychosocial needs of cancer patients and their families during medical student-patientfamily member interviews. This study employed a randomized controlled design.

Two multiple baseline across subjects designs of four baselines each were used for the study. Final-year medical students posted in clinical oncology were randomly assigned to conditions for training and to baselines within each condition. Training conditions were identical in informational content and time requirements. Self-instructional training incorporated two videotapes developed for the study. Through the use of graphs and t-tests, data from the training conditions were analyzed separately, comparatively, and on the basis of overall training effectiveness across all eight subjects.

Overall, majority of the students felt that the intervention influenced their level of empathy (78\%), should be a standard part of medical school curriculums (67\%), and would impact all physicians $(80 \%)$. Faculty-supervised training was found to be more effective than selfinstructional training in increasing responsiveness as measured by (a) observational data from videotaped interviews, (b) patient and family member ratings of interviews, and (c) number of psychosocial needs recognized on medical student dictation reports. Results based on t-tests indicated overall use of training was effective in increasing (a) appropriate use of four interview micro skills, (b) patient and family member interview ratings, and (c) number of psychosocial needs recognized on dictation reports.

Findings suggest that experiential learning methods are well received, feasible, and effective in increasing empathy in medical students and should become a standard part of medical school curriculums. 
CORRESPONDING AUTHOR: Sanghamitra Pati, MD DNHE MPH, Medical Education, SCB Medical College, Bhubaneswar, 751022; sanghamitrapati@yahoo.com

PSW-130

SHORT AND LONG TERM EFFECTS OF USING THE SCREENING INVENTORY OF PSYCHOSOCIAL PROBLEMS (SIPP) ON PSYCHOLOGICAL FUNCTIONING AND QUALITY OF LIFE AMONG CANCER PATIENTS TREATED WITH RADIOTHERAPY

Anna Braeken, MSc, ${ }^{1,2}$ L. Lechner, $\mathrm{PhD},{ }^{2}$ F. van Gils, PhD, MD, ${ }^{3}$ R. Houben, MSc, ${ }^{3}$ D. Eekers, MSc, $\mathrm{MD}^{4}$ and G. Kempen, $\mathrm{PhD}^{1}$ ${ }^{1}$ Health care and nursing science, Maastricht University, Maastricht, Netherlands; ${ }^{2}$ Faculty of Psychology, Netherlands Open University, Heerlen, Netherlands; ${ }^{3}$ Maastricht Radiation Oncology, Maastricht, Netherlands and ${ }^{4}$ Institute Verbeeten, Radiation Oncology, Tilburg, Netherlands.

Background: Cancer often leads to psychosocial distress and may influence health-related outcomes. The SIPP is a short, validated questionnaire to identify distress in cancer patients. Our aim is to test the hypotheses that using the SIPP in physician-patient consultations may prevent underdiagnosis of early distress symptoms resulting in better psychological functioning and quality of life $(\mathrm{QoL})$ in both the short- and long-term. Methods: A cluster randomized controlled trial was conducted. Cancer patients were asked to complete questionnaires measuring sociodemographic characteristics, symptoms of anxiety, depression and psychosocial distress and QoL at baseline before starting radiotherapy (RT) (T1) and post-tests at 3 (T2) and 12 months (T3) after baseline. Results: 569 Cancer patients were included. Preliminary results show at T2 that women of the control groups reported more cognitive dysfunction $(p=0.015)$ than women of the intervention groups. Patients of the control groups with rectum cancer reported more symptoms of depression $(p=0.039)$, and psychosocial distress in general $(\mathrm{p} \leq 0.030)$ than the same patients of the interventions groups. The percentage of patients with prostate $(p=$ $0.045)$ and rectum cancer $(\mathrm{p}=0.044)$ with anxiety were higher in the control groups. At T3, patients of the control groups with breast cancer reported more symptoms of anxiety $(p=0.012)$ and psychosocial distress $(p=0.048)$. The percentage of lung cancer patients with psychosocial distress was higher in the control groups $(p=0.030)$.

Conclusion: Cancer patients receiving a minimal psychosocial intervention seem to improve in psychosocial functioning at $\mathrm{T} 2$ and T3 and marginally improve QoL at T2. In depth results will be presented during the congress.

CORRESPONDING AUTHOR: Anna Braeken, MSc, Health care and nursing science, Maastricht University, Maastricht, 6229 HR; v.braeken@zw.unimaas.nl

\section{PSW-131}

MEASURING SYMPTOM BURDEN IN CANCER PATIENTS

Teresa L. Deshields, PhD, Patricia Potter, PhD and Sarah Olsen, BSN Siteman Cancer Center, St. Louis, MO.

Purpose: In this study, we considered the best approach to measuring symptom burden in cancer patients by comparing patients' reports of their symptom experience and their quality of life. Portenoy created the Memorial Symptom Assessment Scale (MSAS) around 1990 to assess symptom frequency, severity, and distress; it has been used extensively with cancer patients. The MSAS scores include the Global Distress Index (GDI), based on the distress scores associated with 6 physical symptoms and the frequency scores of 4 psychological symptoms.

Methods: We sent survey packets (the MSAS and the Functional Assessment of Chronic Illness Therapy (FACT - G), a quality of life measure) to patients diagnosed with the 5 most common cancer diagnoses in our cancer center (breast, colorectal, gynecologic, lung, and prostate cancers). A total of 458 patients returned surveys. We revised the method for calculating the GDI by using the symptom reports of our sample, using the 6 most common physical symptoms (lack of energy, difficulty sleeping, problems with sexual interest/ activity, pain, feeling drowsy, and numbness/ tingling of the hands and feet) and the 4 most common psychological symptoms (worrying, feeling sad, feeling irritable, and feeling nervous). We compared the MSAS Total Score, the GDI, our revised GDI, and the total number of symptoms to determine which best predicts patients' quality of life.

Results: Our sample yielded $X=86.3$ FACT Total score and these MSAS scores: $X=8.7$ symptoms; $X=.5$ Total score, $X=.9$ GDI score, and $X=1.2$ revised GDI score. These compared to Portenoy's $\mathrm{X}=11.5$ symptoms and $\mathrm{X}=1.3 \mathrm{GDI}$ score. Our revised GDI score was highly correlated with the original GDI $(r=.90, p=.01)$. Of the various measures, the MSAS Total score correlated most highly with overall quality of life ( $\mathrm{rs}=.72, \mathrm{p}=.01$ ).

Discussion: The top physical symptoms reported by our patients differed from the original MSAS results, likely due to changes in cancer treatment over the years. While this might suggest the need to revise the method for calculating the GDI, our revised GDI was virtually identical to the original GDI. Our results indicated that the MSAS Total score is most representative of symptom burden.

CORRESPONDING AUTHOR: Teresa L. Deshields, PhD, Siteman Cancer Center, St. Louis, MO, 63110; tld2593@bjc.org

PSW-132

EPIDEMIOLOGIC ASSESSMENT OF ORAL SQUAMOUS CELL CARCINOMA IN SISTAN VA BALOUCHESTAN FROM 1995 TO 2006, IRAN

Maryam Ghanbariha, doctorate ${ }^{1,2}$ and Fatemeh Shahsavari, doctorate ${ }^{1,2}$ ${ }^{1}$ oral pathology, faculty dentistry of zahedan, Zahedan, Islamic Republic of Iran and ${ }^{2}$ oral pathology, faculty dentistry of Rasht, Rasht, Islamic Republic of Iran.

The aim of this retrospective study was to assess the comparative frequency, gender, age, sub-site distribution and histologic differentiation of oral squamous cell carcinoma (OSCC) in Sistan \& Baluchestan province, Iran. The medical records of 3070 patients with a histopathologic diagnosis of malignant neoplasm were reviewed during the 11-years period from 1995 to 2006.Available data suggested a steady increase in prevalence of OSCC during the last decades. .Of 3070 patients with malignancy, 217 cases had oral malignancy. The most common oral malignant neoplasia was OSCC $(n=178,82 \%)$ and then verroucose carcinoma $(n=14,6.4 \%)$. 
During these years oral SCC was one the most common cancer of the body after esophagus malignancy. Peak incidence of OSCC in both sexes was in the 60-69 years age groups and male-to-female ratio was 1.1:1. The lower lip (22.6\%) and mandibular gingiva (22.1\%) were most commonly affected area. Hard palate squamous carcinoma was uncommon (1.8\%). The most common histological grading was well differentiated SCC $(72.5 \%)$. The data suggest that high rate of OSCC in females may be due to rising prevalence of tobacco use consumption among females, so a more intensive programme of prevention is necessary

CORRESPONDING AUTHOR: Maryam Ghanbariha, doctorate, oral pathology, faculty dentistry of zahedan, Zahedan, 7865342; ghanbariha@yahoo.com

\section{EARLY CAREER AWARDEE \\ PSW-133 \\ SELF-EFFICACY, WELL-BEING AND QUALITY OF LIFE IN YOUNG ADULTS WITH CANCER: A LONGITUDINAL STUDY \\ Elisa K. de Castro, PhD, ${ }^{1}$ Clarissa Franco Ponciano, Psychologist, ${ }^{1}$ Bruna Machado Meneghetti, Psychologist, ${ }^{1}$ Marina Kreling, Psychologist ${ }^{1}$ and Carolina Chem, Psychologist ${ }^{2}$ \\ ${ }^{1}$ Health Sciences Center, Universidade do Vale do Rio dos Sinos, São Leopoldo, Brazil and \\ ${ }^{2}$ Psychology Service, Santa Rita Hospital, Porto Alegre, Brazil. \\ Purpose: Assess longitudinally (one-year follow-up) quality of life, self-efficacy and well-being in young adults with cancer. Participants: 48 patients with cancer, average age of 33.35 years ( $\mathrm{SD}=5.60$ ). Instruments: socio-demographic data, WHOQOL-bref, General Perceived Self- Efficacy Scale and General Health Questionnaire 12. Results: Pearson's correlation showed that quality of life in baseline had a significant correlation with self-efficacy $(r=0.358, p<0.05)$ and well-being $(r=-0.550, p<0.01)$ at baseline, and with self-efficacy $(r=757, p<0.01)$ at follow-up. Paired t-test revealed no differences among quality of life, self-efficacy and well being at baseline and follow-up. However, independent t-test showed that males presented better total and physical quality of life and better well-being than females at baseline $(\mathrm{t}=2.515, \mathrm{p}=<0.05)$ and better well-being at follow-up $(\mathrm{t}=3.159, \mathrm{p}=<0.01)$. It was concluded that over time, positive variables like quality of life, well-being and self-efficacy are stable. However, important differences between male and female patients deserve more attention in order to direct specific future interventions with these patients.}

CORRESPONDING AUTHOR: Elisa K. de Castro, PhD, Health Sciences Center, Universidade do Vale do Rio dos Sinos, São Leopoldo, 93022-000; elisa.kerndecastro@gmail.com

\section{PSW-134}

\section{CONSISTENCY OF CANCER PATIENTS' PREFERENCE} FOR INFORMATION AND INVOLVEMENT IN TREATMENT DECISIONS - A PILOT STUDY

B. K. Nielsen, MSc, ${ }^{1}$ C. M. Andersen, BSc, ${ }^{1}$ C. L. Ollars, BSc, ${ }^{1}$ M. Y. Mehlsen, $\mathrm{PhD}, \mathrm{MSc}^{2}$ and R. Zachariae, Professor, Dr Med ${ }^{2}$ ${ }^{1}$ University of Aarhus, Aarhus, Denmark and ${ }^{2}$ Aarhus University Hospital, Aarhus, Denmark.

Background: In recent years there has been an increasing emphasis on cancer patients' information needs and involvement in treatment decisions. Patients are seen as active information seekers who require involvement. Objectives: The purpose of this study was to explore whether patients are consistent in their preference for information and involvement in treatment decisions throughout a cancer journey. Methods: Patients $(\mathrm{N}=22$, Mean $=57$ years, 9 men and 13 women) with various cancers, who were in the end of their treatment, participated in semi-structured interviews concerning information needs. Prior to the interview patients were given a brief questionnaire and asked their preference for information given by the clinician (prefer all, only positive or minimal information) and for participating in decision making (prefer to participate or prefer the clinician to make decisions concerning my treatment). After completing the questionnaire patients were interviewed focusing on the patients experiences of information. The interviews were analysed according to the principles of thematic analysis. Results: Eighteen out of 22 patients reported that they wanted all available information and to participate in decisions about treatment. However, during the interviews some patients expressed different attitudes towards level of information and involvement. Some patients experienced changes in treatment and chose to let the clinician 
decide the treatment. There were also divergent statements concerning the amount of desired information, which could be related to the situational aspects of information needs. Furthermore, patients seemed to prefer positive information from clinicians in the early stages of treatment. Conclusions: Even though patients view themselves as individuals who want a high level of information regarding their cancer and want to be involved in treatment decisions, this study reveals some discrepancy between patients beliefs and their behaviours, which can be influenced by various situational factors.

CORRESPONDING AUTHOR: B. K. Nielsen, MSc, Psychooncology, University of Aarhus, Aarhus C, 8000; beritkn@psy.au.dk

\section{PSW-135}

RELIGIOUS COPING IN A SECULAR SOCIETY: A PRELIMINARY QUALITATIVE STUDY OF THE CONTENT VALIDITY OF RCOPE-14

Heidi F. Pedersen, PhD fellow, ${ }^{1}$ Christina G. Pedersen, PhD Fellow, ${ }^{1}$ Anne C. Sinclair, Student ${ }^{1}$ and Robert Zachariae, Professor ${ }^{2}$

${ }^{1}$ Aarhus University, Aarhus, Denmark and ${ }^{2}$ Aarhus University Hospital, Aarhus, Denmark.

Background: It is still unknown, to what extent religious and spiritual coping with stressful events is used by individuals in secular societies such as the Scandinavian, and validated, culture-sensitive instruments are needed. According to a Swedish study, cancer patients do not use religious coping as described by Pargament et. al. (1998) but prefer a more spiritual way of coping with illness.

Aim: To provide preliminary content-validation of RCOPE-14 and a new spiritual coping dimension added to the original instrument in a Danish sample.

Method: RCOPE-14 was translated and moderated to fit Danish culture. A spiritual dimension was added, in case respondents could not identity with the concept "God". The content validity was explored in 3 focus group discussions with 1) a mixed group of non-believers and secular Christians, 2) a group of spiritual believers, and 3) a group of religious believers.

Results: All groups agreed that the spiritual dimension was an essential addition to the instrument. The spiritual dimension was found to address coping in the group of spiritual believers coping very well, and the religious dimension appears to cover coping in the group of religious believers. In contrast, the group of non-believers and secular
Christians were unable to identity with the ways of coping suggested by the instrument - especially the religious dimension, which according to this group is seen as only relevant to highly religious individuals. However, the group of non-believers and secular Christians agreed that faith was important to them, but only in a very individual, non-religious way.

Conclusion: Adding a spiritual dimension to RCOPE seems essential if the instrument is to be applied in Danish research on faith and health. In addition, the development of items relevant to individuals, who do not see themselves as religious or spiritual, should be considered prior to the application of quantitative validation procedures in a larger sample

CORRESPONDING AUTHOR: Heidi F. Pedersen, PhD fellow, Psychooncology Research Unit, Aarhus University, Aarhus C, 8000; hefpe@psy.au.dk

PSW-136

AFRICAN AMERICAN BREAST CANCER PATIENTS' EXPERIENCES OF CANCER CARE DELIVERY

Alba Nino, MS in Marriage and Family Therapy, Karni Kissil, MA, MEd LMFT, Maureen Davey, PhD, LMFT and Carolyn Y. Tubbs, PhD, LMFT

Couple and Family Therapy, Drexel University, Philadelphia, PA.

This qualitative focus group study describes cancer care delivery for 9 African-American breast cancer patients. Three focus groups captured participants' experiences with the diagnosis and treatment of breast cancer and parenting their children. Spontaneous accounts of factors which shaped the quality of their relationships with oncology staff were reported by all participants. Content analysis revealed 5 themes: interaction with physicians, power and authority of physicians, collaboration between physicians and mental health professionals, navigating the system, and involvement of the family in the treatment process. Clinical recommendations to improve the quality of care for this vulnerable racial minority group are provided.

CORRESPONDING AUTHOR: Alba Nino, MS in Marriage and Family Therapy, Couple and Family Therapy, Drexel University, Philadelphia, PA, 19102; aln38@drexel.com 


\section{TOP FIFTEEN SCORING POSTER}

PSW-137

TELEPHONE BASED COGNITIVE BEHAVIORAL STRESS MANAGEMENT

INTERVENTION IMPROVES COPING, QUALITY OF LIFE AND CORTISOL REGULATION IN MEN UNDERGOING HORMONAL TREATMENT (HT)

FOR ADVANCED PROSTATE CANCER (APC)

Frank Penedo, PhD, ${ }^{1}$ Catherine Benedict, BS, ${ }^{1}$ Eric Zhou, MS, ${ }^{1}$ Bruce Kava, MD, ${ }^{2}$ Mark

Soloway, $\mathrm{MD},{ }^{2}$ Mike Antoni, $\mathrm{PhD}^{1}$ and Neil Schneiderman, $\mathrm{PhD}^{1}$

${ }^{1}$ Psychology, University of Miami, Coral Gables, FL and ${ }^{2}$ Urology, University of Miami, Coral

Gables, FL.

Hormonal treatment (HT) for advanced prostate cancer (APC) often results in side effects that can compromise quality of life (QOL). Limited work has evaluated the efficacy of psychosocial interventions among men undergoing treatment for APC. The current study evaluated the effects of a 10-week telephone-delivered and group-based Cognitive Behavioral Stress Management (CBSM) intervention on coping strategies, QOL and cortisol regulation among APC survivors undergoing HT. CBSM strategies included stress awareness, relaxation training, cognitive restructuring, and enhancing coping, social support and communication skills with an emphasis on managing treatment-related compromises in QOL. The HP condition involved delivery of health promotion information (e.g., benefits of proper diet, physical activity) with no therapeutic or interactive component. Participants $(\mathrm{N}=83)$ were randomized to either $\mathrm{CBSM}$ or a health promotion (HP) control condition and were assessed at two time points (T1; baseline, and T2; about 12 weeks post-randomization into the CBSM or HP conditions). The mean age was 70.0 years $(\mathrm{SD}=9.5)$ and the sample was ethnically diverse (66\% White, $18 \%$ Black, $12 \%$ Hispanic, 4\% Other). Participants had undergone an average of 19.1 months ( $\mathrm{SD}=17.4)$ of HT. Measures included the Expanded PC Index Composite (EPIC) for treatment-related symptoms and QOL; the COPE Inventory (COPE); Measure of Current Status (MOCS) for perceived stress management skills and salivary cortisol. Analyses were conducted using repeated-measures group by time ANOVAs, controlling for relevant covariates. Participation in the CBSM intervention condition, relative to the HP control condition, was associated with improvements in disease-specific (i.e., urinary and bowel function) and general QOL, adaptive coping strategies, stress management skills and cortisol regulation (all p's $<.05$ ). Several mediation models were tested where coping and other stress management strategies were hypothesized to mediate the relationship between group condition and QOL. Both greater acceptance $(\mathrm{p}<.05)$ and less denial $(\mathrm{p}<.01)$ coping were found to be significant mediators of intervention-related improvements in QOL but did not mediate the effect of the intervention on cortisol regulation. These results suggest that participation in a telephone based CBSM intervention may be effective in improving general and disease-specific QOL, stress management skills and cortisol regulation.

CORRESPONDING AUTHOR: Frank Penedo, PhD, Dept. of Psychology, University of Miami, Coral Gables, FL, 33124; fpenedo@miami.edu

\section{PSW-138}

\section{CANCER-RELATED PSYCHOLOGICAL SYMPTOMS:} LONGITUDINAL CHANGES OVER AN 18-MONTH PERIOD

Claudia Trudel-Fitzgerald, BA, ${ }^{1,2}$ Josée Savard, $\mathrm{PhD}^{1,2}$ and Hans Ivers, $\mathrm{PhD}^{1,2}$

${ }^{1}$ School of Psychology, Université Laval, Québec, QC, Canada and

${ }^{2}$ Laval University Cancer Research Center, Québec, QC, Canada.

Cross-sectional studies have revealed high rates of depression, anxiety, insomnia, fatigue and pain in cancer patients. However, much less is known about the natural course of these symptoms throughout the cancer treatment trajectory. Also, the contribution of medical factors such as cancer site, stage and treatments in explaining changes in these symptoms has yet to be established. As part of a larger research, this study investigated longitudinal changes of these symptoms in patients scheduled to undergo surgery for cancer $(n=828)$. The patients completed the Hospital Anxiety and Depression Scale, the Insomnia Severity Index, the Multidimensional Fatigue Inventory, and a pain questionnaire developed by our research team at six time points: at baseline (T1), as well as 2 (T2), 6 (T3), 10 (T4), 14 (T5) and 18 (T6) months later. Analyses of variance showed significant reductions of depression, anxiety and insomnia levels over time ( $\mathrm{ps}<.0001)$, which were mostly marked between T1 and T3. Fatigue levels were fairly stable, whereas pain increased slowly but significantly 
over time $(\mathrm{p}<.0001)$. Prostate cancer patients had significantly lower levels of anxiety, insomnia, fatigue and pain ( $\mathrm{ps}<.001$ or less) compared to patients with other types of cancer, but no significant differences were found across cancer stages over time. Patients having received chemotherapy were those displaying the greatest levels of insomnia and fatigue (ps $<.05$ or less), whereas patients treated by surgery only (no radiation or hormone therapy, or chemotherapy) were those displaying the lowest levels of anxiety and insomnia ( $\mathrm{ps}<.001$ or less) over time. In summary, cancer patients reported an overall decrease in depression, anxiety and insomnia symptoms, stable fatigue and an increase in pain over the 18-month period following surgery. Results also revealed some differences across cancer sites and treatment regimens received. Further longitudinal studies are needed in order to identify additional factors associated with changes in cancer-related symptoms over time.

CORRESPONDING AUTHOR: Claudia Trudel-Fitzgerald, BA, Groupe de recherche en psycho-oncologie, Centre de recherche de 1'Hôtel-Dieu de Québec, Quebec, QC, G1R 2 J6; claudia.trudel-fitzgerald.1@ulaval.ca

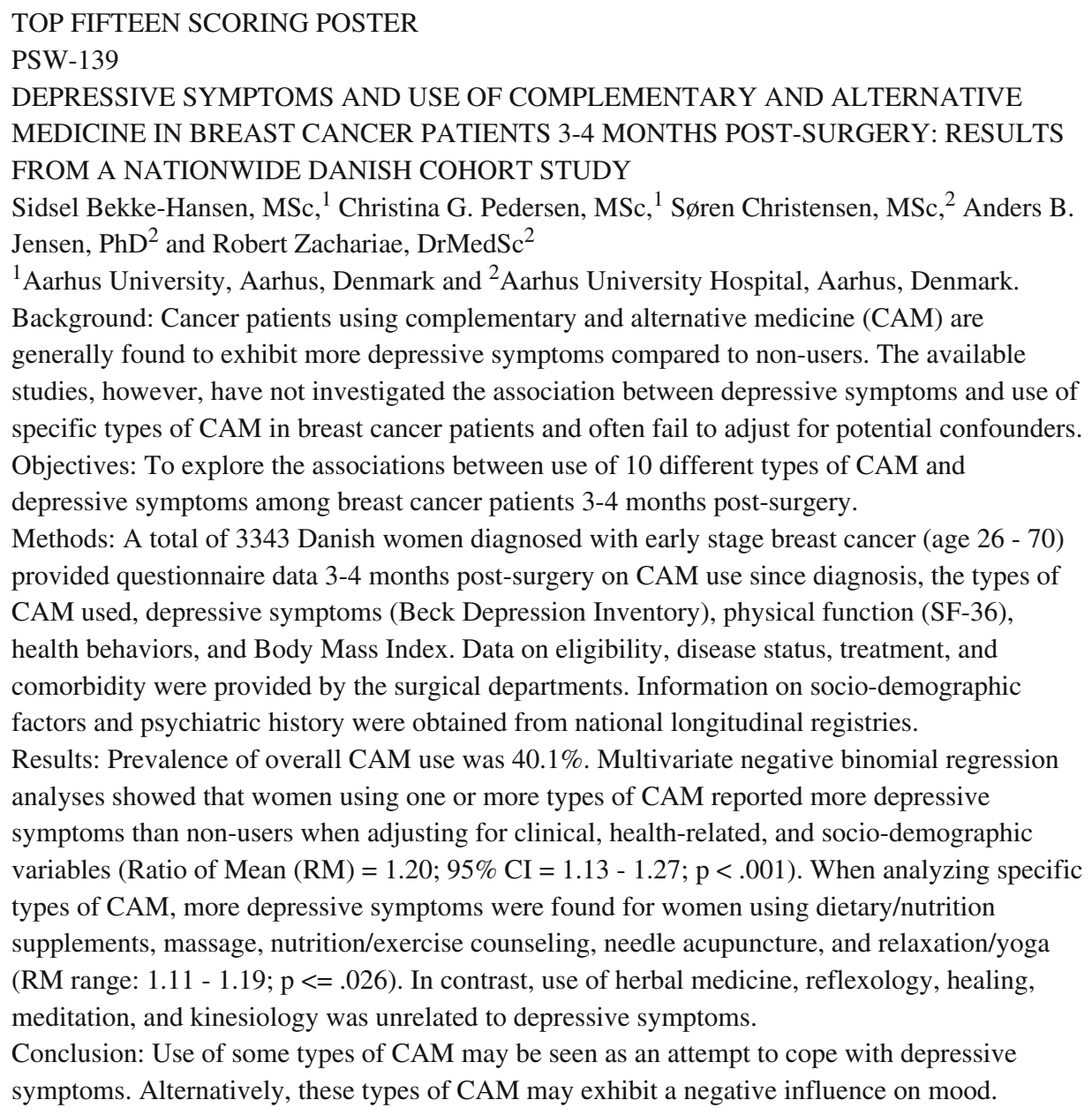

CORRESPONDING AUTHOR: Sidsel Bekke-Hansen, MSc, Psychology, Aarhus University, Aarhus, 8000; sidsel@psy.au.dk

\section{PSW-140}

DIET AND VOLUNTARY EXERCISE AFFECT PERIPHERAL MONOCYTE NUMBERS AND ACTIVITY IN MICE Stephanie Cull, BSc, ${ }^{1,2}$ Rene Figueredo, MSc, ${ }^{2}$ Amy Kossert, MHK, ${ }^{1}$ Harry Prapavessis, $\mathrm{PhD}^{1}$ and James Koropatnick, $\mathrm{PhD}^{1,2}$ ${ }^{1}$ University of Western Ontario, London, ON, Canada and ${ }^{2}$ Lawson Health Research Institute, London, ON, Canada.
Physical activity (PA) and good dietary habits can moderate obesity while enhancing immune status, decreasing cancer risk. Innate immune cells, monocyte/macrophages, respond to and cooperate with other cells and components of the immune system to detect and eliminate abnormal cells.

We report here the effect of physical exercise, with or without a high fat diet, on monocyte/macrophage numbers and activity in mice. Number and function of circulating blood monocytes from C57BL/6 mice were measured after 6 weeks of PA on a voluntary running wheel, compared with sedentary controls (SE). PA and SE groups 
were further subdivided into groups fed a high fat diet (PA-HF and SE-HF) and normal diet (PA-ND and SE-ND). Mice allowed access to a running wheel ran approximately $12 \mathrm{~km} /$ day, and displayed expected decreases in body weight and body fat compared to mice without access to running wheels (SE). Cells were collected from peripheral blood (posterior vena cava) and peritoneum (by lavage). Using flow cytometry, cell numbers (CD11b status), and both basal and chemically-induced activation states (CD11b signal/monocyte) were determined. For HF diet mice, regardless of exercise, the number of circulating monocytes was higher than in similarly-treated ND mice; the fraction of monocytes in the total white blood cell population was $6.4 \%$ vs. $4.6 \%$ (PA-HF and PA-ND, $<<0.001$ ) and $5.9 \%$ vs $4.5 \%$ (SE-HF and SE-ND, $\mathrm{p}<0.001$ ). PA did not affect monocyte numbers in any group, but PA-ND mice had a $20 \%$ higher basal monocyte activity level than PA-HF mice $(p=0.006)$. On the other hand, HF mouse blood monocytes were approximately threefold more responsive to activation compared to sedentary counterparts (SE, $\mathrm{p}=0.023$; PA, $\mathrm{p}=0.002$ ).

Overall, these data indicate that the number of blood monocytes, their basal activity level, and their responsiveness to activation are affected by diet and voluntary PA, and that diet and PA are interdependent in their capacity to affect immune function.

CORRESPONDING AUTHOR: Stephanie Cull, BSc, University of Western Ontario, London, ON, M6A4L6; scull@uwo.ca

\section{PSW-141}

PSYCHOMETRIC PROPERTIES OF THE DUTCH VERSION OF THE MENTAL ADJUSTMENT TO CANCER SCALE IN DUTCH CANCER PATIENTS

Anna Braeken, MSc, ${ }^{1,4}$ G. Kempen, $\mathrm{PhD},{ }^{1}$ M. Watson, $\mathrm{PhD},{ }^{2} \mathrm{~F}$. van Gils, $\mathrm{PhD}, \mathrm{MD}^{3}{ }^{3} \mathrm{R}$. Houben, $\mathrm{MSc}^{3}$ and L. Lechner, $\mathrm{PhD}^{4}$

${ }^{1}$ Health care and nursing science, Maastricht University, Maastricht, Netherlands; ${ }^{2}$ The Royal Marsden NHS Foundation Trust, Psychological Medicine, Surrey, United Kingdom; ${ }^{3}$ Maastricht Radiation Oncology, Maastricht, Netherlands and ${ }^{4}$ Faculty of Psychology, Netherlands Open University, Heerlem, Netherlands.

Background: The measurement of adjustment to cancer is relevant for research purposes and daily practice. Several studies have used the Mental Adjustment to Cancer (MAC) scale to measure adjustment to cancer. Notwithstanding the fact that the MAC scale is considered an adequate tool to measure adjustment responses to cancer, no psychometric analysis has yet been made in Dutch patients. In this study the psychometric properties of the original 5 subscales and the 2 recently proposed summary scales of the MAC scale were examined in Dutch cancer patients treated with radiotherapy.

Methods: Data from 289 cancer patients were assessed with the MAC scale and the Hospital and Anxiety Depression scale (HADS); 259 patients completed the MAC scale for a second time. Results: In total, $85.5 \%$ of the participants completed the full MAC-scale. The internal consistency of the 5 subscales and the summary scales were mostly similar to the original versions. The test-retest reliability of the Fighting Spirit (FS), Helplessness/ Hopelessness (HH), Anxious Preoccupation (AP), Summary
Positive Adjustment (SPA) and Summary Negative Adjustment (SNA) subscales were moderate and the test-retest reliability of the Fatalism (F) and Avoidance (A) subscales were low. Correlations between the original and the 2 summary scales of the MAC scale and the HADS depression and anxiety subscales indicated good convergent validity. The structure of the 5 subscales as well as the structure of the 2 summary scales was adequate as shown by construct validity using confirmatory factor analyses.

Conclusion: The Dutch version of the MAC scale is a feasible questionnaire and appeared to have comparable psychometric properties as demonstrated by studies in the UK. The psychometric properties of SPA and SNA subscales and FS and $\mathrm{HH}$ subscales seem to be acceptable. This supports the cross-national usefulness of the MAC scale.

CORRESPONDING AUTHOR: Anna Braeken, MSc, Health care and nursing science, Maastricht University, Maastricht, 6229 HR; v.braeken@zw.unimaas.nl

PSW-142

PREDICTORS OF LOW-INCOME MOTHERS' INTENTION TO VACCINATE THEIR DAUGHTERS AGAINST HPV

Tiffany Floyd, PhD

Psychology, The City College of New York, New York, NY.

The availability of an efficacious vaccine against human papillomavirus (HPV), the necessary cause of cervical cancer, provides unprecedented opportunity to eliminate income-based disparities in cervical cancer morbidity and mortality. Realization of this potential, however, will depend in part on low-income mothers' decisions to either vaccinate or not vaccinate their daughters against HPV. Using a community-based sample of low-income mothers $(\mathrm{N}=77)$, the present study sought to elucidate psychosocial factors associated with mothers' intentions to vaccinate their age-eligible daughters against HPV. Thirty-five percent of the sample had never heard of HPV. Knowledge about the virus, including its prevalence and relation to cervical cancer, did not relate to mothers' intention to vaccinate their daughters within the next 1 to 2 years; however, intention did relate to attitudes and beliefs about the vaccine. Results from chi-square analyses indicated that intention to vaccinate ones daughter was associated with the belief that the vaccine could prevent future problems for their daughter $(\mathrm{p}<.001)$, wanting to do what their daughter's pediatrician recommends regarding the HPV vaccine $(p=.01)$, and that the vaccine could benefit both men and women $(p=.04)$. Intending to vaccinate ones daughter was also associate with disagreement with the belief that vaccines in general can be dangerous $(p=.05)$ and lower educational attainment $(p=.05)$. Findings provide insight and are discussed in relation to uptake of the HPV vaccine within this high-risk population.

CORRESPONDING AUTHOR: Tiffany Floyd, PhD, Psychology, The City College of New York, New York, NY, 10031; tfloyd@ccny.cuny.edu 
PSW-143

PSYCHOLOGICAL ADJUSTMENT PATTERNS

AND STRATEGIES UTILIZED BY PATIENTS UNDERGOING

HEMATOPOIETIC STEM CELL TRANSPLANTATION:

A MIXED METHODS AND LONGITUDINAL STUDY

OF THE EFFECTS OF AN EXERCISE AND PSYCHOSOCIAL INTERVENTION

Mary Jarden, $\mathrm{PhD},{ }^{1}$ Ellen Boesen, $\mathrm{PhD}^{2}$ and Lis Adamsen, $\mathrm{PhD}^{1}$

${ }^{1}$ University Hospitals Center for Nursing and Care Research, Copenhagen University Hospital, Copenhagen, Denmark and

${ }^{2}$ Institute of Cancer Epidemiology, Danish Cancer Society, Copenhagen, Denmark.

Background: Increasing use of hematopoietic stem cell transplantation (HSCT)and improved survival rates have led to growing concerns regarding the short and long term psychological adjustment of HSCT recipients. Studies applying exercise, relaxation and psychoeducation have each indicated a positive impact on physical performance and/or psychological wellbeing in persons with cancer. These components were incorporated as a multimodal intervention in patients undergoing allogeneic HSCT resulting in physiological and psychological benefits. There is however limited literature about allo-HSCT patients' adjustment process during and after aggressive treatment.

Objective: The aim of the study is to explore the HSCT patients' adjustment strategies and patterns.

Design: 42 adult patients undergoing allo-HSCT were randomized to an intervention or control group. The intervention group $(n=21)$ received standard treatment and care; and a supervised 4-6 week, structured exercise program, progressive relaxation and psychoeducation during hospitalization, $1 \mathrm{hr}$. 5 days/wk. The control group $(n=21)$ received standard treatment, care and physiotherapy. Methods: Semistructured interviews and Mini-Mental Adjustment to Cancer (Mini-MAC) were collected at baseline, post HSCT, and 3 \& 6 months follow up.

Findings: Intergroup analysis of Mini-MAC found no significant differences on adaptation strategies, however, a trend in change score distribution showed a significant decrease in cognitive avoidance for the intervention group at three months, and this trend continued to six months. There were similar intergroup longitudinal patterns revealing that scores at three months were determinants for the adjustment level at six months. Furthermore, improved muscle strength was significantly associated with less cognitive avoidance. Interviews reveal a dynamic adjustment process in both the intervention and control groups where active $\&$ passive adaption strategies and positive \& negative emotions were used to cross over 'transition' periods. Mini-MAC scores reveal greater negative emotion scores in the control group and greater positive emotion scores in the intervention group.

Conclusion: Patients experience both negative \& positive emotions and use active $\&$ passive adaptation strategies across the allo-HSCT treatment trajectory. There were consistent longitudinal adaptation patterns in the intervention and control groups that facilitate the patient through positive and negative transitions. There is indication of adaptation benefits in the intervention group.

CORRESPONDING AUTHOR: Mary Jarden, PhD, UCSF, Copenhagen University Hospital, Copenhagen, 2100; mary@ucsf.dk
PSW-144

CANCER PATIENTS' EXPERIENCES OF A MINDFULNESS BASED STRESS REDUCTION INTERVENTION

Pia Kvillemo, $\mathrm{MSc}^{1}$ and Richard Bränström, $\mathrm{PhD}^{1,2}$

${ }^{1}$ Department of Oncology-Pathology, Karolinska Institute, Stockholm, Sweden and ${ }^{2}$ Osher Center for Integrative Medicine, Karolinska Institutet, Stockholm, Sweden.

There is increasing recognition of mindfulness and mindfulness training as a way to decrease stress and increase psychological functioning. The aim of this study was to examine the perceived effects and experiences of mindfulness stress reduction training as described in semi-structured interviews of the participants $(n=18)$ after the completion of a mindfulness meditation training program. The intervention consisted of an 8-week mindfulness-training course. Participants expressed a number of perceived positive effects of the mindfulness training including increased calm, enhanced sleep quality, more energy, less physical pain, and increased well-being. Some of the participants experienced no effect from the training. Both negative and positive effects of the specific mindfulness training exercises were expressed. Components that were described as of particular importance were the non-judgmental and accepting approach guiding the program and the instructors, the influence of the group process and sharing experiences with patients with similar experiences, and the emphasis on increased awareness of the present moment. The implications for these results on our understanding of the mechanisms behind mindfulness training and positive outcomes are discussed.

CORRESPONDING AUTHOR: Richard Bränström, PhD, Karolinska Institute, New York, NY, 10065; richard.branstrom@ki.se

PSW-145a

PHYSICAL ACTIVITY PREVALENCE AMONG MEN AND WOMEN WITH CANCER IN THE UNITED KINGDOM Clare Stevinson, $\mathrm{PhD},{ }^{1}$ Anne Lydon, $\mathrm{MRes}^{2}$ and $\mathrm{Ziv}$ Amir, $\mathrm{PhD}^{2}$ ${ }^{1}$ School of Sport, Exercise and Health Sciences, Loughborough University, Loughborough, United Kingdom and ${ }^{2}$ School of Nursing, Midwifery and Social Work, University of Manchester, Manchester, United Kingdom.

Despite the evidence on the importance of physical activity for people with cancer, data from other countries indicate that public health guidelines (i.e. $\geq 30$ minutes of moderate/vigorous intensity activity on $\geq 5$ days per week) are only achieved by the minority of this population. PURPOSE: To determine the prevalence of physical activity among adult men and women with cancer in the United Kingdom.

METHOD: As part of a national survey of cancer support group participation, questionnaires including items on physical activity and demographic information were distributed to adult members of 172 support groups by group leaders.

RESULTS: A total of 510 women and 229 men completed questionnaires. The mean number of minutes of moderate/vigorous intensity physical activity performed per week was $74.7 \pm 135.7$. Overall, 395 (52.8\%) participants reported no moderate or vigorous intensity physical activity, while $221(29.5 \%)$ reported some activity but below recommended levels. Only 132 (17.6\%) were meeting public health guidelines. Participants were more likely to be meeting 
guidelines if they were male $(24.5 \%$ vs $14.7 \% ; \chi 2=10.3, \mathrm{df}=1, \mathrm{p}=$ $0.001)$, under 60 years $(22.7 \%$ vs $16.0 \% ; \chi 2=3.996 ; \mathrm{df}=1, \mathrm{p}=0.046)$, lived in the least deprived areas $(21.5 \%$ vs $12.0 \% ; \chi 2=8.795 \mathrm{df}=1, \mathrm{p}=$ $0.003)$, had a post-secondary education ( $22.9 \%$ vs $15.3 \% ; \chi 2=5.563$ $\mathrm{df}=1, \mathrm{p}=0.018)$, and reported good heath $\left(22.6 \%\right.$ vs $10.4 \% ; \chi^{2}=$ $17.993 \mathrm{df}=1, \mathrm{p}<0.001)$. Time since diagnosis, stage of disease, and disease status were not associated with physical activity. Among those not currently meeting guidelines, $59.9 \%$ thought that they ought to be more physically active.

CONCLUSION: Overall levels of physical activity are low among men and women with cancer in the United Kingdom. However, the majority of insufficiently active participants showed awareness of the need to increase their activity, and may be receptive to interventions for promoting physical activity in this population.

CORRESPONDING AUTHOR: Clare Stevinson, PhD, Loughborough University, Loughborough, LE11 3TU; C.D.Stevinson@, lboro.ac.uk

PSW-145b

SYMPTOM COMMUNICATION IN BREAST CANCER: RELATIONSHIPS OF HOLDING BACK AND SELF EFFICACY FOR COMMUNICATION TO SYMPTOMS AND ADJUSTMENT Sara N. Red, BA, ${ }^{1}$ Rebecca A. Shelby, $\mathrm{PhD},{ }^{2}$ Gretchen G. Kimmick, $\mathrm{MD}^{2}$ and Francis J. Keefe, $\mathrm{PhD}^{2}$

${ }^{1}$ Duke University, Durham, NC and ${ }^{2}$ Duke University Medical Center, Durham, NC.

There is growing recognition that symptom communication is important in understanding how patients adjust to cancer and its treatment. This study examined two key aspects of communication: self efficacy for symptom communication and holding back from disclosing cancer related concerns. The sample included postmenopausal breast cancer survivors taking adjuvant hormonal therapy $(\mathrm{N}=35)$. Participants were on average 60.6 years old (SD 7.4), mostly Caucasian (91\%), and over half had at least a college education. Regression analyses were conducted to examine the degree to which self-efficacy for symptom communication and holding back predicted measures of symptoms and adjustment. These analyses controlled for demographic and medical variables that were significantly correlated with outcomes. Results indicated that holding back about discussing cancer related concerns, but not self-efficacy for symptom communication, was a significant predictor of menopausal symptoms, fatigue, intimacy, and functional wellbeing. Specifically, holding back was a significant predictor of physical menopausal symptoms $(\mathrm{t}(30)=4.05, \mathrm{p}=0.000)$, fatigue $(\mathrm{t}(31)=3.47, \mathrm{p}=0.002)$, intimacy $(\mathrm{t}(30)=-2.52, \mathrm{p}=0.017)$, and functional well-being $(\mathrm{t}(31)=-3.77, \mathrm{p}=0.001)$. Patients who reported a greater tendency to hold back from discussing their cancer related concerns had higher levels of menopausal symptoms (such as difficulty sleeping or a decrease in physical strength) and fatigue and lower levels of intimacy and functional well being. Taken together these findings suggest that holding back from disclosing cancerrelated concerns is a significant and consistent predictor of symptoms and adjustment to breast cancer in women taking adjuvant hormonal therapy to prevent recurrence. These findings raise the possibility that interventions designed to address symptom communication might be helpful to those patients who are prone to holding back from discussing their cancer-related concerns.

CORRESPONDING AUTHOR: Sara N. Red, BA, Psychology \& Neuroscience, Duke University, Durham, NC, 27705; sara.red@ duke.edu

PSW-145c

PSYCHOSOCIAL PREDICTORS OF DEPRESSION IN BREAST CANCER SURVIVORS: THE IMPACT OF AGE

Emily A. Meier, MA and Adele S. Rabin, PhD

CSPP, Alliant International University, San Diego, CA.

To date, very little is known about the psychosocial predictors of depression among women with breast cancer (BCA). Even less is known about the psychosocial risk factors for depression across the maturational spectrum. Although premenstrual and postmenstrual women diagnosed with BCA share a substantially elevated risk of developing a major depression; the coping resources, life challenges, and cancer impact may differ markedly for younger and older women. The current study examined coping styles, social support quality, stressful life events, sleep quality, and sexual functioning in 103 women, ages 27 to 69, who had completed treatment for BCA within the previous five years.

Women were sampled from across the U.S. and completed study measures electronically. The mean age of the sample was 46.71 $(\mathrm{SD}=9.88$; median $=46)$. The sample was generally well-educated with $94.6 \%$ of the sample having at least some college education. The majority of respondents were Caucasian (93.2\%) leaving the proportion of minority respondents too small for meaningful analysis. Approximately $71 \%$ were employed either full or part time. The majority were married $(70.9 \%)$ or cohabitating $(4.9 \%)$. Consistent with previous reports, BCA staging and age were inversely and significantly related $(\mathrm{r}=-.225, \mathrm{p}=.024)$ with younger women $(<47$ years) diagnosed with more advanced stage illness. Despite this differential level of life threat, current findings indicate that the emotional impact of BCA is generally equivalent across the maturational spectrum. Both younger and older BCA survivors endorsed significant levels of generalized psychological distress, as measured by the BSI, which was characterized primarily by rumination, anxiety, and hostility. Beck Depression Inventory-2 scores indicated that the sample, on average, experienced mild to moderate levels of depressive symptoms with younger women tending to be more symptomatic than the older women, although the difference did not reach significance $(\mathrm{t}=$ $1.68, \mathrm{p}=.09$ ). Sleep quality was also impaired across the sample with the disruption of sleep deprivation on daily activities more frequently found among younger women $(\chi 2=15.82, \mathrm{p}<.001)$. Data collection is complete and full results will be known well in advance of the conference. The differential experiences, resources, and needs of women struck by BCA at various stages in life are discussed. The identification of age-related risk factors for depression may inform early detection, intervention and prevention efforts.

CORRESPONDING AUTHOR: Adele S. Rabin, PhD, CSPP, Alliant International University, San Diego, CA, 92131; arabin@ alliant.edu 
PSW-145d

THE EFFECTS OF PREFERRED AND NON-PREFERRED SUPPORT ON THE PSYCHOSOCIAL OUTCOME

OF BREAST CANCER PATIENTS

Kimberly Weingart, MA, ${ }^{1}$ Adele S. Rabin, $\mathrm{PhD}^{1}$ and Wayne Bardwell, $\mathrm{PhD}^{2}$

${ }^{1}$ CSPP, Alliant International University, San Diego, CA and ${ }^{2}$ University of California, San Diego, CA.

Spousal support has been shown to influence psychosocial adjustment in breast cancer patients. Recent literature, however, suggests that it is not simply received support that influences psychosocial adjustment but rather the type of support received (i.e., preferred or non-preferred) that may play the most significant role in the psychosocial outcome for women with breast cancer. The primary aim of this study is to better articulate the role of received support and its impact on psychosocial adjustment. We hypothesized that non-preferred support functions as an additional stressor over and above the stress of life threatening illness and, as such, adversely impacts psychosocial adjustment. Conversely, the receipt of preferred support was hypothesized to be positively associated with indicators of healthy psychosocial adjustment.

Preliminary data suggest that there is, in fact, a positive and significant relationship between the frequency with which nonpreferred support is received and levels of stress reported by the recipient $(p=.044)$. Additionally, the data suggest that there is a significant negative correlation between the frequency with which preferred support is received and dysfunctional sexual symptoms associated with cancer $(p=.026)$. Psychoeducational feedback about current levels of preferred and received support was provided to participants in tandem with their primary caregivers with the goal of increasing the probability of receiving higher frequencies of preferred support and lowering the probability of receiving non-preferred support. These data have not yet been analyzed but will be available well in advance of the conference. Findings are discussed in terms of the importance of aligning the social support preferences of the patient with the nature of the support that she receives in order to prevent the burden of additional stress while coping with life threat and to enhance psychosocial adjustment.

CORRESPONDING AUTHOR: Adele S. Rabin, PhD, CSPP, Alliant International University, San Diego, CA, 92131; arabin@ alliant.edu

PSW-145e

RATIONALE, DESIGN AND IMPLEMENTATION OF A STUDY ON PERCEIVED RISK, WORRY AND USE OF OVARIAN CANCER SCREENING

Steven Leadbetter, MS, ${ }^{1}$ Lucy A. Peipins, $\mathrm{PhD},{ }^{1}$ Nikki A. Hawkins, PhD, ${ }^{1}$ Rodriguez L. Juan, MPH, ${ }^{1}$ Lawrence E. Scholl, $\mathrm{MPH},{ }^{2}$ Naomi Freedner, $\mathrm{MPH}^{2}$ and Sharon Hensley Alford, $\mathrm{PhD}^{3}$ ${ }^{1}$ Division of Cancer Prevention and Control, Centers for Disease Control and Prevention, Atlanta, GA; ${ }^{2}$ ICF Macro International, Atlanta, GA and ${ }^{3}$ Henry Ford Health System, Detroit, MI.
We describe the rationale, design, implementation, and sample characteristics of a study on risk perception, worry and use of ovarian cancer screening among women at high, elevated and average risk of ovarian cancer. The key challenges of this study lay in measuring and incorporating affect into the assessment of risk perception and screening behavior, and in finding and recruiting women at high risk of ovarian cancer in the general population. The first challenge was met through developing a scale to access the experience of cancer among friends and relatives. Incorporating both emotion and cognition into a model of perceived risk and screening suggested use of the heuristic processing model and Common-Sense Model of Self-Regulation of Health and Illness as theoretical frameworks. The second challenge led us to conduct the study in an HMO population which provided a defined population setting from which women at high risk could be recruited. The study was composed of a baseline and one-year follow-up survey. From a list of 55,887 managed care enrollees, we conducted a telephone eligibility screening of 20,483 randomly-selected women (screening rate $36.7 \%$ ), with the aim of oversampling women at high risk. Of 16,720 women $(81.6 \%)$ deemed eligible for the study, we selected 3,307 for interview, completing 2,524 interviews (response rate $76.3 \%$ ). A one-year follow-up among 1,910 women (response rate $77.7 \%$ ) who were interviewed at baseline provided information on change in perceived risk and screening behavior. The strengths of this study include a theoretically-based design, a large, racially diverse managed care population, and novel questions designed to assess the experience of cancer among friends or relatives.

CORRESPONDING AUTHOR: Lucy A. Peipins, PhD, Division of Cancer Prevention and Control, Centers for Disease Control and Prevention, Atlanta, GA, 30084; LBP6@CDC.GOV

\section{PSW-145f}

THE IMPACT OF THE CANCER IN JAPANESE CHILDHOOD CANCER PATIENTS AFTER DISCHARGE FROM MEDICAL TREATMENT

Yuko Takei, MA, ${ }^{1}$ Akiko Ogata, $\mathrm{PhD},{ }^{2}$ Miwa Ozawa, $\mathrm{PhD},{ }^{3}$ Atsushi Manabe, $\mathrm{PhD},{ }^{3}$ Hiroshi Moritake, $\mathrm{PhD},{ }^{2}$ Kei Hirai, $\mathrm{PhD}^{4}$ and Shinichi Suzuki, Prof ${ }^{1}$

${ }^{1}$ WASEDA univ., Tokorozawa, Japan; ${ }^{2}$ MIYAZAKI univ., Miyazaki, Japan; ${ }^{3}$ St. Luke's International Hospital, Chuo, Japan and ${ }^{4}$ OSAKA univ., Suita, Japan.

Experience of cancer has been linked to post-traumatic stress disorder for childhood cancer survivors. However, it is also an opportunity for personal growth(Jim \& Jacobsen., 2008). Evaluating the long-term effects of cancer and its treatment, we have to investigate the impact of cancer in life domains. The purpose of this study was to explore the impact of the cancer in Japanese childhood cancer patients after discharge. Subjects were 25 childhood cancer patients attending the pediatric outpatient clinics ( 8 male and 17 female, mean age at survey $=17.0 \pm 3.6$ yrs, mean age at diagnosis $=8.8 \pm 4.1 \mathrm{yrs}$ ). Approximately 18 patients had leukemia and 4 each suffered from malignant lymphoma and bone tumor, respectively. They were asked to participate in a semistructured interview regarding the impact of the cancer after 
discharge from medical treatment. The data was analyzed with the content analysis, chi-square test, and correspondence analysis. A total of 11 attributes were extracted and categorized as follows: changes due to the cancer $(\mathrm{N}=24)$ and perceptions of the cancer $(\mathrm{N}=14)$. The attributes indicated by many of the participants were "having cancer brought my family closer together(N=14)", "I have met various people $(\mathrm{N}=12)$ ", "cancer has been the precious experience of my life $(\mathrm{N}=11)$ ", "I wonder why I got cancer $(\mathrm{N}=9)$ ". Some attributes of positive changes due to the cancer and perceptions of the cancer were statistically significant differences among age. This study identified the important components of the impact of the cancer in Japanese childhood cancer patients after their discharge. Most patients thought that good things have come out of having had cancer. In addition, older patients have more diversified views of the cancer than younger patients because they have had many problems and benefits.

CORRESPONDING AUTHOR: Yuko Takei, MA, WASEDA univ., Tokorozawa, 3591192; yuko-t@akane.waseda.jp

PSW-146

TREATMENT OF ASTHMA IN PUERTO RICAN CHILDREN Elizabeth R. Pulgaron, $\mathrm{PhD},{ }^{1}$ Alan Delamater, $\mathrm{PhD},{ }^{1}$ E. Brooks Applegate, $\mathrm{PhD}{ }^{2}$ Sylvette Nazario, MD ${ }^{3}$ Jesus Casal, MD, ${ }^{3}$ William Rodriguez, $\mathrm{MD},{ }^{3}$ Alfonso Torres-Palacios, $\mathrm{MD}^{3}$ and Adam Wanner, $\mathrm{MD}^{4}$

${ }^{1}$ Pediatrics, University of Miami Miller School of Medicine, Miami, FL; ${ }^{2}$ Educational Leadership, Research and Technology, University of Western Michigan, Kalamazoo, MI; ${ }^{3}$ Internal Medicine, Veterans Affairs Medical Center, San Juan and ${ }^{4}$ Internal Medicine, University of Miami Miller School of Medicine, Miami, FL.

We previously reported high prevalence of asthma (34\% by parent report) in children in Puerto Rico (Nazario et al., 2004). The purpose of this study was to describe the treatment, healthcare utilization, and morbidity of children with asthma in Puerto Rico. This phase of the study involved the administration of a parent interview, spirometry and physician examination, in order to learn more about medical care of children with asthma. Evaluations were conducted in 2001 on a sample of 300 5-10 year-old children enrolled in public schools in San Juan, including both children whose parents reported they had asthma $(n=190)$ and presumed healthy children $(n=110)$. The mean age was 6 years old $(\mathrm{SD}=.9$ years) and $50.2 \%$ were females. Among those with asthma, 32\% were prescribed anti-inflammatory medications, with $49 \%$ receiving only bronchodilators, and $18 \%$ not receiving any asthma medications. Using NHANES criteria, 46\% had mild and 54\% had moderate-severe asthma; of the latter, $13 \%$ of parents reported not having any medication prescriptions for their children. Children with asthma and those without did not differ on environmental risk factors or on forced vital capacity (FVC) measures. However, parents of children with asthma, compared to those without, reported they had significantly more symptoms of asthma in the last 14 days $(\mathrm{p}=.02)$ and missed more school in the last two months due to asthma $(\mathrm{p}=.02)$. Mean number of doctor visits, ED visits, and hospitalizations for asthma in the past year were $2.72,1.64$, and 0.42 , respectively. Forty-one percent of parents of children with asthma reported that they did not have written instructions for taking medications; $96 \%$ were not told to use a peak flow meter; $82 \%$ did not have a written care plan for asthma care at school; and $72 \%$ did not having a written plan for environmental triggers. Of those prescribed nebulizer treatments, $51 \%$ reported not adhering to recommendations. Sixty-seven percent of those who were prescribed medication reported not adhering because of concerns regarding potential side effects. These results indicate that in 2001 children in San Juan were not being optimally treated for asthma. Given the high prevalence and morbidity of asthma in Puerto Rico, there is a need for educational efforts to improve treatment practices.

CORRESPONDING AUTHOR: Elizabeth R. Pulgaron, PhD, Pediatrics, University of Miami Miller School of Medicine, Miami, FL, 33136; epulgaron@med.miami.edu

PSW-147

THE ROLE OF OVERPROTECTION AND PROTECTIVE BUFFERING IN EXPLAINING DISTRESS OF COPD PATIENTS

Gemma A. Maters, MS, ${ }^{1}$ Evelien Snippe, MS, ${ }^{1}$ Johan B. Wempe, $\mathrm{PhD}^{2,3}$ and Robbert Sanderman, $\mathrm{PhD}^{1}$

${ }^{1}$ Health Psychology Section, Department of Health Sciences, University Medical Center Groningen, University of Groningen, Groningen, Netherlands; ${ }^{2}$ Pulmonary Medicine, University Medical Center Groningen, University of Groningen, Groningen, Netherlands and ${ }^{3}$ Center for Rehabilitation, University Medical Center Groningen, University of Groningen, Groningen, Netherlands.

Background/aims: High levels of distress are reported in patients with Chronic Obstructive Pulmonary Disease (COPD). Only a few studies looked into social support as a contributor and suggest that patients' perceptions of support are indicative for their wellbeing. Research in other chronic diseases shows that overprotection (e.g. unnecessary help) and protective buffering (e.g. concealing worries) as reported by partners is often related to distress in patients. We studied overprotection and protective buffering in relation to distress in COPD patients, from a dyadic perspective. Methods: 103 couples were approached at a pulmonary rehabilitation center and an outpatient clinic. The analyses were performed using a sample of the 68 couples who consented (to take part in the study). We assessed distress with the Hopkins Symptom Checklist 25. Patients' and partners' perceptions of partner support were measured with a questionnaire developed by Buunk et al (1996). Data were analysed using bivariate correlations and a stepwise regression analysis.

Results: Mean age of patients was $63.8( \pm 9.5)$ and of partners 62.3 ( \pm 9.7$)$. More male patients participated $(57.4 \%)$ and more patients of the outpatient clinic (55.9\%). Patients were quite distressed $(\mathrm{M}=44.3 \pm 11.4)$. Distress was predicted by patients' perceptions of protective buffering $(\mathrm{R} 2=0.23, \mathrm{p}<0.01)$, partners' report of overprotection $(\mathrm{R} 2=0.10, \mathrm{p}<0.01)$ and differences within a couple in their perception of overprotection $(\mathrm{R} 2=0.08, \mathrm{p}<0.01)$.

Conclusions: Overprotection and protective buffering by partners explain a great deal of the variance in COPD patients' distress. Patient and partner perspectives on partner support, as well as disagreement between the couple, all seem to play an independent role in distress of patients. 
CORRESPONDING AUTHOR: Gemma Maters, Msc, Health Psychology, University Medical Center Groningen, Groningen, 9713 AV; g.a.maters@med.umcg.nl

PSW-148

INTERACTION EFFECT OF SOCIAL SUPPORT

AND COPING STYLES DURING STRESS PROVOCATION: AN EXAMINATION ON CHINESE IMMIGRANTS

Christine Lee, MA, Sonia Suchday, PhD and Judith Wylie-Rosett, EdD

Yeshiva University, New York, NY.

Background: Research has shown that social support and certain coping strategies attenuated cardiovascular reactivity (CVR) to stressful circumstances. This hypothesis has not been tested in an immigrant population. The current study assessed the effects of perceived social support and problem-focused and emotionfocused coping on CVR to stress among Chinese immigrants in the New York City area. Method: First generation Chinese immigrants $(\mathrm{N}=150$; Mean Age $=53$ years old; 86 Females, Average Length of Stay in the United States $=20$ years) were recruited from the New York Downtown Hospital in Chinatown. Following the study description and informed consent procedures, participants completed questionnaires which assessed social support and their use of problem focused and emotion focused coping strategies. Following an 8-minute adaptation period, participants recalled a stress-provoking event related to their immigration experience in a semi-structured interview format. Recovery was monitored for 20 minutes and participants were debriefed. Blood pressure and heart rate were monitored at 2 minute intervals during baseline and recovery, and 1 minute intervals during the interview. Result: Hierarchical multiple regression analysis revealed that the interaction perceived social support and emotion-focused coping is associated with low baseline SBP, $\beta=-.18, \mathrm{t}=-2.19, \mathrm{p}<.03$. However, perceived social support and emotion-focused coping is associated with higher SBP during provocation, $\beta=.23, \mathrm{t}=2.70, \mathrm{p}<.01$. Conclusion: Availability of social support is reassuring when coupled with the use of emotion-focused coping, and when the situation demands low coping resources (as during baseline). However, under conditions of stress where the demand for coping resources is high (e.g., during stress provocation), perceived social support and the use of emotion-focused coping does not help and may actually suggest an increase in CVR to stress. This may be related to the Chinese value of collectivism, which may manifest itself through an unwillingness to burden others with personal needs.

CORRESPONDING AUTHOR: Christine Lee, MA, Yeshiva University, New York, NY, 10075; ysclee@gmail.com

PSW-149

OBESITY MEDIATES THE ASSOCIATION BETWEEN DEPRESSIVE SYMPTOMS AND TRIGLYCERIDE LEVELS IN HEALTHY OLDER ADULTS

Mollie R. Sprung, BS, ${ }^{1}$ Megan M. Hosey, student, ${ }^{1}$ Leslie I. Katzel, $\mathrm{MD}, \mathrm{PhD}^{2,3}$ and Shari R. Waldstein, $\mathrm{PhD}^{1,2}$
${ }^{1}$ Psychology, University of Maryland, Baltimore County, Baltimore, MD; ${ }^{2}$ Medicine, University of Maryland School of Medicine, Baltimore, MD and ${ }^{3}$ Geriatric Research Education and Clinical Center, Baltimore VA Medical Center, Baltimore, MD.

Prospective studies demonstrate relations of subclinical depressive symptoms with enhanced risk for cardiovascular disease, yet little is known about underlying mechanisms. Depressive symptoms are also associated with unhealthy lifestyle behaviors and cardiovascular risk factors. We examined whether lifestyle factors mediated relations of depressive symptoms to cardiovascular risk factors in 205 healthy, community-dwelling older adults ( $55 \%$ male; mean age $=66$ years). Participants underwent a medical history, physical examination, blood chemistries, exercise treadmill testing with maximal oxygen consumption (Vo2max), an oral glucose tolerance test, clinical assessment of blood pressure and body mass index (BMI), completion of the Beck Depression Inventory (BDI), and assessment of smoking status and number of alcoholic drinks consumed per week. Primary outcome variables included systolic blood pressure, diastolic blood pressure, glucose levels at 0 and 120 minutes, waist circumference, triglycerides, and total, HDL, and LDL cholesterol. Potential mediators assessed were number of alcoholic drinks consumed per week, smoking status, Vo2max, and BMI. Multiple regression analyses adjusted for age, education, sex, race, and use of antihypertensives revealed a significant positive association between depression and triglyceride levels $(b=24.2, p=.009)$, waist circumference $(b=9.56$, $\mathrm{p}<.001)$, and BMI $(\mathrm{b}=3.03, \mathrm{p}=.001)$. Subsequent regression models testing for possible mediation indicated that the relation of BDI to triglycerides was mediated by BMI. Thus, in healthy older adults, depressive symptoms are associated with increased body mass, waist circumference, and triglyceride levels, and body mass mediates the relation of depressive symptoms to triglyceride levels. Obesity may be an important mechanism by which depressive symptoms influence cardiovascular risk in older adults.

CORRESPONDING AUTHOR: Mollie R. Sprung, BS, Psychology, University of Maryland, Baltimore County, Baltimore, MD, 21224; mollie.sprung@gmail.com

PSW-150

PSYCHOSOCIAL, COGNITIVE, AND PHYSICAL

FUNCTIONING IN CHRONIC OBSTRUCTIVE PULMONARY DISEASE (COPD) AND IDIOPATHIC PULMONARY

FIBROSIS (IPF)

Kendea N. Oliver, BS, Derek R. Anderson, MA and Charles F. Emery, $\mathrm{PhD}$

Psychology, Ohio State University, Columbus, $\mathrm{OH}$.

Patients with Chronic Obstructive Pulmonary Disease (COPD) and Idiopathic Pulmonary Fibrosis (IPF) display high levels of psychological distress which may be associated with increased mortality, lower functional status, and poor self-care behaviors. The purpose of this study was to characterize the psychosocial, cognitive, physical, and demographic profiles of patients with COPD and IPF with the goal of better understanding similarities and differences among patients with these pulmonary disorders. Patients with COPD $(\mathrm{N}=16$; Female $=5)$ and IPF $(\mathrm{N}=15 ;$ Female $=5)$ were recruited from The Ohio State 
University outpatient pulmonary clinics. Participants completed three visits over the course of twelve months and at each session completed a series of questionnaires and cognitive function tests. At baseline and twelve months, participants completed pulmonary evaluations. IPF patients were significantly older $(M=69.3)$ than COPD patients $(M=$ 58.7) and had better pulmonary functioning (FEV1 \% predicted= $75.7 \%$ in IPF vs. $44.2 \%$ in COPD). All analyses of psychological functioning and cognitive performance were conducted controlling for age. COPD patients indicated significantly more symptoms of depression $(\mathrm{p}<.01$; CES-D) and anxiety $(\mathrm{p}<.05$; BAI) than did IPF patients. COPD patients also reported less social support $(\mathrm{p}<.01$, PSSS). In addition, COPD patients indicated significantly poorer emotional quality of life, as reflected in the Mental Component Score of the SF-36. However, no differences were observed between COPD patients and IPF patients on the Physical Component Score of the SF36 or in symptoms of breathlessness, sleep quality, or cognitive performance. Thus, although perceptions of physical quality of life and breathlessness are similar among COPD and IPF patients, patients with COPD indicate significantly greater symptoms of emotional distress and poorer emotional quality of life than patients with IPF. Heightened distress among patients with COPD is important to consider in treatment of patients. In addition, further evaluation of disease-related processes and biological differences between COPD and IPF patients is needed to understand mechanisms that may explain the observed differences.

CORRESPONDING AUTHOR: Kendea N. Oliver, BS, Psychology, Ohio State University, Columbus, OH, 43215; oliver.231@ buckeyemail.osu.edu

PSW-151

EXPRESSIVE AND SUPPRESSIVE HOSTILITY BEHAVIOR PATTERNS HAVE DIFFERENTIAL INFLUENCES ON CARDIOVASCULAR REACTIVITY AND RECOVERY TO ANGER RECALL TASK

I-Mei Lin, $\mathrm{PhD},{ }^{1,2}$ Chia-Ying Weng, $\mathrm{PhD},{ }^{1}$ Tin-Kwang Lin, $\mathrm{MD}^{3}$ and Chin-Lon Lin, $\mathrm{MD}^{3}$

${ }^{1}$ Department of psychology, National Chung Cheng University, Chiayi, Taiwan; ${ }^{2}$ Department of Psychology, Kaohsiung Medicine University, Kaohsiung, Taiwan and ${ }^{3}$ Department of Internal Medicine, Buddhist Dalin Tzu Chi General Hospital, Chiayi, Taiwan.

Objective: This study investigated a reactivity hypothesis and a vagal brake hypothesis of cardiovascular reactivity and recovery in the expressive and suppressive hostility behavior patterns of coronary artery disease (CAD) patients.

Method: 82 CAD patients (age $=61.50 \pm 10.53 ; 13.40 \%$ were female) participated in this experimental study. Participants were separated into four groups using the Chinese Hostility Inventory_ Short Form: high expressive hostility group, high suppressive hostility group, high expressive with high suppressive hostility group, and control group. Heart rate (HR), systolic blood pressure (SBP), and diastolic blood pressure (DBP) were measured in two-minute intervals under baseline, anger recall task (Time 1, Time 2, Time3), and recovery. Results: After controlling the baseline level to examine reactivity, the results indicated that significant difference between four groups in SBP, DBP and HR; as well an interaction existed among groups and SBP under the anger recall task. Post-hoc analyses indicated that the high expressive hostility group had higher SBP and DBP than the control group in Time 2; the high suppressive hostility group had higher SBP than the control group in Time 3. After controlling the anger level to examine recovery, an interaction existed among groups and SBP, DBP and HR.

Conclusion: This study verified the reactivity hypothesis in the expressive hostility group who had peak SBP and DBP reactivity in the beginning of the anger recall task; however, the suppressive hostility group demonstrated peak SBP reactivity at the end of the anger recall task. This study also extended Proges's vagal brake hypothesis in recovery. Hostility behavior patterns caused different cardiovascular reactivity and recovery that regulated by autonomic nervous system. These reactivity and recovery may predict different psychopathological mechanisms or prognosis pathways in $\mathrm{CAD}$ patients.

CORRESPONDING AUTHOR: I-Mei Lin, PhD, Department of psychology, National Chung Cheng University \& Kaohsiung Medical University, Chiayi, 621; lin1123@gmail.com

PSW-152

EFFECTS OF HEART RATE VARIABILITY BIOFEEDBACK IN CARDIAC PATIENTS

Chao-Fen Chen, MSc, Chia-Ying Weng, PhD, Tin-Kwang Lin, MD, Chin-Lon Lin, MD and Hsin-Ju Chiang, MSc

St. Joesph's Hospital, Yunlin County, Taiwan.

Heart rate variability(HRV) has been related to cardiac mortality and morbidity in cardiac patients. This research is to study whether a 8-week course of interventional program could mediate ANS function in cardiac patients. Participants $(\mathrm{N}=39)$ were randomly assigned to either treatment group consist of eight sessions of cognitive behavior group program, psycho education, breathing retraining and HRV biofeedback(Stress Eraser). The ANS function (SNS) were measured pre-(week 0) and post-(week 8) sessions. HRV biofeedback, ANS function(SNS) were measured by pearson correlation. We found HRV biofeedback and gender has signifi$\operatorname{cant}(\mathrm{r}=., \mathrm{p}<0.05)$. HRV biofeedback and ANS function(SNS) has negative correlation. The interventional program can mediate ANS function and differential in gender.

Key words Heart rate variability; breathing retraining; biofeedback; Stress Eraser.

CORRESPONDING AUTHOR: Chao-Fen Chen, MSc, St. Joesph's Hospital, Yunlin County, 63246; pdsyccf@gmail.com

PSW-153a

CARDIOLOGISTS' BELIEFS ABOUT PSYCHOSOCIAL PROBLEMS FOR PATIENTS WITH IMPLANTABLE CARDIOVERTER DEFIBRILLATORS: A NATIONAL SURVEY Kanako Ichikura, BS, ${ }^{1,3}$ Shiho Matsuoka, BS, ${ }^{1,3}$ Yasuyuki Okumura, $\mathrm{PhD},{ }^{3}$ Mika Kobayashi, MD, $\mathrm{PhD},{ }^{3}$ Shin-ichi Suzuki, $\mathrm{PhD},{ }^{2}$ Kazue Kuwahara, MD, ${ }^{3}$ Hiroto Ito, $\mathrm{PhD},{ }^{3}$ Takashi Noda, $\mathrm{MD},{ }^{4}$ Hiroyuki Yokoyama, MD, ${ }^{4}$ Shiro Kamakura, $\mathrm{MD}^{4}$ and Hiroshi Nonogi, $\mathrm{MD}^{4}$ 
${ }^{1}$ Graduate School of Human Sciences, WASEDA University, Tokorozawa, Japan; ${ }^{2}$ Faculty of Human Sciences, WASEDA University, Tokorozawa, Japan; ${ }^{3}$ National Center of Neurology and Psychiatry, Kodaira, Japan and ${ }^{4}$ National Cerebral and Cardiovascular Center Hospital, Suita, Japan.

Introduction: Implantable cardioverter defibrillators (ICDs) are increasingly used for the prevention of sudden cardiac death in patients with life-threatening ventricular arrhythmias. Most ICD recipients are reported to experience some degree of psychosocial problems. Despite calls by international committees for improving cardiologists' recognition and treatment of psychosocial problems for patients with ICDs, little is known about cardiologists' beliefs for these problems in Asia. The purpose of this study was to identify cardiologists' beliefs about psychosocial problems for ICD recipients in Japan.

Methods: Of all 337 licensed hospitals to implant ICDs in Japan, 145 (40.1\%) participated in our mail survey. The subjects were cardiologists who frequently implanted ICDs in each hospital. The questionnaire consisted of 10 items designed to assess respondents' beliefs about psychosocial problems (i.e., "ICD recipients often have health care issues.") and prior experiences of psychiatric care (i.e., "How often have you prescribed psychotropic medications to patients diagnosed with mental disorders?'). Respondents rated each question on 4-point scales. Results: Most cardiologists (91-96\%) identified "health care issues," "lifestyle issues," and "emotional well-being issues" as concerning factors that could affect the psychosocial problems of ICD recipients. On the other hand, many cardiologists (67-88\%) did not provide mental health care such as pharmacotherapy, psychotherapy, counseling, and listening attentively.

Conclusion: Our results indicate that most cardiologists do not provide mental health care for psychosocial problems of ICD recipients, though they are aware of these problems.

CORRESPONDING AUTHOR: Kanako Ichikura, BS, Graduate School of Human Sciences, WASEDA University, Tokorozawa, 359-1192; ichikura@fuji.waseda.jp

\section{CITATION ABSTRACT}

PSW-154

WEIGHT STATUS AND ASSOCIATED BEHAVIORAL CHARACTERISTICS OF LOWINCOME CHILDREN IN KOREA

Seunghyun Yoo, DrPH, ${ }^{1}$ Hyekyeong Kim, $\mathrm{PhD}^{2}$ and Jungwha Lee, $\mathrm{PhD}^{3}$

${ }^{1}$ Dept. of Health Science \& Services, Seoul National University, Seoul, Republic of Korea;

${ }^{2}$ Health Promotion Research Institute, Korea Association of Health Promotion, Seoul, Republic

of Korea and ${ }^{3}$ Dept. of Preventive Medicine, Northwestern University, Chicago, IL.

Learning objectives

1. Describe the prevalence of abnormal weight among low-income Korean children

2. Explain behaviors associated with abnormal weight status.

Background: A national school health screening program of Korea announces an increase of underweight in school while obesity increases in the country. Low-income children are vulnerable to such discrepancy and subsequent health outcomes. This study is intended to report the prevalence of abnormal weight status and associated behavioral and health characteristics of low-income Korean children.

Methods: A total of 2,033 low-income elementary students were recruited from 106 Community Child Centers in Korea in 2006. Health examination and survey were conducted to detect behavioral characteristics and health conditions. Abnormal weight was determined by using the BMI-for-age and -sex chart of the national School Health Screening Program of Korea. Results: Higher prevalence of underweight $(8.0 \%)$ and overweight/obesity $(18.0 \%)$ is found among low-income elementary children. Differences in eating regularity and variety, smoking, and health education experience are found between abnormal and normal weight children, while previously established factors such as drinking soda and exercise level are not relevant in this study. Underweight children experience higher level of stress with academic performance $(\mathrm{OR}=3.757, \mathrm{p}=0.005)$ than normal weight children, and overweight/obese children are more concerned about their appearance $(\mathrm{OR}=3.881, \mathrm{p}<0.001)$.

Conclusion: These findings highlight the importance of coordinated health promotion programs that integrate school health with services for low-income children. Periodical monitoring and nutritional service should concern preventing and intervening early in order to reduce abnormal weight problems. Research is needed to understand a more comprehensive picture of factors associated with both underweight and overweight/obesity among low-income Korean children. 
CORRESPONDING AUTHOR: Seunghyun Yoo, DrPH, Seoul National University, Seoul, 110-799; syoo@snu.ac.kr

\section{PSW-155}

THE ROLE OF CULTURAL VARIABLE, PERCEIVED BEHAVIORAL CONTROL, AND ATTITUDES ON CHILD MENTAL HEALTH UTILIZATION

Erlanger A. Turner, Psychology (PhD)

Behavioral Psychology, Johns Hopkins University, School of Medicine/Kennedy Krieger Institute, Baltimore, MD.

According to the NIMH (2004), a significant number of children suffer from untreated emotional and behavioral problems. This lack of service use has significant implications considering that mental health $(\mathrm{MH})$ problems during childhood can lead to difficulties later in life. Despite increased efforts to tailor $\mathrm{MH}$ services to the needs of each consumer, ethnocultural disparities in access to and use of these services continue to abound (Yamada \& Brekke, 2008). Findings suggest that ethnic minority populations have a higher unmet need than Non-Hispanic Whites (e.g., Diala et al, 2002). However, efforts to explain disparities in MH services have been limited. Researchers have identified several variables that influence individuals to utilize mental health services such as income, ethnicity, and attitudes (e.g., Cepeda-Benito \& Short, 1998; Turner \& Liew, in press). Obasi \& Leong (2009) hypothesize that it is also important to note that group difference exists, and it is imperative that future research investigate cultural variables (acculturation, traditions, etc.). Previous research has found some ethnic minority groups use coping sources (i.e., friends, family) and practices (i.e., family, social, and religious) that are more consistent with their cultural beliefs instead of those more consistent with traditional MH services (e.g., Harrison et al., 2004; McMiller \& Weisz, 1996). The purpose of the current study is to examine to role of cultural identity, religiosity, perceived behavioral control (PBC), and attitudes on utilization of $\mathrm{MH}$ service among European Americans and non-European Americans. Participants were 150 caregivers ( $89 \%$ female) recruited from the Southwest United States. Mean age of participants was 34.4 years old. Participants completed the following measures a demographic questionnaire, the Parental Attitudes Toward Psychological Services Inventory (PATPSI; Turner, 2006), and selfreported their cultural identity, religiosity, and likelihood of mental health service use. Results indicated that both attitudes and PBC were positively related to utilization, and participants' ratings of cultural identity and religiosity were not correlated with utilization. When examining group differences between European Americans and non-European Americans mean differences were only noted for perceived behavioral control. European Americans reported feeling more control over their ability to seek services if warranted. Finally, both perceived behavioral control and attitudes independently predicted utilization of mental health services.
Implications for future research and applications to families will be discussed.

CORRESPONDING AUTHOR: Erlanger A. Turner, Psychology (PhD), Behavioral Psychology, Kennedy Krieger Institution, Baltimore, MD, 21202; turnerer@kennedykrieger.org

PSW-156

CHILDHOOD HEARING AND ADULT OBESITY

Scott M. Montgomery, PhD Professor, ${ }^{1,3}$ Walter Osika, $\mathrm{PhD},{ }^{2} \mathrm{Ole}$ Brus, $\mathrm{BSc}^{1}$ and $\mathrm{Mel}$ Bartley, $\mathrm{PhD}$ Professor ${ }^{4}$

${ }^{1}$ Clinical Epidemiology and Biostatistics Unit, Örebro University Hospital, Örebro, Sweden; ${ }^{2}$ Stress Research Institute, Stockhom University, Stockholm, Sweden; ${ }^{3}$ Clinical Epidemiology Unit, Department of Medicine, Karolinska Institutet, Stockholm, Sweden and ${ }^{4}$ Department of Epidemiology and Public Health, University College London, London, United Kingdom.

Background: Adult obesity is associated with poorer cognitive function and physical coordination in childhood.

Hypothesis: Hearing impairment in childhood as a marker of childhood function is associated with adult obesity, and is a better marker for neurological function as it is less subject to social confounding and unlikely to influence exercise.

Methods: Among 3288 male and 3527 female members of a longitudinal cohort born in Great Britain in 1970 associations with adult obesity for hearing impairment were assessed. BMI was measured at age 10 years and self-reported at age 34 years. Audiometry was conducted at age 10 years. The dependent variable in logistic regression was minor impairment with bilateral loss as a marker of systemic effects. Adjustment for potential confounding factors included social class, both parents living in the household, maternal education and pubertal development at age 10 years.

Results: Among females, the adjusted odds ratios (95\% CI) for associations observed with hearing impairment at age 10 years were 2.33 (1.36-3.98) for overweight/obesity; and at age 34 years they were $1.71(1.00-2.92)$ for overweight and $2.73(1.58-4.71)$ for obesity. Childhood BMI did not explain the association of childhood hearing impairment with adult BMI. There were no consistent associations among males for hearing impairment and BMI.

Conclusion: The association between bilateral hearing impairment in childhood and subsequent adult-onset obesity might be due to early exposures such as psychosocial stress with chronic activation of glucocorticoid receptors, which may damage hearing through detrimental effects on the central nervous system (i.e. via interactions with estrogen receptors in the cochlea) and also result in obesity.

CORRESPONDING AUTHOR: Walter Osika, PhD, Stress Research Institute, Stockhom University, Stockholm, SE-106 91; walter.osika@stressforskning.su.se 


\section{PSW-157}

SUBSTANCE USE AMONG PORTUGUESE ADOLESCENTS: A NATIONAL STUDY

Mafalda S. Ferreira, PhD Student, Margarida G. Matos, PhD and Celeste Simões, $\mathrm{PhD}$

Technical University of Lisbon, Cruz Quebrada, Portugal.

The purpose of this study was to understand the evolution of substance use, namely tobacco, alcohol and drugs, among Portuguese adolescents.

The data in the study derives from collaborative study with the World Health Organization, integral part of the HBSC study Health Behavior in School-aged Children - developed by the Portuguese team. The sample consists of 17911 adolescents in the 6 th, 8th and 10th grades of the public school system within the average of 14 years old $(\mathrm{SD}=1.81)$. The measure, on which this study is based on, is a self-administration questionnaire, applied in school by teachers and in which some questions regarding substance abuse (tobacco, alcohol and drugs).

The results show a decrease substance use from the study of 2002 to 2006. According to the variants gender, age and social economic status, it shows a bigger user on part of boys in classes of the 10th grade and in lower social economic status, therefore, agreeing with the theoretical results of the literature.

CORRESPONDING AUTHOR: Mafalda S. Ferreira, PhD Student, Tecnhical University of Lisbon, Cruz Quebrada, 1495-688; mafaldaferreira@fmh.utl.pt
TOP FIFTEEN SCORING POSTER
PSW-158
ASSOCIATIONS OF NON-SUPPORTIVE PARENTING BEHAVIORS, CHILD
DEPRESSION, AND SELF-EFFICACY IN CHILDREN WITH TYPE 1 DIABETES
Bridget Armstrong, BA, Eleanor Mackey, $\mathrm{PhD}$ and Randi Streisand, $\mathrm{PhD}$
Children's National Medical Center, Washington, DC, DC.
Parenting involvement is important in children's management of T1D with the quality of involvement being a key factor. For example, negative or critical parenting has been associated with poorer adherence to T1D regimens and worse metabolic control. However, there is little research that has examined the psychosocial impact of non-supportive parenting on youth with T1D. Therefore, the current study examined the relationship of non-supportive parent behaviors and child self-efficacy, hypothesizing child depressive symptoms as a mediator.
Baseline data from an RCT to promote adherence in preadolescents with T1D were used. For the current study, youth self-report was used; including measures of non-supportive parental behavior (Diabetes Family Behavior Checklist), depressive symptoms (Child Depression Inventory; CDI) and self-efficacy for diabetes care (Self-Efficacy for Diabetes Scale).
Participants were 86 youth 8 to 11 years old.
A mediation model was tested (Holmbeck, 2001) controlling for child age, insulin regimen, and ethnicity. None of the demographic variables were significant in the models. More non- supportive parental behavior was associated with higher levels of depressive symptoms $(\mathrm{F}(1,77)=8.058, \mathrm{p}<.01)$. Higher CDI scores were associated with lower self-efficacy $(\mathrm{F}(1,83)=15.316, \mathrm{p}<.0001)$. With all variables entered into the regression equation $(\mathrm{F}(2,69)=7.201, \mathrm{p}=.001)$, the association between non-supportive parental behavior and child self efficacy was no longer significant and was fully mediated by child depressive symptoms, as tested by Sobel's equation $(\mathrm{z}=-2.06)$.
In sum, non-supportive parental behaviors appear to be related to increased child depressive symptoms, which in turn are related to lower self-efficacy for diabetes management. Clinical implications of these findings include encouraging positive parental involvement in children's diabetes management and decreasing negative or critical involvement, as well as assessing and treating depression in youth with T1D in order to improve diabetes self-efficacy and self care.

CORRESPONDING AUTHOR: Bridget Armstrong, BA, Children's National Medical Center, Washington, DC, DC, 20010; barmstro@cnmc.org 
PSW-159

PARENTAL SOCIOECONOMIC POSITION, ADOLESCENT WEIGHT CHANGE AND RISK OF DEVELOPMENT OF OVERWEIGHT

Camilla S. Morgen, Candscientsanpubl, ${ }^{1,3}$ Laust H. Mortensen, $\mathrm{PhD},{ }^{2}$ Mette Rasmussen, $\mathrm{PhD},{ }^{1}$ Anne-Marie N. Andersen, $\mathrm{MD}, \mathrm{PhD}{ }^{2}$ Thorkild I.A. Sørensen, MD, $\mathrm{PhD}^{3}$ and Pernille Due, $\mathrm{MD}^{1}$

${ }^{1}$ Childrens Health, National Institute of Public Health, Copenhagen, Denmark; ${ }^{2}$ Epidemiology, Department of Epidemiology, Faculty of Health Sciences, University of Southern Denmark, Odense, Denmark and ${ }^{3}$ Epidemiology, Institute of Preventive Medicine, Copenhagen University Hospital, Copenhagen, Denmark, Copenhagen, Denmark.

Background: A negative social gradient in overweight among adolescents has been shown in developed countries but few studies have examined whether weight gain and the development of overweight differs among adolescents from different socioeconomic groups in a longitudinal study.

Objective: To identify the possible association between parental socioeconomic position, weight change and the risk of developing overweight among adolescents between the ages 15 to 21 .

Design: Prospective cohort study conducted in Denmark with baseline examination in 1996 and follow-up questionnaire in 2003. A sample of 1,656 adolescents participated in both baseline and follow-up. Of these 1,402 had a body mass index (BMI=weight/ height $2 \mathrm{~kg} / \mathrm{m} 2$ ) below 25 at baseline when adjusted for age and gender according to guidelines from International Obesity Taskforce, and were at risk of developing overweight during the study period. The main outcome measures were change in BMI and development of overweight (from $\mathrm{BMI}<25$ to $\mathrm{BMI}>=25$ ).

Results: Average BMI increased from 21.3 to 22.7 for girls and from 20.6 to 23.6 for boys from age 15 to 21 . An inverse social gradient in overweight was seen for girls at baseline and follow-up and for boys at follow-up. In the full population there was a tendency to a social gradient in the overall increase in BMI for girls, but not for boys. A total of 13.4 percent developed overweight during the follow-up period. Girls of lower socioeconomic position had a higher risk of developing overweight (OR's between 4.72; CI 1.31 to 17.04 and 2.03; CI 1.103.74) when compared to girls of high socioeconomic position. The risk of developing overweight or obesity during the follow-up period was not associated with parental socioeconomic position for boys.

Conclusions: The levels of overweight and obesity among adolescents are high and continue to rise. Results from this study suggest that the social gradient in overweight becomes steeper for girls and emerge for boys in late adolescence (age span 15 to 21 years). Late adolescence seems to be an important window of opportunity in reducing the social inequality in overweight among Danish adolescents.

CORRESPONDING AUTHOR: Camilla S. Morgen, Candscientsanpubl, Childrens Health, National Institute of Public Health, Copenhagen, 1353; csm@niph.dk

\section{CITATION ABSTRACT}

PSW-160

\section{A FAMILY SYSTEMS APPROACH TO UNDERSTANDING SLEEP DISORDERS} IN ADOLESCENTS

Timothy B. Billings, MA, Omer Bangash, MBBS and Linda Berg-Cross, PhD

Clinical Psychology, Howard University, Washington, DC.

Introduction: Adolescent sleep deprivation is an international health crisis affecting learning, high risk behaviors, and mood. Adolescent sleep is impacted by numerous factors; the family sleep environment being one of the most important and most amenable to change. There are currently no valid, reliable measures of family sleep environments. This research developed and validated the Howard Family Sleep Questionnaire (HFSQ). We demonstrated that a) the HFSQ can predict sleep disorders b) gender interacts with family factors to predict the quality, duration and timing of sleep (still analyzing) and c) cultural impacts on the relationship between sleep and family factors (still analyzing).

Methods: The HFSQ, the Pittsburg Sleep Quality Index (PSQI), the Epworth Sleepiness Scale (ESS), the Epworth-Billings Sleepiness scale (EBSS), and questionnaires assessing sleep behaviors were collected from 50 adolescents in various parts of Maryland. An additional 50 adolescents in Karachi, Pakistan were given the test packet.

Results: In the Maryland sample, individuals identified with excessive daytime sleepiness (EDS) either by the ESS or the EBSS scored significantly higher on the HFSQ (ESS t (34) $=-2.509, \mathrm{p}=$ 0.017 ; EBSS $\mathrm{t}(34)=-2.028, \mathrm{p}=.05)$. The HSFQ was significantly correlated with the PSQI total score $(r=.376, p=.05)$ and the PSQI sleep disturbance index $(r=.444, p=.001)$, while the ESS and EBSS scores were not significantly correlated with the HFSQ. Similar analyses on the Pakistan sample is about to begin.

Conclusion: These results suggest that the family sleep environment can significantly predict excessive daytime sleepiness in adolescents. Additionally, the HFSQ gains construct validity by its significant relationship to the PSQI total score (i.e., poor sleepers) and PSQI sleep disturbance index. Hopefully, the HFSQ will be translated and validated in numerous cultures since there is a need for large scale international collaboration on this all important health topic. 
CORRESPONDING AUTHOR: Timothy B. Billings, MA, Clinical Psychology, Howard University, Forest Hill, MD, 21050; tbillin4@gmail.com

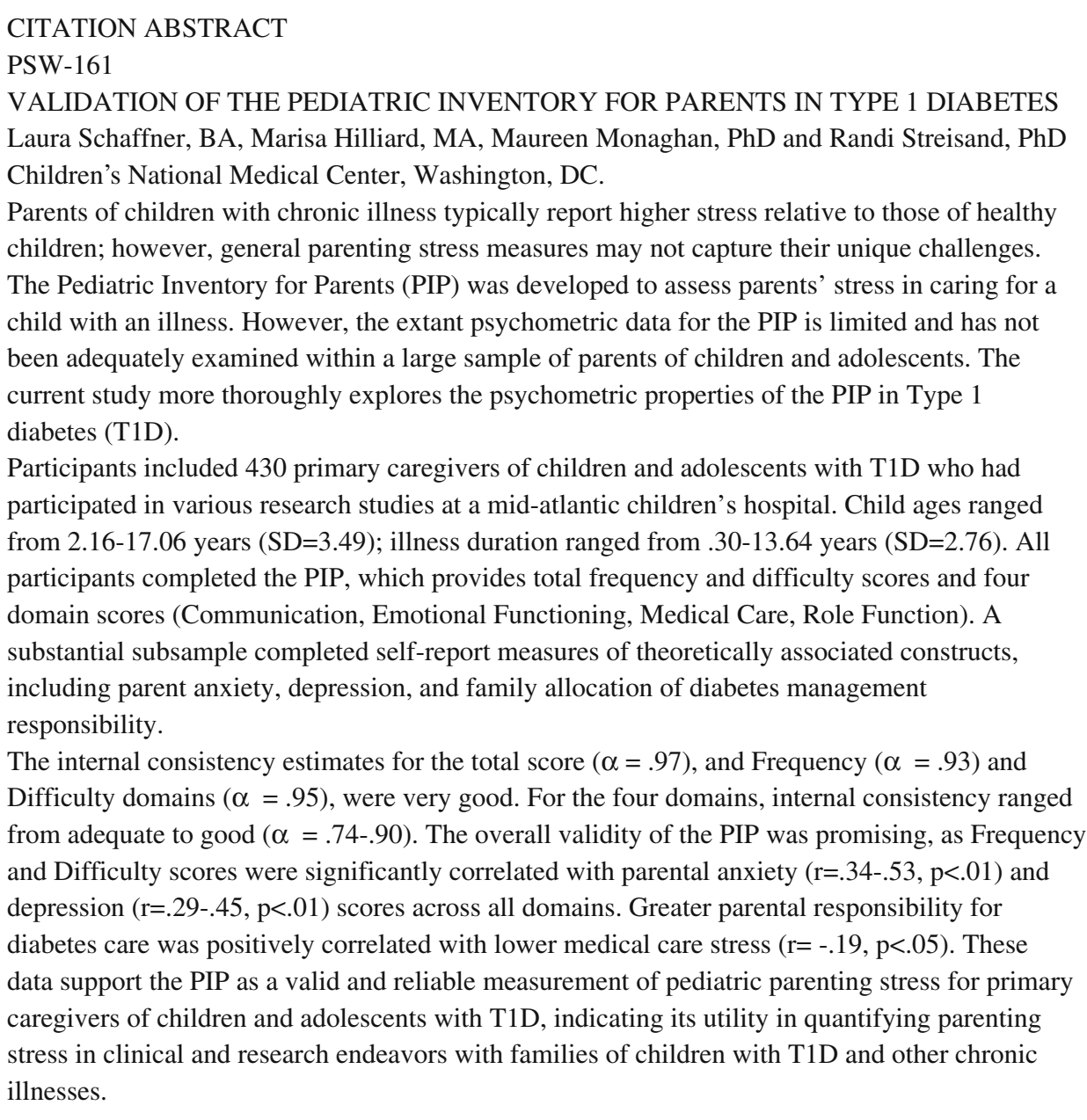

CORRESPONDING AUTHOR: Laura Schaffner, BA, Children's National Medical Center, Washington, DC, 20010; schafflr@ gmail.com

PSW-162

EFFECTS OF PARENTAL CONFLICT ON THE HEALTHRELATED QUALITY OF LIFE OF SPANISH CHILDREN AGED 8-12 YEARS

Mireia Orgilés, $\mathrm{PhD},{ }^{1}$ María D. Samper, $\mathrm{PhD},{ }^{1}$ Tania B. HuedoMedina, $\mathrm{PhD}{ }^{2}$ José A. Piqueras, $\mathrm{PhD}^{1}$ and José $\mathrm{P}$. Espada, $\mathrm{PhD}^{1}$ ${ }^{1}$ MIGUEL HERNÁNDEZ UNIVERSITY, Elche, Spain and ${ }^{2}$ UNIVERSITY OF CONNECTICUT, Storrs, CT.

The objective of this study was to examine the effects of parental conflict on the health-related quality of life in a sample of Spanish children between 8 and 12 years. Participants were 1111 children with a mean age of 10,13 years $(\mathrm{SD}=1,29), 50,3 \%$ boys and $49,7 \%$ girls. The level of parental conflict was assessed by a fivepoint scale, in which children estimated the current relationship between his/her parents as very good, good, fair, poor, very poor. Quality of life was evaluated using the Child Health and Illness Profile-Child Edition that examines the children perceived health in five dimensions: satisfaction (with self and health), comfort (emotional and physical symptoms and limitations), resilience (positive activities that promote health), risk avoidance (risky behaviors that influence future health) and achievement (social expectations in school and with peers). Results show a positive and significant relation in all dimensions, indicating that the more conflictive is the relationship between parents, the worse the quality of life (satisfaction: $\mathrm{F}=26.52, \mathrm{p}=.00$; comfort: $\mathrm{F}=8.44$, 
$\mathrm{p}=.00$; resilience: $\mathrm{F}=30.45, \mathrm{p}=.00 ;$ achievement: $\mathrm{F}=16.39$, $\mathrm{p}=.00$; risk avoidance: $\mathrm{F}=13.77, \mathrm{p}=.00$ ). Post hoc comparisons showed that significant differences were found between children who perceived the relationship between parents very good and those that considered it as good, fair, poor and very poor. However, there were no significant differences between the other groups. The results highlight the importance of the child's perception of a good family atmosphere and the absence of parental conflict for optimal health-related quality of life.

CORRESPONDING AUTHOR: Mireia Orgilés, PhD, MIGUEL HERNÁNDEZ UNIVERSITY, Elche, 03202; morgiles@umh.es

\section{PSW-163}

\section{EXAMINING THE RELATIONSHIP BETWEEN}

SOMATIZATION AND PSYCHOSOCIAL PROBLEMS

IN A PEDIATRIC EMERGENCY DEPARTMENT

Zorash Montaño, BA, ${ }^{1}$ Neda Safvati, MA, ${ }^{1}$ Ilene Claudius, MD, ${ }^{1,2}$ Alan L. Nager, MD, MHA ${ }^{1,2}$ and Jeffrey I. Gold, $\mathrm{PhD}^{1,2}$

${ }^{1}$ Childrens Hospital Los Angeles, Los Angeles, CA and ${ }^{2}$ Keck School of Medicine, University of Southern California, Los Angeles, CA.

Background: Studies have shown that children and adolescents who somatize are at high risk for presenting with underlying psychosocial problems. Objective: To investigate the relationship between somatization symptoms and psychosocial problems in children and adolescents receiving treatment in a pediatric Emergency Department (ED). Method: Three-hundred and fiftyeight patients ( $48.6 \%$ male, $51.4 \%$ female) were recruited from the ED at Childrens Hospital Los Angeles. Patients ranged from 8 to 18 years of age $(\mathrm{M}=12.8, \mathrm{SD}=2.9)$. Investigators conducted an anonymous, cross-sectional, multi-informant (child and caregiver) study. Pearson's product moment correlation coefficient was used to analyze relationships between somatization (pain/weakness, gastrointestinal, and pseudoneurological symptoms) and psychosocial problems (attention, internalizing, externalizing, and total psychosocial problems). Results: Pain/weakness symptoms were significantly correlated with attention $(\mathrm{r}=.292, \mathrm{p}<.01)$, externalizing $(\mathrm{r}=.242, \mathrm{p}<.001)$, internalizing $(\mathrm{r}=.383, \mathrm{p}<.001)$, and total psychosocial problems $(\mathrm{r}=.452, \mathrm{p}<.001)$. Gastrointestinal symptoms also had a significant positive correlation with attention $(\mathrm{r}=.304, \mathrm{p}<.001)$, externalizing $(\mathrm{r}=.242, \mathrm{p}<.001)$, internalizing $(\mathrm{r}=.383, \mathrm{p}<.001)$, and total psychosocial problems $(\mathrm{r}=.452$, $\mathrm{p}<.001)$. Pesudoneurological symptoms also had a positive significant relationship with attention $(\mathrm{r}=.357, \mathrm{p}<.001)$, externalizing $(\mathrm{r}=.211, \mathrm{p}<.001)$, internalizing $(\mathrm{r}=.408, \mathrm{p}<.001)$, and total psychosocial problems $(\mathrm{r}=.455, \mathrm{p}<.001)$. Conclusion: All measures of somatization were associated with psychosocial problems. This demonstrates the importance of screening children and adolescents for somatization symptoms. The pediatric ED can be a useful setting to screen for somatization in order to identify children and adolescent who need referral for mental health assessment and treatment.

CORRESPONDING AUTHOR: Jeffrey I. Gold, PhD, Anesthesiology and Critical Care Medicine, Childrens Hospital Los Angeles, Los Angeles, CA, 90027; jgold@chla.usc.edu

PSW-164

EXPRESSED EMOTION AND ATTRIBUTIONS OF CONTROL IN AN ADOLESCENT OVERWEIGHT POPULATION

Michal Munk, Bachelor of Arts, ${ }^{1}$ Katharine L. Loeb, $\mathrm{PhD}^{1,2}$ and Daniel Le Grange, $\mathrm{PhD}^{3}$

${ }^{1}$ Clinical Psychology, Fairleigh Dickinson University, New York, NY; ${ }^{2}$ Psychiatry, Mount Sinai School of Medicine, New York, NY and ${ }^{3}$ Psychiatry, University of Chicago, Chicago, IL.

Objective: To assess parental attributions of internal locus of control of adolescent obesity as a predictor of Expressed Emotion (EE), specifically Criticism, in parents of obese adolescents.

Background: EE is a measure of family attitudes and feelings towards a symptomatic family member. One theory regarding EE, particularly the criticism facet, is that it stems from attributions of internal control to the afflicted family member. Significant correlations between EE and misattributions have been found in a number of disorders including schizophrenia, anxiety disorders and learning disabilities suggesting that correcting misattributions may in turn modify levels of EE, a positive predictor of relapse rates and a negative predictor of treatment compliance.

Methods: Thirty four parent-adolescent dyads participating in a treatment study targeting adolescent overweight and obesity at Mount Sinai School of Medicine and at the University of Chicago participated in this study. Parents completed the Family Questionnaire to assess EE, and the Multidimensional Health Locus of Control Scale to assess attributions of their child's obesity. Data were collected at baseline assessment.

Results: Linear regression analyses with parental BMI as a covariate indicated that parental internal locus of control regarding adolescent obesity was significant (beta $=-.417, \mathrm{p}=.039$ ) as a unique negative predictor of the criticism factor of EE.

Discussion: Contrary to our hypothesis, increased attributions of obesity to a locus of control internal to the child were associated with reduced parental criticism. Though contradictory to literature on attributions and EE in other disorders and conditions, our findings are consistent with data on parental obesity indicating that overweight parents tend to be less alarmed regarding their overweight child. Notably, the majority $(92.6 \%)$ of parents in our sample were overweight or obese. Given the concordance of overweight or obese status between parents and offspring in our 
sample, our findings may be attributed to parental identification leading to reduced negative expressed emotion.

CORRESPONDING AUTHOR: Michal Munk, Bachelor of Arts, Clinical Psychology, Fairleigh Dickinson University, New York, NY, 10033; MichalMunk@gmail.com

\section{PSW-165}

\section{PREDICTORS OF PSYCHOSOMATIC COMPLAINTS} FOR JAPANESE STUDENTS OF HIGH SCHOOL WITH CORRESPONDENCE COURSES

Yumiko Sakata, $\mathrm{PhD},{ }^{1}$ Ryuta Kanemaru, MA, ${ }^{2}$ Yuriko Takata, $\mathrm{PhD}^{1}$ and Akemi Masuda, $\mathrm{MA}^{3}$

${ }^{1}$ University of Tsukuba, Tsukuba, Japan; ${ }^{2}$ Ibaraki University, Mito, Japan and ${ }^{3}$ Junior College, University of Shizuoka, Shizuoka, Japan.

Introduction: In Japan, we have a unique system of high school education, which is called "Tsushinsei". It means a high school with correspondence courses, and many students enter the school not because of the economical issue but the interpersonal issue. Since most of full-time high schools in Japan make much account of attendance in junior high school for admission, many students who have experienced school refusal select a school with correspondence courses. Thus, the schools have a lot of sensitive students who are easy to have psychosomatic problems, and the teachers need to pay attention to students' early psychosomatic complaints in order to prevent the bigger educational problems such as dropout. This is the critical issue in Japanese "Tsushinsei" high schools with correspondence courses.

Objectives: To clarify the factors affect students' subjective symptoms in high schools with correspondence courses.

Method: The subjects were 836 freshmen in a typical public high school with correspondence courses in Japan, who were surveyed over a 7-year period from 2003 to 2009. The survey was comprised of the Rosenberg Self-Esteem Scale (RSE), TakataSakata Psychosomatic Complaints Scale (PCS) and basic attributes. RSE score was a dependent variable and other scores were independent variables.

Result: Multiple regression analysis revealed bad eating habits (missing breakfast, drinking alcohol, unbalanced diet), bad body image, subjective health state in junior high school period, sex and RSE score were significant predictors of psychosomatic complaints $(\mathrm{R} 2=.579, \mathrm{p}<.001)$.

Conclusion: To prevent severe health and educational problems in high schools with correspondence courses in Japan, we must find early psychosomatic complaints of students and take an appropriate intervention. Paying attention to their eating habits, body image, former subjective health state, sex and self esteem promotes the intervention.

CORRESPONDING AUTHOR: Ryuta Kanemaru, MA, Education, Ibaraki University, Mito, 310-0035; kanemaru@mopera.net
PSW-166

MENSTRUAL-RELATED ATTITUDES AND SYMPTOMS AMONG MULTI-RACIAL ASIAN ADOLESCENT FEMALES

Li-Ping Wong, MSc, $\mathrm{PhD}^{1}$ and Ee-Ming Khoo, MBBS, FAFPM, FAMM, MRCGP ${ }^{2}$

${ }^{1}$ Medical Education \& Research Development Unit, Faculty of Medicine, University of Malaya, Kuala Lumpur, Malaysia and ${ }^{2}$ Department of Primary Care Medicine, Faculty of Medicine, University of Malaya, Kuala Lumpur, Malaysia.

Background: Understanding of factors associated to attitude toward menstruation, and implications of menstrual symptoms on emotional and physical well-being is fundamental to the promotion of adolescents' menstrual health.

Method: This cross-sectional study included 1092 adolescent females from 94 schools in Federal Territory, Kuala Lumpur, Malaysia. This study examined 1) attitudes towards menstruation, 2) symptoms experienced in menstruation, 3) awareness of PMS, and its impact and treatment seeking, 4) information needs.

Results: The results of research show that the attitude mean score was only $2.80(\mathrm{SD} \pm 1.88)$ out of six, $80.7 \%$ and $83.6 \%$ of participants experienced one or more affective and somatic symptoms respectively in premenstrual phase. Irritability, mood swing and tension were the three most frequently reported affective symptoms, while fatigue and menstrual cramps were highly prevalent somatic symptoms in both premenstrual and menstrual phase. Effect on functional impairment and quality of life, in order of importance, include poor class concentration, restriction of social and recreational activities, difficult to mingle with friends, and poor class performance. Despite the evident impact, only $10.3 \%$ consult physician for PMS symptoms, while one-third do nothing about their conditions. There were ethnic differences in treatment seeking for PMS.

Conclusion: The study calls for a menstrual-related educational program to provide information and support, help adolescent cope better with menstrual-related problems, and encourage positive attitudes to menstruation.

CORRESPONDING AUTHOR: Li-Ping Wong, MSc, PhD, Medical Education \& Researc Development Unit, University of Malaya, Kuala Lumpur, 50603; wonglp@ummc.edu.my

PSW-167

VISUAL-MOTOR FUNCTIONING IN CHILDREN FOLLOWING REPAIR OF VENTRICULAR SEPTAL DEFECT IN INFANCY

Julie Simons, PhD and Christian Pizarro, MD

Nemours Cardiac Center, A.I. duPont Hospital for Children, Wilmington, DE.

Studies of children with severe, complex congenital heart disease reveal poorer neurodevelopmental outcome than the general population. Results of a recent meta-analysis revealed that perceptual-organizational abilities and visual-spatial functioning 
are domains of particular vulnerability. There have also been several reports of lower visual-motor drawing ability among children with complex congenital heart disease. Multiple medical and surgical variables have been recognized as impacting neurodevelopmental outcome for these children. Very few studies have specifically examined neurodevelopmental outcome for children with ventricular septal defects (VSDs), which are among the most common congenital heart problems with a rate of approximately one out of 500 births. This population is generally expected to have excellent medical outcome and typical development. As part of a larger study examining mid-term neurodevelopmental outcome for children VSDs surgically repaired in infancy, visualmotor ability was assessed among 31 children ages 3 to 16 years with a commonly used graphomotor drawing task. Whereas many areas of cognitive functioning, including full-scale IQ $(\mathrm{M}=95.6$, $\mathrm{SD}=15.3, \mathrm{p}=.12)$ were comparable to the general population $(\mathrm{M}=$ $100, \mathrm{SD}=15)$, visual-motor ability was an area of weakness $(\mathrm{M}=$ $87.9, \mathrm{SD}=14.2, \mathrm{p}<.001)$. Additional results showed that household income and the presence of a comorbid medical condition were significant predictors of visual-motor ability. Specifically, lower income was associated with lower visual-motor scores $(p=.007)$. The presence of a comorbid medical condition was also associated with lower visual-motor ability $(p=.019)$. Twenty-two additional patient and surgical factors were also examined, with no other variables statistically related to visual-motor ability. This study shows that the weaknesses in visual-motor ability observed among children with more complex, cyanotic congenital heart disease are also found among children with repaired VSD. Furthermore, surgical factors do not appear to affect visualmotor ability among children with VSD.

CORRESPONDING AUTHOR: Julie Simons, PhD, A.I. duPont Hospital for Children, Wilmington, DE, 19803; jsimons@ nemours.org

PSW-168

ADOLESCENT GIRLS ARE MORE BODY DISSATISFIED AND USED MORE COPING STRATEGIES IN STRESSFUL SITUATIONS

Gisele Straatmann, Master and Sebastião S. Almeida, doctor Psychology and Education, University of São Paulo, Ribeirão Preto, Brazil.

This research aimed to investigate aspects of body image, stress and what coping strategies are used by adolescents in a stressful situation that involves body discomfort. A sample of 166 girls and 47 boys, aged 15-18 years, was recruited from public high schools in Ribeirão Preto, state of São Paulo, Brazil. The main outcome measures used were Figure Rating Scale, Lipp Stress Symptoms Inventory and The Ways of Coping Questionnaire. Descriptive analyses were performed. Correlational analyses tested the associations of body image, stress and coping with sex and BMI. Logistic regression analyses were conducted for each of the 8 coping strategies. The confidence intervals used were $95 \%$. The results revealed that both boys and girls were dissatisfied with their body size and the majority overestimated it. The exception was the overweight adolescents that had significantly negative body distortion. Girls showed more body distortion than boys. The correlation between body dissatisfaction and body distortion was negative and moderate $(\mathrm{r}=-.65)$. A large proportion $(55,86 \%)$ of adolescents are found to have significant stress symptoms. A greater proportion of females $(61,40 \%)$ than males $(36,20 \%)$ are found to have stress. Adolescents with stress had more probability using confrontive coping $(\mathrm{OR}=3,14)$, distancing $(\mathrm{OR}=2,09)$, seeking social support $(\mathrm{OR}=3,33)$, self-controlling $(\mathrm{OR}=2,06)$, accepting responsibility $(\mathrm{OR}=4,40)$ and escape-avoidance $(\mathrm{OR}=3,17)$ in a situation that involves body discomfort. Girls significantly employed more self-controlling than boys. In conclusion, negative body image perception and stress were observed in the present sample. Adolescents with stress used more coping strategies.

CORRESPONDING AUTHOR: Gisele Straatmann, Master, Psychology and Education, University of São Paulo, Campinas, 13100-040; giselestr@gmail.com

PSW-169

EFFECTS OF REVISED LIFE ANALYTIC COUNSELING ON PSYCHOSOMATIC COMPLAINTS AND SELF-ESTEEM IN JAPANESE JUNIOR HIGH SCHOOL STUDENTS

Yuriko Takata, $\mathrm{PhD}^{1}$, Yumiko Sakata, $\mathrm{PhD}^{1}$ and Ryuta Kanemaru, $\mathrm{MA}^{2}$ ${ }^{1}$ University of Tsukuba, Tsukuba, Japan and ${ }^{2}$ Ibaraki University, Japan.

Purpose: To clarify the efficacy of the revised life analytic counseling (LAC-R), a set of support skills that aims to teach students self health-care behaviors, on psychosomatic complaints and self-esteem in Japanese junior high school students.

Method: A questionnaire was administered from September 2008 in October the first grade for the third grade of 30 public junior high schools in X Prefecture. The school nurse teachers at each school requested the cooperation of classroom teachers in the study. The questionnaires were distributed and collected by the classroom teachers. The purpose of the study was explained orally at the time of questionnaire administration and informed consent was obtained from students.

LAC-R was then carried out for 3 months in 253 students. After LAC-R, the same questionnaire was administered and the differences in student responses before and after the intervention were analyzed. Using the psychosomatic complaint scale score as a dependent variable and grade level as an independent variable, ANOVA by time (pre- and post-intervention) was performed.

Results: Data for 4367 boys and 4083 girls were analyzed. Significant differences in psychosomatic complaints and selfesteem scale score were observed between boys and girls $(\mathrm{p}<.001)$ and among different grade levels $(\mathrm{p}<.001)$. Moreover, for selfesteem scale score, the interaction between gender and grade level was significant $(\mathrm{p}=.003)$.

Girls reported more psychosomatic complaints than boys, particularly in the higher grades. In addition, the self-esteem scale score for girls was lower than that for boys and decreased with 
increasing grade level. LAC-R was completed by 114 boys and 139 girls. In boys, self-esteem scale score increased after the LAC$\mathrm{R}$ intervention $(\mathrm{p}=.041)$

Conclusion: A 3-month LAC-R intervention increased self-esteem in junior high school boys.

CORRESPONDING AUTHOR: Yumiko Sakata, PhD, University of Tsukuba, Tsukuba, 305-8575; sakata@md.tsukuba.ac.jp

\section{PSW-170}

\section{EFFECTIVENESS OF PARENT TRAINING FOR PARENTS} OF CHILDREN WITH DEVELOPMENTAL DISORDER

Masahiko Inoue, PhD, ${ }^{1}$ Naho Inoue, EdD, ${ }^{1}$ Kazuyo Nomura, EdD ${ }^{1}$ Hideyuki Haraguchi, EdD ${ }^{2}$ and Kosaku Ohno, $\mathrm{PhD}$ in Medicine $^{1}$

${ }^{1}$ Tottori University, Yonago City, Japan and ${ }^{2}$ Tukuba University, Tukuba City, Japan.

This study used parent training (PT) for parents of children with Developmental Disorder. The usefulness of PT was evaluated parent's general health questionnaire (GHQ30 Japanese version) and the number of tasks achieved by the parents at their home setting. Total participants were 141 parents during five years. Each children were diagnosed as autism, asperger syndrome, ADHD or mental retardation. The five training components of the program were: lectures about behavior analytic theory, role playing, group discussion, homework and recording of behaviors about tasks, and feedback to parents from the training staff based on their recordkeeping. We carried out a lecture of applied behavior analysis in the first half of the each session and carried out group work in the latter half. The average scores of GHQ30 were improved. The change was significant. Regarding the behavioral modification of the children, 42.6-64.9\% task achievement rate was obtained.

CORRESPONDING AUTHOR: Masahiko Inoue, PhD, Tottori University, Yonago City, 683-0851; masahiko-inoue@med.tottori-u. ac.jp

PSW-171

CAREER INDECISION AND EGO IDENTITY

Mitsuki Niregi, $\mathrm{PhD},{ }^{1}$ Satoshi Nagai, $\mathrm{PhD},{ }^{1}$ Noriko Nishimatsu, MD, ${ }^{1}$ Hatsue Numa, MA, ${ }^{1}$ Junko Okamoto, MA, ${ }^{1}$ Seiichi Okuno, MA, ${ }^{2}$ Hideyuki Soto, MA, ${ }^{1}$ Kouji Shimoyama, MA, ${ }^{1}$ Hanae Tamura, $\mathrm{MA}^{1}$ and Yoko Sawamiya, $\mathrm{PhD}^{1}$

${ }^{1}$ Clinical Psychology, Rissho University, Tokyo, Japan and ${ }^{2}$ Yamagata University, Yamagata, Japan.

The recent increase in the numbers of NEET young people (not in education, employment or training) is socially anxious problem in the many countries of the world. The purpose of the present study was to investigate the relationship between career indecision and the factors of ego identity. 4 types of mental situations (confusion, anxiety, hesitation and prudence) were constructed by the career indecision scales and 5 factors of adolescent sub-identity(group identity, individual identity, sex role identity, occupational identity and social identity) were constructed by the identity scales A questionnaire was administered to 408 university students, 182 males and 226 females in Japan. The findings by using multiple regression analysis showed that the total of career indecision were greatly influenced by the achievement of total ego identity (R square,.51). The confusion type of career indecision was closer relationship with the individual identity $(\beta,-.352 \mathrm{p}<.01)$. The anxiety types of career indecision was related to sex role identity $(\beta,-.312 \mathrm{p}<.01)$. The scales of occupational identity showed high relationship with total scores of the career indecision $(\beta,-.402$ $\mathrm{p}<.01)$ in male students, although the relationship between the occupational identity and the career indecision in female students was $\operatorname{low}(\beta,-.214 \mathrm{p}<.01)$.

CORRESPONDING AUTHOR: Mitsuki Niregi, PhD, Rissho University, Utsunomiya, 320-0867; mnniregi@yahoo.co.jp

PSW-172

APPLICATION OF GROUP CONTINGENCY

TO HIGH SCHOOL STUDENTS WITH DEVELOPMENTAL DISABILITIES

Kazusa Wakabayashi, master's degree ${ }^{1}$ and Tetusbumi Kato, doctorate degree ${ }^{2}$

${ }^{1}$ The joint graduate school in science of school education, Hyogo university of teacher education, Simokume, Kato, Japan and ${ }^{2}$ Joetsu university of Education, Joetsu, Japan.

In this study, we examined the effectveness of independent and interdependent group contingency to apply to the academic performance of students with developmental disorders enrolled in high school classes. In addition, the study also empirically addressed side effects with regard independent and interdependent group contingency.

As a result, the application of independent and interdependent group contingency was effective in the overall occurrence of academic performance, including students with developmental disorders. On the other hand, when independent group contingency was applyed in writing test scene, some of students gave up the occurrence of academic behavior achieving the criteria to be reinforced. In addition, in the group learning situation, many students were found sabotage behavior after they achieve the criteria. We discussed the way of improbement on these issues and procedure to express positive side effect on group contingency.

CORRESPONDING AUTHOR: Kazusa Wakabayashi, master's degree, The joint graduate school in science of school education, Hyogo university of teacher education, Simokume, Kato, 6731494; j195175m@gmail.com

PSW-173

THE RELATIONSHIP BETWEEN THE TWICE-APPLICATION METHOD OF THE TREE-DRAWING TECHNIQUE AND EGO IDENTITY

Sato Hideyuki, MD and Nagai Satoru, PhD

Rissho University, Shinagawa-ku, Tokyo, Japan. 
The tree-drawing technique is a projective technique that involves the drawing of a tree, and it is one of the most frequently used techniques in Japan. One modified procedure of the technique is "the twice-application method" of the tree drawing technique. The technique is administere by drawing a tree in the first picture and then immediately drawing a tree again in the second picture. It has been demonstrated that through this technique the subject expresses their self-image in two different projection levels - "social attitude" in the first picture, and "internal self-image" in the second. However, little recent studies have addressed the validity of the technique. Therefore, the present study aims to examine the relationship between the twice-application method of the treedrawing technique and ego identity.

The participants were 277 university students who completed the twice-application method of the tree drawing technique and the ego identity status scales. In the present study, we scored the forms of the tree trunk drawing because the relationship between the indices in that element of the drawing and ego status has already been examined. The results of the ego identity status scales demonstrated that the subjects were distributed in identity statuses as follows: 15 identity achievements and 46 identity diffusions. The tree drawings of two groups were compared by chi-square tests. As a result of chi-square tests, we observed that identityachieved individuals who drew a trunk at the center in the first picture with consecutive lines also drew a trunk of medium thickness in the second picture. On the other hand, identitydiffused individuals who drew a trunk on the left side or the right side with a considerably wide trunk base in the first picture also drew a trunk with discontinuous lines and a thin trunk in the second picture. These results reveal that the uncertainty and the conflicts of their self-images were expressed in the twiceapplication method of the tree drawing technique.

CORRESPONDING AUTHOR: Sato Hideyuki, MD, Rissho University, Shinagawa-ku, Tokyo, 141-8602, Japan; satohide@ ris.ac.jp

\section{PSW-174a}

EFFECTS OF MEDIA AND SOCIAL STANDING ON SMOKING BEHAVIORS AMONG ADOLESCENTS IN CHINA

Grace Huang, MPH, ${ }^{1}$ Thomas W. Valente, $\mathrm{PhD},{ }^{1}$ Ping Sun, $\mathrm{PhD},{ }^{1}$ Janet Okamoto, $\mathrm{PhD}$ candidate ${ }^{1}$ and Jennifer Unger, $\mathrm{PhD}^{1,2}$

${ }^{1}$ Institute for Health Promotion and Disease Prevention Research, University of Southern California, Alhambra, CA and ${ }^{2}$ Community and Global Health, Claremont Graduate University, Claremont, CA.

Adolescent smoking in China has become a major public health problem, with initiation rates on the rise, comparable to those in Western countries (Unger et al, 2001). While many studies have documented the associations between peer influences and smoking behaviors in the U.S. (Hoffman et al, 2006) and among Chinese adolescents (Weiss et al., 2008) fewer studies have shown how these influences occur in relation to one's popularity and social status as measured by an individual's position in their social network. Furthermore, recent advances in social media channels now serve as an extension of one's social network and sphere of influence. Online social network sites such as Facebook (QQ in China) and mobile phone text messaging are rapidly gaining popularity. This study examines the interplay between these new media channels, peer influences, and smoking outcomes.

Data were collected from 5,563 students from 24 professional and vocational high schools in Chengdu, the capital of Sichuan Province, China. Exploratory factor analysis of 12 Internet usage items revealed two types of online activity-social and non-social. Students' social status was measured by the number of "wellliked" nominations from classmates and the outcome variable was past-month smoking. Multilevel logistic models were constructed, controlling for school-level random effects and subject-level covariates such as age, gender, SES, friend smoking and friend media use. Results indicated that social Internet activity (gaming, chatting) was a risk factor for smoking $(p=.01)$, while non-social Internet activity (news, forums, homework) had a protective effect $(\mathrm{p}<.01)$ among adolescents. High social status was positively associated with smoking $(\mathrm{p}<.01)$ and was found to significantly moderate the association between mobile phone usage and smoking $(\mathrm{p}=.04)$.

Findings suggest that media usage may have differential effects on risky adolescent behaviors, which may have implications for designing tailored Web or mobile phone-based applications for health promotion interventions.

CORRESPONDING AUTHOR: Grace Huang, MPH, Institute for Health Promotion and Disease Prevention Research, University of Southern California, Alhambra, CA, 90505; grace.huang@usc.edu

PSW-174b

DISPARITIES IN QUALITY OF LIFE AND WELL-BEING AMONG HISPANIC, AFRICAN-AMERICAN, AND WHITE CHILDREN ARE DUE MAINLY TO SOCIODEMOGRAPHIC DIFFERENCES

Jan Wallander, $\mathrm{PhD}$ and Chris Fradkin, MS

University of California, Merced, Merced, CA.

Whereas race/ethnic disparities in health have been widely documented in pediatric populations, there is a dearth of information regarding disparities in quality of life (QOL) of children in the general population. Reflecting the child's wellbeing across multiple domains, QOL captures well the broad meaning of health espoused by WHO. This research therefore examined whether there are race/ethnic disparities in QOL. A multi-site study enrolled 4,824 5th grade children from one of the three major race/ethnic groups in the U.S. (Hispanic $=38 \%$, African American $=36 \%$, White $=26 \%$ ). Children reported their QOL with the PedsQL providing scores for their physical and psychosocial domains, as well as with instruments to measure selfworth and social well-being. Parents rated children's overall health status and provided demographic information on the household. Complex sampling analysis revealed non-White children reporting significantly lower QOL in all domains than White children. However, after controlling for SES differences (parent's education, income), these race/ethnic disparities remained only in the 
domains of school, self-worth, satisfaction with appearance, and social well-being. After also controlling for family contextual differences (family structure, English proficiency), disparities remained in the domain of social well-being only. Findings suggest that disparities across race/ethnicity in children are, mainly, products of sociodemographic differences, rather than being endemic to minority race/ethnic groups. As one of the first studies examining racial/ethnic disparities in QOL of children in the general population, this study provides a basis for further exploration of contextual influences on the QOL experienced by children.

CORRESPONDING AUTHOR: Jan Wallander, PhD, University of California, Merced, Merced, CA, 95343; jwallander@, ucmerced.edu

\section{PSW-174c}

\section{FACTORS AFFECTING THE PSYCHOSOMATIC} COMPLAINTS OF CORRESPONDENCE HIGH SCHOOL STUDENTS

Akemi Masuda, MA, ${ }^{1}$ Yuriko Takata, $\mathrm{PhD},{ }^{2}$ Yumiko Sakata, $\mathrm{PhD}^{2}$ Reiko Sato, Master of Nursing ${ }^{3}$ and Reka Takahashi, Master of Child Studies 4

${ }^{1}$ Nursing, Junior College, University of Shizuoka, Shizuoka city, Japan; ${ }^{2}$ Graduate School of Comprehensive Human Sciences, University of Tsukuba, Tsukuba, Japan; ${ }^{3}$ Doctoral Program in Life System Medical Sciences, Graduate School of Comprehensive Human Sciences, University of Tsukuba, Tsukuba, Japan and ${ }^{4}$ Doctoral Program in Nursing Sciences, Graduate School of Comprehensive Human Sciences, University of Tsukuba, Tsukuba, Japan.

Correspondence education was introduced into Japan from the United States in 1946, and many working young people used it to learn. However, since many of the students today have mental or physical health problems, in this study we conducted a questionnaire survey by mail of 1984 students enrolled in correspondence high schools in two regions of Japan in order to determine the health status of the students and factors that affect it.

1083 students out of 1452 students (reply rate: $73.2 \%$ ), who were included in the analysis, were between 15 and 29 years of age and were not being treated for any diseases and had replied to all of the items on a psychosomatic complaints scale (valid reply rate: $54.6 \%)$.

The chi-square test was used to statistically analyze associations between psychosomatic complaints and both personal attributes and lifestyle habits according to gender, and the t- test was used for age and the self-esteem scale scores. We identified items which has a significant difference, and then performed a multiple logistic regression analysis.

The results for factors that affected psychosomatic complaints among the males showed that low self-esteem $(\mathrm{OR}=0.90,95 \%$ $\mathrm{CI}=0.87-0.94, \mathrm{p}<0.001)$, irregular or skipped breakfasts $(\mathrm{OR}=$ $1.91,95 \% \mathrm{CI}=1.17-3.12, \mathrm{p}=0.01)$, being a fussy eater $(\mathrm{OR}=$ $1.84,95 \% \mathrm{CI}=1.17-2.89, \mathrm{p}=0.008)$, and age $(\mathrm{OR}=1.13,95 \%$ $\mathrm{CI}=1.03-1.24, \mathrm{p}=0.008)$ increased psychosomatic complaints.
Among the females, low self-esteem $(\mathrm{OR}=0.89,95 \% \mathrm{CI}=0.86$ $0.92, \mathrm{p}<0.001)$, poor health status during puberty $(\mathrm{OR}=2.93,95 \%$ $\mathrm{CI}=1.75-4.90, \mathrm{p}<0.001)$, being a fussy eater $(\mathrm{OR}=2.57,95 \% \mathrm{CI}=$ $1.74-3.81, \mathrm{p}<0.001)$, and being a smoker $(\mathrm{OR}=1.95,95 \% \mathrm{CI}=$ $1.17-3.24, \mathrm{p}=0.011$ ) increased psychosomatic complaints. "Low self-esteem" and "being a fussy eater" increased psychosomatic complaints in both sexes. Thus, we would like to make a program which allows correspondence high school students themselves to reconsider their diets by increasing their self-esteem for the future investigation.

CORRESPONDING AUTHOR: Akemi Masuda, MA, Nursing, Junior College, University of Shizuoka, Shizuoka city, 422-8021; masudaa@u-shizuoka-ken.ac.jp

PSW-174d

\section{ADOLESCENT PEER SUPPORT MODERATES} THE RELATIONSHIP BETWEEN WEIGHT STATUS AND BODY IMAGE

Laura J. Caccavale, BA, Tilda Farhat, PhD, MPH and Ronald J. Iannotti, PhD

Prevention Research Branch, Eunice Kennedy Shriver National Institute of Child Health \& Human Development, Washington, DC.

BACKGROUND: US adolescent obesity increased from $5 \%$ to $18.1 \%$ between 1976-1980 and 2007-2008. In addition to health complications (e.g., type 2 diabetes), adolescents who are obese are at risk for developing negative body image which is related to depression and is a risk factor for eating pathology. Having a supportive peer group is an essential part of healthy adolescent development, and for overweight adolescents, could be a protective factor against negative body image.

PURPOSE: To examine whether the association between adolescent weight status and body image varies by peer support.

METHODS: A US nationally-representative sample of 6,909 students in grades 6 -10 with over-sampling of AfricanAmerican and Hispanic students completed the 2006 Health Behavior in School-aged Children survey. Body image, was assessed with 5 items $(\alpha=.87)$ asking for agreement or disagreement with statements about one's body. Weight status, determined from body mass index, was computed from selfreported height and weight and categorized using CDC 2000 growth charts. Peer support was computed as the average of 8 items $(\alpha=.67)$ asking about interactions with peers; higher scores indicated greater peer support. Linear regressions, separately by gender and controlling for age, race/ethnicity and socioeconomic status, were conducted with an interaction term (weight status x peer support).

RESULTS: Adolescents' overweight status was related to poor body image in boys and girls. However, peer support moderated the relationship between weight status and body image for girls but not for boys. Overweight girls with higher peer support were more likely to have a positive body image $(\beta=-0.69, \mathrm{p}<0.000)$ compared to overweight girls with lower peer support $(\beta=-.99$, $\mathrm{p}<0.000$ ). Overweight boys had lower body image compared to their normal weight peers, regardless of the level of peer support. 
CONCLUSION: Encouraging adolescent girls to develop strong healthy relationships with peers may prevent a negative body image. More research is needed to examine whether these findings vary by individual (e.g., race) or familial (e.g., presence of parental support) characteristics.

CORRESPONDING AUTHOR: Laura J. Caccavale, BA, Prevention Research Branch, Eunice Kennedy Shriver National Institute of Child Health \& Human Development, Washington, DC, 20008; caccavalel@mail.nih.gov

\section{PSW-174e}

UNNECESSARY ANTIBIOTIC USE FOR MILD ACUTE RESPIRATORY INFECTIONS IN 28-DAY FOLLOW-UP OF 823 CHILDREN UNDER FIVE IN RURAL VIETNAM Nguyen Quynh Hoa, MPH, ${ }^{1,2}$ Chuc Nguyen TK, PhD, ${ }^{4}$ Phuc Ho $\mathrm{D}, \mathrm{PhD},{ }^{5}$ Mattias Larsson, $\mathrm{PhD}{ }^{2}$ Bo Eriksson, Professor $^{3}$ and Cecilia Stalsby Lundborg, Professor ${ }^{2}$

${ }^{1}$ Department of Pharmacy, Vietnam Cuba Friendship Hospital, Hanoi, Viet Nam; ${ }^{2}$ IHCAR, Karolinska Institutet, Stockholm, Sweden; ${ }^{3}$ Nordic School of Public Health, Gothenburg, Sweden; ${ }^{4}$ Hanoi Medical University, Hanoi, Viet Nam and ${ }^{5}$ Institute of Mathematics, Hanoi, Viet Nam.

Few prospective studies about antibiotic use for mild acute respiratory infections (ARIs) have been conducted in community settings. This paper aims to assess knowledge of child-caregivers and actual antibiotic use for children under five, and to identify associated factors with antibiotic treatment for mild ARIs.

Caregivers in 828 households in Bavi, Vietnam were interviewed using a structured questionnaire regarding the case management of childhood ARI and the selected children's most recent illness assessing both knowledge and practice. Then 823 children were followed for 28 days to collect information regarding symptoms and drug use.

For management of non-febrile common colds, $85 \%$ of caregivers stated correctly that antibiotics are not required. For febrile colds and pneumonia, $45 \%$ and $47 \%$ of cases respectively would require antibiotics. Only 13\% demonstrated correct, in accordance with standard guidelines, overall knowledge for all three situations. The symptoms of the most recent illness were consistent with mild ARI in $79 \%$ of the cases, and antibiotics were used in $71 \%$ of these. During the 28 -day period, $62 \%$ of children had been given antibiotics. Out of all antibiotic courses recorded, $63 \%$ were used for mild ARIs. Half of the mild ARI episodes (528/1048) and 63\% of the children with mild ARIs (392/623) were treated with antibiotics. Most of the unnecessary antibiotic treatment was recommended by healthcare providers $(82 \%)$.

Most of the children had been administered antibiotics for common colds although most caregivers believed that antibiotics were not required. Antibiotics were unnecessarily recommended at health facilities in the area.

CORRESPONDING AUTHOR: Nguyen Quynh Hoa, MPH, Department of Pharmacy, Vietnam Cuba Friendship Hospital, Hanoi, 008443; quynhhoa29@gmail.com
PSW-174f

ADOLESCENT OVERWEIGHT/OBESITY AND PHYSICAL ACTIVITY: MEDIATION BY BODY IMAGE

Tilda Farhat, $\mathrm{PhD}, \mathrm{MPH}$, Ronald J. Iannotti, $\mathrm{PhD}$ and Laura $\mathrm{J}$. Caccavale, BA

NICHD, NIH, Bethesda, MD.

BACKGROUND: The benefits of physical activity (PA) for children and adolescents are numerous and include reduced risk for coronary heart disease, and improved mental health. Physical activity habits that are established during adolescence are likely to be sustained into adulthood. However, the prevalence of PA among adolescents is low, and even lower among overweight/obese (OT) adolescents, who are most in need of the benefits conferred by PA. Body image may mediate this relationship; overweight/obese adolescents are more likely to suffer from poor body image, which could be linked to less engagement in PA.

PURPOSE: To examine the hypothesis that poor body image mediates the relationship between overweight/obesity and PA among adolescents.

METHODS: Data are from the 2006 Health Behaviors in SchoolAge Children survey, a nationally representative sample of students in grades 6-10 during the 2006 school-year. Physical activity was measured by a question asking about the number of days respondents engaged in at least 60 minutes of PA over the last week. Body image was assessed with 5 items $(\alpha=.87)$ asking for agreement/disagreement with statements about one's body. Weight status, determined from body mass index, was computed from self-reported height and weight and categorized using CDC 2000 growth charts. Mediation analysis, stratified on gender and controlling for age, race/ethnicity and SES, was performed to determine whether body image mediated the relationship between weight status and PA.

RESULTS: Overweight was negatively associated with physical activity only among boys whereas obesity was negatively associated with physical activity among boys and girls. Body image was a complete mediator of the association between weight status and PA among girls, and a partial mediator of the association of weight status with PA among boys.

CONCLUSIONS: Mediation analyses demonstrate that body image is a significant mediator of the relationship between overweight/obesity and PA among boys and girls. Encouraging overweight and obese adolescents to develop positive body images may result in greater engagement in PA.

CORRESPONDING AUTHOR: Tilda Farhat, PhD, MPH, NICHD, NICH, Rockville, MD, 20852; farhatti@mail.nih.gov

PSW-174 g

QUALITY OF LIFE IN HISPANIC, AFRICAN AMERICAN, AND WHITE CHILDREN WITH AND WITHOUT COMPROMISED HEALTH STATUS

Jan Wallander, PhD and Chris Fradkin, MS

University of California, Merced, Merced, CA. 
Many children experience compromised health status. Most obvious are chronic health conditions ( $\mathrm{CHC}$ ), but the majority of children only experience temporary health problems (e.g., infection, injury). How do these variations in health status affect quality of life (QL) in children? QL reflects the child's overall well-being across multiple domains. There has been no study examining the relationship between compromised health and QL in children in the general population who do not have a $\mathrm{CHC}$, nor do we know how such variation interacts with race/ethnic status. Our hypotheses are that (1) variation in health status is positively associated with QL regardless of the presence of CHC and (2) Whites report higher QL than African Americans and Hispanics, regardless of health status.

A multi-site study enrolled 4,824 5th grade children from one of the three major race/ethnic groups in the U.S. (Hispanic $=38 \%$, African American $=36 \%$, White $=26 \%$ ). Children reported their QL with the PedsQL providing scores for their physical and psychosocial domains, as well as on instruments to measure selfworth and social well-being. Parents rated their 5th graders' overall health status on the commonly used single item "In general, would you say your child's health is . . . (Excellent/Very Good/Good/Fair/Poor)?"and completed a screener, from which chronic health condition was classified.

Results showed there was a reduction in each QL domain associated with each decrement in overall health status, even in the absence of a CHC. Moreover, African Americans and Hispanics report a lower QL than Whites, when in excellent health as well as at each decrement in health status. This demonstrates for the first time that even minor decrements in health status are associated with concomitant reduction in QL, not only in the physical domain, but also in the emotional and social domains.

CORRESPONDING AUTHOR: Jan Wallander, PhD, University of California, Merced, Merced, CA, 95343; jwallander@ ucmerced.edu

PSW-174 h

EDUCATIONAL GOALS AND SEXUAL RISK BEHAVIORS AMONG MEXICAN ADOLESCENTS

Paula Repetto, $\mathrm{PhD}^{1}$ and Antonia Villarruel, $\mathrm{PhD}^{2}$

${ }^{1}$ P. Universidad Catolica de Chile, Santiago, Chile and ${ }^{2}$ University of Michigan, Ann Arbor, MI.

Several researchers have found that teenage pregnancy is associated with worst educational outcomes among adolescents and that girls who have a child early in life, are less likely to graduate from high school (Molina et al., 2004). Other researchers have proposed that educational expectations do actually predict educational outcomes among youth. They have found that having higher educational expectations is associated with higher educational outcomes later in life (Mello, 2008). However, researchers have not examined how educational expectations may be associated with adolescents' self-reported sexual behaviors. Some researchers suggest that there might be a self-selection process in which adolescents who do worst in school and do not have plans to continue further in their education may also engage in unprotected sexual behavior, putting them at higher risk of becoming pregnant. In the present study we examined the relationship between educational expectations and goals and different sexual behavior outcomes in a sample of 829 Mexican youth. Participants included 829 adolescents between the ages of 14 and 17 (mean 15.18, S.D. $=0.68,55,4 \%$ females) part of an HIV and STDs intervention program. We examine this relationship using time 1 data. Participants completed a self-reported questionnaire that asked about their sexual behavior and explore different psychosocial predictors of sexual behavior. Using a regression analyses we examined whether having plans to complete high school and continue their education predicted ever had sex, age in which girls and boys should have sex for the first time, plans to use condoms when having sex, and perceive that condoms may be effective to protect them of STDs and HIV. Our findings suggest that plans to finish high school predicts only perceptions that condoms may be effective to protect against HIV $(\mathrm{t}=2,06, \mathrm{p}<$ 0.05 ), and not any of the other outcomes. Because we had very few participants who reported ever having sex $(9,5 \%)$, we probably did not have enough power to find further differences. We explored how these variables may predict their sexual behavior at the 12 months follow-up, when $16 \%$ of participants reported ever having sex, and we found that having plans to attend college predicted not having sex among adolescents $(\operatorname{Exp}(\mathrm{B})=$ $0.358, \mathrm{p}<0.01)$. Implications of these findings for intervention and practice are discussed.

CORRESPONDING AUTHOR: Paula Repetto, PhD, P. Universidad Catolica de Chile, Santiago, 00000; prepetto@uc.cl

\section{PSW-174i}

THE EFFECTS OF THE SOCIAL SKILLS

ON THE BEHAVIORAL ACTIVATION LEVEL IN ADOLESCENTS WITH SOCIAL ANXIETY

Masashi Obata, Master, ${ }^{1}$ Tomohiko Muratubaki, Doctor, ${ }^{2}$ Mitimasa Haga, Master, ${ }^{1}$ Tadaaki Tomiie, Assistant Professor $^{3}$ and Yuji Sakano, Professor ${ }^{3}$

${ }^{1}$ Health Sciences University of Hokkaido, Sapporo, Japan; ${ }^{2}$ Behavioral Medicine, Tohoku University Graduate School of Medicine, Sendai, Japan and ${ }^{3}$ Clinical psychology, Health Sciences University of Hokkaido, Sapporo, Japan.

Purposes: Although many reports have suggested a role for the social skill in the treatment of depression, there has been little evidence that the social skill training is effective therapeutic strategies for treating it. The activation level are thought to be an important mediator of the social skill and the depression. Obata et al. (2010) found that the difficulty in the control of social anxiety lead to the inhibition of social activity and the increase of depressive mood in Japanese high school students. We hypothesized that the mechanism for exacerbation of depression may be explained by the behavioral activation and social skill, and these relationships differed with the level of social anxiety. Method: Questionnaires were conducted with 347 Japanese adolescents (16.0 \pm 0.6 years). Questionnaires were composed of Depression 
Self-Rating Scale for Children (DSRS; Birleson, 1981), Assertiveness Skill Scale for High school students (ASS; Takaki et al., 2002), and Behavioral Activation for Depression Scale (BADS; Martell et al., 2001). Higher score group $(n=50)$ and lower score group $(n=50)$ were extracted in subjects on "social anxiety(reverse coded)" in ASS. Data analyses were tested using correlation analysis to examine relationship of between depression, social skill, and behavioral activation, using SPSS 16.0. Main Results: "Activation (reverse coded)" in BADS was significantly associated with "positive interpersonal entry" in ASS(higher score group: $\mathrm{r}=-.37, \mathrm{p}<.01$; lower score group: $\mathrm{r}=-.26$, .n.s.). Conclusion: These results suggest that the control of social anxiety is an important procedure for behavioral activation on depression.

CORRESPONDING AUTHOR: Masashi Obata, Master, Health Sciences University of Hokkaido, Sapporo, 002-8072; obata@, hoku-iryo-u.ac.jp

PSW-174j

FACTOR RELATED TO STATE ANXIETY OF SECONDARY SCHOOL STUDENT IN BANGKOK, THAILAND

Natnaree Aimyong, MSc(Biostatistics)

Biostatistics, Mahidol University, Bangkok, Thailand.

Many people think that the children are not stress, in fact they can stress as the adult but their parent cannot notice or skip it. The objective is to study the association between state anxiety with emotional quotient and strength and difficulty of the secondary school student who also study in Sunday Buddhism and do not study by controlling some potential confounding. The self-report questionnaire was distribution to both type of schools to student age 11-16 years old. The tools of this study are Thai version of state-trait anxiety of Spielberger's; the Strength and Difficulty Questionnaire (SDQ); emotional quotient (EQ). The regression model used for analyzing and the data are centered to reduce the scaling problem. The 303 completed answer questionnaire was returned, the incomplete questionnaire was excluded from the study to reduce the effect of imputation. Mean age of sample is 13.5 years old, $42.24 \%$ is male, and $48.51 \%$ reported that they are also study in the Sunday Buddhism School. The model by stepwise selection presents that trait anxiety, pro-social, selfcontrol, ability to deal with problem, self-esteem, life-satisfaction and interaction between age and emotional problem, the adjusted $\mathrm{R} 2$ is $60.55 \%$ ( $\mathrm{p}$-value $<0.0001$ ). The increasing one unit of trait anxiety, self-control, self-esteem and life-satisfaction increases $0.52,0.10,0.11$ and 0.13 standardize unit of state anxiety, the higher standardize score of ability to deal with problem decrease 0.07 standardize unit of state anxiety. The interaction of emotional and age reduce the standardize score of state anxiety. The higher emotional problem score increased 0.27 standardize unit of state anxiety while age reduce 0.01 unit of state anxiety ( $p$-value $<0.05$ ). The gender and Sunday Buddhism school included into the model to control potential confounding factor, female has higher state anxiety score than male, the student in Sunday Buddhism School have lower state anxiety than non-student ( $p$-value $>0.05)$. The student with high score of self-control, self-esteem and lifesatisfaction have higher level of state anxiety which may cause from they are try to be the best which make them stress. The ability to deal with the problem can reduce state anxiety, the higher age (more maturity) reduce emotional problem and finally reduce state anxiety. The student who is good in some dimension of EQ may cause state anxiety.

CORRESPONDING AUTHOR: Natnaree Aimyong, MSc(Biostatistics), Biostatistics, Mahidol University, Bangkok, 10400; natnaree_a@yahoo.com

\section{PSW-175}

EVALUATING NEW GLOBAL HEALTH ACTORS: THE ROLE OF QUALITATIVE METHODS

Erin Sullivan, PhD, Nayana Dhavan, MSc and Rebecca Weintraub, MD

Global Health Delivery Project, Harvard University, Boston, MA.

The mobilization of significant resources for global health coincides with the emergence of Global Health Initiatives (GHIs) and the rising importance of multilateral and private actors in global health; traditional actors in global health, such as the World Health Organization (WHO), have been joined by a variety of civil society and non-governmental organizations (NGOs), private firms, and private philanthropists. These new actors, which exist alongside traditional arrangements between sovereign states and UN bodies, signal a sea change in the institutional arrangements in global health as well as a change in the norms, expectations and rules relating to the relationships between these new philanthropists.

It has become clear, however, that the new institutions in global health have often failed to adequately evaluate their investments and program implementation. With such enormous investments, it is critical to better understand these new institutions, evaluate their strategies to promote global health and document the successes and failures of these efforts. We propose that rigorous qualitative research methodologies can explore and describe how global health actors and their associated mechanisms interact with complex health systems to yield optimal Results: Qualitative methods, particularly case research, can overcome the three main weaknesses of quantitatively-oriented public health research models: underestimating complexity, failing to incorporate context and an inability to engage in the "why" questions. Qualitative methods can better evaluate if the activities funded by new global health actors are best aligned to meet the objective of delivering high value care to patients. Global health institutions can use this insight to bridge the knowledge-action gap in global health care delivery by translating the current understanding of medical interventions and health systems into evidence-based strategies for care delivery.

CORRESPONDING AUTHOR: Erin Sullivan, PhD, Global Health Delivery Project, Harvard University, Boston, MA, Ma; esulliva@hsph.harvard.edu 
PSW-176

THE IMPACT OF POSITIVE PSYCHOLOGY

ON BEHAVIORAL MEDICINE AND HEALTH

PSYCHOLOGY. A BIBLIOMETRIC OVERVIEW

Gabriel Schui, Dipl-Psych, Clemens Fell, Dipl-Psych and Günter

Krampen, Prof Dr

Leibniz-Institute for Psychology Information (ZPID), Trier, Germany.

Positive Psychology is a relatively new school of thought in Psychology, focusing on human strengths and virtues, and on improving well-being and quality of life. In its aim and scope, it bears special relation to the applied fields of Behavioral Medicine and Health Psychology. Expanding upon a recent bibliometric analysis (Schui \& Krampen, in press), we trace the impact, Positive Psychology had on these larger fields by evaluating the corresponding literature found in the PsycINFO database. Analyses comprise a time span starting with the year 2000 (since when numerous contributions on Positive Psychology are available) until present day. Results show, that even by employing a wide bibliometric definition of Positive Psychology, the overlap with Behavioral Medicine is minimal (also because documents are rarely indexed as such), while that with Health Psychology is considerably larger (ca. 9\%). Further analyses of the overlapping literature include temporal changes of the overlap since year 2000 (it was higher in the early 2000's), the authors' national institutional affiliations, multiple authorships, most frequently used journals, publication types, methodology employed, and the semantic networks opened up by title words and APA-index terms, which are presented as text clouds. In sum, bibliometric results point to a potential for improvement when it comes to establishing Positive Psychology approaches within Health Psychology, and especially Behavioral Medicine. That potential should be realized, since the relationship of Positive Psychology to the health-related fields is already stronger than to other psychological disciplines.

CORRESPONDING AUTHOR: Gabriel Schui, Dipl-Psych, Leibniz-Institute for Psychology Information (ZPID), Trier, 54295; gabriel.schui@zpid.de

\section{PSW-177a}

RELATIONSHIP BETWEEN MEDICAL ERRORS

AND THE PERSONALITY TRAITS OF NURSES

Mayo Suzuki, PhD, ${ }^{1}$ Risa Fukuda, Master, ${ }^{2}$ Yuko Hayashi, PhD, ${ }^{3}$ Chiharu Akazawa, PhD, ${ }^{2}$ Tomoko Kawakami, bachelor, ${ }^{4}$ Mari Azuma, bachelor, ${ }^{4}$ Michio Takemoto, bachelor, ${ }^{5}$ Setsuko Fujimoto, bachelor, ${ }^{6}$ Shigeru Sakuraba, $\mathrm{PhD}{ }^{2}$ Satoshi Sasayama, $\mathrm{PhD}^{2}$ and Chiemi Hayashi, bachelor ${ }^{7}$

${ }^{1}$ Bunri University of Hospitality, Sayama-shi, Japan; ${ }^{2}$ Graduate School of Medicine, Kyoto University, Kyoto-shi, Japan; ${ }^{3}$ Osaka Medical College, Takatsuki-shi, Japan; ${ }^{4}$ Daini Okamoto General Hospital, Uji-shi, Japan; ${ }^{5}$ Kyoto Kagaku co., LTD, Kyoto-shi, Japan; ${ }^{6}$ Pulse Station co., LTD, Minato-ku, Japan and ${ }^{7}$ Mitsubishi Kyoto Hospital, Kyoto-shi, Japan.

Background and Purpose: Nurses are likely to carry out the final role in any given medical procedure. Because some individuals tend to make more errors than others even within the exact same work environment, measures that consider individual characteristics are needed.

This study aimed to elucidate the relationship between medical errors and the personality traits of nurses. We reported on this relationship before; this time, we studied a bigger sample size for greater accuracy.

Methods: Medical errors and near-misses that had occurred in actual medical settings were analyzed, and "administration," which is one of the most common components of a nurse's job, was selected as the theme of this study. For this study, simulation software was developed that enabled nurses to experience all the human errors that could possibly occur in various settings under the broad category of "administration." The software was used to understand the reasons behind the subjects' tendency to commit medical errors. Furthermore, the Kretschmer Type Personality Inventory was administered to the nurses in order to determine their personality traits. This study was approved by the ethics committee of our university.

Results and Conclusions: A relationship was found between the nurses' tendency to commit medical errors and their personality traits (self-repression, self-disclosure, steady-going nature, sensitive nature, unshakable belief, etc.) with a sufficient sample size. Clarifying the relationship between the personality traits of nurses and human errors may be useful for training. Also, this approach may help to prevent human errors by enabling the nurses themselves to become conscious of their actions and objectively aware of their own individual characteristics.

CORRESPONDING AUTHOR: Mayo Suzuki, PhD, Bunri University of Hospitality, Sayama-shi, 350-1336; mayo-suzuki@ bunri-c.ac.jp

\section{PSW-177b}

RELATIONSHIP BETWEEN HUMAN ERRORS AND JOB PERFORMANCE CAPABILITY OF NURSES

Risa Fukuda, MPH, ${ }^{1}$ Mayo Suzuki, $\mathrm{PhD},{ }^{2}$ Chiharu Akazawa, $\mathrm{PhD},{ }^{1}$ Yuko Hayashi, PhD, ${ }^{3}$ Tomoko Kawakami, none, ${ }^{4}$ Mari Azuma, none, ${ }^{4}$ Michio Takemoto, none, ${ }^{5}$ Setsuko Fujimoto, none, ${ }^{6}$ Shigeru Sakuraba, $\mathrm{PhD},{ }^{1}$ Satoshi Sasayama, $\mathrm{PhD}^{1}$ and Chiemi Hayashi, none ${ }^{7}$

${ }^{1}$ Graduate Scool of Medicine, Kyoto University, Kyoto, Japan; ${ }^{2}$ Bunri University of Hospitality, Sayama, Japan; ${ }^{3}$ Osaka Medical College, Takatsuki, Japan; ${ }^{4}$ Daini Okamoto General Hospital, Uji, Japan; ${ }^{5}$ Kyoto Kagaku co., LTD, Kyoto, Japan; ${ }^{6}$ Pulse Station co., LTD, Tokyo, Japan and ${ }^{7}$ Mitsubishi Kyoto Hospital, Kyoto, Japan.

Background: The nurse position is the job position which most contributes to incidents in the medical treatment arena, and nurses generally bear the majority of the final role in providing patients with medical treatment. Therefore, clarifying the relationship between the characteristics of nurses (job performance capability) and human errors would be effective as an aid for educational support and human resource arrangement. 
Purpose: The purpose of this study is to clarify the relationships between human errors and the job performance capability of nurses.

Methods: The subjects were all of the newly hired nurses of five facilities. A simulated case experience software and the UchidaKraepelin Test were used. For data analysis, the subjects were separated into the groups based on the judgment results of their performance on the Uchida-Kraepelin Test. The groups were then analyzed for differences in software scoring and correct answer ratio. In the analysis, SPSS $12.0 \mathrm{~J}$ for Windows was used. This study protocol was approved by the Ethics Committee of Kyoto University.

Results: The analysis subjects numbered 65 people. The average age was $24.9 \pm 4.7$. There were 58 females $(89.2 \%)$. Membership alignment in the groups via the Uchida-Kraepelin Test results were as follows: $72.3 \%$ for the typical group; $35.4 \%, 58.5 \%$, and $55.4 \%$ respectively for the groups with no problems in movability, changeability, and durability; $72.3 \%$ for the group sufficient in efficiency and speed of work; and $67.7 \%$ for the group with no problem in degree of balancing in terms of temperament and behavior.

The software scoring average was $64.3 \pm 29.7$ points, and the correct answer ratio for each error was $36.9 \%$ to $83.1 \%$. For nurses grouped in the job performance characteristic types of atypical nurses and nurses with problems in degree of balancing in terms of temperament and behavior, the software scoring was significantly low. Also, the correct answer ratio for each error was significantly low for nurses grouped in the job performance characteristic types of atypical nurses, nurses with problems in degree of balancing in terms of temperament and behavior, and nurses with problems in durability.

Conclusion: Subjects possessing the job performance characteristics of "atypical type" and "problems in degree of balancing in terms of temperament and behavior" had significantly lower scores of the software than subjects not possessing these characteristics.

CORRESPONDING AUTHOR: Risa Fukuda, MPH, kyoto university, Kyoto, 606-8507; rfukuda@hs.med.kyoto-u.ac.jp

PSW-178

OBESE ADOLESCENT EXERCISE PROGRAMS: WHAT IS THE RIGHT INTENSITY?

A. Justine Wilson, BAH, BPHE, Jacqueline Simatovic, BHSc, Mary E. Jung, PhD, Anita G. Cramp, PhD and Harry Prapavessis, PhD

Kinesiology, University of Western Ontario, London, ON, Canada.

Childhood obesity has rapidly become an epidemic in developed countries globally (WHO, 2000). The development of effective treatment initiatives aimed to help obese youth adopt and maintain healthy lifestyles is prudent. The purpose of this study was to examine the effects of exercise intensity on obese adolescents' enjoyment, satisfaction and program adherence. Thirty obese adolescents (female $=20, B M I \geq 95$ th percentile; 10-16 years of age) were randomly assigned to either a moderate $(H R R=40$
$55 \%)$ or vigorous $(\mathrm{HRR}=60-75 \%)$ supervised 12 -week exercise training program. Satisfaction was measured through self-reported questionnaires assessing satisfaction with outcome progress and current state. Enjoyment was measured using the PACES selfreport questionnaire. Adherence was assessed as a percentage of sessions attended. Repeated measures ANOVAs showed no significant time $\mathrm{x}$ group interaction effects (all ps $>.05$ ). Significant time effects in the expected direction were found for satisfaction with outcome progress $(\mathrm{F}(1,26)=17.62, \eta 2=.40$, $\mathrm{p}<.001)$, satisfaction with current state $(\mathrm{F}(1,26)=5.97, \eta 2=.18$, $\mathrm{p}<.05)$ and enjoyment $(\mathrm{F}(1,26)=9.4, \eta 2=.27, \mathrm{p}<.005)$. No adherence differences were found between intensity groups ( $\mathrm{M}$ for vigorous $=86 \%, \mathrm{SD}=.09 ; \mathrm{M}$ moderate $=85 \%, \mathrm{SD}=.14)$. These data indicate that exercise intensity does not adversely affect satisfaction, enjoyment or adherence. Practical implications of these findings will be discussed.

CORRESPONDING AUTHOR: A. Justine Wilson, BAH, BPHE, Kinesiology, University of Western Ontario, London, ON, N6B 2A6; awils29@uwo.ca

PSW-179

PROJECT IGNITE: LAUNCHING STRONG ATHLETES INTO PEAK PERFORMANCE

Amy Thompson, PhD, Diana Reindl, MA, Karen Gallagher, MA and Debra Boardley, $\mathrm{PhD}$

University of Toledo, Toledo, $\mathrm{OH}$.

Proper nutrition is the foundation for athletic performance. This session introduces a theory based multi-level intervention spanning a convenience sample of 16 sports $(n=250$ athletes) at a Midwestern University. This program provides athletes and staff education about sport-related nutritional issues for performance and recovery. Emphasis of this program is placed on choosing healthy foods and making positive behavior modifications. Several methods of data collection were used for this project. All athletes were given a validated knowledge and dietary practices survey that was based on the health belief model, the EAT 26 survey which screens for disordered eating behaviors was distributed, and body composition was also determined using a Tanita scale. All data was entered into SPSS 16.0. Statistical analysis was conducted using t-tests and descriptive statistics. Changes in baseline knowledge, dietary practices, and body composition data will be reported and discussed. Formative evaluation which includes adherence to program guidelines and recommendations for program improvement will also be reported. Based on the data and information collected Project Ignite shows substantial promise as a model program for improving collegiate athlete dietary practices and knowledge about sport nutrition related issues.

CORRESPONDING AUTHOR: Amy Thompson, PhD, University of Toledo, Toledo, OH, 43606; amy.thompson4@utoledo.edu 
PSW-180

ASSOCIATION BETWEEN HEALTH LOCUS OF CONTROL AND METABOLIC SYNDROME

Atsuko Nakazawa, MD, ${ }^{1}$ Masako Shigeta, MD ${ }^{2}$ Hideko Noaki, Nutritionist, ${ }^{3}$ Katsura Yamakado, Nurse ${ }^{4}$ and Yoshiyuki Watanabe, MD, Professor ${ }^{2}$

${ }^{1}$ Health center, Doshisha University, Kyoto, Japan; ${ }^{2}$ Department of Social Medicine and Cultural Sciences, Kyoto Prefectural University of Medicine, Kyoto, Japan; ${ }^{3}$ Department of Health and Nutrition, Kio University, Nara, Japan and ${ }^{4}$ Division of Health Check-up, Kyoto First Red Cross Hospital, Kyoto, Japan.

Background: Lifestyles that are associated with metabolic syndrome (MS) are affected by individual health locus of control (HLC).

Objective: Association between HLC and the characteristics of MS were examined.

Subjects and methods: Subjects were 200 people who underwent medical checkups. HLC was measured with 11 questions and subjects were asked about their motivation to improve lifestyle and the desire to receive health guidance. The MS components (i.e., blood pressure, waist circumference, glucose, triglycerides, and high-density lipoprotein cholesterol) were measured. The subjects were graded into 4 levels by the number of MS components (i.e.,0,1,2,3>). We reviewed the association between the levels of MS and HLC.

Result: There were no significant differences in HLC between the levels of MS. In response to the item "I become sick because of luck of appropriate physical activity or diet", Level "3>" showed the highest tendency toward internals. In response to the item "I think I have reasons to be sick", Level " 0 " showed the highest tendency toward internals. Level "0" was significantly less willing to receive health guidance and had lower motivation to improve lifestyle than other levels. Among females, the higher the MS level was, the stronger the motivation to improve lifestyles. Among males, Level "2" had the highest motivation.

Discussion: Those with Level " 0 " appear to make decisions themselves and they do not need to improve their lifestyles because they have no components of MS. However, our research showed that they have high awareness of not only preventing MS but also overall health. It is a problem that male with Level "3>" had lower motivation to improve lifestyle, possibly due to resignation or denial. We should raise awareness of overall health for the prevention of MS onset and encourage people who already have MS to make lifestyle changes.

CORRESPONDING AUTHOR: Atsuko Nakazawa, MD, Doshisha University, Kyoto, 602-0023; aak83071@pop21.odn.ne.jp

PSW-181

FINNISH CONSCRIPTS' CHANGES IN EATING

HABITS DURING SIX MONTHS OF MILITARY

SERVICE - INCREASED CONSUMPTIONS OF FIBRE

AND SUGAR CONTAINING FOODS

Clarissa M. Bingham, MSc, Pilvikki Absetz, PhD, Marja

Kinnunen, MSc, Antti Uutela, Prof and Piia Jallinoja, PhD

Lifestyle and Participation, National Institute for Health and Welfare, Helsinki, Finland.
Introduction: In Finland, all men are liable to military service and over $80 \%$ of each age group complete it. Main meals complying nutrition recommendations of the Defence Forces are served but snacks are available for purchase during free time. Research on conscripts' eating habits is limited, suggesting they favor energydense and nutrient-poor foods such as fast food, pastries, soft drinks and confectionery.

Aim: To study conscripts' eating habits before and during military service.

Materials and Methods: In 2007, data on eating habits of 290 1721-year old men were collected by self-administrated questionnaire at two garrisons (in the south and in the north) and at two arrivals (winter, summer) 1 month prior to beginning of military service and at 6 months of service. Three indexes were formed to describe main dimensions of eating habits: Fibre Index (FibI), Sugar Index (SI) and Fat Index (FatI).

Results: At six months of service, the weekly consumption frequencies of rye bread $(p=0.02)$ and fruit and berries $(p=0.05)$ increased. This explained the increase in FibI (change $0.65 \pm 5.1$, $\mathrm{p}=0.03)$. Weekly consumption frequencies of French fries $(\mathrm{p}=$ $0.001)$ and hamburgers and hot $\operatorname{dogs}(\mathrm{pp}=0.005)$ decreased. However, this did not result in a significant temporal change in FatI. Finally, weekly consumptions of sweet pastries $(p=0.002)$, desserts $(p<0.001)$, sweets $(p<0.001)$ and chocolate $(p=0.01)$ increased. This resulted in a significant increase in SI (change $2.0 \pm 4.8, \mathrm{p}<0.001)$. This change was greater $(\mathrm{p}<0.001)$ from summer to winter compared to winter from summer, and also higher $(\mathrm{p}=0.02)$ in the south than in the north.

Conclusions: These results indicate that during military service men prefer eating both fibre and sugar containing foods. This may be due to an overall increase in energy intake. Still, the increase of sugary foods offers an opportunity to intervene also as seasonal and regional differences exist.

CORRESPONDING AUTHOR: Clarissa M. Bingham, MSc, National Institute for Health and Welfare, Turku, 20720; Clarissa. Bingham@thl.fi

\section{PSW-182}

MODIFICATION OF CHARACTERISTICS OF PERSONALITY AND THE REDUCTION OF BODY MASS INDEX IN OBESE Gerardo Leija Alva, master, ${ }^{1}$ Victor Aguilera Sosa, Master ${ }^{2}$ and Juan Daniel Rodriguez Choreño, Master ${ }^{3}$

${ }^{1}$ Psicology, Instituto Politecnico Nacional, Distrito Federal, Mexico; ${ }^{2}$ Psychobiology, CICS. UST, DISTRITO FEDERAL, Mexico and ${ }^{3}$ Psychobiology, Centro Interdisciplinario de Ciencias de la Salud, DISTRITO FEDERAL, Mexico.

Reduce the body mass index (BMI) and modify personality characteristics in an obesity women sample. MATERIALS AND METHODS: 35 women with obesity Phase 1, range of age: 19 40, mean: 40.2, Std. deviation: 8.2 The obesity level and personality characteristics were determined. In the Phase 2, Cognitive-behavior group therapy. Phase 3 , subsequent measuring. RESULTS: The BMI average reduction was $1.9 \%$ and weight was $5 \mathrm{Kg}$. The $85 \%$ of women showed positive attitudes to the 
treatment. Although $15 \%$ showed negative attitudes to the treatment, their BMI and weight were reduced also. CONCLUSION: The cognitive-behavioral group therapy seems to be an adequate strategy to modify irrationals believes and eating behavior, which could derive in an excessive caloric intake and sedentary life.

CORRESPONDING AUTHOR: Gerardo Leija Alva, master, Psicology, Instituto Politecnico Nacional, Distrito Federal, 11340; gleija@ipn.mx

PSW-183

COLLEGIATE ATHLETES AND DISORDERED EATING: IMPLICATIONS FOR POLICY DEVELOPMENT

AND HEALTH EDUCATION

Amy Thompson, PhD, Diana Reindl, MA, Karen Gallagher, MA, Carly Miller, undergraduate and Debra Boardley, PhD

University of Toledo, Toledo, $\mathrm{OH}$.

Research suggests that athletes do not have a higher prevalence of disordered eating behaviors compared to the general population however, their risk of sport related injury is increased when poor nutritional behaviors are present. Project Ignite is a nutrition education program designed to educate 250 collegiate athletes about eating for sport performance and avoiding injury associated with poor nutritional intake. Additionally, data such as body mass index, body-fat (measured with a Tanita scale), and knowledge and perceptions of sport nutrition was measured. All participants of this program completed the EAT 26 survey which has been found to be valid in measuring disordered eating. This presentation will outline 3 case studies of collegiate athletes with disordered eating. For confidentiality reasons all names of participants have been changed. Case studies will include 2 athletes with extremely low body-fat (4\% for female; $2 \%$ male) and 1 athlete with extremely high body-fat $(50 \%$ female). These case examples will discuss the need for health and nutrition policies that guide student-athlete participation in their respective sports when disordered eating is present. Moreover, there is a need for health education regarding proper nutrition for the athlete, coaches, team physicians, and athletic trainers. Further, many universities do not have polices that mandate when a collegiate athlete should be evaluated to determine the extent of any existing disordered eating behaviors or health conditions associated with their nutritional intake. When it is determined that an athlete has disordered eating behaviors or health risks there should be policies in place to determine player eligibility.

CORRESPONDING AUTHOR: Amy Thompson, PhD, University of Toledo, Toledo, OH, 43606; amy.thompson4@utoledo.edu

\section{PSW-184}

PARTICIPANT PERCEPTIONS OF A COMMUNITY-BASED LIFE COACHING AND PHARMACIST COUNSELING DIABETES INTERVENTION

Landry L. Fukunaga, MA and Jean I. Schneider, MEd, SPHR Center on Disability Studies, University of Hawaii at Manoa, Honolulu, HI.
The Live Healthy Work Well (LHWW) project examines whether access to life coaches, pharmacists, and other supports can improve health and prevent the loss of employment and independence due to diabetes-related complications. This poster summarizes treatment participant $(n=128)$ usage and perceptions of intervention components. Data sources include: enrollment survey, service utilization records, satisfaction surveys, and voluntary focus groups.

Six months into a one-year intervention period, 119 (93\%) participants met with a life coach and 98 (77\%) met with a pharmacist. Participants $(n=97)$ responded to a survey assessing satisfaction and perceived effectiveness of the intervention model. Participants expressed satisfaction with life coaching and $91 \%(\mathrm{n}=$ 68) of life coach comments were positive. Of pharmacist comments, $52 \%(n=66)$ were positive and $29 \%$ were negative. A second survey revealed that $85 \%(n=66)$ agreed/strongly agreed that coaching helped improve health, employment, and other areas of life; $75 \%$ agreed that goal setting helped them make positive changes and to continue to set goals; and $94 \%$ would recommend coaching to others. Sixty-eight percent $(n=65)$ agreed/strongly agreed that pharmacists helped them find solutions to diabetesrelated problems and $69 \%$ agreed that sessions with a pharmacist were beneficial and would recommend pharmacist counseling to others.

Treatment participants $(n=47)$ attended voluntary focus groups which were audio recorded, transcribed, and coded to identify emergent themes. Participants were satisfied with support, scheduling, and goal setting with life coaches. Participants suggested that LHWW provide individualized approaches to pharmacist counseling, social supports and coordinated services. Scheduling with pharmacists and additional service providers was perceived as a barrier to program participation.

Findings can be used to guide the development of future programs and health initiatives for employed adults with diabetes and results may be generalizable to employed diabetics across a range of ethnic groups including Asian and Pacific Islanders.

CORRESPONDING AUTHOR: Landry L. Fukunaga, MA, Center on Disability Studies, University of Hawaii at Manoa, Honolulu, HI, 96822; lfukunag@hawaii.edu

PSW-185

PSYCHOLOGICAL PREDICTORS OF WEIGHT REGAIN AFTER A WEIGHT LOSS PROGRAM

Shinji Fujiwara, MD, PhD, ${ }^{1,2}$ Naoki Sakane, MD, PhD, ${ }^{2}$ Yoshiko Sano, BA, ${ }^{2}$ Kokoro Tsuzaki, MA, ${ }^{2}$ Narumi Nagai, $\mathrm{PhD},{ }^{3}$ Toshio Moritani, $\mathrm{PhD}^{4}$ and Yoshinobu Kiso, $\mathrm{PhD}^{5}$

${ }^{1}$ Mima City National Health Insurance Koyadaira Clinic, Mima, Japan; ${ }^{2}$ National Hospital Organization Kyoto Medical Center, Kyoto, Japan; ${ }^{3}$ University of Hyogo, Himeji, Japan; ${ }^{4}$ Kyoto University, Kyoto, Japan and ${ }^{5}$ SUNTORY WELLNESS LTD., Osaka, Japan.

Background: Weight regain is caused by many factors such as environmental, behavioral, and genetic ones. The Three Factor Eating Questionnaire Revised 18-item version (TFEQ-R18) 
measures 3 aspects of eating behavior, cognitive restraint (CR), uncontrolled eating (UE), and emotional eating (EE).

Objective: To identify psychological factors that predict weight regain.

Methods: Observation study after an intervention in which 32 obese female (mean age; $50 \pm 8$ years) lost weight during an 8 -week weight loss intervention program and were followed for a year. TFEQ-R18 was measured by dieticians 3 times at baseline and 8 weeks during the program and at 1 year after the program that included use of meal replacement drinks (diet's; SUNTORY WELLNESS LTD.) under the guidance of dieticians and counseling sessions by dieticians focused on skills of coping with hunger. Enrollment at Kyoto Medical Center occurred November, 2006 and intervention, January, 2007. Data collection was completed in May, 2008.

Results: Mean entry weight was $69.6 \pm 8.0 \mathrm{~kg}$ (BMI $28.4 \pm 3.3 \mathrm{~kg} /$ $\mathrm{m} 2$ ). Participants lost $4.7 \pm 2.0 \mathrm{~kg}$ during the initial 8 -week program and regained $1.6 \pm 0.3 \mathrm{~kg}$ during 1 -year follow-up period. Weight regain was significantly correlated with UE and EE scores at baseline $(\mathrm{r}=0.486 ; \mathrm{p}<0.001$ and $\mathrm{r}=0.531 ; \mathrm{p}<0.001$, respectively) and the scores at 1 year after the program $(\mathrm{r}=0.469 ; \mathrm{p}<0.001$ and $\mathrm{r}=0.401 ; \mathrm{p}<0.001$, respectively).

Conclusion: These findings suggest that UE and EE scores at baseline may predict weight regain of middle-aged women.

CORRESPONDING AUTHOR: Shinji Fujiwara, MD, PhD, Mima City National Health Insurance Koyadaira Clinic, Mima, 777-0302; syfujiwara@jichi.ac.jp

\section{PSW-186}

\section{ETHNIC DIFFERENCES IN PSYCHOSOCIAL AND DISEASE} CARE FACTORS IN YOUTH WITH TYPE 1 DIABETES

Priscilla W. Powell, MS, ${ }^{1}$ Kari L. Struemph, MS, ${ }^{1}$ Kathryn E. Maher, BS, ${ }^{1}$ Elizabeth M. Robinson, BA, ${ }^{1}$ Rusan Chen, $\mathrm{PhD},{ }^{2}$ Anil R. Kumar, $\mathrm{MD}^{1}$ and Clarissa S. Holmes, $\mathrm{PhD}^{1,2}$

${ }^{1}$ Virginia Commonwealth University, Richmond, VA and ${ }^{2}$ Georgetown University, Washington, DC.

Previous research has established demographic differences in metabolic control and chronic disease complications, yet few studies have examined differential demographic effects on psychosocial and disease care correlates of Alc. Group comparisons and regression analyses were conducted to investigate demographic differences in biological, psychosocial and disease care factors in youth with type 1 diabetes. Participants included 349 youth, aged $9-17$ years $(79.9 \%$ Caucasian, $71.3 \%$ lived with two biological parents, M SES=46.24), recruited from endocrinology clinics in two major metropolitan areas. The 24-Hour Diabetes Care Interview was administered to assess specific disease care behaviors and a test of diabetes knowledge was used to measure parent and child disease information. Independent sample t-tests confirmed commonly reported ethnic differences in A1c, where African American youth had significantly higher A1c levels $(M=8.84 \%)$ compared with Caucasian youth $(M=8.25 \%)$. African American youth reported poorer disease care behaviors including fewer intensive insulin regimens, less frequent blood glucose monitoring and poorer dietary composition of carbohydrates and fats. Additionally, African American youth and parents had lower disease knowledge scores than Caucasian youth and parents. However, hierarchical multiple regression analyses that controlled for confounding influences of SES and parent marital status revealed most disease care differences attributed to ethnicity were better explained by SES. The only significant ethnic group difference that remained was a difference in diabetes knowledge; ethnicity explained an additional $8.3 \%$ variance and emerged as a significant predictor of diabetes knowledge $(\beta=-.308, \mathrm{p}<.001)$ after controlling for SES and marital status. Results may inform future interventions for youth at risk of poor metabolic control; lower SES families may benefit from careful attention to diabetes knowledge to facilitate successful implementation of diabetes care.

CORRESPONDING AUTHOR: Priscilla W. Powell, MS, Psychology, Virginia Commonwealth University, Richmond, VA, 23284-2018; priscilla.powell@hotmail.com

\section{PSW-187}

RELATION BETWEEN FEMALE JAPANESE COLLEGE STUDENTS' BINGE EATING/PURGING AND THEIR BODY IMAGE DISSATISFACTION

Keisuke Yamatsuta, $\mathrm{PhD}^{1}$ and Shinobu Nomura, $\mathrm{MD}^{2}$

${ }^{1}$ School of Management and Information Science, SANNO University, Kanagawa, Japan and ${ }^{2}$ Faculity of Human Sciences, Waseda University, Saitama, Japan.

This study examined the relation between abnormal eating behavior, body mass index (BMI), and body image dissatisfaction in 554 female university students (Mean age: 19.22, SD: 1.01). Measures included demographic data, the Body Image Dissatisfaction Scale (BIDS) (Yamatsuta \& Nomura, 2007), and the Abnormal Eating Behavior Scale (AEBS) (Yamatsuta, Nakai, \& Nomura, 2009). BIDS measures the degree of body image dissatisfaction. It contains three lower-rank factors (divided into high and low groups on the basis of one's mean factor score) including "dissatisfaction with body plumpness," "dissatisfaction with other people's opinions of one's own body," and "dissatisfaction with one's own face." AEBS comprises three lower-rank factors including "uncontrollable intake of excessive food," (e.g., binge eating) "purging behavior," and "control of food intake" (e.g., diet behavior). According to the BMI classification, based on that of the Japan Society for the Study of Obesity (1994), 102 subjects $(18.41 \%)$ were thin, $406(73.29 \%)$ were normal, and 46 $(8.30 \%)$ were obese. First, we examined the relation between the BMI and the BIDS and AEBS factor scores; a single-factor analysis of variance of factor scores was conducted on each BMI classification. The results showed a significant main effect of BMI's class in all the factor scores excluding the "uncontrollable intake of excessive food" and "purging behavior" factor scores, and we can infer that the latter two factors have little relation to the BMI levels. Thus, abnormal eating behavior that resembles an eating disorder might have little relation to the actual body type. Second, we examined the relation between the "uncontrollable intake of excessive food," "purging behavior," and the BIDS 
factor scores; a t test on the former two factor scores from among the BIDS lower-rank factor group was conducted. The results indicated high scorers on these two factors in the high "dissatisfaction with body plumpness" group $(\mathrm{t}(531)=|5.08|, \mathrm{p}<.01$; $\mathrm{t}(531)=|2.14|, \mathrm{p}<.05)$; hence, purging behavior might not be only a consciousness of other people's opinions of one's own body but also the problem of self. This work was supported by KAKENHI (20730455).

CORRESPONDING AUTHOR: Keisuke Yamatsuta, PhD, SANNO University, Kanagawa, 259-1197; yamatsuta@pure.ocn. ne.jp

PSW-188a

DOES ATTENDING A SELF-MANAGEMENT PROGRAMME LEAD TO INCREASES IN SELF-MANAGEMENT SKILLS AMONG PATIENTS WITH LONG TERM HEALTH CONDITIONS?

Andy P. Turner, $\mathrm{PhD}$ and Louise Wallace, PhD FBPsS

Applied Research Centre in Health \& Lifestyle Interventions, Coventry University, Coventry, United Kingdom.

Background: Co-Creating Health $(\mathrm{CCH})$ is a national programme commissioned by The Health Foundation, (http://www.health.org. $\mathrm{uk} /$ ) which aims to embed self management support within mainstream health services in order to achieve improvements in long term health (LTH) conditions patients activation. One of the ways $\mathrm{CCH}$ aims to increase activation is through the provision of a clinician and lay delivered self-management programme (SMP). The SMP is a condition specific programme for patients with either depression, pain, COPD or diabetes. The SMP comprises 7 weekly 3 hour sessions during which patients receive training in problem solving, relaxation and collaborative agenda setting, goal setting and follow up in clinical encounters.

Aims: The aim was to determine whether patients report an increase in using a range of self-management skills after attending the SMP

Methods: The Health Education Impact Questionnaire (hei-Q) is a generic eight scale questionnaire designed to measure selfmanagement skills/ outcomes associated with attending health education/self-management courses. Participants are asked to rate items on a 4 point likert scale ranging from "strongly disagree" (1) to "strongly agree" (4). Higher scores represent higher levels of self-management skills. Thirty patients completed the hei-Q prior to and 6 months after attending the SMP.

Findings: After attending SMP patients reported a significant improvements in 4/8 subscales: Positive and active engagement in life; Emotional well-being; Constructive attitudes and approaches (all $\mathrm{p}=<0.01)$ and Skill and Technique Acquisition $(\mathrm{p}=0.03)$.

Discussion: Patients reported higher levels of self-management skills after attending the SMP. Further research involving larger sample sizes will determine whether greater use of self-management skills has a positive impact on health-related quality of life.
CORRESPONDING AUTHOR: Andy P. Turner, PhD, Applied Research Centre in Health \& Lifestyle Interventions, Coventry University, Coventry, CV1 5FB; a.turner@coventry.ac.uk

PSW-188b

USING PUBLIC HEALTH PRACTICE AND PARTICIPATORY RESEARCH PRINCIPLES TO CHANGE NUTRITION ENVIRONMENTS IN PUBLIC, LOW-INCOME SCHOOLS: THE HEALTHY ONES STUDY

Karen J. Coleman, $\mathrm{PhD},{ }^{1}$ Maggie Shordon, $\mathrm{MPH},{ }^{1}$ Magdalena E. Pomichowski, MA, ${ }^{1}$ Christela L. Castro, MPH, ${ }^{1}$ Susan L. Caparosa, MA, ${ }^{1}$ Asherlev T. Santos, MPH, ${ }^{2}$ Ruby Lopez, MPH, ${ }^{2}$ David A. Dzewaltowski, $\mathrm{PhD}^{3}$ and Carol A. Macera, $\mathrm{PhD}^{2}$

${ }^{1}$ Research and Evaluation, Southern California Permanente Medical Group, Pasadena, CA; ${ }^{2}$ Graduate School of Public Health, San Diego State University, San Diego, CA and ${ }^{3}$ Kinesiology, Kansas State University, Manhattan, KS.

Introduction: The Healthy ONES (Options for Nutrition Environments in Schools) study was conducted to determine the effects of changing public school nutrition environments on obesity rates in children.

Intervention: The intervention was designed using systems theory, ecological models, and community based participatory research (CBPR) principals. The following environments were targeted for intervention: before and after school waiting areas, outdoor recreation areas, gyms/cafeterias, break rooms, front offices, classrooms, and administrative offices. Using the Institute for Healthcare Improvement's Plan-Do-Study-Act (PDSA) rapid improvement cycle process, we worked with stakeholder groups within each school to target small, temporary changes in each environment identified for change.

Results: Environmental changes included: a "fruit at recess" program that eliminated outside food during morning recess and substituted cut fruit instead; jog-a-thon and prize walk fundraisers instead of cake walks and bake sales; using treasure chests with nonfood rewards in the classroom and principals' offices; adding vegetables to traditional lunch entrees; and cutting all fruit offered during school meals. In addition to extensive behavioral observation, we measured the heights and weights of 457 children ( $2 \mathrm{nd} /$ 3 rd graders) in year 1 and were able to measure 367 in year 2 (80\% retention rate). Although not significant, obesity rates (> 95th\% BMI) did not change for intervention schools $(22 \%$ to $21 \%$ ) and increased in control schools (21\% to $24 \%)$. Year 3 measures will be done by October, 2010 .

Conclusion: Using a CBPR approach resulted in substantial changes in the school nutrition environment which may lead to meaningful changes in childhood obesity rates.

CORRESPONDING AUTHOR: Karen J. Coleman, PhD, Research and Evaluation, Southern California Permanente Medical Group, Pasadena, CA, 91101; Karen.J.Coleman@kp.org 
PSW-188c

OVERWEIGHT TRAJECTORIES AND PSYCHOSOCIAL

ADJUSTMENT DURING PUBERTAL TRANSITION

Bin Xie, $\mathrm{PhD},{ }^{1}$ Cindy Lin, $\mathrm{BS}^{1}$ and Elizabeth J. Susman, $\mathrm{PhD}^{2}$

${ }^{1}$ School of Community and Global Health, Claremont Graduate University, San Dimas, CA and ${ }^{2}$ Department of Biobehavioral Health, The Pennsylvania State University, State College, PA.

Background: Scant efforts were made to investigate overweight trajectories and their links to multiple psychosocial adjustment problems during the period of pubertal transition. In this study we conducted a secondary analysis with data from the multisite Eunice Kennedy Shriver National Institute of Child Health and Human Development (NICHD) Study of Early Child Care and Youth Development (SECCYD) with the application of state of the art analytic approaches of the group-based growth mixture and mixed-effect models.

Methods: Longitudinal data consisted of repeated measures of weight, height, broad-band behavior problems (i.e. internalizing and externalizing problems) and narrow-band syndrome subscales (i.e. withdrawn, somatic complaints, anxious/depressed, social problems, thought problems, attention problems, delinquent behavior, and aggressive behavior) in 1,364 youths during the period from age nine to age fifteen.

Results: Three trajectory patterns were identified from the whole cohort: never/rarely overweight $(59.5 \%)$, late start/light overweight (12.1\%), and chronically/heavy overweight (28.4\%). After adjustments for gender, ethnicity, income-to-needs ratio based on federal poverty guidelines, and categorical timing of pubertal onset based on two dimensions of boys (genital and pubic hair) and girls (breast and pubic hair) development, youths with chronically/heavy overweight trajectory pattern had significantly worse scores of internalizing problems as well as syndrome subscales of somatic complaints, social problems and withdrawn than youths with never/rare overweight trajectory pattern.

Conclusions: Study findings helped advance knowledge on the distinct developmental trajectory patterns of overweight and their associations with multiple behavior problems in adolescents during pubertal transition.

CORRESPONDING AUTHOR: Bin Xie, PhD, School of Community and Global Health, Claremont Graduate University, San Dimas, CA, 91773; bin.xie@cgu.edu

\section{PSW-188d}

WHAT ARE THE BELIEFS OF HEALTHCARE PHYSICIANS ABOUT OBESITY? A SYSTEMATIC REVIEW

Ângela Maia, Doctorate and Filipa V. Teixeira, master

School of psychology, University of Minho, Braga, Portugal.

Background: Experts affirmed that some health professionals are not taking the issue of obesity as seriously as they should, compromising the success of the treatment of obese people.
Objectives: This study tries to synthesize recent research that has explored the beliefs, attitudes and practices of health professionals about obesity.

Method: The words "healthcare physicians, obesity, beliefs, attitudes, intervention, referral practices, counselling, general practitioners, advices, competency, knowledge and stigma" were entered in databases such as PubMed, Elsevier and PsycINFO. Twenty studies were found from 1999 trough 2009 and included in analyses.

Results: Physicians are negative about their own role in obese treatment. Although they believed it was part of their job to advise obese patients on the health risks of obesity, the majority of doctors thought they had not made any difference in getting their patients to make long term changes in lifestyle. They are sceptical about the effectiveness of available interventions and only few doctors refer patients to behaviour therapy or to obesity surgery.

Conclusions: This issue should be taken in account during physicians' education and in planning multidisciplinary teams of intervention in obesity, because physical doctors should be aware of how their own beliefs and attitudes will influence their behavior on the managing of obesity, compromising therefore the adherence and the successful in obesity treatment.

Key-Words: Beliefs, attitudes, healthcare physicians, obesity

CORRESPONDING AUTHOR: Filipa V. Teixeira, master, School of psychology, University of Minho, Braga, 4710-057; filipa.v. teixeira@gmail.com

PSW-188e

PSYCHOSOCIAL \& CULTURAL VALUES RELATED TO DIET \& EXERCISE

Nafanua Braginsky, $\mathrm{PhD}$, Jillian Inouye, $\mathrm{PhD}$ and Richard Arakaki, MD

Nursing, University of Hawaii Manoa, Honolulu, HI.

Aims: The purpose of this study was to explore psychosocial and cultural values related to dietary and physical activity practices of Asians and Pacific

Islanders (API) with type 2 diabetes.

Methods: Content analysis was conducted with information collected from focus groups that totaled 15 men and women of API descent, ages 18-76, participating in "The Study of Cognitive Behavioral Interventions in Diabetes Self-Management," project. Focus group discussions were recorded and transcribed verbatim. Data was analyzed for categories and themes using the social ecological framework. NVivo 8 computer software was utilized for management of data.

Results: Psychosocial themes related to dietary practices included depression, denial, happiness, self-control, and awareness of complications. Cultural values related to eating, such as upbringing, social events, food variety, food portions, and reciprocity were identified. Underlying medical and physiological conditions limited physical activities of some participants in the study. 
Psychosocial and cultural values that influenced physical activities were motivation, family values and gender.

Conclusions: Results support utilization of the social ecological framework when attempting to understand the health behavior of API. The families,environment, and the local culture greatly affected the dietary and physical activities of participants with type 2 diabetes. Empowerment with knowledge and support would allow individuals and their families to take ownership of their health care and thereby equipping them with tools to develop nutritionally and physically sound health habits to control diabetes.

CORRESPONDING AUTHOR: Nafanua Braginsky, PhD, Nursing, University of Hawaii Manoa, Mililani, HI, 96789; nafanua@, hawaii.edu

\section{PSW-188f}

ELUCIDATING THE PRODIABETIC IMPACT

OF DEPRESSION: A PRELIMINARY TEST OF THE DAIR (DEPRESSION-ASSOCIATED INSULIN RESISTANCE) HYPOTHESIS

Mariska Bot, MSc, ${ }^{2}$ Patrick J. Lustman, $\mathrm{PhD},{ }^{1}$ Kenneth Freedland, $\mathrm{PhD}^{1}$ and Robert Carney, $\mathrm{PhD}^{1}$

${ }^{1}$ Tilburg University, Tilburg, Netherlands and ${ }^{2}$ Psychiatry, Washington University School of Medicine, St. Louis, MO.

Depression and insulin resistance (IR) each are significant predictors of the development and progression of type 2 diabetes (T2DM), and are evident years in advance of the frank hyperglycemia of diabetes. Depression and insulin resistance (IR) each are significant predictors of the development and progression of type 2 diabetes (T2DM) and are evident years in advance of the frank hyperglycemia of diabetes. Depression is associated with factors that promote IR including inactivity, obesity, and hypercortisolemia. The purpose of the present study was to test the hypothesis of depression-associated insulin resistance (DAIR). Seventy-six persons (mean age $52 \pm 10$ years, $76 \%$ female, $39 \%$ Caucasian) with a provisional diagnosis of T2DM (i.e., not taking antihyperglycemic medication and meeting ADA criteria per study OGTT) were enrolled. Major depressive disorder (MDD) was diagnosed using DSM-IV criteria. Depression symptom severity was assessed with the Beck Depression Inventory (BDI). Sixteen patients could be classified as depressed (i.e. DSM-IV MDD and and a BDI score $\geq 16$ ) and 52 could be classified as non-depressed (i.e. no DSM-IV MDD and a BDI score $<16$ ). IR was derived from anthropometric measurements [body mass index (BMI)] and OGTT measurements [HOMA-IR, area under the curve for insulin (AUC-I)]. IR was uniformly higher but not statistically different in the depressed vs. nondepressed groups (BMI: $41.4 \pm 6.6$ vs $37.5 \pm 8.4 \mathrm{~kg} * \mathrm{~m}-2, \mathrm{p}=0.09$; HOMA-IR: $8.0 \pm 5.6$ vs $5.6 \pm 2.8, \mathrm{p}=0.13$; AUC-I: $180 \pm 123$ vs 135 $\pm 65 \mathrm{uU} * \mathrm{~mL}-1 * 2 \mathrm{~h}, \mathrm{p}=0.20)$. In regression analyses with baseline glucose as covariate, depression symptom severity was related to BMI $(p=0.02)$ and trended toward prediction of HOMA-IR, and AUC-I ( $p=0.13$ and $p=0.10$, respectively). These preliminary data support the plausibility of the DAIR hypothesis and the need for larger and more definitive studies.
CORRESPONDING AUTHOR: Patrick J. Lustman, PhD, Psychiatry, Washington University School of Medicine, St. Louis, MO, 63110; lustmanp@wustl.edu

PSW-188g

WHAT MAY EXPLAIN THE UNSUCCESSFULNESS OF THE LAPAROSCOPIC ADJUSTABLE GASTRIC BANDING? A QUALITATIVE STUDY

Ângela Maia, Doctorate and Filipa V. Teixeira, master

School of psychology, University of Minho, Braga, Portugal.

Background: The Laparoscopic Adjustable Gastric Banding has been considered the most effective intervention for morbid obesity, with higher success rates associated with this procedure. However, it appears that the results are not always kept for a long period of time, confirming the results of recent studies that found failure in $20 \%$ of bariatric surgeries.

Objective: This study tries to understand factors related with failures, exploring obese expectations and their understanding of their role along this process.

Method: Ten morbid obese were interviewed about the way they experienced their bariatric surgery, and the reasons and meanings of weight regain. Grounded theory was used to analyze data.

Results: Speech elaboration is poor, emotional words are rare and impersonal mode is frequent. Main categories suggest a psychological "fight" between the individuals and food, a fight that sometimes they win with sacrifices, but other times they loose because their lack of control. Lifestyle changes needed to accomplish the objectives are perceived ambiguously between no responsibility in the treatment and the recognition of the need to be active on the process.

Conclusions: Unsuccessfulness in bariatric surgery seems to be related with defeat in the fight with food which happens in moments of greater emotionality. This could be related with a lack of other coping strategies different from this one, contributing therefore to the regain of weight.

CORRESPONDING AUTHOR: Filipa V. Teixeira, master, School 4872 of psychology, University of Minho, Braga, 4710-057; filipa.v. 4873 teixeira@gmail.com

\section{PSW-188h}

WHAT IS RELATED TO FOOD INTAKE IN REAL DAILY LIFE?: A STUDY USING ECOLOGICAL MOMENTARY ASSESSMENT AND PDA-BASED FOOD RECORDING SYSTEM

Hiroe Kikuchi, MD, PhD, ${ }^{1,2}$ Kazuhiro Yoshiuchi, MD, PhD, ${ }^{3}$ Shuji Inada, $\mathrm{MD},{ }^{3}$ Gen Komaki, $\mathrm{MD}, \mathrm{PhD}^{1}$ and Yoshiharu Yamamoto, $\mathrm{PhD}^{2}$

${ }^{1}$ Department of Psychosomatic Research, National Institute of Mental Health, National Center of Neurology and Psychiatry, Tokyo, Japan; ${ }^{2}$ Educational Physiology Laboratory, Graduate School of Education, The University of Tokyo, Tokyo, Japan and ${ }^{3}$ Department of Psychosomatic Medicine and Stress Sciences, Graduate School of Medicine, The University of Tokyo, Tokyo, Japan.

Eating behavior is important for health education in healthy people as well as for treatment in patients with various diseases. Although 
psychosocial factors have been suggested to affect eating behavior, most previous studies were either in laboratory settings or with recalled self-reports. Therefore, valid relationships between food intake and psychosocial factors in daily life have not been fully understood. Recently, ecological momentary assessment (EMA), in which subjects record their momentary symptoms using portable computers as electronic diaries, has been used as an appropriate method avoiding recall bias. In addition, we have developed and validated personal digital assistant (PDA)based food diary with food photographs. The aim of this study was to investigate the relationships between food intake and psychosocial factors in healthy people in daily life, using EMA and the PDA-based food diary. Twenty subjects (age 23.6 44.2 yo) wore a watch-type computer for a week and recorded their momentary psychological stress, mood states and situational information into it several times a day including just before and after meals. They also recorded into the PDA-based food diary everything that they ate and drank. Multilevel modeling analyses revealed that energy intake when dining out was significantly more than that in the other situations $(p=0.049)$ after controlling for meal types and that momentary depressive mood just before the meal was negatively associated with energy intake only for dinner $(p=0.045)$. The results suggested possible effects of situational and psychological factor on energy intake with ecological validity and nutritional accuracy.

CORRESPONDING AUTHOR: Hiroe Kikuchi, MD, PhD, Department of Psychosomatic Research, National Institute of Mental Health, National Center of Neurology and Psychiatry, Tokyo, 1878553; kikuchih-tky@umin.ac.jp

\section{PSW-188i}

\section{TRENDS IN THE PREVALENCE OF DIABETES}

AND OBESITY IN PENNSYLVANIA ADULTS, 1995 - 2005

Oralia G. Dominic, $\mathrm{PhD}, \mathrm{MS}, \mathrm{MA},{ }^{1}$ Linda A. Wray, $\mathrm{PhD},{ }^{2}$ Eugene J. Lengerich, VMD, ${ }^{1}$ Frank Ahern, $\mathrm{PhD}^{2}$ and Jan $\mathrm{S}$. Ulbrecht, $\mathrm{MD}^{2}$

${ }^{1}$ Public Health Sciences, Penn State Milton S. Hershey, Hershey, PA and ${ }^{2}$ Biobehavioral Health, Penn State, University Park, PA.

Background: This study examined in adult Pennsylvanians from 1995 to 2005 trends in the prevalence and sociodemographic distributions of diabetes; and the associations of diabetes with selected risk factors over time. Methods: BRFSS data collected between 1995 and 2005 were used for this study. Key Measures: Diabetes prevalence was assessed by self-report of physician diagnosis. Obesity was assessed by body mass index (BMI) computed from self-report of height and weight. Analyses: Statelevel data for diabetes and associated obesity prevalence from 1995 to 2005 were reported by each year. The sociodemographic factors (age, sex, race/ethnicity, income, education) and the known disease risk factor obesity were reported for 1995-2005. Logistic regression modeling was used to examine associations between diabetes, sociodemographic and disease risk factors at two time points (1995 and 2005). Results: Diabetes prevalence in Pennsylvania, which increased from $5.7 \%$ in 1995 to $8.1 \%$ in 2005 , followed national trends, but exceeded them each year by approximately $1 \%$. A pattern of obesity becoming a more dominant risk factor for diabetes emerged over this eleven year time period. Conclusion: Results of this study can be used to inform diabetes prevention strategies within the state.

CORRESPONDING AUTHOR: Oralia G. Dominic, PhD, MS, MA, Public Health Sciences, Penn State Milton S. Hershey, Hershey, PA, 17033; odominic@hes.hmc.psu.edu

PSW-188j

CONCERNS ABOUT BODY WEIGHT AND EATING DISORDERS AMONG PEOPLE WITH DIFFERENT BMI

Rocio Hernandez-Pozo, PhD Experimental Psychology

Graduate Studies, UNAM-FES Iztacala, Cuernavaca, Mexico.

Overweight is one of the main concerns in Public Health in Mexico. This study explores whether people of different BMI show different levels of anxiety, as measured with three different questionnaires, a 28-item Fear Checklist (Hernandez-Pozo, Calleja, Castillo, et al 2009), the Spanish translation of the Beck Anxiety Survey (Hernandez-Pozo, Castillo, Mata, et al 2010) and a questionnaire of recent potentially stressing events RPSE (Hernandez-Pozo, Cerezo, Macias, et al, 2010). 60 men and 60 women, ages 15 to 35 , participated in the study, with BMI ranging from 15.05 to 38.28 ; 56 were underweight-normal (G1), and 64 were overweight-obese (G2). A behavioral measure for anxiety towards body weight and eating disorders consisted of a computerized discriminative 8 minutes test based on the emotional version of the Stroop task where thematic and neutral words were used as sample stimulus. Interference was computed by subtracting latencies to neutral words from latencies to thematic words, of the same length and lexical probability. Before and after the Stroop task, blood pressure and heart rate were monitored Additional self-report measures of eating disorders were recorded, by means of the Spanish version of the EAT26, CAR and SCOFF questionnaires. Results showed a significant difference between groups for RPSE $(\mathrm{t}=6.062, \mathrm{p}<.0001)$ with higher scores for the heavier group, and for Beck index $(t=5.159, \mathrm{p}<.0001)$. Combining sex by BMI group significant differences were recorded for all three eating disorders questionnaires, with female heavier group scoring higher on CAR and SCOFF, and male lighter group scoring higher for EAT26. The puzzling finding was that no perceptual bias differences were registered for BMI groups by themselves nor combined by sex, that is, the only differences among them were in verbal self-reports. Heavier females exhibited the highest diastolic reactivity $(\mathrm{F}=8.05, \mathrm{df}=3, \mathrm{p}<.0001)$. We concluded that there is a gap between "talking about obesity and eating disorders" and the actual unconscious bias towards those themes, which might lead to postulate progressive stages of concerns about obesity and eating disorders, described by specific combinations of behavioral, physiological and verbal data. 
CORRESPONDING AUTHOR: Rocio Hernandez-Pozo, PhD Experimental Psychology, Graduate Studies, UNAM-FES Iztacala, Cuernavaca, 62100; herpoz@unam.mx

\section{PSW-188k}

LOW USE OF FRUITS AND VEGETABLES

AMONG ROMANIAN ADOLESCENTS FROM RURAL AREAS Floarea Mocean, MD, PhD ${ }^{1}$ Lucia Lotrean, MD, PhD, ${ }^{1}$ Sorina Poledna, $\mathrm{PhD},{ }^{2}$ Florina Harosa, $\mathrm{MD}^{1}$ and Raluca Andrada Buldus, student $^{2}$

${ }^{1}$ University of Medicine and Pharmacy, Cluj Napoca, Romania and ${ }^{2}$ UBB, Cluj Napoca, Romania.

The objective of this study is to assess the opinions and practices of Romanian students with regard to use of fruits and vegetables (other than potatoes). These information are needed in order to develop future programs for promoting healthy nutrition among Romanian adolescents. Anonymous questionnaires were filled in by 267 school students from rural areas (age 10/11, 12/13, respectively 14/15 years old) of one county of Romania in 2008 . A percentage of $47.4 \%$ of the students eat fruits less than 4 times/ week, while the consume of vegetables less than 4 times/ week was recognized by $62.4 \%$ of the students. Eating fruits and vegetables decreased with age. Moreover the bivariate correlation analyses showed that the lower use of fruits and vegetables was associated with insufficient knowledge regarding the importance of fruits and vegetables and the quantity, which should be consumed each day, as well as with lower intention of consuming more fruits and vegetables in the future. These results underline the importance of developing programs, which promote the use of fruits and vegetables among Romanian adolescents from rural areas, as an important component of a healthy nutrition.

CORRESPONDING AUTHOR: Lucia M. Lotrean, MD, PhD, University of Medicine and Pharmacy, Cluj Napoca, 400340; llotrean@umfcluj.ro

PSW-1881

PARENTAL PERCEPTION ABOUT THE RISK OF DEVELOPING OBESITY IN THEIR CHILDREN

Muntazbano Hassamo, Master Student, ${ }^{1}$ Miguel Trigo, PhD, ${ }^{1}$ Maria João Figueiras, $\mathrm{PhD}^{1}$ and Dália Marcelino, $\mathrm{PhD}$ Student ${ }^{2}$ ${ }^{1}$ Psychology, Instituto Piaget, Almada, Portugal and ${ }^{2}$ Pychology, University of Évora, Évora, Portugal.

Objective: The aim of this study is to assess parental perception against the risk of obesity developing in their children.

Method: This is a cross-sectional study in which 172 parents (143 mothers and 29 fathers) of children aged 2 to 13 , attending in Portuguese paediatric clinics. The measures assess sociodemographic characteristics, parental perception, factors associated with obesity, the BMI of children and parents, and the choice of the body image of the children by their parents.

Results: The results indicate that the majority of the parents perceive the risk of obesity in their children, however, mothers showed greater awareness than fathers. There is a significant correlation between the change in eating habits, the frequency of food intake, the avoidance and attraction to food. The majority of the parents indicated their children's weight as appropriate, which corresponded to their BMI, but they do not recognize the body image of their children. Only $40 \%$ of parents chose the images that corresponded to their children's BMI. The remaining parents chose images ranging from thinness to obesity. The BMI of the majority of the parents and children indicate that the parents and children have appropriate weight.

Conclusions: Most parents recognize the importance of nutrition and physical activity and their own role in eating behaviour and physical exercise habits for their children.

CORRESPONDING AUTHOR: Muntazbano Hassamo, Master Student, Instituto Piaget, Lisboa, 1705-105; mumihassamo@ gmail.com

PSW-188m

ACCURACY OF SELF-REPORTED ENERGY INTAKES IN LOW-INCOME URBAN 4TH GRADE MINORITY CHILDREN Oralia G. Dominic, PhD, MS, MA, ${ }^{1}$ Linda A. Wray, $\mathrm{PhD},{ }^{6}$ Jenny H. Ledikwe, $\mathrm{PhD},{ }^{8}$ Diane C. Mitchell, MS, ${ }^{2}$ Arthur E. Hernandez, $\mathrm{PhD},{ }^{4}$ Zenong Yin, $\mathrm{PhD},{ }^{5}$ Roberto $\mathrm{P}$. Trevino, $\mathrm{MD},{ }^{3}$ Jan $\mathrm{S}$. Ulbrecht, $\mathrm{MD}^{6}$ and Ali K. Ventura, $\mathrm{PhD}^{7}$

${ }^{1}$ Public Health Sciences, Penn State Milton S. Hershey, Hershey, PA; ${ }^{2}$ Nutritional Sciences, Diet Assessment Center, Penn State, University Park, PA; ${ }^{3}$ Bienestar Health Program, The Social \& Health Research Center, San Antonio, TX; ${ }^{4}$ Education, Texas A\&M University at Corpus Christi, Corpus Christi, TX; ${ }^{5}$ Kinesiology and Physical Education, University of Texas at San Antonio, San Antonio, TX; ${ }^{6}$ Biobehavioral Health, Penn State, University Park, PA; ${ }^{7}$ Nutrition, Monell Chemical Senses Center, Phildelphia, PA and ${ }^{8}$ Global Health, University of Washington, Seattle, WA.

We examined the accuracy of self-reported energy intake (rEI) in low-income, urban minority school-aged children at risk for obesity and associated diabetes utilizing a relatively new, simple previously published prediction equation for identifying inaccurate reports of dietary energy intake. Participants included 614 nine year old boys (51\%) and girls (49\%). Three 24-hour dietary recalls were collected. Children's height, weight (used to calculate BMI) and $\% \mathrm{BF}$ were measured. Physical fitness, reported family history of diabetes, and ethnicity were also collected. A previously published prediction equation was used to determine the validity of rEIs in these children to identify under-, plausible-, and overreporters. Additionally, we examined the question of whether there is a difference in reporting by sex, ethnicity, BMI and \%BF. On average, $18 \%$ of the children were at-risk of being overweight, $43 \%$ were already overweight at baseline, yet these children reported consuming fewer calories on average than recommended guidelines. Additionally, reported caloric intake in this cohort was negatively associated with BMI and \% body fat. Using the previously described methods $49 \%$ of participants were identified as under-reporters, while $39 \%$ and $12 \%$ were identified as 
plausible- and over-reporters, respectively. On average, children reported caloric intakes that were almost $100 \%$ of pER when the sedentary category was assigned; Inactivity and excessive energy intake are important contributors to obesity. With the rising rates of obesity and diabetes in children, accurate measures of EI are needed for better understanding of the relationship between energy intake and health outcomes

CORRESPONDING AUTHOR: Oralia G. Dominic, PhD, MS, MA, Public Health Sciences, Penn State Milton S. Hershey, Hershey, PA, 17033; odominic@hes.hmc.psu.edu

\section{PSW-188n}

NUTRITIONAL LABELLING TO PROMOTE HEALTHIER FOOD PURCHASING AND CONSUMPTION: PRELIMINARY RESULTS OF A SYSTEMATIC LITERATURE REVIEW

Rachel A. Crockett, PhD, ${ }^{1}$ Gareth Hollands, $\mathrm{PhD},{ }^{1}$ Susan Jebb, $\mathrm{PhD}^{2}$ and Theresa M. Marteau, $\mathrm{PhD}^{1}$

${ }^{1}$ Institute of Psychiatry, King's College London, London, United Kingdom and ${ }^{2}$ Human Nutrition Research Unit, Medical Research Council, Cambridge, United Kingdom.

There is increasing international concern about diets high in fat, sugar and salt that contribute to the burden of obesity, cardiovascular disease, diabetes and cancer. Nutritional labelling, especially of processed foods, is one population level intervention designed to achieve healthier food purchasing and consumption. A range of nutritional labels varying in information and format are being used but there is currently no consensus on the most effective labelling. A systematic literature review of the impact of nutritional labelling on behaviourally assessed food purchasing and consumption is in progress (Cochrane registration in progress). Pilot literature searches have identified 13 studies assessing behavioural outcomes and preliminary analyses conducted. Eight studies assessed the impact of labelling the calorie content of restaurant menu items and found no effect on purchasing from fast food restaurants but a reduction in calories purchased from non fast food restaurants and canteens. One study found more purchases of low fat options, labelled as such, from vending machines. Two studies assessed supermarkets purchases and, although finding no overall effect of nutritional labels, different effects of different labels were found. Specifically "traffic light" labels highlighting products with high levels of less healthy nutrients had no impact on purchasing compared to unlabelled products. In contrast, while "low trans fat" and "low calorie" labels resulted in more purchases of items thus labelled, "low fat" labels resulted in fewer purchases of items thus labelled. Finally, two related studies assessed the impact of "low fat" labels on consumption finding paradoxically greater consumption of products thus labelled with greater effects in those who were overweight. These results indicate that the impact of nutritional labels on food purchasing and consumption is modified by characteristics of the individual, of the food product labelled and of the label itself. The results further suggest that the purchase of healthier options will not necessarily result in healthier consumption. It is anticipated that the results of the full review will help to elucidate which labels most effectively promote healthy consumption across populations to inform the development of policy internationally.

CORRESPONDING AUTHOR: Rachel A. Crockett, PhD, Health Psychology, King's College London, London Bridge, SE1 9RT; rachel.crockett@kcl.ac.uk

PSW-1880

COACHING IS ASSOCIATED WITH INCREASED UTILIZATION OF HANDHELD COMPUTERS FOR WEIGHT LOSS AMONG LOWER INCOME WOMEN

Claudia R. Pischke, PhD, ${ }^{1}$ Jessica A. Whiteley, $\mathrm{PhD},{ }^{2}$ Sandy Askew, $\mathrm{MPH}^{3}$ and Gary G. Bennett, $\mathrm{PhD}^{1,3}$

${ }^{1}$ Harvard School of Public Health, Boston, MA; ${ }^{2}$ University of Massachusetts, Boston, MA and ${ }^{3}$ Duke University, Durham, NC.

Accumulating scientific evidence demonstrates that e-health strategies such as dietary self-monitoring through the use of handheld computers are effective in assisting individuals to lose weight. However, it is unknown whether interpersonal coaching promotes the utilization of handheld computers to self-monitor health behaviors and whether increased utilization is associated with increased weight loss. The aim of this study was to determine whether interpersonal coaching leads to increased utilization of handheld computers for self-monitoring and weight loss in lower income multi-ethnic women. Participant women, aged $43 \pm 10 \mathrm{yrs}$ among whom $85 \%$ were employed, $51 \%$ had a combined family income $<\$ 40,000,61 \%$ were married/cohabitating, $41 \%$ were African-American, 31\% White, and 28\% Hispanic were randomized to either: a) self-paced use of handheld system (eSolutions, $\mathrm{n}=29$ ); or b) use of the handheld system plus weekly interpersonal coaching sessions (eSolutions-Plus, $n=25$ ). At 12 weeks, tracking frequency was significantly higher in the eSolutions-Plus (mean: 36.6 days, SD: 20.9 days) compared to the eSolutions group (mean: 26.3 days, SD: 24.5 days, $\mathrm{p}<.05$; mean tracking total: 31.6 days, SD: 23.6 days, range: 0-79 days). Regardless of group assignment, higher tracking frequency was also associated with greater weight loss $(\mathrm{r}=-.45, \mathrm{p}<.001$; mean total weight loss: $-3.4 \mathrm{lbs}$, SD: $6.3 \mathrm{lbs}$, range: $-18-12$; e-Solutions: $-3.3 \mathrm{lbs}$, SD: $7.2 \mathrm{lbs}$; eSolutions plus: $-3.5 \mathrm{lbs}$, SD: $5.5 \mathrm{lbs}$; $\mathrm{p}=.919)$. To conclude, interpersonal coaching appears to foster the utilization of handheld computers for weight loss in lower income women.

CORRESPONDING AUTHOR: Claudia R. Pischke, PhD, Harvard School of Public Health, Boston, MA, 02115; claudia_pischke@dfci. harvard.edu

PSW-188p

BEHAVIOUR OF DIABETICS IN ECONOMIC GAMES

Maithili Jog, PhD, ${ }^{1}$ Anuja Joshi, BSc, ${ }^{1}$ Sumedha Kondekar, BSc, ${ }^{1}$ Prajakta Belsare, MSc, ${ }^{2}$ Saroj Ghaskadbi, $\mathrm{PhD}^{2}$ and Milind Watve, $\mathrm{PhD}^{3}$

${ }^{1}$ Dept. of Biotechnology, Abasaheb Garware College, Pune, India; ${ }^{2}$ Dept. of Zoology, University of Pune, Pune, India and ${ }^{3}$ IISER, Pune, Pune, India. 
An upcoming hypothesis about the origins of type II diabetes indicates that it has roots in behaviour rather than diet and metabolism. However, little efforts have been made so far to test whether there are detectable behavioural differences in type II diabetics as compared to age matched healthy controls. Ultimatum Game and its versions have been used to assess economic behaviour such as rationality or fairness in distribution of reward. Prior studies have shown that endocrine status of an individual affects the offers made as well as acceptance levels in the ultimatum game. Individuals with low testosterone levels or high serotonin levels were shown to give as well as accept low offers which is economically more rational. Since both testosterone and serotonin are key players in type
II diabetes, we examined whether the offers and acceptance levels of type II diabetics are different than age matched controls. A survey of 58 patients with type II diabetes and other metabolic syndrome disorders and 60 age matched healthy controls showed that the offers given by patients with type II diabetes, hypertension and cardiovascular diseases were significantly lower than the control. BMI was poorly correlated with the economic behaviour. Further in this sample, the ultimatum game offer was a better predictor of type II diabetes than BMI.

CORRESPONDING AUTHOR: Milind Watve, PhD, IISER, Pune, Pune, 411 021; milind@iiserpune.ac.in

\section{TOP FIFTEEN SCORING POSTER}

PSW-189

HOW QUICK CAN YOU FORGET "TUMOR”? HEALTH ANXIETY AND THE ABILITY TO REMOVE ILLNESS RELATED INFORMATION FROM WORKING MEMORY

Michael Witthöft, PhD, Martha Kuta, Student and Wolfgang Hiller, Prof Dr

Department of Clinical Psychology and Psychotherapy, University of Mainz, Mainz, Germany. Introduction: Persistent rumination about illness is considered as the leading symptom of health anxiety and hypochondriasis. Experimentally, this increased preoccupation with illness related information is reflected in prolonged attention allocation and better recognition memory for illness related stimuli. Yet, the cognitive-psychological mechanisms that underlie these alterations in cognition in health anxiety are poorly understood. In this respect, the ability to quickly and efficiently remove non-longer relevant information from working memory might be crucial. Method: Since a dimensional perspective of health anxiety is psychometrically and empirically superior to a dichotomous view (Ferguson, 2008), the study was based on a sample of college students $(\mathrm{N}=90)$ with varying levels of health anxiety. Cognitive, affective, perceptual, and behavioral aspects of health anxiety were assessed with the Multidimensional Inventory of Hypochondriacal Traits (Longley et al., 2005). The ability to remove illness related information from working memory was measured with the modified Sternberg task (e.g., Oberauer, 2005). In this task, illness related words (e.g., cancer, tumor) and matched neutral words (e.g., lamp, table) served as stimuli. The EZ-diffusion model (Wagenmakers, 2009) was used to simultaneously analyze response speed and accuracy data. Results: The ability to remove illness related words (but not neutral words) from working memory was negatively related to the behavioral facet of health anxiety $(r=-.27)$, i.e., higher levels of hypochondriacal behavior were associated with less efficient inhibition of illness related words. Conclusion: Behavioral and interpersonal aspects of health anxiety (i.e., seeking for reassurance and social support) were found to be associated with deficits in the ability to remove illness related information from working memory. People with higher health anxiety might use behavioral strategies to distract from ongoing cognition about illness. Alternatively, certain behavioral strategies that have been acquired during childhood and adolescence may increase the salience of health threatening information leading to difficulties in their "forgetting". 
CORRESPONDING AUTHOR: Michael Witthöft, PhD, Department of Clinical Psychology and Psychotherapy, University of Mainz, Mainz, 55122; witthoef@uni-mainz.de

\section{PSW-191}

PRELIMINARY DEVELOPMENT OF THE JAPANESE VERSION OF BODY IMAGE CONCERNS INVENTORY Masanori Tanaka, MA, ${ }^{1}$ Jun Tayama, $\mathrm{PhD},{ }^{2}$ Tastuyuki Arimura, $\mathrm{MA}^{3}$ and Masashi Sugawara, $\mathrm{PhD}^{1,4}$

${ }^{1}$ Center for Total Human Education and Child Welfare, Nagasaki University, Nagasaki, Japan; ${ }^{2}$ Center for Health and Community Medicine, Nagasaki University, Nagasaki, Japan; ${ }^{3}$ Department of Psychosomatic Medicine, Graduate School of Medical Sciences, Kyushu University, Fukuoka, Japan and ${ }^{4}$ Faculty of Education, Nagasaki University, Nagasaki, Japan.

The purpose of this study was to develop the Japanese Version of Body Image Concerns Inventory (J-BICI), which is a brief selfrating scale to assess a body dysmorphic concerns. We evaluated the factor structure, reliability and validity of J-BICI. Subjects were 1218 normal undergraduate $($ male $=709$, female $=518$ ) students. They completed the J-BICI and some other related questionnaires (Dysmorphic concerns Examination; DCE, Selfrating Depression Scale; SDS, Drive for Thinness and Bulimia subscale of Eating Disorder Inventory-91; abbreviated EDI-91, Japanese 32item short version of Padua Inventory; J-PI32). Factor analysis using promax lotation method was carried out and identified three interpretable factors. These factors were as follows: safety behavior for one's own appearance flaws (factor1), avoidant behavior from one's own appearance flaws (factor2), negative evaluation for one's own appearance (factor3). These factors had high degrees of internal consistency $(\alpha=.84-.91)$ and good test-retest reliability $(\mathrm{r}=.79-88, \mathrm{p}<.01 ; 3$ weeks interval). Female participants scored significantly higher on these three factors than male ones (factor $1: \mathrm{t}(1225)=-17.69, \mathrm{p}<.001$; factor2: $\mathrm{t}(1225)=-2.23, \mathrm{p}<.05$; factor3: $\mathrm{t}(1225)=-8.60, \mathrm{p}<.001)$. In addition, there were significantly correlation and discrimination between J-BICI and other related questionnaires. In this study, it was shown that J-BICI had good reliability and validity as a measure of body dysmorphic concerns.

CORRESPONDING AUTHOR: Masanori Tanaka, MA, Center for Total Human Education and Child Welfare, Nagasaki University, Nagasaki, 852-8521; m-tnk@nagasaki-u.ac.jp

PSW-192a

INCIDENCE RATE AND PREDICTORS OF IRRITABLE BOWEL SYNDROME: A 7-YEAR COHORT STUDY IN JAPAN

Yasushi Fujii, PhD, Shinobu Nomura, $\mathrm{PhD}$ and Jun Kanno, MA Human Sciences, WASEDA University, Tokorozawa, Japan.

Background: To investigate non-patient irritable bowel syndrome (IBS) change to IBS and to determine factors predictive of the onset of IBS, individual biological factors, psychological factors, behavioral factors, and environmental factors were examined.

Methods: The subjects were 97 non-patient IBS (male $=49$, female $=$ 48, average age:22.31 \pm 2.32 ), including 62 of the diarrheapredominant type and 35 of the constipation-predominant type selected from 1,409 university and technical college students by use of a questionnaire based on the Rome II diagnostic criteria. The subjects were followed for 7 years, and various characteristics and IBS symptoms were serially observed (28 times). The IBS incidence rate was calculated.

Results: During the 7 years, 61 non-patient IBS (59.17\%) changed to IBS: 42 diarrhea-predominant types and 19 constipationpredominant types. All IBS symptoms disappeared in 35 nonpatient IBS subjects $(33.95 \%)$. According to quantification method II (discriminant analysis), seven factors (cognitive appraisal, stressor, stress coping style, three kinds of lifestyle habits and psychologically abuse) were adopted as a predictive model for IBS incidence and were confirmed as predictive of IBS. Conclusions: The results of this research show that non-patient IBS is a changeable state that can change into IBS or persons without symptoms. Most of the non-patient IBS subjects who became asymptomatic had had symptoms for six months or less. Furthermore, the longer a non-patient IBS subject had symptoms, the higher the risk of a change to IBS became. The findings suggest the usefulness of identifying and approaching non-patient IBS as early as possible to prevent the onset of IBS. It must be noted that the persons surveyed in the present study had only the diarrhea-predominant and constipation-predominant types. Therefore, the findings of the present study are limited only these two types. Further study including the mixed type is needed.

CORRESPONDING AUTHOR: Yasushi Fujii, PhD, WASEDA University, Tokorozawa, 359-1192; fu-ya29@aoni.waseda.jp

PSW-192b

THE LIKELIHOOD OF RECEIVING THE DRUG OR PLACEBO DETERMINES THE SIZE OF THE DRUG OR PLACEBO RESPONSE IN IBS TRIALS

Paul Enck, $\mathrm{PhD},{ }^{1}$ Lene Vase, $\mathrm{PhD}^{2}$ and Sibylle Klosterhalfen, $\mathrm{PhD}^{1}$

${ }^{1}$ Psychosomatic Medicine, University Hospitals, Tuebingen, Germany and ${ }^{2}$ Dept. of Psychology, University of Aarhus, Aarhus, Denmark

While placebo controlled trials are common in the evaluation of novel drugs, they have been questioned because of the ethical problems of withholding effective treatment in (pain) patients. As alternative, comparator controlled trials ave been proposed. Both however, differ in the likelihood of providing effective treatment: $50 \%$ and $100 \%$, resp. Methods: We investigated whether the likelihood of receiving drug or placebo determines the size of the drug response and the size of the placebo response (DR, PR) in treatment studies of irritable bowel syndrome (IBS). Results: In 92 published randomized placebo-controlled trials with more than 22.000 patients with IBS, 16 had a drug:placebo ratio of $2: 1$ or greater while the remaining were powered 1:1. Computing the 
overall DR and PR, both responses were lower with higher likelihood of receiving the drug (ratio $2: 1$ or higher), but the difference between DR and PR ("therapeutic gain") decreased. Ten of the 16 studies (4 with a 2:1 ratio, 3 with $3: 1$, and 3 with 4:1; total $n=6484)$ were compared to 10 studies $(n=3782)$ of similar size ( $>80$ patients per study arm) and design (drug trials with novel compounds) based on a standardized patient definition (according to Rome criteria), and with comparable (dichotomous) endpoints. Again, the placebo response decreased with increasing likelihood of receiving a drug from $37.6 \%$ (1:1) to $29.1 \%(4: 1)$. Similarly, the drug response dropped from $49.8 \%$ (1:1) to $42.4 \%$ (4:1) (significant decrease for DR $(\mathrm{p}=.035)$, but not for PR). Discrimination between DR and PR was best for 1:1 trials. Correlation between the drug response and the placebo response across all 20 trials was significant (Pearsons $\mathrm{r}=.781, \mathrm{p}<.001$ ), but was higher in $1: 1$ trials $(n=10 ; r=.91, p<.001)$ than in trials with a higher drug-to-placebo ratio $(\mathrm{n}=10, \mathrm{r}=.69, \mathrm{p}=.027)$. Conclusion: The higher the likelihood of receiving active treatment (a drug as compared to a placebo), the lower the response rate to both placebo and the drug in IBS, at the expense of a poorer discrimination between DR and PR. This is contrary to respective data from depression trials. (Supported by a grant from DFG, En $50 / 25)$.

CORRESPONDING AUTHOR: Paul Enck, PhD, Psychosomatic Medicine, University Hospitals, Tuebingen, 72076; paul.enck@ uni-tuebingen.de

PSW-193

\section{DO BAD RELATIONSHIPS MAKE US UNHEALTHY?} GENDER DIFFERENCES AMONG YOUNG COUPLES

Trace Kershaw, $\mathrm{PhD}$ and Anna Arnold, Mph

Yale University, New Haven, CT.

Background: Much of public health research focuses on individual mechanisms of health (e.g., behavior, genetics), while few studies look at broader contexts of health behavior such as dyadic influences. Romantic partners and relationship factors can influence one's health and well being above and beyond individual factors.

Methods: Preliminary analyses were conducted among 200 couples $(\mathrm{N}=400)$ from low income OB-GYN clinics in the U.S. Women and men were 18.7 and 21.3 years on average, and the average relationship length was 2.3 years. $77 \%$ of participants were Black or Hispanic. We assessed relationship satisfaction, mental health and physical health for both members of a couple. Dyadic analyses using multiple regression assessed actor and partner effects for both men and women. All analyses controlled for confounders.

Results: Men and women shared predictors of relationship satisfaction, including greater relationship equity $(\mathrm{Bm}=.28$, $\mathrm{Bw}=.44, \mathrm{ps}<.05)$, less avoidant attachment $(\mathrm{Bm}=-.32, \mathrm{Bw}=-.22$, $\mathrm{ps}<.05)$, greater feelings of love $(\mathrm{Bm}=.31, \mathrm{Bw}=.14$, $\mathrm{ps}<.05)$, and less partner anxiety attachment $(\mathrm{Bm}=-.14, \mathrm{Bw}=-.14, \mathrm{ps}<.05)$.

Among both men and women, relationship satisfaction related to less depression $(\mathrm{Bm}=-.38, \mathrm{Bw}=-.52$, ps $<.05)$, less stress $(\mathrm{Bm}=$
$-.43, \mathrm{Bw}=-.36, \mathrm{ps}<.05)$, and greater mental quality of life $(\mathrm{Bm}=.39, \mathrm{Bw}=.37, \mathrm{ps}<.05)$. Relationship satisfaction related to greater physical quality of life for men $(\mathrm{B}=.18, \mathrm{p}<.05)$, but not for women $(\mathrm{B}=.08, \mathrm{p}=\mathrm{ns})$. Comparisons showed a stronger association between relationship satisfaction and depression for women $(\mathrm{B}=-.52$ vs. $-.38, \mathrm{p}<.05)$ and physical quality of life for men $(\mathrm{B}=.18$ vs. $.08, \mathrm{p}<.05)$. No partner effects were significant for mental and physical outcomes.

Conclusion: Relationship satisfaction was associated with better mental health outcomes for men and women, and better physical health outcomes for men. While relationship satisfaction had a greater influence on depression among women, it had a greater influence on the physical quality of life for men, suggesting that relationship factors may play a different role in mental and physical functioning across gender.

CORRESPONDING AUTHOR: Trace Kershaw, PhD, Yale University, New Haven, CT, 06510; trace.kershaw@yale.edu

\section{PSW-194}

DETERMINANTS OF PRE- AND POSTPARTUM

CHILDBIRTH FEAR IN A COHORT OF CANADIAN WOMEN Deborah Da Costa, PhD, ${ }^{1,2}$ Nancy Verreault, PhD Candidate, ${ }^{2}$ Maria Dritsa, $\mathrm{PhD}^{2}$ and Samir Khalifé, $\mathrm{MD}^{3}$

${ }^{1}$ Medicine, McGill University, Montreal, QC, Canada; ${ }^{2}$ Division of Clinical Epidemiology, McGill University Health Centre, Montreal, QC, Canada and ${ }^{3}$ Department of Obstetrics and Gynecology, McGill University Health Centre, Montreal, QC, Canada.

Fear of childbirth in pregnant women has been associated with prior and future complicated deliveries, difficulties in the motherinfant relationship and risk of postpartum depression. The aim of this study was to investigate pre- and postpartum levels of childbirth fear in a cohort of pregnant women and examine factors associated with elevated fear. Three hundred sixty-one pregnant women (mean age $=32, \mathrm{SD}=4.5$ ) completed questionnaires measuring: anxiety sensitivity, state-anxiety, fatigue, depression, social support, life events and parental self-efficacy in the third trimester of pregnancy. The Wijma Delivery Expectancy/Experience Questionnaire (W-DEQ) was used to assess childbirth fear at the preand postpartum assessment. Two-hundred sixty-three women returned their questionnaires at the 4-6 weeks postpartum assessment. Demographic variables (i.e. parity, education) and complications in the current pregnancy or in previous deliveries were also collected. Low levels of childbirth fear during pregnancy was reported by $25.8 \%(n=93)$ of the women, with $48.8 \%(n=176)$ moderately fearful and 25.5\% ( $=92)$ highly fearful. At the postpartum assessment $37.6 \%(n=99)$ reported low levels of fear, $44.1 \%(n=116)$ reported moderate fear levels and $18.3 \%(n=48)$ were highly fearful. Overall, fear levels in the postpartum decreased significantly by a mean of 6 points compared to the prepartum assessment $(\mathrm{t}(262)=4.5, \mathrm{p}<.001)$. The regression model examining prepartum childbirth fear showed that lower parental self-efficacy $(p<0.001)$, higher state-anxiety $(\mathrm{p}<0.001)$, more life events $(\mathrm{p}=0.001)$ and greater anxiety 
sensitivity $(p=0.003)$ were significant independent predictors of higher prepartum $\mathrm{W}-\mathrm{DEQ}$, explaining $44 \%$ of the variance. In addition to prepartum WEQ scores $(\mathrm{p}<0.001)$, postpartum childbirth fear was associated with greater anxiety sensitivity $(p=0.001)$, lower parental self-efficacy $(p=0.005)$ and delivery by caesarean section $(p=0.014)$, together explaining $36 \%$ of the variance. Our findings underscore the importance of routine assessment of childbirth fear during pregnancy and highlights areas which can be targeted in order to manage childbirth fear. Women expressing fears may benefit from interventions aimed at improving self-efficacy and reducing stress to enhance the pregnancy and childbirth experience.

CORRESPONDING AUTHOR: Deborah Da Costa, PhD, Medicine, McGill University, Montreal, QC, H3A 1A1; deborah. dacosta@mcgill.ca

PSW-195

GET OUT OF YOUR MIND AND INTO YOUR BODY: MINDFULNESS AND THE TREATMENT OF FEMALE SEXUAL DYSFUNCTION

R. Gina Silverstein, BA and Willoughby Britton, $\mathrm{PhD}$

Brown University, Providence, RI.

Introduction: Recent studies show Female Sexual Dysfunction can be caused by an overemphasis on secondary self-analysis rather than direct experience of arousal. Training in mindfulness has been associated with decreases in self-judgment and enhanced body awareness. This controlled study assesses the impact of mindfulness training on reaction time for ratings of arousal to erotic stimuli and related psychological barriers to female sexual function.

Methods: Fifty-six college students (female=29) participated in either a 15-week course with mindfulness "meditation labs" in addition to didactic class time $(n=35)$ or an active control class $(n=21)$ with a similar format (didactic and experiential laboratory sessions). All participants engaged in computerized neuropsychological performance batteries pre- and post- intervention where they rated erotic and non-erotic photographs in terms of arousal and valence. Scores and reaction times of ratings were recorded. Participants also completed the Five Factors of Mindfulness Questionnaire (Baer et al., 2006), Mindful Attention Awareness Scale (Brown et al., 2003), the Brief Symptom Inventory (Derogatis et al., 1983) and The Self Compassion Scale (Neff, 2003) at the beginning and end of the semester.

Results: At baseline, female participants had a significantly slower reaction time in response to erotic photographs than males, $\mathrm{t}(52)=$ $2.5, \mathrm{p}=.013$. At post-intervention testing, females who participated in the meditation labs significantly faster reaction times, $\mathrm{t}(12)=2.8$, $\mathrm{p}=.016$. This change was more than twice that of females in the control group (723.0 vs. 306.7 milliseconds). Scores related to selfjudgment also decreased significantly more in female meditators than controls, $\mathrm{F}(1,15)=4.8, \mathrm{p}=.045$. The faster reaction times were correlated with this decrease in self-judgment $(r=.70, p=.012)$ as well as an increase in self-kindness $(r=.84, \mathrm{p}=.001)$. Additionally, female meditators showed improved scores in mindfulness, depression, anxiety, and combined clinical symptoms measures (all $\mathrm{p}<.05$ ), but controls did not.

Conclusions: These results suggest that mindfulness training is associated with improvements in many of the psychological barriers to female desire, arousal, and satisfaction. Most notably, it was associated with faster reaction times when reporting arousal that correlated with decreases in self-judgment. Female meditators were ostensibly enabled to react to erotic stimuli with decreased judgment and self-evaluation, thus becoming more engaged in the direct experience of their own arousal. These data strongly support mindfulness as a promising treatment for FSD.

CORRESPONDING AUTHOR: R. Gina Silverstein, BA, Brown University, Providence, RI, 02906; gina_silverstein@brown.edu

PSW-196a

GENDER DIFFERENCES IN ASSOCIATIONS

BETWEEN PSYCHOSOCIAL FACTORS AND ABERRANT CRYPT FOCI AMONG PATIENTS AT RISK FOR COLON CANCER Katherine O'Leary, MA, ${ }^{1}$ Dean G. Cruess, PhD ${ }^{1}$ Caitlin Burbridge, MA, ${ }^{1}$ Devon Pleau, $\mathrm{MS}^{2}$ and Daniel Rosenberg, $\mathrm{PhD}^{2}$ ${ }^{1}$ Psychology, University of Connecticut, Storrs, CT and ${ }^{2}$ Neag Comprehensive Cancer Center, University of Connecticut Health Center, Farmington, CT.

Purpose: The primary aim of this study was to evaluate the association between key psychosocial factors and aberrant crypt foci (ACF), a measure of colonic mucosa and perhaps a putative biomarker of colon cancer risk. We hypothesized that higher levels of depression, stress, and colon cancer worry (and lower levels of social support) would be associated with greater numbers of ACF among individuals at heightened risk for colon cancer. Sample and Methods: Participants were self-referred or referred by physicians from the Gastroenterology Clinic or Colon Cancer Prevention Program at the University of Connecticut Health Center for routine colonoscopy. A total of 93 individuals were consented (51\% women; $49 \%$ men). Within two weeks prior to colonoscopy, participants completed measures assessing the psychosocial factors noted above. Individuals were also examined for $\mathrm{ACF}$ detection at colonoscopy. Regression beta weights were used to examine the association between the psychosocial variables and ACF. Results: Among women, higher levels of depression were associated with greater numbers of ACF $(\beta=.731, p=.032)$ after controlling for age, race/ethnicity, stress, social support, and colon cancer worry. Among men, lower levels of social support were associated with greater numbers of ACF $(\beta=-.398, p=.050)$ after controlling for age and race/ethnicity. These results suggest that although colon cancer affects men and women equally with regards to morbidity and mortality rates, there are important gender differences in how psychosocial factors impact colonic mucosa and perhaps colon cancer risk. Psychosocial interventions aimed at targeting these types of factors are warranted but need to consider the role of gender. 
CORRESPONDING AUTHOR: Katherine O'Leary, MA, Psychology, University of Connecticut, Vernon, CT, 06066; oleary. katie@gmail.com

\section{PSW-196b}

GENDER PATTERNS OF SOCIOECONOMIC DIFFERENCES IN PREMATURE MORTALITY: FOLLOW-UP

OF THE HUNGARIAN EPIDEMIOLOGICAL PANEL

Maria S. Kopp, MD, PhD, Árpád Skrabski, PhD, Krisztina László, $\mathrm{PhD}$ and Imre Janszky, MD, PhD

Inst of Behavioral Sciences, Semmelweis University, Budapest, Hungary.

Background: Gender differences in premature mortality rates and in the size of socioeconomic inequities in mortality vary across countries. We aimed to quantify the gender-socioeconomic status (SES) interaction on premature mortality and to analyse which psychosocial characteristics might explain the different gender patterns of socioeconomic inequalities in premature mortality in this respect in Hungary.

Methods: Men $(n=1130)$ and women $(n=1529)$, aged 40-69 years, participants in the Hungarian Epidemiological Panel (2002) were followed for 3.5 years for total mortality. Cox-proportional hazard models were used to evaluate the association between several socioeconomic measures and death.

Results: During the follow-up 99 men (8.8\%) and 53 women $(3.5 \%)$ died. The age-adjusted hazard ratios and the Rothman's synergy indexes showed that socioeconomic factors (education, blue collar work, personal and family income, subjective social status, lack of car and computer) were more deleterious in men compared to women. Adjustment for severe depression resulted in the highest decrease in the regression coefficients for the association between socioeconomic factors and male premature mortality. There was no indication that depression would mediate between low SES and mortality in women. Work stress measures might partly mediate the effect of socioeconomic deprivation on male mortality, while social support seems to counterbalance the negative aspects of socioeconomic deprivation among women. Conclusions: Middle-aged Hungarian men seem to be considerably more vulnerable to the chronic stress of material deprivation than women. This effect modification by gender might partly be explained by a stronger connection between low SES and depressive symptoms in men.

CORRESPONDING AUTHOR: Maria S. Kopp, MD, PhD, Inst of Behavioral Sciences, Semmelweis University, Budapest, H-1089; kopmar@net.sote.hu

PSW-196c

GI JOE OR AVERAGE JOE? EFFECTS OF AVERAGE-SIZED AND MUSCULAR MALE FASHION MODELS ON MEN'S AND WOMEN'S BODY IMAGE

Phillippa Diedrichs, $\mathrm{PhD}^{2}$ and Christina Lee, $\mathrm{PhD}^{1}$

${ }^{1}$ School of Psychology, University of Queensland, Brisbane, QLD, Australia and ${ }^{2}$ Centre for Appearance Research, University of the West of England, Bristol, United Kingdom.
While the effects on body image of unrealistically thin female fashion models have been extensively investigated, there is very little research on the effects of unrealistically muscular male models. We examined the impact of average-size and muscular male fashion models on men's and women's body image, and on perceived advertising effectiveness. A sample of 619 Australian university students (330 men and 289 women, aged 17-25 years) were systematically assigned to four image exposure conditions: no models, muscular models, average-slim models, and averagelarge models. In each condition, participants viewed eight glamour advertisements created for the purpose of the study, rated the effectiveness of each advertisement, and then completed standardised measures of body image state. Advertisements were matched across conditions for product and general style. Men and women rated average-size male models as equally effective in advertisements as muscular models. Amongst the men, those exposed to average-size models had more positive body image than those viewing no models, with no difference from those viewing muscular models. Similar results were found for women. These findings suggest that average-size male models can promote positive body image and appeal to consumers.

CORRESPONDING AUTHOR: Christina Lee, PhD, School of Psychology, University of Queensland, Brisbane, QLD, 4072; c.lee@ psy.uq.edu.au

\section{PSW-196d}

WORK AND NON-WORK RELATED EXPLANATIONS FOR GENDER DIFFERENCE IN THE USE OF PSYCHOACTIVE DRUGS

Mikko Laaksonen, $\mathrm{PhD}$, Ossi Rahkonen, $\mathrm{PhD}$ and Eero Lahelma, $\mathrm{PhD}$

Department of Public Health, University of Helsinki, Helsinki, Finland.

Background: Mental health problems are often found to be more common among women than among men. We examined physical working conditions, psychosocial working conditions and familyrelated factors as explanations for gender differences in the use of psychoactive medication in an ageing employee cohort.

Methods: Data on the explanatory factors were derived from mail surveys conducted in 2000-2002 among municipal employees of the City of Helsinki aged 40-60 ( $N=8960$, response rate $67 \%$ ). These data were linked with the prescription register of the Social Insurance Institution of Finland. Cox regression analysis was used to calculate hazard ratios (HR) with $95 \%$ confidence intervals (95\% CI) for women as compared to men for the use of all psychoactive drugs (ATC codes in classes N05 and N06, excluding medication for dementia), antidepressants (N06A) and sleeping pills and sedatives (N05B and N05C) among younger (40-50 y) and older (>50 y employees) during three subsequent years.

Results: Among younger women the risk for using psychoactive drugs was only slightly larger than in men (HR 1.24, 95\% CI 1.01-1.54). Adjusting for physical working conditions or psychosocial working conditions somewhat increased the difference. 
There was no association for gender with antidepressants or sleeping pills among the younger. Among the older, the HR for psychoactive drugs among women was 1.70 (95\% CI 1.28-2.27), and it slightly increased after adjustment for physical working conditions. For antidepressant use, the female excess (HR 1.57, 95\% CI 1.12-2.22) was slightly increased after adjusting for physical working conditions. The higher HR for the use of sleeping pills among women 2.28 (95\% CI 1.50- 3.47) was slightly increased after the adjustment for physical working conditions and slightly attenuated by adjustment for familyrelated factors.

Discussion: The female excess in the use of psychoactive drugs was larger in the older than in the younger employees. Physical working conditions, psychosocial working conditions and family related factors in general did not explain the differences. On the contrary, adjusting for physical working conditions rather tended to increase the difference.

CORRESPONDING AUTHOR: Mikko Laaksonen, PhD, Department of Public Health, University of Helsinki, Helsinki, 00014; mikko.t.laaksonen@helsinki.fi

PSW-197

\section{FACILITATING FACTORS AND BARRIERS} OF MULTI-ETHNIC PARTICIPANTS' DECISIONS TO PARTICIPATE IN GENETIC BIOBANKING: PROJECT DIVERSITY GATHER

Georita M. Frierson, $\mathrm{PhD},{ }^{1}$ Marissa C. Perales, $\mathrm{PhD},{ }^{1}$ Margo Simmons, BS, ${ }^{2}$ Benjamin Willis, MD; $\mathrm{MPH}^{2}$ Celette Sugg Skinner, $\mathrm{PhD}^{3}$ and Laura DeFina, $\mathrm{MD}^{2}$

${ }^{1}$ Psychology, Southern Methodist University, Dallas, TX; ${ }^{2}$ The Cooper Institute, Epidemiology, Dallas, TX and ${ }^{3}$ Clinical Sciences, UTSW, Dallas, TX.

Background: The importance of conducting genetic biobanking research about genetic factors that influence major chronic diseases is critical in disease management. Participants eligible for this line of research may not be aware of genetic biobanking research and reasons to participate. This disconnect between potential eligible participants and the importance of genetic biobank research may hinder progress in disease management. Qualitative studies are needed to identify factors that influence a multi-ethnic sample to participate in genetic biobanking research. Purpose: Project Diversity GATHER (Group discussions Around Themes on HEalthful genetic Research) examines health behaviors and factors that influence women's and men's participation in genetic biobanking research. A multi-ethnic sample was recruited to identify factors influencing research participation. Methodology: 85 women and 50 men from Texas completed an 89-item sociodemographic questionnaire and 60-minute non-ethnically or gendered matched focus group. Wegner's translation process model (1993) was used to indentify intra-and inter-cultural themes that influence participants' participation in research. Participants' characteristics were analyzed through descriptive analyses and qualitative themes categorized on 3 key focus group questions:1) research perceptions, 2) facilitating factors, and 3) barriers to participate into research. Results: 135 participants (63\% female; mean age $=46.67, \mathrm{SD}=17.17$, mean education $=14.86 ; \mathrm{SD}=2.49$ ) participated in 28 focus groups. The sample was $49.2 \%$ AfricanAmerican, 36.2\% White, 8.5\% Other, 1.5\% Asian, 2.3\% Native Hawaiian/Pacific Islander, and 2.3\% American Indian/Alaska native; $19.3 \%$ of participants reported a Latino heritage. $81.3 \%$ participants never heard of the term genetic biobanking. $67.4 \%$ of participants indicated that they would participate in genetic biobanking research vs. $93.6 \%$ before and after the group, respectively, $(\mathrm{F}(1,94)=7.23, \mathrm{p}=.015)$. Two, 5, and 5 central themes were identified for research perceptions, facilitating factors, and barriers to research, respectively. All participants reported mistrust, privacy, and lack of knowledge as research barriers. The majority of participants reported addressing importance of research and (non)monetary incentives to increase research participation. Conclusions: Multi-ethic focus group themes highlight that educating the community about the importance of genetic biobanking research may increase research participation.

CORRESPONDING AUTHOR: Georita M. Frierson, PhD, Psychology, Southern Methodist University, Dallas, TX, 75275; frierson@smu.edu

PSW-198

PSYCHOSOCIAL STRESS AFFECTS THE CIRCADIAN RHYTHM: INFLUENCES ON THE GENE EXPRESSION OF CLOCK GENES IN HUMANS

Elvira A. Abbruzzese, Drdes, Noëlle Amstad, Master, Muriel Meyer, Master, Annina Klingmann, Master, Jovita Schuler, Master, Magdalena Skupnjak, Master and Ulrike Ehlert, Professor Clinical Psychology and Psychotherapy, University of Zurich, Zurich, Switzerland.

The circadian clock is a self-sustained time-keeping system, which controls rhythmic behavioral, biochemical and physiological processes. Vital functions such as the timing of cell cycles or apoptosis depend on this intrinsic rhythm. In mammals, the cogwheels of this clock are contained in every single cell. These so-called clock genes control their own expression and the expression of clock-controlled genes via several feedback loops. One of these genes is Per1, a clock gene that disposes of a glucocorticoid-responsive element and is therefore most likely influenced by glucocorticoids. In humans, stress is associated with an increase in the glucocorticoid cortisol. Simultaneously, psychosocial stress is seen as a major factor in the etiology of numerous mental health problems. For this reason, we aimed to investigate first, the association of cortisol and hPER1 as well as hPER2, and second, the putative cortisol-mediated influence of acute and chronic psychosocial stress on the gene expression of the same two clock genes in thirty-one healthy men. Our findings suggest that first, cortisol strongly interacts with hPER1 and hPER2, and second, psychosocial stress influences the expression of hPER1 and hPER2. These results implicate a strong clinical relevance of stress on the human circadian rhythm. 
CORRESPONDING AUTHOR: Elvira A. Abbruzzese, Drdes, Clinical Psychology and Psychotherapy, University of Zurich, Zurich, 8005; e.abbruzzese@psychologie.uzh.ch

\section{PSW-199}

RELIABILITY AND PREDICTIVE VALIDITY

OF A MEASURE OF MOTIVATIONS FOR GENETIC TESTING FOR COMMON DISEASE RISK

Suzanne O'Neill, $\mathrm{PhD},{ }^{1}$ Isaac M. Lipkus, $\mathrm{PhD},{ }^{2}$ Saskia C. Sanderson, $\mathrm{PhD},{ }^{3}$ James Shepperd, $\mathrm{PhD},{ }^{4}$ Sharron Docherty, $\mathrm{PhD}^{2}$ and Colleen M. McBride, $\mathrm{PhD}^{5}$

${ }^{1}$ Georgetown University, Washintgon, DC; ${ }^{2}$ School of Nursing, Duke University, Durham, NC; ${ }^{3}$ Mount Sinai School of Medicine, New York, NY; ${ }^{4}$ University of Florida, Gainesville, FL and ${ }^{5}$ Social and Behavioral Research Branch, National Human Genome Research Institute, Bethesda, MD.

Most measures of motivation to seek genetic susceptibility testing were developed for the context of predictive genetic testing where mutations confer high risk for disease occurrence. It is unclear whether these measures will capture motives for seeking genetic susceptibility testing where gene variants are associated with small increases in disease risk. In a factorial study examining methods to convey low genetic risk for lung cancer to young smokers, we evaluated a 20-item measure of motives for or against genetic testing in 128 college smokers. Items were adapted from those used in other scales and unique items were generated by a panel of experts to assess the following domains: health behavior motivation/demotivation, altruism, uncertainty reduction, impact on perceived risk and worry, and curiosity about oneself and science. Eight items were correlated significantly with intentions to get tested $(p<.05)$. Exploratory factor analysis of these eight items yielded two correlated factors $(\mathrm{r}=.22, \mathrm{p}<.01)$ : maintaining personal health and curiosity about self and technology. These items formed a reliable scale $($ alpha $=.70)$ that correlated with testing intentions $(\mathrm{r}=.63, \mathrm{p}<.001)$. We also asked participants open-ended questions to assess additional reasons for and against testing. Overall, themes paralleled those in our quantitative survey, though participants offered the novel point that they would not want to be tested because the awareness that would come from testing would lead to less enjoyment of life. Prior scales used to assess motivations for testing did not include the construct of curiosity about oneself and technology. This may be unique to intentions to seek new susceptibility tests. Understanding the role of curiosity in test uptake could be informative for this and other novel technologies where health utility has yet to be established.

CORRESPONDING AUTHOR: Suzanne O'Neill, PhD, Georgetown University, Washintgon, DC, 20007; sco4@georgetown.edu
PSW-200

5-HTTLPR POLYMORPHISM, EDUCATION, AND STRESS COPING IN A SAMPLE OF JAPANESE WORKERS

Norito Kawakami, MD ${ }^{1}$ Akiomi Inoue, MHSc, ${ }^{1}$ Masao Ishizaki, $\mathrm{MD},{ }^{2}$ Akihito Shimazu, PhD,${ }^{1}$ Masao Tsuchiya, $\mathrm{PhD},{ }^{1}$ Mitsuyo Kuroda, MPH, ${ }^{1}$ Akiko Kitazume, MHSc, ${ }^{1}$ Miki Akiyama, $\mathrm{PhD}^{3}$ and Masaji Tabata, $\mathrm{MD}^{4}$

${ }^{1}$ Graduate School of Medicine, University of Tokyo, Tokyo, Japan; ${ }^{2}$ Social and Environmental Medicine, Kanazawa Medical University, Uchinada, Japan; ${ }^{3}$ Faculty of Healthcare, Tokyo Healthcare Universit, Tokyo, Japan and ${ }^{4}$ Ishikawa Health Service Association, Kanazawa, Japan.

Aims: A previous studies have reported that the long variant of the serotonin transporter-linked promoter region (5-HTTLPR) polymorphism is associated with problem solving style of stress coping among males (Wilhelm et al. 2007). However, the findings do not have been replicated. In addition, socioeconomic status, such as education, might interact with the genotype to determine stress coping style. This cross-sectional study aimed to examine the interactive effect of education and the 5-HTTLPR polymorphism on coping style in a sample of Japanese workers.

Methods: A total of $241(80 \%)$ out of all employees of a manufacturing factory in Japan completed a mailed questionnaire which included a scale of stress coping, the Brief Scale for Coping Profile (BSCP) (Kageyama et al., 2004). Genomic DNA abstracted from leukocyte in their peripheral venous blood samples. The 5-HTTLPR polymorphism region was amplified using polymerase chain reaction (PCR), and classified into short/short $(\mathrm{s} / \mathrm{s})(\mathrm{n}=153)$, short/long $(\mathrm{s} / \mathrm{l})(\mathrm{n}=\neg 78)$, and long/long $(1 / 1)(\mathrm{n}=6)$, excluding those who had extra long variants $(n=4)$. The respondents were also classified into high school graduates and university graduates. Analyses of covariance (ANCOVA) were applied to compare six subscales of BSCP among the three 5-HTTLPR groups, as well as test the interaction of the polymorphism and education, separately for men and women.

Results: Compared to the s/s group, the $s / 1$ and $1 / 1$ groups had a higher score of the subscale of "seeking help for solution" among male university graduates ( $\mathrm{p}$ for trend $=0.01$ ); Subscale scores of "changing mood" were significantly different among three polymorphism groups, with a low score in the $1 / 1$ group $(\mathrm{p}<$ 0.01 ). The interaction between the polymorphism and education was significant for the subscales of "seeking help for solution" and "changing mood" among men ( $p=0.08$ and $p=0.01$, respectively). Discussion: The study replicated that long variant of 5-HTTLPR polymorphism was associated with problem solving (measured as "seeking help for solution" in this study) among men, while the 1/ 1 group seems less likely to use "changing mood" coping. 5HTTLPR polymorphism, education, and sex seem to interact to determine the problem solving stress coping. 
CORRESPONDING AUTHOR: Norito Kawakami, MD, School of Public Health, University of Tokyo, Tokyo, 113-0033; kawakami@m.u-tokyo.ac.jp

PSW-201a

BELIEFS ABOUT GENETICS RESEARCH

AND ITS APPLICATIONS AMONG BIOBANK

PARTICIPANTS RECRUITED FROM A DIVERSE

COMMUNITY

Samantha Streicher, MSc, ${ }^{1}$ Saskia Sanderson, $\mathrm{PhD},{ }^{1}$ Lynne Richardson, MD, ${ }^{2}$ Ethylin Wang Jabs, MD ${ }^{1}$ Michael Diefenbach, $\mathrm{PhD}^{3}$ and Margaret Smirnoff, $\mathrm{RN}^{4}$

${ }^{1}$ Genetics and Genomic Sciences, Mount Sinai School of Medicine, New York, NY; ${ }^{2}$ Emergency Medicine, Mount Sinai School of Medicine, New York, NY; ${ }^{3}$ Urology, Mount Sinai School of Medicine, New York, NY and ${ }^{4}$ Pediatrics, Mount Sinai School of Medicine, New York, NY.

DNA Biobanks require thousands of participants from diverse backgrounds to donate their blood for research, yet little is known about what participants understand and feel about this kind of genetics research and its applications. The Biobank at Mount Sinai Medical Center (MSMC) in New York City has recruited almost 10,000 individuals from the diverse communities served by MSMC. Our overarching objective was to evaluate Biobank participants' beliefs, knowledge and information needs regarding genetics research and genetic testing. A topic guide was developed and 6 focus groups were conducted with MSMC Biobank participants. The topic guide included questions on understanding of genetics, beliefs about genetics research, and attitudes towards personal and childhood genetic testing. Each focus group was recorded and subsequently transcribed for data analysis. The 6 focus groups comprised 43 participants: 21 were Hispanic, 18 African-American, 2 Caribbean/West Indian, 1 Asian, 1 White; ages ranged from 27 to 76 ; the median yearly household income was $<\$ 20,000$. Participants' reasons for participating in the Biobank included helping future generations, learning more about personal health and disease, and helping the community. Understanding of genetics research and genetics were limited. Participants were generally in favor of personal genetic testing, but expressed mixed views regarding genetic testing of children. Participants expressed that they wanted more information about genetics, and suggested various venues such as streets fairs and community centers as a means for disseminating such information. The results indicate that Biobank participants recruited from a predominantly low income, minority community hold positive views, but have low understanding, of genetics research and its applications. These findings highlight the need for appropriately targeted genetics education initiatives and community engagement in DNA Biobanks and other genetics research.

CORRESPONDING AUTHOR: Saskia Sanderson, PhD, Genetics and Genomic Sciences, Mount Sinai School of Medicine, New York, NY, 10029; saskia.sanderson@mssm.edu
PSW-202

THE INFLUENCE OF SOCIOTROPIC COGNITION

ON MULTIPLE HEALTH RISK BEHAVIORS

C. Teal Pedlow, PhD

University of Massachusetts Dartmouth, N. Dartmouth, MA.

Background: Sociotropic cognition is a set of beliefs associated with social dependency and a high need for approval. Earlier studies with women found that high sociotropic cognition was associated with increased cardiovascular reactivity and sexual risk behavior. However, few studies have included men and the influence of sociotropy on other health risk behaviors has not been evaluated. Purpose: To examine the influence of need for approval, measured by sociotropic cognition, on health risk behaviors, including sexual risk, alcohol, drug, and tobacco use, and disordered eating in young men and women.

Method: Participants were 236 college students who completed self-report measures of sociotropic cognition and health risk behaviors. It was hypothesized that higher sociotropic cognition would be associated with more alcohol, drug, and tobacco use, sexual risk behavior, and disordered eating. Multiple regression was used to examine these relationships.

Results: Sociotropic cognition predicted sex-related alcohol expectancies $(\beta=.16, t(201)=2.24, p<.05)$ and negative consequences from drinking $(\beta=.14, \mathrm{t}(209)=2.11, \mathrm{p}<.05)$. Sociotropic cognition was associated with being pressured into having $\operatorname{sex}(\mathrm{t}(227)=2.19, \mathrm{p}<.05)$, but not to recent intercourse or condom use. Sociotropic cognition predicted recent amphetamine $(\beta=.21, t(229)=3.26, \mathrm{p}<.001)$ and inhalant use $(\beta=.14, \mathrm{t}(229)=2.20, \mathrm{p}<.05)$, but was negatively associated with alcohol $(\beta=-.13, \mathrm{t}(229)=-2.01, \mathrm{p}<.05)$ and marijuana use $(\beta=-.14, \mathrm{t}(229)=-2.09, \mathrm{p}<.05)$. There was no association with tobacco use. Sociotropic cognition predicted more body dissatisfaction $(\beta=.32, \mathrm{t}(225)=5.09, \mathrm{p}<.001)$ and more disordered eating behavior $(\beta=.25, \mathrm{t}(210)=3.81, \mathrm{p}<.001)$, and was not associated with BMI or physical activity.

Conclusions: Sociotropic cognition appears to be an important correlate of interrelated health risk behaviors for men and women. Need for approval influences disordered eating behavior, drug use, problems with alcohol use, and some sexual risk behaviors. Cognitive and behavioral intervention strategies may be useful to address need for approval and its effect on these health risk behaviors.

CORRESPONDING AUTHOR: C. Teal Pedlow, PhD, Psychology, University of Massachusetts Dartmouth, N. Dartmouth, MA, 02747; tpedlow@umassd.edu

\section{PSW-203}

IS THE RELATIONSHIP BETWEEN BMI AND EXERCISE AVOIDANCE MEDIATED BY WEIGHT STIGMA

IN A SAMPLE OF COLLEGE WOMEN?

Angela Coppola, BA Psychology, ${ }^{1,2}$ Rose M. Ward, $\mathrm{PhD}^{1}$ and Sarah Novak, $\mathrm{PhD}^{2}$

${ }^{1}$ Kinesiology \& Health, Miami University, Oxford, OH and ${ }^{2}$ Psychology, Hofstra University, Hempstead, NY. 
Weight stigma involves the negative comments or actions towards an individual because they are perceived as overweight. Previous research has suggested that weight stigma influences health behaviors. Vartanian \& Shaprow (2008) found that weight stigma was associated with exercise avoidance. Whereas positive comments can enhance a person's physical and mental well-being, negative comments from others, in the form of weight stigma, can be harmful. The sample consisted of 120 female students from a mid-sized university (78\% Caucasian). Their ages ranged from 18-22. The average $\mathrm{BMI}$ of the participants was $24.09(\mathrm{SD}=4.34$, range 16.50 to 38.70 ). The online survey was hosted on the secure, encrypted website. Participants completed a questionnaire measuring mental health, social support, weight bias, diet and exercise habits, and diet and exercise-avoidance motivation.

A meditational model was hypothesized. The three constructs of interest were significantly correlated (BMI and exercise avoidance, $.47^{* * *}$; BMI and weight stigma, $.50 * * *$; weight stigma and exercise avoidance, . $51^{* * *}$ ). The primary model examined hypothesized that weight stigma would mediate the relationship between exercise avoidance and BMI. The model testing the relationship between the IV (BMI) and the DV (exercise avoidance) fit the data well. The models testing the relationship between the mediator (weight stigma) and the DV, and the relationship between the IV and the mediator, also fit the data well. The final model testing weight stigma's meditational role between BMI and exercise avoidance, found that weight stigma completely mediated the relationship between the IV and DV.

The results indicated that as BMI increased exercise avoidance increased. Similar to other studies with college females, as BMI increased perceptions of weight stigma also increased. Not only were BMI, exercise avoidance, and weight stigma all related in college females, but weight stigma mediated the relationship between BMI and exercise avoidance, indicating that weight stigma may predict adverse health behaviors. Alternative models and implications will be discussed.

CORRESPONDING AUTHOR: Angela Coppola, BA Psychology, Miami University, Oxford, OH, 45056; angela.coppola1@ gmail.com

\section{PSW-204}

PERSONALITY AND AFFECTIVE PREDICTORS

OF HEALTH DISCOUNTING AMONG COLLEGE STUDENTS

Erin A. Kellogg, Bachelor of Arts and Charles F. Emery, PhD

Clinical Psychology, The Ohio State University, Columbus, OH.

Previous studies examining social determinants of health have found that individuals who tend to discount the future are more likely to engage in negative health behaviors such as smoking and substance use. Due to high rates and detrimental health consequences of negative health behaviors, it is important to identify factors influencing discounting rates. Personality dimensions and depressive symptoms have been identified as potential predictors of discounting rates, but past studies have utilized monetary discounting measures when examining health outcomes rather than health discounting measures. This study examined personal- ity and affective predictors of health discounting in a sample of 97 undergraduate students (41 women) who completed the following questionnaires as participants in a study for course credit: (1) a hypothetical health choice task (Chapman, 1996), (2) the NEO Five-Factor Inventory (NEO-FFI), and (3) the Patient Health Questionnaire (PHQ-9). Hypothetical health choice responses were summed, with higher scores reflecting less discounting of future health rewards. Data were analyzed with Pearson correlations evaluating factors associated with health discounting. Results indicated that higher extraversion was associated with greater likelihood of choosing delayed rewards $(\mathrm{r}=.26, \mathrm{p}=.01)$. However, analysis of sex differences indicated that the association was only significant among men $(r=.29, p=.03)$. Discounting was not associated with any other personality dimension, but it was negatively associated with depressive symptoms $(r=-.32$, $\mathrm{p}=.002$ ), indicating that greater depressive symptoms were associated with choosing immediate rewards. Analysis of sex differences indicated that the association was significant among men $(\mathrm{r}=-.37, \mathrm{p}=.005)$, but not among women. Unlike previous studies using monetary measures of discounting, these results indicate that extraversion among men may be associated with reduced risk of engaging in negative health behaviors, and that depressive symptoms among men may be associated with increased risk of engaging in negative health behaviors.

CORRESPONDING AUTHOR: Erin A. Kellogg, Bachelor of Arts, Clinical Psychology, The Ohio State University, Columbus, OH, 43210; kellogg.62@osu.edu

\section{PSW-205}

\section{DETERMINANTS OF HELP-SEEKING BEHAVIOR} AMONG JAPANESE UNIVERSITY STUDENTS: CAN HELPSEEKING INTENTIONS PREDICT ACTUAL HELP-SEEKING BEHAVIOR?

Nagai Satoru, PhD and Sato Hideyuki, Master in Psychology Rissho University, Shinagawa-ku, Japan.

Many studies have indicated that many people who experience psychological and interpersonal problems don't seek any help. Many researchers have tried to understand the reasons underlying people's decisions to seek or not to seek help. In these studies, measures of help-seeking intentions have been often used instead of measuring actual help-seeking behavior, because it is assumed that help-seeking intentions associate with actual help-seeking behavior. However, there is little evidence of this association. The purpose of this study is to examine whether help-seeking intentions can predict actual help-seeking behavior among Japanese university students. One hundred and eighty-five Japanese university students answered a questionnaire which included social support and help-seeking intentions (Time1). Four weeks later (Time2), participants answered another questionnaire which included psychological distress and actual help-seeking behavior. The correlation between help-seeking intentions at Time1 and actual help-seeking behavior at Time2 was moderate $(\mathrm{r}=.65 \mathrm{p}<.01)$. The result of multiple regression analysis indicated that help-seeking intentions associated with actual help-seeking 
behavior $(\beta=.42 \mathrm{p}<.01)$. Social support at Time1 $(\beta=.18 \mathrm{p}<.05)$ and psychological distress at Time $2(\beta=.44 \mathrm{p}<.01)$ also associated with actual help-seeking behavior. These variables accounted for $57.2 \%$ of the variance in actual help-seeking behavior. Thus, it was revealed that help-seeking intentions can predict actual helpseeking behavior among Japanese university students.

CORRESPONDING AUTHOR: Nagai Satoru, PhD, Rissho University, Shinagawa-ku, 141-8602, JAPAN; nagai-st@ris.ac.jp

\section{PSW-206}

EFFECTS OF EXPRESSIVE WRITING INTERVENTION (EWI) ON INFERTILITY-RELATED SYMPTOMS

IN COUPLES UNDERGOING IN VITRO FERTILIZATION (IVF) TREATMENT - A FEASIBILITY STUDY

Yoon Frederiksen, MSc Psychology, ${ }^{1}$ Signe M. Ebbesen, MSc Psychology, ${ }^{1}$ Mimi Y. Mehlsen, MSc psychology, Phd, ${ }^{1}$ Robert Zachariae, Professor, Drmed, ${ }^{1,2}$ Berit K. Nielsen, MSc Psychology, ${ }^{1}$ Lone Schmidt, drmed, $\mathrm{Phd}^{3}$ and Hans J. Ingerslev, Professor, drmed $^{4}$

${ }^{1}$ Department of Psychology, Aarhus university, Aarhus C, Denmark; ${ }^{2}$ Department of Oncology, Aarhus University Hospital, Aarhus C, Denmark; ${ }^{3}$ Department of Social Medicine, University of Copenhagen, Copenhagen, Denmark and ${ }^{4}$ The Fertility Clinic, Aarhus University Hospital, Aarhus, Denmark.

Background: Suffering from infertility may cause significant stress both on a personal level as well as in the relationship. Expressive writing intervention (EWI) has been shown to be an efficient method for reducing psychological symptoms in both healthy and clinical populations. However, research using this method with infertile couples is limited. Our aim was to investigate the effects of EWI on infertility-related psychological symptoms in a small preliminary sample of patients undergoing In-Vitro Fertilization (IVF).

Methods: 37 couples were included and 8 women were included without their partners $(\mathrm{N}=82)$. Participants were randomized to home-based EWI or a neutral-writing control group. Both groups completed repeated questionnaires measuring infertility-related stress, perceived relationship quality, and dispositional anxiety. Data were collected before the intervention, 3 weeks after the intervention and 6 weeks after the intervention.

Results: No statistically significant effects $(p<0.05)$ of time nor of intervention group emerged from repeated measures ANOVA for infertility-related stress $(\mathrm{F}=2.918, \mathrm{p}=0.07)$, relationship quality $(\mathrm{F}=1.889, \mathrm{p}=0.17)$ and anxiety $(\mathrm{F}=1.921, \mathrm{p}=0.17)$. Effects of EWI corresponding to moderate to large effect sizes were found: Cohens $\mathrm{d}=0.91$ (infertility-related stress), 0.78 (relationship quality) and 0.74 (anxiety).

Conclusion: Although statistically non-significant, the results indicated moderate to large effects in the expected direction, suggesting the relevance of further testing of this method as a potentially useful tool to alleviate stress in infertile couples.

CORRESPONDING AUTHOR: Yoon Frederiksen, MSc Psychology, Department of Psychology, Aarhus university, Aarhus C, DK-8000; yoonf@hotmail.com
PSW-207

DIET AND EXERCISE BEHAVIOUR: SELF-REGULATION CAPACITY AND THE INTENTION-BEHAVIOUR LINK

Tamasin A. Taylor, Msc and Linda D. Cameron, PhD

Psychology, University of Auckland, Auckland, New Zealand.

Despite strong intentions to improve their diet or engage in regular exercise, individuals frequently fail to achieve these health goals. According to Temporal Self-Regulation Theory (Hall \& Fong, 2007), executive function (including working memory and inhibitory control capacities) and prepotent behaviour (e.g., habits) may influence self-regulation abilities. The aim of this study was to replicate and extend initial evidence (Hall, Fong, Epp, \& Elias, 2008) that, within the domains of diet and exercise, executive function moderates the intention-behaviour relationship. At baseline, university students and staff $(\mathrm{N}=123$; ages $17-73 ; 33$ men $)$ completed an $\mathrm{n}$ back task (working memory), a Go/NoGo task (inhibitory control), and measures of dichotomous thinking and weight-cycling. They completed weekly measures of diet and exercise intentions and behaviours (including a modified version of the US National Cancer Institute Fruit and Vegetable screener over three weeks. Diet behaviours included eating five fruit and vegetables a day, low-fat foods, low-sugar foods, and a reduced-calorie diet. Regression analyses revealed interaction effects of executive function and intentions on reduced-calorie diet at Time $2(\beta=.14, \mathrm{p}<.05)$, and on fruit and vegetable intake and low-sugar intake at Times 2 and 3 ( $\beta$ 's $>.18$, $\mathrm{p}$ 's $<.05$ ). Intentions were more predictive of behaviour when executive functions was high than when it was low. Weight-cyclers (compared to those with no history of weight cycling) reported a higher dichotomous thinking style $(\mathrm{t}=2.09, \mathrm{p}<.04)$ and exhibited lower levels of inhibitory control $(\mathrm{t}=2.14, \mathrm{p}<.04)$. The findings support hypotheses that health goal achievement is influenced by self-regulation abilities with a significant biological basis. Research is needed to identify ways to increase these self-regulation capacities or develop alternative techniques for enabling individuals with low executive function to fulfil their health behaviour intentions.

CORRESPONDING AUTHOR: Tamasin A. Taylor, Msc, Psychology, University of Auckland, Auckland, 1022; tamasinataylor@ hotmail.com

PSW-209

MODELING DECISIONS TO UNDERGO GENETIC TESTING FOR SUSCEPTIBILITY TO COMMON HEALTH

CONDITIONS: AN ANCILLARY STUDY

OF THE MULTIPLEX INITIATIVE

Barbara B. Biesecker, MS, ${ }^{1}$ Christopher Wade, $\mathrm{PhD},{ }^{1}$ Shoshana Shiloh, PhD, ${ }^{2}$ Samuel Woolford, $\mathrm{PhD},{ }^{3} \mathrm{~J}$ Scott Roberts, $\mathrm{PhD},{ }^{4}$ Sharon Alford, $\mathrm{PhD}^{5}$ and Theresa M. Marteau, $\mathrm{PhD}^{6}$

${ }^{1}$ National Human Genome Research Institute, National Institutes of Health, Bethesda, MD; ${ }^{2}$ Dept of Psychology, Tel Aviv University, Tel Aviv, Israel; ${ }^{3}$ Dept. of Mathmatical Sciences, Bentley University, Bentley, MA; ${ }^{4}$ Health Behavior and Health Education, Univ. of Michigan School of Public Health, Ann Arbor, MI; ${ }^{5}$ Dept. of Biostatistics and Research Epidemiology, Henry Ford Health System, Detroit, MI and ${ }^{6}$ Dept of Psychology, Institute of Psychiatry Kings College, London, United Kingdom. 
Well-established theories on health behavior have typically been applied to understanding decisions pertaining to one health condition or risk. However, new genetic technologies facilitate testing that can reveal personal genetic risk information about many different conditions simultaneously. These tests are already available to the public despite little understanding of the cognitive and affective factors that affect peoples' decisions to take them. This study was conducted with 270 participants recruited from a parent study, the Multiplex Initiative, who were offered a multiplex genetic test that assessed risk for eight conditions: type 2 diabetes, osteoporosis, and several types of cancer and cardiovascular conditions. Data was collected from a baseline survey, a web-based decisional survey, and at the time of testing. We aimed to understand relationships among predictor variables that originated from a conceptual model; an amalgamation of the Theory of Planned Behavior and the Protection Motivation Theory. Structural equation modeling identified response efficacy as a predictor $(\beta=0.30, \mathrm{p}<0.001)$ of attitudes toward multiplex genetic testing, which in turn predicted $(\beta=1.17, \mathrm{p}<0.001)$ intentions toward testing. Older age had a positive effect on testing intentions $(\beta=0.60, p=0.040)$, while marginally significant effects on intentions were seen for higher educational levels $(\beta=$ $0.34, p=0.078)$ and African Americans $(\beta=-0.56, p=0.054)$. Furthermore, intentions to undergo testing were a strong predictor of testing behavior $(\beta=2.87, \mathrm{p}<0.001)$. These findings support the use of variables from the Theory of Planned Behavior in modeling decision-making about a genetic test for multiple health conditions.

CORRESPONDING AUTHOR: Barbara B. Biesecker, MS, National Human Genome Research Institute, National Institutes of Health, Bethesda, MD, 20892-2073; barbarab@mail.nih.gov

\section{PSW-210}

\section{UNDERSTANDING THE FRUIT AND VEGETABLE} CONSUMPTION BEHAVIOURS OF UNDERGRADUATES

Emily J. Kothe, Master of Applied Science (Health Psychology) and Barbara A. Mullan, PhD

School of Psychology, The University of Sydney, Sydney, NSW, Australia.

Introduction: Inadequate fruit and vegetable consumption is linked to a large number of adverse health outcomes including obesity, heart disease, cancer, diabetes, and asthma (Van Duyn \& Pivonka, 2000). Data suggests that young Australian adults consistently fail to consume as much fruit and vegetables as they should (Australian Institute of Health and Welfare, 2001). In order to develop interventions appropriate for this group it is important to develop an understanding of the factors that motivate and inhibit healthy eating in this population.

Methods: Forty first year psychology students (aged 18-30) from the University of Sydney participated in focus group interviews lasting approximately one hour. Sessions explored factors which inhibit and motivate healthy eating.

Results: Consistent themes described by participants were lack of understanding of current fruit and vegetable consumption guidelines, difficulty incorporating fruits and vegetables into the existing diet, concerns about the costs associated with a diet high in fruit and vegetables, lack of cooking skills, and food preferences. Participants felt that health concerns were the primary motivating factor for increasing fruit and vegetable consumption. Participants also shared ideas for program content and delivery.

Conclusions: The information from focus groups provides valuable information for the planning and implementation of fruit and vegetable interventions for this group. This methodology is a useful and important first step in the development of appropriate, evidence-based, interventions in this population.

CORRESPONDING AUTHOR: Emily J. Kothe, Master of Applied Science (Health Psychology), School of Psychology, The University of Sydney, Sydney, NSW, 2006; pc.miss@gmail. com

\section{PSW-211}

THE RELATIONSHIP BETWEEN CHILDHOOD ABUSE AND THEORETICAL ANTECEDENTS OF HEALTH RISK BEHAVIOR, CONDOM USE, AND PROBLEM DRINKING IN COLLEGE STUDENTS

Bryce DuBois, MA and C. Teal Pedlow, PhD

Psychology, University of Massachusetts Dartmouth, North Dartmouth, MA.

Background: Childhood sexual abuse has been associated with sexual risk behavior and alcohol abuse in adults. However, the relationship of other forms of childhood abuse and neglect to these health risk behaviors has been less studied.

Aim: The purpose of this study was to examine the influence of childhood abuse on theoretical antecedents of health risk-taking, sexual risk behavior, and alcohol use.

Methods: Participants $(\mathrm{N}=184)$ were recruited from a mid-sized northeast university and completed self-report questionnaires to assess childhood experiences with abuse and neglect. Outcome measures included alcohol use, drinking consequences, and sexual risk behavior. Theoretical antecedents to sexual risk behavior also were assessed, including attitudes, norms, self-efficacy, perceived behavioral control, and intentions to use condoms. Data were analyzed with hierarchical multiple regression.

Results: Males more often reported experiencing physical abuse $(\mathrm{t}(180)=2.03, \mathrm{p}<.05)$, whereas only women reported sexual abuse. For females, sexual abuse predicted more heavy episodic drinking $(\mathrm{t}(90)=-2.33, \mathrm{p}<.05)$ and negative consequences from alcohol use $(\mathrm{t}(94)=3.04, \mathrm{p}<.01)$. Heavy episodic drinking was associated with greater frequency of intercourse $(\mathrm{t}(149)=2.00$, $\mathrm{p}<.05$ ). Childhood trauma was not associated with condom use in the past 3 months. Findings from theoretical antecedents of risk behavior showed that childhood trauma was associated with less self-efficacy $(\mathrm{F}(4,174)=3.81, \mathrm{p}<.01)$ and less perceived behavioral control $(\mathrm{F}(4,177)=3.20, \mathrm{p}<.05)$, but was not associated with intentions to use condoms. Childhood physical abuse predicted decreased intentions to use condoms for both men $(\mathrm{t}(76)=-2.14, \mathrm{p}<.05)$ and women $(\mathrm{t}(80)=-2.35, \mathrm{p}<.05)$.

Discussion: Our findings highlight the importance of examining different forms of child abuse and their relationship to future 
health risk behavior. Prevention interventions can target individuals with history of physical or sexual abuse.

CORRESPONDING AUTHOR: Bryce DuBois, MA, University of MA at Dartmouth, Bristol, RI, 02809; bdubois@umassd.edu

PSW-212

\section{FACTORS ASSOCIATED WITH SOCIAL} PROBLEM-SOLVING IN WEIGHT LOSS PARTICIPANTS Jing Wang, BSN, PhDc, ${ }^{1}$ Linda E. Ewing, $\mathrm{PhD},{ }^{3}$ Susan $\mathrm{M}$. Sereika, $\mathrm{PhD},{ }^{1,2}$ Judith T. Matthews, $\mathrm{PhD},{ }^{1}$ Eileen R. Chasens, $\mathrm{DSN}^{1}$ and Lora E. Burke, $\mathrm{PhD}^{1,2}$

${ }^{1}$ University of Pittsburgh School of Nursing, Pittsburgh, PA; ${ }^{2}$ University of Pittsburgh Graduate School of Public Health, Pittsburgh, PA and ${ }^{3}$ University of Pittsburgh School of Medicine, Pittsburgh, PA.

No study has yet examined factors associated with social problemsolving among overweight and obese individuals seeking behavioral weight loss treatment. To develop predictive models of social problem-solving (SPS) in this group, we conducted a secondary analysis using baseline data from an ongoing randomized clinical trial on weight loss. The total score and five subscale scores (rational and negative problem orientation [RPO, NPO], rational problem solving [RPS], impulsive/careless style [ICS], and avoidance style $[\mathrm{AS}]$ ) of the Social Problem-Solving InventoryRevised (SPSI-R) were used to assess SPS. Guided by the literature, we examined the following predictors of SPS: age, gender, education, marital status, employment status, income, mental health quality of life (MHQOL), perceived stress, and barriers to healthy eating (BHE). Predictive models were developed using all possible subsets regression in SAS. The sample was predominantly white $(84.8 \%)$, female $(78.1 \%)$, and middle-aged (average 46.80 years). Age, baseline weight, MHQOL, BHE, and perceived stress were jointly identified as predictors of the total score of SPSI-R $(\mathrm{R} 2=.40, \mathrm{~F}=26.4, \mathrm{p}<.0001)$. This same group of factors, with the addition of being single, formed a significant predictive model of NPO ( $22=.19, \mathrm{~F}=24.7, \mathrm{p}<.0001)$. Higher levels of income and education, fewer perceived stresses, and history of weight cycling predicted RPS ( $\mathrm{R} 2=.34, \mathrm{~F}=24.7$, $\mathrm{p}<.0001$ ), while a higher income, fewer barriers and perceived stresses predicted RPO $(\mathrm{R} 2=.22, \mathrm{~F}=23.8, \mathrm{p}<.0001)$. Less education, more eating barriers and perceived stress predicted an impulsive style $(\mathrm{R} 2=.18, \mathrm{~F}=24.7, \mathrm{p}<.0001)$, while being older, having poor mental health and perceiving more eating barriers and stress together predicted an avoidant style (R2=.34, $\mathrm{F}=24.7$, $\mathrm{p}<.0001$ ) of problem solving. Taken together, age, income, education, mental health, perceived stress, and barriers to healthy eating were associated with participants' social problem-solving. These factors need to be considered when developing interventions to address problem-solving ability related to managing lifestyle changes and weight reduction.

CORRESPONDING AUTHOR: Jing Wang, BSN, PhDc, University of Pittsburgh, Pittsburgh, PA, 15261; jiw38@pitt.edu
PSW-213b

WALK AWAY FROM THE HABIT: OVERCOMING

NICOTINE DEPENDENCE THROUGH EXERCISE

Sarah E. Linke, MS, MPH

Behavioral Medicine, SDSU/UCSD Joint Doctoral Program in Clinical Psychology, La Jolla, CA.

Tobacco use and physical inactivity are the leading causes of preventable death in the US, and persistent smokers tend to remain inactive. Most smokers who quit relapse within three months, suggesting that mainstream cessation assistance options do not work well for many smokers. Interventions using sustained, aerobic exercise as a smoking cessation technique have resulted in success rates comparable to conventional cessation methods. No trial to date has prescribed intermittent bouts of brief exercise in response to nicotine cravings, despite evidence suggesting its potential efficacy as a cessation tool.

This randomized controlled trial attempts to replace smoking with exercise when cravings arise among generally healthy, inactive adult smokers who desire to quit. The first wave of participants $(\mathrm{N}=26$; mean age 42.4[12.2] years; 50\% female) were randomly assigned to a standard care Internet group $(n=13)$ or an Internet + exercise group $(n=13)$ for the three-month intervention phase. The attrition rate $(34.6 \%)$ was comparable to other smoking cessation trials and did not differ between groups. Self-reported quit rates among retained $(29.4 \%)$ and total enrolled (19.2\%) participants were higher than and comparable to, respectively, those attained by trials using traditional quit assist methods such as nicotine replacement therapy. Reduction rates among retained (49\%) and total enrolled (37.4\%) participants were substantial. Quit rates did not differ between groups, but reduction rates were twice as high among participants in the control (52\%) vs. intervention (26\%) group. From baseline to post-intervention, mean FTND scores (5.1 [2.7] vs. 2.9[3.2]) and mean body mass (80.8[20.7] kg vs. 78.9 $[18.3] \mathrm{kg}$ ) decreased among retained participants and did not differ between groups.

Preliminary results from the first wave of participants in this lowcost, web-based trial are comparable to more resource- and timeconsuming cessation programs, suggesting that future iterations of this program may be quite promising. Further analyses will examine the potential roles of exercise adherence, selfmonitoring adherence, and self-efficacy, among other variables, in the smoking cessation and reduction rates associated with this program.

CORRESPONDING AUTHOR: Sarah E. Linke, MS, MPH, Behavioral Medicine, SDSU/UCSD Joint Doctoral Program in Clinical Psychology, La Jolla, CA, 92037; slinke@ucsd.edu

PSW-213c

UNDERSTANDING DIABETES SELF MANAGEMENT BEHAVIOURS AND COPING SKILLS IN MALAY TYPE 2 DIABETES

Salmiah Mohd Ali, B Pharma; PhD

Clinical Pharmacy, Universiti teknologi MARA, Puncak Alam, Malaysia. 
With growing number of people diagnosed with diabetes, there is a lack of diabetic control among people with diabetes in Malaysia and we need to understand why this is. This study set out to explore the perspectives of patients' management of Type 2 diabetes and provide recommendations that aim to enhance adherence to treatment and help patients to improve their selfmanagement skills. In-depth interviews were carried out on a purposeful sample of patients and their health care professional (HCPs). Interviews were recorded, transcribed and audiotapes were analysed using NVivo software to identify emerging themes and code according to categories. Interviews were conducted in an Endocrinology clinic in Malaysia with 18 Malay patients (1575 years, 9 males and 9 females) and 13 HCPs. Themes that emerged from interviews with patients' were problems with integrating the treatment regimen and difficulty developing coping skills to achieve the desired blood glucose level. Patients lacked knowledge about their medicines, dietary control, medicine taking, complexities of treatment, and side effects of medications. They needed to integrate many treatment requirements such as diet, medications, blood glucose monitoring and exercise into their daily routine posed problems. Other barriers such as ethnicity, beliefs (culture and financial) proved to be constraints in their ability to good control diabetes. Education and knowledge related to diabetes that influenced understanding of the disease were also reasons for non-adherence to treatment regimen. According to pharmacists, patients' ethnicity and education level played a substantial role in their attitudes toward diabetes management. Their beliefs, cultural and social needs shaped their attitudes in controlling or adherence to treatment management. Patients were willing to discuss their problems about self-managing diabetes if these barriers were addressed during consultations with the pharmacists. More positive approaches are needed in self-management of diabetes and health care professionals involved in the management of diabetes need to understand their patients' beliefs about their diabetes and their constraints to promote more awareness and to foster greater control of diabetes and improve health outcomes.

CORRESPONDING AUTHOR: Salmaih Mohd Ali, BPharm, $\mathrm{PhD}$, Clinical Pharmacy, Universiti teknologi MARA, Puncak Alam, 42300; drsalmiah@salam.uitm.edu.my

\section{PSW-213d}

PREVALENCE OF CO-MORBID MENTAL AND BEHAVIORAL RISK FACTORS IN A HEALTH PLAN POPULATION

Jennifer McClure, PhD, Evette Ludman, PhD, Sheryl Catz, PhD, Karin Riggs, MSW, Jackie St. John, BA, Julie Richards, MPH and Lou Groathuas, MS

Group Health Research Institute, Seattle, WA.

Most major chronic diseases are caused by more than one behavioral risk factor and risk factors additively contribute to morbidity and mortality. Risk behaviors also tend to co-occur, but data on the prevalence of co-occurring mental and behavioral risk factors are scarce. Most prevalence reports focus on single risk factors. Better understanding the co-occurrence of risk factors, however, could help inform the feasibility and design of multiple risk factor (MRF) interventions, which potentially can be more cost-effective than delivering interventions targeted at single behaviors. We report on the co-occurrence of three primary disease risk factors (depression, low physical activity, and smoking) in a representative health plan population $(n=66,901)$ in the U.S. Pacific Northwest. Data was collected during an online health risk assessment offered to all health plan members. Twothirds of respondents $(66.6 \%)$ were female and $86 \%$ were white. Mean age was 47 years. Approximately 9\% reported current smoking, $8.2 \%$ were moderate to severely depressed based on the PHQ-8, and 24.5\% reported low physical activity per the IPAQ. While $33.1 \%$ of smokers had low physical activity and $18.8 \%$ reported significant depressive symptoms, the overall prevalence of all three behaviors was much lower. As a proportion of the entire sample, $<2 \%(n=1089)$ met criteria for smoking and depression; $2.5 \%(n=1695)$ met criteria for smoking and low physical activity; and 3.2\% $(n=2137)$ met criteria for depression and low physical activity. Fewer $(0.6 \% ; n=405)$ met criteria for all three behaviors. The results highlight a challenge of MRF interventions. While many people report single risk factors, far fewer meet criteria for MRF's, even for common risk factors such as smoking, depression, and inactivity. Given this, MRF interventions may need to be easily disseminable across large populations in order to be cost-effective and have maximum impact. Implications for MRF treatment design will also be discussed.

CORRESPONDING AUTHOR: Jennifer McClure, PhD, Group Health Research Institute, Seattle, WA, 98101; mcclure.j@ghc.org

\section{PSW-213e}

CHANGES IN DIET IMPACT SUBJECTIVE, BUT NOT OBJECTIVE, HEALTH OUTCOMES IN HEART FAILURE PATIENTS

Andrew J. Wawrzyniak, PhD, ${ }^{1}$ Kristie M. Harris, MA, ${ }^{1}$ Stephen S. Gottlieb, MD, ${ }^{2}$ Sarah M. Godoy, MA, ${ }^{1}$ Kerry S. Whittaker, MA, ${ }^{1}$ Nadine S. Bekkouche, BA, ${ }^{1}$ Joanne P. Marshall, RN, ${ }^{2}$ Willem J. Kop, $\mathrm{PhD}^{2}$ and David S. Krantz, $\mathrm{PhD}^{1}$

${ }^{1}$ Medical \& Clinical Psychology, Uniformed Services University of the Health Sciences, Bethesda, MD and ${ }^{2}$ Cardiology, University of Maryland Medical Center, Baltimore, MD.

Background: Dietary intake can impact health and have important effects in heart failure (HF) patients. A goal of the BETRHEART Study is to determine how changes in diet such as fluid and salty food intake and poor ingredient awareness impact subjective and objective health outcomes in HF patients.

Methods: Sixty-three HF patients (mean $=54.4+11.5$ years, ejection fraction $\leq 40$ ) were examined at baseline and 3 months later. The Kansas City Cardiomyopathy Questionnaire (KCCQ) measuring subjective symptom reporting and a standardized Six Minute Walk Test (6MWT) assessing functional status were administered. Hospitalizations were noted for up to 12 months after baseline; plasma levels of b-type natriuretic peptide (BNP), a marker of ventricular wall stretch, were determined. A Dietary Compliance Survey assessed how often patients avoided salty food, read food labels for sodium content, drank fluids, and recently ate salty foods. 
Results: No significant differences between baseline and 3 months were found in the number of times patients avoided salty food $(6.00+8.5$ vs. $4.24+3.7)$, label reading $(2.60+1.8$ vs. $2.4+1.6)$, or salty food intake $(6.89+10.2$ vs. $4.24+3.7)$. The number of times that patients drank less than 2 quarts of water significantly decreased $(3.92+1.3$ vs. $3.08+1.7, p=.009)$. After controlling for covariates (age, body mass index, gender, smoking status, diabetic status, serum creatinine, and prior myocardial infarction), increases in salty food intake were significantly related to worsening of subjective HF symptoms (KCCQ Overall Summary Symptom score, standardized $\beta=-0.34, \mathrm{p}<.05$, adjusted R2=.22). Associations between changes in dietary measures and changes in BNP, the 6MWT, or any other KCCQ subscales were not significant.

Conclusions: Baseline dietary behaviors did not predict future hospitalizations. Salt and fluid intake may influence cardiovascular functioning although the HF patients in this study only reported a worsening of overall subjective HF symptoms in association with increased salt intake over a 3 month period. These findings may be explained in part by imprecise self-reporting of actual eating behavior or by socially desirable responding.

CORRESPONDING AUTHOR: Andrew J. Wawrzyniak, PhD, Medical \& Clinical Psychology, Uniformed Services University of the Health Sciences, Bethesda, MD, 20814; andrew.wawrzyniak. ctr@usuhs.mil

\section{PSW-213f}

IS PERCEIVED REGULARITY OF BEHAVIOUR A USEFUL PREDICTOR OF INTENTION TO ADOPT BEHAVIOUR?

Camille Gagné, $\mathrm{PhD},{ }^{1}$ Danielle Boucher, $\mathrm{MSc}^{1,2}$ and Françoise Côté, $\mathrm{PhD}^{1}$

${ }^{1}$ Laval University, Quebec, QC, Canada and ${ }^{2}$ University of Quebec, Quebec, QC, Canada.

Introduction: The theoretical framework of Raghubir and Menon (2005) suggests that intention to behave in the future will be more favourable when individuals recall positive experiences and these experiences are easy to retrieve (such as when experiences occur on a regular basis). The aim of this study is to verify if perceived regularity in the adoption of behaviour adds to the prediction of intention, taking into account the constructs of the theory of planned behaviour (Ajzen, 1991) and constructs of other relevant social cognitive theories that aim to explain intention.

Method: This is a descriptive correlational design in which 385 college students in Quebec (Canada) volunteered to complete a self-administered questionnaire measuring psychosocial constructs (intention, attitude, subjective norm, descriptive norm, perceived behavioural control, self-efficacy) regarding the consumption of at least five portions of vegetables and fruit every day during the next three months. Consumption of vegetables and fruit in the last seven days and the regularity of this consumption were also measured. The Cronbach alpha of each construct was satisfactory, with values ranging from .62 to .94 . A hierarchical multiple regression was used to analysis the data.

Findings: The findings indicate that attitude, descriptive norm, perceived behavioural control, self-efficacy and past behaviour explain $77 \%$ of the variance in intention. When perceived regularity is added to the model, $79 \%$ of the variance in intention is explained $\mathrm{F}(6,378)=247.16, \mathrm{p}=.00$. In this latter model, perceived regularity is the second most important predictor of intention, after perceived behavioural control.

Conclusion: The findings of this study indicate that perceived regularity of behaviour contributes significantly to the prediction of intention after the influence of psychosocial constructs of main social cognitive theories have been controlled. It should be verified, in future studies, if perceived regularity of behaviour is useful to predict intention to adopt other types of behaviour.

CORRESPONDING AUTHOR: Camille Gagné, PhD, Faculty of nursing, Laval university, Quebec, QC, G1V 0A6; camille. gagne@fsi.ulaval.ca

\section{PSW-213g \\ EXPLORING POTENTIAL BEHAVIORAL MECHANISMS OF THE RELATION BETWEEN TRAIT AFFECT AND HEALTH STATUS IN PERSONS LIVING WITH HIV DISEASE \\ Karen E. Stewart, MS, ${ }^{1}$ Kirk W. Brown, $\mathrm{PhD}^{1}$ and Nancy L. McCain, $\mathrm{DSN}^{2}$ \\ ${ }^{1}$ Psychology, Virginia Commonwealth University, Richmond, VA and ${ }^{2}$ Department of Adult Health and Nursing, Virginia Com- monwealth University, Richmond, VA.}

Research supports an association between negative psychosocial functioning and adverse health outcomes in persons living with HIV. One study has reported reduced mortality in persons with HIV disease reporting high positive affect; however, little is known about mechanisms of this effect. The broaden-and-build model of positive emotions holds that positive emotions promote psychological resource development (e.g. coping skills). The present correlational study conceptualized health behavior practices as coping responses to living with HIV disease. Specifically, medication adherence and health behaviors (smoking, alcohol use, physical activity, and nutrition) were hypothesized to mediate relations between trait positive and negative affect and general and HIV-specific health indicators in persons living with HIV. HIV + participants $(\mathrm{N}=53)$ completed an interview about their emotions, health behaviors, and general health status. Clinical staff provided HIV symptom ratings. Virulogic and immunologic indicators were collected by chart review. High trait negative affect was associated with poor medication adherence, and percent adherence mediated the relation between high trait negative affect and low CD4+ percent $\left(z^{\prime}=-1.33, p<.01\right)$ and high viral load $\left(z^{\prime}=1.61, p<.01\right)$. Mediation hypotheses for health behaviors were not confirmed; however, high trait positive affect was associated with decreased alcohol intake $(\mathrm{F}=3.10, \mathrm{R} 2=.26, \beta=-0.35, \mathrm{p}<.05)$, increased physical activity $(\mathrm{F}=3.89, \mathrm{R} 2=.24, \beta=0.39, \mathrm{p}<.01)$, and better nutrition habits $(F=3.68, \mathrm{R} 2=.22, \beta=0.34, \mathrm{p}<.05)$. Because the current sample was relatively young (average age 44, range 2256), these behaviors may not yet had demonstrable effects on participants' health status; however, future longitudinal studies or 
studies that target older populations may bear out these mediating hypotheses. The current findings provide preliminary evidence on potential mechanisms by which trait affect could impact health status in persons with HIV.

CORRESPONDING AUTHOR: Karen E. Stewart, MS, Psychology, Virginia Commonwealth University, Richmond, VA, 23220; stewartke@vcu.edu

PSW-213h

AN INTERVENTION TO INCREASE WALKING REQUIRES BOTH MOTIVATIONAL AND VOLITIONAL COMPONENTS: A REPLICATION AND EXTENSION

David P. French, $\mathrm{PhD},{ }^{1}$ Andrew Stevenson, $\mathrm{MSc}^{2}$ and Susan Michie, $\mathrm{PhD}^{2}$

${ }^{1}$ Applied Research Centre in Health and Lifestyle Interventions, Coventry University, Coventry, United Kingdom and ${ }^{2}$ Research Dept of Clinical, Educational \& Health Psychology, University College London, London, United Kingdom.

Objectives: We previously showed that a single intervention session had large effects on the objectively assessed walking behavior of $\mathrm{N}=130$ English adult volunteers $(\mathrm{d}=0.90)$, mediated by self-efficacy. The present research aims to establish (a) whether this effect is generalisable (when delivered by a different person (namely the second author), and (b) whether either of the motivational or volitional components of the intervention are alone sufficient to alter walking behaviour, or whether both components are required.

Methods: Adult volunteers $(n=35)$ randomly received one of three interventions: (i) a "combined" intervention containing motivational and volitional components in the same session, (ii) a "motivation first" intervention, where the motivational components were followed by volitional components a week later, and (iii) a "volition first" intervention, where the volitional components were followed by the motivational components a week later. Results: The "combined" intervention produced a large $(\mathrm{d}=1.06)$ and significant $(\mathrm{p}=0.036)$ increase in walking behavior, in contrast to both other interventions (time $\mathrm{x}$ groups interaction, $\mathrm{p}=0.003$ ). The "combined" intervention also produced a significant increase in self-efficacy, relative to the two other interventions.

Conclusions: This demonstrates generalisability of previous large intervention effects and suggests that neither the motivational nor volitional components appear to work effectively in isolation.

CORRESPONDING AUTHOR: David P. French, PhD, Applied Research Centre in Health and Lifestyle Interventions, Coventry University, Coventry, CV1 5FB; david.french@coventry.ac.uk

PSW-213i

\section{EDUCATION \& TRAINING IN BEHAVIORAL MEDICINE} IN LIBYA

Eman A. Bouni, MB of Pathology, University of AL-Fatah, Tripol, Libyan Arab Jamahiriya.

Eman Ahmed Bouni, department of pathology, faculty of medicine, Al Fatah university .Tripoli. Libya
Psychology in general \& behavioral sciences \& behavioral medicine in particular as sciences are less than 133 years old. Behavioral medicine, it is said, as Ebbinghous stated about psychology (( has along past but short history)). Behavioral medicine has been defined as interdisciplinary field that integrates behavioral \& medical knowledge and applies that knowledge to health \& disease. In Libya behavioral medicine is a pretty unknown field of knowledge and as has been defined here is not exist due to many and multiple factors, therefore it is not surprising that you would not find a teaching curriculum in medical schools for psychology or behavioral medicine at any university in Libya before 1988, we can say that the teaching of behavioral sciences started in 1988 in the faculty of medicine in Tripoli, University of Al-Fatah as apart of the community medicine course and is taught in the fourth year. in the faculty of medicine in Benghazi ,psychology was part of pre medical curriculum but behavioral medicine taught in the 4th year as apart of community medicine course. In this paper. the author highlight the historical development of behavioral medicine and behavioral sciences in Libyan medical schools and suggestion to include behavior medicine in the Libyan medical curriculum which should be a compulsory and not an optional subject. The main objective and the primary massage of this paper is to emphasize that modern and successful physicians needs two types of knowledge \& practices: 1-medical \& biological knowledge. 2 - psychological and sociological \& practices knowledge. This means that physicians as has been stated should see beyond the molecular substances and perceive human being again in holistic approach or view.

CORRESPONDING AUTHOR: Eman A. Bouni, MB, of Pathology, University of AL-Fatah, Tripol, 00218; emanbuni@yahoo. com

PSW-213j

DEVELOPING A NOMENCLATURE FOR BEHAVIOUR CHANGE INTERVENTIONS

Marie Johnston, $\mathrm{PhD},{ }^{1}$ Susan Michie, $\mathrm{PhD},{ }^{2}$ Jill Francis, $\mathrm{PhD},{ }^{1}$ Martin Eccles, $\mathrm{MD},{ }^{3}$ Charles Abraham, $\mathrm{PhD},{ }^{4}$ Wendy Hardeman, $\mathrm{PhD}^{5}$ and Diane Dixon, $\mathrm{PhD}^{6}$

${ }^{1}$ Health Psychology, University of Aberdeen, Aberdeen, United Kingdom; ${ }^{2}$ UCL, London, United Kingdom; ${ }^{3}$ Newcastle University, Newcastle, United Kingdom; ${ }^{4}$ Sussex University, Brighton, United Kingdom; ${ }^{5}$ Cambridge University, Cambridge, United Kingdom and ${ }^{6}$ Strathclyde University, Glasgow, United Kingdom.

To date, there has been no shared language for describing the content, especially the 'active ingredients' of behavior change interventions; by contrast, biomedical interventions are precisely specified. This limits the possibility of replicating effective interventions, synthesising evidence, and understanding the causal mechanisms underlying behavior change. Our ultimate aim is to develop a reliable method of specifying behavior change techniques (BCTs), link them to relevant theory and specify the behaviors necessary to implement them. In several stages, we 
have generated lists of BCT labels based on a) systematic reviews of behavior change interventions b) systematic text-book search c) expert brainstorming. We have generated definitions a) from 9 textbooks, b) from dictionaries (including APA Dictionary of Psychology) and c) by reframing to specify the behavioral competencies required of the person implementing the BCT. Two judges had $74.7 \%$ agreement on definitions extracted from textbooks for 53 BCTs. When judging links between BCTs and theoretical constructs, four judges had $71.7 \%$ agreement (385 judgments, based on 35 BCTs and 11 theoretical constructs). To date, we have agreed behavioral specification for 89 BCTs relevant to changing health behaviors. However this is a very early stage in the process and we have recently acquired UK Medical Research Council support to develop an international collaboration to refine and develop a BCT nomenclature, based on extensive Delphi, consensus and judgment processes, and then to test the value of the developed system in increasing the reliability of a) coding of published interventions and b) reporting of interventions.

CORRESPONDING AUTHOR: Marie Johnston, PhD, Health Psychology, University of Aberdeen, Aberdeen, AB; m.johnston@ abdn.ac.uk

PSW-213k

BEYOND 'LIFESTYLES' FACTORS: A BROAD APPROACH TO PREVENTION OF NON-COMMUNICABLE DISEASES IN PERI-URBAN SOUTH AFRICA

Thandi M. Puoane, Dr PH, Ruth Stern, Dr PH and Lungiswa P. Tsolekile, MPH

School of Public Health, Univeristy of the Western Cape, Cape Town, South Africa.

Introduction: Non-communicable diseases are increasing in developing countries, exacerbated by growing urbanization. Contributory factors include limited access to affordable healthy food, readily available cheap unhealthy foods and limited scope for physical activity. Also significant are cultural beliefs and practices. This study examined the experiences and perceptions of people migrating from rural to urban areas in Cape Town, South Africa, and the impact these have had on the risk factors of noncommunicable diseases.

Methods: The setting is an impoverished peri-urban township with a non-communicable disease prevention program that includes weekly health clubs. The program was initiated by a local university that also undertook the research. A qualitative approach was adopted, using a purposive sample of club members and nonclub members. Data was collected through in-depth interviews, participatory reflection and focus group discussions.

Results: Significant changes in respondents' eating patterns and levels of physical activity were described, with accompanying increase in obesity. This was due to both socio-economic and environmental constraints. However, the respondents were not concerned about these changes. Despite many hardships, they were pleased with their new urban lifestyle. Furthermore, they approved of their weight gain as culturally it signified dignity and respect. Those attending health clubs found them both informative and socially and emotionally supportive.

Conclusion: The study highlighted the complexity of the risk factors of non-communicable diseases, and the importance of developing prevention strategies that extend beyond the traditional lifestyle approach that focus on diet and exercise.

CORRESPONDING AUTHOR: Thandi M. Puoane, Dr PH, School of Public Health, Univeristy of the Western Cape, Cape Town, 7535; tpuoane@uwc.ac.za

PSW-214

A PREVENTIVE INTERVENTION PROGRAM

FOR SELF-COMPLEXITY AND DEPRESSIVE SYMPTOMS AMONG JAPANESE COLLEGE STUDENTS

Junko Kawahito, MD, ${ }^{1}$ Masashi Hori, $\mathrm{PhD}^{2}$ and Yasumasa Otsuka, $\mathrm{PhD}^{3}$

${ }^{1}$ Graduate School of Education, Hiroshima University, Hiroshima, Japan; ${ }^{2}$ Graduate School of Education, Hiroshima University, Hiroshima, Japan and ${ }^{3}$ Graduate School of Education, Hiroshima University, Hiroshima, Japan.

An intervention program for improving self-complexity (SC; Linville, 1987) was developed, and its effect on SC, positive self-complexity (P-SC; Woolfolk, Novalany, Gara, Allen, \& Polino, 1995), negative self-complexity (N-SC; Woolfolk et al., 1995), and depressive symptoms among Japanese college students was examined. SC was defined as a function of both the number of self-aspects and the degree to which the self-aspects are differentiated from each other. Linville (1987) has suggested that high self-complexity serves as a buffer against the onset of depressive symptoms following negative events because it partly determines the impact of such events on thoughts and feelings about various self-aspects. Participants were randomly assigned to either the intervention group $(n=20)$ or the control group $(n=20)$. The intervention group received one session of psycho-education on SC and a paper-and-pencil exercise designed to calculate the number of their self-aspects (social roles, interpersonal relationships, activities, traits, etc.) and to evaluate each self-aspect using an adjective checklist. After the education and exercise, daily records of their self-aspects were assigned for one week. Selfreported questionnaires were completed three times in both groups (pre, post, and follow-up). The questionnaire was comprised of the Center for Epidemiologic Studies Depression Scale, the Positive and Negative Affect Schedule, and the Rosenberg Self-Esteem Scale. SC, P-SC, and N-SC, which were assessed using H, a statistical measure of dispersion that represents number of independent dimensions underlying a given trait-sorting, were measured by Linville's (1987) and Sato's (1999) trait sorting task. The Research Ethics Committee of the Hiroshima University Graduate School of Education reviewed and approved the study protocol. In the intervention group, the analysis of variance indicated that P-SC was significantly increased at the post-test and marginally increased at the follow-up test when compared to the pre-test. No significant differences in P-SC were found in the control group. Structured equation modeling revealed that in the 
intervention group, high P-SC at the post-test was related to lower levels of a depressed mood at the post-test and follow-up test. Results demonstrate that this program increases P-SC, which might ameliorate subsequent depressed mood among college students.

CORRESPONDING AUTHOR: Junko Kawahito, MD, Graduate School of Education, Hiroshima University, Hiroshima, 7390151; j-kawahito@hiroshima-u.ac.jp

\section{PSW-215}

\section{INTERDISCIPLINARY ETHICS: CASE STUDIES} PRESENTED WITHIN THE LEVELS OF PREVENTION MODEL FOR A PILOT NUTRITION PROGRAM AIMED AT INTERCOLLEGIATE ATHLETES

Amy Thompson, PhD, Diana Reindl, MA, Elizabeth Greer, undergraduate, Karen Gallagher, MA and Debra Boardley, PhD The University of Toledo, Toledo, $\mathrm{OH}$.

As collaboration between disciplines continues to emerge so do the variety of ethical dilemmas faced. Many vocations have a professional code of ethics to guide acceptable and appropriate behavior however with multiple disciplines collaborating the priority level of the ethical situation may vary. Additionally, depending on the collaborators and the ethical quandaries that arise, diverging viewpoints may develop when determining how to handle the dilemma. This is likely due to the preparation and training of the separate disciplines involved in collaborative efforts. Within the field of Health Education ethical considerations develop in various settings and provide challenging issues to address. Ethical dilemmas originate from the primary, secondary, and tertiary levels of the prevention model. This presentation will outline Ignite, a multidisciplinary approach to nutrition education created for intercollegiate students athletes and designed to enhance nutrition knowledge, perceptions, and behaviors of student athletes $(n=250)$. Through collaboration from athletic staff and health educators, Ignite was crafted to address all levels of the prevention model. This presentation presents ethical dilemmas observed in Ignite within the levels of prevention model. Finally, discussion questions to encourage dialogue regarding ethical behavior and interdisciplinary ethics are included.

CORRESPONDING AUTHOR: Diana Reindl, MA, The University of Toledo, Toledo, OH, 43606; diana.reindl@rockets.utoledo. edu

PSW-216

AN ANALYSIS OF HIGH SCHOOL STUDENTS' KNOWLEDGE AND ATTITUDES TOWARDS HIV/AIDS IN SAUDI ARABIA: IMPLICATIONS FOR HEALTH EDUCATION

Saad Alghanim, PhD

King Saud University, Riyadh, Saudi Arabia.

Objectives: To investigate the knowledge and attitude of high (secondary) school students concerning HIV/AIDS in Riyadh City, Saudi Arabia.
Methods: This is a descriptive study using a questionnaire to investigate students' knowledge of and attitudes to HIV/AIDS. A total of 600 questionnaires were distributed randomly to students at four high schools (two girls' and two boys'), of which 567 $(94.5 \%)$ were returned and valid for analysis. The questionnaire was designed to collect data on a number of variables about HIV/ AIDS epidemic including students' general knowledge and attitudes, its mode of transmission and students' source of information about the disease. Data were analyzed in a descriptive fashion.

Results: Despite the majority of high school students correctly identified the main modes of HIV/AIDS transmission, there was a relative deficiency in their knowledge about the disease. Students' attitudes were consequently defective towards HIV/AIDS infected people. Their main source of information about the infection was the media, particularly TV. The results indicated that the contribution of schools, health staff and relatives in providing high school students with knowledge about HIV/AIDS was the minimal.

Conclusion: There is need to provide students with correct information on HIV/AIDS infection. School teachers and health care providers should work together to help students acquire adequate knowledge and appropriate attitudes towards HIV/AIDS. This will help not only in correcting misconceptions about the disease, but also in preventing the spread of the infection among this young group of the Saudi society.

CORRESPONDING AUTHOR: Saad Alghanim, PhD, King Saud University, Riyadh, 11352; sagksu@gmail.com

\section{PSW-217}

\section{WORK ABILITY AMONG PORTUGUESE NURSES} IN HOSPITAL WARDS

Teresa Cotrim, $\mathrm{PhD}^{1}{ }^{1}$ Anabela Simões, $\mathrm{PhD}^{2}$ and Carlos Silva, $\mathrm{PhD}^{3}$

${ }^{1}$ Faculty of Human Kinetics / Technical University of Lisbon, Lisbon, Portugal; ${ }^{2}$ High Institute for Education and Science, Lisbon, Portugal and ${ }^{3}$ University of Aveiro, Aveiro, Portugal.

Introduction: At hospitals, high physical demands of patient handling tasks, but also the poor design of workplaces and the absence or inadequacies of equipments, influence the work practices of nurses and their work ability perception. The objective of this study was the investigation of the work ability of nurses working in hospital wards.

Methodology: The methodology encompassed the work ability analysis using the Portuguese version of Work Ability Index. Our sample consisted of 238 nurses working in 14 hospital wards of a central hospital. Participants were aged between 22 and 63 years (mean $=34,24 ; \mathrm{sd}=10,02), 82,4 \%$ were female, $50,4 \%$ were single and on average had spent 10 years with the current hospital $(\mathrm{min}=$ $0,5 ; \max =33 ; \mathrm{sd}=9,5)$.

Results: The average results of the work ability index for all nurses were $38,7(\min =18 ; \max =49 ; \mathrm{sd}=5,84)$ corresponding to a good work ability. The proportion of nurses with «good» work ability was the largest $(47,1 \%)$, followed by the group with «moderate» work ability $(27,3 \%)$. The results by age group show a decrease of work ability with 
age. To test if work ability differences do exist between age groups an one way Anova was used. The results show that significant differences exist in work ability index by age groups $(\mathrm{F}(238 ; 3)=19,76 ; \mathrm{p}=, 000)$. The work ability median for the group aged above 45 years was 35,5 , belonging to the «moderate» category.

Discussion: The average work ability index have been found to decrease with age what is in agreement with several studies. Slightly under half of the sample had a good index $(47,1 \%)$, but there was a substantial prevalence $(32,3 \%)$ of decreased WAI (poor and moderate categories) what is in concordance with the results of other studies with healthcare workers. These results can be explained by the nature of the work in hospital wards. People working in physically demanding jobs usually have lower average WAI than people working in mentally demanding jobs. Besides this fact, among nursing personnel the decrease of work ability can be accelerated due to several factors present in the workplace, namely the level of dependency of patients, the number of staff, the availability of mechanical lifts, the rooms layout, the shift work and others.

Conclusions: The study results imply the need for programs in the workplace in order to promote wellbeing. Work ability is the foundation of employability and employment which is getting more importance nowadays in order to prevent the discrimination of older nurses at the hospitals. The work ability index is an important tool for bringing together the working capabilities of ageing employees, identifying their needs and sustaining the choices of occupational health services in the development of programs to enable ageing employees to work.

CORRESPONDING AUTHOR: Alexandra Pereira, Master, University of Aveiro, Aveiro, 3800; alexandra.pereira@ua.pt

\section{PSW-218}

THE DEVELOPMENT OF A LIFESTYLE INTERVENTION IN ORDER TO IMPROVE OLDER WORKERS' VITALITY BY USING THE INTERVENTION MAPPING PROTOCOL Jorien E. Strijk, MSc, ${ }^{1}$ Karin I. Proper, $\mathrm{PhD},{ }^{1,2}$ Allard J. van der Beek, $\mathrm{PhD}^{1,2}$ and Willem van Mechelen, $\mathrm{PhD}^{1,2}$

${ }^{1}$ Department of Public and Occupational Health, EMGO + Institute, Amsterdam, Netherlands and ${ }^{2}$ TNO-VUmc, Body@ Work, Research Center for Physical activity, Work and Health, Amsterdam, Netherlands.

Purpose: The purpose of this study is to develop and evaluate a lifestyle intervention to improve older workers' vitality.

Methods: Using the Intervention Mapping (IM) protocol, a lifestyle intervention was developed based on information obtained from 1) literature, 2) a short lifestyle questionnaire aimed at indentifying the lifestyle behaviour among the target group, and 3) 5 focusgroup (FG) interviews among 36 older workers (aged $45+$ years). The FGs were aimed at identifying: a) key determinants of lifestyle behaviour, b) definition of vitality and, c) ideas about how vitality can be improved by lifestyle.

Results: Based on the short questionnaire, the main lifestyle problems identified were: insufficient levels of leisure time physical activity, sedentary behaviour, and insufficient intake of fruit and vegetables.
Using information from both literature and FG interviews, vitality appears to consist of a mental and a physical component. The interviewees suggested to improve the mental component of vitality by means of relaxation exercises (eg. yoga); physical vitality could be improved by aerobic exercises involving aerobic endurance, strength and flexibility. Based on the information mentioned above, the intervention will consist of three visits to a Personal Vitality Coach combined with a Vitality Exercise Programme, consisting of supervised yoga and an aerobic workout, each once per week, and lasting 45 minutes. Moreover, fruit will be offered at the workplace to meet the recommended intake of fruit.

Conclusion: The lifestyle intervention will be evaluated in a RCT among 698 older workers of two major academic hospitals in the Netherlands.

CORRESPONDING AUTHOR: Jorien E. Strijk, MSc, Department of Public and Occupational Health, EMGO+Institute, Amsterdam, 1081 BT; je.strijk@vumc.nl

PSW-219

EFFECTS OF A PREVENTION PROGRAM

ON THE KNOWLEDGE AND ATTITUDES TOWARDS HIV: DIFFERENCES BETWEEN SEXUALLY ACTIVE ADOLESCENTS AND NON-ACTIVE

Jose P. Espada, $\mathrm{PhD},{ }^{1}$ Yolanda Sanz, MA, ${ }^{1}$ Roberto Secades, $\mathrm{PhD},{ }^{2}$ Eduardo Remor, $\mathrm{PhD},{ }^{3}$ Rafael Ballester, $\mathrm{PhD},{ }^{4}$ Mireia Orgiles, $\mathrm{PhD},{ }^{1}$ Tania B. Huedo-Medina, $\mathrm{PhD}^{5}$ and Cristina Cassa, $\mathrm{MA}^{4}$

${ }^{1}$ Health Psyhology, Universidad Miguel Hernandez, Elche, Spain; ${ }^{2}$ Universidad de Oviedo, Oviedo, Spain; ${ }^{3}$ Universidad Autónoma de Madrid, Madrid, Spain; ${ }^{4}$ Universidad Jaume I, Castellon, Spain and ${ }^{5}$ University of Connecticut, Storrs, CT.

The main goal of this study was to evaluate the effectiveness of an intervention for preventing HIV transmission in adolescents, analyzing the differences between sexually active and those who are not. The sample consisted of 591 Spanish adolescents $(40.4 \%$ boys and $59.6 \%$ girls) with a mean age of $15.75(\mathrm{SD}=.88)$. The level of knowledge about HIV transmission was assessed using the HIV-KS scale (Espada et al., 2009), and the attitudes towards HIV/AIDS and risk practices was assessed using the CASIPR scale (Espada et al. 2009). A quasi-experimental design with two experimental conditions was carried out, with an experimental group $(n=371)$ and a waiting-list control group $(n=220)$. Each school was randomly assigned to one of the conditions. The intervention consisted of 5 one-hour sessions that included the following components: information and cognitive restructuring, social skills training, problem solving training and covered behavioral rehearsal. The effects of the interventions was evaluated through a immediate posttest assessment controlling for the baseline differences. The results point to a greater change in knowledge about HIV/AIDS in individuals who are not sexually active $(\mathrm{OR}=1.86, \mathrm{p}<.01)$ than those who are $(\mathrm{OR}=$ $1.81, \mathrm{p}<.01)$. With regard to attitudinal change, not sexually active adolescents have a higher change $(\mathrm{OR}=1.21, \mathrm{p}<.01)$ than the sexually active $(\mathrm{OR}=1.10, \mathrm{p}<.01)$. The highest efficacy of the 
intervention was observed among those who were not sexually active in both outcomes, knowledge and attitudes.

CORRESPONDING AUTHOR: Jose P. Espada, PhD, Health Psyhology, Universidad Miguel Hernandez, Elche, 03202; jpespada@umh.es

\section{PSW-220}

PSYCHICAL HEALTH STATE OF THE ADULT ROMANIAN POPULATION

Zoltan Abram, PhD

Public Health, UMF Targu Mures, Targu Mures, Romania.

The aim of our research is to study the psychical health state of the Romanian adults in three counties placed in the middle part of the country. We also have followed the lifestyle and the role of some psychological and social factors.

The study is based on a complex questionnaire, which was completed by a representative sample among adult population.

After our results depression is the most common psychical illness with $9,1 \%$ diagnosis, but in much higher rate exists depressive symptoms. According to Beck score, it was established a significant difference in the favour of female, elderly people, lower educational level, urban population. The percentage of suicide attempt among the studied population was $2,9 \%$. According to WHO Positive life quality test, in two-third majority the adult population consider that it is psychically healthy.

In our study it is underlined the role of religious feelings and the importance of health promotion and education to improve the psychical living standards of the population.

CORRESPONDING AUTHOR: Zoltan Abram, PhD, Public Health, UMF Targu Mures, Targu Mures, 540139; abramzoltan@, yahoo.com

PSW-221a

THE ROLE OF FEEDBACK IN A WEB-BASED GAME TARGETING MALARIA RISK REDUCTION

DECISION-MAKING

Laurie B. Hartjes, RN, MS, PNP-BC, ${ }^{1}$ Jeffrey B. Henriques, $\mathrm{PhD}^{1,2}$ and Linda C. Baumann, $\mathrm{PhD}^{1}$

${ }^{1}$ Nursing, University of Wisconsin-Madison, Verona, WI and ${ }^{2}$ Psychology, University of Wisconsin-Madison, Madison, WI.

Malaria is an excellent exemplar for a risk reduction intervention due to geographic fluctuations in its transmission range, lifethreatening consequences, and the vital need for adherence to personal protection measures. This study compared three versions of a web-based game targeting the malaria risk reduction decisionmaking of study abroad students. This population is in jeopardy due to the rapid expansion of programs in malaria endemic regions and the low level of acquired immunity and malaria knowledge among US citizens. Constructs from the self-regulation model and the cognitive theory of multimedia learning informed the creation and evaluation of this game. A three-condition experimental design was used to compare feedback strategies for game decisions: baseline corrective (right/wrong) feedback and explanatory feedback that is either provided automatically with each decision or only when players click on a link to access it. Primary outcome measures were malaria knowledge and player satisfaction. Study abroad email lists at seven Midwestern campuses were used to recruit participants without incentive. Of the 1428 participants who entered the study protocol and were randomly assigned to condition, 918 (64\%) completed the pre-survey, 540 (38\%) completed the game, and 482 (34\%) completed the postsurvey. Only the automatically provided explanatory feedback condition produced a significantly higher knowledge score on the post-test when adjusted for pre-test score. There were no significant differences in player satisfaction between the three feedback conditions, while $65 \%$ of participants rated game satisfaction as 'very' to 'extremely' satisfying. Analysis of secondary outcome measures suggests that students prefer simplicity and efficiency within this interactive learning environment. Future research will examine the impact of a risk reduction game intervention on actual behavior.

CORRESPONDING AUTHOR: Laurie B. Hartjes, RN, MS, PNP-BC, Nursing, University of Wisconsin-Madison, Verona, WI, 53593; 1hartjes@wisc.edu

\section{PSW-221b}

THE CORRELATION BETWEEN ACUPUNCTURE AND MOXIBUSTION DIAGNOSIS AND PERCEIVED MENTAL HEALTH USING CORNELL MEDICAL INDEX (CMI)

Yasuhiro Honda, Student, ${ }^{1}$ Akira Tsuda, Professor, ${ }^{2}$ Deng Ke, Student, ${ }^{3}$ Horiuchi Satoshi, Student ${ }^{4}$ and Hisayoshi Okamura, Assistant Professor ${ }^{5}$

${ }^{1}$ Kurume University, Kurume, Japan; ${ }^{2}$ Kurume University, Kurume, Japan; ${ }^{3}$ Kurume University, Kurume, Japan; ${ }^{4}$ Kurume University, Kurume, Japan and ${ }^{5}$ Kurume University, Kurume, Japan.

Introduction: Today it is important to maintain and promote mental health, since we are facing a lot of stress experiences. Acupuncture and moxibustion are closely related to behavioral medicine based on preventive medicine. Acupuncture and moxibustion medicine include diagnostic systems which enable us to detect sophisticated changes of the body and the mind as abnormalities of the meridians from a phase of pre-sickness. This study examined relationships between acupuncture and moxibustion medical assessments and perceived mental health states using subscores of Cornell Medical Index(CMI). The CMI used widely in the world was selected as the medical and psychiatric health questionnaire.

Method: 235 healthy volunteer college students participated in this study. The cervical meridian test was introduced as a medical diagnosis of acupuncture and moxibustion which is easy to estimate objectively. Subjects were asked whether they felt pain, stretched feeling, dullness or malaise when they flex the neck backward, right side, left side, or forward, as well as perceived 
mental health using CMI. We compared to mental health related symptoms between individuals with and without perceived pain or stretching by the cervical meridian test.

Results: Individuals with such feelings by flexing the neck forward and with such feelings by flexing the neck right and left sides tended to show higher most of scores on mental health related scores. Those with such feelings by flexing the neck backward tended to indicate higher scores on all subscores.

Discussion: The cervical meridian test induced "pain"or "stretched feeling" and reflected perceived sensitively reflect mental health states. Acupuncture and moxibustion medical assessment can successfully be applied to mental health domain.

CORRESPONDING AUTHOR: Yasuhiro Honda, Student, Kurume University, Kurume, 830-0011; ya-honda@dmr.ac.jp

\section{PSW-221c}

THE RELATIONSHIP BETWEEN THE CHARACTERISTICS OF INDIVIDUAL PERCEPTIONS OF ILLNESS AND MISUSE OF OVER-THE-COUNTER MEDICINES

\section{IN UNDERGRADUATE STUDENTS}

Takanori Honda, BA, ${ }^{1}$ Shiho Matsuoka, BA ${ }^{1}$ and Shin-ichi Suzuki, $\mathrm{PhD}^{2}$

${ }^{1}$ Graduate school of Human Sciences, Waseda university, Tokorozawa, Saitama, Japan and ${ }^{2}$ Faculty of Human Sciences, Waseda university, Tokorozawa, Saitama, Japan.

Background: Over-the-counter (OTC) medicines are increasingly used for self-medication, but such products are subject to misuse. Although it is suggested that psychological factors may play a role in OTC drug misuse, the main factors that might be associated with one's manner of drug intake are unknown. Objective: The purpose of this study was to explore the illness perceptions and beliefs about taking OTC medicines and to investigate the differences between users and misusers of OTC medicines. Method: The subjects were 108 undergraduate students (age mean $=20.27$, S.D. $=1.67$ ). They retrospectively completed selfreport measures addressing (a) illness perceptions, (b) beliefs about taking OTC medicines, (c) how to take OTC medicines, (d) common cold symptoms, and (e) frequency of intake. Result: The analysis of covariance indicated that misuse of OTC medicines was related to an individual's perceptions that concern consequences of illness $(\mathrm{F}(1,107)=5.85 ; \mathrm{p}<.05)$ and controllability $(\mathrm{F}(1,107)=6.57, \mathrm{p}<.05)$. However, no other factors are associated with misuse of OTC medicines. Conclusion: This study revealed that consumers' perceptions are associated with their manner of drug intake. These findings indicate that the tendency to misuse OTC drugs can be evaluated using a selfreport questionnaire of illness perception. Further studies are needed in order to develop brief questions to evaluate and examine if illness perceptions predict one's manner of drug intake.

CORRESPONDING AUTHOR: Takanori Honda, BA, Graduate school of Human Sciences, Waseda university, Tokorozawa, Saitama, 359-1192; whs.be_a_cp144@asagi.waseda.jp
PSW-221d

DESIGN, IMPLEMENTATION AND EVALUATION

OF A MOURNING-TASK WORKSHOP

AFTER DISSOLUTION OF AN INTIMATE RELATIONSHIP

IN COLLEGE STUDENTS

Judith de la Serna, student

Psychology, Universidad Nacional Autónoma de México, Mexico City, Mexico.

Breakups are usually distressing and generate different consequences (emotional, cognitive, behavioral and physiological) that can be associated to poor social relationships, physical/mental disorders and may eventually lead to suicide.

The dissolution of a relationship implies a transitional crisis and is considered a relational loss that generates a grieving process. After breaking up with someone, the person must achieve mourning tasks, redefine the relationship, rebuild self-esteem and readjust to adaptive social patterns.

A workshop was designed for vulnerable people, who had been detected with the Detection Questionnaire of Feelings, Emotions and Consequences of Loss (de la Serna, 2009), as a psychotherapeutic approach towards the mourning-task following the dissolution of a relationship. This study consisted of a preexperimental pretest-postest design with 7 participants, all female students aged between 18 and 26 in a public University in Mexico City. The workshop consisted of 10 weekly sessions in which theoretical information and cognitive-behavioral strategies (cognitive restructuring, training in social skills and problem solving) were taught in order to help participants understand their loss, work their grief and increase their self esteem and assertiveness in order to establish healthier social relationships.

Three instruments were used for pre-post evaluation of the workshop and statistically significant differences $(p \leq .10)$ were obtained indicating its effectiveness. Mourning tasks were achieved with significant results in both sections of the detection questionnaire: feelings/emotions $(\mathrm{t}=2.012 ; \mathrm{p}=.091)$ and consequences $(t=4.621 ; p=.004)$. Significant differences $(t=-3.746$; $\mathrm{p}=.010$ ) were also found in the Self Esteem Scale (Reidl, 2005) indicating an increase in participants' self esteem. Finally, assertive behavior also showed significant differences in the Assertion Inventory (Gambrill \& Richey, 1975) with a decrease in the degree of discomfort $(\mathrm{t}=5.024 ; \mathrm{p}=.002)$ and an increase in the probability responding assertively $(\mathrm{t}=3.533 ; \mathrm{p}=.012)$.

CORRESPONDING AUTHOR: Judith de la Serna, student, Psychology, UNAM, Mexico City, 04326; psicjsn@hotmail.com

PSW-221e

PSYCHOSOCIAL FACTORS INFLUENCING

A MULTI-ETHNIC SAMPLE'S WILLINGNESS

TO PARTICIPATE IN GENETIC TESTING: PROJECT GATHER Marissa C. Perales, BA, ${ }^{1}$ Georita M. Frierson, $\mathrm{PhD},{ }^{1,2}$ Margo Simmons, BA, ${ }^{2}$ Celette Sugg Skinner, $\mathrm{PhD},{ }^{3}$ Benjamin Willis, $\mathrm{MD}^{2}$ and Laura DeFina, $\mathrm{MD}^{2}$

${ }^{1}$ Psychology, Southern Methodist University, Dallas, TX; ${ }^{2}$ Cooper Institute, Dallas, TX and ${ }^{3}$ UT Southwestern, Dallas, TX. 
Genetic testing is useful for primary, secondary and tertiary prevention. Patient characteristics and preconceived notions of genetic testing may create barriers in obtaining genetic testing. Multi-ethnic, mixed-methods studies are needed to assess these factors that influence willingness to participate in genetic testing for multiple chronic diseases.

Project Diversity GATHER (Group discussions Around Themes on HEalthful genetic Research) examines sociodemographic and psychosocial factors that may influence willingness to participate in genetic testing.

Participants $(\mathrm{N}=135,63 \%$ female; mean age $=46.67, \mathrm{SD}=17.17$, range $=18-87$ ) completed sociodemographic and psychosocial questionnaires and a one-hour focus group. Participants self-identified as African-American (49.2\%), White (36.2\%), Native Hawaiian or other Pacific Islander (2.3\%), American Indian/Alaska Native (2.3\%), Asian (1.5\%) and Other (8.5\%); $19.3 \%$ of participants were Latino. Univariate and bivariate descriptive analyses were performed where appropriate; univariate and multivariate logistic regression models were applied to compare differences in (un)adjusted odds of who was willing to participate in genetic testing vs. not.

Univariate regression analyses included gender $(\mathrm{OR}=0.94$, $95 \% \mathrm{CI}=0.44-1.99)$, race/ethnicity $(\mathrm{OR}=0.41,95 \% \mathrm{CI}=0.32$ $1.60)$, level of education $(\mathrm{OR}=0.40,95 \% \mathrm{CI}=0.19-0.84)$, employment status $(\mathrm{OR}=0.20,95 \% \mathrm{CI}=0.20-0.87)$, type of insurance $(\mathrm{OR}=0.45,95 \% \mathrm{CI}=0.20-1.00)$, health and psychosocial beliefs $(\mathrm{OR}=1.05,95 \% \mathrm{CI}=0.99-1.11)$, and distress concerns $(\mathrm{OR}=0.95$, $95 \% \mathrm{CI}=0.90-0.99)$. Multivariate analyses confirmed education $(\mathrm{OR}=0.34,95 \% \mathrm{CI}=0.14-0.87)$, employment status $(\mathrm{OR}=0.38$, $95 \% \mathrm{CI}=0.15-0.96)$, health and psychosocial beliefs $(\mathrm{OR}=1.06$, $95 \% \mathrm{CI}=1.00-1.13)$, and distress concerns $(\mathrm{OR}=0.92,95 \% \mathrm{CI}=$ $0.86-0.98)$ as significant predictors of willingness to participate in genetic testing. Participants with higher levels of education, who are employed and who associate helpful or anxiety-reducing outcomes with genetic testing are more willing to participate in genetic testing. Alternatively, those with fears or stress associated with genetic testing were less willing to participate in genetic testing. Findings from this study may have implications on areas to target when educating patients about undergoing genetic testing.

CORRESPONDING AUTHOR: Marissa C. Perales, BA, Psychology, Southern Methodist University, Dallas, TX, 75251; mperales@smu.edu

\section{PSW-221f}

DEVELOPMENT OF JAPANESE LANGUAGE VERSION

OF THE TRANSTHEORETICAL MODEL (TTM)-BASED STRESS MANAGEMENT PROGRAM FOR COLLEGE STUDENTS

Akira Tsuda, $\mathrm{PhD},{ }^{1}$ Satoshi Horiuchi, $\mathrm{PhD},{ }^{1}$ Toru Morita, $\mathrm{PhD},{ }^{1}$ Euiyeon Kim, MA, ${ }^{1}$ Ke Deng, MA, ${ }^{1}$ Ayumi Togawa, MA, ${ }^{1}$ Hisayoshi Okamura, MA, ${ }^{1}$ Yoshiyuki Tanaka, MA, ${ }^{2}$ Shigeko Tsuda, MA, ${ }^{3}$ Kerry M. Evers, $\mathrm{PhD}^{4}$ and Janice M. Prochaska, $\mathrm{PhD}^{4}$ ${ }^{1}$ Psychology, Kurume University, Kurume, Japan; ${ }^{2}$ Child Studeis, Tokyo University and Graduate School of Social Welfare, Isezaki, Japan; ${ }^{3}$ Nursing, Ibaraki Christian University, Hitachi, Japan and ${ }^{4}$ Pro-Change Behavior Systems, Inc., West Kingston, RI.
Background: Managing stress on a population basis is one of the promising approaches to enhancing health and reducing health care costs. This study are aims twofold. First, it is to translate and modify a transtheoretical model (TTM)-based stress management program developed originally for the US population for use in a different cultural context, Japan. Second, it is to report on 6-month outcomes of a randomized clinical trial (RCT) of this TTM-based stress management program from Pro-Change Behavior Systems, Inc., West Kingston, RI, using the internet.

Methods: We translated and modified Pro-Change TTM-based stress management intervention program into Japanese and adapted it for use in a Japanese cultural setting. Stage-based expert systems were applied to reduce stress and enhance effective stress management behavior which is defined as setting aside approximately $20 \mathrm{~min}$ each day for a healthy activity such as talking with others, physical activity, or regular relaxation to manage stress. 456 university students were randomly allocated to either using the TTM self-help stage-based workbook for stress management followed by three individualized minimum reports at 0,3 and 6 month) (i.e., workbook + feedback group) or the workbook followed by the same 3 individualized reports plus detailed prescriptions for processes of change (i.e., workbook + prescription group).

Results: Results indicate that the stage-matched self-help learning for effective stress management behaviours followed by stagebased expert systems had significant effects on stress alleviation and progression of stage for stress management behaviours in both groups, irrespective of with and without individualized and tailored prescription for processes of change feedback reports.

Conclusions: Stage-matched TTM expert systems for self-help stress management behaviour workbook can reduce stress for students irrespective of with and without individualized and tailored prescription for processes of change feedback reports. This study confirmed the long-term positive effects of this modified TTM-based stress management program in a different cultural context.

CORRESPONDING AUTHOR: Akira Tsuda, PhD, Kurume University, Kurume, 8395-8502; tsuda_akira@kurume-u.ac.jp

\section{Thursday \\ August 5, 2010 SYMPOSIA SESSIONS 8:30 AM-10:00 AM}

SS01

THE ROLE OF PATIENTS' BELIEFS ABOUT THEIR ILLNESS AND ABOUT MEDICINES IN ADHERENCE TO TREATMENT

Alexandra Martin, $\mathrm{PhD}$

Psychosomatic Medicine and Psychotherapy, University Hospital of Erlangen, Erlangen, Germany.

Non-adherence to medication prescription is a serious problem in many chronic illnesses that require long-lasting treatment. There is 
considerable research showing that multiple factors such as patient's characteristics (e.g., age), medication related aspects (e.g., side effects) as well as environmental factors (e.g., availability) do affect adherence. In addition, the self-regulatory theory underlines the importance of subjective perceptions and beliefs guiding individual coping behaviour.

The symposium addresses the interaction of illness perceptions and of treatment related beliefs with adherence to treatment from various perspectives. First, Nestoriuc et al. (1) present an epidemiologic study showing the relevance of medication beliefs in the general population and the psychometric foundation of the widely used Beliefs about Medicines Questionnaire (BMQ). Next, two studies are presented about the association of illness perceptions and of medication beliefs with non-adherence in clinical samples. Broadbent et al. (2) present evidence on the effect of both on adherence to immunosuppressant medication in liver transplant patients; while the results of Martin et al. (3) suggest that medication beliefs are associated with the intake of antihypertensive eye drops in patients with glaucoma, whereas illness perceptions do not additionally explain the degree of adherence.

As both, the efficacy of a medication and patient adherence to the therapeutic regiment, influence the effectiveness of a treatment, it appears necessary to improve adherence in many illnesses, e. g. by psychological interventions. Petrie et al. (4) suggest a brief and economic intervention - a tailored text message programme - to change patients' illness perceptions with the aim to improve treatment adherence. Their controlled trial show promising results in asthma patients.

Based on the presentations the symposium will offer a discussion about present evidence and perspectives regarding the role of patients' beliefs and perceptions on adherence.

CORRESPONDING AUTHOR: Alexandra Martin, PhD, Psychosomatic Medicine and Psychotherapy, University Hospital of Erlangen, Erlangen, 91054; alexandra.martin@uk-erlangen.de

\section{SS01a}

TARGETED TEXT MESSAGING DESIGNED TO CHANGE PERCEPTIONS IMPROVES ADHERENCE TO ASTHMA PREVENTER MEDICATION

Keith Petrie, $\mathrm{PhD},{ }^{1}$ Kate Perry, $\mathrm{PhD},{ }^{2}$ John Weinman, $\mathrm{PhD},{ }^{3}$ Hamish Franklin, BS $^{2}$ and Jonny Duder, BS $^{2}$

${ }^{1}$ University of Auckland Medical School, Auckland, New Zealand; ${ }^{2}$ Atlantis Health Care, Auckland, New Zealand and ${ }^{3}$ Kings College, London, United Kingdom.

Background: While effective preventative medication is readily available in asthma, adherence is a major problem due to patients' beliefs about their illness and medication. Many non-adherent patients are reluctant to take their preventer inhaler when they are not experiencing symptoms and have concerns about their inhalers. Aim: We investigated whether a text message programme targeted at changing patients' illness perceptions would improve adherence in asthma patients.

Methods: 212 patients aged between 16 and 45 on asthma medication were recruited from medication package inserts and health web groups. Participants were randomized to receive individually tailored text messages based on their belief profile over 18 weeks or no text messages. The type and number of text messages designed to promote beliefs associated with higher adherence. Adherence rates were assessed by phone calls to participants at 6,12 and 18 weeks.

Results: Overall adherence levels at baseline were low at $46 \%$ with only $12 \%$ taking over $80 \%$ of their prescribed doses. The data showed the targeted text group significantly improved adherence over the follow-up period compared to the control group with a relative average increase in adherence of $17 \%$. The percentage taking over $80 \%$ of prescribed inhaler doses over the follow-up period increased by $20 \%$ in the intervention group compared to the control group.

Conclusions: A targeted text message programme increases adherence to asthma preventer inhaler and may be useful for other illnesses where adherence is a major issue.

CORRESPONDING AUTHOR: Keith Petrie, PhD, University of Auckland Medical School, Auckland, 10024; kj.petrie@auckland. ac.nz

$\mathrm{SS} 01 \mathrm{~b}$

NON-ADHERENCE IN LIVER TRANSPLANT PATIENTS IS ASSOCIATED WITH MEDICATION BELIEFS AND ILLNESS PERCEPTIONS

Elizabeth Broadbent, PhD, ${ }^{1}$ Mary Kung, BSc, ${ }^{1}$ Liz Painter, DipClinPsych $^{2}$ and Val Honeyman ${ }^{2}$

${ }^{1}$ Psychological Medicine, The University of Auckland, Auckland, New Zealand and ${ }^{2}$ Auckland District Health Board, Auckland, New Zealand.

Background: Non-adherence to immunosuppressant medications can contribute to medical complications in transplant patients. A recent pilot study showed that non-adherence is associated with liver transplant patients' beliefs that that the transplant has severe consequences and is distressing, as well as concerns about the harmful effects of medication.

Aim: This study aimed to investigate associations between illness perceptions, medication beliefs, and adherence in liver transplant patients, extending previous work to a larger sample.

Method: 193 liver transplant patients completed a postal questionnaire. Adherence was assessed using self reports (Immunosuppressant Therapy Adherence Instrument) and blood assays. Perceptions were assessed using the Brief Illness Perception Questionnaire, and the Beliefs about Medication Questionnaire.

Results: $50 \%$ of the sample self-reported some form of nonadherence. Lower self-reported adherence was associated with lower education, lower beliefs about medication necessity, higher beliefs that medication was harmful, and had adverse effects, lower beliefs that immunosuppressants could prevent rejection, poorer understanding of the transplant, and higher distress about the condition. A higher percentage of drug test levels below the therapeutic range was associated with less time since transplant, and lower beliefs that medication could prevent rejection.

Conclusion: This research provides further evidence that illness perceptions and medication beliefs are associated with non- 
adherence to immunosuppressant medication in liver transplant patients. Addressing patients' beliefs about their medication, understanding and distress about their transplant may help to improve adherence.

CORRESPONDING AUTHOR: Elizabeth Broadbent, PhD, Psychological Medicine, The University of Auckland, Auckland, 1142; e.broadbent@auckland.ac.nz

\section{$\mathrm{SS} 01 \mathrm{c}$}

RELEVANCE OF ILLNESS PERCEPTIONS AND BELIEFS ABOUT MEDICINES FOR ADHERENCE IN GLAUCOMA PATIENTS

Alexandra Martin, PhD, ${ }^{1}$ Vanessa Roehricht, candpsych ${ }^{1}$ and Ulrich-Christoph Welge-Luessen, $\mathrm{MD}^{2}$

${ }^{1}$ Department of Psychosomatic Medicine and Psychotherapy, University Hospital of Erlangen, Erlangen, Germany and ${ }^{2}$ Department of Ophthalmology, University Hospital of Erlangen, Erlangen, Germany.

Non-Adherence with intraocular pressure lowering eye drops is an important risk factor for going blind in glaucoma patients. Previous research evidenced a relationship between adherence to treatment and patients' illness and treatment related beliefs in a range of chronic illnesses.

Objectives: The aim of this study was to investigate the associations between illness perceptions according to the "common sense model of self-regulation", medication beliefs, and adherence in subjects with glaucoma.

Methods: 145 glaucoma patients with a mean age of $64(\mathrm{SD}=$ 12.7) completed a standardised questionnaire containing the revised Illness Perception Questionnaire [IPQ-R], the Beliefs about Medicines Questionnaire [BMQ], the Adherence to Refills and Medications Scale [ARMS] and a visual analogue scale on adherence [VAS-AD]. Clinical characteristics (e. g., ophthalmologic examination results) were recorded.

Results: Unlike the expectation illness perceptions and disease severity indices did not correlate with adherence. Lower selfreported adherence was associated with the intensity of side effects and the medication related beliefs "General Harm", "General Benefit", "Specific Necessity", "Specific Concerns" [ARMS/ VAS-AD; p's<.05]. Multivariate analysis showed that the beliefs about medicines provide a useful model $(\mathrm{R} 2=.14 ; \mathrm{F}=3.9 ; \mathrm{p}<.01)$ to predict eye drop taking behaviour with the two specific belief dimensions having the strongest predictive power $(\beta=-.21 / .21)$. Conclusions: This study suggests that beliefs about medicines seem to be useful predictors of adherence in glaucoma patients. By contrast the illness perceptions do not seem to affect adherence. The reasons for this result may lie in special characteristics of glaucoma and/ or the assessment methods used. The relevance of patients' beliefs will be discussed in the context of further factors impairing adherence in glaucoma patients.

CORRESPONDING AUTHOR: Alexandra Martin, PhD, Psychosomatic Medicine and Psychotherapy, University Hospital of Erlangen, Erlangen, 91054; alexandra.martin@uk-erlangen.de
SS01d

DOES THE WAY WE THINK ABOUT MEDICATIONS

DETERMINE HOW WE USE THEM? VALIDATION

OF THE BELIEFS ABOUT MEDICINES QUESTIONNAIRE

IN THE GENERAL POPULATION IN GERMANY

Yvonne Nestoriuc, PhD, ${ }^{1}$ Julia A. Glombiewski, PhD, ${ }^{1}$ Heide Glaesmer, $\mathrm{PhD},{ }^{2}$ Rob Horne, $\mathrm{PhD},{ }^{3}$ Elmar Braehler, $\mathrm{MD}^{2}$ and Winfried Rief, $\mathrm{PhD}^{1}$

${ }^{1}$ Clinical Psychology and Psychotherapy, Philipps-University Marburg, Marburg, Germany; ${ }^{2}$ Medical Psychology and Sociology, University of Leipzig, Leipzig, Germany and ${ }^{3}$ Centre for Behavioral Medicine, The School of Pharmacy, University of London, London, United Kingdom.

Individual treatment perceptions are relevant for the self-regulation of health and illness behavior. In people with chronic illnesses, beliefs about medicines have been shown to influence patients' treatment decisions, adherence and side effect reporting. However, to interpret these results epidemiologic data about medication beliefs is essential. In this study, a German translation of the Beliefs about Medicines Questionnaire (BMQ) was psychometrically evaluated. A representative sample of the German population $(\mathrm{N}=2512)$ was screened for medication use, adherence, generic side effects, and beliefs about medicines. Reference scores for beliefs about medicines from the general population were calculated and compared for different drug classes. Moreover, to investigate criterion validity their influence on adherence was determined. A principal component analyses with oblique factors confirmed the original 6 factor structure of the BMQ. Further psychometric analyses revealed moderate to high internal consistencies and good construct validity. In a stepwise linear multiple regression, adherence was significantly predicted by participants' age, sex, current medication, side effects, and beliefs about medicines (adjusted $\mathrm{r}^{2}=.25, p<.001$ ). Specifically, beliefs about medicines explained an additional significant proportion of $20 \%$ of variance, after controlling for relevant demographic characteristics, medication intake and side effects. The German version of the BMQ is a reliable and valid self rating instrument for the assessment of peoples' perceptions of medication. Adherence in the general population is determined by sociodemographic characteristics, current medication intake, experienced side effects, and to a unique and substantial proportion by medication beliefs.

CORRESPONDING AUTHOR: Yvonne Nestoriuc, PhD, Clinical Psychology and Psychotherapy, Philipps-University Marburg, Marburg, 35037; yn@staff.uni-marburg.de

$\mathrm{SS} 02$

PSYCHOLOGICAL, BIOLOGICAL AND BEHAVIORAL ASPECTS OF CORONARY ARTERY DISEASE

INTERVENTION

Hans-Christian Deter, MD, ${ }^{1}$ Manfred E. Beutel, MD, ${ }^{2}$ Kristina Orth-Gomer, $\mathrm{MD}^{3}$ and Neil Schneiderman, $\mathrm{PhD}^{4}$

${ }^{1}$ University Medical Center, Berlin, Germany; ${ }^{2}$ University Medicine, Mainz, Germany; ${ }^{3}$ Karolinska Institute, Stockholm, Sweden and ${ }^{4}$ University of Miami, Coral Gables, FL. 
Several behavioral intervention studies for coronary artery disease (CAD) have been published during the last decade in Belgium, Canada, Sweden and the United States. Many of the studies focused upon reducing CAD risk in patients already showing CAD manifestations. Hans-Christian Deter will examine the rationale and targets of the new German SPIRR-CAD trial, which is a combined psychodynamic and cognitive behavior group therapy intervention aimed at decreasing depressive symptoms, Type D behavior and CAD risk profile and events in CAD patients. Manfred Beutel will describe the diagnostic and therapeutic processes involved as well as the psychological quality management program used to insure a high quality of comparable intervention fidelity at the 10 centers collecting data in this SPIRR-CAD trial. Kristina Orth-Gomér will discuss the reduced mortality produced by psychosocial intervention in the Stockholm Women Intervention Trial for Coronary Heart Disease (SWITCHD) and how tailoring the intervention to the gender related needs of women may have contributed to the outcome. Neil Schneiderman will then describe biological and behavioral factors that appear to have been involved in those randomized controlled trials that have successfully used psychosocial interventions to reduce the recurrence of major adverse coronary events (MACE).

CORRESPONDING AUTHOR: Hans-Christian Deter, MD, University Medical Center, Berlin, 12200; deter@charite.de

\section{$\mathrm{SS} 02 \mathrm{a}$}

DESIGN AND RATIONALE OF A NEW GERMAN RANDOMIZED CONTROLLED TRIAL: STEPWISE PSYCHOTHERAPY INTERVENTION FOR REDUCING RISK IN CORONARY ARTERY DISEASE (SPIRR-CAD)

Hans-Christian Deter, MD, ${ }^{1}$ Christian Albus, $\mathrm{MD}^{2}{ }^{2}$ Manfred Beutel, MD, ${ }^{3}$ Kurt Fritzsche, MD, ${ }^{4}$ Harald Gündel, MD, ${ }^{5}$ Peter Joraschky, MD, ${ }^{6}$ Jochen Jordan, MD, ${ }^{7}$ Jana Jünger, MD, ${ }^{8}$ Karl-Heinz Ladwig, MD, ${ }^{9}$ Michael Mück-Weymann, $\mathrm{MD},{ }^{10}$ Wolfgang Söllner, MD ${ }^{11}$ and Christoph Herrmann-Lingen, $\mathrm{MD}^{12}$ ${ }^{1}$ University Medical Center, Berlin, Germany; ${ }^{2} \mathrm{U}$ Clinic Cologne, Cologne, Germany; ${ }^{3} \mathrm{U}$ Medicine Mainz, Mainz, Germany; ${ }^{4} \mathrm{U}$ Clinic Freiburg, Freiburg, Germany; ${ }^{5}$ Hannover Medical School, Hannover, Germany; ${ }^{6}$ Dresden U, Dresden, Germany; ${ }^{7}$ Kerckhoff Reha Center, Bad Nauheim, Germany; ${ }^{8} \mathrm{U}$ Clinic Heidelberg, Heidelberg, Germany; ${ }^{9}$ TU München, München, Germany; ${ }^{10} \mathrm{U}$ Clinic Dresden, Dresden, Germany; ${ }^{11}$ Clinic Nord Nürnberg, Nürnberg, Germany and ${ }^{12} \mathrm{U}$ Clinic Göttingen and the SPIRRCAD- study group, Göttingen, Germany.

Depressive symptoms are highly relevant in patients with (CAD). Unfortunately, recent psychotherapy trials showed only small effects on depression. However, combined treatment may be more efficacious than a behavioural or interpersonal treatment alone. In our new multicenter trial, funded by the German Research Foundation, we address negative affectivity and social inhibition in addition to depression to enhance treatment efficacy. Men and women $(n=569$, age 18-75 y) with HADS depression scores $>7$ hospitalized for any manifestation of $\mathrm{CAD}$, are randomized into the intervention or control group. Initially, patients in intervention receive 3 sessions of supportive individual psychotherapy on a weekly basis. After reevaluation of depression 4 weeks after inclusion, patients with persisting symptoms of depression receive 25 sessions of combined psychodynamic and group cognitive behavior therapy (CBT) over 10 months. The control group receives one counselling session. Routine cardiac care is offered to both groups. Primary efficacy endpoint is change from baseline to year 1 in HADS depressive symptoms. Secondary endpoints include remission of depression, Type D pattern, health-related quality of life, cardiovascular risk profile, neuroendocrine and inflammatory activation, heart rate variability, cardiac events, and health care utilization, up to 24 months after inclusion. Preplanned subgroup analyses will assess gender, and genetic predictors of treatment success.

CORRESPONDING AUTHOR: Hans-Christian Deter, MD, University Medical Center, Berlin, 12200; deter@charite.de

\section{$\mathrm{SS} 02 \mathrm{~b}$}

PSYCHOLOGICAL BASIS OF THE PSYCHODYNAMIC INTERVENTION IN SPIRR-CAD

Manfred E. Beutel, MD, ${ }^{1}$ Jörg Wiltink, MD, ${ }^{1}$ Matthias Michal, $\mathrm{MD},{ }^{1}$ Christian Albus, MD, ${ }^{2}$ Hans-Christian Deter, MD, ${ }^{3}$ Kurt Fritzsche, MD, ${ }^{4}$ Christiane Waller, $\mathrm{MD},{ }^{5}$ Katja Petrowski, $\mathrm{PhD},{ }^{6}$ Jochen Jordan, $\mathrm{PhD},{ }^{7}$ Jana Jüunger, $\mathrm{MD}{ }^{8}$ Karl-Heinz Ladwig, $\mathrm{PhD},{ }^{9}$ Michael Mück-Weymann, MD, ${ }^{10}$ Wolfgang Söllner, $\mathrm{MD}^{11}$ and Christoph Herrmann-Lingen, $\mathrm{MD}^{12}$

${ }^{1}$ Psychosomatic Clinic, U Medicine, Mainz, Germany; ${ }^{2}$ Psychosomatics, U Clinic Cologne, Cologne, Germany; ${ }^{3}$ Psychosomatics, U Medical Center, Berlin, Germany; ${ }^{4}$ Psychosomatics, U Clinic Freiburg, Freiburg, Germany; ${ }^{5}$ Psychosomatics, Medical School, Hannover, Germany; ${ }^{6}$ Psychosomatics, U Clinic Dresden, Dresden, Germany; ${ }^{7}$ Psychocardiology, Kerckhoff Reha Center, Bad Nauheim, Germany; ${ }^{8}$ Gen. Internal and Psychosomatic Med, U Clinic Heidelberg, Heidelberg, Germany; ${ }^{9}$ Psychosomatics, TU Munich, Munich, Germany; ${ }^{10}$ Psychosomatics, U Clinic Dresden, Dresden, Germany; ${ }^{11}$ Psychosomatics, Klinikum Nord Nürnberg, Nürnberg, Germany and ${ }^{12}$ Psychosomatics, U Clinic Göttingen on behalf of SPIRR-CAD Study Group, Göttingen, Germany.

Psychotherapeutic interventions in the SPIRR-CAD trial are characterized by (1) a stepwise combination of individual and group treatment, (2) integration of behavioural skills and education into a psychodynamic group framework focusing on negative affect and social inhibition. In order to assure a high quality of comparable interventions at the 10 sites of the multicenter trial, systematic analyses of adherence and competence of therapists are performed on videotape recordings of all treatments. Based on rating manuals for psychodynamic and behavioral intervention, specific rating manuals were devised and raters were trained. Moderate to good interrater reliabilities were obtained between raters based on 18 randomly selected individual sessions $(0.48-0.69)$. Therapists are trained according to manual, undergo regular supervision and obtain written feedback on their performance. One out of two individual sessions is evaluated. Out of 25 group sessions, 3 are randomly selected from early, middle and termination phase. 
According to preliminary results, adherence and competence are unrelated to the helping alliance as judged by patient and therapist. Implications for the identification of effective treatment components (e.g. therapist performance) are discussed.

CORRESPONDING AUTHOR: Hans-Christian Deter, MD, University Medical Center, Berlin, 12200; deter@charite.de

\section{$\mathrm{SS} 02 \mathrm{c}$}

GENDER, FAMILY, WORK AND STRESS REDUCTION IN BEHAVIORAL INTERVENTION

FOR CARDIOVASCULAR DISEASE

Kristina Orth-Gomér, $\mathrm{MD}, \mathrm{PhD},{ }^{1,3}$ Neil Schneiderman, $\mathrm{PhD},{ }^{2}$ H. Wang, $\mathrm{PhD},{ }^{1}$ M. Blom, $\mathrm{PhD},{ }^{1}$ C. Walldin, $\mathrm{PhD}^{1}$ and T. Jernberg, $\mathrm{MD}^{1}$

${ }^{1}$ Karolinska Institutet, Stockholm, Sweden; ${ }^{2}$ University of Miami, Miami, FL and ${ }^{3}$ University Medical Center, Berlin, Germany.

Stress at work has been shown to influence both incidence and progression of cardiovascular disease (CVD), but psychosocial interventions to reduce stress and CVD have produced inconsistent Results: Moreover, meta-analysis has indicated that psychosocial interventions have been more effective in reducing CVD in men than in women. One possible explanation is that men and women have different needs in terms of stress reduction. Thus, it appears that family problems constitute stronger emotional stressors and provoke more depressive feelings in women than men, and that women, even when working outside the home are more endangered by family stress than by work stress. Therefore, the Stockholm Women's Intervention Trial (SWITCHD) was specifically designed to meet the needs of women CVD patients in terms of tailored stress reduction. This randomized clinical trial decreased CVD mortality in women receiving the intervention compared to women receiving standard care, providing evidence that an intervention designed to satisfy the gender related needs of women may prolong life in women with CVD.

CORRESPONDING AUTHOR: Hans-Christian Deter, MD, University Medical Center, Berlin, 12200; deter@charite.de

\section{SS02d}

BEHAVIORAL AND BIOLOGICAL FACTORS

IN PSYCHOSOMATIC/BEHAVIORAL MEDICINE INTERVENTIONS FOR CORONARY ARTERY DISEASE (CAD)

Neil Schneiderman, $\mathrm{PhD}^{1}$ and Kristina Orth-Gomér, $\mathrm{MD}^{2}$

${ }^{1}$ University of Miami, Miami, FL and ${ }^{2}$ Karolinska Institutet, Stockholm, Sweden.

Randomized controlled trials (RCT) that have used behavioral interventions designed to reduce morbidity and/or mortality in patients who have had major adverse coronary events (MACE) have reported positive, null and negative Results: In general RCT that have been successful used group based interventions, behavior change strategies, at least 20 sessions during the course of a year, and have followed patients for at least several years.
Some successful studies (e.g., Stockholm Women's Intervention Trial for Coronary Heart Disease: SWITCHD) have employed relaxation training, suggesting that stress reduction is important. SWITCHD also found that the interaction between behavioral intervention and the use of statins also appeared to decrease mortality. And in research conducted in animals we have found that social affiliation can attenuate the development of atherosclerosis by decreasing inflammation and oxidative stress. Because patients with CAD typically are prescribed multiple medications, medication adherence may be a useful intervention target. Similarly, because most CAD patients are overweight, physically inactive, and may have metabolic syndrome, behavioral intervention strategies to increase weight loss and improve fitness could be useful for addressing these issues. This presentation will compare and contrast RCT in terms of the biological and behavioral bases of the intervention strategies used to treat CAD patients.

CORRESPONDING AUTHOR: Hans-Christian Deter, MD, University Medical Center, Berlin, 12200; deter@charite.de

\section{$\mathrm{SS} 03$}

EXPLORING THE NATURE, CAUSES

AND CONSEQUENCES OF "HIKIKOMORI" (SOCIAL WITHDRAWAL): A RECENT ADVANCES IN EPIDEMIOLOGY AND QUALITATIVE RESEARCH

Norito Kawakami, MD

School of Public Health, University of Tokyo, Tokyo, Japan.

The "hikikomori" (social withdrawal) describes a psychopathological and sociological phenomenon in which people, and especially younger generations, become completely withdrawn from society for six months or longer. The "hikikomori" was first reported in Japan, and had been thought as a culture-bounded psychopathology. But cases of "hikikomori" were reported also in Oman and Spain. Recently, a large-scale community-based epidemiology study in Japan, the World Mental Health Japan Survey, as well as other studies, reported that the prevalence, psychiatric comorbidity, and childhood risk factors of "hikikomiri". Also a qualitative study from Hong Kong reported that "hikikomiri" was a psychopathology with difficulties in interacting with others and it was not unique to the Japanese society. In this symposium, each speaker will present their recent research findings. The advances in epidemiology and qualitative research could provide a new perspective of the nature of "hikikomori" as a unique psychopathology developed in the context of the society.

CORRESPONDING AUTHOR: Norito Kawakami, MD, School of Public Health, University of Tokyo, Tokyo, 113-0033; kawakami@m.u-tokyo.ac.jp

SS03a

EXPLORING HIKIKOMORI - A MIXED METHODS

QUALITATIVE APPROACH

Roseline Yong, MPH

School of Public Health, University of Hong Kong, Hong Kong, Hong Kong. 
In Japanese, Hikikomori are people who voluntarily withdraw into social isolation for over six months . Etiology and epidemiology are poorly understood, and evidence for interventions is lacking. The purpose of this study is to describe onset and maintenance of hikikomori. Direct recruitment, snowballing and participant observation in on-line chat rooms were used to recruit participants and obtain data, which was then subject to grounded theory analysis. Of 168 participants several were unconnected with Japan, the oldest being 60 years old. Three major themes emerged of hikikomori as passive coping, trust and existence. Each theme comprised one or more categories, which in turn evidenced a number of different elements. Definitive characteristics of hikikomori were obtained from respondents through virtual participant observation. The emergent theoretical framework and the list are mutually supportive in the results obtained from this study, which suggested emotional pain exists in hikikomori in relation to human relationships. The results of the present study suggest that hikikomori is characterized by more diffuse features, including difficulties in coping with people or tasks, difficulties with trust, unhappiness about life and poor concept of time. There was no evidence of violent or aggressive behaviours. We conclude that Hikikomori is not unique to Japanese youth, but more widespread and diverse anomic coping style for dealing with social disaffection seen cross-culturally.

CORRESPONDING AUTHOR: Roseline Yong, MPH, Tokyo University, Tokyo, 113-0033; yangjinhong@gmail.com

\section{$\mathrm{SS} 03 \mathrm{~b}$}

\section{PREVALENCE AND PSYCHIATRIC COMORBIDITY} OF "HIKIKOMORI" IN A COMMUNITY POPULATION IN JAPAN

Asuka Koyama, $\mathrm{PhD},{ }^{2}$ Norito Kawakami, $\mathrm{MD}^{1}$ and World Mental Heath Japan Survey Group, None ${ }^{1}$

${ }^{1}$ School of Public Health, University of Tokyo, Tokyo, Japan and

${ }^{2}$ National Center of Neurology and Psychiatry, Kodaira, Japan.

Aims: Epidemiology of "hikikomori (acute social withdrawal)" in a community population is not clear, although it has been noted for the decade in Japan. The objective of this study is to clarify the prevalence of "hikikomori" and to examine the relation between "hikikomori" and psychiatric disorders.

Methods: Face-to-face household survey was conducted of community residents $(n=4,134)$. We defined "hikikomori" as a psychopathological phenomenon in which people become completely withdrawn from society for six months or longer. We asked all respondents whether they had any children currently experiencing "hikikomori". For respondents aged 20-49 years old $(n=1,660)$, we asked whether they had ever experienced "hikikomori". Also respondents were asked another question if they had a child with "hikikomori".

Results: A total of $1.2 \%$ had experienced "hikikomori" in their lifetime. Among them, $54.5 \%$ had also experienced a psychiatric (mood, anxiety, impulse control, or substance-related) disorder in their lifetime. A total of $78 \%$ of the "hikikomori" cases in our study reported that they had felt worried or irritable about their "hikikomori" situation. Most of the "hikikomori" cases did not feel the desire to go to work or attend school even if they still had such a duty. There was no case in this study who reported violent behaviors during "hikikomori". Respondents who experienced "hikikomori" had 6.1 times higher the risk of mood disorder. Among respondents, $0.5 \%$ currently had at least one child who had experienced "hikikomori". On the other hand, about $0.5 \%$ of respondents reported that they had a child who had or was experiencing "hikikomori".

Discussion: The study suggests that "hikikomori" is common in the community population in Japan. Approximately 232,000 households are estimated to currently have a child with "hikikomori" in Japan. While psychiatric disorders were often comorbid with "hikikomori", a half of the cases seem to be the "primary hikikomori" without a comorbid psychiatric disorder.

CORRESPONDING AUTHOR: Norito Kawakami, MD, School of Public Health, University of Tokyo, Tokyo, 113-0033; kawakami@m.u-tokyo.ac.jp

$\mathrm{SS} 03 \mathrm{c}$

ASSOCIATION OF CHILDHOOD ADVERSITIES AND FAMILY ENVIRONMENTS WITH THE RISK OF SOCIAL WITHDRAWAL ("HIKIKOMORI")

IN A COMMUNITY SAMPLE OF JAPAN

Maki Umeda, MA, MPH, Norito Kawakami, MD and World Mental Health Japan Survey Group, none

Department of Mental Health, School of Public Health, the University of Tokyo, Bunkyo-ku, Japan.

"Hikikomori", a form of social withdrawal among youths who retreat from social interaction for protracted periods of time, is widely acknowledged in Japan and some other countries. This study examines childhood adversities and family environments as childhood risk factors for "hikikomori" using the retrospective data derived from a population-based survey, the World Mental Health Japan. A random sample of residents aged 20 or older in nine communities in Japan was used, and interviews were conducted using the WHO Composite International Diagnostic Interview 3.0 $(n=4,134$, response rate $=51 \%)$. The subjects of this study were a subsample who responded to the second part of the interview (Part $2)$, and were aged between 20-49 years $(n=708)$. Multiple logistic regression was used to explore the association between the lifetime experience of "hikikomori" and childhood risk factors; social class, parental psychopathology, parents' child rearing style, and other adverse events during a respondent's childhood. The statistical model was adjusted for sex, age, and respondents' experience of common mental disorders using a sampling weight for Part 2. Among the 708 respondents, 15 experienced "hikikomori" in their lifetime ( $\mathrm{n}=9.7$ or $1.2 \%$ in a weighted analysis). Father's high educational level $(\mathrm{OR}=6.0, \mathrm{CI}=1.6-22.9)$, mother's common mental disorders $(\mathrm{OR}=6.5, \mathrm{CI}=1.1-38.0)$, and mother's panic disorders $(\mathrm{OR}=6.6, \mathrm{CI}=1.1-39.1)$ were significantly and positively associated with the experience of "hikikomori" after controlling for respondents' sex, age, and history of mental disorders. Significant associations were not found for parents' child rearing styles or childhood adversities. Our findings suggest that "hikikomori" cases are more likely to occur in families where the parents have high levels of education. Maternal panic disorder may influence the development of "hikikomori" among children, possibly due to reactions of children to their mothers' disorder. 
CORRESPONDING AUTHOR: Maki Umeda, MA, MPH, Mental Health, School of Public Health, the University of Tokyo, Bunkyo-ku, 113-0033; makiumeda-tky@umin.ac.jp

SS03d

MENTAL HEALTH CARE AND EDUCATION IN JAPAN

AS CULTURAL CONTEXTS FOR EXPLORING

THE PHENOMENON OF HIKIKOMORI

Amy Borovoy, Phd

East Asian Studies, Princeton University, Princeton, NJ.

Most observers agree that the category of hikikomori encompasses a wide range of problems and provocations. Furthermore, recent studies have found that a substantial portion of the hikikomori population reports symptoms of mental illness (Kawakami, forthcoming). Taking a cultural anthropological approach, this paper explores the social context that allows withdrawing from society to be a prevalent and viable coping mechanism in Japan. The paper draws on ethnographic, participant-observation data from three clinical facilities in the Tokyo area which treat hikikomori populations, and four special education programs which treat children who missed school for long periods due to learning disabilities and social maladjustment. The paper concludes by reviewing recent literature on mental health in Japan in order to hypothesize about whether Japanese psychiatry will move away from relying so heavily on families and towards psychopharmacological treatments as the dominant method and perceived "cure" for addressing psychopathology.

CORRESPONDING AUTHOR: Amy Borovoy, Phd, East Asian Studies, Princeton University, Princeton, NJ, 08544-1008; aborovoy@princeton.edu

\section{Thursday \\ August 5, 2010 PAPER SESSIONS 8:30 AM-10:00 AM}

OS01

\section{8:30 AM-8:48 AM}

OS01-A

THE DEVELOPMENT OF SOCIOECONOMIC HEALTH DIFFERENCES IN CHILDHOOD: RESULTS OF THE DUTCH LONGITUDINAL PIAMA BIRTH COHORT

Annemarie Ruijsbroek, Msc, ${ }^{1}$ Alet Wijga, $\mathrm{PhD},{ }^{1}$ Marjan Kerkhof, $\mathrm{PhD},{ }^{2}$ Gerard H. Koppelman, $\mathrm{PhD},{ }^{3}$ Henriette A. Smit, prof ${ }^{1}$ and Mariël Droomers, $\mathrm{PhD}^{1}$

${ }^{1}$ Centre for Prevention and Health Services Research (PZO), RIVM, Bilthoven, Netherlands; ${ }^{2}$ Department of Epidemiology and Bioinformatics, University of Groningen, Groningen, Netherlands and ${ }^{3}$ Department of Pediatric Pulmonology and Pediatric Allergology, University Medical Centre Groningen, Groningen, Netherlands.
Objective: To prospectively study the development of socioeconomic health differences in the Netherlands and to investigate possible explanations for socioeconomic variation in childhood health.

Methods: The PIAMA study follows a birth cohort of 3,963 Dutch children during their first 8 years of life. Common childhood health problems, i.e. eczema, asthma symptoms, general health, frequent respiratory infections, overweight, and obesity were assessed yearly using questionnaires. Maternal educational level was used to indicate socioeconomic status. Possible explanatory lifestyle determinants (breastfeeding, smoking during pregnancy, smoking in the first 3 months and the use of day-care facilities) and biological determinants (maternal age at birth, birth weight and older siblings) were analyzed using General Estimating Equations (GEE).

Results: Socioeconomic differences in a broad range of health problems are present early in life and persist during childhood. Socioeconomic differences in maternal age at birth, breastfeeding, and day-care center attendance contribute substantially to childhood socioeconomic health disparities.

Conclusion: This study shows that health disparities already occur in the very early years of life, also in the Netherlands. The less healthy parental lifestyle among lower socioeconomic groups explains most of the observed socioeconomic health differences in childhood.

CORRESPONDING AUTHOR: Annemarie Ruijsbroek, Msc, RIVM, Bilthoven, 3521CN; annemarie.ruijsbroek@rivm.nl

\section{8:48 AM-9:06 AM}

OS01-B

INTERVENING ON THE COMMUNITY LEVEL TO BUILD SOCIAL CAPITAL - A LONGITUDINAL STUDY

OF THE HEALTH EFFECTS IN A LOW-INCOME

AND ETHNICALLY DIVERSE NEIGHBOURHOOD

Per-Olof G. Ostergren, MD, PhD, Elizabeth Cantor-Graae, $\mathrm{PhD}$, Eva Lindbladh, PhD, Cecilia Fernbrant, MPH and Mahnaz Moghaddassi, MSc

Dpt Clinical Sciences in Malmo, Lund University, Social Medicine and Global Health, Malmo, Sweden.

Background: Several studies have shown that social capital in terms of social participation and trust are linked to better health. However, there are few studies on the effect of community interventions attempting to benefit from this.

Methods: A large scale intervention by means of participatory methods, aiming at improving the physical and social environment in an inner city area characterised by a low-income and an ethnically diverse population was launched in a midsize city in southern Sweden. Interviews concerning environmental, sociodemographic factors (including social capital), and health (including mental health measured by GHQ12) were made with 630 randomly selected individuals at baseline. A follow-up with an identical interview was made with those who still lived in the area after three years. The response rate was $66 \%$. 
Results: Bridging social capital had increased significantly, but in a pattern determined by type of social capital, gender, educational level and ethnicity. Bridging social participation had increased among men, individuals born in Sweden and the low educated. Bridging trust on the other hand, increased among women, individuals born outside Sweden and the high educated. Selfrated health did not change in any group between baseline and follow-up. However, mental health improved significantly among individuals born outside Sweden. This seemed to be mediated by social capital components.

Conclusion: Following the community-based intervention, bridging social capital increased among inhabitants who lived in the area for the whole three-year period in a pattern determined by gender, level of education and ethnicity. Mental health improved among individuals born outside Sweden, which may have been mediated by improvement of bridging social capital.

CORRESPONDING AUTHOR: Per-Olof G. Ostergren, MD, $\mathrm{PhD}$, Dpt Clinical Sciences in Malmo, Lund University, Social Medicine and Global Health, Malmo, SE 20502; per-olof. ostergren@med.lu.se

9:06 AM-9:24 AM

OS01-C

LONGITUDINAL EFFECTS OF SOCIAL INTEGRATION ON CO-OCCURRENCE OF DEPRESSION AND CENTRAL OBESITY AMONG MIDDLE AGED ADULTS

Noriko Cable, $\mathrm{PhD},{ }^{1}$ Tarani Chandola, $\mathrm{PhD}^{1}$ and Amanda Sacker, $\mathrm{PhD}^{2}$

${ }^{1}$ Epidemiology and Public Health, University College London, London, United Kingdom and ${ }^{2}$ Institute for Social and Economic Research, University of Essex, Colchester, United Kingdom.

Depression and obesity are the among the most common health problems globally and may share common social determinants. Social integration is suggested to protect adults from ill health; this study examines whether the protective effect of social integration is seen longitudinally and is present in the case of the cooccurrence of depression and central obesity.

We used 3 sweeps of a prospective cohort study, the National Child Development Study, when the participants were aged 33, 42 , and 45 (Men=3,043, Women=3,153). Co-occurrence of depression and central obesity was assessed at age 45 , by the presence or absence of depression (scores over 2 on the depression subscale of the Clinical Interview Schedule) and central obesity (a waist to hip ratio greater or equal to 0.9 for men, 0.85 for women). Social integration was assessed at age 42 using information about cohabitation, employment status, and frequency of attendance at religious or socially organised groups. Psychological health, longstanding illness, and social position assessed at age 33 are treated as confounders and men and women were analysed separately.

Findings using multinomial logistic regression showed the significance of employment as the key protective element of social integration among middle aged adults. Working full time at age 42 was less likely to be associated with depression at age 45 even along with central obesity, but not with central obesity alone. This effect was independent of the effects from the confounders and greater among men (Depression: $\mathrm{OR}=0.15,95 \% \mathrm{CI}=0.07-$ 0.32 ; Comorbdity: $\mathrm{OR}=0.25,95 \% \mathrm{CI}=0.14-0.45$ ). Although considered a confounder rather than an explanatory variable, it is worth noting that psychological ill health at age 33 was linked to depression at age 45 in men and women; however, the degree of the association is greater when central obesity is present, confirming the complex mechanism of co-occurrence of depression and central obesity. It appears employment, may protect middle aged adults against comorbid depression and central obesity through its relationship with depression.

CORRESPONDING AUTHOR: Noriko Cable, PhD, Epidemiology and Public Health, University College London, London, WC1E6BT; n.cable@ucl.ac.uk

9:24 AM-9:42 AM

OS01-D

THE HOPE PROJECTS: ADDRESSING ECONOMIC

AND SOCIAL DETERMINANTS OF HEALTH AMONG LOW INCOME, ETHNICALLY DIVERSE WOMEN IN RURAL NORTH CAROLINA

Salli Benedict, MPH, ${ }^{1}$ Marci K. Campbell, PhD, MPH, $\mathrm{RD}^{2}$ and Barbara Harris, $\mathrm{MA}^{1}$

${ }^{1}$ UNC Center for Health Promotion and Disease Prevention, University of NC, Chapel Hill, NC and ${ }^{2}$ Nutrition, UNC, Chapel Hill, NC.

The four HOPE Projects build on over 16 years of community based research projects with low income women in rural counties in North Carolina. The Community-based participatory research process led us to develop interventions to address women's health within the context of the economic and social circumstances of their lives: poverty, unemployment, racism and lack of access to resources. HOPE (Health, Opportunities, Partnerships, Empowerment) Works (2004-2009) was a successful obesity prevention intervention that drew upon development strategies including loan circles, microenterprise development, and American Indian talking circles. Community women were trained to organize and facilitate HOPE Circles of 8-12 women from their social networks. Circles met twice a month for 6 months: women set goals and supported each other to make health and social/economic improvements. Threads of HOPE (www.threadsofhopenc.org) is a spinoff from HOPE Works: it is a small business that produces sustainable conference bags, and a non-profit whose mission is to train low income women in business development and financial literacy. Seeds of HOPE (2009-2014) will disseminate the HOPE Works model through American Indian and African American churches and community health clinics. It will have a stronger emphasis on microenterprise development and financial literacy. HOPE Accounts for Women (2009-2011) is funded by the American Recovery and Reinvestment Act through the National Institutes of 
Health. This innovative program uses the HOPE Works model but enhances the economic empowerment/financial literacy training by assisting low income women in saving money for starting a business or furthering education through a matched savings incentive. Data from the first 3 cohorts (159 Intervention, 118 Comparison) in the HOPE Works study show that $75 \%$ of women were African American and most were low income: outcome analysis demonstrated that HOPE Circle participants lowered BMI more than the comparison group with average weight loss difference of 4 pounds. Intervention women also increased hope, physical activity and fruit and vegetable intake.

CORRESPONDING AUTHOR: Salli Benedict, MPH, UNC Center for Health Promotion and Disease Prevention, University of NC, Chapel Hill, NC, 27599-7426; salli_benedict@unc.edu

\section{9:42 AM-10:00 AM}

OS01-E

SOCIOECONOMIC DIFFERENCES IN WEIGHT-CONTROL BEHAVIORS AMONG A POPULATION-REPRESENTATIVE GROUP OF MID-AGED ADULTS

Jessica Siu, BHSci (Maj Nutr \& Diet), Katrina Giskes, PhD and Gavin Turrell, $\mathrm{PhD}$

School of Public Health, Queensland University of Technology, Brisbane, QLD, Australia.

Introduction: It has been well documented that socioeconomicallydisadvantaged groups are more likely to be overweight/obese than their more-advantaged counterparts (this trend is seen more consistently among women). These differences may be due (in part) to socioeconomic differences in weight-control behaviors. Methods: Data was obtained from 1012 men and women aged 45 to 60 years residing in Brisbane, Australia (69.6\% response rate). Data were collected by a postal questionnaire, which provided information on past and current weight-control behaviors. Socioeconomic position was characterised by highest attained education, household income and occupation.

Results: Socioeconomically-disadvantaged participants were less likely to report engaging in weight-control in the past 12 months. Those with secondary school or lower education $(\mathrm{OR}=0.58$, $95 \%$ C.I. $0.41-0.82)$, working in a blue-collar profession $(\mathrm{OR}=$ 0.36 , 95\% C.I $0.22-0.60)$, not in the work force $(\mathrm{OR}=0.62$, $95 \%$ C.I. $0.41-0.94)$, or who belonged in the 1 st quartile of equivalised household income $(\mathrm{OR}=0.64,95 \%$ C.I. $0.41-0.98)$ were less likely to report trying to lose weight and/or avoid weight gain in the past 12 months. There were no significant differences between socioeconomic groups in engaging in potentially health-promoting or health-damaging weight-control behaviors.

Conclusion: Socioeconomic differences in weight status may be due to differences in engaging in weight-control behaviors. However among those who do wish to maintain or lose weight, there are no socioeconomic differences in the nature of the strategies they employ.
CORRESPONDING AUTHOR: Jessica Siu, BHSci (Maj Nutr \& Diet), School of Public Health, Queensland University of Technology, Brisbane, QLD, 4059; jessica.siu@qut.edu.au

OS02

8:30 AM-8:48 AM

OS02-A

THE IMPORTANCE OF LONGITUDINAL ASSESSMENT OF HEALTH BEHAVIOURS IN EXPLAINING SOCIAL INEQUALITIES IN MORTALITY: THE BRITISH WHITEHALL II COHORT STUDY

Silvia Stringhini, Ms, ${ }^{1}$ Séverine Sabia, $\mathrm{PhD},{ }^{1}$ Martin Shipley, Ms, ${ }^{2}$ Eric Brunner, $\mathrm{PhD},{ }^{2}$ Hermann Nabi, $\mathrm{PhD},{ }^{1}$ Mika Kivimaki, $\mathrm{PhD}^{2}$ and Archana Singh-Manoux, $\mathrm{PhD}^{1,2}$

${ }^{1}$ U687, INSERM, Villejuif Cedex, France and ${ }^{2}$ Department of Epidemiology and Public Health, University College London, London, United Kingdom.

Background: Previous studies may have underestimated the contribution of unhealthy behaviours to social inequalities in mortality because of the assessment of health behaviours at only one point in time, resulting in a failure to capture long-term exposure.

Methods: This study used 4 repeated assessments of smoking, alcohol consumption, diet and physical activity over 24 years of follow-up to examine their role in mediating the association between socioeconomic position, measured using occupational position, and mortality from all-causes and specific causes in 9 590 men and women aged 35 to 55 years at baseline.

Results: 654 participants died during the follow-up. Those with lowest occupational position had 1.60 times higher risk of death than those with highest position in analysis adjusted for sex and year of birth. For all-cause mortality, this association was attenuated by $42 \%$ (95\% Confidence Interval (CI) $21 \%$, 94\%) when health behaviours assessed at baseline were entered into the model and by $72 \%$ (95\% CI $42 \%, 154 \%)$ when health behaviours were entered as time dependent covariates. The corresponding attenuations were $29 \%(95 \%$ CI $11 \%, 54 \%)$ and $45 \%(95 \% \mathrm{CI}$ $24 \%, 79 \%$ ) for cardiovascular mortality and $61 \%$ (95\% CI $16 \%$, $425 \%)$ and $94 \%$ (95\% CI 35\%, 595\%) for non-cancer noncardiovascular mortality. There was no association between occupational position and cancer mortality so no further analyses were undertaken. The difference between the baseline and longitudinal assessments was mostly due to an increased explanatory power of diet, physical activity and to a lesser extent, alcohol consumption. The role of smoking, the strongest mediator in these analyses, did not much differ when using baseline or repeat assessments.

Conclusions: Health behaviours may be a more important explanatory mechanism for inequalities in mortality than previously estimated.

CORRESPONDING AUTHOR: Silvia Stringhini, Ms, U687, INSERM, Villejuif Cedex, 94807; silvia.stringhini@inserm.fr 


\section{8:48 AM-9:06 AM}

OS02-B

REGIONAL AND FACILITY VARIATIONS IN USE

OF MATERNAL HEALTH SERVICES IN SOUTH AFRICA

Jana Fried, PhD, ${ }^{1}$ Stephen Birch, DPhil, ${ }^{1}$ John Eyles, PhD, ${ }^{1}$ Jenny Zhan, $\mathrm{BHSc}^{1}$ and Sheetal Silal, $\mathrm{BA}^{2}$

${ }^{1}$ McMaster University, Hamilton, ON, Canada and ${ }^{2}$ University of Cape Town, Cape Town, South Africa

According to South Africa's new constitution, access to health care is a fundamental right. In this paper we analyse the utilisation of ante-natal care services under the public health system of South Africa in order to inform policy concerned with equity of access about factors associated with the distribution of current services. We conceptualize access to care as covering three distinct but interacting dimensions of availability, affordability and acceptability. Each dimension reflects aspects of both the demand for and supply of services. We adopt WHO guidelines concerning the need for ante natal care (ANC) visits and explain variations in the number of visits among women giving birth in four selected communities, two urban and two rural.

The results indicate that more marginalised women (i.e., young, unmarried, low levels of education) and those with no previous deliveries were significantly less likely to have the WHO recommended number of 5 ANC visits. Further analysis revealed variations between facilities in the determinants of sufficient ANC visits. These findings show inequalities in utilisation which may suggest that inequities in access remain.

CORRESPONDING AUTHOR: Jana Fried, PhD, McMaster University, Hamilton, ON, L8N 3Z5; friedja@mcmaster.ca

\section{9:06 AM-9:24 AM}

OS02-D

GLOBAL SYSTEMATIC REVIEW OF PEER SUPPORT FOR COMPLEX HEALTH BEHAVIOR

Emily A. Elstad, MPH, Renee I. Boothroyd, PhD, Amy L. Henes, BA, Gary R. Maslow, MD, Katherine Nelson, MPH and Edwin B. Fisher, $\mathrm{PhD}$

Health Behavior and Health Education, University of North Carolina at Chapel Hill, Chapel Hill, NC.

Extant reviews of peer and social support interventions are disease- and approach-specific (e.g., use of community health workers in diabetes management). Because there is limited evidence within any one disease, such reviews do not provide strong evidence for peer support. In contrast, a more general review of peer support across a range of health behaviors can lead to more solid conclusions about its effectiveness. We conducted a systematic review of research on peer support for complex health behavior (e.g., cardiovascular risk reduction) across a variety of health problems. An initial PubMed search of papers published between 1/1/2001 and 8/31/2009 and using cognates of "coach," "promotora," "peer support," etc. identified 902 peer-reviewed articles. Of these, 595 articles were excluded as their titles indicated that they were not patient behavior programs (e.g. coaching for career growth among health professionals). The remaining 307 were reviewed to fit a broad definition of peer support: provided by a nonprofessional and addressing support for multiple health behaviors over time. Exclusion criteria consisted of: Interventions focused on isolated or single behaviors (e.g. cancer screening); interventions evaluating educational classes; and those without outcome data. The resulting 40 papers addressed: breastfeeding ( 6 papers); diabetes ( 5 papers), depression (5 papers), and asthma (4 papers), along with a variety of other health issues, and represented 8 different countries. Provisional rating of outcomes was: No evidence of benefit; Modest evidence of benefit (e.g., changes on self report measure of health behaviors or quality of life); and Strong evidence of benefit (e.g. statistically significant changes on objective clinical measure such as blood pressure or blood glucose, or on well validated psychological measure such as the CES-D). Of the 40 papers, 7 were scored as No, 15 as Modest, and 18 as Strong Evidence of Benefit. Further evaluation of effect sizes and weighting by sample sizes and adequacy of controls will be included in presentation at the Congress. This systematic review indicates that peer support is effective in promoting complex health behaviors across a variety of diseases and national settings.

CORRESPONDING AUTHOR: Emily A. Elstad, MPH, Health Behavior and Health Education, University of North Carolina at Chapel Hill, Chapel Hill, NC, 27599; elstad@email.unc.edu

\section{9:24 AM-9:42 AM \\ OSO2-E}

PRO-POOR HEALTH POLICY IN NEPAL : ENLARGING PEOPLE'S CHOICES

Jhabindra Bhandari, MPH

Health Research, SUCHDEF Nepal, Kathmandu, Nepal.

Background: Nepal is one of the least developed countries in the world. Poverty, illiteracy, poor public health system are tremendously impacting on majority of people's lives who are living in rural areas and limited access to primary health care services. The government of Nepal declared the free health care policy from early 2008 with a view to improve access to and utilization of health services for people who are poor, socially disadvantaged and marginalized communities. The new health policy has highlighted the needs to expand health services in rural areas where there is acute lack of human resources, irregular supplies of drugs and equipment and poor health infrastructure. The pro-poor health policy aims to narrow the wider gaps of disparities in health care by ensuring health equity and the rights of people to their health and life.

Objective: The objective of this study is to explore the effects of pro-poor health care policy at the community levels.

Methods: It was a cross-sectional study with primary focus on qualitative research methodology. The methods for data collection were focus group discussion and in-depth interviews with service providers in local health facilities, community groups, key 
informants and the beneficiaries. Besides, the data from health management information system at local health facilities were reviewed to analyze the trend of health service utilization over the period of two years.

Results and Conclusions: The pro-poor health policy has been positively impacting on people's access to and utilization of health services. There is 15 to $20 \%$ increment in health facility utilization by poor, socially disadvantaged and marginalized communities. There is significant level of community awareness of free health care policy and provision. The supplies of drugs and other logistics at health facilities are gradually improved in the rural areas where as human resources for health are still lacking and not adequate compared to the health care needs of the people. The pro-poor health policy has been instrumental in accessing and utilizing the services by the poorest of the poor as it is free of charge and available in local health facilities.

CORRESPONDING AUTHOR: Jhabindra Bhandari, MPH, Health Research, SUCHDEF Nepal, Kathmandu, 977-1; jh_bhandari@ yahoo.com

OS03

\section{8:30 AM-8:48 AM}

OS03-A

SOCIAL SUPPORT BUFFERS SYMPATHETIC REACTIVITY TO ACUTE PSYCHOSOCIAL STRESS

Roberto La Marca, PhD, ${ }^{1}$ Pearl Ghaemmaghami, MSc, ${ }^{1}$ Mélanie Tripod, MSc, ${ }^{1}$ Hanna Thörn, MSc, ${ }^{1}$ Patricia Waldvogel, BSc, ${ }^{1}$ Jens C. Pruessner, $\mathrm{PhD}^{2}$ and Ulrike Ehlert, $\mathrm{PhD}^{1}$

${ }^{1}$ Clinical Psychology and Psychotherapy, University of Zurich, Zurich, Switzerland and ${ }^{2}$ Department of Psychiatry, and Neurology and Neurochirurgy, McGill University \& Douglas Hospital Research Centre, Montreal, QC, Canada.

Social support is thought to be a health protective resource, however, the underlying mechanisms are elusive. One possible mechanism is that social support has a buffering effect on the sympathetic stress response. The salivary enzyme alpha-amylase (sAA), reflecting the activity of the sympathetic nervous system (SNS), seems to be a promising indicator for stress research. The main purpose of the present study was to test the effect of perceived social support (PSS) on acute sAA stress reactivity.

Thirty-three healthy, young male subjects participated in a computerized stress task consisting of arithmetic problems that had to be solved under time-pressure and social evaluation. Cortisol, sAA, salivary flow rate (SFR), heart rate (HR), and respiratory sinus arrhythmia (RSA) were assessed repeatedly, while perceived social support, chronic stress and rated stress reactivity were assessed using different questionnaires.

Higher PSS was negatively correlated with the stress response of sAA $(\mathrm{r}=-.551, \mathrm{p}<.01)$ and $\mathrm{HR}(\mathrm{r}=-.344, \mathrm{p}<.05)$, but was unrelated to cortisol, SFR, and RSA alterations. Furthermore, higher levels of PSS were negatively associated with ratings of chronic stress $(\mathrm{r}=-.384$, $\mathrm{p}<.05)$ and habitual stress reactivity $(\mathrm{r}=-.372, \mathrm{p}<.05)$.
This is the first study examining the relationship between PSS and acute sAA stress reactivity. The results indicate that social support indeed buffers the effect of psychosocial stress on the SNS activity, and is further inversely associated with ratings of chronic stress and stress reactivity. In addition, our findings emphasize the relevance of sAA for stress and social support research.

CORRESPONDING AUTHOR: Roberto La Marca, PhD, University of Zurich, Zurich, 8050; r.lamarca@psychologie.uzh.ch

\section{8:48 AM-9:06 AM \\ OS03-B \\ THE EFFECT OF MUSIC ON NEUROENDOCRINE, AUTONOMIC, COGNITIVE, AND EMOTIONAL STRESS RESPONSES}

Myriam V. Thoma, MSc, Rebecca Brönnimann, MSc, Ulrike Ehlert, $\mathrm{PhD}$ and Urs M. Nater, PhD

University of Zurich, Zurich, Switzerland.

Music listening has been suggested to beneficially impact health via stress-reducing effects. However, the exact mechanisms through which music exerts its positive consequences on the body are poorly understood. It was the aim of the current study to address this gap in knowledge and to examine the underlying mechanisms of music effects across neuroendocrine, autonomous, cognitive, and emotional domains of the human stress response. Sixty healthy female volunteers (mean $=25.27$ years) were exposed to a standardized psychosocial stress test after having been randomly assigned to one of three different conditions prior to the stressor: 1) relaxing music ('Miserere', Allegri) (RM), 2) sound of rippling water (SW) and 3) rest without acoustic stimulation (R). Salivary cortisol and alpha-amylase (sAA), anticipatory cognitive appraisal, subjective stress perception and anxiety were repeatedly assessed in all subjects. We hypothesized that listening to music prior to the stressor, compared to SW or R would result in an attenuated stress reaction. The stressor caused significant changes in all measurements in all three groups over time. The three conditions significantly differed regarding cortisol responses $(p=0.014)$, with highest values in the RM and lowest values in SW. sAA recovery delta showed a statistical trend $(p=$ 0.060) in favor of the RM. Psychological measures did not significantly differ between groups during the experiment. Our findings indicate that music listening differentially impacts the psychobiological stress system. Listening to music prior to a psychological stressor increases rather than attenuates subsequent endocrine stress responses. In contrast, listening to water sound seems to result in an attenuated endocrine response to stress compared to no auditory stimulation. Listening to music seems to increase autonomic recovery more efficiently than listening to water sound or resting in silence. These findings bear potential to explain the effects of music on the human body.

CORRESPONDING AUTHOR: Myriam V. Thoma, MSc, University of Zurich, Zurich, 8055; m.thoma@psychologie.uzh.ch 
9:06 AM-9:24 AM

OS03-C

SHIFT WORK, CORTISOL, REACTION TIME TEST

AND HEALTH AMONG OFFSHORE WORKERS

Anette Harris, MSc, ${ }^{1,2}$ Siri Waage, MSc, PhD Student, ${ }^{2,4}$ Ursin Holger, MD, Professor Emeritus, ${ }^{2}$ Åse Marie Hansen, $\mathrm{PhD},{ }^{3}$ Bjørn Bjorvatn, $\mathrm{MD}, \mathrm{PhD}^{4,5}$ and Hege R. Eriksen, $\mathrm{PhD}^{1,2}$

${ }^{1}$ Reaearch Center for Health Promotion, Faculty of Psychology, University of Bergen, Bergen, Norway; ${ }^{2}$ Uni Health, University Research Bergen, Bergen, Norway; ${ }^{3}$ National Research Centre for the Working Environment, Copenhagen, Denmark; ${ }^{4}$ Department of Public Health and Primary Health Care, University of Bergen, Bergen, Norway and ${ }^{5}$ Norwegian Competence Center for Sleep Disorders, Haukeland University Hospital, Bergen, Norway.

The aim was to study if health, reaction time, and the diurnal rhythm of cortisol were negatively affected when a group of shift workers changed their work schedule from ordinary night-day shift to "swing shift". This was tested on 19 healthy workers on a Norwegian oil rig in the North Sea. They worked two weeks offshore followed by 4 weeks off work. The ordinary schedule was to work 12-hour day shift during one work period (14 days), and 12-hour night shift on the next work period (14 nights)(fixed-shift). "Swing shift" involves night shift during the first week, then day shift the second week, for every working period. The advantage of swing shift is that the workers are readapted to a normal day-night rhythm when they start their 4 week off work period. The disadvantage is that the workers have to adjust their biological rhythms twice every work period instead of once every other work period. All participants worked ordinary night-day shift when baseline data were collected (questionnaires, saliva cortisol, and reaction time during work). After collection of baseline data the workers changed their work schedule to "swing shift", one year later the same data were collected. "Swing shift" did not give any negative health effects or any negative changes in reaction time during the day they shifted from night work to day work. During swing shift the cortisol rhythm went slowly back to a normal rhythm in the second week, but it was not fully returned to normal values when they returned home for the 4 weeks off period. When working swing shift the cortisol rhythms were readapted to normal values after one week at home. For personnel returning home directly from 14 consecutive night shifts, diurnal cortisol adaptation was not complete after one week at home.

CORRESPONDING AUTHOR: Anette Harris, MSc, Faculty of psychology, University of Bergen, Bergen, 5020; anette.harris@uib.no

9:24 AM-9:42 AM

OS03-D

RELATION BETWEEN VAGAL FUNCTION AND CORTISOL STRESS RESPONSE

Roberto La Marca, PhD, ${ }^{1}$ Patricia Waldvogel, BSc, ${ }^{1}$ Hanna Thörn, MSc, ${ }^{1}$ Mélanie Tripod, MSc, ${ }^{1}$ Jens C. Pruessner, $\mathrm{PhD}^{2}$ and Ulrike Ehlert, $\mathrm{PhD}^{1}$

${ }^{1}$ Clinical Psychology and Psychotherapy, University of Zurich, Zurich, Switzerland and ${ }^{2}$ Department of Psychiatry, and Neurology and Neurochirurgy, McGill University \& Douglas Hospital Research Centre, Montreal, QC, Canada.
Low vagal function is related to several disorders. One possible underlying mechanism linking the vagus nerve and disorders is the HPA axis, although literature referring to this association is inconsistent. The main purpose of the present study was to examine the relation between vagal function and the biopsychological reactivity to acute psychosocial stress.

Healthy male subjects $(\mathrm{N}=33)$ participated in a stress task (Montreal Imaging Stress Task, MIST; Dedovic et al., 2005), while heart rate (HR), respiratory sinus arrhythmia (RSA), salivary cortisol and mood were assessed. Vagal function was determined using baseline, MIST-induced inhibition and Cold Face Test (CFT)-induced stimulation.

The stress task induced a significant increase in cortisol and HR (both $\mathrm{p}<.001$ ) and a decrease in RSA and $\operatorname{mood}($ both $\mathrm{p}<.001)$. A linear regression model with CFT response predicted $17.9 \%$ of observed variance in cortisol AUCI (mood: 38.9\%; tiredness: $19.8 \%$ ) in response to the stressor.

The results indicate that faster or stronger CFT response is associated with reduced cortisol increase, increased mood and wakefulness after acute stress. Our data support an inverse relationship between vagal function and the HPA axis.

CORRESPONDING AUTHOR: Roberto La Marca, PhD, University of Zurich, Zurich, 8050; r.lamarca@psychologie.uzh.ch

\section{9:42 AM-10:00 AM}

OS03-E

GENERALIZED AND FOOT ULCER-SPECIFIC EMOTIONAL DISTRESS ARE ASSOCIATED WITH ELEVATED LOCAL LEVELS OF INTERLEUKIN-6 IN PATIENTS WITH DIABETIC FOOT ULCERS

Loretta Vileikyte, MD, PhD, ${ }^{1,3}$ Biing-Jiun Shen, $\mathrm{PhD},{ }^{2}$ Matthew Hardman, $\mathrm{PhD},{ }^{1}$ Robert Kirsner, $\mathrm{MD}, \mathrm{PhD},{ }^{3}$ Andrew Boulton, $\mathrm{MD}, \mathrm{FRCP}^{1,3}$ and Neil Schneiderman, $\mathrm{PhD}^{3}$

${ }^{1}$ Medicine, University of Manchester, Manchester, United Kingdom; ${ }^{2}$ Psychology, University of Southern California, Los Angeles, CA and ${ }^{3}$ Psychology, University of Miami, Miami, FL.

Emotional distress-induced up-regulation of Interleukin-6 (IL-6) has been found to be detrimental to health. As diabetic foot ulcers are characterized by chronic inflammation, it is plausible that emotional distress could delay the healing of such ulcers by upregulating and sustaining high ulcer tissue-specific levels of IL-6. Here we report a cross-sectional study of 41 patients with type 2 DM (76\% male; mean age 55 yrs) and plantar neuropathic diabetic foot ulcers (University of Texas Classification: $82 \%$ grade 1A; $12 \% 1 \mathrm{~B} ; 3 \% 2 \mathrm{~A}$; and $3 \% 2 \mathrm{~B}$ ). The relationship between patient self reported generalized emotional distress, foot ulcer-specific emotional responses and ulcer biopsy IL-6 levels was investigated. Generalized emotional distress was assessed with the Perceived Stress Scale (PSS: $1.3 \pm 0.8$ ) and with two Hospital Anxiety and Depression Scales: HADS-Anxiety (13.5 \pm 4.4$)$ and HADSDepression (11.7 \pm 5.0$)$. Foot ulcer-specific emotional responses were assessed with three subscales: Patient Interpretation of Neuropathy (PIN)-Amputation Worry (4.1 \pm 0.8$)$, PIN-Anger at Practitioners $(2.3 \pm 1.0)$ and Neuropathy and Foot Ulcer-Specific Quality of Life (NeuroQoL)-Interpersonal Burden (3.7 \pm 1.2 ). 
Ulcer-specific IL-6 levels were determined via quantification of immunohistochemical wound biopsy tissue localization. Additionally, assessed was the physiologic marker of emotional distress, 9 am and 12noon serum cortisol $(458.7 \pm 125.8 \mathrm{nmol} / \mathrm{l}$ and $625 \pm$ $369.7 \mathrm{nmol} / 1$, respectively). In the bivariate analysis, more severe generalized and foot-ulcer-specific emotional distress were each associated with higher levels of ulcer biopsy log-transformed IL-6 (raw mean: $608.3 \pm 545.5$ cells $/ \mathrm{mm} 2)$ : HADS-Anxiety $(\mathrm{r}=0.44 ; \mathrm{p}=$ $0.031)$, PSS $(\mathrm{r}=0.44 ; \mathrm{p}=0.034)$, PIN-Amputation Worry $(\mathrm{r}=0.54$; $\mathrm{p}=0.007)$ and NeuroQoL-Interpersonal Burden $(\mathrm{r}=0.60 ; \mathrm{p}=$ $0.004)$. No significant relationship was noted between serum cortisol and IL-6. These preliminary findings identify a strong link between subjective measures of emotional distress and wound specific IL-6 up-regulation. Whether this relationship contributes to non-healing of diabetic foot ulcers is under investigation.

CORRESPONDING AUTHOR: Loretta Vileikyte, MD, PhD, Medicine, University of Manchester, Manchester, M13 0JE; lvileikyte@med.miami.edu

\section{OS04}

\section{8:30 AM-8:48 AM}

OS04-A

SELF-REGULATORY DEFICITS IN FIBROMYALGIA

AND TEMPOROMANDIBULAR DISORDERS

Lise Solberg Nes, PhD, ${ }^{1,2}$ Charles R. Carlson, $\mathrm{PhD},{ }^{2}$ Leslie J. Crofford, $\mathrm{MD}^{2}$ Reny De Leeuw, DDS, $\mathrm{PhD}^{2}$ and Suzanne C. Segerstrom, $\mathrm{PhD}^{2}$

${ }^{1}$ Mayo Clinic, Rochester, MN and ${ }^{2}$ University of Kentucky, Lexington, KY.

Chronic multi-symptom illnesses such as Fibromyalgia Syndrome (FMS) and Temporomandibular Disorders (TMD) are accompanied by complex interactions of cognitive, emotional, and physiological disturbances. Such conditions are complicated and draining to live with, and successful adaptation may depend on ability to self-regulate. Self-regulation involves capacity to exercise control and guide or alter reactions and behavior. Research indicates that self-regulatory strength is a limited source that can be depleted or fatigued, however, and patients with FMS and TMD may, when trying to adapt to the many demands of their illnesses, deplete or fatigue their self-regulatory resources. The current study aimed to show that patients with FMS and TMD, compared with healthy controls, are more vulnerable to selfregulatory fatigue as a consequence of their condition, and that self-regulatory fatigue has specific physiological markers. Research has found self-regulatory effort to lead to self-regulatory fatigue, indicated by less capacity to persist on a subsequent task. Patients $(\mathrm{N}=50)$ and matched controls $(\mathrm{N}=50)$ were exposed to an experimental self-regulation task, followed by a persistence task. Controls in the low self-regulation condition displayed continued high persistence as expected. However, patients in the same condition displayed similar persistence as patients and controls in the high self-regulatory condition, indicating that patients with FMS and TMD may in fact be suffering from chronic selfregulatory fatigue. Baseline heart rate variability, heart rate, blood glucose, and cortisol predicted persistence, more so for controls than for patients, and more so in the low versus high selfregulation condition. Impact of illness on self-regulatory effort was mediated by pain, but not by any other factors. The current study shows that patients with chronic multi-symptom illnesses likely suffer from chronic self-regulatory fatigue, and that selfregulatory capacity needs to be taken into account when aiming to treat and help these patients through behavioral interventions.

CORRESPONDING AUTHOR: Lise Solberg Nes, PhD, Psychiatry and Psychology, Mayo Clinic, Rochester, MN, 55905; solbergnes.lise@mayo.edu

\section{8:48 AM-9:06 AM \\ OS04-B}

IS APPRECIATION OF WRITTEN EDUCATION RELATED TO CHANGES IN ILLNESS PERCEPTIONS AND QUALITY OF LIFE IN PATIENTS WITH FIBROMYALGIA?

Miriam van Ittersum, MSc, ${ }^{1,2}$ Paul van Wilgen, Dr, ${ }^{2,3}$ Johan Groothoff, Prof $\mathrm{Dr}^{2}$ and Cees van der Schans, Dr ${ }^{1,2}$

${ }^{1}$ Research and Innovation Group in Health Care and Nursing, Hanze University of Applied Sciences Groningen, Groningen, Netherlands; ${ }^{2}$ Department of Health Sciences, University Medical Centre Groningen, University of Groningen, Groningen, Netherlands and ${ }^{3}$ University Centre for Sport, Exercise and Health, University Medical Centre Groningen, University of Groningen, Groningen, Netherlands.

Objective: To investigate the appreciation of written education about pain neurophysiology in patients with fibromyalgia (FM) and its effects on illness perceptions and quality of life.

Method: A booklet explaining pain neurophysiology (i.e. central sensitisation) was sent to participants with FM. Appreciation about the information in the booklet was assessed by 10 questions addressing perceived relevance (0-30) and perceived reassurance (030). Participants filled in the Revised Illness Perception Questionnaire (IPQ-R), the Pain Catastrophizing Scale (PCS) and the Fibromyalgia Impact Questionnaire (FIQ) at baseline (T0), after a 2 week control period (T1) and 6 weeks after the intervention (T2). Differences between T0, T1 and T2 were analysed using a repeated measures design. Pearson's correlation coefficients were used to reveal relationships between appreciation and changes in outcome. Results: Mean (SD) scores for relevance and reassurance were 21.6 (5.6) and 18.7 (5.7), respectively. Illness coherence, emotional representations, pain and fatigue changed significantly between $\mathrm{T} 0$ and T2, pain also before intervention. Correlations between appreciation and changes in illness perceptions, catastrophizing and quality of life ranged between $r=.000$ and $r=.338$.

Conclusions: Although a majority of our subjects with FM appreciated the written information about pain neurophysiology, it did not have clinically relevant effects on illness perceptions, catastrophizing and quality of life. Appreciation of the information was not related to changes in outcome.

CORRESPONDING AUTHOR: Miriam van Ittersum, MSc, Research and Innovation Group in Health Care and Nursing, Hanze University of Applied Sciences Groningen, Groningen, 9701 DC; m.w.van.ittersum@pl.hanze.nl 
9:06 AM-9:24 AM

OS04-C

DO THE ILLNESS PERCEPTIONS OF FIBROMYALGIA PATIENTS AND INJURED ATHLETES DIFFER?

Cornelis $\mathrm{P}$. van WIlgen, $\mathrm{PhD},{ }^{1}$ Mirjam van Ittersum, $\mathrm{MsC}^{2}$ and $\mathrm{Ad}$ Kaptein, $\mathrm{PhD}^{3}$

${ }^{1}$ Movement sciences, University Centre of Sports, Exercise \& Health, University Medical Centre Groningen, University of Groningen, Groningen, Netherlands; ${ }^{2}$ University of Applied Sciences, School of Health Care Studies, Hanze University Groningen, Groningen, Netherlands and ${ }^{3}$ Unit of Psychology, Leiden University Medical Centre (LUMC), Leiden, Netherlands.

Objective: Fibromyalgia patients are perceived by clinicians to be 'difficult' and not easy to manage. Injured athletes are considered as much less difficult to manage clinically. According to Leventhal's Common Sense Model these differences are related to the illness perceptions of these groups. The objective of this study, therefore, was: What are the illness perceptions of patients with fibromyalgia and injured athletes?

Method: 196 fibromyalgia patients and 95 injured athletes participated in the study. In both groups the Illness Perception Questionnaire Revised, adapted for fibromyalgia and sport injuries, respectively, was used. The samples were recruited form physical therapy practices in the Netherlands.

Results: The fibromyalgia group consisted of 172 (88\%) female and $24(12 \%)$ male patients with a mean age of 49 years (SD 11), familiar with the diagnosis since 7 years (SD 6). The athletes sample consisted of 31 (41\%) female and 64 (59\%) males; mean age was 30 (SD 12). The duration of the injury was in 47 athletes (49\%) longer than 6 months; all were injured due to participating in a broad range of sports.

Significant differences were found for illness identity, timeline acute chronic, timeline cyclical, consequences, treatment control and illness coherence.

Fibromyalgia patients have a strong illness identity, perceive more symptoms (pain, fatigue, stiffness), have negative beliefs about the consequences of fibromyalgia, their faith in treatments is low and they do not understand their fibromyalgia and associated symptoms, they have a strong belief in the chronic and cyclical nature of the condition.

Injured athletes have a weak illness identity associated with a high controllability; they see their injury as not chronic, and with minimal serious consequences. They understand the nature of their injury, given their high illness coherence and do not have a strong emotional representation.

Conclusions: The illness perceptions in the two samples studied differ significantly, in a way that substantiates clinical impressions. These differences imply tailoring approaches by health care professionals. Assessing illness perceptions therefore seems useful for diagnostic and therapeutic decision making in patients with chronic conditions.

CORRESPONDING AUTHOR: Cornelis P. van WIlgen, PhD, Movement sciences, University Centre of Sports, Exercise \& Health,
University Medical Centre Groningen, University of Groningen, Groningen, 9700 RB; c.p.van.wilgen@sport.umcg.nl

9:24 AM-9:42 AM

OS04-D

A RANDOMISED CLINICAL TRIAL OF STRESS

MANAGEMENT IN WOMEN WITH THE FIBROMYALGIA SYNDROME (FMS)

Bo Karlsson, $\mathrm{MD},{ }^{1}$ Gunilla Burell, $\mathrm{PhD},{ }^{2}$ Ulla-Maria Anderberg, $\mathrm{MD}^{2}$ and Kurt Svardsudd, $\mathrm{MD}^{1}$

${ }^{1}$ Dept of Public Health and Caring Sciences, Family Medicine and Clinical Epidemiology, Uppsala, Sweden and ${ }^{2}$ Dept of Public Health and Caring Sciences, Social Medicine, Uppsala, Sweden.

Aim: To evaluate the effect of cognitive behavioral therapy (CBT) based stress management on FMS patients regarding pain, stress, and well-being.

Study population and method: 48 women, 27-62 years of age were randomly allocated to two groups, an early treatment group and a waiting list group.

A modified protocol for CBT originally designed for treating women after myocardial infarction was used with key components: education, self-monitoring, skills training, cognitive reconstructing and spiritual development. Brief relaxation was trained. The patients were treated in groups of 5-7 during three hours once week during half a year. During the next 6 months three "booster" sessions were held.

Two experienced clinical psychologists with competence in CBT were responsible for the therapy.

Outcome evaluation are based on Multidimensional Pain Inventory, the Type A behavior instrument, Vital exhaustion, Visual Analogue Scales measured pain, wellbeing and stress.

Results: Perceived "life control" level increased during the intervention in the early treatment group from 3.1 to 3.5 , but decreased in the waiting list group from 3.2 to 2.8 . $(p=0.01)$. There were similar but less pronounced differences for a number of other dimensions, but none was significant.

Stress decreased from 53.7 to $35.9 \mathrm{~mm}$ in the early treatment group, but increased from 46.5 to 52.2 in the waiting list group $(p=0.02)$. Pain and well-being showed similar trends but the differences were non-significant.

Vital exhaustion decreased in the early treatment group from 24.9 to 22.0 , but increased somewhat from 21.5 to 21.8 in the waiting list group $(\mathrm{p}=0.02)$.

Stress behaviour decreased somewhat more in the early treatment group than in the waiting list group,

Conclusions: CBT stress management given to female patients with fibromyalgia increased life control and decreased stress and vital exhaustion. The experience of pain was not significantly reduced. CBT has the potential to improve well-being among patients with cronic widespread pain.

CORRESPONDING AUTHOR: Bo Karlsson, MD, Dept of Public health and Caring Sciences, Uppsala, SE-75122; bo. karlsson@pubcare.uu.se 


\section{CITATION ABSTRACT \\ 9:42 AM-10:00 AM \\ OS04-E \\ PSYCHOLOGICAL TREATMENTS ARE EFFECTIVE IN FIBROMYALGIA: A META- ANALYSIS}

Julia A. Glombiewski, PhD, ${ }^{1,2}$ Alice T. Sawyer, Msc, ${ }^{2}$ Winfried Rief, $\mathrm{PhD}^{1}$ and Stefan G. Hofmann, $\mathrm{PhD}^{2}$

${ }^{1}$ Psychology, University of Marburg, Marburg, Germany and ${ }^{2}$ Psychology, Boston University, Boston, MA.

Treatment of fibromyalgia (FM), a chronic pain disease characterizes by widespread pain is regarded as challenging and the prognosis for recovery is poor. Psychological interventions are known to be effective in other pain disorders and therefore could be a promising treatment of FM. But only few qualitative reviews with divergent conclusions and no meta-analysis on this subject exist.

The aims of the present analysis were to investigate the short- and long-term efficacy and treatment moderators of psychological interventions for fibromyalgia. A literature search identified 23 eligible studies with 30 psychological treatment conditions and 1396 patients. Meta-analytic integration resulted in a significant small effect size $(\mathrm{g}=0.37,95 \%$ confidence interval (CI): 0.27 - 0.48) for short-term pain reduction and a significant small-to-medium effect sizes for long-term pain reduction over an average follow-up phase of 7.4 months $(g=0.47,95 \%$ CI: $0.3-0.65$ ) for any psychological intervention. Psychological treatments also proved effective in reducing sleep problems $(\mathrm{g}=0.46,95 \%$ CI: $0.28-0.64)$ and depression $(\mathrm{g}=0.3,95 \%$ CI: 0.17 - 0.34). These effects remained stable at follow-up. Moderator analyses revealed cognitivebehavioural treatment to be significantly better then other psychological treatments in short-term pain reduction ( $g=0.6,95 \%$ CI: $0.46-0.76$ ) and cognitive-behavioural treatment and relaxation treatments in short-term sleep problems reduction $(\mathrm{g}=0.68,95 \% \mathrm{CI}$ : 0.39 - 0.97). Higher treatment dose was associated with better outcome. Publication-bias analyses demonstrated that the effect sizes were robust.

It is concluded, the effects of psychological treatments for fibromyalgia are promising and comparable to those reported for other pain symptoms or drug treatment in FM. Psychological interventions of sufficient length should be included in FM treatment programmes.

CORRESPONDING AUTHOR: Julia A. Glombiewski, PhD, University of Marburg, Marburg, 35037; glombiew@staff. uni-marburg.de

\section{OS05}

8:30 AM-8:30 AM

OS05-A

HEALTH PROFESSIONAL TRAINEES' USE OF LIFESTYLE BEHAVIOUR CHANGE COMMUNICATION SKILLS: AN EXPERIMENTAL STUDY

Jo Hart, PhD and Sarah Peters, PhD

University of Manchester, Manchester, United Kingdom.

Background: Obesity is a leading cause of morbidity and mortality. Nearly $1 / 4$ of the UK population are obese, and by 2050 this is predicted to reach $60 \%$. This has enormous cost implications for the NHS. Consequently, tackling obesity is a Government priority. An evidence-base exists of theoretically-informed behaviour change techniques for weight loss, however, in routine practice, doctors more commonly use theoretically-unfounded communication strategies (e.g. information giving). It is not known if the current focus on communication skills teaching during undergraduate training adequately prepares future doctors for this growing challenge.

Objectives: To examine 1) what behaviour change techniques medical trainees use to facilitate behaviour change in obese patients and 2) how this impacts on patient behavioural intentions.

Methods: Forty-eight students in their clinical years of a UK medical school were recruited to perform two simulated consultations each. Both consultations involved an obese patient where weight loss was indicated. Simulated patients (SPs) were used to standardise patient variables (e.g. barriers to behaviour change) and presentation of scenario order was counterbalanced. Following each consultation, students assessed the techniques they perceived themselves to have used. SPs rated the extent to which they intended to make behavioural changes. Anonymised transcripts of the audiotaped consultations were coded by independent assessors, blind to student and SP ratings, using a validated behaviour change taxonomy (Abraham \& Michie, 2008).

Results: Students reported using a wide range of evidence-based techniques. In contrast, codings of observed communication behaviours were more limited, focusing on information-giving strategies. SPs behavioural intentions also varied and was explained with reference to students' communication. 
Conclusions: It is suggested that current skills-based communication programmes inadequately prepare future doctors for the growing task of facilitating weight loss. Findings are discussed in relation to the social cognitive basis of behaviour change and communication training needs for health professionals.

CORRESPONDING AUTHOR: Jo Hart, PhD, University of Manchester, Manchester, M14 5NP; jo.hart@manchester.ac.uk

\section{8:30 AM-8:48 AM}

OS05-B

EFFECTIVE WAYS OF TALKING WITH PATIENTS ABOUT BEHAVIOR CHANGE: A QUALITATIVE ANALYSIS OF THE CHALLENGES PERCEIVED BY DOCTORS AND NURSES

Sarah Peters, PhD, ${ }^{1}$ Anna Chisholm, MRes, ${ }^{1}$ Christopher Keyworth, $\mathrm{BSc}^{1}$ and Jo Hart, $\mathrm{PhD}^{2}$

${ }^{1}$ Psychology, University of Manchester, Manchester, United Kingdom and ${ }^{2}$ Medical School, University of Manchester, Manchester, United Kingdom.

Clinicians are increasingly confronted with illness associated with health behaviors (e.g. diet and exercise). Helping patients to adopt healthier behaviors therefore is an important component of medical management and disease prevention. Communication training is a core feature of medical and nursing training. However, recent research reveals that communication training inadequately equips new health professionals for this growing clinical challenge and practitioners remain unconfident about how to discuss behavior change usefully with patients. The study aims to explore how doctors and nurses manage behavior change and identify what barriers operate to prevent effective behavior change management. Semi-structured interviews were conducted with a purposive sample of 14 medical and 14 nursing practitioners at a range of stages during their training (undergraduate and postgraduate). Interviews were transcribed verbatim and analysed thematically using principles of constant comparison to categorise emergent and recurring themes within and between transcripts. Thematic categories arising in initial interviews were explored subsequently and disconfirmatory evidence was sought until thematic saturation arose. Findings reveal that trainees and practitioners consider behavior change to be a fundamental part of medical practice, yet one they are not formally trained in. Participants had developed a range of strategies to encourage behavior change through experiential learning, but were unconvinced of their efficacy. Further barriers to implementing behavior change included the uncertainty over whose responsibility it is, the influence of patient authority within consultations, and desire to protect the doctor-patient relationship. Evidently current communication training is inadequate to equip health practitioners to motivate behavior change. Barriers arise from clinician-patient interactions as well as the context of health care services and have important implications for medical education.

CORRESPONDING AUTHOR: Sarah Peters, PhD, Psychology, The University of Manchester, Manchester, M13 9PL; sarah. peters@manchester.ac.uk
8:48 AM-9:06 AM

OS05-D

IMPLEMENTING A LIFESTYLE INTERVENTION TOOL INTO PRIMARY HEALTH CARE: IDENTIFICATION OF KEY FACTORS THAT INFLUENCE ADOPTION

Siw Carlfjord, Doctoral Student, ${ }^{1}$ Agneta Andersson, $\mathrm{PhD},{ }^{2}$ Preben Bendtsen, Professor, ${ }^{1}$ Per Nilsen, Associate Professor ${ }^{1}$ and Malou Lindberg, $\mathrm{PhD}^{2}$

${ }^{1}$ Department of Medical and Health Sciences, Linköping University, Linköping, Sweden and ${ }^{2}$ R\&D Department of Local Health Care, County Council of Östergötland, Linköping University, Linköping, Sweden.

Background: Lifestyle-related diseases are increasing worldwide, and it is important to address lifestyle issues in primary health care. In order to facilitate this, a tool for lifestyle intervention was developed and implemented in primary health care in Sweden. Implementing new methods, guidelines or tools into routine care, however, is a slow and unpredictable process, and the factors that play a role in the adoption process are not yet fully understood.

Objective: To identify key factors influencing the adoption of a lifestyle intervention tool being introduced in primary health care (PHC) in Sweden.

Methods: A computer-based test for lifestyle intervention was implemented at six PHC units in Sweden. Two different implementation strategies, implicit or explicit, were used. Contextual factors and organizational climate were measured prior to implementation. Outcome in terms of staff referral rates to the lifestyle test was measured after six months of operation. A qualitative study where staff at the units participated in focus group interviews was performed. Interviews were analyzed using the method qualitative content analysis.

Results: A positive organizational climate combined with an explicit implementation strategy did predict a positive implementation outcome when a lifestyle intervention tool was introduced in PHC. Adoption was positively influenced by positive expectations among staff, perceptions of the tool being compatible with existing routines and perceived advantages of the new tool. Organizational changes and staff shortage coinciding with implementation seemed to be obstacles for the adoption process.

Conclusion: Organizational climate and staff expectations should be assessed in advance when a new tool is to be introduced in PHC. Explicit implementation strategy is preferable, and coincidence with major organizational changes should be avoided.

CORRESPONDING AUTHOR: Siw Carlfjord, Doctoral Student, Department of Community Medicine, Linköping University, Institution for Medical end Health Sciences, Linköping, SE58183; siw.carlfjord@liu.se

9:06 AM-9:24 AM

OS05-E

ILLNESS COHERENCE AND YOUNG WOMEN'S UPTAKE OF THE CERVICAL CANCER VACCINE

Kerry A. Sherman, PhD and Danielle Moore, BA (Hons)

Psychology, Macquarie University, Sydney, NSW, Australia. 
The cervical cancer vaccine provides a safe and effective means of reducing the incidence and mortality rate from this disease. Given that uptake is less than optimal, despite the availability of no cost vaccination, there is a need to identify psychosocial factors associated with this preventive behaviour. Applying the Common Sense Model, we first identified the illness representations associated with the acceptance and non-acceptance of the cervical cancer vaccine among young Australian women aged 18-26. We then assessed whether information regarding the link between the viral causes of cervical cancer and the effect of the preventive vaccine can function as a facilitator of future vaccination behaviours. Participants $(\mathrm{N}=132)$ initially completed measures of vaccination status, intentions to be vaccinated, illness representations, perceived risk, perceived vaccine effectiveness and cervical cancer knowledge. Then we experimentally manipulated illness coherence relating to links between the Human Papillomavirus, cervical cancer and the vaccine, by randomly assigning participants to receive either a brief or detailed information condition, to ascertain the effect of this manipulation on vaccination intentions for non-vaccinated women. Higher levels of illness coherence, identity, and perceived vaccine effectiveness, having had a Pap smear in the past, and lower beliefs in the treatment cure/ controllability of cervical cancer were associated with vaccination uptake. Higher intentions to be vaccinated among non-vaccinated women were associated with higher levels of illness coherence and perceived risk for cervical cancer. Post-manipulation measures indicated that the detailed condition of the experimental manipulation resulted in significant increases in illness coherence. The identification of illness cognitions and the role of illness coherence influencing the acceptance of this vaccine adds to the paucity of information available on this underrepresented sample and provides direction for the design of future intervention strategies aimed at modifying illness cognitions to increase vaccine uptake.

CORRESPONDING AUTHOR: Kerry A. Sherman, PhD, Psychology, Macquarie University, North Ryde, NSW, 2109; kerry. sherman@mq.edu.au

\section{OS06}

\section{8:30 AM-8:48 AM \\ OS06-A}

APPLICATION OF THE PRECAUTION ADOPTION PROCESS MODEL TO THE PROMOTION OF CONSUMPTION OF AN UNFAMILIAR NUTRIENT

Philip Mohr, PhD, ${ }^{1}$ Sinéad Quinn, BPsych (Hons), ${ }^{1}$ Matthew Morell, $\mathrm{PhD}^{2}$ and David Topping, $\mathrm{PhD}^{1}$

${ }^{1}$ Food Futures National Research Flagship, CSIRO, Adelaide, SA, Australia and ${ }^{2}$ Food Futures National Research Flagship, CSIRO, Canberra, ACT, Australia.

Although levels of consumption of dietary fibre (DF) are high in Australia, recent evidence indicates the need for increased intakes of the lesser known fibre form, resistant starch (RS), which has been implicated in the reduction of risk of serious diet-related health conditions. The purpose of the present study was to identify the kinds of health messages and the kinds of foods likely to be most acceptable to consumers. Data were obtained by means of a nationwide postal survey $(\mathrm{N}=849)$. Ratings of the importance of various $\mathrm{RS}$ health and functional claims and receptiveness to different foods as RS delivery vehicles were analysed according to the respondents' level of fibre engagement, as classified under the Precaution Adoption Process Model (PAPM) of Health Behaviour (Weinstein \& Sandman, 1992). Significant gender differences were found, with women found to be more fibre engaged and also more receptive than men to RS and its potential benefits. The pattern of association between fibre engagement and self-perceived risk of serious health conditions was consistent with previous observations of changes in risk perception at different PAPM stages. Participants' ratings revealed a general preference for healthy staples over indulgences as foods for the delivery of RS, with the margin in preference increasing markedly with increased dietary fibre engagement. This application of the PAPM illustrates its potential usefulness as a segmentation tool for the exploitation of community engagement with a relatively familiar health concept or behaviour in the promotion of a novel, related concept or behaviour.

CORRESPONDING AUTHOR: Philip Mohr, PhD, CSIRO, Adelaide, SA, 5000; phil.mohr@csiro.au

\section{8:48 AM-9:06 AM}

OS06-B

MULTIPLE BEHAVIOR CHANGE IN DIET AND ACTIVITY: THE MAKE BETTER CHOICES (MBC) TRIAL

Bonnie Spring, $\mathrm{PhD},{ }^{1}$ Kristin Schneider, $\mathrm{PhD},{ }^{2}$ Donald Hedeker, $\mathrm{PhD},{ }^{3}$ H. G. McFadden, BA, ${ }^{1}$ Arlen Moller, $\mathrm{PhD}^{1}$ and Leonard Epstein, $\mathrm{PhD}^{4}$

${ }^{1}$ Preventive Medicine, Northwestern University, Chicago, IL; ${ }^{2}$ University of Massachusetts, Worcester, MA; ${ }^{3}$ University of Illinois, Chicago, IL and ${ }^{4}$ SUNY Buffalo, Buffalo, NY.

Unhealthy diet and inactive lifestyle reflect prevalent, correlated behaviors that heighten chronic disease risk. Intervening on more than one risk behavior simultaneously could be efficient, but the optimal goal framing remains unknown. MBC was designed to test which combination of two behaviors (one dietary, one activity) and two goal frames (increase healthy, decrease unhealthy) maximizes healthy behavior change across all four risk behaviors. $\mathrm{N}=200$ participants with all four risk behaviors [high saturated fat intake (Fat), low fruit and vegetable intake (FV), low physical activity (PA), high sedentary leisure screen time (Sed)] were randomized to one of four goal prescriptions: $\uparrow F V \uparrow P A ; \downarrow$ Fat $\uparrow \mathrm{PA} ; \uparrow F V \downarrow$ Sed; $\downarrow$ Fat $\downarrow$ Sed. They used handheld technology and coaching to change one diet and one activity behavior simultaneously, while change in untargeted (collateral) behavior was also measured. The Familiarity hypothesis predicted best outcome from usual dieting behaviors ( $\downarrow$ Fat $\uparrow$ PA). The Optimal Substitution hypothesis, based on behavioral economic theory, predicted best outcome from substituting healthful for unhealthful behaviors ( $\uparrow F V \downarrow S E D)$. The Low Inhibitory Demand hypothesis, based on Baumeister's self-regulatory strength model, predicted best outcome from minimizing demand on self-control resources ( $\uparrow F V \uparrow P A)$. To scale all behaviors to a common metric, the primary outcome was healthy lifestyle change expressed as a $\mathrm{z}$ score reflecting improvement relative 
to the sample baseline for each behavior and averaged to yield a composite score. As predicted by behavioral economic theory, mixed linear models showed that $\uparrow \mathrm{FV} \downarrow$ Sed maximized healthy lifestyle change compared to alternative interventions $[\mathrm{t}(345)=3.3, \mathrm{p}=.001]$, producing a sustained $1 \mathrm{s.d}$. improvement in saturated fat intake as well as in both targeted behaviors. The untargeted decrease in saturated fat intake complemented the targeted reduction in recreational screen time $(\mathrm{r}=.29, \mathrm{p}=.04)$. Paralleling observed behavior changes, $\uparrow \mathrm{FV} \downarrow$ Sed improved self-efficacy for changing FV $(p<.05)$, Sed $(p<.01)$, and, marginally, Fat $(\mathrm{p}=.09)$. Conversely, the traditional dieting regimen ( $\downarrow$ Fat $\uparrow$ PA) produced less healthy lifestyle change than alternative prescriptions [ $\mathrm{t}(345)=-2.09, \mathrm{p}=.04$ ], yielding sustained 1 s.d. improvement only in saturated fat. Relatedly, $\downarrow$ Fat $\uparrow$ PA failed to increase self-efficacy for targeted behaviors and decreased efficacy for FV and Sed (both $\mathrm{p}<.05$ ). Increasing healthy low-rate eating behavior and decreasing unhealthy high-rate physical activity produced greater and unexpectedly sustained healthy lifestyle change than alternative prescriptions.

CORRESPONDING AUTHOR: Bonnie Spring, PhD, Preventive Medicine, Northwestern University, Chicago, IL, 60610; bspring@northwestern.edu

\section{9:06 AM-9:24 AM}

OS06-C

MY HEALTH MATTERS: A COMMUNITY-LED

INTERVENTION TO REDUCE HEALTH INEQUALITIES RELATED TO PHYSICAL ACTIVITY \& HEALTHY EATING IN STOKE-ON-TRENT, UK

Gemma Hurst, PhD Behaviour Change - Physical Activity, ${ }^{1}$ Rachel Davey, $\mathrm{PhD}^{1}$ and Graham Smith, $\mathrm{MSc}^{2}$

${ }^{1}$ Faculty of Health, Staffordshire University, Stoke-on-Trent, United Kingdom and ${ }^{2}$ Institute for Environment, Sustainability \& Regeneration, Staffordshire University, Stoke-on-Trent, United Kingdom.

Background: Research has demonstrated a strong link between the built environment, health outcomes \& inequalities. Environmental elements can negatively impact lifestyle choices. The 'My Health Matters' project was designed to build partnership with members of the health economy to meet the challenge of increasing physical activity \& healthy eating in three deprived areas of Stoke-onTrent, UK. The project aims to develop \& evaluate an intervention to reduce health inequalities by increasing physical activity \& healthier eating as defined by the community. Results from phase I of the project will be reported including baseline mapping of the built environment using Geographical Information Systems (GIS) \& a community survey. Methods: Environmental factors including convenience \& proximity of physical activity spaces, greenspace $\&$ leisure facilities, neighbourhood connectivity/walkability, land use mix $\&$ population density, traffic, safety $\&$ crime, food outlets $\&$ restaurants, were measured using GIS mapping (calculated around each residential address). A community postal survey of randomly selected addresses $(n=343)$ across the three areas was undertaken. The Neighbourhood Walkability Scale was used to assess residents' perceptions of their neighbourhoods. Perceived health (SF12), social capital, fruit \& vegetable intake \& levels of physical activity (International Physical Activity Questionnaire) were also measured. Results: GIS mapping illustrated a lack of local access to fresh food outlets but easy access to fast food outlets. $76-96 \%$ of residents were within $300 \mathrm{~m}$ walking distance of green space, but these spaces were of poor quality e.g. not functional. Physical activity facilities were within walking distance for some neighbourhoods but not for others. The community survey demonstrated that self-reported health was lower than average compared to England. Negative perceived characteristics of the neighbourhood included; aesthetics (e.g. lack of trees), traffic hazards \& crime. Most people surveyed enjoyed living in their area $\&$ on average had lived there for over 20 years. However, there was low perception of trust \& problems with teenagers \& vandalism. $70-80 \%$ of the sample were not eating the recommended portions of fruit \& vegetables per day \& $70 \%$ were sedentary i.e. not reaching 30 minutes of moderate physical activity 5 days per week. Conclusion: In general, the environments of all three areas were not supportive of healthy living, did not facilitate physical activity or help to promote healthy eating. Recommendations for community consultation \& action (phase II) $\&$ directions for policy change will be discussed.

CORRESPONDING AUTHOR: Gemma L. Hurst, MSc Health Psychology, Faculty of Health, Staffordshire University, Stoke-onTrent, ST10 1EH; g.1.hurst@staffs.ac.uk

\section{9:24 AM-9:42 AM \\ OS06-D \\ META-ANALYSIS OF WORKPLACE PHYSICAL ACTIVITY AND DIETARY BEHAVIOR INTERVENTIONS ON WEIGHT OUTCOMES}

Lisanne M. Verweij, MSc, Jennifer Coffeng, MSc, Willem van Mechelen, $\mathrm{PhD}$ and Karin I. Proper, $\mathrm{PhD}$

Public and Occupational Health, EMGO Institute for Care and Health Research, Amsterdam, Netherlands.

The worldwide increasing prevalence of overweight and obesity is a cause for concern as the overweight-related morbidity, mortality and health care costs concurrently increase. The objective of our meta-analytic review is to critically examine the effectiveness of workplace physical activity and dietary behavior interventions on weight outcomes. Six databases were searched for English-written studies published between 1980 and November 2009. Two authors independently selected 43 studies which met inclusion criteria. Data could be extracted from 22 studies for meta-analysis. Methodological quality was assessed using a predefined checklist. The GRADE approach was used to determine the level of evidence for each pooled outcome measure. Results show there is moderate quality of evidence that workplace physical activity and dietary behavior interventions have a significant positive effect on body weight (14 studies; Mean Difference (MD) $-1.17 \mathrm{~kg}$ [95\% Confidence interval (CI) -1.54 to -0.80$])$. There is low quality of evidence for a significant positive effect on body mass index (14 studies; MD $-0.3 \mathrm{~kg} / \mathrm{m} 2$ [95\% CI -.45 to -0.15$]$ ) and on percentage body fat ( 7 studies; MD $-0.86 \%[95 \%$ CI -1.52 to -0.2$])$. No effects were found for waist circumference 
(4 studies; MD $-0.67 \mathrm{~cm}$ [95\% CI -1.96 to 0.63$]$ ), waist-hip ratio (4 studies; MD $0 \mathrm{~cm}[95 \% \mathrm{CI}-0.03$ to 0.03$]$ ), and sum of skinfolds (2 studies; MD $-0.01 \mathrm{~mm}$ [95\% CI -0.04 to 0.02$]$ ). Subgroup analyses showed interventions using an environmental component had a greater reduction in body weight (3 studies; MD $-1.50 \mathrm{~kg}[95 \% \mathrm{CI}-1.82$ to -1.17$])$. Preventing weight gain by improving physical activity and dietary behavior among workers is recommended as the clinical relevance of the pooled effects are substantial on a population level.

CORRESPONDING AUTHOR: Lisanne M. Verweij, MSc, Public and Occupational Health, EMGO+Institute for Care and Health Research, Amsterdam, 1081 BT; 1.verweij@vumc.nl

\section{9:42 AM-10:00 AM}

OS06-E

INCREASED COGNITIVE AND AFFECTIVE RISK PERCEPTIONS ARE RELATED TO LOWER FRUIT/ VEGETABLE CONSUMPTION AND PHYSICAL ACTIVITY

Rebecca A. Ferrer, PhD, David B. Portnoy, PhD and William M. Klein, $\mathrm{PhD}$

BRP DCCPS, National Cancer Institute, Rockville, MD.

Introduction: Risk perceptions are a key component in many health behavior theories. However, such theories often do not differentiate between cognitive and affective risk perceptions (or the way in which they interact), and it is generally assumed that any increased risk perceptions of either type are associated with preventive behaviors. The present study aims to differentiate between cognitive (perceived risk) and affective (worry) risk perceptions and explore their direct and interactive relationships with two health-related behaviors. Methods: Combined data from the 2003 and 2007 Health Information National Trends Surveys were analyzed using logistic regression with perceived cancer risk, cancer worry, and their interaction independently predicting two dichotomous variables: a) fulfilling the 5-a-day fruit /vegetable consumption guidelines $(\mathrm{n}=7966)$ and $\mathrm{b})$ engaging in any exercise in the past month $(n=8069)$. Results: Neither perceived risk nor worry was significantly related to fruit/ vegetable consumption. However, their interaction was significant such that those who had both the highest perceived risk and worry were the least likely to fulfill the guidelines (OR: .77, $\mathrm{p}<.05$ ). With respect to exercise, worry $(\mathrm{OR}=1.77, \mathrm{p}<.01)$, but not perceived risk, was associated with exercise. More importantly, the interaction was again significant, such that those high in both perceived risk and worry were the least likely to report exercise $(\mathrm{OR}=.77, \mathrm{p}=.01)$. Conclusion: Counter-intuitively, reporting both high perceived risk and high worry is associated with less exercise and produce consumption. This research may have important implications for interventions, which often do not differentiate between targeting cognitive and affective risk perceptions and may even strive to heighten both types of risk perceptions in efforts to prompt behavior change. In addition, this research may inform communication of risk information; optimal changes in health behaviors may be achieved when cognitive risk perceptions are lower, and affective risk perceptions are higher.
CORRESPONDING AUTHOR: Rebecca A. Ferrer, PhD, BRP DCCPS, National Cancer Institute, Rockville, MD, 20878; ferrerra@mail.nih.gov

\section{Thursday August 5, 2010 SYMPOSIA SESSIONS 10:30 AM-12:00 PM}

\section{SS04}

DISEASE ENDPOINTS IN RANDOMIZED CONTROLLED TRIALS USING GROUP BASED COGNITIVE BEHAVIOR THERAPY FOR BREAST CANCER OR CARDIOVASCULAR DISEASE

Neil Schneiderman, $\mathrm{PhD},{ }^{1}$ Barbara L. Andersen, $\mathrm{PhD},{ }^{2}$ Kristina Orth-Gomer, $\mathrm{MD}^{3}$ and Gunilla Burell, $\mathrm{PhD}^{4}$

${ }^{1}$ University of Miami, Coral Gables, FL; ${ }^{2}$ Ohio State University, Columbus, OH; ${ }^{3}$ Karolinska Institute, Stockholm, Sweden and ${ }^{4}$ Uppsala University, Uppsala, Sweden.

Behavioral interventions have not yet been firmly established as being efficacious for reducing nonfatal or fatal cancer or coronary disease recurrence. Three separate randomized clinical trials using group based cognitive behavior therapy (CBT) however, have recently observed positive changes in disease endpoints in patients who had experienced breast cancer or adverse coronary disease events. Group CBT included 20-26 two hour sessions over one year. The trials all used strategies to reduce stress, improve mood, alter health behaviors and maintain adherence to medical treatment. These three studies all included women, used intentto-treat analyses and adjusted for relevant covariates. Andersen and colleagues randomized 227 women who had been surgically treated for regional breast cancer and found after a median of 11 years follow-up, a reduced risk of breast cancer recurrence $(\mathrm{HR}=.55, \mathrm{p}=.034)$, breast cancer mortality $(\mathrm{HR}=.44 ; \mathrm{p}=.016)$ and all-cause-mortality $(\mathrm{HR}=.51 ; \mathrm{p}=.028)$. Orth-Gomér and collaborators randomized 237 women with acute coronary syndrome and found after a median of 7 years, a reduced risk of mortality $(\mathrm{HR}=.31 ; \mathrm{p}=.009)$. Burell and colleagues randomized 362 women and men with acute coronary syndrome and found after a median of 8 years, a reduced risk of recurrent myocardial infarction $(\mathrm{HR}=.55 ; \mathrm{p}=.007)$. Discussion will focus upon the implications of these results and the need for conceptual replication in large multicenter trials.

CORRESPONDING AUTHOR: Neil Schneiderman, PhD, Psychology, University of Miami, Coral Gables, FL, 33124-0751; nschneid@miami.edu

SS04a

A BIOBEHAVIORAL INTERVENTION REDUCES RISK FOR BREAST CANCER RECURRENCE

Barbara L. Andersen, PhD

Ohio State University, Columbus, $\mathrm{OH}$. 
A randomized clinical trial was designed to test the hypothesis that cancer patients coping with a cancer diagnosis but receiving a psychological intervention would have reduced risk for disease progression (1994). Patients $(\mathrm{N}=227)$ surgically treated for regional breast cancer participated. Before beginning adjuvant cancer therapies, patients were assessed with psychological and behavioral measures, had a health evaluation, and a $60 \mathrm{~mL}$ blood sample was drawn. Patients were randomized to Psychological Intervention or Assessment only arms. The intervention was psychologist led, conducted in small groups, and included strategies to reduce stress, improve mood, alter health behaviors, and maintain adherence to cancer treatment. Earlier papers (2004, 2007) showed that, compared to the Assessment arm, the Intervention arm improved across all of the latter secondary outcomes. T cell blastogenesis was also enhanced. An interim paper (2007) clarified treatment mechanisms in the Intervention arm. First, patients were satisfied with the intervention, but it was group cohesion that related to better outcomes. Second, offering patients a stress conceptualization (Selye) and teaching them multiple coping strategies related to better health. Third, three relationships between treatment utilization and outcomes are noted: 1) Relaxation training was associated with both distress reduction and symptom lowering. 2) Patients' use of assertive communication with health care providers was associated with better health outcomes. 3) Intervention strategy use was also associated with fewer signs/symptoms and cancer treatment toxicities. After a median of 11 years follow-up, recurrence occurred for 62 of 212 (29\%) women and death for 54 of 227 (24\%). Intent to treat hazard analyses showed, as predicted, Intervention arm patients had a reduced risk of recurrence [Hazard Ratio $(\mathrm{HR})=0.55, \mathrm{P}=.034]$ and breast cancer death $(\mathrm{HR}=0.44, \quad \mathrm{P}=.016)$. Follow up papers $(2009)$ have examined the covariation of inflammation and distress during the period of intervention delivery (2009) and in the months prior to recurrence diagnosis (2008).

CORRESPONDING AUTHOR: Neil Schneiderman, PhD, Psychology, University of Miami, Coral Gables, FL, 33124-0751; nschneid@miami.edu

\section{$\mathrm{SS} 04 \mathrm{~b}$}

STRESS MANAGEMENT PROLONGS LIFE FOR CHD PATIENTS: A RANDOMIZED CLINICAL TRIAL ASSESSING THE EFFECTS OF GROUP INTERVENTION ON ALL CAUSE MORTALITY, RECURRENT CARDIOVASCULAR DISEASE, AND QUALITY OF LIFE

Gunilla K. Burell, PhD, Kurt Svardsudd, MD and Mats

Gulliksson, MD

Uppsala University, Uppsala, Sweden.

Psychosocial factors are independently associated with increased risk of cardiovascular disease (CVD) morbidity and mortality, but the outcome effect of intervention for these factors on these endpoints has so far been uncertain. 362 men and women, aged 75 or less, discharged from hospital after a coronary heart disease (CHD) event within the past 12 months, were randomized to cognitive behavioral therapy (CBT) focused on stress management during one year $(n=192)$, or to usual care $(n=170)$. Median attendance rate to the intervention program was $85 \%$. Risk factor and quality of life data were measured at baseline and after 6, 12, 18, 24 months. Hospital admission data and survival data were obtained from national registers. During 8 years of follow-up the intervention group had $41 \%$ less fatal and non-fatal first recurrent CVD rate, and 45\% less recurrent acute myocardial infarction (AMI) rate than the reference group. The study was powered to examine the combined endpoint of fatal and nonfatal recurrent coronary event rate and found a positive significant difference between groups (HR 0.59, $95 \%$ CI $0.42-0.83, \mathrm{p}=0.003)$. Subsequent analyses found a significantly reduced nonfatal recurrent acute myocardial infarction rate (HR $0.55,95 \%$ CI $0.36-0.85, \mathrm{p}=0.007)$, but no significant difference in allcause-mortality (HR 0.72, 95\% CI 0.40-1.30, $\mathrm{p}=0.28$ ). During the first 2 years of follow-up there were no significant group differences in quality of life, but the intervention group had a significantly stronger optimism about the future. The CBT stress intervention decreased the risk of recurrent CVD and recurrent AMI. A dose-response relationship between therapy attendance rate and risk was observed. This has implications for targeting and strengthening of secondary preventive programs in CHD patients.

CORRESPONDING AUTHOR: Neil Schneiderman, PhD, Psychology, University of Miami, Coral Gables, FL, 33124-0751; nschneid@miami.edu

\section{$\mathrm{SS} 04 \mathrm{c}$}

COGNITIVE BEHAVIOR THERAPY MAY PROLONG LIVES OF WOMEN WITH CORONARY DISEASE.

THE STOCKHOLM WOMEN'S INTERVENTION TRIAL FOR CORONARY HEART DISEASE (SWITCHD)

Kristina Orth-Gomér, MD, $\mathrm{PhD},{ }^{1}$ Neil Schneiderman, $\mathrm{PhD},{ }^{2}$ N. Wang, $\mathrm{PhD},{ }^{1}$ H. Blom, $\mathrm{PhD},{ }^{1} \mathrm{M}$. Walldin, $\mathrm{PhD}^{1}$ and $\mathrm{T}$. Jernberg, MD ${ }^{1}$

${ }^{1}$ Karolinska Institutet, Stockholm, Sweden and ${ }^{2}$ University of Miami, Miami, FL.

Psychosocial stress may accelerate atherosclerosis progression and worsen prognosis in women with coronary heart disease (CHD). In Stockholm women, we found marital stress to be a stronger predictor than job stress, but behavioral interventions to reduce such psychosocial stressors in women had not been identified. Based on previous findings, we implemented a cognitive behavior therapy (CBT) program for women and investigated its ability to improve survival in women coronary patients. Consecutive women patients, aged 75 years or younger, hospitalized for acute myocardial infarction, coronary artery bypass grafting, or percutaneous coronary intervention were randomized to group-based CBT or to usual care. Intervention groups of 4 to 8 women met for a total of 20 sessions that were spread over a year. We provided education about risk factors, relaxation training techniques, methods for self-monitoring and cognitive restructuring, with an emphasis on coping with stress exposure from family and work, self-care and compliance with medical advice. From randomization until end of follow-up (mean duration, 7.1 years), 25 women $(20 \%)$ in the usual care and 8 women $(7 \%)$ in the CBT condition died, yielding an almost three fold protective effect of the 
intervention $(\mathrm{HR}=0.33 ; 95 \% \mathrm{CI}, 0.15$ to $0.74 ; \mathrm{P}=0.007)$. Controlling for relevant demographic and medical prognostic factors in multivariate models, confirmed the unadjusted results. CBT improved survival independently of medication with statins. Additionally, an interactive effect of statins with behavioral intervention was suggested. In women who received both treatments, only one woman died, $(1.5 \%)$ as compared to 15 out of 70 $(22 \%)$ women who received none of the treatments $(p=0.007)$. We concluded that a group-based CBT program for women with coronary disease may prolong their lives. This effect may be independent of clinical prognostic factors.

CORRESPONDING AUTHOR: Neil Schneiderman, PhD, Psychology, University of Miami, Coral Gables, FL, 33124-0751; nschneid@miami.edu

\section{SS05}

CORRELATES OF SEXUAL RISK BEHAVIORS

AMONG HIGH SCHOOL STUDENTS IN COLORADO: ANALYSIS AND IMPLICATIONS FOR SCHOOL-BASED HIV/AIDS PROGRAMS

Stephen Nkansah-Amankra, $\mathrm{PhD}, \mathrm{MPH}, \mathrm{MA}$ and Adoulaye Diehiou, $\mathrm{PhD}, \mathrm{MD}$

School of Human Sciences/ Colorado School of Public Health, University of Northern Colorado, Greeley, CO.

To evaluate correlates of sexual risk behaviors associated with HIV/AIDS infection among adolescents participating in 2005 Colorado Youth Risk Behavioral Survey, since previous studies have produced mixed findings. The State mandates abstinenceonly program across all public schools. Multinomial logistic regression was used to evaluate relationships among dependent variables and independent variables. We found no significant effect of having received in-school HIV/AIDS education on all outcome measures. Compared with females, males were more likely to initiate sex at a relatively younger age, report unprotected sex with multiple partners, and to drink alcohol before sexual intercourse. Efforts to control the HIV/AIDS epidemic among adolescents may need to focus on targeted interventions aimed at addressing gender- and racial/ethnic-specific risk exposures among this population group, including risk behaviors linked with lifetime sexual abuse. The need to re-examine the role of in-school HIV prevention programs to build adequate competencies among students, parents and community leaders is recommended.

CORRESPONDING AUTHOR: Stephen Nkansah-Amankra, $\mathrm{PhD}$, MPH, MA, School of Human Sciences/ Colorado School of Public Health, University of Northern Colorado, Greeley, CO, 80639; snamankrasc@1ycos.com

\section{$\mathrm{SS} 06$}

WOMENS' HEALTH AND IRRITABLE BOWEL SYNDROME Ulrike Ehlert, $\mathrm{PhD}$ and Paul Enck, $\mathrm{PhD}$

Clinical Psychology and Psychotherapy, University of Zurich, Zurich, Switzerland.
Irritable bowel syndrome is a highly prevalent functional gastrointestinal disorder (FGD) in western countries. Often, the disorder occurs in comorbidity with additional functional symptoms like chronic pelvic pain, fatigue or psychiatric disorders like anxiety and depression. Females suffer at least twice as often from FGD than men. Irritable bowel syndrome (IBS) is the most prevalent FGD and research shows a strong association between chronic stress and trauma in these patients. Due to the increasing knowledge on psychobiological mechanisms regulating adaptive processes to stress, research focuses on functional syndromes, which seem to be associated with maladaption of biological stress regulatory systems. This symposium refers to the different biological factors contributing to the etiology and maintenance of IBS. Besides prevalence rates on the high number of females with IBS, naturally occurring variation of female life conditions like menstrual cycle will be discussed with respect to the occurrence of IBS symptoms. Additionally, data on different biological systems like the HPA axis, pain perception, serotonergic pathways will be presented with reference to IBS and possible treatment approaches.

CORRESPONDING AUTHOR: Ulrike Ehlert, PhD, Clinical Psychology and Psychotherapy, University of Zurich, Zurich, 8050; u.ehlert@psychologie.uzh.ch

SS06a

PSYCHOBIOLOGICAL STRESS RESPONSIVENESS TO NATURALLY OCCURRING AND LABORATORY INDUCED PSYCHOSOCIAL STRESS IN WOMEN WITH IRRITABLE BOWEL SYNDROME

Kerstin A. Suarez-Hitz, MS, ${ }^{1}$ Werner Schwizer, MD, ${ }^{2}$ Michael Fried, $\mathrm{MD}^{2}$ and Ulrike Ehlert, $\mathrm{PhD}^{1}$

${ }^{1}$ Clinical Psychology and Psychotherapy, University of Zurich, Zurich, Switzerland and ${ }^{2}$ Division of Gastroenterology and Hepatology, University Hospital of Zurich, Zurich, Switzerland.

Background \& Aims: Stress has been considered as an important etiological factor in IBS. The physiological mechanisms between stress and gut disturbances, however, are incompletely understood. The current studies aimed to investigate the association between different stressors and functional gastrointestinal (FGI) complaints in female IBS patients and healthy controls.

Methods: In an Internet-based survey conducted in a large number of students, the prevalence of FGI symptoms and perceived stress (chronic stress, coping strategies, stress reactivity) were measured. In a second trial basal and stimulated HPA axis activity was assessed in 57 women with IBS and 20 matched controls. IBS diagnosis was made according the Rome III criteria and psychiatric comorbidity was assessed using a clinical interview. Salivary morning cortisol and diurnal profile were obtained and a standardized psychosocial stress test (TSST) was applied. Cortisol and ACTH were measured before and within one hour following the stressor.

Results: Two third of the online study sample $(n=668)$ reported FGI complaints which were significantly predicted by female gender, high levels of chronic stress, dispositional stress reactivity, and use of maladaptive coping. The biological results indicated that female IBS 
patients have intact circadian rhythmicity of the HPA axis. However, women with diarrhea predominant IBS (IBS-D) exhibited substantially heightened cortisol at awakening and nearly no morning increase. In response to the laboratory stressor significantly blunted cortisol $(\mathrm{p}<$ 0.05 ) and slightly attenuated ACTH secretion was observed in patients compared to controls. In the recovery period ACTH levels were significantly lower in patients than in healthy subjects $(p<0.05)$. Women with IBS perceived higher general and situation specific stress susceptibility.

Conclusions: FGI syndromes, including IBS, seem to be common in apparently healthy subjects whereas female gender is a predictor. Several naturally occurring stressors are associated with the presence of FGI symptoms. In both studies psychological stress measures were overall increased in the IBS group and propose heightened stress susceptibility. Biological data indicate an association between basal HPA axis activity and predominant bowel habit. Furthermore, the downregulated HPA axis reactivity in women with IBS following the laboratory stressor (TSST) suggests an attenuated sensitivity of the endocrine system. The strong relationship between stress experience and the occurrence of IBS indicates that stress-reducing interventions may be beneficial in such individuals.

CORRESPONDING AUTHOR: Kerstin A. Suarez-Hitz, MS, Clinical Psychology and Psychotherapy, University of Zurich, Zurich, 8050; k.suarez@psychologie.uzh.ch

\section{SS06b}

COGNITIVE AND EMOTIONAL MODULATION OF VISCERAL PAIN PROCESSING IN IBS PATIENTS AND HEALTHY SUBJECTS

Sven Benson, $\mathrm{PhD},{ }^{1}$ Christina Rosenberger, $\mathrm{PhD},{ }^{1,2}$ Manfred Schedlowski, PhD, ${ }^{1}$ Michael Forsting, MD, ${ }^{2}$ Elke R. Gizewski, $\mathrm{MD}^{2}$ and Sigrid Elsenbruch, $\mathrm{PhD}^{1}$

${ }^{1}$ Inst. of Medical Psychology and Behavioral Immunobiology, University Hospital Essen, Essen, Germany and ${ }^{2}$ Institute of Diagnostic and Interventional Radiology and Neuroradiology, University Hospital Essen, Essen, Germany.

Background: In this set of fMRI studies, our goal was to establish experimental pain paradigms to address emotional and cognitive modulation of the behavioural as well as neural response to visceral pain.

Method: The blood oxygen level-dependent (BOLD) response to painful rectal distensions was assessed at baseline, in a negative emotional context induced by psychological stress (emotional modulation study: carried out in 15 female IBS patients and 12 healthy women) and in a visceral placebo analgesia paradigm (cognitive modulation study: carried out in 36 healthy subjects). For visceral placebo analgesia, a deceptive study paradigm was used in which expectation of analgesia was manipulated by giving subjects different instructions regarding an intravenous infusion of either "a highly potent analgesic drug" or "an inert substance", when in reality all subjects received a placebo (saline) during painful rectal distensions.

Results: Distensions delivered in a negative emotional context increased distension-induced discomfort and urge to defecate in IBS patients but not controls. At the same time, IBS patients demonstrated more pronounced stress-induced modulation of neural activation during rectal stimulation in the insula, midcingulate cortex, and ventrolateral prefrontal cortex. The placebo analgesia paradigm, carried out in healthy subjects, revealed that manipulation of expectation resulted in significantly decreased subjective pain ratings. Placebo responders demonstrated significantly greater activation of the dorsolateral prefrontal cortex during expectation of analgesia compared to non-responders.

Discussion: Pain amplification and hypervigilance in IBS may be related to altered affective-cognitive modulation of the pain response.

CORRESPONDING AUTHOR: Sven Benson, PhD, Inst. of Medical Psychology and Behavioral Immunobiology, University Hospital Essen, Essen, 45122; sven.benson@uk-essen.de

$\mathrm{SS} 06 \mathrm{c}$

FOOD, MOOD, GENES, AND GENDER

Paul Enck, $\mathrm{PhD}$ and Sibylle Klosterhalfen, $\mathrm{PhD}$

Psychosomatic Medicine, University Hospitals, Tuebingen, Germany.

The importance of serotonin in regulation of central (mood, hunger) and peripheral (intestinal) functions has long been established. Nutritive interventions however, e.g. the increase in dietary tryptophan - the metabolic precursor of serotonin - for the treatment of eating disorders (anorexia), mood disorders (depression), migraine, and the irritable bowel syndrome have not produced significant Results: The unbalanced population prevalence of these disorders in men and women allows concluding a contribution of gender to the efficacy such interventions. Shortterm depletion of tryptophan from food (called acute tryptophan depletion, ATD) has been shown to provoke the respective symptoms both in healthy volunteers as well as in patients, but seems to work predominantly in women. Therefore, gender differences must exist in the serotonin metabolism and its utilization in the brain and in the periphery. In addition, genetic predisposition has been shown to exist that determines - among others - the effectiveness of serotoninergic interventions in patients, with drugs as well as with nutrients. We will review the current evidence for the contribution of gender on food, mood, and genetic determinants of serotonin utilization.

CORRESPONDING AUTHOR: Paul Enck, PhD, Psychosomatic Medicine, University Hospitals, Tuebingen, 72076; paul.enck@ uni-tuebingen.de

\section{SS06d}

MENSTRUAL CYCLE AND IRRITABLE BOWEL SYNDROME: DOES IT MAKE A DIFFERENCE?

Margaret Heitkemper, PhD, RN and Monica E. Jarrett, PhD, RN BNHS, University of Washington, Seattle, WA.

In the U.S. and other western industrial countries more women than men seek health care services for irritable bowel syndrome (IBS). In addition, the health care impact of IBS also appears to be 
greater in women as compared to men. Women with IBS commonly report other somatic and visceral symptoms. Muscle and joint pain and disturbed sleep patterns are among the most common and the most distressing. Gender differences exist in types of GI symptoms (women report greater problems with constipation while men more frequently report diarrhea) as well as other extra-intestinal symptoms. The role of menstrual cycle in IBS symptom reporting is based on observations that women with IBS frequently report cyclic changes in their symptom severity. Women with IBS often report other menstrual cycle linked conditions including migraine headache, dysmenorrhea and premenstrual dysphoria distress syndrome.

Gender differences in IBS begin to emerge at puberty. In children, the rubric of recurrent abdominal pain (RAP) includes IBS, functional abdominal pain, and likely, dyspepsia. Prior to puberty there is an equal proportion of boys and girls with a diagnosis of RAP. The factors responsible for the divergence in prevalence after puberty are not clear. Studies suggest that both environmental (i.e., increased risk if a parent has IBS) and gender factors (i.e., changes in hormonal milieu) likely play a role. The incidence of IBS in both men and women appears to decrease in late middle age. The severity of GI symptoms, including abdominal pain, altered bowel habits, and bloating, varies across phases of the menstrual cycle as well as during the menopause transition in some women with or without IBS or other functional GI disorders. A limited number of studies indicate that premenses (days immediately preceding the onset of menses) and menses, compared with other cycle phases, are periods of increased GI symptom distress in women age 19-37. Women with IBS experience a greater impact of menstrual cycle on symptoms as compared to those without IBS. While not extensively studied, oral contraceptives appear to modestly reduce but not eliminate GI symptoms in those with IBS. This paper will present they hypothesis that declining or low ovarian hormone levels underlies the occurrence or exacerbations of abdominal pain/discomfort across the menstrual cycle and the perimenopause-early menopause transition in women with or without IBS.

CORRESPONDING AUTHOR: Margaret Heitkemper, PhD, BNHS, University of Washington, Seattle, WA, 98195; heit@u. washington.edu

\section{SS07}

\section{TRAJECTORIES OF HEALTH AND HEALTH BEHAVIORS} IN RELATION TO RETIREMENT AND SOCIOECONOMIC STATUS

Hugo Westerlund, $\mathrm{PhD},{ }^{1}$ Jussi Vahtera, $\mathrm{MD}, \mathrm{PhD}^{2,3}$ and Archana Singh-Manoux, $\mathrm{PhD}^{4}$

${ }^{1}$ Stress Research Institute, Stockholm University, Stockholm, Sweden; ${ }^{2}$ Department of Public Health, University of Turku and Turku University Hospital, Turku, Finland; ${ }^{3}$ Finnish Institute of Occupational Health, Helsinki, Finland and ${ }^{4}$ U687, INSERM, Villejuif, France.

Increased availalability of data and recent developments in longitudinal modelling improve the possibilities to understand the long-term development of health. The symposium will present four papers exploring trajectories of health and health-related behavior - based on annual measurements over 9-18 years - in relation to retirement, sickness absence, and socioeconomic status. The two first papers show that retirement was associated with a substantial decrease in fatigue, depressive symptoms, and increase in adherence to medical treatment among individuals with chronic hypertension following retirement. Poor adherence before retirement was more common among individuals exposed to high work stress. The third paper shows that the association between diagnosis-specific sickness absences and sustained sub-optimal health varies by socioeconomic position. While the association with self-rated health was significantly stronger for mental disorders than for musculoskeletal disorders in the higher grades, the inverse was true in the lower grades. The fourth paper shows that weight continues to increase in the transition between midlife and old age, and that this increase is greater in the lower socioeconomic groups.

As discussant, research director Archana Singh-Manoux will highlight the methodological possibilities and challenges in understanding the long-term development of health.

CORRESPONDING AUTHOR: Hugo Westerlund, PhD, Stress Research Institute, Stockholm, SE-106 91; hugo.westerlund@ stress.su.se

SS07a

EFFECTS OF RETIREMENT ON MAJOR CHRONIC CONDITIONS AND FATIGUE: THE FRENCH GAZEL OCCUPATIONAL COHORT STUDY

Hugo Westerlund, $\mathrm{PhD},{ }^{1,3}$ Jussi Vahtera, $\mathrm{MD}, \mathrm{PhD}^{2}$ Jane E. Ferrie, $\mathrm{PhD},{ }^{3}$ Archana Singh-Manoux, $\mathrm{PhD}^{4}$ and Mika Kivimäki, $\mathrm{PhD}^{3}$

${ }^{1}$ Stress Research Institute, Stockholm University, Stockholm, Sweden; ${ }^{2}$ Dept. of Public Health, University of Turku, Turku, Finland; ${ }^{3}$ Dept. of Epidemiology \& Public Health, UCL, London, United Kingdom and ${ }^{4}$ U687, INSERM, Villejuif, France.

Objectives: To determine, using longitudinal analyses, if retirement is followed by a change in the risk of incident chronic diseases, depressive symptoms, and fatigue.

Design, Setting, and Participants: A prospective cohort study with multiple repeated annual surveys from seven years before through seven years after retirement in a large French occupational cohort the GAZEL study - of 11,246 men and 2,858 women. Data were collected by questionnaire each year from 1989 through 2007 inclusive.

Main Outcome Measures: Respiratory disease, diabetes, cardiovascular disease and stroke, mental fatigue, and physical fatigue, measured each year of the 15-year observation period, and depressive symptoms, measured at four time points.

Results: Repeated-measures logistic regression with generalized estimating equations showed that the cumulative prevalence of respiratory diseases, diabetes, and cardiovascular disease and stroke increased with age, with no break in the trend around retirement. In contrast, we found retirement to be associated with a substantial decrease in the prevalence of both mental fatigue (odds ratio [OR] for fatigue one year after versus one year before the 
retirement 0.19 ; $95 \%$ CI 0.18 to 0.21 ) and physical fatigue (OR 0.27 ; CI 0.26 to 0.30 ). A slightly smaller decrease was observed in depressive symptoms (OR 0.60 ; CI 0.53 to 0.67 ). The decrease in fatigue around retirement was more pronounced among those with chronic disease before retirement.

Conclusions: Retirement appears not to change the risk of major chronic diseases, but it is associated with a substantial reduction in mental and physical fatigue and depressive symptoms, particularly among participants with chronic disease. Future research should investigate the generalisability of the findings to other countries and settings, examine the consequences of fatigue among older workers, as well as explore various possible determinants of such fatigue.

CORRESPONDING AUTHOR: Hugo Westerlund, PhD, Stress Research Institute, Stockholm, SE-106 91; hugo.westerlund@, stress.su.se

\section{SS07b}

CHANGES IN ADHERENCE WITH MEDICATION AROUND RETIREMENT AMONG PEOPLE WITH CHRONIC HYPERTENSION: THE PROSPECTIVE FINNISH PUBLIC SECTOR STUDY

Jussi Vahtera, MD, PhD, ${ }^{1,2}$ Hugo Westerlund, $\mathrm{PhD},{ }^{3,4}$ Jaana Pentti, $\mathrm{BSc}^{2}$ and Mika Kivimäki, $\mathrm{PhD}^{2,4}$

${ }^{1}$ Dept. of Public Health, University of Turku, Turku, Finland; ${ }^{2}$ Finnish Institute of Occupational Health, Turku, Finland; ${ }^{3}$ Stress Research Institute, Stockholm University, Stockholm, Sweden and ${ }^{4}$ Dept. of Epidemiology \& Public Health, UCL, London, United Kingdom.

Background: Retirement is a major social transition believed to have important consequences for health, but empirical evidence remains contradictory. The effects of retirement on the risk of specific diseases with major public health importance are unknown. Because poor adherence to treatment can increase the risk of severe medical complications, we examined if retirement is followed by a change in the use of antihypertensive medication among individuals with chronic hypertension.

Methods: We linked an occupational cohort of 1820 retired employees in Finland ( $71 \%$ women) entitled to special reimbursement due to chronic hypertension before retirement to national health and prescription registers. The prescription register contains data on the exact dates and defined daily dosages of every purchase of prescription drugs. Annual use of antihypertensive medication was measured for each year during a period covering 4 years before and 4 years after the year of retirement.

Results: Repeated-measures showed that adherence was significantly better in the years after retirement compared to the years before retirement. The ratio of mean number of days not covered by purchased prescribed drugs in the four years following, compared with the four years preceding, retirement was 0.74 (95\% CI 0.67-0.81) after adjustment for sex, age at retirement, occupational status and year of retirement. This improvement in adherence was observed in both sexes, in all occupational grades and irrespective of type of retirement. However, the improvement in adherence following retirement was significantly greater among employees with high job strain before retirement compared to the others: the mean ratio of days not covered by prescribed drugs in the post-retirement years compared to the pre-retirement years $0.58(95 \%$ CI $0.47-0.70)$ in the high strain group and $0.78(95 \%$ CI $0.71-0.87$ ) in the low strain group (test of interaction, $\mathrm{p}=$ 0.0004). Further adjustments for baseline health indicators had little effect on these associations.

Conclusions: Repeated measurements provide evidence for a substantial and sustained increase in adherence to medical treatment among individuals with chronic hypertension following retirement. Poor adherence before retirement is more common among individuals exposed to high work stress. Because inadequate treatment of chronic hypertension increases the risk of severe medical complications, our findings suggest that the retirement-related relief of work-stress may decrease the risk of complications in high-risk populations.

CORRESPONDING AUTHOR: Hugo Westerlund, PhD, Stress Research Institute, Stockholm, SE-106 91; hugo.westerlund@ stress.su.se

\section{$\mathrm{SS} 07 \mathrm{c}$}

SOCIO-ECONOMIC DIFFERENCES IN THE ASSOCIATION BETWEEN DIAGNOSIS-SPECIFIC SICKNESS ABSENCE AND SUBSEQUENT SUSTAINED SUB-OPTIMAL HEALTH: A 14-YEAR FOLLOW-UP IN THE GAZEL COHORT

Jane E. Ferrie, $\mathrm{PhD},{ }^{1}$ Mika Kivimäki, $\mathrm{PhD},{ }^{1}$ Hugo Westerlund, $\mathrm{PhD},{ }^{1,2}$ Jenny Head, MSc, ${ }^{1}$ Marcel Goldberg, $\mathrm{MD}, \mathrm{PhD}^{3}$ and Jussi Vahtera, $\mathrm{MD}, \mathrm{PhD}^{4}$

${ }^{1}$ Dept. of Epidemiology \& Public Health, UCL, London, United Kingdom; ${ }^{2}$ Stress Research Institute, Stockholm University, Stockholm, Sweden; ${ }^{3}$ U687, INSERM, Villejuif, France and ${ }^{4}$ Dept. of Public Health, University of Turku, Turku, France.

Background: Previous studies show a remarkably persistent association between sickness absence and future long-term selfrated health status for the majority of diagnostic categories for sickness absence but it is unclear whether this association differs by socioeconomic position.

Methods. Prospective occupational cohort of 15,320 employees ( $73 \%$ men) aged 37 to 51 , of whom $30 \%$ were in higher, $55 \%$ intermediate, and $15 \%$ in the lower occupational grades. Sickness absence records 1990-1992, including 13 diagnostic categories, were examined in relation to self-rated health measured annually for the years 1993-2006.

Results: Among higher grade employees $9.5 \%$ had $>30$ days of sickness during the 3 -year exposure window. The corresponding figure among intermediate and lower grade employees was $24 \%$ and $40 \%$, respectively. Repeated-measures logistic regression analysis adjusted for age, sex, and chronic diseases showed higher, intermediate, and lower grade employees with $>30$ absence days, compared to those with no absences, to have $2.22(95 \% \mathrm{CI}$ 1.90-2.60), 2.13 (95\% CI 1.95-2.33), and 1.86 (95\% CI $1.58-$ 2.20) times higher odds of suboptimal health over the 14 years of follow-up, respectively. Most of the 13 sickness absence diagnos- 
tic categories independently predicted an increased risk of sustained sub-optimal health in higher and intermediate grades. However, few categories were predictive in the lower grades; mental disorders, diseases of the circulatory, genitourinary, and musculoskeletal systems. While the association with self-rated health was significantly stronger for mental disorders in the higher grades, there was no association with musculoskeletal disorders. Conclusions: The ability of diagnosis-specific sickness absences to predict sustained sub-optimal health differs by socioeconomic position. The remarkably persistent association between sickness absence and future long-term self-rated health status for the majority of diagnostic categories for sickness absence was observable only in higher and intermediate grades, while for lower grade this association was less ubiquitous.

CORRESPONDING AUTHOR: Hugo Westerlund, PhD, Stress Research Institute, Stockholm, SE-106 91; hugo.westerlund@ stress.su.se

\section{SS07d}

DO SOCIOECONONOMIC FACTORS SHAPE WEIGHT AND OBESITY TRAJECTORIES OVER THE TRANSITION FROM MIDLIFE TO OLD AGE?

Aline Dugravot, MSc, ${ }^{1}$ Séverine Sabia, PhD:, ${ }^{1}$ Silvia Stringhini, PhD: ${ }^{1}$ Alice Guéguen, $\mathrm{PhD},{ }^{1}$ Marie Zins, MD, ${ }^{1}$ Hermann Nabi, $\mathrm{PhD}^{1}$ and Archana Singh-Manoux, $\mathrm{PhD}^{1,2}$

${ }^{1}$ U687, INSERM, Villejuif, France and ${ }^{2}$ Dept. of Epidemiology \& Public Health, UCL, London, United Kingdom.

Objectives: To examine socioeconomic differences in trajectories of body mass index (BMI) and obesity between the ages of 45 and 65 . Methods: 13541 men and 4652 women from the French GAZEL study reported their height and weight annually over 18 years, starting in 1990. Occupational position (managers, skilled and unskilled workers) at age 35 was the indicator of socioeconomic circumstances. Changes in Body Mass Index (BMI) and obesity between ages 45 and 50, 50 and 55, 55 and 60 and 60 and 65 were modelled using Linear Mixed Models (Generalized Estimating Equations).

Results: BMI and obesity increased between the ages of 45 and 65 , albeit at a slower pace at older ages. In men, BMI was higher among unskilled workers compared to managers at age 45 , the difference increased from $0.83 \mathrm{~kg} / \mathrm{m} 2$ (95\% confidence interval (CI): $0.66,0.99)$ at 45 years to $1.12 \mathrm{~kg} / \mathrm{m} 2(95 \% \mathrm{CI}: 0.90,1.33)$ at 65 years. Estimated obesity rates were $3.37 \%$ and $7.54 \%$ in managers and unskilled workers at age 45 and $9.36 \%$ and $18.46 \%$ at age 65 , the difference increasing by $4.93 \%$ (95\% CI: $2.81,7.13)$. A similar trend was observed in women.

Conclusions: This study shows that weight continues to increase in the transition between midlife and old age; this increase is greater in the lower socioeconomic groups.

CORRESPONDING AUTHOR: Hugo Westerlund, PhD, Stress Research Institute, Stockholm, SE-106 91; hugo.westerlund@, stress.su.se
SS16

KNOWLEDGE TO ACTION IN THE HEALTH FIELD: INTERNATIONAL DEVELOPMENTS AND CHALLENGES AHEAD

Arja R. Aro, PhD, DSc

Unit for Health Promotion Research, University of Southern Denmark, Esbjerg, Denmark.

Knowledge translation or exchange between health research, practice, and policy has received active attention recently. Experiences and models from the more individual level behavioral or clinical medicine need to be expanded to include complex organizational and political realities of more contextual health promotion and public health fields. The challenge of such expansion of models is increased all the more when moving from a national to a global perspective. The symposium will contribute to the latest international developments and discussions in bridging between research knowledge, practice and policy making both within health care and health promotion. Allan Best will present the results of a Canadian Institutes of Health Researchfunded meta-narrative review of conceptual models for knowledge to action, and a series of international workshops focused on "embedded" research or alternative organizational or network models to foster and support research applications to policy and practice. Arja R Aro will describe the experiences and challenges as well as present the 1st year evaluation results of the universitymunicipality contractual collaboration in health policy planning in Denmark. Edwin Fisher will describe models of peer support developed to support global promotion of peer support programs across differing cultures, populations, and health systems and then describe how that model is employed in global promotion efforts, focusing though at regional initiatives to demonstrate and advocate for peer support as a standard component of health care and prevention. The symposium will provide time for general discussion.

CORRESPONDING AUTHOR: Arja R. Aro, PhD, DSc, Unit for Health Promotion Research, University of Southern Denmark, Esbjerg, 6700; araro@health.sdu.dk

\section{SS16a}

CHALLENGES IN COLLABORATION BETWEEN ACADEMIA, PRACTICE AND POLICY MAKERS IN HEALTH PROMOTION IN DENMARK

Arja R. Aro, PhD, DSc, Maja Larsen, MSc and Gabriel Gulis, $\mathrm{PhD}$

Unit for Health Promotion Research, University of Southern Denmark, Esbjerg, Denmark.

A structural administrative reform in the Danish society in 2007 gave municipalities new responsibilities for preventive and health promotion activities. In this new situation a window of opportunity opened for knowledge exchange between academia, practice and municipal policy makers. Academia was expected to produce research evidence for applicable and cost effective interventions and policies. On the other hand, academia lacked contextual 
information necessary for tailored research evidence. Collaboration between these different stakeholders has proved to be challenging. The Danish model consists of 3 year contracts for junior researcher-practitioner positions, co-funded by the university and municipalities with the aim to carry out scientific projectbased research relevant to municipalities. The 1 st year evaluation of the collaboration with 3 case municipalities has shown as major benefits: increased access and use of research-based working methods in the municipalities; image lift of the municipalities; concrete products such as usable research results and lectures delivered; and support in project implementation. On the other hand, problems or challenges experienced have been: different working traditions and time scales; translating complicated research results into immediate and applicable practical knowhow; lack of time and capacity for knowledge exchange; and sometimes lack of mutual respect and trust between stakeholders. All in all, collaboration and knowledge exchange has already in the 1st year lead to longer-term collaborative health policy planning; increased knowledge exchange; and also to an interest to start an evidence-based knowledge center within the area of health promotion. Challenges ahead lie in developing capacity building among all stakeholder groups; organizational barriers; development of 'service packages' including contextually tailored research information e.g. of health profiles of communities; implementation in practice and policy making as well as longterm follow-up of the implementation.

CORRESPONDING AUTHOR: Arja R. Aro, PhD, DSc, Unit for Health Promotion Research, University of Southern Denmark, Esbjerg,6700; araro@health.sdu.dk

\section{SS16b}

\section{SYSTEMS STRATEGIES FOR KNOWLEDGE TO ACTION} IN HEALTH CARE: REVIEW OF CONCEPTUAL MODELS AND THE CANADIAN CONTEXT

Allan Best, $\mathrm{PhD},{ }^{1,2}$ Craig Mitton, $\mathrm{PhD},{ }^{1,3}$ Neale Smith, MA, ${ }^{1,3}$ Jennifer Bitz, MA, PMP, ${ }^{1,3}$ Jennifer L. Terpstra, MPH ${ }^{1}$ and John Millar, $\mathrm{MD}^{1}$

${ }^{1}$ School of Population and Public Health, University of British Columbia, West Vancouver, BC, Canada; ${ }^{2}$ InSource Research Group, West Vancouver, BC, Canada and ${ }^{3}$ Centre for Clinical Epidemiology and Evaluation, Vancouver Coastal Health Research Institute, Vancouver, BC, Canada.

The way we think about how health research, practice and policy inform and interact with each other dramatically shapes our efforts to improve health and health care. And the ways in which many of us involved in health system improvements think about these interactions have changed markedly over the last five years. Specifically, growing recognition of how systems dynamics and contextual factors affect implementation demands new models, more comprehensive comparative studies, and the development of new measures to evaluate key contextual factors.

This paper reports on a Canadian Institutes of Health Researchfunded systematic review of conceptual models for knowledge translation that employed the metanarrative methodology devel- oped by Greenhalgh for a major UK National Health Services review of diffusion of innovations in health organizations. Key dimensions along which conceptual models vary will be used to frame a discussion of recent Canadian experiences with organizational models that foster and support research applications to policy and practice, and a series of preliminary international workshops focused on the need for prospective comparative case studies to derive generalizable lessons about how best to design systems support for knowledge to action.

The research questions were:

1. What are the distinct research traditions that guide conceptual models for KTA?

2. What is the nature and strength of the theoretical principles that underpin each model?

3. What is the nature and strength of the evidence related to the application of conceptual model(s) in real-world situations?

4. What are the important features of each model that distinguish it from other models?

5. How does each model address contextual factors known to influence KTA processes and outcomes?

6. How has each tradition developed over time as early models have been refined by the addition of new research?

7. What criteria might guide selection and refinement of the best model in a specific decision context?

The presentation will conclude with a summary of key issues and priorities for further development of conceptual models and evaluation tools for international studies examining knowledge to action systems in healthcare.

CORRESPONDING AUTHOR: Allan Best, PhD, School of Population and Public Health, University of British Columbia, West Vancouver, BC, V7W 2T4; allan.best@in-source.ca

SS16c

STANDARDIZATION BY FUNCTION, NOT CONTENT, AND REGIONAL NETWORKS FOR GLOBAL PROMOTION AND DISSEMINATION OF PEER SUPPORT AS STANDARD COMPONENT OF HEALTH CARE

Edwin B. Fisher, $\mathrm{PhD}^{1,2}$ and Renée I. Boothroyd, $\mathrm{PhD}$, MA, $\mathrm{MPH}^{1,2}$

${ }^{1}$ Health Behavior \& Health Education, University of North Carolina at Chapel Hill, Chapel Hill, NC and ${ }^{2}$ Peers for Progress, American Academy of Family Physicians Foundation, Leawood, $\mathrm{KS}$.

Peer support is at once a) a very powerful influence on behavior and well being in general as well as in health, b) at least as old as primates, c) widely provided around the world through formal programs and informal channels, but d) not well articulated in the health literature, e) without a well defined state of the art, and, therefore, f) not recognized as an important part of health care, and g) not systematically available to all who may benefit from it. Peers for Progress of the American Academy of Family Physicians Foundation is dedicated to accelerating the availability of best practices in peer support around the world. A major challenge is how to develop a coherent model of peer support that is applicable 
across widely differing cultural, organizational, and national settings. "Standardization by function, not content" guided development of a coherent yet broadly applicable and flexible model. Through a WHO consultation with experts from 20 different countries, literature review, and work with 14 grantees representing 9 countries, four key functions of peer support have been identified: assistance in daily management, social and emotional support, linkage to clinical care, and ongoing availability of support. These are implemented in varied ways, by telephone, individual meetings, group meetings, etc. but have been found applicable across varied settings. A second challenge is how to promote best practices in something that is as old as primates and widely implemented around the world. This has led to emphasis on promoting a coherent model of support around the key functions described above, but, then, working with existing programs to encourage information and model sharing, knowledge management, and advocacy through networks and collaborations focused at the national and regional level. Eventually, a network of networks would link peer support programs around the world, facilitating their quality improvement, helping them to achieve recognition of peer support as a key component of care through their own health systems, and, thereby, increasing their ability to reach the millions they might benefit.

CORRESPONDING AUTHOR: Edwin B. Fisher, PhD, Health Behavior \& Health Education, University of North Carolina at Chapel Hill, Chapel Hill, NC, 27599-7440; edfisher@unc.edu

\section{Thursday \\ August 5, 2010 \\ PAPER SESSIONS 10:30 AM-12:00 PM}

\section{OS07}

10:30 AM-10:48 AM

OS07-A

DETERMINANTS OF READINESS TO ADOPT REGULAR PHYSICAL ACTIVITY AMONG THAI EMPLOYERS IN WORKPLACES, THAILAND: A TRANSTHEORETICAL MODEL

Kultida Panidchakul, Philosophy of Doctoral in Nursing Science and Suneerat Boonsil, Master in Nursing

Nursing, Boromarajonani College of Nursing, Saraburi, Thailand.

In spite of promoting regular physical activity in healthy workplace, Thai employers who have been either risks of cardiovascular diseases or healthy are becoming less active physically. Identification of effective strategies to enhance regular physical activity is becoming a priority for health care providers. The Transtheoretical Model (TTM) has been successfully applied to promote physical activity in different countries, but has been limited testing in Thailand.

The purpose of this study was to test the TTM constructs of stages of change, processes of change (POC), self-efficacy (SE), and decisional balance (Pros and Cons), for their applicability as determinants of engaging in regular physical activity (PA) among Thai employers in workplaces. This study used a descriptive design with an accidental sampling of 668 Thai employers working in two workplaces in Saraburi provinces, Thailand. All participants completed questionnaires measuring demographic, regular moderate-intensity PA, and variables from the TTM constructs. Data were analyzed by using mean, standard deviation, analysis of variance, and discriminant analysis.

The results of this study indicated that the weekly moderateintensity PA, processes of change, self-efficacy, and pros consistently increased across 5 stages. Finally, the set of variables including TTM constructs and weekly moderate PA was able to correctly classify stage for $37.3 \%$ of subjects. The present study indicated that TTM may be useful to develop strategies to enhance regular PA among Thai Employers in workplaces.

CORRESPONDING AUTHOR: Kultida Panidchakul, Philosophy of Doctoral in Nursing Science, Boromarajonani College of Nursing, Saraburi, 18000; kpanid1@hotmail.com

\section{0:48 AM-11:06 AM}

OS07-B

STRESS BUFFERING EFFECTS OF CONSCIENTIOUSNESS ON DAILY HEALTH BEHAVIOURS IN HIGH AND LOW STRAIN WORK ENVIRONMENTS

Daryl O'Connor, PhD, ${ }^{1}$ Mark Conner, $\mathrm{PhD},{ }^{1}$ Fiona Jones, $\mathrm{PhD},{ }^{1}$ Brian McMillan, $\mathrm{PhD}^{1}$ and Eamonn Ferguson, $\mathrm{PhD}^{2}$

${ }^{1}$ University of Leeds, Leeds, United Kingdom and ${ }^{2}$ University of Nottingham, Nottingham, United Kingdom.

Background: There is a growing body of research that has demonstrated that conscientiousness is associated with longevity and health status. In parallel research, job strain (characterized by high job demands and low job control) has been found to be linked to increased cardiovascular disease risk. Previous work has focused on potential explanatory mechanisms such that conscientiousness has been found to impact on health behaviors. However, little research has explored the extent to which conscientiousness may moderate the job strain-health outcome relationship and has been overly reliant on "snapshot" retrospective measurements of key health behaviors. Therefore, using a multilevel daily diary design, this study investigated the impact of conscientiousness on health behaviors and daily stressors in individuals working in high compared to low strain jobs.

Methods: 420 employees (193 male, 227 female) completed daily diaries over 4-weeks. Day-to-day within-person effects of daily stressors (or hassles) on health behaviors were examined, together with the influence of conscientiousness and job strain.

Results: Using Hierarchical Multivariate Linear Modeling, the results showed in individuals working in high strain jobs, conscientiousness and its facets were associated with lower fat and sugar snack intake, and higher fruit and vegetable consumption. Moreover, the facets of conscientiousness were found to also significantly moderate the impact of daily stressors on a number of health behaviors. In contrast, few significant direct or moderated 
effects of conscientiousness were identified in individuals working in low strain jobs.

Conclusions: Our results show that: i) conscientiousness and its facets may directly buffer the effects of high strain on daily health behaviors and moderate the impact of daily stressors on these behaviors and, ii) the influence of conscientiousness

CORRESPONDING AUTHOR: Daryl O'Connor, PhD, University of Leeds, Leeds, LS2 9JT; pscdbo@leeds.ac.uk

\section{1:06 AM-11:24 AM}

OS07-E

NON-RESPONSE ANALYSIS OF A NATIONWIDE MAIL SURVEY FROM FINLAND

Sakari B. Suominen, MD, PhD,${ }^{1}$ Lauri Sillanmaki, Ms, ${ }^{1}$ Jussi Vahtera, Prof, ${ }^{1}$ Karoliina Koskenvuo, $\mathrm{PhD}^{2}$ and Markku Koskenvuo, Prof $^{2}$

${ }^{1}$ Departement of Public Health, University of Turku, Turku, Finland and ${ }^{2}$ Public Health, University of Helsinki, Helsinki, Finland.

Suominen Sakari, Koskenvuo Karoliina, Sillanmäki Lauri, Vahtera Jussi, Koskenvuo Markku

Non-response analysis of a nationwide mail survey from Finland Response rates in health oriented survey research are declining. The bias caused by this is unclear.

Aim: The aim of the study was to compare all-cause and disease mortality and mortality related to external causes among participants and non-participants of a large mail survey in Finland.

Study design: The study is based on the complete sample ( $N=67$ 644) of the Health and Social Support (HeSSup) prospective mail survey initiated in 1998 with a panel follow up survey in 2003. Individuals and Methods: Of the original sample 67588 individuals could be re-identified in registries comprising 33446 women and 34142 men The original sample represented the concurrent age cohorts of 20-24, 30-34, 40-44 and 50-54 years of age in Finland. The response rate of the initial survey was $40 \%,(\mathrm{~N}=25$ 901). The participants were asked a signed consent for linkage of the survey data to national health and pension registers which was given by $92 \%$. In 2007 , the survey material was completed by Statistics Finland with data on mortality and by the Finnish Centre for Pensions with data on cumulative incidence of disability pensions and rehabilitation allowance from 1998 to 2006. The same data was anonymously delivered for non-respondents. The statistical analysis was performed with Cox proportional Hazard models.

Results: Total mortality was significantly higher for nonrespondent compared to respondent women in age group 5054 years and for non-respondent men in age groups 40-44 and 50-54 years. Violent mortality was higher for non-respondent men in age brackets 30-34 and 40-44 years but not for any age group of women. Disease mortality was higher for non-respondent women in age group 50-54 years but not for any age group of men. A significantly higher incidence of disability pensions among non- respondents was observed, but this was almost totally due to pensions related to mental disorders, especially among women.

Conclusion: Non-response might bias health survey Results: However, the greatest implications probably influence how results can be generalized and not necessary results about particular risk and protective factors.

CORRESPONDING AUTHOR: Sakari B. Suominen, MD, PhD, Departement of Public Health, University of Turku, Turku, 20014; sakari.suominen@utu.fi

11:24 AM-11:42 AM

OS07-C

CAN THE COGNITIVE ACTIVATION THEORY OF STRESS EXPLAIN THE SOCIAL GRADIENT IN HEALTH?

Magnus Odeen, PsyD, ${ }^{1,3}$ Hugo Westerlund, PhD, ${ }^{2}$ Töres Theorell, MedDr, ${ }^{2}$ Hege R. Eriksen, $\mathrm{PhD}^{1,4}$ and Holger Ursin, MedDr ${ }^{1}$

${ }^{1}$ Uni Health, Bergen, Norway; ${ }^{2}$ Stress Research Institute, University of Stockholm, Stockholm, Sweden; ${ }^{3}$ Department of Physical Medicine and Rehabilitation, Kysthospitalet, Vestfold Hospital Trust, Stavern, Norway and ${ }^{4}$ Research Centre for Health Promotion, Faculty of Psychology, University of Bergen, Bergen, Norway.

Background: Socioeconomic differences in health are well established, and stable or increasing in Europe. Psychobiological factors may be mediators of these differences. The differences appear to occur as a gradient, rather than in distinct classes. Explanations offered for these differences may be classified into two possibly interacting categories: structural factors and individual factors. In this study we examine how individually acquired expectancies of being able or unable to handle the stressors and challenges of everyday life relate to individual health and to the socioeconomic gradient. According to the Cognitive Activation Theory of Stress (CATS) learned response outcome expectancies affect health through health behaviours and stress responses.

Methods: A cross-sectional study of response outcome expectancies, socioeconomic status and health in the 2008 follow-up of a representative sample of the Swedish working population in 2003$2005(\mathrm{~N}=11441)$.

Findings: Response expectancy variables (coping, helplessness, and hopelessness) showed clear and monotonous gradients over a Socioeconomic Status (SES) ladder. Multiple regression analysis revealed that coping was related to subjective SES, but could not be explained by age, gender, or objective SES. After controlling for age and gender, response outcome expectancies explained more variance $(\mathrm{r} 2=0.162)$ in self-rated health than subjective and objective socioeconomic status $(\mathrm{r} 2=0.078)$.

Interpretation: The results indicate that there are systematic differences in coping across the socioeconomic ladder. These differences are independent of objective social status and are associated with health, suggesting that psychological and learning factors contribute to the socioeconomic gradient in health.

CORRESPONDING AUTHOR: Magnus Odeen, PsyD, Uni Health, Bergen, 5015; magnus.odeen@uni.no 


\section{1:42 AM-12:00 PM}

OS07-D

ADAPTED HEALTH BELIEF MODEL INSTRUMENTS

FOR USE IN COMMUNITY-BASED COLORECTAL CANCER CONTROL RESEARCH

Cheryl L. Holt, $\mathrm{PhD},{ }^{1}$ Isabel Scarinci, $\mathrm{PhD}, \mathrm{MPH},{ }^{2}$ Michelle Martin, $\mathrm{PhD},{ }^{2}$ Chastity McDavid, $\mathrm{PhD},{ }^{2}$ Mark Litaker, $\mathrm{PhD},{ }^{3}$ Mohamad Eloubeidi, MD, ${ }^{4}$ Martha Crowther, $\mathrm{PhD},{ }^{5}$ John Bolland, $\mathrm{PhD}^{6}$ and Crystal Lee, $\mathrm{MS}^{2}$

${ }^{1}$ Public and Community Health, University of Maryland, College Park, College Park, MD; ${ }^{2}$ Division of Preventive Medicine, University of Alabama at Birmingham, Birmingham, AL; ${ }^{3}$ Diagnostic Sciences, University of Alabama at Birmingham, Birmingham, AL; ${ }^{4}$ Gastroenterology and Hepatology, University of Alabama at Birmingham, Birmingham, AL; ${ }^{5}$ Psychology, University of Alabama, Tuscaloosa, AL and ${ }^{6}$ College of Human Environmental Sciences, University of Alabama, Tuscaloosa, AL.

Colorectal cancer is the third leading cancer in incidence and mortality among US adults, and these rates are higher in the African American community than among other subpopulations. Theory-based health communication interventions aim to increase awareness and screening for this disease. The Health Belief Model is one of the most used models on which behavior change interventions and their evaluations are based. Previous work has been conducted to develop quality assessments of the Health Belief Model constructs in both breast and more recently in colorectal cancer contexts. However, instrument development initiatives have not focused specifically on disparities-affected populations such as African Americans. We present adapted instruments to assess Health Belief Model constructs, that were adapted/enriched based on qualitative research (e.g., focus groups) among African Americans. Two brief scales assess barriers to colonoscopy and fecal occult blood test, specific to the healthcare system. Internal consistencies were modest $(\alpha=.33 ; \alpha=.43$, respectively) and may indicate multidimensionality. A third scale assesses additional areas of colorectal cancer knowledge $(\alpha=78)$, based on the qualitative research findings. Factor structures and other psychometric properties are reported. Scores on the instruments were associated with screening behaviors. This work is consistent with the recent special issue of Health Education and Behavior, on "Behavioral Constructs and Culture for Cancer Screening", in which it is suggested that traditional behavior change theories developed for mainstream US culture may need to be adapted when working with disparities-affected populations such as racial/ethnic minorities.

CORRESPONDING AUTHOR: Cheryl L. Holt, PhD, Public and Community Health, University of Maryland, College Park, College Park, MD, 20742; cholt14@umd.edu
OS08

10:30 AM-10:48 AM

OS08-A

PROGNOSTIC FACTORS FOR SHIFTING BETWEEN RTW

AND MULTIPLE BENEFITS DURING A 5-YEAR

FOLLOW-UP AFTER OCCUPATIONAL REHABILITATION

Irene Oyeflaten, Candsan, ${ }^{1}$ Stein Atle Lie, $\mathrm{PhD},{ }^{2}$ Camilla Ihlebaek, $\mathrm{PhD}^{2}$ and Hege Randi Eriksen, $\mathrm{PhD}^{2}$

${ }^{1}$ The National Centre for Occupational rehabilitation, Rauland, Norway and ${ }^{2}$ Uni Health, Bergen, Norway.

Background: Longitudinal studies are required to understand more about the complex RTW process after rehabilitation and to explore if there are different sick-leave patterns between different sociodemographic subgroups. The aim of this study was to examine if duration of sick-leave before rehabilitation and socio-demographic factors as gender, age, medical diagnosis, and occupation predict different sick-leave patterns or RTW outcomes in a 5-year follow-up period after a 4-weeks inpatient occupational rehabilitation program. Methods: The sample consisted of 584 patients $(66 \%$ females, mean age 44 years $[\mathrm{sd}=9.3]$, long-term sick-listed; mean duration 9.3 months $[\mathrm{sd}=3.4]$ for psychological $(47 \%)$, musculoskeletal (46\%) and unspecific/other diagnosis (7\%). Register data from The National Insurance Administration in Norway was analysed in an 8 state-model: working, graded sick-listed, sick-listed, medical rehabilitation, vocational rehabilitation, part-time disability pension, time-limited disability pension and permanent disability pension. Extended proportional hazards regression models, were applied to analyze socio-demographic prognostic factors for shifting between any of the 8 states during the 5 years follow up. Results: Age; RR=3.30 [95 \% CI: 2.29, 4.73], female gender; $\mathrm{RR}=2.13[1.09,4.16]$ and having an unspecific/other diagnosis; $\mathrm{RR}=3.39[1.70,6.74]$ gave highest risk for shifting to full-time disability pension. Sick leave length before rehabilitation gave an increased risk for going from sick-listed to any benefit $\mathrm{RR}=1.05$ $[1.03,1.07]$, for going back to work $R R=1.02[1.01,1.04]$ and for having new periods of sick leave $\mathrm{RR}=1.04$ [1.01, 1.07]. Bluecollar worker had the highest risk for shifting from the state working.

Conclusions: Our findings indicate different RTW outcomes for different socio-demographic subgroups. Rehabilitation programs, the work place, and the system should increase focus on women, individuals with higher age, unspecific diagnosis and blue-collar workers in the RTW process after long-term sick leave.

CORRESPONDING AUTHOR: Irene Oyeflaten, Candsan, The National Centre for Occupational rehabilitation, Rauland, 3864; irene.oyeflaten@air.no 


\section{CITATION ABSTRACT \\ 10:48 AM-11:06 AM \\ OS08-B \\ WORKPLACE BULLYING AND MENTAL PROBLEMS}

Eero Lahelma, PhD, Tea Lallukka, PhD, Mikko Laaksonen, PhD, Ossi Rahkonen, PhD and

Peppiina Saastamoinen, MSc

Department of Public Health, University of Helsinki, Helsinki, Finland.

Background

Bullying is a common problem at workplaces with potentially long-lasting adverse consequences for the mental health of the bullied victims. We studied whether workplace bullying is

prospectively associated with mental problems as indicated by antidepressant use among middleaged women and men.

Methods

The participants were municipal employees aged 40-60 from the Finnish Helsinki Health Study cohort baseline survey in 2000-02 ( $\mathrm{n}=8960$, response rate 67\%). Workplace bullying was divided into those who reported being currently bullied and those who reported having been previously bullied. Data on antidepressant prescriptions from the Finnish Social Security Institute registers were available before the baseline survey and were followed up until the end of 2007. The prescription data were individually linked to the baseline survey data for those consenting for such linkage (78\%). Hazard ratios (HR) adjusted for age were calculated using Poisson regression analysis, excluding those with antidepressant use 1.5 years prior to the baseline survey.

Results

At baseline 5\% of both women and men reported being currently bullied, and 19\% of women and 13\% of men reported having previously been bullied. Compared to the never bullied (HR 1.00) the currently bullied were more likely use antidepressants during the follow up among women (HR 1.55, 95\% CI 1.09-2.22) as well as men (HR 2.57, 95\% CI 1.46-4.52). The previously bullied were also more likely to use antidepressants but somewhat less so among men.

Conclusions

Victims of workplace bullying are disproportionately prescribed antidepressants, suggesting that they run the risk of developing mental problems as a consequence of bullying. Workplace bullying is an occupational and public health issue of concern which should be tackled in order to safeguard employee mental health and well-being.

CORRESPONDING AUTHOR: Eero Lahelma, PhD, Department of Public Health, University of Helsinki, Helsinki, 00014 Univ Helsinki; eero.lahelma@helsinki.fi

\section{1:06 AM-11:24 AM}

OS08-C

\section{RELATIONSHIP BETWEEN SOCIAL STATUS}

INCONSISTENCY AND SELF-RATED HEALTH: EVIDENCE OF AN INDIRECT PATH VIA OCCUPATIONAL REWARDS Stefanie Braig, MA and Richard Peter, Prof Dr Institute of Epidemiology, University Ulm, Ulm, Germany.

Background: Evidence on the association between social status inconsistency (SI) and self-reported health is limited and mixed.
Moreover, only little research has been done to examine the pathway through which SI affects health. Therefore we analysed the mediating effect of effort-reward imbalance at work (ERI) on this association. Methods: Analyses were based on a random sample of 1111 male employees (25-64 years) from the south-west of Germany. Data were collected by a telephone interview in $2008 / 09$. Information on socio-demographic variables, social status indicators (education, occupational grade and income), occupational strain (ERI), and physical as well as mental health (SF-12) were given. Results: SI was significantly associated to low mental health, both directly and, consistent to our hypothesis, indirectly via low rewards. As SI was also related to low effort, no association was found for the quotient of both scales (ERI) and mental health. The effect of SI on physical health was entirely mediated by low rewards without any direct effect. The inclusion 
of further risk factors (age, BMI) did not attenuate these results. Conclusions: SI is significantly associated to ill health. Moreover, results indicate that at least a part of this relationship is explained by low rewards at work, findings with implications for further research in job strain and health inequality.

CORRESPONDING AUTHOR: Stefanie Braig, MA, Institute of Epidemiology, Ulm University, Ulm, 89081; stefanie.braig@uni-ulm-de

\section{1:24 AM-11:42 AM}

OS08-D

\section{RETURN TO WORK IN PATIENTS WITH ACUTE HAND} INJURIES

Lonneke Opsteegh, MSc, ${ }^{1,2}$ Heleen A. Reinders-Messelink, $\mathrm{PhD},{ }^{3}$ Johan W. Groothoff, $\mathrm{PhD},{ }^{2}$ Klaas Postema, $\mathrm{PhD},{ }^{1,2}$ Pieter U. Dijkstra, $\mathrm{PhD}^{1,4}$ and Corry K. van der Sluis, $\mathrm{PhD}^{1,2}$

${ }^{1}$ Center for Rehabilitation, University Medical Center Groningen, Groningen, Netherlands; ${ }^{2}$ department of Health Sciences, University Medical Center Groningen, Groningen, Netherlands; ${ }^{3}$ Centre for Rehabilitation, Revalidatie Friesland, Beetsterzwaag, Netherlands and ${ }^{4}$ Department of Oral and Maxillofacial Surgery, University Medical Center Groningen, Groningen, Netherlands.

Background: Return to work (RTW) in acutely hand-injured patients is not only influenced by biomedical determinants, but work-related and psychosocial determinants may influence RTW as well. Aim of this study was to investigate which factors determine RTW.

Methods: Patients with acute hand injuries $(n=69)$ answered several questionnaires on biomedical, work-related and psychosocial factors. All patients were between 18 and 65 years, employed, had sustained an acute hand injury and were treated in one of the two participating rehabilitation centers.

Results: Primary outcome measure was RTW, dichotomized into early ( $\leq 10$ weeks) and late RTW ( $>10$ weeks). The majority of the patients $(55 \%)$ returned to work in the late stage. Six percent of the patients did not RTW within one year, and 4\% failed to RTW. Accident location (Chi2:10.101; $\mathrm{p}=.001)$, job independence $(\mathrm{U}=$ $372.000 ; \mathrm{p}=.021)$ and symptoms of Post-Traumatic Stress Disorder (S-PTSD) $(U=257.000 ; p=.001)$ were univariately associated with RTW, and were entered in the logistic regression analysis. Both S-PTSD (having more symptoms) and accident location (accident sustained on the job) were found to statistically predict late RTW in hand-injured patients.

Exploration of S-PTSD: As S-PTSD are predictors of RTW in patients with acute hand-injuries, it is relevant to investigate which patients develop S-PTSD, and which patients do not. Therefore, we investigated which factors predict S-PTSD in this population. Pain $(\mathrm{r}=.530 ; \mathrm{p}<.001)$, satisfaction with hand function $(\mathrm{r}=.-.451 ; \mathrm{p}<.001)$, aesthetics $(\mathrm{r}=-.320 ; \mathrm{p}=.009)$, palliative coping style $(\mathrm{r}=.281 ; \mathrm{p}=.022)$ and avoidant coping style $(\mathrm{r}=.283 ; \mathrm{p}=.022)$ were univariately associated to S-PTSD. By performing a linear regression analysis, pain and aesthetics of the hand were found to statistically predict S-PTSD (adjusted R2: $33.9 \%$ ).

Conclusion: When patients sustained their hand injury on the job, attention should be paid to RTW during rehabilitation. Furthermore, patients should regularly be checked for S-PTSD, especially if the aesthetics of the hand are severely disturbed, or when patients experience pain.

CORRESPONDING AUTHOR: Lonneke Opsteegh, MSc, Center for Rehabilitation, UMCG, Groningen, 9700 RB; 1.opsteegh@, cvr.umcg.nl

\section{1:42 AM-12:00 PM \\ OS08-E}

MANAGING LOW BACK PAIN IN THE WORK PLACE PROBLEMS AND STRATEGIES

Torill H. Tveito, $\mathrm{PhD},{ }^{1,2}$ William Shaw, $\mathrm{PhD},{ }^{2}$ Yueng-hsiang Huang, $\mathrm{PhD}^{2}$ and Gregory Wagner, $\mathrm{MD}^{1}$

${ }^{1}$ Environmental Health, Harvard School of Public Health, Boston, MA and ${ }^{2}$ Liberty Mutual Research Institute for Safety, Boston, MA.

Most working adults with low back pain (LBP) continue to work despite pain, but few studies have assessed self-management strategies in this at-work population. The purpose of this study was to identify workplace challenges and self-management strategies reported by workers remaining at work despite recurrent or persistent LBP, to be used as a framework for the development of a workplace group intervention to prevent back disability. Workers with LBP $(n=38)$ participated in 5 focus groups, and audio recordings of sessions were analyzed to assemble lists of common problems and coping strategies. A separate analysis provided a general categorization of major themes. Workplace pain problems fell within 4 domains: activity interference; negative self-perceptions; interpersonal challenges; and inflexibility of work. Self-management strategies consisted of: modifying work activities and routines; reducing pain symptoms; using cognitive strategies; and communicating pain effectively. Theme extraction identified 6 overarching themes: knowing your work setting; talking about pain; being prepared for a bad day; thoughts and emotions; keeping moving; and finding leeway. To retain workers with LBP, this qualitative investigation suggests future intervention efforts should focus on worker communication and cognitions related to pain, pacing of work, and employer efforts to provide leeway for altered job routines.

CORRESPONDING AUTHOR: Torill H. Tveito, PhD, Environmental Health, Harvard School of Public Health, Boston, MA, 02115; torill.tveito@uni.no 
OS09

10:30 AM-10:48 AM

OS09-A

PREDICTING UNCERTAINTY IN DECISIONS TO UNDERGO

PRENATAL GENETIC TESTING

Cecile Muller, PhD Candidate and Linda Cameron, Professor

University of Auckland, Auckland, New Zealand.

Deciding whether to undergo prenatal testing, to find out about the health of one's unborn child, can create a state of uncertainty (Decisional Conflict).

365 men and women of child-bearing age (18-46 years; Mean Age $=29.39$ years) recruited from community groups completed an online survey. All read a scenario asking them to imagine they/ their partners were pregnant, and describing one of four genetic conditions varying in age of onset (early vs. late) and in severity (fatal vs. nonfatal). Participants rated items (-3: strongly disagree to +3 : strongly agree) assessing Testing Intention, Decisional Conflict, Test Perceived Efficiency, Benefits from Finding out Results, Disease Coherence (understanding the conditions genetic nature) and Worry (about having a disabled child). Participants were later grouped into the 'expectant' (29\%) and 'non-expectant' groups. A t-test showed that the non-expectant group reported greater Testing Intentions $(\mathrm{M}=0.18, \mathrm{SD}=1.49)$ than the expectant group $(\mathrm{M}=-0.22, \mathrm{SD}=1.41, \mathrm{p}<.05)$. However, Decisional Conflict did not differ significantly between these groups (over $52 \%$ in each group reported some Decisional Conflict). Stepwise regression showed that, in the expectant group, Decisional Conflict was predicted by greater Disease Coherence $(\mathrm{r}=.35)$ and increased Worry $(\mathrm{r}=.28)$. In the non-expectant group, however, Decisional Conflict was associated with low Test Efficiency $(r=-23)$ and greater Benefits from Positive Results $(\mathrm{r}=.22)$. These results did not depend on the conditions' characteristics.

While the prenatal testing decision was somewhat difficult for all participants, factors underlying such uncertainty varied with pregnancy status. Coherence and affect predicted uncertainty for expectant individuals, whereas cognitions about the inefficacy and the benefits of the test did so for non-expectant individuals. During pregnancy, information promoting understanding may also increase Decisional Conflict. Resources may be needed to manage these concerns.

CORRESPONDING AUTHOR: Cecile Muller, PhD Candidate, The University of Auckland, Auckland, Australia; cecile_muller@, hotmail.com
10:48 AM-11:06 AM

OS09-B

UK PARENTAL VIEWS ON THE PROVISION OF INFORMED CONSENT FOR EXPANDED NEWBORN SCREENING RESEARCH

Louise Moody, PhD, BSc, Louise Wallace, PhD, Elizabeth Pottinger, MSc Health Psychology and Kubra Choudhry, MSc Health Psychology

ARC-HLI, HLS, Coventry University, Coventry, United Kingdom.

The development of newborn screening is benefitted by technological advances enabling blood spot testing to identify a wider variety of conditions (Stewart 2006). Introduction of these advances through programmes of research delivered in parallel to routine screening requires consideration of the ethical issues involved and appropriate mechanisms for gaining parental consent (Pollitt et al. 1997, Chadwick et al. 1998).

Ponder et al. (2008) argue that ethical review needs to be more flexible in its attitude to the provision of information and consent processes for some types of research. Exploration of parental views on consent practices is needed to inform the debate, and specifically consider whether more flexible research protocols are needed to fit with the public perception of research in this context. This study has been undertaken to explore the views of parents and future parents regarding information provision and consent processes for expanded newborn screening in the UK. Focus groups $(n=29)$ and a web-survey $(n=142)$ were employed to capture parental experiences of routine screening, and perceptions and attitudes to an expanded screening programme which would constitute research.

The results indicate that the provision of information and informed choice for routine screening is variable and generally unsatisfactory. Parents want guaranteed information provision with clear decision making powers and an awareness of the choice available to them. A paper-based information leaflet on expanded newborn screening information was reviewed by parents and found to be adequate for decision making $(90 \%)$. Recommendations are made for how this information can be further developed.

The difference between routine screening provision and expanded screening was not considered to be significant enough by participants to warrant formal written, informed consent. $71 \%$ of the sample deemed the informed choice model adopted within current screening provision to be sufficient, supporting use of existing procedures within a research remit.

CORRESPONDING AUTHOR: Louise Moody, PhD, BSc, ARCHLI, HLS, Coventry University, Coventry, CV1 5FB; L.moody@ coventry.ac.uk 


\section{CITATION ABSTRACT \\ 11:06 AM-11:24 AM \\ OS09-C \\ EVALUATION OF THE IMPACT OF A CULTURALLY SENSITIVE EDUCATIONAL VIDEO ON PROMOTING MAMMOGRAPHY USE AMONG CHINESE AMERICAN WOMEN}

Judy H. Wang, PhD, Marc D. Schwartz, PhD, Wenchi Liang, DDS, PhD and Jeanne S.

Mandelblatt, MD, MPH

Georgetown University, Washington, DC, DC.

Purpose: To examine the preliminary efficacy of a culturally sensitive educational video in promoting mammography use among Chinese-American women (age $\geq 40$ ) who had never or had not been screened in the past 12 months. Methods: 546 Chinese immigrant women were randomized to three groups: 1) viewed a culturally sensitive video; 2) viewed a generic video; and 3) read a fact sheet (control group). Videos, guided by the Health Belief Model, were presented in "soap opera" format, ending with physician recommendations. The cultural video particularly addressed Chinese culturally-based beliefs about cancer and was made in Chinese language. In contrast, the multiethnic generic video targeted common health beliefs about mammography, not specific to Chinese women, and was made in English with Chinese dubs. Our outcome was self-reported mammography use six months post-intervention. Changes in key screening barriers (i.e., knowledge, cultural views, and health beliefs) pre- and post-intervention were evaluated using T-tests and linear regression models. Participants were successfully randomized. Thus, chi-square tests were used to examine group differences in screening outcomes. Results: The cultural video led to a significant 12 percentage point increase in mammography use relative to the control arm $(41 \%$ vs. $29 \%, \mathrm{p}=.02$; baseline screening rate $=0)$. The increased screening rate of the generic video group (38\%) did not significantly differ from that of the control group. The cultural video also significantly increased mammography use among low-acculturated and never screened women relative to the control arm (39\% vs. $26 \%$, $\mathrm{p}=.03$ and $29 \%$ vs. $15 \% \mathrm{p}=.056$, respectively); the generic video did not. Both videos had a greater impact on improving women's knowledge, cultural, and attitudinal barriers to mammography $(\mathrm{p}<.0001)$ than the control arm. Conclusions: A culturally sensitive video program is relatively efficacious in increasing mammography use over a standard intervention program for Chinese women, suggesting that cultural tailoring of intervention programs is needed to promote low screening rates of this growing immigrant population.

CORRESPONDING AUTHOR: Judy H. Wang, PhD, Georgetown University, Washington, DC, DC, 20007; jw235@georgetown.edu

\section{1:24 AM-11:42 AM \\ OS09-D \\ MIXED-METHODS STUDY EXPLORING BARRIERS TO COLORECTAL CANCER SCREENING AMONG OBESE WOMEN}

Lucia A. Leone, BA and Marci K. Campbell, PhD, MPH, RD

Nutrition, University if North Carolina at Chapel Hill, Chapel Hill, NC.

Compared to non-obese women, obese women are at higher risk for colorectal cancer (CRC), but also have lower rates of CRC screening, particularly colonoscopy. We used mixed methods to better understand these disparities. We conducted seven focus groups on CRC prevention with obese (BMI $\geq 30$ ) white and African American women (age $\geq 50$ ) who were not currently adherent to colorectal cancer (CRC) screening guidelines $(\mathrm{N}=31)$. Discussions and coding were guided by the Health Belief Model. Focus groups were followed by an online survey with
109 obese and 89 non-obese women. We used Chi-square to compare weight groups on screening barriers. Focus group revealed that women did not believe they were at risk for CRC and had poor knowledge of CRC prevention; they did not clearly understand the role of weight or screening in cancer prevention. Many were unaware of potential risk factors for CRC and were much more aware of potential screening barriers than benefits. Cost and time were the most frequently cited barriers to screening. Further investigation revealed that women's time and healthcare dollars were focused on more pressing co-morbidities. While most women relied on their physician to bring up important health issues, the internet was their most frequent source of prevention information. Survey data confirmed that obese women were more likely than non-obese women to agree they had too many other health costs $(p=0.049)$ or more important health concerns that took precedence over colonoscopy $(\mathrm{p}=0.045)$. There were no significant differences by weight group in agreement with the more general cost and time barriers to colonoscopy. Furthermore, a higher proportion $(\mathrm{p}=0.03)$ of obese women $(41.1 \%)$ agreed that screening tests were embarrassing compared to on-obese women (28.6\%). Obese women were more than twice as likely as non-obese women to agree that they would not need a 
screening if they did not have symptoms $(p=0.04)$. Treatment of obesity-related co-morbidities may take precedence over cancer prevention in primary care. Messages which focus on prevention and screening benefits may improve healthy behaviors in obese women.

CORRESPONDING AUTHOR: Lucia A. Leone, BA, Nutrition, University if North Carolina at Chapel Hill, Chapel Hill, NC, 27516; 1leone@email.unc.edu

\section{1:42 AM-12:00 PM}

OS09-E

FRAMING INFORMATION ABOUT COLORECTAL CANCER SCREENING AND FECAL OCCULT BLOOD TESTING IMPACT ON SCREENING ATTTUDES AND BEHAVIOR

Ingrid Flight, $\mathrm{MPH},{ }^{1}$ Carlene J. Wilson, $\mathrm{PhD},{ }^{2,3}$ Greg Evans, $\mathrm{PhD},{ }^{4}$ Julie Syrette, $\mathrm{BA}^{4}$ and Jane McGillivray, $\mathrm{DHSc}^{5}$

${ }^{1}$ CSIRO Preventative Health Flagship, Adelaide, SA, Australia; ${ }^{2}$ School of Medicine, Flinders University, Bedford Park, SA, Australia; ${ }^{3}$ Cancer Council South Australia, Eastwood, SA, Australia; ${ }^{4}$ CSIRO Food and Nutritional Sciences, Adelaide, SA, Australia and ${ }^{5}$ School of Psychology, Faculty of Health, Medicine, Nursing and Behavioural Sciences, Deakin University, Burwood, VIC, Australia.

This study investigated whether differential framing of CRC risk and Fecal Occult Blood Test (FOBT) information impacted upon cancer attitudes and screening behaviour.

$\mathrm{N}=660$ people $50-71$ years were assigned to one of 6 intervention groups. They completed a laboratory-based survey measuring cancer attitudes. After reading information about CRC risk statistics (positive or negative framing, two-level variable) and FOBT advantages, disadvantages, or both/balanced (three-level variable), attitudes were re-measured. Participants were given a card to request a free FOBT kit. Return of card within 7 days was measured as intention to screen, and return of completed kit within 35 days was measured as screening uptake. Results were analysed using Chi-square statistics and repeated measures ANOVA to examine the influence of information provision, in its different forms, on attitudes to CRC screening.

From pre-intervention to post-intervention, risk perception scores increased $(\mathrm{F}(1,448)=18.24 ; \mathrm{p}<0.001)$, and optimistic bias decreased $(\mathrm{F}(1,654)=14.50 ; \mathrm{p}<0.001)$. Powerful others locus of control increased $(\mathrm{F}(1,654)=11.22 ; \mathrm{p}<0.001)$, as did Chance locus of control $(\mathrm{F}(1,654)=29.25 ; \mathrm{p}<0.001)$. There were no treatment effects for any of these variables.

There was no significant difference for intention to screen between the $\mathrm{CRC}$ risk information frames or FOBT information conditions. For screening uptake, there was a significant difference between intervention groups $\left(\mathrm{X}^{2}(5)=13.167 ; \mathrm{p}<0.05\right)$. Data partitioning indicated that there was no difference between FOBT uptake for the CRC positive risk information group by FOBT information condition. There were differences between $\mathrm{CRC}$ negative risk information group conditions $\left(X^{2}(2)=11.316 ; p<0.01\right)$. Significantly more participants in the FOBT 'advantages' condition screened than those in the FOBT 'disadvantages' condition $\left(\mathrm{x}^{2}(1)=3.85 ; \mathrm{p}<0.05\right)$ or the 'balanced' condition $\left(\mathrm{X}^{2}(1)=11.001 ; \mathrm{p}<.0001\right)$.

Overall, results suggest that providing information about the risk of $\mathrm{CRC}$ and the nature of the screening procedure can assist people to change their attitudes, regardless of the way this information is framed. Although framing the message around the screening test in terms of the likelihood of not being affected by CRC combined with the advantages of using the FOBT appears beneficial in a sample of people prepared to come to a laboratory testing session, future research is required to test the generalisability of the result.

CORRESPONDING AUTHOR: Ingrid Flight, MPH, CSIRO Preventative Health Flagship, Adelaide BC, SA, SA 5000; ingrid.flight@csiro.au

OS10

\section{0:30 AM-10:48 AM}

OS10-A

MENSTRUAL CYCLE AND IRRITABLE BOWEL SYNDROME: DOES IT MAKE A DIFFERENCE?

Margaret Heitkemper, $\mathrm{PhD}$ and Monica Jarrett, $\mathrm{PhD}$

BNHS, University of Washington, Seattle, WA.

In the U.S. and other western industrial countries more women than men seek health care services for irritable bowel syndrome (IBS). In addition, the health care impact of IBS also appears to be greater in women as compared to men. Women with IBS commonly report other somatic and visceral symptoms. Muscle and joint pain and disturbed sleep patterns are among the most common and the most distressing. Gender differences exist in types of GI symptoms (women report greater problems with constipation while men more frequently report diarrhea) as well as other extra-intestinal symptoms. The role of menstrual cycle in IBS symptom reporting is based on observations that women with IBS frequently report cyclic changes in their symptom severity. Women with IBS often report other menstrual cycle linked conditions including migraine headache, dysmenorrhea and premenstrual dysphoria distress syndrome. Gender differences in IBS begin to emerge at puberty. In children, the rubric of recurrent abdominal pain (RAP) includes IBS, functional abdominal pain, and likely, dyspepsia. Prior to puberty there is an equal proportion of boys and girls with a diagnosis of RAP. The factors responsible for the divergence in prevalence after puberty are not clear. Studies suggest that both environmental (i.e., increased risk if a parent has IBS) and gender factors (i.e., changes in hormonal milieu) likely play a role. The incidence of IBS in both men and women appears to decrease in late middle age. The severity of GI symptoms, including abdominal pain, altered bowel habits, and bloating, varies across phases of the menstrual cycle as well as during the menopause transition in some women with or without IBS or other functional GI disorders. A limited number of studies indicate that premenses (days immediately preceding the onset of menses) and menses, compared with other cycle phases, are periods of increased GI symptom distress in women age 19-37. Women with IBS experience a greater impact of menstrual cycle on symptoms as compared to those without IBS. While not extensively studied, oral contraceptives appear to modestly reduce but not eliminate GI symptoms in those with IBS. This paper will present they hypothesis that declining or low ovarian hormone levels underlies the occurrence or exacerbations of abdominal pain/discomfort across the menstrual cycle and the perimenopause-early menopause transition in women with or without IBS. 
CORRESPONDING AUTHOR: Margaret Heitkemper, PhD, BNHS, University of Washington, Seattle, WA, 98195; heit@ u.washington.edu

\section{0:48 AM-11:06 AM}

OS10-B

ASSOCIATIONS OF THE PREMENSTRUAL SYNDROME (PMS) WITH DEPRESSIVE - AND SOMATOFORM SYMPTOMS

Corinne Spoerri, MS, ${ }^{1}$ Suzana Drobnjak, MS, ${ }^{1}$ Dominique Wueest, MS, ${ }^{1}$ Ulrike Ehlert, $\mathrm{PhD}^{1}$ and Beate Ditzen, $\mathrm{PhD}^{1,2}$

${ }^{1}$ Psychology, Clinical Psychology and Psychotherapy, Zurich, Switzerland and ${ }^{2}$ Dept. of Psychiatry and Behavioral Sciences, Emory University School of Medicine, Atlanta, GA, GA.

Objective: Premenstrual dysphoric disorder (PMDD) and the premenstrual syndrome (PMS) are psychiatric phenomena, which are associated with the menstrual cycle in women. Symptoms of PMS include mood changes, temper outbursts/anger, lack of concentration and abdominal pain. These symptoms also form the diagnostic criteria for psychiatric disorders, such as depression, pain disorder (e.g. chronic pelvic pain) or somatoform disorder, which are unrelated to the menstrual cycle. Our aim was to identify similarities and differences of PMS with these symptoms during different phases of the menstrual cycle.
Methods: A PMS questionnaire which was based on the DSM-IVTR criteria for PMDD, a screening for somatic symptoms (SOMS, Rief 2008), and the German version of the CES-D (Radloff, 1977) were presented online. In a prospective design, questionnaires were presented at baseline (t1) and at a second assessment (t2). T2 was individually scheduled during a different menstrual cycle phase 2 - 4 weeks later. A total of 301 women (age 20 - 40 yrs.) completed the questionnaires at $\mathrm{t} 1,101$ of them at follow up.

Results: PMS scores significantly correlate with somatoform symptoms $(\mathrm{r}=.307)$ and with depressive symptoms $(\mathrm{r}=.400)$. However, whereas PMS symptoms show significant peaks during the luteal phase of the menstrual cycle $(\mathrm{F}=5.67, \mathrm{p}=.001)$, somatoform and depressive symptoms do not.

Conclusion: Beside their overlap with somatoform and depressive symptoms, premenstrual symptoms show a unique fluctuation over the menstrual cycle which might hint to specific underlying psychobiological mechanisms in PMS and PMDD.

References:

Rief W. \& Hiller W. (2008). SOMS - Screening für Somatoforme Störungen - Manual. Bern: Huber.

Radloff, L.S. (1977). Applied Psychological Measurement, 3, 385-401.

CORRESPONDING AUTHOR: Corinne Spoerri, MS, Psychology, Clinical Psychology and Psychotherapy, Zurich, 8050; c.spoerri@ psychologie.uzh.ch

\section{CITATION ABSTRACT \\ 11:06 AM-11:24 AM \\ OS10-C}

PSYCHOTHERAPY VERSUS PSYCHOPHARMACOTHERAPY FOR PREMENSTRUAL SYNDROME: A META-ANALYSIS

Maria Kleinstäuber, Dipl-Psych, Michael Witthöft, $\mathrm{PhD}$ and Wolfgang Hiller, $\mathrm{PhD}$

Dep. of Clinical Psychology and Psychotherapy, Johannes Gutenberg-University, Mainz,

Germany.

Objective: Up to $18 \%$ of women in reproductive age experience premenstrual symptoms (PMS) of sufficient severity to result in disruption of normal functioning. Selective serotonin reuptake inhibitors (SSRIs) are recommended as first-line treatment. Due to the bio-psychosocial etiology of PMS psychotherapy should also be considered as a treatment option. Therefore, the current meta-analysis compares the efficacy of psychotherapy and psychopharmacotherapy for PMS. Methods: We administered a multiple-phase literature search. Studies (controlled trials with randomized or matched samples) examining the efficacy of psychotherapies and psychotropic drugs for adult patients with moderate to severe PMS published until November 2009 were collected. Data were extracted on the basis of a standardized coding scheme. Quality of the studies was assessed by the use of the Jadad-scale. The standardized weighted mean difference based on a random effects model was used as an effect size index. Outcome variables were affective, behavioral, and physical symptoms, as well as functional impairment.

Results: Twenty-three included studies obtained small and moderate effect sizes for psychotherapy (range: $d=0.24-d=0.70$ ) and for SSRIs (range: $d=0.26-d=0.59$ ) at posttreatment. Follow-up assessments were only performed in psychotherapy studies revealing stability of the effects (range: $d=0.46-d=0.74$ ). The risk of publication bias was reduced by the inclusion of unpublished trials. The year of completion of the study turned out as a significant moderator of the effect of SSRIs on physical symptoms $(\beta=-1.30 ; p<.01)$. Conclusions: Results reveal both psychotherapy and SSRIs as effective treatments of PMS. Due to side effects of psychotropic drugs, psychotherapeutic interventions should be paid more attention as effective treatment options for PMS. 
CORRESPONDING AUTHOR: Maria Kleinstäuber, Dipl-Psych, Dep. of Clinical Psychology and Psychotherapy, Johannes Gutenberg-University of Mainz, Mainz, 55122; kleinsta@ uni-mainz.de

\section{1:24 AM-11:42 AM}

OS10-D

PREDICTORS OF TREATMENT OUTCOME AFTER COGNITIVE BEHAVIOUR THERAPY AND ANTISPASMODIC TREATMENT FOR PATIENTS WITH IRRITABLE BOWEL SYNDROME IN PRIMARY CARE Silje E. Reme, PsyD, ${ }^{1,2}$ Tom Kennedy, MD, ${ }^{4}$ Roger Jones, PhD, ${ }^{4}$ Simon Darnley, $\mathrm{PsyD}^{4}$ and Trudie Chalder, $\mathrm{PhD}^{3}$

${ }^{1}$ Department of Education and Health Promotion, University of Bergen, Bergen, Norway; ${ }^{2}$ Uni Health, Uni Research, Bergen, Norway; ${ }^{3}$ Department of Psychological Medicine, King's College, London, United Kingdom and ${ }^{4}$ Department of General Practice, King's College, London, United Kingdom.

Objective: To examine predictors of treatment outcome in IBSpatients who participated in a randomized controlled trial in primary care, where 149 IBS-patients were randomised to mebeverine hydrochloride $(n=77)$ or mebeverine + cognitive behaviour therapy $(\mathrm{CBT})(\mathrm{n}=72)$. CBT offered additional benefit over mebeverine alone. Methods: Regression analyses were used to identify predictors of work and social adjustment (WASA) 12 months after treatment ended. The intervention groups were analysed separately in order to look at the separate effects in each group. Results: Lower levels of psychological distress (anxiety and depression) at baseline predicted a good outcome in the mebeverine group $(\beta=0.388$ (95\%CI: $0.065-0.936)$, $p$-value $=$ 0.025 ) but not in the mebeverine $+\mathrm{CBT}$ group. In the adjusted model for the mebeverine $+\mathrm{CBT}$ group less adaptive IBS related behavioural coping predicted a good outcome $(\beta=0.285(95 \% \mathrm{CI}$ : 0.002-0.210), $\mathrm{p}$-value $=0.045)$. Conclusion: Different factors are associated with outcome depending on the treatment received. At assessment clinicians should assess patients coping styles and may want to consider recommending CBT to those patients with IBS in primary care who are engaging in unhelpful coping behaviour.

CORRESPONDING AUTHOR: Silje E. Reme, PsyD, Department of Education and Health Promotion, University of Bergen, Bergen, 5015; silje.reme@uib.no

\section{1:42 AM-12:00 PM}

OS10-E

\section{DIAGNOSTIC CHALLENGES OF SUBJECTIVE AND COMPOSITE HEALTH COMPLAINTS IN GENERAL PRACTICE}

Silje Maeland, Msc, Erik Werner, PhD, Liv H. Magnussen, PhD, Holger Ursin, PhD and Hege R. Eriksen, PhD

Stress, Health and Rehabilitation, Uni Health, Bergen, Norway.

Background: In Norway, the majority of medically certified sickness absence is granted for musculoskeletal complaints and mental disorders. The challenge for the general practitioners (GP) is that the majority of these cases tend to be unspecific without any clear and significant objective findings. Traditional medical thinking requires a diagnosis, in order to find a specific and efficient therapy or to grant sickness certificate. The challenge is to find the proper diagnoses when the condition is unspecific, with many complaints, and a high level of comorbidity. The International Classification of Primary Care-second version (ICPC-2) is used in Norwegian primary health care.

Aims: Our aims were to investigate if a dramatised case story (presented exactly the same way to, Norwegian GPs), representing a patient with unspecific and/or comorbid complaints get the same diagnosis and decision on sick leave.

Method: Fifty-six GPs watched 10 case stories and diagnosed each case with ICPC-2 diagnosis and made decisions on whether to sick list or not.

Results: Between 15 and 23 ICPC-2 diagnoses were given to the case stories as main diagnosis. Psychological diagnoses were most frequent, followed by musculoskeletal. Three to five different ICPC-2 organ chapters were used for each case. Decisions on sick leave yes/no and different type of insurance benefits varied greatly within each case story.

Discussion: As expected, the Norwegian GPs did not agree on which diagnoses to use for these patients, and suggested a high number of diagnostic labels from the ICPC-2 system. This is, in our opinion, not an indication of lack of competence in the GPs but that the systems used today are unable to classify and describe these complaints. The high number of possible diagnoses offered by experienced GPs suggests that the conditions are composite, with a high level of comorbidity and the diagnostic system is unable to identify this. For this kind of patients there is no consensus on what kind of treatment to offer and we argue that the only available "treatment" today is sick leave. This increases the risk of long term sick leave, and permanent disability.

Conclusion: Case stories with unspecific and comorbid complaints are diagnosed with a large variety of ICPC-2 diagnosis in general practice and they are frequently put on sick leave.

CORRESPONDING AUTHOR: Silje Maeland, Msc, Stress, Health and Rehabilitation, Uni Health, Bergen, 5015; silje. maeland@uni.no

\section{OS11}

10:30 AM-10:48 AM

OS11-A

ADVERSE CHILDHOOD EXPERIENCES, HEALTH RISK BEHAVIOURS AND PSYCHOPATHOLOGY: COMPARATIVE STUDY BETWEEN INCARCERATED WOMEN AND MEN

Angela Maia, phd and Joana Alves, dr

Psychology, University of Minho, Braga, Portugal.

Context: History of childhood adversity, health risk behaviours and psychopathology are prevalent phenomenon in the detained population, but most studies were done with men. Recently WHO (2009) recognized that the study of health issues in female prisoners is a challenge that need to be urgently addressed. Knowing the differences between sexes in what concerns these 
variables, this study intended to characterize and compare adverse childhood experiences, health risk behaviour and psychopathological symptoms in incarcerated male and female.

Method: A group of 42 female and 65 male inmates of a correctional facility located in the north of Portugal filled a Social-demographic and Life History Questionnaire, Health risk Behaviour Questionnaire and Brief Symptom Inventory.

Results: The majority of the women and men describe several adverse experiences during childhood. From a total of ten possible types of adversity, the mean for women was $5.05(\mathrm{SD}=2.63)$ and $2.63(\mathrm{SD}=2.18)$ for men. There was a high frequency of health risk behaviour and psychopathological symptoms for both samples, women reporting significantly more psychopathology and sexual risk behaviours (no significant differences in other risk behaviours)

Conclusion: Detainees had a high frequency of adverse experiences during childhood, have high levels of psychopathology and several risk behaviours. The differences between women and men in what concerns adverse childhood experiences and psychopathological symptoms suggest more adverse childhood histories for incarcerated women. Those differences should be better explored in order to understand if a self-report effect or a fact of their lives. This data suggest a need to take into account the adverse histories because violent behaviour can function as a coping strategy to deal with negative affect and psychopathology related to prior victimization.

CORRESPONDING AUTHOR: Angela Maia, phd, Psychology, University of Minho, Braga, 4700 BRAGA; angelam@psi. uminho.pt

\section{0:48 AM-11:06 AM}

OS11-B

PREDICTORS AND CONSEQUENCES OF INTERPERSONAL VIOLENCE AMONG YOUNG COUPLES

Trace Kershaw, PhD and Anna Arnold, Mph

Yale University, New Haven, CT.

Background: Violence within relationships is associated with affect disorders and poor physical health. Few studies have looked at interpersonal violence among intact couples. This study focuses on predictors and consequences of violence within couples.

Methods: Preliminary analyses were conducted among 200 couples $(\mathrm{N}=400)$ from low income OB-GYN clinics in the U.S. Women and men were 18.7 and 21.3 years on average, and the average length of relationship was 2.3 years. $77 \%$ of participants were Black or Hispanic. Couples were assessed on victimization and perpetration of physical violence within their relationship and on mental health (depression, stress). All analyses controlled for confounders.

Results: $38 \%$ of couples reported physical violence in their relationship. Men were more likely to be violence victims $(32 \%$ vs. $11 \%, \mathrm{p}<.05)$ and report injury from violence $(6.5 \%$ vs. $1.5 \%$, $\mathrm{p}<.05)$ than women $(11 \%$ vs. $3 \%, \mathrm{p}<.05)$. Men and women shared predictors of being violence victims, including low relationship equity $(\mathrm{ORm}=.91, \mathrm{ORw}=.91, \mathrm{ps}<.05)$ and power
$(\mathrm{ORm}=.27, \mathrm{ORw}=.13, \mathrm{ps}<.05)$. Men with physical violence history $(\mathrm{OR}=3.28, \mathrm{p}<.05)$, concurrent partners $(\mathrm{OR}=3.06$, $\mathrm{p}<.05)$, and partners with hostility $(\mathrm{OR}=1.09, \mathrm{p}<.05)$ or conduct problems $(\mathrm{OR}=1.16, \mathrm{p}<.05)$ were more likely to be violence victims. Women with high attachment anxiety $(\mathrm{OR}=1.23, \mathrm{p}<.05)$ and traditional masculinity norms $(\mathrm{OR}=1.08, \mathrm{p}<.05)$ were more likely to be violence victims.

Among couples with violence, male victims were more depressed $(\mathrm{M}=11.8$ vs. $7.5, \mathrm{p}<.05)$ and stressed $(\mathrm{M}=18.1$ vs. 13.9$)$ than males without violence. Female violence perpetrators were also more depressed $(\mathrm{M}=13.2$ vs. $9.2, \mathrm{p}<.05)$ and stressed $(\mathrm{M}=18.1$ vs. $15.8, \mathrm{p}<.05)$ compared to female non-perpetrators. Among couples with violence, female victims were more depressed $(\mathrm{M}=$ 16.4 vs. $9.8, \mathrm{p}<.05)$ and stressed $(\mathrm{M}=21$ vs. 16) than females without violence.

Conclusion: Violence in young expectant couples was prevalent; however men were more likely to be victims. Mental health consequences were associated with violence victimization among all participants. Mitigating stressful circumstances may reduce violence within relationships.

CORRESPONDING AUTHOR: Trace Kershaw, PhD, Yale University, New Haven, CT, 06510; trace.kershaw@yale.edu

11:06 AM-11:24 AM

OS11-C

MENTAL AND PSYCHOSOMATIC HEALTH, UTILIZATION, AND TREATMENT RESPONSE AMONG IRAQI REFUGEES AS COMPARED TO NON-WAR EXPOSED ARAB IMMIGRANTS

Bengt B. Arnetz, MD, PhD, ${ }^{1}$ Matthew Ventimiglia, MA, ${ }^{1,2}$ Hyder Makki, MD, ${ }^{3}$ Rahim Mahmoud, $\mathrm{MD}^{4}$ and Hikmet Jamil, MD, $\mathrm{PhD}^{1}$

${ }^{1}$ Family Medicine, Division of Occupational and Environmental Medicine, Wayne State University, Detroit, MI; ${ }^{2}$ Department of Psychology, University of Detroit Mercy, Detroit, MI; ${ }^{3}$ Psychiatry, Private Psychiatric Clinic, Dearborn, MI and ${ }^{4}$ Internal Medicine, Internal Medicine--Outpatient Clinic, Dearborn, MI.

Objectives: Although refugees are at an increased risk to suffer from mental disorders, as compared to other immigrants, little is known about possible differences in treatment response and service utilization.

Methods: The current study includes data from three separate samples of Iraqi refugees and Arab immigrants. Prevalence of physician-diagnosed mental disorders along with treatment response and utilization data was collected from medical records or by surveys. Data was collected from 307 refugees and 326 immigrant controls.

Results: The prevalence of depression was significantly greater in refugees as compared to immigrants $(54 \%$ vs. $26 \%$; $<$ <.001). There were no differences between groups in diagnosed anxiety disorders. In addition, self reported complaints of depression (33\% vs. $3.1 \%$; $\mathrm{p}<.001)$, anxiety ( $20 \%$ vs. $4 \% ; \mathrm{p}<.001)$, and sleep difficulties $(15 \%$ vs. $2 \% ; \mathrm{p}<.001)$ were more prevalent among refugees than 
immigrants. In general, treatment response was poor for both groups; however, for longer term patients, treatment response was significantly better $(44 \%$ recovery vs. $15 \%$ recovery; $\mathrm{p}<.001)$ for immigrants than refugees. Typical common psychosomatic symptoms were more prevalent and associated with increased health care utilization in refugees as compared to immigrants.

Conclusions: Results suggest that treatment offered to refugees is far from effective. There is a need to review health systems factors in order to promote cost-effective and timely mental health services for refugees, not least with the current influx of Iraqi refugees commonly suffering from mental and adjustment disorders.

CORRESPONDING AUTHOR: Bengt B. Arnetz, MD, PhD, Family Medicine, Division of Occupational and Environmental Health, Wayne State University, Detroit, MI, 48201; barnetz@ med.wayne.edu

\section{CITATION ABSTRACT \\ 11:24 AM-11:42 AM \\ OS11-D}

HEALTH SYMPTOMS, PSYCHOPATHOLOGY, SOCIAL SUPPORT, QUALITY OF LIFE AND FAMILY FUNCTIONING IN PORTUGUESE WAR VETERANS

M. Graça Pereira, PhD, ${ }^{1}$ Cristiana Lopes, Master, ${ }^{3}$ José Machado, PhD, ${ }^{2}$ Marta Pereira, Master ${ }^{1}$ and Susana Pedras, Master ${ }^{1}$

${ }^{1}$ School of Psychology, University of Minho, Braga, Portugal; ${ }^{2}$ Institute of Social Sciences, University of Minho, Braga, Portugal and ${ }^{3}$ Psychiatry Unit, Braga Hospital, Braga, Portugal. Introduction

Around a million Portuguese soldiers participated in the Colonial War. From these, 140.000 veterans present diverse psychological problems (Albuquerque \& lopes, 1977). Trauma has an impact on the veteran and on the family as well. As a result, this study intends to analyse the relationships between physical and psychosocial variables that have an impact on quality of life and family functioning.

Sample

230 veterans participated in the study. Data was collected in a major psychiatry hospital in the North of Portugal. All veterans volunteered to participate in the study.

Instruments

- Clinical and Socio-Demographic Questionnaire

- Traumatic Events Assessment Scale (McIntyre \& Ventura, 1998)

- Family Adaptability and Cohesion Evaluation Scales (FACES III),(Olson, Portner \& Lavee, 1993); Portuguese Version (Curral, et al., 1999)

- Brief Symptom Inventory (BSI),(Derogatis, 1993); Portuguese Version (Canavarro, 1999)

- WHOQOL-Bref (OMS, 1998);Portuguese Version (Canavarro, Simões, Vaz Serra, Pereira, Rijo, et al., 2006)

- Health Symptoms Checklist (HSC),(Proctor, Heeren, White, Wolfe, Borgos, et al., 1998);Portuguese version (Pereira \& Pedras, 2008)

- Satisfaction with Social Support Scale (ESS),(Pais-Ribeiro, 1999)

All instruments were adapted to the Portuguese population.

Results

We found a positive relationship between PTSD and psychopatology, health symptom and negative with quality of life. War veterans presented a high percentage of extreme families particularly those with PTSD.

We also found differences between veterans with and without PTSD on social support, family functioning and quality of life. Predictors of quality of life and mediation analysis were also performed.

According to these preliminary results, psychological intervention, on this population, is necessary to help veterans and their families to better cope with war trauma. 
CORRESPONDING AUTHOR: M. Graça Pereira, PhD, University of Minho, Braga, 4710-057; gracep@psi.uminho.pt

\section{1:42 AM-12:00 PM}

OS11-E

DISCLOSURE AND PTSD SYMPTOM SEVERITY - EFFECTS OF TALKING TO DIFFERENT RELATIVES

Gregor Weissflog, Dipl-Psych and Elmar Braehler, Prof Dr Department of Mental Health, Medical Psychology and Medical Sociology, University of Leipzig, Leipzig, Germany.

Objective: By recent estimates, 200.000 persons were imprisoned for political reasons in the German Democratic Republic (GDR) between 1949 and 1989. Former political prisoners suffer from the experience of persecution also in a long term. Up to $50 \%$ of those persons develop a post-traumatic stress disorder. Verbal disclosure (the revelation of the traumatic events) to the social environment has been shown to have positive effects on PTSD symptom severity. Little is known about whether this effect is moderated by the receiver of the disclosure.

Method: A total of 157 former political prisoners ( $82 \%$ male) from the GDR (mean age: 65 years, from 39 to 86 years old) were assessed with a questionnaire. Sociodemografic data, intrafamilial communication about the traumatic imprisonment and symptoms of the posttraumatic stress disorder were assessed by self-reports. IES-R (with the subscales intrusion, avoidance and hyperarousal) was used for the assessment of PTSD symptoms.

Results: PTSD symptom severity depends on who is the receiver of disclosure in the family. Persons who report that the actively disclosed their traumatic experience to their partners have lower intrusion (score in the subscale: 18.2. vs. 21.8; $\mathrm{p}=.02$ ) and lower hyperarousal symptoms (score in the subscale: 20.4 vs. $24.5 ; p=.05$ ). The same applies to active disclosure to their children (score in the subscale intrusion: 17.8 . vs. $21.4 ; \mathrm{p}=.02$ and score in the subscale hyperarousal: 19.5 vs. $24.5 ; \mathrm{p}=.01$ ). Disclosure to parents and to grandchildren is not connected with lower PTSD symptoms.

Conclusion: The results provide evidence that the concrete receiver of the disclosure in the family should be taken more into consideration. Future research on effects of disclosure should observe that.

CORRESPONDING AUTHOR: Gregor Weissflog, Dipl-Psych, Department of Mental Health, University of Leipzig, Leipzig, 04157; gregor.weissflog@medizin.uni-leipzig.de

\section{OS12}

10:30 AM-10:48 AM

OS12-A

OPINIONS AND ATTITUDES OF MEDICAL STUDENTS TOWARDS THE 2009 FORTHCOMING MEXICAN SMOKE-FREE POLICY

Claudia Infante, DSc, Guadalupe Garcia de la Torre, MSc, Lizbeth Diaz, MD and Gabriela de la Guardia, MD

School of Medicine, National University of Mexico, Mexico City, Mexico.
A study about the opinions, behaviors and psychosocial attitudes of medical students towards the 2009 forthcoming Mexican smoke-free policy was undertaken in Mexico City. A 69 item questionnaire was answered by 1022 first and second year medical students of the National University of Mexico. Our objective was to identify the relationship between the future physicians' smoking habits and their opinions regarding the expected smoke-free areas compulsory measures. Results: The smoking prevalence was $12 \%$ ( 1 to 5 cigarettes per day) with no sex differences. Overall $80.5 \%$ reported exposure to secondhand smoke at the classroom or work place. Statistical differences were found between smokers $(97.9 \%)$ and non smokers $(92.8 \%)$ with respect to their knowledge of the negative effect that smoking has on quality of life $(p=0.02) .90 .8 \%$ of smokers and $96.9 \%$ of non smokers $(p=0.004)$ agreed that smoking and non smoking areas should be established. $27.3 \%(\mathrm{p}=0.000)$ of non smokers thought that cigarette smoking should not be prohibited in all school areas, compared to only $8 \%$ of smokers. Compared with smokers $(64.9 \%)$, non smokers $(87.3 \%)$ are more likely to accept that the School of Medicine should be declared a tobacco free School $(p=0.000)$. Future research in medical students after the full implementation of the new anti-tobacco legislation is needed to identify changes in smoking patterns and their relation to compliance with the new law.

CORRESPONDING AUTHOR: Claudia Infante, DSc, School of Medicine, National University of Mexico, Mexico City, 14000; claudiainfante@prodigy.net.mx

10:48 AM-11:06 AM

OS12-B

SMOKEFREE LEGISLATION IN ENGLAND: IMPACT ON QUIT ATTEMPTS AND IMPLICATIONS FOR SMOKING CESSATION SERVICES

Lucy E. Hackshaw, BSc, MSc, ${ }^{1}$ Linda Bauld, $\mathrm{PhD},{ }^{1}$ Andy McEwen, $\mathrm{PhD}^{2}$ and Robert West, $\mathrm{PhD}^{2}$

${ }^{1}$ Department of Social and Policy Sciences, University of Bath, Bath, United Kingdom and ${ }^{2}$ Department of Epidemiology \& Public Health, University College London, London, United Kingdom.

Introduction: On July 1st 2007 England implemented smokefree legislation (SF). The primary aim of SF is to protect workers and the public from exposure to second hand smoke. However, emerging evidence suggests that SF can also encourage cessation. This paper examines links between SF and quitting behaviour, including findings from studies conducted in the UK, the USA and Ireland. It outlines findings from new research which explores the impact of SF on the uptake of services to support smokers to quit, conducted in England in 2007 and 2008.

Methods: National household surveys were conducted in England between January 2007 and December 2008. The sample (10,560 adults) was weighted to match census data and included those who reported having smoked within the past year. Data was collected on quit attempts made in the past 12 months and future intentions to quit. 
Surveys of smoking cessation service managers were conducted in England between March - May 2007 (pre-SF; n=125) and between May - June 2008 (post-SF; n=86). Data explored preparation for SF, anticipated and actual impact of SF.

Interviews were conducted with smokers to explore the impact of SF on quitting behaviour.

Results: A greater percentage of smokers reported making a quit attempt in July and August $2007(8.6 \%, n=82)$ compared with July and August 2008 (5.7\%, n=48) (Fisher's Exact=0.022). In the five months following SF $19 \%(n=75)$ of smokers making a quit attempt reported that they had done so in response to SF. The mean percentage increase in smokers attending smoking cessation services in the run up to $\mathrm{SF}(16.2 \%, \mathrm{CI}=13.2-19.2)$ was lower than the mean anticipated demand prior to $\mathrm{SF}(42.5 \%, \mathrm{CI}=37.4-$ 47.8) (Chi-squared=73.35, p<.0001).

Smokers reported that SF encouraged them to reduce consumption, maintain abstinence and make a quit attempt.

Conclusions: SF in England was associated with a significant temporary increase in the percentage of smokers attempting to stop, this corresponded with an increased demand for smoking cessation services. Implications for jurisdictions planning to implement SF are discussed.

CORRESPONDING AUTHOR: Lucy E. Hackshaw, BSc, MSc, Social and Policy Sciences, University of Bath, Bath, BA2 7AY; L.E.Hackshaw@bath.ac.uk

11:06 AM-11:24 AM

OS12-C

A CROSS-NATIONAL COMPARISON OF SMOKING AMONG GIRLS AND YOUNG WOMEN IN THE ASEAN REGION

Kin Foong, $\mathrm{PhD}^{1}$ and Yen Lian Tan, $\mathrm{MA}^{2}$

${ }^{1}$ National Poison Centre, Universiti Sains Malaysia, Georgetown, Malaysia and ${ }^{2}$ Southeast Asia Tobacco Control Alliance, Bangkok, Thailand.

Objective: To compare smoking rates among girls and young women in seven ASEAN countries (Cambodia, Indonesia, Lao PDR, Malaysia, Philippines, Thailand and Vietnam) and sociocultural factors associated with it.

Methods: A regional study on smoking among females was conducted in 2007/08. A quasi-random sample of about 3,000 female respondents between the ages of 13 and 25 from secondary schools and colleges/universities from the capital city of each country together with one rural district was surveyed using a standardized self-administered structured questionnaire. Each country data set was processed and analyzed using SPSS version 12. Logistic regression analysis was used to determine sociocultural factors significantly associated with current smoking (in the last month).

Results: Ever and current smoking rates varied substantially between these countries, highest in the Philippines (38 vs. 20\%) followed by Indonesia ( $20 \%$ vs. $7 \%$ ), Malaysia (19\% vs. $7 \%$ ), Thailand (13\% vs. $7 \%$ ) and lowest in Lao PDR and Cambodia
( $2 \%$ vs. $1 \%$ ). Socio-cultural factors such as parental and peer tobacco use and exposure to direct and indirect pro-tobacco advertising are important predictors of smoking in all countries. For example, Malaysian female students are four times more likely to smoke if their mothers smoke. Having close friends who smoke is another significant predictor (range between four to over ten times more likely to smoke). Indonesian and Philippines female students exposed to tobacco advertising and promotion are twice as likely to smoke compared to females without such exposure. The study also found that females with pro-smoking attitudes, positive image of smoking and a perception that smoking is the norm among people their age are more likely to smoke. Tobacco control policies in these countries are found to influence female smoking rates.

Conclusion: To protect girls and women as well as boys and men, all ASEAN countries should implement the WHO Framework Convention on Tobacco Control, prioritizing raising of taxes on all tobacco products, introduction of comprehensive ban on tobacco advertising and promotion and prohibition of smoking in public places.

Learning Objectives:

At the end of this presentation participants will be able to:

i. Learn about female smoking behaviour in ASEAN countries

ii. Obtain an understanding of social and cultural factors that influence female smoking within ASEAN countries

CORRESPONDING AUTHOR: Kin Foong, PhD, National Poison Centre, Universiti Sains Malaysia, Penang, 11800; kfoong@, usm.my

11:24 AM-11:42 AM

OS12-D

TOBACCO SMOKING REDUCTION AMONG ADOLESCENTS IN NIGERIA

Seye O. Omiyefa, BSC

Programs, Youth Action On Tobacco Control and Health, Agege, Nigeria.

BACKGROUND: For over twenty years, the tobacco industry has made marketing and distribution of tobacco products to youths a core of its marketing strategy in Nigeria. From the internal documents of the tobacco industry which was forced into public domain in the United States of America, it was revealed that the tobacco industry had deliberately marketed to young and underage persons in Nigeria. It was also revealed that British American Tobacco (BAT) had conducted several surveys to determine the rate of youth smoking in Nigeria. In one of such survey minors were referred to as YAUS (Young and Underage Smokers).

METHODS: Experience Freshness was a recent promotion of the British America Tobacco in August 2008. This event was organized to promote the Pall Mall brand. It was organized in all the six geo political zones in the country and was heavy on young people. Invitation cards were secretly given out in secondary schools and colleges. It was a heavy all night smoking party. Research and experience has showed various trick and strategies of the tobacco industries. It is therefore appropriate to implement 
laws that will curb every secret parties and advert used to promote its new product "Pall Mall" in developing countries especially Nigeria. There is also the need to build a strong alliance/network in high school thereby making them Students Action against Tobacco Smoking (SAATS).

RESULTS: Increase awareness on the dangers of smoking and the deadly component in cigarettes thereby saying NO to the tobacco industries souvenirs, baseball cap, t-shirt and sponsorships. The method will also implement laws especially in Nigeria to curb both direct and indirect advertisement of the tobacco multinationals, thereby winning more adolescents for SAATS.

CONCLUSION: Since the tobacco multinationals are recruiting more teenagers and youths everyday, thereby killing millions to make billions, efforts should be made in monitoring the tobacco industries to reduce both direct and indirect advertisement by the tobacco companies and engage more teenagers and youths in tobacco control activities.

CORRESPONDING AUTHOR: Seye O. Omiyefa, BSC, Programs, Youth Action On Tobacco Control and Health, Agege, 23401; omiyefaseye@yahoo.com

\section{1:42 AM-12:00 PM}

OS12-E

PATTERN OF CIGARETTE SMOKING AMONG THAI

ADOLESCENTS : A LONGITUDINAL STUDY

FROM INTERNATIONAL TOBACCO CONTROL

POLICY - THAILAND

Tawima Sirirassamee, MD, ${ }^{1}$ Buppha Sirirassamee, $\mathrm{PhD},{ }^{2}$ Ron Borland, $\mathrm{PhD}^{3}$ and Geoffrey T. Fong, $\mathrm{PhD}^{4}$

${ }^{1}$ Srinakarinwirot university, Nakornnayok, Thailand; ${ }^{2}$ Institute for Population and Social Research, Bangkok, Thailand; ${ }^{3}$ The Cancer Council Victoria, Melbourne, VIC, Australia and ${ }^{4}$ University of Waterloo, Waterloo, ON, Canada.

Background: Adolescents are a major target group of tobacco companies. Tobacco companies spend substantial amounts of money to promote smoking in adolescents. In Thailand, adolescents are considered the primary target for smoking prevention by the government.

Objective: To determine the pattern of cigarette smoking among Thai adolescents over a period of three years.

Design/Methods: A longitudinal study was conducted among adolescents between the ages of 13-18 sampled from 5 regions of Thailand using stratified multistage sampling. Wave 1 survey involved 1,000 adolescents was conducted during January to March 2005, wave 2 survey involved 962 adolescents was conducted during July to September 2006 and wave 3 survey involved 1,096 adolescents was conducted during January to March 2008. Self administered questionnaires were employed for data collection. Data were analyzed and compared using descriptive statistics.

Results: Overall, smoking prevalence has increased from $11.3 \%$ in wave 1 to $13.7 \%$ in wave 2 and $18.3 \%$ in wave 3 . Consistent with other Asian countries, smoking prevalence in young males were more than 10 times higher than young females. Among youth smokers, more than $70 \%$ smoked factory-made cigarette. The total amount of tobacco use per day increased from wave 1 to wave 3 . In wave 1 , no one reported that they smoked more than 20 cigarettes per day but in wave 2 and wave 3 surveys $6.9 \%$ of youth smokers reported that they smoked more than 20 cigarettes per day. In wave 1, approximately half (47.9\%) of youth smokers reported that they obtained cigarette from friends with only $38 \%$ reported that they bought cigarette for themselves. The proportion of young smokers who reported that they bought cigarettes for themselves were significantly increased in wave $2(61.3 \%)$ and wave $3(68.2 \%)$ as compare to wave 1 . More than half of young smokers reported that they tried to quit smoking within the past month. The proportion of youth smokers who reported that they never tried to quit smoking were slightly decreased from wave 1 as compare to wave 2 and wave $3(20.7 \%$ in wave $1,19.7 \%$ in wave 2 and $17.4 \%$ in wave 3 ).

Conclusions: Cigarette smoking among Thai youth remains the important problem and needs further tobacco control policies to prevent and control tobacco consumption.

CORRESPONDING AUTHOR: Tawima Sirirassamee, MD, Srinakarinwirot university, Nakornnayok,26120; tawima_s@yahoo. com

\section{Thursday August 5, 2010 SYMPOSIA SESSIONS 2:30 PM-4:00 PM}

\author{
SS08
}

UNDERSTANDING BEHAVIOURAL RESPONSES

TO GENETIC RISK INFORMATION

Theresa M. Marteau, $\mathrm{PhD},{ }^{1}$ Celeste Condit, $\mathrm{PhD},{ }^{2}$ Winfried Rief, $\mathrm{PhD}^{3}$ and Linda Cameron, $\mathrm{PhD}^{4}$

${ }^{1}$ Psychology, Kings College London, London, United Kingdom; ${ }^{2}$ Speech Communication, University of Georgia, Athens, GA; ${ }^{3}$ Pyschology, University of Marburg, Marburg, Germany and ${ }^{4}$ Psychology, University of Auckland, Auckland, New Zealand.

There are high expectations that the communication of genetic risk information will motivate behaviour change. This symposium considers the theoretical and empirical basis for these expectations and the likelihood that they may be realized. Celeste Condit and Lijiang Shen present the results of three large population-based surveys in the US assessing public perceptions of the interaction between genes and behaviour. Interpretations varied with whether genes and behaviour were conceived as health-damaging or health-protecting, suggesting a role for message framing in motivating behaviour change. Winfried Rief presents the results of a study evaluating the impact of presenting genetic risk information to obese patients. Guided by Leventhal's Common-Sense Model, Linda Cameron presents the results of an internet-based experiment assessing whether information regarding links between physiological 
processes underlying genetic risk, disease development, and behavior enhances beliefs motivating protective behavior and alters willingness to buy tests. Finally, Theresa Marteau presents the results of a randomized controlled trial of using genotype to tailor nicotine replacement therapy in smokers attempting to stop, testing the hypothesis based on Leventhal's CommonSense Model of Self-Regulation of Health and Illness that genetic risk information increases the use of medication as a coping response.

The discussion will focus upon the understanding that these four studies contribute, both theoretically and empirically, to explaining and changing behaviour in the context of genetic risk information.

CORRESPONDING AUTHOR: Theresa M. Marteau, PhD, Psychology, Kings College London, London, SE1 9RT; theresa. marteau@kcl.ac.uk

\section{SS08a}

PUBLIC UNDERSTANDING OF RISKS FROM GENEENVIRONMENT INTERACTION IN COMMON DISEASES: IMPLICATIONS FOR PUBLIC COMMUNICATIONS

Celeste M. Condit, $\mathrm{PhD}$ and Lijiang Shen, $\mathrm{PhD}$

Dept. of Speech Communication, University of Georgia, Athens, GA.

Background: Public understanding of the relationship between health behaviors and genes is likely to affect the motivational impact of learning information about one's own genes. Extant research has featured difficulty measuring public understandings of this relationship. This essay explores public understanding of the relationship between genes and behavior, especially with regard to the mathematical relationships to risk contribution. It also contributes a psychometrically valid scale for measuring beliefs about gene-behavior relationships. Methods: Three population representative surveys $(\mathrm{N}=633,658,1218)$ were conducted using the Knowledge Networks panel platform. Results: Interpretations of risk vary depending on whether genes and behavior are conceived of as health-damaging (loss frame) or health-protecting (gain frame). In the loss frame, the majority of the population adopts an additive model of the relationship with approximately one third adopting an amplificative model. Scores on the nonmathematically based scale indicate higher belief in the existence of interaction than scores on the more concrete question format. Conclusions: The existence of different interpretations of genebehavior relationships based on gain/loss frame and abstract/ concrete modes indicates the need to select frame and mode carefully in both teaching and research. Research is needed to identify optimal configurations for teaching and presenting this relatively complex material.

CORRESPONDING AUTHOR: Celeste M. Condit, PhD, Dept. of Speech Communication, University of Georgia, Athens, GA, 30602; ccondit@uga.edu
$\mathrm{SS} 08 \mathrm{~b}$

CONSULTATION INCLUDING GENETIC INFORMATION

IN PEOPLE WITH INCREASED RISK FOR OBESITY

AND MC4R-MUTATIONS

Winfried Rief, $\mathrm{PhD}{ }^{1}$ Johannes Hebebrand, $\mathrm{MD}^{2}$ and Anke Hinney, $\mathrm{PhD}^{2}$

${ }^{1}$ Clinical Psychology and Psychotherapy, University of Marburg, Marburg, Germany and ${ }^{2}$ Medical School, University of Essen, Essen, Germany.

There is strong evidence for high heredity for obesity. Moreover, if obesity has developed, it is difficult to control weight, and unsuccessful and disappointing weight reduction programs are frequent. In this study we wanted to investigate whether information about the genetic background of obesity is helpful for affected people to develop realistic goals and reduce negative self-perceptions. After genetic screenings of 1000 obese people, we included 320 for a randomized clinical trial evaluating the effect of genetic information versus general information (without genetic content) in obesity. A subgroup of 16 participants was confirmed for functionally relevant MC4R mutations, and they received consultation with genetic information. Their results were analyzed in detail. The acceptance of a consultation including genetic information was high, and those people with a family history of obesity appreciated to receive genetic information in the consultation. People with MC4R mutations reported less feelings of shame and guilt and more realistic weight loss goals after the intervention. We conclude that consultation for obese people should include information about genetic backgrounds, especially if obesity-relevant genetic mutations are obvious, or if people have a family history of obesity.

CORRESPONDING AUTHOR: Winfried Rief, PhD, Clinical Psychology and Psychotherapy, University of Marburg, Marburg, 35032; rief@staff.uni-marburg.de

SS08c

PRESENTING COHERENT LINKS BETWEEN RISKS AND ACTION: IMPACT ON BELIEFS MOTIVATING BEHAVIOR AND EXPECTED VALUE OF GENETIC TESTS Linda D. Cameron, $\mathrm{PhD},{ }^{1}$ Paul Brown, PhD, ${ }^{1}$ Kerry Sherman, $\mathrm{PhD}{ }^{2}$ Theresa Marteau, $\mathrm{PhD}^{3}$ and William Klein, $\mathrm{PhD}^{4}$

${ }^{1}$ The University of Auckland, Auckland, New Zealand; ${ }^{2}$ Macquarie University, NSW, NSW, Australia; ${ }^{3}$ King's College London, London, United Kingdom and ${ }^{4}$ National Cancer Institute, $\mathrm{NIH}$, Bethesda, MD.

Increasingly, individuals can buy genetic tests to identify disease risks that can be reduced through behavior change. Given the challenges in understanding how behavior can reduce geneticallyconferred risk, there is a need to identify how to present information to optimize comprehension and adaptive behavior. Guided by the Common-Sense Model, we tested whether information about links between physiological processes underly- 
ing genetic risk, disease development, and behavior enhances beliefs motivating protective action and alters willingness to buy tests. We tested the impact of this information for genetic tests versus tests of a non-genetic biomarker, at higher versus lower risk levels, and when presenting risk information in graphic versus numeric formats. In an internet-based factorial design experiment, 782 adults responded to messages about a test for colorectal cancer risk. We varied messages by risk-action link information (provision or no provision of information describing how a low-fat diet reduces cancer risk given a positive result), risk increment $(20 \%, 50 \%$, or $80 \%$ risk given a positive result), risk format (numeric or graphic presentation of risk increments), and test type (genetic or enzyme test). ANOVAs revealed that providing risk-action link information enhanced beliefs of response efficacy (that low-fat diets reduce disease risk) and coherence (understanding how low-fat diets reduce risk), and lowered appraisals of anticipated risk with a low-fat diet (p's $<.0001)$. These effects held across risk increments, risk formats, and test types. For genetic tests, provision of riskaction link information reduced the amount individuals were willing to pay for the test $(\mathrm{p}<.0001)$. Brief explanations of how action can reduce biomarker-detected risks promoted beliefs motivating protective action; paradoxically, by enhancing understanding of behavioral control over the risk, they reduced the value of genetic risk information.

CORRESPONDING AUTHOR: Linda D. Cameron, PhD, Psychology, The University of Auckland, Auckland, 1042; 1.cameron@ auckland.ac.nz

\section{SS08d}

IMPACT OF GENETIC FEEDBACK ON ADHERENCE

TO NICOTINE REPLACEMENT THERAPY:

THE PERSONALISED EXTRA TREATMENT (PET) TRIAL Theresa M. Marteau, PhD, ${ }^{1}$ Marcus R. Munafò, $\mathrm{PhD},{ }^{2}$ A Toby Prevost, $\mathrm{PhD},{ }^{5}$ Paul Aveyard, $\mathrm{PhD},{ }^{3}$ Elaine C. Johnstone, $\mathrm{PhD},{ }^{4}$ David Armstrong, $\mathrm{PhD},{ }^{6}$ Ann-Louise Kinmonth, $\mathrm{PhD}^{7}$ and Stephen R. Sutton, $\mathrm{PhD}^{7}$

${ }^{1}$ Psychology, Kings College London, London, United Kingdom; ${ }^{2}$ Experimental Psychology, University of Bristol, Bristol, United Kingdom; ${ }^{3}$ School of Health and Population Sciences, University of Birmingham, Birmingham, United Kingdom; ${ }^{4}$ Department of Clinical Pharmacology, University of Oxford, Oxford, United Kingdom; ${ }^{5}$ Department of Public Health and Primary Care, Kings College London, London, United Kingdom; ${ }^{6}$ Department of General Practice and Primary Care, Kings College London, London, United Kingdom and ${ }^{7}$ General Practice and Primary Care Research Unit, University of Cambridge, Cambridge, United Kingdom.

Despite growing interest in pharmacogenetics to tailor cessation treatment, the behavioural impact of genetic feedback is untested. Communicating genetic information may increase medication adherence, but may also negatively impact motivation to quit if the initial attempt fails. We investigated the impact of genetic feedback on medication adherence and motivation to quit. We tested (1) whether adherence to NRT is greater following genetic (OPRM1 genotype) compared to phenotypic (heaviness of smoking) feedback, and (2) whether smokers who relapsed showed lower motivation to make another attempt following genetic compared to phenotypic feedback.

The study was an open label, parallel group randomised trial (ISRCTN: 14352545) conducted in primary care. Adult smokers $(n=633)$ were prescribed NRT patch, randomised to a top-up dose of NRT based explicitly either on OPRM1 genotype or heaviness of smoking and followed for 6-months. Outcomes measures were: proportion of prescribed NRT consumed in the first 28 days; and motivation to make another quit attempt among those not abstinent at 6-month follow-up.

There was a significant effect of genetic feedback compared to phenotypic feedback on adherence at 7-day follow-up (75\% vs $69 \%, \mathrm{p}=0.040)$, and a marginally non-significant effect at 28-day follow-up ( $69 \%$ vs $64 \%, \mathrm{p}=0.098)$. There were similar effects on abstinence at six-month follow-up ( $14 \%$ vs $8 \%, \mathrm{p}=0.018)$. Amongst those not abstinent at 6-month follow-up, there was no difference in motivation to make another quit attempt between trial $\operatorname{arms}(\mathrm{p}=0.23)$.

This is the first test of the behavioural impact of pharmacogenetic feedback. Genetic feedback had a measurable impact on shortterm medication adherence and abstinence at 6-month follow-up compared to phenotypic feedback, and appears to offer modest improvements to medication adherence to medication, and NRT effectiveness. This suggests potential for an important population impact.

CORRESPONDING AUTHOR: Theresa M. Marteau, PhD, Psychology, Kings College London, London, SE1 9RT; theresa. marteau@kcl.ac.uk

\section{SS09}

IMPACT OF MALE NORMS ON PROSTATE CANCER DIAGNOSIS AND RECOVERY

Betsy Bates, MA, ${ }^{1}$ Michael Hoyt, $\mathrm{PhD},{ }^{2}$ Sharon Manne, $\mathrm{PhD}^{3}$ and Peter G. Kaufmann, $\mathrm{PhD}^{4}$

${ }^{1}$ Antioch University, Santa Barbara, CA; ${ }^{2}$ Health Psychology, University of California Merced, Merced, CA; ${ }^{3}$ Fox Chase Cancer Center, Philadelphia, PA and ${ }^{4}$ Clinical Applications and Prevention Branch Division, Cardiovascular Sciences, National Heart, Lung, and Blood Institute, Bethesda, MD.

Prostate cancer is the most common form of cancer in men (other than skin cancer). The American Cancer Society estimates that 1 in 5 men will get prostate cancer sometime during his lifetime, and that 1 man in 35 will die of this disease. The society also estimates that more than 2 million men in the United States who have prostate cancer at some point are still alive today. Much of the current treatment for cancer (including surgery and hormone therapy) involves temporary or permanent impotence or urinary 
incontinence which would be distressing for all men but particularly for those who are invested in their sexual performance or being in control as signs of their masculinity. Men may therefore forego treatment or delay it, leading to increasing risk of complications from prostate cancer. Presenters in this symposium will look at the impact of male sex role norms (including emotional restriction, emphasis on sexual ability, and being independent) on diagnosis for prostate cancer as well as recovery from treatment. They will examine the kinds of responses men have to being diagnosed and treated for prostate cancer, the type of support they seek and find useful after treatment, and the role partners can play in helping men cope.

\section{SS09a}

PROSTATE CANCER: MALE NORMS AND THE CONCEPT OF SUPPORT

Betsy Bates, MA

Antioch University, Santa Barbara, CA.

This presentation will summarize recent findings from the literature on psychological issues related to diagnosis, the seeking of psychological support, and threats to male identity posed by treatment options that can interfere with potency and continence. Qualitative studies, for example, point to a number of reasons why men may be diagnosed late, including a reluctance to appear weak (by reporting symptoms); embarrassment, guardedness and denial about vulnerability related to their bodies; powerlessness within the medical system, and the fear of a diagnosis that goes to the deepest core of masculinity, the prostate. A recent literature review highlighted clinically meaningful take-home messages, including the fact that men tend to either retreat inward following the diagnosis of cancer, keeping their feelings and worries to themselves, or rely heavily on a partner for emotional support.

Studies contained in the review demonstrate that both men and women benefit equally from psychosocial support. When asked, men say that they want information rather than emotional support from psychosocial oncology teams; yet in online forums, men's supportive exchanges are clearly emotional, couched in dark humor and unifying battlefield terminology. The fact that men enthusiastically participate in emotional support offered at a distance (often anonymously) raises interesting questions about future directions for cancer support, which will be addressed. Also of significance is mens' highly consistent reliance on partners for support during cancer treatment, a finding that points to the possible unmet needs of men who are not in secure relationships during the diagnosis and treatment of prostate cancer.

CORRESPONDING AUTHOR: Betsy Bates, MA, Antioch University, Santa Barbara, CA, 93103; betsybates@aol.com
SS09b

INTIMACY-ENHANCING PSYCHOLOGICAL INTERVENTION FOR MEN DIAGNOSED WITH PROSTATE CANCER AND THEIR PARTNERS: A PILOT STUDY

Sharon Manne, $\mathrm{PhD}$

Fox Chase Cancer Center, Philadelphia, PA

Objective: The diagnosis and treatment of localized prostate cancer can stress the marital relationship and compromise its quality. Couples may benefit from learning how to best handle cancer-related worries and concerns.

Aims: To assess the effects of Intimacy-Enhancing Therapy (IET) on the psychological and relationship functioning of men diagnosed with localized prostate cancer and their partners. Preintervention levels of psychosocial and relationship functioning were evaluated as possible moderators of intervention effects.

Method: 71 men diagnosed with localized prostate cancer and their partners were assigned to five sessions of IET or usual care (UC). Assessments were given at baseline and two months after baseline.

Results: IET effects were largely moderated by pre-intervention psychosocial and relationship factors. Among patients beginning the intervention with higher levels of cancer concerns, IET significantly reduced concerns and similar results were reported for the effects of IET on self-disclosure, perceived partner disclosure, and perceived partner responsiveness. Among partners beginning the intervention with higher cancer-specific distress, lower global marital satisfaction, lower intimacy, and poorer communication, IET improved these outcomes.

Conclusions: IET holds promise as an effective intervention for couples with greater distress and fewer relationship resources. Subsequent research is needed to replicate these findings with a larger sample and a longer follow-up.

CORRESPONDING AUTHOR: Sharon Manne, PhD, Fox Chase Cancer Center, Philadelphia, PA, 19111; Sharon.Manne@fccc.edu

$\mathrm{SS} 09 \mathrm{c}$

MEN, PROSTATE CANCER, AND EMOTIONAL APPROACH COPING

Michael A. Hoyt, PhD

Health Psychology, University of California Merced, Merced, CA.

Although the psychosocial impact of cancer treatment varies across cancer patients, many experience some psychological distress following diagnosis. In addition to the emotional experience, men with prostate cancer often face myriad physical complications including impotence, urinary and bowel incontinence, and sleep disturbance affecting quality of life. The time immediately following treatment can be a critical period marked by increased feelings of fear, uncertainty, and loss of control. Such emotional responses may be experienced as inconsistent with 
psychological attributes often associated with traditional gender role norms, such as strength, independence, and emotional inexpressiveness. Evidence of the negative impact of suppressing emotions, and positive impact of expressing and processing emotions on psychological distress, physical health, and overall adjustment to cancer is growing. Drawing upon examples from the author's past and current research with men with prostate cancer, the relationship of gender-related influences on emotional approach coping efforts will be discussed within a stress and coping framework. Emotional approach coping (EAC) refers to strategies that involve acknowledging, understanding, and expressing emotions, and has been found to be associated with decreased distress and better adjustment in breast cancer patients, though little work has examined EAC in men with prostate cancer. Observations in healthy populations suggest that EAC varies by gender and has been linked to both better and poorer adjustment in men. Prior research in men with cancer found that gender role norms shape EAC processes, and that the interpersonal environment and age may be important contextual variables characterizing its utility in men with cancer. Current research examining the impact of masculine cancer threat, gender-linked personality factors, and gender role norms on health-related quality of life following treatment for prostate cancer will be highlighted.

CORRESPONDING AUTHOR: Michael A. Hoyt, PhD, Health Psychology, University of California Merced, Merced, CA, 95343; mhoyt@ucmerced.edu

\section{CITATION ABSTRACT \\ SS10 \\ DEPRESSION, ANXIETY, AND QUALITY OF LIFE AMONG CAREGIVERS \\ OF CHILDREN WITH TYPE 1 DIABETES \\ Randi Streisand, $\mathrm{PhD}$ and Maureen Monaghan, $\mathrm{PhD}$ \\ Psychology, Children's National Medical Center, Washington, DC. \\ Management of type 1 diabetes (T1D) requires constant, daily attention to blood glucose levels, insulin administration, food intake, and physical activity in order to maintain near normal metabolic control. Caring for a child or adolescent with T1D can be demanding and research suggests that parents of children with diabetes are at greater risk for symptoms of depression, anxiety, and stress. The dynamic relationship between parent emotional functioning and daily diabetes responsibilities and care is not yet well understood and parent well-being may significantly influence critical child health behaviors and outcomes. \\ This symposium provides a cohesive picture of the behavioral impact of parent emotional functioning on child health care in T1D. The symposium chair will highlight developmental differences and key risk and protective factors associated with parent well-being in a chronic illness population. Ongoing promising interventions, including interventions focusing on parenting strategies and support, parent-child communication, and internet-based support, will be discussed. The bidirectional impact of diabetes care and parent depression, anxiety, and well- being is a rich area for discussion and can inform future research.}

CORRESPONDING AUTHOR: Maureen Monaghan, PhD, Psychology, Children's National Medical Center, Washington, DC, 20037; maureen.monaghan@gmail.com

\section{SS10a}

ANXIETY AND DEPRESSION IN PARENTS OF YOUNG CHILDREN WITH TYPE 1 DIABETES

Maureen Monaghan, $\mathrm{PhD},{ }^{1}$ Marisa Hilliard, MA, ${ }^{1}$ Cynthia Ronzio, $\mathrm{PhD},{ }^{2}$ Fran R. Cogen, $\mathrm{MD}^{3}$ and Randi Streisand, $\mathrm{PhD}^{1}$ ${ }^{1}$ Psychology, Children's National Medical Center, Washington, DC; ${ }^{2}$ Clinical and Community Research, Children's National Medical Center, Washington, DC and ${ }^{3}$ Department of Endocrinology and Metabolism, Children's National Medical Center, Washington, DC.

Type 1 diabetes (T1D) management in young children requires significant parent effort and attention to a complex regimen.
Among parents of children with chronic illnesses, symptoms of anxiety and depression are more prevalent; however, less is known about the impact of parent emotions on diabetes care behaviors. This study assesses anxiety and depression in parents of young children with T1D and explores its relationship to diabetes management, including parent reported overall dietary adherence, recall data on frequency of $\mathrm{BG}$ checks and diet/meals, $\mathrm{BG}$ variability, and $\mathrm{A} 1 \mathrm{c}$.

As part of an ongoing RCT of parenting support, 60 primary caregivers (90\% female; $75 \%$ Caucasian) of children age 2-6 with T1D $(\mathrm{M}$ age $=5.86 \pm 1.13 \mathrm{yrs})$ completed baseline assessment of parent anxiety and depression and daily T1D management.

Using the STAI, $31 \%$ of parents scored over the clinical cutoff for symptoms of state anxiety and, on the CES-D, $21 \%$ of parents met criteria for mild $(8 \%)$ or moderate $(13 \%)$ depression. Anxiety and depression symptoms were not significantly associated with frequency of BG checks or BG variability; however, increased 
anxiety was associated with poorer parent-reported dietary adherence $[\mathrm{F}(4,55)=10.09, \mathrm{p}<.01, \mathrm{R} 2=.38]$ and more frequent child snacks/meals $[\mathrm{F}(2,57)=5.39, \mathrm{p}<.01, \mathrm{R} 2=.13]$. Controlling for marital status, education, and income, higher levels of anxiety were associated with poorer metabolic control (A1c) in children [F $(4,54)=4.67, \mathrm{p}<.01, \mathrm{R} 2=.20]$. Depressive symptoms were not significantly related to $\mathrm{A} 1 \mathrm{c}$ and the effect of anxiety on $\mathrm{A} 1 \mathrm{c}$ remained with the inclusion of depression scores.

Parental anxiety appears to be a salient factor in T1D management in young children and treatment of parent anxiety may improve child health outcomes. The impact of anxiety on dietary management may be related to hypoglycemia fear and can provide a specific avenue for intervention. The direction of the relationship between anxiety and A1c is not presently known yet may be better understood with the completion of the RCT.

CORRESPONDING AUTHOR: Maureen Monaghan, PhD, Psychology, Children's National Medical Center, Washington, DC, 20037; maureen.monaghan@gmail.com

\section{SS10b}

\section{MATERNAL DEPRESSIVE SYMPTOMS AND CHILDREN} WITH TYPE 1 DIABETES

Kari L. Struemph, MS, ${ }^{1,3}$ Rusan Chen, $\mathrm{PhD},{ }^{2}$ Priscilla Powell, MS, ${ }^{1}$ Kathryn Maher, BA, ${ }^{1}$ Elizabeth Robinson, BA, ${ }^{1}$ Anil Kumar, $\mathrm{MD}^{1}$ and Clarissa S. Holmes, $\mathrm{PhD}^{1,2}$

${ }^{1}$ Virginia Commonwealth University, Richmond, VA; ${ }^{2}$ Georgetown University, Washington, DC and ${ }^{3}$ Children's National Medical Center, Washington, DC.

Parental involvement is identified as a key factor in optimal management of pediatric diabetes. Further, research shows mothers are often primary care givers such that maternal psychological adjustment and its relation to children's disease care management are surprisingly understudied.

Baseline data were analyzed from 179 mothers of generally middle-class young adolescents with T1D in a multi-site RCT. Mothers completed questionnaires and were interviewed about family functioning and diabetes management. On average, mothers were 42 years old and completed 2 years of post secondary education. Half were employed full time outside the home and $25 \%$ had no outside work. On average, children were 12.9 years old (range $11-14$ years), were generally in fair metabolic control $(\mathrm{HbA} 1 \mathrm{c}=8.7 \%)$ and had diabetes for about 5 years.

A significant portion, $23 \%$ of the sample, had clinically elevated (mild to severe) depressive symptoms. Mothers who endorsed depressive symptoms $(\mathrm{N}=41)$ were matched on SES and compared to nondepressed maternal caregivers. More mothers with depressive symptoms were employed full time $(60 \%)$ and were single parents (35\%) than others ( $46 \%$ and $22 \%$, respectively). No significant group differences were found in maternal age or education, child age, HbA1c, or disease onset age. However, mothers who endorsed clinically elevated symptoms also reported more fear of hypoglycemia and lower self-efficacy for diabetes tasks, although youths' metabolic control did not differ between groups.
Mothers with higher depressive scores appear able to help their youth achieve comparable levels of metabolic control as those without significant symptoms. However, elevated depressive symptoms occurred more often in single mothers who worked full time suggesting these mothers may have less time for and help with disease care. As youths become more autonomous maternal fears of hypoglycemia may magnify and perceptions of selfefficacy may decline. Screening and identification of mothers with high depressive symptoms and referral for supportive counseling may help them cope better and help ensure long-term optimal youth disease care.

CORRESPONDING AUTHOR: Kari L. Struemph, MS, Virginia Commonwealth University, Silver Spring, MD, 20903; morgank1 2@ vcu.edu

SS10c

DEPRESSION, ANXIETY, AND OBSERVED PARENTING IN MOTHERS OF ADOLESCENTS WITH TYPE 1 DIABETES

Sarah S. Jaser, PhD and Margaret Grey, PhD, RN

School of Nursing, Yale University, New Haven, CT.

Research has begun to highlight the negative effects of maternal depression and anxiety on youth with type 1 diabetes (T1D), including poorer family functioning, increased symptoms of depression, and poorer quality of life. However, researchers have typically used self-report measures of family functioning and child adaptation. The current study extends previous research by using observational methods to examine the relationship between maternal symptoms of depression and anxiety and specific parenting behaviors.

In this cross-sectional pilot study, we collected questionnaire (i.e. self-report measures of depression and anxiety), clinical (i.e., HbA1c), and observational data from adolescents with T1D (ages 10-16) and their mothers. Adolescents $(n=30,52 \%$ girls, mean age $=12.6,74 \%$ White $)$ and their mothers engaged in a 15 -minute discussion of diabetes-related stress, which was coded for parenting behaviors.

In our sample, $23 \%$ of mothers were above the clinical cutoff for depression, and $13 \%$ for anxiety. Bivariate correlations indicated that maternal symptoms of anxiety were related to lower levels of observed child-centered behavior (displays of sensitivity) ( $\mathrm{r}=-.50$, $\mathrm{p}=.005)$ and listener responsiveness $(\mathrm{r}=.38, \mathrm{p}=.038)$, higher levels of antisocial behavior $(\mathrm{r}=.54, \mathrm{p}=.002)$, and somewhat higher levels of hostility $(\mathrm{r}=.33, \mathrm{p}=.073)$. Similarly, maternal symptoms of depression were related to lower levels of childcentered behavior $(\mathrm{r}=-.45, \mathrm{p}=.012)$, somewhat lower levels of listener responsiveness $(\mathrm{r}=-.33, \mathrm{p}=.080)$, and higher levels of antisocial behavior $(\mathrm{r}=.46, \mathrm{p}=.012)$. Observed parenting behaviors, in turn, were associated with poorer metabolic control $(\mathrm{r}=$ -.53 for child centered behavior, $\mathrm{r}=-.34$ for listener responsiveness, $r=.54$ for antisocial behavior, and $r=.45$ for hostility, all $\mathrm{p}<.05)$.

Future studies are needed to examine specific parenting behaviors as potential mediators of maternal psychosocial distress and adolescent adaptation. Interventions aimed at teaching effective 
parenting strategies may be helpful in improving adaptation in adolescents with T1D.

CORRESPONDING AUTHOR: Maureen Monaghan, PhD, Psychology, Children's National Medical Center, Washington, DC, 20037; maureen.monaghan@gmail.com

\section{SS10d}

PREVALENCE AND CORRELATES OF POOR EMOTIONAL WELL-BEING IN PARENTS OF TEENAGERS WITH TYPE 1 DIABETES

Maartje de Wit, PhD and Frank J. Snoek, PhD

Department of Medical Psychology, VU University Medical Center, Emgo Institute for Health and Care Research, Amsterdam, Netherlands.

Aim: Dealing with a teenager with diabetes is often not easy and can put pressure on the family and parental well-being. We aim to develop an internet course for parents of teens with diabetes including information, parenting and emotional support. For this course, we first examined prevalence and correlates of poor wellbeing in parents of teens with diabetes.

Methods: Parents of teens (12-19 years) were recruited through the Dutch Diabetes Patient Association and completed questionnaires on the internet about their own well-being (WHO-5 well-being index, parental diseased-related stress (Pediatric Inventory for Parents), family functioning (Diabetes Family Responsibility and Conflict scale, Diabetes Family Behaviour Checklist and Parenting Scale) and demographic and diabetes related characteristics (age, gender, education, family status, child's diabetes onset, duration and latest $\mathrm{HbA} 1 \mathrm{c})$.

Results: 255 parents ( $80 \%$ mothers) completed the questionnaires. Mean age was $46.3 \pm 4.6$ years and $88.6 \%$ was married. Mean age of their teens $(52 \%$ boys) was $15.0 \pm 2.1$ years. Mean diabetes duration was $5.4 \pm 3.6$ years; most recent $\mathrm{HbA} 1 \mathrm{c}$ reported by the parent was $8.1 \% \pm 1.4$.

More non-supportive behavior $(p<.001)$, conflicts $(p<.001)$ and parental stress $(p=.023)$ were related to higher HbA1c levels.

On the WHO-5, 75\% of parents reported satisfactory well-being, $18 \%$ moderate well-being and $7 \%$ had an indication of likely major depression, higher than in the general population.

Better well-being was associated with male gender $(\mathrm{p}=.03)$, being married $(\mathrm{p}=.02)$, higher education $(\mathrm{p}=.003)$, fewer conflicts $(\mathrm{r}=$ $-.25, \mathrm{p}<.001)$, less parental responsibility $(\mathrm{r}=-.26, \mathrm{p}<.001)$, less parental stress $(\mathrm{r}=-.55, \mathrm{p}<.001)$, less over-reactive $(\mathrm{r}=-.33$, $\mathrm{p}<.001)$ and less lax $(\mathrm{r}=-.18, \mathrm{p}=.003)$ parenting styles.

Conclusion: One out of 4 parents of a teenager with diabetes reports moderate to poor well-being, suggesting a need for psychological support particularly for mothers. Addressing family functioning in the internet course could not only improve parental well-being but potentially diabetes outcomes of the teens as well.

CORRESPONDING AUTHOR: Maureen Monaghan, PhD, Psychology, Children's National Medical Center, Washington, DC, 20037; maureen.monaghan@gmail.com
SS11

PSYCHOLOGICAL DISTRESS AND MUSCULOSKELETAL PAIN

Joost Dekker, $\mathrm{PhD}$

Rehabilitation Medicine, VU University Medical Centre, Amsterdam, Netherlands.

Psychological distress is a major factor in musculoskeletal pain. As demonstrated in numerous studies, psychological distress and musculoskeletal pain commonly occur together; psychological distress is associated with a poor prognosis of musculoskeletal pain; and psychological distress is prognostic for a poor response to treatment. Research in this field is in need of better conceptual and theoretical underpinnings: the field is moving from empirical demonstrations of the impact of distress on pain towards better understanding of how psychological distress affects musculoskeletal pain and how psychological distress can be addressed during treatment. The overall goal of this symposium is to contribute to the theoretical understanding of the role of psychological distress in musculoskeletal pain, by summarizing recent developments in research and treatment.

Dr. Keefe will summarize the current state of science on pain coping in osteoarthritis patients. He will discuss the theoretical background of pain coping and then continue to critically review the research and intervention studies in this area. Dr. Crombez will review research on hypervigilance and attentional bias in chronic pain. He will discuss implications for the understanding of chronic pain and for future research. Dr. Steultjens will summarize current literature showing that psychological distress is a major predictor of poor outcome of multidiciplinary rehabilitation in chronic pain and fibromyalgia. He will discuss theoretical interpretations and practical implications of these findings. Dr. Denison will focus on recent treatment approaches aiming at improvement of function and reduction of distress in patients with musculoskeletal pain. Treatment builds on basic motor and behavior learning principles and cognitive-behavioral principles.

CORRESPONDING AUTHOR: Joost Dekker, PhD, Rehabilitation Medicine, VU University Medical Centre, Amsterdam, 1007 MB; j.dekker@vumc.nl

\section{SS11a}

PAIN COPING IN OSTEOARTHRITIS: CURRENT STATE OF THE SCIENCE

Francis J. Keefe, PhD

Department of Psychiatry and Behavioral Sciences, Duke University Medical Center, Durham, NC.

This presentation provides an overview and update on the literature on pain coping in osteoarthritis patients. The presentation is divided into four sections. In the first section, the conceptual background for studies of pain coping in osteoarthritis is reviewed. In the second section, recent studies of pain coping are critically reviewed including studies on the role of pain 
catastrophizing, pain communication in osteoarthritis, pain coping in morbidly obese osteoarthritis patients, and brain correlates of pain coping. In the third section, interventions designed to enhance pain coping are highlighted, including studies of partner-assisted coping skills training, communication skills training, and combined pain coping skills training/lifestyle behavioral weight management. In the final section, the strengths and limitations of current research are summarized and important directions for future research described.

CORRESPONDING AUTHOR: Francis J. Keefe, PhD, Duke University Medical Center, Durham, NC, 27705; keefe003@mc. duke.edu

\section{SS11b}

PREDICTING REHABILITATION TREATMENT OUTCOME IN CHRONIC WIDESPREAD PAIN

Martijn Steultjens, PhD, ${ }^{1,2}$ Aleid de Rooij, $\mathrm{PT},{ }^{2}$ Leo Roorda, PhD, $\mathrm{MD}, \mathrm{PT}^{2}$ and Dekker Joost, $\mathrm{PhD}^{1,2}$

${ }^{1}$ VU University Medical Centre, Amsterdam, Netherlands and

${ }^{2}$ Jan van Breemen Institute, Amsterdam, Netherlands.

Chronic widespread pain (CWP) and fibromyalgia (FM) are common syndromes characterized by widespread musculoskeletal pain with no obvious underlying pathology. Although multidisciplinary rehabilitation treatment has been shown to be effective in CWP and FM, on average effect sizes are small. One possible area of improvement is referring those patients for treatment in whom a clinically relevant treatment effect can be expected. To be able to do this, patient characteristics associated with treatment success need to be identified.

This presentation will first provide results from a systematic review of current literature on predictors of improvement following multidisciplinary rehabilitation treatment. Four outcome domains were studied: pain, physical functioning, emotional functioning, and general improvement / quality of life. In general, the available evidence suggests that cognitive and emotional factors are predictive of treatment outcome. For both pain and physical functioning, poor treatment outcome was predicted by the presence of emotional problems, interpersonal distress, and depression. Apart from these cognitive and emotional factors, evidence for baseline pain intensity and impact of pain as predictors of treatment outcome were found.

Additionally, data will be presented from a prospective cohort study on health outcomes in CWP and FM, for which analyses are currently in progress.

Based on the findings from the systematic review and prospective cohort study, pathways for successful rehabilitation treatment in CWP and FM will be discussed.

CORRESPONDING AUTHOR: Martijn Steultjens, PhD, VU University Medical Centre, Amsterdam, 1007 MB; m.steultjens@, vumc.nl
SS11c

USING AN INTEGRATED BEHAVIORAL MEDICINE

AND PHYSICAL THERAPY APPROACH TO IMPROVE

FUNCTION AND REDUCE DISTRESS IN PATIENTS

WITH MUSCULOSKELETAL PAIN MANAGED IN PRIMARY

HEALTH CARE

Eva Denison, $\mathrm{PhD}$

${ }^{1}$ Department of Evidence-Based Health Care, The Norwegian Knowledge Centre for the Health Services, Oslo, Norway and ${ }^{2}$ Uppsala University, Department of Public Health and Caring Sciences, Uppsala, Sweden.

A majority of patients with musculoskeletal pain (MSP) are managed within primary health care (PHC). While physicians are involved in the initial assessment of patients' problems, physical therapists typically carry out interventions to reduce pain and improve function. There is now ample evidence that psychosocial factors such as selfefficacy, fear of pain and/or movement/re-injury, catastrophizing, and social reinforcement contingencies contribute to the development of persistent pain and disability. In our research group at Uppsala University we have developed an integrated behavioral medicine and physical therapy treatment approach for patients with MSP that builds on basic motor and behavior learning principles and cognitivebehavioral principles. The approach comprises identification of problems and goals, monitoring of problems, functional behavioral analyses, basic and applied skills acquisition, generalization of skills, and relapse prevention. Evaluation of the approach involves clinical studies at both the individual level and group level and there are promising results at both short-term and long-term follow-up. Recent studies have focused on 1) targeting and tailoring of treatment based on screening of psychosocial factors and individual functional analyses, and 2) screening for psychosocial risk factors in telephone counseling. An emerging research area concerns implementation of the integrated approach in general clinical practice within PHC. Pilot work in our research group indicates that physical therapists may have greater difficulties in adopting this integrated approach than anticipated, lack of theoretical knowledge being one possible explanation. Further implementation efforts involve research as well as systematic integration of the approach in physical therapy curricula, the latter being done at two Swedish universities.

CORRESPONDING AUTHOR: Eva Denison, PhD, Department of Evidence-Based Health Care, The Norwegian Knowledge Centre for the Health Services, Oslo, N-0130; eva.denison@kunnskapssenteret.no

\section{SS11d}

HYPERVIGILANCE AND ATTENTIONAL BIAS

IN CHRONIC PAIN PATIENTS: QUO VADIS?

Geert Crombez, $\mathrm{PhD}$

Experimental Clinical and Health Psychology, Ghent University, Gent, Belgium.

Hypervigilance to pain and attentional bias are considered as key processes in understanding chronic pain, distress and suffering (Crombez et al., 2005; Pincus \& Morley, 2001; Vlaeyen et al., 1995). 
Results are however contradictory. We performed a meta-analysis of attentional bias to pain-related information to address this issue, and in particular to investigate the role of pain-related fear, anxiety (trait and state) and depressive mood upon attentional bias to pain.

Method: 61 studies (2739 participants) were identified using an emotional stroop paradigm, dot probe paradigm and exogeneous cueing paradigm. We coded methodological quality (internal and external), sample characteristics (chronic pain, experimental pain, procedural pain), and procedural characteristics. We obtained the original full dataset ( $80 \%$ response rate) to analyze the effects of our theory-based moderators.

Results: The methodological quality of studies varied widely and is on average of moderate quality. Initial analyses revealed a significant attentional bias to pain-related information for whom pain was a current concern $(\mathrm{d}=0.127)$ in comparison with a control group $(\mathrm{d}=0.025)$. The effect was significant for the chronic pain group, the experimental pain group and the procedural pain group. Further analyses revealed that the attentional bias to sensory pain words was signficant, but not to affective pain words, stimuli related to antecedents of pain (e.g. injury or blood) or to stimuli related to consequences of pain (e.g. wheelchair). We are currently performing the moderator analysis focusing upon the role of pain-related fear, anxiety and depressive mood.

In conclusion, the methodological quality of attentional bias studies is moderate and can be improved. An attentional bias to sensory pain words exist in those who are concerned about pain. The implications for future research and for understandings of chronic pain, distress and suffering will be discussed.

CORRESPONDING AUTHOR: Geert Crombez, PhD, Experimental Clinical and Health Psychology, Ghent University, Gent, 9000; geert.crombez@ugent.be

\section{SS33}

IVR ASSESSMENT AND BRIEF INTERVENTION CONCERNING HAZARDOUS AND HARMFUL ALCOHOL USE

Anne H Berman, $\mathrm{PhD}$

Clinical Neuroscience, Karolinska Institutet, Huddinge, Sweden.

Risky use of alcohol is a serious public health problem in Sweden and the U.S., as well as other countries. Despite organized efforts to screen for risky use, only a small proportion of individuals with hazardous and harmful use are screened, and an even smaller proportion receive appropriate tailored help to eliminate risky alcohol use and improve their quality of life. Research on automated telephonic screening and intervention in the form of Interactive Voice Response (IVR) among primary care patients, students, and other risk groups in the population, suggests that this technology could make a significant contribution towards increasing awareness of risky alcohol use among individuals that are otherwise difficult to reach. This symposium, with participants from the U.S. and Sweden, presents recently implemented research on IVR and alcohol as well as currently ongoing and planned projects.

CORRESPONDING AUTHOR: Anne H Berman, PhD, Clinical Neuroscience, Karolinska Institutet, Huddinge, S-14104; anne.h. berman@ki.se
SS33a

SCREENING FOR RISKY ALCOHOL AND DRUG USE VIA INTERACTIVE VOICE RESPONSE (IVR) AND INTERNET IN A SWEDISH RANDOM POPULATION SAMPLE

Anne $\mathrm{H}$ Berman, $\mathrm{PhD},{ }^{1,2}$ Kristina Sinadinovic, $\mathrm{BSc}^{1,2}$ and Peter Wennberg, $\mathrm{PhD}^{1,2}$

${ }^{1}$ Clinical Neuroscience, Karolinska Institutet, Huddinge, Sweden and ${ }^{2}$ Stockholm Center of Dependency Disorders, Stockholm, Sweden.

Objectives: Randomized population surveys on risky alcohol use have been carried out in Sweden using paper versions of the AUDIT and the DUDIT. Earlier response rates have ranged from $80 \%$ to $74.5 \%$. Hazardous alcohol consumption has ranged between 13.2 and $15.1 \%$ and harmful alcohol consumption has been identified for 2.6 to $2.9 \%$. Hazardous and harmful drug consumption was identified at $3.1 \%$.

The increasing general availability and accessibility of information and communication technologies (ICT) over the past decade has made the use of IVR and the Internet in such studies a natural continuing step to take. The aim of this study was to investigate the feasibility of IVR and Internet modes of administration in a population survey and to assess psychometric robustness.

Methods: A random population sample of 5000 individuals 1680 years old was extracted from the Swedish Population Registry. Two thousand individuals were invited to participate via IVR, 2000 via the Internet, and 1000 individuals were offered a choice between IVR and Internet administration. Individuals indicating risky alcohol and drug use were followed up 12 months later using the same administration method.

Results: The overall response rate was $37.6 \%$, via the Internet it was $37.8 \%$ and via IVR $33.8 \%$. The Internet-IVR choice group had a response rate of $46.2 \%$ to $43 \%$, higher when the Internet was the first alternative listed. Hazardous or harmful alcohol use was indicated by $20.7 \%$ with hazardous or harmful drug use at $2.8 \%$. Cronbach $\alpha$ coefficients $\alpha=0.80$ for the Internet version of the AUDIT and $\alpha=0.77$ for the IVR version. For the DUDIT, the Cronbach $\alpha$ coefficient for Internet version was 0.86 and for the IVR version it was 0.85 .

Conclusions: Population surveys using the ICT modes of administration are feasible and reliable. Mixed mode administration increases the response rate. Future research in the near future should, however, include paper questionnaires among the various modes of administration available to respondents.

CORRESPONDING AUTHOR: Anne H Berman, PhD, Clinical Neuroscience, Karolinska Institutet, Huddinge, S-14104; anne.h. berman@ki.se

\section{SS33b}

INTERACTIVE VOICE RESPONSE (IVR): FOR AUTOMATED ALCOHOL SCREENING AND BRIEF INTERVENTION (SBI) IN A PRIMARY CARE CLINIC

John E. Helzer, MD

Health Behavior Research Center, University of Vermont, Burlington, VT. 
Objectives: Our research team is testing the clinical potential of Interactive Voice Response. IVR is a simple, automated technology usable anywhere touch-tone phones are available. We have previously shown that IVR can ascertain valid clinical information, convey information to patients, and enhance interventions. We are currently testing IVR as a method for alcohol screening and delivering an automated Brief Alcohol Intervention (BI) in primary care offices.

Methods: We implemented an IVR system to do a brief screening questionnaire when patients arrive at the clinic for their appointment. The screen includes the AUDIT-C and general health questions. It requires less than one minute to complete; results are printed immediately for review by the provider and discussion with the patient. Patients who screen positive for at risk drinking are offered an IVR-based BI.

Results: Clinic arrivals $(\mathrm{N}=122)$ were invited at random to do the IVR-Screen using a touch-tone phone in the waiting room, $98 \%$ consented. No patient hung up during the screening interview; nearly all expressed satisfaction with the method and question items. Patients and physicians found IVR to be a useful means of screening. Of those screened, $16 \%$ were identified as candidates for an alcohol BI. Of these, $72 \%$ agreed to do the IVR-BI and $91 \%$ of those who initiated the IVR-BI completed it. The mean duration of the IVR-BI was 5 minutes; no caller felt this was too long and $68 \%$ said it increased their awareness of how heavily they drink. No patient expressed discomfort with the IVR-BI; only 5\% expressed any kind of negative reaction.

Conclusions: It is well established that alcohol SBI in primary care clinics is an effective intervention. However providers are reluctant to do SBI because of time constraints and other impediments. This study has demonstrated that IVR-based screening and brief intervention are both feasible and acceptable to patients and providers in a primary care setting. We are currently seeking funding to test the efficacy of an IVR- BI in primary care settings.

CORRESPONDING AUTHOR: Anne H Berman, PhD, Clinical Neuroscience, Karolinska Institutet, Huddinge, S-14104; anne.h. berman@ki.se

\section{$\mathrm{SS} 33 \mathrm{c}$}

USING INTERACTIVE VOICE RESPONSE (IVR) TO TREAT PROBLEM DRINKERS IN THE COMMUNITY

Amy Rubin, PhD

Department of Medicine, Boston University School of Medicine, Boston, MA.

Objectives: People who drink in a problematic or risky manner comprise a larger group than people who are alcohol dependent, yet treatment for problem drinkers is much less prevalent in the $U$. $\mathrm{S}$. Using technological solutions to bring interventions to problem drinkers promises to make appropriate treatment options more widely available. Interactive Voice Response (IVR) with speech recognition is an underused technology that is accessible to anyone with a telephone. IVR is being used to develop and evaluate an evidence-based automated treatment program based on Behavioral Self-control Training. This program is being tested in a randomized clinical trial for problem drinkers.
Methods: Miller and Munoz' book, "Controlling Your Drinking: Tools to Make Moderation Work for You" (2005), was used as the basis for an IVR program with an enhanced Motivational Interviewing component. The program is being compared to a comparison group that receives an informational pamphlet with resources for self-referral. The entire project takes place over the phone, including baseline and follow-up interviews.

Results and Conclusions: 240 participants are being recruited from the community. Preliminary results will be presented and discussed. This will include ease of recruitment, initial use of the system, and change in alcohol use in experimental and comparison group.

CORRESPONDING AUTHOR: Anne H Berman, PhD, Clinical Neuroscience, Karolinska Institutet, Huddinge, S-14104; anne.h. berman@ki.se

\section{SS33d}

REAL-TIME IVR ASSESSMENT OF STRESS AND USE OF ALCOHOL AND DRUGS AMONG UNIVERSITY STUDENTS AND PAROLED OFFENDERS

Claes Andersson, $\mathrm{PhD},{ }^{1,2}$ Agneta Öjehagen, $\mathrm{PhD}^{1}$ and Mats Berglund, $\mathrm{MD}, \mathrm{PhD}^{2}$

${ }^{1}$ Department of Clinical Sciences, Division of Psychiatry, Lund University, Lund University Hospital, Sweden, Division of Psychiatry, Lund University, Lund University Hospital, Lund, Sweden and ${ }^{2}$ Clinical Alcohol Research, Department of Health Sciences, Lund University, Malmoe University Hospital, Lund, Sweden.

Objectives: Stress and use of alcohol and drugs often vary from day to day. The goal of these studies was to investigate whether Interactive Voice Response (IVR) could serve as a useful technology for real-time assessments of stress as well as alcohol and drug use.

Methods: Two samples are included. The first one is completed and includes 84 university students who were exposed to real-time IVR assessment three times daily during a total period of seven consecutive days. A second study is ongoing and will include 200 paroled clients to be followed daily during the first thirty days after release from prison. Half of the clients, randomised, will get automatic feedback on their results and simultaneously an e-mail report will be sent to the parole officer in order to provide a basis for further processing.

Results and conclusions: In the first study, all the participating university students were followed throughout the week (100\%). In total, stress and alcohol use was assessed in 1567 out of 1764 (89\%) data collection calls. Preliminary data from the second study show the same high response rate, and $89 \%$ of all daily data collection calls have been answered. IVR methodology thus offers a promising new technology for daily assessments of stress and use of alcohol and drugs in both university students and paroled offenders.

CORRESPONDING AUTHOR: Anne H Berman, PhD, Clinical Neuroscience, Karolinska Institutet, Huddinge, S-14104; anne.h. berman@ki.se 


\section{Thursday}

August 5, 2010

PAPER SESSIONS

2:30 PM-4:00 PM

OS13

\section{2:30 PM-2:48 PM}

OS13-B

\section{LONG-TERM PSYCHOLOGICAL IMPACT OF SARS} ON SURVIVORS

Parvaneh Moallef, MA, ${ }^{1,2}$ Paula Gardner, EdD ${ }^{2}$ and John Patcai, $\mathrm{MD}^{2}$

${ }^{1}$ York University, Toronto, ON, Canada and ${ }^{2}$ St. John's Rehab Hospital, Toronto, ON, Canada.

Little is known about the long-term psychological impact of SARS on patients who survived the illness. The SARS outbreak in 2003 infected more than 8000 people worldwide and killed almost 800 people (WHO, 2003). SARS is an unusual and contagious form of pneumonia, caused by a novel strain of the corona-virus (Peiris et al, 2003). Compared to research available on the biological or physical effects of SARS, there are relatively few published articles on the psychosocial impact of SARS and, to our knowledge, no follow-up studies exist beyond 18 months. The present study examined the early and long-term psychological effects of SARS on patients who received treatment at a rehab hospital in Toronto after contracting the illness in 2003. Method: 40 post-SARS patients completed measures pertaining to their level of well-being and psychological and physical health. Patients completed questionnaires first in 2004, shortly after recovery from the acute phase of SARS, and again in 2007. Results/Conclusion: Our findings indicated that well-being, general health, mental health, and vitality significantly deteriorated $(\mathrm{P}<0.05)$ from 2004 to 2007 in a large portion of the sample. Follow up study showed that, $87.5 \%$ of the sample population fell below average levels on measures of general health (based on the SF-36) in 2007 as compared to $48.6 \%$ in 2007 . Symptoms of anxiety, depression and posttraumatic stress persisted or intensified in a significant portion of post-SARS patients $(32.5 \%$ to $57.5 \%) 4$ years after infection. This apparent persistence or elevation in emotional distress among SARS survivors was both unexpected and concerning. Since patients had seemingly recovered from the acute phase of the illness, one would have expected a decrease in their level of psychological distress over time. A closer look at the relationship between the examined variables may provide further insight as to the underlying causes of patients' persistent symptoms. Regardless of the cause, as it currently stands, SARS appears to be a chronic illness.

CORRESPONDING AUTHOR: Parvaneh Moallef, MA, York University, Thornhill, ON, L4J 0C1; pmoallef@sympatico.ca
2:48 PM-3:06 PM

OS13-C

FACTORS INFLUENCE THE UPTAKE OF 2009 H1N1

INFLUENZA VACCINE IN A MULTIETHNIC ASIAN POPULATION

Li-Ping Wong, MSc, $\mathrm{PhD}^{1}$ and I-Ching Sam, MRCP, MRCPath ${ }^{2}$

${ }^{1}$ Medical Education \& Research Development Unit, Faculty of Medicine, University of Malaya, Kuala Lumpur, Malaysia and

${ }^{2}$ Tropical Infectious Diseases Research and Education Centre, Department of Medical Microbiology, Faculty of Medicine, University Malaya,, Kuala Lumpur, Malaysia.

Background: It has been shown that early vaccination against 2009 H1N1 influenza is cost-effective and may avert more deaths. Thus, whether or not public agree to be vaccinated is a crucial factor in controlling the $2009 \mathrm{H} 1 \mathrm{~N} 1$ influenza. To increase influenza vaccination rates, it is important to understand the motivators and barriers to vaccination. As the 2009 H1N1 influenza was previously referred to as "swine flu", it is not know how the Muslim community would react to the 2009 H1N1 vaccine as the name might lead to the perception that the vaccine may contain animal parts that may not be 'halal' (kosher) or unfit for Muslims to use. This study assessed a predominantly Muslim, multiethnic Malaysian community' attitudes toward the H1N1 influenza immunization. The aim of this study was to determine factors influence the uptake of 2009 H1N1 influenza vaccine in a multiethnic Asian population.

Methods: Cross-sectional, computer-assisted telephone interview. A total of 1025 multiethnic Malaysian adults responded to the survey (response rate 66.8\%). Interviews were conducted between October 6 and December 12, 2009.using a computer-assisted telephone interview (CATI) system.

Results: $70.2 \%(n=720)$ of overall participants indicated willingness to be vaccinated against the 2009 H1N1 influenza. Participants who indicated positive intention to vaccinate against 2009 H1N1 influenza were more likely to have favorable attitudes toward the 2009 H1N1 vaccine. The vast majority of Malay participants (95.1\%) were of the opinion that the H1N1 vaccine must be kosher for them to consume. Additionally, weather the vaccine was kosher was noted the main factor that determine Malay participants' decision to vaccination, whereas safety of the vaccine was the main factor that influenced vaccination decision for both the Chinese and Indian participants. Study identified sociodemographic variables and psychological impact (OR 1.16, 95\% CI 1.09 to $1.23, \mathrm{P}<0.001$ ) as significant predictors for intention to vaccination.

Conclusion: Study highlights the challenges in promoting the 2009 H1N1 vaccine. Findings suggest that ethnic-sensitive efforts may be needed to reach out to and gain acceptance of H1N1 vaccine in country with diverse ethnic communities and religious practices. Health authorities and public media need to initiate considerable amount of psychological impact of the H1N1 pandemic to the public to enhance vaccine acceptance.

CORRESPONDING AUTHOR: Li-Ping Wong, MSc, PhD, Medical Education \& Researc Development Unit, University of Malaya, Kuala Lumpur, 50603; wonglp@ummc.edu.my 
3:06 PM-3:24 PM

OS13-D

CHANGES IN ANXIETY AND INFORMATION SEEKING FOLLOWING THE H1N1 OUTBREAK: AN ANALYSIS OF WEB BLOGS AND WIKIPEDIA VISITS

Kate Faasse, MSc (Health Psych), ${ }^{1}$ Yla R. Tausczik, BA, ${ }^{2}$ James W. Pennebaker, $\mathrm{PhD}^{2}$ and Keith J. Petrie, $\mathrm{PhD}^{1}$

${ }^{1}$ Psychological Medicine, The University of Auckland, Auckland, New Zealand and ${ }^{2}$ Psychology, University of Texas, Austin, TX.

Background: The Influenza A/H1N1 outbreak was announced on April 24, 2009, and quickly made news headlines. This research investigated whether public anxiety about pandemic influenza was reflected in language use in web blogs, and whether the use of anxiety words on internet blogs was related to online informationseeking behaviour.

Methods: This research examined how word usage in web blogs (in particular anxiety, health, death and positive emotion words) differed between blogs that mentioned "swine flu" and control blogs published between April 24 and May 7, 2007. Web-based information-seeking behaviour was examined using the pattern of visits to H1N1-related Wikipedia pages from two weeks before to two weeks after the outbreak was announced. The relationship between information seeking and anxiety word use in web blogs was also investigated.

Results: Blogs mentioning "swine flu" contained significantly higher levels of anxiety, health and death-related words, and lower levels of positive emotion words that control blogs. Use of anxiety words decreased steadily over the two week study period following the announcement of the H1N1 outbreak. The peak in Wikipedia visits to H1N1-related pages was three days after the peak in anxiety word use measured in web blogs. Lagged Pearson's correlations between language use and daily webpage visits three days later showed significant positive correlations between page views and use of anxiety words in web blogs $(\mathrm{r}=.76, \mathrm{p}<.01)$

Conclusions: The announcement of the H1N1 outbreak was associated with initial anxiety which decreased substantially over a two week time frame, and was found to be significantly associated with internet-based information-seeking behaviour. The analysis of web behaviour can provide a useful indicator of the level and changing pattern of public anxiety and informationseeking following an illness outbreak.

CORRESPONDING AUTHOR: Kate Faasse, MSc (Health Psych), Psychological Medicine, The University of Auckland, Auckland, 1142; k.faasse@auckland.ac.nz

\section{3:24 PM-3:42 PM}

OS13-E

DISCLOSURE OF HEPATITIS C POSITIVE STATUS AMONG PEOPLE SEEKING TREATMENT IN THE NEWCASTLE AND HUNTER REGION, AUSTRALIA

Sheree A. Kable, BMed, $\mathrm{PhD}^{1}$ and Gabrielle Murphy, BSW ${ }^{2}$

${ }^{1}$ Clinical Pharmacology, University of Newcastle, Newcastle, NSW, Australia and ${ }^{2}$ John Hunter Hospital, Newcastle, NSW, Australia.
Hepatitis $\mathrm{C}(\mathrm{HCV})$ is a blood borne virus: $75 \%$ of patients develop chronic infection $(\mathrm{HCV}+)$ and are at risk of cirrhosis, liver failure and cancer. While therapy can clear HCV in $50-80 \%$, side effects may be debilitating. Disclosure of $\mathrm{HCV}+$ status is at the discretion of the individual, but may be precipitated by the decision to seek treatment. The aim of this study was to determine whether patient characteristics were associated with patterns of disclosure in $\mathrm{HCV}+$ people seeking treatment.

Method: As part of routine care in a tertiary referral clinic, a database has been maintained which includes key demographic data and details of psychosocial assessments undertaken by a social worker. De-identified data was analysed from 2001 to 2008 inclusive.

Results: There were 548 complete records, mean age 42.5 years, $68 \%$ male. Most patients with a partner (married/de facto) disclosed to them (97\%) but were less likely to disclose to anyone else, compared with other patients $(\mathrm{p}=0.0002)$. Women were more likely to disclose to a friend compared with men $(p=0.0063)$. Younger patients were more likely to disclose to parents or siblings, while older patients were more likely to disclose to their children (Chi2 test for linear trend: $\mathrm{p}<0.0001)$. Of patients with dependent children (62\%), single parents were less likely to disclose to children than others $(p=0.027)$.

In multivariate analysis, patients were more likely to disclose to at least one person (other than a partner) if they were divorced, separated or widowed (OR 6.17, CI 1.46, 26.00) and if they been diagnosed for $>5$ years (OR 4.18, CI 2.09, 8.38), and those who lived in their own home were less likely to disclose (OR $0.45, \mathrm{CI}$ $0.25,0.80$ ). Patients were less likely to disclose to an employer or work colleague if they were single (OR 0.17, CI 0.04, 0.73).

Conclusions: Patient characteristics are associated with patterns of disclosure in an $\mathrm{HCV}+$ population seeking treatment.

CORRESPONDING AUTHOR: Sheree A. Kable, BMed, PhD, Clinical Pharmacology, University of Newcastle, Newcastle, NSW, 2308; sheree.kable@newcastle.edu.au

\section{OS14}

\section{2:30 PM-2:48 PM}

OS14-A

NEGATIVE EMOTIONAL PROCESSING

WITHOUT AWARENESS INCREASES BLOOD PRESSURE

Jos F. Brosschot, PhD and Wopke Hoeben, MSc

Psychology, Leiden University, Leiden, Netherlands.

There is growing evidence that conscious types of perseverative cognition (PC), such as worry and rumination, can increase healthrelevant physiological parameters, and that $\mathrm{PC}$ may mediate the prolonged physiological effects of psychological stress. There are, however, several indications that part of PC is unconscious and may be responsible for a considerable part of daily prolonged physiological activity. For example, worry itself appears to be associated with prolonged physiological effects, both during nocturnal sleep and in daily life, up to two hours after worry episodes. The existence of unconscious PC (UPC) is likely 
because most cognitive processes are (partly or entirely) unconscious. Moreover, several studies using subliminal (under awareness threshold) negative emotional stimuli suggest that unconscious emotional cognition increase amongst others amygdala activity and skin conductance level. We hypothesized that subliminal negative stimuli also increase more substantial, healthrelated parameters such as blood pressure. Showing this would imply crucial support for the notion that UPC can have healthrelevant physiological effects.

Eighty college students anticipating an examination in the next two weeks, were shown either (1) subliminal negative words, such as "failure" and "stupid", (2) subliminal neutral words, (3) supraliminal (above awareness threshold) negative words, or (4) neutral supraliminal words, during a neutral computer task. For subliminal stimulation as masking procedure was used. Blood pressure was measured continuously using a Portapres Model-2. An awareness check ascertained that no subject could consciously perceive the subliminal stimuli

Results indicated that while negative words decreased systolic blood pressure marginally significantly across awareness conditions $(\mathrm{F}(1,77)=2.265, \mathrm{p}<.10)$, they did so clearly and significantly in the subliminal condition $(\mathrm{p}<.05)$, but hardly and nonsignificantly in the subliminal condition. Diastolic effects were in the same direction but nonsignificant. High trait anxiety but not high trait worry augmented these differential effects of awareness of negative stimuli. The effects could not be explained by conscious PC or mood.

Concluding, the results suggest that unconscious but not (or to a lesser extent) conscious negative emotional information can increase blood pressure. This lends credibility to the hypothesis that unconscious emotional processing (UPC) may have physiological consequences, and warrants further studies, including real life studies, using available measures of unconscious emotion, to examine robustness and magnitude of similar effects in real life.

CORRESPONDING AUTHOR: Jos F. Brosschot, PhD, Psychology, Leiden University, Leiden, 2300RB; brosschot@ffsw.leidenuniv.nl

\section{2:48 PM-3:06 PM}

OS14-B

RELATION OF QT-VARIABILITY INDEX AND EJECTION FRACTION TO T-WAVE ALTERNANS IN DEFIBRILLATOR PATIENTS DURING EXERCISE AND MENTAL STRESS

Anna Ghambaryan, ms md, ${ }^{1}$ Mark C. Haigney, MD, ${ }^{1}$ Heather Rogers, PhD, ${ }^{1}$ Robert E. Goldstein, MD, ${ }^{1}$ Willem J. Kop, $\mathrm{PhD}^{2}$ and David S. Krantz, PhD ${ }^{1}$

${ }^{1}$ Uniformed Services University, Bethesda, MD and ${ }^{2}$ University of Maryland, Baltimore, MD.

Variability of ventricular repolarization (VVR) is associated with increased risk for malignant arrhythmias. Little is known about the pathophysiological mechanisms involved in mental stress (MS)induced arrhythmias in coronary patients with an implantable cardioverter defibrillator (ICD). T-wave alternans (TWA) is an established VVR marker. High levels of resting QT variability index (QTVI) and low levels of resting ejection fraction (EF) are other markers of increased arrhythmic risk. MS and Exercise (EX) differ in central and autonomic cardiac activation patterns. We hypothesized that ICD patients with high levels of TWA would have high levels of QTVI and lower levels of EF and that these linkages would be more pronounced during EX compared to MS. QTVI, TWA and EF were assessed at rest and TWA was also measured during MS (anger recall), and during bicycle ergometry in 43 ICD patients. HR rise during EX was higher than during MS $(\mathrm{p}<.001)$. After adjusting for HR, TWA during EX was significantly greater than TWA during MS, $\mathrm{p}<.05$. Resting QTVI was associated with TWA during MS $(\mathrm{r}=.52, \mathrm{p}<.01)$ and TWA during EX $(r=.48, p<.01)$. Similarly, EF was negatively associated with TWA during MS $(\mathrm{r}=-.43, \mathrm{p}<.01)$ and EX $(\mathrm{r}=-.38, \mathrm{p}<.01)$. The strength of the associations between TWA and QTVI or EF did not differ between EX and MS, $(p>0.8)$. These findings indicate that abnormalities in QTVI and EF at rest may identify similar augmentation of VVR as TWA during EX and MS in ICD patients. The similar influence of MS and EX on indices of VVR suggests that both stressors are potentially pro-arrhythmic, yet the performance characteristics of HR-adjusted TWA are different across the stressors, consistent with the different underlying physiology of the two challenge tasks.

CORRESPONDING AUTHOR: Anna Ghambaryan, ms md, USUHS, Chevy Chase, MD, 20815; aghambaryan@usuhs.mil

\section{3:06 PM-3:24 PM}

OS14-C

25-HYDROXYVITAMIN D DEFICIENCY IS ASSOCIATED WITH ELEVATED DEPRESSIVE SYMPTOMS: RESULTS FROM THE MULTI-ETHNIC STUDY

OF ARTHEROSCLEROSIS (MESA)

Jonathan A. Shaffer, PhD, ${ }^{1}$ Karina W. Davidson, $\mathrm{PhD},{ }^{1}$ Steven Shea, MD, MS, ${ }^{1}$ Bryan Kestenbaum, MD, MS, ${ }^{2}$ David S. Siscovick, MD, $\mathrm{MPH}^{2}$ and Ian De Boer, $\mathrm{MD}, \mathrm{MS}^{2}$

${ }^{1}$ Medicine, Columbia University Medical Center, New York, NY and ${ }^{2}$ Medicine, University of Washington, Seattle, WA.

Background: Depressive symptoms have multiple putative etiologies, and low 25-hydroxyvitamin D [25(OH)D] levels may be one, but this has been tested only once in older, Caucasian European adults. We tested if deficient or insufficient 25(OH)D levels are associated with elevated depressive symptoms in an ethnically and racially diverse sample of adults. Methods: MESA participants with low depressive symptoms (Center for Epidemiologic Studies-Depression Scale $($ CES-D) $<5$; $n=408)$ or elevated depressive symptoms (CES-D $\geqq 10 ; n=244)$ but without chronic kidney disease had levels of $25(\mathrm{OH}) \mathrm{D}$ assessed. Potentially confounding (i.e., age, sex, race, income, education, body mass index, season of data acquisition, study site, amount of moderate and vigorous physical activity) and exploratory causal covariates were also measured (smoking within the past 30 days, self- 
reported history of diabetes mellitus, total cholesterol (mmol/L), low-density lipoprotein $(\mathrm{mmol} / \mathrm{L})$, systolic blood pressure $(\mathrm{mmHg})$, diastolic blood pressure $(\mathrm{mmHg})$, and anti-depressant medication (tricyclic and non-tricyclic use). Results: Deficient levels of $25(\mathrm{OH}) \mathrm{D}(<15 \mathrm{ng} / \mathrm{mL})$ were present in $24.7 \%$ and insufficient levels $(\geqq 15-<30 \mathrm{ng} / \mathrm{mL})$ were present in $59.4 \%$ of participants. Those with $25(\mathrm{OH}) \mathrm{D}$ deficiency were nearly twice as likely to have elevated depressive symptoms adjusting for confounds (odds ratio, 1.94; 95\% confidence interval [CI], 1.05 to $3.58, \mathrm{P}=. .035$ ), and for exploratory covariates (odds ratio, 2.07; $95 \% \mathrm{CI}, 1.08$ to $3.96, \mathrm{P}=.028$ ). Results were invariant across sex, race, income, education, study site, season of data acquisition, body mass index, amount of moderate and vigorous physical activity and all exploratory covariates, although with low power to detect such differences. Conclusion: Vitamin D deficiency in diverse, apparently healthy adults is associated with a significant risk of elevated depression symptoms.

CORRESPONDING AUTHOR: Jonathan A. Shaffer, PhD, Medicine, Columbia University Medical Center, New York, NY, 10032; js3742@columbia.edu

\section{3:24 PM-3:42 PM}

OS14-D

\section{PLASMA INTERLEUKIN-6 LEVELS AND PTSD HISTORY} IN MIDLIFE WOMEN

Tamara L. Newton, $\mathrm{PhD},{ }^{1}$ Rafael Fernandez-Botran, $\mathrm{PhD},{ }^{2}$ James J. Miller, PhD, ${ }^{2}$ Alexander C. Cambon, MEng, ${ }^{3}$ Vicki Ellison Burns, $\mathrm{PhD}^{4}$ and Kristen E. Allison, $\mathrm{MA}^{1}$

${ }^{1}$ Dept. of Psychological \& Brain Sciences, University of Louisville, Louisville, KY; ${ }^{2}$ Department of Pathology and Laboratory Medicine, University of Louisville, Louisville, KY; ${ }^{3}$ School of Public Health and Information Sciences, University of Louisville, Louisville, KY and ${ }^{4}$ School of Nursing, University of Louisville, Louisville, KY.

Many who develop posttraumatic stress disorder (PTSD) ultimately recover. Recovered persons may, however, display "biological vigilance," or physiological reactions to trauma cues. We examined this using measures correlated with aging-related morbidity and mortality: circulating levels of C-reactive protein and plasma interleukin-6 (IL-6), along with stimulated IL-6 production. Apparently healthy, postmenopausal women with divorce histories completed interviews to assess current and lifetime PTSD in response to past intimate partner violence, or other traumatic events. Women, all without current syndromal PTSD, were classified by history of PTSD diagnosis: none (PTSD-; $\mathrm{n}=33$ ), subthreshold (PTSD+s; $n=18$ ), or syndromal (PTSD+; $n=12$ ). Women attended two research visits; for each, a blood draw was followed by questionnaires that were either benign (Baseline Visit 1), or trauma- and PTSD-related, plus a diagnostic interview (Visit 2 Trauma Assessment). Mixed effects models tested effects for PTSD History, Visit, and the PTSD History $\mathrm{x}$ Visit interaction. For circulating IL-6 levels only, there was a trend for a PTSD History $x$ Visit interaction, $F(2,59.4)=2.89, p=.06$. Interleukin-
6 responses to the two visits differed for PTSD- and PTSD+ women $(p=.03)$. For PTSD- women, IL-6 levels were higher at Baseline than Trauma Assessment $(p=.05)$. In contrast, for PTSD+ women, IL-6 levels were higher at Trauma Assessment than Baseline, although this difference did not reach statistical significance $(\mathrm{p}=.16)$. Further, at Baseline only, there was a trend $(p=.06)$ for lower IL-6 levels among PTSD + and PTSD $+s$ women, compared to PTSD- women. These preliminary results, limited by sample size, require replication. Tentatively, however, the pattern suggests that women with past syndromal PTSD may be characterized by baseline IL-6 hypoarousal, along with plasma IL-6 increases in anticipation of confronting trauma reminders. Supported by NIH R21AG024902.

CORRESPONDING AUTHOR: Tamara L. Newton, PhD, Dept. of Psychological \& Brain Sciences, University of Louisville, Louisville, KY, 40292; tlnewton@louisville.edu

\section{3:42 PM-4:00 PM}

OS14-E

NEURAL CORRELATES OF TINNITUS RELATED DISTRESS Dennis Golm, Dipl-Psych, ${ }^{1}$ Anne Meinhardt-Renner, Dipl-Psych, ${ }^{1}$ Tobias Moser, Prof Dr, ${ }^{2}$ Peter Dechent, PD $\mathrm{Dr}^{3}$ and Birgit Kroener-Herwig, Prof $\mathrm{Dr}^{1}$

${ }^{1}$ Clinical Psychology and Psychotherapy, University of Goettingen, Goettingen, Germany; ${ }^{2}$ Dept. of Otorhynolaryngology, University of Goettingen, Goettingen, Germany and ${ }^{3} \mathrm{MR}-\mathrm{Re}-$ search in Neurology and Psychiatry, University of Goettingen, Goettingen, Germany.

About $4 \%$ of the German population experience chronic tinnitus, but only a minority suffers from it to an extent affecting quality of life. In order to explain tinnitus related distress, the neurophysiological model predicts associations of the tinnitus signal with an activation in limbic structures in distressed compared to nondistressed tinnitus patients. The aim of the study was to test this hypothesis by identifying neural correlates of tinnitus annoyance. High and low distressed chronic tinnitus patients and healthy controls were examined in an emotional sentence task using functional magnetic resonance imaging (fMRI). During the task, participants evaluated the personal relevance of tinnitus related, general distress related and neutral sentences.

As a result high but not low distressed tinnitus patients showed activations in the Gyrus cinguli and Insula in reaction to tinnitus related vs. neutral sentences. Similar effects were found comparing distress related and neutral sentences. Additionally high distressed tinnitus patients showed an activation in the Gyrus cinguli in favor of tinnitus related compared to distress related sentences.

The effect seems to be a specific reaction to tinnitus related and not to generally distressing stimuli. So the results are in line with the neurophysiological model.

CORRESPONDING AUTHOR: Dennis Golm, Dipl-Psych, Georg-Elias-Mueller Institute of Psychology, University of Goetingen, Goettingen, 37073; dgolm@gwdg.de 
OS15

2:30 PM-2:48 PM

OS15-A

SOCIOECONOMIC VARIABLES ASSOCIATED

TO GEOGRAPHICAL VARIATIONS OF YOUTH HOMICIDE IN MEXICO

Guillermo J. Gonzalez-Perez, PhD and Maria G. Vega-Lopez, PhD Social Sciences, University of Guadalajara, Guadalajara, Mexico.

This paper analyzes the trend of the youth homicide rate (age groups 15-24 and 25-34) in Mexico in last 30 years and seeks to identify the socioeconomic variables that better explain the geographical variations of youth homicide rate in Mexico in recent years. The basic information for this study was obtained from official sources; trends of homicide rates by age groups 1524 and 25-34 between 1979 and 2007 were analyzed; for each Mexican state, homicide rates by each age groups analyzed in triennial 2005-2007 were calculated; through the use of multiple regression analysis (stepwise method) the variables that better explained the interstate variations in the homicide rates were identified. The results show that the homicide rate in both age groups have diminished in last 30 years, however these rates are relatively high in the international context. Furthermore, although the male homicide rates are clearly higher in both age groups, this excess of male mortality has been notoriously reduced. Moreover impunity, drug trafficking, social exclusion and firearms possession are key elements to understand the geographic variations of the youth homicide mortality in Mexico in the analyzed triennial. In particular, drug trafficking has a great importance to analyze the variations of the homicide rate in 25-34 years old group. The found regression model equations explain more of $50 \%$ of the geographic variations of the homicide rate in both age groups. In this context, findings suggest that if Mexican government wants to reduce the geographical disparities in youth homicide rates, needs to develop new strategies to combat drug trafficking, establish programs to restrict firearm possession, and implement structural reforms to reduce poverty, social marginalization and socioeconomic disparities among states.

CORRESPONDING AUTHOR: Guillermo J. Gonzalez-Perez, $\mathrm{PhD}$, University of Guadalajara, Guadalajara, 44630; ggonzal@ cencar.udg.mx

\section{2:48 PM-3:06 PM}

OS15-B

ADOLESCENT AGGRESSORS IN HIGH SCHOOL IN GUADALAJARA, MEXICO

Maria G. Vega-Lopez, PhD and Guillermo J. Gonzalez-Perez, PhD Social Sciences, University of Guadalajara, Guadalajara, Mexico.

The present paper seeks to establish the prevalence and type of violence among adolescents enrolled in public high schools and to analyze the social and cultural factors associated with aggressor's condition. This is a cross-sectional, analytic type study. The data were gathered by questionnaire applied to 661 students enrolled in public high schools, during the period from December of 2008 to June of 2009. In the sample selection approach - random and multi-stage - it was considered the entirety of state's high schools of the metropolitan area of Guadalajara, Mexico.

According to obtained data, $48 \%$ of students were involved in some kind of violent event; $25 \%$ as victims, and $15 \%$ within a double role: eventually either as victim or aggressor, it was found that 8 out of 100 students were aggressors. Within the aggressors group around $30 \%$ were women and $70 \%$ were men. In the multivariate analysis for both genders, three are the variables that better explain aggressor's status: gender (OR 1.8), to be victimized for the family (OR 2.6) and to be involved in situations that unchain a police detention (OR 3). Conclusion: Before entering school the adolescent has blended a series of family experiences (interfamilial violence) and peer relationships (participation in gangs exhibiting criminal records) -that allow to forecast what could be the way of relating with schoolmates. Although the school becomes the new scenario where the adolescent has the opportunity to play a role already learned, in turn, this could become the field where the necessary transformation of risk behaviors and/or the learning of styles for social affront are mediated.

CORRESPONDING AUTHOR: Maria G. Vega-Lopez, PhD, Social Sciences, University of Guadalajara, Guadalajara, 44630; mgvega.lopez@gmail.com

\section{3:06 PM-3:24 PM}

OS15-C

COGNITIVE DIMENSIONS RELATED TO COUPLE

VIOLENCE OF WOMEN THAT ARE INVOLVED IN IT

Dolores Mercado Corona, PhD, Laura Somarriba, MA, Dulce Alejandra Guerrero, Psic, Michelinne Aviles, Psic and Cuevas Corina, $\mathrm{PhD}$

Psychology School, National University of Mexico, Mexico, Mexico.

In this paper are presented some cognitive dimensions related to couple violence studied in a group of women involved in it.

Violence is a major social problem in the current life around the world. Family violence is the most common and continual type and couple violence has the highest prevalence.

In any case it is a risk for health, it produces stress that for long periods of time affecting the functioning of the Immunological System.

Violence as an aversive situation, is expected to have an avoidance or escape reaction. But as a world mean, it takes a woman near to 15 years to go out of a violent relationship.

Staying in a violent partner relationship, is considered codependency with the aggressor.

Multiple factors are involved for staying in a violent partner relationship: cultural, social and personal.

Is it possible to identify the cognitive and social factors related to couple violence in women that are involved in a violent relationship? Could these factors explain women codependency with the aggressor? 
To aim these objectives, it was developed a self report inventory based on cognitive, affective, and personality theories.

441 women that have being involved in a violent relationship and that look for governmental support programs answered the inventory. Their ages were from 18 to 62 years old. The Socioeconomic and educative levels were from low to middle.

The responses were grouped into 6 dimensions: Strength for coping with the violent situation, Feelings of harassment or fear, Anticipation of consequences, Emotional dependency, Self efficacy, and Self confidence.

These show elements of cognitive structure in women that suffer violence as a result of the social and cultural variables an o the violence experience.

In the next research phase, it will be to identify the dimensions that show which dimensions produce differences between women that stay in the relationship and women that are out of it. These results will help to develop programs to support them.

CORRESPONDING AUTHOR: Dolores Mercado Corona, PhD, Psychology School, Ntional University of Mexico, México, D.F., 14140; doloresm@servidor.unam.mx

\section{3:24 PM-3:42 PM}

OS15-D

ACUTE STRESS DISORDER, PTSD AND PARTIAL PTSD AMONG ROAD TRAFFIC ACCIDENT VICTIMS

Tânia S. Pires, PhD Student and Ângela Maia, Professor

Psychology, University of Minho, Braga, Portugal.

Background: Road traffic accidents (RTA) are an usual traumatic event. In case of serious accidents, behind physical injuries, victims can develop psychological symptoms and problems s (e.g. Acute Stress Disorder (ASD) and PTSD) that interfere with quality of life. Literature shows recent discussion on predictors of ASD and PTSD in these victims.

Methods: 124 RTA victims (34 women and 90 male) were evaluated 6 days (T1) and 4 months (T2) after the accident. At T1 participants filled a demographic questionnaire that asked about risk perception, Standford Acute Stress Reaction Questionnaire, Peritraumatic Dissociative Experiences Questionnaire. At T2 participants filled a 17 items PTSD scale.

Findings: At T1 $64,5 \%$ had ASD. At T2 58,9\% had PTSD and $21 \%$ had partial PTSD. PTSD symptoms were positively correlated with risk perception $(\mathrm{rsp}=.266, \mathrm{p}<.01)$, dissociative experiences $(\mathrm{rsp}=.407, \mathrm{p}<.01)$ and acute stress symptoms $(\mathrm{rsp}=.460, \mathrm{p}<.01)$. In a regression analysis gender, risk perception, dissociative experiences and ASD symptoms accounted for $27,6 \%$ of PTSD symptoms variance. Women report more ASD symptoms and PTSD symptoms than men.

Discussion: The participants in this study were victims all of victims of serious accident, and the number of subjects with PTSD is unusually high, suggesting the need for psychological evaluation and intervention. The predictors could be use to identify the ones that will be more disturbed after these events.
CORRESPONDING AUTHOR: Tânia S. Pires, PhD Student, University of Minho, Vila Nova de Gaia, 4430-225; taniasfpires@ hotmail.com

\section{3:42 PM-4:00 PM}

OS15-E

NEIGHBOURHOOD \& INTERPERSONAL VIOLENCE: GENDERED IMPACT, SECONDARY TRAUMA \& CHRONIC STRESS AMONG YOUNG MALAYSIANS IN URBAN LOW COST HOUSING

Yut Lin Wong, DrPH and Sajaratulnisah Othman, MBBS

MERDU, Faculty of Medicine, University of Malaya, Kuala Lumpur, Malaysia.

Rapid urbanization has led to increasing violence, psychosocial and psychiatric morbidity, particularly in low income/poor communities. Such emerging violence amidst rising prosperity in Malaysia is posing serious challenges to behavioural medicine and healthcare.

In-depth qualitative interviews were conducted with 25 purposively sampled young respondents from 12 low-cost housing neighbourhoods within Kuala Lumpur to explore their perceptions and experiences of violence, and health impact.

The 25 multi-ethnic respondents, (12 men and 13 women) aged between 18-25 years, were residents of public low-cost high density housing where neighbourhood violence, viz. vandalism, street fights, robbery, drug dealing and homicide, was common. Perceptions of violence were varied and gender differentiated. Men tended to perceive crime as violence and serious violence to involve bleeding, hospitalization or death. Women regarded fights, injuring others and crime as violence and ranked murder and rape to be serious violence. Women and men were reluctant to intervene in domestic violence because they perceived it as a private matter although several respondents felt that a husband has no right to beat his wife. Men, more than women, had direct experience with violence. Some men had been robbed, threatened and beaten. Several men had perpetrated violence: selling drugs during teen years, involved in illegal motorcycle racing, and assaulting a neighbour. A couple of women had been robbed. None had suffered any physical injuries/disability due to violence or reported symptoms of severe psychological consequences viz. disturbed sleep, appetite and moods. Yet, women were more affected by high anxiety and pervasive fear of being victims of crime. Men were more concerned about safety of their families, especially female members. Girl child kidnapping, rape and murder happening in and around the neighbourhoods had created secondary traumatisation and strong sense of vulnerability to such violence among women. Women particularly experienced chronic stress in coping with the daily crime and violence in their neighbourhoods.

Gendered experiences and impact of violence with regards to secondary traumatisation and chronic stress point to more sensitive and specific interventions, considering both behavioural and social factors, among low income/poor urban communities. 
CORRESPONDING AUTHOR: Yut Lin Wong, DrPH, MERDU, Faculty of Medicine, University of Malaya, Kuala Lumpur, 50603; wongyl@um.edu.my

OS16

2:30 PM-2:48 PM

OS16-A

A LIFESTYLE INTERVENTION FOR WORKERS WITH AN ELEVATED CARDIOVASCULAR DISEASE RISK: OPENING THE BLACK BOX

Iris Groeneveld, MSc, Karin I. Proper, PhD, Saida Absalah, MSc, Allard J. van der Beek, Professor, $\mathrm{PhD}$ and Willem van Mechelen, Professor, PhD, MD

Public and Occupational Health, VU Medical Center, Amsterdam, Netherlands.

Introduction: Usually, at the end of a lifestyle intervention trial, investigators present the predefined intervention strategy as well as the study Results: In practice, the intervention may not have been performed as intended. In the Health under Construction Study, 27 occupational health professionals (counselors) performed a six-month motivational interviewing-based lifestyle intervention for construction workers. The intervention was aimed at changing dietary, physical activity, and smoking behavior, and thereby lowering cardiovascular disease risk. The purpose of this process evaluation was to evaluate the counselors' adherence to the intervention protocol, their competence, and the associations between adherence, competence and body weight at follow up.

Methods: The number of sessions and prescribed items discussed in the first counseling session were registered by the counselors. Counselors' competence in listening, supporting, motivating, and informing was rated by both participants and counselors. Adherence to motivational interviewing was determined by expert scoring of 19 audio-taped fragments. Associations between reach, dose, and competence, and body weight at follow-up were determined by linear regression analyses.

Results: Two-thirds of all participants attended five or more sessions, and $38.5 \%$ attended all seven sessions. In $90.2 \%$ of all cases, the counselor discussed all four prescribed items in the first session. Adherence to motivational interviewing was concluded from only one audio-taped fragment. 86.3 percent of all participants agreed with the counselor being competent. Neither counselors' competence, nor number of sessions or items discussed, was significantly associated with body weight loss.

Conclusions: Performing five sessions and discussing four prescribed items was feasible for the counselors, whereas adhering to motivational interviewing was not. Still, participants were positive about the counselors' competence and willing to attend the counseling sessions. Investigators are encouraged to report the evaluation of their intervention process, in order to improve future lifestyle interventions in research or in practice.

CORRESPONDING AUTHOR: Iris Groeneveld, MSc, Public and Occupational Health, VU Medical Center, Amsterdam, 1081 BT; iris.groeneveld@vumc.nl

\section{2:48 PM-3:06 PM}

OS16-B

EFFICACY OF A SPIRITUALLY-BASED INTERVENTION

TO INCREASE COLORECTAL CANCER AWARENESS

AND SCREENING IN AFRICAN AMERICAN CHURCHES

Cheryl L. Holt, $\mathrm{PhD},{ }^{1}$ Michelle Martin, $\mathrm{PhD},{ }^{2}$ Isabel C. Scarinci, $\mathrm{PhD}, \mathrm{MPH}^{2}{ }^{2}$ Chastity McDavid, $\mathrm{PhD},{ }^{2}$ Sandre McNeal, MS, ${ }^{3}$ Mark Litaker, $\mathrm{PhD},{ }^{3}$ Mohamad Eloubeidi, MD, ${ }^{4}$ John Bolland, $\mathrm{PhD},{ }^{5}$ Martha Crowther, $\mathrm{PhD}^{6}$ and Crystal Lee, $\mathrm{MS}^{2}$

${ }^{1}$ Public and Community Health, University of Maryland, College Park, College Park, MD; ${ }^{2}$ Division of Preventive Medicine, University of Alabama at Birmingham, Birmingham, AL; ${ }^{3}$ Diagnostic Sciences, University of Alabama at Birmingham, Birmingham, AL; ${ }^{4}$ Gastroenterology and Hepatology, University of Alabama at Birmingham, Birmingham, AL; ${ }^{5}$ College of Human Environmental Sciences, University of Alabama, Tuscaloosa, AL and ${ }^{6}$ Psychology, University of Alabama, Tuscaloosa, AL.

Colorectal cancer is the third leading cancer in incidence and mortality among US adults, and these rates are higher in the African American community than among other subpopulations. Community-based approaches have grown in popularity, in an effort to fight these cancer disparities. Though church-based approaches have been included among these initiatives, few are "spiritually-based" in nature, using spiritual/religious themes and writings to support the core health message. Such an approach may increase the cultural relevance of a health communication, and perhaps even its efficacy. The efficacy of a Community Health Advisor-led intervention to increase awareness and screening for colorectal cancer was examined using a randomized controlled trial, in which a spiritually-based approach was compared against a non-spiritual (e.g., secular) comparison intervention of same structure and content. Trained Community Health Advisors led two educational sessions on colorectal cancer. Study participants $(\mathrm{N}=358)$ completed baseline, 1-month, and 12-month follow-up surveys. A $90 \%$ retention rate was achieved over the course of the study period. Within group analyses suggest that the spiritually-based intervention resulted in significant increases from baseline to one-month follow-up in colorectal cancer knowledge $(\mathrm{p}<.001)$, perceived benefits to screening $(\mathrm{p}<.001)$, and significant decreases in perceived barriers to screening $(\mathrm{p}<.001)$. Comparisons between the spiritually-based and non-spiritual intervention indicate that the spiritually-based intervention was more efficacious than the non-spiritual for increasing perceived benefits to screening $(p<.001)$, while the non-spiritual was more effective for increasing knowledge $(\mathrm{p}<.001)$. Data are also examined among demographic subgroups such as men and women. Screening data at the 12-month followup are also reported. These findings suggest that the spirituallybased and non-spiritual approaches to health communication may have different impacts depending on the outcome and demographic subgroup being examined.

CORRESPONDING AUTHOR: Cheryl L. Holt, PhD, Public and Community Health, University of Maryland, College Park, College Park, MD, 20742; cholt14@umd.edu 
3:06 PM-3:24 PM

OS16-C

HUMOR USAGE IN MEDICINE

Gurprit Lamba, MD, ${ }^{1}$ Michael Henry, MD, ${ }^{1}$ Elizabeth Koby, $\mathrm{MD}^{1}$ and Vijay Aswani, $\mathrm{MD}^{2}$

${ }^{1}$ St. Elizabeth's Medical Center, Boston, MA and ${ }^{2}$ Marshfield Clinic, Marshfield, WI.

Background: Humor in medicine is not a speciality at present but is gaining recognition as a clinical tool.

Purpose: The purpose of this study is to review humor in a holistic manner and explore its various angles from its evolution to usage in the field of medicine.

Method: A literature search was conducted using PubMed restricting to the statements of "Laughter Therapy" and "Humor and Laughter." Additional articles were sourced from reference list of relevant articles. These were critically analysed to identify use of humor in medicine.

Results: This paper provides an overview of the field of humor focusing of what is known and not known about humor in medicine. This information serves as an important ingredient to reinforce the development of formal training in humor.

Conclusions: Based on this review we argue that knowledge of humor should be imparted to care givers as a potential therapeutic tool in the field of medicine.

CORRESPONDING AUTHOR: Gurprit Lamba, MD, St. Elizabeth's Medical Center, Boston, MA, 02135; gslamba@yahoo. com

\section{3:24 PM-3:42 PM}

OS16-D

FEASIBILITY OF USING TELECOMMUNICATIONS TECHNOLOGY IN A CARDIAC-DIABETES

SELF-MANAGEMENT PROGRAM

Chiung-Jung (Jo) Wu, Doctor of Health Sciences

School of Nursing \& Midwifery, Institute of Health \& Biomedical Innovation, Queensland University of Technology, Brisbane, QLD, Australia.

Background: Evidence demonstrates self-management programs are an effective approach to assist patients with chronic diseases such as type 2 diabetes or cardiac conditions to modify their lifestyle for better managing their conditions. Using information technology (IT) has great potential to support self-management programs and assist patients to fulfill their goals in managing their conditions more efficiently and effectively. Examples of different types of technology used in self-management programs that have limited research support include: text messages, telephone followup, web-based programs, and other internet-assisted education. But little is known about the applicability and feasiability of different forms of technology for patients with chronic diseases such as those with type 2 diabetes and critical cardiac conditions. Furthermore, although there is some evidence of the benefits of using IT in supporting self-management programs, further research on the use of IT in such programs is recommended.
Objective: To develop and pilot test an integrated CardiacDiabetes Self-Management Program (CDSMP) incorporating telephone and text-message follow-up.

Methods: A pilot study using randomised controlled trial is conducted in the coronary care unit (CCU) in a Brisbane metropolitan hospital in Australia to collect data on patients with type 2 diabetes admitted to CCU. The main outcomes included self-efficacy levels, knowledge, and quality of life.

Results: Initial results reveal that patients with diabetes admitted to the CCU in the experimental group did improve their self-efficacy, and knowledge levels.

Acknowledgements: This Project is funded by QUT Early Career Researcher Research Grant

CORRESPONDING AUTHOR: Chiung-Jung (Jo) Wu, Doctor of Health Sciences, School of Nursing \& Midwifery, Queensland University of Technology, Brisbane, QLD, 4059; c3.wu@qut.edu. au

\section{3:42 PM-4:00 PM}

OS16-E

DIFFERENCES IN USER ENGAGEMENT WITH DESIGN FEATURES OF INTERNET-BASED HEALTH BEHAVIOUR INTERVENTIONS

Leanne G. Morrison, MSc, ${ }^{1}$ Judith Joseph, PhD, ${ }^{1}$ Lucy Yardley, $\mathrm{PhD},{ }^{1}$ Panayiota Andreou, $\mathrm{MSc}^{2}$ and Paul Little, MB, MD, FRCGP ${ }^{2}$ ${ }^{1}$ School of Psychology, University of Southampton, Southampton, United Kingdom and ${ }^{2}$ School of Medicine, University of Southampton, Southampton, United Kingdom.

Previous research has identified two important user reactions to Internet-based health behaviour interventions: users trust in an intervention and the extent to which users respond to the intervention as if it were a person (e.g. anthropomorphically). The aim of this study was to examine how these user reactions might be linked to users' engagement with five specific design features of the 'Internet Doctor', an Internet-based intervention to promote the self-care of cold and 'flu symptoms. The five design features were: social context and support; visual appearance; credibility; tailoring; and usability. Twenty six adults aged 18 to 63 participated in 'think-aloud' interviews. Inductive thematic analysis and techniques from grounded theory were used to identify recurring patterns within the data. Three user reactions were identified: users' trust of the 'Internet Doctor'; users' anthropomorphic responses to the 'Internet Doctor'; and users' preference for human involvement in their healthcare. These reactions appeared to be linked to differences in user engagement with four of the five design features (social context and support; visual appearance; credibility; and tailoring). For example, some users who trusted the 'Internet Doctor' expressed positive views of social context and support features (e.g. a question and answer format that was analogous to face-to-face dialogue; information about the 'real' doctor behind the advice) - using them as justification to trust the website's diagnosis and advice. In contrast, participants who expressed a desire for human interaction and were aware of their own anthropomorphic responses to the 'Internet Doctor' were uneasy about features that provided social context and 
support. Different user reactions were hence associated with different types of engagement with the same design feature. The implications of these findings for the optimal design of Internet-based health behaviour interventions will be discussed.

CORRESPONDING AUTHOR: Leanne G. Morrison, MSc, School of Psychology, University of Southampton, Southampton, SO17 1BJ; 1m904@soton.ac.uk

\section{OS17}

2:30 PM-2:48 PM

OS17-A

IMPACT OF EXPOSURE TO CRITICAL INCIDENTS

IN HEALTH AND PSYCHOLOGICAL WELL-BEING

OF EMERGENCY AMBULANCE PERSONNEL

Dalia Marcelino, Master ${ }^{2}$ and Maria J. Figueiras, $\mathrm{PhD}^{1}$

${ }^{1}$ Psychology, University of Evora, Evora, Portugal and ${ }^{2}$ Psychology, Instituto Piaget - ISEIT, Almada, Portugal.

Objectives: The aim of this study is to investigate the influence of post-traumatic stress disorder (PTSD), peritraumatic dissociation, psychological distress and subjective health complaints in the psychological well-being of the emergency ambulance personnel. Methods: This is a cross-sectional study in which 250 ambulance personnel; both sexes completed a self-administered questionnaire, with measures of post-traumatic stress disorder, peritraumatic dissociation, psychological distress, subjective health complaints, psychological well-being and socio-demographic variables.

Results: The results indicated that ambulance personnel have on average some PTSD symptoms, while $10 \%$ have a clinical diagnosis of PTSD, peritraumatic symptoms are also significant, as well as psychological distress and health complaints. Correlational analyses indicated a negative significant association between symptoms and psychological well-being. The peritraumatic dissociation, the subjective health complaints, psychological distress and sex were found to be predictors of the post-traumatic stress disorder. There were significant differences concerning the socio-demographic variables in relation to the dependent variables.

Implications: The results of this study highlight relevant indicators of the need to develop and implement intervention programs to alleviate the psychological suffering, promoting the adjustment and the physical and psychological well-being in emergency ambulance personnel.

Conclusions: This study presents a contribution to understanding the psychological consequences of daily exposure to traumatic incidents, and its impact on health and well-being of emergency ambulance personnel.

CORRESPONDING AUTHOR: Maria J. Figueiras, PhD, Psychology, Instituto Piaget - ISEIT, Almada, 2800-305; mfigueiras@ almada.ipiaget.org
2:48 PM-3:06 PM

OS17-B

OCCUPATIONAL HEALTH AND HYDRATION STATUS AMONG WORKERS IN DEVELOPING COUNTRY

Rajan P. Bhandari, $\mathrm{MA}^{1}$ and Kalpana Panday, BN,BA ${ }^{2}$

${ }^{1}$ Community Health and Environmental Society Nepal, Kathmandu, Nepal and ${ }^{2}$ College Of Medical Sciences, Chitwan, Nepal.

Background: Nepal has different climate zones from ice freeze cold to hot in plain tarai regions. The maximum temperature is around 45-52o C. Implicit in all indices used for risk assessment in the prevention of heat stress is the assumption that workers are healthy and well hydrated; studies in Nepal cement, iron factory workers where workers are poorly hydrated, the level of protection offered by management strategies based primarily on environmental monitoring is compromised.

Objectives: To investigate the hydration status of expatriate workers during summer in a range of works as large numbers of expatriate workers are employed as manual labourers in industries under extreme heat stress conditions where heat illness is a significant concern. The aim was to ascertain whether the generally inadequate hydration status, is also an issue in these workers and make practical recommendations for control.

Methods: Studies were carried out at four sites to document the hydration status of exposed workers in different workplaces using urine specific gravity at three time points over two different work shifts.

Results: Although the workers were found in general to be better hydrated than their other zone counterparts, a high proportion were still found to be inadequately hydrated both on presentation for work and throughout the shift. Workers take less drinking water and more alcohol, smoking.

Conclusions: Interventions are required to ensure that workers in extreme heat stress conditions maintain adequate levels of hydration. Behavior change among workers to drink plenty of water, fluids and avoid alcohol, smoking, adequate sleep and rest. Failure to do so reduces the protection afforded by heat stress indices based on environmental monitoring. Regular Health assessment and behavior change programs in those areas is important for safety and reduce occupation health hazards as well.

CORRESPONDING AUTHOR: Rajan P. Bhandari, MA, Community Health and Environmental Society Nepal, Kathmandu, n/a; bhandari.rp@gmail.com

3:06 PM-3:24 PM

OS17-C

HEALTH BEHAVIOUR AND SAFETY AMONG INDUSTRIAL WORKERS IN DEVELOPING COUNTRY

Sanjit R. Joshi, MBA ${ }^{1}$ and Padam P. Prasain, $\mathrm{MA}^{2}$

${ }^{1}$ Jagdamba Spinning mills Pvt. Ltd, Kathmandu-35, Nepal and

${ }^{2}$ Jagdamba Cement Industries Pvt. Ltd, Kathmandu, Nepal. 
Background: Industrial Workers' health behaviour includes habits or actions related to physical exercise, nutrition, smoking, and drug or alcohol consumption. Unhealthy behaviour, and especially alcohol consumption, has been considered a source of accidents and injuries among construction workers. However, unhealthy behaviour can also be seen as a result of the safety and risk conditions of these jobs.

Methods: The purpose of this paper is to contrast the role of unhealthy behaviour as a source or as an outcome of safety and risk in the industrial sector. Data was collected from 180 workers belonging to a Industrial and construction company. Two path models representing these two hypotheses were tested. The model in which unhealthy behaviour is an antecedent of injuries did not fit the data (Chi square $=73.798, \mathrm{df}=3, \mathrm{p}<0.001)$.

Results: It supports the hypothesis of unhealthy behaviour as a result of safety and risk factors through the mediating effect of the experience of tension (Chi-square $=4.507, \mathrm{df}=2, \mathrm{p}=.212$ ). This model not only corroborates the stressful nature of exposure to risk and the absence of supervisors' safety response, but it also makes it possible to consider injuries as a cause of tension that, in turn, affects the employees' unhealthy behaviour.

CORRESPONDING AUTHOR: Sanjit R. Joshi, MBA, Jagdamba Spinning mills Pvt. Ltd, Kathmandu-35, n/a; joshi.sanjit@gmail. com

\section{3:24 PM-3:42 PM}

OS17-D

EFFORT-REWARD IMBALANCE AND ALL-CAUSE MORTALITY IN THREE POST-COMMUNIST COUNTRIES: THE HAPIEE STUDY

Hynek Pikhart, $\mathrm{PhD},{ }^{1}$ Martin Bobak, MD, PhD ${ }^{1}$ Anne Peasey, $\mathrm{PhD},{ }^{1}$ Andrzej Pajak, MD, ${ }^{2}$ Ruzena Kubinova, MD, ${ }^{3}$ Sofia Malyutina, $\mathrm{MD}^{4}$ and Michael G. Marmot, $\mathrm{MD}, \mathrm{PhD}^{1}$

${ }^{1}$ Epidemiology and Public Health, UCL, London, United Kingdom; ${ }^{2}$ Epidemiology and Population Studies, Jagiellonian University, Krakow, Poland; ${ }^{3}$ National Institute of Public Health, Prague, Czech Republic and ${ }^{4}$ Institute of Internal Medicine, RAMS, Novosibirsk, Russian Federation.

Background: Psychosocial factors at work have been previously shown to be related to different health outcomes in different populations but the evidence from transitional countries of Central and Eastern Europe is very limited. This presentation tries to fill this gap and focuses on the relationship between effort-reward imbalance (ERI), overcommitment and all-cause mortality in three middle-aged populations of Central and Eastern Europe in the HAPIEE Study.

Materials and Methods: 13,660 men and women in three population samples aged 45-69 years in Russia, Poland and the Czech Republic completed a questionnaire survey in 2002-2005 that included questions on the effort and reward at work, overcommitment, physical health, and a range of other social, demographic and behavioral indicators. All individuals were then followed for mortality through population mortality registers.
Mortality data are available in all 3 countries until the end of 2008. Cox regression modeling was used to estimate the association between ERI, overcommitment and all-cause mortality. Only men and women who were full-time working at the time of the interview were used for the analysis.

Results: After controlling for age, sex and range of possible confounding variables, the effort-reward ratio and overcommitment were significantly related to the all-cause mortality. Adjustment for socioeconomic position did not substantially change the Results: We did not find strong evidence for any interaction between socioeconomic measures and work-related psychosocial indicators.

Conclusions: Stress at work expressed by effort-reward imbalance and overcommitment is related to all-cause mortality in these three middle-age Central and Eastern European populations.

CORRESPONDING AUTHOR: Hynek Pikhart, PhD, Epidemiology and Public Health, UCL, London, WC1E 6BT; h.pikhart@ ucl.ac.uk

\section{3:42 PM-4:00 PM}

OS17-E

PSYCHOLOGICAL WELL-BEING AND REPEATED UNEMPLOYMENT EVENTS: ADAPTATION OR SENSITIZATION?

Cara L. Booker, PhD and Amanda Sacker, PhD

Institute for Social and Economic Research, University of Essex, Colchester, United Kingdom.

Throughout the course of a person's labour force participation, bouts of unemployment may be experienced. Studies have examined the relationship between single bouts of unemployment and psychological well-being as well as single and multiple bouts of unemployment with life satisfaction. This study examines the effect of multiple bouts of unemployment on psychological wellbeing. Specifically, whether people became sensitized, i.e. Psychological well-being becomes worse with each bout of unemployment, or adapted, i.e. Psychological well-being is worst with the first bout of unemployment and the impact of each additional period of unemployment is less than the first. Data came from the British Household Panel Survey, a large-scale longitudinal representative panel study. Psychological well-being was measured using the GHQ and participation in the labour force was self-reported; data were examined separately for males and females using multilevel modelling. The GHQ score at the first unemployment bout was used as the reference category. These scores were compared to scores from the period previous to the first unemployment bout, and at subsequent unemployment and re-employment bouts. Up to three unemployment and reemployment periods were allowed per individual.

Results show that there are definite gender differences in reactions to unemployment. Among males and females, psychological wellbeing worsened significantly during the period up to the first unemployment bout (difference scores 0.89 , p-value $<0.0001$ and 0.90 , p-value $<0.0001$ for males and females, respectively). Psychological well-being significantly improved during the first 
re-employment stage for both males and females (difference scores $0.95, \mathrm{p}$-value $<0.0001$ and 1.04 , $\mathrm{p}$-value $<0.0001$ for males and females, respectively). Psychological well-being scores were significantly lower for the second unemployment bout compared to the first for males $(0.74, \mathrm{p}$-value $<0.05)$ but not for females (0.02, p-value $>0.05)$. A test for linear trend was significant for both males and females; however differences between reactions became smaller with each unemployment period for males, but larger for females.

There were differences in response to multiple bouts of unemployment between males and females in this study. Males experienced adaptation in that the response to the first bout of unemployment was significantly greater than the response to subsequent bouts (trend test $\mathrm{p}$-value $<0.0001$ ). Females, however, experienced sensitization in that response increased with each bout of unemployment (trend test $\mathrm{p}$-value $<0.0001$ ). The findings suggest that males are able to adjust to multiple unemployment bouts while females' psychological well-being becomes worse.

CORRESPONDING AUTHOR: Cara L. Booker, PhD, Institute for Social and Economic Research, University of Essex, Colchester, CO4 3SQ; cbooker@esses.ac.uk

\section{Thursday \\ August 5, 2010 \\ POSTER SESSION (PST) 6:30 PM-9:00 PM}

\section{PST-100}

\section{FACTORS AFFECTING DURATION OF UNTREATED PSYCHOSIS IN JAPAN}

Kazuhito Yokoyama, MD, DMSc, ${ }^{1}$ Noriko Hagi, MN, ${ }^{2,1}$ Mitsuyuki Takamura, $\mathrm{MD}^{3,1}$ and Kayoko Urakawa, MA, RN, $\mathrm{PhD}^{4,1}$

${ }^{1}$ Epidemiology and Environmental Health, Juntendo University School of Medicine, Tokyo, Japan; ${ }^{2}$ Yokkaichi Nursing and Medical Care University, Yokkaichi, Japan; ${ }^{3}$ Research Project for Occupational Medicine, Mie University Graduate School of Medicine, Tsu, Japan and ${ }^{4}$ Stress Science, Mie University Graduate School of Medicine, Tsu, Japan.

A questionnaire survey regarding the factors affecting duration of untreated psychosis was conducted among 346 members of the association of family of psychosis patients in Mie Prefecture, Japan. Completed answers were returned from 138 members (118 parents, one spouse, 6 siblings and 13 patients themselves). Duration between the suspected first episode of psychosis and the first visit to 'physicians' (including primary care physicians, psychiatrists or others) was 18.5 months on average (SD 40.2). Forty-five subjects consulted family members or relatives first before visit to 'physicians'. It took 9.8 months on average (SD 11.2) for them to decide to visit, which were significantly shorter than the duration for subjects who consulted other than family members or relatives (22.8 months on average with 48.9 of SD) $(\mathrm{p}<0.05)$. Similarly, it took 40.2 months on average (SD 63.0) for 18 subjects who consulted teachers at school, which was significantly longer than the period for those who did not (15.1 months on average with 35.5 of SD) $(p<0.05)$. In case that reasons for visit to 'physicians' were physical symptoms (sleep disturbance etc), the mean span before the visit was 9.3 months (SD 12.1, 48subjects), which was significantly earlier than the visit because of other reasons (24.1 months on average, SD 50.1, 82 subjects) $(\mathrm{p}<0.05)$.

Stigma or prejudice is supposed to be a major barrier to medical care, considering more than half of subjects (71) were anxious about prejudice against psychosis before the visit.

CORRESPONDING AUTHOR: Kazuhito Yokoyama, MD, DMSc, Epidemiology and Environmental Health, Juntendo University School of Medicine, Tokyo, 113-8421; kyokoya@ juntendo.ac.jp

PST-101a

AN OBJECTIVE TEST OF TRAINING NEEDS OF HEALTH STAFF WORKING TO SUPPORT BREASTFEEDING

Louise Wallace, PhD C Psychol, Carol Bryce, MA, Susan Law, MSc and Damian Stanley, PhD

Coventry University, Coventry, United Kingdom.

Background: Breastfeeding rates in the USA and UK are among the lowest in the world, yet breastfeeding is known to be the most healthy feeding method for infant and mother. Staff training is an essential element for organisations wishing to achieve the evidence based UNICEF Baby Friendly Initiative (BFI) and since it is known that those who volunteer for training are often the most competent, an objective training needs analysis is required. A breastfeeding training programme is being delivered to healthcare staff in the West Midlands, UK. Prior to the training staff undertake an online objective evaluation of their breastfeeding knowledge.

Objective: To assess the levels of breastfeeding knowledge of healthcare staff working with mothers in England. To assess if training needs differed according to prior recent training in breastfeeding, years of service, and profession.

Methods: Breastfeeding knowledge was measured before the training programme was undertaken. A web based assessment tool Coventry University Breastfeeding Assessment (CUBA) was used to assess healthcare staff in eight sub scales relating to breastfeeding knowledge and practice.

Results: 248 healthcare staff completed the online assessment. Overall, knowledge levels in relation to the sub scale breastfeeding difficulties and positioning and attachment were highest with $93.1 \%$ and $95.2 \%$ respectively achieving $50 \%$ or higher. Knowledge of who breastfeeds and the challenges to breastfeeding scored the lowest with $54.5 \%$ and $46.4 \%$ respectively scoring less than $50 \%$. In six out of the eight categories $30 \%$ or more staff scored less than $50 \%$. T tests showed scores relating to positioning and attachment, difficulties, challenges, support and total scores were significantly higher for those who had received training within the last year whereas no differences were found by length of service. Midwives had significantly higher mean scores for supporting breastfeeding and practices compared to health visitors. 
Conclusions: These results indicate clear gaps in the knowledge levels of healthcare staff in the West Midlands of England. Knowledge levels vary across the eight subscales and it is possible to identify clearly the areas where further training is required. A training programme is being implemented to address these needs.

CORRESPONDING AUTHOR: Louise Wallace, PhD C Psychol, Coventry University, Coventry, CV1 5FB; hsx201@coventry.ac. uk

\section{PST-101b}

\section{MEASURING THE EFFICIENCY OF PRIMARY CARE/MENTAL HEALTH INTEGRATION WITHIN PRIMARY CARE AND THE IMPACT ON SPECIALTY MENTAL HEALTH UTILIZATION \\ Sarah L. Hartley, PhD and David Buyck, PhD \\ Mental Health Integration, VAMC Salem, Salem, VA.}

Integration of mental health and primary care services has made impressive strides in the past decade. Over the past three years, MH/PC integration has received unprecedented support within Veterans Affairs. This poster will detail how collaboration among primary care and mental health services at one VA has evolved into a successful integration program. The presentation/poster will include information about co-location, integration, and open-access as well as data regarding efficacy and model fidelity. Quantitative data will be presented regarding our universal suicide/homicide risk screening practice in primary care, our substance use co-integration initiative, and our integration project to address diabetes, HTN, and dyslipidemia. Qualitative data addressing primary care staff satisfaction with the program will also be presented. Four indicators of efficiency will be offered: (1) promptness of mental-health consultant availability, (2) market penetration (i.e., what percentage of patients seen are new patients), (3) brief visits to ensure rapid availability, and (4) efficient/ cost-effective utilization of specialty mental health resources (e.g., psychiatry, psychology, social-work clinics). With regard to efficiency/ cost-effectiveness, an integration program is considered more effective when more mild-moderate cases are treated in primary care, fewer referrals are sent to specialty mental health care and a greater proportion of specialty referrals are completed. These efficiency/cost-effectiveness markers offer an indication of whether a patient was appropriately triaged within primary care.

CORRESPONDING AUTHOR: David Buyck, PhD, Mental Health Integration, VAMC Salem, Salem, VA, 24153; david. buyck@va.gov

\section{CITATION ABSTRACT \\ TOP FIFTEEN SCORING POSTER}

PST-102

RESOURCE CHANGES AND THE EFFECT OF LIFE STRESS ON PSYCHOSOMATIC SYMPTOMS IN A 10-YEAR FOLLOW-UP

Olli Kiviruusu, MSocSc, ${ }^{1}$ Taina Huurre, $\mathrm{PhD},{ }^{1}$ Ari Haukkala, $\mathrm{PhD}^{2}$ and Hillevi Aro, MD, PhD, prof $^{1}$

${ }^{1}$ National Institute for Health and Welfare, Helsinki, Finland and ${ }^{2}$ University of Helsinki,

Helsinki, Finland.

This study examined the modifying role of psychosocial resources in the impact of life stress on psychosomatic symptoms in a 10-year follow-up period using a dynamic model of psychosocial resources in the stress coping framework. Subjects $(\mathrm{N}=1262)$ were participants in two follow-up surveys (1989/22yrs; 1999/32yrs) of a Finnish cohort study. The self-report measures included life stress (number of negative life events), psychosomatic symptoms (index of 17 somatic and mental complaints), and psychosocial resources (self-esteem, meaningfulness, locus of control). Analyses were done using change scores of life stress and psychosocial resources in structural equation modeling and regression analysis. Increase in life stress across 10 years was inversely related (standardized regression weight $=-.25, \mathrm{p}<.001$ ) to the change in psychosocial resources. Further, increase in psychosocial resources was inversely related $(-.38, \mathrm{p}<.001)$ to psychosomatic symptoms at 32 years controlling for the baseline symptom level. The total effect of change in life stress on psychosomatic symptoms at follow-up was decomposed to a significant direct effect $(.12, \mathrm{p}<.001)$ and a significant indirect effect through changes in psychosocial resources $(.10, \mathrm{p}<.001)$, indicating a case of partial mediation. The resource change moderated the effect of increasing life stress on psychosomatic symptoms ( $\mathrm{p}=.001)$, the effect being significantly smaller among those with increasing resources as compared to those with resource losses. The results lend support to the conservation of resources theory and emphasize the importance of dynamic conceptualization of psychosocial resources in the stress process: resource losses and gains form an important mediational path between life stress and health. The results also suggest that resource gains can compensate or buffer the negative effects of life stress on health even in the case of increasing life stress. 
CORRESPONDING AUTHOR: Olli Kiviruusu, MSocSc, Department of Mental Health and Substance Abuse Services, National
Institute for Health and Welfare, Helsinki, 00271; olli.kiviruusu@ thl.fi

\section{TOP FIFTEEN SCORING POSTER \\ PST-103 \\ EMOTIONAL STATE AND ILLNESS PERCEPTIONS PREDICT PERCEIVED FUNCTIONALITY IN PORTUGUESE BREAST CANCER PATIENTS \\ Susana M. Fernandes, $\mathrm{PhD}^{1}$ and Teresa McIntyre, $\mathrm{PhD}^{2}$ \\ ${ }^{1}$ Universidade Lusíada, Porto, Portugal and ${ }^{2}$ University of Houston, Houston, TX.}

This study tested whether illness perceptions and emotional state would be significant predictors of perceived functioning in women with breast cancer, after surgery and during adjuvant treatments. Participants were 74 Portuguese breast cancer patients (stages 0 to III), recruited in an Oncology Hospital. All women were treated with surgery, lumpectomies (55.4\%) or mastectomies (44.6\%). Age range was 23-73 (M=50.6, $\mathrm{SD}=11.21)$. Emotional state, illness perceptions (IP) and functionality were evaluated 15 days after surgery, and at four months, during adjuvant treatments. Emotional state, IP and functionality were assessed by the Hospital Anxiety and Depression Scale, the Brief Illness Perceptions Questionnaire, and the Sickness Impact Profile, respectively. Hierarchical multiple regression analyses were conducted. As expected, emotional state and IP were significant predictors of perceived functional limitations after surgery, in the areas of Locomotion, Mobility, Alertness and Social Activities (joint variance explained ranged from 17 to $28 \%$ ). Depression predicted perceived locomotion $(\beta=.26)$, mobility $(\beta=.35)$ and social activities $(\beta=.36)$, illness perceptions of Identity predicted perceived locomotion $(\beta=.37)$, mobility $(\beta=.24)$ and alertness $(\beta=.34)$, and IP of Consequences predicted perceived mobility $(\beta=.35)$, social activity $(\beta=.25)$ and alertness $(\beta=.36)$. The results during adjuvant treatments showed that emotional state and IP predicted self-reported limitations in Personal Care, Mobility, Alertness and Sleep ((joint variance explained ranged from 14 to $39 \%$ ). At both evaluations, higher depression, higher symptom endorsement and the perception of more negative consequences of the illness were associated with more perceived functional limitations. The results suggested that emotional state and illness perceptions after surgery and during adjuvant treatment play an important role in concurrent perceived functional limitations among Portuguese breast cancer patients and point to the need to consider emotional and cognitive factors in early intervention.

CORRESPONDING AUTHOR: Susana M. Fernandes, PhD, Universidade Lusíada, Porto, 4050-145; susanamrfernandes@, gmail.com

\section{PST-104}

MEDICINE USE FOR HEADACHE: THE EFFECT OF SOCIAL CLASS, STRESS AND SENSE OF COHERENCE

Vibeke Koushede, midwife MPH, ${ }^{1}$ Bjørn Holstein, MagScient Soc, ${ }^{2}$ Anette Andersen, MD, $\mathrm{PhD}^{2}$ and Ebba Hansen, MSc (Pharm) ${ }^{1}$

${ }^{1}$ Department of Pharmacology and Pharmacotherapy, Section for social pharmacy, Faculty of Pharmaceutical Sciences, University of Copenhagen, Copenhagen, Denmark and ${ }^{2}$ National Institute of Public Health, Southern University of Denmark, Copenhagen, Denmark.

Background: Studies show that social class and stress are associated with use of over-the-counter medicine, but the underlying mechanisms are not well understood. The first aim of this study was to examine the combined effect of social class and perceived stress on medicine use for headache among 25-44 year old men and women. The second aim of this study was to examine if sense of coherence (SOC) modifies the effect of social class and stress on medicine use for headache.

Methods: National cross-sectional study in Denmark. The study population consisted of men and women aged $25-44, n=955$. The survey was conducted by using web-based questionnaires and telephone interviews. The outcome measure was medicine use for headache within the past 14 days. The independent variables were perceived stress, social class and SOC. Headache prevalence and severity as well as response method were included as co-variates. Results: Among those who often felt stressed the odds for medicine use were $0.89(0.35-2.21)$ in the high social class, 1.98 (1.02-3.88) in the middle social class and 3.98 (1.66-9.56) in the low social class. The odds for medicine use among participants who often felt stressed with high SOC were $1.36(0.75-2.49)$ in the 
high social class and $4.37(1.57-2.17)$ in the low social class. The equivalent odds for participants with low SOC were 5.83 (1.87$18.23)$ in the high social class and $10.21(2.27-45.87)$ in the low social class.

Conclusion: There is a combination effect of social class and stress on medicine use. The lower the social class the steeper the association between stress and medicine use. Sense of coherence modifies the effect of social class and stress on medicine use for headache.

CORRESPONDING AUTHOR: Vibeke Koushede, midwife MPH, Department of Pharmacology and Pharmacotherapy, Section for social pharmacy, faculty of Pharmaceutical Sciences, Copenhagen, 2100; vik@farma.ku.dk

\section{PST-105}

\section{DEPRESSION AND SELF-MANAGEMENT BEHAVIORS} OF PATIENTS WITH T2DM AND STAGE 3 CKD

Teresa J. Sakraida, PhD, RN, Masako Terada, Doctoral Student and Rebecca C. Carron, Doctoral Student

College of Nursing, University of Colorado Denver, Aurora, CO.

Aim: Our overall aim was to characterize self-management (SM) of patients with type 2 diabetes mellitus (T2DM) and with stage 3 chronic kidney disease (CKD). We assessed depression as a correlate of SM.

Background: $\mathrm{T} 2 \mathrm{DM}$ is the leading cause of $\mathrm{CKD}$, a pre-dialysis, staged kidney disorder. $20-40 \%$ of adults with T2DM progress to CKD. Silent symptoms typify early CKD stages making their selfmanagement needs less obvious. It is known that T2DM regimen adherence may forestall glomerular damage, thereby slowing or even halting progression to kidney failure. $30-40 \%$ of patients with diabetes report elevated depressive symptoms. T2DM-CKD stressors associated with elevated depression rates include complications with treatment additions, poor glycemic control, and insulin use. Depression interferes with self-management behaviors

Methods: In this IRB-approved study, convenience sampling was used and subjects were enrolled from medical outpatient clinics. $\mathrm{N}=29$ completed measures. Inclusion criteria entailed: (1) T2DM, (2) 18 to 85 years, and (3) stage 3 CKD based on eGFR calculation. Beck's Depression Inventory (BDI-II) and the Summary of Diabetes Self-Care Activities (SDSCA) survey were completed. A BDI cut score of $\geq 13$ defined depression. Using SPSS v. 17, descriptive analyses of the sample and surveys were performed. Pearson R correlations were conducted to examine depression as a correlate of SM.

Results: These clinic-experienced subjects included 23 men and 6 women. The mean age was 65.6 years $(\mathrm{SD}=9.8 ; \mathrm{Mdn}=69.0)$ with 11 minority and 17 non-minority subjects. The mean education level was 13.8 years $(\mathrm{SD}=2.1$; $\mathrm{Mdn}=14)$. The mean $\mathrm{HbA1c}$ was $6.5(\mathrm{SD}=1.1)$. Most subjects $(\mathrm{n}=25)$ had minimal to no depression, while three scored as mild to moderate. The BDI total mean was $6.46(\mathrm{SD}=5.3)$ with symptoms of energy loss, sleep pattern change, and tiredness/fatigue most often reported. SM behaviors performed less often were exercise, physical activity, eating vegetables, and blood sugar checks. BDI total score correlated significantly with feet checking $(\mathrm{r}=-.53, \mathrm{p}=.004)$. Loss of energy correlated significantly with feet checking $(\mathrm{r}=-.40, \mathrm{p}=$ $.032)$ and exercise $(r=-.46, p=.014)$. Sleep pattern change correlated significantly with feet soaking $(\mathrm{r}=.56, \mathrm{p}=.002)$.

Implications: Depression symptoms of loss of energy and sleep pattern change were associated with decreased SM behaviors. Focused assessment to differentiate patient reasons for fatigue is warranted.

CORRESPONDING AUTHOR: Teresa J. Sakraida, PhD, College of Nursing, University of Colorado Denver, Aurora, CO, 80045; Teresa.Sakraida@ucdenver.edu

\section{PST-106}

PSYCHOSOCIAL ISSUES IN BLADDER CANCER SURVIVORS: RESULTS OF PATIENTS' INTERVIEWS Nihal E. Mohamed, PhD and Michael A. Diefenbach, PhD Department Of Urology, Mount Sinai Medical Center, New York, NY.

Background: The American Cancer Society estimates that in 2009 about 70,980 new cases of bladder cancer (BL Ca)will be diagnosed and 14,330 Americans will die of this disease. Surgery to remove the bladder is the standard therapy when bladder cancer is muscle-invasive. Removal of the bladder necessitates the diversion of urine following three reconstructive techniques. Although all types of urinary diversions offer similar recurrencefree survival rates, the three procedures can have significantly different effects on a patient's urinary and sexual functioning, social activities, and body-image. The present study explored BL $\mathrm{Ca}$ treatment side effects and difficulties in health care among patients treated for invasive bladder cancer.

Materials and Methods: Tape-recorded semi-structured interviews were conducted with eight patients treated with invasive bladder cancer (50\% women). Patients were on average 61.5 years of age and 2 years and 5 months post diagnosis and treatment. Three patients have illial conduit, one patient has continent reservoir, and 4 patients have neobladder. The interviews focused on: a) patients' understanding of invasive bladder cancer and treatment options; $b$ ) treatment side effects, social and psychological impact of treatment and side effects, and problems patients have/had with stoma and neobladder care following treatment.

Results: All patients reported having very little knowledge about treatment side effects and did not know what to expect after surgery. Four patients $(50 \%)$ followed their physicians' treatment recommendations and three patients $(37.5 \%)$ indicated that only one treatment option was discussed and recommended. Before treatment, all patients with neobladder and continent reservoir did not know how to empty the pouch or use a catheter. The three patients with ileal conduit reported difficulty using stoma appliances following treatment.

Conclusions: Invasive bladder cancer patients lack full information of treatment side effects and less likely to be prepared for stoma and neobladder care. Results emphasize the need for educational interventions to help patients cope with treatment side effect and improve post-treatment health care. 
CORRESPONDING AUTHOR: Nihal E. Mohamed, PhD, Department Of Urology, Mount Sinai Medical Center, New York, NY, 10029; Nihal.Mohamed@mountsinai.org

PST-107

STIGMA AND DISEASE: AIDS AND LUNG CANCER Lauren N. Hollrah, MS ${ }^{1,2}$ and Jennifer Antick, $\mathrm{PhD}^{2}$

${ }^{1}$ School of Professional Psychology, Pacific University, Hadley, MA and ${ }^{2}$ School of Professional Psychology, Pacific University, Hilsboro, OR.

The present study was an examination of the stigma of two chronic illnesses, AIDS and lung cancer. Stigma of both diseases is influenced by attitudes toward homosexuality, conservatism and traditional gender roles. The results of social stigma can be experienced at an individual level such as isolation, at the level of family, as well as community and society levels. Allocation of resources can be affected by stigma, thus reducing care available for people with stigmatized illnesses. This study surveyed participants on attitude toward people with chronic illness, homosexuality, conservatism and interaction styles. Five hypotheses examined factors affecting stigma toward people with AIDS as compared to people with lung cancer. Data were collected in survey form and analyzed. Results indicate that, within the study sample, AIDS was not as stigmatized as lung cancer, though disease in general remains stigmatized. Variables such as negative attitude toward homosexuality, conservatism, and willingness to interact with people contributed to attitude toward people with AIDS or lung cancer.

CORRESPONDING AUTHOR: Lauren N. Hollrah, MS, School of Professional Psychology, Pacific University, Hadley, MA, 01035; laurenh@pacificu.edu

\section{PST-108}

\section{LINGUISTIC MARKERS OF POSITIVE TREATMENT} RESPONSE TO MINDFULNESS-BASED COGNITIVE THERAPY FOR DEPRESSION

Maria Capecelatro, BS, Anne-Catharine Brown, BS 2012, Julia Bond, BS 2012, Amitte Rosenfeld, BS 2012, Evangeline KurtzNelson, BA 2011, Caroline Anderson, BS and Willoughby Britton, $\mathrm{PhD}$

Brown University, Providence, RI.

Recent studies have suggested that patterns of spontaneous word use may be sensitive markers of psychological state (Mehl, 2006) and psychological change (Cohn, Mehl and Pennebaker 2004). The purpose of this study was to investigate linguistic markers of depressed mood and positive treatment response to Mindfulness-Based Cognitive Therapy for depression. Fifty partially remitted depressed individuals were randomly assigned an 8-week MBCT or control condition. Before and after treatment, participants rated 27 photographs ( 9 neutral, 9 positive, 9 negative) from the International Affective Picture System (Lang, Bradley and Cuthbert ,1999) on dimensions of valence and arousal. Following a delay, participants were asked to recall as much detail as possible for each photo. Verbatim responses were analyzed with LIWC2007 for 5 categories of depression-related content (positive emotion, negative emotion, sex, appetite, and death). Participants completed the Beck Depression Inventory (BDI: Beck \& Steer, 1987) and the Mindful Attention Awareness Scale (MAAS; Brown and Ryan, 2003) before and after treatment phase completion. At baseline, only words related to appetite were related to depression scores, while emotion, sex and death-related word use were uncorrelated. Use of appetite words were negatively associated with BDI scores $(\mathrm{r}=$ $-.32, \mathrm{p}=.02)$ so that the actively depressed used less appetite words than those in remission $(\mathrm{BDI}<10),[\mathrm{t}(50)=1.8 \mathrm{p}=.01]$. BDI scores decreased significantly more in the MBCT group than controls $[\mathrm{F}(1,43)=9.3, \mathrm{p}=.004, \mathrm{~d}=.89]$, and improvements in depression were associated with increased appetite word use $(\mathrm{r}=-.54, \mathrm{p}=.006)$. MAAS mindfulness scores increased more in the MBCT group than controls $[\mathrm{F}(1,43)=8.6$, $\mathrm{p}=.001, \mathrm{~d}=1.02]$ and increases in appetite word use was associated with increases in mindfulness scores $(r=.41$, $\mathrm{p}=.05$ ). Results from this study suggest that spontaneous use of appetite-related words in response to photographs may be a sensitive indicator of the appetitive deficits that characterize depression

CORRESPONDING AUTHOR: Maria Capecelatro, BS, Brown University, Providence, RI, 02912; maria.capecelatro@gmail.com

\section{PST-109}

\section{AGE INFLUENCES ILLNESS PERCEPTIONS} AMONG PATIENTS WITH CHRONIC OBSTRUCTIVE PULMONARY DISEASE

Risa N. Long, BA and Charles F. Emery, PhD

Psychology, The Ohio State University, Columbus, OH.

Chronic Obstructive Pulmonary Disease (COPD) is the fourth leading cause of death in the United States. Physical impairment in COPD may contribute to reduced health-related quality of life (QoL). Increased symptoms of anxiety and depression among patients with COPD also may lead to reduced QoL (Di Marco et al., 2006). In addition, prior research has indicated that illness perceptions, specifically increased awareness of illness symptoms and greater perceived consequences, are associated with poorer QoL (Scharloo et al., 2007); and research suggests that a patient's age may influence attributions that patients make about symptoms with older patients tending to attribute illness symptoms to aging (Leventhal \& Crouch, 1997). No prior studies have examined the relationship between age and illness perceptions among patients with COPD. The present study evaluated 75 COPD patients (mean age $=63.24, \mathrm{SD}=9.37$ years) upon entry into an outpatient pulmonary rehabilitation program. All patients completed pulmonary function testing (FEV1, FVC), six-minute walk test, and self-report questionnaires, including the Illness Perception Questionnaire (IPQ; Weinman, Petrie, Moss-Morris \& Horne, 1996). Data were analyzed with Pearson correlations. Results indicated that age was inversely associated with two dimensions of the IPQ, identity $(\mathrm{r}=-.24$, $\mathrm{p}=.0452)$ and consequences $(\mathrm{r}=-.44, \mathrm{p}=.0002)$. These associations remained significant after controlling for disease severity (FEV1). 
Thus, younger age was associated with increased attention to COPD symptoms and greater likelihood of attributing illness consequences to disease severity. These results suggest that beliefs regarding illness may be more salient among younger patients with COPD than among older patients who are likely to have had more time to adjust to the disease.

CORRESPONDING AUTHOR: Risa N. Long, BA, Psychology, The Ohio State University, Columbus, OH, 43210; long.713@osu. edu

\section{PST-110}

PERSONALITY TRAITS, SELF-EFFICACY AND DEPRESSION IN PATIENTS AWAITING KIDNEY TRANSPLANT

Elisa K. de Castro, PhD and Caroline V. Thomas, MsC Health Sciences Center, Universidade do Vale do Rio dos Sinos, Porto Alegre, Brazil.

Purpose: To assess personality factors, self-efficacy and depression in patients awaiting kidney transplant. Participants: 65 patients under hemodialysis treatment in Brazil. Instruments: Big Five Questionnaire, General Self-Efficacy Scale, and Beck Depression Inventory. Results: Pearson correlation showed positive associations among the factors Surgency $(r=0,35, \mathrm{p}<0,01)$, Conscientiousness $(\mathrm{r}=0,41, \mathrm{p}<0,01)$ and self-efficacy; Neuroticism had negative correlation with self-efficacy $(\mathrm{r}=0,38, \mathrm{p}<0,01)$ and with total depression level $(\mathrm{r}=0.68, \mathrm{p}<0,01)$, psychological $(\mathrm{r}=0,67$, $\mathrm{p}<0,01)$ and somatic $(\mathrm{r}=0,62, \mathrm{p}<0,01)$; and Conscientiousness $(\mathrm{p}<0,05)$ and Agreableness $(\mathrm{p}<0,01)$ have also positively and significantly correlated with age and diagnostic age. In the linear regression model, neuroticism was a predictor in significant ways $(\mathrm{R} 2=0,47, \beta=5.35, \mathrm{p}<0,01)$ of depressive symptoms, whereas the Conscientiousness, Neuroticism and Surgency were predictors of the self-efficacy $(\mathrm{R} 2=0,33, \beta=0,23, \mathrm{p}<0,05)$. Conclusions: Personality factors should be considered in patients who are in a transplant list, because they seem to have an impact on patients' behavior and depression levels.

CORRESPONDING AUTHOR: Elisa K. de Castro, PhD, Health Sciences Center, Universidade do Vale do Rio dos Sinos, São Leopoldo, 93022-000; elisa.kerndecastro@gmail.com

\section{PST-111}

\section{DIFFERENCES IN THE QUALITY OF LIFE}

\section{BEFORE AND AFTER ADMISSION TO INTENSIVE CARE} UNIT

Rafael Ballester Arnal, doctorate in psychology, ${ }^{1}$ Sandra Gomez, Postgraduate, ${ }^{1}$ Beatriz Gil, Postgraduate, ${ }^{1}$ Ricardo Abizanda, $\mathrm{PhD}^{2}$ and Amparo Bernat, Postgraduate ${ }^{2}$

${ }^{1}$ Psicología Básica, Clínica y Psicobiología, Universitat Jaume I, Castellon, Spain and ${ }^{2}$ Hospital General Universitario, Castellon, Spain.

The medical experience of intensive care unit (ICU) is associated with many negative conditions such as isolation, loneliness, immobility or dependence, which have significant impact on patient status. Therefore, it is not surprising that patients report worse quality of life in both physical status and depression, anxiety and discomfort once being discharged from the unit (Garcia et al, 2003). Taking into account this fact and the importance of quality of life related to health, the aim of this study was to evaluate the differences in perception of quality of life reported by patients before and after admission to ICU. The study was based in 57 patients admitted to the ICU at General Hospital in Castellón. Of these patients, $70.5 \%$ were male and $29.5 \%$ female, with a mean age of 53.6 years. From this study, we excluded people who had any communicating problem, and also people who suffer a prior psychological disorder. The participants, whilst staying in ICU and after giving their consent, were administered various questionnaires. One of them was the EuroQol in its Spanish version (Badia et al, 1999). The overall results show lower quality of life after admission to the unit $(\mathrm{t}=13.65, \mathrm{p}<$ $0.00)$. When evaluating the dimensions separately, significant differences are observed in all of them (mobility, self care, ability to perform usual activities, pain/discomfort and anxiety/depression) for the total sample. When we analyzed the results by gender, we noticed differences before and after admission in all dimensions for men, whereas in women, no differences have been found in self care and anxiety/depression. Our results support findings in previous studies (Badia et al, 2008) suggesting that, although women have a worse quality of life before admission than men, these differences become smaller after the admission, showing a similar level of quality of life in both genders.

This work was supported by Spanish Ministry of Science and Education (PSI2008-01642/PSIC)

CORRESPONDING AUTHOR: Rafael Ballester Arnal, doctorate in psychology, Psicología Básica, Clínica y Psicobiología, Universitat Jaume I, Castellon, 12071; gomezs@psb.uji.es

PST-112

\section{CORRELATES OF ILLNESS BEHAVIOR} IN SCHIZOPHRENIA PATIENTS

Alexandra I. Mihailescu, MD, ${ }^{1}$ Oana Danciu, student, ${ }^{2}$ Silvia Ghilinschi, student ${ }^{2}$ and Cristian Mihailescu, $\mathrm{Dr}^{3}$

${ }^{1}$ Medical Psychology and Behavioral Sciences, University of Medicine and Pharmacy Carol Davila, Bucharest, Bucharest, Romania; ${ }^{2}$ University of Medicine and Pharmacy Carol Davila, Bucharest, Bucharest, Romania and ${ }^{3}$ Lotus Medica, Bucharest, Romania.

Background: Illness behavior in schizophrenia patients implies illness denial (lack of insight). Different studies of insight in schizophrenia have led to inconsistent findings and conflicting results alternatively stating that acknowledgement of one's mental illness is a detriment and a key to successful adaptation.

Objective: This cross-sectional study investigates the relationship between illness denial in schizophrenia and its influence on frequency of hospitalization and relapse, as well as the way insight relates to other important variables, such as depression, quality of life and optimism, patient's social context and available support system in Romania 
Methods: All patients diagnosed with chronic schizophrenia (DSM-IV-R), and having a history of relapse and admitted in a six month period were asked to participate in the study. Patients who gave consent (45 patients) were evaluated using the Quality of Life Enjoyment and Satisfaction Questionnaire (QLESQ-18), The Life Orientation Test-Revised (LOT-R), and the Perceived Stress Scale (PSS), Results to PANSS item G12 were used for the assessment of insight. Admission charts and interview were used for the assessment of social and familial support.

Results: The patients who had insight had also reduced optimism, a high rate of perceived stress, these being associated with a high frequency of hospital admissions and with a lower quality of life. 32 subjects, or $71 \%$, had severe insight deficits or even lack of insight. These findings were associated with higher optimism, and a lower rate of perceived stress. Patients who benefited of support system and had an adequate social context had fewer hospital admissions.

Conclusion: We could advance the premise that the incapacity to benefit from this higher insight is due to the poor quality of the supporting system in our country. It is vital to be able to connect the capacity of "being aware" that the patient has, with a proper supporting system and social context, in order to obtain a maximum of efficiency.

CORRESPONDING AUTHOR: Alexandra I. Mihailescu, MD, Medical Psychology and Behavioral Sciences, University of Medicine and Pharmacy "Carol Davila", Bucharest, Bucharest, 031723; andrast79@yahoo.com

PST-113

DEPRESSION AND PLACE - A DANISH FOLLOW-UP STUDY OF THE EFFECT OF MUNICIPALITY ON CHANGES IN THE MDI-SCORE

Ingelise Andersen, PhD, Charlotte Hougaard, MSc, Finn Diderichsen, Dr Med Sci, Karsten Thielen, MD, Margit Kriegbaum, MSc, Else Nygaard, $\mathrm{PhD}$ and Ulla Christensen, $\mathrm{PhD}$

Social Medicine, Public Health, Copenhagen University, Copenhagen K, Denmark.

Background: Individual risk-factors for depressive disorder are documented in several studies whereas neighbourhood etiologic factors for depression are relatively understudied.

Objective: The aim of this study is in a longitudinal design by means of hierarchical models to investigate whether wellestablished neighbourhood indices such as material deprivation and social fragmentation, respectively, as well as economical indicators of inequality at municipality level have an impact on the scoring at the MDI-scale (Major Depressive Inventory) after controlling for individual confounders.

Material: In March 2000 and in October 2006 a random sample of 5,953 individuals, $58 \%$ females, and aged $40-50$ years answered a range of questions on physical and mental health, demographic and socioeconomic factors, social relations, health behaviours and depression. Information on contextual components in 275 municipalities was obtained from was obtained from StatBank Denmark Results: The Congdon index on social fragmentation shows that the most deprived municipality has a z-score of -7.17, whereas the score for the most affluent is 17.9. The Townsend Material Deprivation Score Index is correspondingly skewed and range from -5.31 to 16.09 . The initial single item analyses showed a strong association between changes in the MDI-score and single resident households from the Congdon index and no car ownership from the Townsend index, respectively. In a gender stratified linear regression model the Townsend index for men significantly increases the MDI-score by $0.15(\mathrm{p}<0.01)$, the Congdon index increases by $0,08(\mathrm{p}<0.05)$ whereas every $20.000 \$$ increase in equivalent income, decrease the MDI score by $-1.25(\mathrm{p}<0.05)$. No significant results were found among women.

Conclusion: The study indicates that Congdon and Townsend indices and equivalent income are separately able to explain differences in the MDI-score at municipality level among men, but not significantly among women

CORRESPONDING AUTHOR: Ingelise Andersen, PhD, Public Health, Copenhagen University, Copenhagen K, 1014; inan@ sund.ku.dk

\section{PST-114a}

EMOTIONAL CLOSENESS PREDICTS IMPROVEMENT IN DEPRESSION AND ANXIETY FOLLOWING LIVER TRANSPLANTATION

Anne Eshelman, $\mathrm{PhD},{ }^{2}$ Marwan Abouljoud, $\mathrm{MD},{ }^{2}$ Tina Meyer, $\mathrm{PhD},{ }^{2}$ Diane Fischer, $\mathrm{PhD},{ }^{2}$ Dilip Moonka, $\mathrm{MD},{ }^{2}$ Daniel Paulson, $\mathrm{MA}^{1,2}$ and Kimberly Brown, $\mathrm{MD}^{2}$

${ }^{1}$ Institute of Gerontology/Dept. of Psychology, Wayne State University, Detroit, MI and ${ }^{2}$ Henry Ford Health System, Detroit, MI.

Background: Depression and anxiety symptoms are common among patients with end-stage liver disease (ESLD), symptoms which improve after liver transplant. Depression adversely affects subjective and objective evaluations of recovery following medical interventions. The present research tests the hypothesis that emotional closeness facilitates an adaptive positive affective reaction, denoted by reduction of depression and anxiety, following liver transplantation.

Methods: The sample included 74 liver transplant surgery candidates and their primary caregivers. Patients ranged in age (18-74) and were $62.1 \%$ male. Data was collected prior to transplant and at 6-month follow-up. Caregivers rated the "degree of closeness you feel in your relationship to the [patient]" on a Likert-type scale ranging from 0-7. The sample was divided into groups with caregivers reporting maximum closeness $(7 ; n=44)$ or less closeness $(0-6 ; n=30)$.

Results: Two repeated-measures ANOVAs were used to evaluate the influence of liver transplant and closeness on change in 
anxiety and depression as measured by the Hospital Anxiety and Depression scale. Both depression $(\mathrm{F}=23.12, \mathrm{p}<.001)$ and anxiety $(\mathrm{F}=13.33, \mathrm{p}<.001)$ scores decreased following transplant and borderline significant and significant interaction terms, respectively suggested greater improvement in depression $(\mathrm{F}=3.31, \mathrm{p}=.069)$ and anxiety $(\mathrm{F}=3.34, \mathrm{p}=.041)$ for patients with emotionally close caregivers. The closeness variable was confounded by gender $(\mathrm{X} 2=13.66, \mathrm{p}<.001)$ prompting post-hoc analyses which suggested that emotional closeness was critical for affective improvement in men, but less so for women, though interpretation is limited by a small sample size.

Conclusions: For patients with ESLD, depression and anxiety decline sharply after liver transplant, but declines are attenuated for individuals with emotionally distant caregiving relationships. These findings suggest caregiving relationships as a target for psychotherapeutic intervention among patients with ESLD.

CORRESPONDING AUTHOR: Daniel Paulson, MA, Institute of Gerontology/Dept. of Psychology, Wayne State University, Detroit, MI, 48202; paulson@wayne.edu

\section{PST-114b}

JAPANESE DIALYSIS PATIENTS' VIEWS OF AVANTAGES AND DISADVANTAGES IN HEMODIALYSIS

\section{AND PERITONEAL DIALYSIS}

Nanako Nakamura, $\mathrm{PhD}$

Clinical and Research Center for Human Development, Hyogo University of Teacher Education, Kato-shi, Japan.

Background and purpose: Despite a recent increase in the attention given to improving communication of chronic kidney disease (CKD) patients' decision-making (Murray, et al., 2009), understanding of patients view is still lacking in Japan. The purpose of this study was to describe Japanese dialysis patients' views about advantages and disadvantages of their dialysis treatments (CHD: in-center hemodialysis, CAPD: continuous ambulatory peritoneal dialysis, APD: automated PD).

Methods: Forty-one participants, including 17 CHD, 17 CAPD, and 7 APD patients (mean age: 58.5 year old $(\mathrm{SD}=13.6)$, mean dialysis period: 43.5 months $(\mathrm{SD}=29.2)$ ), were requested to undergo a semi-structured interview, and the data obtained were qualitatively analyzed.

Results: The participants considered that their dialysis modalities had its advantages and disadvantages. In terms of advantage, "less trouble for the people around me" was the most common answer of all dialysis modalities. "Independence", "less hospital visit", and "flexibility" were advantages of CAPD and APD patients. On the other hand, CHD patients consider the advantages of the treatment were "professional care" and "dialysis-free days". As for disadvantage, "catheter care" and "making trouble for the people around me" were the common answers of CAPD and APD patients. For CHD patients, "making trouble for the people around me", "restriction of diet and fluid", and "feeling bad after carrying out dialysis" were common answers.
Conclusions: Flexibility, autonomy, and professional care are key factors in subjective advantages for dialysis patients and these domains of advantages of dialysis are apparently similar to results in Western previous studies. In addition, results of this study indicate that Japanese patients' decision making needs to consider whether or not the dialysis modality make trouble for the people around patients.(This work was supported by KAKENHI 21730549.)

CORRESPONDING AUTHOR: Nanako Nakamura, PhD, Clinical and Research Center for Human Development, Hyogo University of Teacher Education, Kato-shi, 673-1429; nanako@ hyogo-u.ac.jp

PST-114c

ADDITIONAL APPROACHES TO BEHAVIORAL INTERVENTION FOR PATIENTS WITH A MENTAL HANDICAP

Takao Suzuki, Master's ${ }^{1}$ and Gosei Aoki, MD $^{2}$

${ }^{1}$ Waseda University, Tokorozawa, Japan and ${ }^{2}$ Tobu Maruyama Hospital, Satte, Japan.

In this descriptive study, we will demonstrate therapeutic processes of clinical cases of maladaptive patients with moderate mental retardation. Although behavioral interventions are one of the most effective treatments to such populations, the relationship often encounters difficulties and the therapy becomes unprogressive. It is partly because of their limitation of ability to use language which enables them to understand and express their thoughts, feelings and understandings. The aim of this descriptive study is to show some of the examples of inventive approaches which thought to be helpful to the progression of the therapies for the patients with a mental handicap.

Method (process of treatment): The patients were male $20 \mathrm{~s}$ and female $30 \mathrm{~s}$, both were unmarried and lived with their families. Three years of outpatient therapy, conducted once every two to three weeks, took place at the psychiatric hospital. Medication and behavioral interventions, including management of use of social welfare services and psycho-education, were given. In addition, the following approaches were developed in the course of treatment: (1) Psychiatrist and psychologist worked as a team to deal with the patients, and discussed about emotional burden that had been experienced. (2) The patients were asked to come to therapy by themselves and with their families alternatively. (3) Importance of attending work at the welfare facility was emphasized at each session and patients' refusals of work were disputed thoroughly. The patients gradually have become to hold back complaining and maladaptive behaviors decreased.

Discussions: In order to maintain the therapy relationships and prevent drop out, three approaches listed above seemed useful. Approach (1) may enable therapists to manage negative feelings toward patient which are often underestimated in the case of patient with mental handicap. Approach (2) may give patients the room for privacy and it may also enable to observe and approach family problems at the same time. Approach (3) may set strong therapeutic framework which enables both patients and therapists 
to realize small progresses that have been achieved through course of therapy. Quantitative research and experimental study will be needed to specify the effect of these approaches.

CORRESPONDING AUTHOR: Takao Suzuki, Master's, Waseda University, Tokorozawa, 359-1145; suz-takao@aoni.waseda.jp

\section{PST-115}

\section{RECRUITMENT AND RETENTION IN INTERVENTION} TRIALS: LISTENING TO HIV-POSITIVE MOTHERS' EXPERIENCES IN THE MOMS PROGRAM

Katharine E. Stewart, PhD, MPH, ${ }^{1}$ Michael K. Stracener, MSW, Lucy Annang, $\mathrm{PhD}^{3}$ and Susan L. Davies, $\mathrm{PhD}^{2}$

${ }^{1}$ Fay W. Boozman College of Public Health, University of Arkansas for Medical Sciences, Little Rock, AR; ${ }^{2}$ School of Public Health, University of Alabama at Birmingham, Birmingham, AL and ${ }^{3}$ Arnold School of Public Health, University of South Carolina, Columbia, SC.

Clinical and behavioral research is commonly challenged by difficulties in recruitment and retention, particularly for many underrepresented or vulnerable populations, including HIVpositive individuals, minority individuals, and individuals with low socioeconomic status. Indeed, recruitment rates in recent published reports of behavioral interventions focused on health improvement in such populations range from as low as $13.5 \%$ to as high as $59 \%$. Methods: We conducted post-intervention focus groups with 15 participants in the Making Our Mothers Stronger (MOMS) Project, a randomized behavioral intervention. MOMS participants were randomly assigned to either the experimental condition of a parenting skills intervention or to an attentioncontrol condition of a health related behaviors intervention. Two focus groups were conducted with participants from each intervention condition. A focus group guide ensured consistency between groups and covered participants' response to the MOMS Project as well as their perceptions and recommendations about recruitment and retention. Three coders developed a codebook using constant comparison to identify major themes and sub-themes. Results: $87 \%$ of the focus group participants were African-American; their mean age was 41 years and 53\% were single/never married. Identified themes included approaches to and venues for recruitment success, the role of key staff, effective and ineffective incentives, the impact of group process on recruitment and retention, and program resources that are needed. Discussion: Specific recommendations that emerged from the focus groups include recruiting in trusted venues, employing a persistent and encouraging recruiter who is sensitive to stigma issues, ensuring acceptability of recruitment materials through field-testing, ensuring social benefits of the intervention are immediate, and providing programs that are highly salient and practical.

CORRESPONDING AUTHOR: Katharine E. Stewart, PhD, MPH, Health Behavior \& Health Education, UAMS College of Public Health, Little Rock, AR, 72211; kestewart@uams.edu
PST-116

STEP: A HARM REDUCTIONIST APPROACH TO HIV TREATMENT ADHERENCE

Katie Querna, MSW

Treatment, Lifelong AIDS Alliance, Seattle, WA.

A. Introduction:

Taken consistently, highly active antiretroviral therapy (HAART), has demonstrated success in inhibiting HIV viral load replication and reducing morbidity and mortality for people living with HIV. However, adherence to HAART is often suboptimal, compromising the effectiveness of treatment. The Seattle Treatment Education Program (STEP) aims to mitigate treatment adherence barriers by providing education and support through empirically driven, strengths-based, behavior change interventions.

B. Description:

The STEP program is based upon a harm-reductionist framework, within a larger agency which ascribes to congruent ideology. Interventions are strengths-based and grounded in Rogerian, Motivational Interviewing and Solution Focused clinical orientations. Clients meet with a Treatment Education Coordinator (TEC) to assess their specific treatment-related questions, concerns, and barriers. The TEC and client strategize to create concrete steps to maintain adherence. Evidence-based tools, including symptom and barrier assessors, incentives, and regular follow ups, are utilized. Monitoring and evaluating outcomes is completed regularly through use of a specialized database, and qualitative surveys distributed to clients and referring parties.

C. Lessons Learned:

Eighty percent of STEP participants maintained or achieved the 95\% HAART adherence rate suggested for optimal treatment outcomes. The literature suggests that the grounding theories in which STEP is based are efficacious in brief treatment settings, and our outcomes data supports this assertion. Limitations of the intervention include participant attrition and insufficient access to comprehensive mental health and substance use management resources.

D. Further Recommendations:

For many, maintaining treatment adherence is challenging. Implementing programs that offer unlimited sessions of mental health counseling and substance use treatment or harm-reduction strategies for people living with HIV could greatly increase treatment adherence. Additionally, public health policy could be modified with the aim of de-stigmatizing those living with HIV/ AIDS. Effective legislation could include acts recognizing and legitimizing same sex couples. Comprehensive, accessible health care should be provided to anyone, regardless of socioeconomic status. These changes could help mitigate the shame reported by some people living with HIV/AIDS, potentially leading to greater help seeking behaviors and lower transmission rates. Additionally, embracing HIV testing as a routine component of primary care could help to reduce HIV incidence and minimize stigma, potentially leading to a number of positive personal and public health outcomes, including increased treatment adherence.

CORRESPONDING AUTHOR: Katie Querna, MSW, Lifelong AIDS Alliance, Seattle, WA, 98122; katieq@1laa.org 
PST-117

THE ASSOCIATIONS BETWEEN HIV RISK PERCEPTIONS (GLOBAL VS. CONDITIONAL ESTIMATES) AND RISK BEHAVIORS AMONG SEXUALLY ACTIVE CHINESE INJECTING DRUG USERS

Hi Yi Tsui, PhD Candidate, ${ }^{1}$ Joseph Lau, PhD, ${ }^{1}$ Renfan Wang, $\mathrm{MD}^{2}$ Hongyao Chen, MD, ${ }^{2}$ Chuliang Liu, $\mathrm{MD}^{3}$ and Jun Liu, $\mathrm{MD}^{3}$

${ }^{1}$ Centre for Health Behaviours Research, School of Public Health and Primary Care, The Chinese University of Hong Kong, Hong Kong, China; ${ }^{2}$ Dazhou Center for Disease Control and Prevention, Dazhou, Sichuan, China and ${ }^{3}$ Hengyang Center for Disease Control and Prevention, Hengyang, Hunan, China.

Risk perception is central to HIV research and intervention. Yet, empirical findings concerning the perception-behavior link have been inconsistent. Despite the efforts made by quite a few researchers in identifying inherent conceptual and methodological limitations in the literature, only a few studies have explicitly addressed these issues. Among others, one such issue related to the use of global (unconditional) and conditional measure of risk perception. In this study, the significance of the associations between HIV-related risk behaviors and perceived risk of contracting HIV measured by two approaches (global and conditional sexual/injecting risk estimates) was tested and compared among a group of sexually active male injecting drug users in China. Of all 456 respondents, $82.5 \%$ had unprotected sex and $11.2 \%$ shared syringes with others for drug injection in the past six months. In response to the global risk perception, $12.5 \%$ perceived high chance of HIV infection. When the risk perception is conditional on unprotected sex and syringe-sharing respectively, the corresponding figures were $38.8 \%$ and $71.9 \%$. Logistic regression analyses revealed that syringe-sharing was positively associated with global risk perception (Adjusted odds ratio $(\mathrm{AOR})=4.29 ; 95 \% \mathrm{CI}=2.07,8.92)$ but negatively associated with conditional injecting risk perception $(\mathrm{AOR}=0.53 ; 95 \% \mathrm{CI}=$ $0.29,0.97$ ). Unprotected sex was not associated with global risk perception but was negatively associated with conditional sexual risk perception $(\mathrm{AOR}=0.46 ; 95 \% \mathrm{CI}=0.27,0.76)$. There are some common and different predictors for the three measures of risk perceptions. The results highlight and confirm the importance of the choice of measures in assessing HIV risk perception. Overall, risk perception should remain an essential target of HIV intervention efforts for IDU.

CORRESPONDING AUTHOR: Hi Yi Tsui, PhD Candidate, The Chinese University of Hong Kong, Hong Kong, (na); hytsui@ cuhk.edu.hk

PST-118

HOMELESS ADOLESCENT/YOUTHS WITH HIV IN IBADAN NIGERIA

Seye O. Omiyefa, BSC

Programs, Youth Action On Tobacco Control and Health, Agege, Nigeria.
Authors:

1. MR. SEYE OMIYEFA, YOUTH ACTION ON TOBACCO CONTROL AND HEALTH (YATCH)

2. MR PETER AKINPELUMI, Africa Consortium for the Displaced (ACD)

Background: The human immunodeficiency virus (HIV) is the causative agent of AIDS. Intravenous drug use and sexual activity are known risk factors associated with HIV transmission. Adolescents have experiment with drugs and sex which increased their risk for HIV infection. Although teenagers account for only one percent of the total cases of AIDS, adults between the ages of 20 and 29 years account for 20 percent of all reported AIDS cases which is caused by HIV.

Methods: Since the time interval between exposure to HIV and the development of AIDS is close to 10 years, it is likely that these young adults were exposed to HIV when they were teenagers; this has rendered many of them homeless and hopeless. A study should be conducted in Ibadan Nigeria for the homeless youths living with HIV. This study will determine the knowledge, attitudes and behaviours of homeless youths regarding HIV infection. Self administered semi structured questionnaire would be administered by $70 \%$ of the homeless youth with HIV. A focus group discussion will also be used to get responses from parents/ siblings of victims.

Results: This method will creatively educate, sensitize and enforce behavioral change among the homeless youth living with HIV. Percentage of homeless youth with HIV in Ibadan Nigeria would also be revealed, it will also reveal various means through which the victims are affected with HIV in Ibadan Nigeria.

Conclusion: Educational, social and medical programs are needed to reduce the risk of infection in this population. There is need for more training to empower this group to take a leading role in the community to prevent the spread of HIV and make them Volunteers against HIV (VAH). This will reduce numbers of homeless youths living with HIV in Ibadan Nigeria.

CORRESPONDING AUTHOR: Seye O. Omiyefa, BSC, Programs, Youth Action On Tobacco Control and Health, Agege, 23401; omiyefaseye@yahoo.com

\section{PST-119}

PREDICTORS OF PTSD IN SARS SURVIVORS

Niloufar Assar, BA, ${ }^{2,1}$ Parvaneh Moallef, MA, ${ }^{1}$ Paula Gardner, $\mathrm{EdD}^{1}$ and John Patcai, $\mathrm{MD}^{1}$

${ }^{1}$ St. John's Rehab Hospital, Toronto, ON, Canada and ${ }^{2}$ University of California, Berkeley, CA.

The authors examined the relationship between symptoms of Posttraumatic Stress Disorder (PTSD) and potential mediating factors among 40 severe acute respiratory syndrome (SARS) patient survivors. Participants were assessed twice within a 3 year time period: initially in 2004, shortly after they had recovered from the acute phase of SARS, and again 3 years later in 2007. Prevalence of PTSD among patients was $30 \%$ in 2007 compared to $15.6 \%$ in 2004 . The association between PTSD symptoms and 
demographic variables (i.e., age at infection, age at testing, gender, education, marital status, and living arrangement), job type, severity of disease (i.e., ICU requirement, respiratory failure, low oxygen saturation, cardiac failure), duration of hospitalization, and duration of quarantine was identified using Spearman rank-order correlations. A stepwise regression analysis was performed for each of the PTSD symptoms of re-experiencing, avoidance, and hyperarousal. All potential mediating factors (those identified in univariate analyses with a significance of $\mathrm{P}<0.05$ were entered into the analysis). Results indicated that age at infection significantly contributed to the variance in PTSD symptoms of hyperarousal and re-experiencing in 2004 (at $\mathrm{P}<.01$ levels) and had an inverse relationship with these two variables. Duration of hospitalization significantly increased the $\mathrm{R}$ square value in predicting symptoms of hyperarousal in 2004. Interaction of age at infection and duration of hospitalization explained $33.5 \%$ of the variance in symptom of hyperarousal in $2004(\mathrm{~F}=7.06 ; \mathrm{df}=2,28$; $\mathrm{P}=.003)$. Severity of disease was the best predictor of chronic Posttraumatic Stress Disorder $(\mathrm{F}=3.76 ; \mathrm{df}=3,34 ; \mathrm{P}=.020)$ and symptoms of re-experiencing, avoidance and hyperarousal in 2007. In conclusion, the findings of this study have important implications for the understanding, prevention and treatment of long-term psychological effects of infectious diseases.

CORRESPONDING AUTHOR: Niloufar Assar, BA, UC Berkeley, Thornhill, ON, L4J 0C1; niloufar.assar@yahoo.com

\section{PST-120}

WOMEN, THE INTERNET, AND SEXUAL RISK BEHAVIOR Eric G. Benotsch, PhD, ${ }^{1}$ David W. Seal, PhD, ${ }^{2}$ Sheana S. Bull, $\mathrm{PhD}^{3}$ and Aaron Martin, $\mathrm{MA}^{1}$

${ }^{1}$ Psychology, Virginia Commonwealth University, Richmond, VA; ${ }^{2}$ Medical College of Wisconsin, Milwaukee, WI and ${ }^{3}$ University of Colorado Denver, Denver, CO.

Previous research has documented that using the Internet for romance or to find sexual partners is associated with HIV risk behavior. Most of the previous work has been conducted with men, especially gay and bisexual men. Relatively few studies have examined the use of the Internet for romance among women. Even fewer have had a strong theoretical base. In the present study, we recruited women $(\mathrm{N}=255)$ from MySpace and other Internet venues to complete measures assessing demographic information, sexual behavior, and components of the Information-MotivationBehavioral Skills (IMB) model. Overall, 31\% of women reported that they had had sex with someone they initially "met" online. Women who used the Internet for romance reported more partners in the previous 3 months $(\mathrm{M}=2.74, \mathrm{SD}=3.86)$ than women who had not $(\mathrm{M}=1.47, \mathrm{SD}=2.55)$, a significant difference (MannWhitney $\mathrm{Z}=3.54, \mathrm{p}<.001)$. Two components of the IMB model were associated with sexual risk behavior. Perceptions of peer norms for sexual activity (a motivational construct) were associated with higher numbers of sexual partners $(\mathrm{rho}=0.27, \mathrm{p}<.01)$.
Higher self-efficacy for condom use (a behavioral skills component) was associated with fewer sexual partners (rho $=-0.16$, $\mathrm{p}<.05$ ). In this study, the IMB component of information was not predictive of risk. Results suggest that risk associated with using the Internet to find partners is not restricted to men. Women in the present study may be at risk for a variety of negative outcomes of sexual activity, including contracting HIV or other sexuallytransmitted infections, and unwanted pregnancy.

CORRESPONDING AUTHOR: Eric G. Benotsch, PhD, Psychology, Virginia Commonwealth University, Richmond, VA, 23284; ebenotsch@VCU.edu

PST-121

KNOWLEDGE AND ATTITUDES IN REGARD TO PANDEMIC INFLUENZA A(H1N1) IN A MULTIETHNIC COMMUNITY

Li-Ping Wong, MSc, $\mathrm{PhD}^{1}$ and I-Ching Sam, MRCP, MRCPath ${ }^{2}$

${ }^{1}$ Medical Education \& Research Development Unit, Faculty of Medicine, University of Malaya, Kuala Lumpur, Malaysia and ${ }^{2}$ Tropical Infectious Diseases Research and Education Centre, Department of Medical Microbiology, Faculty of Medicine, University of Malaya, Kuala Lumpur, Malaysia.

Objective: To investigate the level of knowledge and attitudes towards the influenza $\mathrm{A}(\mathrm{H} 1 \mathrm{~N} 1)$ outbreak across various ethnic groups and socio-demographic backgrounds in Malaysia.

Design: A cross-sectional, population-based, computer-assisted telephone interview exploring knowledge and attitudes regarding influenza $\mathrm{A}(\mathrm{H} 1 \mathrm{~N} 1)$ was conducted in Malaysia. Between July 11, to September 12, 2009, a total of 1050 respondents were interviewed (response rate 69.3\%).

Results: The mean total knowledge score for the overall sample was $7.30(\mathrm{SD} \pm 1.961)$ out of a possible score of 13 (Chinese had the highest scores, followed by Indians, then Malays). Some erroneous beliefs about the modes of transmission were identified. A considerable number of participants $(n=329,31.3 \%)$ erroneously believed that the $\mathrm{A}(\mathrm{H} 1 \mathrm{~N} 1)$ influenza can be transmissible to people through eating improperly cooked pork and pork products, the majority of whom were of Malay ethnicity $(47.8 \%)$. The majority of the participants $(73.8 \%)$ perceived the $\mathrm{A}(\mathrm{H} 1 \mathrm{~N} 1)$ infection as often deadly. Despite the overestimation of the severity of $\mathrm{A}(\mathrm{H} 1 \mathrm{~N} 1)$ infection, high confidence in preventing infection and low perceived susceptibility of infection were reported. Influenza $\mathrm{A}(\mathrm{H} 1 \mathrm{~N} 1)$ related stigma was prevalent and exhibited differences across ethnic groups.

Conclusions: Findings suggest that provision of education and clear information are essential to correct the misconceptions, and increase perceived susceptibility to infection so that the general public will take precautions against $\mathrm{A}(\mathrm{H} 1 \mathrm{~N} 1)$ infection.

CORRESPONDING AUTHOR: Li-Ping Wong, MSc, PhD, Medical Education \& Researc Development Unit, University of Malaya, Kuala Lumpur, 50603; wonglp@ummc.edu.my 
PST-122

PSYCHOPATHY, DISCLOSURE, AND RISK BEHAVIOR IN HIV-POSITIVE ADULTS

Eric G. Benotsch, PhD, ${ }^{1}$ Aaron Martin, MA, ${ }^{1}$ Shannon Perschbacher, MA, ${ }^{2}$ Marisa Green, $\mathrm{MA}^{2}$ and Mark Thrun, $\mathrm{MD}^{3}$

${ }^{1}$ Psychology, Virginia Commonwealth University, Richmond, VA; ${ }^{2}$ University of Colorado Denver, Denver, CO and ${ }^{3}$ Denver Public Health, Denver, CO.

Previous work has shown that most, but not all, HIV-positive adults disclose their HIV status to their sexual partners. Factors associated with non-disclosure include stigma, fear of rejection, fear of violence, and privacy concerns. In work with populations at risk for HIV, psychopathy (a personality characteristic associated with antisocial behavior and egocentrism) has been associated with substance use and sexual risk behavior. Little work has explored associations between psychopathy, disclosure, and risk in HIV-positive adults. In the present study, HIV-positive men and women $(\mathrm{N}=310)$ were recruited from the waiting room of an infectious disease clinic. Participants completed a self-administered questionnaire assessing demographic information, disclosure of HIV status, psychopathy, and sexual risk behavior. The majority (74\%) of participants disclosed their HIV status to all sex partners in the past 3 months. Individuals who did not disclose their HIV status to all partners scored significantly higher in psychopathy $(\mathrm{M}=24.00, \mathrm{SD}=5.22)$ than those who had disclosed $(\mathrm{M}=21.02, \mathrm{SD}=5.91 ; \mathrm{F}=7.98, \mathrm{p}<.001)$. Higher psychopathy scores were associated with higher rates of having sex after drinking ( $r h o=0.20)$, having sex after using drugs $(r h o=0.24)$, the number of sexual partners $(\mathrm{rho}=0.13)$ and unprotected vaginal and anal sex acts in the past 3 months ( $r h o=0.13$; all ps $<.05$ ). Higher psychopathy scores were also associated with lower intentions to use condoms with future partners $(\mathrm{rho}=-0.22, \mathrm{p}<.001)$. Findings suggest that psychopathy is associated with risk behavior among HIVpositive men and women. Disclosure of HIV status has the potential to help reduce the spread of HIV. Interventions focused on increasing disclosure need to take into account associations between psychopathy and non-disclosure.

CORRESPONDING AUTHOR: Eric G. Benotsch, PhD, Psychology, Virginia Commonwealth University, Richmond, VA, 23284; ebenotsch@VCU.edu

PST-123

\section{THE ROLE OF PERSONALITY IN PERCEIVED RISK OF HIV INFECTION}

Rafael Ballester Arnal, doctorate in psychology, ${ }^{1}$ Maria Dolores Gil, $\mathrm{PhD},{ }^{2}$ Estefanía Ruiz, Postgraduate ${ }^{1}$ and Cristina Giménez, $\mathrm{PhD}^{1}$

${ }^{1}$ Psicología Básica, Clínica y Psicobiología, Universitat Jaume I, Castellon, Spain and ${ }^{2}$ Psicología evolutiva y de la educación, Universidad de Valencia. Estudi General, Valencia, Spain.

The spread of HIV/AIDS has increased the effort to explain why people maintain unsafe sexual behaviors. Studies have suggested that personality traits have an influence on sexual behaviors to facilitate the HIV infection. Moreover, the perception of risk is one of the main factors to consider in sexual prevention of HIV. The aim of this study was to analyze the influence of personality traits on "perceived risk of HIV infection", in a sample of 120 Spanish university students with a mean age of $19.79(\mathrm{SD}=1.33)$. Data were collected from the "AIDS Prevention Questionnaire" (Ballester et al., 2004), which evaluates relevant variables to HIV prevention. Specifically, we have used the questions related to the component "perceived risk": perceived severity, probability of infection risk and perceived fear. Secondly, the Personality Inventory NEO-PI-R (Costa \& McCrae, 1992) was used to measure personality traits. Results showed that perceived probability of infection correlated positively and significantly with Neuroticism $(\mathrm{r}=0.257, \mathrm{p} \leq 0.005)$ and with three of its traits: Depression $(\mathrm{r}=0.242, \mathrm{p} \leq 0.008)$, Impulsivity $(\mathrm{r}=0.185, \mathrm{p} \leq 0.044)$ and Vulnerability $(\mathrm{r}=0.243, \mathrm{p} \leq 0.008)$. Regarding to perceived fear, was observed a significant positive correlation with Impulsivity $(\mathrm{r}=0.182, \mathrm{p} \leq 0.048)$ (Ns trait) and Gregariousness $(\mathrm{r}=0.204$, $\mathrm{p} \leq 0.026$ ) (Es trait). Finally, perception of severity got a significant positive correlation with Modesty $(\mathrm{r}=0.222, \mathrm{p} \leq 0.015)$ (As trait). That is, people with higher scores on traits related to psychological distress, tend to perceive greater likelihood of HIV infection. While people with higher scores on traits related to social interaction, tend to perceive greater fear and severity toward the disease. In conclusion, personality traits are really relevant to understand significant variables of HIV transmission, as well as to understand risky sexual behavior and more vulnerable population.

Research was supported by Universitat Jaume I-Fundació Bancaixa (P1 1B2006-19) and Foundation for Research and prevention of AIDS in Spain (exp. 36639/07)

CORRESPONDING AUTHOR: Rafael Ballester Arnal, doctorate in psychology, Psicología Básica, Clínica y Psicobiología, Universitat Jaume I, Castellon, 12071; gomezs@psb.uji.es

\section{PST-124}

PERSEVERANCE, SUBSTANCE USE, AND SEXUAL RISK BEHAVIOR IN GAY AND BISEXUAL MEN

Eric G. Benotsch, PhD and Aaron Martin, MA

Psychology, Virginia Commonwealth University, Richmond, VA.

Positive psychology seeks to understand the human strengths and virtues that enable people to succeed and to thrive. Relatively little research in health psychology and behavioral medicine has examined relationships between the constructs of positive psychology and health-relevant behaviors. The present study assessed associations between perseverance, substance use and sexual risk behavior in gay and bisexual men. Participants attending a gay pride celebration $(\mathrm{N}=$ 350) completed measures of perseverance, demographics, substance use and sexual risk behavior. Perseverance was associated with years of formal education $(\mathrm{r}=0.27, \mathrm{p}<.01)$ and income $(\mathrm{r}=0.22)$ but was unrelated to age, employment status, or HIV status. Perseverance scores were negatively correlated with the use of marijuana $(\mathrm{rho}=$ $-.30)$ ecstasy $(\mathrm{rho}=-0.19)$, cocaine $(\mathrm{rho}=-0.18)$, ketamine $(\mathrm{rho}=$ $-0.14)$, methamphetamine $(\mathrm{rho}=-0.15)$ and poppers $(\mathrm{rho}=-0.12)$ (all ps<.05). Perseverance scores were also associated with lower rates of using drugs (rho=-.23) and alcohol $(\mathrm{rho}=-.16)$ in conjunction with sexual activity. Interventions designed to reduce 
HIV risk behavior in gay and bisexual men may benefit from efforts to promote perseverance and other human strengths.

CORRESPONDING AUTHOR: Eric G. Benotsch, PhD, Psychology, Virginia Commonwealth University, Richmond, VA, 23284; ebenotsch@VCU.edu

PST-125

SARS SURVIVORS: A REVIEW OF THE PSYCHOLOGICAL IMPACT

Paula Gardner, EdD ${ }^{1}$ and Parvaneh Moallef, $\mathrm{MA}^{2,1}$

${ }^{1}$ Back on Track Program, St. John's Rehab Hospital, Toronto, ON, Canada and ${ }^{2}$ York University, Toronto, ON, Canada.

Introduction: This paper reviews the existing literature on the psychological impact of Severe Acute Respiratory Syndrome (SARS) on patients who contracted the illness following the outbreak of this atypical pneumonia condition. SARS was an infectious illness that spread to 29 countries in 2002/2003, infecting over 8,000 people, 774 of whom died. Method: A literature search was conducted on available electronic databases, including MEDLINE, PsycINFO, CINAHL, and Cochrane Library. The search produced only a small number of articles published pertaining to the psychological symptoms and experience of patients (total of 28 original studies and 3 early reviews). No studies were found that assessed the emotional impact beyond 18 months post-infection. Studies used various methods of appraisal: self-report, standardized measures or questionnaires of original design, and a small number of studies employed hospital chart reviews. For the purpose of this study, the research results were divided into two recovery stages: early stages ( $<3$ months post infection), and later stages ( $>3$ months post-infection). Results: Early study variables that emerged included "worry about infecting others," "elevated emotional distress," "changes in mood," "symptoms of acute stress disorder," "feelings of stigmatization," and "psychiatric complications," such as the presence of psychotic symptoms (possibly secondary to steroid toxicity). Later stage variables included "emotional distress", "depressed mood", and "posttraumatic stress symptoms." Evidence of psychological problems developing in the later stages was also found in a small proportion of survivors. Conclusion: The limited number of published studies available for review consistently reported high rates of emotional distress in patients in both the early stages and later stages of recovery up to 18 months post-infection, with worry about infecting others, feelings of stigmatization, and psychotic symptoms emerging earlier in the recovery process. The levels of psychological distress found in these studies are similar to those reported in other comparable chronic physical illnesses. SARS remains a new disease, and the course of its development, both physically and emotionally, is still unfolding as time progresses. Longer term follow-up to determine the course of SARS patients' psychological status will help to guide treatment interventions. This, in turn, will help to inform the needs for future emergent, possibly similar, viral infections.

CORRESPONDING AUTHOR: Paula Gardner, EdD, Back on Track Program, St. John's Rehab Hospital, Toronto, ON, M2M 2G1; pgardner@stjohnsrehab.com
PST-126

DOES DEPRESSION INFLUENCE ON HIV RISK BEHAVIOR OF YOUTH?

Rafael Ballester Arnal, doctorate in psychology, ${ }^{1}$ Maria Dolores Gil, $\mathrm{PhD}^{2}$ Estefanía Ruiz, Postgraduate ${ }^{1}$ and Cristina Giménez, $\mathrm{PhD}^{1}$

${ }^{1}$ Psicología Básica, Clínica y Psicobiología, Universitat Jaume I, Castellon, Spain and ${ }^{2}$ Psicología evolutiva y de la educación, Universidad de Valencia. Estudi General, Valencia, Spain.

The severity of AIDS epidemic mainly affects young people. In this context, different programs of prevention have been developed successfully, although there have been some barriers to achieve changing risk behavior. In this experience, dispositional characteristics have been revealed really important. Thus, considering the complexity of risk behaviors, we should improve our knowledge about mediator variables in HIV behaviors. For example, depression has revealed some influences related to HIV risk on different population but this has not been specified yet in youth. Our study analyzed the influence of depression on significance variables associated with HIV transmission, such as knowledge, attitudes, behavior and behavior intention. Participants were 170 students recruited from University of Castellon (Spain), 18-21 years old,. They were administered anonymously two questionnaires: NEO-PIR (Costa \& McCrae, 1992) related to personality traits and AIDS Prevention Questionnaire (Ballester, Gil, Guirado \& Bravo, 2004) about HIV/AIDS. Correlation analyses showed with significance statistical that more levels of depression are associated with higher personal risk perception $(\mathrm{r}=0.242, \mathrm{p} £ .008)$, more condom use in regular partner $(\mathrm{r}=0.229, \mathrm{p} £ .021)$ and less intention to have a sexual relation without condom $(\mathrm{r}=-0.193, \mathrm{p} £ .038)$. Depression factor is closed to reveal statistical significance related to information about HIV ( $\mathrm{r}=0.179, \mathrm{p} £ .051)$ but analyses have not showed any relation to confidence about condom $(\mathrm{r}=0.026, .777)$, fear perceived $(\mathrm{r}=0.107$, $\mathrm{p} £ .246)$ or condom use in sporadic partner $(\mathrm{r}=-0.071, \mathrm{p} £ .536)$. These findings suggest a complex influence of depression in youth risk in different levels such as attitudes or behavior. In order to improve prevention effectiveness, we should accurate our study about factors such as depression that have an influence depending on criteria variables and people profile.

Research was supported by Universitat Jaume I-Fundació Bancaixa (P1 1B2006-19) and Foundation for Research and prevention of AIDS in Spain (exp.36639/07)

CORRESPONDING AUTHOR: Rafael Ballester Arnal, doctorate in psychology, Psicología Básica, Clínica y Psicobiología, Universitat Jaume I, Castellon, 12071; gomezs@psb.uji.es

\section{PST-127a}

INTENTIONS TO USE CONDOMS IN A SAMPLE OF HIV+ OLDER ADULTS: THE ROLE OF PAST CONDOM USE BEHAVIORS

Heather A. Piper, BA, Eric J. Geyer, BA, Liana C. Hawkins, BA, Elisabeth A. Kinsley, BA, Blake A. Elliott, BA and Travis I. Lovejoy, MS, MPH

Psychology, Ohio University, Athens, $\mathrm{OH}$. 
Background: Constructs from the Theory of Planned Behavior have been used to develop and refine health behavior interventions. However, few studies have examined the role of past behaviorabove and beyond attitudes, social norms, and self-efficacy-in predicting future behavioral intentions.

Participants and Procedures: Participants were 100 HIV-positive adults $45+$ years of age enrolled in a randomized controlled trial testing the efficacy of telephone-delivered motivational interviewing to reduce risky sexual practices. All participants reported engaging in one or more occasions of unprotected anal and/or vaginal intercourse in the past 3 months. At baseline, participants completed a telephone interview that assessed recent sexual behaviors, as well as attitudes and social norms about the use of protection, condom use self-efficacy, and intentions to use protection during sex in the next three months. Results: We employed structural equation modeling to test two models predicting intentions to use condoms during anal and/or vaginal intercourse in the next three months. The first model examined direct contributions of attitudes, social norms, selfefficacy, and past sexual behaviors in predicting behavioral intentions. This model provided adequate fit to the data, $\chi 2(3)=5.92$, $\mathrm{p}=.12(\mathrm{AIC}=41.92, \mathrm{BIC}=88.82)$ and accounted for $67 \%$ of the variance in behavioral intentions. The second model examined direct effects of past behavior on future intentions, as well as indirect effects of past behavior via attitudes, social norms, and self-efficacy. This model also provided adequate fit to the data, $\chi 2(6)=10.51, p=.11$ $(\mathrm{AIC}=40.51, \mathrm{BIC}=79.59)$ and accounted for $78 \%$ of the variance in behavioral intentions.

Conclusions: Although attitudes, social norms, and self-efficacy are important constructs to consider when developing and refining health behavior interventions, the role of past behavior in forming these cognitive impressions must also be taken into consideration.

CORRESPONDING AUTHOR: Travis I. Lovejoy, MS, MPH, Psychology, Ohio University, Athens, OH, 45701; t1399805@ ohio.edu

\section{PST-127b}

SELF-REPORT VS. CLINICAL DIAGNOSTIC SCREENING FOR MENTAL HEALTH NEEDS IN YOUNG PEOPLE LIVING WITH HIV

Ricardo D. LaGrange, PhD, Maria Lewis, MPH and Jessica Gaines, BA

Children's Research Intitute, Children's National Medical Center, Washington, DC, DC.

Young people living with HIV (YPLH) face a variety of difficult challenges while negotiating the already demanding developmental tasks and changes associated with adolescence. Not surprisingly, mental health needs frequently rise to the forefront of concerns clinicians must address prior to, or alongside, medical treatment. A better understanding of how to assess the psychosocial needs of this vulnerable population can help inform interventions to improve selfcare and health outcomes. The preliminary data presented in this study includes 114 participants who have completed their initial visits of a study assessing coping styles as predictors of adherence in an urban sample of YPLH in the United States. The sample consisted of patients ranging from 13-22 years old. Using an Audio Computer Assisted Survey Instrument (ACASI), study participants responded to questions from a self-report mental health assessment instrument. Results indicated that only $10-15 \%$ showed signs of mental health needs that approached clinical levels. However, an examination of mental health screening records provided a clearer picture of the diagnostic profile of the ACASI respondents. When screened by a psychologist, nearly half of the participants were diagnosed with at least one mental health condition. Of these, 44\% were diagnosed with a depressive disorder while $8 \%$ an anxiety disorder and $24 \%$ an attentive disorder. Several had multiple diagnoses. Clinicians treating YPLH should rely on methods beyond self-report tools to screen for mental health needs in order to develop a treatment plan and make referrals as appropriate.

CORRESPONDING AUTHOR: Ricardo D. LaGrange, PhD, Children's Research Intitute, Children's National Medical Center, Washington, DC, DC, 20010; rlagrang@cnmc.org

PST-127c

APPLICATION OF THE THEORY OF REASONED ACTION IN PARENTS INTENTION TO VACCINATE

THEIR DAUGHTERS AGAINST HPV

Corina Cuevas, $\mathrm{PhD}$ and Florencia Romero, student

Psicología Experimental, Universidad Nacional Autónoma de México, México, Mexico.

In Mexico, cervical cancer is the most common cancer among women; it is also the second cause of death between 15 and 49 years old. The HPV (Human Papilomavirus) is a sexually transmitted virus which mathematical modeling and economic analysis have shown the maximum benefits of the vaccine as an effective method to prevent cervical cancer. This research was addressed from the Deliberate Processing Model using the Theory of Reasoned Action (TAR) (Fishbein, M. \& Ajzen, 2006). The importance is that, since 2008, the government of Mexico City is carrying out a public health program for girls between 9 and 12 years old to get vaccinated, but this program has not succeeded due to the negative attitudes of the parents to get them vaccine. Method

Objective: Build a model based on the theory of reasoned action by Fishbein to predict the behavior of parents towards vaccinating their daughters against HPV.

Population: 713 parents (men and/ or women) with non-married daughters between 12 to 17 years old, who live in Mexico City and have not been vaccinated against HPV. An instrument was built to know attitudes, behavioral intention and subjective norms of parents towards the HPV.

Results: The instrument with 16 items about attitudes and subjective norms had a reliability coefficient: Cronbach=.65. Its validity with three factors reached a $67 \%$ of the explained variance. Taking as dependent variable "intention to vaccinate", the regression model was: $\mathrm{Y}=1.903+.102$ subjective norms -.283 attitudes $+\varepsilon$

$\mathrm{R}=.113$

Conclusion: The results showed the need to work on the behavioral cognitive and medicine approaches on negative attitudes and subjective norms that limit public health programs to reach the target population. 
Finally it is possible to say that the $\mathrm{R}$ value in the model came out very low due to a lack of information about what is HPV, how it spreads, the vaccine's consequences and the parents' fear about this issue.

CORRESPONDING AUTHOR: Corina Cuevas, PhD, Psicología Experimental, Universidad Nacional Autónoma de México, México,03920; corinacr@servidor.unam.mx

\section{PST-128}

\section{REPRESENTATIVENESS AND DETERMINANTS}

OF ATTRITION IN A LARGE DANISH YOUTH COHORT

Mathilde Vinther-Larsen, Candscientsanpubl, ${ }^{1}$ Mette Riegels, candscientsanpubl, ${ }^{1}$ Morten H. Rod, candscientanth, ${ }^{1}$ Michaela Schiotz, candscientsanpubl, ${ }^{2}$ Tine Curtis, magart, phd, ${ }^{1}$ Pernille Due, candmed ${ }^{1}$ and Morten Groenbaek, candmed, phd, drmed ${ }^{1}$

${ }^{1}$ The child health programme, National Institute of Public Health, Copenhagen, Denmark and ${ }^{2}$ Institute of Public Health, University of Copenhagen, Copenhagen, Denmark.

Background: The Danish Youth Cohort (DYC) is a recently established longitudinal cohort studying predictors of health behaviour and health outcomes in adolescence and young adulthood. . Over the years decreasing rates of participation have been reported in population based studies. Consequently, differences between participants and nonparticipants in distribution of the exposure to an outcome may be present. This question, whether results from population based studies can be generalized to the background population.

Results: Participants born in $1991(\mathrm{~N}=10,666)$ were compared with adolescents born in 1991 who did not participate in The Danish Youth Cohort $(\mathrm{N}=56,619)$ through registries in The Statistics Denmark. We found that non-participants were more likely to be of lower socio-economic groups.

Further, participants at baseline with follow-up in 2007 (N=3777) were compared with participants at baseline with no follow-up $(\mathrm{N}=8721)$. We found that adolescents who at baseline had tried drinking one unit of alcohol ( $\mathrm{OR}=0.87$, CI: 0.81-0.94), had experienced being drunk $(\mathrm{OR}=0.85$, CI: 0.77-0.94), and who smoked $(\mathrm{OR}=0.65, \mathrm{CI}$ : 0.49-0.88) have lower risk of follow-up two years after baseline.

Conclusions: Our study confirms what already is known from many other previous study cohorts; that lower socio-economic groups are underrepresented in epidemiological studies. Methods to account for low participation rates in cohort studies have been introduced. Future analysis in the Danish Youth Cohort may overcome the rather low participation rate, and hereby the skewed sample, by for example weighting some characteristics of non-participation.

Regarding attrition, only $25 \%$ was followed up from baseline in 2005 to second follow-up in 2007. Loss to follow-up was only associated with drinking and smoking, while the factors sex, FAS-score, diet, physical activity, and bullying were not associated with attrition. Data from the Danish Youth Cohort can be used to investigate research question that require longitudinal data. However, in future research on alcohol or smoking the high attrition should be considered.

CORRESPONDING AUTHOR: Mathilde Vinther-Larsen, Candscientsanpubl, The child health programme, National Institute of Public Health, Copenhagen, 1353; mvl@niph.dk
PST-129

ASSOCIATIONS BETWEEN VO2MAX AND VITALITY

IN OLDER WORKERS: THE VITAL@WORK STUDY

Jorien E. Strijk, MSc, ${ }^{1}$ Karin I. Proper, $\mathrm{PhD},{ }^{1,2}$ Allard J. van der Beek, $\mathrm{PhD}^{1,2}$ and Willem van Mechelen, $\mathrm{PhD}^{1,2}$

${ }^{1}$ Department of Public and Occupational Health, EMGO+ Institute, Amsterdam, Netherlands and 2TNO-VUmc, Body@Work, Research Center Physical activity, Work and Health, Amsterdam, Netherlands.

Background: Vitality is related to health and is characterised by a perceived energy level, feelings of fatigue and feeling fit. These subjective factors can be positively affected by physical activity. Since vigorous physical activity is strongly related to aerobic fitness (VO2max), it is hypothesised that VO2max is related also to vitality. Therefore, the aim of this study was to investigate the association between vitality and estimated VO2max in older workers.

Methods: Participants $(n=427)$ were aged 45 years and over, and worked for at least 16 hours a week at a large hospital in the Netherlands. VO2max was estimated at baseline using the 2-km UKK walk test. Vitality was measured by both the UWES Vitality Scale and the RAND-36 Vitality Scale. Associations were analysed using linear regression analyses. Significance level was defined as $\mathrm{p}<0.05$.

Results: The linear regression models, adjusted for age, showed a significant association between $\mathrm{VO} 2 \mathrm{max}$ and vitality measured with the RAND-36 Vitality Scale ( $\beta=0.446$; 95\% CI: 0.220-0.673). There was no significant association between $\mathrm{VO} 2 \mathrm{max}$ and vitality measured with the UWES ( $\beta=-0.005 ; 95 \%$ CI:-0.019 - 0.008), after adjusting for age, gender and chronic disease status.

Conclusions: This study showed an association between VO2max and vitality measured by the RAND-36 Vitality Scale. However, there was no association found between VO2max and vitality measured by the UWES Vitality Scale. These results suggest that the two measurements of vitality (i.e. UWES and RAND-36) measure different constructs of vitality: e.e. a mental component (UWES Vitality Scale) and a physical component (RAND-36 Vitality Scale). These findings deserve further exploration in future research.

CORRESPONDING AUTHOR: Jorien E. Strijk, MSc, Department of Public and Occupational Health, EMGO+ Institute, Amsterdam, 1081 BT; je.strijk@vumc.nl

\section{PST-130}

DEVELOPMENT OF HEALTH LITERACY SCREENING TOOLS FOR CHINESE IMMIGRANTS

Jing Wang, BSN, PhDc, ${ }^{1}$ Ady S. Oster, MD, ${ }^{2}$ Stella G. Lu, MAA, ${ }^{2}$ Allison L. Knopf, BSNc ${ }^{1}$ and Judith T. Matthews, $\mathrm{PhD}^{1}$

${ }^{1}$ University of Pittsburgh School of Nursing, Pittsburgh, PA and ${ }^{2}$ Charles B. Wang Community Health Center, New York City, NY.

Approximately half of 3.3 million Chinese Americans do not speak English, and Chinese is the second most common language spoken by Americans who do not speak English well or at all. Poor health literacy in English has been observed among foreign-born Chinese, yet evaluation of health literacy has not been attempted in their primary language. To ensure the cultural appropriateness of health literacy tools for this population, we involved linguists, health educators, and clinicians in the design of new tools in Chinese, 
employing the same methods used to develop the Rapid Estimates of Adult Literacy in Medicine (REALM) and Test of Functional Health Literacy in Adults (STOFHLA), rather than direct translation of these established tools in English. We developed the Rapid Estimate of Adult Health Literacy in Chinese (REAHLC) scale, a 10-item word recognition test. We used the modified cloze procedure to design the Chinese Short Test of Functional Health Literacy in Adults (CSTOFHLA), consisting of two paragraphs drawn from existing Chinese health education materials with 35 multiple choice questions. Higher scores on both tools indicated higher health literacy. We pilot tested these tools with 32 adult Chinese immigrants (68.8\% female; mean age: 49.6 years) recruited from a Chinese community health center in New York City. Participants completed the REAHLC, CSTOFHLA, a Chinese literacy test with 10 items in Chinese (CLT), and a demographic questionnaire. REAHLC and CLT scores were significantly correlated $(\mathrm{r}=.40, \mathrm{p}<.05)$, though none of the tools' scores were associated with CSTOFHLA scores ( $\mathrm{ps}>.05$ ). Our findings provide beginning evidence that word literacy and functional health literacy may be different in Chinese. REAHLC scores correlated with years of education in China $(\mathrm{r}=.38, \mathrm{p}<.05)$, while CSTOFHLA scores were negatively associated with age $(\mathrm{r}=-.50$, $\mathrm{p}<.01$ ), respectively suggesting that individuals with higher education in China have higher word literacy and younger adults tend to have higher functional health literacy. Our study designed the first health literacy screening tools in Chinese that are culturally appropriate. However, further testing of these tools is needed in a larger, more diverse Chinese immigrant population.

CORRESPONDING AUTHOR: Jing Wang, BSN, PhDc, University of Pittsburgh, Pittsburgh, PA, 15261; jiw38@pitt.edu

\section{PST-131}

\section{EVALUATION OF INTERVENTIONS TO REDUCE SICKNESS} ABSENCE

Stein Atle Lie, $\mathrm{PhD}$

Uni Health, Uni Research, Bergen, Norway.

How should one measure the effect of interventions to reduce sick leave, which survey methodology and study designs should one use and what statistical methods should be applied? Various designs provide different information and quality of information, and this should be put in a perspective in the analyses and in the interpretation of the results from available data. Depending on how an outcome (e.g. return to work) is measured various statistical methods are natural. This presentation discusses the use of extended event history analyses in multi-state models for the analyses of sick leave data. Using multi-state models, one can exploit more of the data available in the evaluation of interventions to reduce sickness absence or the effect after rehabilitation programs. We show the advantage of multi state models uses two datasets as examples. The first data is from a randomised controlled trial (RCT) on 256 individuals with low back pain, while the second data is an uncontrolled study on 577 individuals with different diagnosis after an 8 week stay at a rehabilitation clinic. For the RCT we show that the intervention increases the number of days in work with 46 day during a period of three years, while for the uncontrolled study we find that $60 \%$ of the participants return to work.
CORRESPONDING AUTHOR: Stein Atle Lie, PhD, Uni Health, Uni Research, Bergen, 5015; stein.lie@uni.no

PST-132

THE SPANISH VERSION OF THE SUBJECTIVE HEALTH COMPLAIN INVENTORY

Eduardo Remor, $\mathrm{PhD}$

Psychobiology and Health Psychology, Universidad Autonoma de Madrid, Madrid, Spain.

The Subjective Health Complaint Inventory (SHC; Eriksen, Ihlebaek and Ursin, 1999), consists of 29 items concerning subjective somatic and psychological complaints experienced during the last 30 days. Severity of each complaint included in the inventory is rated on a 4point scale $(0=$ none, $1=$ some, $2=$ much, $3=$ severe $)$. Each complaint is also scored for duration (number of days) during the last 30 days. The product of severity and duration can be used to obtain a total score $(0-90)$ indicating the degree of health problems (absence of positive health). The SHC inventory include 5 Subscales (flu, pain, allergy, anxiety and gastrointestinal) and a total health complain score is possible to get as well. The SHC is available in several languages, and psychometric properties were previously described for different language versions. The present work describe the adaptation process for the Spanish language and preliminary psychometrics.

Method: The English version of the SHC inventory was translated to Spanish by two experts, the Spanish version were reviewed by a third colleague to check the accuracy of the translation. This version was backtranslated to English by an independent translator. Backtranslated version was contrasted with the English original by the two initial experts. The Spanish version was then piloted with a small group of health adults to check comprehension of the items and wording. No suggestions were made by pilot subjects. Then final version of the SHC inventory in Spanish was considered achieved. These steps were followed by a psychometric study $(n=83)$. Evidences of reliability (internal consistency), validity (construct validity, CFA) and sensitivity to change (after an intervention) were assessed.

Results and conclusions: Results will be described in detail in the poster. Good internal consistency was achieved for the inventory $(>.70)$, structure of the questionnaire was confirmed, and inventory show change in scores after participation in a stress related intervention. The SHC inventory shows be a useful, reliable, valid and sensible measure to assess subjective health complains in Spanish adults, and is available to use in research in Spain.

CORRESPONDING AUTHOR: Eduardo Remor, PhD, Psychobiology and Health Psychology, Universidad Autonoma de Madrid, Madrid, 28049; eduardo.remor@uam.es

PST-133

"ROLE LETTERING THERAPY" AND THE DESCRIPTION CODES

Shuhei Kaneko, $\mathrm{PhD}$

Graduate School of Medical Sciences, Tottori University, Yonago city, Japan. 
Purpose: A writing psychotherapy, "Role Lettering Therapy(RLT)" was developed in Japanese reformatory in 1983. The procedure is almost same as empty chair technique, but written language is used instead of spoken. Haruguchi (1987) indicated several original effects of RLT. That is, 1) clarification of emotions, 2) self counseling, 3) catharsis, 4) confrontation and acceptance, 5) acquisition of both sides' viewpoints, etc.

In empirical RLT studies, increase of self-esteem, "adult" and "nurturing parent" (in egogram)" was pointed out (Hosoi et al., 2002; Okamoto, 2006). And in case studies, expressed emotions, insight, self-awareness were suggested as significant indicators of this therapy. But it is necessary so that qualitative studies with objective summary tool clarify long sentences. In this study, description codes for RLT are structured, and the applications are discussed.

METHOD: Sentences extracted from 12 articles and 2 technical books about RLT were categorized by using KJ method. The codes were examined two steps of validity (correspondence, usability, frequency, clinical importance) by 3-5 researchers. And the reliability was confirmed by concordance rate of the coding by 7 researchers and graduate students.

RESULT: As a result of classification, 15 codes for "letters to another" and 14 codes for "letter to self" were structured. The former consists of "anger/dissatisfaction", "dependence", "need/hope", "reflection/awareness", "care/thoughtfulness", "gratefulness", etc. The latter consists of "complaint/blame", "enmeshment", "advice/ opinion", "expectation/wish", "care", "comprehension/regard", etc. A rate of agreement in 2 people's cording (in arbitrary 3 in 7) is $82.2 \%$, therefore, these cords need the use in more than 3 people.

DISCUSSION: The codes are in practical use stage, and have following utilizations. a) Discovering patterns in letters, b) convert a description into variables, c) comparison of technique. Furthermore, it can be applied to RLT as a supporting tool for understanding. For example, ascertaining a fluctuation in the number of the cords leads to understanding of client's power of expression.

CORRESPONDING AUTHOR: Shuhei Kaneko, PhD, Graduate School of Medical Sciences, Tottori University, Yonago city, 6838503; skaneko@med.tottori-u.ac.jp

\section{EARLY CAREER AWARDEE}

PST-134

THE VALIDITY OF DEPRESSION DIAGNOSIS OF THE MINI INTERNATIONAL NEUROPSYCHIATRIC INTERVIEW (MINI) ACCORDING TO THE STRUCTURE CLINICAL INTERVIEW DIAGNOSTIC (SCID) AS A SUPPORT TO THE INDONESIA BASIC HEALTH RESEARCH 2010

Sri Idaiani, Dr

Ministry of Health Republic of Indonesia, National Institute of Health Research and Development, Bekasi, Indonesia.

The Indonesia Basic Health Research measures several health status and strategies. It needs a short and easy questionnaire to measure each health status. The main objective of this study was to conduct the validity of MINI as support to the Indonesia Basic Health Research. MINI is a short questionnaire, developed in France and USA according to Diagnostic Statistical Manual III-R (DSM IIIR) and has used in many mental health surveys in the world. We only describe depression validity due to other data analysis of this study is still in processing. This study is a diagnostic study, conducted in 2009 at 5 psychiatric centers in 5 cities (Jakarta, Bandung, Yogjakarta, Surabaya and Makassar). The participants were minimum 18 years, were excluded if had severe dementia and mental retardation. All participants were administered by the MINI and followed by SCID as a reference test. The SCID interviewers were general doctor and who were following the postgraduate of psychiatry, the MINI interviewer were nursing student and nurse. Both of interviewers were blind about the respondent's diagnosis except for respondent from population. The data processing used computer with SPSS version 15 statistical program. There were 82 data analyzed in this study, consisted of 45 patients and 27 non patients from population, 41 males and 41 females. The sensitivity of depression is 0.66 and the specificity is 0.89 . The positive predicted value is 0.33 , the negative predictive value is 0.97 and positive likelihood ratio is 6.3 , negative likelihood ratio is 0.37 . The median duration of interview was 30 minutes for a completed MINI and 60 minutes for completed SCID. The validity of depression diagnosis in this study is good but it needs comprehensive interpretation. It seems that even though the sensitivity is above 0.50 , the questions are more specific to diagnose depression. The positive predictive value is low due to lack of patients. The next analysis will be better by completed data from all sites. 
CORRESPONDING AUTHOR: Sri Idaiani, Dr, Ministry of Health Republic of Indonesia, National Institute of Health Research and Development, Bekasi, 17134; sriidaiaini@yahoo. com

\section{PST-135a}

NEW METHOD OF BEHAVIORAL MEDICINE RESEARCH FROM THE CLIENT'S PERSPECTIVE

Hideko Itoh, MA

National Institute of Multimedia Education, Tokyo, Japan.

The experiences of the author, a psychologist, regarding clinical depression were analyzed in order to develop new research methods for behavioral medicine. The analysis focused on self-investigation, quantitative and qualitative approaches, and an evidence-based approach for the individual. In Period I (1994-2000), a female client was treated with cognitive behavior therapy (CBT) by a clinical psychology professor. In Period II (2000-2005), pharmacotherapy was conducted by a psychosomatician. In Period III (2005-present), CBT and holistic medical treatment were conducted by another psychosomatician. In the second year of Period III (Period III-2), to facilitate self-regulation, Self-Efficacy and Self-Evaluation Scales were developed with the following items: 1. Absorb fulfilling experiences, 2. Keep good memories, 3. Achieve the research goals, 4. Calmly express feelings, 5. Change one's mood to cope with or avoid stress, 6. Regulate drowsiness, 7. Assert own opinion, 8. Ward off unimportant things, 9. Keep appropriate distance from others, and 10. Keep good personal relationships. Items 1-6 were common to Periods III-2-III-5, whereas items 7-9 were used in Periods III-2 and III-3 and integrated into item 10 in Periods III-4 and III-5. In each session, the client rated her pre-practice self-efficacy and postpractice self-evaluation. Average self-efficacy and selfevaluation scores descended in the order of items $1,2,3,5$, 6, and 4. Self-efficacy and self-evaluation scores on items 7-9 increased from Period III-2 to Period III-3. Most of the problems occurred in the family and relatives area. The top three reasons for positive discrepancy of the scores (evaluation - efficacy) include fewer problems occurred, or there was less sensitivity to the problems, executed things in workplace or personal areas, and maintained good communications. Correlations among self-efficacy scores on items 1-3 and 5 were significant in Periods III-4 and III-5. These results suggest that the client became able to cope with the problems and stress. The significance of this research is discussed in relation to "clients as researchers."
CORRESPONDING AUTHOR: Hideko Itoh, MA, National Institute of Multimedia Education, Tokyo, 169-0051; hideko.lisa. itoh@gmail.com

PST-135b

VALIDATION OF A KNOWLEDGE, ATTITUDE AND BEHAVIOUR QUESTIONNAIRE FOR DIETARY EDUCATION IN MALAYSIAN DIABETIC PATIENTS

Amutha Ramadas, MSc, ${ }^{1}$ Carina K. Chan, $\mathrm{PhD},{ }^{1}$ Kia Fatt Quek, $\mathrm{PhD},{ }^{1}$ Hussein Zanariah, $\mathrm{MBBS}^{3}$ and Brian Oldenburg, $\mathrm{PhD}^{2}$

${ }^{1}$ Monash University (Sunway Campus), Bandar Sunway, Malaysia; ${ }^{2}$ Monash University, Australia, Melbourne, VIC, Australia and ${ }^{3}$ Hospital Putrajaya, Putrajaya, Malaysia.

Introduction: Diabetes education improves knowledge and selfmanagement skills, and leads to better blood glucose control. Diabetes-related knowledge deficits and inadequate self-care practices has been identified among the majority of Malaysian diabetic patients with suboptimal glycaemic control. Rationale \& Objective: The evidence available on management of diabetes mellitus via dietary education, improving the knowledge, attitude and behaviour (KAB) of the patient is rather limited. Existing validated questionnaires measure general diabetes knowledge, which are not specifically meant to measure $\mathrm{KAB}$ related to diet and there is no published data on a validated dietary $\mathrm{KAB}$ questionnaire unique to Malaysian diabetic patients. There is a need for a $\mathrm{KAB}$ questionnaire that is explicitly developed for Malaysians as dietary habits are unique and very closely related to the culture of the population. Thus, this study aims to develop and validate a dietary KAB questionnaire for diabetic patients in Malaysia. Methods: The content of the KAB questionnaire was developed, and its face and content validity was determined by a panel of experts. In total, 36 questions were administered in 30 type 2 diabetic patients attending out-patient clinics in 3 public hospitals. Internal consistency was determined using Cronbach's alpha (CA) coefficient. Intraclass correlation coefficient (ICC) was used to determine the reliability. The KAB scores were used to determine the sensitivity of the questionnaire. Results \& Discussion: The Flesch Reading Ease score was $74.6 \%$ suggesting the questions were reasonably easy to understand. The CA was 0.72 , while the ICCs were between 0.71 and 0.92 . There was no significant difference in test-retest mean KAB score, indicating the questionnaire is sensitive enough to detect the changes in patients' response. Conclusion: A reliable KAB measure of dietary education for Malaysian diabetic patients has been developed and validated. 
CORRESPONDING AUTHOR: Carina K. Chan, PhD, Monash University (Sunway Campus), Bandar Sunway, 46150; carina. chan@med.monash.edu.my

\section{PST-135c}

EXAMINATION OF NEW METHODS TO MEASURE FATIGUE AND DEPRESSIVE MOOD BY USING PHYSICAL ACTIVITY LEVEL

Jinhyuk Kim, MSc, ${ }^{1}$ Hiroe Kikuchi, $\mathrm{MD}, \mathrm{PhD}^{2}$ and Yoshiharu Yamamoto, $\mathrm{PhD}^{1}$

${ }^{1}$ Educational Physiology Laboratory, Graduate School of Education, The University of Tokyo, Tokyo, Japan and ${ }^{2}$ Department of Psychosomatic Research, National Institute of Mental Health, National Center of Neurology and Psychiatry, Tokyo, Japan.

A limitation of self-reports for disease symptoms is recall bias including retrospective distortions of the respondents' experiences. To overcome this concern, ecological momentary assessment (EMA) and the day reconstruction method (DRM) have recently been used, but the validity of these methods has not been objectively examined. Therefore, the aim of this study was to investigate the validity of EMA and DRM by using physical activity level (PAL) as an objective external criterion. Twenty-two healthy undergraduates (age 21.9 $\pm 2.6 \mathrm{yrs}$ ) wore a watch-type computer and recorded visual analog scales of fatigue and depressive mood at different EMA design; recorded momentary symptoms every two hours (time-based) and recorded the symptoms during the behavioral episode which was just finished when switching one behavior to another (episode-based). They also recorded the symptoms afterward according to the series of behavioral episodes they reconstructed (DRM) about the same days. PAL was obtained using an actigraph built into the watch-type computer. Multilevel modeling showed that the association between depressive mood recorded with time-based EMA and PAL averaged over 60 minutes around EMA recording was significantly negative $(p=$ 0.002); however, depressive mood recorded with episode-based EMA and DRM had no association with PAL averaged over the period of corresponding episodes. As for fatigue, none of the methods showed significant association with averaged Pal. These results suggest that time-based EMA might have the validity against the objective PAL when assessing depressive mood in contrast to episode-based EMA and DRM. Though averaged PAL alone failed to confirm the validity of all three methods used in this study as assessment of fatigue, incorporating other PAL measures than a simple average might distinguish the validity of them and need to be investigated.

CORRESPONDING AUTHOR: Jinhyuk Kim, MSc, Graduate School of Education, The University of Tokyo, Tokyo, 113-0033; grefur@gmail.com
PST-135d

FURTHER VALIDATION OF THE TYPE D PERSONALITY SCALE (DS-14) IN HONG KONG CHINESE COMMUNITYCONFIRMATORY FACTOR ANALYSIS

Yi-jie Wang, PhD candidate, Siu-man $\mathrm{Ng}, \mathrm{PhD}$ and Ted, C.T. Fong, mphil candidate

Department of Social Work \& Social Administration, The University of Hong Kong, Hong Kong, Hong Kong.

Background: The Chinese version of the Type D Personality Scale (DS-14), a two-factor 14-item self-reported questionnaire for evaluating Type D personality has been validated in Beijing. Objective: To further validate DS-14 by confirmatory factor analysis (CFA) with Hong Kong Chinese sample. Methods: A cross-sectional questionnaire survey was conducted based on a random community sample of 755 adults. Results: The goodnessof-fit indices revealed that DS-14 was at the 'mediocre' fit level $(\mathrm{CFI}=.912$; $\mathrm{RMSEA}=.0097$; $\mathrm{SRMR}=.080)$. The overall prevalence of Type D personality was $29.4 \%$.The prevalence increased from $34.8 \%$ among participants aged 18 through 25 years to $43.5 \%$ for participants aged 26 through 35 years, but decreased to $27.5 \%$, $26.7 \%$ and $25 \%$ for participants aged 36 through 45 years, aged 46 through 55 years and aged above 56 years, respectively. The prevalence was lower in male $(27.5 \%)$ than in female $(32.3 \%)$. Type $\mathrm{D}$ personality was positively correlated with anxiety $(\mathrm{r}=.47, \mathrm{p}<.01)$ and depression $(\mathrm{r}=.37, \mathrm{p}<.01)$, but negatively correlated with age $(\mathrm{r}=-.11, \mathrm{p}<.01)$ Conclusion: DS-14 scale possesses basic factorial and conduct validity in measuring Type $\mathrm{D}$ personality.

CORRESPONDING AUTHOR: Yi-jie Wang, PhD candidate, Department of Social Work \& Social Administration, The University of Hong Kong, Hong Kong, nil; yukiwang@hkusua. hku.hk

\section{PST-135e}

A STUDY FOR FACTORIAL VALIDITY OF THE SHORT VERSION OF THE SELF-EFFICACY FOR SOCIAL PARTICIPATION FOR PEOPLE WITH PSYCHIATRIC DISABILITIES SCALE

Manami Amagai, PhD, ${ }^{1}$ Mayo Suzuki, PhD ${ }^{2}$ Chika Shimizu, bachelor, ${ }^{1}$ Mayumi Nitta, Master, ${ }^{1}$ Makiko Takahashi, bachelor ${ }^{1}$ and Fumie Shibata, bachelor ${ }^{3}$

${ }^{1}$ National College of Nursing, Japan, Kiyose-shi, Japan; ${ }^{2}$ School of Nursing, Bunri University of Hospitality, Sayama-shi, Japan and ${ }^{3}$ Kubota Clinic, Sumida-ku, Japan.

Purpose: This study examined the factorial validity of the short version of the Self-Efficacy for Social Participation for People with Psychiatric Disabilities scale (SESP). To develop a short version to measure self-efficacy for social participation was possible to use the instrument easily for people with severe mental illness (SMI). 
Methods: A total of 140 community-dwelling individuals with SMI completed an anonymous self-report questionnaire consisting of questions for background information and the original 27-item SESP on a 4-Likart scale. The first data analysis was an exploratory factor analysis (principal component method with varimax rotation). Then we mainly chose ten items which were big factor loading in the factor structure of the 27-item SESP. We assumed them the short version of SESP (SESP10). And we performed confirmatory factor analysis to inspect the factorial validity of SESP10.

Results: From results of confirmatory factor analysis, SESP10 was kept structure of 4 interpretable factors: Trust for Social Self, Self Management, Social Adaptability, and Mutual Support. It became clear that SESP10 had enough constructive validity. In addition, Internal consistency of the total SESP10 was excellent $(\alpha=.91)$.

Discussion: It was proved that SESP10 had a sufficient validity. It will be to review the predictive and criterion-related validity of SESP10 in future. The short version of the Self-Efficacy for Social Participation for People with Psychiatric Disabilities scale has potential as a reliable and valid scale for measurement of self-efficacy of people who are afflicted with severe mental illnesses and who live in the community.

CORRESPONDING AUTHOR: Manami Amagai, PhD, National College of Nursing, Japan, Kiyose-shi, 204-8575; amagaim@adm. ncn.ac.jp

\section{PST-137}

THE STRESS SYSTEMS IN WOMEN WITH FIBROMYALGIA ARE DYSREGULATED

Roberto Riva, PhD student, ${ }^{1}$ Paul J. Mork, Associate Professor, ${ }^{2}$ Rolf H. Westgaard, Professor ${ }^{2}$ and Ulf Lundberg, Professor ${ }^{1,3}$

${ }^{1}$ Psychology, Stockholm University, Stockholm, Sweden; ${ }^{2}$ Human Movement Sciences Programme, Faculty of Social Sciences and Technology Management, Norwegian University of Science and Technology, Trondheim, Norway and ${ }^{3}$ Centre for Health Equity Studies (CHESS), Stockholm University, Stockholm, Sweden.

Fibromyalgia syndrome (FMS) is a disease of unknown pathogenesis characterized by chronic musculoskeletal pain. FMS also includes other symptoms like poor quality sleep, morning stiffness, fatigue, anxiety, depression and altered endocrinological responses, but findings are inconsistent. The aim of the present study was to investigate free salivary cortisol levels, urinary catecholamines (noradrenaline, adrenaline and dopamine), heart rate, self-reported pain, and psychological features in FMS patients compared with healthy controls.

Twenty-nine female FMS patients and 29 age-matched healthy female controls were recruited. Questionnaires measuring pain levels, sleeping problems, perceived stress and personality were administered to the participants. Salivary cortisol samples were collected 8 times: in the afternoon when subjects arrived at the hospital, after stress provocation, in the evening, before they went to sleep, upon awakening, 30 and 60 minutes later, and during the afternoon of the second day. Urinary catecholamines were repeatedly measured from the corresponding 24-hours period.

Results revealed significantly more pain, perceived stress, sleeping problems, anxiety and depression among FMS patients compared with controls. Patients with FMS had significantly lower cortisol levels during the day, most pronounced in the morning (Cortisol Awakening Response). Moreover, FMS patients had significant lower adrenaline and dopamine levels compared with healthy controls, but similar noradrenaline levels. Furthermore, FMS patients showed increased heart rate values and lower sleep quality.

Results support the hypothesis of a dysfunction in the regulation of the stress systems in FMS patients, and increased pain perception and sleeping problems.

CORRESPONDING AUTHOR: Roberto Riva, PhD student, Psychology, Stockholm University, Stockholm, 10691; roberto. riva@psychology.su.se

\section{PST-138}

EFFECTS OF USING A HEAD-MOUNTED DISPLAY HELMET FOR VIRTUAL REALITY DISTRACTION ON COLD PRESSOR PAIN IN COLLEGE STUDENTS

Bridget Armstrong, BA, Emily Law, MA, Soumitri Sil, MA and Lynnda M. Dahlquist, PhD

Psychology, University of Maryland, Baltimore County, Baltimore, MD.

Emerging evidence suggests that immersive Virtual Reality (VR) distraction is an efficacious intervention for procedural pain. VR distraction offers an alternative to pharmacological treatments that may cause adverse side effects. Although VR distraction has been demonstrated to be effective in laboratory and clinical settings, the method by which it works to combat pain remains unclear. McCaul and Malott (1984) hypothesize that pain processing requires conscious attention. A highly immersive distractor should leave less attention available for pain processing. This study examined whether a VR head-mounted display helmet increases the analgesic effects of interactive distraction. A head-mounted display occludes extraneous sights and sounds, helping a person feel 'present' in the virtual world, leaving less attention available for pain sensations originating outside of the virtual world. Seventy-three college students between the ages of 18 and 25 were exposed to cold pressor pain without any distraction intervention (baseline) and then two cold pressor trials during which they played a Nintendo ${ }^{\circledR}$ Wii videogame. They viewed the game on a 21 " TV or through a VR head-mounted display unit. The study used a within-subjects repeated-measures design, with the two videogame distraction trials presented in counterbalanced order following baseline. LOG-10 transformation was conduced on all pain tolerance scores to correct for skew and kurtosis. Results showed that pain tolerance during the baseline trial $(M=1.46$, $\mathrm{SD}=.254$ ) was significantly lower than pain tolerance during the helmet-on condition $(\mathrm{M}=1.72, \mathrm{SD}=.329), \mathrm{t}(72)=-11.24, \mathrm{p}<.001$, and the helmet-off condition $(\mathrm{M}=1.68, \mathrm{SD}=.32), \mathrm{t}(72)=-10.69$, $\mathrm{p}<.001$. Participants showed the greatest increases in pain tolerance in the helmet-on condition, $t(72)=2.82, p<.01$. Further research is needed to determine whether these results could be replicated in a clinical setting. 
CORRESPONDING AUTHOR: Bridget Armstrong, BA, Children's National Medical Center, Washington, DC, DC, 20010; barmstro@cnmc.org

PST-139

ASSESSMENT OF COGNITION IN OLDER ADULTS

WITH CHRONIC, NON-MALIGNANT PAIN IN RESPONSE

TO SUSTAINED RELEASE OPIOID MEDICATION

Ashley J. Holder, BS, ${ }^{1,2}$ Monique Cherrier, PhD, ${ }^{1,2}$ Danny Shen, $\mathrm{PhD},{ }^{2}$ Linda Risler, BS, ${ }^{2,4}$ Steven Overman, MD ${ }^{3}$ Mark Hawley, $\mathrm{PhD}^{5}$ and Anna Du Pen, ARNP

${ }^{1}$ Psychiatry and Behavioral Sciences, University of Washington, Seattle, WA; ${ }^{2}$ Pharmacy and Pharmaceutics, University of Washington, Seattle, WA; ${ }^{3}$ Northwest Hospital, Seattle, WA; ${ }^{4}$ Fred Hutchinson Cancer Research Center, Seattle, WA; ${ }^{5}$ Veterans Administration Puget Sound Health Care System, Seattle, WA and ${ }^{6}$ Swedish Medical Center, Seattle, WA.

Persistent pain, sufficient to cause significant impairment in daily functioning affects up to 50 percent of older adults. Analgesics are the most common therapy used to manage pain, with opioids playing an increasingly important role in pharmacological treatment of chronic disabling pain in older adults. The efficacy of medication therapy needs to be balanced against potential side effects, including the risk of neurocognitive impairment. In this study we objectively examined neurocognitive effects of methadone in older adults ( $>65$ years) suffering from daily chronic pain (osteo-arthritis). Older participants (mean age 76 years) were evaluated twice at baseline (prior to the start of medication) and again 24hours after initial dose and again after 5 and 21 days of medication. Subjective ratings of pain along with objective testing of memory, attention and concentration were measured. Physiologic measurement of medication effects (pupil size) were also obtained. Results indicate a slight decline in weekly pain ratings (NS) and a modest drop in pupil size (NS). Neurocognitive tests indicated no significant change from baseline on measures of memory (HVLT), concentration (D2), reaction time or global cognitive measures (MMSE). There was an improvement on a measure of working memory $(\mathrm{p}<.05)$ and a trend toward improvement on an attention task $(\mathrm{p}<.06)$. Global measures of functioning did not change. Taken together, results suggest that older adults suffering from chronic non-malignant pain do not experience significant decrements in cognitive functioning in response to methadone. This may be consistent with findings of improved cognition with improved pain control observed in younger samples.

CORRESPONDING AUTHOR: Ashley J. Holder, BS, Psychiatry and Behavioral Sciences, University of Washington, Seattle, WA, 98108; holdea@uw.edu

PST-141

THE CONTEXTUAL EFFECTS ON CHRONIC PAIN WHEN MEASURED IN A DOCTOR-OFFICE VERSUS IN A HOMESETTING

Egil A. Fors, $\mathrm{MD} / \mathrm{PhD}^{1}$ and Øyvind Bakke, $\mathrm{PhD}^{2}$

${ }^{1}$ Pain and Psychiatry, St Olavs Hospital, Trondheim, Norway and

${ }^{2}$ Mathematics, Norwegian University of Science and Technology,

Trondheim, Norway.
Aim of investigation: There is limited knowledge about vulnerability factors for pain in fibromyalgia (FM), but low education, hereditary influences and mood are known, while contextual triggers and personality factors are dubious. Our aim was to study how FM pain differed in two contexts.

Methods: Fiftyfive female FM patients scored pain (VAS 0-100), demographic data (age, education, and single status), mood (depression/BDI and anxiety/STAI-T), Cluster C personality traits (avoidance, OCD, dependence by MCMI-I), sleep (VAS), TCA-use dummy and FM-duration. Main outcome was Pain-difference (VAS) between "pain measured in a doctor's office" and "pain measured alone at home" one hour later. Secondary outcomes were vulnerability and resilience factors for pain in those two contexts.

Results: "Pain office" (VAS=50.6) versus "Pain home" $(\mathrm{VAS}=43.4)$ showed a significant difference $=7.2$ by a paired $t$-test $[\mathrm{t}=2.4 ; \mathrm{df}=54$; $\mathrm{p}=0.022]$. Generalized Linear Methods (GLM) formula end-model: "Pain office" Anxiety + Education showed that Anxiety $(\mathrm{t}=3.15$, $\mathrm{p}<0.01)$ facilitated the "pain office" experience, while Education $(\mathrm{t}=-2.43, \mathrm{p}<0.05)$ impeded it. Avoidance $(\mathrm{t}=2.66, \mathrm{p}=0.01)$ and "poor sleep" ( $\mathrm{t}=2.55, \mathrm{p}<0.05)$ worsened the "home-context pain". Conclusions: Pain was perceived weaker at home than in the doctor-office. Avoidance and poor sleep were vulnerability factors for pain at home, while trait-anxiety worsened pain in the office. Education was a resilience factor against pain in the doctor-office, while no resilience factors were found at home except the home context itself.

CORRESPONDING AUTHOR: Egil A. Fors, MD/PhD, Pain and Psychosomatic Center, St Olavs Hospital, Trondheim, 7006; eafors@online.no

\section{PST-142}

FEASIBILITY AND EFFECTS OF PHYSICAL EXERCISE IN DAILY LIFE FOR PATIENTS WITH IRRITABLE BOWEL SYNDROME

Toyohiro Hamaguchi, PhD, ${ }^{1,2}$ Kunihiko Shimizu, MS, ${ }^{3}$ Jun Tayama, $\mathrm{PhD},{ }^{4}$ Tadaaki Tomiie, $\mathrm{PhD},{ }^{5}$ Motoyori Kanazawa, $\mathrm{PhD}, \mathrm{MD},{ }^{2}$ Kohji Sakurai, PHD, $\mathrm{MD}^{3}$ and Shin Fukudo, PHD, $\mathrm{MD}^{2}$

${ }^{1}$ Occupational Therapy, Saitama Prefectural University, School of Health and Social Services, Koshigaya, Japan; ${ }^{2}$ Behavioral Medicine, Tohoku University, Graduate School of Medicine, Sendai, Japan; ${ }^{3}$ Department of Occupational Therapy, Niigata University of Health and Welfare, Graduate School of Health Science, Niigata, Japan; ${ }^{4}$ Center for Health and Community Medicine, Nagasaki University, Nagasaki, Japan and ${ }^{5}$ Department of Psychology, Health Sciences University of Hokkaido, School of Psychological Science, Sapporo, Japan.

Symptoms of Irritable bowel syndrome (IBS) are often aggravated by stress, which alters colonic motility and visceral perception. We reported the possibility of improving IBS pathophysiology by passive abdominal muscle stretching as indicated by chromogranin A ( $\mathrm{CgA})$, a biochemical index of the activity of the sympathetic / adrenomedullary system. Although the degree to which patient intervention in the areas of exercise, and management of daily activity can improve symptoms of IBS through healthier lifestyle behaviors is unknown. 
The aim of this study was to determine the effects of intervention to non-patient with IBS on the short and long term outcomes, and the association between physical exercise and symptoms.

The present case controlled study was conducted on 30 adult subjects with mild or moderate symptom of IBS who were allocated randomly to either the exercise (intervention) group $(n=15)$ or the wait-listed control group $(n=15)$. Four-week intervention consisted of components: self record, stretch of abdominal muscle, physical exercise of pelvis, and $15 \mathrm{~min}$ working. Measurements (symptom-limited daily life performance, psychological status, $\mathrm{CgA}$, heart rate variability and regional cerebral oxy-hemoglobin change) were performed before and after intervention. Spearman rank correlations were used to assess the association between psycho-physiological data and symptom scores. Wilcoxon rank sum tests compared changes in scores versus their baseline values.

After the intervention, tendency of improvements were found in symptom-limited performance of daily life and psychological status. The four-week intervention had immediate beneficial effects on physiological variables and some neurological factors in patients with IBS. The findings of this study could be used as a reference for intervention, education, and physical health policies in IBS.

CORRESPONDING AUTHOR: Toyohiro Hamaguchi, PhD, Occupational Therapy, Saitama Prefectural University, Koshigaya, 343-8540; hamaguchi-toyohiro@spu.ac.jp

PST-143

AN APPROACH TO THE ILLNESS BEHAVIOR OF FIBROMYALGIA PATIENTS

Maria Dolores Gil Llario, PhD, ${ }^{1}$ Estefanía Ruiz, Postgraduate, ${ }^{2}$ Rafael Ballester, $\mathrm{PhD}^{2}$ and Cristina Gimenez, $\mathrm{PhD}^{2}$

${ }^{1}$ Departamento de Psicología evolutiva y de la educación, Universidad de Valencia, Valencia, Spain and ${ }^{2}$ Psicología Básica, Clínica y Psicobiología, Universitat Jaume I, Castellón, Spain.

Fibromyalgia is one of the chronic pain syndrome most prevalent worldwide, but there are serious doubts about its origin and its causes yet. Its complexity difficults the diagnosis and, especially, the sick role experience of patients. In this context, showing a maladaptive illness behavior will produce a negative interference in daily lives of these people, increasing their subjective distress. To improve our knowledge about this topic, our pilot study compares the responses between a group of fibromyalgia patients $(\mathrm{N}=16)$ and a control group $(\mathrm{N}=13)$, all of them women, with a mean age of 44 . All participants belonged to a local association (Association of Fibromyalgia of Onda). The evaluation instrument was a Spanish adaptation of Illness Behavior Questionnaire (IBQ - Pillowsky and Spencer, 1983) by Ballester (1992), which evaluates attitudes and feelings about the disease, the perception of the reactions of others, and their own perception about the psychosocial situation. Data show higher scores on IBQ factors of all patients with fibromyalgia compared with controls. We got statistically significant in: hypochondriasis $(\mathrm{t}=2.247, \mathrm{p} \leq 0.032)$, disease conviction $(\mathrm{t}=2.121, \mathrm{p} \leq 0.042)$, psychologic vs. somatic focus $(\mathrm{t}=3.042, \mathrm{p} \leq$ $0.005)$, affective disturbance $(\mathrm{t}=2.814, \mathrm{p} \leq 0.008)$, denial of psychosocial problems $(\mathrm{t}=3.865, \mathrm{p} \leq 0.001)$ and Whiteley Index of Hypochondriasis $(t=3.271, p \leq 0.003$. These results indicate that people who are suffering fibromyalgia have higher predisposition to somatization and seek medical treatment. Moreover, they also show a tendency toward depression and anxiety as a result of the disease. This information will facilitate the comprehension and diagnosis of abnormal illness behaviours to treat fibromyalgia patients with more effectiveness

CORRESPONDING AUTHOR: Maria Dolores Gil Llario, PhD, Departamento de Psicología evolutiva y de la educación, Universidad de Valencia, Valencia, 46022; dolores.gil@uv.es

PST-144a

PREDICTING ACTIVITY LIMITATIONS IN ORTHOPAEDIC PATIENTS FROM BIOMEDICAL AND BEHAVIOURAL MODELS: REPLICATION AND EXTENSION

Marie Johnston, $\mathrm{PhD},{ }^{1}$ Francis Quinn, BSc, ${ }^{1}$ Diane Dixon, $\mathrm{PhD},{ }^{2}$ Derek Johnston, $\mathrm{PhD}^{1}$ and Beth Pollard, $\mathrm{PhD}^{1}$

${ }^{1}$ Health Psychology, University of Aberdeen, Aberdeen, United Kingdom and ${ }^{2}$ Strathclyde University, Glasgow, United Kingdom.

The WHO International Classification of Functioning and Disability (ICF) has been widely accepted as a conceptual model of disablement, but further specification of behavioral factors is required. We proposed conceptualizing disability, or activity limitations, as behavior and integrating biomedical and behavioral determinants. We previously tested a model including constructs from ICF and Social Cognitive Theory (SCT) using structural equation modelling; it fitted better and explained more variance than ICF or SCT alone. The current study replicated that study with a new sample from the same population (orthopedic patients awaiting joint replacement), and extended the tests to after surgery. Two weeks before surgery, 342 orthopedic patients with chronic pain (most having arthritis) completed a questionnaire assessing impairment and activity limitations plus SCT variables (SelfEfficacy and Outcome Expectancy). One year after surgery, when both impairment and activity limitations were reduced, 228 completed the questionnaire again. We imposed the statistical models found in our previous study (before and after surgery) using structural equation modelling, with NNFI, CFI, RMSEA fit indices) to predict activity limitations. Before surgery, findings were very similar to our previous findings, with all models accounting for significant variance and fitting well, but the integrated model fitted better and accounted for more variance. (ICF: 35\%; SCT: 60\%; Integrated model: 64\%). Each model showed stronger predictions after surgery, with the combined model accounting for most variance (ICF: 52\%; SCT: 79\%; Integrated model: $82 \%$ ). On each occasion, all three predictor variables had significant coefficients. The integrated model provides a good explanation of disability in this population, superior to a biomedical or behavioral model alone, and identifies targets for intervention to reduce disability.

CORRESPONDING AUTHOR: Marie Johnston, PhD, Health Psychology, University of Aberdeen, Aberdeen, AB; m.johnston@ abdn.ac.uk

\section{PST-145}

POTENTIAL MEDIATORS OF CHANGE IN CHILDREN'S PHYSICAL ACTIVITY

Helen L. Brown, BApp Sci (health promotion), Jo Salmon, PhD and Clare Hume, $\mathrm{PhD}$

Centre for Physical Activity and Nutrition Research, Deakin University, Burwood, VIC, Australia. 
The purpose of this study was to conduct an in-depth exploration of potential mediators of change in children's physical activity and the feasibility of strategies targeting these potential mediators.

A qualitative study of 143 children aged 10-12 years including eight focus groups from eight schools and teachers, and one-onone interviews with 12 parents were conducted. Common mediators of physical activity were identified from the literature and behavioural theories and a semi-structured interview explored the potential mediators of change in children's physical activity. Thematic analysis identified key and emergent themes.
Several new potential mediators as well as previously identified mediators were identified (eg. fear of the unknown and competitiveness). Most of the potential strategies were seen to be feasible; however, differing views existed among children, parents and teachers. These findings will inform the development of effective strategies for promoting children's physical activity by identifying the most appropriate potential mediators to be targeted.

CORRESPONDING AUTHOR: Helen L. Brown, BApp Sci (health promotion), School of Exercise and Nutrition Sciences, Deakin University, Burwood, VIC, 3124; hbrown@deakin.edu.au

\section{CITATION ABSTRACT \\ TOP FIFTEEN SCORING POSTER}

PST-146

AN OUTCOME \& PROCESS EVALUATION OF A FINANCIAL INCENTIVE SCHEME AIMED AT ENCOURAGING PARTICIPATION IN PHYSICAL ACTIVITY IN SANDWELL IN THE WEST MIDLANDS REGION OF THE UK

Gemma Hurst, PhD Behaviour Change - Physical Activity, ${ }^{1}$ Rachel Davey, PhD, ${ }^{1}$ David ClarkCarter, $\mathrm{PhD}^{2}$ and Sarah Grogan, $\mathrm{PhD}^{2}$

${ }^{1}$ Faculty of Health, Staffordshire University, Stoke-on-Trent, United Kingdom and ${ }^{2}$ Faculty of Sciences, Staffordshire University, Stoke-on-Trent, United Kingdom.

Background: Given the many benefits of physical activity \& the current levels of physical inactivity in England, it is important that effective interventions that promote the adoption \& maintenance of an active lifestyle are well designed, implemented \& evaluated. There is growing interest in the potential use of direct incentives to encourage individuals to adopt healthy lifestyle behaviours. The focus of this project was to investigate a community-based approach aimed at encouraging residents in Sandwell to increase their lifestyle physical activity levels using financial incentives, town competition \& an end state goal of collectively walking the distance to Beijing. Methods: A pre-, post-intervention, cohort design was employed with 1678 participants receiving a pedometer, health assessment, financial incentive, tailored health advice \& an activity programme run by Sandwell Primary Care Trust. Incentives allocated were based upon improvement in objective measures (BMI, waist-hip ratio, waist-circumference \& blood pressure) \& self-reported measures (Stage of Change (TTM), SF12, EQ5D \& Theory of Planned Behaviour (TPB)) over a 3-month intervention period. Incentives were given in the form of cash vouchers. A total of 92 individuals took part in semi-structured interviews $(n=34) \&$ focus groups $(\mathrm{n}=13)$ at 3-, 6- \& 12-months follow up. Idiographic experiences of the project $\&$ its design \& delivery were discussed. Data was analysed using Thematic Analysis. Results: The "Walk to Beijing" programme was successful in achieving statistically significant $(\alpha-$ level of $0.05)$ positive changes in objective $\&$ subjective measures of health status at 3- \& 6-month follow-up compared to baseline. A significant decrease in waist-hip-ratio, waist circumference \& diastolic blood pressure was found. In addition, there were positive changes in TTM stage of change \& in perceived health status, as measured by the EQ5D \& SF12 at 3-month follow-up. There were also significant positive changes in the intention, perceived behavioural control \& attitude subscales of the TPB. The majority of participants received the gold or silver award \& on average, were walking over 5,500 steps per day throughout the intervention period. Conclusions: This research supports the hypothesis that a scheme employing the use of financial incentives can increase individual physical activity levels. Qualitative findings suggested that providing a financial incentive within the behaviour change programme helped with uptake. This project provided the 'incentive' needed in order to initialise a healthier lifestyle by increasing levels of physical activity.

CORRESPONDING AUTHOR: Gemma L. Hurst, MSc Health Psychology, Faculty of Health, Staffordshire University, Stoke-onTrent, ST10 1EH; g.1.hurst@staffs.ac.uk 


\section{PST-147}

THE EFFECTS OF SELF EFFICACY ON THE OUTCOME OF GROUP COGNITIVE-BEHAVIORAL INTERVENTION INCLUDING THE SELF-MONITORING METHOD

Jun Tayama, $\mathrm{PhD},{ }^{1}$ Kazuki Nishiura, $\mathrm{PhD},{ }^{2}$ Toyohiro Hamaguchi, $\mathrm{PhD},{ }^{3}$ Tadaaki Tomiie, $\mathrm{PhD},{ }^{4}$ Mami Tamai, MD, PhD, ${ }^{1}$ Hironori Yamasaki, MD, PhD, ${ }^{1}$ Masaki Hayashida, MD, PhD, ${ }^{1}$ Susumu Shirabe, $\mathrm{MD}, \mathrm{PhD}^{1}$ and Shin Fukudo, $\mathrm{PhD}^{5}$

${ }^{1}$ Center for Health and Community Medicine, Nagasaki University, Nagasaki, Japan; ${ }^{2}$ Developmental and Clinical Studies, Miyagi Gakuin Women's University Faculty of Liberal Arts, Sendai, Japan; ${ }^{3}$ Occupational Therapy, Saitama Prefectural University, School of Health and Social Services, Koshigaya, Japan; ${ }^{4}$ Department of Psychology, Health Sciences University of Hokkaido, School of Psychological Science, Sapporo, Japan and ${ }^{5}$ Department of Behavioral Medicine, Tohoku University Graduate School of Medicine, Sendai, Japan.

The purpose of this study was to investigate whether degrees of self efficacy (SE) are associated with physical activities or psychological factors after self-monitoring intervention. The subjects found in this study consisted of 49 female college students. We put the subjects in three groups, according to ones degree of SE: high SE group, middle $\mathrm{SE}$ group and low SE group. We conducted an intervention study using group cognitive-behavioral intervention including the selfmonitoring method. We performed a psychological assessment in order to exam SE, self esteem and stress responses, while also applying experimental protocol in the pre, intervention and post period stages. ANOVA of exercise volume disclosed significant group $\times$ period interaction. The significant period effect was disclosed in exercise volume and total energy expenditure. In the post period, low SE group showed a significantly lower exercise volume than high SE group. Low SE group had a significantly lower exercise volume, total energy expenditure and step counts in the post period compared in the intervention period. It should be noted that this pilot study was conducted without a follow up period. In the future, we need to examine whether self-monitoring intervention effects physical activities in a follow up period. In conclusion, after we applied group cognitive-behavioral intervention including the self-monitoring method, we discovered that high SE promoted the habituation of exercise while also inhibiting the risk of rebound. Apathy was decreased in only the subjects categorized with low SE using group cognitivebehavioral intervention including the self-monitoring method.

CORRESPONDING AUTHOR: Jun Tayama, PhD, Center for Health and Community Medicine, Nagasaki University, Nagasaki, 852-8521; jtayama@nagasaki-u.ac.jp

\section{PST-148}

\section{DETERMINANTS OF EXERCISE STAGE}

OF CHANGE AMONG INDIVIDUALS

WITH SPONDYLOARTHROPATHY

Deborah Da Costa, PhD, ${ }^{1}$ Michel Zummer, $\mathrm{MD}^{2}$ and Mary-Ann Fitzcharles, $\mathrm{MD}^{3}$

${ }^{1}$ Medicine, McGill University, Montreal, QC, Canada; ${ }^{2}$ Rheumatology, Hopital Maisonneuve-Rosemont, Montreal, QC, Canada and ${ }^{3}$ Rheumatology, McGill University Health Center, Montreal, QC, Canada.
Spondyloarthropathies (SpA) consist of a group of chronic inflammatory diseases characterized by symptoms of back pain, stiffness, fatigue, and sleep disturbance. While regular exercise has been shown to be beneficial and is recommended as part of patient selfcare, little is known regarding intention to initiate, alter, or maintain exercise in this patient population. This study examined the distribution of exercise stages of change and the biopsychosocial factors which differentiate readiness to exercise among individuals with SpA. Demographic and clinical data (comorbid disease, disease duration), along with self-report standardized questionnaires assessing functional disability, disease activity, depressed mood, perceived stress, leisure time physical activity, exercise self-efficacy, exercise pros and cons and exercise stage of change were collected crosssectionally from a sample of 125 individuals with $\mathrm{SpA}$. Data were analyzed by frequency analysis, ANOVA and chi-square. Fifty-two percent $(52 \%)$ of the participants were in the action/maintenance stages for exercise adoption and $49 \%$ in the preaction stages (precontemplation $=20.8 \%$; contemplation $=13.6 \%$, preparation $13.6 \%)$. Patients in the preaction stages had more disease activity $(p<.005)$, more functional disability $(p<.001)$, higher perceived stress $(p p=.008)$ and reported lower self-efficacy $(p=.003)$ and less barriers for exercise $(p=.008)$. Gender, age, socioeconomic status (i.e. education, income) and exercise pros did not differ across the stages. These findings suggest that strategies designed to improve exercise self-efficacy, reduce exercise barriers and emphasize the functional and emotional (i.e. stress reducing) health benefits associated with physical activity may be useful for patients with SpA who are less motivationally ready to engage in regular exercise.

CORRESPONDING AUTHOR: Deborah Da Costa, PhD, Medicine, McGill University, Montreal, QC, H3A 1A1; deborah. dacosta@mcgill.ca

\section{PST-149}

HABITUAL BEHAVIOR PATTERNS AMONG REGULAR EXERCISERS: A SURVEY STUDY USING THE SELFREPORT HABIT INVENTORY

Karyn Tappe, $\mathrm{PhD},{ }^{1}$ Alison C. Sweeney, MA, ${ }^{2}$ Megan E. Brault, $\mathrm{BS}^{2}{ }^{2}$ Deirdra Frum, MA, ${ }^{2}$ Jayme Oltarzewski, MA, ${ }^{2}$ Ellen Tarves, $\mathrm{BA}^{2}$ and Maureen Endres, $\mathrm{MS}^{2}$

${ }^{1}$ Department of Emergency Medicine Research, Cooper University Hospital, Camden, NJ and ${ }^{2}$ Psychology, LaSalle University, Philadelphia, PA.

Regular leisure-time physical activity behavior is achieved by fewer than $50 \%$ of Americans. If it were possible to decrease the cognitive load associated with deciding to exercise, it might be easier to adhere to a regular exercise routine, as it becomes an automatic activity that does not require decision making; this type of behavior would be termed by theorists to be a "habit." However, empirical evidence is lacking as to whether those individuals who have successfully instituted a regular exercise routine have such aspects of "habit" in their behavior patterns. The present study reports the level of habit formation among regular exercisers using a survey format.

The Self-Report Habit Inventory (SRHI) (Verplanken \& Orbell, 2003) measures the subjective sense of habit formation. The present exploratory study surveyed a sample of individuals $(n=$ 
176) who attended a fitness club in a metropolitan area using the SRHI (with a 7-point Likert scale), as well as objective questions about regularity of behavior (frequency and constancy of time, place, and weekly and seasonal patterns).

Results indicated a median SRHI-average score of 5.17 (range: 1.257.00). Even though this sample was presumed to represent regular exercisers, it was notable that there was a substantial subsample whose scores on the SRHI were quite low. Therefore, a median split was calculated, and participants with low versus high scores were compared on the other (non-SRHI) survey items. Low-SRHI participants exercised significantly fewer days per week $(p<0.001)$ and their exercise patterns were more likely to change seasonally than highSRHI scorers $(\mathrm{p}<0.05)$. However, the groups did not differ in regularly of time $(p=0.52)$, location $(p=0.14)$, specific exercise behavior $(p=$ $0.41)$, or weekly consistency of exercise $(p=0.36)$. Correlational and Fisher's exact test analyses suggested that individuals who demonstrated constancy on one objective measure of habit were likely to score high on other objective measures of habit as well $(\mathrm{ps}<0.05)$. Findings suggest that subjective self-concept of "habit" may not strongly correspond to all objective measures of consistency of behavior, and that only some individuals performing regular exercise behavior do so through a habit formation processes.

CORRESPONDING AUTHOR: Karyn Tappe, PhD, none, Sewell, NJ, 08080; karyn.tappe@gmail.com

\section{PST-150}

FUZZY TRACE THEORY EXPLAINS THE EFFECTS OF EXPERTISE ON DECISION MAKING OF BIKERS Efrat Neter, PhD, Moti Gabay, BA and Erez Chayun, B A Ruppin Academic Center, Emeq Hefer, Israel.

Background: Fuzzy Trace Theory (FTT) is a cognitivedevelopmental theory on representation and memory recently applied to decision making in health domains (Renya, 2007). It posits two processes/representations: 'Gist', which is bottom-line meaning of information, vague and qualitative, as opposed to 'Verbatim' representation which is the exact surface form (literal), precise and quantitative. The paper applied this theory to examine differences in decision processes of novices and experts in biking. It was hypothesized that experts, who rely on gist processing and less on knowledge, will use fewer information dimensions, reach unequivocal decisions with starker separations among categories, yet make high-quality decisions (as compared to a gold standard). Methods: 90 participants, who were novices (up to a year), experienced (one-two years of experience) and expert bikers (two to five years) filled out a self-administered questionnaire comprised of knowledge, intuition, and nine scenarios depicting challenging biking situations. Two recognized experts' responses served as a gold standard.

Findings: Significant differences were found between the groups, so that experts were higher in the knowledge and intuition from the experienced and novices. Experts also relied in their decisions on fewer dimensions of information, provided starker and unequivocal judgments, and made more similar decisions to the golden standards than experienced and novice bikers. Finally, their judgments were based more on intuition (=gist) than on knowledge.
Conclusion: Experts in sports think differently than novices, and are higher in their use of both knowledge and intuition.

CORRESPONDING AUTHOR: Efrat Neter, PhD, Ruppin Academic Center, Emeq Hefer, 40250; neter@ruppin.ac.il

\section{PST-151a}

SOCIAL FACILITATION WHILE CYBERCYCLING WITH AVATARS AFFECTS EXERCISE BEHAVIOR: DIFFERENTIAL EFFECTS OF COMPETITIVENESS

Amanda Snyder, BS anticipated, ${ }^{1}$ Arielle Gartenberg, BS anticipated, ${ }^{1}$ Vadim Yerokhin, BS anticipated, ${ }^{1}$ Ciro Griffiths, BS anticipated, ${ }^{1}$ Tracey Rocha, BS, ${ }^{1,2}$ Joseph Nimon, BS, ${ }^{1}$ Paul Arciero, $\mathrm{PhD}^{3}$ and Cay Anderson-Hanley, $\mathrm{PhD}^{1}$

${ }^{1}$ Psychology, Union College, Schenectady, NY; ${ }^{2}$ Counseling Psychology, University at Albany, Albany, NY and ${ }^{3}$ Exercise Science, Skidmore College, Saratoga Springs, NY.

This study evaluated the effects of social facilitation and competiveness on exercise behavior among older adults participating in the Cybercycle Study. Social facilitation is defined as an increase in response due to the presence of others (Allport, 1924). Several theories, such as the Self-Determination Theory (SDT) and the Cognitive Evaluation Theory (CET), have been developed to explain exercise behavior and the influences of intrinsic motivation on performance (Fredrick \& Ryan, 1995). Some studies have suggested that competitive environments fostered by social facilitation enhance performance on motor tasks (Zajonc, 1965). It was hypothesized that a personality factor, such as competitiveness could influence the effect of social presence. Thirteen older adults (age $=78.6)$ participating in the Cybercycle Study (a randomized clinical trial investigating the neuropsychological, physiological, and behavioral effects of a videogame-enhanced stationary bike) were assigned to ride an exergaming bike while competing against virtual riders. Participant performances during one month (frequency and intensity of rides) were compared with the previous month's performances when no other avatars had been present. Results show that with the introduction of on-screen riders, as competitiveness increased, ride frequency decreased $(\mathrm{r}=-.67 ; \mathrm{p}=.01)$, while riding intensity increased $(\mathrm{r}=.55 ; \mathrm{p}=.05)$. Social facilitation appears to interact with an exerciser's level of competitiveness such that it could enhance or detract from exercise goals, depending on the desired outcomes. This finding has interesting implications for prescribing various types of exercise according to personality style and desired exercise goals.

CORRESPONDING AUTHOR: Cay Anderson-Hanley, PhD, Psychology, Union College, Schenectady, NY, 12866; andersoc@ union.edu

\section{PST-151b}

PSYCHOSOCIAL DETERMINANTS OF THE INTENTION OF DAYCARE WORKERS TO MAKE CHILDREN AGED 3 TO 5 YEARS MOVE

Camille Gagné, $\mathrm{PhD}$

Faculty of nursing, Laval university, Quebec, QC, Canada. 
Introduction: Data available in the literature show that children in daycare centres spend most of their time in sedentary activities that could have an incidence on their health. To the best of our knowledge, this is the first study to use a combination of theoretical frameworks (theory of planned behaviour, theory of interpersonal behaviour, process model on ease of retrieval) to identify psychosocial factors underlying the intention of daycare workers to make children aged 3 to 5 years move. It is important to study intention, because findings of meta-analyses indicate that this variable is the best determinant of behaviour.

Method: This study is based on a descriptive correlational design. Daycare centres in the City of Quebec (Canada) were chosen at random. Daycare workers were then invited to complete a questionnaire measuring their intention to make children move for at least two hours a day in the next 30 days, as well as the following variables: attitude, subjective norm, perceived behavioural control, self-efficacy, descriptive norm, past behaviour, habit, perceived moral obligation and perceived regularity at which they make the children move. The Cronbach alpha of each construct was satisfactory, with values ranging from .82 to .97 . Multiple regression analysis was used to analyze the data.

Findings: Preliminary findings obtained among 37 daycare workers from 15 daycare centres indicate that perceived behavioural control $(b=.35)$, subjective norm $(b=.26)$, perceived moral obligation $(b=.23)$, attitude $(b=.20)$ and the regularity with which daycare workers perceive they make children $(b=.14)$ move explain $87 \%$ of the variance in intention $F(5,31)=51.23, p=.00$. Conclusion. These preliminary findings indicate that a large part of the intention of daycare workers to make children move can be explained by factors in the theoretical frameworks selected. These factors could eventually be used to develop an intervention aimed at improving or consolidating the intention of daycare workers. However, the findings first need to be confirmed in a larger sample.

CORRESPONDING AUTHOR: Camille Gagné, PhD, Faculty of nursing, Laval university, Quebec, QC, G1V 0A6; camille. gagne@fsi.ulaval.ca

\section{PST-151c}

THE CAT TEST AND PREDICTING HEALTH BEHAVIOR IN OLDER ADULTS: RESULTS FROM THE CYBERCYCLE STUDY Joseph Nimon, BS, ${ }^{1}$ Tracey Rocha, Doctoral Candidate, ${ }^{1,3}$ Ellie Lakritz, BS (in progress), ${ }^{1}$ Benjamin Wantman, BS (in progress), ${ }^{1}$ Elana Vetrano, BS (in progress), ${ }^{1}$ Paul Arciero, PhD, Exercise Science $^{2}$ and Cay Anderson-Hanley, PhD, Psychology ${ }^{1}$

${ }^{1}$ Psychology, Union College, Schenectady, NY; ${ }^{2}$ Exercise Science, Skidmore College, Saratoga Springs, NY and ${ }^{3}$ Counseling Psychology, University at Albany, Albany, NY.

It has been documented that global attitudes are very poor predictors for outcomes of singular behavioral responses toward a given psychological object (Ajzen and Cote, 2008). In addition to the psychological object, we must also know its underlying factors; chiefly action, context, and time. When trying to assess an attitude toward a health behavior, it is important to specify as many of the details surrounding the health behavior as possible, otherwise the attitude may be too generalized, or global, to be predictive of actual engagement in the health behavior under question.

In this two-year multi-site, randomized trial, 75 participants (mean age $=78.99 ; \mathrm{SD}=8.49)$ were enrolled and assessed using the Theory of Planned Behavior (Ajzen, 2007) to help determine how personality, disposition toward health behaviors, and environment affect a person's choice to engage in cybercycling. However, many of the measures used in the Cybercycle Study help to assess only global attitudes towards exercising and health behaviors, and for this reason we decided to develop our own measure specifically tailored to the act of cybercycling, called the Cybercycling Attitudes Test (CAT).

Our hypothesis is that the CAT would lead participants to take into account the previously mentioned factors of action, context, and time while completing the questionnaire, such that it would be more predictive than the other measures for successful engagement in cybercycling. We aim to demonstrate the reliability and validity of the CAT, as well as to offer suggestions for replication in future behavioral health research. We hope that our results will add to the literature in suggesting more stringent development criteria for future measures assessing health behavior attitudes.

CORRESPONDING AUTHOR: Joseph Nimon, BS, Psychology, Union College, Schenectady, NY, 12305; nimonj@gmail.com

\section{PST-151d}

ELECTROPHYSIOLOGICAL AND NEUROPSYCHOLOGICAL EFFECTS OF A SINGLE BOUT OF EXERCISE

Vadim Yerokhin, BS anticipated, ${ }^{1}$ Mina Dunnam, PhD, ${ }^{2}$ Paul Arciero, $\mathrm{PhD}^{3}$ and Cay Anderson-Hanley, $\mathrm{PhD}^{1}$

${ }^{1}$ Psychology, Union College, Schenectady, NY; ${ }^{2}$ Psychology, Stratton VA Hospital, Albany, NY and ${ }^{3}$ Exercise Science, Skidmore College, Saratoga Springs, NY.

INTRODUCTION: Exercise has been shown to have a significant impact on cognitive function in older adults and it is thought to offset declines in cognitive performance with advancing age (Colcombe et al., 2004; Larson et al., 2006). Exercise has also been shown to increase nutrient supply to capillaries, to decrease neuronal death and to promote an overall better cognitive health (Kramer et al., 2007). Nevertheless, very little research has been done to clarify the impact of exercise on the brain using neuroimaging techniques such as electroencephalography (EEG).

METHODS: The current study aims to establish normative EEG and neuropsychological data in a sample of adults before and after a single bout of exercise. The level of cognitive state will be measured before and after exercise using a neuropsychological battery focusing on executive functions (e.g., Stroop, Trails, etc). Participant's neurophysiological state will be measured using EEG. Resting amplitude variability in frequencies will be measured and analyzed.

RESULTS: It is hypothesized that a single bout of exercise will enhance cognitive function, especially executive functions. It is expected that there will be a significant relationship between the performance on executive functions and the power of Alpha and Beta waves. This research will provide clarify regarding the neurophysiological and neuropsychological impact of exercise in normals and will be useful in additional research with impaired populations. Of particular concern, is Alzheimer's which is on the 
rise bringing devastating social and economic impacts (Brookmeyer, 2007). It is hoped that the potential neurophysiological and neuropsychological benefits of interventions such as exercise can be further clarified and utilized in the fight against cognitive decline.

CORRESPONDING AUTHOR: Cay Anderson-Hanley, PhD, Psychology, Union College, Schenectady, NY, 12866; andersoc@ union.edu

\section{PST-151e}

\section{DEMOGRAPHIC AND LIFESTYLE CHARACTERISTICS} OF RESPONDENTS TO A MAIL-BASED ACCELEROMETER SURVEILLANCE STUDY

Shigeru Inoue, $\mathrm{PhD},{ }^{1}$ Yumiko Ohya, $\mathrm{PhD},{ }^{1}$ Yuko Odagiri, MD, $\mathrm{PhD},{ }^{1}$ Tomoko Takamiya, MD, PhD, ${ }^{1}$ Shigeho Tanaka, PhD, ${ }^{2}$ Catrine Tudor-Locke, $\mathrm{PhD}^{3}$ and Teruichi Shimomitsu, MD, $\mathrm{PhD}^{1}$ ${ }^{1}$ Department of Preventive Medicine and Public Health, Tokyo Medical University, Tokyo, Japan; ${ }^{2}$ Health Promotion and Exercise Program, National Institute of Health and Nutrition, Tokyo, Japan and ${ }^{3}$ Walking Behavior Laboratory, Pennington Biomedical Research Center, Baton Rouge, LA.

BACKGROUND: Differences in characteristics between respondents and non-respondents to an objective physical activity assessement survey could indicate selection bias. The aim of this study was to identify the demographic and lifestyle characteristics of respondents to accelerometry in a field-based mail survey.

METHODS: A cross-sectional mail survey was sent to 4,000 residents aged 20-69 years old and 50\% male, randomly selected from the registry of residential addresses of four cities in Japan. There were 1,508 respondents (Responding subsample) to the initial questionnaire. Seven hundred eighty-six participants from the Responding subsample also agreed to wear an accelerometer for 7 days (Accelerometer subsample). Age and sex were compared between the Accelerometer subsample and all 3,214 Non-respondents (including those not responding to initial questionnaire). In addition, the Accelerometer subsample and the 722 respondents who participated only in the questionnaire survey but not in the accelerometry survey (Questionnaire Only subsample) were compared for demographic and physical activity differences using chi-square tests.

RESULTS: The Accelerometer subsample included significantly more women and middle-to-older age individuals compared with all Non-respondents. Comparisons between Accelerometer and Questionnaire Only subsamples revealed that persons who reported walking $>=150$ minutes/week for any purposes $(p<0.01)$ or walking for leisure $(p<0.001)$ tended to participate in the accelerometry survey.

CONCLUSIONS: The response pattern in this study reveals potential for selection bias in mail-based accelerometry surveillance studies. It is important to develop strategies to increase overall response rate and address this bias.

CORRESPONDING AUTHOR: Shigeru Inoue, PhD, Department of Preventive Medicine and Public Health, Tokyo Medical University, Tokyo, 1608402; inoue@tokyo-med.ac.jp
PST-151f

EFFECTS OF EXERCISE FREQUENCY ON ADHERENCE AND PSYCHOLOGICAL OUTCOMES: A RANDOMIZED CONTROLLED TRIAL

Lynda-Maree Bavin, Masters (Currently doing PhD) and Linda D. Cameron, $\mathrm{PhD}$

Psychology, University of Auckland, Auckland, New Zealand.

Understanding which exercise prescriptions are best suited for increasing adherence and exercise levels in previously sedentary adults is critical for efforts to enhance wellbeing through exercise program recommendations. Improvements in psychological wellbeing have been found with short (15-minute) exercise bouts, and it is possible that a regimen involving short bouts of exercise in a day is superior to one involving a single, long bout of exercise in a day because: (1) the anxiety-reducing effects of any single exercise bout reportedly last 4 to 6 hours, and (2) adherence may be greater because participants find it easier to fit short bouts into their daily schedules. This study investigated changes in levels of exercise participation, mood, anxiety and perceived stress in 82 sedentary adults who were randomly assigned to one of three conditions: a prescription of twelve 15-minute sessions of walking exercise per week (15Minx12), a prescription of four 45-minute sessions of walking exercise per week (45Minx4), or a waitlist control condition. Measures of exercise participation and psychological wellbeing were assessed at baseline and [daily/weekly] over an eight-week intervention.

Participants in the $15 \mathrm{Minx} 12$ group completed more walking minutes for every week of the intervention, and significantly more so, or bordering on significance, in three of the eight weeks ( $\mathrm{t}$ 's $\geq 2.00$, p's $\leq .05$ ), relative to participants in the $45 \mathrm{Minx} 4$ group. Although group differences in psychological outcomes fell short of statistical significance, regression analyses demonstrated that, for some weeks, walking minutes was an independent predictor of psychological well-being.

These findings support that a 15 minute twice daily prescription may yield greater exercise participation in previously sedentary adults, relative to a regime involving less frequent, longer bout sessions. This enhanced exercise participation may, in turn, promote psychological well-being.

CORRESPONDING AUTHOR: Lynda-Maree Bavin, Masters (Currently doing PhD), Psychology, University of Auckland, Auckland, 2102; lyndambavin@yahoo.co.nz

\section{PST-151g}

\section{THE THEORY OF PLANNED BEHAVIOR}

AND VIDEOGAME-ENHANCED EXERCISE FOR OLDER ADULTS: RESULTS FROM YEAR 2 OF THE CYBERCYCLE STUDY

Tracey Rocha, BA, ${ }^{1,3}$ Joseph Nimon, BS, ${ }^{1}$ Amanda Snyder, undergraduate, ${ }^{1}$ Arielle Gartenberg, undergraduate, ${ }^{1}$ Allison Seiler, undergraduate, ${ }^{1}$ Paul Arciero, $\mathrm{PhD}^{2}$ and Cay Anderson-Hanley, $\mathrm{PhD}^{1}$ ${ }^{1}$ Healthy Aging \& Neuropsychology Laboratory, Union College, Schenectady, NY; ${ }^{2}$ Exercise Science Laboratory, Skidmore College, Saratoga Springs, NY and ${ }^{3}$ Counseling Psychology, University at Albany, Albany, NY. 
INTRODUCTION: The Theory of Planned Behavior attempts to explain how people's attitudes and beliefs influence their engagement in health-related behaviors (Ajzen, 1985). Selfefficacy is one type of control belief in the model, and personality factors may also play a role in predicting behavior (Ajzen \& Cote, 2008). This study examined self-efficacy, along with competitiveness and frustration intolerance as two personality factors that may influence older adults' exercise behavior.

METHODS: Thirty-nine older adults completed the 3-month experimental exercise phase in Year 2 of the "Cybercycle Study," a randomized clinical trial investigating the neuropsychological, physiological, and behavioral effects of a videogame-enhanced stationary bike. Personality and behavioral measures were administered pre-intervention. Self-efficacy for sticking to exercise was measured by the Self-Efficacy for Physical Activities Scale (Sallis, 1988). Competitiveness was measured by the Competitiveness Index (Smither, 1992). Discomfort intolerance was measured using the Frustration Discomfort Scale (FDS; Harrington, 2005). Frequency was measured by the number of occasions participants exercised during the study period.

RESULTS: A hierarchical regression was performed with competitiveness and discomfort intolerance entered in step one, and self-efficacy entered in step 2. Frequency was the dependent variable. Results indicated that self-efficacy accounted for significant increases in variance above personality factors for exercise frequency $(\Delta \mathrm{F}(1,35)=31.44, \mathrm{p}=.00, \Delta \mathrm{Rsq}=.37)$. The overall model accounted for $59 \%$ of the variance in exercise frequency, indicating that self-efficacy is an important factor in predicting older adults' exercise behavior.

CORRESPONDING AUTHOR: Tracey Rocha, BA, Psychology, Union College, Schenectady, NY, 12308; tracey.rocha@gmail.com

\section{PST-152}

POOR SLEEP PREDICTS DEPRESSIVE SYMPTOMS

Tellervo Korhonen, $\mathrm{PhD},{ }^{1,2}$ Tiina Paunio, $\mathrm{PhD},{ }^{1,2}$ Christer Hublin, $\mathrm{MD},{ }^{3}$ Markku Partinen, $\mathrm{MD},{ }^{4}$ Markku Koskenvuo, $\mathrm{MD}^{2}$ and Jaakko Kaprio, $\mathrm{PhD}^{2,1}$

${ }^{1}$ National Institute for Health and Welfare, Helsinki, Finland; ${ }^{2}$ University of Helsinki, Helsinki, Finland; ${ }^{3}$ Finnish Institute of Occupational Health, Helsinki, Finland and ${ }^{4}$ Helsinki Sleep Clinic, Helsinki, Finland.

We have previously identified poor sleep as a risk factor for incidence of depressed mood, while depressed mood does not add the risk for incidence of poor sleep (Paunio et al., 2009). Here, we proceeded in the research by investigating if poor sleep quality predicts more comprehensively depressive symptoms as defined by the Beck Depression Inventory (BDI). We used questionnaire data from surveys in 1975, 1981 and 1990 in the Finnish Twin Cohort. In 1990 12,063 persons had data on BDI in 1990 (response rate 77\%). We excluded those who were in 1975 retired due to chronic disease or work disability, unemployed, used hypnotics or tranquilizers, or did night work. Our sample included 9,529 twins with no missing values on main variables. Based on self-reported sleep quality (five responses dichotomized as Good or Poor) in 1975 and 1981, we categorized the subjects into 4 groups: Good-Good; Poor-Good; Good-Poor; Poor-Poor. Out of BDI items we focused on the dimension Negative Attitudes Toward Self (NATS), including the items on self-dislike, sense of failure, guilt feelings, mood, selfaccusation, pessimism, sense of punishment, lack of satisfaction, suicidal wishes, and crying (score 0-25). To examine sleepdepression-association, logistic regressions were computed for 7,476 persons without baseline life dissatisfaction. In 1990 14\% of subjects had NATS depression (score $>3$ ). When adjusting for twinship, sex, age, smoking, alcohol, somatic health, social network, emotional support, father relationship, and life events, poor sleep predicted depression significantly (Good-Poor OR $=2.0,95 \% \mathrm{CI} 1.4-$ 2.7, $\mathrm{p}<.001$;Poor-Poor $\mathrm{OR}=1.9,95 \% \mathrm{CI} 1.1-3.3, \mathrm{p}<.05)$. These results were replicated when controlling for familial factors among 38 twin pairs discordant for sleep and depression. We conclude that poor sleep independently predicts depressive symptoms. Whether this association is causal calls for further attention.

CORRESPONDING AUTHOR: Tellervo Korhonen, PhD, Department of Public Health, University of Helsinki, Helsinki, 00014; tellervo.korhonen@helsinki.fi

\section{PST-153}

SLEEP DISTURBANCES IN INDIVIDUALS DIAGNOSED WITH RESPIRATORY DISEASES; ASTHMA, BRONCHIECTASIS, COPD AND ASBESTOSIS

Daljinder Virk, $\mathrm{PhD},{ }^{1}$ R. Stores, $\mathrm{PhD}^{1}$ and A. J. Chauhan, $\mathrm{PhD}$, FRCP $^{2}$

${ }^{1}$ School of health Sciences and Social Work, University of Portsmouth, Portsmouth, United Kingdom and ${ }^{2}$ Department of Respiratory Medicine, Portsmouth Hospitals National Health Service Trust, Queen Alexandra Hospital, Portsmouth, United Kingdom.

Introduction: Sleep disturbance is a common complaint in various respiratory diseases (Lewis, 1999). Although this area has been the topic of some research, certain respiratory disorders, such as sleep apnoea and chronic obstructive pulmonary disease (COPD), have received more attention than others. In addition, much of the research has been physiological in nature investigating sleep disturbance in terms of objective measures rather than assessing the sleep complaints of the individuals themselves.

Aims: The aims of the present research were to describe in detail than in previous investigations the occurrence and nature of sleep problems and behaviours in patients diagnosed with respiratory diseases compared to healthy controls.

Design: A cross-sectional questionnaire survey of the prevalence and range of sleep disorders was investigated in individuals diagnosed with respiratory diseases, these being asthma $(n=46)$, Chronic Obstructive Respiratory Disease (COPD) $(n=18)$, bronchiectasis $(n=16)$ and asbestos and related diseases $(n=11)$ and their matched healthy controls. The Basic Nordic Sleep Questionnaire (BNSQ) was used to collect data on sleep disorders.

Individuals with respiratory diseases were recruited from the outpatients Respiratory Service at Portsmouth Hospitals NHS Trust, and healthy matched controls were recruited through various sources including University, Portsmouth council and hospital staff. Results: 
Patients diagnosed with asthma, COPD, bronchiectasis showed a significantly greater number of sleep problems and behaviours than the healthy controls. Different sleep problems and behaviours were seen in the three respiratory disease groups.

Conclusions: Generally individuals diagnosed with respiratory diseases reported greater disturbances in sleep compared to their healthy controls.

CORRESPONDING AUTHOR: Daljinder Virk, PhD, University of Portsmouth, Coventry, CV6 7NJ; dallyvirk@hotmail.com

\section{PST-154a}

THE DISPARITY BETWEEN SUBJECTIVE AND OBJECTIVE MEASURES OF INSOMNIA IN A POPULATION OF OLDER ADULTS WITH COMORBID INSOMNIA

Hannah G. Lund, BA, Bruce Rybarczyk, PhD, Laurin Mack, BS and Andrea Shamaskin, BA

Psychology, Virginia Commonwealth University, Richmond, VA.

Background: Clinical research on insomnia has observed that many individuals with this sleep disorder exhibit a significant disparity between their subjective reports and objective measures of symptom severity. This subjective/objective disparity poses potential challenges for diagnosis and treatment of insomnia and adds complexity to our conceptualization of the disorder. Insomnia is most common, severe, and impairing in adults over 60 years of age (Lichstein, 2004) and additional impairments may be present in older adults with insomnia that is comorbid with other psychiatric or medical conditions. This population is also at particular risk for making inaccurate subjective impressions of their insomnia symptoms due to a higher endorsement of unrealistic sleep expectations or worry over the effect of compromised sleep on daytime functioning (Morin, 1993). Despite these points, the subjective/objective disparity has not yet been assessed or addressed in this population.

Method: This study examined the subjective/objective disparity in older adults with comorbid insomnia through a secondary analysis of previously collected data from an R01 grant from the National Institutes of Health (NIH) comparing Cognitive Behavioral Therapy for Insomnia (CBT-I) to a Stress Management and Wellness (SMW) therapy placebo condition in 92 older adults with comorbid insomnia (Rybarczyk, 2005). Original data on sleep parameters was collected using sleep diaries and polysomnography at pre-treatment and post-treatment.

Results: A repeated measures multivariate analysis of variance (MANOVA) revealed significant differences for measurement method, Wilks' $\lambda=.59, \mathrm{~F}(1,64)=43.8, \mathrm{p}<.001$, multivariate $\eta 2=.41$, indicating a significant disparity between subjective (diary) and objective (polysomnography) measures of insomnia.

Conclusions: This finding reveals that older adults with comorbid insomnia are inaccurate in their reporting of insomnia symptoms, which poses a challenge to the diagnosis and treatment of the disorder.

CORRESPONDING AUTHOR: Hannah G. Lund, BA, Psychology, Virginia Commonwealth University, Richmond, VA, 23220; lundhg@vcu.edu
PST-154b

SELF-MONITORING COGNITIVE PERFORMANCE DURING SLEEP RESTRICTION AND RECOVERY DAYS Mikael Sallinen, $\mathrm{PhD},{ }^{1,2}$ Katriina Tirkkonen, $\mathrm{Ms},{ }^{2}$ Jussi Onninen, $\mathrm{Mr}^{2}$ Marja-Leena Haavisto, MA, ${ }^{1,3}$ Pertti Mutanen, MA, ${ }^{1}$ Mikko Härmä, MD, ${ }^{1}$ Jussi Virkkala, $\mathrm{MA}^{1}$ and Tarja Porkka-Heiskanen, $\mathrm{MD}^{4}$ ${ }^{1}$ Finnish Institution of Occupational Health, Helsinki, Finland; ${ }^{2}$ University of Jyväskylä, Jyväskylä, Finland; ${ }^{3}$ Technical Research Centre of Finland, Helsinki, Finland and ${ }^{4}$ University of Helsinki, Helsinki, Finland.

Introduction: Shift workers' sleep is restricted to $4-5 \mathrm{~h}$ a day for several days in association with consecutive early morning shifts and night shifts. After sleep restriction workers try to recover by sleeping at least $8 \mathrm{~h}$ a night. We examined the consequences of this kind of sleep-wake pattern on the ability to self-monitor one's own cognitive performance.

Methods: A total of 20 healthy men (19-29 yr) participated in a laboratory study. 13 of them attended a sleep restriction (SR) condition including a baseline (BL) day ( $8 \mathrm{~h}$ in bed per night), five SR days ( $4 \mathrm{~h}$ in bed), and two recovery (RC) days ( $8 \mathrm{~h}$ in bed). Seven controls slept 7-8 h each night. Each participant had two $50 \mathrm{~min}$ multitask sessions daily. After an extensive training phase task difficulty was set individually. Before and after each task session the participants estimated their performance ( $\%$ of all available scores). Results: Both performance and subjective performance estimates were affected by the group-by-day interaction effect $(\mathrm{p}<.001)$. In the SR group, these outcomes were at a lower level during the last three SR days than during the BL day $(\mathrm{p}<.01)$. In addition, the SR affected selectively the pre-task performance estimates and actual performance: the pre-task estimates decreased less than actual performance during the last two SR days $(\mathrm{p}<.05)$. The recovery days restored both the subjective performance estimates and actual performance to their baseline levels. In the control group, neither performance nor the subjective performance estimates showed a decrease over the experimental days.

Conclusions: Cumulative sleep restriction makes one to prospectively underestimate the actual degree of cognitive impairment. This metacognitive effect of sleep restriction may play a role in an increased accident risk in shift work. Recovery sleep after sleep restriction seems to restore the relationship between subjective performance estimates and actual performance to its baseline level.

CORRESPONDING AUTHOR: Mikael Sallinen, PhD, Brain and Work Research Centre, Finnish Institution of Occupational Health, Helsinki, 00250; mikael.sallinen@ttl.fi

\section{PST-155}

THE IMPACT OF RELIGIOSITY ON HEALTH BELIEFS ABOUT BREAST CANCER AWARENESS IN SOUTH ASIAN MUSLIM WOMEN

Anna Baker, PhD, Samarah Hashmi, MSc, Jo Skinner, BSc and Eileen O'Keefe, $\mathrm{PhD}$

London Metropolitan, London, United Kingdom. 
Breast cancer screening uptake is lower in ethnic minority women compared to white women. In particular South Asian women are less respondent to invitations for screening but also have higher breast cancer risk. This study looked at beliefs about breast cancer awareness in South Asian Muslim women pre-mammography age (29-49 years). The focus was to see how South Asian women conceptualised their beliefs in relation to healthy breast awareness and to determine the role of religion and body image in intention to be breast aware.

Women (mean age 37 years) were recruited through mosques and community forums in the UK. They completed a brief questionnaire looking at constructs from the Health Belief Model (Champion, 1999), body image and religiosity.

Results showed that higher religiosity was associated with the following: greater perceived barriers to both breast cancer awareness and mammography screening, reduced confidence in breast self awareness, and less perceived benefits in mammography. In contrast positive body image was found to have a positive impact on breast health motivation, breast cancer awareness and finding out more about breast cancer. Good breast cancer awareness was associated with higher perceived benefits and lower barriers as expected. Examination of the predictors of intention to self-examination showed that higher body image and educational level had a positive impact on confidence, although no direct relationship to intentions.

The findings indicate that the constructs of the health belief model are relevant for South Asian Muslim women. Issues around body image and religiosity are important to acknowledge in building up confidence for breast cancer awareness in order to improve the likelihood of behavioral actions. The role of religion does not have a direct impact on actions but may inhibit development of positive beliefs systems about breast cancer awareness and mammography. This is particularly important for second generation South Asian women nearing the age for screening.

CORRESPONDING AUTHOR: Anna Baker, PhD, London Metropolitan, London, E1 7NT; a.baker@londonmet.ac.uk

\section{PST-156}

\section{AN EASY AND QUICK SCREENING FOR EARLY ALZHEIMER DISEASE}

Shinya Takeda, MA, ${ }^{1}$ Kayo Tajime, $\mathrm{MD}^{2}$ and Kazuyuki Nakagome, $\mathrm{MD}, \mathrm{PhD}^{3}$

${ }^{1}$ Department of Clinical Psychology, Tottori University Graduate School of Medical Sciences, Yonago, Japan; ${ }^{2}$ Department of Psychiatry, Tottori Seikyo Hospital, Tottori, Japan and ${ }^{3}$ Division of Brain and Neuroscience, Department of Neuropsychiatry, Tottori University Graduate School of Medical Sciences, Yonago, Japan.

In this study, we developed the Takeda Three Colors Combination Test (TTCC) as a simplified screening test for early detection of AD and examined its effectiveness. 166 senior persons participated in the research; 91 with mild $\mathrm{AD}$ and 75 healthy volunteers represented the control group. The TTCC, which is a colored cards configuration memory task, was examined for sensitivity and specificity as well as reliability and validity. The sensitivity of the TTCC in detecting mild AD was $84 \%$ with a specificity of $87 \%$. The positive predictive value was $89 \%$, the negative predictive value was $82 \%$ and the predictive accuracy was $86 \%$. The odds ratio of the incorrect response to TTCC with control group as a standard was 32.0 (95\%CI,13.1-78.1, p < 0.0001 ) for mild $\mathrm{AD}$ group. Including the results of the re-tests, the Phi coefficient indicated $0.76(\mathrm{p}<0.0001)$ and the consistency percentage between first and second trials was $88 \%$. Spearman's rank correlation analysis, using MMSE as an external standard, revealed a significant correlation between MMSE and the TTCC results $(\rho=0.58, p<$ 0.0001). Conducting the TTCC (including instruction and evaluation) was accomplished within 2 minutes for all subjects. No refusal or resistance to this test was observed among any of the subjects. The TTCC has proven to be of adequate sensitivity and specificity as a screening test for early detection of AD. Furthermore, administration time is short and requires no special training or skills. Thus, we believe the TTCC shows great potential for use as an AD screening test by a general practitioner in communities worldwide.

CORRESPONDING AUTHOR: Shinya Takeda, MA, Department of Clinical Psychology, Tottori University Graduate School of Medical Sciences, Yonago, 683-8503; takedas@med.tottori-u.ac.jp

\section{PST-157}

\section{WHY AFRICAN-AMERICANS DECIDE TO PARTICIPATE} IN COLON CANCER SCREENING RESEARCH

Anuli Njoku, MPH, ${ }^{1}$ Desiree Burgh, MEd, ${ }^{1}$ Melissa DiCarlo, $\mathrm{MPH},{ }^{1}$ Nancy Brisbon, MD, ${ }^{2}$ Randa Sifri, $\mathrm{MD}^{2}$ and Ronald E. Myers, $\mathrm{PhD}^{1}$

${ }^{1}$ Department of Medical Oncology, Thomas Jefferson University, Philadelphia, PA and ${ }^{2}$ Department of Family and Community Medicine, Thomas Jefferson University, Philadelphia, PA.

Background: Although colorectal cancer (CRC) screening can prevent $\mathrm{CRC}$ and save lives, too few African-Americans get screened. As a result, African-Americans have higher CRC incidence and mortality than other racial/ethnic groups. Although research studies are underway to learn how to increase CRC screening, African-American participation in such studies is limited. Little is known about factors that influence African-American participation in CRC screening research studies.

Objective: To understand reasons that influence African-American participation in a study designed to increase CRC screening in primary care.

Methods: As part of a larger randomized, controlled behavioral intervention, we administered a baseline telephone survey to 134 African-American primary care patients ages 50 to 79 . The survey included sociodemographic background measures and perceptions about CRC and screening. We also asked consenting participants why they joined the study. The research team performed content analysis of responses to this question and tallied categories.

Results: $75 \%$ of respondents were female and $25 \%$ male. Ages ranged from 50 to 79 with $72.4 \%$ of participants between ages 51 60. In this sample, $38 \%$ had a HS/GED education, $19 \%$ had a college degree or more, and $17 \%$ had a technical degree. Primary reasons for participation included: desire to support research (45\%), contact by study staff (36\%), and a desire to maintain health $(34 \%)$. 
Conclusions: As this was an African-American study sample, desire to support research as a primary participation reason is noteworthy. Contact by study staff as a major reason for participation is also significant. Importantly, respondents' desire to maintain their health was another principal reason cited for participation. These findings provide insight into reasons why African-American patients decide to participate in prevention research. We will add to these data through the analysis of responses from 896 individuals targeted for recruitment.

CORRESPONDING AUTHOR: Anuli Njoku, MPH, Department of Medical Oncology, Thomas Jefferson University, Philadelphia, PA, 19107; anuli.njoku@jefferson.edu

\section{PST-158a}

BARRIERS TO COLORECTAL CANCER SCREENING AMONG LATINO/HISPANIC ADULTS IN URBAN AND RURAL PENNSYLVANIA: RESULTS FROM THE APPALACHIA COMMUNITY CANCER NETWORK (ACCN)

Oralia G. Dominic, PhD, MS, MA, ${ }^{1}$ Eugene J. Lengerich, VMD, $\mathrm{MS},{ }^{1,2}$ Linda Wray, $\mathrm{PhD},{ }^{3}$ Roxanne Parrott, PhD, ${ }^{4}$ John Ochoa, $\mathrm{PhD},{ }^{5}$ Brenda Kluhsman, $\mathrm{PhD},{ }^{1}$ Besty Aumiller, PhD,${ }^{1}$ Carlos Renderos, Assoc $^{6}$ and Mark Dignan, $\mathrm{PhD}^{7}$

${ }^{1}$ Public Health Sciences, Penn State Milton S. Hershey, Hershey, PA; ${ }^{2}$ Penn State Cancer Institute, Penn State Milton S. Hershey Medical Center, College of Medicine, Hershey, PA; ${ }^{3}$ Biobehavioral Health, Penn State Milton S. Hershey Medical Center, College of Medicine, Hershey, PA; ${ }^{4}$ Communication Arts and Sciences, Penn State University, University Park, PA; ${ }^{5}$ Spanish, Italian, Portuguese and Comparative Literature, Penn State University, University Park, PA; ${ }^{6}$ Community Leader, CBO Juniata County, Mifflintown, PA and ${ }^{7}$ Internal Medicine, University of Kentucky, Lexington, KY.

We examined barriers to colorectal cancer (CRC) screening among urban and rural Pennsylvania Latino/Hispanic adults. A total of 82 Latinos/Hispanics engaged in one of eight focus groups stratified by sex and geography offered; and completed a 25-item survey eliciting their demographic information and CRC cancer screening behaviors. After the focus group, participants viewed a sevenminute American Cancer Society's published Spanish DVD entitled, "Get Tested for CRC. Here's How" and reassemble to answer questions about their preference and acceptability of the CRC screening options. Focus group data were audio taped, transcribed and grouped into thematic units using content analysis. We found substantial barriers by sex and geography, including urban residents received screenings during annual check-ups, while rural residents received screenings in response to symptoms. Of the 57 total barriers reported, four barriers were unique to urban Latino/Hispanic residents; and 13 barriers were unique to rural Latino/Hispanic residents. Low levels of health literacy, knowledge and awareness of CRC risk and screening were reported barriers across groups. The family unit and strong social support were also factors reported as influencing their CRC screening behavior. Participants identified barriers to CRC screening that fit into five categories: (a) physical environment, (b) structural factors, (c) sociocultural factors, (d) individual factors, and (e) physician-related barriers. Latino/Hispanic participants also identified potential strategies to overcome each reported barrier. These finding suggest that a targeted CRC screening intervention utilizing a physician- recommended home fecal immunochemical test with instructions is preferred among Latino/Hispanics over a non-targeted approach.

CORRESPONDING AUTHOR: Oralia G. Dominic, PhD, MS, MA, Public Health Sciences, Penn State Milton S. Hershey, Hershey, PA, 17033; odominic@hes.hmc.psu.edu

\section{PST-158b}

PREDICTING STAGE OF READINESS TO SCREEN FOR COLORECTAL CANCER AMONG AFRICAN

\section{AMERICANS}

Susan M. Rawl, PhD, RN, FAAN, ${ }^{1}$ Celette Sugg Skinner, $\mathrm{PhD},{ }^{3}$ Victoria L. Champion, DNS, RN, FAAN, ${ }^{1}$ Connie Krier, BS, ${ }^{1}$ Kathleen Russell, DNS, RN, ${ }^{1}$ Susan Perkins, $\mathrm{PhD},{ }^{2}$ Yan Tong, $\mathrm{PhD},{ }^{2}$ Netsanet Gebregziabher, $\mathrm{MS},{ }^{2}$ Broderick Rhyant, $\mathrm{MD},{ }^{2}$ Thomas Imperiale, $\mathrm{MD}^{2}$ Deanna Willis, MD, $\mathrm{MBA}^{2}$ and Laura Jones Myers, $\mathrm{PhD}^{4}$

${ }^{1}$ School of Nursing, Indiana University, Indianapolis, IN; ${ }^{2}$ School of Medicine, Indiana University, Indianapolis, IN; ${ }^{3}$ Clinical Sciences, University of Texas Southwestern Medical Center, Dallas, TX and ${ }^{4}$ Health Services Research \& Development, Roudebush VA Medical Center, Indianapolis, IN.

African Americans are more likely to be diagnosed with, and to die from, colorectal cancer (CRC) than any other group. Approximately half of $\mathrm{CRC}$ deaths could be prevented if screening were consistently implemented. The Transtheoretical Model postulates that behavior change occurs over time with people moving through stages of readiness to adopt the behavior. The aims of this study were to: 1) examine distribution across stages of readiness to screen for CRC among African American primary care patients; and 2) determine whether demographic and health belief variables predict stage of readiness to screen. Baseline data were collected via telephone interviews from 311 patients who were due for screening and enrolled in a RCT testing efficacy of a computer-based intervention designed to increase CRC screening. Ordinal logistic regression models were used to examine demographic variables and Health Belief Model constructs (perceived risk, benefits, barriers and self-efficacy) as predictors of stage of readiness to screen for CRC. The sample was $56 \%$ male and a mean age of 58 years. For FOBT, $51.8 \%$ of patients were precontemplators, $37 \%$ were contemplators, and $11.3 \%$ were in preparation. Those at more advanced stages of readiness to screen with $\mathrm{FOBT}$ were male $(\mathrm{OR}=3.49$; $95 \% \mathrm{CI}=$ $2.20,5.53)$, had a lower BMI $(\mathrm{OR}=0.96,95 \% \mathrm{CI}=0.93,0.99)$, higher perceived risk for $\mathrm{CRC}(\mathrm{OR}=1.43,95 \% \mathrm{CI}=1.10,1.87)$, lower perceived barriers to $\mathrm{FOBT}(\mathrm{OR}=0.72,95 \% \mathrm{CI}=0.53,0.98)$ and higher perceived self-efficacy for FOBT $(\mathrm{OR}=2.27 ; 95 \% \mathrm{CI}=$ $1.45,3.55)$. For colonoscopy, $39.7 \%$ were precontemplators, $51.6 \%$ were contemplators, and $8.7 \%$ were in preparation. Those 
at more advanced stages for colonoscopy had higher perceived risk for $\mathrm{CRC}(\mathrm{OR}=1.29,95 \% \mathrm{CI}=0.99,1.68)$, higher perceived benefits of colonoscopy $(\mathrm{OR}=1.62,95 \% \mathrm{CI}=1.18,2.22)$ and higher self-efficacy for colonoscopy $(\mathrm{OR}=1.71,95 \% \mathrm{CI}=1.17$, 2.51). Receipt of a recommendation from their provider was the most significant predictor of advanced stage for both FOBT $(\mathrm{OR}=$ $4.16,95 \% \mathrm{CI}=2.63,6.59)$ and colonoscopy $(\mathrm{OR}=2.90,95 \% \mathrm{CI}=$ $1.85,4.55)$. About half were thinking about being screened for CRC, with more considering colonoscopy than FOBT. Results provide evidence for strategies to move patients to action. Computer-based interventions that incorporate these strategies have great potential to move primary care patients forward in their stage of readiness to screen for CRC.

CORRESPONDING AUTHOR: Susan M. Rawl, PhD, RN, FAAN, School of Nursing, Indiana University, Indianapolis, IN, 46202; sraw1@iupui.edu

\section{PST-158c}

\section{CONTENT VALIDITY OF A NEW TOOL DEVELOPED} TO HELP PALLIATIVE CARE NURSES TO ASSESS ORAL HEALTH

Camille Gagné, $\mathrm{PhD}^{1}$ and Isabelle Martineau, $\mathrm{MSc}^{2}$

${ }^{1}$ Faculty of nursing, Laval university, Quebec, QC, Canada and ${ }^{2}$ Maison Michel-Sarrazin, Quebec, QC, Canada.

Introduction. A number of cancer patients in palliative care have oral health problems. A tool, in French, was developed to guide nurses in the care of these patients. After a literature review, 17 oral health problems and their corresponding clinical signs (67 in total) were identified. The aim of this study is to present the content validity of the tool.

Method. A dentist and three general practitioners in palliative care in Quebec (Canada) were invited to evaluate the relevance and sufficiency of each of the clinical signs identified. The relative importance of clinical signs was also assessed for certain oral health problems. As suggested by Polit, Tatano Beck and Owen (2007), an I-CVI of at least .75 was used to conclude about the relevance of a given clinical sign.

Findings. The I-CVI was satisfactory for 62 of 67 clinical signs. There was disagreement between the experts concerning the relevance of using pain/burning pain of mucosa and pain/ burning pain of tongue to detect both pseudomembranous candidiasis and erythematous candidiasis. Opinions were also shared concerning the use of tooth sensitivity to cold and that of nearby tissues as a clinical sign of pulpitis and dental abscess. Finally, the experts considered that some clinical signs were more important than others to detect erythematous candidiasis, hyperplasic candidiasis, mucositis/stomatite, infections of viral origin and xerostomia. Thus, it will be necessary to develop a scoring method that takes this aspect into account.

Conclusion. The content validity of most of the clinical signs is satisfactory. Only minor adjustments to the tool are necessary. The next step is to demonstrate the convergent validity of the tool.

CORRESPONDING AUTHOR: Camille Gagné, PhD, Faculty of nursing, Laval university, Quebec, QC, G1V 0A6; camille. gagne@fsi.ulaval.ca 


\section{TOP FIFTEEN SCORING POSTER \\ PST-159 \\ SOCIOECONOMIC STATUS PREDICTS PHYSIOLOGIC \& PSYCHOSOCIAL INDICATORS OF CARDIOVASCULAR RISK AMONG FIRST GENERATION CHINESE IMMIGRANTS IN NYC}

Sonia Suchday, $\mathrm{PhD},{ }^{1}$ Natania Wright, $\mathrm{PhD},{ }^{2}$ Jing Fang, MD, ${ }^{3}$ Sun Hoo Foo, $\mathrm{MD}^{4}$ and Judith Wylie-Rosett, EdD ${ }^{5}$

${ }^{1}$ Clinical Psychology Ph.D. (Health Emphasis) Program, Ferkauf Graduate School of Psychology/Albert Einstein College of Medicine/Institute of Public Health Sciences/Yeshiva University, Bronx, NY; ${ }^{2}$ Department of Epidemiology \& Population Health, Albert Einstein College of Medicine, Bronx, NY; ${ }^{3}$ Department of Epidemiology \& Population Health, Albert Einstein College of Medicine, Bronx, NY; ${ }^{4}$ Downstate Hospital, New York, NY and ${ }^{5}$ Department of Epidemiology \& Population Health, Albert Einstein College of Medicine/Institute of Public Health Sciences, Bronx, NY.

Purpose: Assess whether socioeconomic status (SES; income, education) predicts cardiovascular risk factors (CRF) and psychosocial characteristics in a first generation Chinese immigrant community in NYC. Sample \& Methods: In a study assessing SES and health among first generation Chinese immigrants ( $\mathrm{N}=2072$, Females $=53 \%$; Mean Age $=52.7, \mathrm{SD}=13.8$ years), $33.7 \%$ of the sample had greater than high school education and $80.3 \%$ of the sample had an income less than $\$ 30,000 /$ year. Participants' cardiovascular health was assessed using anthropometric measures and blood pressure. Measures, translated into Chinese and administered via semi-structured interviews, included demographics, the MacArthur SES ladder-perceived SES, PHQ-depression, Spielberger Trait Anger Scale, and self-reported health status. Results: Linear regression equations were computed controlling for age and gender. SES predicted perceived SES (income: $\beta=.19, \mathrm{t}(2071)=8.72, \mathrm{p}<.001$; education: $\beta=.19, \mathrm{t}(2071)=8.64, \mathrm{p}<.001$ ), BMI (education: $\beta=-.11, \mathrm{t}(2071)=-4.91, \mathrm{p}<.001$ ), systolic blood pressure (education: $\beta=-.09, \mathrm{t}$ (2071) $=-4.93, \mathrm{p}<.001$ ), diastolic blood pressure (education: $\beta=-.12, \mathrm{t}(2071)=-5.43, \mathrm{p}<.001$ ), waist circumference (education: $\beta=-.11, \mathrm{t}(2071)=-4.86, \mathrm{p}<.001$ ), self reported health (income: $\beta=.13, \mathrm{t}(2071)=-5.77, \mathrm{p}<.001$; education: $\beta=-.11, \mathrm{t}(2071)=-4.86, \mathrm{p}<.001$ ), anger (income: $\beta=.05$, $\mathrm{t}(2071)=2.45, \mathrm{p}<.001$; education: $\beta=.06, \mathrm{t}(2071)=2.67, \mathrm{p}<.008$ ), and depression (income: $\beta=$ $-.09, \mathrm{t}(2071)=-3.93, \mathrm{p}<.001)$. Discussion: SES predicts physiologic CRF and psychosocial indicators including self-rated health, perceived status, anger, and depression among Chinese immigrants. It appears that education is a more robust predictor of physiologic CRF compared to income. Given that education may reflect status prior to migration (Average Age at Migration $=39$ years), the interaction between status and physiologic indicators of health may be important to assess throughout the lifespan in future studies. It is also possible that Education has a higher range than income with almost $80 \%$ of the sample earning less than $\$ 30,000 /$ year.

CORRESPONDING AUTHOR: Sonia Suchday, PhD, Clinical Psychology Ph.D. (Health Emphasis) Program, Ferkauf Graduate School of Psychology/Albert Einstein College of Medicine, Bronx, NY, 10461; Sonia.Suchday@einstein.yu.edu

PST-160

SOCIOECONOMIC STATUS, SUBJECTIVE SOCIAL CLASS, AND PSYCHOLOGICAL DISTRESS IN JAPAN AND THE US Norito Kawakami, MD, Maki Umeda, MPH, Keiko Sakurai, MPH and World Mental Health Japan Survey Group, None

School of Public Health, University of Tokyo, Tokyo, Japan.

Aims: Investigating the gradient of mental health along social class among countries with different social and cultural background might contribute to the further understanding of social class disparity in mental health. The study aimed to explore a difference in the association of socioeconomic status (SES) and subjective social class with psychological distress between the US and Japan.

Methods: Database from community-based epidemiologic studies of common mental disorders in the US and Japan, i.e., the US National Comorbidity Survey Replications $(n=9,282)$ and the World Mental Health Japan Survey $(n=4,130)$, were analyzed. In both studies, interviewers collected information on education, household income subjective social class (SSS, 10 rung ladder scale), and psychological distress (K6) during the worst 30 days in the past 12 months. Respondents were classified into quartiles based on the distribution of each indicators of SES or social class. Cases with psychological distress were defined as having 13 or greater scores of K6. The analyses were conducted of the part 2 samples for which these data were available ( $n=5,692$ and 1,682 for the US and Japan, respectively), adjusting gender, age, marital 
status, and employment status, with using weights for the sampling design.

Results: In the US sample, education, household income and SSS were significantly and inversely associated with psychological distress $(\mathrm{p}<0.05)$. In the Japanese sample, respondents with high education or low household income had a non-significant but slightly higher prevalence of psychological distress; furthermore, those with the highest or lowest level of SSS had a higher prevalence of psychological distress $(\mathrm{p}<0.05)$. A significant interaction between the country and education or SSS was observed $(\mathrm{p}<0.05)$.
Conclusions: While a linear association between any social class indicator and psychological distress was observed in the US, the association between subjective social class and psychological distress seems $U$ shaped in Japan, probably reflecting the fact that those with high education or low income had a higher prevalence.

CORRESPONDING AUTHOR: Norito Kawakami, MD, School of Public Health, University of Tokyo, Tokyo, 113-0033; kawakami@m.u-tokyo.ac.jp

\section{TOP FIFTEEN SCORING POSTER \\ PST-161 \\ POWER OF THE PROCESS: EVALUATING THE IMPACT OF THE FRAMEWORK CONVENTION ON TOBACCO CONTROL NEGOTIATIONS}

Grace Huang, MPH and Heather Wipfli, PhD

Preventive Medicine, University of Southern California, Los Angeles, CA.

Background. Despite a number of papers on the FCTC negotiation process and final text, there have not been attempts to test the usefulness of treaty process by quantifying its global impact on the adoption of domestic tobacco control policies. This paper suggests that the negotiation process provided a platform for increased communication and international learning through which tobacco control information spread to many countries; and that this simultaneous learning process accelerated the adoption of internationally promoted tobacco control policies by countries around the world.

Objective. The present study quantifies the impact of the FCTC negotiation process on the global diffusion of domestic tobacco control policies.

Methods. Country characteristics, including income, population, region, democracy, tobacco production, smoking prevalence and network participation were analyzed to determine their effects on the frequency, type and strength of tobacco control policy adoption among WHO Member States. Bivariate analyses were conducted for each country characteristic and used to compare the frequency and strength of control policies adopted between pre-negotiation and negotiation periods. Multivariate regression analyses were performed to determine the predictive nature of these variables.

Findings. The frequency of policy adoption escalated between 2002 and 2003, the years in which the FCTC negotiations were most intense. The strength of policies adopted also shifted significantly towards policies promoted by WHO. The average strength of policies adopted varied significantly with population size, region, democracy levels and network participation. All characteristics, with the exception of total and male smoking prevalence, were significantly associated with the number of policy types adopted.

Conclusion. This study suggests that investments in formal international legal processes can be effective and appropriate, even when the outcomes are unclear from the start. The FCTC negotiation process coincided with a rise in domestic policy adoption in the direction advocated by WHO. However, there remains the need to improve outreach and diffusion to lower-income countries in the area of tobacco control, and likely other areas of chronic disease control.

CORRESPONDING AUTHOR: Grace Huang, MPH, Institute for Health Promotion and Disease Prevention Research, University of Southern California, Alhambra, CA, 90505; grace.huang @usc.edu 
PST-162

SOCIAL CLASS, MENTAL AND PHYSICAL HEALTH, AND HELP SEEKING OF THE UNEMPLOYED WHO LOOK FOR A JOB IN JAPAN

Masumi Okamoto, none, Norito Kawakami, MD and World Mental Health Japan Survey Group, none

Graduate School of Medicine, The University of Tokyo, Tokyo, Japan.

Aims: The number of the unemployed has been increasing in Japan, as well as in other countries, due to the economic turn-down in 2008. The unemployed might have lower socioeconomic status (SES), thus have poor mental health, and difficulty in seeking help for mental health problems. This study aimed to clarify subjective social class, mental health, and help seeking behaviors among the employed who looked for a job, comparing with those currently employed.

Methods: A community-based epidemiologic study of common mental disorders, the World Mental Health Japan 2002-2006 Survey, were conducted of random samples of residents in 11 municipalities $(\mathrm{N}=4,130)$, with using the WHO-CIDI 3.0. A subsample of the respondents (the part 2 sample, $\mathrm{N}=1,682$ ) was asked to determine their social class, self-rated health, psychological distress (K6), 12- month DSM-IV mental disorders, and help-seeking behaviors for mental health problems. Analyses were conducted, comparing these variables between the currently unemployed who look for a job $(\mathrm{N}=$ 35 ) and the currently employed ( $\mathrm{N}=997)$, adjusting for sex, age, and education, with considering the sampling weight.

Results: The unemployed had significantly lower education and household income than the employed $(\mathrm{p}<0.05)$. The unemployed reported poorer physical and mental health status than the employed $(p=0.08$ and $p=0.06$, respectively), while there was no significant difference in prevalence of psychological distress or mental disorders. The proportion of those who sought help for mental health problems was not significantly different between the two groups; however, as a reason of the delay in seeking help, the unemployed more tended to concern "how much money the treatment would be cost" $(p=0.07)$. Conclusions: The unemployed were at lower SES, had poor physical and mental health status, and delayed their help seeking because of their concern on treatment fee.

CORRESPONDING AUTHOR: Masumi Okamoto, none, medicine, Tokyo university, Tokyo, 120-0036; okamoto510@gmail. com 


\section{EARLY CAREER AWARDEE \\ TOP FIFTEEN SCORING POSTER \\ PST-163 \\ MUNICIPAL SOCIOECONOMIC STATUS IN RELATION TO CAUSE-SPECIFIC MORTALITY AMONG JAPANESE MIDDLE AGED MEN AND WOMEN: A MULTILEVEL STUDY IN JAPAN}

Kaori Honjo, $\mathrm{PhD},{ }^{1}$ Hiroyasu Iso, $\mathrm{MD},{ }^{1}$ Nobuo Nishi, MD,${ }^{2}$ Yoshiharu Fukuda, $\mathrm{MD}^{3}$ and Akiko Tamakoshi, $\mathrm{MD}^{4}$

${ }^{1}$ Osaka University Graduate School of Medicine Public Health, Suita, Japan; ${ }^{2}$ National Institute of Health and Nutrition, Tokyo, Japan; ${ }^{3}$ Yamaguchi University School of Medicine, Ube, Japan and ${ }^{4}$ Aichi Medical University School of Medicine, Nagakute, Japan.

Backgrounds and purpose

Limited evidence has been available for regional socio-economic status (SES) and health in Asian countries. Whether regional SES influences individual cause-specific risk of death remained to be examined. The aim of this multilevel study is to evaluate whether regional SES influences cause-specific mortality independently from individual SES.

Methods

We used the data from the Japan Collaborative Cohort (JACC) Study in 1988-1990, including 22,638 men and 30,518 women who aged 40 to 64 with no medical history of cancer and cardiovascular diseases at baseline in 35 municipalities. They were followed up to determine mortality of various diseases by the end of 2005. All deaths were ascertained by death certificates from public health centers. The ICD 9th and 10th revisions were used to determine cause-specific mortality.

Proportion of college graduates, per capita income, unemployment rate, per capita bank deposit, and proportion of households receiving welfare were used as indicators of regional SES. We adjusted individual SES (education level, occupation) and possible confounding variables. Gender-specific adjusted odds ratios for specific causes of deaths were estimated according to regional SES by multilevel logistic regression.

Results

Regional SES indicators were associated with risk of mortality independently from individual SES. The effect of regional SES was statistically significant for cardiovascular disease, especially stroke among men, while it was apparent for respiratory diseases or external causes of death among women.

Conclusions

This multilevel study showed that SES of municipalities has independent influence on risk of mortality among individuals living in these areas. Not only individual but also regional SES

could be social determinants of health. 
CORRESPONDING AUTHOR: Kaori Honjo, PhD, Osaka University Graduate School of Medicine Public Health, Suita, 5650871; khonjyo@pbhel.med.osaka-u.ac.jp

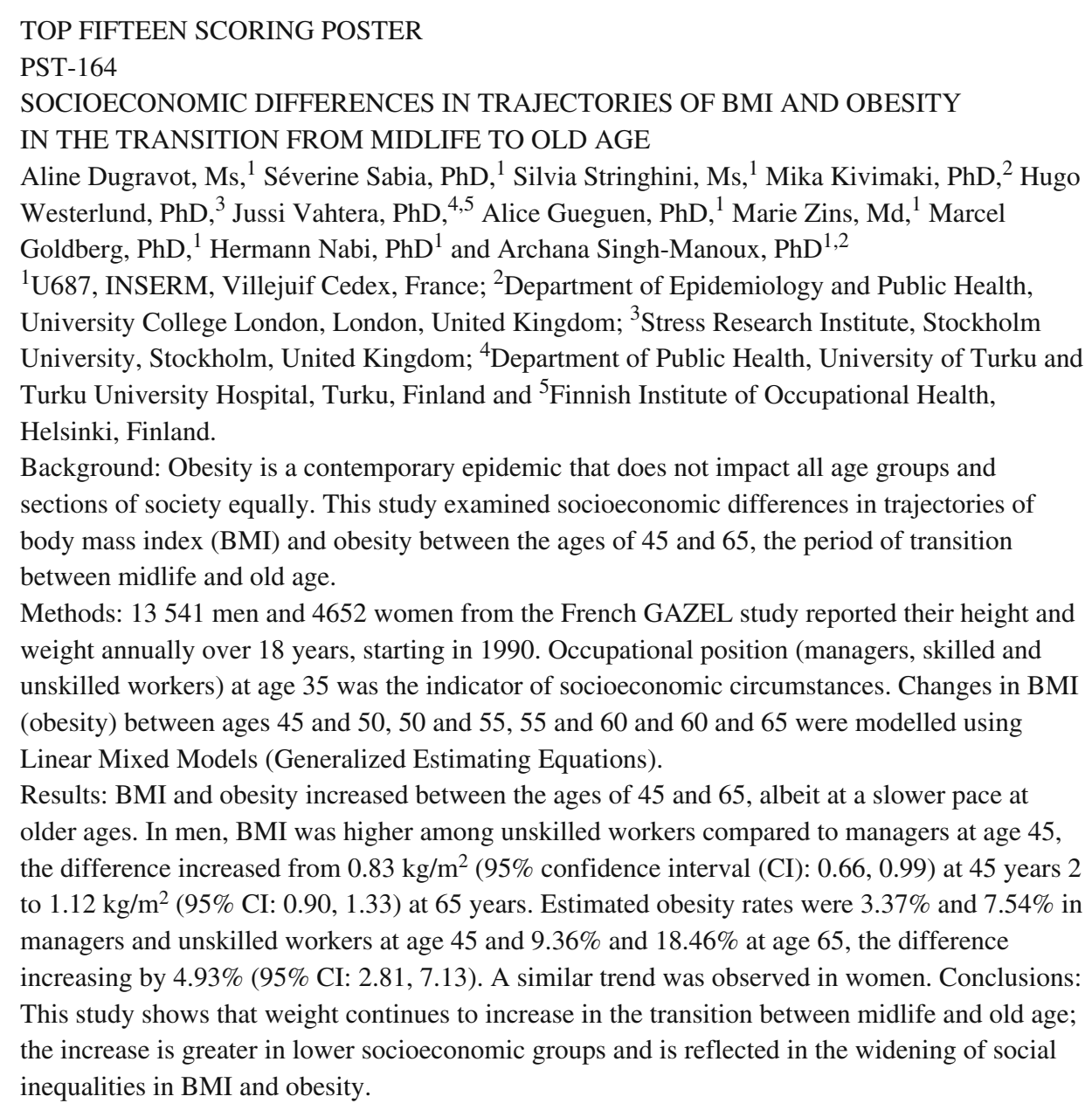

CORRESPONDING AUTHOR: Aline Dugravot, Ms, U687, INSERM, Villejuif Cedex, 94807; aline.dugravot@inserm.fr

\section{PST-165}

SUBJECTIVE SES AS A PREDICTOR OF VITAL EXHAUSTION WITHIN A CARDIOVASCULAR SAMPLE Miriam Katzenstein, BA, Elina Spektor, MA, Maria Dziok, MS, $\mathrm{MA}$ and Sonia Suchday, PhD

Ferkauf Graduate School of Psychology, Yeshiva University, Bronx, NY.

Introduction: Cardiovascular disease (CVD) is associated with high morbidity and mortality rates in the U.S. Negative associations between CVD and socioeconomic (SES) are well established. Socioeconomic status, generally defined by factors such as income, education and occupation, can be measured subjectively via-self report using a scale representing a ten rung ladder.
Recently, subjective SES has shown to be more predictive of health and cardiac outcomes than actual SES. Vital exhaustion (VE) is another risk factor associated with CVD and is characterized by feelings of excessive fatigue and demoralization, as well as in increase in irritability. To our knowledge, socioeconomic differences, both subjective and objective, with respect to VE, have not been explored. Therefore, the current study examined the associations between subjective SES and VE among cardiac patients.

Methods: A sample of 119 cardiac patients was recruited from hospitals and an associated private cardiology practice in New York City. The sample comprised of $45.4 \%$ women and the mean age was $59(\mathrm{SD}=11.63)$. Subjective SES was assessed using the MacArthur Scale of Subjective SES and VE was assessed using the Maastricht Questionnaire (revised scale version).

Results: Mean score on VE was $47(\mathrm{SD}=16.89)$. Within the community comparison of social standing, the mean score was $7.08(\mathrm{SD}=1.78)$ and within the USA comparison of social 
standing the mean score was $5.91(\mathrm{SD}=1.89)$. Vital exhaustion was negatively correlated with both community comparison $(r=$ $-.29, \mathrm{p}<.001)$ and USA comparison of social standing $(\mathrm{r}=-.31$, $\mathrm{p}<.001)$. Subjective SES compared to others in the U.S., but not others within the community, was an independent predictor of vital exhaustion when controlling for gender, race/ethnicity, and age $(\beta=-.22, p=.03)$. These negative associations have implications for identifying a population at risk for heart disease.

Conclusion: Our findings indicate that among U.S. cardiac patients, perception of social standing is associated with being Vitally Exhausted. Moreover, perception of social standing is an independent predictor of Vital Exhaustion.

CORRESPONDING AUTHOR: Miriam Katzenstein, BA, Ferkauf Graduate School of Psychology, Highland Park, NJ, 08904; miriam.katzenstein@gmail.com

\section{PST-166}

\section{WHAT COULD HELP TO DIE IN PEACE FROM YOUNG} PEOPLE'S PERSPECTIVE?

Maria Dolores Gil Llario, $\mathrm{PhD},{ }^{1}$ Rafael Ballester, $\mathrm{PhD},{ }^{2}$ Beatriz Gil, Postgraduate, ${ }^{2}$ Maria Pilar Ibañez, Postgraduate ${ }^{2}$ and Sandra Gomez, Postgraduate ${ }^{2}$

${ }^{1}$ Departamento de Psicología evolutiva y de la educación, Universidad de Valencia, Valencia, Spain and ${ }^{2}$ Psicología Básica, Clínica y Psicobiología, Universidad Jaume I, Castellón, Spain.

Death is a natural and inevitable phenomenon that everyone has to face it. Nevertheless, our society seems to refuse everything that could be related to illness, suffering and death. Daily we overhear news about unexpected deaths, which highlights the importance of being aware of one's death as a part of the cycle of life. The aim of this study was to analyze the main factors that could help to face the own death from young people's perspective. The sample was made up of 60 young people, aged between 18 and 27. All participants were evaluated by the "Helping to Die in Peace Questionnaire" (CAMP) by Bayés et al., (2000), which is composed of two major items. The first one assesses the aspects that could help people to die in peace, answering to eleven sentences according to a 5 points scale. In relation to the second one, participants have to select the two most important factors when death is close, through the eleven previous statements. Results showed the following factors as the most relevant to young people: "Being able to be close, communicate and strengthen my bonds with loved ones" $(\mathrm{X}=3,1 ; \mathrm{SD}=0,8)$, "To think that my life has had some meaning" $(\mathrm{X}=3,0 ; \mathrm{SD}=1,1)$, "To think that my death won't cause an unbearable burden to loved ones" ( $X=2,9 ; S D=1,1)$. On the other hand, the less important aspects were: "Believing in other life after death" $(\mathrm{X}=1,5 ; \mathrm{SD}=$ $1,5)$, "To think that I may die at home" $(\mathrm{X}=1,8 ; \mathrm{SD}=1,3)$, "To think that my dying process will be short in case of suffering" ( $\mathrm{X}=$ $2,3 ; \mathrm{SD}=1,4)$ and "Not to feel guilty about past personal conflicts" $(X=2,3 ; \mathrm{SD}=1,4)$. The rest of the items reached intermediate values. According to these results we can conclude that young people give priority to emotional and existential aspects when they think about their own death, supporting other study carried through health professionals (Bayés, 2000).

This work was supported by the Spanish Ministry of Science and Education (PSI2008-01642/PSIC) and by a predoctoral fellowship from the Generalitat Valenciana, Spain (BFPI/2008/129).

CORRESPONDING AUTHOR: Maria Dolores Gil Llario, PhD, Departamento de Psicología evolutiva y de la educación, Universidad de Valencia, Valencia, 46022; dolores.gil@uv.es

\section{PST-167}

\section{FAMILY NEED IN AN INTENSIVE CARE UNIT}

Maria Dolores Gil Llario, $\mathrm{PhD},{ }^{1}$ Rafael Ballester Arnal, $\mathrm{PhD},{ }^{2}$ Beatriz Gil, Postgraduate, ${ }^{2}$ Sandra Gómez, Postgraduate ${ }^{2}$ and Ricardo Abizanda, $\mathrm{PhD}^{3}$

${ }^{1}$ Departamento de Psicología evolutiva y de la educación, Universidad de Valencia, Valencia, Spain; ${ }^{2}$ Psicología Básica, Clínica y Psicobiología, Universidad Jaume I, Castellón, Spain and ${ }^{3}$ Hospital General Universitario, Castellón, Spain.

Family members play a fundamental role in the recovery of the hospitalized patients, even more in the intensive care units (ICUs) (Hidalgo et al, 2007). Allowing them to collaborate in the care of patients in ICUs may improve the quality of their experience (Azoulay et al, 2003). The aim of this study was to analyze family needs in an ICU. The sample was made up of 55 family members of patients admitted to the ICU at General Hospital (Castellón-Spain). In order to achieve our purpose, all participants were evaluated using the Spanish Adaptation of the Critical Care Family Needs Inventory (Johnson et al, 1998) by Ballester et al (2004).

Results showed family satisfaction in descending order, so family members were more pleased in relation to "satisfaction with medical care to patients" $(81,5 \%)$, "best care given to patients" (77,8\%), "be called with some significant change in patients' condition" $(75,9 \%)$ "care about the patients from hospital personnel" (74,1\%), "understanding about patient's situation and procedures applied" $(74,1 \%)$ and "honesty of the information provided" $(70,9 \%)$. Lesser degree, they were satisfied in "explanations about patient's condition", "staff members attention to family" and "loneliness/isolation in the waiting area" (61-67\%). Finally, the results pointed as the fewest satisfied aspects those connected with "comfort about visiting patients in ICU" (47,3\%) "interest showed by staff members"(32,7\%), "explanation about the equipment used" $(25,9 \%)$ and "comfort of waiting room" $(11,5 \%)$.

These findings show that family members satisfaction to their ICU's experience was quite good in general. It is necessary taking into account those improvable aspects in order to provide them the best stay in our ICUs.

This work was supported by the Spanish Ministry of Science and Education (PSI2008-01642/PSIC) and by a predoctoral fellowship from the Generalitat Valenciana, Spain (BFPI/2008/129).

CORRESPONDING AUTHOR: Maria Dolores Gil Llario, PhD, Departamento de Psicología evolutiva y de la educación, Universidad de Valencia, Valencia, 46022; dolores.gil@uv.es 
PST-168a

RELATIONSHIP BETWEEN SOCIAL STATUS

INCONSISTENCY AND SELF-RATED HEALTH: EVIDENCE OF AN INDIRECT PATH VIA OCCUPATIONAL REWARDS

Stefanie Braig, MA and Richard Peter, Prof Dr

Institute of Epidemiology, Ulm University, Ulm, Germany.

BACKGROUND: Evidence on the association between social status inconsistency (SI) and self-reported health is limited and mixed. Moreover, only little research has been done to examine the pathway through which SI affects health. Therefore we analysed the mediating effect of effort-reward imbalance at work (ERI) on this association. METHODS: Analyses were based on a random sample of 1111 male employees (25-64 years) from the south-west of Germany. Data were collected by a telephone interview in 2008/09. Information on socio-demographic variables, social status indicators (education, occupational grade and income), occupational strain (ERI), and physical as well as mental health (SF-12) were given. RESULTS: SI was significantly associated to low mental health, both directly and, consistent to our hypothesis, indirectly via low rewards. As SI was also related to low effort, no association was found for the quotient of both scales (ERI) and mental health. The effect of SI on physical health was entirely mediated by low rewards without any direct effect. The inclusion of further risk factors (age, BMI) did not attenuate these Results: CONCLUSION: SI is significantly associated to ill health. Moreover, results indicate that at least a part of this relationship is explained by low rewards at work, findings with implications for further research in job strain and health inequality.

CORRESPONDING AUTHOR: Stefanie Braig, MA, Institute of Epidemiology, Ulm University, Ulm, 89069; stefanie.braig@ uni-ulm.de

\section{PST-168b}

\section{SOCIAL ADAPTATION AND DEPRESSION}

Heather Glubo, BA, ${ }^{1}$ Hillary Lewin, BA, ${ }^{1}$ Maureen Almeida, MA, MPhil, ${ }^{2}$ Sonia Suchday, $\mathrm{PhD}^{1}$ and Pejman Sehatpour, MD, $\mathrm{PhD}^{1}$

${ }^{1}$ Ferkauf Graduate School of Psychology, New York, NY and ${ }^{2}$ St Xaviers College, Mumbai, India.

Selection of a spouse reflects the cultural values of society. Spouse selection in the west is based primarily on falling-in-love whereas in India, selecting a spouse has been traditionally arranged. As a result of globalization, western influence in India is on the rise requiring a traditional society to modify its cultural norms. While arranged marriage is still prevalent in India, a new mode of marriage, love marriage, is becoming increasingly common. This transition introduces new social stressors, which may impact health. We hypothesize that traditional individuals will feel conflicted by the shift in cultural norms toward a more nontraditional system and possibly experience symptoms of depression. To test the hypothesis, a group of 350 college students in Mumbai, a major metropolis of India undergoing rapid cultural change in recent years were surveyed. We administered questionnaires measuring the influence exerted by tradition on mate selection and its health impact. Logistic regression analysis determined that traditional influence predicted the choice of arranged marriage ( $\%$ Variance accounted for $=-.25 ; \mathrm{p}<.005)$. Thus traditional individuals are more likely to choose an arranged marriage. Based on our hypothesis these individuals would be more vulnerable to cultural shifts and manifest symptoms of depression more than those choosing love marriages. The Mann-Whitney test results indicated significantly $(p<0.04)$ higher depression among individuals who chose arrange marriage. These results suggest that traditional individuals are more susceptible to feeling dysphoric secondary to the cultural changes taking place in urban India. This discontent could impact their health.

CORRESPONDING AUTHOR: Heather Glubo, BA, Ferkauf Graduate School of Psychology, New York, NY, 10028; heather glubo@gmail.com

\section{PST-168c}

WEALTH, HEALTH AND SPIRITUALITY

Hillary Lewin, MA, ${ }^{1}$ Heather Glubo, MA, ${ }^{1}$ Maureen Almeida, $\mathrm{MA}^{2}$ Pejman Sehatpour, PhD, $\mathrm{MBBS}^{1}$ and Sonia Suchday, $\mathrm{PhD}^{1}$ ${ }^{1}$ Psychology, Yeshiva University, New York, NY and ${ }^{2}$ Psychology, St. Xavier's College, Mumbai, India.

Subjective socioeconomic status (SES) impacts health independently of objective SES. However, little is known about variables that contribute to perception of social standing other than objective SES, which is only modestly correlated with subjective SES. Spirituality also impacts on health and may synergistically interact with perceived SES to predict health. It is hypothesized that Subjective SES will correlate positively with Spirituality. And spirituality and subjective SES will predict health outcomes after controlling for objective SES. Urban college students $(\mathrm{N}=568$; Mean Age: 19 years; 73.1\% Female; Religion: 46.3\% Christian; 37.3\% Hindu; Other: 16.4\%) in Mumbai, India completed questionnaires on Spiritual Well-Being (SWB) measuring Existential (EWB) and Religious Well-Being (RWB), somatic symptoms (CHIPS), subjective SES and objective SES. Results indicated that objective SES and subjective SES were positively but modestly correlated $(\mathrm{r}=.20, \mathrm{p}<.001)$. There was a negative relationship between objective SES and SWB, RWB and CHIPS $(\mathrm{r}=-.11, \mathrm{p}<.05 ;-.20, \mathrm{p}<.001 ;-.10, \mathrm{p}<.05$, respectively) and between subjective SES and RWB and CHIPS ( $\mathrm{r}=-.12, \mathrm{p}<.005$; $.20, \mathrm{p}<.001$, respectively). These data indicate that high SES perceived and actual - is associated with lower engagement in prayer and relationship with a higher spiritual power and with fewer somatic symptoms than low SES. There was no correlation found between objective SES and EWB and subjective SES and EWB were positively correlated $(r=.20, p<.001)$ indicating that perceived social standing is associated with life satisfaction and stability. These data indicate the complexity of variables that contribute to material and spiritual well-being and its interaction with health where subjectively poor people engage more in religious practice and do not experience existential satisfaction and report more somatic symptoms. 
CORRESPONDING AUTHOR: Hillary Lewin, MA, Hillary Lewin, Yeshiva University, New York, NY, 10024; hillary. lewin@gmail.com

\section{PST-168d}

PROJECT GATHER: CAN HEALTH CARE PERCEPTIONS FACILITATE DECISIONS OF A MULTI-ETHNIC SAMPLE TO PARTICIPATE IN A GENETIC BIOBANK?

Marissa C. Perales, BA, ${ }^{1}$ Georita M. Frierson, $\mathrm{PhD},{ }^{1,2}$ Margo Simmons, BA, ${ }^{2}$ Celette Sugg Skinner, $\mathrm{PhD},{ }^{3}$ Benjamin Willis, $\mathrm{MD}^{2}$ and Laura DeFina, $\mathrm{MD}^{2}$

${ }^{1}$ Psychology, Southern Methodist University, Dallas, TX; ${ }^{2}$ Cooper Institute, Dallas, TX and ${ }^{3}$ UT Southwestern, Dallas, TX.

Discussions about medical information banks may influence decisions to participate in genetic biobanks. Project Diversity GATHER (Group discussions Around Themes on HEalthful genetic Research) examines demographics and health care perceptions that may affect willingness to participate in genetic biobanking.

Participants $(\mathrm{N}=135,63 \%$ female; mean age $=46.67, \mathrm{SD}=17.17$, range $=18-87)$ completed sociodemographic and psychosocial questionnaires and a one-hour focus group. Participants selfidentified as Black (49.2\%), White $(36.2 \%)$, Pacific Islander (2.3\%), American Indian (2.3\%), Asian (1.5\%) and Other (8.5\%); $19.3 \%$ of participants were Latino. Univariate and bivariate descriptive analyses and t-tests were performed where appropriate. Univariate and multivariate logistic regressions were applied to compare differences in (un)adjusted odds of who was willing to participate in biobanking vs. not controlling for confounds.

Participants unwilling to participate in genetic biobanking had higher health care distrust than willing participants $[\mathrm{t}(126)=$ $-3.14, p<.01]$. Logistic regression models compared participants willing to participate in genetic biobankIng vs. not. Univariate logistic regressions noted significant relationships for race/ethnicity $(\mathrm{OR}=0.30,95 \% \mathrm{CI}=0.12-0.75)$, primary care physician status $(\mathrm{OR}=0.51,95 \% \mathrm{CI}=1.03-1.18)$, and health care system beliefs $(\mathrm{OR}=1.11,95 \% \mathrm{CI}=1.02-1.18)$, with gender, age, and education level yielding nonsignificant relationships. Multivariate analyses confirmed race/ethnicity $(\mathrm{OR}=0.25,95 \% \mathrm{CI}=0.09-0.70)$ and health care beliefs $(\mathrm{OR}=1.10,95 \% \mathrm{CI}=1.02-1.18)$ to be significant predictors. Participants characterized as racial/ethnic minorities or who are distrustful of the health care system may be less likely to participate in genetic biobanking.

Our findings support previous research in this area and may help to explain why medical research often underrepresents racial/ ethnic minority groups. The relationship between health care distrust and unwillingness to participate in genetic biobanking highlights potential recruitment barriers in genetic testing. Clinicians and researchers may wish to assess and attend to distrust in patients for whom genetic testing is clinically indicated.

CORRESPONDING AUTHOR: Marissa C. Perales, BA, Psychology, Southern Methodist University, Dallas, TX, 75251; mperales@smu.edu
PST-168e

SOCIAL SUPPORT IN THE PREDICTION OF DAILY BLOOD PRESSURE AND HEART RATE

Lauri Parkkinen, MSc, ${ }^{1}$ Martti T. Tuomisto, $\mathrm{PhD}^{2,3}$ and Jyrki Ollikainen, $\mathrm{MSc}^{4}$

${ }^{1}$ Department of Extension Studies, University of Tampere, Tampere, Finland; ${ }^{2}$ Department of Psychology, University of Tampere, Tampere, Finland; ${ }^{3}$ Department of Psychiatry, University of Tampere, Tampere, Finland and ${ }^{4}$ Department of Mathematics and Statistics, University of Tampere, Tampere, Finland.

Our purpose was to predict systolic and diastolic blood pressure (SBP, DBP) and heart rate (HR) during 24-hour ambulatory BP monitoring using respective diagnostic values of $\mathrm{BP}$ and $\mathrm{HR}$, and measures of social support as predictors in a hierarchical regression analysis. The predicted periods of the ambulatory BP monitoring were 24-hours, morning, evening, and night. The participants were 95 healthy men with different BP levels from the Tampere Ambulatory Blood Pressure Study. The participants could be treated as one group, because the diagnostic BPs were normally distributed. The measures of social support were the total score of the Interview for Social Interaction (ISSI; Henderson, Byrne, \& Duncan-Jones, 1981) and five subscales of it. The first sets in the hierarchical regression analysis had the respective diagnostic values of BP and HR as predictors. These were all significant predictors of daily cardiovascular measures as expected. In the second series of sets, the total score of ISSI and the subscale scores of it were added to the models, respectively. The prediction of ambulatory cardiovascular measures improved by up to $3 \%$ by adding the Adequacy of Social Integration (ADSI), a subscale of ISSI to the diagnostic SBPs. In conclusion, one specific measure of social support, the Adequacy of Social Integration (but not others) improved the prediction of cardiovascular measures in daily life. These results are in line with previous findings emphasizing the function of the quality of social support as a buffer in daily life.

CORRESPONDING AUTHOR: Lauri Parkkinen, MSc, Department of Extension Studies, University of Tampere, Halikko as, 24910; parkkinen.lauri@gmail.com

PST-168f

LOWER EDUCATION LEVELS AND HIGHER SYSTOLIC BLOOD PRESSURE (SBP) IN THE NATIONAL LONGITUDINAL STUDY OF ADOLESCENT HEALTH (ADD HEALTH)

Beverly H. Brummett, PhD, ${ }^{1}$ Ilene C. Siegler, $\mathrm{PhD},{ }^{1}$ Eric A. Whitsel, MD, ${ }^{2,3}$ Jon M. Hussey, $\mathrm{PhD},{ }^{3}$ Liana J. Richardson, $\mathrm{PhD},{ }^{3}$ Glen H. Elder, $\mathrm{PhD}^{4}$ and Redford Williams, $\mathrm{MD}^{1,3}$

${ }^{1}$ Psychiatry, Duke University Medical Center, Durham, NC; ${ }^{2}$ Medicine, University of North Carolina, School of Medicine, Chapel Hill, NC; ${ }^{3}$ UNC Gillings School of Public Health, Chapel Hill, NC and ${ }^{4}$ Sociology, University of North Carolina, Chapel Hill, NC. 
Recent findings (Chaix et al. Hypertension, 2010) suggest that central adiposity and heart rate account for a sizable amount of the inverse association between individual education and SBP in a French cohort: body mass index (BMI) and waist circumference explained $28 \%$ and resting heart rate, $15 \%$ of this association. Psychological factors and health behaviors also helped account for the education-SBP association, but to a much smaller degree. We used data on a nationally representative U.S. sample of 15,701 young adult participants aged 24-32 years in Add Health, Wave IV, to determine whether a similar education-SBP association exists in a U.S. cohort and to examine several potential mediators of it. Individual education level was associated with SBP $(\mathrm{p}<$ 0.0001 ) as follows: Some high school or less, $\mathrm{N}=1212$, $\mathrm{SBP}=$ 126.0+14.1(S.D.)mmHg; Graduated high school, $\mathrm{N}=2453, \mathrm{SBP}=$ $126.6+13.9 \mathrm{mmHg}$; Some college or vocational tech, $\mathrm{N}=6710$, $\mathrm{SBP}=124.7+13.8 \mathrm{mmHg}$; Bachelors degree, $\mathrm{N}=2942, \mathrm{SBP}=$ $123.2+13.1 \mathrm{mmHg}$, and; Graduate school or more, $\mathrm{N}=1845$,
$\mathrm{SBP}=122.0+12.7 \mathrm{mmHg}$. When the education-SBP association was adjusted for BMI, the $4.0 \mathrm{mmHg}$ difference in SBP between the lowest and highest education groups was reduced to $2.7 \mathrm{mmHg}$. Further adjustment for gender, race/ethnicity, physical activity, alcohol consumption, smoking, marital status, employment status, income, presence of financial strain and home ownership reduced the SBP difference to $0.6 \mathrm{mmHg}$. These findings replicate in a U.S. cohort the finding in a French cohort of a robust association between lower education level and increased SBP. In the larger Add Health sample, this association was nearly completely accounted for by BMI, race/ethnicity, gender, marital status and lifestyle characteristics. (Supported by NICHD grant 2 P01 HD031921-11A1)

CORRESPONDING AUTHOR: Redford Williams, MD, Psychiatry, Duke University Medical Center, Durham, NC, 27710; redfordw@duke.edu

\section{TOP FIFTEEN SCORING POSTER}

PST-169

STRESS REDUCTION IN SHAVASAN (YOGIC RELAXATION IN DEAD BODY POSITION) DURING UPPER GASTROINTESTINAL ENDOSCOPY Mool R. Kotwal, MB,BS,MD,MRCP,FRCP,FACG ${ }^{1,2}$ and Chewang Z. Rinchen, MB,BS ${ }^{1,2}$

${ }^{1}$ Gastroenterology, Sir Thutob Namgyal Memorial Hospital, Tibet Road Gangtok, India and

${ }^{2}$ Home \& Health, Government Of Sikkim, Gangtok, India.

Stress reduction in shavasan (Yogic relaxation in dead body position) during upper gastrointestinal endoscopy.

M.R.Kotwal 1, 2, 3. CZ Rinchhen 2. Home Department Government of Sikkim 1. STNM Hospital Department of Health \& Family Welfare Government of Sikkim 2. Sikkim Manipal Institute of Medical sciences 3.

Introduction: Many patients fear upper gastrointestinal endoscopies. Natural anxiety may be aggravated by horror stories from friends or inappropriate remarks by endoscopy staff. Shavasan is probably the best-known relaxation exercise. Through relaxation of muscle tension, an anxiety free sate is reached. Objective: The study was to examine effects of relaxation in Shavasan posture in patients undergoing upper GI endoscopies. Method: Study was conducted on 63 consecutive patients between ages of 16 to 86 years. Patients were randomly assigned to two groups regardless of sex, age and underlying disease. Thirty one patients relaxed in shavasan, before and during procedure, while 32 patients did not. No patients were given sedation or local anesthetic spray. Blood pressure, heart and respiratory rate were recorded. Perception of procedure using a 5- point attitude scale was assessed. Results: Paired T-test was used to analyze the data. Test group results showed significant difference in three parameters i.e. blood pressure, systolic, heart and respiratory rate in the difference of values at the beginning and end of procedure. Control group did not show statistically significant difference in any parameters. However, data comparison in two groups showed statistically significantly difference in the heart and respiratory rate. More patients in test group reported decrease in distress. Conclusion: This preliminary study shows the effect of relaxation in Shavasan posture. Repeated practice is necessary to attain quick de-stressed state in distress. We suggest that this exercise could be applied to any other medical situations as well, which tend to generate undue psychological stress and anxiety. 
CORRESPONDING AUTHOR: Mool R. Kotwal, MB,BS,MD, MRCP,FRCP,FACG, Gastroenterology, STNM Hospital, Gangtok, 737101; rajkotwal@gmail.com

\section{PST-170}

SEEKING SOCIAL SUPPORT AND WISHFUL THINKING MODERATE LOW SELF ESTEEM INDICATORS OF PERCEIVED STRESS FOR AFRICAN AMERICANS

Veronica Y. Womack, Bachelor's and Lloyd Sloan, PhD

Psychology, Howard University, Washington DC, DC.

Problem: Coping resources can be of great assistance when one is placed in stressful situations because they influence whether or not the stressor is directly approached or avoided (Taylor \& Stanton, 2007). This research proposes that self esteem, a key dispositional factor will reduce perceived stress for African Americans. Further, effective coping strategies may moderate the impact of self esteem on stress. This research hypothesizes that lower perceived stress will be predicted by higher self esteem and that effective coping strategies will increase this relationship.

Procedure: 146 African American college students in small groups completed measures of self esteem, coping strategies and perceived stress.

Results: As expected, low self esteem $(\mathrm{r}=-.29 \mathrm{p}<.01$; Rsquare $=$ $.09 \beta=-.29, \mathrm{t}=-3.56 \mathrm{p}<.01)$ predicted higher perceived stress. In addition, seeking social support(Rsquare $=.15, \beta=.23 \mathrm{t}=2.63$ $\mathrm{p}<.01$ ), an active coping strategy, and wishful thinking (Rsquare $=$ $.25, \beta=-.21 \mathrm{t}=-2.78 \mathrm{p}<.01$ ), an avoidant coping strategy moderated the relationship between self esteem and perceived stress. Low social support seekers and high wishful thinkers showed stronger impacts of lower self esteem on increased stress. The coping strategy of seeking social support was not independently correlated with perceived stress but "wishful thinking" $(\mathrm{r}=.35 \mathrm{p}<.01)$ was.

Conclusion: This study found that low self esteem is directly related to increased perceived stress among African Americans. The inclination to seek social support reduces low self esteem individual's perception of a situation as stressful. However, wishful thinking increased the likelihood that an individual with low self esteem would perceive an environment as stressful. These findings suggest that cognitive-behavioral prevention programs that seek to alleviate stress among African Americans who have low self esteem should emphasize the importance of creating trusted social support systems and realistically assessing the magnitude of a problem in an effort to better manage one's stress responses.

CORRESPONDING AUTHOR: Veronica Y. Womack, Bachelor's, Psychology, Howard University, Washington DC, DC, 20011; veronica_w20@hotmail.com

PST-171

INFLUENCE OF OCCUPATIONAL STRESS ON MENTAL HEALTH AMONG INDUSTRIAL WORKERS

Sanjit R. Joshi, MBA

Jagdamba Spinning mills Pvt. Ltd, Kathmandu-35, Nepal.
AIM: To explore the influence of occupational stress on mental health in Industrial worker. METHODS: A cross-sectional survey was conducted among 561 workers. The workers were invited to fill in a self-administered questionnaire exploring their socio-demographic characteristics, occupational stress levels, and 12-item general health questionnaire. A hierarchical multiple regression procedure was used to assess the effects of occupational stress on mental health. RESULTS: After controlling for age, educational level, marital status and years of offshore work, poor mental health was found to have a significant positive association with seven of the nine identified sources of occupational stress. They were: conflict between job and family/ social life, poor development of career and achievement at work, safety problems at work, management problems and poor relationship with others at work, poor physical environment of the work place, uncomfortable ergonomic factors at work, and poor organizational structure at work. All of these occupational stress sources together explained $19.9 \%$ of the total variance. CONCLUSIONS: The results confirmed that occupational stress was a major risk factor for poor mental health among industrial workers. Reducing or eliminating occupational stressors at work would benefit workers' mental health in developing country like Nepal. There should be some meditation program, behovior change and rest for workers to reduce the occupational Health

CORRESPONDING AUTHOR: Sanjit R. Joshi, MBA, Jagdamba Spinning mills Pvt. Ltd, Kathmandu-35, n/a; joshi.sanjit@gmail. com

\section{PST-172}

FATALISTIC CONTROL EXPECTATIONS AFFECT CARDIOVASCULAR STRESS RESPONSE TO STANDARDIZED MEDICAL PROCEDURES IN PREGNANCY:

\section{A PILOT STUDY}

Pearl Ghaemmaghami, MSc, ${ }^{1}$ Sara Dainese, MSc, ${ }^{1}$ Roberto La Marca, $\mathrm{PhD},{ }^{1}$ Nadia Fiabane, cand lic phil, ${ }^{1}$ Leandra Gurzan, cand lic phil, ${ }^{1}$ Maria Rigozzi, cand lic phil, ${ }^{1}$ Roland Zimmermann, $\mathrm{MD}^{2}$ and Ulrike Ehlert, $\mathrm{PhD}^{1}$

${ }^{1}$ Department of Psychology, University of Zurich, Zurich, Switzerland and ${ }^{2}$ Department of Gynecology, University Hospital of Zurich, Zurich, Switzerland.

Undergoing medical procedures, such as an amniocentesis, is stressful for pregnant women because they are often in a state of heightened anxiety about the well-being of their unborn child and experience uncertainness and possibly also increased physical discomfort.

The aim of the pilot study was to evaluate the impact of an amniocentesis during pregnancy on cardiovascular stress response.

Twelve healthy pregnant women in the second trimester of pregnancy (age: $\mathrm{M}=38.1, \mathrm{SD}=2.8$ ), underwent an amniocentesis during which heart rate (HR) and Respiratory Sinus Arrhythmia (RSA) were measured continuously, beginning 20 minutes 
before the examination and lasting until 30 minutes after termination of the examination. Additionally, a questionnaire was distributed to measure the participants' general control conviction in life.

Analyses revealed a significant decrease of HR $(\mathrm{p}<.001)$ and a concomitant increase of RSA $(p=.036)$. Furthermore, a general fatalistic control expectation attitude was associated with a smaller decrease in HR $(\mathrm{r}=.616, \mathrm{p}=.077)$ and a smaller increase in RSA $(\mathrm{r}=-.735, \mathrm{p}=.024)$ after the procedure.

Our findings reveal a harmful anticipatory cardiovascular stress response in pregnant women prior to an invasive medical procedure with an anticipatory increase of HR primarily mediated by a decrease in cardiovagal activity as indexed by RSA. Furthermore, a fatalistic control conviction seems to dampen stress reactivity. Based on these findings, adjuvant stressprotective psychological intervention and counseling with special focus on control conviction should be considered for pregnant women undergoing medical procedures.

CORRESPONDING AUTHOR: Pearl Ghaemmaghami, MSc, University of Zurich, Zurich, 8050; p.ghaemmaghami@psychologie. uzh.ch

PST-173

CONTROLLED BREATHING AFFECTS AMYGDALA AND INSULA ACTIVATION IN RESPONSE TO AVERSIVE PICTURES: A PRELIMINARY STUDY WITH FMRI Ko Chia Chen, MS, ${ }^{1,2}$ Kuan-hao Cheng, MS, ${ }^{2}$ Hsu-Huei Weng, $\mathrm{MD}, \mathrm{PhD},{ }^{3}$ Chia-Ying Weng, $\mathrm{PhD}^{2}$ and Sigmund Hsiao, $\mathrm{PhD}^{2}$

${ }^{1}$ St. Martin De Porres Hospital, Chiayi, Taiwan; ${ }^{2}$ National Chung Cheng University, Chiayi County, Taiwan and ${ }^{3}$ Chang Gung Memorial Hospital, Chiayi Country, Taiwan.

Objective: Breathing control therapy may reduces emotion, but the neural underpinning of this effect is unknown. We induced emotion with aversive pictures in 20 subjects and using a breath control procedure to test a hypothesis that (a) Breathing control alters the visceral feedback as reflected in the insula activation (that reflects interoception), and (b) This leads to reduced fear as reflected in amygdala activation. Method: Subjects went through fMRI scanning while observing Neutral Pictures, Emotional Pictures and then practiced breathing control (Exp Group) or no breathing control (Control group). Results: (a) Aversive pictures activated left amygdale and right insula in the control group. (b) The activations at the same areas were altered in the experimental group that practiced the breathing control procedure. Discussion: Intervention of emotion-related breathing can modulate left amygdala activation indicative of reduced fear and this effect cooccurred with altered activation at the right insula cortex indicative of altered interceptive feedback. Conclusion: Breathing control therapy involves altered activation of insula and amygdala.

CORRESPONDING AUTHOR: Ko Chia Chen, MS, National Chung Cheng University, Chiayi County, 62102; puni.chen@ gmail.com
PST-174

IMMUNOMODULATORY EFFECTS IN ANXIOUS

AND DEPRESSED PATIENTS AFTER A MINDFULNESS MEDITATION PROGRAM

Juan M. Manzaneque, PhD, ${ }^{1}$ Francisca M. Vera, $\mathrm{PhD},{ }^{1}$ Yelba A. Godoy, Psychology Graduate, ${ }^{2}$ Natalia S. Ramos, $\mathrm{PhD},{ }^{2}$ Francisco M. Rodriguez, Biology Graduate, ${ }^{1}$ Maria J. Blanca, $\mathrm{PhD},{ }^{1}$ Ana Fernandez, Medicine Graduate, ${ }^{3}$ Alfredo Enguix, Medicine Graduate $^{3}$ and Maria R. Iglesias, Medicine Graduate ${ }^{4}$

${ }^{1}$ Department of Psychobiology and Methodology, Faculty of Psychology, UNIVERSITY OF MALAGA, Malaga, Spain; ${ }^{2}$ Department of Personality, Assessment and Psychological Treatment, Faculty of Psychology, UNIVERSITY OF MALAGA, Malaga, Spain; ${ }^{3}$ Clinical Biochemistry Service, "Virgen de la Victoria" UNIVERSITY HOSPITAL, Malaga, Spain and ${ }^{4}$ Delicias Health Center, MALAGA SANITARY DISTRICT, Malaga, Spain.

Mindfulness meditation is an ancient meditation technique with a modern name based on the concept of mindfulness, a relatively recent psychological construct. This form of meditation is rooted in Buddhist Vipassana and Zen practices, and involves momentby-moment, detached awareness and observation of the continually changing field of perception and its contents. Albeit the interest to verify the clinical efficacy of mindfulness practice has grown considerably in the last years, its specific psychobiological profile has been scarcely investigated, particularly with respect to the immune system, and this research does not seem to have been conducted, integratively, in clinical population. Thus, the present study was carried out to examine the effects of a mindfulness meditation program on immune parameters in a clinical sample. 16 patients with depression and anxiety symptoms participated in the study; these patients underwent a mindfulness meditation program for the period of eight weeks. For the quantification of immune parameters (leucocytes, immunoglobulins and complement), blood samples were taken before and at the end of the training program. T-test and Wilcoxon signed-rank test were performed as statistical analyses. After completing the mindfulness meditation program, patients displayed significantly lower levels of monocytes and $\operatorname{IgM}$, and higher levels of $\mathrm{C} 3$ and $\mathrm{C} 4$, as compared with pre-training. Therefore, these results show that mindfulness meditation may exert a significant immunomodulatory action in anxious and depressed patients, which may hold clinical relevance. Future studies should confirm these findings, as well as fully assess their clinical implications.

CORRESPONDING AUTHOR: Juan M. Manzaneque, PhD, Psychobiology, University of Malaga, Malaga, 29071; manzaneque@ uma.es

\section{PST-175}

\section{EFFECT OF LPS AND FOOT SHOCK STRESS} ON PRO-INFLAMMATORY CYTOKINE IL1 $\beta$ IN RATS

Eun Yee Jung, MS, Kyung-Soo Kim, MD and Insop Shim, PhD Integrative Medicine, The Catholic University of Korea, Seoul, Republic of Korea. 
It is known that pro-inflammatory cytokine such as IL- $1 \beta$ plays an important role in behavioral and physiological alterations produced by exposure to immune stimulation or exposure to stressors. Similarities between the neurochemical, endocrine, and behavioral consequences of immune challenge and stressors have been described frequently but the impact of immune stimulation such as lipopolysaccharide (LPS) or stressors on central or peripheral IL- $1 \beta$ has not been systematically explored. To establish whether treatment of LPS or exposure to the foot shock stress (FS) leads to a changes in central or peripheral production of IL-1 $\beta$ and what types of cells express IL- $1 \beta$ in paraventricular nucleus (PVN) and hippocampus were investigated in rats. After treatment of LPS $(100 \mathrm{ug} / \mathrm{kg}$ ) or exposure to the FS (ten, $0.8-\mathrm{mA}$, foot shock for $5 \mathrm{sec}$ each and $90 \mathrm{sec}$ interval), fever were assessed and plasma, spleen, brains were collected to measure IL-1 $\beta$ levels and brains were perfused for immunohistochemical study. LPS produced widespread increase IL-1 $\beta$ in the brain, spleen and plasma, but FS produced significant increase in IL-1 $\beta$ only in the brain but not in spleen and plasma. In confocal immunofluorescence study, LPS and FS increased IL-1 $\beta$ immunoreactivities in the PVN and in the hippocampal $\mathrm{CA} 1$ and $\mathrm{CA} 3$ regions and the IL- $1 \beta$ immunoreactivities were colocalized with NeuN. Our results indicate that in brain both LPS and FS increase IL-1 $\beta$ but in periphery only LPS increases IL- $1 \beta$ and that IL-1 $\beta$ immunoreactivities on neuron may play an important role in the hypothalamus and the hippocampus regions.

CORRESPONDING AUTHOR: Insop Shim, PhD, Integrative Medicine, The Catholic University of Korea, Seoul, 137-701; ishim@catholic.ac.kr

PST-176

RESTING HEART RATE VARIABILITY IS DIFFERENTIALLY ASSOCIATED WITH UNDERLYING HEMODYNAMICS

IN AFRICAN AMERICAN MEN AND WOMEN

LaBarron K. Hill, MA, ${ }^{1}$ Jay Van Bavel, PhD, ${ }^{2}$ Dixie D. Hu, BA, ${ }^{1}$ Dewayne P. Williams, BA, ${ }^{1}$ William A. Cunningham, PhD,${ }^{1}$ John J. Sollers, $\mathrm{PhD}^{1,3}$ and Julian F. Thayer, $\mathrm{PhD}^{1}$

${ }^{1}$ Psychology, The Ohio State University, Columbus, OH; ${ }^{2}$ Psychology, New York University, New York, NY and ${ }^{3}$ Psychological Medicine, University of Auckland, New Zealand, Auckland, New Zealand.

Heart rate variability (HRV) is largely regarded as a useful marker of cardiovascular functioning (Task Force, 1996). Despite disparities in cardiovascular disease prevalence, especially hypertension, researchers have also suggested that African Americans (AA) actually display greater resting HRV compared to Whites (Li et al., 2009). One proposed explanation for the greater rates of hypertension in African Americans is a greater peripheral resistance in the systemic vasculature (Dorr et al., 2007). In the present study, we sought to examine the relationships among HRV, and central (i.e. Cardiac Output|CO) and peripheral (i.e. Total Peripheral Resistance|TPR) hemodynamic measures. As part of a larger study, continuous measurements of HRV and hemodynamics were obtained for 34 AA participants (22 females, Mage=19.8,
$\mathrm{SD}=2.7$ ), during a five minute resting baseline period. Initial correlational analysis revealed a moderate association between resting $\mathrm{HRV}$ and resting $\mathrm{CO}(\mathrm{r}=.29, \mathrm{p}=.08)$. Analyses were then conducted separately for each gender. Interestingly, this association was stronger for AA females $(r=.47, p=.02)$, but weaker and in the opposite direction for AA males $(r=-.09, p=.79)$. Separate analyses also revealed a strong relationship between resting HRV and TPR in AA men $(r=.60, p=.04)$, that was not observed for AA women $(r=.13, p=.61)$. In follow-up regression analysis the effect for AA women was attenuated when BMI was added to the model; results for men were not diminished when controlling for age and BMI. These results suggest that important gender differences may modify the association between autonomic nervous system activity and hemodynamic parameters associated with cardiovascular disease.

CORRESPONDING AUTHOR: LaBarron K. Hill, MA, Psychology, The Ohio State University, Columbus, OH, 43210; hill.871@gmail.com

\section{PST-177}

EFFECTS OF SENSE OF COHERENCE (SOC)

ON PSYCHOLOGICAL RESPONSES TO JOB STRESSORS AND PERCEIVED HEALTH AMONG JAPANESE FACTORY WORKERS

Kayoko Urakawa, PhD, MA, $\mathrm{RN}^{1,2}$ and Kazuhito Yokoyama, $\mathrm{MD}, \mathrm{DMSc}^{2}$

${ }^{1}$ Stress Science, Mie University Graduate School of Medicine, Tsu, Japan and ${ }^{2}$ Epidemiology and Environmental Health, Juntendo University Faculty of Medicine, Bunkyo-ku, Japan.

Sense of Coherence (SOC) scale measures the stress coping ability, viz comprehensibility, manageability, and meaningfulness. To investigate the relationships of SOC scores to perceived health status and job stress, self-administered questionnaires were distributed 1968 workers in some industries. Complete answers were recovered from 295 workers $(14.9 \%, 192$ males and 103 females) and used for the analysis(179 office clerks, 28 sales representatives, 21 technical engineers, and 67 others). Work stressors, psychological and somatic responses, social support, and job satisfaction were assessed by Brief Questionnaire for Job Stress (BQJS). Perceived health were evaluated by asking subjects to give 1-4 points to their own status (1 very healthy, 2 rather healthy, 3 not so healthy, 4 unhealthy). Ages were 49.3 years on average (SD11.4)for all respondents, and were 50.7 (SD 11.6) and 46.4(SD 10.7) for males and females, respectively. SOC scores were 56.8(SD6.1)on average, without significant differences between two genders $(\mathrm{p}>0.05)$. Multiple regression analysis indicated that, for both males and females, tension scores (BQJS) were inversely related to SOC scores, whereas were positively related to job load and stress by human relations assessed by BQJS $(p<0.05)$; tension scores were also inversely related to supervisor's support and job satisfaction (BQJS). Similarly, in males, depression scores (BQJS) were related inversely to SOC, job satisfaction and supervisor's support, and positively to job load and stress by human relations $(p<0.05)$. The similar 
relationships of depression scores to job load, supervisor's support and stress by human relations were observed in females $(\mathrm{p}<0.05)$. Perceived health was not significantly associated with SOC scores in the multiple regression analysis $(\mathrm{p}>0.05)$. It is thus suggested that SOC can reduce adverse responses to job stress(job load and stress by human relations).

CORRESPONDING AUTHOR: Kayoko Urakawa, PhD, MA, RN, Stress Science, Mie University Graduate School of Medicine, Tsu, 514-8507; adiemus@nurse.medic.mie-u.ac.jp

\section{PST-178}

SALIVARY ALPHA-AMYLASE IN A POPULATION BASED SAMPLE. ASSOCIATIONS WITH PSYCHOSOCIAL FACTORS, SELF RATED HEALTH AND INFLAMMATORY MARKERS

Peter Garvin, $\mathrm{PhD},{ }^{1}$ Anke Suska, $\mathrm{PhD},{ }^{2}$ Margareta Kristenson, MD $\mathrm{PhD},{ }^{1}$ Ingemar Lundström, $\mathrm{PhD}^{2}$ and Jan Ernerudh, $\mathrm{MD} \mathrm{PhD}^{3}$

${ }^{1}$ Dep of Medical and Health Sciences, Linköping University, Sweden, Linköping, Sweden; ${ }^{2}$ Dep of Physics, Chemistry and Biology, Linköping University, Sweden, Linköping, Sweden and ${ }^{3}$ Dep of Clinical and Experimental Medicine, Linköping University, Sweden, Linköping, Sweden.

Objective: In recent years, salivary alpha-amylase (sAA) has been proposed as a reliable proxy for sympathetic activity. This study aimed at testing the association between sAA to a broad range of psychosocial factors, self rated health, cardiovascular risk factors and inflammatory markers in a normal population sample.

Methods: 30 participants, all men between 50 and 54 years old, were randomly selected from a normal population based study. Saliva samples were collected at awakening, 30 minutes after awakening and just before going to bed. sAA was measured by a calorimetric method using Phadebas amylase test. Linear regression models were used to test associations between sAA levels and a broad spectrum of psychosocial factors (e.g. depressive symptamology, vital exhaustion, mastery and sense of coherence) self rated health and inflammatory markers (e.g. C-reactive protein). Adjustments were made for physical exercise, smoking, blood lipids and time point when sample was collected.

Results: sAA levels at awakening were positively associated with depressive symptamology $(\mathrm{p}=0.046)$, vital exhaustion $(\mathrm{p}=0.025)$ and negatively associated with sense of coherence $(p=0.034)$. It was further associated positively associated with levels of C-reactive protein $(p=0.024)$ and negatively associated with self reported general health $(p=0.010)$. Samples taken just before going to bed were showing similar results, whereas samples taken 30 minutes after awakening only showed a few significant associations.

Conclusions: The associations found give further support for the use of salivary alpha amylase as a psychoneuroendocrinological biomarker. Assessment just after awakening or just before going to bed seems to be more reliable than samples 30 minutes after awakening.
CORRESPONDING AUTHOR: Peter Garvin, PhD, Dep of Medical and Health Sciences, Linköping University, Sweden, Linköping, SE-581 83; peter.garvin@liu.se

PST-179g

COGNITIVE-AFFECTIVE AND TOTAL DEPRESSION SYMPTOM SEVERITY PREDICT CHANGES

IN C-REACTIVE PROTEIN (CRP) IN PATIENTS

WITH ACUTE CORONARY SYNDROMES (ACS)

Jonathan A. Shaffer, PhD, ${ }^{1}$ Donald Edmondson, $\mathrm{PhD},{ }^{1}$ William F. Chaplin, PhD ${ }^{3}$ Joseph E. Schwartz, PhD ${ }^{1}$ Padmini Iyer, BS, ${ }^{1}$ Matthew M. Burg, PhD, ${ }^{1}$ Nina Rieckmann, $\mathrm{PhD},{ }^{2}$ Daichi Shimbo, $\mathrm{MD}^{1}$ and Karina W. Davidson, $\mathrm{PhD}^{1}$

${ }^{1}$ Medicine, Columbia University Medical Center, New York, NY; ${ }^{2}$ Berlin School of Public Health, Charite University Medical Center, Berlin, Germany and ${ }^{3}$ Psychology, St. John's University, Jamaica, NY.

Background: Depression symptom severity contributes independently to risk for recurrent cardiac events, and inflammation has been suggested as a mechanism by which this risk is conferred. Evidence regarding the directionality of the depressioninflammation relationship is equivocal however, and indicative of possible complex and bidirectional associations. Methods: At the time of their index event (baseline), and then again 1- and 3months later, 163 post-Acute Coronary Syndrome (ACS) patients completed the Beck Depression Inventory (BDI), a measure of depression symptom severity with cognitive and somatic components. C-Reactive Protein (CRP) level was also assessed at these visits, as were known correlates of depression and CRP (i.e., age, sex, body mass index, education, raceethnicity, mean arterial pressure [MAP], smoking status, history of rheumatic disease, and history of diabetes). Path analyses were conducted to evaluate the directionality of the depressionCRP relation and whether directionality varied by sex and/or specific symptom dimensions of depression. Results: Baseline total depression symptom severity predicted an increase in CRP from baseline to 1 -month $(\beta=0.23, \mathrm{p}<0.001)$ controlling for all covariates, as did baseline cognitive-affective depression symptom severity $(\beta=0.28, p=0.02)$. In contrast, baseline somaticvegetative depression symptom severity did not predict an increase in CRP $(\beta=-0.01, p=0.92)$. CRP did not significantly predict 1 - or 3-month change in total, cognitive-affective, or somatic-vegetative depression symptom severity. Results did not differ for men and women. Conclusion: Cognitive-affective and total depression symptom severity at the time of a cardiac event predicted 1-month increases in CRP for both men and women, but there was no evidence that earlier CRP predicts later depression symptoms.

CORRESPONDING AUTHOR: Jonathan A. Shaffer, PhD, Medicine, Columbia University Medical Center, New York, NY, 10032; js3742@columbia.edu 
PST-179b

SOCIAL NETWORK DEVELOPMENT AND SALIVARY

CORTISOL IN HEALTHY OLDER PEOPLE

Julian C. Lai, $\mathrm{PhD},{ }^{1}$ Sik Hung $\mathrm{Ng}, \mathrm{PhD},{ }^{1}$ Alice Chong, $\mathrm{PhD},{ }^{1}$

Oswald Siu, DSc, ${ }^{2}$ Phil Evans, $\mathrm{PhD}^{3}$ Cecilia Chan, $\mathrm{PhD}^{4}$ and Rainbow $\mathrm{Ho}, \mathrm{PhD}^{4}$

${ }^{1}$ Applied Social Studies, CIty University of Hong Kong, Hong Kong, China; ${ }^{2}$ SCOPE, City University of Hong Kong, Hong Kong, China; ${ }^{3}$ Psychology, University of Westminster, London, United Kingdom and ${ }^{4}$ Center on Behavioral Health, University of Hong Kong, Hong Kong, China.

This study examined the impact of social network variables on diurnal rhythm of cortisol levels among a group of 84 older people (45 men) whose ages ranged from 59 yrs to 86 yrs (mean= 73.02 yrs). Eight saliva samples were collected from the participants for two days at specific times after waking: immediately, 15 minutes, 30 minutes, 45 minutes, 3 hours, 6 hours, 9 hours, and 12 hours. Cortisol data of the two days were aggregated for analysis. The two components of diurnal rhythm, namely, awakening response and diurnal decline were examined separately. For the cortisol awakening response, two separate indices reflecting the mean level of secretion and the rise from immediately to 45 minutes post-awakening, AUCG and AUCI, were computed using the trapezoid formula. Similar procedure was applied to arrive at two indices of decline: AUCG-d and AUCI-d, from the peak level in the morning to that of 12 hours post-awakening. The relation of each of the two indices within each diurnal component to the availability, use and development of social network was examined in a multiple regression analysis while controlling for the effect of gender, age, socioeconomic status, waking time, humor, and self-esteem. Results indicated that higher scores in social network development were associated with a steeper rise in the morning $(\operatorname{Beta}=.32, \mathrm{t}=2.24, \mathrm{p}<.05)$ and $\mathrm{a}$ faster decline over the day $(\mathrm{Beta}=.34, \mathrm{t}=2.38, \mathrm{p}<.05)$. Participants having higher scores on this variable exhibited a stronger motivation to develop and extend their social relationships. Its effect on cortisol might stem from the positivity underlying the intention, which is consistent with what prior data suggest. None of the other variables included in the regression analysis had significant impact on cortisol levels. Findings of the present study suggest that the hypothalamic-pituitary-adrenocortical (HPA) axis is the major pathway whereby positive psychosocial factors exert their health effects in the aging population.

CORRESPONDING AUTHOR: Julian C. Lai, PhD, Applied Social Studies, CIty University of Hong Kong, Hong Kong, n/a; ssjulwin@cityu.edu.hk
PST-179c

SALIVARY IL-6 AND CRP INCREASE IN RESPONSE TO ACUTE PSYCHOSOCIAL STRESS

Shuhei Izawa, $\mathrm{PhD},{ }^{1,2}$ Nagisa Sugaya, MA, ${ }^{2}$ Kenta Kimura, $\mathrm{PhD},{ }^{3}$ Namiko Ogawa, MA, ${ }^{2}$ Kosuke C. Yamada, $\mathrm{PhD},{ }^{4,2}$ Kentaro Shirotsuki, PhD, ${ }^{5}$ Ikuyo Mikami, BA, ${ }^{2}$ Kanako Hirata, BA, ${ }^{2}$ Yuichiro Nagano, $\mathrm{PhD}^{6}$ and Shinobu Nomura, $\mathrm{PhD}^{2}$

${ }^{1}$ National Institute of Occupational Safety and Health, Japan, Kawasaki, Japan; ${ }^{2}$ Waseda University, Tokorozawa, Japan; ${ }^{3}$ Nagoya University, Nagoya, Japan; ${ }^{4}$ National Institute of Advanced Industrial Science and Technology, Koto-ku, Japan; ${ }^{5}$ Tokai Gakuin University, Kakamigahara, Japan and ${ }^{6}$ Bunkyo Gakuin University, Fujimino, Japan.

Although inflammatory activity is mostly measured in the blood, recent studies suggested that some inflammatory activity could be measured in the saliva. This study investigated the effects of acute psychosocial stress on two salivary inflammatory biomarkers, Interleukin-6 (IL-6) and C-Reactive Protein (CRP). Fifty-seven male and female students (mean age $21.4 \mathrm{yrs}$.) were subjected to the psychosocial stress test "Trier Social Stress Test" (TSST), in which the participants were asked to deliver a speech and perform a mental arithmetic in front of two audiences. Saliva samples were taken before, during, and after TSST. Salivary IL-6 and CRP concentrations significantly increased by an average of $71 \%$ and $56 \%$, respectively, compared to each baseline value. Both concentrations peaked during stress tasks and IL-6 and CRP levels remained elevated for 20 and 10 minutes, respectively, after the cessation of stress tasks. IL-6 response was moderately correlated to CRP response $(\mathrm{r}=.305, \mathrm{p}<.05)$. These results indicated that both salivary inflammatory biomarkers are stresssensitive and could be applied for stress research.

CORRESPONDING AUTHOR: Shuhei Izawa, PhD, National Institute of Occupational Safety and Health, Japan, Kawasaki-shi, 214-8585; izawa0810@gmail.com

\section{PST-179d}

PRENATAL ADVERSITY - A RISK FACTOR

IN BORDERLINE PERSONALITY DISORDER

Cornelia E. Schwarze, MSc, ${ }^{1,2}$ Bettina Pallasch, MD, ${ }^{1}$ Gregor Hoppe, MD ${ }^{1}$ Matthäus Kurz, MD,${ }^{1}$ Arian Mobascher, Dr, ${ }^{1}$ Dirk H. Hellhammer, Prof ${ }^{2}$ and Klaus Lieb, Prof ${ }^{1}$

${ }^{1}$ Klinik für Psychiatrie und Psychotherapie, Universitätsmedizin der Johannes Gutenberg-Universität Mainz, Mainz, Germany and ${ }^{2}$ Klinische und Physiologische Psychologie, Universität Trier, Trier, Germany. 
It has been reported that unfavourable prenatal conditions - such as prenatal maternal stress, drugs, tobacco smoking or medical complications - affect the intrauterine development of the fetus, and may be associated with an increased risk for physical and mental disorders in the offspring.

Severe maternal stress during pregnancy affects the offspring's hypothalamic-pituitary-adrenal axis (HPA) and may result in longterm diseases like metabolic syndrome, type 2 diabetes, hypertension or psychiatric disorders. This phenomenon is called 'prenatal programming'.

We here investigated 'prenatal adversity' as a potential risk factor in the etiophathogenesis of Borderline Personality Disorder.

We assessed whether patients with Borderline Personality Disorder experienced more pre-, peri- and postnatal adverse life events than healthy controls, and tested the predictive value of these factors.

One hundred patients with a primary DSM-IV diagnosis of Borderline Personality Disorder and 100 healthy controls underwent semi-structured interviews about the course of pregnancy, severe maternal stress, birth complications and childhood trauma. Further information was gathered from birth records, self-rating questionnaires and from close relatives of the participants.

Statistical analyses revealed that mothers of patients with Borderline Personality Disorder experienced significantly more adverse life events during pregnancy compared to mothers of healthy controls (e.g. traumatic stress $\mathrm{p}=.029$, low social support $\mathrm{p}=.007$, severe familial conflicts $\mathrm{p}=.008$ or medical complications $\mathrm{p}=.017$ ).

Logistic regression analyses revealed that prenatal risk factors explain $30.9 \%$ of variance in Borderline Personality Disorder. In particular tobacco smoking $(\mathrm{p}=.003)$ and medical complications $(p=.006)$ seem to mark important risk factors. Furthermore patients with a history of early adversity show significantly more somatic comorbidities than controls $(\mathrm{p}<.001)$.

Our findings suggest that pre- and perinatal adversity constitute important risk factors in the etiopathogenesis of Borderline Personality Disorder.

CORRESPONDING AUTHOR: Cornelia E. Schwarze, MSc, Klinik für Psychiatrie und Psychotherapie, Universitätsmedizin der Johannes Gutenberg-Universität Mainz, Mainz, D-55116; schwarze@psychiatrie.klinik.uni-mainz.de

\section{PST-179e}

\section{SOCIAL ADVERSITY IN ADOLESCENCE INCREASES} THE PHYSIOLOGICAL VULNERABILITY TO JOB STRAIN IN ADULTHOOD: A PROSPECTIVE POPULATION-BASED STUDY

Hugo Westerlund, PhD, ${ }^{1}$ Per E. Gustafsson, $\mathrm{PhD},{ }^{2}$ Töres Theorell, $\mathrm{MD}, \mathrm{PhD}$, Profem, ${ }^{1}$ Urban Janlert, MD, PhD, Professor ${ }^{2}$ and Anne Hammarström, MD, PhD, Professor ${ }^{2}$

${ }^{1}$ Stress Research Institute, Stockholm University, Stockholm, Sweden and ${ }^{2}$ Dept of Public Health and Clinical Medicine, Umeå University, Umeå, Sweden.

Background: Social conditions during childhood have been mentioned as a possible confounder in the relationship between job strain and myocardial infarction risk. However, stress theory also suggests that early experiences may modify the individual's vulnerability to later stress, for instance through learned helplessness or hopelessness.

Methods: In a prospective population-based cohort (effective $\mathrm{n}=$ $771 ; 72 \%$ ), we examined the association between on the one hand exposure to an adverse social environment in adolescence, measured at age 16, and job strain measured with the DemandControl Questionnaire (DCQ) at age 43, and on the other hand allostatic load at age 43. Adversity in adolescence was operationalised as an index comprising residential mobility and crowding, parental loss, parental unemployment, and parental physical and mental illness (including substance abuse). Allostatic load was operationalised as an index comprising body fat, blood pressure, inflammatory markers, glucose metabolism, blood lipids, and cortisol area under curve.

Results: Adversity in adolescence was associated with higher adult allostatic load in women $(\beta=0.170, \mathrm{p}=0.001)$. There was also a significant interaction between adversity in adolescence and job strain in the whole cohort $(\beta=0.081, p=0.026)$, indicating that the ability to cope with the demands in working life may be negatively affected by exposures in early life.

Conclusion: Exposure to an adverse social environment in adolescence was associated with increased vulnerability to job strain in mid-life, indicating that sensitivity to stress and social inequalities in health may both be partially determined by material factors in early life.

CORRESPONDING AUTHOR: Hugo Westerlund, PhD, Stress Research Institute, Stockholm, SE-106 91; hugo.westerlund@ stress.su.se

\section{PST-179f}

RELIGIOUSNESS IS ASSOCIATED WITH SLOWER DISEASE PROGRESSION IN HIV BALBIN E, IRONSON G, STUETZLE R, FLETCHER MA, SCHNEIDERMAN N. UNIVERSITY OF MIAMI

Elizabeth Balbin, MA

Psy \& Behav Med, University of Miami, Coral Gables, FL.

Meta analyses have shown that religiousness, reflected in behavior such as frequently attending church, has documented health advantages. However, this has not been examined in HIV, nor has it been determined whether advantages of church attendance may be independent of spirituality. This study examined whether frequent church attendance is related to disease progression markers (CD4 and Viral Load (VL)) over time in people with HIV, and whether these effects may be independent of spirituality. Methods: One hundred diverse participants with HIV (men, women, African American, Caucasian, and Hispanic) answered questions about church attendance and spirituality as part of a psychosocial battery. Participants were followed every 6 months for CD4 and VL changes over 4 years. Hierarchical Linear Modeling assessed whether frequency of church attendance was associated with slope changes in CD4 or VL (log) over time 
controlling for baseline CD4, VL, age, gender, ethnicity, education, and spirituality. Results: Church attendance was significantly associated with slower CD4 decline $(\mathrm{t}=3.52 \mathrm{p}=.001)$ but not VL. Note that the impact of church attendance was significant even above spirituality. Conclusions: Church attendance predicts slower CD4 decline in people with HIV, and these effects seem to be independent of spirituality. A discussion of why there may be benefits independent of spirituality will be discussed.

CORRESPONDING AUTHOR: Elizabeth Balbin, MA, Psy \& Behav Med, University of Miami, Coral Gables, FL, 33124; ebalbin@miami.edu

\section{PST-179g}

THE CONTROL STUDY OF BEHAVIORAL AND EMOTIONAL PROBLEM BETWEEN LOCAL CHILDREN AND VICTIMIZED CHILDREN MIGRATED OUT OF DISASTER AREA ONE YEAR AFTER WENCHUAN EARTHQUAKE

Gongying $\mathrm{Li}, \mathrm{PhD}$, Zhiyin Yang, $\mathrm{MD}$ and Bo Bai, $\mathrm{PhD}$

Jining Medical University, Institute of Behavioral Medicine, Jining, China.

Objective: To investigate the behavioral and emotional status of the migrated children one year after they were victimized from earthquake in Wenchuan.

Method: Using Achenback Child Behavior Checklist (CBCL), the Screen for Child Anxiety Related Emotional Disorders (SCARED) and Depression Self-rating Scale for Children (DSRSC), we investigated the behavioral, anxious and depressive dimensions of 513 victimized children as pupils studying in Rizhao, Shandong province ,to which they were migrated from Wenchuan, Sichuan province. The controls were local children $(n=475)$ coming from the same schools as the victimized subjects.

Results: We got 487 answered files that were validated. There were significant differences between the victimized and migrated children and the local children in $\mathrm{CBCl}$ 's total scores, and its withdrawal, somatization complaining and anxiety-depression scores, as well as its factors such as social problem, attentional problem, attack behavior, internalizing behavior, social communication and school scores. On the SCARED and DSRSC, the two groups were significantly different in total scores of anxiety and depression as well as in such factors as somatization, generalized anxiety, separated anxiety.

Conclusion: Though migrated out of the earthquake area, the victimised children still suffer obviously from behavioral problems, and anxious and depressive emotion one year after the earthquake, making it necessary to continue psycho-behavioral intervention in them.

Key words: earthquake; children; behavior; anxiety; depression.

CORRESPONDING AUTHOR: Gongying Li, PhD, Jining Medical University, Institute of Behavioral Medicine, Jining, 272013; ligongying2005@126.com
PST-179h

THE RELATIONSHIP BETWEEN PERCEIVED LONELINESS AND CORTISOL AWAKENING RESPONSE ON WEEKDAYS AND WEEKENDS

Hisayoshi Okamura, Assistant professor, ${ }^{1}$ Akira Tsuda, Proffesor $^{2}$ and Jumpei Yajima, $\mathrm{Dr}^{3}$

${ }^{1}$ Kurume University, Kurume, Japan; ${ }^{2}$ Kurume University, Kurume, Japan and ${ }^{3}$ Beppu University, Beppu, Japan.

Background: Pressman (2005) found that high levels of momentary or experiences of loneliness were associated with elevated morning and evening cortisol. Recently, it is thought that the salivary cortisol awakening response (CAR) can serve as a reliable marker of HPA axis to respond to stress. The CAR is greater in people under chronic work stress and on the work day than the weekend. We recently demonstrated that the difference on this weekday and weekends is an adequate responsiveness to adapt to stress. However, there are no studies that investigate the relationship between the CAR and loneliness on the work days and the weekends in women. The present study was to investigate the relationship between the CAR and loneliness on the work days and the weekends in women.

Methods: The participants were healthy 90 women (20-50 years old). Loneliness was evaluated using the revised UCLA loneliness scale (Russell et al., 1980) and participants were classified into high (average $+1 \mathrm{SD})(\mathrm{n}=13)$ or low loneliness groups (average 1SD) $(n=13)$. The saliva samples were collected over the work days and the weekends. Participants were instructed to collect saliva on six occasions each day: before sleep, immediately on awakening, 30 minutes after awakening, and then as near as possible to 10:00 am, 12:00 pm, and 3:00 pm.

Results: CAR was larger on weekends in the high loneliness group than the low loneliness group. However, there were no significant differences in CAR on workdays between subjects with higher loneliness level and with lower one. In addition, there were significant differences in CAR on low loneliness group between workdays and weekends but not in high loneliness group.

Discussion: Loneliness was associated with a promoted CAR, especially on weekends. These results indicated that the CAR and cortisol levels on high loneliness people showed no significant differences between work days and weekends due to allsotaic load that the HPA system is always activated.

CORRESPONDING AUTHOR: Hisayoshi Okamura, Assistant professor, Kurume University, Kurume, 830-0011; okamura hisayoshi@med.kurume-u.ac.jp

\section{PST-179i}

MEMORY AND POSSIBLE CAUSES OF REDUCED MEMORY FUNCTION IN PREGNANCY

Ilona Fridman, MA, ${ }^{1}$ Edan Yodfat, $\mathrm{MD}^{3}$ and Shira Mor, $\mathrm{MA}^{2}$

${ }^{1}$ Brooklyn College, Brooklyn, NY; ${ }^{2}$ Columbia Business School, New York, NY and ${ }^{3}$ Maimonides Medical Center, Brooklyn, NY. 
Fridman Ilona1, Yodfat Edan2, MD, Mor Shira3

1. Brooklyn College - City University of New York

2. Maimonides Medical Center, Brooklyn, NY

3. Columbia Business School.

Background: It is a common stereotype, that women experience decrease in memory function during pregnancy. Previous studies about memory changes associated with pregnancy showed inconsistent Results: Working memory is a type of a short-term memory that is responsible for monitoring, keeping and applying information. A decrease of working memory function may lead to unexpected mistakes, absentmindedness and as a result to anxiety stress and decrease in self-esteem. Current research assesses the changes in working memory of healthy pregnant women. We also evaluate the influence of other variables such as depression, sleep changes, and negative self-beliefs on working memory.

Materials and methods: Healthy pregnant women going for routine prenatal care were enrolled in the study. Overall 25 women were approached and agreed for participation. Control was matched for age and consisted of 10 non-pregnant women. Validated neurocognitive tests battery (Symbol-Digit Modalities Test and E-prime computer based test) was used to evaluate working memory function. Results: There was no significant difference between the groups in scores of memory encoding and retrieval. Depression and insomnia were not associated with changes of the working memory. Negative self-perception on Symbol-Digit Modalities Test was associated with decrease in working memory function $(\mathrm{r}=-.43)$.

Conclusion: We failed to prove that pregnancy causes changes in the working memory. Self-perception reduces memory performance in both control and experimental groups.

Key words: memory test, pregnancy

CORRESPONDING AUTHOR: Ilona Fridman, MA, Brooklyn College, Brooklyn, NY, 11219; svitina@gmail.com

PST-181

THE DEVELOPMENT OF AN OCCUPATIONAL HEALTH GUIDELINE TO IMPROVE WORKERS' PHYSICAL ACTIVITY AND DIETARY BEHAVIOUR IN ORDER TO PREVENT WEIGHT GAIN

Lisanne M. Verweij, MSc, ${ }^{1}$ Karin I. Proper, $\mathrm{PhD},{ }^{1}$ Carel Hulshof, $\mathrm{PhD}^{2}$ and Willem van Mechelen, $\mathrm{PhD}^{1}$

${ }^{1}$ Public and Occupational Health, EMGO+ Institute for Care and Health Research, Amsterdam, Netherlands and ${ }^{2}$ Netherlands Society of Occupational Medicine, Amsterdam, Netherlands.

The prevalence of overweight and obesity has reached epidemic proportions, also in the Netherlands. Weight gain prevention interventions have shown to be feasible within the occupational health services, but are hardly being implemented by occupational physicians (OPs) due to a lack of methods and materials. The aim of this study is to 1) develop, 2) evaluate, and 3) implement a weight gain prevention guideline to be used by OPs. Following the standard template of the Netherlands Society of Occupational Medicine and using the Intervention Mapping protocol, a draft guideline was developed based on literature, interviews with employers, OPs and employees, input from an external project group consisting of OPs and lifestyle experts, and input from independent lifestyle experts. Based on all sources mentioned above, the developed guideline consists of clear cut recommendations for OPs to 1) advise employers to promote employees' physical activity and diet and 2) counsel employees to adopt a physically active and healthy diet behaviour. Tools to implement recommendations consist of a minimal intervention strategy to counsel employees, and a scan to assess the obesogenic environment. The guideline is now being evaluated in a RCT among 30 OPs and 350 employees. If proven effective, this weight gain prevention guideline will be implemented on a larger scale within the occupational health services in the Netherlands.

CORRESPONDING AUTHOR: Lisanne M. Verweij, MSc, Public and Occupational Health, EMGO+ Institute for Care and Health Research, Amsterdam, 1081 BT; 1.verweij@vumc.nl

\section{PST-182}

WHAT ARE THE KEY ELEMENTS

OF THE CO-PRODUCTION OF HEALTH IN CLINICIANPATIENT CONSULTATIONS AIMING

TO SELF-MANAGEMENT SUPPORT IN LONG TERM HEALTH CONDITIONS?

Alba Realpe, MSc, ${ }^{1}$ Louise M. Wallace, Professor, ${ }^{1}$ Ann Adams, $\mathrm{Dr}^{2}$ and Jane Kidd, $\mathrm{Dr}^{2}$

${ }^{1}$ Applied Research Centre for Health and Lifestyle Interventions, Coventry University, UK, Coventry, United Kingdom and ${ }^{2}$ Warwick Medical School, University of Warwick, Coventry, United Kingdom.

Background: Health policy in the UK and the USA supports the design of health services for people with long term conditions (LTC) to be conducive to self-management support. The Wagner's chronic care model suggests that self-management support needs to be embedded in the system. Coote suggests that co-production can be applied to health care. It is a model in which true partnership is based on the active input of recipients in the provision of their own care however it is at best 'too flexible' to guide practice.

Aims: To define the key observable behaviours that clinicians and patients require for co-productive interactions in LTC consultations. Methods: A convenient sample of eleven UK and US experts in self-management support and communication skills were interviewed for an hour using a semi-structured format. The transcripts were analyzed thematically.

Results: Clinicians and patients need to enter in a partnership, recognizing mutual expertise within a long term relationship; consultations should include discussions about self-management strategies used to develop a highly personalized solution; shared decision making should develop e.g. over health priorities. This means negotiating an agenda, setting goals and agreeing in a followup; the development of rapport is important to engage patients in behaviour change and co-production of health requires a system that facilitates joint decision making. The final model will help to develop a measurement tool using the Roter Interaction Analysis System to assess co-production of health in video-recordings of real life clinician-patient consultations across the UK. 
Discussion: This study adds to the understanding of clinical practices and communication behaviours in consultations for people with LTC. The results will be submitted to a further eDelphi consultation process with the same experts $(n=11)$ and also a wider group of health care professionals to reach a reasonable level of agreement and to confirm the model.

CORRESPONDING AUTHOR: Alba Realpe, MSc, Coventry University, UK, Coventry, CV1 5FB; A.Realpe@coventry.ac.uk

\section{PST-183}

TRANSLATING FAMILY HEALTH PROGRAM HEALTH PROFESSIONAL PERCEPTIONS TO IMPROVE CHILD HEALTH IN VESPASIANO, BRAZIL

Lilian Perez, MPH, ${ }^{1}$ Priscila Saleme, MD Candidate, ${ }^{2}$ Bernardo Patrus, MD Candidate, ${ }^{2}$ Jose A. Ferreira, $\mathrm{PhD}^{2}$ and Juan Leon, $\mathrm{PhD}, \mathrm{MPH}^{1}$

${ }^{1}$ Global Health, Rollins School of Public Health, Atlanta, GA and ${ }^{2}$ Faculdade da Saúde e Ecologia Humana (FASEH), Vespasiano, Brazil.

The Family Health Program (FHP) was implemented across Brazil in 1994 to deliver primary healthcare services, such as diarrhea treatment and prevention, through health teams that include physicians, nurses, nurse assistants and community health workers (CHWs). Several studies have pointed to the impacts of the FHP on improving child survival in Brazil. Few studies, however, have examined the mechanisms underlying the successful delivery of services in the community, particularly from the perspectives of the health professionals. The goal of this study, therefore, was to evaluate the perceptions of FHP professionals of Vespasiano, Brazil on training, workplace challenges, and practices for child health. This cross-sectional study involved interviews with 77 professionals using a mixed-methods approach. Data was doubleentered and analyzed in Epi Info version 3.5.1. Open-ended responses were stratified by professional category, translated from Portuguese to English, and analyzed using a thematic coding approach. The results show that $92 \%$ of professionals received training in their first 12 months in the program but the majority found the training was limited in quantity, content, methods of delivery, and overall quality. Challenges in the workplace include insufficient government support, poor infrastructure, and lack of resources. In spite of these limitations, over $70 \%$ of CHWs reported promoting important health messages to caretakers and $87 \%$ of professionals were satisfied with the FHP child diarrhea services. In addition, the majority of professionals perceived the FHP is created for the needs of the community (83\%) and is accepted by the users $(81 \%)$. Translation of this research will be important for policies and program developments to improve on the limitations and promote the strengths of the FHP in order to advance Brazil's healthcare model to address leading causes of disease among the Brazilian pediatric population

CORRESPONDING AUTHOR: Lilian Perez, MPH, Global Health, Rollins School of Public Health, Atlanta, GA, 30306; lgperez12@gmail.com
PST-184

THE HEALTH IMPACT OF CHILDHOOD MALTREATMENT: HEALTH VARIABLES IN RELATION TO OFFICIAL

AND SELF REPORT DATA

Angela Maia, phd and Ricardo Pinto, dr

Psychology, University of Minho, Braga, Portugal.

Aims: Childhood maltreatment had been considered a risk factor for psychopathology and health problems, including health complaints, health risk behaviors and diseases. Studies usually use retrospective methodologies and clinical samples. However, some authors have been discussing the validity of the associations these studies usually found suggesting that recall bias can explain the relation between maltreatment and symptoms. The aim of this study was to characterize current psychological and health functioning in young adults that were victims of maltreatment as children and compare the self report with official records from Child Protection Services. Method: Official records of 109 young adults that were identified as victims by protection services during childhood ( 55 female and 54 male) were compared with self-reported maltreatment history data using Childhood History Questionnaire. Participants also filled Brief Symptoms Inventory and Rotterdam Symptoms Checklist.

Results: Subjects that report more adverse experiences have more psychopathology $(\mathrm{r}=.48 ; \mathrm{p}=.000)$ and health complaints $(\mathrm{r}=.36$; $\mathrm{p}=.000)$. Some subjects $(21 \%)$ do not report any maltreatment experience, $36 \%$ report only some of the experiences they were victims and $44 \%$ do accurate reports. These groups are not different in relation to psychopathology $(\mathrm{F}(2,104)=.618$; $\mathrm{p}=.541)$ or health complaints $(\chi 2(2)=4.40 ; \mathrm{p}=.111)$

Conclusion: Self-report false negatives for adverse childhood experiences are very high and most of the subject's don't report all the experiences they were victims. Some authors had suggested that the relation between reported childhood experiences and symptoms could be interpreted as a record bias for participants that are symptomatic in the evaluation moment, but this data does not confirm this hypothesis since there were no symptoms differences between the groups. Reasons for not report and relation between adversity and health should be better understood in future research.

CORRESPONDING AUTHOR: Angela Maia, phd, Psychology, University of Minho, Braga, 4700 BRAGA; angelam@psi. uminho.pt

\section{PST-185}

SCREENING FOR HIV-RELATED PTSD: SENSITIVITY AND SPECIFICITY OF THE 17-ITEM POSTTRAUMATIC STRESS DIAGNOSTIC SCALE (PDS) IN IDENTIFYING HIV-RELATED PTSD AMONG A SOUTH AFRICAN SAMPLE Lindi Martin, MA, Dylan Fincham, $\mathrm{PhD}$ and Ashraf Kagee, $\mathrm{PhD}$ Stellenbosch University, Matieland, South Africa.

Objectives: The identification of HIV-positive patients who exhibit criteria for Posttraumatic Stress Disorder (PTSD) and related trauma symptomatology is of clinical importance in the maintenance of their overall wellbeing. This study assessed the sensitivity and specificity of the 17-item Posttraumatic Stress Diagnostic Scale (PDS), a self- 
report instrument, in the detection of HIV-related PTSD. An adapted version of the PTSD module of the Composite International Diagnostic Interview (CIDI) served as the gold standard.

Method: 85 HIV-positive patients diagnosed with HIV within the year preceding data collection were recruited by means of convenience sampling from three HIV clinics within primary health care facilities in the Boland region of South Africa.

Results: A significant association was found between the 17-item PDS and the adapted PTSD module of the CIDI. A ROC curve analysis indicated that the 17-item PDS correctly discriminated between PTSD caseness and non-caseness $74.9 \%$ of the time. Moreover, a PDS cut-off point of $\geq 15$ yielded adequate sensitivity $(68 \%)$ and 1 -specificity $(65 \%)$. The 17 -item PDS demonstrated a PPV of $76.0 \%$ and a NPV of $56.7 \%$.

Conclusion: The 17-item PDS can be used as a brief screening measure for the detection of HIV-related PTSD among HIVpositive patients in South Africa.

CORRESPONDING AUTHOR: Ashraf Kagee, PhD, Stellenbosch University, Matieland, 7602; skagee@sun.ac.za

\section{PST-186}

\section{CONTEMPORARY SOCIAL CONFLICT THEORY:} IMPLICATIONS FOR HEALTH RELATED COMMUNICATION AND BEHAVIOR

Michael Van Slyck, $\mathrm{PhD}^{1}$ and Marilyn Stern, $\mathrm{PhD}^{2}$

${ }^{1}$ Virginia Comonwealth University, Richmond, VA and ${ }^{2}$ Virginia Commonwealth University, Richmond, VA.

Research documents the impact of social environments on health related communication and behavior, and actual health. However, one aspect of the environment along with its related area of scientific investigation and professional application, all with clear implications for health issues, has received less attention than is warranted. Specifically, we are referring to Interpersonal conflict as described by Contemporary Social Conflict Theory (CSCT), which is not well known or understood. The result is that Its implications for health issues are underestimated or ignored.

Conventional views equate conflict with behavior (arguing/fighting), view it as "bad," and advocate avoidance as a response to prevent violence. CSCT views conflict as a cognitive process based on beliefs concerning needs and the expectations for satisfying them. Conflict, defined as "perceived divergence of interest" is regarded as inevitable, ubiquitous and necessary for change. For CSCT, contention (fighting) is one of five response modalities - accommodation, avoidance, compromise, and problem solving - are the other "Conflict Management Styles (CMS's), each with predictable consequences.

Conventional views of conflict limit us to consideration of the contentious CMS which has implications for health ranging from covert effects of stress from using this CMS on a regular basis to overt effects of violence that can result from this CMS. However, other CMS's have implications for health outcomes, raising a variety of research questions, e.g., who gets better treatment, the demanding "contentious" patient or the compliant "accommodating" patient? Does an avoidant CMS result in lessened ability to address important health care issues with family or healthcare providers? Does a problem solving CMS result in proactive health behavior, better communication with HCP's, and higher rates of compliance?

The purpose of this poster is to explicate the principles and practices of CSCT and suggest their potential practical use - especially those involving decision making activities about treatment.

CORRESPONDING AUTHOR: Michael Van Slyck, PhD, Virginia Comonwealth University, Richmond, VA, 23282; mrvanslyck@vcu. edu

\section{PST-187a}

SPIRITUAL WELLBEING MEDIATES THE EFFECTS

OF A PSYCHO-SPIRITUAL MANTRAM PROGRAM

ON PTSD SYMPTOM SEVERITY IN VETERANS

WITH MILITARY-RELATED PTSD

Jill E. Bormann, PhD, RN, ${ }^{1,2}$ Steven Thorp, $\mathrm{PhD},{ }^{1,3}$ Lin Liu, $\mathrm{PhD},{ }^{3,4}$ Julie L. Wetherell, $\mathrm{PhD},{ }^{3,4}$ Dale Glaser, $\mathrm{PhD}^{2}$ and Ariel $\mathrm{J}$. Lang, PhD MHP ${ }^{1,3}$

${ }^{1}$ Center of Excellence in Stress and Mental Health, VA San Diego Healthcare, San Diego, CA; ${ }^{2}$ San Diego State University, San Diego, CA; ${ }^{3}$ University of California San Diego, San Diego, CA and ${ }^{4}$ VA San Diego Healthcare System, San Diego, CA.

Purpose: A mantram (sacred word) intervention has demonstrated significant reductions in PTSD symptom severity when applied as a complementary treatment for Veterans with military-related trauma compared to usual care controls. Little is known, however, about mechanisms for this improvement. Prior studies of the mantram intervention have demonstrated increased spiritual wellbeing (SWB) and decreased psychological distress attributable to this intervention. Therefore, we hypothesized that increases in SWB may mediate reductions in PTSD symptom severity.

Methods: Path analysis was conducted on data from a randomized clinical trial in which 66 Veterans with chronic military-related PTSD completed a 6-week (90-minutes/week) mantram group intervention and were compared to 70 usual care controls. The dependent variable was the pre- to post-treatment change scores on the PTSD Checklist (PCL). The mediator variable was the Functional Assessment of Chronic Illness Therapy-Spiritual Well-Being scale (FACIT-SWB). The sample was 97\% male; 59\% Caucasian, 24\% African American, 7\% Hispanic and 10\% other. They ranged in age from 25 to $83(\mathrm{M}=57, \mathrm{SD}=9.26)$, averaged 14 years $(\mathrm{SD}=2.1)$ of education and experienced an average of 13 months $(\mathrm{SD}=10.0)$ of combat.

Results: The hypothesis that SWB partially mediates reductions in PTSD symptom severity was supported. The mantram intervention predicted post-treatment changes in PCL $(b=3.71, p<.05)$. There was a significant and positive, indirect effect in the path from the mantram intervention to SWB $(b=6.37, p<.05)$ and a significant, negative effect from SWB to PCL $(b=.32, p<.0002)$. Similar results were found using the Baron and Kenny statistical approach. The Sobel test was significant at $2.55(\mathrm{p}<.01)$.

Conclusion: Findings suggest that one potential mechanism by which the mantram intervention reduces PTSD symptom severity in Veterans is by promoting beliefs about meaning and/or purpose 
in life and feelings of peace and harmony. Spiritual wellbeing may be an important factor to consider when treating PTSD.

CORRESPONDING AUTHOR: Jill E. Bormann, PhD, RN, Nursing \& Patient Care, VA San Diego Healthcare System, San Diego, CA, 92161; jill.bormann@va.gov

\section{PST-187b}

\section{CHILD PHYSICAL ABUSE AND PHYSICAL HEALTH} CONSEQUENCES IN ADULTHOOD: HOW STRONG

\section{IS THE EVIDENCE?}

Ângela Maia, PhD, Vanessa Azevedo, Master and Carla Martins, $\mathrm{PhD}$

School of Psychology, Braga, Portugal.

Only recently researchers became interested in studying the physical health consequences in adulthood that result from physically abusive experiences during childhood, but now is a promising area of research.

The state of the art about the topic is quite interesting and researchers deal with many challenges. One of them is the difficulty to establish a clear relationship between the experience of physical violence and health variables, mainly due to methodological issues. In order to overcome this concern some epidemiological guidelines were defined, namely: major criteria (temporal relationship; biological plausibility; consistency), alternative explanations (confounding variables), other considerations (dose-response relationships; strength of association; and cessation of exposure).

Regarding these criteria, we proceeded to a systematic review of nineteen studies about the long-term physical health impact of child physical abuse in community samples. These researches included at least one of seven health categories: sexual risk-behaviors, substance dependence, alcohol consumption, drug consumption, nicotine consumption, current general health and physical health condition.

Our review revealed that results are inconclusive and mixed for all categorical groups. However, it seems to be a positive association between "being victim of child physical abuse" and "sexual riskbehaviors" and "alcohol consumption" in men; "physical health condition" in women; and "drug consumption" in mixed samples.

Based on our results, we cannot draw an unambiguous conclusion between these variables and therefore this issue should be further analyzed.

CORRESPONDING AUTHOR: Vanessa Azevedo, Master, School of Psychology, Braga, 4710-057; vazevedo@psi.uminho.pt

\section{CITATION ABSTRACT \\ PST-188}

A RANDOMISED CONTROLLED TRIAL OF IMAGERY TECHNIQUES FOR PROMOTING QUALITY SLEEP

Marisa H. Loft, MSc (Health Psychology) and Linda D. Cameron, PhD

Psychology, University of Auckland, Auckland, New Zealand.

Sleep deprivation is a significant problem in the modern workplace and has negative consequences for employee health. This trial assessed the effects of two mental imagery techniques, one inducing implementation intentions so as to close the intention-to-action gap in sleep promoting behaviours and the other inducing relaxation so as to reduce arousal prior to going to sleep. We recruited 72 employees from local businesses who reported difficulty sleeping. Following screening, randomization and a training session, they were asked to practice brief imagery exercises involving either arousal reduction imagery, implementation intention imagery (i.e., specifying context and action), a combination of the two, or imagery of their normal night routine (control) twice a day over 21 days. They completed an online, daily diary of sleep behaviours and, at baseline and day 21, an online questionnaire that included the Pittsburgh Sleep Quality Index (PSQI), Pre-Sleep Arousal scale, Sleep Hygiene Index, Dysfunctional Sleep Beliefs Scale, and measures of state anxiety and sleep-related planning. Repeated measures ANOVAs showed that, relative to the groups not using implementation intention imagery, the implementation intention groups had a greater reduction in negative sleep habits $(\mathrm{p}<.01)$ and greater improvements in the sleep-related actions targeted in the imagery exercise $(p<.01)$. Increases in sleep motivation $(\mathrm{p}<.001)$ and response efficacy for sleep behaviours $(\mathrm{p}<.001)$ were found across all groups, along with decreases in anxiety $(\mathrm{p}<.001)$, dysfunctional sleep beliefs $(\mathrm{p}<.001)$ and pre-sleep arousal $(\mathrm{p}<.001)$. Improvement in all groups was also shown for total PSQI scores $(\mathrm{p}<.001)$ and level of detail in plans for positive sleep behaviour $(\mathrm{p}<.001)$. Mixed modeling analyses of the daily data indicated that those using implementation intention imagery had faster times of falling asleep $(\mathrm{p}<.01)$ and earlier times of lights out $(\mathrm{p}<.01)$ compared to those not using this imagery. These brief, low-cost techniques appear to provide a promising alternative to the use of medication for the improvement of sleep in the general population. 
CORRESPONDING AUTHOR: Marisa H. Loft, MSc (Health Psychology), Psychology, University of Auckland, Auckland, 1051;m.finn@auckland.ac.nz

\section{PST-189}

A MULTI-STATE MODEL ON OFFICIAL REGISTERED SICK-LEAVE DATA - APPLIED ON A 5-YEAR FOLLOW-UP AFTER OCCUPATIONAL REHABILITATION IN NORWAY Irene Oyeflaten, Candsan, ${ }^{1}$ Stein Atle Lie, $\mathrm{PhD},{ }^{2}$ Camilla Ihlebaek, $\mathrm{PhD}^{2}$ and Hege Randi Eriksen, $\mathrm{PhD}^{2}$

${ }^{1}$ The National Centre for Occupational rehabilitation, Rauland, Norway and ${ }^{2}$ Uni Health, Bergen, Norway.

Background: RTW is a complex process, where the individual may be in multiple and recurrent states. The aim of this study was to describe the evolving RTW-process in a 5-year follow-up period after a 4-weeks inpatient occupational rehabilitation program.

Methods: The sample consisted of 584 patients $(66 \%$ females, mean age 44 years $[\mathrm{sd}=9.3]$, long-term sick-listed; mean duration 9.3 months $[\mathrm{sd}=3.4]$ for psychological $(47 \%)$ and musculoskeletal (46\%) diagnosis. Register data from The National Insurance Administration in Norway over a 5-year follow-up period was analysed. Outcome was measured in an 8 state-model: working, part-time sick-listed, sick-listed, medical rehabilitation, vocational rehabilitation, part-time disability pension, time-limited disability pension and permanent disability pension. Extended statistical tools for multistate models were used to calculate transition probabilities between the different states.

Results: During the 5-year follow-up period the patients had on average 4.6 [range 1-19] shifts between the 8 different states. During the first 30 days after rehabilitation $9 \%$ returned to full time work, $23 \%$ were graded sick-listed, $45 \%$ were full-time sicklisted, $15 \%$ were on medical rehabilitation, $6 \%$ on vocational rehabilitation and $2 \%$ on graded disability pension. After 5 years $58 \%$ had returned to full-time work, $0 \%$ was sick-listed or on medical rehabilitation, $10 \%$ were on vocational rehabilitation, $14 \%$ on part-time disability pension, $5 \%$ on time-limited disability pension and $13 \%$ received permanent disability pension. Conclusions: The RTW-process for long-term sick-listed individuals is long and complex. Despite poor RTW during the first 30 days after occupational rehabilitation, the long-term RTWprospects seem advantageous. It is of interest to examine what role environmental and legal system factors and of the work-place and health-care providers play in this prolonged RTW-course.

CORRESPONDING AUTHOR: Irene Oyeflaten, Candsan, The National Centre for Occupational rehabilitation, Rauland, 3864; irene.oyeflaten@air.no

\section{PST-190}

\section{SELF-EFFICACY ASSOCIATED TO INTENTION}

TO CHANGE AND RETURN-TO-WORK BEHAVIOR

Sandra Brouwer, $\mathrm{PhD}^{1}$ and Renee-Louise Franche, $\mathrm{PhD}^{2}$

${ }^{1}$ university medical center groningen, Groningen, Netherlands and

${ }^{2}$ Occupational Health and Safety Agency for Healthcare, Vancouver, BC, Canada.
Background: the influence of self-efficacy in the RTW process has been emphasized; however, a comprehensive understanding of the role of self-efficacy on RTW behavior is still lacking. Aim of the study is to investigate the association between self-efficacy for RTW (SERTW) and: a. the intention to change, and b. RTW status, in a cohort of injured workers with work-related musculoskeletal conditions.

Methods: A baseline telephone interview was conducted in 632 claimants one-month post-injury and 446 claimants (six-month post-injury). Return-to-Work Self-Efficacy (RTWSE) was measured with a validated questionnaire consisting of three subscales: RTWSE with regard to 1. Controlling pain at work; 2. Obtaining help from supervisor and 3. Obtaining help from co-workers. Intention to change was assessed with the validated Readiness for Return-To-Work (RRTW) Scale, reflecting closely the five stages described in the Readiness for RTW model $[1,3]$. RTW status was measured based on the workers' responses to the question whether they returned to work at one- and six-month follow-up. ANOVA tests were conducted to compare relationships for each of the three RTWSE subscales with the stages of change and the RTW status. Results: with regard to intention to change all univariate F-tests were significant at both measures in time, except for the co-worker RTWSE subscale at one-month follow-up. RTWSE is at its peak in workers who are actively preparing to return to work, however, it decreases significantly for people who start working again on all three RTWSE subscales. Comparing RTWSE with RTW status significant differences were found only for the Pain SERTW subscale at both time measures. Workers who returned to work showed higher Pain RTWSE scores compared to those who were still sick-listed. Conclusion: a strong association between SE-RTW and Readiness for RTW stages was found, however the association between SERTW and RTW status was only strong for RTWSE. Our findings suggest that self-efficacy is more strongly associated with intention/motivation for the behavior (internalized processes of behaviour), than strictly behavioural ones.

Message:

1. Self-efficacy for RTW is significantly associated to the intention to change but not to the actual behavior, i.e. RTW status.

2. Self-efficacy for RTW changes over time during RTW. It is at its peak in workers who are actively preparing to return to work, however it decreases significantly for people who start working again.

3. Our findings suggest that self-efficacy is more strongly associated with intention or motivation for the behavior, than strictly behavioural ones.

CORRESPONDING AUTHOR: Sandra Brouwer, $\mathrm{PhD}$, university medical center groningen, Groningen, 9700 AD; sandra.brouwer@ med.umcg.nl

PST-191

THE IMPACT OF SMOKING ON DISABILITY PENSION AMONG MIDDLE-AGED PUBLIC SECTOR EMPLOYEES Ossi Rahkonen, PhD, ${ }^{1}$ Jouni Lahti, MSc, ${ }^{1}$ Mikko T. Laaksonen, $\mathrm{PhD},{ }^{1}$ Olli Pietiläinen, MSc, ${ }^{1}$ Sakari Karvonen, $\mathrm{PhD},{ }^{2}$ Tea Lallukka, $\mathrm{PhD}^{1}$ and Eero Lahelma, $\mathrm{PhD}^{1}$

${ }^{1}$ Department of Public Health, University of Helsinki, Helsinki, Finland and ${ }^{2}$ National Institute for Health and Welfare, Helsinki, Finland. 
Background: Smoking and work disability are major public health and welfare problems. The health consequences of smoking are well-documented but less is known about the independent effect of smoking on disability pension of various diagnoses. The aim of this study was to examine the impact of smoking on subsequent disability pensions among middle-aged public sector employees.

Methods: Baseline survey was collected in 2000-2002 among middle-aged employees of the City of Helsinki $(n=6373,80 \%$ women). Data on disability pensions were obtained from the Finnish Centre for Pensions (2000-2004) and were linked to the questionnaire data. Cox regression analysis was used to calculate hazard ratios (HR) for disability pension. To examine the independent effect of smoking age, socioeconomic position, working conditions, alcohol drinking, physical activity and body mass index were adjusted for.

Results: There were 232 (4\%) disability pension events during the follow-up. Age adjusted smoking strongly predicted disability pensions (HRs 1.5-1.6) among women and men. Risk for disability pension increased with number of smoked cigarettes. Especially strong was the effect among those who smoked more than 20 cigarettes per day. Adjusting for confounders attenuated the effect but it remained.

Conclusions

To support employees quitting smoking may prevent work disability pensions.

CORRESPONDING AUTHOR: Ossi Rahkonen, PhD, Department of Public Health, University of Helsinki, Helsinki, 00014; ossi.rahkonen@helsinki.fi

\section{PST-192}

\section{A MULTINATIONAL STUDY OF THE RELATIONSHIP BETWEEN EMPLOYMENT STATUS AND HEALTH IN IRAQI REFUGEES}

Hikmet J. Jamil, MD, DIH, MSc, $\mathrm{PhD}^{1}$ and Bengt B. Arnetz, MD, MPH, MscEpi, PhD, CIME ${ }^{1,2}$

${ }^{1}$ Family Medicine \& Public Health Sciences, Division of Occupational \& Environmental Health, Wayne State University, Detroit, MI and ${ }^{2}$ Dept. of Public Health, Uppsala University, Uppsala, Sweden.

Background: Refugees are at a dramatically increased risk to suffer from mental \& somatic disorders. Research has focused on pre-displacement trauma. However, many refugees find themselves unemployed or underemployed in their new country. Employment is known to be critical for social integration \& well-being. This study focuses on the relationship between postdisplacement skills utilization among Iraqi refugees and health.

Methodology: Iraqis throughout the world were contacted using web-pages commonly used by Iraqis and by emails. Inclusion criteria were bachelor or above and having left Iraq as refugees 1990 or later. Potential participants were referred to a Monkey
Survey Link, \& following consenting to the study, they responded to a survey with 34 questions concerning socio-demographic, employment, stress, psychosomatic, mental \& somatic complaints. All respondents $(n=408)$ were classified according to employment status; Unemployed (G1, 42\% of total), Professional working at their skills level (G2, 27\%), \& Professionals working below their skills level (G3, 31\%), representing 65 countries. Significant group differences are presented. WSU HIC approved the study. Results: Participants in the G1 group rated their health the highest (Excellent to Good, 69\%), versus $80 \%$ in G3, and $86 \%$ in G2. Stress, sleep difficulties, depression and psychosomatic symptoms were the highest in G1, intermediate in G3, \& the lowest in G2. $90 \%$ of G2 enjoyed their work significantly more as compared to G3 (10\%). The major barriers to getting a job were: language, financial limitation for training, \& insufficient professional skills. Logistic regressions showed that G1 rated their health better versus unemployed (OR 2.6; 95\% C.I. 1.4-5.2). There were no differences between G2 and G3.

Conclusions: Professional refugees allowed working at their skills level, fair better than underemployed following forced displacement. Results have implications for refugee policy.

CORRESPONDING AUTHOR: Hikmet J. Jamil, MD, DIH, MSc, PhD, Family Medicine \& Public Health Sciences, Division of Occupational \& Environmental Health, Wayne State University, Detroit, MI, 48201; hjamil@med.wayne.edu

\section{PST-193}

DEVELOPMENT OF A SOLUTION-FOCUSED OCCUPATIONAL GROUP THERAPY FOR PATIENTS WITH ACUTE HAND INJURIES

Lonneke Opsteegh, MSc, ${ }^{1,2}$ Heleen A. Reinders-Messelink, $\mathrm{PhD},{ }^{3}$ Corry K. van der Sluis, $\mathrm{PhD}^{1,2}$ and Ant T. Lettinga, $\mathrm{PhD}^{1,2}$

${ }^{1}$ Center for Rehabilitation, UMCG, Groningen, Netherlands;

${ }^{2}$ department of Health Sciences, Graduate school for Health Research, UMCG, Groningen, Netherlands and ${ }^{3}$ Center for Rehabilitation, Revalidatie Friesland, Beetsterzwaag, Netherlands.

Background: Diverging return to work (RTW) interventions can be found in literature, designed for specific populations. However, no such intervention exists for patients with acute hand injuries, while these injuries can be devastating and severely influence a person's ability to work. Regular rehabilitation interventions' focus is on functional recovery of the hand, including tasks necessary to perform the job. However, also psychosocial and work-related factors influence RTW strongly. Therefore, we developed a psychosocial intervention for patients with acute hand injuries, aimed at to facilitate RTW in this population.

Methods: A combination of qualitative research techniques was used, comprising a mixture of literature study, survey, individual and focus group interviews with patients, rehabilitation practitioners and work counseling experts, and translation methodology. 
Results: Some preliminary ideas about the nature of the intervention came to fore. The RTW intervention should: 1) take into account the acute character of the injury; 2) be short of duration; 3) be non-invasive, as most patients do not consider themselves sick; 4) be aimed at RTW; 5) reduce costs and therefore group sessions are favored.

Solution-Focused Therapy (SFT) met most of the aforementioned criteria. However, as SFT originates in family therapy, its principles and practices had to be translated to the problems of patients with acute hand injuries targeted on RTW. SFT therapists use a number of specific questioning techniques that invite clients to co-construct a vision of a preferred future and draw on their past successes, strengths, and resources to make that vision a reality. Examples of questioning techniques are the miracle question, scaling questions and coping questions.

The SFT treatment ingredients were incorporated in a new intervention, consisting of one individual meeting, and four group meetings. In every group meeting one topic is discussed in a SF manner. Topics were: Law and Legislation, Social Support, Medical \& Cognitive Problems, and Strength \& Weaknesses. To evaluate the effect of the intervention on RTW, a cohort study is performed.

CORRESPONDING AUTHOR: Lonneke Opsteegh, MSc, Center for Rehabilitation, UMCG, Groningen, 9700 RB; 1.opsteegh@cvr. umcg.nl

PST-195

\section{ORGANIZATIONAL JUSTICE AND WORKPLACE} BULLYING AMONG CIVIL SERVANTS IN JAPAN

Kanami Tsuno, MPH, Akiomi Inoue, MS and Norito Kawakami, $\mathrm{MD}, \mathrm{PhD}$

Department of Mental Health, Graduate School of Medicine, The University of Tokyo, Tokyo, Japan.

Background: The association between organizational justice and workplace bullying has not yet been investigated, while the workplace atmosphere is supposed to responsible for it. The objective of the study was to explore the crosssectional association of organizational justice, as well as job strain (the ratio of job demands to job control) and effortreward imbalance, with workplace bullying in a sample of civil servants in Japan.

Methods: The study sample consisted of 807 males and 735 females of the civil servants in the Kanto region in Japan (response rate, 46.7\%). They were asked to fill in a selfadministered questionnaire including scales of workplace bullying, organizational justice (interactional justice and procedure justice), job demands and job control, effortreward imbalance (ERI), supervisor and coworker support, and other covariates (sex, age, marital status, occupational status, employment contract, work shift, overtime in the past month, unit size, and sex ratio of the unit). Workplace bullying was measured by the Negative Acts Questionnaire-
Revised, and was defined as those experiencing at least one of 22 negative acts weekly or more frequently during the past six months. Each job stressor variable was categorized into the tertiles. Odds ratio of workplace bullying associated with each job stressor was estimated by using logistic regression analysis, using the lowest tertile as the reference, adjusting for the covariates.

Results: After adjustment for the covariates, high interactional justice (odds ratio $0.23,95 \% \mathrm{Cl} 0.14-0.39$ ) and high procedure justice (odds ratio $0.21,95 \% \mathrm{Cl} \quad 0.13-0.34$ ) were negatively associated with workplace bullying. High job strain, high effortreward imbalance, low supervisor support, and low coworker support were also significantly associated with workplace bullying in an expected direction $(\mathrm{p}<0.05)$.

Conclusion: Low organizational justice may be a risk factor for workplace bullying among Japanese civil servants. The study also confirmed that job strain, ERI, and low worksite social support were associated with bullying.

CORRESPONDING AUTHOR: Kanami Tsuno, MPH, Department of Mental Health, The Graduate School of Medicine, The University of Tokyo, Japan, Tokyo, 113-0033; ktsuno@ m.u-tokyo.ac.jp

\section{PST-196}

OCCUPATIONAL CLASS AND DEPRESSION: A CROSS-SECTIONAL ANALYSIS

Akizumi Tsutsumi, MD

University of Occupational and Environmental Health, Kitakyushu, Japan.

To investigate 1) whether socioeconomic state such as income, education attainment, and occupational class are associated with employees' depression, and 2) whether occupational stress would explain the associations in Japanese working populations, we developed a cohort of employees of 3 different manufacturing workplaces and sent a self-administered questionnaire as the baseline data.

A total of 3767 employees responded to the survey (response rates $75-96 \%$ ). Associations of income level (lower or higher than $¥ 5,000,000$ of annual household income), educational attainment (lower or higher than the level of high school education) and occupational class (managerial or not) with depression were examined. Based on a recent validation test among Japanese workers using the Mini International Neuropsychiatric Interview, depression was defined as a dichotomize variable with a cut point of the CES-D scores 19 or above. Occupational stress was evaluated using Japanese versions of demand-control questionnaire and effort-reward questionnaire. Using the sex-specific median values, we produced a quadrant scheme with 4 exposure categories, with low job demand and high job control representing a relax job, high job demand and high job control representing an active job, low job demand and low job control representing a passive job, and high job demand and low job control representing 
a strain job. According to Siegrist, we defined a ratio of the scales 'effort' and 'rewards', weighted for number of items where a value $>1$ as the critical 'high cost / low gain' condition.

Univariate analyses showed that depression was prevalent among the lower socioeconomic groups than the counterpart groups. Strain and passive jobs were prevalent among lower socioeconomic groups, whereas effort-reward imbalance tended to be prevalent among higher socioeconomic groups. Multivariate analyses revealed that socioeconomic indices were not associated with depression but the occupational stressors were significantly associated with depression. The association between occupational class and depression appeared to be explained by occupational stress, in particular, low job control.

In conclusion, the current cross-sectional analyses in our Japanese sample did not reveal independent associations between socioeconomic state of interests and depression. Although the two occupational stressors were associated with socioeconomic indices differently, they were consistently associated with depression.

CORRESPONDING AUTHOR: Akizumi Tsutsumi, University of Occupational and Environmental Health, Kitakyushu, 807-8555; tsutsumi@med.uoeh-u.ac.jp

\section{TOP FIFTEEN SCORING POSTER \\ PST-197 \\ THE ASSOCIATION OF WORK STRESS WITH HEART RATE VARIABILITY OVER THE WORKING DAY \\ Gyongyver Salavecz, $\mathrm{MsC},{ }^{1}$ Maria Kopp, $\mathrm{PhD}^{1}$ and Andrew Steptoe, $\mathrm{PhD}^{2}$ \\ ${ }^{1}$ Institute of Behavioral Sciences, Semmelweis University, Budapest, Hungary and ${ }^{2}$ Department of Epidemiology and Public Health, University College London, London, United Kingdom. \\ Background: There is increasing evidence that chronic diseases are associated with work stress. The mechanisms underlying these associations remain porly understood, but disturbances in cardiac autonomic control may be involved. \\ Aims: To investigate associations between work stress, in terms of job strain and job control, and heart rate variability (HRV) over the working day. \\ Methods: Job strain and control was measured by the Whitehall II Study job strain questionnaire, which was developed to test Karasek's job strain hypothesis of the demand-control model. HRV was measured using spectral analysis from Actiheart recordings obtained over the working day from 169 women working in Budapest, Hungary. \\ Results: Linear regression analysis adjusted for age, educational level, BMI, social support, smoking status, alcohol consumption, physical activity, sleep quality, working hours was used to determine the relation between job strain and job control and HRV. We found a significant positive relationship between job control and high frequency to low frequency ratio (HF/LF ratio) adjusted for covariates $(\mathrm{p}<0.05)$ and a positive tendency with high frequency power during work $(\mathrm{p}<0.1)$. Heart rate variability (high frequency power) after work was negatively associated with job strain $(\mathrm{p}<0,05)$. \\ Conclusions: The results suggest that low job control and high job strain may contribute to the development of chronic disease through the dysregulation of highly integrated physiological systems. The findings implicate autonomic deregulation under job strain conditions, providing a powerful explanation of heart disease, as well a potential explanation of other stress-related chronic diseases. However, further studies are needed to explicitly demonstrate how the cardiac regulation measured by HRV serves as the cardiovascular disease pathway for work stress.}


CORRESPONDING AUTHOR: Gyongyver Salavecz, MsC, Semmelweis University, Institute of Behavioral Sciences, Budapest, H-1084; salavecz@gmail.com

\section{PST-198}

GROUP COHESION AND ORGANIZATIONAL COMMITMENT: PROTECTIVE FACTORS FOR NURSE RESIDENTS?

Angela Li, BA, ${ }^{1}$ Sean F. Early, $\mathrm{PhD},{ }^{2}$ Nicole E. Mahrer, BA, ${ }^{1}$ Jessica Klaristenfeld, RN, BSN ${ }^{1}$ and Jeffrey I. Gold, $\mathrm{PhD}^{1}$

${ }^{1}$ Childrens Hospital Los Angeles, Los Angeles, CA and ${ }^{2}$ Versant LLC, Los Angeles, CA.

Background: Nurses are frequently exposed to work-related stressful situations. This often makes it difficult for hospitals to recruit nurses and to retain experienced staff. Current research has found that new nurses may be particularly vulnerable to job stress, and that stress levels among nurses are inversely related to job satisfaction. Purpose: To determine if nurse resident's (NRs) sense of group cohesion and organizational commitment serve as protective factors against the negative effects of preexisting trauma and current stress on job satisfaction, burnout, and fatigue. Methods: A longitudinal study of 178 nurse residents (15 male, 163 female) from Childrens Hospital Los Angeles was conducted across three time points. Levels of preexisting trauma, current stress, compassion satisfaction, burnout, compassion fatigue, job satisfaction, group cohesion and organizational commitment were measured. A range of regression models was developed to examine the relationships between the predictor variables (pre-existing stress and current stress), the moderating variables (group cohesion and organizational commitment), and outcome variables of interest (job satisfaction, fatigue, burnout, and compassion satisfaction). Results: Higher organizational commitment scores provided a 'protective' effect against pre-existing trauma with respect to compassion satisfaction $\left(\mathrm{R}^{2}=.35, \mathrm{p}<.0001\right)$. Group cohesion was found to be a predictor of job satisfaction, and was found to have protective effects for job satisfaction from pre-existing trauma and current stress $\left(\mathrm{R}^{2}=.25, \mathrm{p}<.0001\right)$; it also provided direct 'protection' against burnout, which was affected by current stress $\left(\mathrm{R}^{2}=.24, \mathrm{p}<0.0001\right)$. Pre-existing trauma was a strong predictor of fatigue, and accounted for $50 \%$ of observed variance in fatigue scores with $\left(\mathrm{R}^{2}=.50, \mathrm{p}<0.0001\right)$. Conclusion: Results from the study suggest that group cohesion and organizational commitment serve as protective factors against the negative effects of pre-existing trauma and current stress. The findings have implications for hospital-based interventions structured to address nurse stress, while enhancing group cohesion and organizational commitment.

CORRESPONDING AUTHOR: Jeffrey I. Gold, PhD, Anesthesiology and Critical Care Medicine, Childrens Hospital Los Angeles, Los Angeles, CA, 90027; jgold@chla.usc.edu
PST-199

THE EFFECTS OF EMPOWERING IN-SERVICE TRAINING PROGRAM ON HEALTH CARE PROBLEMS

AMONG NURSE AIDES IN LONG TERM CARE

FACILITIES IN TAIWAN

Mei hsinag Lin, EdD, ${ }^{1}$ Hsiu chin $\mathrm{Hsu}, \mathrm{PhD}^{1}$ and Chin ying Lee, $\mathrm{BSN}^{2}$

${ }^{1}$ Nursing, Chang Gung Institiute of Technology, Tao-yuan, Taiwan and ${ }^{2}$ Nursing, Li Shin Hospital, Tao- yuan, Taiwan.

The purpose of this study was to explore the effects of empowering in-service training program on health care problems among nurse aides in long term care facilities. A non-equivalent control group design was conducted. Purposeful sampling was employed. A total of 104 nurse aides subjects were recruited from Tao-yuan area, and divided to experimental and control groups. The empowering inservice training program involved the curriculum content which include the cognition of workplace difficulties, social interaction and communicates, the pressure manages and refuses technique etc. Both experimental and control groups took pre-test as baseline, and only the experimental group obtained 6-weeks classes regarding to the prevention of smoking. The experimental group took post-test immediately after intervention in order to evaluate the instant effect of teaching programs. Data was analyzed by using descriptive statistics and t-test. The results showed as following: 1 . In the task pressure part, there was a significance difference in experimental group after the empowering in-service training program; 2. In the effect of job satisfaction aspect, the average scores had increased but there was no significant statistical significance after the training program; 3 . From the quality of working life perspective, the average scores had increased but there was no significant statistical significance after the training program, however, there was a significant clinic explanation on the intervention. These results could provider information as supplementary for aboriginal nurse aids in-service in long- term care facilities, and to increase their job satisfaction.

CORRESPONDING AUTHOR: Mei hsinag Lin, EdD, Nursing, Chang Gung Institiute of Technology, Tao-yuan, 333; mhlin@ mail.cgit.edu.tw

\section{PST-200}

THE RELATIONSHIPS OF OBESITY TO JOB STRESS, EATING BEHAVIOR, PHYSICAL ACTIVITY AND SOCIAL SKILLS IN PART-TIMERS IN JAPAN

Tomohiko Muratsubaki, doctoral course, ${ }^{1}$ Tadaaki Tomiie, associate professor, ${ }^{2}$ Jun Tayama, associate professor, ${ }^{3}$ Yuji Sakano, professor $^{2}$ and Shin Fukudo, professor ${ }^{1}$

${ }^{1}$ Behavioral Medicine, Tohoku University Graduate School of Medicine, Sendai, Japan; ${ }^{2}$ School of Psychological Science, Health Sciences University of Hokkaido, Sapporo, Japan and ${ }^{3}$ Mental counseling center for Health \& Community Medicine, Nagasaki University, Nagasaki, Japan. 
Objective: To examine a possible relationship between obesity, job stress, eating behavior, physical activity, and social skill in Japanese part-timers.

Method: Questionnaires on job stress (Brief Job Stress Questionnaire; Shimomitsu et al., 1998), eating behavior (Eating Behavior Scale; Sakata, 1997), self-efficacy in controlling situational appetite (Situational Appetite Measure; Shimai et al., 2000), physical activity (Physical Activity Assessment Scale; Wakui \& Suzuki, 1997), and social skill (W-KiSS-16; Tanaka, 2007) were conducted with 254 part-timers aged $19-72$ years $(46.96 \pm 11.41$ years $)$ in a food industry in Japan. The relation between obesity, job stress, eating behavior, physical activity and social skills were analyzed between 52 obese subjects $(\mathrm{BMI} \geq 25.0 \mathrm{~kg} / \mathrm{m} 2), 185$ normal range subjects $(18.5 \mathrm{~kg} / \mathrm{m} 2 \leq \mathrm{BMI}<25.0 \mathrm{~kg} / \mathrm{m} 2)$, and 17 underweight subjects $(\mathrm{BMI}<18.5 \mathrm{~kg} / \mathrm{m} 2)$. Statistical analyses included correlation analysis and multivariate regression analysis.

Result: In Obese subjects, eating behavior was significantly associated with job stress response of physical stress response $(\beta=.42, \mathrm{p}<.001)$, self-efficacy in controlling situational appetite of reward $(\beta=-.37, p<.01)$, relax $(\beta=-.25, p<.05)$, and physical activity was significantly associated with job stress response of fatigue $(\beta=-.46, p<.001)$. In normal range subjects, eating behavior was significantly associated with job stress response of physical stress response $(\beta=.17, \mathrm{p}<.01)$, self-efficacy in controlling situational appetite of negative feeling $(\beta=-.23, p<.01)$, relax $(\beta=-.30, p<.001)$, and physical activity was significantly associated with job stress factor of qualitative workload $(\beta=.16, \mathrm{p}<.05)$, family and friend support $(\beta=-.19, \mathrm{p}<.05)$.

Conclusion: The present study suggests that obese part-timers tend to be in a stressful state in the workplace. Such stressful conditions may eat much and contribute to obesity. Stress management might be necessary in workplace for prevention and treatment of obesity among part-timers.

CORRESPONDING AUTHOR: Tomohiko Muratsubaki, doctoral course, Behavioral Medicine, Tohoku University Graduate School of Medicine, Sendai, 980-8575; tomo_muratsu@yahoo.co.jp

PST-201

ASSOCIATION BETWEEN THE LEVEL OF FATIGUE ACCUMULATION AND THE WORKING CONDITIONS IN JAPANESE SYSTEM ENGINEERS OF INFORMATION SERVICES Toshiko Niwa, Bachelor, ${ }^{1}$ Chu-Hyang Oh, Master ${ }^{2}$ and Emiko Saito, $\mathrm{PhD}^{2}$ ${ }^{1}$ Tokyo Metropolitan Matsuzawa Hospital, Tokyo, Japan and

${ }^{2}$ Tokyo Metropolitan University, Tokyo, Japan.

Objectives: During six years from 2003 to 2008, the number of employees of information services increased from 567,000 to 858,000 in Japan. Their health problems have increased over the last 10 years. In the information services, it was reported that the stress factors of their work environment was associated with their health than their personal relationships on business and their life event. The purpose of this study was to identify the association between the level of fatigue accumulation and the working conditions in Japanese system engineers of information services.
Methods: We conducted an anonymous self-report questionnaire in 160 system engineers of information services in 2008. A checklist of the level of fatigue accumulation developed by the Ministry of Health, Labor and Welfare in Japan was used. Items of questionnaire were degree of a burden of working hours, work contents, eye strains, sleeping hours and situations of daily life. A description of entry by voluntary agreement was attached to questionnaires. We obtained approval from respondents because of submitting their questionnaires.

Results: Responses were obtained from 120 people (75\%). One hundred $(83 \%)$ people for men and they had most $30 \mathrm{~s}$ in 59 people $(49 \%)$. The number of people with 45 hours or more overtime work in month was 26 people $(22 \%)$. The number of low risk group of fatigue accumulation was 53 people (44\%) and highrisk group was 67 people (56\%). The high risk group had a significantly higher percentage of 60 minutes or more of commuting time, seconded staff and 45 hours or more of overtime hours than low risk group. The high risk group had a significantly higher percentage of the burdens in working hours, amount of work, difficulty of work, time pressure on the appointed date of delivery, troubles after the delivery of the order, communication problems, uncomfortable setting, eyes or neck pain and fatigue. Conclusion: This study revealed that the level of fatigue accumulation of Japanese system engineers related their work environment. It was suggested that nurses in business setting need to approach the level of fatigue accumulation depending on characteristics of the work condition and environment of system engineers.

CORRESPONDING AUTHOR: Emiko Saito, PhD, Tokyo Metropolitan University, Tokyo, 116-8551; saito@hs.tmu.ac.jp

\section{PST-202}

DO (CHANGES IN) WORK CONDITIONS AND HIGHER ORDER GOALS AFFECT QUALITY OF WORK AND WELLBEING AMONG HEALTH CARE EMPLOYEES? Hennie Koelewijn, Drs, ${ }^{1}$ Vera van Mourik, MSc, ${ }^{1}$ Nynke Kuipers, $\mathrm{Drs}^{1}$ and Stan Maes, Prof Dr ${ }^{2}$

${ }^{1}$ PCC Health Promotion, Amersfoort, Netherlands and ${ }^{2}$ Leiden University, Leiden, Netherlands.

Objectives: The aim of this longitudinal study is to evaluate wellness effects of a tailored problem solving process intervention program for health care employees.

Methods: Socio demographic variables, work conditions, higher order goal facilitation as well as health related outcomes were assessed from the experimental group $(\mathrm{N}=461)$ as from the control group ( $\mathrm{N}=246$; Total: $\mathrm{N}=707)$ at a first measurement moment (T1) and a second T2 (3 years later). Regression analyses were used to explore the changes between T1 and T2 and the effects on health related outcome variables.

Results: Generally, the results indicate that positive changes in work conditions and higher order goals significantly affect health and wellbeing of health care employees positively. The effects are stronger in the experimental health care centres compared to the effects in the total sample group. 
Conclusions: These findings suggest that a tailored problem solving process intervention based on motivational regulation techniques, such as goal setting, action planning and training in communication- and cooperation skills positively affect quality of work and wellbeing in health care employees.

CORRESPONDING AUTHOR: Hennie Koelewijn, Drs, PCC Health Promotion, Amersfoort, 3824MP; healthpromotion@ pccnederland.nl

PST-203

OVERCOMMITMENT AS RELATED TO POOR NEXT-DAY RECOVERY AND FATIGUE AMONG WOMEN

Ulrica von Thiele Schwarz, PhD

Department of Psychology, Stockholm university, Stockholm, Sweden.

Recovery from work stress is crucial to avoid stress-related illhealth. The aim of this longitudinal study was to investigate whether processes that prolong the mental exposure to work stressors, e.g. overcommitment (OC), along with psychosocial work conditions, predicts fatigue and poor next-day recovery six months later. 160 women working in dental care completed selfratings in questionnaires at two time points, six months apart. Psychosocial work conditions and OC were used to predict fatigue and poor next-day recovery respectively, in multiple regression analyses. Results show that having children living at home, high job demands and high $\mathrm{OC}$ were associated with fatigue six months later. Job demands and OC, but not having children at home, predicted poor next-day recovery. Sobel's test and bootstrapping procedure indicated that $\mathrm{OC}$ partly mediated the relationship between job demands and fatigue and between job demands and next-day recovery. These findings highlight the importance of considering OC, e.g. inability towithdraw from work, in relation to fatigue and recovery from work and that such factors should be included in interventions targeting workrelated stress.

CORRESPONDING AUTHOR: Ulrica von Thiele Schwarz, PhD, Department of Psychology, Stockholm university, Stockholm, 10691; uvt@psychology.su.se

\section{PST-204a}

RACIAL DISPARITIES IN JOB STRAIN: AN EXPLORATION FROM THE WORK, FAMILY AND HEALTH NETWORK

David Hurtado, MSc, ${ }^{1}$ Karen Ertel, ScD, ${ }^{1}$ Buxton Orfeu, $\mathrm{PhD}^{3,4}$ and Lisa F. Berkman, $\mathrm{PhD}^{2,1}$

${ }^{1}$ Deparment of Society, Human Development and Health, Harvard School of Public Health, Boston, MA; ${ }^{2}$ Harvard Center for Population and Development Studies, Cambridge, MA; ${ }^{3}$ Division of Sleep Medicine, Harvard Medical School, Boston, MA and ${ }^{4}$ Department of Medicine, Brigham \& Women's Hospital, Boston, MA.

Objective: To examine racial disparities in the psychosocial work environment in a sample of Non-Hispanic White (NHW) and Non-Hispanic Black (NHB) Registered Nurses (RN), Licensed
Practical Nurses (LPN) and Certified Nursing Assistants (CNA) from four extended care facilities in Massachusetts.

Methods: Cross-sectional study design. Structured interviews were conducted among 109 NHW and 131 NHB nursing staff between September 2006 and July 2007. Demographic and occupational information was self-reported. We used questions from the Job Content Questionnaire (JCQ) to assess three aspects of the psychosocial work environment: job demands, job control and social support at work. We dichotomized each JCQ measure at the median to create high and low groups. Job strain was defined as the combination of reporting high demands and low control. We first examined racial differences in JCQ measures among all nursing staff. Next we contrasted RN/LPN (38.2\%) vs. CNA (61.2\%). Potential confounders included age, gender, marital status, education, income, occupation, facility and duration at current job.

Results: NHB nursing staff were more likely to report high strain jobs compared to NHW (49.1\% vs. $7.9 \%$, p <0.001). After adjusting for potential confounders, NHB nursing staff were more likely to report high strain, compared to NHW peers (Odds Ratio [OR]: 6.1, 95\% Confidence Interval $[\mathrm{CI}] 2.4-15.1, \mathrm{p}<0.001)$. Stratified analysis showed that NHB CNA were more likely to report job strain than NHW CNA (OR: 12.0, 95\% CI: 3.1-46.1, p<0.001). $\mathrm{RN} / \mathrm{LPN}$ reported less job strain overall (7 out of 85 nurses), limiting the power to detect potential disparities. There were no differences regarding social support at work in either occupation.

Conclusion: NHB nurses, compared to NHW nurses, were six times more likely to report job strain. The effect was higher among CNA. There are several possible reasons for these differences, including confounding by an unmeasured variable such as skill level or differential self-report by race. These differences may also reflect unmeasured organizational and interpersonal behaviors, including discrimination based on race. Further research should consider these alternative explanations for racial disparities in selfreported job strain.

CORRESPONDING AUTHOR: David Hurtado, MSc, Harvard School of Public Health, Cambridge, MA, 02139; hurtado.d@ gmail.com

\section{PST-204b}

A PROJECT FOR PREVENTING SUICIDES : SCREENING FOR DEPRESSION (2)

Reiko Hori, $\mathrm{PhD},{ }^{1}$ Hikari Furui, $\mathrm{PhD}^{2}$ and Fumio Kobayashi, $\mathrm{PhD}^{1}$

${ }^{1}$ Health \& Psychosocial Medicine, Aichi Medical University School of Medicine, Aichi, Japan and ${ }^{2}$ Psychology, Aichi Shukutoku University, Aichi, Japan.

Background and purpose: We have launched the project of screening for depression among workers in local area from 2007. The results of the first year are shown in the other paper, which shows that job involved factors may relate to a depressive mood of workers. The purpose of this year survey is to verify the previous results and examine if job involved factors increase depressive mood. Methods and Results: We performed screening of depression for 428 male and 249 female workers, who work in some small enterprises, during their annual medical checkup 
in 2008. They answered self-reported questionnaire including items screening for depression, living alone or not, work arrangement, working time, job demand, physical loads, job control, friendly work place or not, and job satisfaction. Following the first screening, 92 men and 63 women were determined as highly depressive. Among them, 87 men and 63 women were interviewed using CIDI-SS-RR as a second screening. Finally, 20 men and 12 women were detected as ones to be recommended for medical care. Highly depressive men by the first screening were more likely to live alone $(\mathrm{p}<0.01)$, and had more job demand $(\mathrm{p}<0.05)$, more physical loads $(\mathrm{p}<0.05)$, less friendly work place $(\mathrm{p}<0.05)$, less job satisfaction $(\mathrm{p}<0.01)$ than non-highly depressive men. Highly depressive women by the first screening had more job demand $(\mathrm{p}<0.05)$, less job control $(p<0.05)$, and less job satisfaction $(p<0.05)$ more than non-highly depressive women. Men that were recommended for medical care were more likely to live alone $(\mathrm{p}<0.05)$ and had more physical loads $(\mathrm{p}<0.05)$, less job satisfaction $(\mathrm{p}<0.01)$, and less friendly work place $(\mathrm{p}<0.01)$ than non-highly depressive men by the first screening. Women that were recommended medical care had more job demand $(\mathrm{p}<0.05)$ than non-highly depressive women by the first screening. Depressiveness of 18 men and 14 women increased in 2008. There was the trend to increase in physical loads and decrease in work place friendship or job satisfaction among these men, and that to increase in physical loads and decrease in job control or job satisfaction among these women. Conclusion: These results will support our previous report, in which job involved factors may relate to depressive mood of workers, and these factors may relates to increase depressive mood.

CORRESPONDING AUTHOR: Reiko Hori, PhD, Aichi Medical University School of Medicine, Aichi, 480-1195; rhori@aichimed-u.ac.jp

\section{PST-204c}

THE EFFECTS OF THE MENTAL-ROSAI WEB-BASED MENTAL HEALTH CHECK SYSTEM AMONG JAPANESE MALE EMPLOYEES

Sakurako Ito, $\mathrm{MA},{ }^{1}$ Haruyoshi Yamamoto, $\mathrm{MD}^{1}$ and Akira Tsuda, $\mathrm{PhD}^{2}$

${ }^{1}$ Workers' Mental Health Research Center, Yokohama Rosai Hospital, Yokohama, Japan and ${ }^{2}$ Kurume University, Kurume, Japan.

OBJECTIVES: To examine the effect of web-based mental health check system for workers (MENTAL-ROSAI hereinafter referred to as the "M-R"). M-R provides self-check questionnaires on stress symptoms and work and lifestyle related stressors, and a computer-tailored personal report based on the check results via internet. This study tested the effect of individually computertailored messages designed to maintain mental wellness and reduce work and lifestyle related stressors.
METHODS: Employees of a Japanese IT company were surveyed using M-R at baseline, and 317 males, who were not under treatment for mental illness and having few depressive symptoms $(\mathrm{CES}-\mathrm{D}<16)$, were randomly assigned to Group $\mathrm{C}$ in which the participants receive a simple report containing scores and charts only or Group M in which participants receive individually tailored stress-management messages added to the simple report. The change in stress symptoms and work and lifestyle related stressors were examined on 206 participants who answered both 2- and 4-month follow-up surveys using M-R. At 2-month survey, those participants exhibited higher depressive symptoms (CES-D $\geqq 16$ ) received an additional message from a medical doctor.

RESULTS: At 2 months, maintenance of mental wellness and improvement of lifestyle were observed in $7 \%$ more participants in the Group M, but the difference was not statistically significant. Of those who scored over 16 on CES-D at 2 months and received a doctor's message, the CES-D score significantly decreased at 4 months in Group M only $(\mathrm{p}<.01)$. Somatic stress symptoms also tended to decrease at 2 months in Group $\mathrm{M}$ and it maintained up to 4 months. Significantly more participants in Group M (79\%) than Group C (68\%) maintained mental wellness all through 3 surveys $(\mathrm{p}<.001)$. No significant change in work and lifestyle related stressor was observed.

CONCLUSIONS: Adding tailored messages to the simple report of M-R was more effective in maintaining mental wellness and improving depressive symptoms. However, to support improvement in work or lifestyle related stressors, complementing the concise report of M-R by extending our center's health care services may be needed.

CORRESPONDING AUTHOR: Sakurako Ito, MA, Yokohama Rosai Hospital, Yokohama, 222-0036; tksacra@hotmail.com

\section{PST-204d}

A PROJECT FOR PREVENTING SUICIDES : SCREENING FOR DEPRESSION (1)

Reiko Hori, PhD, ${ }^{1}$ Hikari Furui, Professor, ${ }^{2}$ Misuzu Watanabe, $\mathrm{PhD}^{3}$ and Fumio Kobayashi, Professor ${ }^{1}$

${ }^{1}$ Health \& Psychosocial Medicine, Aichi Medical University School of Medicine, Aichi, Japan; ${ }^{2}$ Psychology, Aichi Shukutoku University, Aichi, Japan and ${ }^{3}$ Institute \& Occupational Health Science, Aichi Medical University, Aichi, Japan.

Background and purpose. More than thirty thousand people commit suicide in Japan every year from 1998. Therefore, preventing suicides is one of important issues to be addressed in Japan. Our prefectural government also launched a project of screening for depression among workers in local area from 2007 in order to prevent suicides. Methods and Results: For the project, we performed screening of depression for 475 male and 284 female workers, who work in some small enterprises, during their annual medical checkup in 2007. They answered self-reported question- 
naire including items screening for depression, living alone or not, work arrangement, working time, job demand, physical loads, job control, friendly work place or not, and job satisfaction. Following the first screening, 203 men and 129 women were determined as highly depressive. Among them, 138 men and 97 women were interviewed using CIDI-SS-RR as a second screening. Finally, 21 men and 22 women were detected as ones to be recommended for medical care. Highly depressive men by the first screening were more likely to live alone $(p<0.05)$, worked longer $(p<0.05)$, and had more job demand $(\mathrm{p}<0.01)$, more physical loads $(\mathrm{p}<0.01)$, less friendly work place $(p<0.01)$, less job satisfaction $(p<0.01)$, and lower job control $(\mathrm{p}<0.05)$ than non-highly depressive men. Highly depressive women by the first screening were younger $(\mathrm{p}<0.05)$ and had more job demand $(\mathrm{p}<0.01)$, more physical loads $(\mathrm{p}<0.05)$, less friendly work place $(\mathrm{p}<0.01)$, and less job satisfaction $(\mathrm{p}<0.01)$ more than non-highly depressive women. Men that were recommended for medical care were more likely to live alone $(\mathrm{p}<0.05)$ and had more job demand $(\mathrm{p}<0.01)$, more physical loads $(\mathrm{p}<0.05)$, less job satisfaction $(\mathrm{p}<0.01)$, and less friendly work place $(\mathrm{p}<0.01)$ than non-highly depressive men in the first screening. Women that were recommended for medical care were younger $(p<0.01)$ and had more job demand $(\mathrm{p}<0.01)$, more physical loads $(\mathrm{p}<0.01)$, and less job satisfaction than non-highly depressive women in the first screening. Conclusion: These results suggest that job involved factors relate to a depressive mood of workers.

CORRESPONDING AUTHOR: Reiko Hori, PhD, Aichi Medical University School of Medicine, Aichi, 480-1195; rhori@, aichi-med-u.ac.jp

\section{PST-204e}

\section{WORK PHYSICAL ENVIRONMENTS TO QUALITY}

OF WORK LIVES: NEGATIVE AND POSITIVE ATTRACTORS FOR HOSPITAL PHYSICIAN EXECUTIVES Blossom Yen-Ju Lin, PhD, ${ }^{1}$ Yung-Kai Lin, MD, MBA, ${ }^{3}$ ChengChieh Lin, MD, PhD, ${ }^{1,2}$ Li-Hua Huang, MS, ${ }^{4}$ Edward Chen, $\mathrm{MS}^{4}$ and Kao-Chi Cheng, $\mathrm{MD}^{2}$

${ }^{1}$ Health Services Administration, China Medical University, Taichung, Taiwan; ${ }^{2}$ Family Medicine, China Medical University Hospital, Taichung, Taiwan; ${ }^{3}$ Division of Cardiovascular Surgery, Taichung Veterans General Hospital, Taichung, Taiwan and ${ }^{4}$ TaiLin Community Pharmacy, Taipei County, Taiwan.

Objectives: In healthcare, architects, interior designers, engineers, and healthcare administrators have paid attention to the construction and design of healthcare facilities. Research is needed to better understand how health professionals and employees perceive their work environment, in order to improve the physical environment in which they work. This study was aimed to examined the negative and positive attractors (designs) in the physical environments of hospital settings and examine their effects on employees' quality of work lives.
Methods: Developed, structured and validated questionnaire was released by mails to hospital physician executives in the period of Aug-Nov 2009 and 737 respondents returned in this study with a response rate of $32.83 \%$. Physical environments in hospital settings included negative attractors of visual, hearing, touch, space, functional, clearance, thermal, private and safety dimensions, and positive attractors of plants, waters, music, arts and exhibitions, private spaces, religious areas, gardens, coffee shops, leisure areas, and so on. Work lives were measured as work and career satisfaction, health status and illness, substance uses, and psychological work and life moods. Personal characteristics and work status of hospital physician executives were also collected. Descriptive analyses, factor analyses, and multiple regressions were performed.

Results: Controlling the personal characteristics and work status of hospital physician executives, it was found that negative touch, noise, safety, and thermal environments were related to worse work and career satisfactions, health, illness symptoms, substance uses, and psychological moods to some extents however, positive attratcors such as window sunshine, plants, art and exhibition, private space, religious areas, coffee shops and leisure areas within hospital settings played important but various effects on employees' quality of work lives.

Conclusions and implications: The designs of physical environments deserve attention in order to create more appropriate and healthier environments for hospital employees. In this study, it revealed that the negative and positive attractors of hospital physical environments were related to quality of work lives. Further research should be devoted to trace more psychological or physiological responses to the physical environment from a longitudinal perspective.

CORRESPONDING AUTHOR: Blossom Yen-Ju Lin, PhD, Health Services Administration, China Medical University, Taichung, 404; yenju1115@hotmail.com

\section{PST-204f}

\section{PSYCHOLOGICAL IMPACT OF EMERGENCY}

\section{AMBULANCE WORK: A 4-MONTHS FOLLOW-UP}

Dália Marcelino, Master, PhD Student, ${ }^{1}$ Maria João Figueiras, $\mathrm{PhD}^{2}$ and Adelaide Claudino, $\mathrm{PhD}^{1}$

${ }^{1}$ Psychology, University of Évora, Évora, Portugal and ${ }^{2}$ Psychology, Instituto Piaget, Almada, Portugal.

Objective: The aim of this study is to investigate what are the predictors of symptomatology associated with critical incidents among emergency ambulance personnel.

Methods: This is a prospective study in which 425 Portuguese emergency ambulance personnel completed a questionnaire at two time points: July 2009 (T1) and November 2009 (T2). The measures were post-traumatic stress disorder (PTSD), peritrau- 
matic dissociation, mental health, subjective health complaints and socio-demographic variables.

Results: The majority of the ambulance workers had experienced critical incidents in their work. Over time there was a decrease in PTSD symptoms and peritraumatic dissociation, an increase of health complaints, and a significant improvement in psychological well-being. However, correlational analyses indicated a negative significant association between symptoms and psychological wellbeing at the two time points. The predictors of post-traumatic stress disorder were traumatic incident-related, peritraumatic dissociation, psychological distress and subjective health complaints at both assessments.

Conclusions: Ambulance personnel are at risk to develop health symptoms due to work related stressors. However, the results suggest that shifts in symptomatology may be associated with the time of the year in which the assessments took place. Further research should explore whether these symptoms increase in peak times of critical incidents over the year.

CORRESPONDING AUTHOR: Dália Marcelino, Master, PhD Student, Psychology, University of Évora, Corroios, 2855-589; daliamarcelino@gmail.com

\section{PST-204g}

WHAT ARE THE CRITICAL ASPECTS OF WORK TIME ARRANGEMENT FOR SLEEP AND INSOMNIA IN SHIFT WORK?

Sampsa Puttonen, $\mathrm{PhD},{ }^{1}$ Katriina Viitasalo, $\mathrm{MD}^{2}$ and Mikko Härmä, MD, professor ${ }^{1}$

${ }^{1}$ Finnish Insitute of Occupational Health, Helsinki, Finland and ${ }^{2}$ Finnair Health Services, Vantaa, Finland.

Poor or insufficient sleep is a risk factor of accidents and several illnesses including cardiovascular diseases. We aimed to clarify aspects of shift arrangements which are important for sleep in shift work.

The participants were 305 (248 men) aged 25-60 yrs. shift workers in a Finnish airline company. Sleep length, insomnia symptoms and work arrangements were self-rated by the participants. The considered aspects of shifts were: frequency of night shifts, the number of consecutive night shifts, mean and the maximum shift length, frequency of shifts starting before 06:00, quick returns (less than 11 hours between shifts), less than 28 hours of free time after the last nigh shift, a single free day between shift periods, and the regularity of shift system with a question on the direction of rotation (backward vs. forward). Sex and age were included as covariates in all analyses and additional adjustments were done for education and health habits (alcohol use, physical activity, and smoking).
The number of years in shift work and possibility to influence the shift schedule were significant predictors of insomnia $(\mathrm{p}<.001)$ but were unrelated to sleep length. Insomnia was related to a high number of consecutive night shifts $(\mathrm{p}=.006)$, less than 28 hours of free time after the last nigh shift $(\mathrm{p}=.013)$, and backward rotating shift system $(p=.019)$. Shorter sleep was associated with early starting morning shifts $(p=.030)$ and a high number of single free days between two shift periods $(p=.047)$ and marginally with the maximum length of shift $(\mathrm{p}=.067)$. Quick returns and early morning shifts were marginally significantly associated with insomnia (p-values .091, .069). Additional adjustments for education and life habits did not change the Results: The present results suggest that the sleep of shift workers would benefit from employee control over working times and shift systems that properly take into account the extra need for recovery in shift work.

CORRESPONDING AUTHOR: Sampsa Puttonen, PhD, Finnish Insitute of Occupational Health, Helsinki, 00250; sampsa.puttonen@ ttl.fi

\section{PST-204h}

RELIABILITY AND VALIDITY OF THE JAPANESETRANSLATED SWEDISH DEMAND-CONTROL-SUPPORT QUESTIONNAIRE

Junji Mase, phD, ${ }^{1}$ Atsuhiko Ota, $\mathrm{phD},{ }^{1}$ Ken Inoue, $\mathrm{phD},{ }^{1}$ Tadayuki Iida, $\mathrm{phD},{ }^{1}$ Akizumi Tsutsumi, $\mathrm{phD}^{2}$ and Yuichiro Ono, $\mathrm{phD}^{1}$

${ }^{1}$ Department of Public Health, Fujita Health University School of Medicine, Toyoake, Japan and ${ }^{2}$ Occurational Health Training Center, University of Ocuupational and Environmental Health, Kitakyushu, Japan.

Objectives: Karasek's Job Content Questionnaire (JCQ) has been adopted to assess psychosocial work environment according to the Demand-Control-Support (DCS) model. Meanwhile, for instance, the Demand-Control-Support Questionnaire (DCSQ) is also used in Scandinavian countries. We translated it into Japanese, i.e., the Japanese-translated DCSQ (J-DCSQ), and investigated its reliability and validity.

Methods: The subjects were volunteer 239 workers in 19 nursery schools in Japan. Psychological demands (PD: calculated with 5 items), decision latitude (DL: 6 items), and social support (SS: 6 items) were assessed with the J-DCSQ. One-week test-retest reliability and internal consistency were estimated by means of intra-class correlation coefficient (ICC) and Cronbach's alpha, respectively. Concurrent validity was evaluated with Pearson's correlation coefficient for which the Japanese-version JCQ (J-JCQ) was used as the reference. Factorial validity was evaluated with factor analysis.

Results: ICCs were $0.80,0.83$, and 0.81 for PD, DL, and SS, respectively. Correspondingly, Cronbach's coefficient alphas and 
Pearson's correlation coefficients were $0.65,0.63,0.87$ and 0.72 , $0.61,0.57$ for PD, DL, SS. Factor analysis suggested a 4-factor construction which explained the variance as much as $50 \%$. The first factor was constructed of 4 DL items. The second factor included the whole of SS items. The third factor was composed of 3 PD items and 2 DL items. The last factor was constituted of 3 DL items and 1 PD item.

Conclusions: The J-DCSQ exhibited as equivalent reliability as the J-JCQ. The present findings suggested adequate concurrent validity of the J-DCSQ. Factor analysis did not present factor structure of the J-DCSQ as theoretically hypothesized. Previous studies also reported similar factor structure of the J-JCQ. Further studies are needed to better identify psychosocial properties among larger samples.

CORRESPONDING AUTHOR: Junji Mase, phD, Public Health, mase@fujita-hu.ac.jp, Toyoake, 470-1192; mase@fujita-hu.ac.jp

\section{PST-204i}

ARE THE EFFECTS OF LONG WORKING HOURS

ON FATIGUE AND DEPRESSIVE SYMPTOMS VARIED AMONG DIFFERENT INDUSTRIES OR OCCUPATIONS? Yasumasa Otsuka, PhD, ${ }^{1}$ Kenji Iwasaki, PhD, ${ }^{2}$ Takeshi Sasaki, M $\mathrm{Sc},{ }^{2}$ Masaya Takahashi, $\mathrm{PhD},{ }^{2}$ Tomohide Kubo, $\mathrm{PhD},{ }^{2}$ Ippei Mori, $\mathrm{M} \mathrm{D}^{3}$ and Masashi Hori, $\mathrm{PhD}^{4}$

${ }^{1}$ Graduate School of Education, Hiroshima University, Higashihiroshima, Japan; ${ }^{2}$ National Institute of Occupational Safety and Health, Kawasaki, Japan; ${ }^{3}$ The Institute for Science of Labour, Kawasaki, Japan and ${ }^{4}$ Center for the Advancement of Higher Education, Tohoku University, Sendai, Japan.

Although previous studies revealed that long working hours predicted fatigue and depressive symptoms, little is known about how these relationships vary among different industries or occupations. This study examined the effect of working hours on fatigue and depressive symptoms among particular industries and occupations. Longitudinal data on 1,588 Japanese daytime workers (575 women and 1,013 men) were used to estimate the effect of working hours on fatigue and depressive symptoms. Working hours per week at baseline was divided into three categories: "less than 60 h" $(\mathrm{N}=1,346,84.8 \%)$, "61-65 h" $(\mathrm{N}=87,5.5 \%)$, and "66 h or more" $(\mathrm{N}=155,9.8 \%)$. Participants completed the SelfDiagnosis Check List for Assessment of Worker's Accumulated Fatigue developed by the Japan Ministry of Health, Labour, and Welfare, and the Center for Epidemiologic Studies Depression Scale at 1-year follow-up. Industries were divided into 13 categories based on the International Standard Industrial Classification of All Economic Activities, Rev. 4 by the United Nations. Occupations were divided into 6 categories based on the International Standard Classification of Occupations- 88 by the International Labour Office. Analyses of variance were carried out separately for industries and occupations. Multiple comparisons with Bonferroni method indicated that in the manufacturing industry and professionals, the " $66 \mathrm{~h}$ or more" group had a higher level of fatigue than the "less than $60 \mathrm{~h}$ " group $(\mathrm{P}<0.05)$. In the human health and social work activities industry, the "61-65 h" and " $66 \mathrm{~h}$ or more" groups had a higher level of fatigue than the "less than 60 h" group $(\mathrm{P}<0.05)$. No significant association was found between working hours and depressive symptoms in any industry or occupation. Although reducing the number of working hours is essential to ameliorate workers' fatigue, special attention should be paid to workers in manufacturing or human health and social work activities industries and professionals who work long hours. Especially, workers employed in the human health and social work activities industry who worked at least 61 hours per week, showed higher scores of fatigue. This suggests that the association between working hours and fatigue is varied among different industrial sectors.

CORRESPONDING AUTHOR: Yasumasa Otsuka, PhD, Graduate School of Education, Hiroshima University, Higashi-hiroshima, 739-8524; yasumasa-otsuka@hiroshima-u.ac.jp

PST-204j

ARE GENERAL MENTAL ABILITY AND PSYCHOSOCIAL WORK CHARACTERISTICS RELATED TO MID-LIFE HEALTH IN WORKING WOMEN AND MEN?

Cornelia Wulff, PhD student, ${ }^{1}$ Petra Lindfors, Ass professor ${ }^{1}$ and Magnus Sverke, professor ${ }^{1,2}$

${ }^{1}$ Stockholm University, Psychology, Stockholm, Sweden and ${ }^{2}$ WorkWell: Research Unit for People, Policy and Performance, North-West University, North-West, South Africa.

Psychosocial work conditions including high demands, lack of control and support have been linked to poor health. Yet, the influence of individual factors such as general mental ability (GMA) remains to be examined. The present study set out to investigate how childhood mental ability and psychosocial work characteristics relate to different health indicators in a cohort of working women( $(n=271)$ and men $(n=291)$. Specifically, childhood GMA and self-reports of job demands, job control and social support were linked to two positive health indicators (sense of coherence and self-rated health) and two negative health indicators (musculoskeletal problems and anxiety in midlife). Considering the gendered labor market and variations in health patterns between women and men, gender specific analyses were performed. Results revealed no linkages between childhood GMA and the health indicators included. Further, there were no significant interactions between GMA and the psychosocial factors. The overall impact of occupational level was low and controlling for occupational level did not change the results much. These findings are likely to result from the study cohort being 
fairly homogeneous and the women and men being in good health.

CORRESPONDING AUTHOR: Cornelia Wulff, PhD student, Psychology, Stockholm University, Stockolm, 10691; cwf@ psychology.su.se

\section{PST-204k}

\section{EARLY SYMPTOMS OF MENTAL HEALTH PROBLEMS} IN EMPLOYEES AND THEIR SUPPORT NEEDS Mayo Suzuki, $\mathrm{PhD},{ }^{1}$ Suguru Okubo, $\mathrm{PhD},{ }^{2}$ Iori Tani, $\mathrm{PhD},{ }^{3}$ Wakaha Ikeda, $\mathrm{PhD},{ }^{4}$ Kazuhito Yokoyama, $\mathrm{PhD}^{4}$ and Fumihiko Kitamura, $\mathrm{PhD}^{4}$

${ }^{1}$ Bunri University of Hospitality, Sayama-shi, Japan; ${ }^{2}$ Graduate School of Medicine, The University of Tokyo, Bunkyo-ku, Japan; ${ }^{3}$ Hamamatsu University School of Medicine, Hamamatsu-shi, Japan and ${ }^{4}$ untendo University Faculty of Medicine, Bunkyo-ku, Japan.

Background: Mental health problems of employees have become a vital issue. More and more employees have taken sick leave due to mental health problems and have been diagnosed with depression, anxiety disorders and so on. Many employers have implemented mental health care programs or employed mental healthcare professionals. Early detection of and early intervention in mental health problems are expected to alleviate employees' mental health problems and improve the productivity of companies.

Purpose: The purpose of this study is to show the early symptoms of mental health problems in employees and the support needs perceived by employees with mental health problems, and to consider preventive measures in regard to the mental health of employees.

Method: We conducted research of industrial physicians and medical staff into typical patterns of the initial symptoms of mental health disorders in the workplace. We also conducted research of employees with mental health problems in regard to support they wished they had. This research was approved by the Institutional Review Board of Mie University, Faculty of Medicine.

Results and Conclusions: The most common early symptom in the typical patterns for initial symptoms of mental health disorders in the workplace was work absence, followed by a decrease in work efficiency and insomnia. In bringing together the results of our survey of employees with mental health problems as to 'what support they wished they had', we found the content of that desired support to differ according the timing of the employee's situation. They were the preventive stage, the stage where the person does not know about their disorder and the stage where the person knows about their disorder. The results of our research showed us one direction we can take toward guidelines regarding preventive measures for the mental health of employees.
CORRESPONDING AUTHOR: Mayo Suzuki, PhD, Bunri University of Hospitality, Sayama-shi, 350-1336; mayo-suzuki@ bunri-c.ac.jp

PST-2041

WORK STRESS TO QUALITY OF WORK LIVES: MODERATING ROLES OF LABOR ACTIVITIES FOR HOSPITAL PHYSICIAN EXECUTIVES

Cheng-Chieh Lin, MD, PhD, ${ }^{1,2}$ Yung-Kai Lin, MD, MBA, ${ }^{3}$ Li-Hua Huang, MS, ${ }^{4}$ Edward Chen, $\mathrm{MS}^{4}$ and Blossom Yen-Ju Lin, $\mathrm{PhD}^{1}$

${ }^{1}$ Health Services Administration, China Medical University, Taichung, Taiwan; ${ }^{2}$ Family Medicine, China Medical University Hospital, Taichung, Taiwan; ${ }^{3}$ Division of Cardiovascular Surgery, Taichung Veterans General Hospital, Taichung, Taiwan and ${ }^{4}$ Tai-Lin Community Pharmacy, Taipei County, Taiwan.

Objectives: Few studies have been devoted to exploring hospital physicians' work stresses to their quality of work lives. This study was aimed to explore the relationship between work stress and the quality of work lives, especially under the circumstances of given work stress, the moderating effects of labor activities on the work stress and quality of work lives.

Methods: Developed, structured and validated questionnaire was released by mails to hospital physician executives in the period of Aug-Nov 2009 and 737 respondents returned in this study with a response rate of $32.83 \%$. Measures included work stressors as financial-oriented and norm-oriented, and work lives as work and career satisfaction, health status and illness, and substance uses. Labor activities were measured as body labors and leisure activities. Personal characteristics and work status of hospital physician executives were also collected. Descriptive analyses, factor analyses, and multiple regressions were performed.

Results: Controlling the personal characteristics and work status of hospital physician executives, it was found that perceived financial-oriented and norm-oriented stresses by hospital physician executives had lower work satisfaction and career satisfaction, more illness symptoms, and worse perceived health status; however, more labor activities had positive effects on quality of work lives. Especially, body labors could moderate financialoriented stress to better career and work satisfaction, better perceived health status, less muscular illness, and reduced sleeping disorders. Also, leisure activities moderated norm-oriented stress to reduced nose and throat illness and moderated financialoriented stress to higher alcohol assumptions.

Conclusions and implications: We verified the negative relationships of work stress to quality of work lives for hospital physician executives. Moreover, labor activities played important roles in moderating the work stress to work lives. Under the given work stresses in the health care work environments, hospital administrators could recognize the values of body labors and leisure activities into health promotion programs for their health care professionals to create healthy work environments. 
CORRESPONDING AUTHOR: Blossom Yen-Ju Lin, PhD, Health Services Administration, China Medical University, Taichung,404; yenju1115@hotmail.com

\section{Friday \\ August 6, 2010 \\ SYMPOSIA SESSIONS 8:30 AM-10:00 AM}

\section{SS12}

\section{CARDIOVASCULAR REGULATION, REACTIVITY,} AND RECOVERY

Martti T. Tuomisto, PhD, ${ }^{1}$ Marc Gellman, $\mathrm{PhD},{ }^{2}$ Mark Hamer, $\mathrm{PhD}^{3}$ and Ydwine J. Zanstra, $\mathrm{PhD}^{4}$

${ }^{1}$ Department of Psychology, University of Tampere, Tampereen yliopisto, Finland; ${ }^{2}$ Behavioral Medicine Research Center, University of Miami, Miami, FL; ${ }^{3}$ Department of Epidemiology and Public Health, University College London, London, United Kingdom and ${ }^{4}$ Psychiatry, University of Pittsburgh, Pittsburgh, PA.

Cardiovascular reactivity has been intensively studied for some decades as a potential risk factor or risk marker of cardiovascular disorders and diseases. Among other end-points hypertension, coronary heart disease, and stroke have been possible to predict in research using reactivity measures. However, these predictions have not always been successful. Progress needs to be made. One of the possibilities is using cardiovascular recovery in addition to reactivity in the prediction of pathological processes of hemodynamics and neuroendocrinology as well as direct disorders and diseases. New steps towards applying the research results in clinical practice are necessary to realize the promises of cardiovascular behavioral research. This symposium is one step in this direction.

In this symposium researchers will present papers regarding different aspects of cardiovascular regulation, reactivity, and recovery. The symposium starts with Dr Gellman who has comprehensively studied the relationship among hostility, metabolic syndrome, and cardiac structure \& function. Dr. Gellman's research is relevant for many of the most common life style factors. The second paper will be Dr. Hamer's presentation regarding psychophysiological responses and their relationship with sub-clinical coronary heart disease. This topic will help particularly in the planning of coronary heart disease prevention. Third, Dr. Tuomisto will illuminate different patterns of cardiovascular reactivity and recovery in intra-arterial blood pressure of normotensive and hypertensive individuals. The results may promote research in the prediction of cardiovascular risks. The last presentation will be Dr. Zanstra's study of challenge appraisals' role in the prediction of a myocardial reaction pattern in anticipation of and reactivity to a naturalistic stressor. The study has interest especially for those who do research in real world circumstances. All these presentations will have both indirect and direct relevance for clinical research in the prediction and prevention of cardiovascular diseases in addition to more basic theoretical issues.

CORRESPONDING AUTHOR: Martti T. Tuomisto, PhD, Department of Psychology, University of Tampere, Tampere, FI-33014; martti.tuomisto@uta.fi

SS12a

RELATIONSHIPS AMONG HOSTILITY, METABOLIC SYNDROME, AND CARDIAC STRUCTURE \& FUNCTION Marc Gellman, PhD, P. Wachowiak, PhD, M. Gutt, PhD, M. Llabre, PhD, R. Goldberg, $\mathrm{PhD}$ and N. Schneiderman, $\mathrm{PhD}$

Behavioral Medicine Research Center, University of Miami, Miami, FL.

Risk factors associated with Metabolic Syndrome (MetS) have been implicated in cardiovascular disease (CVD). The present study examined associations between cynical hostility (CynHo), MetS factors, and cardiac structure and function in $186(81.7 \%$ Hispanic) post-myocardial infarction (MI) patients (69.4\% men) with a mean age of $53.3(\mathrm{SD}=8.8)$ years. Structural equation modeling (SEM) was used to test a model of MetS. Primary MetS variables included waist circumference (WC), insulin resistance (HOMA-IR), glucose area under the curve (G-AUC), triglycerides (TRIG), high-density lipoprotein cholesterol (HDL-C), and diastolic blood pressures (DBP). Secondary MetS variables included plasminogen activator inhibitor-1 (PAI-1) and a latent inflammation variable comprised of C-reactive protein and interleukin-6. Cardiac function variables were fractional shortening (FS), a measure of cardiac contractility, E/A ratio, a measure of cardiac compliance, and rate-pressure product (RPP), a measure of myocardial oxygen demand. Cardiac structure variables were left ventricular mass index, left ventricular posterior wall thickness at diastole, and interventricular septal thickness at diastole, which together constituted the latent variable cardiac mass $(\mathrm{CM})$. The final structural model had good model fit $(\chi 2(102)=100.65, p=$ $0.52, \mathrm{CFI}=1.00, \mathrm{RMSEA}=0.00$, and $\mathrm{SRMR}=0.04)$. Direct paths were supported between WC and CM and all MetS factors except TRIG and G-AUC. WC was indirectly associated with DBP via CM. WC demonstrated indirect relationships with the cardiac function measures. The model supported positive direct paths between HOMA-IR and G-AUC, TRIG, and PAI-1, but not inflammation or HDL-C. With respect to cardiac function, HOMA-IR demonstrated a direct positive association with RPP and direct inverse associations with FS and E/A ratio. CynHo had a direct positive relationship with HOMA-IR but none with WC. The relationships observed suggest that central adiposity and IR play a primary role in cardiac structural and functional impair- 
ment. The findings suggest that CynHo could promote the progression of metabolic dysfunction and cardiac disease via factors that influence the efficiency of glucose metabolism.

CORRESPONDING AUTHOR: Martti T. Tuomisto, PhD, Department of Psychology, University of Tampere, Tampere, FI-33014; martti.tuomisto@uta.fi

\section{$\mathrm{SS} 12 \mathrm{~b}$}

\section{PSYCHOPHYSIOLOGICAL RESPONSES}

\section{AND SUB-CLINICAL CORONARY HEART DISEASE}

Mark Hamer, PhD and A. Steptoe, DPhil

Department of Epidemiology and Public Health, University

College London, London, United Kingdom.

Acute psychophysiological stress testing, involving measurement of cardiovascular and biological responses to laboratory-induced mental stress, is an important tool to investigate mechanisms that might account for the association between psychosocial stress and cardiovascular diseases (CVD). The present study was designed to examine associations of disturbed psychophysiological responses with sub-clinical atherosclerosis. Participants were 543 healthy men and women (mean age $=62.9 \pm 5.7 \mathrm{yrs}$ ), without history or objective signs of CVD, drawn from the Whitehall II epidemiological cohort. Various cardiovascular (beat-to-beat blood pressure, heart rate variability) and biological measures (inflammatory and haemostatic markers, salivary cortisol) were assessed during and following mental stressors, consisting of a 5-min Stroop task and a 5-min mirror tracing task. Coronary artery calcium (CAC), a measure of subclinical coronary atherosclerosis, was measured using electron beam computed tomography. The tasks induced significant increases in blood pressure, cortisol, interleukin-6, and fibrinogen. Cortisol responders were at higher risk of having clinically relevant levels of CAC (odds ratio $=2.17,95 \%$ CI, 1.37 - 3.41) after adjustments for age, gender, baseline cortisol, social status, depressive symptoms, and conventional risk factors. Cardiovascular responses were not associated with CAC. Basal levels of inflammatory markers were associated with $\mathrm{CAC}$ although the stress responses were not. These data support the notion that cortisol stress reactivity, an index of hypothalamic pituitary adrenal function, is one of the possible mechanisms through which psychosocial stress may influence the risk of CHD.

CORRESPONDING AUTHOR: Martti T. Tuomisto, PhD, Department of Psychology, University of Tampere, Tampere, FI33014; martti.tuomisto@uta.fi
$\mathrm{SS} 12 \mathrm{c}$

REACTIVITY AND RECOVERY OF SYSTOLIC AND DIASTOLIC BLOOD PRESSURE AND HEART RATE IN BEHAVIORAL STRESS

Martti T. Tuomisto, $\mathrm{PhD},{ }^{1,2}$ J. Ollikainen, $\mathrm{MSc}^{3}$ S. Majahalme, $\mathrm{MD},{ }^{4} \mathrm{M}$. Kähönen, $\mathrm{MD}^{5,6}$ and $\mathrm{V}$. Turjanmaa, $\mathrm{MD}^{5,6}$

${ }^{1}$ Department of Psychology, University of Tampere, Tampere, Finland; ${ }^{2}$ Department of Psychiatry, Tampere University Hospital, Tampere, Finland; ${ }^{3}$ Department of Mathematics and Statistics, University of Tampere, Tampere, Finland; ${ }^{4}$ Appleton Heart Institute, Appleton, WI; ${ }^{5}$ Department of Clinical Physiology. Medical School, University of Tampere, Tampere, Finland and ${ }^{6}$ Department of Clinical Physiology, Tampere University Hospital, Tampere, Finland.

Cardiovascular reactivity to behavioral stress tasks has been shown to be associated with pathological hemodynamic and neuroendocrinological processes. Later, recovery from such stress has been studied for similar reasons. Our purpose was to study cardiovascular reactivity from a baseline condition, a relaxation to behavioral stress tasks and recovery from the tasks to a relaxation condition after the tasks. As data, we used the Tampere Ambulatory Hypertension Study data bank of intraarterial blood pressure (BP) from the 1990's. The participants were 95 newly detected, WHO-classified, age-matched normotensive $(\mathrm{NT} ; \mathrm{n}=33)$, borderline hypertensive $(\mathrm{BHT} ; \mathrm{n}=30)$ and hypertensive (HT; $n=32)$ men recruited through routine health examinations. They underwent a 5-minute cue-controlled relaxation (R1) followed by 8 standardized behavioral challenges: a video game, arithmetic, social problem solving, imagery, a habituation test, a comic film, and the cold pressor test. After the tasks, a 10-minute relaxation period (R2) was started. Systolic and diastolic BP (SBP, DBP) and heart rate (HR) and were analyzed. First, in a mixed-design ANOVA, Time factor, R1 (the reactivity baseline) and R2 (the recovery baseline) was studied together with the groups. The Time $\mathrm{x}$ Group interactions did not differ for SBP, DBP and HR: Fs $(2,92)<1.04$. However, the direction of recovery was different for BPs and HR. During the recovery baseline, HR was lower than during the reactivity baseline. The reverse was true for both SBP and DBP. Second, $\mathrm{R} 2$ variables were compared in ANCOVA using R1 variables as covariates. Following main effects were obtained: $\mathrm{SBP}, \mathrm{F}(2,91)=$ $3.14, \mathrm{p}<.05$ and DBP, $\mathrm{F}(2,91)=5.98, \mathrm{p}<.005$. In Bonferroni controlled group contrasts, the recovery baseline measures showed differences between the hypertensive groups in DBP $(\mathrm{p}<.05,95 \%$ confidence interval $=0.8-6.59)$ while reactivity measures had shown DBP differences between these groups only in the cold pressor test. In conclusion, intra-arterial BP and 
HR in NT and newly detected HT groups showed different patterns in reactivity respective recovery. These results are in accordance of some earlier research and show that optimizing the use of cardiovascular data requires that these different patterns are taken into account in the predictions of health risks.

CORRESPONDING AUTHOR: Martti T. Tuomisto, PhD, Department of Psychology, University of Tampere, Tampere, FI33014; martti.tuomisto@uta.fi

\section{SS12d}

CHALLENGE APPRAISALS PREDICT A MYOCARDIAL REACTION PATTERN IN ANTICIPATION OF AND REACTIVITY TO A NATURALISTIC STRESSOR

Ydwine J. Zanstra, $\mathrm{PhD}^{1}$ and D. W. Johnston, DPhil ${ }^{2}$

${ }^{1}$ Psychiatry, University of Pittsburgh, Pittsburgh, PA and ${ }^{2}$ Psychology, University of Aberdeen, Aberdeen, United Kingdom.

The current study examined the changes in parameters underlying blood pressure in relation to anticipation, reactivity and recovery to stress. Further, it was examined how these vascular and myocardial parameters relate to cognitive appraisal in a real-life stressful situation. 24 men aged 19-28 (mean age: 23.5; SD: 2.5) participated in this study. Ambulatory blood pressure was recorded on a beat-to-beat basis before and during performance of a real life speech. Vascular and myocardial hemodynamic parameters (total peripheral resistance (TPR) and cardiac output (CO), respectively) were derived from the blood pressure waveform. Participants' appraisals were obtained prior to the stressor. Evidence was obtained suggesting a myocardial reaction pattern during the stressor. This finding is consistent with the hypotheses as the stressor is an active coping task. The anticipation was hypothesized to be a relatively passive situation and therefore expected to engender a vascular response. However, the reaction pattern during anticipation was myocardial. Finally, recovery was slower in the vascular than in the myocardial variables. Multilevel regression models (MLwiN v2.2) were used to analyze the relationships between appraisal and CO and TPR. During the anticipation period prior to the stressor, increased challenge was associated with decreased vascular resistance $(Z=2.70 \mathrm{p}<.01)$ and increased myocardial reactivity $(Z=2.43 \mathrm{p}<.01)$. During the stressor increases in challenge were associated with further increases in myocardial responding $(Z=2.03 \mathrm{p}<.05)$ but relationships between appraisal and vascular resistance were not significant $(\mathrm{Z}=1.82$, n.s.). This study is among the first to examine the hemodynamic reactivity and recovery patterns to a real life stressor in conjunction with stressor appraisals. Furthermore, our findings suggest that challenge appraisals to real-life stressors contribute to an, arguably healthier, myocardial reaction pattern. This ambulatory study was followed up by a study aimed at changing appraisals and appraisal related hemodynamic reactivity patterns.
CORRESPONDING AUTHOR: Martti T. Tuomisto, PhD, Department of Psychology, University of Tampere, Tampere, FI33014; martti.tuomisto@uta.fi

SS30

HARNESSING IMAGERY PROCESSES TO MOTIVATE HEALTH BEHAVIORS

Linda D. Cameron, $\mathrm{PhD},{ }^{1}$ Gerry Humphris, $\mathrm{PhD},{ }^{2}$ Gareth Hollands, $\mathrm{PhD},{ }^{3}$ Susan Persky, $\mathrm{PhD}^{5}$ and Brian Williams, $\mathrm{PhD}^{4}$

${ }^{1}$ The University of Auckland, Auckland, New Zealand; ${ }^{2}$ University of St. Andrews, St Andrews, United Kingdom; ${ }^{3}$ King's College London, London, United Kingdom; ${ }^{4}$ University of Dundee, Dundee, United Kingdom and ${ }^{5}$ National Human Genome Research Institute, Bethesda, MD.

With advances in visual media technologies, health communications and interventions can now incorporate a variety of sophisticated images and pictures. Optimal use of these technologies requires that we advance our understanding of how individuals respond to health imagery, particularly since information processes for imagery differ markedly from those involved with linguistic information. This symposium reviews recent research on techniques using visual images to motivate behavior change. The speakers represent multidisciplinary teams of experts in psychology, sociology, communications, graphics, and information technology. Gerry Humphris will present research on using graphic warning labels on cigarette packets to motivate smoking cessation. Using data from a large community survey, his team found that images evoking disgust responses are potent triggers of motivations to quit. Gareth Hollands will present research on an intervention in which aversive images are used to condition responses that reduce motivations to eat unhealthy foods. This technique, based on implicit cognition and evaluative conditioning principles, altered implicit attitudes about foods and increased healthy food choices. Susan Persky will present research in which immersive virtual environment (IVE) technologies are used to motivate protective behavior. The studies demonstrate how IVEs can instill experiential knowledge of behavioral consequences and enhance understanding of complex concepts such as genebehavior interactions. Brian Williams will present research on a framework for developing theoretically-guided interventions involving visual media. The four-stage process was tested in the development of an intervention incorporating 3D animation about links between obesity and arteriosclerosis. Throughout the symposium, discussion will address how the research informs theory on visual imagery processes in health behavior as well as the use of imagery in interventions.

CORRESPONDING AUTHOR: Linda D. Cameron, PhD, Psychology, The University of Auckland, Auckland, 1042; 1.cameron@, auckland.ac.nz 


\section{CITATION ABSTRACT \\ SS30a \\ GETTING GRAPHIC: EMBEDDING BEHAVIOURAL THEORY IN ANIMATION \\ TO FACILITATE BEHAVIOUR CHANGE}

Brian Williams, BSc, PhD, Annie Anderson, BSc, PhD and Karen Barton, BSc

Social Dimensions of Health Institute, University of Dundee, Dundee, United Kingdom.

Background: Health promotion interventions frequently employ visual images to influence

knowledge, beliefs, attitudes, and thus behaviour. However, such interventions are often highly

pragmatic and lack clear theoretical underpinning. The stages of development are often also

poorly documented.

Objectives: To develop a visual intervention informed by behavioural theory and concepts in order to increase understanding of arteriosclerosis and intentions to behaviour change.

Methods: A multidisciplinary group applied the first two stages of the MRC Framework for the

Development and Evaluation of Complex Interventions. This was operationalised in 4 subphases.

1. Establishing a theoretical basis: the creation of conceptual content

2. Establishing structure: creating a visual narrative

3. Establishing the "look": visual rendering of narrative and concepts

4. Establishing interpretation and impact: creating evidence

Initial testing of the animation included in-depth interviews with 15 patients and 5 health

professionals. Understanding of the animation was explored, followed by emotional reaction,

acceptability and suggestions for improvement. Interviews were recorded, transcribed and

analysed using the "Framework" method.

Results: Phases 1 to 3 established an animation of the body and heart that sought to increase knowledge, perceived severity, susceptibility, self-efficacy and coherence. In phase 4 testing all but one participant provided an accurate and unprompted interpretation of the animation. Increases in knowledge, perceived susceptibility, self-efficacy and coherence were reported. Numerous participants indicated that the animation moved illness representations from the abstract to the concrete. An increase in anxiety and worry was reported but regarded as acceptable by all participants.

Conclusions: We have established a detailed stepwise process to develop and document the creation of visual-based, theory-informed behavioural interventions. The digital animation appears acceptable to staff and patients, and to influence intended behavioural concepts.

CORRESPONDING AUTHOR: Brian Williams, BSc, PhD, Social Dimensions of Health Institute, University of Dundee, Dundee, DD1 4SY; b.y.williams@cpse.dundee.ac.uk

\section{SS30b}

USING IMAGES OF THE BODY IN AN EVALUATIVE CONDITIONING INTERVENTION TO ENHANCE HEALTHY FOOD PREFERENCES

Gareth J. Hollands, PhD and Theresa M. Marteau, PhD

Psychology Department, King's College London, London, United Kingdom.

Background: A growing body of research suggests that communicating associations between aversive health-related images and a given behaviour may diminish the likelihood of that behaviour. However, there is limited experimental evidence of this relationship. The current study examines the behavioural and attitudinal effects of communicating images of energy-dense snacks, paired with images of the body indicative of health risk.
Methods: Participants $(n=132)$ were randomly allocated to either a conditioning procedure pairing images of snack foods with images related to cardiovascular disease, or a control condition featuring images of snack foods alone. They completed a measure of implicit attitudes (pre and post-intervention) and two behavioural choice tests in which they chose between fruit or snack products (post-intervention).

Results: The conditioning intervention made implicit attitudes towards energy-dense snacks more negative $(\mathrm{F}(1,129)=4.666$, $\mathrm{p}=.033, \mathrm{r}=.19$ ) with this effect greater for those with relatively more favourable implicit attitudes towards these snacks preintervention $(\mathrm{F}(1,63)=12.717, \mathrm{p}=.001, \mathrm{r}=.39)$. Participants who received the conditioning intervention showed a significantly greater preference for fruit over snacks than controls on the tests of behavioural choice $(\mathrm{t}(131)=-4.854, \mathrm{p}<.001, \mathrm{r}=.39)$. Changes in implicit attitudes partially mediated the effects of the intervention on these tests $(\beta=.06, p=.027)$.

Conclusion: Presenting aversive images of the body with those of specific foodstuffs can change implicit attitudes and impact on food choice. 
CORRESPONDING AUTHOR: Gareth J. Hollands, PhD, King's College London, London, SE1 9RT; gareth.hollands@kcl.ac.uk

\section{SS30c}

HEIGHTENING THE IMPACT OF IMAGERY THROUGH USE OF IMMERSIVE VIRTUAL ENVIRONMENTS

Susan Persky, PhD

Social and Behavioral Research Branch, National Human Genome Research Institute, Bethesda, MD.

Recent work has demonstrated that visual imagery is an important tool for shaping attitudes and eliciting health-promoting behaviors. Though many characteristics impact the effectiveness of imagerybased health communication, presentation medium may be particularly influential on engagement with image content. Advances in media technology are providing new tools which hold the capacity to make imagery more impactful. Key among these is immersive virtual environment (IVE) technology (i.e., virtual reality).

Above all, use of IVEs allows individuals to interact with health imagery in an immersive and experiential fashion, as opposed to being passive viewers. Several research studies have demonstrated that such immersion uniquely captures individuals' attention and can increase engagement with message content. Furthermore, IVEs are typically interactive, opening the door to more detailed understanding and personalization of visual information.

These features could heighten many types of health messages conveyed via visual imagery. For example, a major purpose for invoking imagery is to demonstrate consequences of one's behavior (e.g., illustrating detrimental effects of smoking). These consequences can be experienced firsthand in an IVE, and an interactive component could include practicing and building efficacy for cessation behaviors while observing how these behaviors mitigate health consequences. Indeed, an imagery-based IVE for smoking cessation was recently created by Canadian researchers.

Another use of imagery involves conveying health concepts, often via metaphor (e.g., a ladder as a metaphor for varying risk levels). In IVEs individuals can directly experience a visual metaphor. They might also manipulate situational variables to gain nuanced understanding of the processes a metaphor conveys. Our group created and tested immersive visual metaphors based on an elevator and a bridge for conveying genetics concepts. Going forward, IVEs are poised to become an innovative medium for imagery provision and a vital research tool for exploring issues around image effectiveness.

CORRESPONDING AUTHOR: Susan Persky, PhD, Social and Behavioral Research Branch, National Human Genome Research Institute, Bethesda, MD, 20015; perskys@mail.nih.gov
SS30d

DOES DISGUSTING IMAGERY AID HEALTH PROMOTION CAMPAIGNS?

Gerry Humphris, $\mathrm{PhD}^{1}$ and Brian Williams, $\mathrm{PhD}^{2}$

${ }^{1}$ Health Psychology, University of St Andrews, St Andrews, United Kingdom and ${ }^{2}$ Public Health, University of Dundee, Dundee, United Kingdom.

Recent research has highlighted the possibility that the emotion of disgust can act as a significant driver to initiate behaviour change as a separate or even additional component to traditional fear appeal approaches. Pictorial images may be particularly effective in generating disgust emotion. Therefore the aim of this investigation was to test the specific image qualities associated with reported disgust and rating of potential success in promoting behaviour change. Data were obtained from a public consultation exercise (Department of Health, England and Wales, 2006) that requested, through a web-based answering system, participant preferences of 42 images that might be effective to encourage tobacco cessation in smokers. Initial results indicated preferences for effectiveness of cessation message were rank ordered according to the level of disgust emotion generated, i.e. images that might persuade smokers to quit tobacco consumption appeared revolting. To confirm that disgust emotion generated by the UK tobacco packet images was associated to the public's selection of possible effective images; three cross-sectional opinion surveys were conducted. These included students from medicine and psychology disciplines, and a section of the public. The web opinion consultation database was utilised for secondary analysis. A total of 291 participants were involved in the three convenience surveys and 19812 participants gave complete replies to the public consultation website. The pictorial Disgust Sensitivity Scale was used to assess the individual's rating of every image on a five category rating ranging from 'extremely disgusting' to 'not disgusting'. Significant correlations (ranging from 0.91 to 0.94) existed between the image rank order aggregated preference ratings from the original public consultation and the average final score of the disgust ratings for specific items for the three groups. The correlations were very similar regardless of smoking status of respondents (in both public preference and opinion surveys). The emotion of disgust may be a possible intervening variable to explain the initial reactions to health promotion materials and smoking cessation. Future research needs to explore the strength of disgust emotions and fear generated by images of this nature with actual behaviour change and other intervening constructs.

CORRESPONDING AUTHOR: Gerry Humphris, PhD, Health Psychology, University of St Andrews, St Andrews, KY16 9TS; gmh4@st-and.ac.uk 


\section{SS13}

THE ROLE OF SALIVA CORTISOL MEASUREMENTS IN HEALTH AND DISEASE - A MATTER OF THEORY AND METHODOLOGY

Margareta Kristenson, MD $\mathrm{PhD},{ }^{1}$ Petra Lindfors, $\mathrm{PhD},{ }^{2}$ Ulf Lundberg, $\mathrm{PhD},{ }^{2}$ Anette Harris, $\mathrm{PhD},{ }^{3}$ Åse M. Hansen, $\mathrm{PhD},{ }^{4}$ Peter Garvin, $\mathrm{PhD}^{1}$ and Holger Ursin, $\mathrm{MD} \mathrm{PhD}^{3}$

${ }^{1}$ Dep of Medical and Health Sciences, Linköping University, Sweden, Linköping, Sweden; ${ }^{2}$ Dep of Psychology, Stockholm University, Sweden, Stockholm, Sweden; ${ }^{3}$ Dep of Psychology, BergenUniversity, Norway, Bergen, Norway and ${ }^{4}$ National Work environment resarch inst, Copenhagen University, Copenhagen, Denmark.

Over the last decade, the technique of using ambulatory saliva sampling has become increasingly popular in field research and clinical studies. The non-invasive method is easy to administer and analyze, and therefore allows implementation in large scale study designs.

However, this large interest in use of saliva cortisol measurement is paralleled with frustrations on opposing Results: This symposium is based on a critical evaluation of existing literature on salivary cortisol, aiming to evaluate the utility of salivary cortisol as a biomarker in various settings and how we can understand cortisol reactivity using evidence of experiences from different study designs.

The work is compiled by the Scandinavian Stress and Cortisol Network, a network financed by the Swedish Research Council, and one main question asked was: is it possible that different results of studies involving cortisol assessments are functions of differences in the theoretical assumptions made and methods used?

In particular the symposium will focus on how the many different ways of evaluating levels and dynamics of salivary cortisol (i.e. with regards to time points of assessment and different algorithms based on multiple time points) may have an impact on the interpretation of cortisol measurements in various contexts.

Salivary cortisol has been studied in relation to the following topics: demographic variables, psychosocial work environment, psychological resources (e.g. mastery) and outcomes (eg burnout), sleep quality, biological markers (markers of cardiovascular risk, inflammation and metabolism) and somatic outcome. The following topics shall be covered at the symposium; where all data shall refer to how results of analyses can differ depend on methods used.

CORRESPONDING AUTHOR: Peter Garvin, PhD, Dep of Medical and Health Sciences, Linköping University, Sweden, Linköping, SE-581 83; peter.garvin@liu.se

\section{SS13a}

INTRODUCTION, WHY THIS REVIEW ON SALIVARY CORTISOL MEASUREMENTS?

Ulf Lundberg, PhD

Dep of Psychology, Stockholm University, Sweden, Stockholm, Sweden.
Measurement of cortisol in saliva is a very convenient method and has become popular in various stress-related investigations. However, results from different studies are often inconsistent. The aim of the present symposium is to critically analyze the literature with regard to the use of saliva cortisol as a biomarker of stress and stress-related disorders. The analysis is focused on definitions and measurements of stress and stress-related disorders, methods for saliva sampling and statistical analysis of cortisol measures and the treatment of confounders.

The following areas will be covered in the different presentations:

Saliva Cortisol and Work environment

Saliva Cortisol and cardiovascular risk factors

Saliva Cortisol and immunology

Saliva Cortisol and Somatic outcome

CORRESPONDING AUTHOR: Peter Garvin, PhD, Dep of Medical and Health Sciences, Linköping University, Sweden, Linköping, SE-581 83; peter.garvin@liu.se

\section{SS13b}

SALIVA CORTISOL IN PSYCHOSOCIAL WORK ENVIRONMENT STUDIES

Petra Lindfors, $\mathrm{PhD}$

Dep of Psychology, Stockholm University, Sweden, Stockholm, Sweden.

Over the last decade, the technique of using ambulatory saliva sampling has become increasingly popular in field research and clinical studies. The non-invasive method is easy to administer and analyze, and therefore allows implementation in large scale study designs.

However, this large interest in use of saliva cortisol measurement is paralleled with frustrations on opposing Results: This presentation is based on a critical evaluation of existing literature on salivary cortisol, aiming to evaluate the utility of various measures of salivary cortisol as a biomarker in relation to psychosocial work stressors.

Various measures of salivary cortisol were studied in relation to psychosocial work stressors. Twenty-seven papers including psychosocial work stress in terms of the Job Demand-ControlSupport model or the Effort-Reward Imbalance model were reviewed. The presentation focuses on how salivary cortisol relates to work stressors, and discusses how these results may vary depending on measurement and data analysis.

CORRESPONDING AUTHOR: Peter Garvin, PhD, Dep of Medical and Health Sciences, Linköping University, Sweden, Linköping, SE-581 83; peter.garvin@liu.se

SS13c

SALIVA CORTISOL AND ITS CORRELATIONS WITH CARDIOVASCULAR RISK FACTORS

Peter Garvin, $\mathrm{PhD}$

Department of Medical and Health Sciences, Linköping University, Linköping, Sweden. 
This presentation is based on a literature search using PubMed, aiming to evaluate the utility of various measures of salivary cortisol as a biomarker in relation to cardiovascular risk factors.

In particular, the presentation focuses on a discussion on how these results may vary depending on measurement and data analysis.

Background: The altered cortisol is sometimes regarded as a warning reaction essential part of the survival strategy of living organisms. An often asked question is "Is it unhealthy to have an increased or decreased cortisol awakening response?" In particular, correlations between altered cortisol levels and cardiovascular risk factors have been discussed.

Aim: To summarize existing knowledge on associations between measures of cortisol in saliva and cardiovascular risk factors (e.g. HDL, LDL and total cholesterol, Apolipoproteins A and B, Glucose, Insulin resistance, $\mathrm{HbA} 1 \mathrm{c}$ ) and endogenous hormones (e.g. Cortisone, Melatonin, Adrenaline, Noradrenaline, Prolactin, Testosterone, Dehydroepiandrosterone)

Methods: In a first step, an on-line search of the NCBI PubMed database (National Library of Medicine, National Institutes of Health, Bethesda, MD, USA-http:/www.ncbi.nlm.nih.gov/ PubMed) was conducted. The search covered the time period up to October 2009. Search terms are selected with reference to relevant PubMed terms and key words such as salivary cortisol and other biological markers. Studies are only included in this review if the topic are covered by the aim, study group is somatic healthy human adults, and the paper is written in English.

Results: A literature search in PubMed gave a total of 74 references. The included studies were both cross sectional and prospective studies. We did not find a clear association between derived cortisol measures (AUC, CAR, concentration) used in the included papers. The included studies used different sampling protocols and different way of calculating AUC and CAR.

Discussion: The inconclusive literature may be due to different sampling protocols and different ways of calculating AUC and CAR.

CORRESPONDING AUTHOR: Peter Garvin, PhD, Dep of Medical and Health Sciences, Linköping University, Sweden, Linköping, SE-581 83; peter.garvin@liu.se

\section{SS13d}

\section{SALIVA CORTISOL AND ITS CORRELATIONS WITH INFLAMMATORY MARKERS}

Peter Garvin, $\mathrm{PhD}$

Dep of Medical and Health Sciences, Linköping University, Sweden, Linköping, Sweden.

The anti-inflammatory properties of exogenous cortisol are well described. However, empirical data on endogenous cortisol and its correlations with inflammatory markers are few.

This presentation is based on a literature search using PubMed, aiming to evaluate the utility of various measures of salivary cortisol and its correlation with various inflammatory markers such as interleukines, acute phase proteins, and growth factors. The presentation focuses on a discussion on how these results may vary depending on measurement and data analysis.
In particular, the results would be discussed in the light of a possible down-regulation of glucocorticoid receptors.

CORRESPONDING AUTHOR: Peter Garvin, PhD, Dep of Medical and Health Sciences, Linköping University, Sweden, Linköping, SE-581 83; peter.garvin@liu.se

SS13e

LEVELS OF SALIVA CORTISOL AS PREDICTOR FOR SOMATIC OUTCOMES

Margareta Kristenson, MD PhD

Dep of Medical and Health Sciences, Linköping University, Sweden, Linköping, Sweden.

Salivary cortisol has been suggested to be an important stressrelated biomarker. However, while the use of salivary cortisol is still emerging in various settings, empirical studies linking saliva cortisol with somatic outcome are few.

This presentation is based on a literature search using PubMed, aiming to evaluate the utility of various measures of salivary cortisol and its correlation with various severe somatic outcomes such as myocardial infarction, cancer and auto-immune diseases. The presentation focuses on a discussion on how these results may vary depending on measurement and data analysis.

CORRESPONDING AUTHOR: Peter Garvin, PhD, Dep of Medical and Health Sciences, Linköping University, Sweden, Linköping, SE-581 83; peter.garvin@liu.se

\section{SS14}

NEW RESEARCH IN INSOMNIA ASSOCIATED

WITH CANCER: FROM DIAGNOSIS AND TREATMENT

TO SURVIVORSHIP

Oxana Palesh, PhD, MPH

University of Rochester, Rochester, NY.

Insomnia, a type of sleep disruption, is an extremely common and troublesome condition associated with cancer. The diagnosis of cancer represents a threat to survival; consequently, both patients and physicians believe that transient sleep dysfunction in response is normal. Currently, relatively little is known about the etiology and evolution of insomnia in cancer patients. Emerging evidence suggests that a significant minority of cancer patients develop a chronic persistent form of insomnia as a result of the diagnosis and treatment of their cancer. Chronic insomnia is a substantial problem; in the general population insomnia is a risk factor for additional morbidity (e.g., depression, fatigue, heart disease). This symposium will first discuss the prevalence of insomnia and its progression over an 18-month period in a large sample of newly diagnosed cancer patients. The second presentation will describe the sleep architecture of cancer patients undergoing chemotherapy. Polysomnographic sleep findings revealing the prevalence of slow wave sleep (SWS) in cancer patients before treatment, following treatment, and at later follow-up will be discussed. Finally, results 
from a national randomized clinical trial of YOCAS ${ }^{\circledR}$ Yoga will show how a brief, community-based intervention of 4 weeks' duration can significantly reduce insomnia and use of sleep medication in cancer survivors, suggesting that persistent insomnia responds quickly to a behavioral exercise intervention and can be treated easily and effectively. The goal of this symposium is to disseminate exciting recent findings in the areas of sleep and cancer and to generate new ideas and an awareness of chronic insomnia in cancer patients so that its negative effects can be addressed.

CORRESPONDING AUTHOR: Oxana Palesh, PhD, MPH, Radiation Oncology, University of Rochester, Rochester, NY, 14642; oxana_palesh@urmc.rochester.edu

\section{SS14a}

\section{EPIDEMIOLOGY OF INSOMNIA COMORBID} WITH CANCER: RESULTS OF AN 18-MONTH LONGIDUTINAL STUDY

Josée Savard, PhD, ${ }^{1,2}$ Julie Villa, MPs, ${ }^{1}$ Aude Caplette-Gingras, $\mathrm{BA},{ }^{1,2}$ Hans Ivers, $\mathrm{PhD}^{1,2}$ and Charles M. Morin, $\mathrm{PhD}^{2}$

${ }^{1}$ Laval University Cancer Research Center, Université Laval, Québec, QC, Canada and ${ }^{2}$ Psychology, Université Laval, Québec, QC, Canada.

The goal of this large scale epidemiological study was to assess the prevalence and incidence of insomnia comorbid with cancer over an 18-month period. All patients scheduled to undergo surgery after a first diagnosis of non-metastatic cancer were solicited at their pre-operative visit. Among the 3196 patients approached, 1681 were found eligible and 962 (57\%) accepted to participate. The participants completed a semi-structured interview for insomnia at baseline (T1), 2 (T2), 6 (T3), 10 (T4), 14 (T5) and 18 months (T6). The prevalence of the insomnia syndrome (T1: 28\%; T2: 26\%; T3: 25\%; T4: 23\%; T5: 21\%; T6: 22\%) decreased progressively and significantly over the 18 -month period, $\mathrm{F}(5,3725)=7.58, \mathrm{p}<.01$. Similarly, the prevalence of insomnia symptoms (including patients with an insomnia syndrome) decreased steadily and significantly over time (T1: 60\%; T2: 48\%; 46\%; T3: 46\%; T4: 41\%; T5: 38\%; T6: 37\%), F(53725) = 43.77, $\mathrm{p}<.01$. Simple effects indicated that the decrease was significant between $\mathrm{T} 1$ and $\mathrm{T} 2$ and between $\mathrm{T} 3$ and $\mathrm{T} 4(\mathrm{ps}<.01)$. Prevalence rates of insomnia symptoms were the highest in breast cancer patients $(42-69 \%)$ and the lowest in prostate cancer patients (25-38\%). The overall reduction in prevalence rates was reproduced for each cancer site except for prostate cancer patients in which the decline between $\mathrm{T} 1$ and $\mathrm{T} 4$, was followed by a resurgence of insomnia symptoms. Based on mixed models analyses, the incidence of insomnia among good sleepers at the previous time assessment was $20 \%$ at $\mathrm{T} 2,22 \%$ at $\mathrm{T} 3,17 \%$ at $\mathrm{T} 4,17 \%$ at $\mathrm{T} 5$, and $14 \%$ at $\mathrm{T} 6$. Insomnia is highly prevalent in cancer patients, particularly at the time of cancer surgery and then decreases progressively over time. Despite this overall pattern, insomnia symptoms develop in a significant proportion of patients during the cancer care trajectory, thus suggesting distinct patterns of evolution across patients.
CORRESPONDING AUTHOR: Josée Savard, PhD, Laval University Cancer Research Center, Université Laval, Québec, QC, G1R 2 J6; josee.savard@psy.ulaval.ca

SS14b

SLEEP ARCHITECTURE AND SLOW-WAVE SLEEP

PREVALENCE FROM 102 POLYSOMNOGRAPHY

ASSESSMENTS IN PATIENTS PRIOR TO AND FOLLOWING CHEMOTHERAPY

Joseph A. Roscoe, $\mathrm{PhD},{ }^{1}$ Michael L. Perlis, $\mathrm{PhD},{ }^{2}$ Oxana G. Palesh, PhD ${ }^{1}$ Gary R. Morrow, $\mathrm{PhD}^{1}$ and Wilfred R. Pigeon, $\mathrm{PhD}^{3}$

${ }^{1}$ Cancer Center, University of Rochester, Rochester, NY; ${ }^{2}$ Behavioral Sleep Medicine Program, University of Pennsylvania, Philadelphia, PA and ${ }^{3}$ Psychiatry, University of Rochester, Rochester, NY.

Background: Sleep disturbance is prevalent among cancer patients undergoing chemotherapy. Our previous work showed that nearly $80 \%$ self-report disturbed sleep during treatment. However, little objective evidence has been gathered on the patterns of sleep before and following chemotherapy.

Method: 26 Patients scheduled to receive chemotherapy were recruited. Sleep architecture was assessed by in-lab polysomnography (PSG) for two consecutive nights prior to first chemotherapy (T1), approximately two weeks following the patient's last chemotherapy or radiation treatment (T2) and three months following the last treatment. Most subjects (20) were female breast cancer patients. Five patients ( 3 males) had colorectal cancer and one (female) had lymphoma. The mean age of the 23 women was 52.4 and for the three men was 56.7. Sleep efficiency (SE), Stage 1, Stage 2, Slow-Wave Sleep (SWS), and REM sleep percentages were recorded with the two nights for each sleep variable averaged at each time point.

Results: On average, patients had good SE ( $84.1 \%$ at T1, $86.2 \%$ at $\mathrm{T} 2$ and $84.0 \%$ at T3. Findings of interest were that the proportion of SWS, unlike in healthy adults, was not correlated with age at any time point, (all, Rs $<1.8, \mathrm{Ps}>0.40$. SWS was also was notably less prevalent at all time points $(\mathrm{T} 1=5.3 \%, \mathrm{~T} 2=4.8 \%, \mathrm{~T} 3=5.3 \%)$ than the $20 \%$ level typically observed in healthy adults. Paired ttests revealed no significant changes over time in any of the sleep parameters (all, Ps $>0.20$ ).

Conclusion: Five key components of sleep architecture showed no changes from pre- cancer treatment to post-treatment. SWS was notably lower at all time points for patients compared to normal values. This suggests a relationship between cancer and SWS, which is often referred to as deep sleep and generally considered to be a restorative phase of sleep, that predates actual treatment. Further research is warranted.

Supported by ACS grant MRSG-04-233-01-CPPB.

CORRESPONDING AUTHOR: Joseph A. Roscoe, PhD, Cancer Center, University of Rochester, Rochester, NY, 14642; joseph roscoe@urmc.rochester.edu 
$\mathrm{SS} 14 \mathrm{c}$

YOCAS ${ }^{\circledR}$ YOGA IMPROVES INSOMNIA AMONG 410

CANCER SURVIVORS

Karen Mustian, PhD, MPH, ${ }^{1}$ O. Palesh, $\mathrm{PhD}, \mathrm{MPH},{ }^{1}$ L. Sprod, $\mathrm{PhD},{ }^{1}$ L. Peppone, PhD,${ }^{1}$ M. Janelsins, PhD,${ }^{1}$ C. Heckler, PhD,${ }^{1}$ B. Frizzell, MD, ${ }^{2}$ R. Gaur, MD ${ }^{3}$ J. Ruzich, $\mathrm{MD}^{4}$ and G. Morrow, $\mathrm{PhD}^{1}$

${ }^{1}$ University of Rochester Medical Center, Rochester, NY; ${ }^{2}$ SCCC CCOP, Winston-Salem, NC; ${ }^{3}$ Kansas City CCOP, Kansas City, KS and ${ }^{4}$ Columbia River CCOP, Portland, OR.

Insomnia is one of the most prevalent side effects experienced by cancer survivors and is associated with poorer prognosis and impaired quality of life. We conducted a nationwide, multi-site, phase II/III randomized, controlled, clinical trial examining the efficacy of yoga for improving insomnia. Non-metastatic, cancer survivors suffering from moderate or greater sleep disruption between 2-24 months after completing adjuvant therapy with no participation in yoga during the previous 3 months were randomized into 2 arms: 1) standard care monitoring and 2) standard care plus the 4-week (wk) yoga intervention (2 x's/wk; $75 \mathrm{~min} . / \mathrm{session})$. The yoga intervention utilized the Yoga for Cancer Survivors $\left(\right.$ YOCAS $\left.^{\circledR}\right)$ program consisting of pranayama (breathing exercises), 18 gentle Hatha and Restorative yoga asanas (postures) and meditation. Insomnia was assessed preand post-intervention using the Insomnia Severity Index (ISI). 410 survivors were accrued (96\% female, mean age $=54,75 \%$ breast cancer). ANCOVAs with baseline covariates revealed significant differences in insomnia (ISI total score; $\mathrm{p}<0.05$ ) between groups at post-intervention with the yoga group exhibiting greater reductions in insomnia ( $\mathrm{CS}=$ change score; $\mathrm{CS}=-3.49$, standard error $=\mathrm{SE} ; \mathrm{SE}=0.37$ ) compared to the control group $(\mathrm{CS}=-0.89, \mathrm{SE}=0.29)$. ANCOVAs also demonstrated significant differences between groups in difficulty falling asleep, difficulty staying asleep, waking up too early, sleep satisfaction, sleep interference, sleep-related distress and sleep problems noticeable to others (all $\mathrm{p}<0.05$ ) at postintervention with the yoga group improving the most. ANCOVAs also revealed significant differences in sleep medication use $(\mathrm{p}<0.05)$ with the yoga group reducing medication $(\mathrm{CS}=$ $-0.21, \mathrm{SE}=0.09)$ while the control group increased medication $(\mathrm{CS}=0.04, \mathrm{SE}=0.07)$. The brief community-based $\mathrm{YOCAS}^{\circledR}$ yoga intervention significantly improves insomnia and sleep components while reducing sleep medication use among survivors. Funding: NCI U10CA37420 and K07CA120025.

CORRESPONDING AUTHOR: Karen Mustian, PhD, MPH, University of Rochester Medical Center, Rochester, NY, 14642; karen_mustian@urmc.rochester.edu

\section{SS15}

\section{ADOLESCENTS' MEDICINE USE: A RESPONSE} TO STRAIN?

Bjørn E. Holstein, Mag scient soc, ${ }^{1}$ Ebba H. Hansen, Professor, ${ }^{2}$ Ron Iannotti, $\mathrm{PhD},{ }^{3}$ Anette Andersen, $\mathrm{PhD}^{1}$ and Hilde Tobi, $\mathrm{PhD}^{4}$
${ }^{1}$ National Institute of Public Health, University of Southern Denmark, Copenhagen, Denmark; ${ }^{2}$ Department of Pharmacology and Pharmacotherapy, University of Copenhagen, Copenhagen, Denmark; ${ }^{3}$ National Institute of Child Health and Human Development, Washington D.C., DC and ${ }^{4}$ Wageningen University $\&$ Research centre, Wageningen, Netherlands.

Adolescents' medicine use: a response to strain?

Hansen EH1, Andersen A2, Holstein BE2, Iannotti R3, Tobi H4

1 Department of Pharmacology and Pharmacotherapy, University of Copenhagen, Denmark

2 National Institute of Public Health, University of Southern Denmark, Copenhagen, Denmark

3 National Institute of Child Health and Human Development, Washington, USA

4 Department of Social Sciences, Wageningen University \& Research centre, Wageningen, Netherlands

Research about medicine use among adolescents is scarce. However, studies have shown that medicine use for common complaints is widespread among adolescents although there are major differences across countries. Medicine use is a public health concern. First, medicines are toxic and have adverse side effects. Second, medicine use in childhood and adolescence tracks into adulthood. Third, among adults medicine use is the most common response to ill health. Therefore, it is important to identify the main determinants of medicine use in adolescence.

A few studies have suggested that adolescents' medicine use is a response to a range of stressors, e.g. exposure to bullying, belonging to a lower socio-economic group or an ethnic minority, and poor self-rated health.

The objective of this symposium is to present a scientific rationale for the study of adolescents' medicine use, to provide an overview of recent empirical studies of adolescents' medicine use, to discuss methodological challenges in this area of interest, and to present new empirical findings from the international Health Behaviour in School-aged Children (HBSC) study.

CORRESPONDING AUTHOR: Bjørn E. Holstein, Mag scient soc, National Institute of Public Health, University of Southern Denmark, Copenhagen, DK-1353; bho@niph.dk

SS15a

ADOLESCENT MENTAL HEALTH AND MEDICINE USE IN SEVEN COUNTRIES

Ronald J. Iannotti, $\mathrm{PhD}^{1}$ and Kate Levin, $\mathrm{BSc}, \mathrm{MSc}^{2,3}$

${ }^{1}$ Eunice Kennedy Shriver National Institute of Child Health and Human Development, Bethesda, MD; ${ }^{2}$ University of Edinburgh, Edinburgh, United Kingdom and ${ }^{3}$ HBSC Medicine Use Writing Group, N/A, United Kingdom.

Adolescent mental health has been related to a variety of physical health problems and adolescents can have considerable independence in their use of medicines to treat acute health problems. The relationship between adolescent mental health and medicine use 
(MU) has not received sufficient attention. The present analyses examine relationships of adolescent mental health and MU and whether mental health contributes to MU beyond the effect of experiencing symptoms of those health problems. Self-reported mental health, MU for four common health problems (headache, stomachache, difficulties sleeping, and nervousness) and symptoms for common health problems were assessed in a nationallyrepresentative sample of 7,611 U.S. students in grades 6 through 10 and in 26,111 11-, 13-, and 15-year-old students in six countries participating in the Health Behavior in School-Aged Children study: Austria, Luxembourg, Germany, Switzerland, Macedonia, and Scotland. A brief measure of depression was included in the US HBSC survey and a broader mental health index (KIDSCREEN) was included in the surveys of the six European nations. In US adolescents, depression predicted MU for each of the four health problems but when both depression and symptoms were in the regression models depression added to the prediction for difficulties sleeping $(p=.0071)$ and nervousness $(\mathrm{p}<.0001)$ only. These findings were replicated in the European sample; using hierarchical linear modeling, there no significant effects due to country. MU for each of the four health problems was predicted by mental health; however, MU for headache and stomachache were no longer significant when symptoms were added to the models. Adolescents with poorer mental/psychological health are more likely to experience symptoms of common health problems and to take medicine for these problems. However, self-reported symptoms may partially mediate the relationship between adolescent mental health and MU.

CORRESPONDING AUTHOR: Bjørn E. Holstein, PhD, National Institute of Public Health, University of Southern Denmark, Copenhagen K, 1353; bho@niph.dk

\section{$\mathrm{SS} 15 \mathrm{~b}$}

\section{DIFFERENT SOCIOECONOMIC MEASURES SHOW} DIFFERENT ASSOCIATIONS WITH MEDICINE USE FOR HEADACHE AMONG ADOLESCENTS: AN INTERNATIONAL COMPARATIVE STUDY

Anette Andersen, MD, PhD

National Institute of Public Health, University of Southern Copenhagen, Copenhagen, Denmark.

Aim: To use comparable data from many countries to examine the associations between medicine use for headache among adolescents and two different aspects of socioeconomic position (SEP): family affluence and family occupational class.

Methods: We used data from the international study, Health Behaviour in School-aged Children (HBSC) 2005/2006. 17 countries provided data on 98907 11-, 13- and 15-year old students from nationally random samples of schools in Europe. The outcome measure was self-reported medicine use for headache within the last month. We included two measures of socioeconomic position; family affluence measured by the Family Affluence Scale (FAS) and family occupational class. Analyses stratified by country were controlled for gender, age and the prevalence of headache.
Results: The distribution of children categorised in high, medium and low FAS varied between countries. High FAS varied between $12.7 \%$ in Romania and $55.9 \%$ in Sweden. Children categorised in high family occupational class varied as well, from $5.8 \%$ in Austria to $49.2 \%$ in United Kingdom. Family affluence showed no association with medicine use for headache in any of the countries. In analyses combined for all countries, OR for medium FAS $=0.98$ (CI: 0.95-1.02), OR for low FAS=0.99 (CI: 0.94-1.03). Family occupational class showed significant associations with medicine use for headache in ten out of 17 countries, e.g. OR for low family occupational class in Denmark=1.42 (CI: 1.22-1.67).

Conclusions: It seems that different aspects of SEP influence medicine use among adolescents differently. Affluence in the family seems not to influence medicine use for headache, whereas family occupational class, reflecting occupational and educational level in the family, seems to have an influence among adolescents.

CORRESPONDING AUTHOR: Bjørn E. Holstein, PhD, National Institute of Public Health, University of Southern Denmark, Copenhagen K, 1353; bho@niph.dk

\section{$\mathrm{SS} 15 \mathrm{c}$}

METHODOLOGICAL CHALLENGES AND OPPORTUNITIES IN THE STUDY OF ADOLESCENTS' MEDICINE USE

Hilde Tobi, $\mathrm{PhD}, \mathrm{MSc}^{1}$ and Lenneke Vaandrager, $\mathrm{PhD}, \mathrm{MSc}^{2}$

${ }^{1}$ Research Methodology, Department of Social Sciences, Wageningen University \& Research centre, Wageningen, Netherlands and ${ }^{2}$ Public Health and Society, Department of Social Sciences, Wageningen University \& Research centre, Wageningen, Netherlands.

First methodological challenges and opportunities in the study of adolescents' medicine use will be discussed for the constituting parts 'adolescents' and 'medicine use'. These parts will then be combined in the context of the study of ADHD medication use in adolescents.

The very word 'adolescence' draws attention to the developmental perspective of any study involving adolescents. Advances in developmental neuroscience can help decide on age boundaries. However, the developmental perspective requires more than age boundaries due to the social and cultural aspects and expectations in this transitional phase, particularly in multi-national adolescents' medicine use studies. Consequences of this developmental perspective for data collection and data analysis will be discussed. With regard to medication use, prescription medication and overthe-counter medication are often distinguished. Another distinction is on-label, off-label and unlicensed medication. A special issue in adolescence is the study of compliance since adolescents seem more reluctant to take medicine according to instructions. Over-the-counter drugs and the use of illegal drugs also require specific attention when we study adolescents' medicine use, as does the difference between incidental and frequent or prolonged medicine use. These differences have consequences for data collection and data analysis.

Adolescents' use of ADHD medication is usually on-prescription. Methodological issues in the study of prolonged monitoring of adolescents' ADHD medication use as hindered by factors 
belonging to the developmental perspective and as supported by technological opportunities, will be discussed. Finally, some issues and opportunities in the study of non-prescribed ADHD medication will be presented.

CORRESPONDING AUTHOR: Bjørn E. Holstein, PhD, National Institute of Public Health, University of Southern Denmark, Copenhagen K, 1353; bho@niph.dk

\section{SS15d}

MENARCHE AND MEDICINE USE FOR PAIN

AND PSYCHOLOGICAL PROBLEMS AMONG GIRLS: NATIONAL CROSS-SECTIONAL SURVEY

Bjørn E. Holstein, $\mathrm{PhD},{ }^{1}$ Ebba Holme Hansen, MSc (pharm), ${ }^{2}$ Anette Andersen, $\mathrm{MD}, \mathrm{PhD},{ }^{1}$ Candace Currie, $\mathrm{BSc}, \mathrm{PhD},{ }^{3}$ Agnes Nemeth, PhD, ${ }^{4}$ Saoirse nic Gabhainn, BA, MA, PhD, CPsychol, CSci, AFBPsS ${ }^{5}$ and Pernille Due, MD $^{1}$

${ }^{1}$ National Institute of Public Health, University of Southern Denmark, Copenhagen K, Denmark; ${ }^{2}$ Department of Pharmacology and Pharmacotherapy, University of Copenhagen, Copenhagen, Denmark; ${ }^{3}$ Child \& Adolescent Health Research Unit (CAHRU), University of Edinburgh, Edinburgh, United Kingdom; ${ }^{4}$ National Institute of Child Health, Budapest, Hungary and ${ }^{5}$ Department of Health Promotion, National University of Ireland, Galway, Ireland.

Objective: To examine the association between menarche and use of medicine for common complaints (headache, stomach ache, difficulties in getting to sleep, nervousness) in a national representative sample of 11- and 13-year-old girls.

Methods: This cross-sectional school survey included all girls in the fifth (mean age 11.6 years) and seventh (mean age 13.6 years) grade in a random sample of Danish schools, $n=2,423$. Medicine use was measured by self-reports. We used logistic regression analysis with menarche as the independent variable and medicine use as the dependent variable, adjusted for social class and prevalence of the symptom for which the medicine was used.

Results: Menarche had been achieved by $8.4 \%$ of the 11 -year-old girls and $40.5 \%$ of the 13 -year old girls. During the previous month, $21.7 \%$ had used medicine for stomach ache, $45.8 \%$ for headache, $3.5 \%$ for difficulties in getting to sleep, and $3.4 \%$ for nervousness. Multivariate analyses showed that menarche among the 11-year-old girls was associated with medicine use for stomach-ache, OR $(95 \% \mathrm{CI})=1.97(1.18-3.30)$, medicine use for headache, 1.50 (1.00-2.27), medicine use for difficulties in getting to sleep, $1.45(0.56-3.78)$ and medicine use for nervousness, 1.97 (0.86-4.52). Among 13-year-old girls menarche was associated with medicine use for all these complaints but only significantly for medicine use for stomach ache and headache.

Conclusion: Menarche was independently associated with medicine use for headache, stomach ache, difficulties in getting to sleep, and nervousness among young adolescent girls.

CORRESPONDING AUTHOR: Bjørn E. Holstein, PhD, National Institute of Public Health, University of Southern Denmark, Copenhagen K, 1353; bho@niph.dk

\section{Friday \\ August 6, 2010 \\ PAPER SESSIONS \\ 8:30 AM-10:00 AM}

OS18

8:30 AM-8:48 AM

OS18-A

STRUCTURAL MODELS. RISK FACTORS OF BULIMIC BEHAVIOR IN WOMEN AND MEN COLLEGE SAMPLES

Gilda Gomez-Peresmitre, $\mathrm{PhD},{ }^{1}$ Gisela Pineda, $\mathrm{PhD}^{2}$ and Silvia Platas, $\mathrm{MA}^{3}$

${ }^{1}$ Faculty of Psychology, UNAM, Mexico, D.F., Mexico; ${ }^{2}$ Faculty of Sciences, UAB, Ensenada B.C, Mexico and ${ }^{3}$ Faculty of Psychology, UNAM, MEXICO D.F., Mexico.

The dual pathway model (DPM) of bulimic pathology represents an integrative etiological theory that contemplates the mediational mechanisms by which the risk factors might work together to promote bulimic behavior (BB). The internalization of the thin ideal contributes to body dissatisfaction (BD); increased $\mathrm{BD}$, in turn, fosters dieting and negative affect (depression and stress, in this study), which increase the risk for BB. Individuals may initiate BB because of either extreme dieting or chronic negative affect. In order to gain a better comprehension of $\mathrm{BB}$ and the main risk factors (thin ideal, body dissatisfaction, restrained dieting, and negative affect) two structural models were built and compared by sex (the DPM of women vs. the DPM of men) in order to detect which one of the group's models: a) shows more risk factors for BB, and which one of them b) provides a better understanding (higher explained variance) of the predictive role of the risk factors for BB. The total sample was formed by $\mathrm{N}=196$ college students, comprised of women (121) and men (75). The total mean age was $\mathrm{M}=19.35(\mathrm{SD}=1.75)$. The data collection used measures with acceptable psychometric properties. Among the main results, it was found that the DPM of women, when compared to the DPM of men, explained bigger amounts of variance (R2) of bulimic behavior (.48 vs. .26 ), restrained dieting (.52 vs. .27 ), and stress (.30 vs. .16), as well as higher beta weights in the interrelations of the risk predictive factors of $\mathrm{BB}$. The structure of both models, i.e., the DPM of women and the DPM of men, allowed: a) confirmation of the theoretical proposition, which states that restrictive dieting is a predictive risk factor for $\mathrm{BB}$, and $\mathrm{b}$ ) to evaluate specifically which factor of the negative affect (stress/depression) contributes to a better understanding of the dependent variable, i. e., to gain a greater explained variance of $\mathrm{BB}$.

Key Words. bulimic behavior, men and women, structural models, depression, stress.

CORRESPONDING AUTHOR: Gilda Gomez-Peresmitre, PhD, Faculty of Psychology, National University at Mexico, Mexico, D.F., 04510; gildag@servidor.unam.mx 


\section{8:48 AM-9:06 AM}

\section{OS18-B}

SOCIAL COHESION INCREASES FRUIT AND VEGETABLE CONSUMPTION IN OBESE ETHNIC MINORITY WOMEN Rebecca Lee, $\mathrm{PhD},{ }^{1}$ Renae Smith-Ray, MA, ${ }^{2}$ Scherezade K. Mama, $\mathrm{MPH},{ }^{1,3}$ Ashley V. Medina, BS, ${ }^{1}$ Jacqueline Y. Reese-Smith, PhD, ${ }^{4}$ Daniel O'Connor, $\mathrm{PhD}^{1}$ and Paul A. Estabrooks, $\mathrm{PhD}^{5}$

${ }^{1}$ Texas Obesity Research Center, University of Houston, Houston, TX; ${ }^{2}$ School of Public Health, University of Illinois at Chicago, Chicago, IL; ${ }^{3}$ School of Public Health, University of Texas, Houston, TX; ${ }^{4}$ University of Kansas, Lawrence, KS and ${ }^{5}$ Virginia Tech, Blacksburg, VA.

Background: Poor dietary habits may contribute to higher rates of obesity in ethnic minority women compared to all other populations subgroups in the US. Group dynamics based, social cohesion intervention approaches may improve adherence and participation, but little is known about ethnic minority samples. This study investigated the role of social cohesion variables in a longitudinal study to increase fruit and vegetable consumption in obese ethnic minority women. Method: African American $(n=204)$ and Hispanic or Latina $(n=105)$, apparently healthy, obese $(M=34.5 \mathrm{~kg} / \mathrm{m} 2)$ women ( $\mathrm{M}=46 \mathrm{yrs})$ were recruited from community sites and randomly assigned to a 6-month social cohesion intervention to promote increased vegetable and fruit consumption (VF) or comparison group. Participants completed six content-intensive social cohesion building sessions focusing on small team based activities, shared goal setting and achievement and take home activities. Sessions were conducted separately by ethnic group. Interviewer administered and self completed questionnaires measured baseline (T1) and post-intervention (T2) sociodemographic, social cohesion and dietary habits variables. BMI was measured by trained health assessors. Results: Women in the VF group reported significant increases in servings ( $\mathrm{T} 1 \mathrm{M}=2.7, \mathrm{~T} 2 \mathrm{M}=4.9)$ compared to comparison group ( $\mathrm{T} 1 \mathrm{M}=3.0, \mathrm{~T} 2 \mathrm{M}=3.2 ; \mathrm{p}<.01$ ), regardless of ethnicity or dose. Participants also experienced significant increases in all domains of social cohesion measures $(p<.001)$; however, this was not associated with outcomes. Conclusions: Obese ethnic minority women responded to dietary habits and social cohesion based interventions, regardless of ethnicity. Intervention participants consumed two servings more after treatment. Further research is needed to understand complex relationships among social cohesion variables and their change over time and how these can influence dietary habits and group adherence. Funded by 1R01CA109403.

CORRESPONDING AUTHOR: Rebecca Lee, PhD, University of Houston, Houston, TX, 77004; releephd@yahoo.com

\section{9:06 AM-9:24 AM}

OS18-C

VALIDATION OF A SURVEY INSTRUMENT TO MEASURE COLLEGIATE ATHLETES'KNOWLEDGE, PERCEPTIONS, AND DIETARY PRACTICES: PROJECT IGNITE

Diana Reindl, MA, Amy Thompson, PhD, Karen Gallagher, MA and Debra Boardley, PhD

University of Toledo, Toledo, $\mathrm{OH}$.
There is no doubt that nutrition affects athletic performance. Unfortunately, few collegiate sports programs include a comprehensive nutrition component. The purpose of this study was to determine stability-reliability of a 29 item instrument created to provide baseline data that would be used to develop a multi-level nutrition program for collegiate student-athletes. This survey also included demographic questions and was based on the Health Belief Model. Questions included measures of self-efficacy, perceived susceptibility, benefits and barriers. In addition, the Stages of Change Model was used to assess current nutritional activities. The survey was administered to a convenience sample of 41 collegiate-athletes and yielded 41 matched pairs used for analysis. A single item measuring Stages of Change resulted in a moderate correlation $(\mathrm{r}=.6)$, and 5 items on self- efficacy ranged from $r=.33-.68$, for an overall moderate correlation $(r=.6)$. There were 6 items that assessed benefits of receiving sport nutrition education (range $\mathrm{r}=.2-1.0$ ) and 7 items that measured barriers to healthy eating (range $r=.4-1.0$ ). These items yielded a total correlation score of .6 and .7 respectively. There were 15 items that measured nutritional knowledge. Because these items were dichotomous, a Kuder Richardson 20 (KR 20) test was used. Correlation coefficients for this subscale ranged from .03 to 1.0, for an overall correlation coefficient of .52. Items with correlation scores less than .06 were modified to yield more consistent responses and thus this survey shows promise as a potential valid and reliable assessment of collegiate athletes' knowledge, perceptions, and dietary practices. This information is essential when designing nutrition education programs and interventions for this population.

CORRESPONDING AUTHOR: Amy Thompson, PhD, University of Toledo, Toledo, OH, 43606; amy.thompson4@utoledo.edu

\section{9:24 AM-9:42 AM}

OS18-D

DEPRESSIVE SYMPTOMS, EMOTIONAL EATING

AND OBESITY: ARE THE ASSOCIATIONS SIMILAR ACROSS SOCIODEMOGRAPHIC GROUPS?

Hanna Konttinen, MSocSc, ${ }^{1}$ Karri Silventoinen, PhD, ${ }^{1}$ Sirpa Sarlio-Lähteenkorva, $\mathrm{PhD},{ }^{2}$ Satu Männistö, $\mathrm{PhD}^{3}$ and Ari Haukkala, $\mathrm{PhD}^{1}$

${ }^{1}$ Department of Social Studies, University of Helsinki, Helsinki, Finland; ${ }^{2}$ Department of Public Health, University of Helsinki, Helsinki, Finland and ${ }^{3}$ Department of Chronic Disease Prevention, National Institute for Health and Welfare, Helsinki, Finland.

Relationships between depression and obesity have been investigated extensively in the recent decades with increasing evidence of positive relationship, but few studies have examined the possible mechanisms explaining this association. The present study investigated direct and indirect associations between depressive symptoms, emotional eating and adiposity indicators across genders, and age and education groups, including whether emotional eating accounts for the association between depression and adiposity. The participants were Finnish men $(n=2325)$ and women $(n=2699)$ aged 25-74-year-old from the FINRISK 2007 
Study (DILGOM substudy). Structural equation modelling was used to test the hypothesised associations. Depressive symptoms and emotional eating were modelled as latent factors with indicators derived from the Center for Epidemiological Studies Depression Scale and Three-Factor Eating Questionnaire R-18, respectively. Participants' body mass index (BMI), waist circumference and fat percentage were measured in a health examination. The mediation model had an adequate fit with the data with respect to all adiposity indicators in both genders. Higher depressive symptoms were related to higher levels of emotional eating ( $\beta=.39$ in men and $\beta=.31$ in women), and emotional eating was related to higher BMI, waist circumference and fat percentage $(\beta=.26-.30$ in men and $\beta=.22-.29$ in women). The positive bivariate associations between depressive symptoms and adiposity indicators became non-significant in the models including emotional eating. Multigroup analyses indicated that there were no significant differences between genders, and age and education groups. Findings from this population-based sample indicate that emotional eating may be one factor explaining the positive relationship between depressive symptoms and obesity across different sociodemographic groups.

CORRESPONDING AUTHOR: Hanna Konttinen, MSocSc, Department of Social Studies, University of Helsinki, University of Helsinki, 00014; hanna.konttinen@helsinki.fi

\section{9:42 AM-10:00 AM}

OS18-E

CONSCRIPTS' ATTITUDES TOWARDS HEALTH

AND EATING - CHANGES DURING THE MILITARY

SERVICE AND ASSOCIATIONS WITH EATING PATTERNS

Piia Jallinoja, PhD,${ }^{1}$ Pilvikki Absetz, PhD ${ }^{1}$ Clarissa Bingham, MSci, ${ }^{1}$ Antti Uutela, Professor, ${ }^{1}$ Hely Tuorila, Professor ${ }^{2}$ and Nelli Hankonen, LicSocSci ${ }^{1}$

${ }^{1}$ National Institute for Health and Welfare, Helsinki, Finland and

${ }^{2}$ University of Helsinki, Helsinki, Finland.

In total institutions such as the military, meal times serve as release from daily routines. In Finland, all men are called for military service and $80 \%$ complete. Main meals are served as part of the service, but snacks are available for purchase during free time. The aim of the study was to analyse young men's attitudes towards health and eating before and at military service and their associations with eating patterns. A total of 290 men (age 18-21) participating in the DefenceNutri project filled in questionnaires before service and at 6th month of service. Four sub-indexes of Health and Taste Attitude scale (HTAS) were measured: General health interest (GHI); Craving for sweet foods (CSF); Using food as a reward (REW); and Pleasure (PLE), each with 4-6 statements rated from strongly disagree to strongly agree. The food consumption was measured as the number of days/week 6 sweet foods (Sweet food index) and 6 fatty foods (Fatty foods index) were consumed. Changes of HTAS indexes were analysed with paired sample t-test and associations with eating patterns with ANOVA.
GHI remained stable throughout the follow-up. CSF (3.0-3.6,p $<$ 0.000), REW (3.3-4.0,p $<0.000)$, and PLE $(3.9-4.1, \mathrm{p}<0.000)$ increased. Before entering military service eating of sweet foods was associated negatively with GHI $(\mathrm{p}=0.033)$ and positively with CSF $(p<0.000)$ and REW $(p<0.000)$; and eating of fatty foods negatively with GHI $(p=0.003)$ and positively with REW $(p=$ 0.001). At the end of 6th month of service eating of sweet foods was explained again by GHI $(p=0.002), \operatorname{CSF}(p=0.001)$ and REW $(p=0.021)$, but eating of fatty foods was not associated any of the attitude scales. PLE was not associated with eating of fatty or sweet foods in neither measurement.

Military service increases men's tendency to view food as a source of reward and pleasure, and to crave for sweet foods. In civilian life, health pursuit decreases eating of sweet and fatty foods but during service only of sweet foods. During service eating of fatty foods is not related to pleasure pursuit.

CORRESPONDING AUTHOR: Piia Jallinoja, PhD, National Institute for Health and Welfare, Helsinki, 00271; piia.jallinoja@ thl.fi

\section{OS19}

\section{8:30 AM-8:48 AM}

OS19-A

SOCIO-ECONOMIC AND DISEASE-RELATED PREDICTORS FOR ATTRACTION TO THE "READY TO ACT" PROGRAM IN PRIMARY CARE AIMING AT ACTION COMPETENCE FOR LIVING WITH DYSGLYCAEMIA

Helle T. Maindal, PhD, MPH, Mette V. Skriver, Statiscian, Torsten Lauritzen, Professor, MD and Annelli Sandbaek, PhD, MD

General Practice, University of Aarhus, School of Public health, Aarhus, Denmark.

Introduction: Screening for type 2 diabetes is increasingly being offered in general practice. Once the diagnosis is made healthcare services are likely to offer health promotional and preventive initiatives. Preventive and treatment recommendations are fully developed, but how we motivate people to make use of these initiatives and offer the right ones remains less clear.

Aim: To investigate socio-economic and disease-related predictors for participating in the "Ready to Act" program dealing with support to action competence for living with dysglycaemia. We hypothesize that the possibility of participating in this particular program will increase in accordance with a higher level of education, cohabitant and no competing diseases.

Design: A cohort study was embedded in the randomized controlled trial of a primary care/general practice screening and intervention programme. The study population $(n=320)$ were adults with screendetected prediabetes or with type 2 diabetes recruited by a mailed invitation after the screening procedure. The "Ready to Act" program, which consisted of 2 individual interviews and 8 group meetings delivered over 12 weeks, was offered to the intervention group by a multidisciplinary team of health professionals, as a supplement to intensive treatment in general practice. In total $45 \%(n=144)$ accepted to participate in the program and $38 \%(n=123)$ fulfilled. 
Study plan: A comparison between those who accepted the program and those who did not will be conducted according to the following outcomes: socio-economic: age, sex, cohabitant and education and disease-related: diagnosis, duration of diagnosis, comorbidity (cancer, CHD, COPD).

Perspective: The evidence of predictors for participation in health promoting programs is of great value for the future planning of target group differentiated health service initiatives.

CORRESPONDING AUTHOR: Helle T. Maindal, PhD, MPH, General Practice, University of Aarhus, School of Public health, Aarhus, 8000; htm@alm.au.dk

\section{8:48 AM-9:06 AM}

OS19-B

EFFECTS OF A LIFE COACHING AND PHARMACY COUNSELING MODEL FOR INDIVIDUALS

\section{WITH DIABETES}

Rebecca Rude Ozaki, PhD, Jean Isip Schneider, MEd, SPHR and Landry Fukunaga, MA

Center on Disability Studies, University of Hawaii, Honolulu, HI.

Many working adults with diabetes face major barriers to effective disease self management. The Hawaii Demonstration to Maintain Independence and Employment was a randomized controlled trial providing treatment group participants with individual life coaching and a pharmacist counseling while tracking health and employment outcomes. Self-reported diabetes self-efficacy, quality of life, functioning, hours worked, work productivity, and use of government services data were obtained at baseline, 6, and 12 months. Additional health information was obtained from healthcare providers. Generalized linear models for repeated measures are being used to evaluate the effect of the intervention. Surveys and focus groups were used to obtain participant perceptions about the intervention. 190 employed individuals diagnosed with diabetes or with indication of uncontrolled blood sugar were enrolled. Preliminary results show that body mass index and diabetes self-efficacy changes were significantly improved in the treatment group $(\mathrm{p}<0.05)$. Treatment group participants reported greater work productivity improvements. Diabetes management improvements were frequently attributed to increased accountability and goal setting with a life coach, as well as nutrition and diabetes education provided by pharmacists. This presentation will discuss how participant engagement, perceptions, and intervention components may have influenced employment and health indicators. A diabetes management intervention was well received by participants and showed the potential to delay functional decline and reliance on government assistance programs. Findings have implications for employers, health care providers, and professionals using early intervention models intended to prevent disability and support the ongoing employment of individuals with chronic conditions. The use of Life Coaching and Pharmacists as counselors is an emerging service delivery approach. Further RCT research is warranted to determine the viability of this model for chronic disease management. Cost effective analysis would also be valuable.
CORRESPONDING AUTHOR: Jean Isip Schneider, MEd, SPHR, Center on Disability Studies, University of Hawaii, Honolulu, HI, 96822; isip@hawaii.edu

9:06 AM-9:24 AM

OS19-C

THE IMPACT OF A PSYCHOEDUCATIONAL PROGRAM (SWEEP) IN DEPRESSED WOMEN WITH DIABETES

Sue Penckofer, $\mathrm{PhD},{ }^{1}$ Mary Byrn, BSN, ${ }^{1}$ Patricia Mumby, $\mathrm{PhD},{ }^{2}$ Carol Ferrans, $\mathrm{PhD},{ }^{3}$ Patrick Lustman, $\mathrm{PhD}^{4}$ and Patrick Harrison, $\mathrm{PhD}^{5}$

${ }^{1}$ School of Nursing, Loyola University Chicago, Maywood, IL;

${ }^{2}$ School of Medicine, Loyola University Chicago, Maywood, IL;

${ }^{3}$ College of Nursing, University of Illinois, Chicago, IL; ${ }^{4}$ School of Medicine, Washington University St. Louis, St. Louis, IL and ${ }^{5}$ Department of Psychology, Loyola University Chicago, Chicago, IL.

Depression is a serious problem affecting $25 \%$ of persons with diabetes, and the risk of depression is doubled in women compared to men with diabetes. Other moods (e.g., anxiety, anger) often accompany depression and negatively impact diabetes outcomes. We tested the feasibility of the Study of Women's Emotions and Evaluation of a Psychoeducational (SWEEP) program to assess the effectiveness of group cognitive behavioral therapy (CBT) for treating depression and dysphoric symptoms in women with type 2 diabetes. Seventy four women with diabetes and co-morbid depression were randomized to the SWEEP program $(n=34)$ or usual care $(n=36)$. The treatment included 8 weekly sessions targeted at depression, anxiety, and anger. Sixty women $(81 \%)$ completed baseline, 3 and 6 month measurements. Findings indicated a greater decrease in depression (CES-D) for the treatment $(\mathrm{T} 1=26.8, \mathrm{~T} 2=15.2, \mathrm{~T} 3=12.6)$ as compared to the control group $(\mathrm{T} 1=28.9, \mathrm{~T} 2=23.3, \mathrm{~T} 3=21.5), \mathrm{p}=.03$. Similarly, there was a greater decrease in trait anxiety (STAI) $(\mathrm{T} 1=49.9$, $\mathrm{T} 2=41.2, \mathrm{~T} 3=36.8)$ for the treatment group as compared to the control group $(\mathrm{T} 1=50.5, \mathrm{~T} 2=46.6, \mathrm{~T} 3=45.3), \mathrm{p}=.01$. For anger (STAXI), although there were no significant differences between the treatment $(\mathrm{T} 1=20.0, \mathrm{~T} 2=17.3, \mathrm{~T} 3=15.4)$ and the control conditions at any point $(\mathrm{T} 1=18.0, \mathrm{~T} 2=16.2, \mathrm{~T} 3=16.0)$, there was a significant decrease in anger scores overall, $\mathrm{p}<.001$. There was a clinically significant decrease in HBA1C in the treatment group from baseline to 3 months $(\mathrm{T} 1=7.6, \mathrm{~T} 2=7.2)$ however, it was not statistically significant and the improvement was not sustained $(\mathrm{T} 3=7.4)$. There were minimal changes in HBA1c in the control group $(\mathrm{T} 1=7.9, \mathrm{~T} 2=7.9, \mathrm{~T} 3=7.8)$. Overall, the SWEEP program significantly improved depression and anxiety outcomes in depressed women with type 2 diabetes. There were also favorable effects on anger and HBA1C. These data provide the first demonstration that depression, a common co-morbidity in women with type 2 diabetes, can be effectively treated in a group setting using CBT techniques.

CORRESPONDING AUTHOR: Sue Penckofer, PhD, School of Nursing, Loyola University Chicago, Maywood, IL, 60153; spencko@luc.edu 


\section{9:24 AM-9:42 AM}

OS19-D

SHORT- AND LONG TERM EFFECTS OF A MOTIVATIONAL INTERVIEWING-BASED LIFESTYLE INTERVENTION FOR CONSTRUCTION WORKERS WITH AN ELEVATED RISK OF CARDIOVASCULAR DISEASE

Iris Groeneveld, MSc, Karin I. Proper, PhD, Allard J. van der Beek, Professor, $\mathrm{PhD}$, Vincent H. Hildebrandt, $\mathrm{PhD}$ and Willem van Mechelen, Professor, $\mathrm{PhD}$

Public and Occupational Health, VU Medical Center, Amsterdam, Netherlands.

Introduction: More than $60 \%$ of male construction workers in the Netherlands are overweight or obese and more than a quarter is at risk for cardiovascular disease (CVD). The aim of this study was to evaluate the effectiveness of a motivational interviewing-based lifestyle intervention in this population.

Methods: 816 Male construction workers with an elevated CVD risk were individually randomized into the intervention or control group. The 6-month intervention consisted of three face-to-face and four telephone contacts with an occupational health professional, in which behavior change was discussed in the style of motivational interviewing. Participants chose to aim at either energy balance or smoking behavior. The control group received usual care. At 6 and 12 months, dietary intake, physical activity, and smoking behavior were assessed by means of a questionnaire. Moreover, body weight, cholesterol, blood pressure, and $\mathrm{HbAlc}$ were measured according to a standardized protocol. In order to determine the intervention effects, linear and logistic regression analyses were performed in the total study population as well as in the energy balance and the smoking subgroups.

Results: The intervention had a statistically significant beneficial effect on fruit intake (pieces per week: $\beta=1.7,95 \% \mathrm{CI} 0.6 ; 2.9$ ) and smoking at 6 months $(\mathrm{OR}=0.3,95 \% \mathrm{CI} 0.1 ; 0.7)$, and snack intake (pieces per week) at $6(\beta=-1.9,95 \% \mathrm{CI}-3.7 ;-0.02)$ and 12 months ( $\beta-1.9,95 \%$ CI $-3.6 ;-0.03)$. No intervention effect was found for physical activity. In the energy balance subgroup, a significant intervention effect was found for body weight at 6 months $(\beta=-2.1,95 \% \mathrm{CI}-2.9 ;-1.3)$, which was sustained until 12 months $(\beta=-2.2,95 \%$ CI $-3.1 ;-1.3)$. At 12 months, a significant effect was found for HDL cholesterol as well $(\beta=$ $0.05,95 \%$ CI $0.01 ; 0.10$ ).

Conclusions: This lifestyle intervention was effective in lowering CVD risk. Implementation in the occupational health setting is therefore recommended.

CORRESPONDING AUTHOR: Iris Groeneveld, MSc, Public and Occupational Health, VU Medical Center, Amsterdam, 1081 BT; iris.groeneveld@vumc.nl
9:42 AM-10:00 AM

OS19-E

MINDFULNESS IN THE MAINTENANCE OF WEIGHT LOSS: A RANDOMIZED CONTROLLED TRIAL

OF THE EMPOWER PROGRAM

Janna L. Fikkan, $\mathrm{PhD},{ }^{1}$ Michael Baime, $\mathrm{MD},{ }^{2}$ Linda Sanders, MS, ${ }^{4}$ Jeffrey Greeson, $\mathrm{PhD},{ }^{1}$ Webb Jennifer, $\mathrm{PhD},{ }^{5}$ Jessica Wakefield, MA, ${ }^{1}$ Lawrence Ladden, $\mathrm{PhD},{ }^{2}$ Jeanne Gresko, MA, ${ }^{1}$ Mara Wai, $\mathrm{MEd},{ }^{2}$ Catherine Stoney, $\mathrm{PhD}^{3}$ and Ruth Wolever, $\mathrm{PhD}^{1}$

${ }^{1}$ Duke Integrative Medicine, Duke University Medical Center, Durham, NC; ${ }^{2}$ Penn Program for Mindfulness, University of Pennsylvania, Philadelphia, PA; ${ }^{3}$ National Center for Complementary \& Alternative Medicine, National Institutes of Health, Washington, DC; ${ }^{4}$ General Internal Medicine, Duke University Medical Center, Durham, NC and ${ }^{5}$ Department of Psychology, University of North Carolina at Charlotte, Charlotte, NC.

Although weight loss programs tend to produce clinically meaningful though modest weight loss, most individuals steadily regain their weight. The current study compared the efficacy of a standard behavioral weight loss maintenance intervention (SBWLM) to a novel 12-week mindfulness-based intervention plus coaching tailored to WLM (Enhancing Mindfulness for the Prevention Of WEight Regain: EMPOWER). Community members who had lost a minimum of $9 \%$ of their body weight in the previous three years were recruited and randomized. Both groups received identical information about nutrition, physical activity, the importance of stress management, and values and goal setting. The EMPOWER group, however, was presented the information in the context of learning mindfulness skills to facilitate change. EMPOWER participants were also taught meditation, mindful eating, and mindfulness strategies to reduce stress-related reactivity while SBWLM participants spent equivalent time reading and discussing relevant material. Both groups were carefully structured to ensure parity in numerous factors (e.g., time invested, peer and interventionist support, positive expectancy). Mixed models determined that incremental weight regain rates did not differ by group. On average, both groups maintained their baseline weight loss 16 months later, one year post intervention (EMPOWER: $\downarrow 1.5 \mathrm{~kg}$; SBWM: $\uparrow 0.9 \mathrm{~kg}$ ). Weight regain rates were equivalent to or lower than those reported in other large scale studies (e.g., Svetkey et al., 2008, Dansinger et al., 2007). Only the EMPOWER group reported increases in intuitive eating $(\mathrm{p}<.0001)$, a trait previously associated with better physical health (Hawks, et al., 2005) and lower eating pathology (Tylka \& Wilcox, 2006). Further analyses will determine whether metabolic and inflammatory biomarkers were impacted differentially by group. These data support the use of the EMPOWER program as a novel and effective approach for supporting individuals in the maintenance of weight loss.

CORRESPONDING AUTHOR: Janna L. Fikkan, PhD, Psychiatry \& Behavioral Sciences, Duke University Medical Center, Durham, NC, 27710; Janna.Fikkan@duke.edu 
OS20

8:30 AM-8:48 AM

OS20-A

BEHAVIORAL ORIGINS OF METABOLIC SYNDROME DISORDERS

Milind Watve, $\mathrm{PhD},{ }^{1,4}$ Prajakta Belsare, $\mathrm{MSc}^{2}$ and Maithili Jog, $\mathrm{PhD}^{3}$

${ }^{1}$ IISER,Pune, Pune, India; ${ }^{2}$ Zoology, University of Pune, Pune, India; ${ }^{3}$ Biotechnology, Abasaheb Garware College, Pune, India and ${ }^{4}$ Anujeeva Biosciences Pvt Ltd, Pune, India.

Behavioral origins of metabolic syndrome disorders

Milind watve1, Prajakta Belsare2, Maithili Jog3

1. Indian Institute of Science Education and Research, Pune

2. Department of Zoology, University of Pune

3. Department of Biotechnology, Abasaheb Garware College, Pune

4. Anujeeva Biosciences Pvt Ltd., Pune

The metabolic syndrome disorders (MSD) including type 2 diabetes, hypertension, hypercholesterolemia, atherosclerosis and cardiovascular disease are known to be triggered by lifestyle change. We state and support a hypothesis here that changes in behavior rather than changes in diet and metabolism are central to MSD. We provide and discuss evidence in support of the following sequence of arguments. (i) Diet, thriftiness and obesity centered paradigm is inadequate to explain most of the physiological and immunological changes associated with metabolic syndrome disorders (MSD). (ii) Comparative studies show that relative obesity rather than absolute obesity is strongly associated with various parameters of MSD and further perception of calories without actual change in intake of calories can induce physiological changes. (iii) Alternative behavioral strategies co-exist in animal populations which are associated with characteristic metabolic states and some of these states show close resemblance with MSD in humans. (iv) Obesity and MSD are strongly and cross culturally associated with certain behavioral traits. (v) Deficiencies of certain behaviors that evolved with hunter gatherer life as adaptive behaviors but that are deficient in modern life lead to corresponding dysfunctions of neuro-endocrine mechanisms. Through a meta-analysis study we show that deficiency of physical aggression is strongly associated with MSD as seen by neuro-endocrine mechanisms as well as epidemiological data. This raises a possibility that the effects of behavioral deficiencies may be reversed by behavioral supplementation. (vi) On a pilot scale, behavioral interventions substantially ameliorated many components of MSD without significantly changing body weights. All the evidence collec- tively indicates that MSD originates due to behavioral deficiencies rather than metabolic thriftiness and therefore behavioral supplementation may be effective in reversing the chronic conditions.

CORRESPONDING AUTHOR: Milind Watve, PhD, IISER, Pune, Pune, 411 021; milind@iiserpune.ac.in

\section{8:48 AM-9:06 AM \\ OS20-B \\ PSYCHOSOCIAL FACTORS OVER A LIFE COURSE AND RISK OF THE METABOLIC SYNDROME: A PROSPECTIVE COHORT STUDY}

Jolene Masters Pedersen, master, ${ }^{1}$ Ingelise Andersen, $\mathrm{PhD},{ }^{1}$ Rikke Lund, $\mathrm{MD}, \mathrm{PhD},{ }^{1}$ Eva Prescott, $\mathrm{MD}^{2}$ and Naja Hulvej Rod, $\mathrm{PhD}^{1}$ ${ }^{1}$ Department of Social Medicine, University of Copenhagen, Copenhagen, Denmark and ${ }^{2}$ Department of Cardiology, Bispebjerg University Hospital, Copenhagen, Denmark.

Background: The prevalence of the metabolic syndrome is steadily increasing and stress has been suggested to play an important role in its etiology. The objective of this study was to assess the individual and cumulative effects of being exposed to psychosocial stress over a life course on the risk of developing the metabolic syndrome.

Material and Methods: The study population consisted of 5008 men and women aged 21-87 years from the Copenhagen City Heart Study, a prospective cohort study with over 10 years followup. Stress measured over a life course included information on major life events in childhood, adult, and work life, vital exhaustion, economic hardship, lack of social network, lacking a confidant, dissatisfaction with social network and the daily use of tranquilizers and/or sleeping pills. Analyses were adjusted for age, education, civil status, family history of ischemic heart disease, physical inactivity, tobacco smoke and alcohol consumption.

Results: In women, vital exhaustion was associated with a higher risk of the metabolic syndrome in a dose-response manner $(\mathrm{p}=0.01)$, so that women with high vital exhaustion had a higher risk of the metabolic syndrome $(\mathrm{OR}=1.71 ; 95 \%$ CI: 0.96-3.05) compared with women with low vital exhaustion. Dissatisfaction with social network (1.53; 1.11-2.12), lacking a confidant (1.98; 1.08-3.60) and an increased accumulated stress score $(1.94 ; 1.25-3.01)$ were also associated with a higher risk of the metabolic syndrome in women. In men, daily use of tranquilizers $(3.39 ; 1.53-7.53)$ was associated with a higher risk of the metabolic syndrome.

Conclusion: This longitudinal study supports a causal relationship between some psychosocial factors and the metabolic syndrome.

CORRESPONDING AUTHOR: Jolene Masters Pedersen, master, Department of Social Medicine, University of Copenhagen, Copenhagen, 1014; jope@sund.ku.dk 
9:06 AM-9:24 AM

OS20-C

HEALTH-RELATED QUALITY OF LIFE, SUBJECTIVE HEALTH COMPLAINTS, PSYCHOLOGICAL DISTRESS AND COPING IN PAKISTANI IMMIGRANT WOMEN WITH AND WITHOUT THE METABOLIC SYNDROME. THE INVADIAB-DEPLAN STUDY ON PAKISTANI IMMIGRANT WOMEN LIVING IN OSLO, NORWAY Victoria T. Hjellset, PhD, ${ }^{1,3}$ Camilla M. Ihlebæk, Prof, ${ }^{2,3}$ Benedikte Bjørge, Student ${ }^{4}$ and Hege R. Eriksen, Prof ${ }^{3}$

${ }^{1}$ Department of Preventive medicine, University of Oslo, Oslo, Norway; ${ }^{2}$ Health and Quality of Life, University of Life Sciences (UMB), Ås, Norway; ${ }^{3}$ Uni Health, University of Bergen, Bergen, Norway and ${ }^{4}$ Department of Nutrition, University of Oslo, Oslo, Norway.

Background: The increasingly high number of immigrants from non-Western countries represents an important challenge for the public health sector. One particular concern is the high prevalence of The Metabolic Syndrome (MetS) among immigrants from South-East Asia. The aim of the present work was to describe health-related quality of life, subjective health, psychological distress, and coping in Pakistani immigrant women, with and without MetS.

Methods: As a part of an intervention study in Oslo, Norway, female Pakistani immigrants $(n=198$, age 25 to 63) answered questionnaires regarding health related quality of life, subjective health complaints, psychological distress, and coping. To determinate metabolic syndrome, venous blood samples were drawn from an antecubital vein, and a standardized oral glucose tolerance test (OGTT) was performed.

Results: About $40 \%$ of the participants had MetS, and this group showed significantly lower general health, lower physical function, and more bodily pain, than those without MetS. They also had more subjective health complaints, depression and higher levels of somatisation, and scored significantly lower on the coping strategy of active problem solving than participants without MetS.

Conclusion: Pakistani immigrant women living in Oslo, Norway, seem to have a high prevalence of subjective health complaints and psychological distress, especially those with MetS.

Key words: Pakistani immigrant women, immigration, health, coping, metabolic syndrome.

CORRESPONDING AUTHOR: Victoria T. Hjellset, PhD, University of Oslo, Oslo, 0363; v.t.hjellset@medisin.uio.no

\section{9:24 AM-9:42 AM}

OS20-D

BODY COMPOSITION AND CLINICAL RISK FACTORS AMONG MALE CONSCRIPTS AT ENTRANCE AND AFTER 6 MONTHS OF SERVICE IN FINLAND

Antti Uutela, $\mathrm{PhD},{ }^{1,2}$ Pilvikki Absetz, Dr Psych, ${ }^{1,2}$ Clarissa M. Bingham, MSc, ${ }^{1}$ Marja Kinnunen, MSocSc, ${ }^{1}$ and Piia Jallinoja, DrSocSc ${ }^{1,3}$

${ }^{1}$ Lifestyle and Participation, National Institute for Health and Welfare THL, FI-00271 Helsinki, Finland; ${ }^{2}$ Department of Health,
University of Tampere, Tampere, Finland and ${ }^{3}$ Department of Sociology, University of Helsinki, Helsinki, Finland.

Introduction: All eligible young male citizens in Finland are conscripted in the Defence Forces (DF) for 6-11 months. DF has been stressing physical training but is currently emphasizing healthy nutrition increasingly for physical performance, too, as weight gain is a cohort trend. In 2007 THL and DF started a 3-year intervention study (DefenceNutri) to find instruments to improve the conscripts' diet, including a control (in 2007) and two consecutive intervention phases (to be activated in 2008 and 2009).

Subjects and measures: Subjects were 551 volunteering male conscripts (79\% of those targeted), typically aged 19-20 years, serving either in the Armoured Brigade in the South or the Kainuu Brigade in the North of Finland in 2007. Subjects answered questionnaires and participated in standardized clinical risk factor and anthropometric measurements at entrance and at 6 months just before private soldiers finished their service.

Results: Subjects showed the following average weight-related characteristics at entrance (SD): $\mathrm{BMI}=23.5 \mathrm{~kg} / \mathrm{m}^{2}$ (3.8), waist circumference $(\mathrm{WC})=84.3 \mathrm{~cm}(10.4)$, muscle mass $(\mathrm{MM})=$ $35.0 \mathrm{~kg}(4.3)$, and fat mass $(\mathrm{FM})=13.3 \mathrm{~kg}(8.4)$. By 6 months their BMI was down by 0.2 units $(\mathrm{p}<.05)$, WC down by $1.7 \mathrm{~cm}$ $(\mathrm{p}<.001)$, and FM down by $0.9 \mathrm{~kg}(\mathrm{p}<.001)$ while their MM was up by $0.5 \mathrm{~kg}(\mathrm{p}<.001)$. Clinical risk factors at entrance were in average (SD): SBP 126.1 hgmm (10.5), DBP=68.5 hgmm (6.9), total cholesterol=3.7 mmol/1 (0.8), LDL=2.2 mmol/1 (0.7), triglycerides $=0.7 \mathrm{mmol} / 1(0.3)$, and fasting glucose $=5.3 \mathrm{mmol} / \mathrm{l}(0.4)$. At 6 months SBP was lowered in average by $2.1 \mathrm{hgmm}(\mathrm{p}<.001)$, DBP and LDL were kept maintained, but triglycerides and fasting glucose were increased by $0.4 \mathrm{mmol} / \mathrm{l}(\mathrm{p}<.001)$, respectively. Low-educated subjects had somewhat more detrimental characteristics at start, but differences between educational groups largely levelled-off during the service.

Conclusions: While the DF provided nutrition already follows guidelines there is a need for supply/demand targeted nutrition intervention related especially to saturated fat and sugar containing snacks among conscripts.

CORRESPONDING AUTHOR: Antti Uutela, PhD, Lifestyle and Participation, National Institute for Health and Welfare THL, FI00271 Helsinki, FI-00271; antti.uutela@thl.fi

\section{9:42 AM-10:00 AM}

OS20-E

EFFECT OF A CULTURALLY ADAPTED INTERVENTION PROGRAM ON COMPONENTS OF THE METABOLIC SYNDROME IN PAKISTANI IMMIGRANT WOMEN LIVING IN OSLO, NORWAY

Victoria T. Hjellset, PhD, ${ }^{1,3}$ Marte Råberg, PhD student, ${ }^{2}$ Margareta Wandel, Prof ${ }^{2}$ and Hege R. Eriksen, prof ${ }^{3}$

${ }^{1}$ Institute of Preventive Medisine, University of Oslo, Oslo, Norway; ${ }^{2}$ Institute Nutrition, University of Oslo, Oslo, Norway and ${ }^{3}$ UniHealth, University of Bergen, Bergen, Norway. 
Background: Type 2 diabetes (T2D) is prevalent among South Asian immigrants in Western countries, including Norway. The metabolic syndrome (MetS) is a cluster of risk factors for T2D, and also for coronary heart diseases.

Aim: To reduce the risk of MetS by increasing the level of physical activity (PA) and change unhealthy diet habits in Pakistani immigrant women, living in Oslo, Norway. Method: Using a multi recruitment strategy, we included 198 women, who were randomised into a control and an intervention group. For $7 \pm$ 1 months we carried out a culturally adapted intervention program, consisting of 6 group sessions on diet and physical activity, using knowledge about Pakistani lifestyle, and focusing upon understanding of blood glucose regulation and significance. Fyll ut mer her Several blood variables were determined before and after the intervention, e.g. plasma glucose, HbA1c, insulin, and lipids.

Results: Among those attending $\geq 4$ of the group sessions, we found reduced fasting values of plasma glucose $(p=0.002)$ and $2 \mathrm{~h}$ values after an OGTT $(\mathrm{p}=0.004)$, and reduced incremental area under the blood glucose vs. time curve (IAUC) $(\mathrm{p}=0.028)$, as well as lowered diastolic blood pressure $(p=0.030)$ and BMI $(p=$ 0.002 ). The improvements in the intervention group was significantly different from the control group for fasting plasma glucose $(p=0.020)$ and insulin $(p=0.021)$, triglycerides $(p=0.045)$ and BMI ( $p=0.035)$. At baseline, almost half of the women had MetS. After the intervention, a significantly $(\mathrm{p}=0.003)$ larger proportion in the control group (45\%) than in the intervention group (19\%) had developed MetS.

Conclusions: Our culturally adapted health education approach may reduce MetS and possible T2D in the target group by improving diet and increasing the level of physical activity, but further research is needed to explore whether the observed effects are sustained.

CORRESPONDING AUTHOR: Victoria T. Hjellset, PhD, University of Oslo, Oslo, 0363; v.t.hjellset@medisin.uio.no

\section{OS21}

\section{8:30 AM-8:48 AM \\ OS21-B}

\section{SUSTAINABILITY AND HEALTH SAFETY} OF AN INTERDISCIPLINARY SECONDARY PREVENTION PROGRAM FOR INDUSTRIAL WORKERS

Sanjit R. Joshi, MBA ${ }^{1}$ and Padam P. Prasain, $\mathrm{MA}^{2}$

${ }^{1}$ Jagdamba Spinning mills Pvt. Ltd, Kathmandu-35, Nepal and ${ }^{2}$ Industrial unit, Jagdamba Cement Industries Pvt. Ltd, Kathmandu, Nepal.

OBJECTIVE: Two hundred and fifteen industrial workers suffering from occupational skin diseases (OSD) have attended a 6months combined dermatological and educational prevention program with an education and counseling scheme as well as an intervention The aim of this program, conducted from 2007-2009, was to enable the affected worker to remain at work without suffering from major OSD.
METHODS: To assess the sustainability of this interdisciplinary medical, behavior and educational training program, the intervention group ( $\mathrm{IG}, \mathrm{N}=215)$ and a control group (CG, Industrial worker with OSD who solely received dermatological treatment, $\mathrm{N}=85$ ) were followed up 9 month and 2 years after their individual project participation by a standardized questionnaire. A subcohort of the intervention group ( $\operatorname{IG}(2007$,$) participants in$ 2007, $\mathrm{N}=62$ ) was followed up again 2 years after their participation. RESULTS: The follow-up survey 9 months after the beginning of the program (response rate: $\mathrm{IG}$ : $\mathrm{N}=163,75.8 \%$; CG: $N=80,94.1 \%)$ showed that $71.8 \%(\mathrm{~N}=117)$ of the intervention group could remain in work as opposed to $60.0 \%$ $(\mathrm{N}=48)$ in the control group. In the intervention group $14.7 \%$ gave up work due to OSD versus $22.5 \%$ in the control group (no statistically significant effect). In the 2-years follow-up (response rate: IG: $\mathrm{N}=172,80 \%$; $\mathrm{CG}: \mathrm{N}=55,64.7 \%) 58.7 \%(\mathrm{~N}=101)$ of the IG remained at work versus $29.1 \%(\mathrm{~N}=16)$ of the CG. In the IG $12.8 \%$ had stopped work because of OSD versus $27.3 \%$ in the CG $(p<0.001$, Cramer's V=0.26). Two years after intervention, the follow-up showed a stabilization of the effects shown by the 1-year follow-up Results: In addition to the criteria 'remaining' or 'not remaining at work' the results show that our interdisciplinary intervention program led to an increased and sustained knowledge on OSD and more adequate prevention at the work place in the IG. The results confirm that interdisciplinary training can be successful in effecting self-protection, behavior change against workplace hazards by using positive approaches that include the learning of "safe" behavior and insuring transferability to real workplace settings ("empowerment"). Combined preventive measures as studied in this program have recently become the standard offered by different statutory accident liability insurances for patients in different wet work occupations with OSD.

CORRESPONDING AUTHOR: Sanjit R. Joshi, MBA, Jagdamba Spinning mills Pvt. Ltd, Kathmandu-35, n/a; joshi.sanjit@gmail. com

\section{8:48 AM-9:06 AM}

OS21-C

WORKPLACE INTERVENTIONS TO IMPROVE

THE COPING SKILLS OF THE EMPLOYEES

Adrienne Stauder, PhD, Zoltán Cserháti, MD, András Székely, MA, Szilvia Ádám, PhD, Barna Konkoly Thege, PhD and Mária S. Kopp, DSc

Institute of Behavioural Sciences, Semmelweis University Budapest, Budapest, Hungary.

We compared the impact of two stress management interventions delivered at the workplace.

Workplaces from divers background were randomly selected either to receive a 16 lesson (16x45') coping skills training, or a 4 lesson interactive presentation on stress and coping.

166 employees, selected by their superiors, participated in the long, and 188 in the short program. At 3 month follow up, 
questionnaires were completed by $63,2 \%$ and $61,2 \%$ of the participants. Work related stress was measured by the Siegrist Effort-Reward (ERI) and the Karasek Job Content (JCQ) questionnaires. Stress and outcome indicators included Cohen Perceived Stress Scale (PSS10), Carlson Work-Family Conflict (WFC), Spielberger Trait Anxiety Inventory (STAIT), shortened Beck Depression Inventory (BDI), Subjective somatic symptoms (PHQ15), WHO Well-being (WWB5), Rahe Life Meaning (LM). During the study period, there was no change in job stress (JCQ) or effort-reward imbalance (ERI). Compared to baseline, in both intervention groups there was significant decrease in stress level (PSS10, WFC), a reduction in symptom scores (STAIT, PHQ15) and an increase in well being (WWB5, LM). Overcommitment decreased only in the long intervention group. Employees working in the human sector (teachers, social workers, health care providers) benefited more, than industrial workers or those working in law enforcement.

Our results confirm the benefits of the stress management interventions at the workplace. The short, 4 lesson program was found nearly as effective as the longer 16 lesson one. These results suggest the feasibility of short interventions, and the importance of focusing on overcommitment, a key factor in work-related stress. Further analysis are needed to clarify the magnitude of the beneficial effects in connection with personal and work stress characteristics of the participants.

Research supported by the Hungarian Ministry of Health.

CORRESPONDING AUTHOR: Adrienne Stauder, PhD, Institute of Behavioural Sciences, Semmelweis University Budapest, Budapest, 1089; staadr@net.sote.hu

\section{EARLY CAREER AWARDEE \\ 9:06 AM-9:24 AM \\ OS21-D}

OVERCOMMITMENT AT WORK DECREASED AFTER STRESS MANAGEMENT TRAINING

Zoltan Cserhati, Medical doctor, Szilvia Adam, PhD, Adrienne Stauder, PhD, Barna Konkoly Thege, PhD, Andras Szekely, economist and Maria S. Kopp, Professor

Institute of Behavioural Sciences, Semmelweis University Budapest, Hungary, Budapest, Hungary.

Aim: Overcommitment is one of the key factors of work-related stress. We examined its association with other stress indicators. We carried out individual-focused interventions and examined their effect on overcommitment and other parameters.

Methods: 474 employees from different economic sectors took part in our study.

Overcommitment was measured by Siegrist Effort-Reward Imbalance (ERI) Overcommitment subscale. Stress and quality of life indicators as Cohen Perceived Stress Scale (PSS10), Carlson Work-Family Conflict, Spielberger Trait Anxiety Inventory (STAI-T), Subjective Health Complaints (PHQ15), and WHO Well-being (WWB5) were also taken.

283 persons participated in a two-day stress management training where communication skills, assertiveness, empathy and various coping strategies were taught. 191 employees took part in a short program of 4 hours. At 3 month follow up 73\% of the participants ( $N=229$ and 117) completed our questionnaire.

Results: Controlled for age and gender, overcommitment in work showed strong correlation with effort-reward imbalance, perceived stress, anxiety, WHO well-being, work-related work-family conflict.

Overcommitment decreased significantly after intervention in the two-day training group $(p<0,001)$ and showed no significant change in the short program group.

In the two-day training group we found significant decrease in perceived stress $(\mathrm{p}<0,001)$, anxiety $(\mathrm{p}<0,001)$, work-related work-family conflict $(\mathrm{p}<0,01)$, subjective health complaints $(\mathrm{p}<0,001)$ and significant increase in WHO well-being $(\mathrm{p}<0,01)$ among employees with high baseline overcommitment score $(\mathrm{N}=132)$. Among participants with low overcommitment scores $(\mathrm{N}=97)$ there were no significant changes in these parameters.

Conclusion: Stress-management trainings can be effective to decrease overcommitment at work and are more beneficial for participants with higher overcommitment. Further investigations are needed to clarify the role of overcommitment in work-related stress and quality of life.

Research supported by Hungarian Ministry of Health. 
CORRESPONDING AUTHOR: Zoltan Cserhati, Medical doctor, Institute of Behavioural Sciences, Semmelweis University Budapest, Hungary, Budapest, 1089; csirzol@yahoo.com

\section{9:24 AM-9:42 AM}

OS21-E

WORK ABILITY INDEX IN PORTUGUESE WORKERS: THE ROLE OF DEMOGRAPHIC FACTORS

Carlos Silva, PhD, ${ }^{1}$ Anabela Pereira, PhD,${ }^{1}$ Vânia Amaral, Master, ${ }^{1}$ Alexandra Pereira, Master, ${ }^{1}$ Gustavo Vasconcelos, Master, ${ }^{1}$ Vítor Rodrigues, $\mathrm{PhD},{ }^{3}$ Jorge Silvério, $\mathrm{PhD}^{2}$ and Paulo Nossa, $\mathrm{PhD}^{2}$

${ }^{1}$ Educational Sciencies, University of Aveiro, Aveiro, Portugal;

${ }^{2}$ University of Minho, Braga, Portugal and ${ }^{3}$ University of Coimbra, Coimbra, Portugal.

Ilmarinen has developed the Work Ability Index (WAI) in 1991 as a tool for evaluation of the concept of work ability. According to the results obtained by this author and his colleagues at the Finnish Institute of Occupational Health through a longitudinal study of 11 years, the WAI is presented as a good predictor of early retirement due to disability and validated support for preventive interventions or rehabilitation. Promote the ability to work in the labour force is even more important to allow individuals to maintain their health and functional capacity in the reform period.

Considering the foregoing, the present study aims to evaluate the work ability index in Portuguese workers in various professions and see how the ability to work is linked to demographic characteristics.

A cross-sectional study was carried out, including 1955 workers (40,3\% males) from chemical and metal-mechanic industry, nurses, teachers and public administration. Subjects responded to self-administered questionnaires evaluating demographic characteristics and work ability index. The causal relationship between the sociodemographic characteristics and the WAI was quantified by calculating the odds ratio and confidence intervals of $95 \%$.

In conclusion, it was found that the vast majority of workers analyzed present an ability to work fairly well. And consistent with the literature, it appears that the younger the better their individual work capacity. Similarly, this study shows that male workers and married workers have a better capacity for work compared to female workers and unmarried ones.

It is an instrument of great importance to occupational health, in that it permits evaluation of the working capacity of the individual, the perception of the current state of work ability compared to his best, allowed the identification of areas of health injured and potentiate loss of working capacity and thus corrective measures, allows the evaluation of the effectiveness of these measures in overall capacity for work.
CORRESPONDING AUTHOR: Vânia Amaral, Master, Educational Sciencies, University of Aveiro, Aveiro, 3810-193; vania. amaral17@gmail.com

OS22

8:30 AM-8:48 AM

OS22-A

PREVALENCE OF PSYCHIATRIC COMORBIDITY

IN CHRONIC LOW BACK PAIN PATIENTS

Silje E. Reme, PsyD, ${ }^{1,3}$ Tone Tangen, $\mathrm{PhD},{ }^{2,3}$ Hege R. Eriksen, $\mathrm{PhD}^{2,1}$ and Trygve Moe, $\mathrm{MD}^{2,4}$

${ }^{1}$ Department of Health Promotion and Development, University of Bergen, Bergen, Norway; ${ }^{2}$ Uni Health, Uni Research, Bergen, Norway; ${ }^{3}$ Department of psychiatry, Haukeland University Hospital, Bergen, Norway and ${ }^{4}$ Friskvernklinikken, Oslo, Norway.

Background and Aim: In chronic low back pain (CLBP) there is a high degree of comorbidity, and the prevalence of comorbid psychiatric disorders is between $40 \%-100 \%$. The aim of this study was to assess the prevalence of psychiatric comorbidity in a population of CLBP patients, using a psychiatric diagnostic interview. Methods: 400 patients sick listed between 2 and 10 months for unspecific LBP were included in the study. All were recruited as part of an ongoing trial in secondary care, and were assessed with the Mini-International Neuropsychiatric Interview (MINI), which is a short structured diagnostic interview for DSM-IV and ICD-10 psychiatric disorders.

Results: The prevalence of current psychiatric disorders was $30 \%$. The diagnoses included a wide range of psychiatric disorders, with the most common being major depressive disorders (4\%), substance abuse $(4.3 \%)$, anxiety disorders (10\%), and somatoform disorders $(18 \%)$. There were no gender differences in prevalence of psychiatric disorders, and no differences in mean duration of back pain between those with and without a psychiatric disorder.

Discussion: The results show that CLBP patients present with more psychiatric disorders than the general population, but less compared to previous studies of psychiatric comorbidity in CLBP. The difference could be a result of different compensational systems, different settings were the interviews were conducted, or different characteristics of the populations in the comparative studies.

Conclusion: In a large population of CLBP patients, $30 \%$ fulfilled the criteria for at least one current psychiatric disorder when measured with a diagnostic interview. The results imply that screening LBP patients for psychopathology in secondary care is important and should involve a wide range of psychiatric disorders since psychopathology might have consequences for prognosis and treatment.

CORRESPONDING AUTHOR: Silje E. Reme, PsyD, Department of Education and Health Promotion, University of Bergen, Bergen, 5015; silje.reme@uib.no 


\section{8:48 AM-9:06 AM}

OS22-B

\section{PROGNOSTIC FACTORS FOR THE TWO-YEAR COURSE}

OF ACTIVITY LIMITATIONS IN EARLY OSTEOARTHRITIS

OF THE HIP AND/OR KNEE: RESULTS FROM THE CHECK COHORT

Jasmijn Holla, MSc, ${ }^{2}$ Martijn Steultjens, PhD, ${ }^{1,2}$ Leo Roorda, $\mathrm{PhD}, \mathrm{MD}, \mathrm{PT}^{2}$ and Joost Dekker, $\mathrm{PhD}^{1,2}$

${ }^{1}$ VU University Medical Centre, Amsterdam, Netherlands and

${ }^{2}$ Jan van Breemen Institute, Amsterdam, Netherlands.

Objective: To predict the two-year course of activity limitations in patients with early knee and/or hip osteoarthritis.

Methods: The CHECK cohort comprising participants $(\mathrm{N}=1002)$ with early osteoarthritis-related knee and/or hip symptoms was followed for two years. Participants completed questionnaires, and underwent physical, laboratory and radiographic examination. Regression models were used to examine whether baseline variables predicted the course of activity limitations as measured with the WOMAC. Analyses were performed separately for participants with knee symptoms and participants with hip symptoms.
Results: After two-years of follow-up activity limitations slightly decreased in participants with knee symptoms, and were not significantly changed in participants with hip symptoms. Large between subject variation was observed in WOMAC change scores. In participants with knee symptoms, few activity limitations at baseline, non-western or Indonesian ethnicity, low educational level, bilateral hip pain, high comorbidity count, high BMI, pain on palpation of the joint line, high bodily pain and poor general health perception were associated with a two-year increase in activity limitations. In participants with hip symptoms, few activity limitations at baseline, low educational level, bilateral hip pain, high comorbidity count, no paid employment, few physical activity during leisure, restricted active hip flexion, poor general health perception, and frequent use of the pain coping strategy transformation were associated with a two-year increase in activity limitations.

Conclusion: After two years of follow-up large between subject variation was observed in the course of activity limitations. Risk factors for an unfavorable course of activity limitations are identifiable already at an early stage of the disease.

CORRESPONDING AUTHOR: Martijn Steultjens, PhD, VU University Medical Centre, Amsterdam, 1007 MB; m.steultjens@vumc.nl

\section{CITATION ABSTRACT \\ 9:06 AM-9:24 AM \\ OS22-C \\ CHANGING PAIN TOLERANCE WITH UNCONSCIOUS ILLNESS MEMORY}

Jos F. Brosschot, PhD, Esther Meerman, MSc and Bart Verkuil, PhD

Psychology, Leiden University, Leiden, Netherlands.

Medically unexplained symptoms (MUS) are an economic and humanitarian burden. Amongst

them, pain complaints without organic pathology are the most prevalent. Theoretically, activated

illness-related memory may cause reporting of symptoms by changing perception and

interpretation of bodily signals. In the current study, we used a subliminal priming technique to

test whether activating memory related to illness without conscious awareness leads to increased reporting of pain. The subliminal priming task consisted of a simple computer task during which words were shown for a short duration $(34 \mathrm{~ms})$. Participants were divided over four different conditions, with prime words describing either (a) health complaints, to activate an illnessrelated memory, or three control categories: (b) neutral words, (c) words describing bodily sensations and (d) negative valence words. The latter two conditions were added to test whether reduced pain tolerance would already be observed after the semantic activation of these two components or correlates of health complaints. For pain stimulation, we used a cold pressor task (CPT). We hypothesized that participants primed with health complaint words would show less pain tolerance (PT) compared to participants primed with neutral words. Participants were healthy students ( $\mathrm{n}=66$; mean age $=21.57$ years, $\mathrm{SD}=3.45 ; 84.8 \%$ female). Participants primed with health complaint words $(\mathrm{n}=15$; mean $\mathrm{PT}=77.9 \mathrm{~s}, \mathrm{SD}=79.7)$ showed reduced pain tolerance compared to participants primed with neutral words $(\mathrm{n}=17$; mean $\mathrm{PT}=127.2 \mathrm{~s}, \mathrm{SD}=92.5), \mathrm{t}(30)=-$ $1.963, \mathrm{p}=.027$ (one-tailed). Participants primed with either sensation words ( $\mathrm{n}=17$; mean $\mathrm{PT}=95.9 \mathrm{~s}, \mathrm{SD}=75.3$ ) or negative valence words ( $\mathrm{n}=17$; mean $\mathrm{PT}=95.4 \mathrm{~s}, \mathrm{SD}=77.8)$ did not significantly differ in pain tolerance from participants primed with neutral words, respectively $\mathrm{t}(32)=-0.581, \mathrm{p}=.282$ (one-tailed) \& $\mathrm{t}(32)=-0.859, \mathrm{p}=.197$ (one-tailed). Thus, pain complaints can be involuntary produced by activated illness-related memory. This memory is thought to be chronically over-activated in MUS patients. This would explain why they have complaints without observable bodily pathology and thus without a medical explanation. 
CORRESPONDING AUTHOR: Jos F. Brosschot, PhD, Psychology, Leiden University, Leiden, 2300RB; brosschot@fsw.leidenuniv.nl

\section{9:24 AM-9:42 AM}

OS22-D

DECREASES IN CATASTROPHIZING ARE ASSOCIATED WITH DECREASES IN DISABILITY OVER BEHAVIORAL MIGRAINE TREATMENT

Elizabeth K. Seng, MS, Annette Magyar, HSDG, Kenneth A. Holroyd, PhD, Jana B. Drew, PhD and Constance C. Cottrell, PhD Psychology, Ohio University, Athens, $\mathrm{OH}$.

Background: Catastrophizing is an integral concept in the fearavoidance model of chronic pain, which has only recently been applied to headache disorders. Thorn (2007) found that behavioral treatment decreases pain-related catastrophizing. Holroyd (2007) found that lower levels of catastrophizing were associated with lower levels of disability. The relationship between changes in catastrophizing and changes in disability in the treatment of headache disorders has not been examined.

Methods: After receiving one month of optimal acute therapy, 232 severe migraine sufferers $(79 \%$ female) were randomized into a 2 [Behavioral Migraine Management (BMM) vs. No BMM] X 2 (Propranolol vs. Placebo) treatment design. Participants received 4 months of BMM and/or dose adjustment, and were followed for an additional twelve months. During office visits, participants completed The Pain Catastrophizing Scale (PCS) and two measures of headache-related disability [Headache Disability Inventory (HDI); Migraine Specific Quality of Life Questionnaire (MSQL)].

2 (BMM vs. No BMM) X 2 ( $\beta$-blocker vs. Placebo) mixed models for repeated measures analyses were conducted to examine changes in PCS. Within-group mixed models for repeated measures analyses were used to examine relationships over time between changes in PCS and changes in headache-related disability (HDI, MSQL) across multiple assessment points. The 176 participants who received treatment, completed the BMM administration and/or medication dose adjustment period were included in analyses.

Results: PCS decreased during BMM, BMM X Time $\mathrm{F}(1,1075)=$ $20.85, \mathrm{p}<.01$. The $\beta$-blocker effect was not significant, $\mathrm{p}>.20$. Within-BMM analyses revealed that decreases in PCS were associated with decreases in both MSQL, $F(1,551)=126.03$, $\mathrm{p}<.05$, and HDI, $\mathrm{F}(1,551)=165.24, \mathrm{p}<.05$.

Conclusions: BMM decreased catastrophizing in severe migraine sufferers. For individuals who received BMM, decreases in catastrophizing were associated with decreases in headacherelated disability. Catastrophizing may be a useful target for behavioral migraine treatment.

CORRESPONDING AUTHOR: Elizabeth K. Seng, MS, Psychology, Ohio University, Athens, OH, 45701; es842306@ohio.edu

\section{9:42 AM-10:00 AM}

OS22-E

DETERMINANTS OF CHANGE IN PERCEIVED DISABILITY OF PATIENTS WITH NON-SPECIFIC CHRONIC LOW BACK PAIN

Audy P. Hodselmans, PT,dr, ${ }^{1}$ Pieter U. Dikstra, ProfDr, ${ }^{2}$ Jan H. Geertzen, profdr ${ }^{2}$ and Cees P. Schans, Profdr ${ }^{1}$

${ }^{1}$ Physical Therapy applied sciences, Hanze University Groningen, Groningen, Netherlands and ${ }^{2}$ Center for Rehabilitation Unversity medical center groningen, University Groningen, Groningen, Netherlands.

\section{DETERMINANTS OF CHANGE IN PERCEIVED DISABILITY OF PATIENTS WITH NON-SPECIFIC CHRONIC LOW BACK PAIN}

AP.Hodselmans, PU.Dijkstra, JHB.Geertzen, HR.Schiphorst

Preuper, CP van der Schans

Introduction: In the rehabilitation of non-specific chronic low back pain (CLBP) patients the emphasis has shifted from biomedical to bio-psychosocial rehabilitation. These rehabilitation programs reduce perceived disability. However it is unclear which determinants contribute to this reduction. Change in psychophysical capacity, calculated as the ratio between physical capacity and perceived effort, may be a determinant of change in perceived disability.

Aim: The aim of this historical cohort study was to identify determinants for change in perceived disability measured with the Roland Morris Disability Questionnaire (RMDQ) in patients with non-specific CLBP after a cognitive somatic rehabilitation program.

Method: Data of 84 outpatients with non-specific CLBP who participated in a rehabilitation program were gathered. Aerobic capacity, physical lifting capacity, perceived lifting effort, psychophysical capacity (psychophysical static leg lift, psychophysical static trunk lift, psychophysical dynamic lifting capacity), and perceived disability (RMDQ) were assessed. Associations between change in RMDQ and potential determinants were calculated. Variables significantly changed with the change in RMDQ were entered in a multivariate linear regression analysis (backward).

Results: Change in psychophysical static trunk lift $(\mathrm{r}=-0.51)$ and psychophysical dynamic lifting capacity $(\mathrm{r}=-0.53)$ and psychophysical static leg lift capacity $(r=-0.23)$ were significantly associated with change in RMDQ. The RMDQ score at baseline $(\beta=-0.438)$, change in psychophysical dynamic lifting capacity ( $\beta=-0.109)$, psychophysical static trunk lift capacity $(\beta=-0.038)$, psychophysical static leg lift capacity $(\beta=-0.012)$ and static leg lift capacity $(\beta=0.007)$ all contributed significantly to the regression model $(\mathrm{r} 2=52 \%)$.

Conclusion: Improvements in psychophysical lifting capacity are determinants for a reduction of perceived disability after rehabilitation in non-specific CLBP patients. 
Keywords: Determinants of change in perceived disability, nonspecific chronic low back pain

CORRESPONDING AUTHOR: Audy P. Hodselmans, PT,PhD, Physical Therapy applied sciences, Hanze University Groningen, Groningen, 9714 CE; a.p.hodselmans@pl.hanze.nl

OS23

\section{8:30 AM-8:48 AM}

OS23-A

OUTCOMES OF COGNITIVE BEHAVIORAL INTERVENTION IN TWO ETHNIC GROUPS WITH TYPE 2 DIABETES

Jillian Inouye, $\mathrm{PhD},{ }^{1}$ Richard Arakaki, $\mathrm{MD}^{2}$ and James Davis, $\mathrm{PhD}^{2}$ ${ }^{1}$ Nursing and Dental Hygiene, University of Hawaii at Manoa, Honolulu, HI and ${ }^{2}$ John A Burns School of Medicine, University of Hawaii at Manoa, Honolulu, HI.

Aims: Because there is little information on interventions for Asians and Pacific Islanders (API) the purpose of this study was to 1) examine the effects of cognitive behavioral intervention (CBI) on improving health, psychosocial, and behavioral outcomes in API with type 2 diabetes; and to 2) determine variations between the groups.

Methods: API participants who met the inclusion and exclusion criteria were enrolled and randomized into two groups. Groups met for 6 successive weekly meetings that averaged 2 hours, with group sizes ranging between 2-6 participants and their significant others. Participants in the CBI group were provided 6 modules on mood management, relaxation, biofeedback, cognitive restructuring, values clarification, and cultural responses to life styles. The diabetes educational/support (DES) group participants were provided modules of a refresher course on diabetes education topics and allowed to express their issues and needs. They all completed a demographic form, and various measures on depression, quality of life, self efficacy, self-care activities, health beliefs and adherence measure. Results: The 207 participants between the ages of 18-76 years had a mean age of 58 years. Three-fourths of the participants were Asians and one fourth primarily PIs. Pre and post intervention data indicated that the CBI group on the CESD scored 1.95 units lower than the DES group $(p<0.02)$, a $19.3 \%$ decrease relative to the baseline average. For the SF-36 subscale on emotional well being, the CBI group scored 3.92 units higher than the DES group $(\mathrm{p}<$ 0.0457 ), a $4.9 \%$ increase relative to the baseline average. On the Fatigue subscale, the CBI group scored 4.72 units higher than the DES group $(p<0.03)$, a $7.9 \%$ increase relative to the baseline average.

Conclusions: Initial intervention results indicate efficacy of CBI for depression and some quality of life measures. The important long term effects of CBI will be assessed with the soon to be completed one-year follow up data.

CORRESPONDING AUTHOR: Jillian Inouye, PhD, School of Nursing and Dental Hygiene, University of Hawaii, Honolulu, HI, 96822; jinouye@hawaii.edu

\section{8:48 AM-9:06 AM}

OS23-B

BEHAVIORAL RESPONSE TO THE INFLUENZA A H1N1 EPIDEMIC IN MEXICO

Claudia Infante, DSc, Liliana Giraldo, MSc and Rosalba Casas, PhD

School of Medicine, National University of Mexico, Mexico City, Mexico.

The 23rd of April, the Mexican population woke up to an unfamiliar influenza epidemiological alarm. The Mexican government implemented vigorous pandemic control measures. The objective of the present study was to describe the psycho-social reactions to the epidemic, risk perception behaviors and compliance with the recommended control measures. An online questionnaire that was posted at the National University's Web page in May 2009, was answered by 13,690 students and employees. Knowledge, beliefs, perceptions, attitudes and behaviors in regard to both, the epidemic and the contingency, were studied. Results: $23 \%$ of the population believed that the epidemic was not real but in fact political manipulation, while $34 \%$ thought it was a new influenza strain. Worry was the most commonly reported feeling in the academic employees $(74 \%)$, while students felt mainly confused or anxious (40\%). The measurement of the chronological tendency of these feelings showed that at the beginning the population was mainly worried and anxious, then they felt confused, isolated and depressed, and finally they felt discriminated. Information was the most widely expressed need during the contingency and the University Web page was the most trusted source. 55\% thought that the epidemic was more severe in Mexico than in other countries. The higher the perceived severity of the problem, the higher the acceptance of vigorous population control measures. Those that personally knew someone affected by the influenza A H1N1 virus were more likely to have sought the seasonal flu vaccine (OR 2.1, $\mathrm{p}<0.0001)$. Contingency had the following social impacts on daily life: impossibility to assist to public entertainment places which were closed (68\%), interruption of academic work $(60 \%)$, increased time with the family $(50 \%)$, more frequent hand washing (91\%) and mask wearing (66\%). Immediate behavioral research must be considered within epidemic preparedness plans.

CORRESPONDING AUTHOR: Claudia Infante, DSc, School of Medicine, National University of Mexico, Mexico City, 14000; claudiainfante@prodigy.net.mx 
9:06 AM-9:24 AM

OS23-C

AN INTERVENTION TO ENCOURAGE ACTIVITY

AND HEALTHY EATING IN PEOPLE RECOVERING

FROM RECENT-ONSET PSYCHOSIS

Alison J. Wearden, $\mathrm{PhD},{ }^{1}$ Karina Lovell, $\mathrm{PhD},{ }^{2}$ Max Marshall, $\mathrm{PhD},{ }^{3}$ Timothy Bradshaw, $\mathrm{PhD},{ }^{2}$ Nusrat Husain, $\mathrm{PhD}^{3}$ and Jeff Warburton, $\mathrm{MSc}^{4}$

${ }^{1}$ School of Psychological Sciences, University of Manchester, Manchester, United Kingdom; ${ }^{2}$ School of Nursing Midwifery and Social Work, University of Manchester, Manchester, United Kingdom; ${ }^{3}$ School of Medicine, University of Manchester, Manchester, United Kingdom and ${ }^{4}$ Early Intervention Service, Lancashire Care NHS Trust, Preston, United Kingdom.

People with psychosis are at increased risk of weight gain, heart disease and diabetes. While part of this risk is attributable to medication, the impact of psychosis on life style also plays a role. We aimed to design and test an intervention to encourage exercise and healthy eating in this group.

Our development work included a systematic review of previous randomised controlled trials of healthy living interventions, to determine which aspects of interventions had been successful previously. We interviewed service-users and case-managers to determine preferences, accessibility and feasibility of different models. We performed a systematic synthesis of the outputs of these exercises, and used this to design a culturally sensitive intervention incorporating theory-based intervention techniques.

Our systematic review suggested that our intervention should last longer than a few months, and that it should include some structured activities. Some service users expressed a preference for group activities while others preferred to meet with a therapist on a one-to-one basis. Our intervention employs a mix of one-to-one sessions with a trained support-time-recovery (STR) worker, and the opportunity to join activities and groups facilitated by exservice-users. It starts by eliciting service users' beliefs and knowledge about their weight gain, their motivations to change and their goals. Service users and STR workers then collaboratively develop action plans with cues for action, strategies for overcoming barriers, regular reviews and feedback.

The intervention is currently being piloted. The potential strengths and limitations of our development process will be discussed.

CORRESPONDING AUTHOR: Alison J. Wearden, PhD, School of Psychological Sciences, University of Manchester, Manchester, M13 9PL; alison.wearden@manchester.ac.uk

\section{9:24 AM-9:42 AM}

OS23-D

SLEEP PROBLEMS AS A PREDICTOR OF WEIGHT GAIN

Tea Lallukka, PhD, Peppi Lyytikäinen, student, Ossi Rahkonen, $\mathrm{PhD}$ and Eero Lahelma, Professor

Department of Public Health, University of Helsinki, Helsinki, Finland.

Purpose: Sleep problems and obesity are prevalent public health problems. However, the contribution of sleep problems to weight gain is poorly understood. We examined sleep problems as predictors of subsequent weight gain.

Methods: Data were derived from baseline (2000-2002, response rate 67\%) and follow-up (2007, response rate 83\%) postal questionnaires surveys ( $n=7330$, aged $40-60$ years). At baseline, all participants were employed by the City of Helsinki, Finland. The follow-up questionnaire surveys were mailed to all those who returned the baseline questionnaire. Weights and heights were self-reported. Those who had gained at least five kilograms during the follow-up were examined as reporting major weight gain. Sleep problems were measured by problems maintaining and initiating sleep, and non-restorative sleep during the previous month. Logistic regression models were fitted, adjusting for age, baseline body mass index, socio-demographic background, health behaviours, health status, sleep duration, and shift work.

Results: Altogether $26 \%$ of women and $24 \%$ of men had gained at least five kilograms during the follow-up. Sleep problems at least three times a week ( $20 \%$ of women and $17 \%$ of men) were associated with major weight gain among women after adjusting for age (OR 1.41; CI 95\% 1.13-1.75). The adjustments for baseline body mass index, marital status, education, heavy drinking, current smoking, physical activity, limiting long standing illness, sleep duration, and shift work had practically no effect on the association. However, adjustment for baseline mental disorders attenuated the association. Among men, none of the associations were statistically significant.

Conclusions: Sleep problems remained associated with weight gain among women after pertinent risk factors have been accounted for. Associations among men and the effects of co-occurrence of mental health problems need to be confirmed in further studies. Promoting better sleep and preventing sleep problems might help prevent weight gain and subsequent health risks.

CORRESPONDING AUTHOR: Tea Lallukka, PhD, Department of Public Health, University of Helsinki, Helsinki, 00014; tea. lallukka@helsinki.fi

\section{9:42 AM-10:00 AM \\ OS23-E \\ EVALUATION OF A SELF-HELP PROGRAM IN ACUTE TINNITUS}

Nele Nyenhuis, Dipl-Psych, ${ }^{1}$ Sarah Zastrutzki, Dipl,-Psych, ${ }^{2}$ Cornelia Weise, Dr, ${ }^{2,3}$ Burkard Jaeger, Dr $^{2}$ and Birgit KroenerHerwig, $\mathrm{Dr}^{1}$

${ }^{1}$ Department of Clinical Psychology and Psychotherapy, GeorgAugust-Universität Göttingen, Göttingen, Germany; ${ }^{2}$ Department of Psychosomatics and Psychotherapy, Hannover Medical School, Hannover, Germany and ${ }^{3}$ Department of Behavioural Sciences and Learning, Linköping University, Linköping, Sweden.

Tinnitus can lead to a substantial decline in psychological wellbeing. Existing treatments focus mainly on chronic tinnitus-sufferers, but treatment in the acute phase seems also desirable to prevent a decompensation. The aim of this study was to develop a self-help program for acute tinnitus-sufferers and to evaluate its efficacy. Participants were 18 to 75 years old and had suffered a minimum of two weeks and a maximum of 6 months from subjective tinnitus. 
They were randomly assigned to either a CBT-based self-help treatment (internet- or paper-based, $n=68)$ or a control group $(n=38)$. Some basic information about tinnitus was given to members of the control group, none of which was relevant to treatment.

Tinnitus distress and coping abilities were measured as main outcomes. Assessments were performed via self-report inventories at entry and after 3 months. Results of a one-way repeated-measures ANOVA showed that overall tinnitus distress improved in both groups over time $(\mathrm{F}(104)=40.27, \mathrm{p}=.000, \mathrm{Eta} 2=.28)$. In addition, the intervention condition led to more extended improvement than the control condition $(\mathrm{F}(104)=12.56, \mathrm{p}=.001$, Eta2 $=.11)$. In both groups use of helpful self-instructions became more likely over time $(\mathrm{F}(99)=5.86, \mathrm{p}=.017, \mathrm{Eta} 2=.06)$ while unhelpful self-instructions decreased $(\mathrm{F}(99)=15.59, \mathrm{p}=.000, \mathrm{Eta} 2=.17)$. Between-group comparison showed that participants in the self-help program reported more helpful self-instructions $(\mathrm{F}(99)=5.66$, p. $=.019$, $\mathrm{Eta} 2=.05)$ and fewer unhelpful self-instructions $(\mathrm{F}(99)=5.78$, $\mathrm{p}=.018$, Eta2 $=.06)$ than controls. Post-hoc tests revealed no differences between internet-based and paper-based intervention and the control condition.

The results indicate that the self-help manual leads to additional improvement regarding tinnitus distress and enhances copingabilities. To further investigate the long-term effects of the program on tinnitus distress a 6-month follow-up measurement is planned.

CORRESPONDING AUTHOR: Nele Nyenhuis, Dipl-Psych, Clinical Psychology and Psychotherapy, Georg-AugustUniversität Göttingen, Göttingen, 37073; nnyenhu@gwdg.de

\section{Friday \\ August 6, 2010 SYMPOSIA SESSIONS 10:30 AM-12:00 PM}

\section{CITATION ABSTRACT}

SS17

COGNITIVE BEHAVIOURAL THERAPY AND MECHANISMS OF CHANGE FOR FATIGUE IN CHRONIC ILLNESS

Rona Moss-Morris, $\mathrm{PhD}$

School of Psychology, University of Southampton, Southampton, United Kingdom.

Fatigue is a symptom common to many chronic illnesses. It is also an unpleasant side-effect of medical interventions such as curative treatment for cancer. Even when fatigue is not one of the key presenting symptoms, it if often described by patients as one of the most disabling and difficult to manage. This symposium will present a series of treatment trials which have looked at cognitive behavioural therapy (CBT) treatment packages for fatigue in three different patient groups, cancer, multiple sclerosis (MS) and chronic fatigue syndrome (CFS). Four key questions will be addressed during the symposium talks and discussion 1. Is CBT an effective treatment for fatigue across conditions? 2. Can CBT for fatigue be offered in a more cost-effective and potentially widely available web-based format? 3 . What are the key mediators of CBT in the treatment of fatigue? 4. How can our understanding of mechanisms of change in CBT help maximise our treatment effects?

The first talk presents data from a randomised controlled trial of CBT versus a brief nursing intervention for the reduction of fatigue during curative cancer treatment. CBT is shown to be the only effective treatment. Interestingly, increases in physical activity do not mediate the treatment effect. The second study shows that therapist driven CBT is useful for the treatment of fatigue in MS. Mediation analysis suggests that the key mediator of change in fatigue is change in patients' negative beliefs about fatigue. The third study discusses the development of a web-based intervention to treat MS fatigue. Data from a pilot RCT suggests that this approach can substantially reduce fatigue and is economical, but the effect size is smaller than therapist delivered CBT. The final presentation looks at both mediators and predictors of the effectiveness of CBT in a sample of patients undergoing treatment at a specialised fatigue clinic.

Catastrophising about symptoms is the key treatment mediator, while extreme behavioural or cognitive avoidance predicts worse treatment outcome. The symposium discussion will focus on the best way to package CBT for different patient groups. How the findings from these talks can help us understand models of fatigue and treatment processes will also be addressed.

CORRESPONDING AUTHOR: Rona Moss-Morris, PhD, University of Southampton, Southampton, SO171RE; remm@soton.ac.uk 


\section{CITATION ABSTRACT}

SS17a

WHAT MEDIATES THE EFFECT OF COGNITIVE BEHAVIOUR THERAPY ON CHRONIC FATIGUE IN MS PATIENTS?

Hans Knoop, $\mathrm{PhD},{ }^{2,1}$ Kirsten Van Kessel, $\mathrm{PhD},{ }^{3}$ Trudie Chalder, $\mathrm{PhD}^{4}$ and Rona Moss-Morris, $\mathrm{PhD}^{1}$

${ }^{1}$ School of Psychology, University of Southampton, Southampton, United Kingdom; ${ }^{2}$ Expert Centre Chronic Fatigue, Radboud University Nijmegen Medical Centre, Nijmegen, Netherlands; ${ }^{3}$ Department of Psychological Medicine, University of Auckland, Auckland, New Zealand and

${ }^{4}$ Academic Department of Psychological Medicine, Guy's, King's and St Thomas' School of Medicine, London, United Kingdom.

Background

The majority of patients with multiple sclerosis (MS) report fatigue that is often chronic and interferes with daily life. Although fatigue in MS is poorly understood, cognitions and behaviours in relation to symptoms and fatigue appear to play a role in the maintenance of MS fatigue. An intervention was developed on the basis of a cognitive-behavioural model of MS fatigue. The efficacy of this cognitive behaviour therapy (CBT) was tested in a randomised controlled trial. CBT resulted in a greater reduction in MS fatigue than relaxation training directly following treatment. In this study the data of this RCT were reanalysed to address the following questions:

1) Does CBT lead to significant change in the cognitions and behaviours hypothesised to perpetuate MS fatigue when compared to relaxation therapy?

2) Do any of these cognitive-behavioural variables mediate the effect of CBT on MS fatigue?

Method

70 patients were randomised to $\mathrm{CBT}(\mathrm{n}=35)$ or relaxation training $(\mathrm{n}=35)$. Patients completed the Cognitive and Behavioural Responses to Symptoms Questionnaire, the Brief Illness Perception Questionnaire (B-IPQ) which was modified to measure beliefs about fatigue, and the Fatigue Scale, pre- and post-therapy. Multiple mediation analysis using bootstrapping was used to determine which of the variables mediated the change in fatigue brought on by CBT.

Results

There was a significant change in several cognitive variables directly following CBT. Patients focused less on MS symptoms and their interpretation of MS symptoms became less negative (embarrassment, catastrophising and damage beliefs decreased). Also, the overall perception of fatigue, measured by the B-IPQ, became more positive. Mediation analysis showed that the change in fatigue severity in the CBT group was fully mediated by the change in the perception of fatigue. Change in anxiety also co-variated with the reduction in fatigue.

Discussion

Changing beliefs about fatigue seems an important factor in CBT for MS fatigue. The change in fatigue is also closely related to a reduction in anxiety. The results of the present study are comparable to those investigating the effects of $\mathrm{CBT}$ for chronic fatigue in other conditions.

CORRESPONDING AUTHOR: Hans Knoop, PhD, Expert Centre Chronic Fatigue, Radboud University Nijmegen Medical Centre, Nijmegen, 6500 HB; j.knoop@nkcv.umcn.nl 


\section{CITATION ABSTRACT \\ SS17b \\ HOW IMPORTANT IS INCREASING PHYSICAL ACTIVITY AIMING TO REDUCE FATIGUE DURING CURATIVE CANCER TREATMENT? A RANDOMISED CONTROLLED TRIAL OF A BRIEF NURSING INTERVENTION AND COGNITIVE BEHAVIOUR THERAPY}

Gijs Bleijenberg, PhD, ${ }^{1}$ Martine Goedendorp, MSc, ${ }^{1}$ Hans Knoop, PhD, ${ }^{1}$ Stans Verhagen, MD,${ }^{2}$ Marlies Peters, $\mathrm{MSc}^{2}$ and Marieke Gielissen, $\mathrm{PhD}^{1}$

${ }^{1}$ Expert Centre Chronic Fatigue, Radboud University Nijmegen Medical Centre, Nijmegen, Netherlands and ${ }^{2}$ Medical Oncology, Radboud University Nijmegen Medical Centre, Nijmegen, Netherlands.

Most patients experience fatigue during cancer treatment. The assumption is that physical activity is essential to reduce fatigue. However, the mediating role of physical activity in fatigue interventions during cancer treatment has never been demonstrated.

Our aim was to evaluate two interventions during curative cancer treatment in a RCT and to determine the role of physical activity.

Patients $(\mathrm{N}=220)$ with various malignancies receiving cancer treatment with curative intent were assessed before (T1) and two months after cancer treatment (T2) and randomized to a brief nursing intervention (BNI), cognitive behaviour therapy (CBT) or usual care (UC). Fatigue was measured with the Checklist Individual Strength, physical activity with actigraphy and questionnaires.

The BNI ( 2 sessions) focused solely on increasing and maintaining physical activity during cancer treatment. The CBT (6 sessions) focused additionally on regulating the sleep-wake cycle, accepting the consequences of cancer and treatment, managing the experience of cancer in relation with others, and challenging to make future plans.

The CBT group was significantly less fatigued after cancer treatment compared to the UC, but no difference was found between BNI and UC (ANCOVA). Mediation analysis showed that none of the physical activity measures mediated the reduction in fatigue realised by CBT.

Conclusion: $\mathrm{CBT}$ proved to be effective in reducing fatigue during cancer treatment. Physical activity had no mediating role in the effect of CBT. Thus, our findings indicate that with CBT it was possible to realize a significant reduction in fatigue without a lasting increase in physical activity.

Funding provided by the Dutch Cancer Society.

CORRESPONDING AUTHOR: Gijs Bleijenberg, PhD, Expert Centre Chronic Fatigue, Radboud University Nijmegen Medical Centre, Nijmegen, 6500 HB; G.Bleijenberg@nkcv.umcn.nl

\section{SS17c}

THE POTENTIAL EFFICACY AND COST-EFFECTIVENESS OF A WEB-BASED COGNITIVE BEHAVIOURAL THERAPY PROGRAMME FOR MULTIPLE SCLEROSIS FATIGUE: THE MS-INVIGOR8 PILOT TRIAL

Rona Moss-Morris, PhD, ${ }^{1}$ Kirsten van Kessel, $\mathrm{PhD},{ }^{4}$ Lucy Yardley, $\mathrm{PhD},{ }^{1}$ Gary Wills, $\mathrm{PhD}^{2}$ and Paul McCrone, $\mathrm{PhD}^{3}$

${ }^{1}$ School of Psychology, University of Southampton, Southampton, United Kingdom; ${ }^{2}$ Electronics and Computer Sciences, University of Southampton, Southampton, United Kingdom; ${ }^{3}$ Institute of Psychiatry, King's College London, London, United Kingdom and ${ }^{4}$ University of Auckland, Auckland, New Zealand.

Background: Fatigue is a common, distressing and disabling symptom associated with multiple sclerosis (MS). Therapist delivered, manualised cognitive behavioural therapy (CBT) has been shown to be effective in treating MS fatigue. The purpose of this study was to develop and pilot the efficacy and costeffectiveness of a web-based version of this CBT package.

Methods: The MS-Invigor8 programme was developed over 9 months by a team of software developers, psychologists and people with MS. 40 patients with MS fatigue were then randomised to the web intervention in conjunction with 3 therapy phone calls $(n=23)$ or a wait list control group $(n=17)$. Patients were assessed using standardised self-report measures of fatigue severity and impact prerandomisation and 10 weeks later. They also completed measures of health care use and quality of life for the economics analysis. Patients in the intervention group were interviewed to see how they had experienced the treatment approach.

Results: Intention-to-treat analysis showed that patients randomised to MS-Invigor8 reported significantly greater reductions on the Fatigue Scale $(\mathrm{F}(3,36)=16.59, \mathrm{p}<.001)$ and the Modified Fatigue Impact Scale $(\mathrm{F}(3,34)=17.15, \mathrm{p}<.001)$ at 10 weeks than those on the wait list. The QALY gain based on the EQ-5D was 0.015 higher in the intervention group $(p=0.038)$, but there were no differences in service 
use between the groups. The mean number of sessions completed in the intervention group was 5 out of 8. Qualitative data suggested that most patients found the programme and telephone sessions helpful, had made lifestyle modifications to manage their fatigue, felt they were more in control of their fatigue and had greater understanding of this symptom. Modifications were suggested to further improve the programme.

Discussion: This pilot study suggests that computerised CBT may be an effective, accessible and acceptable intervention for managing fatigue in MS. The improvements in quality of life and the relatively low cost of the intervention highlight the potential for it to be a costeffective way of offering CBT to this group.

CORRESPONDING AUTHOR: Rona Moss-Morris, PhD, University of Southampton, Southampton, SO171RE; remm@soton.ac.uk

\section{SS17d}

ASSESSING PREDICTORS OF OUTCOME AND MEDIATORS OF CHANGE IN CHRONIC FATIGUE SYNDROME AFTER COGNITIVE BEHAVIOUR THERAPY

Trudie Chalder, $\mathrm{PhD}$, MSc

Psychological Medicine, King's College London, London, United Kingdom.
Background: Cognitive behaviour therapy (CBT) is an effective treatment for chronic fatigue syndrome (CFS). The process of change in cognitive behaviour therapy for chronic fatigue syndrome (CFS) is potentially complex.

Methods: First we examined whether cognitive behavioural responses changed after a course of CBT. Second, we investigated which cognitive and behavioural responses were important in mediating change in the treatment of CFS/ME and examined factors which predicted treatment outcome in a specialist clinic in the UK.

Findings: Three hundred and fifty six patients entered the study and commenced CBT. Change in catastrophic thinking mediated change in social adjustment in CFS/ME. Extreme behavioural and cognitive avoidance predicted worse social adjustment and fatigue.

Discussion: Implications of these findings for understanding treatment process and mechanisms of change in a range of disorders will be discussed.

CORRESPONDING AUTHOR: Trudie Chalder, PhD, MSc, King's College London, London, SE5 9RJ; trudie.chalder@kcl. ac.uk

\section{CITATION ABSTRACT \\ SS18 \\ METHODS TO INCREASE COLON CANCER SCREENING IN AUSTRALIA, CANADA, AND THE UNITED STATES \\ Ronald E. Myers, $\mathrm{PhD}$ \\ Medical Oncology, Thomas Jefferson University, Philadelphia, PA. \\ Colon cancer (CRC) screening rates in Australia, Canada, and the United States are sub-optimal. \\ Investigators in these countries are developing behavioral interventions, grounded in the \\ Preventive Health Model (PHM), that can be used to increase CRC screening. In Australia, the \\ Adelaide Colorectal Cancer Collaborative has developed a web-based, interactive decision support tool that utilizes empirical evidence about the predictors of screening to deliver tailored information designed to improve readiness to screen. In several Canadian provinces, the Institute for Health Research Team is carrying out pilot studies of invitation letter promotions related to stool blood testing and colonoscopy, initiating tailored-navigation intervention research, and developing CRC screening management software useful for multiple cancer screening programs. In the United States at the Kimmel Cancer Center of Thomas Jefferson University, preference- based tailored navigation methods are being tested in diverse populations served by primary care practices. In each setting, developmental research had has been completed and randomized controlled trials of PHM-based interventions are underway. The symposium will elaborate the theoretical foundation for the international research initiatives; describe tailored navigation interventions and provide data on implementation results; and summarize lessons learned to date.}


CORRESPONDING AUTHOR: Ronald E. Myers, PhD, Medical Oncology, Thomas Jefferson University, Philadelphia, PA, 19107; ronald.myers@jefferson.edu

\section{SS18a}

USING TAILORED NAVIGATION TO INCREASE CRC SCREENING IN PRIMARY CARE PRACTICES

IN THE UNITED STATES

Ronald E. Myers, PhD

Medical Oncology, Thomas Jefferson University, Philadelphia, PA.

Background: Colorectal cancer (CRC) screening is underutilized. Effective methods to increase screening use are needed. This study sought to determine the impact of tailored navigation on CRC screening in primary care.

Methods: The study included 154 primary care practice patients who were 50 or more years of age, were eligible for CRC screening, and had an office visit within two years prior to study initiation. Baseline telephone survey data were collected on participant sociodemographic characteristics, psychosocial factors, and screening test (fecal occult blood test (FOBT) or colonoscopy) decision stage. By comparing decision stage data, we identified that test with the highest decision stage (i.e., preferred screening test). Participants who preferred FOBT were sent an FOBT kit and a reminder. Those preferring colonoscopy were sent colonoscopy instructions. After this mailing, a study patient navigator made a telephone call to guide participants towards screening. Six-month endpoint survey and medical records data were obtained. Univariable and multivariable analyses were performed to identify predictors of screening and of change in preferred screening test decision stage.

Results: At endpoint, 63 (41\%) study participants had screened. From baseline to endpoint, overall screening preference increased for $75(63 \%)$ participants. Age and perceived salience and coherence (i.e., screening is important and sensible) were positive, significant predictors of screening use $(p=0.02$ and $p=0.05$, respectively); while only age predicted change in overall screening preference $(\mathrm{p}=0.03)$

CORRESPONDING AUTHOR: Ronald E. Myers, PhD, Medical Oncology, Thomas Jefferson University, Philadelphia, PA, 19107; ronald.myers@jefferson.edu

\section{$\mathrm{SS} 18 \mathrm{~b}$}

\section{POPULATION-BASED SCREENING - LESSONS} IN CANADA, FROM SALIENT MESSAGES TO SOFTWARE Paul Ritvo, $\mathrm{PhD}$

Division of Preventive Oncology, Cancer Care Ontario, Toronto, ON, Canada.

In 2007, Ontario (population, 12.9 million) launched a provincewide CRC screening program, ColonCancerCheck, offering FOBT to average risk individuals and colonoscopy to individuals with first degree family members affected by CRC. Also in 2007, the Alberta Colorectal Cancer Screening Program was launched, an initiative recommending all provincial residents be tested annually with FOBT (beginning at age 50) while in 2008, the Forzani and MacPhail Colon Cancer Screening Centre, opened in Calgary ( Alberta) the only non-hospital Canadian colon cancer screening facility. In Canada, only $40 \%$ report being up-to-date CRC screening using FOBT or endoscopy, with FOBT compliance especially suboptimal. The Canadian Institute for Health Research Team in Population-based Colorectal Cancer Screening was accordingly funded to investigate CRC screening activities in several provinces and to originate multiple activities, notably focus groups aimed at ascertaining optimal methods for promoting FOBT-CRC and Targeted and Tailored- Navigated Intervention Trials that directly test specific approaches to increasing screening uptake. In this presentation, a range of findings will be shared, as related to regionally-diverse Focus Group Studies, pilot studies of invitation letter promotions on a provincial basis (Ontario) and to individuals awaiting colonoscopies (Alberta); and TailoredNavigation interventions directed at attendees of a large primary care practice where patient navigators undertake promotion/ information related to screening promotion. Findings will also be shared from efforts in Saskatchewan, Canada to develop CRC screening management software.

CORRESPONDING AUTHOR: Ronald E. Myers, PhD, Medical Oncology, Thomas Jefferson University, Philadelphia, PA, 19107; ronald.myers@jefferson.edu

SS18c

OPTIMIZING PARTICIPATION IN A POPULATION-BASED BOWEL CANCER SCREENING PROGRAM - LESSONS LEANED FROM LARGE SCALE TRIALS IN AUSTRALIA Carlene Wilson, $\mathrm{PhD}, \mathrm{MBA}^{1}$ and Ingrid Flight, $\mathrm{MA}^{2}$

${ }^{1}$ Cancer Prevention, Flinders University, Adelaide BC, SA, Australia and ${ }^{2}$ Preventative Health Research, CSIRO, Adelaide BC, SA, Australia.

In 2006, the Australian government implemented the National Bowel Cancer Screening Program. Invitations were sent to people turning 55 and 65 years. They offered the opportunity to screen for Bowel Cancer with the Faecal Immunochemical Test. Participation rate was $39.7 \%$. This less than optimal uptake highlights the size of public health challenge. How do we optimize participation rates utilizing publicly delivered rather than clinician-based interventions?

The Adelaide Colorectal Cancer Collaborative has undertaken trials designed to identify solutions. Results indicate improved participation associated with use of an advance notification letter, reminder letters, medical practitioner endorsement (including personalized letters), the removal of dietary restrictions from the requirements of testing, and minimally "distasteful” sampling technology.

Recent behavioral studies have further highlighted the importance of addressing attitudinal and cognitive barriers. These studies indicate that the Australian population is influenced by the way messages are framed, confidence in their capacity to use the kit (self-efficacy), perceived salience and coherence of screening, cancer worries, response efficacy (i.e., belief in the effectiveness of screening), social influences and perceived susceptibility to bowel cancer. 
The collaborative has also developed a web-based, interactive decision support tool that utilizes empirical evidence about the predictors of screening to deliver tailored information designed to improve readiness to screen. Pilot data highlight the potential utility of this approach. They document the prevalence of internet use and acceptability in the target age group, establish the validity of the social cognitive variables included in the decision support tool as correlates of Australians' readiness to screen for bowel cancer, and demonstrate within the laboratory, the greater effectiveness of tailored compared with generic messages.

CORRESPONDING AUTHOR: Ronald E. Myers, PhD, Medical Oncology, Thomas Jefferson University, Philadelphia, PA, 19107; ronald.myers@jefferson.edu

\section{SS19}

\section{THE FUTURE OF CLASSIFICATION AND TREATMENT OF SOMATOFORM DISORDERS}

Winfried Rief, PhD

Clinical Psychology and Psychotherapy, University of Marburg, Marburg, Germany.

Medically unexplained physical symptoms are a major challenge not only for the general health care system, but also for the development of reliable and valid classification approaches and effective interventions. A revision of the classification of somatoform disorders seems necessary, although there is continuing discussion what should be revised in detail. Joel Dimsdale, the speaker of the DSM-V work group, will present the latest DSM-V proposal for the classification of somatic symptoms causing significant psychological distress, and empirical data supporting this new classification approach. Per Fink and colleagues will provide data from Denmark indicating one new approach to tackle diagnostic problems of somatoform disorders, and its empirical validation. New frontiers of interventions are reported by $\mathrm{A}$. Schröder who focus on functional somatic symptoms. The presentation of Arthur Barsky will finally address the different nature of somatic symptoms, and especially the distinction between nonpathological bodily discomfort versus psychopathological features that need to be diagnosed. The symposium will result in a discussion of future options for classification and treatment of this prevalent and costly medical condition.

CORRESPONDING AUTHOR: Winfried Rief, PhD, Clinical Psychology and Psychotherapy, University of Marburg, Marburg, 35032; rief@staff.uni-marburg.de

\section{SS19a}

RETHINKING SOMATIZATION; WHAT'S NORMAL AND WHAT'S NOT

Arthur Barsky, MD

Brigham \& Women's Hospital, Boston, MA.

Medically unexplained somatic symptoms may result from many sources other than psychiatric disorder, and somatization therefore is not by definition psychopathological. Such symptoms are a final common pathway for the expression of situational, intrapsychic, behavioral, interpersonal and sociocultural distress. Symptoms without a serious medical cause are a nearly universal human experience, arising in the daily lives of perfectly healthy people. Some are a non-verbal interpersonal communication in which the individual seeks the acknowledgment from others that they are in distress and suffering, hoping thereby to secure special attention, support, and consideration. For some patients, a lifetime of medically unexplained complaints can be best conceptualized as a stable personality trait, one that has been associated with other enduring personality characteristics. Somatization can also be understood as a form of illness and sick role behavior - not something an individual has, but rather something he/she does. Alternatively, emotional distress, particularly when combined with certain early childhood experiences of trauma, adversity or deprivation, can generate somatic symptoms. Preoccupied and fearful attachment styles that are learned in childhood can also lead to somatization in adulthood.

The presentation of medically unexplained symptoms is also subject to socio-cultural influences. Cultural norms and values encourage the expression of distress in certain forms and discourage it in others; these cultural forces shape the experience and reporting of bodily symptoms by influencing how they are identified, interpreted, described and reported. Finally, the search for neurobiological mechanisms of somatization is under way, implicating the hyperresponsivity of centrally acting proinflammatory cytokines, dysregulation of central and peripheral serotonergic systems, and dysregulation of the hypothalamic-pituitary-adrenal axis.

It is only when these medically unexplained symptoms are recurrent, especially distressing, severe, prolonged, and disabling that they may be considered psychopathological.

CORRESPONDING AUTHOR: Arthur Barsky, MD, Brigham \& Women's Hospital, Boston, MA, 02115; abarsky@partners.org

\section{SS19b}

DOES THE BODILY DISTRESS SYNDROME DIAGNOSIS UNIFY THE FUNCTIONAL SOMATIC SYNDROMES AND SOMATOFORM DISORDERS?

Per Fink, DMSc, PhD and Andreas Schröder, MD

The Research Clinic for Functional Disorders and Psychosomatics, Aarhus University Hospital, Aarhus, Denmark.

Background: In order to clarify the classification of physical complaints not attributable to verifiable, conventionally defined diseases, a new diagnosis of Bodily distress syndrome was introduced. The aim of this study was to test if patients diagnosed with one of six different functional somatic syndromes or a DSMIV somatoform disorder were captured by the new diagnosis.

Method: A stratified sample of 978 consecutive patients from neurological $(n=120)$ and medical $(n=157)$ departments and from primary care $(n=701)$ was examined applying post-hoc diagnoses based on the SCAN diagnostic instrument. Diagnoses were assigned only to clinically relevant cases, i.e. patients with impairing illness. 
Results: Bodily distress syndrome included all patients with Fibromyalgia $(n=58)$, Chronic fatigue syndrome $(n=54)$ and Hyperventilation syndrome $(n=49), 98 \%$ of those with Irritable bowel syndrome $(n=43)$ and at least $90 \%$ of patients with Noncardiac chest pain $(n=129)$, Pain syndrome $(n=130)$ or any somatoform disorder $(n=178)$. The overall agreement of Bodily distress syndrome with any of these diagnostic categories was $95 \%$ (95\% CI [93.1; 96.0]; kappa 0.86, p<0.0001).

Conclusion: Bodily distress syndrome covers most of the relevant "somatoform" or "functional" syndromes presenting with physical symptoms not explained by well-recognised medical illness, thereby offering a common ground for the understanding of functional somatic symptoms. This may help unifying research efforts across medical disciplines and facilitate delivery of evidence-based care, given that very similar treatments have been shown to be effective in various functional somatic syndromes and somatoform disorders.

CORRESPONDING AUTHOR: Per Fink, DMSc, PhD, The Research Clinic for Functional Disorders and Psychosomatics, Aarhus University Hospital, Aarhus, 8000; per.fink@aarhus.rm.dk

\section{CITATION ABSTRACT \\ SS19c}

A NOVEL TREATMENT APPROACH FOR PEOPLE WITH SEVERE FUNCTIONAL SOMATIC SYNDROMES (STRESS-1): RANDOMIZED TRIAL

Andreas Schröder, MD, ${ }^{1}$ Emma Rehfeld, MD ${ }^{1}$ Eva $\emptyset$ rnbøl, MSc, ${ }^{1}$ Michael Sharpe, Professor, ${ }^{3}$ Rasmus W. Licht, MD, $\mathrm{PhD}^{2}$ and Per Fink, DMSc, $\mathrm{PhD}^{1}$

${ }^{1}$ The Research Clinic for Functional Disorders and Psychosomatics, Aarhus University Hospital, Aarhus C, Denmark; ${ }^{2}$ Mood Disorders Research Unit, Aarhus University Hospital, Aarhus C, Denmark and ${ }^{3}$ Psychological Medicine Research, School of Molecular and Clinical Medicine, University of Edinburgh, Edinburgh, United Kingdom.

Background: Functional somatic syndromes (FSS) such as fibromyalgia or irritable bowel syndrome are highly prevalent and may be persistent and disabling for patients and costly to services. We assessed the efficacy of a cognitive behavioral group treatment (STreSS) for patients with a variety of severe FSS in improving physical health.

Methods: Patients aged 20-45 year with chronic, disabling FSS or somatoform disorders who met criteria for the unifying diagnosis of severe, multi-organ Bodily distress syndrome were eligible for this randomized trial at a General University Hospital. 120 patients were randomly assigned by means of a computer-assisted block protocol to a cognitive behavioral group treatment (54 patients) or to enhanced usual medical care (66 patients). The primary outcome was the difference in change from baseline to 16 months in the SF-36 Perceived Physical Health (PPH) score between the two groups. Analysis was by intention to treat.

Results: 44 patients in the STreSS group and 50 in the comparison group completed follow-up at 16 months. By use of repeated measures mixed effects modeling and multiple imputation, all 120 participants were included in the analysis. The adjusted mean difference in PPH score change from baseline to 16 months was 4.1 (95\% CI 1.5-6.7, $\mathrm{p}=0.002)$, a moderate effect size $(0.51$; [0.19-0.83]). The number needed to treat to achieve one additional treatment response was 5 (353, $\mathrm{p}=0.03)$.

Conclusion: Specialized Treatment for Severe Bodily Distress Syndromes (STreSS) provides a promising model for the management of patients with various severe FSS. Our approach may be a feasible alternative to the current organization of care in many different medical specialties.

CORRESPONDING AUTHOR: Andreas Schröder, MD, The Research clinic for Functional Disorders and Psychosomatics, Aarhus University Hospital, Aarhus C, 8000; malene.skjoeth@, aarhus.rm.dk

\section{SS19d}

\section{SOMATIC SYMPTOM DISORDERS IN DSM V}

Joel Dimsdale, MD

University of California, San Diego, La Jolla, CA.
Major efforts are under way to develop and test a new version of DSM. One of the workgroups has been charged to review somatoform disorders, a group of disorders of special relevance to behavioral medicine. Feedback from the audience is sought concerning the proposed changes.

Somatoform disorders have been problematic in the current diagnostic schema. The term itself is confusing. Somatoform disorders are rarely diagnosed although they are frequently encountered in medical practice. The diagnosis tends to emphasize constructs such as "medically unexplained symptoms," thereby 
basing the diagnosis on negative rather than positive manifestations. Patients regard the terminology as offensive. It is difficult to understand the prevalence and treatment of such disorders as long as a hierarchical decision model prevents diagnosing these disorders when other psychiatric disorders are present.

The workgroup feels that the hallmark of such disorders is the presence of somatic symptoms associated with significant distress and/or dysfunction. These disorders typically present first in nonpsychiatric settings. Somatic symptoms are common in every day life \& may be initiated, exacerbated or maintained by combinations of biological, psychological and social factors. Somatic symptom disorders can accompany diverse general medical as well as psychiatric diagnoses. When criteria are fulfilled, for example, for major affective disorder and for complex somatic symptom disorder, both diagnoses should be coded.

Major changes are summarized below:

1. move "Psychological Factors Affecting a Medical Condition" from "other factors that may be of interest."

2. introduce a new diagnosis "Complex Somatic Symptom Disorder." This disorder is characterized by multiple somatic symptoms, as well as misattributions, excessive concern or preoccupation with symptoms \& illness. The workgroup feels that this rubric accurately describes disorders such as somatization disorder, hypochondriasis, undifferentiated somatoform disorder, and pain disorder.

3. The workgroup proposes changes to criteria for conversion disorders. Most notably, we recommend removing the requirement for psychological factors to be associated with symptom onset. This matter is difficult to establish. There is currently discussion regarding whether conversion disorder is most usefully aligned with somatic symptom disorders or anxiety disorders.

CORRESPONDING AUTHOR: Joel Dimsdale, MD, University of California, San Diego, La Jolla, CA, 92093-0804; jdimsdale@, ucsd.edu

\section{Friday \\ August 6, 2010 \\ PAPER SESSIONS \\ 10:30 AM-12:00 PM}

OS24

10:30 AM-10:48 AM

OS24-A

ELABORATING THE TARGETS FOR A PRACTICE INTERVENTION TO IMPROVE OPPORTUNISTIC CHLAMYDIA SCREENING BY GP PRACTICES

Louise M. Wallace, Professor, ${ }^{1}$ Angela Hogan, BSc, ${ }^{2}$ Julie Bayley, MSc, ${ }^{1}$ Cliodna McNulty, $\mathrm{Dr}^{2}$ and Jananee Jesuthasan, MSc ${ }^{1}$

${ }^{1}$ Applied Research Centre in Health and Lifestyle Interventions Faculty of Health and Life Sciences, Coventry University, Coventry, United Kingdom and ${ }^{2}$ Health Protection Agency Primary Care Unit, Health Protection Agencey, Gloucester, United Kingdom.
Background: Chlamydia is a common sexually transmitted infection and is often asymptomatic, so early treatment can be difficult. The National Chlamydia Screening Programme aimed to increase screening of sexually active 15-24 year olds. Half of PCT's are under-performing against the Department of Health's target for all Primary Care Trusts (PCTs) to screen $18 \%$ of this population. Therefore there is a need to improve opportunistic screening in GP practices. As part of a wider project to develop an intervention this study assesses individual staff attitudes and intentions to screening, and informs the content of the intervention.

Method: A theory based survey was developed from previous qualitative and quantitative data from practice staff. The survey was administered to clinical and non-clinical staff in GP surgeries; of which 466 had been returned

Findings: Intention to offer Chlamydia screening was high (mean 5.50, $\mathrm{SD}=1.07)$. Subjective norms and $\mathrm{PBC}$ scores were also high (mean 6.01, $\mathrm{SD}=1.16$; mean $6.24, \mathrm{SD}=.82$ respectively), with attitude similarly positive $(5.97(\mathrm{SD}=.75)$. Linear regression analysis showed that intention significantly predicted approximately $13 \%$ variation in behaviour in clinical staff $(F=34.708, \operatorname{AdjR} 2=.131$, $\mathrm{p}<.001)$ but was insignificant for non-clinical staff. Attitude and subjective norms were the most significant predictors of intention $(\mathrm{p}<.005)$ whilst PBC did not significantly predict intention.

Discussion: Intention to offer screens is directly related to actual behaviour, and those who have more positive attitudes and perceive their colleagues to be more approving are more likely to offer screens. The lack of predictive power with non-clinical staff may reflect their view that offering information is beyond their role, and thus it is important to raise awareness of the value of doing so.

CORRESPONDING AUTHOR: Louise M. Wallace, Psychology, Coventry University, Coventry, CV1 5FB; 1.wallace@coventry.ac.uk

\section{0:48 AM-11:06 AM}

OS24-B

A STUDY OF GERIATRIC MENTAL HEALTH SERVICES TRAINING NEEDS AMONG PRIMARY CARE PERSONNEL IN ORISSA, INDIA

Sanghamitra Pati, MD DNHE MPH, Sandipana Pati, MBBS PGDGM and Saumyakanta Das, MD (Psychiatry)

Geriatric Medicine, Unit IX Hospital, Bhuabneswar, India.

The challenge of increasing numbers of elderly in need of mental health services in the developing world will not be adequately met without effective undergraduate education for all health professionals caring for elderly patients. There is a need for continuing professional training to keep the skills, attitudes and performance of care providers appropriate and update for recognizing and treating old age mental health disorders. There is also a need for more research on effective training approaches. Keeping this in mind, the present study sought to investigate the level of psychogeriatric knowledge and attitudes towards care of the elderly among primary care personnel of Orissa, India and an assessment of psycho-geriatric training needs in primary health care.

The specific aims of the study were to explore the present knowledge of geriatric psychiatry among primary care physicians, 
nurses and social workers and compare it with that of psychiatry residents and nurses and to explore the attitudes of the same groups toward the elderly. It was expected that the study results would provide relevant inputs towards developing continuing professional education methods for primary care professionals and to enable them to care for old age mental problems

The participants of this study were primary care health workers, nurses and physicians. The reference group for comparison comprised psychiatry residents and nurses. All were administered knowledge and attitude questionnaire. Background questionnaire covering previous psycho-geriatric education, work experience and need for further training was collected and the data were assessed as group mean comparisons. Psycho-geriatric knowledge was measured by a 40-item knowledge test on psychopharmacology, clinical syndromes, assessment, old age and psychotherapy. The attitudes were measured by a 30-item Likert-type questionnaire.

The geriatric psychiatry knowledge level and the reported attitudes toward care of the elderly of the primary care groups were significantly lower than the reference group. The discrepancy was attributed to lack of adequate training and undergraduate education. Most of the participants felt that they could benefit from suitable tailor-made continuous multi-professional education. The focus areas for training were clinical assessment and psychopharmacology. The multi professional approach was favored in order to optimize the benefits of care for elderly patients with multiple problems.

There is a need for continuing professional education in old age mental health for primary care personnel and its incorporation into extant primary health care. Resources provided by guidelines and modern technology should be available for professionals working with elderly.

CORRESPONDING AUTHOR: Sanghamitra Pati, MD DNHE MPH, Medical Education, SCB Medical College, Bhuabneswar, 751022; drspati@yahoo.com

\section{1:06 AM-11:24 AM}

OS24-C

SELF-EFFICACY IN DIABETES SELF-MANAGEMENT AND SELF-EFFICACY IN CHANGING SPECIFIC BEHAVIORS IN RELATION TO REGIMEN ADHERENCE

Jing Wang, BSN, PhDc, ${ }^{1,2}$ Susan M. Sereika, $\mathrm{PhD},{ }^{1,2}$ Linda M. Siminerio, $\mathrm{PhD}^{3}$ Denise Charron-Prochownik, $\mathrm{PhD},{ }^{1,2}$ Janice $\mathrm{C}$. Zgibor, $\mathrm{PhD},{ }^{2}$ Ronald E. LaPorte, $\mathrm{PhD}^{2}$ and Judith T. Matthews, $\mathrm{PhD}^{1}$ ${ }^{1}$ University of Pittsburgh School of Nursing, Pittsburgh, PA; ${ }^{2}$ University of Pittsburgh Graduate School of Public Health, Pittsburgh, PA and ${ }^{3}$ University of Pittsburgh Department of Medicine, Pittsburgh, PA.

Little is known about the impact of self-efficacy (SE) in changing specific diabetes self-management (DSM) behaviors on patient adherence to a DSM regimen, compared to general SE in DSM. We used data from the American Association of Diabetes Educators Outcome System, implemented in eight DSM education programs in western Pennsylvania, to examine the bivariate relationship between adherence to the DSM regimen and SE in changing specific behaviors and general SE in DSM among patients with type 2 diabetes. DSM regimen adherence was measured as the frequency with which patients followed their recommended DSM regimen, including medication taking, diet, exercise, and self-monitoring of blood glucose [SMBG]). Behavior-specific SE was measured by asking patients their level of confidence in making changes in these four DSM behaviors. Higher scores indicated greater adherence or confidence. General SE in DSM was measured by asking "How sure are you that you can manage your diabetes?" with four responses (A lot/ some/ a little/ not at all). We used correlation and simple linear regression to analyze the data. The sample $(\mathrm{N}=709)$ was $88.3 \%$ white and $53.8 \%$ female, with a mean age of 58.2 years and an average of 7.9 years since diagnosis of diabetes. We observed a stronger relationship with $\mathrm{SE}$ in changing diet $(\mathrm{r}=.29, \mathrm{p}<.001)$ and exercise $(\mathrm{r}=.21, \mathrm{p}<.05)$ and the corresponding adherence behaviors than we found between DSM self-efficacy and adherence to $\operatorname{diet}(\mathrm{r}=.14, \mathrm{p}<.001)$ and exercise $(\mathrm{r}=.10, \mathrm{p}<.05)$. These associations remained significant after controlling for age, gender, ethnicity, education, family history, and time since diagnosis. Medication adherence was weakly correlated with SE in DSM $(\mathrm{r}=.09, \mathrm{p}=.02)$ but not medication-specific SE $(\mathrm{r}=.25, \mathrm{p}=.08)$. No associations were found between general or specific SE measures and SMBG (ps>.05). Further evidence is needed to confirm our results in a research setting. Our findings suggest that promoting patients' confidence in managing their diabetes, especially with respect to making changes in diet and exercise could improve patient adherence to their recommended diabetes self-management regimen.

CORRESPONDING AUTHOR: Jing Wang, BSN, PhDc, University of Pittsburgh, Pittsburgh, PA, 15261; jiw38@pitt.edu

11:24 AM-11:42 AM

OS24-D

UK CLINICIANS' MOTIVATION TOWARDS USING SELF MANAGEMENT SUPPORT PRACTICES WHEN WORKING WITH PATIENTS WITH LONG TERM CONDITIONS

Louise M. Wallace, Professor, Joanna Kosmala-Anderson, PhD and Andy Turner, $\mathrm{PhD}$

Applied Research Centre for health and Lifestyle Interventions, Coventry University, Coventry, United Kingdom.

Background: Training in self management support (SMS) for long term conditions (LTC) were offered to 367 clinicians as a part of The Health Foundation's Co Creating Health Initiative. We applied the Self Determination Theory to explain how SMS behaviours are formed and sustained.

Aims: Explore the relationship between motivational regulation to support self management and clinicians' practices in SMS.

Method: We obtained 78 responses . To assess the use of SMS practices we applied the Practices in Self Management Support questionnaire (PSMS) developed by the authors. The questionnaire comprises 3 subscales: Clinical Self Management Support (CSMS), Patient Centeredness (PC) and Organization of Services to Support 
Self Management (OSMS). We adapted the Self-Regulation Questionnaire developed from previous SDT research protocols.

Results: Clinicians, who before attending ADP training were intrinsically motivated for SMS were more likely to engage in CSMS, PC and OSMS pre and post ADP (pre ADP $\mathrm{r}=.53$; $\mathrm{p}<.001 ; \mathrm{r}=.36 ; \mathrm{p}=.02$ and $\mathrm{r}=.42 ; \mathrm{p}=.007$; post ADP: $\mathrm{r}=.66$; $\mathrm{p}<.001 ; \mathrm{r}=.45 ; \mathrm{p}=.005$ and $\mathrm{r}=.36 ; \mathrm{p}=.05$ respectively). Strong integrated motivation for SMS increased engaging in CSMS pre and post $\operatorname{ADP}(\mathrm{r}=.63 ; \mathrm{p}<.001$ and $\mathrm{r}=.53 ; \mathrm{p}=.001$ respectively) and PC pre and post ADP $(\mathrm{r}=.31 ; \mathrm{p}=.05$ and $\mathrm{r}=.39 ; \mathrm{p}=.01$ respectively), but not OSMS. Identified motivation positively influenced applying CSMS both pre and post ADP $(r=.46 ; \mathrm{p}=.003$ and $\mathrm{r}=.47 ; \mathrm{p}=.003$ respectively).

Conclusion: Our findings support the importance of building an ethos of SMS and provide support for clinicians working with patients with LTC. Clinicians' attitudes and motivation to support self management is as important as attending professional training, thus organization should invest not only in staff development but also create supportive environment for the practice of SMS.

CORRESPONDING AUTHOR: Joanna Kosmala-Anderson, PhD, ARCHLI, Coventry University, Coventry, CV1 5FB; j.kosmalaanderson@coventry.ac.uk

11:42 AM-12:00 PM

OS24-E

APPLIED BEHAVIORAL MEDICINE IN PRIMARY CARE: AN INTER-PROFESSIONAL COURSE FOR ADVANCED UNDERGRADUATE STUDENTS OF MEDICINE, CLINICAL PSYCHOLOGY AND PHYSIOTHERAPY

Anne $\mathrm{H}$ Berman, $\mathrm{PhD}^{1}$ and Gabriele Biguet, $\mathrm{MSc}^{2}$

${ }^{1}$ Clinical Neuroscience, Karolinska Institutet, Huddinge, Sweden and ${ }^{2}$ Neurobiology, Care Sciences and Society, Karolinska Institutet, Huddinge, Sweden.

Background and purpose: Stress-related illness with psychosocial antecedents has increased globally. Patients seeking help in primary care often exhibit a wide variety of problems with both somatic and psychological symptoms. The role of primary care has also changed in relation to psychiatry in that it now constitutes the first-order treatment for psychiatric disorders. A biopsychosocial perspective, increased knowledge of behavioural medicine and improved inter-professional collaboration all offer a viable response to this situation. Inter-professional courses at the advanced undergraduate level provide opportunities for interprofessional learning, a learning about, and with one another. At Karolinska Institutet, a cross-program elective course in applied behavioural medicine has been offered since the fall of 2006, first for medical and psychology students, and since 2008 also for physiotherapy students. This presentation describes the course structure and presents evaluation results from the five-week interprofessional course between 2006 and 2009.

Results and discussion: In 2006 and 2007 the course was held with medicine and psychology students; in 2008, only physiother- apy and clinical psychology students were recruited. Medical students did not apply due to new program changes. All three groups have been very satisfied with the course, experiencing it as an important complement to their undergraduate education. It has been rewarding to train themselves to communicate and interact around the patient's problems from each separate perspective. Individual professional roles have become clearer, and all indicate their conviction of the need for inter-professional collaboration. Evaluation of attitudes toward other professional groups showed that only psychology students changed their perceptions of physicians and physiotherapists during the course.

Conclusion: Inter-professional courses at the advanced undergraduate level offer a useful meeting between the different professions and can therefore be an important step in applying bio-psychosocial model to work in practice. Results will also be presented from the 2009-10 courses where registered and professionally active physiotherapists, doctors and psychologists have also been recruited as course participants alongside the undergraduate students.

CORRESPONDING AUTHOR: Anne H Berman, PhD, Clinical Neuroscience, Karolinska Institutet, Huddinge, S-14104; anne.h. berman@ki.se

\section{OS 25}

10:30 AM-10:48 AM

OS25-A

THE EFFECTS OF PARENTING BEHAVIOR ON MENTAL HEALTH OF DISADVANTAGED CHILDREN

AND ADOLESCENTS

Luke Yang, $\mathrm{PhD}$

Department of Social Welfare, Hsuan Chuang university, Hsinchu, Taiwan.

Disadvantaged children and adolescents need more supportive care, and most studies show that parenting plays a significant role in explaining mental health among them. This research is to evaluate connections between supportive behavior and the need of mental care for disadvantaged boys and girls in Taiwan. By collecting the data of thirty ordinary families and thirty disadvantaged families, parenting and psychological conditions of children are categorized and compared. Major parenting behavior produces results similar to or lower in quality than those acquired from ordinary families; however, according to the analysis of scale, disadvantaged boys and girls face more conflicts and difficulties in mental condition. Even though parenting affects the mental health of young kids, it seems that other factors must be considered. Findings provide empirical support for further research and practice in supportive parenting. Additionally, the attitude of children and adolescents on feeling supportive behavior is also a key aspect to understand their mental health.

CORRESPONDING AUTHOR: Luke Yang, PhD, Department of Social Welfare, Hsuan Chuang university, Hsinchu, 300; lhy@hcu.edu.tw 
10:48 AM-11:06 AM

OS25-B

SOCIALLY DISADVANTAGED PARENTS OF CHILDREN TREATED WITH ALLOGENEIC HAEMATOPOIETIC STEM CELL TRANSPLANTATION (HSCT) IN A WELFARE BASED SOCIETY

Hanne B. Larsen, RN, MScSoc, ${ }^{1,6}$ Carsten Heilmann, MD, ${ }^{2}$ Christoffer Johansen, $\mathrm{MD}^{4,5}$ and Lis Adamsen, RN, MSc, Soc ${ }^{3}$ ${ }^{1}$ BMT unit 4042, Rigshospitalet, Copenhagen, Denmark; ${ }^{2} \mathrm{BMT}$ unit 5061, Rigshospitalet, Copenhagen, Denmark; ${ }^{3}$ The University Hospitals Center for Nursing and Care Research, Rigshospitalet, Copenhagen, Denmark; ${ }^{4}$ Department of Psychosocial Cancer Research, The Institute of Cancer Epidemiology, Copenhagen, Denmark; ${ }^{5}$ National Centre for Cancer Rehabilitation, Research, Southern Danish University, Copenhagen, Denmark and ${ }^{6}$ Faculty of Health Sciences, University Hospital of Copenhagen, Copenhagen, Denmark.

Introduction: A child's HSCT is distressing for both parents and children. The child experiences side effects to treatment, infections, Graft versus Host Disease under isolation conditions. A psychosocial intervention study aimed at reducing parents and child's level of distress identified a group of parents with an immediate, extended need for psychosocial intervention.

Patients: Parents to 25 HSCT children were included in a psychosocial intervention study during the child's admission. The intervention included daily psychosocial support.

Methods: Case study combined interviews and observational study.

Results: Parents to 8 of the 25 children had complex psychosocial problems which impaired their ability to support and care for their child during hospitalisation and after discharge. The parent's were characterised by: 1) low level income 2) low level education 3) low level support 4) marginal relationship to the work market 5) physical illness 6) psychiatric illness 7) nonwestern ethnicity and 8) Danish as a second language. Parents of the 8 children had 4 or more of the 8 characteristics, which place them at risk of social exclusion. These parents needed individual interventions such as 1) help to understand the child's HSCT treatment 2) the child's reactions 3) extended emotional support 4) help to facilitate communicate with the welfare system 5) additional economical support and 5) additional help to care for the child.

Conclusion: Parents' prior psychosocial situation in conjunction with the strain of caring for a HSCT child exceeds the parent's resources, being the reason why psychosocial interventions are required so the child's health is not placed at an additional risk.

Parents of HSCT children at risk of social exclusion experience difficulties with manoeuvring within the welfare-based system, which increases their risk of social exclusion.
Psychosocial screening and interventions to parents of HSCT children is recommended.

CORRESPONDING AUTHOR: Hanne B. Larsen, RN, MScSoc, BMT unit 4042, Rigshospitalet, Copenhagen, 2100; hanne@baekgaard. com

11:06 AM-11:24 AM

OS25-C

ASSOCIATIONS BETWEEN PARENTING PRACTICES, PARENTAL CARE AND HEALTH BEHAVIOURS OF FINNISH CHILDREN AGED 10-11

Carola Ray, MSc, PhD student, ${ }^{1,2}$ Mirjam Kalland, Adj Prof ${ }^{1}$ and Eva Roos, Adj Prof ${ }^{1,2}$

${ }^{1}$ Folkhälsan Research Center, Helsinki, Finland and ${ }^{2}$ Hjelt Institute, University of Helsinki, Helsinki, Finland.

Introduction: Parenting style, including parental care, is a general interaction between parent and child, whereas through parenting practices parents perform their parental duties like having practices about bed time or screen time. The aim of the study is to examine whether there are differences in children's health behaviours if there exist parenting practices, parental care or simultaneously both.

Methods: Cross-sectional study in Helsinki region in 2006. 1271 children aged $10-11$ (response rate $79 \%$ ) and 816 parents (64\%) answered questionnaires. 801 matching child parent pairs used in analysis. Children's questionnaire included questions about children's health behaviours, parenting practices and parental care. Parents reported additional parenting practices, education level and employment status. Logistic regression was used in analyses. Results: Reporting simultaneously high amount of parenting practices and parental care compared to few practices and no parental care was among children associated with a higher probability of frequent nutrient-dense food intake, having a regular meal pattern, sleeping at least 10 hours per night, and having less than 2 hours daily screen time. The odds ratio for less screen time was highest when reporting a high amount of practices and no parental care. The combination few practices and parental care increased the odds for higher frequent intake of nutrient-dense food. No associations were found between parenting practices, parental care and intake of energy-rich food.

Conclusion: A high amount of parenting practices concerning daily routines and simultaneously parental care seem to be associated with more favorable health behaviours in children.

CORRESPONDING AUTHOR: Carola Ray, MSc, PhD student, Folkhälsan Research Center, Helsinki, 00250; carola.ray@ folkhalsan.fi 


\section{1:24 AM-11:42 AM}

OS25-D

MATERNAL DEPRESSION AND DISRUPTIVE BEHAVIOR: GENDER DIFFERENCES IN EARLY CHILDHOOD

Caroline M. Coope, MPH, Yvonne Kelly, PhD, Anne McMunn, $\mathrm{PhD}$ and Saffron Karlsen, $\mathrm{PhD}$

Epidemiology and Public Health, University College London (UCL), London, United Kingdom.

Background: A higher prevalence of disruptive behavior is found in boys than in girls. The causes and pathways to these problems, such as socio-economic deprivation and maternal depression, remain unclear, particularly in relation to potentially different pathways by gender and at younger ages.

The objective of this paper is to examine the relationship between maternal depression and disruptive behavior and potential pathways via socio-economic circumstance and parenting behaviours in boys and girls in early childhood.

Data used were from the UK Millennium Cohort Study (MCS) made up of 18,552 families recruited between 2000-2001 when the cohort children were aged 9 months old, with follow ups at ages 3 years old $(n=15,590)$ and 5 years old $(n=15246)$.

Findings: At aged $312 \%$ of boys and $10 \%$ of girls had a high conduct score; whilst $11 \%$ of boys and $7 \%$ of girls had a high hyperactivity score. Boys with a depressed mother had a 4.5 times higher odds (unadjusted) of having a high conduct score $(\mathrm{p}<0.0001)$ and a two and half times higher odds (unadjusted) of a high hyperactivity score $(\mathrm{p}<0.0001)$ compared with boys whose mothers were not depressed. Girls with a depressed mother had a 4 times higher odds (unadjusted) of a high conduct score $(\mathrm{p}<0.0001)$ and a three-fold higher odds (unadjusted) of a high hyperactivity score $(p<0.0001)$ compared with girls whose mothers were not depressed. The relationship was tested using logistic regression models controlling for child and parent characteristics, socio-economic circumstance and parenting behaviors. The odds for both boys and girls of depressed mother having a high conduct score remained significantly higher compared to boys and girls of non-depressed mothers but were attenuated to two-fold higher odds. The odds of boys with a depressed mother having a high hyperactivity score remained significantly higher compared to boys of nondepressed mothers but decreased to a one and half times higher odds, whilst for girls the relationship was no longer significant.

Conclusion: The relationship between maternal depression and a high conduct and hyperactivity score in 3 year old boys and a high conduct score in girls is not wholly accounted for by socio-economic circumstance or parenting behaviors and therefore other mechanisms are likely to be additionally important.

CORRESPONDING AUTHOR: Caroline M. Coope, MPH, Epidemiology and Public Health, University College London (UCL), London, WC1E 6BT; caroline.coope@ucl.ac.uk
11:42 AM-12:00 PM

OS25-E

MATERNAL EMPLOYMENT AND CHILD

SOCIO-EMOTIONAL BEHAVIOR: LONGITUDINAL

EVIDENCE FROM THE UNITED KINGDOM

Noriko Cable, PhD, Yvonne Kelly, PhD, Anne McMunn, PhD, MPH and Mel Bartley, PhD

Epidemiology \& Public Health, University College London, London, United Kingdom.

Background: Mothers of young children are increasingly combining paid work with childrearing. Empirical evidence on the effects of maternal employment on children is contradictory and has little work has considered the impact of maternal employment within the context of the employment patterns of both parents.

Objectives and methods: This study examines the effects of parental employment in the early years on child socio-emotional behavior at age 5 in a recent birth cohort study in the United Kingdom. Participants of the Millennium Cohort Study were born between September 2000 and January $2002(n=18,819)$. Data on parental employment across three sweeps of data collection (infancy, age 3 and age 5) were used to investigate: (i) whether children whose mothers were in paid work during their first five years were more likely than children whose mothers were at home full-time to display adverse behavioral symptoms at age 5, independent of maternal education, mental health or economic position; (ii) whether effects of maternal employment on child socio-emotional development were cumulative in nature, or whether children were more sensitive to the effects of maternal employment during their first year; and (iii) the effects of different types of parental work arrangements on child socio-emotional behavior at age 5. Child socio-emotional behavior was measured using the Strengths and Difficulties Questionnaire with clinically relevant cut-points for problem behaviors.

Results: No evidence of detrimental effects of maternal employment in the early years on subsequent child socio-emotional behavior was seen. There were significant gender differences in the effects of parental work arrangements on behavioral outcomes. Girls whose mothers were not in paid work during their first five years were $77 \%(95 \% \quad \mathrm{CI}=1.21-2.57)$ more likely to have behavioral difficulties at age 5 than girls whose mothers were in paid work throughout their early years, independent of maternal characteristics and household income. For boys this was not the case, but boys in two-parent households in which their father was not in paid work for at least one period during their first five years were at an increased risk for behavioral problems at age 5. The most beneficial working arrangement for both girls and boys was that in which both mothers and fathers were present in the household and in paid work, independent of parental educational attainment and household income.

CORRESPONDING AUTHOR: Anne McMunn, PhD, MPH, Epidemiology \& Public Health, University College London, London, WC1E 6BT; a.mcmunn@ucl.ac.uk 


\section{CITATION ABSTRACT \\ OS26 \\ 10:30 AM-10:48 AM \\ OS26-A}

LONG WORK HOURS AND PHYSICAL FITNESS - 30-YEAR RISK OF ISCHEMIC HEART DISEASE AND ALL-CAUSE MORTALITY

Ole S. Mortensen, MD, Phd, ${ }^{1}$ Andreas Holtermann, PhD, ${ }^{2}$ Hermann Burr, PhD, ${ }^{2}$ Karen Soegaard, PhD, ${ }^{3}$ Finn Gyntelberg, DrMedSci ${ }^{1}$ and Poul Suadicani, DrMedSci ${ }^{1}$

${ }^{1}$ Occupational Medicine, Bispebjerg University Hospital, Copenhagen NV, Denmark; ${ }^{2}$ National Research Centre for the Working Environment, Copenhagen, Denmark and ${ }^{3}$ cInstitute of Sport Science and Clinical Biomechanics, University of Southern Denmark, Odense, Denmark. Background: No previous long-term studies have examined if workers with low physical fitness have an increased risk of cardiovascular mortality due to long work hours. We tested this hypothesis.

Methods: 30-year follow-up in the Copenhagen Male Study of 5,249 gainfully employed men aged 40-59 years. 274 men with cardiovascular disease were excluded from the follow-up. Physical fitness (maximal oxygen consumption, VO2Max) was estimated using the Åstrand bicycle ergometer test, and number of work hours was obtained from questionnaire items; 4,943 men were eligible for the incidence study.

Results: 587 men (11.9\%) died due to Ischemic heart disease (IHD). Cox analyses adjusted for blood pressure, smoking, alcohol, body mass index, diabetes, hypertension, physical work demands, and social class, showed that working more than 45 hours per week was associated with an increased risk of IHD mortality in the least fit (VO2Max range 15-26; HR: 2.28,CI:1.104.73), but not intermediate (VO2Max range 27-38; HR:0.94,CI:0.59-1.51) and most fit men (VO2Max range 39-78; HR:0.91,CI:0.41-2.02) referencing men working less than 40 hours per week.

Conclusion: The findings indicate that men with low physical fitness are at increased risk for IHD mortality from working long hours. Men working long hours should be physically fit.

CORRESPONDING AUTHOR: Ole S. Mortensen, MD, Phd, Occupational Medicine, Bispebjerg University Hospital, Copenhagen NV, DK-2400; o.mortensen@dadlnet.dk

\section{0:48 AM-11:06 AM \\ OS26-B}

THE EFFECT OF COGNITIVE-BEHAVIOR GROUP INTERVENTION ON BLOOD COAGULATION FUNCTION OF CORONARY HEART DISEASE PATIENTS:

\section{A CONTROLLED STUDY}

Chia-Ying Weng, $\mathrm{PhD},{ }^{1}$ Tin Kwang Lin, $\mathrm{MD},{ }^{2}$ Hsin-Hung Chiang, $\mathrm{BS},{ }^{3}$ Chiu-Tien Hsu, MSc, ${ }^{1}$ Kao-Chia Chen, MSc, ${ }^{1}$ Chao-Fen Chen, MSc, ${ }^{1,4} \mathrm{Su}-Y a$ Hseueh, $\mathrm{BS}^{3}$ and Chin-Lon Lin, $\mathrm{MD}^{2}$

${ }^{1}$ Psychology, National Chung Cheng University, Chia-Yi, Taiwan; ${ }^{2}$ Division of Cardiology, Department of Internal Medicine, The Buddhist Dalin Tzu Chi General Hospital, Chia-Yi, Taiwan; ${ }^{3}$ Health Management Center, The Buddhist Dalin Tzu Chi General Hospital, Chia-Yi, Taiwan and ${ }^{4}$ St. Joesh Hospital, Yun-Lin, Taiwan.

The purpose of this study is to develop a cognitive-behavior group intervention program to reduce the hostility levels of coronary heart disease (CHD) patients and to examine its effect on blood coagulation function. A case control study with matched age, sex and education, based on a two group pre- and post-test design was adopted. Participants of the experimental group were 9 CHD patients (mean age $=56.33 \pm 10.45$, male 67\%) who attended a weekly two-and-a-half hour session and did homework for two months. The intervention foci included psycho-education, biofeedback-assistant relaxation training, and cognitive-behavior strategies to reduce the levels of hostility affect, hostility cognition, and hostility behavior. Participants of the control group were $13 \mathrm{CHD}$ patients (mean age $=59.23 \pm 6.80$, male $69 \%$ ) who did not receive any psychological treatment until they finished the three-month waiting status. After intervention, participants showed a significant reduction in hostility level $(\mathrm{t}=4.27, \mathrm{p}<.01)$ and respiration rate $(\mathrm{t}=3.06, \mathrm{p}<.01)$, and a significant increase in prothrombin time $(\mathrm{t}=-4.75, \mathrm{p}<.001)$. The results of the repeated measure of ANOVA indicated that the experimental group revealed a significantly higher reduction of respiration rate $(\mathrm{F}=$ $8.76, \mathrm{p}<.01)$, and a marginally higher increase of prothrombin time $(\mathrm{F}=-3.66, \mathrm{p}=.06)$ than the waiting control group. In conclusion, the results of this study imply that psychological intervention is efficient to reduce the biological risk of disease recurrence for $\mathrm{CHD}$ patients.

CORRESPONDING AUTHOR: Chia-Ying Weng, PhD, Psychology, National Chung Cheng University, Chia-Yi, 62102; psycyw@ ccu.edu.tw 
11:06 AM-11:24 AM

OS26-C

QIGONG FOR HYPERTENSION: A SYSTEMATIC REVIEW

Myeong Soo Lee, $\mathrm{PhD},{ }^{1,2}$ Tae-Young Choi, MD, PhD, ${ }^{1}$ Sun-Mi Choi, OMD, $\mathrm{PhD}^{1}$ and Edzard Ernst, $\mathrm{MD}, \mathrm{PhD}^{2}$

${ }^{1}$ Division of Standard Research, Korea Institute of Oriental Medicine, Daejeon, Republic of Korea and ${ }^{2}$ Complementary Medicine, Peninsula Medical School, Universities of Exeter \& Plymouth, Exeter, United Kingdom.

Complementary therapies are popular and frequently used by patients with CVD. Survey data indicate that herbal therapies and mind-body therapies are most commonly used. One such treatment option is qigong. Several reviews have claimed that qigong has therapeutic effects on blood pressure in patients with hypertension. However, these reviews are non-systematic and therefore open to bias. To systematically assess the clinical evidence of qigong for hypertension. Databases were searched up to December 2009. All randomised clinical trials testing qigong in patients with hypertension of any origin and assessing clinically relevant outcomes were considered. Trials using any type of control intervention were included. The selection of studies, data extraction and quality assessment were performed independently by at least two reviewers. Methodological quality was evaluated using the Jadad score. 12 RCTs could be included. Seven of these RCTs tested qigong in combination with antihypertensive drugs compared with antihypertensive drugs alone. The meta-analysis of four trials reporting adequate data suggested beneficial effects in favour of qigong (weighted mean difference, systolic blood pressure $(\mathrm{mmHg})-12.06,95 \%$ confidence interval (CI) -17.12 to -7.00 ; diastolic blood pressure -8.46 , 95\% CI -12.55 to -4.37 ). Qigong alone was compared with waiting list control in 2 RCTs and was found to significantly reduce systolic blood pressure (weighted mean difference $(\mathrm{mmHg})-18.46,95 \% \mathrm{CI}-23.07$ to -13.86). In further three RCTs comparisons made were: qigong combined with antihypertensive drugs versus muscle relaxation combined with antihypertensive drugs; qigong as a sole treatment versus exercise and/or antihypertensive drugs, all reported positive results in at least some of the relevant outcome measures. The methodological quality of the original studies was low. There is some encouraging evidence to suggest that qigong is effective for lowering systolic blood pressure. However, the conclusiveness of these findings is limited. Rigorously designed trials seem warranted to confirm these results.

CORRESPONDING AUTHOR: Myeong Soo Lee, PhD, Division of Standard Research, Korea Institute of Oriental Medicine, Daejeon, 305-811; drmslee@gmail.com

\section{1:24 AM-11:42 AM}

OS26-D

EFFECTIVENESS OF A DEPRESSION MANAGEMENT AND SECONDARY PREVENTION PROGRAM ON FUNCTIONING AND EMPLOYMENT OUTCOMES OF PATIENTS FOLLOWING MYOCARDIAL INFARCTION: THE MOOD-CARE TRIAL
Adrienne O’Neil, BA Hons, ${ }^{1}$ Kristy Sanderson, BSc (Hons) $\mathrm{PhD},{ }^{2}$ Bianca Chan, BSc BA MPH ${ }^{1}$ and Brian F. Oldenburg, BSc (Hons) $\mathrm{MA} \mathrm{PhD}{ }^{1}$

${ }^{1}$ International Public Health Unit, Monash University, Melbourne, VIC, Australia and ${ }^{2}$ Menzies Research Institute, Hobart, TAS, Australia.

Background: One in five people suffer from depression after myocardial infarction (MI) or heart attack. Depression impedes important indicators of recovery including Quality of Life (QOL) and employment outcomes. Yet depression continues to remain under-recognised and under-treated in cardiac patients. We are conducting a randomised controlled trial of a state-of-the-art, telephone-delivered counselling and educational program (MOOD-CARE) for MI patients diagnosed with depression and investigating its effectiveness in relation to mental and physical functioning and employment outcomes.

Method: Newly diagnosed MI patients admitted to five hospitals in Australia are being screened for depression using the Patient Health Questionnaire (PHQ-9) $(n=1619)$. Those with PHQ9=5-19 (mild-moderately severe depression) at discharge who are still symptomatic 2 weeks later will be randomly assigned to a Usual Care (UC) or Intervention (MOOD-CARE) group, stratified by current DSM-IV diagnosis of depression versus subthreshold depression. MOOD-CARE includes up to 10 telephone counselling sessions over 6 months, delivered by clinical psychologists using Cognitive Behaviour Therapy (CBT) and coronary heart disease (CHD) risk factor management.

Results: Presentation will describe study methods, baseline recruitment and related findings. Conclusion: MOOD-CARE has the potential to improve psychosocial, vocational and health outcomes of depressed MI patients. The study will provide valuable clinical, vocational and economic information about the value of the program and its potential translation into the health system.

CORRESPONDING AUTHOR: Adrienne O'Neil, BA Hons, Epidemiology and Preventive Medicine, Monash University, Melbourne, VIC, 3004; adrienneEO@yahoo.com

\section{1:42 AM-12:00 PM \\ OS26-E \\ QUALITY OF LIFE CHANGES ASSOCIATED \\ WITH PARTICIPATION IN A CARDIAC \\ REHABILITATION PROGRAM: A LONGITUDINAL STUDY OF COLOMBIAN PATIENTS WITH CONGESTIVE HEART FAILURE}

Heather Rogers, $\mathrm{PhD}, \mathrm{MPH},{ }^{1}$ Claudia Maria Navas, $\mathrm{MD},{ }^{2}$ Sergio Daniel Ortiz, MD, ${ }^{2}$ Carlos Aguilera, $\mathrm{MD}^{3}$ and Luz Helena Lugo, $\mathrm{MD}^{3}$

${ }^{1}$ Department of Social and Behavioral Health, Virginia Commonwealth University, Richmond, VA; ${ }^{2}$ Clinica de las Americas, Medellin, Colombia and ${ }^{3}$ Antioquia University, Medellin, Colombia.

Congestive Heart Failure (CHF) affects more and more individuals in developed and developing countries alike. In Latin American, $\mathrm{CHF}$ is one of the main causes of cardiovascular mortality. In 
Colombia, it is the third leading cause of cardiovascular death. Cardiac rehabilitation programs aimed at individuals with $\mathrm{CHF}$ are designed to decrease morbidity and mortality and improve quality of life (QOL). Little data is available on the impact of cardiac rehabilitation programs on QOL in CHF patients in Colombia. The SF-36, a self-report instrument validated in Spanish with 8 subscales and a question regarding Health Expectations, was used to measure QOL in $35 \mathrm{CHF}$ patients participating in a cardiac rehabilitation program at St. Vincent de Paul Hospital in Medellín, Colombia at baseline, 3-month, and 6-month follow-ups. The rehabilitation program consisted of 12-session treatment protocol including exercise, occupational therapy, and psycho-education. One-way repeated measures ANOVAs were conducted for each QOL sub-scale. Results indicated that CHF patients participating in cardiac rehabilitation significantly improved their Physical Performance sub-scale scores and Health Expectations score over time $(\mathrm{p}<0.001$ and $\mathrm{p}<0.01$ respectively). Cardiac rehabilitation CHF patients also tended to have improved General Health subscale scores and Physical Functioning sub-scale scores over time $(p=0.05$ and $p=0.09$ respectively). Scores on the remaining SF-36 sub-scales (Pain, Emotional Performance, Social Functioning, Mental Health, and Vitality) did not vary over time $(p=N S)$. A cardiac rehabilitation directed at CHF patients appears to improve specific physical health-related domains of QOL, although future research involving a control group would strengthen these Results: The findings suggest the need for psychologists, nurses, and other health professionals to integrate psychosocial aspects into existing cardiac rehabilitation program in order to potentially improve other domains of QOL in Colombian patients with CHF.

CORRESPONDING AUTHOR: Heather Rogers, PhD, MPH, Department of Social and Behavioral Health, Virginia Commonwealth University, Richmond, VA, 23233; hlrogers@vcu.edu

\section{OS27}

\section{0:30 AM-10:48 AM}

OS27-A

IMPLEMENTATION TRIAL OF AN AUTOMATED TELEPHONE SYSTEM TARGETING DIABETES

\section{SELF-MANAGEMENT}

Dominique Bird, MD,${ }^{1}$ Brian Oldenburg, $\mathrm{PhD},{ }^{1}$ Mandy Cassimatis, Grad Dip Psych, ${ }^{2}$ Anthony Russell, $\mathrm{PhD}^{2}$ and Robert H. Friedman, $\mathrm{MD}^{3}$

${ }^{1}$ Monash University, Melbourne, VIC, Australia; ${ }^{2}$ University of Queensland, Brisbane, QLD, Australia and ${ }^{3}$ Medical Information Systems Unit, Boston Medical Center, Boston, MA.

Delivery of the regular and long-term patient support required to improve diabetes self-management remains a challenge for health systems. This study is evaluating the health outcomes and costeffectiveness of a program using an automated interactive telephone system, the Diabetes Telephone Linked Care (TLC) Australia, designed to promote and support behaviour change and disease management.
Participants $(n=120)$ were adults from Brisbane, Australia, aged 18 to 70 , diagnosed with type 2 diabetes at least 3 months prior, with sub-optimal glycemic control (Haemoglobin A1C (HbA1c) $\geq 7.5 \%$ ). Participants (79 males, mean age $=57.47$ years, $\mathrm{SD}=$ 8.26) were randomised to a usual care group $(n=60)$ or to the intervention group. For 6 months, participants in the latter group upload their past week's blood glucose levels to the TLC system's database via a cell phone link prior to calling the system weekly to "converse" on one or more of the following topics: blood glucose monitoring, nutrition, physical activity and medication taking. Primary outcomes are $\mathrm{HbA1C}$ and quality of life at 6- and 12-month follow-up. Secondary outcomes include self-care behaviours and measures of depression and social support.

Baseline HbA1C ranges from 7.5 to $15.0 \%(\mathrm{M}=8.8, \mathrm{SD}=1.4)$. Participants' average BMI is 33.62 ( $\mathrm{SD}=6.89$ ); $56.7 \%$ meet the Australian physical activity recommendations; $82.5 \%$ and $65.8 \%$ have a normal score on the HADS depression and anxiety scales respectively. The average duration of a call to the TLC system is 10.77 ( $\mathrm{SD}=1.60)$ minutes and to date participants have made on average $81.7 \%$ of their weekly calls. The presentation will include 6-month follow-up Results: This study is examining the effectiveness of a telehealth program targeting essential diabetes self-care behaviours and holds potential for addressing barriers faced in the delivery of long-term diabetes self-management support.

CORRESPONDING AUTHOR: Dominique Bird, MD, Monash University, Brisbane, QLD, 4029; dbird@coh.uq.edu.au

\section{0:48 AM-11:06 AM}

OS27-B

TRAINING PEERS TO PROVIDE SUSTAINED DIABETES SELF-MANAGEMENT SUPPORT (DSMS): A PILOT STUDY Tricia S. Tang, PhD, Martha M. Funnell, MS,RN,CDE, Mary Lou Gillard, MS,RN,CDE and Robin Nwankwo, MPH, CDE, RD Medical Education, University of Michigan Medical School, Ann Arbor, MI.

Background: Interventions involving peer support offer a promising approach for providing diabetes self-management support (DSMS) that is ongoing, culturally responsive, and low cost.

Purpose: The objectives of this study were (1) to pilot a program training peer leaders (PLs) to facilitate ongoing, empowermentbased, DSMS interventions, (2) to determine the feasibility of graduating PLs who achieve the pre-established competency criteria, and (3) to assess program satisfaction.

Methods: The Peer Leader Training (PLT) pilot program recruited eight African-American adults. The 12-week program utilized a wide variety of instructional methods and addressed three major components including self-management education, communication skills, and facilitation skills. To graduate, participants were required to meet the pre-established criteria for four competency domains including diabetes-related knowledge, active listening skills, empowerment-based facilitation skills, and self-efficacy. Participants were given three attempts to pass all four domains. We also assessed 
program satisfaction and perceived efficacy of training using qualitative (i.e., focus group) and quantitative approaches.

Results: All participants achieved the criteria for all four competency domains. On the first attempt $75 \%, 75 \%, 63 \%$, and $75 \%$ passed the diabetes knowledge, empowerment-based facilitation skills, active listening skills, and self-efficacy, respectively. Participants who failed at least one competency domain on the first attempt successfully passed on the second attempt. Participants were generally satisfied with the program length, training session length, balance between content and skills development, and preparation for leading group and individual-based support activities.

Conclusion: Findings suggest that it is feasible to train and to graduate PLs with the diabetes-related knowledge, communication skills, and facilitation skills needed to lead ongoing DSMS interventions. Subsequent research should examine the impact of this peer-led DSMS model on improving patients' diabetes-related health outcomes.

CORRESPONDING AUTHOR: Tricia S. Tang, PhD, Medical Education, University of Michigan, Ann Arbor, MI, 48109-5201; tangts@umich.edu

\section{1:06 AM-11:24 AM}

OS27-C

IN PURSUIT OF CONTROL AND HAPPINESS:

THE PSYCHOLOGICAL WAY TO A LOWER BODY MASS INDEX, BUT HOLD THE DIETING!

Angel Chater, PhD and Erica Cook, MSc

Psychology, University of Bedfordshire, Bedfordshire, United Kingdom.

Background: Obesity continues to present a major public health concern (NHS, 2008). This study aimed to explore the relationship between body mass index (BMI) and the affective states: happiness, anxiety and depression, alongside self efficacy and eating behaviours. It further aimed to identify if these components influence overeating behaviour, a factor that is empirically linked to a high BMI (Macht, 2008).

Method: Data was collected from 104 adults ( $76 \%$ female), mean age $38.48(\mathrm{SD}=12.47)$ years, who completed questionnaires which measured happiness, depression, anxiety, generalised self efficacy, dietary restraint, emotional eating and binge eating. Height and weight were also taken to calculate BMI, which ranged from 16.41-44.38 with a mean BMI of $26.92(\mathrm{SD}=5.98)$.

Results: Findings confirm that there are significant relationships between personal control, affective states, eating behaviours and BMI. Generalised self efficacy and happiness were revealed as predominant psychological factors, correlating with all variables $(p<.001)$. Controlling for age and gender, generalised self efficacy explained the variance in emotional eating by $16 \%$, binge eating by $12 \%$ and BMI by $19 \%$. Dietary restraint accounted for $66 \%$ of the variance in binge eating behaviour and was also the highest predictor of BMI $\left(\mathrm{R}^{2}=.45\right)$, with depression explaining a further $9 \%$. Finally, investigating the influence of emotional eating, negative affect $\left(\mathrm{R}^{2}=.32\right)$ and dietary restraint $\left(\mathrm{R}^{2}=.07\right)$ were revealed to be the most significant predictors.

Conclusion: Evidence presented here highlights the importance for behavioural medicine to consider the influence of self efficacy and affect when tailoring weight loss interventions, while also exercising caution over the negative influence of dieting behaviour.

CORRESPONDING AUTHOR: Angel Chater, PhD, Psychology, University of Bedfordshire, Bedfordshire, LU1 3JU; angel.chater@beds.ac.uk

11:24 AM-11:42 AM

OS27-E

CULTURAL CONSIDERATIONS IN TRANSLATING

"DIABETES CARE IN AMERICAN SAMOA"

Rochelle K. Rosen, PhD, ${ }^{1,2}$ Judy DePue, EdD, MPH, ${ }^{1,2}$ Nicole Bereolos, PhD, MPH, ${ }^{3}$ Michael G. Goldstein, MD, ${ }^{1,2}$ John Tuitele, MBBS, $\mathrm{MS}^{4}$ and Stephen T. McGarvey, PhD, $\mathrm{MPH}^{2,5}$

${ }^{1}$ Behavioral Medicine, The Miriam Hospital, Providence, RI; ${ }^{2}$ Alpert Medical School of Brown University, Providence, RI; ${ }^{3}$ School of Public Health, University of Texas, Dallas, TX; ${ }^{4}$ Tafuna Family Medical Center, Tafuna, American Samoa and ${ }^{5}$ International Health Institute, Brown University, Providence, RI.

Overview: The global challenge of translational behavioral research requires acute attention to the cultural context into which interventions are translated and awareness of the culturallyspecific needs of targeted patients. Formative qualitative research is often used to translate interventions developed for one population to another. We review the role of qualitative methods in "Diabetes Care in American Samoa" a clinical trial of a CHWdelivered intervention.

Methods: Formative research included 14 individual interviews (IDI) with medical clinic staff, 6 focus groups (FG) with 39 diabetes patients and 10 cognitive interviews (CI), also with diabetes patients. IDIs and FGs inquired on barriers or facilitators of diabetes care and sought input on planned intervention components. CIs explored the effectiveness and cultural relevancy of a subset of quantitative survey items. NVivo software was used for data management; relevant themes were identified and applied to the Precede-Proceed model to adapt intervention materials.

Results: Analysis identified barriers to diabetes self care including preferences for high fat foods, consumption of large quantities at social gatherings, and social expectations to eat large amounts. Facilitators included extended family networks, respect for doctors, and strong religious faith used in support of self care. CIs confirmed linguistic translations, with accommodations for relevant cultural examples within items.

The research practice of identifying barriers and facilitators of healthy behavior does not view those cultural elements the way participants do. Therefore, we have also sought to understand how participants see these same behaviors, including their variability.

Conclusions: Identifying the cultural meaning of self care-related behaviors is important to designing interventions to address them; qualitative methods are extremely valuable for identifying key cultural features on which to adapt intervention delivery models, materials and measures.

CORRESPONDING AUTHOR: Rochelle K. Rosen, PhD, Behavioral Medicine, The Miriam Hospital and Brown University, Providence, RI, 02903; rrosen@lifespan.org 


\section{CITATION ABSTRACT \\ OS28 \\ 10:30 AM-10:48 AM \\ OS28-A}

DEVELOPMENTAL TRAJECTORIES OF CIGARETTE USE AND ASSOCIATIONS WITH MULTI-LAYERED RISK FACTORS IN CHINESE EARLY ADOLESCENTS

Bin Xie, $\mathrm{PhD}$

School of Community and Global Health, Claremont Graduate University, San Dimas, CA.

Background: This study represents the first longitudinal analysis effort identifying

developmental trajectories of cigarette use as well as risk factors associated with the distinct developmental courses of smoking in Chinese early adolescents from age 12 to 16.

Methods: Analysis was conducted with secondary data from a longitudinal, prospective cohort, which consisted of 3,521 Chinese adolescents randomly selected from 4 rural and 7 urban middle schools in Wuhan, China. A group-based growth mixture modeling approach was adopted to identify the developmental trajectories of cigarette use. Multi-layered intrapersonal (e.g. attitudes toward smoking, grade point average, subjective academic performance, school bonding, and depressive symptoms) and inter-personal (e.g. parental smoking, perceived parental disapproval of smoking, parent-child relationships, family disharmony, perceived norms of peer smoking, good friend smoking, troubles with teachers) risk factors selected from an ecological perspective were prospectively linked to the identified patterns of smoking trajectory.

Results: Three trajectory patterns were identified from the whole cohort: non/experimental smokers (53.8\%), light/occasional smokers (43.9\%), and heavy/regular smokers (2.3\%). After adjustments for gender, urban residence, and family socioeconomic status, adolescents with higher levels of problems in parent-child relationships and family disharmony, higher perceived norms of peer smoking, higher proportion of good friend smoking, having more troubles with teachers, poorer academic performance and reporting more frequent depressive symptoms were significantly more likely to be in the trajectory groups of either light/occasional smokers or heavy/regular smokers than in the group of non/experimental smokers. The probability of being in the heavy/regular smoking trajectory group was positively and significantly related to parental smoking and lack of school bonding.

Conclusions: Study findings helped advance knowledge on the distinct developmental courses of smoking behavior and their associations with multi-layered risk factors in Chinese early adolescents.

CORRESPONDING AUTHOR: Bin Xie, PhD, School of Community and Global Health, Claremont Graduate University, San Dimas, CA, 91773; bin.xie@cgu.edu

\section{0:48 AM-11:06 AM}

OS28-B

PROSPECTIVE PREDICTORS OF QUITTING BEHAVIORS AMONG ADULT SMOKERS IN CHINA

Lin Li, PhD, ${ }^{1}$ Hua H. Yong, $\mathrm{PhD},{ }^{1}$ Guoze Feng, $\mathrm{MD},{ }^{2}$ Yuan Jiang, $\mathrm{MD},{ }^{2}$ Geoffrey T. Fong, $\mathrm{PhD}^{3}$ and Ron Borland, $\mathrm{PhD}^{1}$

${ }^{1}$ VicHealth Centre for Tobacco Control, Cancer Council Victoria, Melbourne, VIC, Australia; ${ }^{2}$ National Tobacco Control Office, Chinese Center for Disease Control and Prevention, Beijing, China and ${ }^{3}$ Department of Psychology, University of Waterloo, Waterloo, ON, Canada.

This paper uses longitudinal data from the International Tobacco Control Policy Evaluation (ITC) China Survey to examine prospective predictors of smoking cessation among adult smokers in China, and compares them with those found in previous research in two Southeast Asian countries (Malaysia and Thailand) and four Western countries (Australia, Canada, the UK and USA) that are also part of the broader ITC project.

In April 2006 a total of 4732 adult smokers were first surveyed in Beijing and other 5 cities in China. Of these, 3863 were successfully followed up in the second Wave in late 2007 (with a retention rate of $81.6 \%$ ). Baseline measures of sociodemographics, dependence and interest in quitting were used prospectively to predict both making quit attempts and quit success.

Overall, 979 out of the 3863 (25.3\%) Chinese smokers reported having made at least one quit attempt between Waves 1 and 2; of these, $212(21.7 \%)$ were still stopped at Wave 2. Independent predictors of making quit attempts included having higher quitting self-efficacy, previous quit attempts, some intention to quit, disagreeing that $\mathrm{s} / \mathrm{he}$ enjoyed smoking too much to quit, and having very negative opinion of smoking. Independent predictors of quit success among those who attempted were having longer previous abstinence from smoking $(7$ months or 
more), and having greater interest in quitting (planning to quit within 1 month).

Compared to other countries fewer adult smokers in China attempt to quit and predictors vary: with measure of nicotine dependence less predictive and, like in Malaysia and Thailand, interest in quitting more predictive of success. These findings indicate that existing knowledge from Western countries about smoking cessation are not necessarily readily generalizable to China, which has different social-economic conditions and tobacco control environment.

CORRESPONDING AUTHOR: Lin Li, PhD, VicHealth Centre for Tobacco Control, Cancer Council Victoria, Melbourne, VIC, 3053; lin.li@cancervic.org.au

\section{1:06 AM-11:24 AM \\ OS28-C \\ BELIEVING TO RECEIVE NICOTINE ACCELERATES REACTION TIME IN MALE SMOKERS BUT NOT IN NON-SMOKERS IRRESPECTIVE OF GENDER}

Paul Enck, PhD, Bjoern Horing, Dipl-Psych, Julian Stuermer, candmed, Nicole Oetama, RN, Katja Weimer, Dipl-Psych and Sibylle Klosterhalfen, $\mathrm{PhD}$

Psychosomatic Medicine, University Hospitals, Tuebingen, Germany.

Separation of the "expected" from the "true" drug effect in placebocontrolled drug trials can be achieved by informing prior to testing half of the subjects in each group (drug, placebo) correctly that they receive the drug or placebo, while the others are misinformed (deception) about the drug they received; this is called the "balanced placebo design" (BPD). A half-BPD provides no drug but only placebo. Methods: Sixty-four healthy subjects (32:32 males:females, 24 years) balanced for smokers and never-smokers were made to believe that a chewing gum they received may or may not contain $4 \mathrm{mg}$ nicotine to improve vigilance and reaction time, while in fact all received placebo. The experimenter conducting the study was also made to believe this was a fully BPD to maintain double-blindness. Immediately prior to neurocognitive function testing (N-back tasks with three levels of difficulties and two consecutive runs) they were informed that they belong to the nicotine group or to the placebo group. Dependent measures were reaction time (RT, ms) and number of false go (GO) and no-go (NGO) responses. Results: A significant $(p=.028)$ interaction of all five factors (within: level of difficulty, runs; between: information, smoking status, gender) and age as covariate was found indicating that the information to receive nicotine shortened RT in male, but not in female smokers, while in nonsmokers, the information prolonged RT, specifically in males. The placebo information produced no differences in RT in smokers/ nonsmokers and in males/females. The differential effects on RT were more pronounced with a high cognitive demand, and during the second run. No effect of any of the factors was found for the number of GO and NGO errors. Conclusion: The known effects of nicotine on $\mathrm{RT}$ can be mimicked by the belief to receive nicotine, but males are more prone then females to respond to such information. Neversmokers, in contrast experience opposite effects. These effects may be regarded as conditioned responses. (Supported by a grant from Volkwagen Foundation, Germany)

CORRESPONDING AUTHOR: Paul Enck, PhD, Psychosomatic Medicine, University Hospitals, Tuebingen, 72076; paul.enck@ uni-tuebingen.de

11:24 AM-11:42 AM

OS28-D

GENDER INTERACTION EFFECTS ON BEHAVIORAL INTENTIONS TO QUIT SMOKING AMONG KOREAN AMERICAN SMOKERS

Sun Kim, PhD, ${ }^{1}$ Seognho Kim, $\mathrm{MA}^{2}$ and Sona Dolan, $\mathrm{PhD}^{1}$

${ }^{1}$ Psychiatry, University of Massachusetts Medical School, Worcester, MA and ${ }^{2}$ School of Social Services, Fordham University, New York, NY.

Tobacco dependence is a leading cause of increased morbidity and mortality and current tobacco smoking is estimated will cause about 450 million deaths worldwide during the next 50 years. Tobacco dependence has also dramatically impacted Asian countries and subsequently Asian Americans who migrated to the United States. Korean male immigrants in the US have been known for the highest smoking prevalence and highest smoking-attributable cancer death fractions. The study is designed to test the applicability of Theory of Planned Behavior to Korean Americans' smoking cessation. Its main hypothesis is that perceived risks and benefits of quitting, social norms for quitting, self-efficacy in smoking cessation, and nicotine dependence will predict behavioral intentions to quit smoking among Korean American smokers. A nationwide sample of Korean American smokers is currently recruited into this one-time telephone survey. Subjects are randomly selected from the online website InfoSpace.com by their Korean surname such as "Kim", "Lee", "Choi", etc. The sample size of each city was weighted by the Korean population in the 2000 U.S. Census data. Three cities such as Duluth, GA that lately have the large influx of Korean Americans were added to the list of cities. Preliminary findings with 181 subjects (150 males and 31 females) revealed that gender moderates the relationships between perceived risks of quitting and behavioral intentions to quit smoking in Korean American smokers. Perceived family social norm for quitting was the only significant factor associated with Korean male smokers' behavioral intentions to quit smoking, whereas, perceived risks of quitting, and perceived family and friend social norms for quitting were the factors associated with Korean female smokers' behavioral intentions to quit smoking. However, perceived risks of quitting showed the strongest relationship. Data collection will be continued till May 31, 2010 and final results will be presented.

CORRESPONDING AUTHOR: Sun Kim, PhD, Psychiatry, University of Massachusetts Medical School, Worcester, MA, 01652; sun.kim@umassmed.edu 


\section{CITATION ABSTRACT}

11:42 AM-12:00 PM

OS28-E

EVALUATION OF A COGNITIVE SMOKING CESSATION INTERVENTION DESIGNED TO COMMUNICATE THE NEGATIVE REINFORCEMENT EXPLANATION FOR SMOKING: A CLUSTER-RANDOMISED CONTROLLED TRIAL Mairtin McDermott, MSc, BSc, ${ }^{1}$ Matthew Hankins, BSc, PhD, ${ }^{1}$ Peter Hajek, MA, $\mathrm{PhD}^{2}$ and Theresa M. Marteau, $\mathrm{PhD}^{1}$

${ }^{1}$ Health Psychology Section, King's College London, Institute of Psychiatry, London, United Kingdom and ${ }^{2}$ Tobacco Dependence Research Unit, Bart's and The London School of Medicine and Dentistry, London, United Kingdom.

Background

The negative reinforcement explanation for smoking comprises a set of ideas derived from popular self-help books which aim to change the way smokers perceive the benefits of smoking and the post-quit withdrawal discomfort. Acceptance of these ideas is hypothesised to facilitate smoking cessation, possibly through a reduction in post-cessation urges to smoke.

Aims

To assess the impact of a cognitive intervention aimed at communicating the negative reinforcement explanation for smoking upon participants' reported urges to smoke measured at one-week post cessation.

Design

A two group, cluster-randomised controlled trial (ISRCTN: 65854845) was conducted in which groups of smokers received either the experimental intervention, comprised primarily of a 20 minute presentation accompanied by group discussion and a self-monitoring task, or a control intervention which involved watching a video on the health risks of smoking. Both interventions were offered in addition to 'standard care'. The primary outcome was participants reported urges to smoke at one week post-cessation. Potential cognitive mediators assessed were participants' levels of acceptance of the negative reinforcement explanation for smoking, positive outcome expectations for the affective benefits of smoking and self-efficacy. Participants $(n=205)$ were volunteers drawn from smokers attending for treatment at a specialist smoking cessation clinic (SSCC).

Results

The intervention failed to have a significant effect on any of the proposed cognitive mediators. There was also no significant difference between the groups on post-cessation urges to smoke (Adjusted expt. group mean $=2.78$, Control group mean $=3.00,(F(1,100)=1.17, p=.28)$. Conclusions The experimental intervention based on a set of ideas commonly communicated in popular selfhelp titles, failed to alter smokers' cognitions or lead to a reduction in post-cessation urges to smoke. The intervention may have lacked sufficient power to bring about the intended cognitive shift or these highly conditioned beliefs about smoking may be resistant to change by socialcognitive means alone. Implications for smoking cessation treatments will be discussed.

CORRESPONDING AUTHOR: Mairtin McDermott, MSc, BSc, Health Psychology Section, King's Colege London, Institute of Psychiatry, London, SE1 9RT; mairtin.mcdermott@kcl.ac.uk

\section{OS29}

10:30 AM-10:48 AM

OS29-A

INCREASING RATES OF ORGAN DONATION - THE ROLE

OF THE "ICK FACTOR" AND ANTICIPATED REGRET
Ronan O'Carroll, PhD, Catherine Foster, BSc, Grant McGeechan, MSc and Kayleigh Sandford, BSc

University of Stirling, Stirling, United Kingdom.

Background: There are over 100,000 people in the USA and 10,000 in the UK awaiting organ transplants. Over $90 \%$ of the general population state that they are in favour of organ donation, however only $26 \%$ of the UK population are registered on the organ donor register. There is an urgent need to identify barriers, and find ways of increasing the number of organ donors.

Objectives: This research tested the role of emotional barriers to organ donation such as the "ick factor" (a basic disgust reaction to 
the idea of organ donation). In addition we tested the potential role of manipulating anticipated regret to increase intention to donate in people who are not yet registered as organ donors

Methods: In 3 Experiments involving 635 members of the UK general public, participants were invited to complete questionnaire measures tapping the emotional factors such as "ick", the desire to retain body integrity after death and medical mistrust. Registered organ donors were compared with non-donors. Non-donors were randomly allocated to an anticipated regret manipulation (2 questions) versus a control condition, and the impact on intention to donate was tested.

Results: In all 3 experiments non-donors scored significantly higher than donors on the ick factor and bodily integrity scales. Traditional rational/cognitive factors such as knowledge, attitude and subjective norm failed to distinguish doors from non-donors. For non-donors, the anticipated regret manipulation led to a significant increase in intention to register as an organ donor in future.

Conclusions: Negative emotional factors are important barriers for people becoming organ donors. In particular a basic disgust reaction when contemplating donation and the desire to retain bodily integrity after death are key areas to target to test if they are changeable. In addition, a simple anticipated regret manipulation has the potential to significantly increase donation rates.

CORRESPONDING AUTHOR: Ronan O'Carroll, PhD, University of Stirling, Stirling, FK9 4LA; reo1@stir.ac.uk

10:48 AM-11:06 AM

OS29-B

KNOWLEDGE, ATTITUDES, PRACTICES AND BEHAVIORS REGARDING DECEASED ORGAN DONATION

AND TRANSPLANTATION IN MALAYSIA'S MULTI-ETHNIC SOCIETY

Li-Ping Wong, MSc, PhD

Medical Education \& Research Development Unit, University of Malaya, Kuala Lumpur, Malaysia.

Background: Malaysia's organ and tissue donation rates are amongst the lowest in the world. Having the three ethnic groups (namely the Malays, Chinese and Indian) with the most pronounced cultural and religious prohibitions to organ donation, the shortage of organ donations continues to raise extreme concern. As evident, the rate of procurement (per million population) in Malaysia was only 1.01 in year 2006. From year 1997 until 2006, only a total of 162 organ procurements were carried out. Its rich ethnic diversity allow for a comprehensive understanding of a variety of factors associated to organ donation. Methods: A cross-sectional, population-based, computer-assisted telephone interview exploring multi-ethnic participants' knowledge, attitudes, practices and behavioral on deceased organ donation and transplantation was conducted during February to April 2009.

Results: Although only $5.5 \%$ of the total participants $(\mathrm{N}=1174)$ reported that they have registered to be organ donor, a further $35.2 \%$ of those who have not register for organ donation indicated willingness to donate one's own. In particular, Malays (20.7\%) indicate lower willingness to donate organs compared to the Chinese $(36.6 \%)$ and Indians $(51.4 \%) \quad(\mathrm{P}<0.001)$. In multivariate logistic regression analysis, willingness to donate one's own organ was associated with knowledge score $(\mathrm{OR}=1.17,95 \% \mathrm{CI}=1.13-1.22)$, attitude score $(\mathrm{OR}=1.17,95 \% \mathrm{CI}=1.05-1.31)$, secondary school education $(\mathrm{OR}=$ $1.46,95 \% \mathrm{CI}=1.05-2.02)$ and Malay ethnicity $(\mathrm{OR}=0.18,95 \% \mathrm{CI}=$ 0.03-0.94). Significant socio-demographic disparities with respect to knowledge and attitudes scores were observed. Study showed marked ethnic differences in attitudes towards organ donation. Some knowledge inaccuracies and misconception identified in this study need particular attention in future educational campaign.

Conclusion: Findings assist organ donation and transplantation organizations to reach out the diverse socio-demographic and ethnic communities with cultural-specific information about organ donation. The data also highlight the importance of educational efforts to enhance knowledge and cultivate supportive attitudes toward organ donation.

CORRESPONDING AUTHOR: Li-Ping Wong, MSc, PhD, Medical Education \& Researc Development Unit, University of Malaya, Kuala Lumpur, 50603; wonglp@ummc.edu.my

\section{1:06 AM-11:24 AM}

OS29-C

THE ACCEPTABILITY OF PERSONAL FINANCIAL INCENTIVES(PFI) FOR REDUCING ANTENATAL SMOKING Marita Lynagh, $\mathrm{PhD},{ }^{1}$ Ian Symonds, $\mathrm{PhD}$, BMed, ${ }^{1,2}$ Rob SansonFisher, $\mathrm{PhD}$, Laureate Prof ${ }^{1}$ and Billie Bonevski, $\mathrm{PhD}^{1}$

${ }^{1}$ School of Medicine \& Public Health, University of Newcastle, Newcastle, NSW, Australia and ${ }^{2}$ Obstetrics \& Gynaecology, John Hunter Hospital, Newcastle, NSW, Australia.

The use of personal financial incentives (PFI) to encourage people to change their behaviour is an emerging model of health intervention being trialled internationally. Recent reviews conclude that PFI can be efective in promoting behaviour change, but this is tempered by provisos. Women who smoke during pregnancy, particularly women of low SES, have been resistant to traditional smoking cessation initiatives. Smoking in pregnancy leads to significantly poorer health outcomes for mothers and their babies. There is an urgent need to develop effective smoking cessation interventions that can readily be adopted into routine antenatal care. This paper reports on the acceptability of PFI for reducing smoking in pregnant women among both patients and antenatal care providers.

CORRESPONDING AUTHOR: Marita Lynagh, PhD, School of Medicine \& Public Health, University of Newcastle, Newcastle, NSW, 2291; Marita.Lynagh@newcastle.edu.au

\section{1:24 AM-11:42 AM}

\section{OS29-D}

\section{COMMUNITY-BASED PARTICIPATION RESEARCH} ON THAI PEOPLE WITH TYPE 2 DIABETES: PRELIMINARY KEYS TO MOBILIZE AND CHANGE BEHAVIORS

Pimsupa Chandanasotthi, PhD, ${ }^{1}$ Tassanee Rawiworrakul, PhD, ${ }^{1}$ Naruemon Auemaneekul, $\mathrm{PhD},{ }^{1}$ Napaporn Sowattanangoon, $\mathrm{PhD},{ }^{1}$ Wimonrut Boonsatean, $\mathrm{MS},{ }^{2}$ Rachanee Sunsern, $\mathrm{PhD},{ }^{3}$ Surapun Wichitnak, $\mathrm{MD}^{4}$ and Pornthep Ritthuredee, $\mathrm{BPh}^{5}$

${ }^{1}$ Department of Public health Nursing, Mahidol University, Bangkok, Thailand; ${ }^{2}$ Faculty of Nursing, Rangsit University, 
Bangkok, Thailand; ${ }^{3}$ Faculty of Nursing, Burapa University, Chonburi, Thailand; ${ }^{4}$ Pamok Hospital, Angthong, Thailand and ${ }^{5}$ Pamok District Public Health Office, Angthong, Thailand.

Background: To assess situations, problems, needs, and ways of life of the diabetic people in each community, which tailored by culture, religion, socioeconomic, health care service system, and political system is crucial before mobilizing stake-holders and changing behaviors of these diabetes patients, family and community. Method: The integration of Sufficiency Economy(SE), Innovative Care for Chronic Conditions(ICCC) and Community-Based Participation Research(CPBR) was employed to five communities of Pamok district, Angthong province. Five stake-holders(diabetes patients, diabetes risk group, health care volunteers, health officers, and local administrators) were invited to participate in this study. The process in each community consisted of focus group discussion using Appreciation Influence Control(AIC), semi-formal interview, and informal interview. Results: The similarity of diabetes problem situations in each stake-holder were; less knowledge or no awareness on diabetes, unhealthy food consumption, lack of exercise, and non-adherence treatment. The likeness vision of diabetes in each community were; self care management, empowerment health volunteer, health promotion activities exercise, local wisdom of herb and alternative medicine, and integrate all stake-holder together. After discussing and priority setting of the health promotion project for diabetes and risk group under concepts of SE, ICCC, and CBPR, five different health promotion projects were initiated. Conclusion: Community-based participation research was an apparatus to build relationship and trust among the stake-holders and researchers which were the preliminary keys to raise awareness, responsibility, and empowerment to develop health promotion behaviors to reduce complication and prevention diabetes in the community.

CORRESPONDING AUTHOR: Pimsupa Chandanasotthi, PhD, Department of Public health Nursing, Mahidol University, Bangkok, 10400; too19@hotmail.com

\section{1:42 AM-12:00 PM \\ OS29-E \\ MODEL DEVELOPMENT ON RISK COMMUNICATION FOR PREVENTING AND CONTROLLING DIABETES MELLITUS}

Kaysorn Sumpowthong, PhD

Faculty of Public Health, Thammasat University, Patumtani, Thailand.

Diabetes mellitus is currently a significant health problem in Thailand and in many countries. To solve this problem, health care providers have to provide not only curative care, but also, prevention, promotion and rehabilitation services. Risk communication is a crucial process to help the people to maintain and improve their health through understanding risk issues and theirs context. The different perceptions on risks and their relevant factors among stakeholders make it difficult to implement effective risk communication.

This study aims at developing a model on risk communication for preventing and controlling diabetes mellitus. A six-month study was conducted in March to September 2008. Literature reviewing from both national and international articles provided a conceptual framework and a process for developing risk communication model which was preparing, assessing and working with the community. Stakeholders were approached to involve in all steps of the study. The setting of the study was in Mugdhaharn Province, Thailand.

The conceptual framework of the risk communication was developed from literature review comprising two major components: 1) risk assessment and 2) risk management. In relation to risk assessment, qualitative and quantitative techniques were applied. The target groups of risk assessment were people at risk of diabetes mellitus, family members, and related persons. The issues to be assessed covered risk factors, risk perception, strengths and weaknesses of self care and risk contexts. The results of the risk assessment led to the guidelines and strategies for coping with the risk factors. Regarding the risk management, the guideline and strategies were initially implemented by focusing on the engagement of the stakeholders which resulted in learning and better understanding among related people. The proposed model reflecting two significant components and its process was accepted by stakeholders and further implemented by local health care team in the study area.

CORRESPONDING AUTHOR: Kaysorn Sumpowthong, PhD, Faculty of Public Health, Thammasat University, Patumtani, 10210; kaysorns@tu.ac.th

\section{Friday \\ August 6, 2010 SYMPOSIA SESSIONS 2:30 PM-4:00 PM}

\section{SS20}

EVALUATION OF HIV PREVENTIVE INTERVENTIONS WITH ADOLESCENTS

Jose P. Espada, PhD

Health Psychology, Universidad Miguel Hernandez, Elche, Spain.

HIV prevention programs with adolescents use different strategies with varying degrees of success. Behavioral programs have been effective in reducing risk factors, although data on the most effective components are needed. This symposium is addressed from an international point of view different experiences of program evaluation focused specifically on HIV, applying experimental methodology, with high-risk and general population, and combining the evaluation of empirical studies, the quantitative review and proposed new challenges.

In the first presentation, by Espada et al, a behavioral program implemented by experts and peers is evaluated. Griffin will present a proposal for joint intervention on substance abuse and HIV transmission. Orgiles et al. address HIV prevention with young people under the stressor of the divorce of their parents. Huedo-Medina will show a meta-analytic review of the effectiveness of prevention programs with adolescents.

It thus seeks to answer outstanding questions about HIV prevention with adolescents, the degree of the overall effectiveness of behavioral programs, the better alternatives to applicators, the relationship between HIV prevention and other risk behaviors, and intervention with high risk populations served shortly. 
CORRESPONDING AUTHOR: Jose P. Espada, PhD, Health Psyhology, Universidad Miguel Hernandez, Elche, 03202; jpespada@umh.es

\section{CITATION ABSTRACT \\ SS20a}

INTERVENTIONS TO REDUCE SEXUAL RISK FOR HIV IN ADOLESCENTS: A META-ANALYSIS

Blair T. Johnson, $\mathrm{PhD},{ }^{1}$ Lori A. Scott-Sheldon, $\mathrm{PhD},{ }^{2}$ Tania B. Huedo-Medina, $\mathrm{PhD}^{1}$ and Michael P. Carey, $\mathrm{PhD}^{2}$

${ }^{1}$ Center for Health, Intervention, and Prevention, University of Connecticut, Storrs, CT and

${ }^{2}$ Center for Health and Behavior, Syracuse University, Syracuse, NY.

The main objective of this work was to provide an updated review of the efficacy of behavioral interventions to reduce sexual risk of HIV among adolescents. We searched electronic databases, leading public health journals, and the document depository held by the Synthesis of HIV/AIDS Risk Reduction Project. Studies that fulfilled the selection criteria and were available as of December 31, 2008 were included. Studies were included if they investigated any behavioral intervention advocating sexual risk reduction for HIV prevention, sampled adolescents (age range, 11-19 years), measured a behavioral outcome relevant to sexual risk, and provided sufficient information to calculate effect sizes. Data from 67 studies and 98 interventions $(\mathrm{N}=$ 51,240 participants) were included. Independent raters coded participant and intervention characteristics as well as methodological features. Weighted mean effect sizes, using both fixedand random-effects models, were calculated; positive effect sizes indicated greater risk reduction.

Relative to controls, interventions succeeded at reducing incident STIs, increasing condom use, reducing or delaying penetrative sex, and increasing skills to negotiate safer sex and to acquire prophylactic protection. Initial risk reduction varied depending on sample and intervention characteristics but did not decay over time. Comprehensive behavioral interventions reduce risky sexual behavior and prevent transmission of STIs. Interventions are most successful when administered in larger doses.

CORRESPONDING AUTHOR: Jose P. Espada, PhD, Health Psyhology, Universidad Miguel Hernandez, Elche, 03202; jpespada@umh.es

\section{SS20b}

\section{SKILLS-BASED HEALTH EDUCATION TO PREVENT} MULTIPLE RISK BEHAVIORS: FOCUS ON SUBSTANCE USE AND HIV RISK BEHAVIORS

Kenneth W. Griffin, PhD, MPH

Weill Medical College, Cornell University, New York, NY.

School-based drug abuse prevention programs for adolescents are an efficient way to deliver prevention services to young people before they become regular substance users or abusers. Contemporary prevention programs focus on social refusal skills training along with training in more generic "life skills" using cognitive, behavioral, and affective skills training techniques. This type of programming teaches principles of cognitive reframing, behavioral self-control, coping, and relaxation skills, along with how to apply these skills outside of the classroom in high risk situations. This presentation will review the concepts and empirical findings from the "Life Skills Training" prevention program, including short and long-term effects on related risk behaviors. Life Skills Training (LST) is a theoretically-driven evidence-based drug abuse prevention program for middle school students that emphasizes social resistance skills as well as more generic skills such as communication, assertiveness, decision-making, goal-setting, selfcontrol, and coping skills. Over 30 peer-reviewed articles with analyses of 18 separate cohorts of students have demonstrated that the LST program is effective with a variety of populations, producing behavioral effects on alcohol, tobacco, illicit drug use, as well as aggression and delinquency. Because LST targets etiologic factors common to both drug abuse and HIV risk and teaches skills that have a broad application, we examined the extent to which the intervention may reduce later HIV risk behavior. This presentation reviews findings demonstrating that the intervention reduced drug use and HIV risk behaviors in a sample of 2,042 predominantly white, suburban young adults who had received the preventive intervention in junior high school. Additional findings from a sample of 2,122 predominantly minority young adults will be presented demonstrating that several key skills measures (e.g., cognitive and behavioral self-control skills) that are targeted by the intervention predict various forms of substance use and HIV risk behavior in a similar fashion. These findings are presented in order to frame a discussion on the opportunities for developing competence-enhancement prevention programs to address both substance use and HIV risk behaviors, along with the challenges associated with addressing multiple risk behaviors in a single intervention. One 
goal of the presentation is to suggest that broad-based competence enhancement prevention programs may be an efficient and effective way to prevent a variety of negative outcomes during adolescence.
CORRESPONDING AUTHOR: Jose P. Espada, PhD, Health Psyhology, Universidad Miguel Hernandez, Elche, 03202; jpespada@umh.es

\section{CITATION ABSTRACT \\ SS20c \\ LONG-TERM EVALUATION OF TWO MODALITIES OF A PROGRAM FOR HIV PREVENTION WITH ADOLESCENTS: PEERS AND EXPERTS}

Jose P. Espada, $\mathrm{PhD},{ }^{1}$ Eduardo Remor, $\mathrm{PhD},{ }^{2}$ Daniel Lloret, $\mathrm{MA},{ }^{1}$ Roberto Secades, $\mathrm{PhD}^{3}$ and Rafael Ballester, $\mathrm{PhD}^{4}$

${ }^{1}$ Health Psyhology, Universidad Miguel Hernandez, Elche, Spain; ${ }^{2}$ Psicologia, Universidad Autonoma de Madrid, Madrid, Spain; ${ }^{3}$ Psychology, Universidad de Oviedo, Oviedo, Spain and ${ }^{4}$ Psicologia, Universidad Jaume I, Castellon, Spain.

Primary prevention of HIV sexual transmission among adolescents is a well-based strategy to prevent new infections. Controlled studies for the evaluation of preventive programs show its effectiveness, identify effective interventions and improve the cost/benefits balance. After previous short-term evaluations, this presentation analyzes the long-term effectiveness of "Preventing AIDS", a preventive program of sexual HIV transmission among adolescents. The sample included 1,216 Spanish adolescents with a mean age of 15.83 years $(\mathrm{SD}=.78)$. Participants were residents in 5 Spanish provinces. Six hundred and three (49.6\%) were students of 4th grade of ESO and 613 (50.4\%) were students of 1st grade of BAT. Five hundred and twenty four (43.1\%) were boys and $692(56.9 \%)$ girls. To assess the level of knowledge the scale HIV-KS (Espada et al., 2009) was used, and to measure attitudes toward HIV/AIDS and risk behaviors the questionnaire CASIPR (Espada et al., 2009) was employed. A quasi-experimental design with three experimental conditions was carried out: program implemented by experts, program implemented by peers, and a waiting-list control group. Each participating center was randomly assigned to one condition. The intervention consisted of 5 sessions of one hour with the following components: information and cognitive restructuring, social skills training, problem solving training, and covert behavioral rehearsal. Three measures were conducted: pretest, posttest and 12-months follow-up.

After the implementation of the program have shown improvement in the degree of knowledge and a significant attitudinal change. Comparisons between groups indicated that significant differences exist between the programs implemented by experts vs. the control group, and the program applied by peers. The effect size comparing the two types of applications was low. It shows how an intervention based on cognitive behavioral techniques succeeded in improving the level of knowledge about HIV / AIDS and change attitudes towards the disease, risk behavior and condom use. The program implemented by experts who performs better when implemented in pairs.

CORRESPONDING AUTHOR: Jose P. Espada, PhD, Health Psyhology, Universidad Miguel Hernandez, Elche, 03202; jpespada@umh.es

\section{SS20d}

EFFECTS OF A BEHAVIORAL INTERVENTION ON HIV RISK AMONG ADOLESCENTS WITH DIVORCED PARENTS Mireia Orgiles, $\mathrm{PhD},{ }^{1}$ Blair T. Johnson, $\mathrm{PhD}^{2}$ and Tania $\mathrm{B}$. Huedo-Medina, $\mathrm{PhD}^{2}$

${ }^{1}$ Health Psyhology, Universidad Miguel Hernandez, Elche, Spain and ${ }^{2}$ Center for Health, Intervention, \& Prevention, University of Connecticut, Storrs, CT.

This study aims to evaluate the effectiveness of a behavioral intervention on HIV risk implemented by experts in adolescents with divorced parents. Participants were 76 adolescents with a mean age of 15.78 years $(\mathrm{SD}=.76), 31.6 \%$ boys and $68.4 \%$ girls. Fifty-three adolescents took part in the experimental group and 23 in the control group. The average number of sessions attended was 4.5. Results show significant differences between study groups, with better results in attitudes and knowledge about AIDS in the group that received the intervention program. Specifically, statistically significant differences were found in knowledge about risk $(\mathrm{t}=-2.70, \mathrm{p}=.003)$, knowledge about the effects of HIV $(\mathrm{t}=-2.95, \mathrm{p}=.004)$, total knowledge $(\mathrm{t}=-4082$, $\mathrm{p}=.000)$, attitudes toward barriers $(\mathrm{t}=-2.30, \mathrm{p}=.024)$, attitudes toward condom use $(\mathrm{t}=-2.57, \mathrm{p}=.012)$ and total attitudes $(\mathrm{t}=-2.62, \mathrm{p}=.011)$.

CORRESPONDING AUTHOR: Jose P. Espada, PhD, Health Psyhology, Universidad Miguel Hernandez, Elche, 03202; jpespada@umh.es 
SS21

\section{LEVELS OF INTERVENTION IN THE WORKPLACE}

Jac van der Klink, PhD, MD

Health Sciences, University Medical Centre Groningen, Groningen, Netherlands.

Job control (autonomy and decision latitude) is an important aspect of stress theories. In the Job-demand-control model of Karasek and Theorell it is the single important factor that compensates for high demands. In the more recent Job-demand-resources model control is an important factor besides other resources.

The level of control that employees experience - a powerful resource - can be threatened at three levels: 1) the function in itself can, regarding organizational structure and task embedding, offer too little possibilities of decision latitude and be at a 'wrong' level of control, 2) the function is all right but the department has a supervisor who cannot delegate 'job control' or decision latitude 3) 1 en 2 are well but the employee himself cannot see and use the decision latitude and autonomy that is there.

Stress management programms or other interventions are seldom targeted as a result of a thorough (organisational) diagnostic proces. Most of the time interventions are mainly indicated by the specific orientation of the provider and most of the interventions are entirely focused on the individual level. In this symposium we will present a study in which the explained variance on the different organisational levels will be presented. Besides that, we will present the present state of knowledge on the three levels of intervention: function redesign, supervisor focused interventions and individual focused interventions.

CORRESPONDING AUTHOR: Jac van der Klink, PhD, MD, Health Sciences, University Medical Centre Groningen, Groningen, 9713 AV; j.j.l.van.der.klink@med.umcg.nl

\section{CITATION ABSTRACT \\ SS21a \\ WORKPLACE INTERVENTION: A POSSIBLE MECHANISM THROUGH INCREASED JOB CONTROL}

Akizumi Tsutsumi

University of Occupational and Environmental Health, Kitakyushu, Japan.

Recent trends in workplace intervention include comprehensive and integrative approaches. An important element in these interventions is the participatory approach, which implies control and empowerment for those involved. Greater participation in the process was found to be associated with increased levels of job control and communication and consequently reduced symptoms and time loss at work.

In a cluster randomized controlled trial, the effect of a participatory intervention for workplace improvement on workers' mental health and productivity was evaluated. Eleven assembly lines in a medium-sized company producing electrical devices were randomly allocated to six intervention and five control lines (47 and 50 workers, respectively). The team-based, problemsolving intervention was based on active employee involvement, shared work-related goals, and action planning to improve the work environment for stress reduction. The intervention focused on environment improvement or job redesign rather than behavioral change in symptoms of ill health. A participatory approach has several principles which include 1); to build on local practice (starting from real problems of the enterprise that exist at the site where employees work and need improvement), 2) to focus on achievements (good practices already available), 3) to link working conditions with other management goals (ie, productivity), 4) to encourage exchange of experience, 5) to promote employee involvement, and 6) to use learning-by-doing. General Health Questionnaire scores significantly deteriorated in the control lines, whereas the score remained at the same level in the intervention lines. Health and Work Performance Questionnaire scores increased in the intervention lines, but decreased in the control lines, yielding a significant intervention effect.

The process evaluation revealed that job control was improved in some lines which was supposed to bring about beneficial effect. For example, in a line where a variety of small parts were dealt with and the tasks had not been standardized, the workers made themselves a plan and the small units they worked on were adjusted according to the specific task.

The realization of workplace intervention requires the understanding and cooperation of employers or administrators, because these measures depend largely on organizational redesigns. Researchers are required to ensure that administrators and other key stakeholders have a clear understanding of their roles and responsibilities, and for the workers, to encourage them to sustain the autonomous activities for workplace improvement. 
CORRESPONDING AUTHOR: Akizumi Tsutsumi, University of Occupational and Environmental Health, Kitakyushu, 807-8555; tsutsumi@med.uoeh-u.ac.jp

\section{CITATION ABSTRACT \\ SS21b PERFORMANCE: A META ANALYSIS \\ Norito Kawakami, MD \\ School of Public Health, University of Tokyo, Tokyo, Japan.}

EFFECTS OF SUPERVISOR TRAINING ON WORKER MENTAL HEALTH AND WORK

Aims: Attitude and behaviors of managers and supervisors are expected to have an important effect on job stress and mental health among their subordinate workers. The aim of this presentation is to provide an updated meta-analysis on the effects of supervisor training on worker mental health and work performance.

Methods: We conducted a literature search using exiting database (PubMed and Web of Knowledge) with keywords "(supervisor*[ti] or manager*[ti]) AND (education* or training) AND (clinical trial OR quasi-experimental) AND stress)", as well as a hand search; 18 studies were found. We included only studies which tested the effect on stress and mental health among subordinate workers, excluding ones on supervisor's knowledge and attitude. We identified one quasi-experimental study (QS) (Tsutsumi et al. 2005; Ikegami et al. 2008), three controlled trials (CTs) (Hamaguchi et al., 1994; Theorell et al., 2001; Kawakami et al., 1997) and three randomized controlled trials (RCTs) (Kawakami et al, 2005 \& 2006; Takao et al 2007). Studies were conducted in Japan, except for one from Sweden. Data were entered into and analyzed by STATA.

Results: In all studies except one, supervisor training included provision of basic knowledge of psychosocial factors at work; In most studies from Japan the training focused on providing skills to communicate with subordinates (such as "active listening" skills); in one study from Sweden, they put more focus on redesign and improvement of psychosocial work environment. A onetime session was applied for most studies; multiple sessions with two-week intervals during one year were provided in one study. One RCT used a small group discussion; two RCTs used a web-based training method. Most studies used three-month follow-up. A significant overall effect of supervisor training was found on mental health among subordinate workers (Effect size, $-0.13 ; 95 \% \mathrm{CI},-0.26$ to $0.00 ; \mathrm{p}=0.05)$. A marginally significant effect was observed on job control (Effect size, $0.100 ; 95 \%$ CI, -0.01 to $0.21 ; \mathrm{p}=0.06$ ). No significant effect was observed on job demands, supervisor or coworker support, or work performance $(\mathrm{p}>0.05)$.

Conclusions: The meta-analysis indicated the effect of supervisor training on mental health among their subordinate workers, while the effect size was small. Supervisor training may enhance worker mental health through improving their job control. Supervisor training does not seem to have an effect on increasing work performance.

CORRESPONDING AUTHOR: Norito Kawakami, MD, School of Public Health, University of Tokyo, Tokyo, 113-0033; kawakami@m.u-tokyo.ac.jp

\section{SS21c}

\section{LEVELS OF CONTROL IN THE WORKPLACE} Jac van der Klink, PhD, MD, ${ }^{1}$ Corné Roelen, Dr, ${ }^{1,2}$ Sandra Brouwer, $\mathrm{Dr}^{1}$ and Ute Bültmann, $\mathrm{Dr}^{1}$

${ }^{1}$ Health Sciences, University Medical Centre Groningen, Groningen, Netherlands and ${ }^{2}$ ArboNed, Zwolle, Netherlands.

Context: Job control is considerated to be an important resource in organisations enabling employees to cope with a high workload.
Dutch employees with a high level of job control in combination with social support from supervisor and colleagues, show low levels of sickness absence, whatever the workload.

Older employees and employees with a chronic health condition need high levels of job control and support for a sustainable participation. Little is known about levels in the organisation that influence job control. Interventions are mainly focussed on enhancing the utilization of job control on the individual level. Aim of the present study is to identify levels in the organisation that can account for job control and other stress related variables. Methods: The management of an insurance company offered a health survey to their employees in 2006. The health survey questionnaire included valid and reliable scales about mental health, work character- 
istics and coping behaviour. The explained variance at the departmental and the occupational levels on mental health, work characteristics and coping was assessed using Anova's.

Results: 90 employees working in departments which were headed by one supervisor, were eligible for this study. The variance explained by function was $38 \%$ for autonomy, $36 \%$ for skill discretion, $14 \%$ for job demands, $27 \%$ for support by supervisor, $10 \%$ for coworker support, $17 \%$ for distress and for anxiety, $22 \%$ for depression, $22 \%$ for active coping, $10 \%$ for passive and $15 \%$ for distraction. The variance explained by departmetn (supervisor level) was 31\% for autonomy, 29\% for skill discretion, $28 \%$ for job demands, 39\% for support by supervisor, $31 \%$ for coworker support, $14 \%$ for distress, $16 \%$ for anxiety, $9 \%$ for depression, $20 \%$ for active coping, $20 \%$ for passive and $25 \%$ for distraction.

Conclusions: a considerable amount of the variance in experienced control and other stress related vaiables is explained by the function (occupational level) and by the department (supervisor level). This might indicate for interventions on these levels while most interventions are aimed at the individual level

CORRESPONDING AUTHOR: Jac van der Klink, PhD, MD, Health Sciences, University Medical Centre Groningen, Groningen, 9713 AV; j.j.1.van.der.klink@med.umcg.nl

\section{SS21d}

RESTORING WORK CAPACITY AFTER SICKNESS LEAVE DUE TO PSYCHOLOGICAL COMPLAINTS: PERSPECTIVES FROM INDIVIDUAL FOCUSED INTERVENTIONS

Roland Blonk, $\mathrm{PhD}$

Work participation, TNO Quality of Life, Hoofddorp, Netherlands.

The present paper deals with predictors of the duration to full return to work of employees on sick leave due to psychological complaints. Research we recently conducted revealed that two factors may be predictive for the duration to full return to work (RTW). These factors are early partial RTW and work related selfefficacy. This conclusion is based on three individual focused intervention studies that have been conducted in recent years. The first study was a randomized controlled study with two experimental interventions and one no treatment condition. One hundred self-employed were randomly assigned to one of the conditions. Psychological complaints, professional efficacy and other variables were assessed before, after 4 months and after 10 months. Partial and full RTW was assessed continuously. The second study was a cluster randomized comparative intervention study with an experimental condition and a care as usual condition. One hundred sixty eight employees absent form work due to psychological complaints enrolled in the study. Psychological complaints, work related self-efficacy and other variables were assessed at three times, before, after 6 months and after 12 months. Partial and full RTW were assessed continuously. The third study was a longitudinal cohort study with 177 employees absent form work due to psychological complaints. Partial and full RTW were assessed continuously. Further, psychological complaints, work related self-efficacy and other variables were assessed at a three months time interval during one year.
Both partial and work related self-efficacy appeared to be strong predictors of full RTW. This result fits the theoretical notion of graded activity as part of an individual focused treatment and the concept of self-efficacy as a moderating or facilitating factor in restoring an individual's work capacity after sick leave due to work related psychological complaints.

CORRESPONDING AUTHOR: Roland Blonk, PhD, TNO Quality of Life, Hoofddorp, 2130 AS; roland.blonk@tno.nl

\section{SS22}

HOW DIFFERENT OR SIMILAR ARE ETHNIC/RACIAL DISPARITIES IN CHILD DEVELOPMENT ACROSS THE US AND UK?

Yvonne Kelly, $\mathrm{PhD},{ }^{1}$ Amanda Sacker, $\mathrm{PhD},{ }^{2}$ Pamela Davis-Kean, $\mathrm{PhD},{ }^{4}$ Sharon Simonton, $\mathrm{PhD},{ }^{4}$ James Nazroo, $\mathrm{PhD},{ }^{3}$ Laia Becares, $\mathrm{PhD}^{1}$ and Samantha Worzalla, $\mathrm{MSc}^{4}$

${ }^{1}$ Department of Epidemiology and Public Health, University College London, London, United Kingdom; ${ }^{2}$ University of Essex, Colchester, United Kingdom; ${ }^{3}$ University of Manchester, Manchester, United Kingdom and ${ }^{4}$ University of Michigan, Ann Arbor, MI.

Ethnic/racial disparities in early childhood health and development are evident in the US and UK. To date little cross-national comparative work has been carried out in this area, but with the availability of similar data collections in the US and UK such comparative work is now possible. Data from two nationally representative population based surveys, the Early Childhood Longitudinal Study in the US and the Millennium Cohort Study in the UK allow questions on the origins of ethnic/racial disparities to be addressed.

This symposium examines how racial/ethnic disparities in childhood health and development vary across groups and national contexts. Presentations consider the underlying pathways to a diverse set of child health and developmental markers: growth, socioemotional difficulties, mental and motor development, infant feeding and obesity.

Paper 1 given by Prof Sacker will examine whether weight at birth is important for subsequent child growth and development and how patterns might vary across ethnic groups. Paper 2 given by Dr DavisKean examines relationships between the development of early motor skills and later mental and motor development. Paper 3 given by Dr Kelly considers whether experience of racism translates into poor developmental outcomes in childhood, and paper 4 given by Dr Simonton examines the links between unwanted pregnancy and the likelihood of breastfeeding initiation across ethnic/racial groups. Important to our understanding of ethnic/racial disparities are factors relating to the drivers for migration to the US and UK and national policy contexts for health and social care and these will be discussed. This symposium explores issues on ethnic/racial disparities in an interdisciplinary manner from the joint perspectives of psychology, epidemiology and sociology.

CORRESPONDING AUTHOR: Yvonne Kelly, PhD, Department of Epidemiology and Public Health, University College London, London, E11 1BZ; y.kelly@ucl.ac.uk 
SS22a

BETTER OR WORSE? ETHNIC DIFFERENCES IN GROWTH IN EARLY CHILDHOOD

Amanda Sacker, $\mathrm{PhD}^{1}$ and Yvonne Kelly, $\mathrm{PhD}^{2}$

${ }^{1}$ Institute for Social and Economic Research, University of Essex, Colchester, United Kingdom and ${ }^{2}$ Department of Epidemiology and Public Health, University College London, London, United Kingdom.

There are clear differences in birth weight by ethnic group, but whether these disparities are replicated in later markers of physical development is unknown. We examine evidence for ethnic disparities in children's height at 3 and 5 years. Data are from the UK Millennium Cohort Study, constructed to over-represent children from ethnic minority, giving us reasonable sample sizes for Indian, Pakistani, Bangladeshi, Black African and Black Caribbean groups. Mean birth weight of ethnic minority children was significantly lower than that of the ethnic majority (range $3.06-3.34 \mathrm{~kg}$ versus $3.41 \mathrm{~kg}$ ). By contrast, ethnic minority children were taller than the White majority at ages 3 and 5, significantly so in the case of Pakistani, Black Caribbean and Black African children (by $0.5 \mathrm{~cm}, 1.4 \mathrm{~cm}$. and $3.5 \mathrm{~cm}$ at 5 years). Controlling for parental height to take account of intergenerational effects did not affect height differentials. Two mechanisms were hypothesised: (a) a poorer intrauterine environment given the short stature of some minority children's mothers resulted in catch-up postnatal growth and (b) conditions during the parents' childhood led to a reduced capacity to reach their own height potential. A reparameterization of parent heights showed that mother's height contributed more to predicting child height than mean parental height. Adding birth weight to the model showed that height was positively related to birth weight and attenuated the extra contribution from mothers' heights to non significance. The interaction of mean parental height with ethnicity was significant in models at age 3 and age 5. For all ethnic minority groups, mean parental height was more weakly related to child height than in White majority families, although only significantly different in the largest Pakistani group. In conclusion, we found evidence of catch-up growth in ethnic minority children and that ethnic minority parents had not reached their height potential. There was no suggestion that the lower birth weight of ethnic minority children has adverse consequences for their growth in the preschool years.

CORRESPONDING AUTHOR: Yvonne Kelly, PhD, Department of Epidemiology and Public Health, University College London, London, E11 1BZ; y.kelly@ucl.ac.uk

\section{$\mathrm{SS} 22 \mathrm{~b}$}

IS EXPERIENCE OF RACISM RELATED TO EARLY CHILD DEVELOPMENT IN THE UK?

Yvonne Kelly, $\mathrm{PhD},{ }^{1}$ Laia Becares, $\mathrm{PhD}^{1}$ and James Nazroo, $\mathrm{PhD}^{2}$

${ }^{1}$ Department of Epidemiology and Public Health, University College London, London, United Kingdom and ${ }^{2}$ School of Social Sciences, University of Manchester, Manchester, United Kingdom.

Background: Previous work has shown that experience of racism is related to poor health outcomes in adults, but little is known about whether such experiences translate into developmental outcomes in children.

Objectives: 1. to examine the relationship between parental experience of racism and markers of child development 2. to assess whether family socioeconomic position and neighbourhood level disadvantage explain observed relationships.

Methods: Data from the UK Millennium cohort study on ethnic minority participants of Indian, Pakistani, Bangladeshi, Caribbean and African descent were used $(\mathrm{N}=2157)$. Questions on parental interpersonal experience of racism, and the perception of racist attacks in the residential neighbourhood were asked when cohort members were aged 5 years. A summary 'objective' score for residential perception of racism was created. Markers of child development at age 5 were obesity and overweight, socioemotional difficulties, and cognitive ability scores.

Results: $43 \%$ of parents reported any experience of interpersonal racism, and $12 \%$ reported that racist attacks or insults were common in their residential neighbourhood. It appeared that parental reports of interpersonal racism were not strongly associated with poor developmental outcomes. Conversely, perception of racism in the area of residence was significantly associated with socioemotional difficulties (coeff 1.4, $\mathrm{p}<0.001$ ) and spatial ability (coeff $-1.4, \mathrm{p}<0.01$ ). A trend was observed between the objective marker of area racism and an increased risk of obesity and lower verbal and non-verbal ability scores. The objective measure of area racism was found to be associated with lower spatial ability score. Statistical adjustment for socioeconomic factors attenuated associations, but area and objective measures remained independently associated with socioemotional difficulties and spatial ability.

Conclusions: Parental perceptions of racism are linked to poor developmental outcomes in children. Economic disadvantage among ethnic minority groups appears to partly explain some of these relationships.

CORRESPONDING AUTHOR: Yvonne Kelly, PhD, Department of Epidemiology and Public Health, University College London, London, E11 1BZ; y.kelly@ucl.ac.uk

\section{$\mathrm{SS} 22 \mathrm{c}$}

DOES UNINTENDED PREGNANCY EXPLAIN RACIAL/ ETHNIC DISPARITIES IN THE INITIATION OF BREASTFEEDING IN THE US?

Sharon Simonton, $\mathrm{PhD}$ and Pamela Davis-Kean, $\mathrm{PhD}$

University of Michigan, Ann Arbor, MI.

Background: Rates of breastfeeding in the US vary across racial/ ethnic groups and levels of socioeconomic position (SEP). Unintended pregnancy, associated with reduced odds for breastfeeding (BF), is more prevalent among African American and Hispanic women and those having lower levels of SEP.

Objectives: 1.To examine breastfeeding initiation by ethnicity and maternal nativity status and 2. To assess the extent to which unintended pregnancy may mediate observed relationships.

Methods: Data for white, Hispanic, American Indian, Asian/ Pacific Islander (API) and black and African American mothers and children included in the Early Childhood Longitudinal Study- 
Birth Cohort (ECLS-B), a nationally representative sample of US children born in 2001, were used $(n=7577)$. Nativity status was measured by reported country of birth. Pregnancy intention was assessed when children were 9-months-old by whether the mother had wanted to have a baby at the time of pregnancy. A pregnancy was defined as unwanted if the mother had not wanted to have a baby at time of pregnancy. Models controlled for child low birth weight, number of siblings, household structure and income (quintiles), and maternal age and education.

Results: Compared with US-born-white mothers, non-US-born white, black, Hispanic and API mothers were more than twice as likely to have initiated BF. African American women had lower odds for $\mathrm{BF}$ [odds ratio $(\mathrm{OR})=0.52,95 \%$ confidence interval (95\% CI): $0.43,0.61]$ and US-born Hispanic women were more likely to have $\mathrm{BF}(\mathrm{OR}=1.43,95 \% \mathrm{CI}: 1.13,1.81)$. Odds for $\mathrm{BF}$ for American Indian mothers did not differ from those for USBorn white women. $10 \%$ of mothers reported that their pregnancy had been unwanted. Effects of race/ethnicity on BF were slightly attenuated when pregnancy intention was added to the model. Mothers who had had an unwanted pregnancy were less likely to $\mathrm{BF}(\mathrm{OR}=0.70,95 \% \mathrm{CI}: 0.56,0.87)$.

Conclusion: Unintended pregnancy is significantly and independently related to breastfeeding but does not explain ethnic disparities in the initiation of breastfeeding in the US.

CORRESPONDING AUTHOR: Yvonne Kelly, PhD, Department of Epidemiology and Public Health, University College London, London, E11 1BZ; y.kelly@ucl.ac.uk

\section{SS22d}

PREDICTORS OF MOTOR SKILLS IN INFANTS: DOES EARLY EXPERIENCE MATTER?

Pamela Davis-Kean, PhD and Samantha Warzalla, MSc

University of Michigan, Ann Arbor, MI.

Recent research has shown that motor skills in children may be an important predictor in achievement in the elementary school years (Grissmer, et al, 2009). In this study we test whether early gross motor skills and early mental skills in infants and toddlers are predicted by early motor movements such as pulling up or walking with the assistance of other. These early skills may be important for indicating brain development that may be promoting later motor development and problem solving.

In order to examine our model we use the first wave (9 months old) of the Early Childhood Longitudinal Study-Birth Cohort (ECLS-B), which is a nationally representative sample of children born in the US in 2001. For the analyses, 10, 680 children were examined. We controlled for child's age, being foreign born, multiple births, low birth weight, highest parental education in the home, and household income. We examined whether children were breastfeed as a parental nutrition indicator, and age child pulled themselves to standing, age of walking with help as child as indicators of early motor skills/early brain development.

Using hierarchical regressions we find that low birth weight, age of pulling oneself to a standing position and walking with help are negative predictors of early gross motor skills $(\mathrm{B}=-.07,-.07,-.02$ respectively). Being Black, is a positive predictor of gross motor skills (.03). These variables with controls account for $70 \%$ of the variance in gross motor skills. For early mental skills, being Black and household income is associated with a .02 positive increase and age walking with help (.06) was also positive. Being Asian $(-02)$, low birth weight (-.04), and ever breastfeed (-.02), are negative predictors. Similar levels of variance are accounted for (.70) with children's age being the largest predictor of both gross motor and mental skills (.82, .84 , respectively).

Though the effects are small, there is some indication that early motor and mental skills may be related to early breastfeeding and physical motor skills.

CORRESPONDING AUTHOR: Yvonne Kelly, PhD, Department of Epidemiology and Public Health, University College London, London, E11 1BZ; y.kelly@ucl.ac.uk

\section{Friday \\ August 6, 2010 \\ PAPER SESSIONS \\ 2:30 PM-4:00 PM}

OS30

2:30 PM-2:48 PM

OS30-A

NEIGHBORHOOD DESIGN, PHYSICAL FUNCTION, AND HEALTHFUL LIFESTYLES IN OLDER ADULTS: RESULTS FROM THE SENIORS NEIGHBORHOOD QUALITY OF LIFE STUDY

Abby King, PhD, ${ }^{1}$ James Sallis, $\mathrm{PhD},{ }^{2}$ Lawrence Frank, PhD, ${ }^{3}$ Brian Saelens, $\mathrm{PhD},{ }^{4}$ David Ahn, $\mathrm{PhD},{ }^{1}$ Terry Conway, $\mathrm{PhD}^{2}$ and Kelli Cain, MS $^{2}$

${ }^{1}$ Stanford University School of Medicine, Los Altos, CA; ${ }^{2}$ SDSU, San Diego, CA; ${ }^{3}$ UrbanDesign4Health, Inc, Atlanta, GA and ${ }^{4}$ Seattle Children's Hospital, Seattle, WA.

Automobile-oriented urban design is linked with increasingly sedentary lifestyles, which are associated with an array of chronic diseases as people age. Yet little work has occurred on the relationships among built environment features and active transport and other physical activity (PA) forms among older adults. 709 adults ages 66 yrs and above living in Seattle or Baltimore regions were recruited from GIS-enumerated neighborhoods identified as high or low affluent and high or low 'walkable' based on land use mix, street connectivity, and other features. Variables included reported active transport (walking/bicycling for errands), general PA (via accelerometry and self-report), and reported BMI and mobility impairments. Persons in less affluent neighborhoods reported generally less PA and higher BMI. After controlling for individual and neighborhood-level sociodemographic and regional factors, walkability was positively associated with active transport $(\mathrm{p}<.0001)$, total PA $(\mathrm{p}=.036)$, leisure walking/cycling/jogging $(\mathrm{p}<.008)$, accelerometry-derived minutes/day of moderate or vigorous PA $(p=.056)$, and lower BMI $(p=.02)$. A significant walkability-mobility impairment 
interaction was observed for active transport only; as mobility increased, differences in minutes/week of active transport among those living in low vs. high walkable neighborhoods increased $(p=.001)$. Persons in the lowest mobility tertile living in high walkable neighborhoods had active transport levels comparable to persons in the two higher mobility tertiles but living in less walkable neighborhoods (means $=35 \mathrm{mins} / \mathrm{wk}$ vs. $27 \mathrm{mins} / \mathrm{wk}$, respectively, $\mathrm{p}>.13$ ). Results suggest the potential importance of environmental features in facilitating active transport and other healthful patterns among older adults across mobility levels, with potential benefits related to both health and independence as people age.

CORRESPONDING AUTHOR: Abby King, PhD, Stanford University School of Medicine, Los Altos, CA, 94024; king@ stanford.edu

\section{2:48 PM-3:06 PM}

OS30-B

THE INFLUENCE OF URBAN FORM AND PERCEPTION ON YOUTH TRAVEL TO SCHOOL

Lawrence D. Frank, PhD, ${ }^{1}$ Michael J. Greenwald, $\mathrm{PhD},{ }^{2}$ James E. Chapman, $\mathrm{MS}^{2}$ and Sarah E. Kavage, MUP ${ }^{2}$

${ }^{1}$ University of British Columbia, Vancouver, BC, Canada and

${ }^{2}$ Urban Design 4 Health, Inc., Seattle, WA.

Objective: The project's aim was to analyze built environment and parental perception factors that influence youth travel mode to school. Methods: Data from a sample of Atlanta youth ages 5-18 was used along with objective measures of urban form surrounding home and school and along the route from home to school. The large sample size $(n=5890$ trips) allowed stratified analysis by age group (5-10, 11-15, 16-18) and distance to school (0-1.5 miles, 1.5-3 miles, over 3 miles), while controlling for sociodemographic factors known to influence travel.

Results: Using a nested logit model of travel mode choice, we observed more sidewalks, higher residential densities and interconnected street networks along the route to school and higher parental perception of neighborhood school quality were positive, significant $(\mathrm{p}<=.05)$ predictors of higher probabilities of walking to school for shorter distances (0-1.5 miles) and younger children (ages 5-10). Further analysis indicates small marginal changes can substantially improve probabilities of selecting walking - the probability of walking after the increase in sidewalk coverage to 60 th percentile is $118.44 \%$ of the probability of walking at median sidewalk coverage. However, it also appeared that the influence of urban form can be overwhelmed if other factors (parental perceptions of school quality, traffic safety and crime, or even other urban form characteristics) are not supportive. Land use mix, a consistently significant predictor of adult travel behavior, was consistently not associated with youth school trips.

Discussion: Travel mode may be predetermined by parents or constrained by other circumstances, and it may only be under certain circumstances - youth located in communities safe from crime and traffic that are close to school - where urban form may influence travel mode. There are indications that school quality may have an indirect impact on physical activity through its impact on neighborhood school choice.
CORRESPONDING AUTHOR: Lawrence D. Frank, PhD, University of British Columbia, Vancouver, BC, V6T 1Z2; ldfrank@ interchange.ubc.ca

\section{3:06 PM-3:24 PM \\ OS30-C \\ APPLYING URBAN FORM, PHYSICAL ACTIVITY AND ACTIVE TRANSPORTATION ANALYSIS TO A PLANNING TOOL}

Lawrence D. Frank, PhD, ${ }^{1}$ Mark Bradley, MS, ${ }^{2}$ Sarah E. Kavage, MUP, ${ }^{3}$ James E. Chapman, MS, ${ }^{3}$ James Sallis, $\mathrm{PhD},{ }^{4}$ Brian E. Saelens, $\mathrm{PhD}^{5}$ and Gordon Garry, MCRP ${ }^{6}$

${ }^{1}$ University of British Columbia, Vancouver, BC, Canada; ${ }^{2}$ Mark Bradley Research \& Consulting, Santa Barbara, CA; ${ }^{3}$ Urban Design 4 Health, Inc., Seattle, WA; ${ }^{4}$ San Diego State University, San Diego, CA; ${ }^{5}$ Seattle Childrens' Hospital, Seattle, WA and ${ }^{6}$ Sacramento Area Council of Governments, Sacramento, CA.

Objective: To apply an analysis of urban form and physical activity relationships to a land use planning tool in King County WA (Seattle region).

Method: Regional travel survey data was used to measure active transportation (number and miles of bicycle or walk trips) in King County ( $\mathrm{n}=2699$ households); objectively measured data from the Neighborhood Quality of Life Study (NQLS, n=1200 households) was used to measure daily minutes of moderate and vigorous physical activity (PA). Urban form was measured at the $1 \mathrm{~km}$ network buffer level around each household using GIS parcel data. A multiple regression model which controlled for sociodemographic factors was used to estimate coefficients, which were subsequently incorporated into I-PLACE3S, a sketch planning tool developed by the California Energy Commission. All statistically significant variables $(p<=.05)$ were integrated into I-PLACE3S; urban form variables of particular policy relevance that did not attain $\mathrm{p}<=.05$ were included in the final models for incorporation into I-PLACE3S if they were logically signed.

Results: Residential density, retail density, and presence of a park within the buffer were significantly related $(\mathrm{p}<=.05)$ to PA. Intersection density approached but did not attain statistical significance on PA, and was left in the model for incorporation into I-PLACE3S. Of the urban form variables, intersection density had the strongest association to active transportation trips and miles $(\beta=.022$ and $\beta=.023$, respectively; $p<.01)$. Retail density was also significantly related to active transportation trips and miles $(\beta=1.876$ and $\beta=2.888$, respectively; $p<.01)$. The number of retail/food-related parcels significantly increased the number of nonmotorized trips, but did not significantly change number of miles.

CORRESPONDING AUTHOR: Lawrence D. Frank, PhD, University of British Columbia, Vancouver, BC, V6T 1Z2; ldfrank@ interchange.ubc.ca 
3:24 PM-3:42 PM

OS30-D

EXPLORING THE FACTORS ASSOCIATED

WITH MODERATE-INTENSITY PHYSICAL ACTIVITY -

A PRELIMINARY REPORT FOR COMMUNITY-DWELLING JAPANESE ELDERLY PEOPLE -

Kousuke Maeba, master ${ }^{1}$ and Koji Takenaka, $\mathrm{PhD}^{2}$

${ }^{1}$ Graduate School of Human Sciences, Waseda University, Tokorozawa-City, Japan and ${ }^{2}$ Faculty of Human Sciences, Waseda University, Tokorozawa-city, Japan.

Introduction: According to Physical Activity Guidelines for Americans (2008), older adults should aim to do at least 150 minutes of moderate-intensity physical activity (MPA) a week. Despite the known benefits of MPA, over $60 \%$ of older adults do not engage in it (Center for Disease Control and Prevention, 2006). Exploring the effective way to keep older adults engaging in MPA is quite important to improve or maintain their health. This study examined what factors could be associated with engaging in MPA for Japanese elderly people.

Methods: Participants were 180 community-dwelling Japanese elderly people $($ men $=93$, women $=87$ : mean age $=74.67 \pm 7.66)$. We conducted individual interview for each participant to ask the questions, including demographic factors, previous falling history within a year, falls self-efficacy, current physical activity level (Japanese version of International Physical Activity Questionnaire-Short Version [IPAQ-SV] ; Murase et al., 2002). We divided participants into two groups (MPA group and nonMPA group) on the basis of the result of IPAQ-SV. To reveal the differences between groups, we conducted statistical analyses including t-test, chi-square test, and Mann-Whitney $U$ test. Statistical analysis was conducted using PASW Statistics 17.0.

Results: Compared to non-MPA group, participants in MPA group didn't have chronic pain $(\chi 2=4.87, \mathrm{p}<.05)$ or activity restriction $\left(\chi^{2}=\right.$ $6.93, \mathrm{p}<.01)$, and didn't experience falls within a year $(\chi 2=5.64$, $\mathrm{p}<.05)$. Further, their falls self-efficacy was higher $(\mathrm{t}=-6.15, \mathrm{p}<.01)$, and their walking time per week was longer $(\mathrm{t}=-2.15, \mathrm{p}<.05)$. In addition, they had better perceived health $(\mathrm{Z}=-4.14, \mathrm{p}<.01)$.

Conclusion: It is suggested that maintaining light-intensity physical activity such as walking or enhancing confidence to falls could be important to keep older adults engaging in MPA. Further interventions should be focused on such factors.

CORRESPONDING AUTHOR: Kousuke Maeba, master, Graduate School of Human Sciences, Waseda University, TokorozawaCity, 359-1165; comaeba@yahoo.co.jp

\section{OS31}

\section{2:30 PM-2:48 PM}

OS31-A

\section{UNDERSTANDING GLOBALIZATION \& ITS IMPACT} ON HEALTH

Sonia Suchday, $\mathrm{PhD},{ }^{1,2}$ Pejman Sehatpour, MBBS/PhD, ${ }^{1,2}$ Rosy Chhabra, PsyD, ${ }^{1,2}$ Hillary Lewin, BA, ${ }^{1,2}$ Heather Glubo, BA, ${ }^{1,2}$ Maureen Almeida, MA, MPhil ${ }^{3}$ and Ruby Pavri, MA $^{3}$
${ }^{1}$ Clinical Psychology Ph.D. (Health Emphasis) Program, Ferkauf Graduate School of Psychology/Albert Einstein College of Medicine, Bronx, NY; ${ }^{2}$ Clinical Psychology (Health Emphasis) Ph.D., Institute of Public Health Sciences/, Bronx, NY and ${ }^{3}$ Psychology, St. Xavier's College/Mumbai University, Mumbai, India.

In addition to economic factors, globalization has an impact on cultural, social, and psychological aspects of functioning. These changes require adaptation and are often a source of stress. The current study assessed the stress experienced due to globalization. Undergraduate participants from St. Xavier's College in Mumbai, India $(\mathrm{N}=56$; Mean Age= 19 years; 3 Men; 50\% Christian \& 28\% Hindu) reported on the impact of globalization on their lives and on the culture and the society where they lived. Two independent raters identified common themes and three additional independent raters reviewed the themes for overlapping content and modified the themes listed until they had consensus among them on 32 themes that described the experience of globalization. The Impact of Globalization Scale (IGS) was created with each theme identified as an item. Respondents $(\mathrm{N}=542$; Females $=437$; Mean Age $=19$ years; $47.4 \%$ Christian \& $36.3 \%$ Hindu) from the same institute stated the frequency with which each of the themes identified were a source of stress to them on a 4-point Likert scale ranging from "almost never" to "almost always". Exploratory factor analysis with promax rotation identified the presence of 4 factors. Based on an examination of item content the factors were named: Egalitarian Values, which described a healthy challenge to traditional norms/roles (Eigenvalue $(\mathrm{e})=16.21$; \% Variance Accounted For $(\%)=50.66$; Internal Reliability Alpha $(\mathrm{A})=.97)$; Unhealthy Habits or an increase in alcohol/smoking, etc $(\mathrm{e}=2.53$; $\%=7.90 ; \mathrm{r}=.83$ ); Challenging Traditions or declining traditional value $(\mathrm{e}=1.18 ; \%=3.69 ; \mathrm{A}=.76)$; and Growth of Competitiveness \& Materialism $(\mathrm{e}=1.07 ; \%=3.33 ; \mathrm{A}=.36)$. Correlations of these factors with measures of anger, anxiety, rumination, symptom report (SR), and stress revealed that Egalitarian values was positively associated with SR $(\mathrm{r}=.12, \mathrm{p}<.008)$; Unhealthy habits correlated with $\mathrm{SR}(\mathrm{r}=.17$, $\mathrm{p}<.001)$, anger $(\mathrm{r}=.11, \mathrm{p}<.05)$, rumination $(\mathrm{r}=.11, \mathrm{p}<.01)$; Challenging Traditions correlated with SR $(\mathrm{r}=.16, \mathrm{p}<.001)$, anger $(\mathrm{r}=.10$, $\mathrm{p}<.03)$, rumination $(\mathrm{r}=.13, \mathrm{p}<.003)$; Competitiveness correlated with $\mathrm{SR}(\mathrm{r}=.14, \mathrm{p}<.003)$, stress $(\mathrm{r}=.10, \mathrm{p}<.02)$, anxiety $(\mathrm{r}=.10, \mathrm{p}<.02)$, rumination $(\mathrm{r}=.10, \mathrm{p}<.03)$. Conclusion: Clearly, globalization has a positive and negative impact on rapidly changing social systems and structures and is experienced as stressful and anger/anxiety provoking.

CORRESPONDING AUTHOR: Sonia Suchday, PhD, Clinical Psychology Ph.D. (Health Emphasis) Program, Ferkauf Graduate School of Psychology/Albert Einstein College of Medicine, Bronx, NY, 10461; Sonia.Suchday@einstein.yu.edu

\section{2:48 PM-3:06 PM}

OS31-B

ROLE OF BUDDHIST PRACTICES ON EMOTIONAL DISTRESS IN STROKE SURVIVORS

Napaporn Sowattanangoon, PhD in Health Psychology, ${ }^{1}$ Vimonwan Hiengkaew, $\mathrm{PhD}^{2}$ Mantana Vongsirinavarat, $\mathrm{PhD}^{2}$ and Jiraporn Chompikul, $\mathrm{PhD}^{3}$ 
${ }^{1}$ Department of Health Education and Behavioral Sciences, Faculty of Public Health, Mahidol University, Bangkok, Thailand; ${ }^{2}$ Faculty of Physical Therapy, Mahidol University, Nakhon Pathom, Thailand and ${ }^{3}$ ASEAN Institute for Health Development, Mahidol University, Nakhon Pathom, Thailand.

Purpose: Emotional distress often follows a stroke and can impede the process of rehabilitation in stroke survivors. The aim of this study was to examine the association of mental state, functional status, Buddhist values and Buddhist practices, respectively, with emotional distress among Thai patients after having a stroke. Methods: We surveyed 190 stroke survivors who participated in a home health care physical therapy service. Mini Mental State Examination (MMSE) and Barthel index (BI) were used to assess mental state and functional status, respectively. The stroke survivors completed a questionnaire measuring Buddhist values, Buddhist practices, and Thai Hospital Anxiety and Depression Scale (HADS). Results: We found HADS scores were related to MMSE $(r=-.27, p=.000)$, BI $(r=-.32, p=.000)$, Buddhist values $(r=-.16, p=.030)$, and Buddhist practices $(r=-.25, p=.001)$. In a hierarchical regression analysis, Buddhist practices were significantly associated with HADS scores when controlling for MMSE and BI $\left(R^{2}\right.$ change $\left.=.04, F(2,182)=4.36, p=.014\right)$. No relationship was found between Buddhist values and HADS scores in this hierarchical regression model. Conclusion: In Thai stroke survivors, Buddhist practices seem to improve emotional status. Therefore, stroke rehabilitation programs should include religious practices.

CORRESPONDING AUTHOR: Napaporn Sowattanangoon, PhD in Health Psychology, Health Education and Behavioral Sciences, Mahidol University, Pomprap, 10100; nsow001@yahoo.com

3:06 PM-3:24 PM

OS31-C

THE INFLUENCE OF MOTHERS-IN-LAW IN ANTENATAL CARE DECISION-MAKING IN NEPAL: A QUALITATIVE STUDY

Bibha Simkhada, $\mathrm{PhD},{ }^{1}$ Maureen Porter, BSc $\mathrm{MSc}^{\mathrm{PhD}^{1}}$ and Edwin R. van Teijlingen, MA MEd PhD ${ }^{2}$

${ }^{1}$ University of Aberdeen, Aberdeen, United Kingdom and ${ }^{2}$ Bournemouth University, Bournemouth, United Kingdom.

Antenatal care (ANC) has been recognised as a way to improve health outcomes for pregnant women and their babies. Only 29\% of pregnant women receive the recommended four antenatal visits in Nepal and reasons for such low utilisation of ANC are poorly understood. The main aims of this paper are to explore the mother-in-law's role in ANC uptake and decision-making about using ANC services in Nepal. In-depth interviews were conducted with 30 purposively selected prenatal or postnatal mothers (half users, half non-users of ANC), 10 husbands and 10 mothers-in-law in two communities.

Our findings suggest that mothers-in-law are pivotal family members who often make decisions about ANC check ups for their daughters-in-law. Sometimes this influence is positive, encouraging women to seek ANC, but more often it is negative. A number of mothers-in-law had not used ANC themselves and did not see any benefits. The main factor leading mothers-in-law not to support/ encourage ANC check ups were workload for pregnant women, mothers-in-law's perceptions of benefits deriving from ANC, their control over resources, their own past experiences and power relations between mothers-in-law and daughters-in-law.

Mothers-in-law have a strong influence on the use of ANC. Health promotion and education interventions to improve the use of ANC should target both women and their family members, particularly mothers-in-law.

CORRESPONDING AUTHOR: Bibha Simkhada, PhD, University of Aberdeen, Aberdeen, AB25 3AU; b.simkhada@abdn.ac.uk

\section{3:24 PM-3:42 PM}

OS31-D

ANTIBIOTIC USE, RESISTANCE DEVELOPMENT AND ENVIRONMENTAL FACTORS: A QUALITATIVE STUDY AMONG HEALTHCARE PROFESSIONALS IN ORISSA, INDIA

Krushna C. Sahoo, MSc, ${ }^{1}$ Ashok J. Tamhankar, PhD, ${ }^{2}$ Eva Johansson, $\mathrm{PhD}^{3}$ and Cecilia Stålsby Lundborg, $\mathrm{PhD}^{1}$

${ }^{1}$ Div of Global Health, IHCAR, Dept of Public Health Sciences, Karolinska Institutet, Stockholm, Sweden; ${ }^{2}$ Department of Environmental Medicine, R.D. Gardi Medical College, Ujjain, India and ${ }^{3}$ Public health, Nordic School of Public Health, Gothenburg, Sweden.

Objective: To explore the perceptions of healthcare providers on antibiotic use and resistance development in relation to environmental factors i.e. physical, natural, social and behavioural factors. Methods: A qualitative interview study was conducted using faceto-face, semi-structured interviews among registered allopathic doctors, veterinarians and drug dispensers in Orissa, India. The interview transcripts were analyzed using content analysis.

Results: The main findings of this study relate to two themes: 'Interrelationship between antibiotic use, resistance development and environmental factors' and 'Antibiotic handling contributing to the development and spread of resistance'. Changes in the natural and physical environment i.e. climate variability, physiography and population growth; the socioeconomic environment affecting healthseeking behaviour and noncompliance with medication; a lack of healthcare facilities and poor professional attitudes; and ineffective law enforcement on medicine dispensing, manufacturing and disposal were viewed as possible contributors to resistance development.

Conclusion and implication: Our study indicates that although behavioural and social environmental factors are major contributors to resistance development, changes in the physical and natural environment exacerbate these problems, and suggests further quantitative studies. The study also indicates a lack of information about and awareness of what constitutes prudent use of antibiotics and thus emphasizes the need for comprehensive action including information, education, dissemination and proper implementation and enforcement of legislation at all levels of drug delivery and disposal in order to rationalize antibiotic use.

CORRESPONDING AUTHOR: Krushna C. Sahoo, MSc, Div of Global Health, IHCAR, Dept of Public Health Sciences, Karolinska Institutet, Stockholm, SE 171 77; krushna.sahoo@ki.se 


\section{3:42 PM-4:00 PM}

OS31-E

THE PORTUGUESE VERSION OF COPENHAGEN

PSYCHOSOCIAL QUESTIONNAIRE

Carlos Silva, $\mathrm{PhD},{ }^{1}$ Anabela Pereira, $\mathrm{PhD},{ }^{1}$ Alexandra Pereira, Master, ${ }^{1}$ Vânia Amaral, Master, ${ }^{1}$ Vitor Rodrigues, $\mathrm{PhD},{ }^{3}$ Jorge Silvério, $\mathrm{PhD}{ }^{2}$ Paulo Nossa, $\mathrm{PhD}{ }^{2}$ Gustavo Vasconcelos, $\mathrm{Dr}^{1}$ and Teresa Cotrim, $\mathrm{PhD}^{4}$ ${ }^{1}$ University of Aveiro, Aveiro, Portugal; ${ }^{2}$ University of Minho, Braga, Portugal; ${ }^{3}$ University of Coimbra, Coimbra, Portugal and ${ }^{4}$ Faculty of Human Kinetics / Technical University of Lisbon, Lisbon, Portugal.

The aim of this study is to study the Portuguese translation of the Copenhagen Psychosocial Questionnaire (COPSOQ). This questionnaire developed in 2000 by the National Institute of Occupational Health in Copenhagen, is considered one of the most powerful tools in the evaluation of important psychosocial dimensions, such as cognitive skills and emotional rewards, interpersonal conflict, stress and harassment stemming from occupation. The questionnaire seems to be comprehensive and to include most of the relevant dimensions according to several important theories on psychosocial factors at work. Is presented in three different lengths for assessing psychosocial factors at work, stress, and the well-being of employees and some personality factors. The purpose of the COPSOQ concept is to improve and facilitate research, as well as practical interventions at workplaces. The Portuguese COPSOQ validation study is conduct by a project group headed by de Foundation of Science and Technology (FCT). The validation study comprised some working steps like a performance of a pilot study with an adaptation of the instrument. The development of the pilot study was based on a survey on a sample of 400 Portuguese employees aged 20-59 years. The values obtained in this sample of Portuguese workers will be analysis and compared with international data taking as an important starting point for the assessment and intervention in occupational health.

We adapted this instrument to the Portuguese population, and intend to pass it by promising and innovative nature of the evaluation of the psychosocial work environment, promoting communication between researchers, employers and occupational health and evaluation of interventions on the welfare and health work.

CORRESPONDING AUTHOR: Alexandra Pereira, Master, University of Aveiro, Aveiro, 3800; alexandra.pereira@ua.pt

\section{CITATION ABSTRACT \\ OS32 \\ 2:30 PM-2:48 PM \\ OS32-A}

TRAIT ANGER INTERACTS WITH BOTH POSITIVE AND NEGATIVE EMOTIONS IN RELATION TO AMBULATORY BLOOD PRESSURE AND HEART RATE

George D. Bishop, PhD, ${ }^{1}$ Francis Ngau, MS, ${ }^{1}$ E Shyang Tai, $\mathrm{MB} \mathrm{ChB}{ }^{2,3}$ and Jeannette Lee, MBBS (Hons) ${ }^{3}$

${ }^{1}$ Psychology, National University of Singapore, Singapore, Singapore; ${ }^{2}$ Medicine, National University of Singapore, Singapore, Singapore and ${ }^{3}$ Epidemiology and Public Health, National University of Singapore, Singapore, Singapore.

Objective: This study tested the hypothesis that emotional arousal interacts with trait anger in its relationship to cardiovascular parameters.

Method: Three hundred twenty participants (174 females, 148 Chinese, 86 Malays, 86 Indians) aged 24 to 64 years $(M=48.2)$ were drawn from a population based epidemiological cohort study and participated in $24 \mathrm{hr}$ ambulatory BP \& HR monitoring with measurements taken every 30 minutes during waking hours. All participants took the STAXI II and answered a computerized diary, including items on both positive and negative emotional experiences, after each BP measurement.

Results: As predicted individuals high in Trait Anger (TA) showed increased SBP $(\mathrm{p}<.06) \&$ HR $(p<.002)$ when reporting negative emotions whereas this was not true for those low in TA. For SBP this interaction was significant for Chinese $(\mathrm{p}<.04)$ but not for Malays and Indians. TA also interacted with positive emotions such that for males with high TA higher levels of positive emotions were associated with increased SBP $(p<.05)$ whereas this was not true for males with low TA or for females. In addition, Indian males with high TA showed reduced HR with higher levels of positive emotion whereas this was not true for those with low TA $(\mathrm{p}<.01)$. This interaction was not found for Chinese or Malays.

Conclusions: These data provide further evidence for the interaction of TA with negative emotions in cardiovascular reactivity and also obtained ethnic and gender differences in this relationship. However, the fact that TA also interacts with positive emotions in its effects on cardiovascular parameters suggests that individuals with high TA, particularly if they are Chinese and/or male, may be more generally reactive to emotional experiences, irrespective of valance. 
CORRESPONDING AUTHOR: George D. Bishop, PhD, Psychology, National University of Singapore, Singapore, 117570; psygb@nus.edu.sg

\section{2:48 PM-3:06 PM}

OS32-B

MULTIPLE REWARDING SOCIAL ROLES LIMIT THE PROGRESSION OF CORONARY CALCIUM IN MIDLIFE WOMEN

Imke Janssen, $\mathrm{PhD},{ }^{1}$ Lynda $\mathrm{H}$. Powell, $\mathrm{PhD},{ }^{1}$ Steven Hollenberg, $\mathrm{MD},{ }^{3}$ Karen A. Matthews, $\mathrm{PhD},{ }^{2}$ Kim Sutton-Tyrrell, $\mathrm{DrPH},{ }^{2}$ John Cursio, $\mathrm{MS}^{1}$ and Susan A. Everson-Rose, $\mathrm{PhD}^{4}$

${ }^{1}$ Rush University Medical Center, Chicago, IL; ${ }^{2}$ University of Pittsburgh, Pittsburgh, PA; ${ }^{3}$ Cooper University Hospital, Camden, $\mathrm{NJ}$ and ${ }^{4}$ University of Minnesota, Minneapolis, MN.

Background: Studies of protective psychosocial factors for women focus mainly on factors such as social support and optimism. Little attention has been directed toward the impact of rewarding social roles on physical health.

Methods: To examine the link between rewarding social roles and progression of coronary calcification (CAC) among women at midlife, psychosocial assessments and CT scans were compared as part of the Study of Women's Health Across the Nation (SWAN) Heart ancillary study conducted from 2001-2005 in Chicago, IL and Pittsburgh, PA. Data from 337 women (31\% black, $69 \%$ white; mean age, $50.8 \pm 2.8$ ) years) with 2 assessments an average of 2.3 years apart were analyzed. Four roles - spouse, parent, employee, and caregiver were assessed and rated on a 5-point scale from not rewarding to extremely rewarding. CAC was assessed via electron beam computed tomography; progression was defined as an increase in $\mathrm{CAC}$ of $>10$ Agatston units and analyzed using relative risk (RR) regression.

Results: White women had more roles than black women (mean $2.8 \pm 0.7$ vs. $2.6 \pm 0.7, p=0.045$ ) and found their roles more rewarding (mean $3.9 \pm 0.7$ vs. $3.7 \pm 0.6, p=0.007$ ). In a multivariable model adjusted for baseline calcium, age, time between CT scans, race, education, BMI, blood pressure, statin use, smoking, menopausal status, HT use, and HDL cholesterol, rewarding social roles significantly predicted $\mathrm{CAC}$ progression. Each 1-point higher reward score was associated with decreased risk $[\mathrm{RR}=0.66(95 \% \mathrm{CI}=0.47-0.93), \mathrm{p}=.018]$. The effect also was independent of depression, social support and optimism.

Conclusions: Rewarding roles may be a novel and independent protective factor for subclinical disease progression in midlife women. More attention to this protective factor is needed. Supported by NIH/DHHS (grants AG012505, AG012546, HL065581, HL065591, HL089862).

CORRESPONDING AUTHOR: Imke Janssen, PhD, Preventive Medicine, Rush University Medical Center, Chicago, IL, 60612; Imke_Janssen@rush.edu
3:06 PM-3:24 PM

OS32-C

QUALITY OF LIFE DIFFERENCES IN COLOMBIAN CORONARY HEART DISEASE PATIENTS AND CONTROLS: A LONGITUDINAL FOLLOW-UP

Mariantonia Lemos Hoyos, MS, ${ }^{1}$ Juan C. Arango, $\mathrm{PhD}^{2}$ and Heather Rogers, $\mathrm{PhD}, \mathrm{MPH}^{2}$

${ }^{1}$ CES University, Medellin, Colombia and ${ }^{2}$ Virginia Commonwealth University, Richmond, VA.

The prevalence of Coronary Heart Disease (CHD) is rising in many developing countries. In Colombia, cardiovascular disease is the leading cause of morbidity and mortality. Individuals with chronic diseases have poorer-quality of life (QOL), however, little is known about the QOL of Colombians with CHD or QOL evolution over time. The SF-36, a self-report instrument validated in Spanish with 8 sub-scales, was used to measure QOL in 19 CHD patients from the Clínica Cardiovascular in Medellín, Colombia and 33 controls at baseline and 6-month follow-up. The CHD Patients were significantly older than the Controls $(61.9$ yrs vs 55.0 yrs, $\mathrm{p}<0.05)$ and more likely to be male $(84 \%$ vs $18 \%, \mathrm{p}<0.001)$. Individual 2 (group: patient vs control) x 2 (time: baseline vs 6 months) ANCOVAs were conducted for each QOL sub-scale, controlling for age and gender. Results indicated a main effect for group on 4 subscales, such that, compared to Controls, CHD Patients had significantly lower Physical Role Limitation $(p<0.001)$, Vitality $(p<0.05)$, Mental Health $(p<0.01)$, and Pain $(p<0.001)$ scores across both time points. For the Physical Functioning and Emotional Role Limitations sub-scales, there was a main effect for group $(p<0.001 ; p<0.01)$ and a group $X$ time interaction $(p<0.01$; $\mathrm{p}<0.05)$ such that CHD Patients had lower scores than Controls, but significantly increased their scores from baseline to follow-up, while the Controls stayed the same. For the Social Functioning and General Health sub-scales, there were marginally significant differences for group such that CHD Patients tended to have lower scores than Controls across both time points. CHD patients in Medellín report having poorer QOL across all SF-36 domains, improving in 2 areas. These findings suggest the need for psychologists, nurses, and other health professionals to develop and implement culturally-appropriate interventions to improve QOL in Colombian patients with CHD.

CORRESPONDING AUTHOR: Heather Rogers, PhD, MPH, Department of Social and Behavioral Health, Virginia Commonwealth University, Richmond, VA, 23233; hlrogers@vcu.edu

\section{3:24 PM-3:42 PM}

OS32-D

COGNITIVE REPRESENTATIONS OF HEART FAILURE AND THEIR ASSOCIATIONS WITH TREATMENT ADHERENCE

Lana A. Jago, MSc and Linda D. Cameron, PhD

Department of Psychology, The University of Auckland, Auckland, New Zealand.

Despite considerable research supporting the effectiveness of heart failure (HF) treatments, adherence remains low. Preliminary research suggests that misconceptions about HF and its treatment 
may underlie difficulties in self-management. However HF patients are an understudied population and little is known about their illness and treatment beliefs. Guided by the Common-Sense Model, we examined the illness and treatment representations of HF patients, and their relationships with adherence. HF patients $(\mathrm{N}=79)$ completed a questionnaire assessing illness beliefs (IPQ$\mathrm{R}$ ), treatment beliefs (TRI), and adherence to medications and lifestyle behaviours. Descriptive analyses showed general uncertainty or low awareness of HF as a chronic (versus acute or cyclic) condition and uncertainty or doubts regarding potential for control. There was significant misidentification of symptoms, with only $60 \%$ of participants correctly identifying 5 or more of the key symptoms (breathlessness, weight change, fatigue, wheeziness, sleep difficulties, loss of strength, swelling). Regression analyses, controlling for demographic factors, identified several illness and treatment beliefs as predictors of adherence. Stronger beliefs in a chronic timeline predicted greater dietary adherence $(\mathrm{t}=3.31, \mathrm{p}=.001)$. Greater adherence to lifestyle changes was predicted by lower levels of cyclical timeline beliefs $(\mathrm{t}=-3.74, \mathrm{p}=.000)$. Higher beliefs in the treatment's value predicted better medication adherence $(\mathrm{t}=-2.72, \mathrm{p}=.008)$. Illness and treatment beliefs may play an important role in promoting treatment adherence and healthy lifestyle practices in individuals with HF. Many HF patients appear to lack a comprehensive understanding of their condition and its treatment, resulting in vague representations and potentially contributing to poor treatment adherence. Improving understanding of HF may strengthen representations, leading to improvements in adherence.

CORRESPONDING AUTHOR: Lana A. Jago, MSc, Psychology, The University of Auckland, Auckland, 1142; 1.jago@auckland. ac.nz

\section{3:42 PM-4:00 PM}

OS32-E

THE ROLE OF EXECUTIVE FUNCTION ON ADHERENCE IN PATIENTS WITH HEART FAILURE

Sebastian Kohlmann, MSc, ${ }^{1}$ Susanne Brenner, MD, ${ }^{2}$ Bettina Hamann, MD, ${ }^{1}$ Winfried Rief, $\mathrm{PhD}^{3}$ and Stefan Stoerk, $\mathrm{MD}, \mathrm{PhD}^{2}$ ${ }^{1}$ Department of Psychiatry, Psychosomatics \& Psychotherapy, University's Medical Centre Wuerzburg, Wuerzburg, Germany; ${ }^{2}$ Department of Internal Medicine, University's Medical Centre Wuerzburg, Wuerzburg, Germany and ${ }^{3}$ Department of Clinical Psychology, University of Marburg, Marburg, Germany.

Theory: Memory dysfunction is correlated with reduced adherence in various patients groups. However, non-adherence is not only caused by memory dysfunction, as adherence is a complex behavioural regimen including planning, forming intentions and implementing actions. Neurocognitive executive functioning is a precondition for these action-plans and therefore serves as a base for adherence. Based on a model of executive function in medical illnesses this study investigates the link between executive function and adherence to medication in heart failure patients. Method: Executive function in patients $(\mathrm{N}=130)$ with heart failure is tested using Trail-Making test, Go/noGo-, Visual Set-Shifting- and n-Back task; other neurocognitive tests include digit-span (forward/backward), word recall (short/long-term), alertness and focussed attention. Self-reported adherence is assessed using the Medication Adherence Rating Scale adjusted for social desirability. As possible confounders age, years of education, beliefs about medicines, illness perception, and depression are analyzed. To define health status quality of life, NYHA-class and relevant biological health markers are measured.

Results: Preliminary results indicate that non-adherent patients showed worse performance on Trail-Making test and n-Back task $(\mathrm{p}<.05)$. Results from multiple regression analyses are presented and a neurocognitive model of adherence is introduced.

Discussion: The role of executive function on adherence to medication has not been investigated yet. As medication taking involves complex regimens, executive dysfunction could explain non-adherent behaviour. Patients with executive dysfunction may benefit from an early screening and guided medication taking procedure to overcome this barrier.

CORRESPONDING AUTHOR: Sebastian Kohlmann, MSc, Psychiatry, Psychosomatics \& Psychotherapy, University's Medical Centre Wuerzburg, Wuerzburg, 97080; Kohlmann_S1@klinik. uni-wuerzburg.de

OS33

\section{2:30 PM-2:48 PM}

OS33-A

WHIPLASH INJURY AND POSTTRAUMATIC STRESS: A ANALYSIS OF THE IMPACT OF COMPENSATION ON RECOVERY TRAJECTORIES

Justin Kenardy, $\mathrm{PhD}$ and Michele Sterling, $\mathrm{PhD}$

CONROD, University of Queensland, Herston, QLD, Australia.

This study aimed to identify distinctive trajectories for pain/ disability and posttraumatic stress disorder (PTSD) symptoms following whiplash injury and to examine the effect of injury compensation claim lodgement on the trajectories. In a prospective study, 155 individuals with whiplash were assessed at $<1$ month, 3, 6 and 12 months post injury. Outcomes at each time point were: Neck Disability Index (NDI) and the Posttraumatic Stress Diagnostic Scale (PDS). Group based trajectory analytical techniques were used to identify outcome profiles. The analyses were then repeated after including third party compensation claim lodgment as a binary time-changing covariate. Three distinct NDI trajectories were determined: 1) Mild: mild or negligible pain/ disability for the entire 12 months (45\%), 2) Moderate: initial moderate pain/disability that decreased to mild levels by 3 months (39\%) and 3) Chronic-severe: severe pain/disability persisting at moderate/severe levels for 12 months (16\%). Three distinct PTSD trajectories were also identified: 1) Resilient: mild symptoms throughout $(40 \%), 2)$ Recovering: initial moderate symptoms declining to mild levels by 3 months (43\%) and 3) Chronic moderate-severe: persistent moderate/severe symptoms throughout 12 months (17\%). Claim submission had a detrimental effect on 
all trajectories $(\mathrm{P}<0.001)$ except for the Chronic-severe NDI trajectory $(\mathrm{P}=0.098)$. Following whiplash injury, there are distinct pathways of recovery for pain/ disability and PTSD symptoms. Management of whiplash should consider the detrimental effect of compensation claim on psychological recovery and those with mild to moderate pain/disability levels. However claim lodgement has no significant influence on those following a more severe pain and disability trajectory.

CORRESPONDING AUTHOR: Justin Kenardy, PhD, CONROD, University of Queensland, Herston, QLD, 4006; j.kenardy@uq. edu.au

\section{2:48 PM-3:06 PM}

OS33-B

LEARNING TO SCREEN FOR YELLOW FLAGS IN BACK PAIN A TAILORED SKILLS TRAINING INTERVENTION IN PHYSIOTHERAPY TELEPHONE CONSULTATIONS Ingrid Demmelmaier, $\mathrm{PhD}$, Eva Denison, $\mathrm{PhD}$, Per Lindberg, $\mathrm{PhD}$ and Pernilla Åsenlöf, PhD

Uppsala University, Uppsala, Sweden.

Background: Guidelines for back pain in primary health care recommend early screening of psychosocial prognostic factors for disability. Early screening is performed to a low extent in clinical practice. Barriers for change in caregivers' behaviours have been identified as lack of agreement with guidelines recommendations, organisational constraints and conflicting patient expectations.

Aims: To study the effect of a tailored skills training intervention on physiotherapists screening of psychosocial prognostic factors in initial telephone consultations regarding back pain.

Methods: A quasi-experimental single-subject design was used, with repeated measurements during baseline, intervention and postintervention. Four physiotherapists in primary health care participated. Primary outcomes were number of screened factors and consultation time used for screening. The intervention included individual goalsetting, identification of and tailoring to barriers of change.

Results: There was a positive effect on screening behaviour in all four participants. The number of screened factors increased and time spent on detailed discussions of pain symptoms decreased. Conclusions: The results support the use of interventions based on social cognitive theories and identification of barriers for behavioural change.

CORRESPONDING AUTHOR: Ingrid Demmelmaier, $\mathrm{PhD}$, Public Health and Caring Sciences, Uppsala University, Uppsala, 751 22; ingrid.demmelmaier@pubcare.uu.se

\section{3:06 PM-3:24 PM}

OS33-D

JUSTICE MISCARRIED: LEGAL SEQUELAE OF OCCUPATIONAL BACK INJURIES

Raymond C. Tait, $\mathrm{PhD}$ and John T. Chibnall, $\mathrm{PhD}$

Neurology \& Psychiatry, Saint Louis University, St. Louis, MO.
Study Design: Population-based telephone survey and longitudinal database analysis in Missouri.

Objective: To compare legal difficulties experienced by African American and Caucasian workers' compensation claimants presettlement versus post-settlement.

Summary of Background Data: Retrospective studies suggest that workers with occupational back injuries experience financial and personal duress following claim settlement. This study examined these issues by comparing financial and domestic court actions for the five years pre-settlement against five years post-settlement. Sociodemographic differences also were examined.

Methods: Missouri judicial records were reviewed for African American $(n=580)$ and non-Hispanic Caucasian $(n=892)$ workers' compensation claimants to determine the frequency with which four types of cases occurred: general financial, domestic financial, residence financial, and domestic behavior. Average annual level of judicial activity during the five years prior to claim settlement was compared to activity for each of five post-settlement years; significance of change was evaluated with univariate and multivariate repeated measures analyses.

Results: Statistically significant $(\mathrm{p}<.001)$ post-settlement increases in legal cases were noted for each of the four categories of cases. There were significant interactions between race and time for general financial and domestic financial cases. A significant interaction between age and time occurred for general financial cases. Significant three-way interactions (race $\mathrm{x}$ income change $\mathrm{x}$ time) emerged for general and domestic financial cases.

Conclusions: The results confirm that workers with occupational back injuries, especially African American and younger adults, encounter long-term financial and domestic duress that appears to escalate with each passing year following claim settlement. This pattern suggests that short-term studies under-estimate postsettlement difficulties, particularly among selected demographic cohorts.

CORRESPONDING AUTHOR: Raymond C. Tait, PhD, Neurology \& Psychiatry, Saint Louis University, St. Louis, MO, 63104; taitrc@slu.edu

\section{3:24 PM-3:42 PM}

OS33-E

QUALITY OF LIFE DIFFERENCES IN COLOMBIANS WITH CORONARY HEART DISEASE, TRAUMATIC BRAIN INJURY, OR SPINAL CORD INJURY

Mariantonia Lemos Hoyos, MS, ${ }^{1}$ Heather Rogers, PhD, MPH, ${ }^{2}$ Juan C. Arango, PhD ${ }^{2}$ Silvia Leonor Olivera, MS, ${ }^{3}$ Carlos Jose de los Reyes, BS, Jose Libardo Perdomo, $\mathrm{BS}^{3}$ and Jose Arango, $\mathrm{BS}^{3}$ ${ }^{1} \mathrm{CES}$ University, Medellin, Colombia; ${ }^{2}$ Virginia Commonwealth University, Richmond, VA; ${ }^{3}$ Surcolombian University, Neiva, Colombia and ${ }^{4}$ North University, Barranquilla, Colombia.

In many developing countries, prevalence rates of chronic diseases are growing. In Colombia, cardiovascular disease is the leading cause of morbidity and mortality; Traumatic Brain Injury (TBI) 
and Spinal Cord Injury (SCI) are top causes of disability. Individuals with chronic diseases have poorer quality of life (QOL), however, little is known about the differences in QOL of Colombians with Coronary Heart Disease (CHD), TBI, and SCI. The Patient Health Questionnaire (PHQ-9) was used to measure depression and the SF-36 and the Satisfaction with Life Scale (SWLS) was used to measure QOL in 116 CHD patients from the Clínica Cardiovascular in Medellín, 30 individuals with TBI living in Barranquilla, 40 individuals with SCI living in Neiva, and 168 controls from the three cities. All analyses controlled for age, gender, and education. Individuals with TBI had sig higher PHQ-9 scores than the other 3 groups $(\mathrm{p}<0.05)$. Compared to Controls, individuals with SCI had sig higher, and those with CHD tended to have higher, PHQ-9 scores $(p<0.05 ; p=0.06)$. Individuals with SCI had sig lower SWLS scores than the other groups $(p<0.001)$. Compared to Controls, individuals with CHD had sig lower SF-36 sub-scale scores across all 8 domains $(\mathrm{p}<0.01)$, those with TBI had sig lower scores in all domains except vitality ( $\mathrm{p}=\mathrm{NS}$ ), and those with $\mathrm{SCI}$ in all except vitality $(p=N S)$ and emotional well-being $(p=N S)$. Individuals with SCI had sig lower general health $(\mathrm{p}<0.01)$ and physical functioning ( $p<0.001)$ scores than the other 3 groups. Those with CHD had sig lower physical functioning scores than those with TBI $(p<0.001)$ and those with TBI had sig lower emotional role limitation scores than those with SCI $(\mathrm{p}<0.05)$. Colombians with TBI are more depressed than Controls and those with SCI or CHD, but those with SCI have the lowest satisfaction with life. Individuals with chronic diseases, especially CHD, have lower QOL than Controls, yet those with SCI appear most affected among those with chronic disease. Further investigation into specific determinants of QOL for Colombians with chronic diseases is warranted.

CORRESPONDING AUTHOR: Heather Rogers, PhD, MPH, Department of Social and Behavioral Health, Virginia Commonwealth University, Richmond, VA, 23233; hlrogers@vcu.edu

\section{OS34}

\section{2:30 PM-2:48 PM}

OS34-A

REBELLIOUSNESS: ITS MEASUREMENT AND ROLE IN SOCIOECONOMIC DIFFERENCES IN HEALTH IN OLDER PEOPLE

Gonnie Klabbers, MA, ${ }^{1}$ Hans Bosma, $\mathrm{PhD},{ }^{1}$ Marjan van den Akker, $\mathrm{PhD}^{2}{ }^{2}$ Gertrudis I. Kempen, Prof ${ }^{3}$ and Jacques T. van Eijk, Prof ${ }^{1}$ ${ }^{1}$ Social Medicine, CAPHRI, Maastricht, Netherlands; ${ }^{2}$ General Practice, CAPHRI, Maastricht, Netherlands and ${ }^{3}$ Health Care and Nursing Science, CAPHRI, Maastricht, Netherlands.

Rebelliousness is about feeling compelled to do something contrary to that required by some external agency. More than hostility (a known psychological risk indicator for heart disease), rebelliousness might be strongly related to health and heart disease via rebellious persons opposing and obstructing health campaigns aimed at modifying unhealthy behaviours. We set out to examine, firstly, the psychometric properties of a rebelliousness question- naire and, secondly, the extent to which rebelliousness and hostility compete in the prediction of incident heart disease and in the contribution to socioeconomic differences in health. Using Dutch data on older men and women $(n=4,485)$, we found moderate reliability (Cronbach's $\alpha$ ranging between 0.50 and 0.60 ) and moderate (construct) validity, as indicated by rebelliousness being (moderately) associated with low control beliefs, hostility, and unhealthy behaviours. Using longitudinal data ( $\mathrm{n}>$ 2,000), we will further report on the extent to which rebelliousness is more common (than hostility) in lower socioeconomic status groups, the extent to which rebelliousness is more common (than hostility) in the etiology of heart disease, and the extent to which rebelliousness more strongly (than hostility) affects heart disease through poor health behaviours. The findings add to the body of evidence on the psychological pathways involved in how socioeconomic structure gets under the skin.

CORRESPONDING AUTHOR: Hans Bosma, PhD, Social Medicine, CAPHRI, Maastricht, 6200 MD; hans.bosma@ socmed.unimaas.nl

\section{2:48 PM-3:06 PM}

OS34-B

OBJECTIVELY ASSESSED PHYSICAL ACTIVITY

AND HEALTH AMONG ELDERLY: THE IMCA-ACTIFE STUDY

Richard Peter, PhD, ${ }^{1}$ Jochen Klenk, PhD,${ }^{1}$ Sebastian Franke, Psychologist, ${ }^{1}$ Michael Denkinger, $\mathrm{MD}^{2}{ }^{2}$ Kilian Rapp, $\mathrm{MD},{ }^{3}$ Gudrun Weinmayr, $\mathrm{PhD},{ }^{1}$ Thorsten Nikolaus, $\mathrm{MD}^{2}$ and IMCA ActiFe Study Group, MD ${ }^{1}$

${ }^{1}$ Epidemiology, Ulm University, Ulm, Germany; ${ }^{2}$ Bethesda Hospital, Ulm, Germany and ${ }^{3}$ Robert-Bosch Hospital, Stuttgart, Germany.

Physical activity is a powerful predictor of health in older ages. However, in most population-based studies on this topic physical activity has been measured retrospectively with the help of questionnaires. Accordingly, bias may occur. The present investigation applies an ambulatory objective measurement of physical activity which helps to avoid bias.

The baseline screening of a prospective study is currently conducted in a random sample of the older population in the greater Ulm area, Germany. It is planned to investigate 1500 men and women aged 65 to 90 years. Recently, about 1000 are included. Extensive information on cognitive and physical functioning, chronic disease, quality of life and socio-demographic variables is derived from well-tested questionnaires and tests. Information on medication and falls is assessed prospectively. Lung function is measured according to international standards. Blood samples are taken to determine biomarkers. Furthermore, physical activity is assessed ambulatory with the help of accelerometers. The accelerometer information is combined with an activity diary.

First preliminary results show associations between physical activity as measured by the accelerometers and health. Individuals with type II diabetes are significantly less active as compared to elderly without this disease even after adjustment for age and gender. Over a period of at least 5 days subjects with diabetes are on 
an average one hour per day longer inactive as compared to the healthy group. Further analyses will include a number of additional variables presumably influencing these associations like depression, lung function, and cognitive as well as physical functioning. First preliminary results of the IMCA-ActiFe study indicate the validity of objective measurement of physical activity and show associations of inactivity with type II diabetes. For the future a more clear and detailed picture of daily activity among elderly can be expected from accelerometer measurement. Accordingly, interventions better tailored to individual needs can be developed.

CORRESPONDING AUTHOR: Richard Peter, PhD, Epidemiology, Ulm University, Ulm, DE-89081; richard.peter@uni-ulm.de

\section{3:06 PM-3:24 PM}

OS34-C

ROLE REWARD, PREPAREDNESS, ANXIETY AND DEPRESSION IN FAMILY CAREGIVERS WITH DIFFERENT LEVELS OF RESOURCEFULNESS

Elizabeth W. Gonzalez, PhD, ${ }^{1}$ Carol F. Lippa, $\mathrm{MD}^{2}$ and Marcia Polansky, ScD, MSW ${ }^{3}$

${ }^{1}$ College of Nursing \& Health Professions, Drexel University, Philadelphia, PA; ${ }^{2}$ College of Medicine, Drexel University, Philadelphia, PA and ${ }^{3}$ School of Public Health, Drexel University, Philadelphia, PA.

Background: Despite the evidence that providing care to a person with dementia has adverse health consequences on caregivers, there are individual variations in their responses to the caregiving role. Approximately 10.4 million family caregivers of people with Alzheimer's and other dementias provide care to their loved ones in the community. The purpose of this study was to examine the differences between highly resourceful and less resourceful family caregivers on role reward, preparedness for caregiving, anxiety and depression.

Method: A comparative descriptive design was used on baseline data of 84 family caregivers who were living with and caring for person diagnosed with Alzheimer's disease. The data is part of an ongoing intervention study to enhance resourceful skills in family caregivers. Instruments: Resourcefulness was with the 36-item Self-Control Schedule (SCS: Rosenbaum, 1980). Depression was measured with the 20-item CESD scale. Preparedness was measured using the 8-item Family Preparedness Scale (Archbold \& Stewart, 1994). Anxiety was measured using the Anxiety Scale on the State Trait Anxiety Inventory (Speilberger et al, 1970). Role reward was measured with Family Role reward Scale (Archbold \& Stewart, 1994), a 15-item Likert-type tool with a 5 point scale. Descriptive statistics and t-test were used to analyze the data.

Findings: The results showed that family caregivers with low SCS scores (less than 25) were significantly less prepared $(\mathrm{t}=3.03, \mathrm{df}=$ $83, \mathrm{p}<.004)$, more anxious $(\mathrm{t}-3.055, \mathrm{df}=83, \mathrm{p}, .004)$ and reported less role reward $(\mathrm{t}=3.59, \mathrm{df}=83, \mathrm{p}<.001)$ in caregiving than those who were highly resourceful. There were no difference in depression, and appraisal of behavior of behavior problems between groups. The implication is to assess and enhance caregivers' resourcefulness on those caregivers with low resourceful skills.
Funded by NINR (R15-NR009490-01A1) PI: Elizabeth W. Gonzalez, $\mathrm{PhD}$

CORRESPONDING AUTHOR: Elizabeth W. Gonzalez, PhD, College of Nursing \& Health Professions, Drexel University, Philadelphia, PA, 19102; ewg22@drexel.edu

3:24 PM-3:42 PM

OS34-D

TRAUMATIC EXPERIENCES, POSTTRAUMATIC SYMPTOMATOLOGY AND IT'S COMORBIDITY WITH DEPRESSION AND SOMATISATION IN THE GERMAN ELDERLY POPULATION Heide Glaesmer, Dr and Elmar Brähler, Prof Dr University of Leipzig, Leipzig, Germany.

Background: The German elderly population experienced more traumatic experiences across their life-span than the younger cohorts. Even sixty years after the WW II, this cohort is significantly more often affected by posttraumatic symptomatology compared to the younger cohorts (Maercker et al., 2008). The present study is addressing prevalence rates of posttraumatic symptomatology and it's comorbidity with depression and somatization. Methods: The study examines PTSD according to DSM-IV, partial PTSD, depression and somatisation in a randomly selected sample of the German general population aged 60 to 85 years $(\mathrm{N}=1.659)$ using self rating instruments (PHQ, PDS). Results: One-month prevalence rates were $2.8 \%$ for DSM-IV PTSD, another $11.9 \%$ fulfilled the criteria of partial PTSD. This points out, that sub threshold symptomatology plays a major role in the elderly. $11.5 \%$ of the persons affected by PTSD symptomatology fulfil the criteria of a somatoform syndrome, $8.5 \%$ fulfil the criteria of Major Depression and $10.4 \%$ fulfil the criteria of other depressive syndromes according the Patient Health Questionnaire. 5.6\% report both - depression and somatoform disorder. Discussion: Even sixty years after WW II, posttraumatic symptomatology is more common in the elderly in Germany compared to the younger cohorts. It is often accompanied by other comorbid mental disorders like depression or somatisation. Implications of these findings for health and health care in the elderly will be discussed.

CORRESPONDING AUTHOR: Heide Glaesmer, Dr, University of Leipzig, Leipzig, 04103; Heide.Glaesmer@medizin.uni-leipzig.de

\section{OS35}

\section{2:30 PM-2:48 PM}

OS35-A

"WE ARE SURVIVORS TOO": AFRICAN AMERICAN YOUTHS' EXPERIENCES OF COPING WITH PARENTAL BREAST CANCER

Karni Kissil, MA, M Ed, Alba Nino, MS in Marriage and Family Therapy, Maureen Davey, PhD and Carolyn Y. Tubbs, PhD

Couple and Family Therapy, Drexel University, Philadelphia, PA. 
Objective: To explore how African American youth cope with the diagnosis and treatment of parental breast cancer and to identify culturally sensitive ways to recruit and sustain participation of this vulnerable population in intervention programs.

Methods: Three qualitative focus groups which were part of a larger study were conducted with 12 African American youth between the ages of 11 and 18 currently coping with parental breast cancer from the northeastern part of the United States. Interviews were audiotaped and transcribed verbatim, and analyzed using content analysis. Results: African American youth described fear and uncertainty about the mortality of their parent, their unpredictable future, and discomfort in negotiating breast cancer's relationship with the entire family. Four primary themes emerged which were coping with cancer, surviving cancer too, changes in family function, and growth through pain. African American youth described feeling overlooked by their families and oncology staff treating their parents, often being in the role of protecting their parents physically and emotionally.

Conclusions: This study suggests that clinicians can improve the care of African American breast cancer patients and their adolescent children by being more family-centered. Adolescents need more developmentally appropriate preparation for the family changes likely to occur when a parent is diagnosed and treated for breast cancer. Developing a support group comprised of other youth coping with parental breast cancer from diagnosis throughout treatment was described as a preferred intervention to promote a shared understanding in order to overcome feelings of isolation, worry, and fear.

CORRESPONDING AUTHOR: Alba Nino, MS in Marriage and Family Therapy, Couple and Family Therapy, Drexel University, Philadelphia, PA, 19102; aln38@drexel.com

\section{2:48 PM-3:06 PM}

OS35-B

USE OF DIETARY OR VITAMIN SUPPLEMENTS IS A RISK FACTOR FOR DEPRESSIVE SYMPTOMS AT 1-YEAR FOLLOW-UP AFTER PRIMARY SURGERY FOR EARLY BREAST CANCER. RESULTS FROM A NATIONWIDE DANISH COHORT STUDY

Christina G. Pedersen, MSc, ${ }^{1,2}$ Søren Christensen, MSc, ${ }^{1,2}$ Anders Bonde Jensen, $\mathrm{Dr}^{2}$ and Robert Zachariae, MSc Dr Med ${ }^{1,2}$

${ }^{1}$ Psychooncology Research Unit, Aarhus Univeristy, Aarhus C, Denmark and ${ }^{2}$ Department of Oncology, Aarhus University Hospital, Aarhus, Denmark.

Background: Previous studies suggest that use of complementary and alternative medicine (CAM) may have a beneficial influence on emotional well-being of cancer patients. Most existing studies, however, are based on small non-representative samples and have not differentiated between different types of cancer and specific types of CAM.

Objective: To investigate if use of specific types of CAM measured 3 months after surgery predicted the level of depressive symptoms at follow-up 15-16 months after surgery when controlling for baseline depression and sociodemographic-, health-, disease-, and treatment related confounders.
Methods: In a nationwide cohort of 3343 Danish women (age 2670) (response rate: $68 \%$ ), use of CAM since diagnosis, depressive symptoms (Beck's Depression Inventory), and health-related variables were assessed at baseline 3 months post-surgery. Depressive symptoms were re-assessed at follow-up 15 months post-surgery (response rate for disease free women: 94\%). Sociodemographic-, and clinical variables and data on comorbidity were obtained from the surgical departments and national longitudinal registries.

Results: Overall CAM use was associated with more depressive symptoms at follow-up. This was due to users of dietary or vitamin supplements $(\mathrm{N}=895)$ at baseline exhibiting more depressive symptoms at follow-up, compared to non-users in a negative binomial regression analysis fully adjusted for baseline depression and potential confounders (ratio of mean $(\mathrm{RM})=1.11$; $95 \% \mathrm{CI}=1.04-1.19 ; \mathrm{p}=.003)$.

Conclusion: In this prospective study use of dietary or vitamin supplements showed to be a risk factor of depressive symptoms. It remains to be explored if this finding may be explained by a hidden vulnerability for depressive symptoms among women using dietary or vitamin supplements, by interaction effects with conventional treatment, or by a direct negative influence of dietary or vitamin supplements.

Learning objective 1: To gain knowledge of the association between use of CAM and changes in QoL in breast cancer patients.

Learning objective 2: To gain knowledge of the association between specific types of CAM use and changes in QoL in breast cancer patients.

CORRESPONDING AUTHOR: Christina G. Pedersen, MSc, Aarhus Univeristy and Aarhus University Hospital, Psychooncology Research Unit, Aarhus C, 8000; christina@psy.au.dk

3:06 PM-3:24 PM

OS35-C

THE M.A.P. (MIND AFFECTS THE PHYSICAL) PROJECT: UNDERSTANDING THE PSYCHOSOCIAL NEEDS OF BREAST CANCER SURVIVORS

Joanne S. Buzaglo, PhD, Kasey R. Dougherty, BA and Mitch Golant, $\mathrm{PhD}$

Research \& Training Institute, Cancer Support Community, Philadelphia, PA.

Increasingly, breast cancer(BC) is managed as a chronic disease with persistent physical and psychosocial demands. Yet,little research has focused on the long-term psychosocial needs of women across the care continuum. The Cancer Support Community(CSC) fielded a national survey to better understand the current psychosocial needs of BC survivors.

Methods: CSC conducted an online survey of 1,004 breast cancer survivors, 0 to 5 years post-diagnosis. Questions were developed with input from a National Advisory Council of interdisciplinary experts representing advocacy, oncology,psychology,policy,industry and managed care.

Results: $87 \%$ participants were white,with a mean age of $54.45 \%$ graduated from college and $14 \%$ had a graduate degree. $75 \%$ had 
stage II cancer or less and $13 \%$ had a $\mathrm{BC}$ recurrence. $81 \%$ survivors reported some form of physical, emotional or spiritual distress with $67 \%$ reporting emotional distress. 64\% women indicated fear of recurrence, $58 \%$ physical changes, $55 \%$ anxiety, $47 \%$ depression, $46 \%$ financial worries as the leading contributors to distress. Regarding their immediate needs, women wanted support around fears of recurrence (62\%);making healthy lifestyle choices (60\%); financial matters (42\%); and reducing anxiety (41\%). $86 \%$ believe emotional support is important to overall health,while only $52 \%$ had someone from their medical team address their emotional needs. $37 \%$ respondents did not receive a referral for support services. Of those that did receive a referral,30\% did not follow up on any referrals. To reduce the cost of breast cancer, $38 \%$ of respondents delayed seeking counseling or support. $64 \%$ would be likely to seek psychosocial support if it were free of charge.

Discussion: Women with breast cancer recognize the importance of emotional support as part of survivorship care,yet few survivors access the care they need. As part of the M.A.P. Project,these data will inform the development of a Cancer Survivor Registry to identify behavioral trends and gaps in psychosocial care across the survivorship continuum.

CORRESPONDING AUTHOR: Joanne S. Buzaglo, PhD, Research \& Training Institute, Cancer Support Community, Philadelphia, PA, 19131; joanne@cancersupportcommunity.org

\section{3:24 PM-3:42 PM}

OS35-E

BREAST CANCER PATIENTS EVALUATING THEIR

"LIFE NOW" AS BETTER THAN THEIR "PREVIOUS LIFE" MAY EXPERIENCE ADVERSE EFFECTS OF EXPRESSIVE WRITING INTERVENTION

Mimi Mehlsen, PhD, ${ }^{1}$ Mikael B. Jensen-Johansen, MSc, ${ }^{1}$ Søren Christensen, MSc, ${ }^{1}$ Heiddis Valdimarsdottir, $\mathrm{PhD}{ }^{2}$ Sandra G. Zakowski, $\mathrm{PhD},{ }^{3}$ Dana Bovbjerg, $\mathrm{PhD},{ }^{4}$ Anders B. Jensen, $\mathrm{PhD}^{1}$ and Robert Zachariae, MDSci ${ }^{1}$

${ }^{1}$ Aarhus University Hospital, Aarhus, Denmark; ${ }^{2}$ Mount Sinai School of Medicine, New York, NY; ${ }^{3}$ Argosy University, Chicago, IL and ${ }^{4}$ University of Pittsburgh School of Medicine, Pittsburgh, PA.

Background: Several studies have reported beneficial effects of expressive writing intervention (EWI) on emotional distress in patient populations. However, moderating variables are often involved and possible deleterious effects have received little attention

Objectives: To explore whether the cognitive strategy of temporal comparison (i.e. comparing present life with past life) moderates the effect of EWI on negative affect (NA).

Methods: A population based sample of women completing adjuvant treatment for breast cancer were randomized to a EWI group $(n=253)$ writing about the most traumatic experience or to a control group $(n=254)$ writing about daily activities. Both groups completed three home-based writing sessions one week apart. Temporal comparison was an evaluation of "Compared to previously, my life now is..." on a 5-point Likert scale ranging from "much worse" to "much better". NA was measured by the POMS. Both variables were assessed before randomization, 3 months, and 9 months after the first writing session.

Results: The EWI and control group did not differ in use of comparisons or in NA at baseline. Repeated-measures ANOVAs showed no main effects of EWI on NA $(F=0.48, n s)$. When temporal comparison was included in the model, a significant effect of EWI on NA was found $(\mathrm{F}=3.53, \mathrm{p}<0.001)$, together with an interaction between EWI and temporal comparison $(\mathrm{F}=2.13, \mathrm{p}<0.05)$.

Conclusion: The results showed that EWI-women who at baseline evaluated their present life as better than previous periods increased in negative affect, whereas in the control condition, these women showed stable or reduced negative affect. This suggests that EWI could have an adverse effect in individuals who are not settled with their past. Hence, future studies may consider prescreening to optimize outcomes of EWI in patient populations.

CORRESPONDING AUTHOR: Mimi Mehlsen, PhD, Psychooncology Research Unit, Aarhus, Dk8000; mimim@psy.au.dk

OS36

\section{2:30 PM-2:48 PM}

OS36-A

TAKEAWAY FOOD CONSUMPTION AMONG AUSTRALIAN ADULTS: DO SOCIOECONOMIC GROUPS DIFFER IN THEIR TAKEAWAY FOOD CONSUMPTION BEHAVIOUR?

Kyoko Miura, BHSc (Nutrition), Katrina Giskes, $\mathrm{PhD}$ and Gavin Turrell, PhD

School of Public Health and Institute of Health and Biomedical Innovation, Queensland University of Technology, Kelvin Grove, QLD, Australia.

Introduction: Socioeconomic differences in weight status are well documented. These inequalities may be partly due to differences in takeaway food consumption patterns as frequent fast-food consumption is associated with increased risk of weight gain. However, the findings of studies examining socioeconomic position (SEP) and fast-food consumption are inconsistent. The current study examines SEP and takeaway food consumption behaviours.

Method: A cross-sectional postal survey was conducted among 1500 adults aged 25 and 64 years (response rate=63.6\%). SEP was ascertained from participants' highest achieved education level, and takeaway consumption was measured using a detailed food frequency questionnaire of 22 takeaway food and beverage items. Socioeconomic differences in the frequency (whether ate $\geq 2$ times per week) and types of takeaway food consumed were determined by logistic regression.

Results: About $42 \%$ of men and $33 \%$ women consumed takeaway food at least weekly. There were no education differences in the frequency of takeaway food consumption between the highest and lowest education groups (men OR 1.48; 95\% CI 0.68, 3.18; women OR 1.39; 95\% CI 0.69, 2.80). However, lower educated men and women were more likely to make unhealthy takeaway 
choices though this difference only reached statistical significance among women (OR 2.87; 95\% CI 1.51, 5.45).

Conclusion: Takeaway food consumption may be an important contributor to dietary intakes among adults and may contribute to socioeconomic inequalities in dietary intakes and subsequently inequalities in overweight/obesity and chronic diseases.

CORRESPONDING AUTHOR: Kyoko Miura, BHSc (Nutrition), School of Public Health, Queensland University of Technology, Kelvin Grove, QLD, 4059; k.miura@qut.edu.au

\section{2:48 PM-3:06 PM}

OS36-B

PSYCHOLOGICAL DISTRESS, GLUCOSE METABOLISM AND MORTALITY IN ADULTS WITH AND WITHOUT DIABETES

Mark Hamer, PhD, ${ }^{1}$ Emmanuel Stamatakis, $\mathrm{PhD},{ }^{1}$ Mika Kivimaki, $\mathrm{PhD},{ }^{1}$ Andre Pascal Kengne, $\mathrm{PhD}^{2}$ and David G. Batty, $\mathrm{PhD}^{3}$

${ }^{1}$ Epidemiology and Public Health, University College London, London, United Kingdom; ${ }^{2}$ The George Institute for International Health, The University of Sydney, Sydney, NSW, Australia and ${ }^{3}$ Centre for Cognitive Ageing \& Cognitive Epidemiology, University of Edinburgh, Edinburgh, United Kingdom.

Poor mental health is more prevalent among diabetic patients and may be a risk factor for worse prognosis. Worsening glucose metabolism appears to contribute to patient's level of distress and may be an important factor in explaining future prognosis. We examined associations between psychological distress, glucose metabolism and death in adults with and without diabetes. In a representative, prospective study of 18,525 adults $(4.1 \%$ with physician-diagnosed diabetes) we measured levels of psychological distress using the 12-item General Health Questionnaire (GHQ-12) and blood was collected for the assessment of glycated haemoglobin, a marker of glucose metabolism. The main outcome was all cause death. Psychological distress, that was defined as GHQ-12 score of $\geq 4$, was apparent in $19.5 \%$ and $13.9 \%$ of participants with and without diabetes, respectively. Distress was associated with greater cardiovascular morbidity although not with impaired glucose metabolism in diabetics. Among diabetics there were 187 deaths over an average of 6.0 years of follow up. Distress was associated with higher risk of death (multivariate adjusted hazard ratio $=1.95,95 \%$ CI, $1.34-2.84$ ) after adjustment for glucose control, health behaviours, and cardiovascular comorbidity. The synergistic coexistence of diabetes and distress was associated with an elevated risk of all cause death, beyond that due to having either diabetes or distress alone. In summary, psychological distress is an independent risk factor for death in diabetic patients, but this association was not explained through impaired glucose metabolism. Early screening for distress may have important implications for prevention and treatment in diabetics.

CORRESPONDING AUTHOR: Mark Hamer, PhD, Epidemiology and Public Health, University College London, London, WC1E 6BT; m.hamer@ucl.ac.uk
3:06 PM-3:24 PM

OS36-E

“IT JUST CREPT UP ON ME” UNDERSTANDING

THE HEALTH AND WEIGHT EXPERIENCES OF OBESE MEN IN AUSTRALIA: A QUALITATIVE STUDY

Sophie Lewis, BmedSci, ${ }^{1}$ Samantha L. Thomas, BA MA PhD, ${ }^{1}$ R. Warwick Blood, BS PhD, ${ }^{2}$ Kate Holland, $\mathrm{PhD},{ }^{2}$ David J. Castle, MBBS PhD FRANZP ${ }^{3}$ and Jim Hyde, BA MA PhD ${ }^{4}$

${ }^{1}$ Consumer Health Research Group (CHaRGe), Primary Care Research Unit, School of Primary Health Care, Monash University, Melbourne, VIC, Australia; ${ }^{2}$ News Research Group, Faculty of Arts and Design, University of Canberra, Canberra, VIC, Australia; ${ }^{3}$ Department of Psychiatry, St Vincent's Hospital, University of Melbourne, Melbourne, VIC, Australia and ${ }^{4}$ Victorian Department of Health, Melbourne, VIC, Australia.

Background: Research suggests that men may underestimate both their weight and the health risks associated with their weight. However, there is only limited understanding of the health beliefs and health behaviours of obese men. This qualitative study of Australian men with obesity (BMI over 30) aimed to explore their beliefs about their weight, health and wellbeing; their attitudes towards weight loss; and the strategies that they employed to lose weight or improve their health.

Method: In-depth semi-structured telephone interviews were conducted with a community sample of men with a body mass index of 30 or more. A range of community engagement strategies were used to encourage men to take part in the study from different socioeconomic and geographical backgrounds. Data were analysed using a descriptive thematic approach.

Results: Thirty-six men participated. Most men stated that they were not aware of their weight gain and that their weight had increased slowly and steadily over a number of years. Often a significant life event, for example a health scare, motivated men to recognise that their weight was a problem and that they needed to lose weight. Men often blamed themselves for their obesity. They believed that their obesity was primarily caused by a lack of activity rather than their eating behaviours. Most strongly believed that it was their personal responsibility to lose weight and felt confident that they could achieve weight loss without the support of family members or health professionals. However, they wanted to be engaged in strategies that focused on improving their health and fitness, rather than weight loss. They felt that this was a more empowering approach and the most effective way to lose weight and keep it off. Perceived barriers to successful weight loss included commitment to work and family; social pressures to maintain unhealthy behaviours; and the costs of interventions such as gym memberships, personal trainers and commercial diets.

Conclusion: The information provided in this study informs how we can tailor medical and public health strategies for obese men that resonate with their lived experiences. It also highlights the importance of social and contextual factors in understanding the experiences of obese adults.

CORRESPONDING AUTHOR: Sophie Lewis, BmedSci, School of Primary Health Care, Monash University, Melbourne, VIC, 3168; sophie.lewis@med.monash.edu.au 


\section{3:24 PM-3:42 PM}

OS36-D

OBESE ADULTS ATTITUDES' TOWARDS INDIVIDUAL AND POPULATION BASED INTERVENTIONS FOR OBESITY: A QUALITATIVE COMMUNITY STUDY Samantha L. Thomas, BA MA PhD, ${ }^{1}$ Sophie Lewis, BmedSci, ${ }^{1}$ Jim Hyde, BA MA PhD, ${ }^{2}$ David J. Castle, MBBS PhD FRANZP ${ }^{3}$ and Anna Chapman, B App Sc (Health Science)/B Nutrition \& Dietetics $^{4}$

${ }^{1}$ Consumer Health Research Group (CHaRGe), Primary Care Research Unit, School of Primary Health Care, Monash University, Melbourne, VIC, Australia; ${ }^{2}$ Victorian Department of Health, Melbourne, VIC, Australia; ${ }^{3}$ Department of Psychiatry, St Vincent's Hospital, University of Melbourne, Melbourne, VIC, Australia and ${ }^{4}$ Department of General Practice, Monash University, Melbourne, VIC, Australia.

Background: Previous studies of public perceptions of obesity interventions have been quantitative and based on general population surveys. This study aims to explore the opinions and attitudes of obese individuals towards obesity interventions aimed at the population and individual level in Australia.

Methods: Qualitative methods using in-depth semi-structured telephone interviews with a community sample of obese adults (Body Mass Index $\geq 30$ ). Theoretical, purposive and strategic sampling techniques were used to ensure a broad representation of individuals from different demographic and geographical backgrounds. Participants were asked about their attitudes towards three population based interventions (regulation, public health campaigns, and social marketing) and three individual interventions (tailored fitness programs; commercial dieting; and bariatric surgery), and the effectiveness of these interventions.

Results: One hundred and forty two individuals (19-75 years) were interviewed. Participants strongly supported non-commercial interventions that were focused on encouraging individuals to make healthy lifestyle changes (regulation, physical activity programs, and public health campaigns). There was less support for interventions perceived to be invasive or high risk (gastric band surgery), stigmatising (social marketing) or commercially motivated (commercial diets and gastric band surgery). Personal experiences with weight and weight loss attempts strongly influenced attitudes.

Conclusion: Obese adults support non-commercial, nonstigmatising interventions which are designed to improve lifestyles, rather than promote weight loss.

CORRESPONDING AUTHOR: Sophie Lewis, BmedSci, School of Primary Health Care, Monash University, Melbourne, VIC, 3168; sophie.lewis@med.monash.edu.au

\section{3:42 PM-4:00 PM}

OS36-C

SCREENING FOR DEPRESSION IN DIABETES: WHAT HAPPENS NEXT?

Mala McHale, $\mathrm{PhD}$ and Justin Kenardy, $\mathrm{PhD}$

CONROD, University of Queensland, Herston, QLD, Australia.
While it is well established that depression is frequently comorbid with diabetes, it is still unclear whether screening for depression has utility. In this study the aims was to assess the impact of screening for depression in a hospital outpatient diabetes clinic by comparing the outcomes in patients with diabetes and depression following random allocation to three different management options.

1070 outpatients with type 1 and 2 diabetes were screening using the CES-D. Those that screened positive had a diagnosis of a major depressive episode (MDE) confirmed using the CIDI. Thus 178 patients with MDE were randomly allocated to direct referral to the hospital psychology service, information and a letter for patients to give to their primary care practitioner or usual care. Patients were assessed and then followed up six-months later to assess depression and diabetes outcomes. Patients in both the direct referral and the primary care conditions improved significantly on measures of depression compared to those in the usual care condition. However, only those patients in the primary care condition also improved significantly on a measure of diabetes control, $\mathrm{HbA} 1$.

These findings suggest that screening for depression following by primary care is valuable in the management of diabetes.

CORRESPONDING AUTHOR: Justin Kenardy, PhD, CONROD, University of Queensland, Herston, QLD, 4006; j.kenardy@uq. edu.au

\section{Saturday \\ August 7, 2010 SYMPOSIA SESSIONS 8:30 AM-10:00 AM}

\section{SS23}

PEER SUPPORT ACROSS CULTURAL, NATIONAL AND ORGANIZATIONAL SETTINGS: COMMON

\section{FUNCTIONS AND SETTING-SPECIFIC FEATURES}

Edwin B. Fisher, $\mathrm{PhD}^{1,2}$ and Renée I. Boothroyd, $\mathrm{PhD}$, MA, $\mathrm{MPH}^{1,2}$

${ }^{1}$ Health Behavior \& Health Education, University of North Carolina at Chapel Hill, Chapel Hill, NC and ${ }^{2}$ Peers for Progress, American Academy of Family Physicians Foundation, Leawood, $\mathrm{KS}$.

The lack of a well characterized state of the art in peer support interventions impedes their routine inclusion as standard and funded components of health care. A major challenge to efforts to characterize peer support is, in turn, the extent to which it is culturally contingent, that is, how characteristics of support are dependent on differing cultural, social, and organizational standards, norms or values. What is a sensitive offer of help in one culture may be intrusive in another or too reserved and thereby indicative of disinterest in a third. Additionally, varying attitudes toward health, fate, life and death as well as cultural contingencies surrounding key health behaviors such as diet add complexity to efforts to promote peer support across different countries. To 
address these challenges, Peers for Progress, a global program to promote peer support in health care, has followed a strategy of "standardization by function, not content." This identifies key functions of peer support across cultures and settings which, at the same time, allow for considerable flexibility in content of local implementation. Peers for Progress and its grantees have identified as key functions of peer support 1) assistance in daily management, 2) social and emotional support, 3) linkage to clinical care, and 4) ongoing availability of support. This symposium will explore how this approach has been realized in peer support programs in Australia, China, Thailand and Uganda. From Australia, Dr. Oldenburg will describe extension of support groups that Diabetes Australia-Victoria has established for over a decade to improve daily management, social and emotional support, and linkage to clinical care. From Hong Kong, Dr. Chan will describe "Pearl," a program that integrates peer support within "Jade," a program to promote algorithm based, systematic clinical care for diabetes in primary care. From Uganda, Dr. Baumann will describe challenges faced and successes of a peer support program in small, severely under-resourced villages. Finally, Dr. Boothroyd will describe how these and the other eleven project of Peers for Progress have increased understanding of important features of the four key functions of peer support, identified important aspects of implementation, and addressed programmatic, cultural and organizational challenges. Across all of these, identification of common functions guides program development that reflects generalized knowledge while allowing for tailoring to the strengths and characteristics of populations, cultures, and settings.

CORRESPONDING AUTHOR: Edwin B. Fisher, PhD, Health Behavior \& Health Education, University of North Carolina at Chapel Hill, Chapel Hill, NC, 27599-7440; edfisher@unc.edu

\section{SS23a}

JADE AND PEARL IN HONG KONG: THE INTEGRATION OF PEER SUPPORT, TELEHEALTH, AND PRIMARY CARE IN DIABETES MANAGEMENT

Gary Ko, MD, ${ }^{1,2}$ Wing-Yee So, $\mathrm{MD},{ }^{1,2}$ Peter Tong, $\mathrm{PhD},{ }^{1,2}$ Ronald Ma, MBChB, ${ }^{1}$ Alice Kong, $\mathrm{MBChB},{ }^{1}$ Rebecca Wong, RN, DDME, ${ }^{1}$ Hairong Nan, $\mathrm{PhD} \mathrm{MD}^{2,3}$ and Juliana C. Chan, $\mathrm{MD}^{1,2}$

${ }^{1}$ Department of Medicine and Therapeutics, The Chinese University of Hong Kong, Hong Kong, China; ${ }^{2}$ Asia Diabetes Foundation, Hong Kong, China and ${ }^{3}$ Hong Kong Institute of Diabetes and Obesity, The Chinese University of Hong Kong, Hong Kong, China.

Diabetes is a killing and costly disease although many of its complications are preventable and highly treatable. The Joint Asia Diabetes Evaluation (JADE) Program is the first web-based program incorporating a comprehensive risk engine, care protocols, clinical decision and self management support to improve ambulatory diabetes care. It uses state-of-the-art information technology to facilitate healthcare professionals to create a diabetes registry. The JADE e-portal also incorporates an interactive risk engine which stratifies patients into different risk levels based on clinical and biochemical data measured annually. With this risk stratification, the JADE e-portal recommends a care protocol tailored to these risk levels with decision support triggered by various risk factors.

On top of 'High-Tech' accessories, quality diabetes care requires informed-decisions of motivated care providers and diabetes patients. We started the Peer Support, Empowerment And Remote Communication Linked By Information Technology (PEARL) Study in early 2009 in Hong Kong to use peer support and information technology to facilitate care providers to implement structured care and empower diabetes patients acquire selfmanagement skills. PEARL is a multi-component program making use of peer support, JADE Program and the Australasian Telephone Linked Care (TLC) system. TLC system utilizes an automatic, interactive, computer-controlled telephone system to enhance knowledge on diabetes self-management

The PEARL Program involves trained mentors who are themselves diabetes patients with good self care and are motivated to support their peers. Diabetes patients receiving structured care in Hong Kong through the JADE Program will be recruited as mentees. They will receive peer support by the mentors ( 1 mentor to 10 diabetes patients or mentees) through regular phone calls and sharing session. They will also be reminded to use the TLC for knowledge enhancement and motivational support. Effects of various components of peer support on the patients' disease control as well as user acceptability and cost-effectiveness of these programs will be examined.

We believe the combined use of a registry, structured care protocol and peer support will make quality diabetes care more accessible, sustainable and affordable, especially in resource-scare areas.

CORRESPONDING AUTHOR: Hairong Nan, PhD MD, Hong Kong Institute of Diabetes and Obesity The Chinese University of Hong Kong, Hong Kong, N.T.; jh_nan@cuhk.edu.hk

\section{$\mathrm{SS} 23 \mathrm{~b}$}

THE AUSTRALASIAN PEERS FOR PROGRESS DIABETES PROGRAM: IMPORTANT FEATURES AND POTENTIAL FOR TRANSFER TO OTHER COUNTRIES

Brian F. Oldenburg, PhD, ${ }^{1}$ Prasuna Reddy, $\mathrm{PhD},{ }^{2}$ Michaela Riddell, PhD, ${ }^{1}$ Carla Renwick, BSc, ${ }^{1}$ Ralph Audehm, BMBS, ${ }^{3}$ Pilvikki Absetz, PhD, ${ }^{4}$ Anuar Zaini, $\mathrm{PhD}^{5}$ and James Dunbar, $\mathrm{MD}^{2}$

${ }^{1}$ Epidemiology and Preventive Medicine, Monash University, Melbourne, VIC, Australia; ${ }^{2}$ Flinders and Deakin Universities, Warrnambool, VIC, Australia; ${ }^{3}$ Diabetes Australia-Victoria, Melbourne, VIC, Australia; ${ }^{4}$ National Institute for Health and Welfare, Helsinki, Finland and ${ }^{5}$ Monash University, Sunway, Malaysia.

Diabetes Australia-Victoria (DA-Vic) is a very well established NGO that has sustained a community network (ComNet) of support groups for people with diabetes for more than a decade. They aim to convey expert information about diabetes and to provide social support to members. Building on this experience 
over many years, the Australasian Peers for Progress Diabetes Program (PfP-DP) aims to implement and evaluate a peer-led group program to improve daily management, social and emotional support, and linkage to clinical care for people with type 2 diabetes (T2DM). Participants will be recruited from the membership of (1) the National Diabetes Services Scheme, an Australian government initiative for people with diabetes to obtain subsidized consumables, and (2) DA-Vic membership of people with T2DM. The intervention trial involves 12 groups of 8-12 people led by lay peer leader(s), who are receiving training in group facilitation, communication, goal setting and motivational skills. Groups will meet monthly for 12 months, during which time group participants will review difficulties with their diabetes care, successes and achievements. The control (waitlist) arm comprises 12 "virtual" groups of similar composition who do not meet and receive their usual care until after the first 12 months. All study participants, and peer leaders, receive 1-day of basic diabetes self-management education prior to intervention groups commencing. Primary outcome is based on cardiovascular risk score (using the UKPDS equations) at baseline, 12 and 18 month follow up. Secondary outcomes include self-care behaviors, psychosocial functioning and quality of life. The program will also undergo an economic evaluation. The program partnership with an NGO and program delivery by volunteer, lay peer leaders trained to support behavior change in people with T2DM could enhance program transfer to other countries within the Asia Pacific region, where lay workers and community health workers are already integral to delivery of health services.

CORRESPONDING AUTHOR: Brian F. Oldenburg, PhD, Epidemiology and Preventive Medicine, Monash University, Melbourne, VIC, 3004; brian.oldenburg@med.monash.edu.au

\section{$\mathrm{SS} 23 \mathrm{c}$}

PEER SUPPORT FOR ADULTS WITH DIABETES IN RURAL UGANDA: CHAMPIONS AND PARTNERS

Linda C. Baumann, PhD, RN, ${ }^{1}$ Frederick Nakwagala, MS, $\mathrm{MD}^{2}$ and Agatha Nambuya, $\mathrm{MD}^{2}$

${ }^{1}$ School of Nursing, university of wisconsin-madison, Madison, WI and ${ }^{2}$ Department of Medicine, Mulago Hospital, Kampala, Uganda.

The purpose of this 3-month pilot intervention was to test the feasibility of a peer support program for persons with type 2 diabetes in a resource-poor setting. Using a pre- post-intervention design, data were collected on A1C, perceptions of social support, coping, and diabetes self-care behaviors. Outcomes were improved daily self-care behaviors, social and emotional support, A1C values, and linkages with healthcare providers. Peer Champions $(n=19)$ were patients who attended the Mityana diabetes clinic, could speak and read English, and agreed to contact another patient with diabetes (Partner) at least weekly. Champions received education in diabetes self-care and in providing supportive communication to peer Partners. Peer Partners $(n=27)$ were patients with diabetes who attended the Mityana Clinic. Champions and Partners met at a community meeting where diabetes self-care education was again provided, and participants were assigned to pairs or triads. Telephones provided were linked to a pre-paid network so that calls were free if they were to another peer or champion, or to a healthcare provider. Participants who completed the intervention (28 females, 13 males) had a mean age of 55 years (range $=32$ to 74 ) and a mean duration of diabetes of 6.5 years. A post-intervention meeting was held with 16 Champions and 25 Partners to complete measurements and share experiences. Sixty-six percent of participants reported peer contact at least weekly. There was no change in scores on a 5-item diabetes coping scale, a 14-item barriers scale, physical activity or missing medications. Perceptions of social support from family and friends and a single item rating on "confidence in managing diabetes" significantly decreased $(p=.03$, and $\mathrm{p}=.02$ respectively). There was improvement in eating behaviors $(\mathrm{p}<.001)$ and $77.8 \%$ reported that they contacted the clinic and healthcare providers more often than prior to the intervention. The most striking finding was a reduction $(\mathrm{p}<.05)$ in mean A1C from $9.8 \%$ to $6.8 \%$ (by IFCC standards). Eighty percent of participants indicated that the most helpful aspects of peer support were 'advice about taking care of diabetes' and 'encouragement to contact the clinic'. Participants especially liked using pre-paid telephones to contact healthcare providers and peers and receiving written material about diabetes self-care; however, most would have preferred materials in the local language and $92 \%$ reported difficulty reading because of poor eyesight. In conclusion, both providers and peers were actively engaged in a program that resulted in improved glycemic control and linkages with healthcare providers; other results may be explained by the resource-poor setting and unique cultural factors.

CORRESPONDING AUTHOR: Linda C. Baumann, PhD, RN, School of Nursing, university of wisconsin-madison, Madison, WI, 53792-2455; 1jbauman@wisc.edu

\section{SS23d}

GENERALIZABLE FUNCTIONS OF PEER SUPPORT

AND LOCAL TAILORING OF PEER SUPPORT INTERVENTIONS: EXAMPLES FROM PEERS FOR PROGRESS Renee Boothroyd, $\mathrm{PhD}, \mathrm{MA}, \mathrm{MPH}, \mathrm{CHES}^{1,2}$ and Edwin B. Fisher, $\mathrm{PhD}^{1,2}$

${ }^{1}$ Health Behavior \& Health Education, University of North Carolina at Chapel Hill, Chapel Hill, NC and ${ }^{2}$ Peers for Progress, American Academy of Family Physicians Foundation, Leawood, KS.

In addition to the programs from Australia, China and Uganda described in this symposium, Peers for Progress has funded 11 other projects representing Argentina, Cameroon, England, South Africa, Thailand, and the United States. Observations from these provide opportunity for identifying ways in which generalizable key functions of peer support (assistance in daily management, social and emotional support, linkage to clinical care, and ongoing support) are represented in programs as well as ways in which programs are tailored to local characteristics or strengths. In particular, the experiences of individual projects have led not to major changes in the four key functions but to a) enhanced understanding of several of them, b) recognition of important 
implementation considerations, and c) attention to challenges in implementation. Enhanced understanding of the important of the functions has included recognition of the importance of attention to medications and adherence in a disease like diabetes, emotional and social support, and assisting individuals in navigating the health care system. Implementation considerations include recognizing varied modes of delivery (face-to-face, individual, group, telephone, etc.), the needs not just of individuals but of their family and caregivers, and the need of support providers for their own ongoing encouragement and support. In implementation, recognition has also grown regarding the importance of integration of understanding of the role of peer support across members of the health care team so that everyone delivers a solid and common message though they may be using different "languages." Challenges have included the widespreaqd and substantial barriers individuals face in transportation, infrastructure (e.g., electricity), and employment, difficulties in negotiating and establishing organizational partnerships, encouraging among peer supporters a stance of "peerage" rather than teaching, and cultural challenges such as those related to roles of women, roles of patients vis a vis professionals, etc. The four functions of peer support that Peer for Progress has emphasized provide important structure to thinking about peer support across national lines, but, nevertheless, leave much room for attention to the kinds of needs and challenges which successful implementation in individual settings must address.

CORRESPONDING AUTHOR: Edwin B. Fisher, PhD, Health Behavior \& Health Education, University of North Carolina at Chapel Hill, Chapel Hill, NC, 27599-7440; edfisher@unc.edu

\section{SS24}

INSOMNIA ACROSS THE LIFE-SPAN

Mari Hysing, $\mathrm{PhD},{ }^{1}$ Paula Salo, $\mathrm{PhD},{ }^{2}$ Arnstein Mykletun, $\mathrm{PhD}^{1,3}$ and Borge Sivertsen, $\mathrm{PhD}^{1,3}$

${ }^{1}$ Faculty of psychology, University of Bergen, Bergen, Norway;

${ }^{2}$ Finnish Institute of Occupational Health, Helsinki, Finland and ${ }^{3}$ Division of mental health, Norwegian Institute of Public Health, Bergen, Norway.

Insomnia is one of the most common complaints in the general population, and is associated with a range of adverse consequences and conditions. In the four presentations in this symposium, we focus on various aspects of insomnia at different stages throughout the life-span. Beginning with a longitudinal study on children with chronic illness, Dr Mari Hysing will present data on the chronicity of insomnia, as well as on behavioral and emotional correlates in this population. Dr Paula Salo will then present data from a Finish cohort examining the effect of insomnia on a range of depression-related outcomes. Dr Arnstein Mykletun will then present data on the association between individual and combined anxiety/depressive symptoms and insomnia, and cause specific mortality over a 3-6 year period. Finally, Dr. Børge Sivertsen will explore the consequences of chronic use of sleep medication among the elderly.

CORRESPONDING AUTHOR: Borge Sivertsen, PhD, University of Bergen, Bergen, 5015; borge.sivertsen@psykp.uib.no
SS24a

SLEEP AND EMOTIONAL PROBLEMS IN CHILDREN

WITH A CHRONIC ILLNESS

Mari Hysing, PhD

Faculty of psychology, University of Bergen, Bergen, Norway.

Background: Children with a chronic illness have an increased rate of sleep problems and this has previously been related to emotional problems. Less is known about what specific aspects of emotional problems that contribute to sleep problems, and if sleep can predict the development of emotional problems in children with a chronic illness.

Aim: The aim of this study was to assess the relation between sleep problems and emotional problems in general and more specifically; anxiety, perfectionism and obsessive compulsive symptoms in children with a chronic illness. Further, we want to examine sleep problems as a predictor of emotional problems from the age of 7-9 to $11-13$.

Methods: Using data from a longitudinal total population study in Norway, The Bergen Child Study, data on sleep problems, chronic illness, emotional and behavioral problems (SDQ; Strength and Difficulties Questionnaire) and anxiety (SCARED) were assessed at ages 7-9 and 11-13.

Results: 496 children with a chronic illness in the age group 11-13 participated in the Bergen Child study, including a 295 of 4025 $(7.3 \%)$ in the longitudinal sample. Children with a chronic illness had a higher rate of sleep problems and anxiety symptoms than their peers $(p<0.001)$. In children with a chronic illness sleep problems were related to anxiety symptoms both based on parent and child report. Sleep problems were independent risk factors of later emotional problems (odd ratio=3.5), also when adjusting for emotional problems at time 1 (odd ratio=3.0).

Conclusions: Emotional problems in general and anxiety more specifically, were related to sleep problems in children with chronic illness. Sleep problems predicted the development of emotional problems over time. Based on the results screening for anxiety is recommended as a part of sleep assessment in children with a chronic illness.

CORRESPONDING AUTHOR: Mari Hysing, PhD, University of Bergen, Bergen, 5009; mari.hysing@psybp.uib.no

\section{$\mathrm{SS} 24 \mathrm{~b}$}

INSOMNIA AS A PREDICTOR OF DEPRESSION

Paula Salo, PhD, ${ }^{1}$ Borge Sivertsen, $\mathrm{PhD},{ }^{2}$ Tuula Oksanen, MD, $\mathrm{PhD},{ }^{1}$ Jaana Pentti, BSc, ${ }^{1}$ Marianna Virtanen, $\mathrm{PhD},{ }^{1}$ Mika Kivimäki, $\mathrm{PhD}^{1,3}$ and Jussi Vahtera, $\mathrm{MD}, \mathrm{PhD}^{1,4}$

${ }^{1}$ Unit of Excellence for Psychosocial Factors, Finnish Institute of Occupational Heatlh, Turku, Finland; ${ }^{2}$ Department of Clinical Psychology, University of Bergen, Bergen, Norway; ${ }^{3}$ Department of Epidemiology and Public Health, University College London Medical School, London, United Kingdom and ${ }^{4}$ Department of Public Health, University of Turku, Turku, Finland.

We examined whether the quantity and quality of insomnia symptoms were associated with risk of new-onset depression in 
a cohort of 47,816 public sector employees who were free of clinical depression during four years preceding the study (mean age $43.9,79 \%$ female). They responded to a survey in 2000-2002 and/or 2004 and their self-reported sleep disturbances were linked to comprehensive individual-level health registers to assess depression from several sources, i.e. medically certified work disability, use of antidepressant medication, and psychotherapy for depression. During a mean follow-up of 3.3 years, 273 participants were granted long-term work disability due to depression, 1611 had new antidepressant treatment and 74 had new commencements of psychotherapy for depression. Any sleep disturbance at least 5 nights per week increased the risk of incident depression to 1.6-1.7-fold after extensive adjustments for baseline age, sex, socioeconomic status, night/shift work, health behaviors, somatic diagnoses, pain killers, psychological distress, and anxiety. Risk of work disability due to depression was 2.9 [95\% confidence interval (CI) 1.9-4.4] in those with difficulties initiating sleep at least 5 nights per week. Too early awakenings and non-refreshing sleep doubled the risk of work disability and difficulties maintaining sleep increased the risk to 1.5 (95\% CI 1.1-2.0). Also moderate sleep disturbances (2-4 nights/week) were associated with increased risk. The results were essentially replicated with antidepressant medication use and psychotherapy. In conclusion, Sleep disturbances seem to precede objective depression-related outcomes. Our results emphasize the importance of early detection of sleep disturbances in preventing subsequent depressive disorders.

CORRESPONDING AUTHOR: Paula Salo, PhD, Unit of Excellence for Psychosocial Factors, Finnish Institute of Occupational Heatlh, Turku, FI-20520; paula.salo@ttl.fi

\section{$\mathrm{SS} 24 \mathrm{c}$}

DEPRESSION, ANXIETY AND INSOMNIA AS PREDICTORS OF MORTALITY. THE HUNT STUDY

Arnstein Mykletun, Professor, PhD, PsyD

Norwegian Institute of Public Health, Bergen, Norway and University of Bergen, Research Centre for Health Promotion, Bergen, Norway.

Background: Depression is reported to be associated with increased mortality, although underlying mechanisms are uncertain. Increased cardiovascular (CVD) mortality and suicide is reported in depression, but the effect of depression on other causes of death is uncertain. The few studies of anxiety or insomnia in relation to mortality have produced inconclusive Results: Aims To investigate associations between individual and combined anxiety/ depression symptom loads (HADS) and insomnia, and cause specific mortality over a 3-6 year period.

Method: We utilized a unique link between a large population survey (HUNT-2, $\mathrm{n}=61,349$ ) and a comprehensive mortality database.

Results: Case-level depression was associated with increased mortality (Hazard Ratio $=1.52$, 95\% CI 1.35-1.72) comparable to that of smoking (Hazard Ratio $=1.59,95 \%$ CI 1.44-1.75), and which was only partly explained by somatic symptoms/conditions.
Depression increased CVD mortality as much as other causes of death. Anxiety comorbid with depression lowered mortality compared to depression alone (anxiety by depression interaction $\mathrm{p}=.017)$. The association between anxiety symptom load and mortality was U-shaped. There was no effect of insomnia on mortality beyond confounding.

Conclusions: Depression is an important and independent risk factor for mortality across common causes of death. Comorbid anxiety reduced mortality compared to depression alone. The relationship between anxiety symptoms and mortality was more complex with a U-shape and highest mortality in those with the lowest anxiety symptom loads.

Declaration of interest None.

CORRESPONDING AUTHOR: Arnstein Mykletun, Professor, $\mathrm{PhD}$, PsyD, University of Bergen, Research Centre for Health Promotion, Nesttun, 5221; arnstein.mykletun@iuh.uib.no

\section{SS24d}

SLEEP AND SLEEP DISORDERS IN OLDER USERS OF SLEEP MEDICATION

Borge Sivertsen, $\mathrm{PhD}$

Division of mental health, Norwegian Institute of Public Health, Bergen, Norway and Faculty of psychology, University of Bergen, Bergen, Norway.

Objective: To examine polysomnographic sleep parameters as well as the prevalence of sleep apnoea and periodic limb movement disorder (PLMD) in older chronic hypnotic users compared to aged-matched drug-free patients complaining of insomnia and good sleepers. Methods: Polysomnographic data were collected at a university-based outpatient clinic for adults and elderly. 19 patients using sleep medications on a daily basis (5-7 days per week) for at least one year were compared with 64 drug-free patients complaining of insomnia and 26 good sleepers. Mean age was 63.8 years $(\mathrm{SD}=7.0)$. Outcome measures were polysomnographic sleep parameters (total sleep time, wake after sleep onset, sleep onset latency, sleep efficiency, slow-wave sleep), and prevalence estimates of sleep apnoea and PLMD. Results: The chronic hypnotic users spent more time awake and had reduced sleep efficiency compared to good sleepers. The amount of slowwave sleep was significantly lower among chronic hypnotic users compared to the other two groups. There were no differences between the chronic hypnotic users and drug-free patients complaining of insomnia on any of the other PSG-parameters. The prevalence rate of sleep apnoea (AHI $>10$ ) was $42 \%$ both among the drug-free patients complaining of insomnia and chronic users of sleep medications, compared to $12 \%$ in the good sleepers group. There were no significant group differences in the prevalence of PLMD between the three groups (37\%-39\%). Conclusions: This study suggests that the sleep of chronic users of sleep medications is no better than that of drug-free patients complaining of insomnia. It is disturbing that $42 \%$ of the patients treated pharmacologically for insomnia also suffers from sleep apnoea. We suggest careful sleep assessment as a prerequisite for long-term prescription of sleep medications. 
CORRESPONDING AUTHOR: Borge Sivertsen, PhD, University of Bergen, Bergen, 5015; borge.sivertsen@psykp.uib.no

\section{SS25}

\section{COMPARATIVE EFFECTIVENESS RESEARCH}

IN CARDIOVASCULAR BEHAVIORAL MEDICINE

Catherine M. Stoney, $\mathrm{PhD}^{1}$ and Karina W. Davidson, $\mathrm{PhD}^{2}$

${ }^{1}$ NHLBI, NIH, Bethesda, MD and ${ }^{2}$ Columbia University, New York, NY.

Comparative effectiveness research (CER) provides evidence for health care providers, consumers, caregivers, and policy makers regarding the relative benefits and risks of two or more prevention, treatment, or diagnostic strategies for specific medical conditions, under specific circumstances, and for particular populations. There are several design strategies that can provide critical comparative effectiveness information, but a balanced portfolio of methodologies will be most informative. In CER research, patients are studied within the context of real-world clinical settings. The Institute of Medicine (IOM) has recently developed national priorities for comparative effectiveness research, and has also established a working definition for CER. The IOM also underscored the importance of identifying infrastructure and resources necessary for sustaining CER research. Importantly, among the national priorities identified, a number of recommendations included behavioral interventions (including but not limited to exercise treatments, social support, family issues in care-giving, dietary interventions, improving the built environment, symptom management, cognitive behavioral therapy, biofeedback, and many others) related to cardiovascular and other diseases and conditions. There are many opportunities for cardiovascular behavioral medicine studies to contribute important information in CER. The purpose of this symposium is to highlight examples of some of the early behavioral medicine studies that have been conducted in CER, for the purpose of encouraging advances.

CORRESPONDING AUTHOR: Catherine M. Stoney, PhD, NIH, Bethesda, MD, 20892; stoneyc@mail.nih.gov

\section{SS25a}

\section{COMPARATIVE EFFECTIVENESS OF DIET AND ACTIVITY} INTERVENTIONS

Bonnie Spring, PhD, ${ }^{1}$ Donald Hedeker, $\mathrm{PhD},{ }^{2}$ Juned Siddique, $\mathrm{PhD},{ }^{1} \mathrm{H}$ Gene McFadden, BA, ${ }^{1}$ Arlen Moller, $\mathrm{PhD}^{1}$ and Kristin Schneider, $\mathrm{PhD}^{3}$

${ }^{1}$ Preventive Medicine, Northwestern University, Chicago, IL; ${ }^{2}$ University of Illinois Chicago, Chicago, IL and ${ }^{3}$ University of Massachusetts, Worcester, MA.

As much as $40 \%$ of premature mortality is attributable to unhealthy lifestyle behaviors. To be maximally effective, therefore, efforts to improve population health need to address health risk behaviors. Poor quality diet and sedentary lifestyle are wellestablished risk factors for cardiovascular disease and cancers.
Four unhealthy behaviors are especially prevalent. in industrialized cultures: eating a high-fat diet, consuming few fruits and vegetables, extensive television viewing, and low moderatevigorous physical activity. Public health guidelines advise correcting all of these unhealthy behaviors, but little guidance is available about the best approach. To address that question, we conducted a head-to-head comparative effectiveness randomized clinical trial $(n=200)$ of four diet and activity interventions, all of which entailed guideline-recommended behavior change. In each condition, participants modified one diet and one activity behavior, while effects on all four behaviors were evaluated as an aggregated healthy lifestyle score. Results indicated that the conventional intervention practice of increasing physical activity and decreasing fat intake produced the least improvement in overall healthy lifestyle. Conversely, a novel approach suggested by behavioral economic theory (increase fruits/ vegetables and decrease recreational screen time) maximized acrossthe-board healthy lifestyle change. To fulfill its full potential, however, comparative effectiveness research needs to extend beyond determining only what works best for the average patient. In the present study, for example, traditional dieting (decrease fat, increase physical activity) minimized overall healthy lifestyle change for females but maximized it for males, suggesting that gender-tailored guidance might be needed. Comparative effectiveness research holds potential to undergird personalized, evidence-based behavioral medicine. The aim is for intervention decisions to be guided by evidence that conveys what works best for particular types of individuals or communities and in different settings and circumstances.

CORRESPONDING AUTHOR: Bonnie Spring, PhD, Preventive Medicine, Northwestern University, Chicago, IL, 60610; bspring@northwestern.edu

\section{SS25b}

EXERCISE AND PHARMACOTHERAPY IN DEPRESSION James Blumenthal, $\mathrm{PhD}$

Psychiatry, Duke University Medical Center, Durham, NC.

Depression is a very common and disabling condition. Prevalence rates of major depressive disorder (MDD) have been estimated to be a high as $25 \%$, with point prevalence rates estimated at up to $45 \%$ for patients with minor depression (mDD) or elevated depressive symptoms. Moreover, depression in cardiac patients has been associated with greater than a 2 -fold increase in risk for adverse clinical events, including death. Although effective treatments for depression are available, the optimal ways to manage depression in cardiac patients are not known. This presentation will briefly review the prior randomized clinical trials for treating depression using cognitive behavioral therapy (CBT) and pharmacotherapy. Recent studies of exercise in treating depression also will be reviewed, and data examining the comparative efficacy of exercise, medication and CBT will be examined. The added physical and mental health benefits of exercise training in treating depression in cardiac patients will be highlighted and directions for future research in the area will be discussed. 
CORRESPONDING AUTHOR: James Blumenthal, PhD, Psychiatry, Duke University Medical Center, Durham, NC, 27710; blume003@mc.duke.edu

\section{$\mathrm{SS} 25 \mathrm{c}$}

BEHAVIOUR CHANGE PLUS PEDOMETER IN INCREASING PHYSICAL ACTIVITY IN SEDENTARY OLDER WOMEN

Derek W. Johnston, $\mathrm{PhD},{ }^{1}$ Falko F. Sniehotta, $\mathrm{PhD},{ }^{1}$ Jacqui Sugden, $\mathrm{PhD},{ }^{2}$ Ishbel Argo, $\mathrm{RGN}^{2}{ }^{2}$ Paul Boyle, $\mathrm{PhD},{ }^{4}$ Peter T. Donnan, $\mathrm{PhD}^{3}$ and Marion E. McMurdo, $\mathrm{MD}^{2}$

${ }^{1}$ Psychology, University of Aberdeen, Aberdeen, United Kingdom; ${ }^{2}$ Ageing and Health, University of Dundee, Dundee, United Kingdom; ${ }^{3}$ Biostatistics, University of Dundee, Dundee, United Kingdom and ${ }^{4}$ Geography and Geosciences, University of $\mathrm{St}$ Andrews, St Andrews, United Kingdom.

Older people are the least active segment of the population. This contributes to their high level of disease (including cardiovascular disease), disability and healthcare usage. Practical and effective interventions are needed to increase activity in this population. A brief behavioural change intervention consisting of goal setting, planning, self monitoring delivered with or without a pedometer was compared with no treatment in a 3 arm prospective study of 204 sedentary community dwelling women aged $\geq 70$ years (average age 77 years). The primary outcome was daily activity counts assessed by accelerometer over 7 days prior to treatment, at 3 months and at 6 months. Secondary outcomes included lower limb function, health related quality of life, anxiety, depression and falls. 179 women completed the trial with most dropouts from the behaviour change alone condition $(15 / 68)$. Over the first 3 months activity increased reliably more in the intervention groups than the control (which did not change). Pedometers did not add to the effects of the behaviour change techniques. The increase in activity was not maintained at the 6 month assessment. The secondary outcomes were not affected by the intervention. The psychological and behavioural processes mediating the change in activity, and the failure to sustain it, will be discussed.

CORRESPONDING AUTHOR: Derek W. Johnston, PhD, psychology, University of Aberdeen, Aberdeen, AB242UB; d.johnston@abdn.ac.uk

\section{SS26}

LESSONS LEARNED FROM BEHAVIORAL MEDICINE RESEARCH THAT CAN BE APPLIED TO RESEARCH IN INTEGRATIVE MEDICINE: SUCH AS QIGONG AND TAI CHI

Anna Rusiewicz, PhD, ${ }^{1,2}$ Guen-Cheng Sun, $\mathrm{PhD},{ }^{5}$ Laura Redwine, $\mathrm{PhD},{ }^{3}$ Sarosh Motivala, $\mathrm{PhD}^{4}$ and Myeong Soo Lee, $\mathrm{PhD}^{6}$

${ }^{1}$ War Related Illness and Injury Study Center, Stop 129, Dept of Veterans Affairs NJ Healthcare System, East Orange, NJ;
${ }^{2}$ Preventive Medicine, Mount Sinai School of Medicine, New York, NY; ${ }^{3}$ Department of Psychiatry and Medicine, University of California, San Diego, San Diego, CA; ${ }^{4}$ Cousins Center for Psychoneuroimmunology, University of California, Los Angeles, Los Angeles, CA; ${ }^{5}$ Bastyr University Research Institute, Bastyr University, Kenmore, WA and ${ }^{6}$ Complementary Medicine, Peninsula Medical School, Exeter, United Kingdom.

Integrative Medicine derived from Eastern philosophy contains a strong preventive element closely linked to the idea of a balance between humankind and nature, and between mind and body. It emphasizes that a sound mind in a sound body is essential to longevity. Recently there is a growing wave of individuals seeking complementary and alternative medicine (CAM) treatments. However, for most of these treatments there are essential questions that remain to be answered through rigorous scientific studywhether they work for the diseases or medical conditions for which they are used. Ancient self-care practices such as qigong and tai chi have been used traditionally in the East for complementing chronic disease management. Qigong and tai chi are each forms of traditional Chinese medicine practice that combine breathing, movement and meditation. Decades of behavioral medicine investigations suggest life-style modifications including exercise and relaxation-related activities are beneficial for health. Thus, through behavioral medicine, integrative medicine and CAM can perhaps find the perfect venue for determining the health benefits of these practices.

CORRESPONDING AUTHOR: Anna Rusiewicz, PhD, War Related Illness and Injury Study Center, Stop 129, Dept of Veterans Affairs NJ Healthcare System, East Orange, NJ, 07018; anna.rusiewicz@va.gov

SS26a

TAI CHI TRAINING ON SOMATIC SYMPTOMS

AND PHYSICAL FUNCTION IN OLDER HEART FAILURE PATIENTS

Laura Redwine, $\mathrm{PhD}$ and Paul Mills, MD

Department of Psychiatry and Medicine, University of California, San Diego, San Diego, CA.

Heart failure (HF) affects millions of individuals and is increasing in prevalence each year as the population ages. Approximately one third of HF patients experience clinical depression symptoms, which are further associated with elevated risk of cardiovascular hospitalization and mortality. However, depression treatments such as cognitive-behavioral therapy (CBT) do not decrease morbidity/mortality in patients with coronary heart disease. This is not surprising since recent evidence suggests somatic but not cognitive/affective depressive symptoms have a greater association with cardiovascular-related mortality and events. Somatic symptoms such as fatigue and sleep disturbances are common among HF patients that may lead to physical inactivity and create a spiraling decline in physical and cardiac function. Indeed, 
physical inactivity is associated with reduced quality of life and greater morbidity and mortality in HF. Meanwhile, Tai Chi practice is observed to increase vigor, and reduce tension, anger and fatigue. Tai Chi emphasizes a holistic approach, both mind and body. It is a meditative exercise originating from China, and with its moderate intensity and relative ease of performance it is well-suited even for those with significant physical impairments. The present study consists of HF patients ( $n=29$ completers; 4383 years old; mean age $=72.9, \mathrm{SD}=5.1$ ) that had Tai chi training $(n=19)$ twice per week for 12 weeks or were in a waitlist control group $(\mathrm{n}=10)$. At baseline and after the 12-week intervention period, patients completed the Beck Depression Index (BDI), subcategorized into somatic (BDI-s) and cognitive (BDI-c) symptoms, and the Multidimensional Fatigue Symptom Inventory - Short Form (MFSI-SF). All subjects also were measured pre- and post- intervention period for physical function, in a 6-min walk task. Compared to controls, HF patients in the Tai Chi group had reduced total BDI scores from pre- to post-intervention $(\mathrm{F}(1,21)=$ $4.1, \mathrm{p}=.055, \mathrm{r}=.40)$ and BDI-s $(\mathrm{F}(1,21)=8.5, \mathrm{p}<.01, \mathrm{r}=.55)$, but not BDI-c $(\mathrm{p}=.50)$. Those in the Tai Chi group also had reduced MFSI total fatigue $(\mathrm{F}(1,18)=4.2, \mathrm{p}=.054, \mathrm{r}=.43)$ and increased MFSI vigor $(\mathrm{F}(1,18)=5.3, \mathrm{p}=.033, \mathrm{r}=.48)$ and greater improvement in distance walked $\mathrm{F}(1,27)=6.1, \mathrm{p}=.02$ in the 6 min walk task compared to controls. These findings suggest that Tai Chi reduces symptoms of depression, due mainly to changes in somatic symptoms, and increases physical function. Larger studies are needed to replicate our findings, to establish Tai Chi as a viable intervention for reducing somatic symptoms of depression and increasing physical function. Future studies should examine whether HF related morbidity and mortality is affected by practicing Tai Chi. NCCAM 1-R21-AT-001910-01A2

CORRESPONDING AUTHOR: Anna Rusiewicz, PhD, War Related Illness and Injury Study Center, Stop 129, Dept of Veterans Affairs NJ Healthcare System, East Orange, NJ, 07018; anna.rusiewicz@va.gov

\section{SS26b}

TAI CHI AS A STRATEGY TO ENHANCE MENTAL HEALTH: IMPROVING SLEEP AND REDUCING STRESS IN OLDER ADULTS

S. J. Motivala, PhD, R. E. Olmstead, PhD, A. J. Tomiyama, PhD, N. Joseph, MS, S. Soleymani, BS, F. Yusuf, BS, E. Ji, BS and M. R. Irwin, MD

UCLA Semel Institute, Cousins Center for Psychoneuroimmunology, Westwood, CA.

Tai Chi is a form of calisthenic exercise that incorporates slow, structured movements with attentional focus. Tai Chi reduces fallrisk and improves balance in older adults and practitioners report that it is relaxing and stress reducing as well. Unfortunately, empirical evidence regarding the effects of Tai Chi on mental health parameters is scarce. This talk will focus on Tai Chi studies conducted at the UCLA Cousins Center for Psychoneuroimmunology as a means of demonstrating some of the avenues through which Tai Chi may promote mental health. We conducted a randomized clinical trial comparing Tai Chi Chih with health education in 112 healthy older adults. We found that in those with difficulties sleeping (indexed by Pittsburgh Sleep Quality Inventory scores $>5$ ), Tai Chi led to significant improvements in sleep whereas health education did not $(\mathrm{p}<.05)$, including improvements in perceived sleep quality and duration (p's<.05). We continued to examine sleep with an ongoing trial of Tai Chi Chih in older adults with insomnia, as well as examining stress in a smaller pilot study with isolated older adults. In this presentation, I will discuss three key themes in conducting such clinical trials: 1) understanding the different schools of Tai Chi; 2) choosing the best type of intervention for a control group; and 3) adjusting Tai Chi for working with populations with mental health issues.

CORRESPONDING AUTHOR: Anna Rusiewicz, PhD, War Related Illness and Injury Study Center, Stop 129, Dept of Veterans Affairs NJ Healthcare System, East Orange, NJ, 07018; anna.rusiewicz@va.gov

SS26c

QIGONG AS A COMPLEMENTARY THERAPY TO REDUCE STRESS AND IMPROVE GLUCOSE CONTROL IN ADULTS WITH TYPE 2 DIABETES

Guan-Cheng Sun, $\mathrm{PhD},{ }^{1}$ Jennifer C. Lovejoy, $\mathrm{PhD},{ }^{2,3}$ Amy L. Putiri, MS, ${ }^{1}$ Sara Gillham, BA, ${ }^{1,4}$ Masa Sasagawa, ND ${ }^{1}$ and Ryan Bradley, $\mathrm{ND}^{1,3}$

${ }^{1}$ Bastyr University Research Institute, Kenmore, WA; ${ }^{2}$ Free \& Clear, Inc., Seattle, WA; ${ }^{3}$ University of Washington School of Public Health, Seattle, WA and ${ }^{4}$ National College of Naturopathic Medicine, Portland, OR.

Qigong is a traditional Chinese energy medicine practice combining breathing, movement, and meditation. Qigong therapy is becoming a popular adjunctive treatment for type 2 diabetes. Previous studies have suggested benefits of Qigong practice for this disease; however, its effectiveness has never been rigorously evaluated in adults diagnosed with type 2 diabetes. We conducted a randomized, controlled 3-arm clinical trial in comparing 12weeks of Qigong, Progressive Resistance Training (PRT), or usual care in 32 adults with type 2 diabetes. Outcome measures included fasting glucose, hemoglobin A1c, insulin, C-peptide, BMI, HOMA-IR, Perceived Stress Scale (PSS) and Beck's depression Index (BDI) before, during and at the end of the interventions. We found that body weight increased in the usual care group, but 
decreased in both Qigong and PRT groups. Additionally, the Qigong group showed significant reductions in fasting glucose $(\mathrm{P}=0.003)$ and PSS $(\mathrm{P}=0.045)$ and also demonstrated trends for improved insulin resistance, as measured by the HOMA-IR Index. In contrast, the PRT group showed a significant reduction in depression scores with an increase in fasting glucose and insulin resistance, which also increased in the usual care group (NS). In this presentation, I will discuss three key themes in conducting comparative efficacy clinical trials: 1) understanding the different schools of Qigong; 2) choosing the best control group for Qigong intervention; and 3) choosing an appropriate patient population with type 2 diabetes for Qigong intervention. (NCCAM: T32 AT000815-06).

CORRESPONDING AUTHOR: Anna Rusiewicz, PhD, War Related Illness and Injury Study Center, Stop 129, Dept of Veterans Affairs NJ Healthcare System, East Orange, NJ, 07018; anna.rusiewicz@va.gov

\section{SS26d}

QIGONG FOR HYPERTENSION: A SYSTEMATIC REVIEW

Myeong Soo Lee, PhD, Tae-Young Choi, MD, PhD, Sun-Mi Choi, OMD, PhD and Edzard Ernst, MD, PhD, FMedSci, FRCP, FRCPEd

Korea Institute of Oriental Medicine, Daejeon, Democratic People's Republic of Korea.

Complementary therapies are popular and frequently used by patients with CVD. Survey data indicate that herbal therapies and mind-body therapies are most commonly used. One such treatment option is qigong. Several reviews have claimed that qigong has therapeutic effects on blood pressure in patients with hypertension. However, these reviews are non-systematic and therefore open to bias. To systematically assess the clinical evidence of qigong for hypertension. Databases were searched up to December 2009. All randomized clinical trials testing qigong in patients with hypertension of any origin and assessing clinically relevant outcomes were considered. Trials using any type of control intervention were included. The selection of studies, data extraction and quality assessment were performed independently by at least two reviewers. Methodological quality was evaluated using the Jadad score. 12 RCTs could be included. Seven of these RCTs tested qigong in combination with antihypertensive drugs compared with antihypertensive drugs alone. The meta-analysis of four trials reporting adequate data suggested beneficial effects in favour of qigong (weighted mean difference, systolic blood pressure $(\mathrm{mmHg})-12.06,95 \%$ confidence interval (CI) -17.12 to -7.00; diastolic blood pressure $-8.46,95 \%$ CI -12.55 to -4.37 ). Qigong alone was compared with waiting list control in 2 RCTs and was found to significantly reduce systolic blood pressure (weighted mean difference $(\mathrm{mmHg})-18.46,95 \% \mathrm{CI}-23.07$ to -13.86). In further three RCTs comparisons made were: qigong combined with antihypertensive drugs versus muscle relaxation combined with antihypertensive drugs; qigong as a sole treatment versus exercise and/or antihypertensive drugs, all reported positive results in at least some of the relevant outcome measures. The methodological quality of the original studies was low. There is some encouraging evidence to suggest that qigong is effective for lowering systolic blood pressure. However, the conclusiveness of these findings is limited. Rigorously designed trials seem warranted to confirm these results.

CORRESPONDING AUTHOR: Anna Rusiewicz, PhD, War Related Illness and Injury Study Center, Stop 129, Dept of Veterans Affairs NJ Healthcare System, East Orange, NJ, 07018; anna.rusiewicz@va.gov

\section{Saturday \\ August 7, 2010 \\ PAPER SESSIONS \\ 8:30 AM-10:00 AM}




\section{CITATION ABSTRACT \\ OS37 \\ 8:30 AM-8:48 AM}

OS37-A

PROJECT L.E.A.N.: AN AFTER-SCHOOL HEALTH EDUCATION AND PHYSICAL ACTIVITY PROGRAM FOR ELEMENTARY SCHOOL CHILDREN IN EL PASO, TEXAS Hendrik de Heer, $\mathrm{PhD}$ MPH, ${ }^{2,1}$ Osvaldo Morera, $\mathrm{PhD}^{2}$ and Rockie Pederson, $\mathrm{PhD}^{3}$

${ }^{1}$ Social and Behavioral Research Branch, National Human Genome Research Institute, Silver Spring, MD; ${ }^{2}$ Psychology, University of Texas at El Paso, El Paso, TX and ${ }^{3}$ College of Health Sciences, University of Texas at El Paso, El Paso, TX.

After-school activities provide valuable opportunities for health promotion activities that do not interfere with the regular school day, especially in minority populations with higher rates of obesity and type 2 diabetes. The current study is an evaluation of an after-school health education and physical activity program conducted in 8 elementary schools along the U.S.Mexico border in El Paso, Texas.

The intervention was a 10-12 week (twice a week) after-school program including a pilot (with 2 experimental schools) and main intervention (6 schools each including control and experimental groups). Each session consisted of health education taught by a Community Health Worker, based on the bilingual BienEstar ('Well-Being') curriculum, and physical activity based on the Coordinated Approach to Children's Health $(\mathrm{CATCH})$ curriculum. Main outcome variables were Body Mass Index (BMI), aerobic capacity health knowledge and healthy intentions.

Participants $(n=1,103)$ were predominantly socio-economically disadvantaged Hispanic 3rd to 5 th graders. At baseline, $21.6 \%$ of children were overweight and another $25.6 \%$ obese. The intervention was successful in recruitment, implementation and retention of participants.

The pilot study ( $\mathrm{n}=172$ ) found a significant BMI reduction and increased aerobic capacity. In the main intervention, experimental group participants $(n=323)$ reduced their BMI, but this reduction was not significantly larger than the control group $(n=608)$. Intervention group participants significantly increased their healthy nutrition intentions compared to control group participants, but not health knowledge.

While the findings in regards to changes in health indicators were modest, all findings were in the expected direction. The current project provides evidence for a strong need and desire to participate in projects that are accessible and address primary prevention of chronic diseases within a population that has a high prevalence of risk factors and limited access to care.

CORRESPONDING AUTHOR: Hendrik de Heer, PhD MPH, Social and Behavioral Research Branch, National Human Genome Research Institute, Silver Spring, MD, 20910; dirk.deheer@, nih.gov

\section{8:48 AM-9:06 AM}

OS37-B

DO PSYCHOSOCIAL FACTORS MEDIATE

THE ASSOCIATION BETWEEN MEDICINE USE AND ADOLESCENTS' MIGRANT BACKGROUND?

Lourdes Cantarero-Arévalo, Economist, MPH, PhD Student, ${ }^{1,3}$ Bjørn E. Holstein, Professor, ${ }^{2,3}$ Annette Andersen, Senior Researcher ${ }^{2,3}$ and Ebba H. Hansen, Professor ${ }^{2,3}$

${ }^{1}$ Institute of Pharmacology and Pharmacotherapy, Section of Social Pharmacy, University of Copenhagen, Faculty of Pharmaceutical Sciences, Copenhagen, Denmark; ${ }^{2}$ National Institute of Public Health, University of Southern Denmark, Copenhagen, Denmark and ${ }^{3}$ Research Centre for Quality in Medicine Use, Copenhagen, Denmark.
Background: Differences in medicine use between adolescents from different foreign backgrounds and native adolescents have been documented, miming other ethnic disparities in health and healthcare. These differences however continue to be poorly understood. The objective of this study is to analyse whether psychosocial factors may mediate the association between medicine and migrant background.

Methods: Data from the 2006 cross-sectional study Health Behaviour in School-aged Children (HBSC). The study population consisted of boys and girls from 11 to 15 years old (508 descendants, 456 migrants and 8480 natives). The data were collected through a selfcompleted questionnaire in school settings. The outcome measure was medicine use for headache, stomach-ache, difficulties in getting to sleep, and nervousness. The independent variable "migration background" were categorized into four levels, namely 1) native, 2) migrant, 3) descendant, and 4) unknown.

Results: Among the boys who took medicine for stomach-ache the odds for medicine use were 2.11 (1.46-3.05) for descendants and 1.55 (1.00-2.39) for first generation migrants. The psychosocial factor "feeling helpless" modifies the effect by reducing the odds to 1.84 
(1.22-2.78) for descendants and $1.46(0.90-2.35)$ for first generation migrants. Among the girls who took medicine for stomach-ache the odds were 1.27 (0.92-1.73) for descendants and 1.50 (1.09-2.05) for migrants. The psychosocial factor "feeling helpless" modifies the effect by reducing the odds to $1.23(0.90-1.69)$ for descendants and 1.48 (1.08-2.04) for first generation migrants.

Conclusion: There is higher risk of using medicine among nonnative populations, and it is statistically significant for stomachache medicines. The psychosocial factors contemplated are associated with medicine use both for boys and for girls and they partially explain the association between medicine use with migration background.

CORRESPONDING AUTHOR: Lourdes Cantarero-Arévalo, Economist, MPH, PhD Student, Institute of Pharmacology and Pharmacotheraty, Section of Social Pharmacy, University of Copenhagen, Faculty of Pharmaceutical Sciences, Copenhagen, 2000; 1ca@farma.ku.k

\section{9:06 AM-9:24 AM}

OS37-C

ECONOMIC VULNERABILITY AND ADOLESCENT HEALTH -FRAGILE FAMILY FINANCES AND HEALTH FUNCTIONING AMONG SWEDISH ADOLESCENTS

Lisa Folkesson, $\mathrm{BsC}{ }^{1,2}$ Petra Lindfors, $\mathrm{PhD}^{1,2}$ and Viveca Östberg, $\mathrm{PhD}^{1}$

${ }^{1}$ Centre for Health Equity Studies (CHESS), Stockholm University/Karolinska Institute, Stockholm, Sweden and ${ }^{2}$ Department of Psychology, Stockholm University, Stockholm, Sweden.

Background: While Adult health is known to vary by SES and gender, less is known about the linkages between socioeconomic circumstances and various aspects of health in adolescents.

Aim: This study investigates how household financial insecurity relates to different aspects of health in adolescent girls and boys, aged 10-18.

Method: Data came from the Swedish Child-LNU and Child-ULF studies of 2000-2003 that include approximately 5400 children aged 10-18 who answered questions relating to health. Parents were also asked to report "cash-margin", a frequently used singleitem measure asking whether parents can access 12.000 SEK in a week's time if they have to.

Results: A majority, $85 \%$ of the parents were able to access 12.000 SEK in a week if necessary while $15 \%$ were unable to do so. Analyses performed separately for girls and boys showed significant main effects of cash margin on somatic health, negative functioning and positive functioning for both groups. There was no significant age $\mathrm{x}$ cash margin interactions. Cash margin was significantly related to stomach ache, insomnia, difficulties concentrating, irritability, short temperedness, sadness, tension/ nervousness, belief in the future, endurance and happy mood in both girls and boys. Girls with no cash margin reported more headaches while boys with a cash margin reported higher levels of self-assurance. No other significant relationships emerged.

Discussion: While most previous studies use symptom indexes and problems, this study covers multiple aspects of health functioning. To conclude, fragile family finances during adolescence as reflected in poor positive functioning and high levels of negative functioning suggests stressful living conditions that may influence academic achievement, life-choices and future health.

CORRESPONDING AUTHOR: Lisa Folkesson, BsC, Centre for Health Equity Studies, CHESS (Stockholm University/Karolinska Institute), Stockholm, 106 91; lisa.folkesson@chess.su.se

\section{9:24 AM-9:42 AM \\ OS37-D}

THE ROLE OF PAID MATERNAL EMPLOYMENT IN CHILDHOOD ON ADOLESCENT HEALTH IN BHPS STUDY Jitka Pikhartova, MD, Yvonne Kelly, PhD, Tarani Chandola, $\mathrm{PhD}$, Annie Britton, $\mathrm{PhD}$ and Anne McMunn, $\mathrm{PhD}$

Epidemiology and Public Health, UCL, London, United Kingdom.

Background: Maternal employment has been shown to influence various child/adolescent outcomes such as cognitive outcomes, educational achievement, behavioural problems, overweight, selfrated health or child fatalities. There is, however, only limited number of studies using UK longitudinal data, and limited number of data using health measures as study outcomes. The aim of this study is to cover these gaps by looking at influence of maternal employment in three periods of childhood on health outcomes among young people aged 16-21 years in British Household Panel Survey (BHPS).

Methods: BHPS is annual panel survey that has started in 1991 with currently completed 17 th wave of data. Response rate to wave 1 was $74 \%$, and response between waves 2 and 17 varied between $84 \%-89 \%$. There are 3,696 individuals for whom selfrated health and/or GHQ-12 at age 16-21 (study outcomes) and maternal employment data prior to age 16 years are available. Other variables, such as gender, maternal age, maternal education and marital status, household income or maternal smoking were used as additional explanatory variables. Regression modeling in STATA 10 was used to estimate the associations between study outcomes and maternal employment.

Results: $19 \%$ of young adults aged 16-21 reported poor self-rated health, and mean GHQ-12 was 10.1. Approximately $40 \%$ of mothers worked at some point during age $0-4$ of their child. This proportion increased to $59 \%$ at age $5-11$ and $68 \%$ at age $12-16$. Children of mothers who were not employed reported OR of poor SRH 1.48, 1.11 and 1.28 for 3 periods of exposure, however these effects were mostly explained when adjusted for maternal education and household income. The differences in GHQ-12 by maternal employment were similarly reduced by indicators of social position.

Conclusions: The associations between maternal employment in childhood and young adults' health exist at least partly because of the generally higher social position and more stable family structure of households with working mothers.

CORRESPONDING AUTHOR: Jitka Pikhartova, MD, Epidemiology and Public Health, UCL, London, WC1E 6BT; j.pikhartova@ ucl.ac.uk 
9:42 AM-10:00 AM

OS37-E

WEIGHT CONTROL BEHAVIORS AND ATTITUDES

IN ADOLESCENTS IN TREATMENT FOR OBESITY VERSUS A COMMUNITY SAMPLE

Eleanor R. Mackey, PhD, Angela Fletcher, PsyD and Randi Streisand, $\mathrm{PhD}$

Psychiatry and Behavioral Sciences, Children's National Medical Center, Washington, DC.

Childhood obesity is widespread and often requires medical intervention. Research has shown that obese youth may be at risk for engaging in maladaptive weight control behaviors, which increase risk for complications and impede weight loss. The current study examined weight control behaviors (WCB) and attitudes among youth in treatment for obesity as compared to community youth, including those matched for weight status and ethnicity to the clinical group, which was primarily $(74 \%)$ African-American (AA).

Participants were from two samples of youth; one from public high schools (community sample; $\mathrm{N}=359$, ages 13-19, $66 \%$ female) and one seen in treatment hospital-based clinic for either obesity or type 2 diabetes (clinical sample; $\mathrm{N}=42$, ages 12-18, $57 \%$ female). Adolescents completed demographic measures and measures assessing body esteem and current WCB.

A MANOVA was conducted examining differences in body esteem and weight satisfaction $(\mathrm{F}(2,396)=66.17, \mathrm{p}<.001)$. Youth in the clinical sample reported lower body esteem than the community group. A MANOVA was also conducted comparing clinical youth to obese community youth (BMI $\geq 95$ th percentile; $\mathrm{N}=41 ; \mathrm{F}(7,73)=$ $14.51, \mathrm{p}<.001)$ and AA community youth $(\mathrm{N}=80 ; \mathrm{F}(2,108)=$ $52.77, \mathrm{p}<.001)$. Clinical youth reported lower weight satisfaction than obese or AA community youth. Via logistic regression, the clinical youth were significantly more likely to report exercise, eating less, and fasting to lose weight than the general or AA community youth. When compared to the obese community youth, clinical youth were more likely to eat less to lose weight.

In sum, the youth in treatment for obesity were significantly more likely to have lower body esteem and weight satisfaction than community youth, even obese or AA youth in the community. When compared to community youth, obese youth also engaged in more weight loss attempts, including maladaptive behaviors such as fasting. Findings suggest that treatment for obesity needs to include assessment and treatment of body esteem and weight dissatisfaction, as well as focus on avoiding maladaptive WCB.

CORRESPONDING AUTHOR: Eleanor R. Mackey, PhD, Psychiatry and Behavioral Sciences, Children's National Medical Center, Washington, DC, 20010; emackey@cnmc.org
OS38

\section{8:30 AM-8:48 AM \\ OS38-A}

FACTORS RELATED TO APPRAISAL AND DIAGNOSTIC DELAY: A QUALITATIVE STUDY OF COLORECTAL CANCER PATIENTS

Heather Rogers, PhD, MPH and Laura A. Siminoff, PhD

Department of Social and Behavioral Health, Virginia Commonwealth University, Richmond, VA.

PURPOSE: To examine factors associated with appraisal delay (time between symptom recognition and seeing a physician [PHYS]) and diagnostic delay (time between first medical visit and diagnosis). METHODS: Individuals diagnosed with colorectal cancer (CRC) in the past 6 months who experienced symptoms prior to diagnosis are recruited to participate in an on-going qualitative research study. Participants are administered a semistructured, cognitive interview about pre-diagnosis events and complete standardized scales. The interview focuses on: 1) symptom recognition, 2) influence of family and friends, 3) social support, 4) ease of access to care, 5) communication with PHYS, and 6) socio-demographic and psychological factors. Qualitative data is themed and coded. Self-report information is verified via chart review. 50 coded interviews (of 200 conducted to date) were analyzed. RESULTS: Compared to individuals in the first tertile of appraisal delay $(n=20)$, individuals in the third $(n=17)$ were more likely to not initially infer serious illness $(\mathrm{p}<0.05)$, decide not to seek medical attention $(\mathrm{p}<0.01)$, and experience behavioral delay in acting on a decision to seek medical attention $(p<0.001)$ due to procrastination $(p<0.01)$ or system barriers $(p<0.05)$. Compared to patients in the first tertile of diagnostic delay $(n=17)$, patients in the third $(n=17)$ were more likely to have their PHYS fail to fully explore symptoms or investigate complaints $(p<0.001)$, have the PHYS believe symptoms were not serious $(p<0.001)$, and experience referral delay $(\mathrm{p}<0.01)$ and behavioral or scheduling delay with respect to referral appointments $(p<0.05)$. CONCLUSION: In this sample, patients with longer delay did not appraise initial symptoms as important or requiring medical attention. Once deciding to see a PHYS, individual- and system-level factors interfered with making an appointment. Upon seeing a PHYS, patient and PHYS factors contributed to diagnostic delay, including not exploring symptoms or investigating patient complaints and delay in making referral appointments. Further investigation into factors moderating delay are warranted.

CORRESPONDING AUTHOR: Heather Rogers, PhD, MPH, Department of Social and Behavioral Health, Virginia Commonwealth University, Richmond, VA, 23233; hlrogers@vcu.edu 


\section{8:48 AM-9:06 AM}

OS38-B

DEMOGRAPHIC AND BEHAVIOURAL DIFFERENCES

BETWEEN THE LATER STAGES OF READINESS

TO SCREEN FOR BOWEL CANCER: IMPLICATIONS FOR RESCREENING INTERVENTIONS

Amy Duncan, BHealth Science Hons, ${ }^{1}$ Carlene Wilson, $\mathrm{PhD},{ }^{2}$ Deborah Turnbull, $\mathrm{PhD},{ }^{1}$ Ingrid Flight, $\mathrm{BA}, \mathrm{MPH},{ }^{3}$ Tess Gregory, $\mathrm{PhD},{ }^{1,2}$ Ian Zajac, B Psychology Hons, ${ }^{2}$ Stephen Cole, Bsc, $\mathrm{MPH}^{4}$ and Graeme Young, MD FRACP ${ }^{2}$

${ }^{1}$ School of Psychology, Adelaide University, Adelaide, SA, Australia; ${ }^{2}$ Medicine, Flinders University, Adelaide, SA, Australia; ${ }^{3}$ Preventive Health Research Flagship, CSIRO, Adelaide, SA, Australia and ${ }^{4}$ Bowel Health Service, Repatriation General Hospital, Daw Park, SA, Australia.

Limited behavioural research is available concerning the differences between those who maintain continued adherence to Colorectal Cancer (CRC) screening guidelines and those who do not. Using the Transtheoretical Model of Behaviour Change (TTM) this study examines the differences between the stages of readiness to screen for $\mathrm{CRC}$, with a focus on the'rescreening stages' (maintenance, relapse and inconsistent), according to demographic, social cognitive and past experiences variables.

A random sample of 1973 people aged 50 to 74 years residing in selected Adelaide Electoral Divisions completed a survey concerning attitudes toward screening using a Faecal Occult Blood Test (FOBT). Univariate analyses were performed to examine significant differences between stages according to survey variables.

Few demographic differences were identified, with lack of private health insurance being the only characteristic to differentiate the relapse stage from the inconsistent and maintenance stages $(\chi 2$ $(18)=55.61, p<.001)$. Similarities were identified between relapse and the earlier precontemplation stage with both showing significantly lower; benefits of screening $(F(6,1786)=69.59$, $\mathrm{p}<.001)$, self efficacy $(\mathrm{F}(6,1301.37)=31.426, \mathrm{p}<.001)$, family history of CRC $(\chi 2(6)=30.43, \mathrm{p}<.001)$ and social influence $(\mathrm{F}$ $(6,1786)=32.215, p<.001)$ and higher belief in chance health $(F$ $(6,1429.76)=8.89, \mathrm{p}<.001)$ compared to all later stages. Relapse and inconsistent screeners differed from the maintenance stage showing greater barriers toward screening $(\mathrm{F}(6,1391.2)=57.31$, $\mathrm{p}<.001)$, less social support $(\mathrm{F}(6,1325.33)=14.12, \mathrm{p}<.001)$ and lower satisfaction with previous screening $(\mathrm{F}(3,482.21)=14.84$, $\mathrm{p}<.001)$. Relapse participants rated their previous screens as less worthwhile $(\mathrm{F}(3,682)=6.753, \mathrm{p}<.001)$ reassuring $(\mathrm{F}(3,700)=$ $7.465, \mathrm{p}<.001)$ and convenient $(\mathrm{F}(3,667)=4.33, \mathrm{p}<.05)$ and more embarrassing $(\mathrm{F}(3,632)=10.717, \mathrm{p}<.001)$ and difficult to complete $(\mathrm{F}(3,738)=9.73, \mathrm{p}<.001)$ compared to those in the action and maintenance stages. Relapse participants also had poorer rescreening knowledge $(\chi 2(12)=150.73, \mathrm{p}<.001)$ than all remaining stages.

Results highlight similarities between relapse and precontemplation supporting the notion that movement through the TTM can be cyclic in nature. Results also indicate that a positive experience of screening is important for screening maintenance. Differences between those in the relapse and inconsistent stages suggest separate interventions are required to increase screening maintenance amongst these groups.

CORRESPONDING AUTHOR: Amy Duncan, BHealth Science Hons, Adelaide.University, Adelaide, SA, 5005; amy.duncan@ adelaide.edu.au

9:06 AM-9:24 AM

OS38-C

MEASURING THE CONTINUUM OF RESISTANCE TO PARTICIPATION IN COLORECTAL CANCER SCREENING: IMPLICATIONS FOR THE DESIGN OF INVITATION AND REMINDER LETTERS

Tess Gregory, $\mathrm{PhD},{ }^{1,2}$ Ingrid Flight, BA MPH, ${ }^{4}$ Carlene Wilson, $\mathrm{PhD},{ }^{1}$ Deborah Turnbull, $\mathrm{PhD},{ }^{2}$ Stephen Cole, $\mathrm{BSc} \mathrm{MPH}^{1,3}$ and Graeme Young, MD FRACP ${ }^{1}$

${ }^{1}$ Medicine, Flinders University, Adelaide, SA, Australia; ${ }^{2}$ Psychology, University of Adelaide, Adelaide, SA, Australia; ${ }^{3}$ Bowel Health Service, Repatriation General Hospital, Daw Park, SA, Australia and ${ }^{4}$ Preventative Health Research Flagship, CSIRO, Adelaide, SA, Australia.

The design of invitation and reminder letters to screen for cancer has been influenced by studies comparing the psychological and attitudinal differences between participants and non-participants. Participation in cancer screening has been thought to follow the continuum of resistance model, which assumes the same factors influence early and late participation. This study investigated the validity of the continuum of resistance model for explaining participation in colorectal cancer (CRC) screening.

People aged 50 to 74 years, recruited from the electoral roll who had completed a baseline survey and were in the early stages of readiness to screen $(n=376)$ were subsequently invited to complete a faecal occult blood screening test. Multivariate analyses examined the predictors of early participation (kit returned in weeks 1-6) and late participation (kit returned in weeks 7 - 12).

Results confirmed that different factors influenced early and late participation. Early participation was associated with employment status and barriers to screening, with retired people more likely to screen early than employed people (relative risk $\mathrm{RR}=1.69, \mathrm{CI}=$ 1.22 to 2.38 ) and people with higher barriers (including faecal aversion) less likely to screen early ( $R R=0.92, C I=0.88$ to 0.97 ). Late participation was associated with higher perceived susceptibility $(\mathrm{RR}=1.26, \mathrm{CI}=1.06$ to 1.49$)$, participating in cancer screening in the past $(\mathrm{RR}=2.17, \mathrm{CI}=1.30,3.63)$, lower internal health locus of control ( $\mathrm{RR}=0.87, \mathrm{CI}=0.79$ to 0.96$)$ and having fewer chronic diseases $(\mathrm{RR}=0.63, \mathrm{CI}=0.46,0.88)$.

Based on the differences between groups, initial invitation letters need to focus on different factors than reminders letters when attempting to increase participation rates. Future research would benefit from investigating the efficacy of enhanced invitation and reminder letters, based on these results, to increase participation rates in CRC screening. 
CORRESPONDING AUTHOR: Tess Gregory, PhD, Medicine, Flinders University of South Australia, Adelaide, SA, 5001; tess.gregory@psychology.adelaide.edu.au

\section{9:24 AM-9:42 AM}

OS38-D

AN INTERNET-BASED PERSONALISED DECISION SUPPORT SYSTEM FOR COLORECTAL CANCER SCREENING: ITS IMPACT ON ATTITUDES AND KNOWLEDGE, AND ITS PERCEIVED USABILITY AND ACCEPTABILITY

Ian Zajac, Honours Psychology, ${ }^{1}$ Katrina Lindblom, Honours Psychology, ${ }^{2}$ Tess Gregory, $\mathrm{PhD}^{2}$ Carlene Wilson, $\mathrm{PhD}^{3}$ and Ingrid Flight, Behavioural Science ${ }^{1}$

${ }^{1}$ Preventative Health Research Flagship, CSIRO, Adelaide, SA, Australia; ${ }^{2}$ Psychology, University of Adelaide, Adelaide, SA, Australia and ${ }^{3}$ Medicine, Flinders University, Adelaide, SA, Australia.

Colorectal cancer (CRC) is a major public health problem. It is the most commonly diagnosed non-cutaneous cancer in Australia, and the second most common cause of cancer related death. The Australian government has introduced mass population screening by faecal occult blood test (FOBT) to reduce CRC incidence and mortality. However, participation in this program has been suboptimal.

Research shows that cancer decision aids increase knowledge about the benefit associated with screening and decrease levels of decisional conflict. This subsequently leads to better behavioural outcomes including participation in cancer screening programmes. The purpose of this study then, was to investigate the utility of an internet delivered CRC decisional support aid. Specific aims were to examine (1) whether changes in knowledge and attitudes after exposure to the PDS were moderated by the perceived usability and acceptability of the tool, and (2) test the degree to which perceived usability was dependent on "technophobia" as measured by computer anxiety and computer self-efficacy.

Thirty-six male $(M=60.5$ years, $S D=6.6)$ and 45 female $(M=$ 58.2 years, $\mathrm{SD}=6.3$ ) members of the general public, aged 50 to 76 years, participated in the study $(n=81)$. Results confirmed that exposure to the PDS led to a significant decrease in cancer worries $(\mathrm{F}(1,80)=7.14, \mathrm{p}=<.01)$ and an increase in CRC knowledge $(\mathrm{F}$ $(1,80)=30.72, \mathrm{p}<.001)$, salience and coherence $(\mathrm{F}(1,80)=6.32$, $\mathrm{p}=.05)$, perceived susceptibility $(\mathrm{F}(1,80)=16.05, \mathrm{p}=<.001)$ and response efficacy $(\mathrm{F}(1,80)=22.71, \mathrm{p}=<.001)$. However, perceived usability and acceptability (PUA) mediated this effect for most factors and participants with low PUA showed stability in their attitudes, whereas people with high PUA showed significant change. Computer anxiety was found to be a significant predictor of perceived usability and acceptability $(\mathrm{r}=-.46, \mathrm{p}<.001)$, supporting the hypothesis that technophobia is an important predictor of the way people perceive the tool, and in turn the degree to which it influences their attitudes towards screening.

The results of this study suggest that internet based decision support tools may improve participatory behaviour in large scale CRC screening programs. However, results also suggest that the utility of computer-based decision aids may be impacted by 'technophobia' in the target population who are generally aged 50 years and older.
CORRESPONDING AUTHOR: Ian Zajac, Honours Psychology, Preventative Health Research Flagship, CSIRO, Adelaide, SA, 5000; ian.zajac@adelaide.edu.au

\section{9:42 AM-10:00 AM}

OS38-E

PERCEIVED RISK OF BREAST CANCER, BELIEFS ABOUT MAMMOGRAPHY, AND PSYCHOLOGICAL DISTRESS IN BREAST CANCER SURVIVORS AND HEALTHY WOMEN AT THE TIME OF MAMMOGRAPHY

Rebecca A. Shelby, $\mathrm{PhD},{ }^{1}$ Francis J. Keefe, $\mathrm{PhD},{ }^{1}$ Dana H. Bovbjerg, $\mathrm{PhD},{ }^{2}$ Celette Sugg Skinner, $\mathrm{PhD},{ }^{3}$ Tamara J. Somers, $\mathrm{PhD},{ }^{1}$ Mary Scott Soo, MD, ${ }^{1}$ Sara Red, BS, ${ }^{1}$ Sandra Stinnett, DPH, ${ }^{1}$ Mary Frances Luce, $\mathrm{PhD}^{1}$ and Jules Sumkin, $\mathrm{DO}^{2}$

${ }^{1}$ Duke University Medical Center, Durham, NC; ${ }^{2}$ University of Pittsburgh Medical Center, Pittsburgh, PA and ${ }^{3}$ UT Southwestern Medical Center, Dallas, TX.

Little is known about how breast cancer and beliefs about mammography relate to psychological distress assessed at the time of mammography. This study examined perceived breast cancer risk and perceived benefits and barriers to mammography in breast cancer survivors $(\mathrm{N}=64)$ and women with no cancer history $(\mathrm{N}=70)$. A key goal of this study was to examine associations between perceived risk and anxiety, depression, and mammography specific distress. In the breast imaging clinic and immediately prior to undergoing mammography, breast cancer survivors rated their perceived risk of breast cancer recurrence $(\mathrm{M}=14, \mathrm{SD}=6)$ and beliefs about mammography. Women with no cancer history rated their perceived risk of developing breast cancer $(\mathrm{M}=12, \mathrm{SD}=6)$. Breast cancer survivors reported higher perceived risk $(p=.05)$ and lower perceived benefits of mammography $(p=.01)$ compared to women with no cancer history. Perceived barriers did not differ across groups. Immediately prior to mammography, women also rated their mammography specific distress, anxiety, and depression. Levels of anxiety and depression did not differ across groups, but breast cancer survivors reported higher mammography specific distress $(\mathrm{p}<.001)$. Multiple linear regression analyses showed that anxiety and mammography specific distress were positively associated with perceived risk in breast cancer survivors, but these associations were not found in women with no cancer history [Anxiety: Group $\mathrm{x}$ Perceived Risk $\mathrm{B}=.27, \mathrm{SE}=.11, \mathrm{p}=.02$; Mammography Specific Distress: Group x Perceived Risk $\mathrm{B}=.62, \mathrm{SE}=.21, \mathrm{p}=.004]$. A similar trend was found for depression [Depression: Group $\mathrm{x}$ Perceived Risk $\mathrm{B}=.15, \mathrm{SE}=.08, \mathrm{p}=.06]$. These findings suggest that breast cancer survivors with high perceived risk are more likely to experience distress during mammography. Strategies to help breast cancer survivors develop more accurate cancer risk perceptions and manage distress during mammography may be beneficial.

CORRESPONDING AUTHOR: Rebecca A. Shelby, PhD, Psychiatry and Behavioral Sciences, Duke University Medical Center, Durham, NC,27705; shelb003@mc.duke.edu 
OS39

\section{8:30 AM-8:48 AM}

OS39-A

\section{TOWARDS A CONCEPT OF UNIVERSAL HEALTH COVERAGE}

Jesse Bump, PhD, MPH

Global Health and Population, Harvard School of Public Health, Boston, MA.

Health systems are among the most important elements of the social contract that defines modern nations. Almost all developed countries have implemented a comprehensive care system to provide medical services to their citizens at low or no cost at the point of service - universal health coverage (UHC) systems. In the past few years, UHC has risen to the forefront of the global health agenda, as reflected by donor pledges, international declarations, and high-profile publications. Under other banners, similar objectives have been advanced at the global level since the WWI era, first by private entities such as the Rockefeller Foundation's International Health Division and after WWII by the main institutions of the international post-colonial architecture, particularly by the WHO.

This paper investigates the historical origins of UHC systems and analyzes the politics surrounding the national decision to implement them. Understanding how, when, and why countries have established UHC systems sheds light on current international assistance strategies to encourage the same transition in developing countries. First, I analyze definitions of UHC to distill the important elements and discuss their primary assumptions, including their commitments to solidarity, redistribution, and government responsibility. I then focus on the development of the two main financing models-social health insurance (Germany) and general taxation (United Kingdom) - and then follow global-level attempts to promote UHC over the past century.

Moving toward UHC is a renegotiation of the social contract and is hotly contested because it changes who gets what and who pays for it. As such it is primarily a matter of domestic politics. I find that international aid agencies have not played a significant role in fostering the spread of UHC systems in the past. Beyond the possibility of fostering international learning and providing resources, there is no clear evidence in support of any particular role for international actors in fostering UHC systems.

CORRESPONDING AUTHOR: Jesse Bump, PhD, MPH, Global Health and Population, Harvard School of Public Health, Boston, MA, 02115; jbump@hsph.harvard.edu

\section{8:48 AM-9:06 AM}

OS39-B

CONSIDERATIONS MADE BY GENERAL PRACTITIONERS WHEN DEALING WITH SICK-LISTING OF PATIENTS SUFFERING FROM MEDICALLY UNEXPLAINED CONDITIONS Liv H. Magnussen, $\mathrm{PhD},{ }^{1,2}$ Erik L. Werner, $\mathrm{PhD}, \mathrm{MD},{ }^{1}$ Silje Mæland, $\mathrm{MSc}^{1}$ and Stein Nilsen, $\mathrm{MD}^{1}$

${ }^{1}$ Uni Health, Uni Research, Bergen, Bergen, Norway and ${ }^{2}$ 2Department of Public Health and Primary Health Care, University of Bergen, Bergen, Norway.
Background: Although we have some knowledge on general practitioners' (GP) practices on sick-listing in general, there is scarce knowledge about how they make their assessments especially in the most complex cases of medically unexplained conditions.

Aims: The aim of this study was to explore on considerations made by GPs when dealing with sick-listing of patients suffering from medically unexplained conditions.

Materials and Methods: Data were drawn from focus-group interviews with 48 general practitioners (GP). The GPs were recruited as they participated on a course in Social Medicine. We established groups with variation regarding age, gender, nationality and work experience. Seventeen women and 31 men (15 with other nationalities) aged 32-65 accepted to participate in the interviews. The doctors had worked in general practice from 1 to 34 years.

Results: Sick-listing patients with medically unexplained disorders was regarded as a very challenging task by many GPs. Trust in the patient's own story and his self-judgement is deemed crucial, but many miss hard evidence of illness and loss of function. Several factors which may influence the decision making regarding sickleave were identified: To which extent the patient manage to present his story, extensive prior knowledge of the patient, but also properties within the doctor himself: own experience as a patient, tendency to avoid conflicts and others. Strategies in dealing with sick-listing would be to initially comply with the patient's request in order to build alliance and then start to motivate for a return to work. Confrontation when they disagreed strongly on a sick note was another strategy mentioned, but at the same time risking that the patient would leave them for a more cooperative colleague. Many judged themselves as having no real power in the decisionmaking concerning sick-listing, evoking frustration in some.

Conclusion: This study shed light on the many challenges that the GPs are confronted to when making decisions regarding sicklisting of patients suffering from medically unexplained disorders.

CORRESPONDING AUTHOR: Liv H. Magnussen, PhD, Uni Health, Uni Research, Bergen, Bergen, N-5020; liv.magnussen@ uni.no

9:06 AM-9:24 AM

OS39-C

GUATEMALA: THE ECONOMIC BURDEN OF ILLNESS AND HEALTH SYSTEM IMPLICATIONS

Diana Bowser, MPH and Ajay Mahal, PhD

GHP, HSPH, Boston, MA.

Objectives: To describe the economic burden of ill health on Guatemala's population and to highlight the characteristics of its health sector that likely explains this burden and to use recent health reform efforts in several developing countries to highlight lessons that Guatemalan policymakers can use to formulate strategies to address the economic consequences of ill health for their population. Methods: The quantitative analysis reported in this paper is based primarily on the 2000 and 2006 Living Standard Measurement Surveys (LSMS or Encuesta Nacional de Condiciones de Vida). The LSMS data is used to estimate health and consumption spending as well as examine certain health access variables. We 
assessed health-related impacts on household poverty using the methodology of van Doorslaer et al. (2006). Similarly, we constructed estimates of the proportion of the Guatemalan population incurring catastrophic levels of health expenditures to assess the financial risk of ill health using the methodology of $\mathrm{Xu}$ et al. (2003). We defined catastrophic health spending (on both inpatient and outpatient) as a level of health spending that exceeds $40 \%$ of a household's ability to pay, although other thresholds are obviously possible.

Results: There is both a high level and inequitable distribution of financial burden due to ill health in Guatemala. This is primarily due to low levels of insurance coverage and a heavy concentration of the uninsured among the less well off and rural populations as well as poor quality and low levels of access to public services for the poor, stagnant or declining government spending on health and increasing privatization that likely has resulted in rising treatment costs.

Conclusions: High levels of catastrophic health spending and poverty co-exist with significant economic inequality and poverty in Guatemala. With health system characteristics and an economic structure similar to many other developing countries, international experience provides useful lessons to help Guatemala devise innovative financing and payment mechanisms to address these concerns.

CORRESPONDING AUTHOR: Diana Bowser, MPH, GHP, HSPH, Boston, MA, 02115; dbowser@hsph.harvard.edu

\section{9:24 AM-9:42 AM}

OS39-D

CONTRACTING HEALTH WORKERS TO STRENGTHEN HEALTH SYSTEMS PERFORMANCE IN MEXICO.

\section{THE CASE OF POPULAR HEALTH INSURANCE}

Gustavo Nigenda, PhD, Elizabeth Aguilar, BA, Jose Arturo Ruiz, BA and Rosa Maria Bejarano, BA

Center for Health Systems Research, National Institute of Public Health, Cuernavaca, Mexico.

By means of two evaluations focusing on managerial process carried out in 2007 and 2009, the paper analyzes the policy that the Mexican Popular Health Insurance(PHI) has followed to hire thousands of workers in order to strengthen the capacity of the program to increase the volume of services provided and improve quality. As part of the Ministry of Health structure, PHI started its activities in 2003 aiming at providing services to 11.2 million families in the country not covered by traditional social insurance institutions. PHI counts with unprecedent financial public resources to carry out its activities. More than 70,000 workers of 17 categories in 32 states have been hired. Although workers were offered initially a temporary contract with no benefits such as social insurance, annual allowance and vacations, the Ministry of Health $(\mathrm{MOH})$ along with the Ministry of Budgeting and the MOH's workers union decided to provide benefits defined by Law starting in 2008. So far, half of all workers are contracted under the new conditions. However, 2009 evaluation using a sample of 9 states, showed that initially the performance of workers in terms of productivity and quality of care has not necessarily improved raising questions about the way Human Resources for Health policies should be designed and applied to really generate improvements for the benefit of those populations covered by the program.

CORRESPONDING AUTHOR: Gustavo Nigenda, PhD, Center for Health Systems Research, National Institute of Public Health, Cuernavaca,62100; gnigenda@insp.mx

\section{9:42 AM-10:00 AM}

OS39-E

APPLICATION OF IMPOSSIBILITY THEOREM: PARETO VERSUS LIBERTY PRINCIPLES IN CONDITIONAL

FOREIGN AID

Yanfang $\mathrm{Su}, \mathrm{ScD}$

Global Health and Population, Harvard School of Public Health, Boston, MA.

The aim of this research is to demonstrate why in global governance Pareto principle from an economic perspective and liberty principle from a political perspective are incompatible. Impossibility Theorem is applied to analyze the international relations among players with different preferences. The theoretical proposition of incompatibility is followed by the example of conditional foreign aid, with distinction of indirect liberty and direct control.

Two rival hypotheses, global Pareto condition and national liberty condition, are tested in the empirical evaluation of the foreign aid conditional on the Washington Consensus from 1989 to 1999. First, small-N design is to compare the pre-treatment and post-treatment situations in 3 representative countries, as the non-complier, partial-complier and complete-complier of the Washington Consensus. Second, large-N regressions are to estimate the effect of foreign aid on social developments measured by health outcomes: (1) foreign aid without controlling for the adoption level of the Washington Consensus, which reflects in liberty principle; (2) foreign aid after controlling for the adoption of the Washington Consensus, which reflects in Pareto principle.

Furthermore, responding to the criticism of "what causes what" in the relationship of underdevelopment and foreign aid, oil price and oil reserve are introduced as instrumental variable (IV) into the model to isolate the causation of foreign aid on social developments, especially health outcomes. Finally, the concept of overlapping consensus from John Rawls is incorporated to interpret why partial-compliers are better off than both non-compliers and complete-compliers given conditionality of foreign aid.

The strategy is to not only work within the domain of the impossibility theorem with empirical evidences but also step out to examine the assumptions that constrain the outcome through the inspiration of overlapping consensus.

CORRESPONDING AUTHOR: Yanfang Su, ScD, Global Health and Population, Harvard School of Public Health, Boston, MA, 02120; ysu@hsph.harvard.edu 


\author{
CITATION ABSTRACT \\ OS40 \\ 8:30 AM-8:48 AM \\ OS40-A
}

POLYMORPHISMS IN FATTY ACID METABOLISM-RELATED GENES

ARE ASSOCIATED WITH DEPRESSIVE SYMPTOMS

Hirohito Tsuboi, MD, PhD,${ }^{1}$ Hiroyuki Sakakibara, $\mathrm{PhD},{ }^{2}$ Kimiko Yamakawa-Kobayashi, $\mathrm{PhD},{ }^{3}$ Asami Tatsumi, PhD, ${ }^{4}$ Masahiro Matsunaga, PhD, ${ }^{1}$ Yoko Kawanishi, BA, ${ }^{1}$ Hiroshi Kaneko, $\mathrm{PhD}^{1}$ and Kayoko Shimoi, $\mathrm{PhD}^{2}$

${ }^{1}$ Department of Neurology \& Psychosomatic Medicine, Fujita Health University School of Medicine, Nagoya, Japan; ${ }^{2}$ Institute for Environmental Sciences, University of Shizuoka, Shizuoka, Japan; ${ }^{3}$ School of Food and Nutritional Sciences, University of Shizuoka, Shizuoka, Japan and ${ }^{4}$ Department of Community Health Nursing, Hamamatsu University School of Medicine, Hamamatsu, Japan.

[Objectives] Depression is a heterogeneous heritable psychological trait, also influenced by environmental factors. Previous studies have found the associations between major depression and gene polymorphisms such as serotonin transporter gene linked polymorphic region (5HTTLPR). Since fatty acid (FA) metabolism appear to contribute to the pathoaetiology of affective disorders, we examined the associations between depressive symptoms and genetic variation in FA metabolism-related genes.

[Methods] A cross-sectional study was conducted on 166 female workers of a hospital and nursing homes. Depressive symptoms and subjective psychological stress were assessed by the Center for Epidemiologic Studies Depression (CES-D) scale and visual analogue scale, respectively. Selected gene polymorphisms associated with FA metabolism as well as 5HTTLPR were analysed.

[Results] Linear regression analysis was performed in which CES-D scores served as a dependent variable, and subjective stress and gene polymorphisms as independent variables: consequently, SS carriers of the 5-HTTLPR gene showed significant higher depressive symptoms in comparison with LL/SL carriers, even after controlling for confounders ( $\mathrm{F}=27.6$, standardized beta $=2.2, \mathrm{p}<0.05$ ). Regression analysis also confirmed some other gene polymorphisms significantly associated with depressive symptoms.

[Conclusion] Some gene polymorphisms associated with FA metabolism may affect depressive symptoms as the 5-HTTLPR does.

CORRESPONDING AUTHOR: Hirohito Tsuboi, MD, PhD, Department of Neurology \& Psychosomatic Medicine, Fujita Health University School of Medicine, Nagoya, 454-8509; tsuboih@fujita-hu.ac.jp

\section{8:48 AM-9:06 AM \\ OS40-B}

\section{METAANALYSIS OF THE EFFECT OF DEPRESSION} ON IN-VITRO FERTILIZATION (IVF) TREATMENT OUTCOME Signe M. Ebbesen, M Sc Psychology, ${ }^{1}$ Robert Zachariae, Professor, Dr Med ${ }^{1,2}$ and Yoon Frederiksen, MSc Psychology ${ }^{1}$ ${ }^{1}$ Psychology, Aarhus University, Aarhus, Denmark and ${ }^{2}$ Dept of Oncology, Aarhus University Hospital, Aarhus, Denmark.

Background: Suffering from involuntary infertility and going through In Vitro Fertilization (IVF) treatment is significant stressor and associated with increased risk of depression. Depression has been hypothesized to reduce the chances of a succesful IVF- outcome, and a number of studies have reported associations between depression and IVF-outcome. Other studies, however, have been unable to replicate these findings, and the strength of a possible association between depressive symptoms and pregnancy chances following IVF remains to be clarified. Our aim was therefore to conduct a statistical quantitative review of published articles reporting on the association between depression and IVFoutcome and to estimate the strength of such an association. Methods: Systematic literature searches were conducted in PubMed, PsychInfo and Web of Science, identifying prospective empirical studies reporting data on associations between depression and IVF-outcome, i.e. pregnancy. Results: The forward database searches resulted in 14 accessible independent studies, providing the necessary and unadjusted data for Effect size calculations. A simple vote count showed a significant effect of depression on IVF-outcome in 4 of these studies. Effect size correlations (ESR) were calculated by two independent researchers for each individual study and then combined to estimate an overall effect size for the depression-IVF-outcome association. 
Due to considerable heterogeneity $(\mathrm{Q}=131,10 ; \mathrm{p}<0.0001)$, a random effects model was applied. The overall effect size found for the association between depression and IVF-outcome was statistically significant, with a higher number of depressive symptoms being associated with reduced pregnancy chances ( $\mathrm{ESr}=-0,06$, failsafe $\mathrm{N}=114, \mathrm{p}<0.05)$. Conclusion. A modest influence of depression on IVF-outcome was found. While the Failsafe-number indicates a relatively robust result, further studies are needed to explore possible moderators of the depression - IVFoutcome effect: e.g. female age, IVF inductees or veterans, duration and timing of presence of depressive symptoms.

CORRESPONDING AUTHOR: Signe M. Ebbesen, M Sc Psychology, Psychology, Aarhus University, Aarhus, 8200; signese@, psy.au.dk

\section{9:06 AM-9:24 AM}

\section{OS40-C}

\section{NEURAL-IMMUNE CORRELATES OF STRESS} DURING PREGNANCY

Mary Coussons-Read, $\mathrm{PhD},{ }^{1}$ Marianne Kreither, MS, ${ }^{1}$ Marci Lobel, $\mathrm{PhD}^{2}$ and Courtney Cage, $\mathrm{BS}^{1}$

${ }^{1}$ University of Colorado Denver, Denver, $\mathrm{CO}$ and ${ }^{2}$ University of Stony Brook, Stony Brook, NY.

Growing evidence shows that that elevated stress during pregnancy may increase the risk of preterm delivery and other pregnancy and birth complications. Neuroendocrine, immune, inflammatory, and cardiovascular factors have been suggested as possible mediators of the relationship between stress and birth outcomes, and recent data suggest that stress-related changes in cytokine production and inflammation may contribute to these effects. Work in our laboratory has focused on quantifying the effects of perceived stress and social support on pregnancy outcome and the degree to which neural-immune interactions may be involved in these effects. Initial work in the lab focused on establishing connections between maternal prenatal stress and low social support and alterations in cytokine balance in the circulation and cytokine production by stimulated peripheral lymphocytes. This work indicated that indeed, elevated stress and lower social support was related to increases in circulating inflammatory mediators, reduction in anti-inflammatory cytokines, and similar changes in the production of cytokines in vitro by mitogenstimulated peripheral blood lymphocytes. Additional studies began to connect these findings to the likelihood of premature delivery and other complications of pregnancy, and have started to demonstrate that indeed, increased stress and distress early in pregnancy is related not only to elevations in inflammatory mediators in the prenatal period, but is also associated with higher frequency of pregnancy and birth complications. Most recently, our work has focused on the role of maternal ethnicity and acculturation in the impact of stress on pregnancy, and on the role of catecholamines in these relationships. Elevated proinflammatory cytokines were lower levels of support and higher stress throughout pregnancy, and higher social support was associated with higher levels of anti-inflammatory cytokines late in pregnancy. Moreover, elevated inflammatory markers were related to higher pregnancy-specific distress early in gestation, as well as lower 1-minute APGAR scores, lower gestational age at birth, and increased occurrence of birth complications including meconium aspiration, nuchal cord, and asphyxia. Together, these studies indicate that psychosocial factors are related to increases in inflammatory mediators and subsequently, increased risk of pregnancy complications and untoward birth outcomes.

CORRESPONDING AUTHOR: Mary Coussons-Read, PhD, University of Colorado Denver, Denver, CO, 80217-3364; Mary. Coussons-Read@ucdenver.edu

\section{9:24 AM-9:42 AM}

\section{OS40-D}

INFLUENCE OF MATERNAL PSYCHOSOCIAL STATUS, DIETARY QUALITY AND PLACENTAL DEVELOPMENT IN THE 1ST TRIMESTER OF PREGNANCY

Eileen Fowles, PhD, RNC-OB

School of Nursing, University of Texas at Austin, Austin, TX.

A pregnant woman's dietary quality during the 1st trimester of pregnancy has a profound effect on placental development, which can result in diminished supply of nutrients to the fetus, thus inhibiting proper fetal growth. However, little is known about lowincome women's dietary quality during the 1 st trimester when placental development is vulnerable to changes in maternal nutrition. The aims of this study were to explore the relationships 1) between maternal psychosocial factors and dietary quality; and 2) between dietary quality and selected placental biomarkers. A descriptive design was used in a clinic sample of low-income women $(\mathrm{N}=30)$. Women were, on average, 24 years old and 8 weeks pregnant. Most were unmarried, uninsured, and Hispanic. Spearman's rho statistic was used to analyze data due to small sample size and skewed data. Women $(\mathrm{N}=30)$ were, on average, 24 years old and 8 weeks pregnant. 1st Study Aim Social support was related to vegetable intake $(r=.37)$ and intake of iron-rich $(\mathrm{r}=.478)$ and folate-rich $(\mathrm{r}=.50)$ foods. Emotional eating in response to anxiety was negatively relationship to intake of fruits $(\mathrm{r}=-.33)$ and calcium-rich $(\mathrm{r}=-.33)$. Depressed women had less social support $(\mathrm{r}=-.43)$, more emotional eating $(\mathrm{r}=.38)$, and decreased intake of foods rich in calcium $(\mathrm{r}=-.38)$, iron $(\mathrm{r}=$ -.60), and folate ( $\mathrm{r}=-.49)$ 2nd Study Aim Vascular Endothelial Growth Factor was positively related to fruit intake $(r=.40)$ and Placental Growth Factor was negatively related to the intake of iron $(\mathrm{r}=-.43)$ and zinc (-.38). partner support partner was related positively to vegetable intake and negatively to intake of iron and grains. Although results should be interpreted with caution, the 
findings suggest that low-income pregnant women who are depressed or engage in emotional eating are likely to have inadequate intake of nutrients that contribute to positive pregnancy outcomes. Placental development in the early weeks of pregnancy may be influenced by maternal dietary intake. More research is needed to explore the relationships among psychosocial status, dietary intake and placental development in the first trimester of pregnancy.

CORRESPONDING AUTHOR: Eileen Fowles, PhD, RNC-OB, School of Nursing, University of Texas at Austin, Austin, TX, 78701-1499; efowles@mail.nur.utexas.edu

\section{CITATION ABSTRACT \\ 9:42 AM-10:00 AM \\ OS40-E}

\section{RACIAL DIFFERENCES IN THE RELATION OF MULTIPLE ROLES TO PROGRESSION} OF CAC IN MIDLIFE WOMEN

Imke Janssen, $\mathrm{PhD},{ }^{1}$ Lynda H. Powell, $\mathrm{PhD},{ }^{1}$ Karen A. Matthews, $\mathrm{PhD},{ }^{2}$ Kim Sutton-Tyrrell, $\mathrm{DrPH},{ }^{2}$ Steven Hollenberg, MD, ${ }^{4}$ John Cursio, $\mathrm{MS}^{1}$ and Susan A. Everson-Rose, $\mathrm{PhD}^{3}$

${ }^{1}$ Preventive Medicine, Rush University Medical Center, Chicago, IL; ${ }^{2}$ University of Pittsburgh, Pittsburgh, PA; ${ }^{3}$ University of Minnesota, Minneapolis, MN and ${ }^{4}$ CooperUniversity Hospital, Camden, NJ.

Background: Psychosocial risk for cardiovascular disease (CVD) in women has focused on individual factors such as marital stress and job stress. Little is known about the joint effects, especially in women and minorities. We examined the relation to two-year progression of coronary calcification (CAC) among women at midlife.

Methods: Data come from 337 women (31\% black, 69\% white; mean age, $50.8(+2.8)$ years) who completed psychosocial assessments and two CT scans, an average 2.3 years apart, as part of the Study of Women's Health Across the Nation (SWAN) Heart ancillary study. SWAN is a longitudinal, multi-site study of health and psychosocial factors in women transitioning through menopause. SWAN Heart was conducted from 2001-2005 in Chicago, IL and Pittsburgh, PA to examine CVD risk factors and subclinical atherosclerosis in women without CVD. Four roles spouse, parent, employee, and caregiver - were assessed on a 5-point scale from not stressful to extremely stressful. CAC was assessed by electron beam tomography; CAC progression was defined as an increase in CAC of $>10$ Agatston units and analyzed using relative risk (RR) regression.

Results: Multiple role stress levels [2.75 \pm 0.79 ] did not differ by race and were modeled continuously; covariates were age, time between CT scans, race, education, BMI, blood pressure, statin use, smoking, menopausal status, HT use, and HDL cholesterol. We observed a race $\mathrm{x}$ stress interaction $(\mathrm{p}<.001)$ in CAC progression. A 1-point higher stress score decreased risk $[\mathrm{RR}=0.65(95 \% \mathrm{CI}=0.46-0.91), \mathrm{p}=.011]$ in white women, but increased risk in black women $[\mathrm{RR}=1.40(95 \% \mathrm{CI}=0.99-1.98, \mathrm{p}=0.059)$. This effect was independent of family income and depressive symptoms in both ethnic groups.

Conclusions: In this cohort of women, stress from multiple roles decreased risk of CAC progression among white women and increased risk in black women independent of known risk factors.

Supported by NIH/DHHS (grants AG012505, AG012546, HL065581, HL065591, HL089862). 
CORRESPONDING AUTHOR: Imke Janssen, PhD, Preventive Medicine, Rush University Medical Center, Chicago, IL, 60612; Imke_Janssen@rush.edu

OS41

\section{8:30 AM-8:48 AM}

OS41-A

DEMOGRAPHIC AND TRANSTHEORETICAL MODEL VARIABLES PREDICT FUTURE ALCOHOL DRINKING BEHAVIORS

Rose Marie Ward, PhD, Angela Coppola, BA and John Ward, PhD Miami University, Oxford, $\mathrm{OH}$.

Alcohol use and abuse on US college campuses is not a new idea. Alcohol abuse has been linked to lower grades, more mental health problems, and failure to meet obligations. University officials implement population based programming to curb these issues. Many of these services are based on the Transtheoretical Model. The current project examined demographic variables and the constructs of the Transtheoretical Model. The goal was to determine if these constructs could predict alcohol behavior over time and changes in alcohol behavior.

Data from 321 first year students ( $70 \%$ female) were collected on the first day of classes and the week before finals at a Midwestern public university. Participants were recruited through a general education course at the university. Multiple structural models, consistent with the TTM, were evaluated and tested. Competing models included demographic variables as well as TTM variables. The model which examined demographic (Body Mass Index, gender, and ethnicity) and TTM variables predicting stage and then predicting future alcohol behavior fit the model well, chisquare $(20)=30.22, \mathrm{p}=.07, \mathrm{CFI}>.99, \mathrm{TLI}=.98, \mathrm{RMSEA}=.04$. Models including both the demographic variables and TTM variables, predicted future drinking behavior very well. Stage of change was the largest predictor of future behavior. In additional models, the TTM variables predicted future behavior without the demographic variables, and fit the data well. The models indicated that stage of change was a good predictor of current alcohol behaviors, future alcohol behaviors, and change in alcohol behaviors. Some additional constructs from the TTM explained additional variance.

These results suggest that interventions aimed at first year college students that concentrate on stage of change, demographic variables, and the cons of alcohol use may be effective in influencing change in the alcohol behaviors. Interventions should consider tailoring both to TTM variables and common demographic variables.

CORRESPONDING AUTHOR: Rose Marie Ward, PhD, Miami University, Oxford, OH, 45056; wardrm1@muohio.edu
8:48 AM-9:06 AM

OS41-B

ALCOHOL USE AS A MEDIATOR OF FORCED SEXUAL INTERCOURSE AND SUICIDALITY IN ADOLESCENT GIRLS

Yen-Chi L. Le, $\mathrm{PhD},{ }^{1}$ Monic P. Behnken, JD, $\mathrm{PhD}^{2}$ and Jeff R. Temple, $\mathrm{PhD}^{3}$

${ }^{1}$ School of Public Health, UT Health Science Center - Houston, Houston, TX; ${ }^{2}$ Dept. of Sociology, Iowa State University, Ames, IA and ${ }^{3} \mathrm{Ob} / \mathrm{Gyn}, \mathrm{UTMB}$, Galveston, TX.

Introduction: Although sexual assault victimization has been shown to predict suicidality, little is known about the mechanisms linking these two factors. Thus, we examined whether alcohol use mediates the relationship between forced sexual intercourse (FSI) and suicidality in adolescent girls. Methods: We analyzed data from the 2007 Youth Risk Behavior Survey, a nationally representative survey of US students conducted by CDC. Responses from Hispanic $(H)(n=1915)$, Caucasian $(W)(n=$ 2928), and African American (AA) $(n=1521)$ females in grades 9 - 12 were examined. FSI history was assessed by asking the following yes/no question: "Have you ever been physically forced to have sexual intercourse when you did not want to?" The outcome variable, suicidality, was measured by a single yes/no item: "During the past 12 months, did you ever seriously consider attempting suicide?" Alcohol use, was assessed by asking, "During the past 30 days, on how many days did you have 1 or more drinks of alcohol?" Responses were dichotomized as "yes/no" to having one or more drinks of alcohol on at least 1 day in the past 30 days. Results: The mean age of girls in the overall sample $(\mathrm{N}=$ $6364)$ was $16(\mathrm{SD}=1.22)$ with a mean grade of 11 th. Overall, $11 \%$ of participants reported a lifetime history of FSI. AA girls (14.2\%) were more likely than $\mathrm{W}(10.5 \%)$ or $\mathrm{H}(11.3 \%)$ girls to report a lifetime history of FSI $\left(\chi^{2}(2)=13.33, p<.01\right)$. Nineteen percent of participants considered suicide in the past year, with $\mathrm{H}(21.5 \%)$ more likely than AA $(18.2 \%)$ and $\mathrm{W}(18.2 \%)$ participants to report this behavior $\left(\chi^{2}(2)=9.18, \mathrm{p}<.05\right)$. Additionally, $45 \%$ of participants acknowledged alcohol use, with $\mathrm{W}(47.4 \%)$ and $\mathrm{H}(49.4 \%)$ girls more likely than AA girls (34.6\%) to report alcohol use $(\chi 2(2)=$ $78.08, \mathrm{p}<.001)$. FSI was associated with suicidality $(\beta=1.26$, $\mathrm{SE}=.08$, OR: $3.53,95 \% \mathrm{CI}: 3.00-4.16, \mathrm{p}<.001)$ and alcohol use $(\beta=.69, \mathrm{SE}=.08, \mathrm{OR}: 2.00, \mathrm{CI}: 1.70-2.36, \mathrm{p}<.001)$ for the total sample. Alcohol use was associated with suicidality after controlling for FSI $(\beta=.80, \mathrm{SE}=.07, \mathrm{OR}: 2.22, \mathrm{CI}: 1.93-2.55, \mathrm{p}<.001)$. The path from FSI to suicidality was reduced when accounting for alcohol use $(\beta=1.17, \mathrm{SE}=.09, \mathrm{OR}: 3.22, \mathrm{CI}: 2.70-3.83, \mathrm{p}<.001)$, suggesting that alcohol use partially mediated the relationship between FSI and suicidality (Sobel=9.16, $\mathrm{p}<.001$ ). The overall group results held for each ethnicity: AA (Sobel test $=3.95, \mathrm{p}<.001$ ), W (Sobel $=5.55$, $\mathrm{p}<.001$ ), and $\mathrm{H}$ (Sobel $=4.35, \mathrm{p}<.001)$. Conclusion: Results suggest the need for closer monitoring of adolescent sexual assault victims who also use alcohol to intervene in suicide behaviors. 
CORRESPONDING AUTHOR: Yen-Chi L. Le, PhD, School of Public Health, University of Texas Health Science Center at Houston, Houston, TX, 77030; yen-chi.1.le@uth.tmc.edu

9:06 AM-9:24 AM

OS41-C

DRINKING PATTERNS OF WINNERS AND LOOSERS OF ECONOMIC TRANSFORMATION: THE CZECH HAPIEE STUDY

Hynek Pikhart, PhD, ${ }^{1}$ Martin Bobak, MD, PhD, ${ }^{1}$ Ruzena Kubinova, $\mathrm{MD}^{3}$ and Nico Dragano, $\mathrm{PhD}^{2}$

${ }^{1}$ Epidemiology and Public Health, UCL, London, United Kingdom; ${ }^{2}$ University of Duesseldorf, Duesseldorf, Germany and ${ }^{3}$ National Institute of Public Health, Prague, Czech Republic.

Background: Psychosocial factors have been shown to be related to various health behaviours in the past. In this presentation we focus on the subjective evaluation of social and economic changes in Central European population undergoing fast societal transformation, and its relationship with alcohol consumption.

Methods: The sample of 8,856 men and women aged 4569 years in the Czech part of the Health, Alcohol and Psychosocial Indicators in Eastern Europe (HAPIEE) study completed a questionnaire and examination in 2002-2005 that included rating of social and economic changes since 1989 (changes in occupational position, income, material circumstances and general social position), evaluation of drinking habits (both in terms of absolute consumption and drinking patterns), and a range of other covariates. 4 domains of change were coded into three categories: good change, no change, bad change. The analyses were then conducted for single domains and for a sum score indicating the overall rating of change. The CAGE questionnaire score, the score of drinking-related problems and mean annual intake of alcohol were used as the outcomes, and regression modelling was used to analyze the association between the exposures and the outcomes taking range of covariates into the account. The analysis was done separately for men and women.

Results: After controlling for age, sex and range of possible confounding variables, the persons who rated the changes as negative have more alcohol-related problems and alcohol abuse (odds ratios of a high CAGE score 1.30 for men and 1.76 for women for those reporting negative change compared to those reporting no change). The amount of consumed alcohol is significantly higher among the "winners" (those who rate the changes as good), followed by loosers with those rating changes as neutral drinking the least.

Conclusions: While the alcohol-related problems and alcohol abuse are more likely among those who perceive the social and economic changes as negative, the amount of consumed alcohol is significantly higher among the "winners" of social change.

CORRESPONDING AUTHOR: Hynek Pikhart, PhD, Epidemiology and Public Health, UCL, London, WC1E 6BT; h.pikhart@, ucl.ac.uk
9:24 AM-9:42 AM

OS41-D

FACTORS INFLUENCING ALCOHOL USE

AND BINGE DRINKING AMONG SCHOOL STUDENTS

IN BANGKOK, THAILAND: RESULTS OF A MULTILEVEL ANALYSIS

Somsak Wongsawass, MPH, ${ }^{1}$ Rohan Jayasuriya, MBBS,MBA, ${ }^{1}$ Nicola Man, $\mathrm{PhD},{ }^{1}$ Sally Rooke, $\mathrm{PhD}^{2}$ and Upali Jayasinghe, $\mathrm{PhD}^{3}$

${ }^{1}$ School of Public Health and Community Medicine, University of New South Wales, Sydney, NT, Australia; ${ }^{2}$ National Drug and Alcohol Research Centre, University of New South Wales, Sydney, NSW, Australia and ${ }^{3}$ Centre for Primary Health Care and Equity, University of New South Wales, Sydney, NSW, Australia.

Background: Alcohol consumption among adolescents is an important public health issue. In Thailand, a significant increase among individuals aged 15-24 has been reported. Bangkok Behavioural Surveillance has shown different prevalence of alcohol use between schools. However, many studies on factors influencing alcohol use have not appropriately accounted for school level variation

Method: A random survey of fifteen secondary schools and fourteen vocational schools in Bangkok Metropolitan Administration was conducted in 2007. A total of 2,405 students aged 15-18 participated in the study. Multilevel logistic regression analyses were applied to investigate the significance of explanatory variables in predicting alcohol use and binge drinking in the past month. Explanatory variables included gender, age, school type (secondary or vocational), self-esteem, family relationships, peer relationships, school and neighbourhood connectedness.

Result: Self-reported prevalence of alcohol drinking and binge drinking in the past month were $28.5 \%$ and $5.8 \%$. There was significant variance between schools both in alcohol use and binge drinking. The student and school explanatory variables explained $81 \%$ and $70 \%$ of the school variance in binge drinking and alcohol use respectively. Significant risk factors for binge drinking were being male $(\mathrm{OR}=2.6)$, aged 17-18 (OR=1.5), vocational school $(\mathrm{OR}=3.6)$ and significant protective factors were family bonding $(\mathrm{OR}=0.5)$, school performance $(\mathrm{OR}=0.5)$ and neighbourhood safety (0.6). Similarly, being male $(\mathrm{OR}=1.8)$, aged 17-18 $(\mathrm{OR}=1.3)$, vocational school $(\mathrm{OR}=2.3)$ and peer bonding $(\mathrm{OR}=1.6)$ found to be significant risk factors for currently alcohol use, while school performance $(\mathrm{OR}=0.7)$ and neighbourhood safety $(\mathrm{OR}=0.8)$ played as protective factors. There was no relation between self-esteem and alcohol use or binge drinking.

Conclusion: The findings suggest that factors influencing alcohol use and binge drinking are complex after accounting for school level variance. Effective school intervention is needed to integrate different components of risk factors.

CORRESPONDING AUTHOR: Somsak Wongsawass, MPH, School of Public Health and Communitiy Medicine, University of New South Wales, Sydney, NT, 2052; somsakw@student.unsw. edu.au 
9:42 AM-10:00 AM

OS41-E

THE EFFECTS OF THE YEAR 2004 REDUCTION

IN THE PRICE OF ALCOHOL ON ALCOHOL-RELATED

HARM IN FINLAND - A NATURAL EXPERIMENT BASED ON REGISTER DATA

Kimmo Herttua, PhD

Department of Sociology, University of Helsinki, Helsinki, Finland.

There was a large reduction in the price of alcohol in 2004 in Finland due to a reduction in alcohol taxes by one-third, on the average, and due to the abolition of travellers' duty free allowances from the EU. The present study investigated the effects of this reduction in alcohol prices on alcohol-related and all-cause mortality, alcohol-related hospitalisation, and interpersonal violence.

This study used several population-based register data and statistical Methods: Time series intervention analysis modelling was applied to monthly aggregations of deaths and hospitalisation in 1996-2006 for men and women aged 15 years and over. Socioeconomic differentials in alcohol-related mortality and interpersonal violence data were analysed with a before/after design. Alcohol-related mortality was defined using information on both underlying and contributory causes of death. Hospitalisation related to alcohol was defined as those with a reference to alcohol in the primary diagnosis.

The results of this study indicated that alcohol-related deaths increased substantially among persons aged 50-69 years after the price reduction when trends and seasonal variation were taken into account. The increase was mainly due to liver diseases. In contrast to alcohol-related mortality, mortality due to cardiovascular diseases and all-cause mortality decreased considerably among men and women aged over 69 years. Among persons aged 30-59 years, the increase in absolute terms was largest among the unemployed or early-age pensioners and those with low education, social class, or income. The employed and persons aged below the age 35 did not suffer from increased alcohol-related mortality. Alcohol-related hospitalisation rates in both chronic and acute causes for men increased among those below the age 70, and for women in the 50-69 year-olds. The increase was mainly due to mental and behavioural disorders due to alcohol. The rates in interpersonal violence did not increase.

These findings suggest that reduction in alcohol prices may lead to a substantial increase in alcohol-related mortality and morbidity. However, large population group differences were observed. Those in the older age groups may even benefit from cheaper alcohol in terms of decreased rates of CVD mortality. Moreover, reductions in alcohol prices do not necessarily affect interpersonal violence. The population group differences in the effects of price changes should be acknowledged, and therefore, the actions of policy should be focused on the population subgroups which are primarily responsive to the price reduction.
CORRESPONDING AUTHOR: Kimmo Herttua, PhD, Department of Sociology, University of Helsinki, Helsinki, 00014; kimmo.herttua@helsinki.fi

OS42

\section{8:30 AM-8:48 AM}

OS42-A

EXERCISE ADHERENCE IMPROVES LONG-TERM PATIENT OUTCOME IN PATIENTS WITH OSTEOARTHRITIS OF THE HIP AND/OR KNEE

Martijn Pisters, MSc, PT, ${ }^{1,2}$ Cindy Veenhof, PhD, PT, ${ }^{1}$ François Schellevis, $\mathrm{PhD}, \mathrm{MD},{ }^{1,3}$ Jos Twisk, $\mathrm{PhD},{ }^{3}$ Joost Dekker, $\mathrm{PhD}^{3}$ and Dinny de Bakker, $\mathrm{PhD}^{1,4}$

${ }^{1}$ Netherlands Institute for Health Services Research (NIVEL), Utrecht, Netherlands; ${ }^{2}$ Utrecht School of Health Sciences, Utrecht University Medical Center, Utrecht, Netherlands; ${ }^{3}$ EMGO Institute, VU University Medical Center Amsterdam, Amsterdam, Netherlands and ${ }^{4}$ Tranzo, Tilburg University, Tilburg, Netherlands.

Objective: To determine the effect of adherence within and after an exercise therapy treatment period on patients' outcome on pain, physical function and patient self-perceived effect in patients with osteoarthritis (OA) of the hip and/or knee.

Methods: One hundred fifty patients with OA of the hip and/or knee receiving exercise therapy were followed 60 months. Data were obtained from a randomized controlled trial, with assessments at baseline, 3, 15, and 60 months follow-up. Exercise adherence was defined as the extent to which a person's behavior doing home exercises, home activities and being more physically active - corresponds with agreed recommendations by the patient's physical therapist. The association between exercise adherence and patients' outcome on pain, physical function and selfperceived effect was examined using generalized estimating equations (GEE) analyses.

Results: Adherence to recommended home exercises and being more physically active was significantly associated with better treatment outcome on pain, self-reported physical function, physical performance and self-perceived effect. The association between adherence and outcome was consistent over time. Adherence to home activities was only associated with better self-perceived effeCT.

Conclusion: Better adherence to recommended home exercises as well as being more physically active improves the long-term effectiveness of exercise therapy in patients with osteoarthritis of the hip and/or knee. Both within and after the treatment period better adherence is associated with better patients' outcome on pain, physical function, and self-perceived effect. Since exercise adherence declines over time, future research should focus on how exercise behavior can be stimulated and maintained in the long-term.

CORRESPONDING AUTHOR: Martijn Pisters, MSc, PT, Netherlands Institute for Health Services Research (NIVEL), Utrecht, 3500 BN; m.pisters@nivel.nl 


\section{8:48 AM-9:06 AM}

OS42-B

PHYSICAL ACTIVITY AFTER HIP RESURFACING: HOW MUCH DO PATIENTS ACTUALLY MOVE?

Inge van den Akker-Scheek, $\mathrm{PhD},{ }^{2,1}$ Rik Philips, BSc, ${ }^{2,3}$ Carina L. Gerritsma, MD $\mathrm{PhD}^{2}$ Jos J. van Raay, MD $\mathrm{PhD}^{2}$ Wiebren Zijlstra, $\mathrm{PhD}^{3}$ and Martin Stevens, $\mathrm{PhD}^{1}$

${ }^{1}$ Orthopaedics, University Medical Center Groningen, University of Groningen, Groningen, Netherlands; ${ }^{2}$ Orthopaedics, Martini Hospital Groningen, Groningen, Netherlands and ${ }^{3}$ Center for Human Movement Sciences, University Medical Center Groningen, University of Groningen, Groningen, Netherlands.

Introduction: Osteoarthrosis of the hip joint causes pain, stiffness and loss of joint function, therefore impairing a person in activities of daily life. Total hip replacement is the most common and successful surgical treatment. An alternative to the conventional total hip arthroplasty (THA) is hip resurfacing, which is considered to be a solution for treatment of young and active patients with osteoarthrosis. Aim of the study was to gain a detailed insight into the physical activity behaviour of patients after hip resurfacing and to compare it to the physical activity behaviour of a gender- and age-matched group of total hip arthroplasty (THA) patients and a normative population.

Method: A retrospective cross-sectional cohort study was conducted, including all patients who underwent hip resurfacing one to four years previously. The SQUASH questionnaire was sent to these patients to determine physical activity behaviour. Resurfacing patients were matched on gender and age with patients after THA and a normative population.

Results: Of in total 50 patients after hip resurfacing, 46 patients participated (response rate 92\%). The resurfacing group shows significantly more total physical activity than the THA group and the normative group. Compared to the THA patients, resurfacing patients showed significantly more light and vigorous intensity physical activity. The hip resurfacing group and the normative group show an equal amount of persons fulfilling the international health-enhancing physical activity guidelines $(67.4 \%)$.

Conclusion: Patients after hip resurfacing are more physically active compared to gender and age matched patients after THA and even more active than a gender and age matched normative population. Although hip resurfacing is claimed to be the solution for active patients with hip osteoarthrosis, consequences on the long term of increased physical activity on risks concerning implant failure and wear have to be established yet.

CORRESPONDING AUTHOR: Inge van den Akker-Scheek, $\mathrm{PhD}$, Orthopaedics, University Medical Center Groningen, Groningen, 9700 RB; i.scheek@orth.umcg.nl
9:06 AM-9:24 AM

OS42-C

MANAGING CHRONIC ORO-FACIAL PAIN:

A QUALITATIVE STUDY OF THE EXPERIENCES

OF PATIENTS, DOCTORS AND DENTISTS

Sarah Peters, PhD, ${ }^{1}$ Vishal Aggarwal, PhD, ${ }^{1}$ Joanna Goldthorpe, $\mathrm{MSc}^{2}{ }^{2}$ Cheryl McElroy, MPhil, ${ }^{2}$ King Elizabeth, MRes, ${ }^{1}$ Justin Durham, BDS, $\mathrm{PhD}^{3}$ and Martin Tickle, FDS, $\mathrm{PhD}^{2}$

${ }^{1}$ Psychology, The University of Manchester, Manchester, United Kingdom; ${ }^{2}$ Dentistry, University of Manchester, Manchester, United Kingdom and ${ }^{3}$ Dentistry, University of Newcastle, Manchester, United Kingdom.

Persistent unexplained pain in the face and surrounding tissues are common presentations to dental and medical services. Routine dental treatment of chronic oro-facial pain (OFP) primarily involves using splints to correct malocclusion or teeth-clenching/ grinding. However this mechanistic approach is ineffective. Moreover, such treatments can result in physical and psychological iatrogenesis. In contrast, a growing evidence-base recognises the effectiveness of psychological interventions for OFP, suggesting a role for psychological services within dentistry. In order to translate research into practice, further understanding is needed of patients' and physicians' current experience of OFP and its management. Semi-structured interviews were conducted with a purposive sample of 25 patients and 35 clinicians (comprising medical and dental practitioners from a range of primary and secondary care services). Audiotaped interviews were transcribed and analysed thematically using principles of constant comparison to categorise emergent and recurring themes within and between transcripts. Thematic categories arising in initial interviews were explored subsequently and disconfirmatory evidence sought until thematic saturation arose. Similar themes emerged from both patients and physicians. Whilst both sets of participants recognised the role that psychological factors could play in the development and maintenance of OFP, management and self-management strategies were largely limited to biomedical interventions. Achieving a diagnosis proved problematic but functional for both parties. Frustration at the current inadequacy of OFP management often led to conflict with (or disengagement from) the clinicianpatient relationship. Current management of OFP is ineffective and unsatisfactory for both patients and clinicians and impacts on their relationship. A key barrier to implementing psychological interventions for OFP is the ineffective communication between physicians and patients, and between medical and dental practitioners.

CORRESPONDING AUTHOR: Sarah Peters, PhD, Psychology, The University of Manchester, Manchester, M13 9PL; sarah. peters@manchester.ac.uk 
9:24 AM-9:42 AM

OS42-D

VITALITY AND THE COURSE OF LIMITATIONS

IN ACTIVITIES IN OSTEOARTHRITIS OF THE HIP

OR KNEE

Gabriela M. van Dijk, PhD,${ }^{1}$ Cindy Veenhof, $\mathrm{PhD},{ }^{1}$ Cornelia $\mathrm{H}$. van den Ende, $\mathrm{PhD},{ }^{1}$ Guus J. Lankhorst, $\mathrm{MD}, \mathrm{PhD}^{2}$ and Joost Dekker, $\mathrm{PhD}^{2}$

${ }^{1}$ Rehabilitation Medicine, VU University Medical Centre, Amsterdam, Netherlands and ${ }^{2}$ Netherlands Institute for Health Services Research, Utrecht, Netherlands.

Background: Psychological and social factors are associated with limitations in activities in osteoarthritis of the hip or knee. It is not known, however, whether psychological and social factors have impact on the course of limitations in activities, after controlling for established somatic and cognitive risk factors.

Purpose: To determine whether psychological and social factors have impact on the course of limitations in activities in elderly patients with osteoarthritis of the hip or knee, in addition to established somatic and cognitive risk factors.

Methods: A longitudinal cohort study with a follow-up period of three years was conducted. Patients $(\mathrm{N}=237)$ with hip or knee osteoarthritis were recruited from rehabilitation centers and hospitals. Body functions, comorbidity, cognitive functioning, limitations in activities and psychological and social factors (mental health, vitality, pain coping and perceived social support) were assessed. Statistical analyses included univariate and multivariate regression analyses.

Results: In knee OA, low vitality predicted deterioration of selfreported and performance-based limitations in activities, after controlling for somatic and cognitive factors. In hip OA, psychological and social factors had no additional contribution to the model.

Conclusion: Low vitality has a negative impact on the course of limitations in activities in elderly patients with osteoarthritis of the knee, in addition to established somatic and cognitive risk factors.

CORRESPONDING AUTHOR: Joost Dekker, PhD, Rehabilitation Medicine, VU University Medical Centre, Amsterdam, 1007 MB; j.dekker@vumc.nl

\section{9:42 AM-10:00 AM}

OS42-E

GOALS, GOAL ATTAINMENT AND PATIENT SATISFACTION IN PSYCHOSOMATIC REHABILITATION Fritz A. Muthny, PhD, MD and Matthias Richter, Psychologist University Hospital Muenster, Dept. of Medical Psychology, Muenster, Germany.

Objectives: Reaching the rehabilitation goals the doctor and the patient have agreed on appears to be important, and there is presumed to be a correlation between goal attainment and patient satisfaction. The main goal of the study was the detailed assessment of patient satisfaction and the investigation of the correlation between patient satisfaction and the setting and attainment of goals.
Methods: The cross-sectional questionnaire-based assessment was performed anonymously by 276 patients in psychosomatic rehabilitation at the end of their inpatient stay. In this way patient satisfaction, sociodemographic, disease-related and outcome parameters as well as rehabilitation goals at the beginning of rehabilitation and their attainment at the end were assessed in a standardized way.

Results: The primary goals set by patients were related to symptom reduction and stress coping. Goal attainment scales based on factor analysis showed a high degree of goal attainment with respect to "life style and priorities" and "psychotherapeutic goals".

Patient satisfaction correlated most significantly with the goal attainment level at the end of rehabilitation $(\mathrm{r}=.62)$, followed closely by the subscale "attainment of psychotherapeutic goals" $(\mathrm{r}=.57)$. With regards to the number of attained or non-attained goals it was found, that both had a significant relationship with patient satisfaction, but the non-attaining of goals $(\mathrm{r}=-.55)$ was more closely related with patient satisfaction than the attainment $(\mathrm{r}=.40)$.

Discussion: The results confirm the central role of goal setting and goal attainment in rehabilitation. The explicit consideration of goal setting in the rehabilitation process as well as the assessment of goal attainment appears to be unavoidable.

CORRESPONDING AUTHOR: Fritz A. Muthny, PhD, MD, Dept. of Medical Psychology, University Hospital Muenster, Muenster, 48149; muthny@uni-muenster.de

\section{Saturday August 7, 2010 SYMPOSIA SESSIONS 10:30 AM-12:00 PM}

\section{SS27}

EVIDENCE-BASED PRACTICE IN GLOBAL PERSPECTIVE Bonnie Spring, PhD, ${ }^{1}$ Ross Brownson, PhD, ${ }^{2}$ Geoffrey Setswe, $\mathrm{PhD},{ }^{3}$ Brian Oldenburg, $\mathrm{PhD}^{4}$ and Stephen Weiss, $\mathrm{PhD}^{5}$

${ }^{1}$ Preventive Medicine, Northwestern University, Chicago, IL; ${ }^{2}$ Social Work, Washington University, St. Louis, MO; ${ }^{3}$ Social Aspects of HIV/AIDS and Health, South African Human Services Research Council, Pretoria, South Africa; ${ }^{4}$ Epidemiology and Preventive Medicine, Monash University, Melbourne, VIC, Australia and ${ }^{5}$ Psychiatry, University of Miami, Miami Beach, FL.

Evidence-based practice (EBP) can be defined as accountable health care policy that conscientiously funds those interventions whose effectiveness is supported by research. As applied to particular individuals, communities or populations, EBP uses best available evidence to make decisions about how to promote health. The EBP process integrates three domains of information: (1) best available evidence; (2) client characteristics, including values and preferences; and (3) resources. The process proceeds through a step sequence that includes appraising the quality and contextual relevance of research evidence. 
As the major health professions have embraced evidence-based practice globally, practice and policy guidelines have increasingly become based upon systematic evidence reviews. Dissemination of systematic evidence reviews has become a worldwide enterprise supported by the Cochrane and Campbell Collaborations. However, a number of challenges surround the uptake of evidencebased practices, especially when interventions are introduced into cultures very different than those from which they originated.

After Bonnie Spring introduces the topic, speakers will present lessons learned when implementing globally in diverse real-world settings behavioral medicine interventions that were validated in an Anglo-European context. Ross Brownson will discuss findings derived from introducing public health physical activity campaigns in South America. Geoffrey Setswe will discuss implications for intervention of social aspects of HIV/AIDS and health across the African continent. Brian Oldenburg will share observations based on the spread of diabetes interventions between different countries, cultures, and populations. Discussant, Stephen Weiss will synopsize lessons learned about the process of engaging in evidence-based practice when the relevance of the available research is unclear. Presenters will address three issues:

1) How can an evidence-based intervention best be tailored to a local individual or community?

2) How should an investigator evaluate both the adequacy of contextualization and the success with which active intervention ingredients were preserved?

3) What does treatment fidelity mean when interventions are to be implemented in diverse cultural contexts?

CORRESPONDING AUTHOR: Bonnie Spring, PhD, Preventive Medicine, Northwestern University, Chicago, IL, 60610; bspring@northwestern.edu

\section{SS27a}

UNDERSTANDING AND DISSEMINATING

EVIDENCE-BASED PRACTICES TO PROMOTE PHYSICAL ACTIVITY IN LATIN AMERICA

R. C. Brownson, PhD,${ }^{1,2}$ D. Parra, MPH, ${ }^{1}$ M. Pratt, $\mathrm{MD}, \mathrm{MPH}^{3}$ and L. Ramos, $\mathrm{MD}^{4}$

${ }^{1}$ Prevention Research Center in St. Louis, George Warren Brown School of Social Work, Washington University in St. Louis, St Louis, MO; ${ }^{2}$ Department of Surgery and Alvin J. Siteman Cancer Center, Washington University School of Medicine, Washington University in St. Louis, St. Louis, MO; ${ }^{3}$ Physical Activity and Health Branch, Division of Nutrition and Physical Activity, Centers for Disease Control and Prevention, Atlanta, GA and ${ }^{4}$ Department of Preventive Medicine, Universidade Federal de São Paulo, São Paulo, Brazil.

The majority of developing countries are experiencing a shift from a high prevalence of infectious diseases to a high prevalence of chronic diseases. These shifts are due in part to rapid urbanization and globalization processes, which are contributing to significant changes in behaviors and living situations among populations in these countries. The growing burden of chronic diseases on developing nations is increasing attention on risk factors such as physical inactivity. The U.S. Task Force on Community Preventive Services developed the Guide to Community Preventive Services (the Community Guide) to evaluate the effectiveness of different health promotion interventions, including strategies to increase physical activity in communities. These recommendations have not been systematically examined and applied in developing countries. Project GUIA was initiated in 2005 to examine and promote evidence-based strategies to increase physical activity in Latin America. Its three aims are: (1) to conduct a systematic review of the Latin American literature on physical activity promotion strategies in the community and carefully examine the Community Guide's applicability; (2) to evaluate two ongoing physical activity interventions in Brazil, and (3) to disseminate evidence-based approaches for promoting physical activity in community settings. Coordinated by the Prevention Research Center in St. Louis, this Project involves cross-national collaboration with multiple academic and governmental institutions, including the Centers for Disease Control and Prevention, WHO/PAHO, the Brazil Ministry of Health, Universidade Federal de São Paulo and CELAFISCS. This presentation will highlight the project's aims, results of the literature review, findings from an evaluation in Recife, Brazil that is now being replicated in San Diego, California, and results from a social network analysis that has been conducted among partners promoting physical activity in Latin America. The presentation will highlight key lessons from this cross-national collaboration that include the need for a clearer understanding of context, the role of inter-sectoral partnerships, the need to better understand translation of an effective intervention from one population to another.

CORRESPONDING AUTHOR: Ross Brownson, PhD, Washington University in St. Louis, St. Louis, MO, 63110; rbrownson@wustl.edu

\section{SS27b}

\section{CONTEXTUALIZING HIV PREVENTION INTERVENTIONS} THAT WORK

Geoffrey Setswe, PhD, MPH

Human Sciences Research Council, Pretoria, South Africa.

Introduction: Many HIV prevention interventions have been tested for efficacy or effectiveness. However, in almost three decades of the HIV/AIDS epidemic, we are still not sure which interventions work best in preventing HIV transmission in different communities and cultural settings. This presentation provides evidence of HIV prevention interventions that work and also presents lessons learned about applying them in different contexts.

Methods: A systematic review of randomized controlled trials (RCTs) testing HIV prevention interventions for evidence of efficacy or effectiveness was conducted. The interventions were classified as biomedical, behavioral and structural. The evidence provided by RCTs was graded in four different levels: best $(80 \%$ or more), good $(60-79 \%)$, promising $(30-59 \%)$ and poor or no evidence $(<30 \%)$. We calculated the effect size to show the difference between treated and control conditions.

Findings and Discussion: Using the grading system, male and female condoms and PMTCT were classified as best evidence HIV prevention 
interventions because they had achieved more $80 \%$ level of efficacy or effectiveness. ART, male circumcision, HIV Counseling and Testing for people who test positive were classified as good evidence HIV prevention interventions as they had achieved between 60 and $79 \%$ level of efficacy or effectiveness. Treatment of STI, a microbicide called PRO2000, the RV144 Thailand vaccine trial and a structural community RCT that reduced intimate partner violence were classified as promising evidence HIV prevention interventions as they had achieved between 30 and 59\% level of efficacy or effectiveness. All other interventions that obtained less than $30 \%$ effectiveness were classified as poor or no evidence.

We will present the effect size of these interventions.

Conclusion: There is no "magic bullet" for HIV prevention, particularly as applied in different cultural settings. The lesson learned is that the acceptability of intervention strategies is not the same across most cultures and individuals. A community should choose to use a combination of interventions that best suits their context from among best, good or promising practices.

CORRESPONDING AUTHOR: Bonnie Spring, PhD, Preventive Medicine, Northwestern University, Chicago, IL, 60610; bspring@northwestern.edu

\section{$\mathrm{SS} 27 \mathrm{c}$}

PREVENTION OF TYPE 2 DIABETES

AND ITS COMPLICATIONS: HOW TO STRENGTHEN

THE GLOBAL EVIDENCE BASE FOR EFFECTIVE

PREVENTION?

Brian F. Oldenburg, $\mathrm{PhD},{ }^{1}$ Pilvikki Absetz, $\mathrm{PhD},{ }^{2}$ James Dunbar, $\mathrm{MD},{ }^{3}$ Prasuna Reddy, $\mathrm{PhD}^{3}$ and Lal B. Rawal, $\mathrm{MPH}^{1}$

${ }^{1}$ Epidemiology and Preventive Medicine, Monash University, Melbourne, VIC, Australia; ${ }^{2}$ National Institute for Health and Welfare, Helsinki, Finland and ${ }^{3}$ Greater Green Triangle University Department of Rural Health, Flinders and Deakin Universities, Warrnambool, VIC, Australia.

Type 2 diabetes mellitus (T2DM) is a significant global public health problem which is already affecting more than 250 million people worldwide, with an increasing number of cases in developing countries. Efficacy trials conducted in developed countries have clearly demonstrated that lifestyle change and other kinds of non-pharmacological approaches can significantly reduce diabetes risk. Two key challenges are: (1) How to develop and implement programs that are more feasible for 'real world' implementation and (2) How to extend the reach of such programs to low and middle income countries, as the current trial evidence based in such countries is currently very limited? This presentation considers the development, implementation and evaluation of such programs in Finland and Australia, as well as the recent uptake of lifestyle-related interventions for reducing diabetes risk in other countries. The GOAL study in Finland involved the "real world" evaluation of a moderate-intensity group-based lifestyle change counselling program to prevent type 2 diabetes, adapted from the original Finnish Diabetes Prevention Study (DPS). Drawing on evidence from the Finnish DPS and the short-term results from the GOAL implementation trial, the Australian Diabetes Prevention
Program (DPP), adapted from the GOAL program, achieved similar positive behavioral and clinical outcomes. Currently, more routinized programs, that are building on the evidence from these 'real world' implementation trials, are being implemented and evaluated in Finland and Australia. As key components of the Finnish GOAL program have become more refined over time, successive versions of the program in different countries are aiming to improve the 'fit' between characteristics of the interventions, the adopters, and the implementation environments. Core components of the GOAL program are now also being adapted for use in programs that are currently being evaluated in Malaysia, India and South Africa. There is an urgent need for more collaborative and coordinated approaches that compare and contrast methods for adapting and spreading effective intervention programs between settings, regions, and countries.

CORRESPONDING AUTHOR: Brian F. Oldenburg, PhD, Epidemiology and Preventive Medicine, Monash University, Melbourne, VIC, 3004; brian.oldenburg@med.monash.edu.au

\section{SS28}

INVOLVING AND INFORMING ADULTS MAKING CANCER TREATMENT DECISIONS

Jeffrey K. Belkora, PhD

Institute for Health Policy Studies, University of California, San Francisco, San Francisco, CA.

Adults diagnosed with cancer are often faced with multiple treatment options. Many want to participate with their physicians in the process of deciding which treatment is best for them. This symposium features four projects that are testing various applications of the SCOPED process in different settings, using different delivery models and intervention components. SCOPED is a six step process for helping patients formulate and analyze issues, synthesize insight, and translate the insight into action. The steps include documenting questions and answers about the Situation, Choices, Objectives, People, Evaluation, and Decisions. Each project will present data about using the SCOPED process to inform and involve adults making cancer treatment decisions.

CORRESPONDING AUTHOR: Jeffrey K. Belkora, PhD, University of California, San Francisco, San Francisco, CA, 94121; jeff.belkora@ucsfmedctr.org

SS28a

EARLY RESULTS FROM A PILOT STUDY OF DECISION NAVIGATION FOR BREAST AND PROSTATE CANCER PATIENTS IN SCOTLAND

Sarah Scott, BSc Neuroscience (honours), ${ }^{1}$ Lucy Dickinson, MSc Health Psychology, ${ }^{1}$ Tish Chalmers, BSc Social Sciences (honours), ${ }^{1}$ Louise M. Wallace, PhD Clinical Psychology, ${ }^{2}$ Belinda Hacking, Doctorate Clinical Psychology ${ }^{1}$ and Jeffrey Belkora, $\mathrm{PhD}$ Engineering ${ }^{3}$ ${ }^{1}$ NHS Lothian, Edinburgh, United Kingdom; ${ }^{2}$ Coventry University, Coventry, United Kingdom and ${ }^{3}$ University of California, San Francisco, CA. 
Introduction: The 'Better Cancer Care' campaign launched by the Scottish Government promotes partnership between patients and healthcare professionals. The aim of this study in progress is to evaluate decision navigation using the SCOPED question-listing and note taking process within NHS Scotland.

Methods: Newly diagnosed breast and prostate cancer patients attending the Edinburgh Cancer Centre are invited to participate. Patients are randomized to intervention or control. Decision self efficacy (DSE) is measured in both groups (scale of 0 to10) at baseline (T1) and after the consultation (T3). DSE is additionally measured in the intervention group after the decision navigation session (T2). Intervention patients also receive a service evaluation form at $\mathrm{T} 2$.

Preliminary Analysis: Prostate: $\mathrm{N}=63$ age range 56-78 years. As of November 2009, there are no differences between groups at T1 on the DSE $x=8.39$ in the intervention group and $x=8.3$ in the control group $(\mathrm{p}=.82)$. At $\mathrm{T} 3$ there is a significant difference between groups $x=9.0$ in the intervention group and $x=8.3$ in the control group $(\mathrm{t}(51)=2.059, \mathrm{p}=.045) .90 \%$ of the intervention group $(\mathrm{n}=24)$ found decision navigation 'very helpful' and $10 \%$ $(\mathrm{n}=3)$ 'somewhat helpful'.

Breast: $\mathrm{N}=40$ age range $41-81$ years. $79 \%$ of the intervention group $(\mathrm{n}=15)$ found decision navigation 'very helpful' and $21 \%$ $(n=4)$ 'somewhat helpful' at T2. The intervention and control group do not differ significantly on the DSE.

Feedback from a subset of patients $(n=9)$ stated that the intervention was "supportive" "helped to structure thoughts" and "reason through the decision process".

Discussion: It is premature to draw conclusions from these preliminary data. Eventual differences in impact of decision navigation between breast and prostate patients may be attributable to the greater degree of choice left to prostate patients. In contrast, breast cancer patients are strongly advised to follow set treatment guidelines for adjuvant therapy.

CORRESPONDING AUTHOR: Sarah Scott, BSc Neuroscience, Clinical Psychology Office, NHS Lothian, Edinburgh, EH4 2XU; Sarah.E.Scott@luht.scot.nhs.uk

\section{$\mathrm{SS} 28 \mathrm{~b}$}

OPEN TO OPTIONS AT CANCER SUPPORT COMMUNITY SITES IN PENNSYLVANIA, OHIO, AND CALIFORNIA M. F. Miller, $\mathrm{PhD}, \mathrm{MPH},{ }^{1} \mathrm{~N}$. Blakeney, $\mathrm{MPH},{ }^{2} \mathrm{~K}$. Coyne, MSS, $\mathrm{LCSW}^{3}$ B. Crawford, MSW, ${ }^{4}$ M. Stauffer, MFT, ${ }^{5}$ M. Michaels, $\mathrm{MPH},{ }^{2} \mathrm{M}$. Golant, $\mathrm{PhD}^{1}$ and J. Belkora, $\mathrm{PhD}^{6}$

${ }^{1}$ Cancer Support Community, Washington, DC; ${ }^{2}$ Education Network to Advance Cancer Clinical Trials, Silver Spring, MD; ${ }^{3}$ The Wellness Community, Philadelphia, PA; ${ }^{4}$ The Wellness Community, Cincinnati, OH; ${ }^{5}$ The Wellness Community, San Francisco, CA and ${ }^{6}$ University of California, San Francisco, CA.

Introduction: Open to Options, a pilot project of ENACCT and CSC provides one-on-one support to hematological cancer patients in Philadelphia, PA, San Francisco/East Bay, CA, and Cincinnati, $\mathrm{OH}$.

Methods: Trained CSC facilitators use the SCOPED process to prompt patients to list questions for their providers. Patients complete pre and post session surveys, and follow-up evaluations at 30 and 90 days. The surveys measure distress, anxiety, question self-efficacy, satisfaction and (for a subset), patient use of the question list during a health care provider visit.

Results: Three facilitators have used the SCOPED question-listing process with 42 patients. Among patients, there was a significant decrease in distress $(p=0.044)$ and anxiety $(p=0.045)$ and a significant increase in the patient's question self-efficacy $(\mathrm{p}<$ $0.001)$. To date, 26 have made a treatment decision, and $4(15 \%)$ of these chose treatment through a clinical trial, which is higher than the national participation rate of 3-5\%. Thirteen of 25 respondents reported discussing cancer clinical trials with their oncologist. Satisfaction among participants was high post-intervention (mean= 8.6 out of a maximum of 10) and remained high at $30($ mean $=8.5)$ and 90 (mean=8.7) days follow-up. Fifteen of 16 patients surveyed (94\%) reported bringing their question list to their provider appointment, 13 (81\%) thought the question list contributed to a more productive appointment, and $13(81 \%)$ reported their oncologist answered most of their questions. Participants reported the question list: "gave structure to the meeting like an agenda"; "clarified my thoughts"; and "was empowering."

Discussion: Preliminary findings suggest use of the SCOPED process for question-listing among blood cancer patients in a community-based setting may be associated with improvements on psychosocial measures and with enhanced enrollment in clinical trials.

CORRESPONDING AUTHOR: Melissa F. Miller, PhD, MPH, Cancer Support Community, Fairfax, VA, 22033; melissa@cancersupportcommunity.org

SS28c

DECISION SERVICES AT THE UNIVERSITY

OF CALIFORNIA, SAN FRANCISCO, CA

Shelley Volz, MA, Alexandra Teng, BS, Meredith Loth, BA and Jeffrey Belkora, $\mathrm{PhD}$

University of California San Francisco (UCSF), San Francisco, CA.

INTRODUCTION: At the UCSF Breast Care Center, pre-medical interns contact upcoming clinic visitors by telephone and offer SCOPED question-listing and note-taking services free of charge. METHODS: We used the RE-AIM framework to evaluate our implementation of question-listing and note-taking services. Program years run June to July. For 2009 we report through November.

Reach: We counted the number of visitors receiving services and compared it to premedical intern capacity for 2005-2008.

Effectiveness: We counted the number of questions listed and surveyed visitors in 2009 for self-efficacy and satisfaction ratings. Adoption: We counted the number of visitors contacted and how many accepted versus declined the services in 2009 .

Implementation: We surveyed visitors in 2009 to assess the proportion who reviewed the notes from their consultations within six weeks of their visit.

Maintenance: We applied for renewed funding each year from internal and external sources in 2004-2008. 
Results: Reach: From 2005 to 2008 we doubled our reach from 142 to 285 and capacity exploitation from $28 \%$ to $65 \%$.

Effectiveness: We counted a mean of 9 questions before and 23 questions after 137 question-listing sessions $(\mathrm{p}<0.001)$. On a scale of $0(\mathrm{~min})$ to $10(\max ), 115$ visitors reported (response rate $84 \%$ ) self-efficacy scores of 6.7 before and 8.1 after question-listing $(\mathrm{p}<$ 0.001 ), and median satisfaction of 9 .

Adoption: We contacted 493 clinic visitors. 261 responded and were eligible (53\%). 162 (62\%) accepted and 99 (38\%) declined services. Implementation: 39 out of $44(89 \%)$ survey respondents reviewed the notes from their consultations within 6 weeks of their visit (response rate $48 \%$ ).

Maintenance: We have renewed internal subsidies and an external foundation grant each year for 5 years and are now exploring new sources of financial support from government and private insurers. DISCUSSION: Survey responses indicate that clinic visitors who accept SCOPED question-listing and note-taking services generally are satisfied, experience increases in self-efficacy, identify new questions to ask their doctors, and refer to the notes after their visits.

CORRESPONDING AUTHOR: Shelley Volz, MA, PRL Institute for Health Policy Studies, University of California, San Francisco (UCSF), San Francisco, CA, 94143-0936; shelley.volz@, ucsfmedctr.org

\section{SS28d}

\section{DECISION SUPPORT AT THE CANCER RESOURCE} CENTERS OF MENDOCINO COUNTY, CA

Sara O'Donnell, N/A, ${ }^{1}$ Lauren Franklin, BA Anthropology ${ }^{2}$ and Jeffrey Belkora, $\mathrm{PhD}^{2}$

${ }^{1}$ Cancer Resource Centers of Mendocino County, Ukiah, CA and

${ }^{2}$ Institute for Health Policy Studies, University of California, San Francisco, San Francisco, CA.

INTRODUCTION: CRCMC offers information and support to people facing cancer in a rural, medically underserved area, including SCOPED question-listing and note-taking services. We asked: 1) Are SCOPED services acceptable to minority Native American, Latino, and Frontier (remote) rural residents? and 2) How can we best implement the services in a rural, community setting?

METHODS: In 2004 we interviewed 44 residents (including 27 Native Americans and Latinos) about barriers and facilitators relevant to implementing the SCOPED question-listing service with diverse and remote county residents. In 2005, we interviewed 12 doctors, 12 breast cancer survivors and 10 employees of social services agencies about facilitators and barriers relevant to providing note-taking and related services. In both studies, we inductively categorized examples of facilitators and barriers into themes and adapted the interventions to expressed needs. In 2007, we reviewed 50 patient ratings of decision self-efficacy (scale of minimum 0 to maximum 4) and decisional conflict (scale of minimum 1 to maximum 5) and anxiety (scale of minimum 1 to maximum 10), assessed before and after question-listing.
RESULTS: Participants in our 2004 study endorsed the cultural acceptability of the SCOPED question-listing service. Our 2005 respondents suggested that SCOPED note-taking addresses facilitators and barriers relevant to effective patient-physician consultations. Based on themes from interview data, we made modifications to the delivery of the SCOPED interventions (e.g. training Spanish-English interpreters). In 2007, we observed significant $(\mathrm{p}<0.0001)$ gains in self-efficacy $(2.72$ to 3.53$)$, decisional conflict (3.00 to 1.74 ) and anxiety (7.15 to 5.61).

DISCUSSION: Our rural community, including Native Americans, Latinos, and Frontier residents, welcomed SCOPED question-listing and note-taking services and suggested delivery improvements. We found associated gains in psychosocial measures.

CORRESPONDING AUTHOR: Lauren Franklin, BA Anthropology, Institute for Health Policy Studies, University of California, San Francisco, Albion, CA, 95410; lauren.franklin@ucsfmedctr.org

\section{SS29}

PSYCHOLOGICAL FACTORS AND SOMATIC SYMPTOMS

Birgit Kröner-Herwig, $\mathrm{PhD}$

Psychology, University of Göttingen, Göttingen, Germany.

A wide range of symptoms cannot be understood on the basis of medical explanations alone.

Hiller et al. determined the number of medically unexplained somatic symptoms (MUSS) in two German primary care practices $(n \geq 600)$ and subjective disability. More than two thirds of the symptoms were rated as MUSS and more than $70 \%$ met the criteria for a Somatoform Disorder. Psychological trait variables differentiated MUSS patients with high disability from those not suffering greatly from their symptoms.

No single medical condition has been found to be associated with Chronic Fatigue (CFS). It is often claimed that stress plays a role in the manifestation of CFS. Nater et al. examined more than 500 Ss regarding life stress, coping, psychological traits and cortisol as well as CSF symptoms. SEM models for predicting CSF symptoms revealed that maladaptive personality features were the strongest predictors, partly mediated by stress.

Barke et al. focussed their research on the fear avoidance model of non-specific chronic back pain, using fMRI to investigate neural correlates of fear of movement in 30 female chronic low back pain patients and 30 controls. Patients were grouped into high and low fear avoidance groups and viewed photos of neutral and painful movements. The expectation of finding higher activations in areas related to pain and emotion in the high fear avoidance group was not fulfilled. It was concluded that contrary to the suggestion of the fear avoidance model phobic fear of movement may not be the process underlying chronic back pain.

Kröner-Herwig et al. examined the influence of gender role on pain responsivity in an experiment using mechanical pain. Pain intensity, pain threshold, affective and sensory pain were assessed 
in 74 female and male participants, who were grouped according to gender role on the basis of the Bem Sex Role Inventory. Using sex, gender and psychological traits (catastrophizing, fear of pain), it was found that sex, but not gender, strongly predicted pain responsivity in nearly all pain measures and fear of pain was a mediator.

CORRESPONDING AUTHOR: Birgit Kröner-Herwig, PhD, Psychology, University of Göttingen, Göttingen, 37073; bkroene@ uni-goettingen.de

\section{SS29a}

NEURAL CORRELATES OF FEAR AVOIDANCE IN CHRONIC LOW BACK PAIN: AN FMRI STUDY

Antonia Barke, DPhil, ${ }^{1}$ Jürgen Baudewig, $\mathrm{PhD},{ }^{2}$ Peter Dechent, $\mathrm{PhD}^{2}$ and Birgit Kröner-Herwig, $\mathrm{PhD}^{1}$

${ }^{1}$ Institute for Psychology, University of Göttingen, Göttingen, Germany and ${ }^{2}$ MRT Research in Neurology and Psychiatry, University Hospital Göttingen, Göttingen, Germany.

The fear avoidance model postulates that in chronic low back pain (CLBP) a fear of movement is acquired in the acute phase, which leads to subsequent avoidance of physical activity and thereby drives the chronification of the pain syndrome.

In an event-related fMRI study we investigated neural correlates of the hypothesized fear of movement. 60 women (30 CLBP patients, 15 healthy controls and 15 women with spider phobia, mean age: $46.8 \pm 9.8$ years) participated. The CLBP patients were divided on the basis of the Tampa Scale of Kinesiophobia (TSK) into a high and low fear-avoidant group. They viewed 120 photographs depicting neutral and painful movements, generally fear-inducing and neutral objects from the International Affective Picture System (IAPS) and spiders while functional MRI data were acquired. For CLBP patients activations were found in brain areas commonly implicated in emotional processing (cingulate cortex, orbitofrontal cortex) for the contrast of painful $>$ neutral movements. However, in a random effects analysis, CLBP patients with high fear avoidance did not differ from those with low fear avoidance or controls with regard to this contrast. For the contrast fear-inducing IAPS pictures $>$ neutral pictures, CLBP patients with high fear avoidance exhibited an activation in the left anterior insula, which was not found in those with low fear avoidance. The spider phobics showed extensive bilateral activation in the cingulate cortex and the insulae for the contrast spiders $>$ neutral pictures and the random effects analysis revealed marked differences in these areas between the spider phobics and the healthy controls.
On the basis of the fear avoidance model we expected group differences between CLBP patients with high and low fear avoidance as well as between CLBP with high fear avoidance and controls. Regarding neural correlates such no such differences were found, thereby casting doubt on the concept of fear avoidance as a process primarily driven by fear or phobic fear.

CORRESPONDING AUTHOR: Antonia Barke, DPhil, Psychology, University of Göttingen, Göttingen, 37073; abarke@gwdg.de

SS29b

SEX AND GENDER ROLE AS MODERATORS OF RESPONSES TO MECHANICAL PAIN STIMULI

Birgit Kröner-Herwig, PhD, Marie Tromsdorf, Diplpsych and Elfie Zahrend, Diplpsych

Psychology, University of Göttingen, Göttingen, Germany.

We examined the hypotheses that sex and gender, assessed by the Bem Sex Role Inventory, modulate responsiveness to experimentally induced pressure pain. Furthermore various psychological variables assumed to be potential mediators were assessed. 74 students took part in the experiment. Pain threshold (PT), intensity (PI) and unpleasantness (PU) of a standard pressure stimulus were determined as well as sensory and affective pain (SP, AP).

Analysis of variance revealed a significant main effect of sex for nearly all pain variables. Women exhibited a lower PT, rated pain to be more severe and unpleasant, and reported more SP and AP. Against our hypothesis gender role did not influence pain behaviour. Fear of pain (FPQ / PASS) and catastrophizing (PCS) showed significant correlations to all pain parameters except PT. The variable depressive symptoms was almost without influence whereas coping style modulated pain responsiveness. Gender Role Expectations of Pain (GREP) correlated only in a few cases with pain responses. Using sex and gender and all significant psychological variables as predictors in multiple regression analyses showed that essentially sex and fear of pain significantly predicted pain. PT was the only parameter that was not influenced by psychological variables. In summary, our study corroborated a distinct effect of sex regarding mechanical pain stimuli, showing women to be more responsive. In contrast the expected effect of gender role was not confirmed. Fear of pain seems to mediate the effect of sex on pain responsiveness, at least partially.

CORRESPONDING AUTHOR: Birgit Kröner-Herwig, PhD, Psychology, University of Göttingen, Göttingen, 37073; bkroene@ uni-goettingen.de 


\section{CITATION ABSTRACT}

SS29c

PREVALENCE AND PSYCHOLOGICAL FACTORS ASSOCIATED WITH MEDICALLY UNEXPLAINED SOMATIC SYMPTOMS IN PRIMARY HEALTH CARE

Wolfgang Hiller, PhD, Stephanie Körber, DiplPsych and Dirk Frieser, DiplPsych

University of Mainz, Mainz, Germany.

Objective: Patients with medically unexplained somatic symptoms (MUSS) are classified as somatoform disorders (SFDs) whenever the clinical pictures is complicated by disproportionate personal distress or functional impairments. This study examines the prevalence of MUSS and SFDs in the German primary care setting. It was further investigated how psychological factors discriminate between MUSS patients with high and low disability levels.

Methods: Data were collected from 614 consecutive outpatients of two regular general medical practices in Mainz, Germany. A subsample of 308 patients underwent a detailed structured clinical interview assessing symptoms, psychological characteristics and illness-related behaviors. We used the Patient Health Questionnaire PHQ-15 to screen for medically explained and unexplained somatic symptoms. Patients scoring 10 were defined as high-risk patients for SFDs. Symptom-related disability was assessed with a modified version of the Pain Disability Index (mod-PDI). The following seven psychological factors were examined using a SCID-type structured interview: (1) frequent health worries, (2) selective attention on body signals, (3) fear of illness and illness consequences, (4) inadequate symptom attributions, (5) body checking behavior, (6) illness behavior, (7) increased health care utilization.

Results: Patients reported a total of 2838 physical symptoms during the past 12 months. The proportion of symptoms not entirely due to medical factors was $78.2 \%$ (according to systematic ratings of the primary care physicians). $22.9 \%$ of the patients met the DSM-IV criteria for a SFD and $11.3 \%$ for major depression. Patients with MUSS and high disability level had more health worries, reported a stronger focus on body-related signals, attributed their symptoms more often to biomedical factors and showed more avoidance and illness behaviors, as compared to patients with lower disability levels. Psychological factors predicted general psychopathology, number of somatic symptoms and dysfunctional illness perception.

Conclusions: This study confirms that MUSS and SFDs are very common in the primary care setting. It was demonstrated that several psychological factors can be used to characterize and further sub-specify this clinical population. It is recommended to consider these factors for future SFD classification approaches. 
CORRESPONDING AUTHOR: Wolfgang Hiller, PhD, University of Mainz, Mainz, 55122; hiller@mail.uni-mainz.de

\section{SS29d}

THE EFFECT OF STRESS ON CHRONIC FATIGUE SYMPTOMS Urs Nater, $\mathrm{PhD},{ }^{1,2}$ Brian Gurbaxani, $\mathrm{PhD},{ }^{1}$ Christine Heim, $\mathrm{PhD}^{1}$ and William C. Reeves, $\mathrm{MD}^{1}$

${ }^{1}$ Centers for Disease Control and Prevention, Atlanta, GA and ${ }^{2}$ University of Zurich, Zurich, Switzerland.

Chronic fatigue syndrome (CFS) is a debilitating disorder for which no single etiological factor has been identified. Stress has been suggested to play a role in the manifestation and maintenance of CFS. Various studies haven shown associations between different aspects of stress (e.g. childhood trauma, maladaptive coping, adult chronic stress) and fatigue. However, no studies have simultaneously measured and analyzed multiple characteristics of stress in one single study of CFS.

In the current population-based study, 501 participants took part and provided information on early life stress, personality characteristics, chronic stress, coping, cortisol as biological stress marker, and CFS symptoms. Data were analyzed using structural equation modelling (SEM, LISREL).

After comparing various theoretically sound models, the strongest model focused on the combined effect of childhood trauma and personality features. This model shows a strong effect of maladaptive personality features on CFS symptoms, in part mediated by adult chronic stress and coping. The impact of childhood trauma was significant, but the effect was lower than that of personality. SEM analysis indicates a good fit of this model (Chi2 $=855.19, \mathrm{p}=$ $0.0, \mathrm{GFI}=.88 ; \mathrm{AGFI}=.85, \mathrm{CFI}=.95, \mathrm{SRMR}=.05 ; \mathrm{RMSEA}=.071$, $\mathrm{p}(\mathrm{RMSEA})=0.0)$.

Our findings indicate the importance of stress in CFS. We have combined various aspects of stress in our study and were able to test a model encompassing a broad variety of stress characteristics. Future studies should include longitudinal designs in order to test causal relationships. It seems critical to devote research resources to a detailed understanding of the processes that lead from stress to CFS in order to improve current treatment strategies.

CORRESPONDING AUTHOR: Urs Nater, PhD, Centers for Disease Control and Prevention, Atlanta, GA, 30322; unater@emory.edu

\section{Saturday \\ August 7, 2010 \\ PAPER SESSIONS \\ 10:30 AM-12:00 PM}

OS43

10:30 AM-10:48 AM

OS43-A
WORKLOAD, CONTROL OVER WORK, REWARDS AND STRESS AS FACTORS AFFECTING FACULTY MEMBERS AND THEIR INTENTION TO LEAVE THEIR JOBS

Peter H. Sawchuk, PhD and Milosh M. Raykov, PhD

Ontario Institute for Studies in Education, University of Toronto, Toronto, ON, Canada.

Background: Attrition and workload are serious issues for faculty members, faculty associations and university administration. Despite the debates about methods for measuring workload, there is a high level of agreement that faculty members devote large amounts of time to their work, causing high levels of stress, and affecting their well-being and work commitment and. This study explores relatively underexamined questions related to factors affecting faculty members and their intention to leave their jobs, such as work demands, control over work, work-related rewards and stress pertinent to work in academic settings.

Methods: The faculty workload study was carried out using an online survey that completed 637 faculty members. The data on faculty intentions to quit, workload as an indicator of work demands, control over work, rewards, and work-related stress specific to the university setting were collected with a survey developed for this study. This study also collected open-ended, qualitative data that are included in the report, and compared this data with that from similar Canadian surveys, i.e., the Labour Force Survey (LFS) and the Work and Lifelong Learning Survey (WALL).

Findings: The study shows a high faculty workload, as demonstrated by the large number of work hours, significantly exceeding the national average found in the LFS and the WALL surveys (41\% of faculty work more than 60 hours per week), and by the large number of courses taught during the academic year (24\% of faculty teach five to eight courses). The results show significant bivariate associations between faculty's desire to leave their jobs with the majority of indicators related to workload, control over work, rewards, and work-related stress. Multiple logistic regression analysis, controlled for relevant demographic factors (age, sex and years of service), shows that faculty who perceive low control over work demonstrate the strongest likelihood of leaving their jobs (adjusted odds, $\mathrm{AO}=$ $3.775)$, followed by those who perceive lack of rewards $(\mathrm{AO}=$ 2.402), high work-related stress $(\mathrm{AO}=2.363)$, and higher workload $(\mathrm{AO}=2.278)$.

Conclusions: Similarly to the growing number of studies demonstrating that the control-demand and effort-reward models complement each other, this study identifies the important roles of both models of work-related stress, but suggests that among faculty members, control over work plays a highly important role in their intention to quit.

CORRESPONDING AUTHOR: Milosh M. Raykov, PhD, University of Toronto, OISE, Toronto, ON, M5S 1 V6; milosh. raykov@utoronto.ca 


\begin{abstract}
CITATION ABSTRACT
10:48 AM-11:06 AM

OS43-B

USING ECOLOGICAL MOMENTARY ASSESSMENT TO TEST THE ERI MODEL IN THE PREDICTION OF STRESS AND AFFECT AT WORK IN PORTUGUESE HEALTH PROFESSIONALS

Teresa M. McIntyre, $\mathrm{PhD},{ }^{1}$ Scott E. McIntyre, $\mathrm{PhD}^{2}$ and Derek W. Johnston, $\mathrm{PhD}^{3}$

${ }^{1}$ Psychology, University of Houston, Houston, TX; ${ }^{2}$ School of Human Sciences and Humanities, University of Houston-Clear Lake, Houston, TX and ${ }^{3}$ School of Psychology, University of Aberdeen, Aberdeen, United Kingdom.

The Effort-Reward Imbalance (ERI) model of work stress (Siegrist, 1996) has been widely tested using questionnaires. The model predicts that high effort and low reward elicit negative emotions, stress and reduced health, and that positive emotions and well-being are associated with reward. Ecological Momentary Assessment (EMA) is designed to capture phenomena in real time overcoming limitations of self-report instruments. This study tested the feasibility of using EMA methods to test the ERI model, using EMA reports of job characteristics, stress, negative and positive affect (NA, PA) and cardiac reactivity. The longitudinal design involved EMA repeated measures over a shift, using an electronic diary, and the OSCAR II ambulatory recorder for heart rate, systolic and diastolic blood pressure (SBP, DBP). The sample consisted of 92 health professionals who completed 366 observations $(\mathrm{M}=4, \mathrm{SD}=1.19)$. The model was tested using hierarchical regression analyses and multilevel modeling. Effort and reward, independently predicted EMA stress response and affect, and with the ERI ratio explained 5-15\% of the variance. Higher effort was associated with feeling "stressed" and more NA (e.g. tired, angry, sad), whereas higher reward correlated with lower NA and more positive states/affect (e.g. happy, energetic). The ERI ratio predicted NA (2-22\% of variance explained). Multilevel modeling supported these findings (effort predicted NA: Beta $=.115$, ndt $=3.83, \mathrm{p}=.005$, reward predicted PA: Beta $=.093$, ndt=2.82, $\mathrm{p}=.004$ ) but the ERI ratio was not significant. The model was also not supported for cardiac reactivity, except for higher reward being associated with lower SBP (sr= -.31). Overall, the results support the ERI model using EMA measures of stress and affect during a work shift. A contribution of the study was to test the ERI model in the prediction of positive emotions at work.
\end{abstract}

CORRESPONDING AUTHOR: Teresa M. McIntyre, PhD, Psychology, University of Houston, Houston, TX, 77204-5022; teresamcintyreusa@gmail.com

\section{1:06 AM-11:24 AM \\ OS43-C}

WORK-FAMILY CONFLICT, COPING AND CO-WORKER SUPPORT AS PREDICTORS OF HEALTH DISTRESS IN PORTUGUESE NURSES

Clara M. Simães, Graduate, ${ }^{1,2}$ Teresa McIntyre, $\mathrm{PhD}^{2,3}$ and Scott McIntyre, $\mathrm{PhD}^{4}$

${ }^{1}$ School of Nursing, University of Minho, Braga, Portugal; ${ }^{2}$ Department of Psychology, University of Minho, Braga, Portugal; ${ }^{3}$ Department of Psychology, University of Houston, Houston, TX and ${ }^{4}$ School of Human Sciences \& Humanities, University of Houston-Clear Lake, Houston, TX.
The aim of this study was to investigate the role of workfamily conflict, coping confidence and co-worker social support as predictors of health distress among Portuguese nurses. The sample was constituted by 100 female hospital-based nurses (Age: $\mathrm{M}=34.32 ; \mathrm{SD}=9.15$ ), from northern Portugal. Participants were assessed using a Demographic and Professional Form; the General Health Questionnaire (GHQ-12) and the Brief Personal Survey-R (BPS), to evaluate stress responses and coping resources; the "Job Content Questionnaire" (JCQ - SS), to assess co-worker social support; and the "Work-Family Conflict \& Family-Work Conflict" Scales, to measure the interference between work and home domains. Hierarchical regression analyses were computed to determine the significant predictors of health distress. The results of the GHQ-12 show clinical levels of distress in $73 \%$ of the nurses. General levels of distress were predicted by coping confidence (CC, $\beta=-.28$, $\mathrm{p}=.005$ ) and Work-Family Conflict (WFC. $\beta=.30, \mathrm{p}=.004$ ). 
Predictors for specific stress responses (BPS anger, guilt and pressure) were the same and in the same direction. In addition, co-worker social support predicted stress responses of guilt $(\beta=$ $-.19, \mathrm{p}=.04)$ and anger $(\beta=-.19, \mathrm{p}=.04)$. The total variance explained by these predictors in nurses' stress responses ranged from 15 to $27 \%$. Some professional variables (night shift work and daily work load) and family variables (number of children at home) were also significant predictors of reported stress (variance explained ranging from 4 to $8 \%$ ). Findings support the high incidence of distress in Portuguese nurses reported in other studies. Furthermore, the data points to the need to consider the family-home spill over in occupational health intervention programs. Coping resources and co-worker social support constitute key variables in reducing work stress among this population.

CORRESPONDING AUTHOR: Clara M. Simães, Graduate, School of Nursing, University of Minho, Braga, 4710-229; csimaes@ese.uminho.pt

\section{1:24 AM-11:42 AM}

OS43-D

IMPACT OF WORK-RELATED FACTORS, LIFESTYLE, AND WORK ABILITY ON SICKNESS ABSENCE AMONG CEMENT CONSTRUCTION WORKERS

Padam P. Prasain, $\mathrm{MA}^{1}$ and Sanjit R. Joshi, MBA ${ }^{2}$

${ }^{1}$ Jagdamba Cement Industry Pvt. Ltd, Kathmandu, Nepal and

${ }^{2}$ Jagdamba Spinning Mills Pvt. Ltd, Kathmandu, Nepal.

OBJECTIVE: The objective of this study was to evaluate the relative contribution of individual characteristics, lifestyle factors, work-related risk factors, and work ability on the occurrence of short ( $<2$ weeks), moderate ( $2-12$ weeks), and long ( $>12$ weeks) durations of sickness absence.

METHODS: Altogether 5867 cement construction workers with complete sick leave registration were followed from the day of their medical examination in 2007 until the end of 2009. The main outcome of the study was the duration of sickness absence, as registered by an occupational health service. Independent variables consisted of individual characteristics, lifestyle factors, workrelated factors, and the work ability index. We used Poisson regression analyses with repeated occurrence of sick leave to calculate rate ratios (RR) and 95\% confidence intervals of independent variables for the three categories of sick leave duration.

RESULTS: Predictors for sick leave lasting 2-12 weeks and $>12$ weeks were: older age, obesity, smoking, manual materials handling, lack of job control, lung restriction, and a less than excellent work ability. For most predictors, higher RR values were observed with a longer duration of sickness absence. Obesity, smoking, manual materials handling, and lack of job control remained important risk factors for moderate and long durations of sick leave after adjusting for the strong effects of work ability on sickness absence. The highest population-attributable fractions were observed for: age over 50 years (18\%), manual materials handling (20\%), and good (18\%), moderate $(28 \%)$, and poor $(2 \%)$ work ability.

CONCLUSION: This study suggests that a variety of preventive measures targeted at smoking, obesity, physical load, psychosocial work factors as well as work ability will contribute to a reduction in the occurrence of sick leave.

CORRESPONDING AUTHOR: Padam P. Prasain, MA, Jagdamba Cement Industry Pvt. Ltd, Kathmandu, n/a; all.myinbox@ gmail.com 


\section{CITATION ABSTRACT \\ 11:42 AM-12:00 PM}

OS43-E

A PROSPECTIVE STUDY ACROSS DIFFERENT HEALTH CONDITION SUBGROUPS: DIFFERENCES IN ASSOCIATION BETWEEN PSYCHOSOCIAL FACTORS AND RETURN TO WORK OUTCOME

Sandra Brouwer, $\mathrm{PhD},{ }^{1}$ Michiel F. Reneman, $\mathrm{PhD},{ }^{2}$ Ute Bültmann, $\mathrm{PhD},{ }^{1} \mathrm{Jac} \mathrm{J}$. van der Klink, $\mathrm{PhD}, \mathrm{MD}^{1}$ and Johan W. Groothoff, $\mathrm{PhD}^{1}$

${ }^{1}$ Health Sciences, University Medical Center Groningen, Groningen, Netherlands and ${ }^{2}$ Center for Rehabilitation, University Medical Center Groningen, Groningen, Netherlands.

Background: Work attitude, self-efficacy and perceived social support are associated with the time to Return-to-Work (RTW). Aim of the study was to investigate whether associations between these three factors and time to RTW differ across different health condition subgroups. Methods: The study was based on a sample of 926 workers on sickness absence (6-12 weeks). The participants filled out a baseline questionnaire with items about demographic characteristics, work attitude, self-efficacy and perceived social support and were subsequently followed until the tenth month after listing sick. The sample was divided into three subgroups: musculoskeletal, other physical and mental health conditions. We used Anova analyses and Cox regression analyses.

Results: Significant differences were found on the mean scores of the three psychosocial factors between the health condition subgroups, with the highest mean scores in the musculoskeletal subgroup. Moreover, differences were observed with respect to the strength of the associations (HR) in both the uni- and multivariate model between work attitude and perceived social support and time to RTW. In the two physical health condition subgroups better work attitude and more perceived social support are facilitators to RTW (adjusted HR ranging from 1.26-1.51).

However, in the subgroup with mental health problems better work attitude and more perceived social support were actually a barrier to RTW $(H R=0.94$ respectively HR=0.80). Only selfefficacy remained in the multivariate model in all subgroups (adjusted HR ranging from 1.49 to $1.60)$.

Conclusions: The results of this study show that work attitude, self-efficacy and perceived social support are relevant psychosocial predictors with regard to the time to RTW in all types of health conditions, but that important differences are observed in type of factor and strengths of the relationships between physical and mental health conditions.

Message:

1. Important difference exists between physical and mental health condition in accordance to the relevance of work attitude, self-efficacy and perceived social support as psychosocial predictors for time to RTW.

2. In sick-listed workers with mental health problems better work attitude and more perceived social support are actually a barrier to RTW.

3. Different subgroup profiles may have clinical relevance in the development of disease-groupspecific disability management guidelines or may be beneficial to develop disease-specific interventions. 
CORRESPONDING AUTHOR: Sandra Brouwer, PhD, university medical center groningen, Groningen, 9700 AD; sandra.brouwer@ med.umcg.nl

\section{OS44}

\section{0:30 AM-10:48 AM}

OS44-A

GENDER DIFFERENCES IN BEREAVEMENT REACTIONS AMONG ELDERLY PEOPLE

Maja O'Connor, $\mathrm{PhD}^{1}$ and Margaret Stroebe, Professor ${ }^{2}$

${ }^{1}$ Institute of psychology, Aarhus University, Aarhus, Denmark and ${ }^{2}$ Department of Psychology, Utrecht University, Utrecht, Netherlands.

Background: Women generally show higher frequencies of DSM IV symptoms of PTSD, depression, and anxiety than men. Yet, widowers have relatively higher excess mortality rate after the loss than widows compared to non-bereaved counterparts. When nonbereaved controls are not taken into account, widowers and widows show similar frequencies of symptoms and gender differences in coping with spousal bereavement appear to be relatively stable across previous studies.

Objectives: The aim was to investigate the importance of gender in relationship to other risk factors for poor bereavement outcome, using an integrative framework to enable the relative impact of different variables to be established.

Methods: 296 elderly bereaved people (Mean=73 years, 38\% men) were examined at 2, 6, 13, and 18 months post loss using self-report scales measuring posttraumatic stress, coping style, crisis support, and personality factors.

Results: A selection of major risk factors was entered into a hierarchical linear regression. The model predicted $49 \%$ of the variance in long-term traumatic stress (18 months). When conducted for widows and widowers separately, the predictive power of the model rose to $62 \%$ for the widows and $58 \%$ for the widowers. For the widows, emotional coping, emotional loneliness, and satisfaction with social support predicted $62 \%$ of the variance. When early traumatic distress ( 2 months) was entered at the last step of the model, the predictive power of the model remained unchanged. For the widowers, sense of peace and satisfaction with social support predicted $40 \%$ of the variance in long-term traumatic distress. When early distress was entered, the predictive power of the model rose to $58 \%$.

Conclusion: Stable, interpersonal traits such as emotional coping style may predict bereavement outcome well for widows, while strong early distress may be a better predictor for widowers. Frequently, widowers are excluded or combined with widows in research. These results indicate that false conclusions will be reached, particularly regarding widower's reactions, if this is done.

CORRESPONDING AUTHOR: Maja O'Connor, PhD, Institute of psychology, Aarhus University, Aarhus, 8000 C; maja@psy.au.dk

10:48 AM-11:06 AM

OS44-B

SEX DIFFERENCES IN RELATIONS AMONG SUBCLINICAL CEREBROVASCULAR DISEASE, BRAIN ATROPHY

AND HEALTH RELATED QUALITY OF LIFE IN HEALTHY OLDER ADULTS

Megan Hosey, MA, ${ }^{1}$ David M. Lefkowitz, MD, ${ }^{2}$ Leslie I. Katzel, $\mathrm{MD}, \mathrm{PhD},{ }^{3,4}$ Eliot L. Siegel, $\mathrm{MD}^{2,4}$ William F. Rosenberger, $\mathrm{PhD}^{5}$ and Shari R. Waldstein, $\mathrm{PhD}^{1,4}$

${ }^{1}$ University of Maryland, Baltimore County, Baltimore, MD;

${ }^{2}$ Diagnostic Radiology, University of Maryland, School of Medicine, Baltimore, MD; ${ }^{3}$ Medicine, University of Maryland, School of Medicine, Baltimore, MD; ${ }^{4}$ Geriatric Research Education and Clinical Center, Baltimore VA Medical Center, Baltimore, MD and ${ }^{5}$ Statistics, George Mason University, Fairfax, VA.

Age-related subclinical brain pathology is associated with poor health outcomes, though relations with health related quality of life (HRQoL) are poorly understood. This study investigated sex differences in relations of subclinical cerebrovascular disease (SCD), brain atrophy (BA) and HRQoL in 154 healthy older adults $(50.6 \%$ male; mean age $=66$ years $)$ free of major medical, neurologic, and psychiatric disease. Participants completed the Medical Outcomes Survey Short Form-36 (SF36) and underwent brain magnetic resonance imaging (MRI) rated by a neuroradiologist for markers of SCD (i.e. periventricular and deep white matter hyperintensities, number of silent infarcts) and BA (i.e. ventricular enlargement, sulcal widening). We create two rank-sum variables (SCD, BA) to serve as primary predictor variables. Multiple regression analyses (adjusted for age, race, education, blood pressure, body mass index, smoking, and Beck Depression Inventory scores) examined the interaction of sex with SCD and BA on HRQoL. Results revealed that for women, SCD was negatively related to the Mental Component Score of the SF-36 (MCS; $\mathrm{rsq}=-.09, \mathrm{~b}=-4.26, \mathrm{p}=.02$ ). For men, BA was negatively related to the Physical Component Score of the SF-36 (PCS; $\mathrm{rsq}=.06, \mathrm{~b}=-1.58, \mathrm{p}=.04)$. Results suggest that deleterious changes in brain structure (particularly SCD) are related to poorer outcomes in mental health dimensions of older women's quality of life. Conversely, in men, brain atrophy relates to poorer physical function. Thus, there are sex differences in the relations of subclinical brain pathology to HRQoL in older adults. 
CORRESPONDING AUTHOR: Megan Hosey, MA, University of Maryland, Baltimore County, Baltimore, MD, 21250; mhosey1@umbc.edu

\section{1:06 AM-11:24 AM \\ OS44-C}

RELATIONSHIP BETWEEN SELF-REPORTED FUNCTIONAL LIMITATIONS AND PHYSICAL PERFORMANCE STATUS AMONG AGED HOME CARE CLIENTS

Heikki Heinonen, $\mathrm{PhD},{ }^{1}$ Antti Uutela, prof, ${ }^{1}$ Mikael Fogelholm, $\mathrm{PhD},{ }^{2}$ Pilvikki Absetz, $\mathrm{PhD},{ }^{1}$ Raisa Valve, $\mathrm{PhD}^{3}$ and Arja Aro, Prof $^{4}$

${ }^{1}$ National Public Health Institute, Helsinki, Finland; ${ }^{2}$ Academy of Finland, Helsinki, Finland; ${ }^{3}$ University of Helsinki, Helsinki, Finland and ${ }^{4}$ Unit for Health Promotion Research, University of Southern Denmark, Esbjerg, Denmark.

OBJECTIVES: Objective of this study was to investigate relationship between self-reported functional disabilities and measured physical performance among aged homecare clients

DESIGN: Baseline measurement of a physical performance intervention

SETTING: Health centers in six semi-urban and rural municipalities in Finland

PARTICIPANTS: One hundred and seventy-one aged (>mean age 82 yrs.) home care clients

MEASUREMENTS: Functional balance was measured using the Berg Balance Scale (BBS), physical performance by the Short Physical Performance Battery (SPPB), the 10-meter walking time test, the hand grip strength test and self-reported disability in activities of daily living (ADLs) and instrumental activities of daily living (IADLs) by Euro-Reves.

RESULTS: Significant correlations were found between the number of functional limitations and the measured performance status. Self reported disabilities were strongly related to the measures of physical performance and in the hierarchical regression model these variables explained $40 \%$ of the variance of the functional limitations

CONCLUSIONS: Self-reported difficulties in the ADL and IADL activities were in line with previous studies indicating that the progression of physical disability follows a hierarchical pattern, starting with difficulties in mobility, progressing to difficulties with IADLs, and, finally, culminating in problems with ADLs. A threshold of physical performance on/above which people seem to report no/minimal disability was found. However, even a high performance status does not entirely protect a person from having multiple disabilities. Present study indicated that physical performance measures are independent predictors of functional disability and therefore interventions should directly address factors associated with physical performance in order to enhance autonomy of aged persons.

CORRESPONDING AUTHOR: Heikki Heinonen, PhD, National Institute for health and Welfare, Helsinki, 00300; heikki. heinonen@thl.fi
11:24 AM-11:42 AM

OS44-D

SOCIOECONOMIC STATUS AND THE DEVELOPMENT

OF FRAILTY. A TEN-YEAR LONGITUDINAL STUDY

OF DUTCH OLDER MEN AND WOMEN

Martijn Huisman, PhD, ${ }^{1,2}$ Martijn Heijmans, $\operatorname{Dr}^{1}$ and Dorly Deeg, Prof $\mathrm{Dr}^{1}$

${ }^{1}$ EMGO Institute, VU medical center, Amsterdam, Netherlands and ${ }^{2}$ Sociology, VU University, Amsterdam, Netherlands.

Background: Frailty is a geriatric concept that represents a lack of reserve functional capacity in older people. It has practical clinical implications because it constitutes a state of health that is still modifiable but is on the threshold to chronic irreversible health problems. In this study we analysed the development of frailty over a ten-year follow-up period in older Dutch men and women from different socioeconomic groups.

Methods: Data came from a nationally representative cohort of 1,509 men and women aged 55-85 years. Frailty was measured with the following markers: physical activity, and grip strength, BMI, cognitive functioning, vision and hearing problems. A maximum of 4 observations of the degree of frailty were acquired over a ten-year follow-up period. Indicators of socioeconomic position were education level and household income. We conducted several types of linear mixed model analyses to assess socioeconomic inequalities in the development of frailty, including complete case analyses, and with frailty imputed for those who died during follow-up.

Results: Adjusted for age, sex, and a partner being present inside the house or not, primary educated had higher odds of developing frailty than tertiary educated men and women $(\mathrm{OR}=1.76$; 95\%CI: 1.05-2.97), and the lowest income groups had a higher odds than the highest income groups ( $\mathrm{OR}=1.90$; 95\%CI: 1.20-3.02). Interactions of education and income with time of frailty measurement suggested that the association of education and income with frailty increased during follow-up. Analyses with imputations for missing observations due to deaths yielded similar results. Conclusion: Having a high socioeconomic position is still protective against one of the core geriatric health problems; frailty. This suggests that differences in vulnerability late in life may account for socioeconomic inequalities in health at even later stages of life, but also that reversing these inequalities is partly feasible.

CORRESPONDING AUTHOR: Martijn Huisman, PhD, LASA, VU medical center, Amsterdam, 1081 BT; ma.huisman@vumc.nl

11:42 AM-12:00 PM

OS44-E

PREDICTORS OF MILD COGNITIVE IMPAIRMENT

IN ELDERS OF DIVERSE BACKGROUND ATTENDING

AN EYE CLINIC

Patricia C. Heyn, PhD, Exercise Science \& Geront ${ }^{1}$ and Rosa A. Tang, MD, $\mathrm{MPH}^{2}$

${ }^{1}$ Physical Medicine \& Rehabilitation, University of Colorado Denver, Denver, $\mathrm{CO}$ and ${ }^{2}$ University Eye Institute, University of Houston, Houston, TX. 
The purpose of this study is to assess cognitive function in a random population undergoing regular eye exams at a Family Practice Eye Service. The goal of the study is to identify individuals at risk for Mild Cognitive Impairment (MCI) in order to help develop early treatment for the prevention of Alzheimer's disease and other mental disorders.

Study aims:1) To determine the predictors of mild cognitive impairments in older adults of diverse background attending a Family Practice Eye Service; 2) To evaluate ethnic group variations; and 3) To evaluate a standardized computerized screening battery to detect mild cognitive impairment on a random sample of older adults.

Study Hypothesis: A great number of elderly patients attending a community Eye Clinic will present significant Mild Cognitive Impairment (MCI) as measured by the Stroop Test, Reaction Time Test (RTT), Shifting Attention Test (SAT), and Continuous Performance Test (CPT) from the CNS Vital Sign Computerized Battery Neuropsychological Assessment.

Study Design: Feasibility study and cross-sectional.

Outcomes: Demographical, cognitive, mood and visual assessments (one time; cross-sectional).

Statistical Plan: Descriptive statistics (proportion, range, means, and SDs), correlations, test of mean differences.

Results: Hypothesis is supported by cognitive assessment analysis of 143 research participants. These results support that $55 \%$ of the older adults attending the community Eye Clinic showed at least one abnormal score in one of the cognitive tests, indicative of mild cognitive impairment or cognitive impairment. Further data and results will be discussed.

CORRESPONDING AUTHOR: Patricia C. Heyn, PhD, Exercise Science \& Geront, Physical Medicine \& Rehabilitation, University of Colorado Denver, Denver, CO, 80203; patricia.heyn@, ucdenver.edu

\section{OS45}

\section{0:30 AM-10:48 AM}

OS45-A

ONE-YEAR EVALUATION OF A THEORY-BASED

INTERVENTION TO ENHANCE PHYSICAL EXERCISE

Reinhard Fuchs, $\mathrm{PhD}^{1}$ and Wiebke Göhner, $\mathrm{PhD}^{2}$

${ }^{1}$ University of Freiburg, Freiburg, Germany and ${ }^{2}$ Catholic University of Applied Sciences Freiburg, Freiburg, Germany.

Little is known about the long-term effects of group intervention programs targeting physical exercise. This paper reports on the effectiveness of MoVo-LISA, a theory-based (MoVo-concept) standardized intervention program. Participants are taught cognitive-behavioral strategies of goal-setting, action planning, barrier management and self-monitoring.

Methods: $\mathrm{N}=220$ in-patients of an orthopedic rehabilitation clinic were assigned (quasi-experimental design) to the control group (usual care) or the intervention group. Assessments were conducted at five time points.

Results: At 12 month follow-up, level of exercise in the intervention group was $28.5 \mathrm{~min} /$ week higher than in the UCG $(\mathrm{p}=.05)$. Moreover, $50 \%$ of the IG was exercising for at least $60 \mathrm{~min} /$ week, but only $33 \%$ of the UCG $(\mathrm{p}=.01)$. Results also revealed significant intervention effects on cognitive mediator variables: the IG reported enhanced self-efficacy and more positive balance of outcome expectations at six months as well as stronger goal intentions, more elaborated implementation intentions, and optimized strategies of intention shielding at 12 months follow-up.

Conclusions: Results provide evidence that intervention programs based on the MoVo concept lead to long-term improvement in exercise behavior and underlying cognitive factors.

CORRESPONDING AUTHOR: Reinhard Fuchs, PhD, University of Freiburg, Freiburg i. Br., 79102; reinhard.fuchs@sport.unifreiburg.de

\section{0:48 AM-11:06 AM}

OS45-B

\section{LOW TECHNOLOGY TRAINING IN OLDER PEOPLE} WITH FUNCTIONAL LIMITATIONS

\section{Nina Beyer, $\mathrm{PhD}^{1}$ and Margit Vestergaard, $\mathrm{MSc}^{2}$}

${ }^{1}$ Musculoskeletal Rehabilitation Research Unit \& Institute of Sports Medicine Copenhagen, Bispebjerg Hospital, Copenhagen, Denmark and ${ }^{2}$ Danish Workers Sports Association, Copenhagen, Denmark.

Aim of study: This community-based exploratory study investigated the results of supervised low technology training on function, physical activity, and falls in older people with functional limitations.

Methods: Home-dwelling people from four municipalities $(\mathrm{n}=$ 123, age 75-95 yrs) were included. Supervised balance and strength training was performed at home for 16 weeks. External load was $65 \%$ of $1 \mathrm{RM}$. The instructors used the principles of positive reinforcement to enhance motivation for regular physical activity. After the 16 weeks contact was established between volunteer instructors and participants who wanted to continue exercising. Assessments were performed by health professionals who did not train the participants. Function (static balance (SPPB) and Senior Fitness test) was assessed at baseline and after 16, 32 and 52 weeks. Self reported data were collected at baseline and after 52 weeks.

Results: At baseline $86 \%$ used walking aid, $89 \%$ had balance problems and $62 \%$ had fallen during the previous year, and 3\% met the physical activity recommendations. Seventy-six \% completed the intervention. Those who were able to rise from a chair without using the arms $(83 \%)$ improved in static balance, 30 s-chair-stand, $30 \mathrm{~s}$-arm-curl and 8 -feet-Up\&Go on average $16 \%, 50 \%, 34 \%$ and $16 \%$, respectively $(\mathrm{p}<0.001)$. Improvements were preserved at 52 weeks except for the $30 \mathrm{~s}$-chair-stand, but these results were better compared to baseline $(p<0.01)$. No changes were found in participants who needed the arms to rise from a chair, and drop out was high in this group (60\%). At 52 weeks the participants were more physically active with $55 \%$ exercising in groups or with an exercise-friend $(\mathrm{p}<0.0001)$. 
Balance had improved $(\mathrm{p}=0.001)$ and numerically fewer had fallen $(p=0.065)$.

Conclusion: Supervised low technology balance and strength training at home may lead to increased physical activity and counteract decrease in reserve capacity in older people with functional limitations.

CORRESPONDING AUTHOR: Nina Beyer, PhD, Musculoskeletal Rehabilitation Research Unit \& Institute of Sports Medicine Copenhagen, Bispebjerg Hospital, Copenhagen, 2400 NV; ninabeyer@ oncable.dk

\section{1:06 AM-11:24 AM}

OS45-C

A PROSPECTIVE LONGITUDINAL STUDY EXPLORING THE RELATIONSHIP BETWEEN FUNCTIONAL STATUS AND QUALITY OF LIFE (QOL) AFTER TRAUMATIC BRAIN INJURY (TBI)

Dianxu Ren, $\mathrm{PhD},{ }^{1}$ Amy Wagner, $\mathrm{MD}^{2}$ and Emily Rogers, $\mathrm{MS}^{2}$

${ }^{1}$ School of Nursing, University of Pittsburgh, Pittsburgh, PA and

${ }^{2}$ School of Medicine, University of Pittsburgh, Pittsburgh, PA.

Objectives: Traumatic Brain Injury (TBI) is among the most common causes of brain damage in the United States and results in the disturbance of cognitive, behavioral, emotional, and physical functioning that leads to functional impairments, disability, and decreased quality of life. However, there are very few studies to date that have sought to determine the relationship between objective functional status evaluations and subjective QoL measures in individuals after TBI. The purpose of this study was to explore the relationship between functional status and QoL over time controlling for demographics and injury severity after severe TBI.

Methods: This prospective study used longitudinal data collected from patient questionnaires and from medical records of subjects with severe TBI $(\mathrm{N}=46)$. In addition to demographic and injury related data, patient functional status was measured by Glasgow Outcome Scale (GOS), Disability Rating Scale (DRS) and Functional Independence Measure (FIM). Patient QoL was evaluated using the Modified Perceived QoL questionnaire. Data was collected regarding 6 month and 12 month for both functional status and QoL. The baseline QoL was recalled by patients after TBI. A mixed effects model for repeated measures was performed to examine the relationship between each measure of functional status and QoL overall in patients 6 and 12 months after TBI while controlling for age, gender, injury severity and QoL at baseline respectively.

Results: Overall, QoL measure significantly improved over time $(\mathrm{P}<0.05)$. Baseline overall QoL and GOS were both significantly and positively correlated with the overall post-TBI trajectory QoL score $(\mathrm{P}<0.01)$. DRS was significantly and inversely associated with overall post-TBI trajectory QoL score $(\mathrm{P}<0.01)$. There is no relationship between FIM and overall post-TBI trajectory QoL score $(\mathrm{P}>0.10)$. Age, gender and injury severity were not significantly related with overall post-TBI QoL scores $(\mathrm{P}>0.05)$.

Conclusions: As a subjective measure of TBI outcome, the QoL of patients after TBI continues to improve over time. Baseline QoL before TBI and objective global function measures (GOS and DRS) are strongly associated with long term QoL for TBI patients.

CORRESPONDING AUTHOR: Dianxu Ren, PhD, School of Nursing, University of Pittsburgh, Pittsburgh, PA, 15261; dir8@ pitt.edu

11:24 AM-11:42 AM

OS45-D

EFFECTS OF FREQUENCY OF GROUP-BASED EXERCISE AND BEHAVIORAL COUNSELING ON FUNCTIONAL FITNESS AND QOL IN FRAIL ELDERLY PERSONS

Ryosuke Shigematsu, $\mathrm{PhD},{ }^{1}$ Rei Nakanishi, $\mathrm{MS}^{1}$ and Kiyoji Tanaka, Prof ${ }^{2}$

${ }^{1}$ Faculty of Education, Mie University, Tsu, Japan and ${ }^{2}$ Graduate School of Comprehensive Human Sciences, University of Tsukuba, Tsukuba, Japan.

For frail elderly persons, exercise is essential to preserve functional fitness and quality of life (QOL). Municipalities in Japan offer elderly persons interventions consisting of groupbased exercise sessions and behavioral counseling; however, for a variety of reasons, many individuals do not participate in the interventions. Some are physically frail, some have limited means of transportation and others are self-conscious about exercising with friends. Therefore, it is important to confirm the effect of low-frequency exercise participation compared with that of highfrequency. Thirty-nine participants, aged 67-85 years, participated in this study. All were identified as frail elderly persons according to the criteria of the Ministry of Health, Labor and Welfare, Japan. The participants were divided into two groups that were assigned to different intervention schedules. The high-frequency group $(\mathrm{n}=$ 14 ; 68-83 years) met 12 times over a 3 -month period, while the low-frequency group $(n=25 ; 67-85$ years $)$ met 6 times over a 3month period. Each 90-minute session consisted of group-based exercises and behavioral counseling. The exercises, which the participants were encouraged to practice daily at home, included 4 stretching and 6 strengthening exercises. To encourage exercise, 6 behavioral counseling sessions were offered in which illustrated stories or four-panel cartoons were used. After 3 months, members of both groups showed significant improvement in functional fitness as well as in 5 of 8 dimensions of QOL measured by the SF-36. Improvement in the social dimension of QOL was significantly higher in the high-frequency group than in the lowfrequency group. Thus, both low- and high-frequency interventions offer significant positive effects for frail elderly persons. If elderly individuals need a great amount of socialization, a highfrequency intervention is recommended. 
CORRESPONDING AUTHOR: Ryosuke Shigematsu, PhD, Faculty of Education, Tsu, 514-8507; rshige@edu.mie-u.ac.jp

\section{1:42 AM-12:00 PM}

OS45-E

MEDIATORS OF ADHERENCE AFTER A PHYSICAL ACTIVITY INTERVENTION FOR BREAST CANCER SURVIVORS

Laura Q. Rogers, MD, MPH, ${ }^{1}$ Stephen Markwell, MA, ${ }^{2}$ Patricia Hopkins-Price, PhD ${ }^{1}$ Sandy Vicari, PhD ${ }^{3}$ Kerry S. Courneya, $\mathrm{PhD},{ }^{4}$ Karen Hoelzer, $\mathrm{MD}^{5}$ and Steven Verhulst, $\mathrm{PhD}^{2}$

${ }^{1}$ Medicine, SIU School of Medicine, Springfield, IL; ${ }^{2}$ Statistics \& Research Consulting, SIU School of Medicine, Springfield, IL; ${ }^{3}$ Psychiatry, SIU School of Medicine, Springfield, IL; ${ }^{4}$ Physical Education \& Recreation, University of Alberta, Edmonton, AB, Canada and ${ }^{5}$ Hematology \& Oncology, Springfield Clinic, Springfield, IL.

Although physical activity (PA) provides multiple benefits for breast cancer survivors (BCS), most do not exercise regularly. Identifying mediators of PA maintenance is important for assisting BCS in adopting sustainable PA behavior change. Because we have reported continued improvements in PA 3 months after completing a PA behavior change intervention for BCS, our study aim was to determine if the change in social cognitive theory (SCT) constructs during the intervention mediated the effect of our intervention on PA. We randomized $41 \mathrm{BCS}$ to receive a SCTbased 3-month PA behavior change intervention (INT; $n=21$ ) or usual care (UC; $n=20)$. The INT group received 12 supervised aerobic exercise sessions tapered to home-based exercise. Behavioral support was provided in 6 discussion groups and 3 individual face-to-face counseling sessions. The UC group received written materials. PA (i.e., accelerometer) and SCT constructs (i.e., survey) were assessed at baseline, immediately post-intervention (i.e., 3 months), and 3 months post-intervention (i.e., 6 months). The mediation of the intervention effect on PA at 6 months by changes in SCT constructs from baseline to 3 months was examined using the Freedman and Schatzkin approach. Compared to UC, the INT group reported improved barriers interference (mean difference $=-7.8 ; 95 \% \mathrm{CI}=-15.1$ to $-.4 ; \mathrm{d}=-.67 ; \mathrm{p}=.04$ ) and PA enjoyment (mean difference $=.7 ; 95 \% \mathrm{CI}=0$ to $1.5 ; \mathrm{d}=.61$; $\mathrm{p}=.06)$. Barriers self-efficacy $\mathrm{d}$ was $.28(\mathrm{p}=.38)$. Barriers interference and self-efficacy mediated $39 \%(\mathrm{p}=.004)$ and $19 \%$ $(p=.02)$ of the intervention effect on sustained adherence, respectively. In conclusion, only barrier interference and barriers self-efficacy significantly mediated the effect of the intervention on PA 3 months after intervention completion. The increase in enjoyment did not explain sustained PA improvement. Our results suggest that reducing barriers may partially explain the ability of our intervention to demonstrate longer term PA adherence.

CORRESPONDING AUTHOR: Laura Q. Rogers, MD, MPH, Southern Illinois University School of Medicine, Springfield, IL, 62794-9636; 1rogers@siumed.edu
OS46

10:30 AM-10:48 AM

OS46-A

SIX-MONTH DIETARY CHANGES IN OBESE ADOLESCENTS PARTICIPATING IN A MULTIDISCIPLINARY WEIGHT MANAGEMENT PROGRAM

Melanie K. Bean, PhD, ${ }^{1}$ Suzanne E. Mazzeo, $\mathrm{PhD},{ }^{1}$ Marilyn Stern, PhD, ${ }^{1}$ Edmond P. Wickham, MD,${ }^{1}$ Ronald K. Evans, PhD, ${ }^{1}$ Daphne L. Bryan, $\mathrm{MD}^{1}$ and Joseph H. Laver, $\mathrm{MD}^{2}$

${ }^{1}$ Virginia Commonwealth University, Richmond, VA and ${ }^{2}$ St. Jude Children's Research Hospital, Memphis, TN.

Treating pediatric obesity is essential to reduce adult morbidity and mortality and improve the health of obese youth. We report 6month nutrition data from a 2-year multidisciplinary weight management program for obese adolescents, the T.E.E.N.S. Program ( $\mathrm{N}=173$ at baseline, $\mathrm{N}=66$ at 6 months). At baseline, participants were $76 \%$ African American, $65 \%$ female, with a mean age of 13.7. Mean BMI percentile was 99.1 (mean BMI zscore $=2.5)$ at baseline. Repeated measures MANOVAs were used to examine differences from baseline (T1) to 6-month (T2) followup on a 24-hour dietary recall measure. Changes in anthropometrics and blood lipid parameters were also evaluated. At 6months, participants significantly reduced their intake of calories, fat, sodium, and sugar $(\mathrm{p}<.05)$; no changes in calcium or fiber intake were found $(\mathrm{p}>.05)$. Gender stratified models showed that boys had greater increases in fruit and vegetable and vitamin $D$ intake compared to girls $(\mathrm{p}<.05)$. Significant improvements in body mass index and lipid profiles were found in the total sample $(p<.05)$. Multiple regression analysis suggested that caloric reduction from baseline to 6-month was associated with decreases in BMI $\mathrm{z}$-score $(\beta=-0.11, \mathrm{p}<.05)$. However, when parental compliance with nutrition visits was included in the regression, change in calories was no longer significant. Parental compliance became the only significant predictor of 6-month BMI z-score, while controlling for baseline BMI $z$-score $(\beta=-0.16, p<.01)$. Findings suggest that promoting intake of essential nutrients may be more challenging than reducing excessive intake. Additional gender specific strategies for dietary modification may be warranted. Further, increased parental participation in adolescent weight management programs is also recommended to enhance weight loss.

CORRESPONDING AUTHOR: Melanie K. Bean, PhD, Pediatrics and Psychology, Virginia Commonwealth University, Richmond, VA, 23298-0268; mkbean@vcu.edu

\section{0:48 AM-11:06 AM}

OS46-B

DEPRESSION, PAIN, AND PHYSICAL ACTIVITY IN OBESE MALE VETERANS

Patricia H. Rosenberger, $\mathrm{PhD}^{1,2}$ and John Sellinger, $\mathrm{PhD}^{1,2}$

${ }^{1}$ VA Connecticut Healthcare System, West Haven, CT and

${ }^{2}$ Psychiatry, Yale Medical School, New Haven, CT. 
Regular physical activity (PA) is a challenge for obese persons. It is even more challenging for obese persons with either depression or pain, but the impact of these conditions may differ. The present study examined the effects of depression and pain on perception of barriers to PA, confidence about engaging in PA, and current weekly PA in a group of overweight/obese male veterans. Study participants completed the Barriers to Being Active Quiz, Exercise Self-efficacy Scale, and the International Physical Activity Questionnaire. The study sample included 101 male Veterans with $\mathrm{BMI} \geq 25$. The mean age was 61.2 years. Of the sample, $62.4 \%$ reported a painful medical condition (i.e., either arthritic joint pain or back pain) and $48.5 \%$ reported a clinically significant Patient Health Questionnaire-9 depression score $\geq 15$. Reporting a pain condition was marginally associated with depression $(\mathrm{p}=.064)$. Of the study sample, $85.9 \%$ reported weekly PA, ranging from walking to vigorous activity. Amount of activity was brief; only $27.7 \%$ reported $\mathrm{PA} \geq 1$ hour. Two factor ANOVAs assessed the contributions of both pain and depression on barriers to PA and exercise self-efficacy. Results revealed a main effect for depression on three of seven perceived barriers to PA: lack of energy, motivation, and skill level. In contrast, a main effect for pain was revealed for one barrier, fear of injury, as well as for exercise selfefficacy (p's<.05). Veterans with pain (but not depression) were less likely to report engaging in weekly PA $(\mathrm{X} 2=4.0, \mathrm{p}<.05)$. Results suggest that although frequently comorbid in obese Veterans, depression and pain are associated with different perceived PA challenges. Depressed Veterans are more likely to report energy and motivational barriers to PA, while Veterans with pain are specifically concerned about hurting themselves during exercise and are less confident that they could actually engage in exercise under challenging conditions. In turn, veterans with pain are less likely to engage in any weekly activity.

CORRESPONDING AUTHOR: Patricia H. Rosenberger, PhD, VA Connecticut Healthcare System, West Haven, CT, 06516; patricia.rosenberger@va.gov

\section{1:06 AM-11:24 AM}

OS46-C

\section{PERCEIVED HEALTH CARE DISCRIMINATION}

IS ASSOCIATED WITH WEIGHT GAIN AMONG SEVERELY OBESE WOMEN

Lena Hansson, MSc and Finn Rasmussen, Professor

Department of Public Health Sciences, Karolinska Institutet, Stockholm, Sweden.

Aim: We examined whether experience of health care discrimination was associated with weight gain in normal weight, moderate obese and severe obese individuals.

Methods: Men and women with normal weight (BMI 18.5-24.9), moderate obesity (BMI 30-34.9) and severe obesity (BMI $>35$ ) were identified from a yearly population based national survey investigating living conditions. Data on height and weight were available from the years between 1996 and 2006. A questionnaire about experiences of previous health care discrimination and current weight and height was mailed to 5018 individuals in 2008.
Multiple linear regression analyses were performed investigating the association between weight gain and health care discrimination controlling for a range of socio-demographic and psychological variables.

Results: Totally 2633 individuals had complete data on all variables used. The average weight gain in the different weight groups according to sex was: $2.5 \mathrm{~kg}(\mathrm{SD}=6.1)$ for normal weight women, $2.4 \mathrm{~kg}(\mathrm{SD}=10.0)$ for moderate obese women, $-0.3 \mathrm{~kg}$ $(\mathrm{SD}=15.7)$ for severe obese women, $3.7 \mathrm{~kg}(\mathrm{SD}=7.4)$ for normal weight men, $1.4 \mathrm{~kg}(\mathrm{SD}=8.2)$ for moderate obese men and $-1.3 \mathrm{~kg}$ $(\mathrm{SD}=14.4)$ for severe obese men. Health care discrimination was not associated with weight gain in men $(-0.3 \mathrm{~kg} \mathrm{95 \% CI}-1.8 ; 1.1)$. This was regardless of weight status. In severe obese women, experience of health care discrimination was associated with an average weight gain of $6.3 \mathrm{~kg}(95 \% \mathrm{CI} 1.0 ; 11.6)$. No association was found between health care discrimination and weight gain in normal weight women $(-0.3 \mathrm{~kg} 95 \% \mathrm{CI}-1.4 ; 0.8)$ or moderate obese women $(1.2 \mathrm{~kg}, 95 \% \mathrm{CI}-0.6 ; 2.9)$

CORRESPONDING AUTHOR: Lena Hansson, MSc, Department of Public Health Sciences, Karolinska Institutet, Stockholm, 17176; lena.hansson@ki.se

\section{1:24 AM-11:42 AM}

OS46-D

ARE OBESITY MESSAGES INFLUENCING THE HEALTH BELIEFS AND BEHAVIOURS OF OBESE ADULTS?

AN IN-DEPTH QUALITATIVE STUDY OF 142 AUSTRALIANS Samantha L. Thomas, BA MA PhD, ${ }^{1}$ Sophie Lewis, BmedSci, ${ }^{1}$ R. Warwick Blood, BS PhD, ${ }^{2}$ Kate Holland, $\mathrm{PhD},{ }^{2}$ David J. Castle, MBBS PhD FRANZP ${ }^{3}$ and Jim Hyde, BA MA PhD ${ }^{4}$

${ }^{1}$ Consumer Health Research Group (CHaRGe), Primary Care Research Unit, School of Primary Health Care, Monash University, Melbourne, VIC, Australia; ${ }^{2}$ News Research Group, Faculty of Arts and Design, University of Canberra, Canberra, ACT, Australia; ${ }^{3}$ Department of Psychiatry, St Vincent's Hospital, University of Melbourne, Melbourne, VIC, Australia and ${ }^{4}$ Victorian Department of Health, University of Canberra, Melbourne, VIC, Australia.

Background: As a society we are increasingly fixated on the consequences of 'being fat'. Public responses have focused on alerting individuals to the risks associated with obesity and the urgent need for intervention and self-regulation. However, there is limited understanding of the impact of these messages on obese adults. This study sought to explore obese individuals' perceptions and attitudes of population based obesity messaging in Australia, how they applied these messages to themselves and how personal and social contexts and experiences influenced these perceptions. Method: We adopted a qualitative approach which utilised indepth semi-structured telephone interviews with a community sample of obese adults (Body Mass Index 30 and over). Theoretical, purposive and strategic sampling techniques were used to ensure a broad representation of individuals from different demographic and geographical backgrounds. We analysed the data using a descriptive thematic approach. 
Results: One hundred and forty two adults participated in this study. Participants' attitudes were strongly influenced by personal and contextual factors, including personal experiences with weight and weight loss attempts. Participants felt that messages were overly focused on the physical rather than emotional health consequences of obesity i.e. the risk of getting cancer, or dying from obesity. Many, particularly those who were severely obese, described feeling stigmatised and blamed by the simplicity of messages and the lack of realistic solutions available to them. Whilst they trusted government based messages, they acted upon the solutions provided by the commercial weight loss industry. Participants supported non-stigmatising messages from noncommercial sources that were focused on encouraging individuals to make healthy lifestyle changes, rather than promoting weight loss.

Conclusion: This study is the first step in exploring the ways in which we understand how obesity messages resonate with obese individuals in Australia and influence their weight loss behaviours. However, further qualitative and quantitative research is needed before we fully understand the impact of obesity messaging on the health beliefs, behaviours and wellbeing of obese adults.

CORRESPONDING AUTHOR: Sophie Lewis, BmedSci, School of Primary Health Care, Monash University, Melbourne, VIC, 3168; sophie.lewis@med.monash.edu.au

\section{1:42 AM-12:00 PM}

OS46-E

OBESITY, BARIATRIC SURGERY, PHYSICAL AND PSYCHOLOGICAL COMPLAINTS: A LONGITUDINAL STUDY

Susana Silva, PhD Student and Angela Maia, $\mathrm{PhD}$

School of Psychology, University of Minho, Braga, Portugal.

Objective: Bariatric surgery has been indicated as a preferential treatment for morbid obesity and is associated with a set of physical and psychological improvements. The aim of our study was to characterize the current functioning and the physical and psychological health problems of bariatric surgery candidates in the surgery moment, in a follow-up of 6 and 12 months.

Methods: 30 obese subjects filled Childhood History Questionnaire, Brief Symptoms Inventory, Rotterdam Symptoms Checklist, Health Questionnaire and Ways of Coping Questionnaire at the surgery, in a follow-up of 6 and 12 months.

Results: The number of reported adverse childhood experiences does not change over time and the prevalence was low. Preliminary results indicate that at the time of surgery, participants reported more passive coping, more problems and physical health complaints than in the 6 months follow-up. 12 months after, there is an increase of psychopathology, passive coping and physical complaints.

Conclusion: The prevalence of adverse childhood experiences is lower when compared with other studies with this population. There is an improvement on current functioning and quality of life after 6 months, however, these gains are going to be blurring the end of 12 months. Obese seem to use more passive coping strategies and do not actively involve in solving their problems.
These results reinforce the importance of developing interventions aimed at behavioural change allowing the empowerment of patients and learning more active coping strategies. Moreover, it is necessary to follow these subjects longitudinally allowing greater and better understanding of its evolution during treatment.

CORRESPONDING AUTHOR: Susana Silva, PhD Student, University of Minho, Braga, 4730-272; sofiasilva@portugalmail.pt

\section{OS47}

\section{0:30 AM-10:48 AM}

OS47-A

EARLY CBT INTERVENTION FOR TREATMENT OF POSTTRAUMATIC STRESS AND DEPRESSION IN HEAD AND NECK CANCER PATIENTS

Maria Kangas, $\mathrm{PhD},{ }^{1}$ Chris Milross, MBBS, FRACR ${ }^{2}$ and Richard Bryant, $\mathrm{PhD}^{3}$

${ }^{1}$ Centre for Emotional Health, Department of Psychology, Macquarie University, Sydney, NSW, Australia; ${ }^{2}$ Royal Prince Alfred Hospital \& Sydney Cancer Centre, Sydney, NSW, Australia and ${ }^{3}$ University of New South Wales, Sydney, NSW, Australia.

Individuals diagnosed with a head and neck cancer (HNC) are at substantial risk of experiencing disfigurements and dysfunctions as a result of the disease process and medical treatments. Consequently, it is not surprising that a substantial number of studies have revealed that HNC patients are susceptible to experiencing clinical levels of anxiety (including PTSD) and depressive disorders. Surprisingly however, there is a paucity of psychological studies targeting the management of stress and depressive disorders in this population. Similarly, there are only a very limited number of studies that have evaluated the efficacy of psychotherapy programs tailored for other types of oncology populations (including breast, prostate, and gastro-intestinal cancers) specifically suffering from clinically elevated anxiety or mood disturbances. Cognitive Behavioural Therapy (CBT) has been shown to be helpful in managing anxiety and depression in various psychiatric and health populations. Several studies have found the early application of a brief version of CBT, to be a useful treatment in reducing severe acute stress, as well as preventing the development of chronic psychological disorders in survivors following non-cancer traumatic events. Accordingly, the aim of the present RCT study (still in progress) was to test an early, brief (6-session) CBT program in reducing clinically elevated posttraumatic stress and depressive responses in newly diagnosed HNC patients undergoing radiotherapy. The efficacy of the CBT program was compared to a 6-session Supportive Counselling (SC) intervention. Participant inclusion criteria comprised adult individuals (aged 18 - 70 years) who met subthreshold or full diagnostic criteria for cancer-related PTSD and/or comorbid anxiety and depression disorders, and who had been prescribed to receive radiotherapy as part of their medical treatment for their HNC. To date, 25 patients have been randomized to receive either the $\mathrm{CBT}$ or SC intervention, and 16 patients have completed the therapy program. The results to date indicate that the CBT program is effective in reducing both 
posttraumatic stress and depressive symptoms, as well as enhancing social well-being. Findings will be discussed in context of the utility of this early brief intervention in treating acute stress and preventing chronic psychopathology in traumatized cancer patients. Discussion will also focus on logistical considerations in conducting psychotherapy concurrent with medical treatments in oncology populations.

CORRESPONDING AUTHOR: Maria Kangas, PhD, Centre for Emotional Health/Dept of Psychology, Macquarie University, Sydney, NSW, 2109; maria.kangas@mq.edu.au

\section{0:48 AM-11:06 AM}

OS47-B

\section{EFFECTS OF MINDFULNESS TRAINING}

ON PSYCHOLOGICAL WELL-BEING AND SYMPTOMS OF STRESS IN CANCER PATIENTS AT 6-MONTH FOLLOW-UP Richard Bränström, $\mathrm{PhD},{ }^{1,2}$ Pia Kvillemo, MSc, ${ }^{1}$ Yvonne Brandberg, $\mathrm{PhD}^{1}$ and Judith Tedlie Moskowitz, $\mathrm{PhD}^{3}$

${ }^{1}$ Department of Oncology-Pathology, Karolinska Institute, Stockholm, Sweden; ${ }^{2}$ Osher Center for Integrative Medicine, Karolinska Institute, Stockholm, Sweden and ${ }^{3}$ Osher Center for Integrative Medicine, Unversity of California San Fracisco, San Francisco, CA.

There is increasing recognition of mindfulness and mindfulness training as a way to decrease stress and increase psychological functioning. The aims of this study were to examine the effects of mindfulness stress reduction training on perceived stress, psychological well-being and levels of cortisol, and to examine if changes in mindfulness mediate intervention effects on these outcomes. Seventy women and one man with a previous cancer diagnosis (mean age 51.8 years, $\mathrm{SD}=9.86$ ) were randomized into an intervention group or a wait-list control group. The intervention consisted of an 8-week mindfulness-training course. A repeated measures MANCOVA showed that compared to participants in the control group, participants in the mindfulness-training group had significantly decreased perceived stress, post-traumatic avoidance symptoms, and increased coping self-efficacy at 6-month followup $(\mathrm{F} 16,51=1.32, \mathrm{p}<0.05)$. Those who participated in the intervention reported significant increase in mindfulness when compared to controls $(\mathrm{F} 10,57=2.34, \mathrm{p}<0.05)$. The increase in mindfulness mediated the effects of the intervention on perceived stress, and coping self-efficacy. The results indicated and intervention effect on awakening cortisol. Among those with initial low levels of cortisol, there was an increase from baseline to 3-month follow-up, and among those with initial high levels there was a decreased level of cortisol at 3-month follow-up. There was an association between changes in stress symptoms and cortisol levels. This study indicates that increased levels of mindfulness can potentially explain reduction of stress symptoms resulting from mindfulness stress reduction training. The results indicated a normalization of cortisol levels as a result of the intervention. The importance of these findings for future research in the field of mindfulness is discussed.

CORRESPONDING AUTHOR: Richard Bränström, PhD, Karolinska Institute, New York, NY, 10065; richard.branstrom@ki.se

11:06 AM-11:24 AM

OS47-C

AN EXPRESSIVE WRITING INTERVENTION IMPROVED SELF-REPORTED HEALTH AMONG EARLY STAGE BREAST CANCER PATIENTS HIGH IN REPRESSIVE COPING: PRELIMINARY RESULTS

OF A POPULATION-BASED, RANDOMIZED CLINICAL TRIAL Mikael B. Jensen-Johansen, MSc Psychology, ${ }^{1}$ Heiddis Valdimarsdottir, Assistant Professor, ${ }^{2}$ Sandra Zakowski, Associate Professor, ${ }^{3}$ Dana H. Bovbjerg, PhD Director, ${ }^{4}$ Søren Christensen, MSc Psychology, ${ }^{1}$ Anders B. Jensen, $\mathrm{PhD}^{1}$ and Robert Zachariae, Professor, drmed ${ }^{1}$

${ }^{1}$ Psychooncology and Health Psychology Research Unit, Aarhus University Hospital, Aarhus C, Denmark; ${ }^{2}$ Mount Sinai School of Medicine, New York, NY; ${ }^{3}$ Argosy University, Chicago, IL and ${ }^{4}$ Cancer Institute, University of Pittsburgh, Pittsburgh, PA.

Objective: To test the effects of an Expressive Writing Intervention (EWI) on subsequent self-reported health of women treated for early stage breast cancer and to examine repressive coping as a moderator.

Methods: Breast cancer patients $(n=507$; mean age 53.6) were recruited following completion of adjuvant treatment randomly assigned to 3 weekly $20 \mathrm{~min}$ home-based writing exercises involving either: 1) emotional disclosure (EWI) about one of their most traumatic experiences, or 2) a control topic (CTRL) of how they spend their time. The primary outcome was self-reported physical health (Patient Health Questionnaire (PHQ)). Repressive copers $(n=161)$ were identified at baseline by having high defensiveness (Marlowe Crowne Social Desirability Scale) and low negative affectivity (Taylor Manifest Anxiety Scale).

Results: A repeated measures ANOVA showed no effect of EWI (time $\mathrm{x}$ group interaction) $(\mathrm{p}=.31)$ but did reveal a significant time $\mathrm{x}$ group $\mathrm{x}$ repressive coping interaction $(\mathrm{p}=.024)$. Repressive copers in the EWI group had improved PHQ-scores at follow-up (9 mo), while PHQ-scores of repressive copers in CTRL worsened. The results continued to be statistically significant $(p=.045)$ with potential confounders included in the model.

Conclusions: An expressive writing intervention may have positive effects on health outcomes among breast cancer patients high in repressive coping, suggesting the potential utility of targeting the intervention to such individuals in future studies.

CORRESPONDING AUTHOR: Mikael B. Jensen-Johansen, MSc Psychology, Psychooncology and Health Psychology Research Unit, Aarhus University Hospital, Aarhus C, DK-8000; mikajens@rm.dk 
11:24 AM-11:42 AM

OS47-D

CAPITALIZING ON THE TEACHABLE MOMENT:

IMPROVING SELF-HELP SMOKING CESSATION

INTERVENTIONS IN ONCOLOGY HOSPITAL ENVIRONMENT

Sanghamitra Pati, MD DNHE MPH

Medical Education, SCB Medical College, Bhubaneswar, India.

The 'teachable moment' has long being considered a cornerstone of public health intervention planning. The Teachable Moment (TM) model suggests that there are certain times when an individual is especially ready to change behavior and thus, open to receiving messages about behavior change. According to the TM model, experiences that jointly (1) increase risk perceptions, (2) prompt concordant emotional responses, and (3) impact selfconcept may offer a powerful motivational context for promoting behavior change. Further, tailoring smoking cessation interventions on TM components may increase the salience of health messages.

In the present study, TM model was used to examine the desire to quit smoking among family members of lung cancer patients. Further, the relationship between desire to quit and engagement with and reactions to self-help smoking cessation materials was examined. Participants were family members of lung cancer patients recruited for a randomized controlled trial testing a counseling intervention for smoking cessation. The main independent variable was lung cancer diagnosis in a family member and the main dependent variable was intention to quit smoking.

Study results indicated that components of the TM model were related to desire to quit in family members of lung cancer patients. Specifically, increased perceived risk, negative self image, and high subjective norm for quitting were related to high desire to quit. In a final path analysis model that controlled for age, sex, ethnicity, education and smoking rate, the construct of perceived individual risk of cancer was identified as a mediator of the relationship between having a family member with a diagnosis of lung cancer and quitting intention. Findings on engagement with and reactions to materials were mixed. Engagement with materials was not related to baseline desire to quit, but was related to positive reactions to the materials. Those with a higher desire to quit were more likely to report that the information in the tailored booklet applied to them, and was new to them, interesting, trustworthy, and moving. Finally, family members with a higher desire to quit were more likely to say that the information in the booklet make them want to quit smoking.

Evidence from this initial study indicates that the TM model provides a strong conceptual framework for (1) identifying specific determinants of desire to quit among family members of lung cancer patients; and (2) developing effective tailored selfhelp materials for this population. These data suggest that the concept of the teachable moment may be adopted and adapted for the smoking cessation in the oncology context in Indian set up.
CORRESPONDING AUTHOR: Sanghamitra Pati, MD DNHE MPH, Medical Education, SCB Medical College, Bhubaneswar, 751022; sanghamitrapati@yahoo.com

\section{1:42 AM-12:00 PM}

OS47-E

REHABILITATION OF CANCER SURVIVORS IN DENMARK A RANDOMIZED CONTROLLED TRIAL ON THE EFFECT OF A MULTI-FOCUSED PSYCHOSOCIAL REHABILITATION COURSE

Nina Rottmann, MSc, ${ }^{1}$ Hanne Würtzen, MSc, ${ }^{1}$ Susanne O. Dalton, MD, PhD,${ }^{1}$ Jane Christensen, MSc, ${ }^{1}$ Pernille E. Bidstrup, MSc, $\mathrm{PhD},{ }^{1}$ Kirsten Frederiksen, $\mathrm{MSc}, \mathrm{PhD}^{1}$ and Christoffer Johansen, MD, PhD, DMSc ${ }^{1,2}$

${ }^{1}$ Institute of Cancer Epidemiology, Copenhagen, Denmark and ${ }^{2}$ National Centre for Cancer Rehabilitation Research, University of Southern Denmark, Odense, Denmark.

Purpose: Worldwide, the number of cancer survivors is increasing. In the Danish population of 5.4 million people, more than 300000 are cancer survivors. Given the range of physical, psychological and social late effects of cancer and its primary treatment many survivors need tailored rehabilitation interventions. This randomized study evaluates the short term effect of a multi-focused, psychosocial 6-day residential rehabilitation course at the Dallund Rehabilitation Centre in Denmark.

Methods: Breast, prostate or colorectal cancer survivors were randomly assigned to the rehabilitation course (IG) or a standard care control group (CG). Outcome measures included self-reported quality of life (qol), psychological mood and mental adjustment styles and were assessed at baseline, 1-, 6- and 12-month follow up. Multiple linear regression was used in preliminary analyses to compare differences between IG and CG. Global qol at baseline, cancer diagnosis, age, gender and education were examined as confounders. Results: The preliminary analyses included 352 patients (IG $\mathrm{n}=$ 165 and CG $n=187)$. A total of 188 patients had breast cancer, 94 had colorectal cancer and 70 had prostate cancer. Participants were on average 61 years old and 228 were women. No effect of the intervention was found on change in global qol at 1-month follow up $(\beta=0.40 ; 95 \%$ CI $-2.95-3.74)$.

Conclusion: This is the first study to evaluate a residential rehabilitation course among cancer patients in a randomized design. Preliminary analyses could not confirm an effect of the intervention on qlobal qol. This might be due to methodological limitations of the study. However, the effect of the intervention on the different domains of quality of life and other outcome measures at the 6-and 12-month follow up taking into account clinical variables still needs to be examined.

CORRESPONDING AUTHOR: Nina Rottmann, MSc, Department of Psychosocial Cancer Research, Institute of Cancer Epidemiology, Copenhagen, 2100; rottmann@cancer.dk 
OS48

10:30 AM-10:48 AM

OS48-A

LONGTERM EFFECTS OF PRENATAL STRESS

ON PSYCHOBIOLOGICAL REACTIVITY TO ACUTE PSYCHOSOCIAL STRESS IN 10-YEAR OLD CHILDREN

Katja Erni, Msc, Luljeta Emini, Msc and Ulrike Ehlert, PhD

Clinical Psychology and Psychotherapy, University of Zurich, Zurich, Switzerland.

Prenatal stress seem to have a significant impacts on the biological and psychological development in the offspring. In particular, prenatal stress is associated with long-term disturbances in HPA axis function.A sample of 115 10-year old children was examined in a cross-sectional study design. The experimental group $(n=43)$ was characterized by a glucocorticoid treatment of their mothers due to high risk of preterm delivery during the second trimester. Control group A $(n=35)$ consisted of children who were at risk of being born prematurely but did not receive glucocorticoid treatment. In control group B $(n=37)$ children were included born after complication-free pregnancy. A standardized psychosocial stress test for children (TSST-C) was applied. All children were healthy and showed no disparities in their level of development. Salivary cortisol and heart rate were measured during the experiment as well as psychological parameters using self-report questionnaires (EWL-KJ and PASA). The TSST-C induced a marginally significant increase of salivary cortisol $(\mathrm{F}(4.814,269.601)=1.939, \mathrm{p}=.091)$. Significant higher cortisol levels were observed in children of the experimental group as compared to children of control group B. Referring to the data for the heart rate in response to the TSST-C a trend towards significance was revealed $(\mathrm{F}(15.069,836.313)=1.505, \mathrm{p}=.096)$. lower heart rates were found in The experimental group showed lower heart rates than the control groups A and B. Furthermore, stress appraisal and mood significantly differed between the three groups.Even though all children were comparable concerning their level of education and mental abilities, we found subtle differences in their psychobiological stress reactivity. The prenatally stressed offspring demonstrated a stronger glucocorticoid response in reaction to challenging situations than controls. In addition, the results revealed long term effects of prenatal glucocorticoids on psychological and on autonomic reactivity to a standardized psychosocial stress test.

CORRESPONDING AUTHOR: Katja Erni, Msc, Clinical Psychology and Psychotherapy, University of Zurich, Zurich, 8050; k.erni@psychologie.uzh.ch
10:48 AM-11:06 AM

OS48-B

DO MUM AND DAD GET ALONG? FAMILY CONFLICT AND HEALTH AND DEVELOPMENT IN EARLY CHILDHOOD: FINDINGS FROM THE UK MILLENNIUM COHORT STUDY

Yvonne Kelly, PhD, ${ }^{1}$ Anne McMunn, $\mathrm{PhD},{ }^{1}$ Mel Bartley, PhD, ${ }^{1}$ Noriko Cable, $\mathrm{PhD},{ }^{1}$ Amanda Sacker, $\mathrm{PhD}^{2}$ and Scott Montgomery, $\mathrm{PhD}^{3}$

${ }^{1}$ Department of Epidemiology and Public Health, University College London, London, United Kingdom; ${ }^{2}$ Institute for Social and Economic Research, University of Essex, Colchester, United Kingdom and ${ }^{3}$ Department of Medicine, Karolinska Institutet, Stockholm, Sweden.

Background: Previous work suggests that family conflict impacts adversely on child development. But it is not clear to what extent these relationships might hold in a contemporary population setting.

Objectives: 1. to examine the extent to which markers of parental conflict are associated with child development 2. to assess the influence of socioeconomic and psychosocial factors in observed relationships.

Methods: Data from the UK Millennium cohort study were used $(\mathrm{N}=9144)$. Questions on parental conflict - whether they felt they were on the brink of separation and on the experience of physical force/violence in the relationship were answered by both parents when cohort members were age 9 months, 3 and 5 years. Markers of child development at age 5 were height, socioemotional difficulties, and verbal ability.

Results: Girls and boys whose mothers felt they were on the brink of separation from their partner were shorter (girls 109.0 vs 110.3 , boys 110.9 vs $111.3 \mathrm{~cm}$ ), more likely to have socioemotional difficulties (girls 9.7 vs 4.1 , boys 15.7 vs $6.4 \%$ ) and lower verbal test scores (girls 55.5 vs 57.1 , boys 52.9 vs 56.5 ) compared with children where this was not the case. For girls height differences remain statistically different on adjustment for socioeconomic and psychosocial factors, whilst differences in socioemotional difficulties and verbal ability were rendered statistically nonsignificant on adjustment for psychosocial factors. For boys differences in the likelihood of socioemotional difficulties and verbal ability were reduced on adjustment for socioeconomic and psychosocial factors but remained statistically significant.

Conclusion: Children living in homes in which there was likely family conflict had poorer developmental outcomes compared with children living in more favourable circumstances. Gender differences in factors that explained observed patterns were apparent. 
CORRESPONDING AUTHOR: Yvonne Kelly, PhD, Department of Epidemiology and Public Health, University College London, London, E11 1BZ; y.kelly@ucl.ac.uk

\section{1:06 AM-11:24 AM \\ OS48-C}

RISK FACTORS FOR MULTIPLE PAINS IN CHILDREN UND ADOLESCENTS

Birgit Kröner-Herwig, PhD, Nuria Vath, PhD, Jnnifer Gassmann, $\mathrm{PhD}$ and Hester van Gessel, Msc Psychol

Psychology, University of Göttingen, Göttingen, Germany.

Objective: The aim of the study was to ascertain the prevalence of multiple pains (MP) and detect psychosocial risk factors assessed one year prior. Method MP was defined by the number of sites for which pain was reported to occur at least "sometimes" in a sample of 2219 German youths (10-17 years).

Results: More than one site of pain was reported by $37 \%$ of the children. Girls were much more prone to MP than boys. A regression model revealed that prior MP, age, gender, internalizing / externalizing symptoms, dysfunctional coping, and watching TV explained $33 \%$ of the variance: The contribution of the psychosocial factors to the model was small. Separate analyses for boys and girls displayed different risk factor profiles and a higher degree of predictability in girls. Conclusion: It is assumed that the variable MP represents a dispositional, rather stable trait conceived as pain vulnerability.

CORRESPONDING AUTHOR: Birgit Kröner-Herwig, PhD, Psychology, University of Göttingen, Göttingen, 37073; bkroene@ uni-goettingen.de

\section{1:24 AM-11:42 AM}

OS48-D

THE IMPORTANCE OF ADOLESCENCE FOR ADULT HEALTH INEQUALITIES

Pernille Due, MD, ${ }^{1}$ Rikke Krølner, PhD student, ${ }^{1}$ Mette Rasmussen, $\mathrm{PhD},{ }^{1}$ Mogens T. Damsgaard, associate prof, ${ }^{1}$ Hilary Graham, Professor $^{2}$ and Bjørn E. Holstein, Professor ${ }^{1}$

${ }^{1}$ National Institute of Public Health, University of Southern Denmark, Copenhagen, Denmark and ${ }^{2}$ Department of Health Sciences, University of York, York, United Kingdom.

Most lifecourse research concentrates on how early life factors influence adult health through processes enacting within and across generations. Our paper makes the case for the inclusion of adolescence as a distinct stage in life course studies. We introduce a model to capture the most important pathways and mechanisms working over adolescence to develop adult health inequalities, and review and include relevant literature to illustrate the evidence for these pathways and mechanisms.

For each of the four pathways: the health-, the health behaviour-, the relational- and the educational pathway, the paper investigates whether indicators are socially patterned in adolescence, whether they track into adulthood, and whether any socially differential tracking or -vulnerability has been investigated. The paper focuses on examples of indicators from each pathway that is prevalent and/or have serious implications for later life: pains, overweight, depression, low physical activity, low fruit/vegetable intake, smoking, alcohol consumption (behavioural indicators), lone parenting, bullying victimization (relational indicators) and education.

Most health indicators are socially patterned in adolescence and track into adulthood, with higher risks of adverse outcomes among individuals from lower socioeconomic positions. Adolescent health behaviours track into adulthood. Smoking, physical activity and especially fruit and vegetable intake are socially patterned, while evidence for social patterning of alcohol-use is less consistent. Lone parenthood and bullying are socially patterned and track over time, and there are indications of a socially differential vulnerability to the effects of these types of relational strain.

Very little research has investigated the social patterning over time or studied social vulnerability of most of the above indicators from adolescence to adulthood. However, all four mechanisms: socially differential exposure, tracking, socially differential tracking and socially differential vulnerability seem to be active in establishing social differences in adult educational attainment.

CORRESPONDING AUTHOR: Pernille Due, MD, National Institute of Public Health, University of Southern Denmark, Copenhagen, 1353; pdu@niph.dk

\section{Saturday August 7, 2010 SYMPOSIA SESSIONS 2:30 PM-4:00 PM}

\section{SS32 \\ HEALTH OUTCOMES IN CHILDREN AFFECTED BY TRAUMA}

Annette M. LaGreca, PhD

University of Miami, Coral Gables, FL.

In recent years, there has been increased attention to the impact of trauma on children and youth. Evidence suggests that devastating natural disasters (such as hurricanes and bushfires) (La Greca et al. 1996; Yelland et al., in press), injuries resulting from motor vehicle and other types of accidents (Meiser-Stedman et al., 2008), and life-threatening medical conditions such as cancer (Phipps, Jurbergs \& Long, 2009) are associated with the development posttraumatic stress (PTS) symptoms and disorder (PTSD) in children and adolescents. Although existing research has focused on mental health reactions of child trauma victims, very little attention has been devoted to the health effects of traumatic events. This is especially surprising, in that stress has long been known to affect immune functioning and susceptibility to disease. Nevertheless, there has been recent interest in the health effects of children's trauma exposure (Fairbank \& Fairbank, 2009), and this symposium will address this issue. The three papers in the 
symposium address the impact of traumatic stressors on child health. Each of the papers in the symposium focuses on different traumatic stressors and their potential health effects. The first paper by Kenardy, Anderson, and Le Brocquehus focuses on the predictive value of posttraumatic stress on health outcomes in a large sample of children with mild to severe traumatic brain injury. The second paper by La Greca and colleagues will present two recent studies that examined the impact of destructive natural disasters (hurricanes) on children's somatic symptoms. Finally, the third presentation by Llabre and Hadi presents data on two studies of the psychological and health consequences of war exposure on children. All authors will discuss the implications of their findings for assessing children affected by traumatic events.

CORRESPONDING AUTHOR: Annette M. LaGreca, PhD, University of Miami, Coral Gables, FL, 33124; alagreca@miami.edu

\section{SS32a}

THE IMPACT OF NATURAL DISASTERS ON CHILDREN'S PHYSICAL HEALTH

Annette M. LaGreca, PhD, ${ }^{1}$ Betty Lai, MS, ${ }^{1}$ Beth A. Auslander, $\mathrm{PhD},{ }^{3}$ Mary Short, PhD ${ }^{2}$ John F. Thomas, $\mathrm{PhD},{ }^{3}$ Whitney Herge, MS, ${ }^{1}$ Cortney Taylor, $\mathrm{MS}^{1}$ and Wendy K. Silverman, $\mathrm{PhD}^{4}$

${ }^{1}$ University of Miami, Coral Gables, FL; ${ }^{2}$ University of Houston Clear Lake, Clear Lake, TX; ${ }^{3}$ University of Texas Medical Branch, Galveston, TX and ${ }^{4}$ Florida International University, Miami, FL.

Natural disasters have a significant negative impact on children's psychological adjustment. However, little research has examined the health consequences of disasters. This presentation focuses on two studies of the impact of hurricane exposure on children's somatic symptoms. Study 1 examined Hurricane Charley (2004, Category 4); Study 2 examined Hurricane Ike (2008, Category 2). Study 1 included 385 children ( $55 \%$ girls; $M$ age $=8.7$ years). At 9 months postdisaster, children completed measures of hurricane exposure, life events, and PTSD; 21-months postdisaster children reported ongoing stressors, life events, and somatic symptoms. Hierarchical regressions revealed that hurricane experiences accounted for $7.2 \%$ of the variance in somatic symptoms at Time $2(\mathrm{p}=.001)$. Time 1 PTSD also predicted Time 2 somatic symptoms $(p=.001)$, but hurricane experiences were not significant when PTSD symptoms were included. Major life events added to the prediction of Time 2 somatic symptoms $(\mathrm{p}<.001)$. Study 2 included 328 children ( $50 \%$ girls; $M$ age $=8.72$ years) who completed similar measures 9 months post-disaster. Using regression to predict somatic symptoms, demographics were entered first ( $8 \%$ of the variance, $\mathrm{p}<.01)$; then hurricane-exposure $(14 \%$ of the variance, $\mathrm{p}<.001)$; and finally children's PTSD symptoms $(16 \%$ of the variance, $\mathrm{p}<.001)$. In the final step, only PTSD symptoms, child's age $(\beta=-.15)$ and stressors occurring during the hurricane $(\beta=.11)$ remained significant.

These studies are the first to examine health outcomes in children exposed to natural disasters. Children exposed to destructive disasters and who experience disaster-related stressors report significant somatic symptoms 9 and 21 months postdisaster. Findings suggest that children exposed to natural disasters may be vulnerable in terms of their physical health as well as mental health. Further attention the health status of children exposed to natural disasters is important in to address.

CORRESPONDING AUTHOR: Annette M. LaGreca, PhD, University of Miami, Coral Gables, FL, 33124; alagreca@miami. edu

\section{SS32b}

PSYCHOLOGICAL AND HEALTH CONSEQUENCES

OF EXPOSURE TO WAR IN CHILDHOOD

Maria Llabre, $\mathrm{PhD}^{1}$ and Fawzyiah Hadi, $\mathrm{PhD}^{2}$

${ }^{1}$ University of Miami, Coral Gables, FL and ${ }^{2}$ Kuwait University, Kuwait City, Kuwait.

Two studies, conducted in the Middle East, document the psychological and health consequences of war exposure in childhood. One study, conducted in Lebanon after the LebaneseIsraeli war in the summer of 2006, included a population-based sample of over 6000 children in grades 1 through 12 who were assessed for multiple exposures, prior exposure, sociodemographic characteristics, and symptoms of posttraumatic stress (PTS). The prevalence of PTS symptoms was estimated at $27.7 \%$ in children who were in grades 1 to 5 and at $26.4 \%$ in children and adolescents in grades 6 to 12 . Specific symptom clusters had higher prevalence. Between 41-53\% (depending on age) reported difficulty in falling and staying asleep. Older age was associated with increased symptoms. Past and concurrent exposures to warrelated events were the strongest predictors of PTS symptoms. The other study included 151 Kuwaiti children who were 7-10 years old during the first Gulf war, selected for participation based on their level of exposure to war-related events. Participants were assessed two years after the war and again as young adults (1923 years old). As adults they reported on their general health, medical diagnoses, and sleep habits. At both assessments they reported on symptoms of posttraumatic stress, depression, and anxiety. A structural model supported the notion that psychological distress mediates the effect of war exposure on self-rated health, quality of sleep, and obesity.

The session will conclude with a brief discussion with all presenters participating

CORRESPONDING AUTHOR: Maria Llabre, PhD, University of Miami, Coral Gables, FL, 33124; mllabre@miami.edu

$\mathrm{SS} 32 \mathrm{c}$

DOES POSTTRAUMATIC STRESS PREDICT HEALTH OUTCOMES IN CHILDREN WITH TRAUMATIC BRAIN INJURY?

Justin Kenardy, $\mathrm{PhD},{ }^{1}$ Vicki Anderson, $\mathrm{PhD}^{2}$ and Robyne Le Brocque, $\mathrm{PhD}^{1}$

${ }^{1}$ CONROD, University of Queensland, Herston, QLD, Australia and

${ }^{2}$ Murdoch Children's Research Institute, Parkville, VIC, Australia. 
Research on Iraq War veterans who have experienced traumatic brain injuries has found that posttraumatic stress mediated the effect of the brain injuries on health outcomes. The aim of this paper is to explore the predictive value of posttraumatic stress on health outcomes in children with mild to severe traumatic brain injury (TBI).

204 children aged 6 to 14 years were recruited after admission to hospital for TBI. Participants were followed before 2 months and at 3 , 6,12 , and 18 months post injury. Posttraumatic stress was identified via a structured clinical interview (CAPS-CA). Health outcomes were assessed using the Child Health Questionnaire (CHQ).

The presence of PTSD was found to be unrelated to the severity of the TBI. After accounting for the severity of the TBI, PTSD was associated with longer recovery times over the 18 months as assessed by physical outcomes. Of greater concern PTSD was associated with a lack of recovery as assessed by psychosocial outcomes. This has significant implications for the assessment and rehabilitation of pediatric TBI.

CORRESPONDING AUTHOR: Justin Kenardy, PhD, CONROD, University of Queensland, Herston, QLD, 4006; j.kenardy@uq. edu.au

\section{SS34}

SUBJECTIVE HEALTH COMPLAINTS, MODERN HEALTH WORRIES AND COPING

Camilla Ihlebaek, $\mathrm{PhD},{ }^{2,3}$ Kirsti Malterud, $\mathrm{MD}, \mathrm{PhD},{ }^{6}$ Keith Petrie, $\mathrm{PhD},{ }^{1}$ Maria J. Figueiras, $\mathrm{PhD}^{5}$ and Hege R. Eriksen, $\mathrm{PhD}^{3,4}$

${ }^{1}$ University of Auckland, Auckland, New Zealand; ${ }^{2}$ Research group for Nature, Health and Quality of life, Norwegian University of Life Sciences, Aas, Norway; ${ }^{3}$ Stress, Health and Rehabilitation, Uni health, Bergen, Norway; ${ }^{4}$ Hemil, Research Centre for Health Promotion, University of Bergen, Bergen, Norway; ${ }^{5}$ ISEIT, Instituto Piaget, Almada, Portugal and ${ }^{6}$ Research Unit for General Practice, Uni health, Bergen, Norway.

Subjective health complaints, modern health worries and coping. Camilla Ihlebæk

Terms like functional somatic illnesses, unexplained medical symptoms, and somatization disorders have been used on a variety of combinations of multiple symptoms, with diagnostic labels such as fibromyalgia, irritable bowel syndrome, multiple chemical sensitivity, chronic fatigue syndrome, and chronic whiplash syndrome. These conditions are described by common subjective health complaints such as musculoskeletal pain, gastrointestinal discomfort, tiredness, fatigue, allergic and respiratory complaints, depression, and anxiety. Subjective health complaints are common in the general population. For most people the complaints are minor everyday nuisance, however for some it becomes substantial and is given a diagnostic label and professional help is needed. An important question is why and to whom this happens. A possible mechanism for such multiple symptoms could be somatic and cognitive sensitization. Furthermore, two cognitive mechanisms that seem to be closely linked to the process of sensitization are worrying and coping. There is an increasing interest of health in media and in the population. This might lead to worries concerning features of modern life and its affect on health. Worrying could play a role by enhancing biased memory and interest of health related information, leading to sensitization. Therefore, modern health worries could be associated with health complaints and medically unexplained symptoms. Individuals that uses active problem solving coping strategies have been found to have lower levels of subjective health complaints than others in stressful work situations. An essential condition for coping to occur is that the individual expects a positive result of the handling of a stressful situation, i.e. positive response outcome expectancy. The result of no control of the outcome would be learned helplessness and negative response outcome expectancy could be defined as hopelessness. Both hopelessness and helplessness may lead to depression and multiple subjective health complaints. In this symposium the relationships between subjective health complaints, modern health worries and coping will be discussed.

CORRESPONDING AUTHOR: Camilla Ihlebaek, PhD, Research group for Nature, Health and Quality of life, Norwegian University of Life Sciences, Aas, 1432; camilla.ihlebak@umb.no

\section{SS34a}

THE RELATIONSHHIP OF MODERN HEALTH WORRIES TO DEPRESSION AND SYMPTOM REPORTING IN THE GENERAL POPULATION

Keith Petrie, $\mathrm{PhD},{ }^{1}$ Vera Baehr, $\mathrm{MHS}^{1}$ and Winfried Rief, $\mathrm{PhD}^{2}$

${ }^{1}$ University of Auckland Medical School, Auckland, New Zealand and ${ }^{2}$ Philipps University, Marburg, Germany.

Background: Worries about environmental and technical changes affecting people's health have been shown be associated with the use of health care services, health behaviours, mood and reporting of physical symptoms.

Aim: In a representative sample of the German population we examined the relationship of modern health worries to demographic factors. depression, symptom reporting and health-related quality of life.

Methods: 2,524 participants of a representative sample of the German completed the Modern Health Worries Scale, Depression and Somatization Module of the Patient Health Questionnaire, as well as the SF-12 health-related quality of life scale. Socioeconomic status and demographic information were also gathered.

Results: Higher levels of modern health worries were found in females but were not associated with income, age or education. Higher levels of modern health worries were significantly associated with depression, symptom reporting and lower healthrelated quality of life. Hierarchical regression analyses show depression to mediate the relationship between modern health worries and symptom reporting.

Conclusions: In a large general population modern health worries were higher in females but unrelated to other demographic factors. Depression seems to play a more important role than modern health worries in explaining symptom reporting.

CORRESPONDING AUTHOR: Keith Petrie, PhD, University of Auckland Medical School, Auckland, 10024; kj.petrie@auckland. ac.nz 


\section{SS34b}

HEALTH INTEREST, MODERN HEALTH WORRIES

AND HEALTH COMPLAINTS IN THE GENERAL POPULATION

Camilla Ihlebaek, $\mathrm{PhD}^{1,2}$ and Hege R. Eriksen, $\mathrm{PhD}^{2,3}$

${ }^{1}$ Research group for Nature, Health and Quality of life, Norwegian University of Life Sciences, Aas, Norway; ${ }^{2}$ Stress, Health and Rehabilitation, Uni Health, Bergen, Norway and ${ }^{3} \mathrm{Hemil}$, Research Centre for Health Promotion, University of Bergen, Bergen, Norway.

Background: There is an increasing focus and interest of health in media and in the population. The high level of interest and information could lead to worries concerning features of modern life and its affect on health. Worrying could play a role by enhancing biased memory and interest of health related information, leading to sensitization and subjective health complaints.

Aim: To examine the relationship between health interests, modern health worries (MHW) and subjective health complaints (SHC) in the general population.

Methods: A representative sample $(n=1000)$ of the general Norwegian population completed a telephone interview in 2008 about their interest in health and health information, MHW and SHC. Stepwise multiple regression analysis was used to investigate the association between variables.

Results: In the general population $12 \%$ reported not to be interested in health information, $41 \%$ were a little interested, $29 \%$ were moderately interested, and $18 \%$ were very interested. Women were more interested than men $(p<0.000)$ and higher educated were more interested than lower educated $(\mathrm{p}<0.000)$. There were no age differences in health interest. The most common MHW were pesticides in food (76\%), additives in food $(73 \%)$, drug resistant bacteria (71\%), climatic changes $(69 \%)$, air pollution (68\%) and depletion of the ozone layer (68\%). The most common SHC were tiredness (43\%), LBP (38\%), shoulder pain $(33 \%)$, headache $(31 \%)$, sleep disturbances $(31 \%)$, and allergic complaints $(26 \%)$. High levels of SHC and MHW were significantly associated with high interest in health information. There was a significant association between MHW and SHC, even after controlling for gender, age, education and health interest. Conclusion: In the population there seems to be an association between SHC, MHW and health interest. Individuals with high levels of complaints have high levels of worries, and are more interested in health information than the rest of the population. This could have implications for how this group should be informed and met by the health care system.

CORRESPONDING AUTHOR: Camilla Ihlebaek, PhD, Research group for Nature, Health and Quality of life, Norwegian University of Life Sciences, Aas, 1432; camilla.ihlebak@umb.no

$\operatorname{SS} 34 \mathrm{c}$

IDENTITY AND COPING IN PATIENTS WITH CHRONIC FATIGUE SYNDROME (CFS)

Kirsti Malterud, MD PhD and Lillebeth Larun, MPH

Research Unit for General Practice, Uni Health, Bergen, Bergen, Norway.
A metaanalysis of 20 qualitative studies about CFS experiences demonstrated how symptoms and responses from significant others could jeopardise the patients' senses of identity. While patients felt severely ill, they experienced blame and dismissal. The findings illustrated how patients' beliefs and causal attributions may oppose the doctor's understanding of the condition. For the patient, getting a diagnosis and knowing more was necessary for recovery. Doctors were reluctant towards the diagnosis, and struggled to maintain professional authority. For patients, experience of discreditation could lead to withdrawal and disengagement. Identity is challenged when the legitimacy of the illness is questioned. This burden adds to a loss of previously established identity and makes the patient vulnerable beyond symptom perception. CFS patients cope with their condition by trying to know more, by keeping a distance to protect themselves and learning more about their limits. Doctors can support patients' coping by supporting the strong sides of the patients instead of casting doubt upon them. Furthermore, common ground for understanding can be enhanced by presenting disease models suggesting mechanisms of what happens in the body in CFS, such as sustained arousal with subsequent symptom maintenance. The Cognitive Activation Theory of Stress provides a framework for negotiating the impact of stimulus and response expectations and transcends the vicious circles of sensitization. The presentation will demonstrate how patient perspectives and disease models can be connected.

CORRESPONDING AUTHOR: Kirsti Malterud, MD PhD, Research Unit for General Practice, Uni Health, Bergen, Bergen, N-5018; kirsti.malterud@isf.uib.no

\section{SS34d}

MODERN HEALTH WORRIES AND SUBJECTIVE HEALTH COMPLAINTS IN A SENIOR POPULATION: A PILOT STUDY

Maria J. Figueiras, $\mathrm{PhD},{ }^{1}$ Jorge C. Jesuino, Emeritus Professor, ${ }^{2}$ Teresa Cruz, Bsc ${ }^{1}$ and Jennifer Fazendeiro, Bsc ${ }^{1}$

${ }^{1}$ Psychology, Instituto Piaget - ISEIT, Almada, Portugal and ${ }^{2}$ ISCTE-IUL, Lisboa, Portugal.

Background: to explore the perceptions of subjective health complaints (SHC) when modern health worries (MHW) are considered, and to characterize the level of worry concerning features of modern life in a sample of Portuguese senior citizens. Methods: Longitudinal study in which 49 retired participants (both sexes) completed an anonymous questionnaire at two time points (baseline and six weeks later), measuring SHC, MHW, personality measures and socio-demographic information.

Findings: Participants presented an average level of subjective health complaints (musculoskeletal pain) which increased significantly after the introduction of the modern health worries scale. Discussion: There is little research about this topic in older people. However the results are suggestive that features of modern life are likely to influence perceptions of health in senior citizens which may lower the threshold for seeking care and influence health outcomes in this age group. 
CORRESPONDING AUTHOR: Maria J. Figueiras, PhD, Psychology, Instituto Piaget - ISEIT, Almada, 2800-305; mfigueiras@, almada.ipiaget.org

\section{Saturday \\ August 7, 2010 \\ PAPER SESSIONS \\ 2:30 PM-4:00 PM}

OS49

\section{2:30 PM-2:48 PM}

OS49-A

THE EFFECTIVENESS OF AN ADVANCED DEVELOPMENT PROGRAMME FOR CLINICIANS IN SELF MANAGEMENT FOR LONG TERM CONDITIONS IN THE UK

Louise M. Wallace, Professor, Joanna Kosmala-Anderson, $\mathrm{PhD}$ and Andy Turner, PhD

Applied Research Centre for Health and Lifestyle Interventions, Coventry University, Coventry, United Kingdom.

Background: 3 workshops were offered to 367 clinicians working with patients with long term conditions (LTC) (diabetes, COPD, depression and chronic musculoskeletal pain) as a part of The Health Foundation's Co Creating Health Initiative. Workshops teach techniques that impact on the clinician-patient relationship and support patient self-management. The ADP is co delivered by clinical and a lay tutor who has a LTC.

Aims: Assess the overall effectiveness of the ADP in improving clinicians' self management support practices.

Method: 78 clinicians responded, before and after attending the ADP. Self management support (SMS) practices was assessed by the Practices in Self Management Support questionnaire (PSMS) developed by the authors. The questionnaire comprises 3 subscales assessing Clinical Self Management Support (CSMS), Patient Centeredness (PC) and Organization of Services to Support Self Management (OSMS). We also interviewed 14 completers asking them about their experience of ADP.

Results: Paired T Test results showed that after completing ADP clinicians significantly increased $\mathrm{PC}$ practices $(\mathrm{t}=-1.9 ; \mathrm{df}=75 ; \mathrm{p}=$ 0.05). CSMS and OSMS did not change.

Interview data showed that the most useful practices were agenda setting, goal setting, exploring ambivalence and assessing patient's confidence to change. Time restrictions and lack of systems in place to support self management on organizational level are considered the biggest barriers for SMS.

Conclusion: ADP training effectively builds clinicians' SMS practices. Further research is necessary to establish whether applying those practices in clinical consultations influence patients' engagement in self managing their long term condition.

CORRESPONDING AUTHOR: Joanna Kosmala-Anderson, PhD, ARCHLI, Coventry University, Coventry, CV1 5FB; j.kosmalaanderson@coventry.ac.uk

\section{2:48 PM-3:06 PM}

OS49-B

STRATEGIC WORK-PLACE MALE SUPPORT IN UPTAKE OF WOMEN'S BREAST CANCER SCREENING:

TRANSLATION OF ADVOCACY RESEARCH

INTO ORGANISATIONAL \& INDIVIDUAL CHANGE

Yut Lin Wong, DrPH, Li Ping Wong, PhD, Eng Suan Ang, MBBS and Wah Yun Low, PhD

MERDU, Faculty of Medicine, University of Malaya, Kuala Lumpur, Malaysia.

A husband's neglecting/abandoning her if diagnosed with breast cancer is a woman's greatest fear and barrier to screening/early detection, a major reason for rising breast cancer morbidity and mortality in Malaysia. Thus, MENCARE!, focusing on men advocates to empower women for breast cancer screening, has been implemented.

MENCARE! trains men working in public and private corporations to provide support for women's breast cancer screening. Project effectiveness was evaluated by assessing changes in policies and procedures in one public agency and changes in men's knowledge, attitudes on breast cancer, and actions to support women's breast cancer screening, using multistage pre \& post advocacy evaluation design with mixed Methods: One month and one year post-training evaluation of 75 male advocates through questionnaire surveys and 3 focus group discussion (FDG) were conducted. In-depth interviews were conducted with 5 management personnel pre and one-year post advocacy.

Organisationally, while existing health package within the agency still did not include breast cancer screening, it would support all cancer treatment $100 \%$ at the one year post-advocacy. The flexible time-off procedure for male staff needing to support spouse/family for breast cancer screening continued a year later. "Many of them have gone for screening" was observed by one personnel interviewed one year post-advocacy. Request for a mobile mammography clinic on-site was made by management a year later. Individually, the training increased male staff's knowledge of breast health, cancer and screening in certain though not all aspects. The training increased their willingness to be involved in all types of support for women: to discuss/motivate women to get early and complete treatment, to prepare types and levels of support. Yet, men expressed some problems: lack of awareness/ exposure to what is commonly regarded as a women's disease/ problem; embarrassed to talk about women's breasts; lack of screening facilities and medical insurance, especially in rural areas; and men's busy work schedule.

Buying in of public or private corporations and targeting male support at the work-place appears strategic in addressing women's delayed or low uptake of breast cancer screening through changes in organisational policies and procedures as well as individual changes in knowledge, attitudes and support actions.

CORRESPONDING AUTHOR: Yut Lin Wong, DrPH, MERDU, Faculty of Medicine, University of Malaya, Kuala Lumpur, 50603; wongyl@um.edu.my 
3:06 PM-3:24 PM

OS49-C

DEMONSTRATION AND TRANSITION AT SCALE: TRANSLATING EFFECTIVE HIV PREVENTION INTO NATIONAL POLICY IN INDIA

Maria A. May, BA, Rebecca L. Weintraub, MD and Claire B. Cole, MPH

Global Health Delivery Project, Harvard University, Boston, MA.

Once a health intervention is proven effective at a technical level, the steps required to generate a delivery system that produces positive health outcomes are nontrivial and poorly understood. For governments, the political environment often precludes experimentation or data-driven decision-making at the program level. Particularly in HIV, the political processes often fail to serve those most at risk, the socially marginalized.

We explore the Bill and Melinda Gates' Foundation's novel approach of building a large-scale HIV prevention program in India, called the Avahan Initiative, through partnerships with NGOs, small-scale delivery experiments and rigorous data collection, to then transition to the government, to understand its effectiveness as a strategy to yield scientific programming and policy nationally.

In 2009, we interviewed over 70 stakeholders, including key decision-makers at the government, Avahan, and implementing NGOs. Fieldwork also included site visits to various programs and interactions with community members. Data included costing and organizational level metrics.

Commonly mentioned distinctions between Avahan's approach and that of other aid organization and the public sector, include: depth and application of data, capacity building of local NGOs, financing mechanisms, and intensiveness of technical assistance. Avahan's decisions around how to interact with other key players in HIV in India featured prominently in discussions around sustainability. Many of Avahan's strategies, metrics and programmatic structures have been adopted as national guidelines. Assessments of the feasibility and logic of the transition strategy varied widely across stakeholders.

Though it is premature to assess the success of Avahan's transition strategy, our research suggests that it achieved and maintained an effective HIV prevention program and has influenced governmental policy significantly. Large-scale NGOs may be well positioned to conduct iterative delivery experiments and ensure that governments adopt and promote robust public health models.

CORRESPONDING AUTHOR: Maria A. May, BA, Global Health Delivery Project, Harvard University, Boston, MA, 02120; maria_may@hms.harvard.edu

\section{3:24 PM-3:42 PM}

OS49-D

ENFORCEMENT OF THE BAN ON SMOKING IN PUBLIC PLACES

Orna Baron-Epel, PhD MPH, ${ }^{1}$ Carmit Satran, MPH, ${ }^{1}$ Anat DrachZahavy, $\mathrm{PhD}^{1}$ and $\mathrm{S}$. Katharine Hammond, $\mathrm{PhD}, \mathrm{CIH}$ Professor ${ }^{2}$

${ }^{1}$ School of Public Health, Haifa University, Haifa, Israel and

${ }^{2}$ School of Public Health, University of California Berkeley, Berkeley, CA.
Background: Secondhand smoke (SHS) is a major preventable cause of death and disease. Many countries have enacted laws banning smoking in public places as part of their strategy to decrease the ill effects of smoking. In some countries this strategy appears to be successful in reducing smoking and in reducing levels of non-smoker's exposure to SHS. In recent years some countries have banned smoking in pubs and bars, the last public venues where it was still allowed. Subsequent studies measuring SHS have shown dramatic drop in SHS following the ban. In 1983, a law banning smoking in public places in Israel was enacted and amendments to the basic law were added over the years. The latest amendment came into effect in 2007, adding pubs and bars to the list of public places in which it is prohibited to smoke.

Objective: To measure the adherence of bars and pubs with the law banning smoking in them, and to identify variables that may predict adherence to the law.

Methods: This study includes data collection from two sources. (1) Passive airborne nicotine monitoring devices used to measure indoor SHS in pubs and bars in about 20 Jewish-Israeli cities. (2) Face-to-face interviews with bar owners were conducted to identify variables predicting implementation of the law.

Results: Two years after the Israeli smoke-free law has come into effect levels of SHS in most of the pubs and bars remains relatively high, however, in some bars the ban is implemented. Smoking and SHS is higher in bars and pubs in cities where the city-hall does not enforce the law by sending inspectors to inspect bars and pubs and give fines. Bar owners respond directly to the chances of receiving a fine.

Conclusions: Although Israeli non-smoking legislation is progressive, enforcement is lacking, and smoking in public places still exists. Law enforcement is the responsibility of the local authorities, however, not enough has been done to enforce the law and city-halls should be encouraged to enforce the law more successfully as this has a profound effect on SHS in the pubs.

CORRESPONDING AUTHOR: Orna Baron-Epel, PhD MPH, School of Public Health, Haifa University, Haifa, 31905; ornaepel@research.haifa.ac.il

\section{OS50}

\section{2:30 PM-2:48 PM}

OS50-E

STRESSFUL LIFE EVENTS AND THE RISK OF IHD. THE ROLE OF INDUCTION TIME AND ACCUMULATION? Ingelise Andersen, $\mathrm{PhD},{ }^{1}$ Finn Diderichsen, Dr Med Sci, ${ }^{1}$ Eva Prescott, DrMedSci ${ }^{2}$ and Naja H. Rod, $\mathrm{PhD}^{1}$

${ }^{1}$ Social Medicine, Copenhagen University, Copenhagen K, Denmark and ${ }^{2}$ Copenhagen City Heart Study, Bispebjerg Hospital, Copenhagen, Denmark.

Background: Stress is a consequence of different types of stressors that all separately are associated with IHD. It has not been investigated whether the impact of stress on IHD is similar for all kinds of stressors or whether accumulation of stressors increases the risk of IHD/MI. 
Objective: To study the effect of individual and cumulative psychosocial stressors on the risk of incident IHD and MI among men and women.

Material: In 1991-3 a random sample of 9,599 participants, $57 \%$ women, from the Copenhagen City Heart Study answered a range of questions on major life events (LE) in childhood, adulthood and at work, vital exhaustion, lack of social network and daily use of psychotropic medication and were followed in a nationwide hospital discharge register until 2007.

Results: During follow-up 257 experienced an event of MI and 714 an event of IHD. The hazard ratio of IHD among those exposed to life events both in adulthood and at work was HR 1.25 (CI 95\% 0.9-1.7), whereas no association was found between life events in childhood, adulthood or work-related, respectively, nor for all three types of LE accumulated. The association between Vital Exhaustion $\geq 10$ and IHD was HR 1.79 (1.3-2.4), and people lacking a close confident had a HR of IHD of 1.73 (1.0-2.9). We found no association with MI. By means of logistic regression we found an association between accumulated LE in child-, adulthood and work-related and Vital Exhaustion $\geq 10$ with an OR 11.9 (CI 95\% 7.8-18). The association between accumulated LE and lack of a close confident was OR 4.52 (2.2-9.2). We were unable to elucidate any gender difference.

Conclusion: In this population-based cohort study we found that neither Individual nor accumulated LE are associated with IHD/MI, but are associated with other psychosocial risk factors of IHD/MI.

CORRESPONDING AUTHOR: Ingelise Andersen, PhD, Public Health, Copenhagen University, Copenhagen K, 1014; inan@ sund.ku.dk

\section{2:48 PM-3:06 PM}

OS50-B

IS FAMILY HISTORY'S RISK FOR CORONARY HEART DISEASE INCIDENCE MEDIATED BY PREVENTABILITY BELIEFS AND MODIFIABLE RISK FACTORS?

Maya R. Korin, $\mathrm{PhD},{ }^{1}$ William F. Chaplin, $\mathrm{PhD},{ }^{2}$ Mary-Jane Ojie, $\mathrm{BA},{ }^{2}$ Mark J. Butler, BA, ${ }^{2}$ Jonathan A. Shaffer, $\mathrm{PhD}^{1}$ and Karina W. Davidson, $\mathrm{PhD}^{1}$

${ }^{1}$ Medicine, Columbia University Medical Center, New York, NY and ${ }^{2}$ Psychology, St. John's University, Jamaica, NY.

Objectives: To test if health beliefs mediate the relation between family history of coronary heart disease (CHD) and 10-year CHD incidence in a large population-based sample of Canadians ( $\mathrm{n}=$ 2,688), controlling for established CHD risk factors. Background: CHD is the leading cause of death in the United States today, with family history $(\mathrm{FH})$ long known as an important risk factor. We tested the hypothesis that those who believe that CHD is not preventable, possibly because they have a positive $\mathrm{FH}$, do not alter their health behavior (in this case exercise) to be cardioprotective. Methods: Cox proportional hazards models were used to estimate the adjusted hazard ratios (HR) of FH, preventability belief, and exercise for incident CHD. We first constructed a baseline Cox proportional hazards regression model of the association between incident $\mathrm{CHD}$ and $\mathrm{FH}$ controlling for age at baseline and
Framingham risk score (an index of standard CHD risk factors). We then constructed a second Cox proportional hazards regression model to test if preventability belief was related to $\mathrm{FH}$ and a third model to test if belief could be viewed as a mediator of the FH-CHD relation. Finally we evaluated if the preventability belief-CHD relation was itself mediated by exercise .Results: Preventability belief was correlated with family history for both genders $(\beta=.724,95 \%$ confidence interval $[\mathrm{CI}] .592-.885, \mathrm{p}<.005)$, with those that had a family history of heart disease less likely to believe that heart disease can be prevented. Preventability belief was also found to be a mediator between family history and CHD, but for women only $(\beta=2.51,95 \%$ CI 1.65-3.80, $\mathrm{p}<.001)$. Finally exercise mediated this relation, again for women only $(\beta=-.868$, $\mathrm{HR}=.420,95 \% \mathrm{CI}(\mathrm{HR}) 0.770-0.907, \mathrm{p}<.001)$ Conclusion: Health belief in the preventability of CHD was found to be an important mediator in the relation between family history of CHD and incident CHD in women because of its effect on exercise.

CORRESPONDING AUTHOR: Maya R. Korin, PhD, Medicine, Columbia University Medical Center, New York, NY, 10012; mr2035@columbia.edu

\section{3:06 PM-3:24 PM \\ OS50-C}

\section{SOMATIC SYMPTOMS OF DEPRESSION AND IL-6 LEVELS} IN HEART FAILURE

Laura Redwine, PhD, Suzi Hong, PhD and Paul J. Mills, PhD Psychiatry, University of California, San Diego, San Diego, CA.

Heart failure (HF) affects millions of individuals and is increasing in prevalence. Close to a third of HF patients exhibit clinical levels of depressive symptoms. In turn, depression is associated with greater morbidity and mortality in HF. The mechanisms linking depression and worse outcome in HF are unclear. One suggested pathway links depression with heightened inflammation that may then be related to undesirable cardiac remodeling in HF. However, there have been mixed results in the literature connecting depression with inflammation in cardiac patients. Methods: The present study attempts to further delineate relations between depression and inflammatory markers in HF patients by using an exercise task to unmask group differences. Further, components of depression were examined separately to determine if inflammatory markers were differentially associated with somatic or cognitive depression symptoms. 40 NYHA II-IV HF patients and 35 healthy controls performed a moderate 20-min bicycle exercise task and completed the Beck Depression Inventory (BDI), sub-categorized into somatic (BDI-s) and cognitive (BDI-c) symptoms. Plasma levels of IL- 6 were measured at baseline, $10 \mathrm{~min}$ and $30 \mathrm{~min}$ after exercise. Repeated measures ANCOVA were performed, controlling for age, gender, body mass index and physical function. Results: Both HF patients and controls with elevated BDI-s exhibited higher plasma levels of IL- 6 at baseline, which remained elevated until 30 min post- exercise task (time x BDI-s effect, $\mathrm{F}(8$, $65)=4.16, p=.045$ ). Meanwhile, those with lower BDI-s had reduced IL-6 levels across the entire period. In contrast, elevated BDI-c symptoms were associated with low IL- 6 at baseline in both 
HF and non-HF groups, but then an increase in IL-6 in the non-HF controls by 30 min post- exercise (group $\mathrm{x}$ time $\mathrm{x}$ BDI-c effect, $\mathrm{F}(8,65)=7.6, \mathrm{p}=.007)$. Conclusions: Elevated IL-6 levels until 30-min post task were associated with heightened somatic symptoms of depression, whereas HF patients with elevated cognitive symptoms had lower levels of IL-6 through 30 minpost exercise. By further understanding the components of depression associated with elevated inflammatory markers, interventions may be developed to specifically address symptoms of depression that may worsen cardiac outcomes.

CORRESPONDING AUTHOR: Laura Redwine, PhD, Psychiatry, University of California, San Diego, San Diego, CA, 92161; lredwine@ucsd.edu

\section{3:24 PM-3:42 PM}

OS50-D

\section{RISK OF MULTIPLE PSYCHOSOCIAL FACTORS} ON STROKE MORTALITY IN OLDER ADULTS

Kimberly M. Henderson, Bachelor of Arts, ${ }^{1}$ Cari J. Clark, PhD, ${ }^{1}$ Ann M. Brearly, PhD, ${ }^{1}$ Scott Lunos, MS, ${ }^{1}$ Denis A. Evans, MD, ${ }^{2}$ Carlos F. Mendes de Leon, $\mathrm{PhD}^{2}$ and Susan A. Everson-Rose, $\mathrm{PhD}^{1}$

${ }^{1}$ University of Minnesota, Minneapolis, $\mathrm{MN}$ and ${ }^{2}$ Rush University Medical Center, Chicago, IL.

Psychosocial factors are independently related to cardiovascular disease outcomes, but less is known about the effect of these factors on stroke risk. The purpose of this study was to determine the effect of multiple psychosocial factors on stroke mortality in older adults. Participants included 3,656 adults (59.9\% African American, $40.1 \%$ non-Hispanic white; $61.2 \%$ female) with a mean (SD) age of 77.0 (6.2) years from the Chicago Health and Aging Project (CHAP). CHAP is an ongoing, longitudinal study of risk for Alzheimer's Disease and other chronic conditions of the elderly. Mortality was ascertained through 12/31/05 and verified by data from the National Death Index; over 7.7 years of followup, 76 fatal strokes were identified. Prior principal component analysis on nine psychosocial questionnaires revealed three primary factors related to distress (depression, neuroticism, perceived stress, life satisfaction), social connectivity (social engagement, spirituality, social networks), and unfair treatment (hostility, perceived discrimination). Factor scores were computed by summing individual $\mathrm{z}$-scores for each questionnaire loading on the factor. Cox models were conducted for each factor score adjusting for age, race, sex, education, systolic blood pressure, body mass index, physical activity, smoking and chronic conditions. Each 1-point higher distress score related to a significant $10 \%$ increase in stroke mortality $[\mathrm{HR}=1.10 ; 95 \% \mathrm{CI}=1.03-1.18$; $\mathrm{p}<.008$ ]. Results were unchanged further controlling for history of stroke. There were no main effects of social connectivity and unfair treatment on stroke mortality. In this cohort of elderly African American and non-Hispanic white adults, higher levels of distress contributed significantly to greater risk of dying from stroke over nearly 8 years of follow-up. Additional research is needed to identify mechanisms underlying the association between distress and stroke mortality. [Supported by NIH/DHHS (grants HL84209, AG11101, ES10902.)]

CORRESPONDING AUTHOR: Kimberly M. Henderson, Bachelor of Arts, Medicine, University of Minnesota, Minneapolis, MN, 55414; riley245@umn.edu

\section{3:42 PM-4:00 PM}

OS50-A

POSITIVE AND NEGATIVE PSYCHOSOCIAL CONCEPTS AS PREDICTORS OF CARDIOVASCULAR DISEASE: SENSE OF COHERENCE AND DEPRESSIVE SYMPTOMS

Ari Haukkala, PhD, ${ }^{1}$ Hanna Konttinen, MSocSc, ${ }^{1}$ Elviira Lehto, BSocSc, ${ }^{1}$ Tiina Laatikainen, $\mathrm{MD}^{2}$ and Antti Uutela, $\mathrm{PhD}^{2}$

${ }^{1}$ Social Psychology, University of Helsinki, Helsinki, Finland and

${ }^{2}$ National Institute for Health and Welfare, Helsinki, Finland.

There is an increasing research interest on the positive psychosocial factors that are assumed to maintain and enhance health. However, there is discussion whether positive and negative constructs are separate or bipolar opposites of the same phenomenon. High sense of coherence (SOC) refers to a dispositional orientation that includes flexible and adaptive coping with stressors. We examine the relationships between SOC and cardiovascular disease (CVD) and compare these results with a negative construct, depressive symptoms.

Participants included men $(\mathrm{N}=3850)$ and women $(\mathrm{N}=4083)$ aged 25-74 years who had participated in risk factor surveys (The FINRISK Study) conducted in 1992 and 1997. SOC was measures with a 13-item scale. Depressive symptoms were measured with the 21-item Beck Depression Inventory (BDI) and had correlation of -0.60 with SOC. Incident CVD and IHD were derived from hospital records/death certificates. Subjects with a history of CVD or IHD at baseline were excluded.

During a ten- to 15-year follow-up there were 397 nonfatal and fatal CVD and 219 IHD events. The 2nd SOC tertile had a higher risk of CVD (RR 1.32; 95\% CI 1.03-1.69) than the highest SOC tertile after adjustment for age, gender, education, smoking, body mass index, blood pressure, cholesterol and alcohol consumption. The highest BDI tertile (RR 1.31; 1.01-1.70) had a higher risk for CVD compared to the lowest BDI tertile after adjustment for age, gender, education, and smoking. The second SOC tertile had a higher risk of CVD (RR $1.34 ; 1.04-1.72)$ in the age-adjusted model but BDI tertiles in the same model did not differ significantly. Neither SOC nor BDI were related to IHD events.

Sense of coherence was related to CVD events but not to IHD events. However, the association was not linear as was the case with depressive symptoms. Further studies on psychosocial factors in CVD could benefit from parallel comparisons of positive and negative constructs.

CORRESPONDING AUTHOR: Ari Haukkala, PhD, Social Psychology, University of Helsinki, Helsinki, 00014; ari.haukkala@ helsinki.fi 
OS51

\section{2:30 PM-2:48 PM}

OS51-A

INCREASING PREVALENCE OF DIABETES IN NORTHERN SWEDEN, BUT THERE IS A LIGHT IN THE TUNNEL

Bernt Lindahl, MD PhD, Hans Stenlund, Associate Professor and Margareta Norberg, MD PhD

Public Health and Clinical Medicine, Umea University, Umea, Sweden.

Aims: To examine time trends in fasting and 2-hour glucose concentrations, prevalence and 10-year cumulative incidence of diabetes, and the role of education in these trends.

Methods: Each year the Västerbotten Intervention Programme invites all 40, 50 and 60 year old individuals to a health survey includes a cardiovascular risk factor screening and oral glucose tolerance test. The cross-sectional part of the study is based on health examinations conducted between 1990 and $2007(n=102,822)$. The prospective subset (panel dataset) of the study is based on individuals who have had two health examinations 10 years apart and were not defined as having diabetes at their first health examination $(n=23,546)$.

Results: Glucose concentrations and the prevalence of diabetes are increasing in northern Sweden, especially in men. Between 1990 and 2007 , the mean population fasting glucose concentration increased $0.5 \mathrm{mmol} / \mathrm{L}$. Comparing the prevalence in 1990-1995 with 2002-2007 demonstrated a significant 44\% increase in men and a significant $17 \%$ increase in women. Socioeconomic status, here represented by education, clearly influenced both prevalence and incidence of diabetes and glucose concentration. In all time periods and in all age groups, individuals with low education were more likely to have or get diabetes than highly educated people. A large age-specific effect was found, with the 10-year risk of developing diabetes 4-5 times higher in the oldest age group (50 to 60 years) compared with the youngest (30 to 40 years). We also found a $30 \%$ reduction in the 10 -year risk of developing diabetes in women between 2000-2003 and 2004-2007.

Conclusions: Despite a clear increase in glucose concentrations and diabetes prevalence between 1990 and 2007, especially in men, there was a decline in the 10-year risk of developing diabetes in women between 2000-2003 and 2004-2007.

CORRESPONDING AUTHOR: Bernt Lindahl, MD PhD, Public Health and Clinical Medicine, Umea University, Umea, S-90185; bernt.lindahl@envmed.umu.se

\section{2:48 PM-3:06 PM}

OS51-B

PERCEIVED WEIGHT AMONG DIABETES PATIENTS IN AMERICAN SAMOA

Judith DePue, EdD, MPH, ${ }^{1,2}$ Rochelle K. Rosen, PhD, ${ }^{1,2}$ Nicole Bereolos, PhD, MPH, ${ }^{3}$ Michael G. Goldstein, MD, ${ }^{1,2}$ Pratima Kumar, JD, MPH, ${ }^{2}$ John Tuitele, $\mathrm{MD}^{4}{ }^{4}$ Ofeira Nuusolia, $\mathrm{MEd}^{4}$ and Stephen T. McGarvey, $\mathrm{PhD}^{2}$
${ }^{1}$ Ctr for Behavioral \& Preventive Medicine, Miriam Hospital, Providence, RI; ${ }^{2}$ Alpert Medical School, Brown University, Providence, RI; ${ }^{3}$ School of Public Health, University of Texas, Dallas, TX and ${ }^{4}$ Tafuna Family Health Center, Pago Pago, American Samoa.

Background: Prior studies have documented a traditional value of large body size in Samoans and other Pacific Islanders. Obesity rates in the Pacific are among the highest in the world. In 2002, $90 \%$ of American Samoans were overweight or obese, based on Pacific criterion $(\mathrm{BMI} \geq 26)$ and $21 \%$ of adults had type 2 diabetes. These rates have steadily increased over 30 years of study. It is unknown how weight is perceived among diabetes patients whose weight directly impacts their disease.

Methods: Using preliminary baseline data from our study, "Diabetes Care in American Samoa", we used t-tests and chisquare to examine bivariate comparisons and Logistic Regression was used to examine a multivariate model with perceived weight ratings of , "just right"/"too light" vs. "much too heavy"/ "moderately heavy" as the dependent variable, and with BMI, $\mathrm{HbA} 1 \mathrm{c}$, age, and gender entered as independent variables entered into the model in a backward stepwise procedure via SPSS.

Results: The sample $(\mathrm{n}=162)$ was $64 \%$ female, mean age 56 , mean BMI $37(94 \% \geq 26)$, and mean $\mathrm{HbA1c} 9.4$; perceived weights were $4 \%$ "too light", $31 \%$ "just right", $47 \%$ "moderately heavy", $18 \%$ "much too heavy". Bivariate statistics showed younger age $(p<.05)$ and higher BMI $((p<.001)$ were associated with higher perceived weight. Younger age patients also had higher BMI $(p<.03)$. The multivariate model showed only BMI remained significant $(\mathrm{p}<.001 ; \mathrm{CI} .703-.843)$ when other variables were controlled.

Conclusions: It appears that diabetes patients who are overweight do perceive their weight as "moderately heavy" or "much too heavy", suggesting that attitudes about body size in this Pacific Island population may be changing, perhaps due to the influence of modernization, media and/or the current epidemic of diabetes and overweight. This may also be a function of healthcare provider messages. Providers may be able to incorporate these perceptions as part of motivation to make dietary changes necessary for diabetes control.

CORRESPONDING AUTHOR: Judith DePue, EdD, MPH, Ctr for Behavioral \& Preventive Medicine, Miriam Hospital, Providence, RI, 02903; jdepue@lifespan.org

3:06 PM-3:24 PM

OS51-C

LONGITUDINAL RELATIONSHIP OF DIABETES-RELATED SYMPTOM DISTRESS AMONG ELDERLY WITH TYPE 2 DIABETES: THE HOORN STUDY

Rachel Vijlbrief, MSc, ${ }^{1}$ Marcel C. Adriaanse, PhD, ${ }^{1}$ Jacqueline M. Dekker, $\mathrm{PhD},{ }^{2}$ Marjan Alssema, $\mathrm{PhD},{ }^{2}$ Katja van den Hurk, MSc, ${ }^{2}$ Coen D. Stehouwer, MD, PhD, ${ }^{3}$ Frank J. Snoek, $\mathrm{PhD}^{2,4}$ and Giel Nijpels, $\mathrm{MD}, \mathrm{PhD}^{2,5}$ 
${ }^{1}$ Health Sciences, VU university, Amsterdam, Netherlands; ${ }^{2}$ EMGO institute, VU university Medical Centre, Amsterdam, Netherlands; ${ }^{3}$ Internal Medicine, VU university Medical Centre, Amsterdam, Netherlands; ${ }^{4}$ Medical Psychology, VU university Medical Centre, Amsterdam, Netherlands and ${ }^{5}$ General Practice, VU university Medical Centre, Amsterdam, Netherlands.

Objective: Besides general accepted complications, diabetesrelated symptom distress is an important outcome measure to understand the total burden of diabetes. Besides, diabetes-distress severity contributes to the variation in different aspects of quality of life (QOL). We investigated the longitudinal relationship of diabetes-related symptom distress among elderly with type 2 diabetes over a 7-year period.

Research design and methods: The Hoorn Study is a prospective population based cohort study (2000/01 and 2007/08) among elderly in the region of Hoorn, The Netherlands. Complete data on glucose metabolism and diabetes-related symptom distress for both measurements were available for 34 subjects. Changes in total diabetes-related symptom distress and 8 subgroups (psychological fatigue, cognitive, neuropathic pain, sensoric, cardiovascular, ophthalmological, hypoglycaemic and hyperglycaemic distress) were assed by the validated Type 2 Diabetes Symptom Checklist (DSC-R).

Results: Crude analyses showed an increase in total symptom distress (range 0-100) over time, especially in the subgroup psychological fatigue (mean difference 7.90 (95\% CI; -0.66 16.47) p, 0.069). However, these results were not significant.

Conclusion: Due to missing power we were not able to detect significant differences over time in symptom-distress. Further research is needed in larger sample size with inclusion of potential and reasonable influences of co-morbidities and diabetestreatment.

CORRESPONDING AUTHOR: Rachel Vijlbrief, MSc, Health Sciences, VU university, Amsterdam, 1081 HV; rachelvijlbrief@, hotmail.com

\section{3:24 PM-3:42 PM}

OS51-D

POTENTIAL DIETARY CONSEQUENCES OF FOOD INSECURITY AMONG AUSTRALIAN ADULTS RESIDING IN URBANISED DISADVANTAGED AREAS

Rebecca Ramsey, Bachelor of Health Science - Doctor of Philosphy and Katrina Giskes, Doctor of Philosophy

Queensland University of Technology, Brisbane, QLD, Australia.

Introduction: Food insecurity is the limited/uncertain availability, access to or ability to acquire nutritionally-adequate, culturallyrelevant and safe foods. Adults suffering from food insecurity are at risk of inadequate nutrient intakes or, paradoxically, overweight/ obesity and the development of chronic disease. Despite the global financial crisis and rising costs of living, there are few studies investigating the potential dietary consequences of food insecurity among the Australian population. This study examined whether food insecurity was associated with weight status and poorer intakes of fruits, vegetable and takeaway foods among adults residing in socioeconomically-disadvantaged urbanised areas.

Methods: In this cross-sectional study, a random sample of residents $(n=1000)$ were selected from the most disadvantaged suburbs of Brisbane city (response rate 51\%). Data were collected by postal questionnaire which ascertained information on sociodemographic information, household food security status, height, weight, fruit and vegetable intakes and takeaway consumption. Data were analysed using chi-square and logistic regression.

Results: The overall prevalence of food insecurity was $31 \%$. Food insecurity was not associated with weight status among men or women. Associations between food security status and potential dietary consequences differed for men and women. Among women, food security was not associated with intakes of fruit, vegetable or takeaway consumption. Contrastingly, among men food security was associated with vegetable intakes and consumption of takeaway food: men reporting food insecurity had lower intakes of vegetables and were more likely to consume takeaway foods compared to those that were food secure.

Conclusion: Food security is an important public health issue in Australia and has potential dietary consequences that may adversely affect the health of food-insecure groups, most notably men residing in food-insecure households.

CORRESPONDING AUTHOR: Rebecca Ramsey, Bachelor of Health Science - Doctor of Philosphy, Queensland University of Technology, Brisbane, QLD, 4059; rebecca.ramsey@qut.edu.au

\section{3:42 PM-4:00 PM}

OS51-E

RISK FACTORS FOR TYPE 2 DIABETES AMONG FEMALE PAKISTANI IMMIGRANTS: THE INVADIAB-DEPLAN STUDY ON PAKISTANI IMMIGRANT WOMEN LIVING IN OSLO, NORWAY

Victoria T. Hjellset, PhD, ${ }^{1,3}$ Benedikte Bjørge, Student ${ }^{2}$ and Hege R. Eriksen, Prof ${ }^{3}$

${ }^{1}$ Institute of Preventive Medicine, University of Oslo, Oslo, Norway; ${ }^{2}$ Department of Nutrition, Univesrity of Oslo, Oslo, Norway and ${ }^{3}$ UniHealth, University of Bergen, Bergen, Norway.

Background: Pakistani immigrants living in Oslo, Norway, have a high prevalence of Type 2 diabetes (T2D), but there are few data on risk factors for the disease.

Aim: To study the distribution of, and association between, physiological and psychological risk factors for T2D in a group of Pakistani immigrant women living in Oslo.

Methods: Female Pakistani immigrants $(n=198$, aged 25 to 63 years) with increased risk of, or newly diagnosed T2D were interviewed to give information on diet, physical activity, and demographic and psychological data. Anthropometric and 
blood data were also obtained. Additionally, an oral glucose tolerance test (OGTT) was performed, and maximum heart rate and level of physical activity were recorded, using SenseWear Armband.

Results: Ninety eight \% had body mass index (BMI) values higher than the IDF recommendation for South-Asians (BMI>23 kg/m2) and $39 \%$ were obese $(\mathrm{BMI} \geq 30)$. Impaired glucose tolerance (IGT) was found in $37.4 \%$ and T2D in $12.6 \%$. The participants had low energy expenditure, despite acceptable number of steps. According to the "Findrisk" and "Indian risk scores", $29 \%$ and $89 \%$, respectively, had a high risk of T2D. Subjects with the metabolic syndrome reported more pain, reduced physical functioning and more subjective health complaints than those without the syndrome.

Conclusions: T2D risk factors are prevalent in young, female Pakistani immigrants in Oslo, Norway.

CORRESPONDING AUTHOR: Victoria T. Hjellset, PhD, University of Oslo, Oslo, 0363; v.t.hjellset@medisin.uio.no7

\section{OS52}

\section{2:30 PM-2:48 PM}

OS52-A

GENDER DIFFERENCES IN THE EFFECT OF BREAST FEEDING ON ADULT PSYCHOLOGICAL WELL-BEING

Noriko Cable, PhD, Mel Bartley, PhD, Anne McMunn, $\mathrm{PhD}$ and Yvonne Kelly, $\mathrm{PhD}$

Epidemiology and Public Health, University College London, London, United Kingdom.

It is not known whether the positive effect of breast feeding on child physical health extends into adult psychological adjustment. We examined changes in the social distribution of breast feeding and its effect on adult psychological well-being through the pathway of childhood psychological health by using the available cases from the National Child Developmental Study (NCDS, N= 7,750 , born in 1958) and the 1970 British Cohort Study (BCS70, $\mathrm{N}=6,492$ born in 1970).

Psychological well-being was defined in terms of measures of self-efficacy and psychological ill-health. Childhood adversity was measured by a dichotomised index created after tabulating information about parenthood, mother's age, mother's education, and presence of older siblings. Childhood psychosocial adjustment was measured by the Bristol Social Adjustment Guides for the NCDS and the Rutter scale graded by a teacher for the BCS70. We used logistic regression to test if there was an effect of breastfeeding on adult psychological well-being over and above the effects of adversity and psychosocial adjustment in childhood.

The effect of breastfeeding appears to be gender specific. Findings showed that the magnitude of the effect of breastfeeding on psychological well-being was larger in women than in men. After accounting for the effect of childhood social adversity, breastfeed- ing promoted psychosocial adjustment during childhood in girls in NCDS $(\mathrm{OR}=1.25,95 \% \mathrm{CI}=1.05-1.48)$ and in $\mathrm{BCS} 70(\mathrm{OR}=1.44$, $95 \% \mathrm{CI}=1.15-1.81$ ), but not in boys. In adulthood, being breastfed at birth was associated with higher self efficacy $(\mathrm{OR}=1.31,95 \%$ $\mathrm{CI}=1.06-1.61)$ and lower risk of psychological ill health $(\mathrm{OR}=$ $0.76,95 \% \mathrm{CI}=0.61-0.96)$ in women in BCS70 only. Although breastfeeding did not moderate the negative effect of childhood social adversity on childhood or adulthood outcomes in this study, findings suggest that the practice of breastfeeding can be important for women's psychological well-being throughout the life course.

CORRESPONDING AUTHOR: Noriko Cable, PhD, Epidemiology and Public Health, University College London, London, WC1E6BT; n.cable@ucl.ac.uk

\section{2:48 PM-3:06 PM}

OS52-B

SIDE-EFFECTS OF INTRA-UTERINE HORMONAL CONTRACEPTION: ACCEPTABILITY OF UNSCHEDULED VAGINAL BLEEDING

Pamela Warner, PhD, Anna Glasier, PhD, Robert Lee, BSc, Anja Guttinger, $\mathrm{MBChB}$ and Hilary Critchley, $\mathrm{PhD}$

Centre for Population Health Sciences, University of Edinburgh, Edinburgh, United Kingdom.

Hormonal methods of contraception are highly effective, but in some users unscheduled vaginal bleeding occurs. If this is judged unacceptable it can lead to discontinuation, and hence risk of unwanted pregnancy and wasted health care resource. Data from a trial (where test drug had no consistent effect on bleeding) will be utilised to explore acceptability to women of differing patterns of unscheduled bleeding.

Methods: Further analysis of data from a double-blind RCT (funded by NICHD), comparing intermittently administered antiprogestin CBD2914 with placebo, involving 136 new users of an hormonal intrauterine contraceptive implant. Six month follow-up comprised daily bleeding diaries and questionnaires at 3 points.

Results: Days bleeding/spotting decreased markedly across the study, from $65 \%$ of days in the first three weeks to $32 \%$ in the last block ( $p<0.001)$. Side effects most commonly reported at 6 months were irregular bleeding (40\%), mood swings (22\%), bloating (21\%) and skin problems (20\%). By study end, 4 women had had removal of implant, and 10 were considering this in the near future, with main reasons given as weight gain (2 \& 2), and bleeding $(0,2)$. Pattern of bleeding was reported as worrying by $40 \%$, and more inconvenient than before by $41 \%$. Total days bleeding was more strongly associated with 'worrying' than 'more inconvenient', which might be more strongly related to pattern, or individual propensity/tolerance, given that at baseline $26 \%$ of women reported having previously stopped a method of contraception for irregular bleeding. Total days bleeding was most strongly associated with reporting, as more problematic than 
before insertion, bleeding outside menstrual period, too many days bleeding, interruption to sex life, interruption to daily life, irregular periods, extra washing, and 'lose too much blood' $(\mathrm{p}<0.001)$. Further descriptive analyses will be presented.

Conclusion: Better understanding is needed of women's judgements regarding unscheduled bleeding, and other side-effects, so as to discuss concerns and develop appropriate interventions.

CORRESPONDING AUTHOR: Pamela Warner, PhD, Centre for Population Health Sciences, University of Edinburgh, Edinburgh, EH8 9AG; p.warner@ed.ac.uk

3:06 PM-3:24 PM

OS52-C

A HIERARCHICAL MODEL OF PSYCHOLOGICAL WELL-BEING IN TRANSGENDER PEOPLE

Jose M. Rodriguez-Molina, $\mathrm{PhD},{ }^{1,2}$ Nuria Asenjo, Psychologist ${ }^{2}$ and Maria Frenzi Rabito, Psychologist ${ }^{1}$

${ }^{1}$ Biological and Health Psychology, Universidad Autónoma de Madrid, Madrid, Spain and ${ }^{2}$ UTIG, Hospital Ramón y Cajal, -1D, Madrid, Spain.

Backgrounds: Transgenderism is an emergent worldwide phenomenon. There is much talk of this issue in no-scientific journals and meetings. However we know relatively little about the factors that produce a high or low psychological well-being.

On the other hand most research has been focused in simple correlations among variables which do not allow creating a genuine theoretical model. Moreover clinical variables (such as depression or anxiety) are usually chosen as dependent variables. Most transgender people do not suffer clinical problems, so these variables give us little information about their psychological state. Aim: The aim of this study is to create a model of psychological well-being in transgender people.

Method: We have carried out a cross-sectional descriptive study. The sample consists of 110 patients in treatment in the Gender Identity Disorders Unit (GIDU) of Ramón y Cajal Hospital of Madrid (Spain).We have created a model that uses as dependent variables Quality of Life (measured by Goldberg's GHQ), Psychological Well-Being (measured by Ryff's PWS scale), Subjective Happiness (measured by Fordyce's Subjective Happiness Scale) and Satisfaction with Life (measured by the Diener's Scale of Satisfaction with Life). Our model is developed in three steps. Are included in these steps sociodemographic variables (as age), psychosocial (as appearance), pharmacological (as the stage of hormone treatment), surgery (as surgery performed), sex (such as sexual orientation) and psychological (e.g. personality traits, psychopathological personality and others).

Outcomes: Although the model is still provisional, the results show acceptable correlations. Our model can be tentatively accepted as a model of psychological well-being in transgender people.

CORRESPONDING AUTHOR: Jose M. Rodriguez-Molina, PhD, Psicología Biológica Salud, Universidad Autónoma Madrid, Madrid, 28034; jm.rodriguez@uam.es

\section{3:24 PM-3:42 PM \\ OS52-D}

"MODERN HEALTH WORRIES (MHW)" - DO THEY HAVE DIFFERENT MEANINGS IN WOMEN AND MEN?

Fritz A. Muthny, PhD, MD and Birgit Schulze Willbrenning, $\mathrm{PhD}$ Dept. of Medical Psychology, University Hospital Muenster, Muenster, Germany.

Background: Many people worry about features of modern life, f.e. electromagnetic pollution or genetically modified food. But still we have limited data, whether these "Modern Health Worries" (MHW, Petrie at al. 2001) are related to other anxieties and depression (and if so, to what extent). Furthermore we still do not know, whether these worries mean the same in women and men or have gender-specific relationships.

Method: 282 subjects (age 16 to $88,68 \%$ women) completed questionnaires assessing MHW (German adaptation of the MHW), anxiety, depression, somatization and quality of life.

Findings: The majority of the participants confirmed strong worries, primarily with respect to drug resistant bacteria and pesticides in food, with stronger worries in female and older persons. Factor analysis indicatetd a four factors structure: 'Tainted food', 'Environmental pollution', 'Electromagnetic pollution' and 'Toxins in houseold' (Cronbach's alpha .85 to .94). MHW scales were moderately correlated with depression and physical impairments, but not with somatization. Comparing women and men all four subscales have higher means in women. In addition these scores show different correlations with other psychological scales and constructs: While men with high scores in MHW report more other (general) sorrows and stress-related strains, MHW in women are (positively) related to life satisfaction, hedonistic orientation and good psychosocial health status.

Conclusions: The subjective importance of MHW could be demonstrated in this study, but the presumed closeness to somatization could not be confirmed. Furthermore the meaning of MHW seems to be somehow different in the genders, related to other worries and strains in men, but to psychosocial health, hedonism and life satisfaction in women.

CORRESPONDING AUTHOR: Fritz A. Muthny, PhD, MD, Dept. of Medical Psychology, University Hospital Muenster, Muenster, 48149; muthny@uni-muenster.de 
3:42 PM-4:00 PM

OS52-E

GENDER-RELATED PERSONALITY TRAITS, SELF-EFFICACY, AND SOCIAL SUPPORT: HOW ARE THEY ASSOCIATED WITH WEIGHT LOSS SUCCESS?

Nelli Hankonen, M Soc Sc, ${ }^{1}$ Hanna Konttinen, M Soc $\mathrm{Sc}^{2}$ and Pilvikki Absetz, $\mathrm{PhD}^{1}$

${ }^{1}$ Department of Lifestyle and Participation, National Institute for Health and Welfare, Helsinki, Finland and ${ }^{2}$ Department of Social Psychology, University of Helsinki, Helsinki, Finland.

Although gender-role related personality traits agency and communion are positively related to psychological adjustment and physical health in both sexes, they have rarely been studied in the context of health behavior interventions. However, both might contribute to health goal attainment, potentially interplaying with domain-specific psychosocial factors: Agency, mediated by health-related self-efficacy, and communion, moderated by available social support for the goal at hand.

We tested these hypotheses among the participants $(\mathrm{N}=385$; women $73.2 \%$, men $26.8 \%$ ) of the GOAL Lifestyle Implementation Trial to prevent type 2 diabetes. Health-related self-efficacy was measured at baseline (T1) and after the intensive phase of the intervention at 3 months (T2). Perceived social support received during the intervention was measured at $\mathrm{T} 2$. Waist circumference, indicator of abdominal overweight, was measured at T1, at one-year (T3) and three-year (T4) follow-ups. Agency and communion were measured at $\mathrm{T} 4$ with the Personal Attributes Questionnaire (PAQ).

Among women, higher agency was associated with a greater likelihood of at least 5\% waist circumference reduction (WCR) by T3 $(\mathrm{OR}=2.70$, CI 95\% 1.19-6.15); among men, the association was reversed (but only marginally significant $p=.057$ ). Further analyses in women's sample showed that agency and increase in self-efficacy independently predicted T1-T3 WCR (both $\mathrm{p}<.05$ ). High communion was associated with WCR when social support was high ( $\mathrm{p}$-value for interaction term=.034). T1-T4 WCR was only predicted by T1-T2 self-efficacy increase $(\beta=-.17, p=.024)$. One plausible explanation of the results is that agentic women are more successful at weight loss because they are less affected by feminine self-sacrificing role demands. However, also communal women, sensitive to their social environment, succeed well when backed up by social support for their personal goal pursuit.

CORRESPONDING AUTHOR: Nelli Hankonen, M Soc Sc, National Institute for Health and Welfare, Helsinki, 00300; nelli. hankonen@helsinki.fi
OS53

2:30 PM-2:48 PM

OS53-A

"IT'S NOT NASTY IF YOU GOT A CLEAN PARTNER:" PERSPECTIVES ON SEXUAL RISK FROM RURAL

\section{AFRICAN AMERICAN COCAINE USERS}

Katharine E. Stewart, $\mathrm{PhD}, \mathrm{MPH},{ }^{1}$ Patricia B. Wright, $\mathrm{MPH},{ }^{1}$ Brooke E. Montgomery, MPH, ${ }^{1}$ LeaVonne Pulley, PhD, ${ }^{1}$ Donna Gullette, $\mathrm{DSN}^{2}$ and Brenda M. Booth, $\mathrm{PhD}^{3}$

${ }^{1}$ Fay W. Boozman College of Public Health, University of Arkansas for Medical Sciences, Little Rock, AR; ${ }^{2}$ College of Nursing, University of Arkansas for Medical Sciences, Little Rock, AR and ${ }^{3}$ College of Medicine, University of Arkansas for Medical Sciences, Little Rock, AR.

African-Americans experience disparately high rates of HIV and other sexually transmitted infections (STI); these rates are increasing among rural drug-using communities. However, sexual risk reduction interventions for drug users have traditionally focused on urban populations. We used qualitative techniques to understand rural AfricanAmerican cocaine users' perspectives on sexual risk and HIV/STI so that we could tailor an existing intervention to this underserved population. Using purposive sampling, 40 African-American cocaine users from two rural towns were recruited to participate in four focus groups, which were audio-taped and transcribed verbatim. Three investigators coded the data using content analysis and constant comparison. Three major themes emerged: HIV knowledge and beliefs, selling \& buying sex, and perceptions of condom use. Participants held many inaccurate beliefs about HIV, including how to identify high-risk partners. Attitudes about HIV testing varied by gender, with women being more accepting; however, most were doubtful about effective HIV treatments. Most participants had complex beliefs about trading sex, with "survival sex" seen as acceptable but "tricking for drugs" as unacceptable, despite reports that both types were common. Both men and women were socially classified as "dirty" or "nasty" based in large part on sexual trading behaviors. Participants viewed condoms as protective against HIV and STI, but also reported strong resistance to condom use by both men and women. They reported using hygiene as a proxy for gauging a potential partner's risk and therefore the importance of condom use. Significant knowledge deficits, high-risk beliefs, and social pressures appear to converge on rural African-American cocaine users and contribute to increased HIV and STI rates. These findings were incorporated into a social cognitive theory-based intervention to address these challenges and reduce risk behaviors.

CORRESPONDING AUTHOR: Katharine E. Stewart, PhD, MPH, Health Behavior \& Health Education, UAMS College of Public Health, Little Rock, AR, 72211; kestewart@uams.edu 


\begin{abstract}
EARLY CAREER AWARDEE
2:48 PM-3:06 PM

OS53-B

PREDICTING CONDOM USE INTENTION AMONG CHINESE MALE INJECTING DRUG USERS WITH EXCLUSIVE FEMALE REGULAR SEX PARTNER

Hi Yi Tsui, PhD Candidate, ${ }^{1}$ Joseph Lau, PhD, ${ }^{1}$ Renfan Wang, MD, ${ }^{2}$ Hongyao Chen, MD, ${ }^{2}$ Chuliang Liu, $\mathrm{MD}^{3}$ and Jun Liu, MD ${ }^{1}$

${ }^{1}$ Centre for Health Behaviours Research, School of Public Health and Primary Care, The Chinese University of Hong Kong, Hong Kong, China; ${ }^{1}$ Dazhou Center for Disease Control and Prevention, Dazhou, Sichuan, China and ${ }^{3}$ Hengyang Center for Disease Control and Prevention, Hengyang, Hunan, China.

Male injecting drug users (IDU) play a key role in the spread of HIV in mainland China. They face double risk of HIV acquisition through drug using as well as sexual risk behaviors. They also may form a ëbridgeí for transmission of HIV between high risk and low-risk populations such as their non-drug-using female sex partners. Better understanding of the underlying factors of their sexual behaviors is of critical public health importance. The present study examined factors in association with condom use intention among Chinese male IDU, using the Health Action Process Approach (HAPA) as a basic conceptual framework. An anonymous crosssectional survey, measuring background characteristics and specific constructs of the motivational phase of HAPA (conditional sexual risk perceptions (acquisition as well as transmission to others), self-efficacy, outcome expectancies, and behavioral intention), was conducted. Data obtained from 243 non-institutionalized Chinese male IDU with an exclusive female regular sex partner (RSP) in mainland China were reported. Of the respondents, the mean age was 35.2 ( $\mathrm{SD}=5.7$ ). Seventy percent received junior high education or less, $51 \%$ were currently married, $54.7 \%$ injected drugs for 5 years or more, $13.6 \%$ had shared syringes or injection paraphernalia with others and $85.6 \%$ did not use condoms consistently with RSP in the last 6 months. In line with the HAPA, perceived risk of contracting HIV from RSP (Beta $=0.23$, $\mathrm{p}<0.05$ ), condom use negative outcome expectancies (Beta $=-0.37, \mathrm{p}<0.001$ ), and condom use self efficacy (Beta $=0.21, \mathrm{p}<0.001$ ) predicted intention to use condoms with RSP. Neither perceived risk of transmitting HIV to RSP or condom use positive outcome expectancies were significant factors. For the first time, support for the use of HAPA to understand condom use behaviors among Chinese male IDU was found. Study findings inform future design of theorybased intervention programs.
\end{abstract}

CORRESPONDING AUTHOR: Hi Yi Tsui, PhD Candidate, The Chinese University of Hong Kong, Hong Kong, (na); hytsui@, cuhk.edu.hk

\section{3:06 PM-3:24 PM}

OS53-C

HEALTH PROTECTIVE BEHAVIORS AMONG URBAN ADOLESCENTS LIVING WITH HIV/AIDS

Ricardo D. LaGrange, $\mathrm{PhD},{ }^{1}$ Maria M. Lewis, $\mathrm{MPH}^{1}$ and Amita N. Vyas, $\mathrm{PhD}^{2}$

${ }^{1}$ Children's Research Institute, Children's National Medical Center, Washington, DC and ${ }^{2}$ School of Public Health and Health Sciences, George Washington University, Washington, DC.

The growing amount of youth in the United States living with HIV/AIDS is a persistent problem. Not only has the number of newly infected 15 to 29 year olds consistently increased over the last decade, the emergence of antiretroviral therapy has meant that those born with HIV are growing into adolescence and beyond.
Consequently, HIV/AIDS is now being treated more like a chronic illness than a terminal disease. While much focus has been paid to identifying the specific factors that place individuals at risk for acquiring or transmitting HIV, little attention has been directed toward those behaviors that help promote health in the existing population of young people living with HIV (YPLH). These health protective behaviors can assist in prolonging lives. The preliminary data being presented here includes ninety-one participants who have completed their initial visits of a study assessing coping styles as predictors of adherence in urban YPLH. The sample was recruited from two clinics that serve adolescents with perinatal and risk-acquired HIV. The youth range from age 13 22 years. Specific health protective behaviors were identified that either help promote immune functioning or delay disease progression in YPLH. Study participants responded to questions regarding their diet/nutrition, exercise, sleep habits and taking of multivitamins/supplements. Results highlighted some health protective behavior areas of promise and need. Most respondents reported eating vegetables and fruit "some days" of the week ( $71.4 \%$ and $76.9 \%$ respectively). In the month prior to baseline, 
only $32 \%$ reported eating fast food one or more times a week. More than half of the youth $(50.6 \%)$ had at least 20 minutes of exercise less than 3 days a week. While a third of the group (33\%/ 30 pts) walked briskly for at least 10 minutes daily. On the other hand, almost all participants reported eating candy, chips or cookies "some days" (52.7\%) or "every day" (41.8\%). Similarly, $73.7 \%$ had less than 4 days of exercise in the prior month and $43 \%$ received less than the recommended amount of sleep. Health protective behaviors are an important aspect of managing care for urban YPLH.

CORRESPONDING AUTHOR: Ricardo D. LaGrange, PhD, Children's National Medical Center, Washington, DC, 20010; RLaGrang@cnmc.org

\section{3:24 PM-3:42 PM}

OS53-E

ASSESSING THE IMPACT OF THE GLOBAL FINANCIAL CRISIS ON ART PROGRAMS: A BEHAVIORAL FEEDBACK MODEL

Till Baernighausen, $\mathrm{PhD},{ }^{1,2}$ David Bloom, $\mathrm{PhD}^{1}$ and Salal Humair, $\mathrm{PhD}^{1,3}$

${ }^{1}$ Department of Global Health and Population, Harvard School of Public Health, Boston, MA; ${ }^{2}$ Africa Center for Health and Population Studies, University of KwaZulu-Natal, Mtubatuba, South Africa and ${ }^{3}$ School of Science and Engineering, Lahore University of Management Sciences (LUMS), Lahore, Pakistan.

Introduction: The worldwide scale-up of antiretroviral treatment (ART) in recent years ( $>4$ million under treatment in 2009) occurred against a backdrop of strong economic growth. Still, 5 million people needing ART remain uncovered. Further, this progress is threatened by the global financial crisis making it urgent to estimate how funding changes will affect ART programs.

Methods: We quantify the impact of ART funding changes by modeling their effect on ART coverage and incorporating feedback from coverage to mortality, and from coverage to HIV incidence using a behavioral sub-model. We analyze the changes in ART coverage, HIV incidence, and mortality for several funding scenarios to make concrete policy recommendations. We apply our model to South Africa (SA) using published estimates of current ART coverage, ART effectiveness, HIV incidence, HIV prevalence, HIV transmission probabilities and the annual number of sexual partners. Results: Universal ART coverage (UC) can be achieved in SA within a few years if funding increases every year at the rate it increased from 2007-08. In contrast, only maintaining current funding levels will result in declining coverage. Surprisingly, even a $1 / 3$ rd funding decrease over 3 years followed by increases at the 2007-08 rate delays achieving UC by 10 years. Increased prevention effectiveness can substantially reduce time to achieving UC. In contrast, increases in the CD4 count threshold for treatment eligibility could make UC very hard to achieve.

Discussion: Only continued increases in ART funding in coming years can ensure that ART coverage levels can be increased in the future. Policy choices, such as investment in prevention and ART eligibility criteria, will be crucial in ensuring the future success of the ART roll-out.

CORRESPONDING AUTHOR: Salal Humair, PhD, Department of Global Health and Population, Harvard School of Public Health, Boston, MA, 02115; salal@alum.mit.edu

OS54

\section{2:30 PM-2:48 PM}

OS54-A

SENSITIVITY AND SPECIFICITY OF THE DANISH VERSION OF THE DISTRESS THERMOMETER

Pernille E. Bidstrup, PhD, ${ }^{1}$ Birgitte G. Mertz, Nurse, ${ }^{3}$ Isabelle Deltour, MSc, ${ }^{2}$ Susanne Dalton, PhD, ${ }^{1}$ Ninna Rottmann, MSc, ${ }^{1}$ Niels Kroman, DMSc, ${ }^{3}$ Henrik Kehlet, $\mathrm{DMSc}^{4}$ and Christoffer Johansen, DMSc ${ }^{1,5}$

${ }^{1}$ Department of Psychosocial Cancer Research, Institute of Cancer Epidemiology, Danish Cancer Society, Copenhagen, Denmark; ${ }^{2}$ Department of Statistics and Epidemiology, Institute of Cancer Epidemiology, Danish Cancer Society, Copenhagen, Denmark; ${ }^{3}$ Breast Surgery Clinic, Rigshospitalet, Copenhagen, Denmark; ${ }^{4}$ Department of Surgical Pathophysiology, Rigshospitalet, Copenhagen, Denmark and ${ }^{5}$ National Centre for Cancer Rehabilitation Research, University of Southern Denmark,Institute of Public Health, Odense, Denmark.

Purpose: A cancer diagnosis often involves distress which may in the long term develop into clinical depression. Screening instruments identifying highly distressed patients are needed. In Denmark, no validated instrument has been developed to identify cancer patients in need of psychosocial support. We are thus examining the psychometric properties of a Danish version of the single-item Distress Thermometer.

Methods: A total of 434 women newly diagnosed with breast cancer (within 1-2 days) were invited to participate in this study. A total of 362 accepted $(83 \%)$. The accuracy of the single item Distress Thermometer is compared to the longer validated scales of Hospital Anxiety and Depression Scale (HADS) and Impact of Event Scale (IES) using Receiver Operating Characteristic curve analyses.

Results: Preliminary results show that $72 \%$ are distressed according to a cut-score of $\geq 4$ on the Distress Thermometer (11-point scale, $0-10$ ) with a mean of 5.4 and a standard deviation of 3.1 . We plan to present results on the accuracy of the Distress Thermometer compared to the HADS and IES. Psychometric properties including sensitivity, specificity, positive and negative predictive value of the Distress Thermometer will be presented. Also, a cut-point for clinical use will be discussed.

Conclusion: We expect that the psychometric properties of the Distress Thermometer will be satisfactory and that we will be able to determine an optimal cut-point. The results will be important for improved handling of psychosocial problems among breast 
cancer patients in Denmark. By targeting psychosocial support and rehabilitation to those who are most distressed we may be able to tailor the support and hence prevent that some patients develop clinical depression.

CORRESPONDING AUTHOR: Pernille E. Bidstrup, PhD, Department of Psychosocial Cancer Research, Danish Cancer Society, Institute of Epidemiological Cancer Research, Copenhagen, DK-2100; pernille@cancer.dk

\section{2:48 PM-3:06 PM}

OS54-B

DEPRESSION 3 MONTH AFTER SURGERY

IS AN INDEPENDENT PREDICTOR OF TREATMENTRELATED PAIN AT 1 YEAR FOLLOW-UP

IN A NATIONWIDE COHORT

OF WOMEN TREATED FOR PRIMARY BREAST CANCER

Soeren Christensen, MSc, ${ }^{1,2}$ Anders B. Jensen, $\mathrm{MD}, \mathrm{PhD}{ }^{2}$ Susanne Moeller, $\mathrm{MSc}^{3}$ and Robert Zachariae, MSc, MDSci ${ }^{1,2}$

${ }^{1}$ Psychooncology, Aarhus University, Aarhus, Denmark; ${ }^{2}$ Oncology, Aarhus University Hospital, Aarhus, Denmark and ${ }^{3}$ Danish Breast Cancer Cooperative Group (DBCG), Rigshospitalet, Copenhagen, Denmark.

BACKGROUND: Treatment-related pain is an important issue in breast cancer rehabilitation. As most studies in the area are crosssectional, relatively little is known about possible predictors. AIM: To explore the prevalence of pain and the role of depression as predictor in a nationwide prospective study of women treated for breast cancer. METHODS: The study cohort consisted of 3343 Danish women (aged 26-70) treated for primary breast cancer according to standardized guidelines. A questionnaire package including Beck's Depression Inventory was mailed out 3(baseline) and 15-months post-surgery (follow-up). The response rate at follow-up was 94\%. Probable cases of major depression (MD) were identified according to the manual (cutoff $>=17$ ). Frequency and severity of treatment-related pain in breast and arm or shoulder was assessed by 4 items at follow-up (range: "no pain" to "all the time" and "no pain" to "extremely severe"). DBCG and the surgical departments provided data on eligibility, clinical variables and comorbidity. Data on psychiatric history, demographic-, and socioeconomic factors were obtained from national registries. RESULTS: At follow-up, 73\% reported pain during the recent month and $33 \%$ had pain "almost every day" or more often. Twenty-eight percent were "somewhat" or more affected by pain and 11\% were "very" or "extremely" affected. Women with MD at baseline were more likely to experience pain "almost every day" or more often at follow-up (OR=2.63; $(95 \%$ CI, 2.12-3.27)) and more likely to be affected "somewhat" or more by pain $(\mathrm{OR}=3.49$; $(95 \% \mathrm{CI}, 2.80-4.35))$. When adjusted for potential confounders (demographic-, socio-economic-, clinical-, health status-, and health behavior variables), the predictive power of $\mathrm{MD}$ persisted $(\mathrm{OR}=1.93$; (95\% CI, 1.50-2.48), and $\mathrm{OR}=2.31$; (95\% CI, 1.79-2.99)). CONCLUSION: The prevalence of treatment-related pain was substantial with nearly 3 out of 4 experiencing pain 15 months post-surgery and 1 out of 9 reporting to be very or extremely affected. Depression was found to be an important independent predictor of pain.

CORRESPONDING AUTHOR: Soeren Christensen, MSc, Aarhus University Hospital, Aarhus, DK-8000 C; soeren.christense$\mathrm{n} @$ aarhus.rm.dk

3:06 PM-3:24 PM

OS54-C

THE EFFECTS OF POSTTRAUMATIC STRESS ON NEUROCOGNITIVE AND PSYCHOSOCIAL FUNCTIONING IN BRAIN TUMOR SURVIVORS

Maria Kangas, $\mathrm{PhD},{ }^{1}$ Robyn Tate, $\mathrm{PhD},{ }^{2}$ Janet Williams, $\mathrm{CRN}^{3}$ and Robert Smee, MBBS, FRACR ${ }^{3}$

${ }^{1}$ Centre for Emotional Health, Macquarie University, Sydney, NSW, Australia; ${ }^{2}$ University of Sydney, Sydney, NSW, Australia and ${ }^{3}$ Prince of Wales Hospital, Sydney, NSW, Australia.

AIM: Relative to other oncology populations, the brain tumor (BT) population is unique given that both benign and malignant BTs can be life-threatening and can cause severe neurocognitive and psychosocial disturbances. No study to date however, has investigated the effects of BT-related posttraumatic stress (PTSS) on neurocognitive functioning and overall quality of life in adult BT patients. The aim of the present study was to address this question. METHOD: Utilizing a longitudinal design, adult individuals diagnosed with a primary BT were assessed on average, within 6-weeks prior to starting radiotherapy (T1), and re-assessed at 3 months post-radiotherapy (T2). At both assessments, participants were administered a standardized battery of tests measuring BT-related PTSS, mood, quality of life, memory and executive functioning. RESULTS: To date, 64 patients (36 females, 28 males) have been re-assessed at T2. At both T1 and T2, 14\% of the sample reported clinically elevated levels of PTSS pertaining to their BT experience; although, the effects of PTSS fluctuated over time. Whereas $40 \%$ of individuals who reported elevated PTSS prior to commencing radiotherapy continued to report elevated PTSS at T2, 60\% of patients with elevated PTSS at T2 did not experience elevated PTSS at T1. Prior to radiotherapy (T1), individuals with elevated PTSS were found to have significantly reduced functional well-being, elevated BT symptoms and social constraints, as well as heightened depressive, anger, fatigue and confusion symptoms $(p$ 's $<.05)$ compared to patients with low PTSS at T1. Individuals with elevated PTSS also evidenced significantly reduced scores on working memory and executive tests (p's<.05) compared to individuals with low PTSS at T1. Similarly, individuals with elevated PTSS at T2 reported significantly reduced functional well being, vigor, and overall quality of life, as well as elevated BT symptoms, depression, anger, confusion, and social constraints $(p ' s<.05)$ compared to individuals with low PTSS, 3-months post-radiotherapy. Individuals with high PTSS at T2 also performed significantly worse on working memory and executive tests (p's<.05). DISCUSSION: The findings suggest that approximately one in seven individuals 
diagnosed with a primary BT are at risk of suffering from PTSS as a result of their tumor experience. Notably, these results indicate that PTSS compromises neurocognitive and psychosocial functioning including overall quality of life in BT patients undergoing radiation therapy. The findings will be discussed in context of developing and implementing appropriate counseling and rehabilitation services to address the unique needs of BT survivors.

CORRESPONDING AUTHOR: Maria Kangas, PhD, Centre for Emotional Health / Dept of Psychology, Macquarie University, Sydney, NSW, 2109; maria.kangas@mq.edu.au

\section{3:24 PM-3:42 PM}

OS54-D

\section{COGNITIVE FUNCTIONING IN BREAST CANCER} PATIENTS TREATED WITH CHEMOTHERAPY AND TAMOXIFEN

Christna M. Andersen, Bachelor in Psychology, ${ }^{1}$ Mimi Y. Mehlsen, PhD in Psychology, ${ }^{1}$ Anders D. Pedersen, MSc, ${ }^{2}$ Anders B. Jensen, $\mathrm{MD}^{3}$ and Robert Zachariae, $\mathrm{MDSci}^{3}$

${ }^{1}$ Department of Psychology, Aarhus University, Aarhus, Denmark; ${ }^{2}$ Aarhus University Hospital, Hammel, Denmark and ${ }^{3}$ Aarhus University Hospital, Aarhus, Denmark.

Background: Oestrogen has been shown to influence cognitive functioning, and recent studies have focused on the effects of endocrine therapy on cognitive functioning in breast cancer patients. The current results, however, have been inconclusive.

Objectives: To examine the effects of endocrine therapy (tamoxifen) on cognitive functioning in breast cancer patients.

Methods: 20 patients receiving adjuvant chemotherapy followed by tamoxifen and 12 patients receiving chemotherapy only were assessed with neuropsychological tests before and again 4-6 weeks after completion of the chemotherapy.

Results: Group differences were only found for one out of 21 neuropsychological tests. When controlling for age, patients treated with both chemotherapy and tamoxifen scored significantly lower on the letter-number sequence test (WAIS-III LN) measuring working memory $(\mathrm{P}=0.013)$ than patients treated with chemotherapy only. Of the 21 tests, 5 showed differences corresponding to an effect size $>0.3$ (Cohen's d). These 5 tests were WAIS-III LN (letter-number sequence), WAIS-III A (Arithmetic), TMA (Trail-making A), RAVL (Rey Auditory Verbal learning) and WF-N-words (Word Fluency $\mathrm{N}$-words) representing the cognitive domains of working memory, processing speed and verbal fluency.

Conclusion: While we cannot conclude that tamoxifen causes cognitive impairment in breast cancer patients, our results do not exclude the possibility. Our finding that $25 \%$ of the neuropsychological tests showed effect sizes greater than 0.3 are in agreement with results of previous studies. The small samples and subsequent low statistical power of existing studies suggest the need for largescale studies. If tamoxifen causes cognitive decline in breast cancer patients, this knowledge could be relevant for the patients' choice of treatment.
CORRESPONDING AUTHOR: Christna M. Andersen, Bachelor in Psychology, Psykologisk Institut, Aarhus, 8000; christinamaar. andersen@gmail.com

\section{3:42 PM-4:00 PM \\ OS54-E \\ CREATING A SURVIVORSHIP PROGRAM-WHAT DOES IT TAKE TO BE SUCCESSFUL?}

Marci K. Campbell, MPH, PhD, ${ }^{1}$ Donald Rosenstien, MD, Alison Jones, $\mathrm{ND},{ }^{8}$ Barbara Andersen, $\mathrm{PhD},{ }^{6}$ Karen Syrjala, $\mathrm{PhD},{ }^{4}$ Scott Baker, MD, ${ }^{4}$ Kenneth Miller, MD ${ }^{3}$ Linda Jacobs, $\mathrm{PhD}, \mathrm{RN},{ }^{2}$ Mary McCabe, RN, MA, ${ }^{5}$ Patricia Ganz, MD, ${ }^{7}$ Mindy Gellin, $\mathrm{RN},{ }^{1}$ Irene Tessaro, $\mathrm{PhD}^{1}$ and Michelle Manning, $\mathrm{MOH}^{1}$ ${ }^{1}$ UNC-Lineberger Comprehensive Cancer Center, Chapel Hll, NC; ${ }^{2}$ Abramson Cancer Center, University of Pennsylvania, Philadelphia, PA; ${ }^{3}$ Dana-Farber Cancer Institute, Boston, MA; ${ }^{4}$ Fred Hutchinson Cancer Research Center, Seattle, WA; ${ }^{5}$ Memorial Sloan-Kettering Cancer Center, NY, NY; ${ }^{6}$ Ohio State University Comprehensive Cancer Center - James Cancer Hospital and Solove Research Institute, Columbus, OH; ${ }^{7}$ UCLA's Jonsson Comprehensive Cancer Center, Los Angeles, CA and ${ }^{8}$ University of Colorado Cancer Center, Denver, CO.

Introduction: This study examined models of clinical survivorship care and outreach, organizational factors, and center evolution over time in the eight participating centers in the Lance Armstrong Foundation LIVESTRONG Centers of Excellence (COE) Network, to provide a set of recommendations to aid other institutions in the utilization of "best practices and processes" in the development of successful comprehensive cancer survivorship programs.

Method: Guided by the Chronic Care Model this mixed methods study used both quantitative and qualitative approaches to characterize institutional barriers, models of care, community outreach and education among the COEs. The three phase approach included document review, in-depth key informant telephone interviews, quantitative surveys and site visits over a one year time period.

Result: Findings identified several overarching themes, lessons, and recommendations for developing and sustaining survivorship care: one size does not fit all-each program is unique with different organizational and environmental factors that inhibit and facilitate progress ; developing survivorship models of care is an ongoing process of trial and error; leadership and institutional commitment are essential to provided needed resources and support; lack of awareness of the need for survivorship care and resistance to change are critical barriers to address in order to institute survivorship care in oncology practice; and establishing community-based centers has the potential to advance the field of survivorship beyond comprehensive cancer centers.

Conclusion: Several key components of the Chronic Care Model are essential for the development of a successful quality, comprehensive cancer survivorship program such as those represented in the LIVESTRONG Centers of Excellence in Cancer Survivorship Network. 
CORRESPONDING AUTHOR: Marci K. Campbell, MPH, PhD, Department of Nutrition, UNC-Lineberger Comprehensive Cancer Center, Chapel H1l, NC, 27599; mindy_gellin@med.unc.edu

\section{OS55}

\section{2:30 PM-2:48 PM}

OS55-B

FATALISTIC BELIEFS AND CHILD RESTRAINT IN MOTOR VEHICLES AMONG ARABS IN ISRAEL

Orna Baron-Epel, PhD MPH and Hir Omri, MPH

School of Public Health, Haifa University, Haifa, Israel.

Background: Comparatively, a higher rate of Arab Children in Israel are victims of car accidents compared to Jewish children, this may be a result of low compliance with the law regarding child restraint in cars among Arabs. One possible explanation for the low use of car restraints may be fatalistic beliefs, common in the Arab community. These beliefs suggest that health is a result of luck and beyond an individual's control.

Objectives: To asses levels of fatalistic beliefs and their association with child restraint in motor vehicles among Arabs in Israel. Methods: A cross sectional observational study of child restraint in motor vehicles was conducted. Four hundred cars with 835 children under the age of 8 in them were observed while entering a parking lot. The drivers were interviewed regarding beliefs and attitudes towards child restraint and in addition their fatalistic beliefs towards road accidents and fatalistic beliefs in general. The observations were conducted within an Arab town and seven Arab villages in the northern part of Israel.

Results: Of the 400 drivers, $63 \%$ were restrained while driving the car while only $32 \%$ of the children in the cars were restrained. A higher percent of the children under one year old were restrained (64\%) compared to $41 \%$ of the children aged 1 to 3 and $9 \%$ of children aged $3-8$. Around $60 \%$ of the drivers reported high fatalistic beliefs. High fatalist beliefs were associated with lower education and were more frequent among women. After adjustment for gender, age of driver, education, number of children and driver's restraint, fatalism was associated with child restraint. Those with high fatalistic beliefs restrained their children more often.

Conclusion: High levels of fatalism may inhibit people from use of child restraint in motor vehicle among Arabs in Israel. Targeting these beliefs may increase levels of compliance with the law.

CORRESPONDING AUTHOR: Orna Baron-Epel, PhD MPH, School of Public Health, Haifa University, Haifa, 31905; ornaepel@ research.haifa.ac.il
2:48 PM-3:06 PM

OS55-C

PREDICTORS OF PARENTAL SUPPORT OF PHYSICAL ACTIVITY AMONG PREDOMINANTLY HISPANIC SCHOOL CHILDREN

Christina R. Esperat, $\mathrm{PhD},{ }^{1} \mathrm{Du}$ Feng, $\mathrm{PhD}^{1,2}$ and Zhen Cong, $\mathrm{PhD}^{2}$

${ }^{1}$ The School of Nursing, Texas Tech University Health Sciences Center, Lubbock, TX and ${ }^{2}$ College of Human Sciences, Texas Tech University, Lubbock, TX.

Purpose: The lack of physical activity (PA) is associated with obesity among school-aged children. Although parental support has been found to increase children's PA, few studies tested predictors and effects of parental support for PA among young Hispanic children. The main purpose of this study is to examine the effect of a multi-component intervention program on parental support for PA among predominantly Hispanic children from $\mathrm{K}$ to 2nd grade, accounting for parents' characteristics such as acculturation, education, and age.

Design and setting: Quasi-experimental research design was used to evaluate a multi-component intervention program, in which five waves of data were collected from two sites in West Texas.

Subjects. Data are collected from predominantly Hispanic and low-income parents/guardians $(\mathrm{N}=442)$ and their children of 5 to 9 years $(\mathrm{N}=848)$.

Measures: Children's anthropometric measures were obtained, and their overweight status was determined based on their age-andgender adjusted BMI. A demographic questionnaire, an acculturation scale (Brief ARSMA II), and a parent survey were used among parents who reported on home environmental factors (e.g., whether there is a TV in the study child's bedroom) and children's behaviors (e.g., physical activity, TV viewing, sweetened beverage intake, family meals).

Analysis: Descriptive statistics were obtained, and random coefficient regression was used to test the hypotheses.

Results: Intervention increases parental support for PA (intercept). Time alone does not influence parental support (slope). The more acculturated parents provide more parental support (intercept, main effect). More acculturated parents are less influenced by the intervention (negative interaction between intervention and acculturation level), but not enough to counterbalance the effect of the intervention. Also, parents who have higher level of education are more supportive of PA, and older parents give less support for PA. Children's age and gender are not significant covariates, and neither is the site where the intervention is delivered.

Conclusion.... More acculturated parents are more likely to provide support for PA. Intervention is important in raising parents' awareness of problems of children's obesity and mobilizing parents to provide more support for PA. 
CORRESPONDING AUTHOR: Christina R. Esperat, PhD, The School of Nursing, Texas Tech University Health Sciences Center, Lubbock, TX, 79430; christina.esperat@ttuhsc.edu

3:06 PM-3:24 PM

OS55-D

YOUTH AND HOW THE COMMUNITY RESPONSES

TO REPRODUCTIVE HEALTH AND SEXUALITY

BEHAVIOR IN DEVELOPING COUNTRY

Shambhu D. Joshi, MBBS ${ }^{1}$ and Rajan P. Bhandari, BA ${ }^{2}$

${ }^{1}$ Public Health, Primary Health Center, Kailali, Nepal and

${ }^{2}$ Community health, Community Health and Environmental Society Nepal, KTM, Nepal.

Issues: Like many developing countries, Nepal has facing difficult problem to youth knowledge, awareness, behavior and attitude on their reproductive health and sexuality.. But, on the other hand, today's nepalese young girls and boys are more tolerant toward premarital sexual relations. The youth reproductive health services is then directed to assist youth to possess knowledge, awareness, behavior and attitude of responsible reproductive health through promotion by information, education, and communication, counseling, services for youths particularly those with particular problems, and supporting positive activities for youths.

Methods: This paper aims to examine the result of the youth reproductive health services in nepal through explore the realty of their knowledge, awareness, behavior, and attitude of sexual and reproductive health from 2007 to mid 2009. To examine this reality this paper using the data from the Survey of High Risk Behavior of Adolescent Including Sexuality and HIV/AIDS Prevention with Implication on Reproductive Health which interviewed 1956 youths in 5 districts; 310 school and colleges. The data were edited and anaysed by EPI info software program. Results: The summary of the result found that the reality of the knowledge, awareness, behavior, and attitude of sexual and reproductive health is still low. For the knowledge of reproductive health: most (53,6 percent) do not know basic meaning of reproduction, 78 percent of female in rural do not know the cycle of menstruation period, 64.8 percent never discussed reproductive health and even, 51 percent never discussed it with family, and 64.6 percent didn't getting family planning lesson. But, on the other hand, there are 49.1 percent kissing, 45.8 percent embracing/ hugging, and 2.6 percent having sexual intercourse during dating. This realty implicated on enhancing effort to create and promote more attractive program in informing reproductive health information and launching friendly service concern to the young girls and boys.
CORRESPONDING AUTHOR: Shambhu D. Joshi, MBBS, Primary Health Center, Kailali, n/a; dr.sdjoshi@yahoo.com

3:24 PM-3:42 PM

OS55-E

EFFICACY OF A BRIEF HIV PREVENTION INTERVENTION FOR YOUNG PEOPLE CONSULTING SEXUALLY

TRANSMITTED DISEASE CLINICS IN LOMé, TOGO. A RANDOMIZED CONTROLLED TRIAL

Lonzozou Kpanake, $\mathrm{PhD},{ }^{1}$ Akouda Patassi, $\mathrm{MD}^{2}$ and Komlantsè M. Gossou, $\mathrm{PhD}^{3}$

${ }^{1}$ McGill University, Montreal, QC, Canada; ${ }^{2}$ University of Lomé Teaching Hospital, Lomé, Togo and ${ }^{3}$ University of Lomé, Lomé, Togo.

Context and objectives: With over $80 \%$ of those currently living with AIDS aged between 15 and 24 and $75 \%$ of these youth living in sub-Saharan Africa, young people in sub-Saharan Africa have been worst affected by the AIDS pandemic. Since HIV in Togo the most common cause of death, representing $17 \%$ of all deaths is spread primarily through unprotected sex, safe sex practices such as condom use can reduce HIV spread significantly. We examined the efficacy of a behavioral intervention to promote condom use among Togolese young people at high risk for HIV infection. Methods: We randomized - without known HIV infection - 446 Togolese people aged 16 to 24 years consulting 3 sexually transmitted disease clinics in Lomé, who had recently had unprotected sex, to a 1-hour behavioral intervention or a didactic control condition. At baseline and 6 months, participants underwent interviews about their sexual behaviors and consistent condom use and testing for HIV. Results: We observed a $51 \%$ decline in HIV infection incidence $(\mathrm{P}=.03)$ in the intervention group. Incidence density for the intervention versus control groups was 1.6 versus 3.27 per 100 person-years $(\mathrm{P}<.001)$. Participants in the intervention group reported using condoms more consistently in the 30 days preceding the 6-month assessment (unadjusted analysis, intervention, $74.2 \%$ vs control, $48.7 \%$ ). Participants in the intervention group reported using condoms more consistently over the entire 6-month period (adjusted odds ratio, 2.30; 95\% CI, 1.51-3.50; $\mathrm{P}=.001)$. Conclusions: This brief behavioral intervention shows promise in reducing HIV risk behaviors among Togolese young people and may be transferable to other subSaharan African countries.

CORRESPONDING AUTHOR: Lonzozou Kpanake, PhD, McGill University, Montreal, QC, H3T 1E2; lonzozou.kpanake@ mail.mcgill.ca 
3:42 PM-4:00 PM

OS55-A

CORRELATES OF SEXUAL RISK BEHAVIORS AMONG

HIGH SCHOOL STUDENTS IN COLORADO: ANALYSIS

AND IMPLICATIONS FOR SCHOOL-BASED HIV/AIDS

PROGRAMS

Stephen Nkansah-Amankra, PhD, MPH, MA and Adoulaye Diehiou, $\mathrm{PhD}, \mathrm{MD}$

School of Human Sciences/Colorado School of Public Health, University of Northern Colorado, Greeley, CO

SUMMARY: To evaluate correlates of sexual risk behaviors associated with HIV/AIDS infection among adolescents participating in 2005 Colorado Youth Risk Behavioral Survey, since previous studies have produced mixed findings. The State mandates abstinence-only program across all public schools. Multinomial logistic regression was used to evaluate relationships among dependent variables and independent variables. We found no significant effect of having received in-school HIV/AIDS education on all outcome measures. Compared with females, males were more likely to initiate sex at a relatively younger age, report unprotected sex with multiple partners, and to drink alcohol before sexual intercourse. Efforts to control the HIV/AIDS epidemic among adolescents may need to focus on targeted interventions aimed at addressing gender- and racial/ethnic-specific risk exposures among this population group, including risk behaviors linked with lifetime sexual abuse. The need to re-examine the role of inschool HIV prevention programs to build adequate competencies among students, parents and community leaders is recommended. STUDY DESIGN AND DATA SOURCE: We used cross-sectional data from the 2005 Colorado Youth Behavioral Risk survey (YRBS). Details of the survey design and psychometric validity of questionnaire items are found in other publications. Briefly, the YRBS is a self-reported paper-based survey of high school students conducted every two years by the Centers for Disease Control and Prevention (CDC) in collaboration with Colorado local implementing partners. The survey monitors risk behaviors and other characteristics associated with mortality and morbidity among students in grades 9 through 12. In 2005, the survey was administered to a representative sample of 1,498 students from 29 public high schools throughout the state, and the overall response rate was $60 \%$. Surveyed schools and classes were selected systematically with probability proportional to enrollment based on grade levels.

OUTCOME VARIABLES: Sexual Risk Behavior was a threelevel variable created from items suggested in previous studies, and categorized as: no risk (never had sexual intercourse or engaged in any sexual risk behavior at the time of survey), low risk (had sex with one partner, used condom and did not drink alcohol in the last sexual intercourse, and used a method of contraception) and high risk (had sex with multiple partners, did not use condoms and/or drank alcohol before the last sexual intercourse, and used no method of contraception). Age at first sexual intercourse was categorized based on respondents' age at their first sexual intercourse as: no sexual intercourse (participants reported as not having ever had sexual intercourse at the time of survey irrespective of age), young adolescents (11-14 years), and older adolescents ( $\geq 15$ years). Number of current sexual partners was assessed based on the reported number of sexual partners in the 3 months preceding the survey and categorized as following: never had sexual intercourse, had 1 partner, and had $\geq 2$ sexual partners. These variables were selected because of their known association with transmission of HIV infections (Mullings, Marquart \& Brewer, 2000).

COVARIATES: HIV/AIDS education, substance use score, physically forced sex, alcohol use. Potential confounding variables selected include age, gender, number of hours watching TV, feeling sadness or hopelessness, and cigarette smoking because of their documented relationships with sexual risk behavior.

STATISTICAL ANALYSIS: Characteristics of respondents participating in the survey were compared according to race and gender using Rao-Scott $\chi 2$ tests for categorical variables. To further assess the relationship between independent and outcome variables, all independent variables that attained a statistical significance of $\mathrm{P} \leq .25$ in bivariate analyses were entered into multinomial logistic regression models in a manual stepwise fashion, starting with those with the lowest P-value. This method allows dependent variable to have more than two categories, thereby providing a single estimate of odds ratio (OR) for the association between the independent variables and each combination of the dependent variables, while controlling for potential confounders. Odds ratios (OR) were calculated and used as estimates of risk ratios. The likelihood ratio test was used to compare models to determine which variables were retained in multivariate models at a significance level $\alpha=.05$. Age and grade level were not included in the same models because of potential collinearity. All analyses were performed with SAS 9.1.3 (SAS Institute, Cary, NC) and the survey weight variable was used to account for the complex sampling design of the survey to provide estimates representative of the high school student population across the state.

RESULTS: Nearly $64 \%$ of respondents were non-Hispanic whites, Hispanics 24\% while Blacks and other racial/ethnic groups represented $12 \%$. These proportions mirror the approximate distribution of racial groups across the state. Both genders are equally represented in this sample with older adolescents (ages 15-18 years) being the predominant group. Nearly $85 \%$ of students reported receiving voluntary in-school HIV/AIDS education. In adjusted analyses, predictors significantly associated with high risk sexual behaviors included being male, Hispanic, and having had physically forced sex. Likewise, light and binge drinking, smoking, and illegal substance use showed significant effects on sexual risk behaviors. The highest risk for early sexual 
debut was observed among Blacks, and Hispanics. Similarly, there was an 8-fold increase in the odds of early sexual debut among adolescents reporting physically forced sex. A dose-response relationship was observed between illegal substance use and age of sexual debut, with the strongest effect among young adolescents. Likewise, current or past smoking was associated with increased odds of early sexual debut. Statistically significant correlates of having one or more sexual partners included being Latino and high user of illegal substances, reporting forced sex, drinking alcohol, and current smoking. Associations appeared more marked among respondents using two or more drugs and having $\geq 2$ sexual partners.

CORRESPONDING AUTHOR: Stephen Nkansah-Amankra, $\mathrm{PhD}, \mathrm{MPH}, \mathrm{MA}$, School of Human Sciences/Colorado School of Public Health, University of Northern Colorado, Greeley, CO, 80639, United States; snamankrasc@lycos.com 